UCID--1932\%

DES4 006272

\title{
DISCLAIMER
}

This report was prepared 25 an sconunt of work sponuoned by an agency of the United States Government. Neither the United Stales Cowernment nor any agency theroof, nor any of their employes, makes any watranty, express or inplied, or ssumes any legal liability or responsibility for the scouracy. completences, or usefulnes of - i information, epporatus, peoduct, or proces: diaclosed, of represents that its use would not inge privately owned rights. Reference terein to any specific conmercial product, process, service hy trade anme, trademark, manufucturer, or aberwise does not neceasarity constilute or imply its endorsement, recommendation, or favoring by the Unitad States Govemment or any agency thereol. The views and opinions of autbors expresued herein to not nocess iy state or rellect those of the United States Government or any atency thereof.

\section{A TANDEM MIRROR TECHNOLOGY DEMONSTRATION FACILITY}

\author{
Lawrence Livermore National Laboratory \\ Fusion Engineering Design Center \\ TKW Energy Development Group \\ General Dynamics/Convair Division \\ University of Wisconsin-Madison \\ Bechtel Group, Inc. \\ Science Applications, Inc. \\ Los Alamos National Laboratory \\ University of California at Los Angeles \\ Massachusetis Institute of Technology
}

Manuscript aate: October, 1983
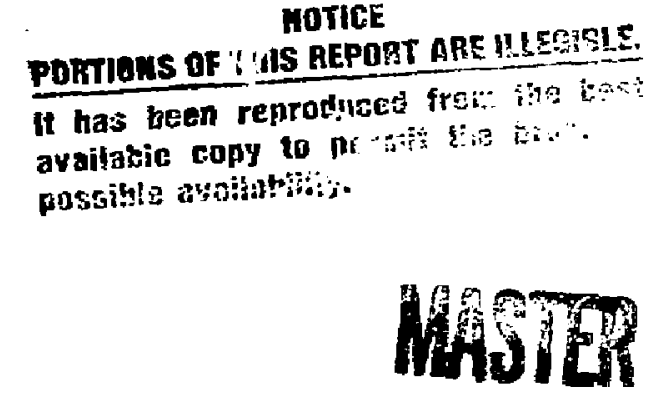

T. H. Batzer
R. H. Bulmer
J. N. Doggett
A. I. Golaner

B. A. Cramer

B. A. Engnolmi

P. J. Fogarty

G. M. Fuller

S. K. Ghose

G. E. Gorker

H. E. Boehmer

R. B. Campbell

D. L. Chesshire

A. J. Cole

S. A. Freije

K. L. Agarwal

R. W. Baldi

R. Uharmarajan

Ni. W. Liggett

J. F. Parmer
J. A. Kerns
B. G. Logan
J. E. Osner
H. H. Otsuk $i$

\section{FUSION ElIGINEERING DESIGN CENTER}
J. R. Haines
P. T. Spampinato
B. L. Hunter
V. C. Srivastava
D. H. Metzler
F. W. Wiffen
W. D. Nelson
M. G. Willey
M. Sek i
G. E. Smith

TRW ENERGY UEVELOPMENT GROUP
C. Garner
J. A. Maniscaico
D. Goebel
S. Mortenson
J. D. Gordon
S. Salem
D. J. Grady
J. Vetrovic

GENERAL OYNAMICS/CONVAIR DIVISION
c. M. Pawers
D. J. Tait
d. L. Saunders
H. E. Toffolo
C. A. Sink
J. I. Valerio
J. D. Sutliff
P. G. Vanoer Kraats
R. A. Sutton
J. C. Yu 


\section{UNIVERSITY OF WISCOISIN}
G. L. Kulcinski
J. F. Santarius
L. J. Perkins
M. E. Sawan

BECHTEL GROUP, INC.

S. L. Thomson

SCIENCE APPLICATIONS, INC.

3. E. Glancy and D. T. Fence

LOS ALAMOS NATIONAL LABORATORY

J. L. Anderson

UNIVERSITY OF CALIFORNIA AT LOS ANGELES

R. W. Conn and M. M. Ghoniem

MASSACHUSETTS INSTITUTE OF TECHNOLOGY

J. Schultz 


\section{ABSTRACT}

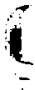

This report describes a facility for generating engineering data on the nuclear technologies needed to build an engineering test reactor (ETR). The facility, based on a tandem mirror operating in the Kelley mode, could be used to produce a high neutron flux $\left(1.4 \mathrm{mw} / \mathrm{m}^{2}\right)$ on an $8-\mathrm{m}^{2}$ test area for testing fusion blankets. Runs of more than $100 \mathrm{~h}$, with an average availability of $30 x$, would produce \& fluence of $5 \mathrm{mw} / \mathrm{yr} / \mathrm{m}^{2}$ and give the necessary experience for successful operation of an ETR. 


\section{ACKNOWLEDGHENTS}

As indicated by the list of contributors, this design study was undertaken by a large group of organizations, including national laboratories, industrial firms, and universities. We would like to thank these organizations and their individual participants for their patience and cooperation in bringing together the efforts of contributors working in geographically separated areas with varying corporate priorities and constraints.

We are particularly indebted to the industrial contributors whose independent R\&D work in several technology areas is reported here in order to form a clear and complete picture of the TDF system.

Finally, we especially thank the LLML Technical Information Department editorial and Magnetic Fusion Energy production staff, along with the clerical staff of each of the contributors, for getting all these words and arawings assembled into a readable document. 


\section{CONTENTS}

1.0. Executive Summary

1.1. Introouction

1.2. Mission . . . . . . . . . . . . . . . 1-2

1.3. Physical Description . . . . . . . . . . . . . $1-5$

1.4. Physics Description . . . . . . . . . . . . 1-8

1.5. Engineering Description . . . . . . . . . . . . . 1-12

1.6. Conclusions . . . . . . . . . . . . . . . . 1-19

References--Section l . . . . . . . . . . . . . . . . . 1-20

2.0. Pnysics

2.1. Introduction . . . . . . . + . . . . . . . . . 2-1

2.2. TDF Equilibrium Parameters . . . . . . . . . . . . 2-2

2.2.1. Overview . . . . . . . . . . . . . . . . 2-2

2.2.2. Code Base for Calculations . . . . . . . . 2-8

2.2.3. Startup Scenario . . . . . . . . . . . 2-10

2.3. Pinsical Requirements for Stream

Stabilized Operation . . . . . . . . . . 2-13

2.3.1. Heating and Fueling with

Neutra]-Baam Injection . . . . . . . . . . . 2-15

2.3.2. Pellet Fueiing for Strean Stabilization . . . . . 2-17

2.3.3. Neutral Beam Pumping and ECAH

for Barrier Formation . . . . . . . . . . 2-18

2.3.4. Vacuum and Halo Requirements . . . . . . . . . . 2-23

2.4. Design Selection for Stability . . . . . . . . . . . 2-24

2.4.1. Magnetic-Field irrangement and Barrier

Profile Used to Provide Stability from

Flute and Ballooning . . . . . . . . . . 2-24

2.4.2. Neutral-Beam Orientation Necessary to Avoid

Alfven Ion Cyclotron Instabi]ities . . . . . . 2-26

2.4.3. Stream-Stabjlization of Drift-Cyclotron-

Loss-Cone Modes . . . . . . . . . . . . . 2-26

2.4.4. Tandem Mirror Magnetic and Barrier/Plug

Combination Used to Avoid Trapped Particle Modes . . . 2-27 
2.5. Effect of Full Thermal-Barrier Operation . . . . . . 2-28

2.6. Fusion Reactor Condition Wall Loading . . . . . . . . 2-31

2.E.1. D-T Neutron Yieló and Wall Loading . . . . . . . . 2-31

2.6.2. First Wall Thermal Loading . . . . . . . . . 2-32

Appendix A Code Formulation for TOF Case . . . . . . . . . . . 2-34

Appendix $B$ An Example of Pĩasma Startup in Programed Steps . . . . . 2-38

Appendix C Factors Related to Neutrat Beans . . . . . . . . . . 2-42

Appendix D A Monte Carlo Study of Charge-Exchange First wall

Power Densities for the TDF Central Cell Beams . . . . 2-47

Appendix $E$ Pellet Fueling Calculations . . . . . . . . . . . 2-60

Appendix $F$ Vacuum and Halo Requirements . . . . . . . . . . . 2-67

Appendix G Impurity Buildup in TDF . . . . . . . . . . . . . 2-88

Refarences--Section 2 . . . . . . . . . . . . . 2-90

\subsection{Engineering}

3.1. Configuration Overview . . . . . . . . . . . 3-1

3.1.1. Introduction and umary . . . . . . . . . . 3-1

3.1.2. Design Approach and Requirements . . . . . . . . 3-4

Modular Design . . . . . . . . . . . 3-6

Access . . . . . . . . . . . . . 3-6

Systems Integration . . . . . . . . . . . 3-7

3.1.3. Selected Physical Characteristics . . . . . . . 3-7

Overall Device . . . . . . . . . . . 3-9

Central Cell . . . . . . . . . . 3-10

Anchor cell . . . . . . . . . . . . 3-10

End Cell . . . . . . . . . . . . . 3-11

\subsection{Magnets}

3.2.1. Overview . . . . . . . . . . . . . . 3-12

Magnet Field Requirements . . . . . . . . 3-12

Magnet Configuration . . . . . . . . . 3-12

External Fjeld . . . . . . . . . . . . 3-12

Conductor Fields . . . . . . . . . . . 3-12

Stored Energy . . . . . . . . . . . 3-18

Halo Clearance . . . . . . . . . . 3-18

Magnetic Forces . . . . . . . . . . . 3.18 
3.2.2. Summary Description of the Centra) [e]1

and Croke Coil system . . . . . . . . . . . 3-18

Central Cell Solenoias. . . . . . . 3-18

Choke Coils . . . . . . . . . . . . . 3-23

3.2.3. Central Cell solenoias. . . . . . . . . . . 3-23

Lesign Considerations and Requirements . . . . 3-23

Engineiring Trace-off Studies . . . . . . . 3-24

Choice of Coolant . . . . . . . . . . 3-26

$E_{i}^{*}$ fect of Neutron Shielding on Cryoplant Cost . . . 3-26

Selerted Design Approach . . . . . . . . . . 3-30

Quencl Analysis . . . . . . . . . . . 3-47

3.2.4. Choke Coils . . . . . . . . . . . . . . 3-48

Uesign Considerations and Requirements . . . . 3-48

Engineering Trade-0ff Studies . . . . . . 3-51

Selected Design Approach . . . . . . . . 3-56

Alternative Design Approaches . . . . . . 3-67

3.2.5. Anchar Cell . . . . . . . . . . . . 3-70

Design Requirements . . . . . . . . . 3-70

Engineering Trade-Off Studies. . . . . . . 3-75

Selected Design Approach . . . . . . . . . 3-80

3.2.6. Internally Water-Cooled Kesistive Coil . . . . . 3-118

Theory . . . . . . . . . . . . . .3-118

Results and Discussion . . . . . . . . . .3-124

3.3. Neutral Beams

3.3.1. Summary . . . . . . . . . . . . . . . .3-128

3.3.2. Central Cell Neutral-Beam Requirements . . . . .3-130

3.3.3. Central Cell Beams . . . . . . . . . . .3-132

Approäch . . . . . . . . . . . . . . .3-132

Component Design . . . . . . . . . . . .3-139

Ion Source . . . . . . . . . . . . .3-139

Neutralizer . . . . . . . . . . .3-149

Reflection Magriet . . . . . . . . . . .3-154

Drift Duct . . . . . . . . . . . . . 3-157

Power Absorbing Surfaces . . . . . . . . .3-178

Vacuum System . . . . . . . . . . . . .3-204 
Magnetic Shield . . . . . . . . . . . . 3-213

Neutron Shielo . . . . . . . . . . . +3-221

Systems Design . . . . . . . . . . . . . . . . 223

Instrumentation, Control, and

Data Acquisition . . . . . . . . . . . . .3-223

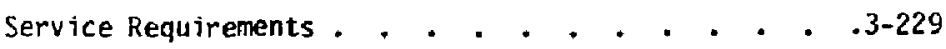

Installation and Servicing . . . . . . . . . 3-233

Key Technical Issues . . . . . . . . . . . .3-239

3.3.4. End Cell Beam Requirements . . . . . . . . . .3-240

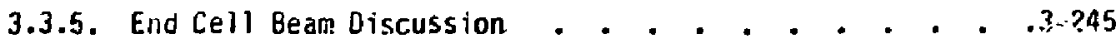

Approach . . . . . . . . . . . . . . . 3-245

Component Design . . . . . . . . . • . . . 3-245

Transition Pumping Neutral-beam Injector . . . . . .

Anchor Cell Pump Beam . . . . . . . . . 3-256

Anchor Cell Sloshing Beam . . . . . . . . . 3-268

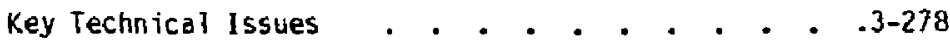

Anchor Cel1 Beam Access Requires Low

Divergence and Collimation . . . . . . . . 3-278

Direct Recovery Development Risk

is Worthwhile. . . . . . . . . . . . . 3-286

Magnet ic Shielding Requirements for

Low Divergence . . . . . . . . . . . .3-287

Drift-Dict Design Optimization . . . . . . . . . . •-287

Neutra1-Beam Injector Sizing for

Acceptable Availability . . . . . . . . . . -29l

\subsection{Microwave Systens}

3.4.1. Overview . . . . . . . . . . . . . . . .3-293

3.4.2. Requirements . . . . . . . . . . . . . . j-293

3.4.3. Design Description . . . . . . . . . . . . 3-293

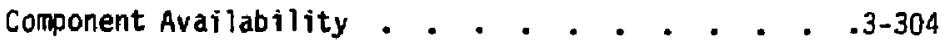

Window Survivability . . . . . . . . . . . . . . . . . . . .

Power Control . . . . . . . . . . . . 3-305

3.5. Low-Energy Deuterium Injection

3.5.1 Gas Injectors . . . . . . . . . . . . . . .307

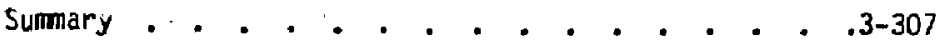


Requirements . . . . . . . . . . . . . . . . . . . . . . . . . .

Design Description . . . . . . . . . . . . . . . . . . . . . . . . . .

Key Issues . . . . . . . . . . . . . . . . . . . . . .

3.5.2. Plasma-Gun Injectors . . . . . . . . . . . . 3-312

Sumary . . . . . . . . . . . . . . . . . . . . . . . . . . .

Requirements . . . . . . . . . . . . 3-312

Design Description . . . . . . . . . . . . . . . . . . . . . . . . .

Key Issues . . . . . . . . . . . . . . . . . . . . . . . . . .

3.5.3. Pellet injectors . . . . . . . . . . . . 3-319

Sumary . . . . . . . . . . . . . 3-319

Requirements . . . . . . . . . . . . . . . . . . . . . . . . .

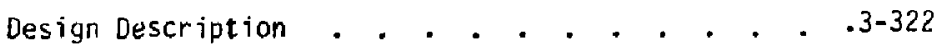

key issues . . . . . . . . . . . . . . 3-326

\subsection{Vacuum Vesse?}

3.6.1. Ma in Vacuum Vesse1. . . . . . . . . . . . . . . . . . . . . . . . . .

3.6.2. Manufacturing . . . . . . . . . . . . . . . $3-330$

Fabrication . . . . . . . . . . . . . . . . . . . . . . . . . .

Magrıets . . . . . . . . . . . . . . . 3-330

End Cells . . . . . . . . . . . . 3-330

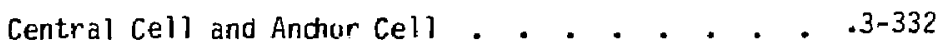

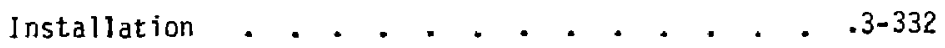

Magnets . . . . . . . . . . . . . . 3-332

Ena Cells , . . . . . . . . . . . . 3-333

Central Cell and Anchor Cell . . . . . . . . 3-333

Structural Analysis. . . . . . . . . . . . 3-334

Centra) Cel] Magnet ( $S-0$ and $S-1$ )

Vacuum Vesse1 . . . . . . . . . . . 3-334

External Vacuum Vessel . . . . . . . . . . . . - . 335

Materials. . . . . . . . . . . . . . 3-335

3.6.3. First Wall--Mechanical . . . . . . . . . . 3-335

Design . . . . . . . . . . . . . . . . - . 335

Manufacturing . . . . . . . . . . . . . . . 339

Fabrication . . . . . . . . . . . . . . . . . 339

Installation . . . . . . . . . . . . . . . . . . • • •

First-wall Material Properties . . . . . . .3-34l

3.6.4. Structural Support . . . . . . . . . . 3-34] 
Manufacturing . . . . . . . . . . . . . . . - . 342

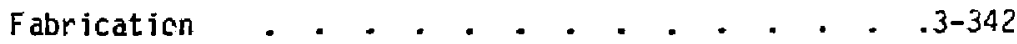

Installation . . . . . . . . . . . . . . - .

Structural Analysis for TDF End Cel?

and Central Cell Support: Weight Loading . . . . .3-343

Preliminary Stress Analys is of the

Anchor Cell Vacuum-Vessel Support Structure . . . .3-344

3.7 Shielding

3.7.1. Shield--Mechanical . . . . . . . . . . . .3-345

Manufacturisiy . . . . . . . . . . . . 3-345

Fabrication. . . . . . . . . . . . . . . . . .

Installation . . . . . . . . . . . . . - . 346

Thermodynamics. . . . . . . . . . . . 3-346

3.7.2. First Wall . . . . . . . . . . . . . . . .3-348

Summary . . . . . . . . . . . . . . . . . . - . 348

Requirements . . . . . . . . . . . . 3-348

Discussion . . . . . . . . . . . . . . . . . . . 350

3.7.3. Bulk Shiela . . . . . . . . . . . . . . 3-355

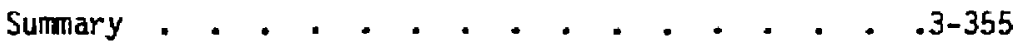

Requirements. . . . . . . . . . . . . . . . . . .

Discussion . . . . . . . . . . . . . . 3-356

3.7.4. Streaming and Shielding Analys is for the

Neutral Bearn Injection Duct . . . . . . + . +. 3-361

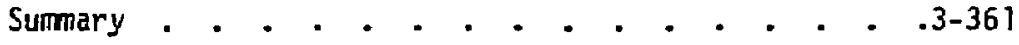

Requirenents. . . . . . . . . . . . .3-364

Discussion . . . . . . . . . . . . . . . . . . . . . . . . .

3.7.5. End Cell Magnet Shielding . . . . . . . . . . . . . . . . . . . . . . .

Sumbary . . . . . . . . . . . . . . . . . 373

Requirements . . . . . . . . . . . . . . . . . . . . . .

Discussion . . . . . . . . . . . . . . 3-373

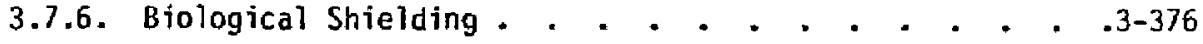

Requirements . . . . . . . . . . . . 3-376

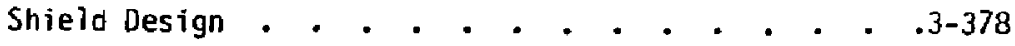

Reactor . . . . . . . . . . . . . . . . - . - . . . .

Building . . + . . . . . . . . . . .3-379 


\subsection{Test Facilities}

3.8.1. Sumary . . . . . . . . . . . . . . . . .3-380

3.8.2. Requirements . . . . . . . . . . . . . .3-38?

3.8.3. Discussion . . . . . . . . . . . . . . .3-386

3.8.4. Key Technical Issues . . . . . . . . . . . . .3-399

3.9. Vacuum Pumping Subsystem Sumary

3.9.1. General Vacuum Requirements . . . . . . . . . .3-402

3.9.2. Discussion . . . . . . . . . . . . . . 3-403

3.9.3. Design . . . . . . . . . . . . . . . .34403

3.10. Particle Dumps . . . . . . . . . . . . . .3-419

3.10.1. Overview . . . . . . . . . . . . . . .34419

3.10.2. Requirements . . . . . . . . . . . . .3-424

3.10.3. Neutral Beam Dump Design . . . . . . . . . .3-424

3.10.4. Neutral-Beam Dump--Startup Analysis . . . . . . .3-441

3.10.5. Central Cell Bean Dumps- Trade Study and Off-Normal Conditions . . . . . . . . . . .3-456

3. 10.6. Beam Dumps for the Transition Region . . . . . . .3-459

3.10.7. First Wall of the Central Cell . . . . . . . . .3-473

3.10.8. Blistering of the Beam Dumps . . . . . . . . . .3-480

3.10.9. Plasma End Dump . . . . . . . . . . . . . .3-481

3.11. Tritium Studies

3.11.1. Summary . . . . . . . . . . . . .3-502

3.11.2. System Requirements . . . . . . . . . .3-502

3.11.3. Tritium Material Balance . . . . . . . . . 3-503

3.11.4. Fuel Cleanup System . . . . . . . . . . . .3-506

3.17.5. Isotope Separation Systein . . . . . . . . . . .3-510

3.11.6. Fuel Preparation System . . . . . . . . . . $3-513$

3.11.7. Tritiated-Waste Treatment System . . . . . . . .3-513

3.11.8. Tritiated-Water Recovery System . . . . . . . .3-514

3.11.9. Facility Air Detritiation System . . . . . . . .3-518

3.11.10. GTovebox Gaseous Treatment System . . . . . . . .3-518

3.11.11. Tritium Systems Data Acquisition. . . . . . . . .3-519 
3.11.12. Response of Tritiun Systems to Accidents..... . . 3-519

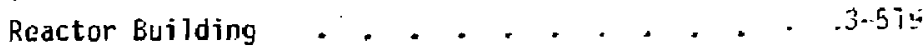
Tritium Processing Building . . . . . . . . . . . . . . . . Hot Cell . . . . . . . . . . . . . . . . . . . . . . Giovebox Gaseous Treatment System . . . . . 3-5.24

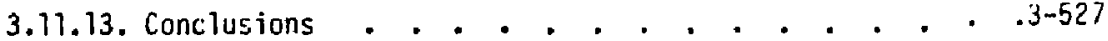

3.1!. 14. Uncertain Technical Issues . . . . . . . . . 3-527 3.12. Plant Fa:ilities

3.12.1. introduction and Summary . . . . . . . . . . 3-529

3.12.2. Facility Requirements . . . . . . . . . . 3-532

3.12.3. Design Description . . . . . . . . . . . .3-543

Site Facilities . . . . . . . . . . . 3-543

Mechanical Systens . . . . . . . . . . 3-545

Buildings and Structures . . . . . . . . . 3-558

AC Power System . . . . . . . . . . . . . . . . . . . . . . .

3.12.4. Key Issues . . . . . . . . . . . . . . . 3-587

3.13. Instrumentation And Control System

3.13.1. Sumiary . . . . . . . . . . . . . .3-591

3.13.2. Requirements . . . . . . . . . . . . . . 3-591

3.13.3. Discussion . . . . . . . . . . . . . . . . . . . . . .

The Supervisory control and

Diagnostic Systen . . . . . . . . . . . 3-592

LCIS Interface . . . . . . . . . . . . . 3-595

Diagnostics . . . . . . . . . . . . . 3-595

Operator-Machine Interface . . . . . . . . . . . -598

Exception Handling .' . . . . . . . . . . .3-598

Local Control and Instrumentation Sysiem . . . . . 3-599

Computer-Automated Measurement

and Control . . . . . . . . . . . . 3-600

Safety Inter jock Systen . . . . . . . . . . 3-603

3.14. Cryoplant . . . . . . . . . . . . . . . . . . . . . . . . . .

3.14.1. Summary . . . . . . . . . . . . . . . . 3-606

3.14.2. Requirement Survey and Analysis . . . . . . . . . 3-606

Magnet Cryogenic Requirements . . . . . . . 3-606 
Vacuum Plimping System: . . . . . . . . 3-oii

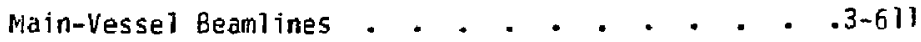

Tritium Systems/Processing. . . . . . . . . . . - 611

Pellet Injection. . . . . . . . . . . 3-61l

3.14.3. Cryoplant System Concepts . . . . . . . . 3-612

Heliurn System . . . . . . . . . . . . . - . biź

Cold Box (Refrigerator) . . . . . . . . . . . - .

Compressors . . . . . . . . . . . . . . . . . . .

Helium Sturage ana Supply Dewars . . . . . . 3-6́ls

Nitrogen Systern. . . . . . . . . . . . . . . . . . . .

builuing/Utility Requirenents . . . . . . . . . . . - .

3.25. Magnet Power Conversion and Protection

3.15.1. Sumnary. . . . . . . . . . . . .3-613

3.15.2. Requirements . . . . . . . . . . . . 3-61b

3.15.3. Design Uescription . . . . . . . . . . .3-017

3.15.4. Key Issues . . . . . . . . . . . . . . . 3-626

3.16. Maintenance

3.16.1. Overview . . . . . . . . . . . . . 3-628

3.16.2. Requirements and Performance . . . . . . . . . 3-624

Contact and Remote Majintenance . . . . . . 3-629

Component Disassembly . . . . . . . . . . . . . . . . . . . .

Remote Maintenance Equipment Technology . . . . . .3-629

3.16.3. Maintenance Equipment . . . . . . . . . . . . 3-632

3.16.4. Scheruled ana Unscheduled Uperations . . . . . . .3-632

3.16.5. Component Replacenents . . . . . . . . . . .3-b38

Test Module. . . . . . . . . . . . . . . .

Choke Coil . . . . . . . . . . . . . . . . . . . .

Central Cell Beam Dump . . . . . . . . . . . . . . . . . .

Anchor-Siloshing beamline . . . . . . . . .3-cal

Central Cell Beamline . . . . . . . . . . . . . . . . . . . . . . . .

Cryopanel . . . . . . . . . . . . 3-64b

Particle bump . . . . . . . . . . . . . . . . 645

Hel ium Pump . . . . . . . . . . . . . . . . . . 


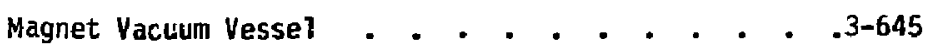

Heferences--Section 3 . . . . . . . . . . . . . . . . . . . . 1

4.0 Safety Assessment

4.1. Introduction . . . . . . . . . . . . . . 4-1

4.2. Safety Criteria and Requirements. . . . . . . . . 4-3

4.3. Potential Radiological Consequences. . . . . . . . . 4-10

4.4. Safety Features . . . . . . . . . . . . . . 4-24

4.5. Environmental Considerations - . . . . . . . . . 4-27

4.6. Key Safety Issues. . . . . . . . . . . . . . . 4-28

References--Section 4 . . . . . . . . . . . . . . . 4-29

5.0. Cost Estimates

5.1. Introduction . . . . . . . . . . . . . . .5-1

5.2. Total Capital Costs . . . . . . . . . . . . 5-1

5.2.1. Direct Costs . . . . . . . . . . . . . 5-1

5.2.2. Indirect Costs . . . . . . . . . . . . . . 5-6

5.2.3. Discussion of Estimate . . . . . . . . . . . . 5-7

References--Section 5... . . . . . . . . . . . 5-13 


\section{LIST OF ILLUSTRATIONS}

1-7. Cross section of TDF machine

1-2. Central portion of TDF, showing the two

blanket module test locations, scheme for

removing choke coils, beamline penetration

th: ough shieiding, and bean dump access for repair . . . . . . 1-7

1-3. Profiles of magnetic field, potential, and density along the machine axis ............. . 1-10

$1-4$. Details of the blanket test area showing how mucules could be readily removed . . . . . . . . . . 1-15

1-5. Azimuthat view of the four beamlines

at each end of the central cell.,. . . . . . . . . . 1-16

1-6. An individual beamline with four sources, one of them a spare to improve reliability . . . . . . . . 1-17

2-1. Hase case axial magnetic field, plasma potential, and density profiles . . . . . . . . . . . . . . . 2-9

$2-2$. Phase space plat for the $Z \sim 6.5 \mathrm{~m}$ position of the transition section

2-3. The B.rcorrected phase space plot for the anchor midplane . . . . . . . . . . . . . . . 2-22

2-4. Marginal flute and ballooning stability boundaries calculated by $R$. Hong with the TEBASCO code under base case conditions . . . . . . . . . 2-25

2-5. The line source neutron yield calculated for $D-T, D-D$, ano $T-T$ reactions, as a function of $Z$. . . . . . . 2-33

$\mathrm{C}-1$. Average $0^{0}$ energy for an $80-k V$ NBI system with a $D_{2}$ gas fill in the charge-exchange cell . . . . . . . . 2-44

$0+$. Polar plot of charge-exchange wall flux in the $x-y$ plane for one of four D-T beams on D-T plasma . . . . . . 2-52

$D-2$. Composite polar plot of charge-exchange wall flux in the $x-y$ plane for all four D-T beams on D-T plasma . . . . . . 2-53

D-3. Plot of an 80-keV beam on D-T plasma . . . . . . . . . . . 2-55 D-4. D-T beams on D-T plasma . . . . . . . . . . . . . . . 2-58 
E-1. Pellet . epositior in the TDF plasma where the

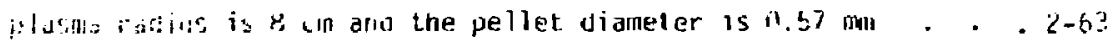

E-2. Pellel. ofios li int in the lof plasma vhere the

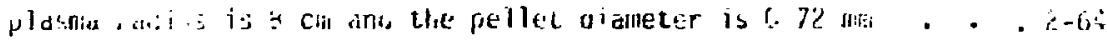

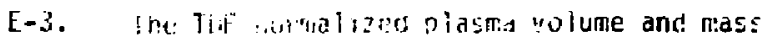

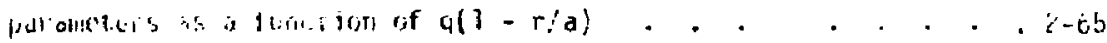

E-4. The petlet "illocity and plasula peretiation,

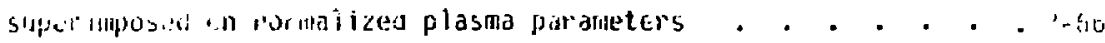

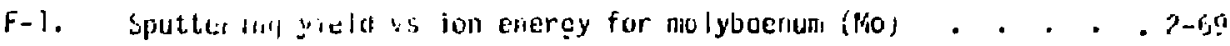

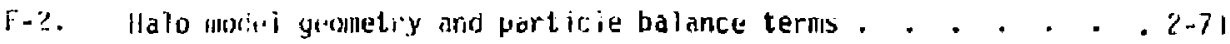

F-3. Attenuation of neutral gas vs the halo line aensity. . . . 2 1-74

F-4. Lur rough nowel for the reionization if charge-exchanged neutrals, shoring (a) the model ano (b) three possible special cases. . . . . . . . . . 2-79

F-5. Halo density and temperature vs neutral density at the first wali . . . . . . . . . . . . 2-85

F-6. Halo density and beam attenuation us relative transport rate from the central cell . . . . . . 2-87

3-1. The TDF configuration: an evolved design that meets the requirements of all system components . . . . 3-2

3-2. Side elevation and end views of the nuclear engineering aevice . . . . . . . . . . . . . . 3-2

3-3. The three areas of the machine: the central, ar:cirm, and end cells. . . . . . . . . . . . 3-5

3-4. Five conponents integrateo into a limited portion of the central cell . . . . . . . . . . . 3-b

3-5. Center-line field profile... . . . . . . . . . 3-14

3-6. Reference magnet configuration . . . . . . . . . . . 3-15

3-7. TOF external fields. . . . . . . . . . . . . . . 3-16

3-8. Flux bundle through the choke . . . . . . . . . . . 3-20

3-9. Baseline magnet system forces . . . . . . . . . . . 3-21

3-10. JIJF magnet arrangement . . . . . . . . . . . . . . . 3-22

3-11. Coil configurations for Hel and Hell cooling schemes . . . 3-27 
3-12. Cross section and characteristics of the S-0 solenoid . . . 3-32

3-13. Cross secition and characteristics of the $S-1$ solenoid . . . 3-33

3-14. Solenoid magnets, TDF-010 . . . . . . . . . . . 3-37

3-14. Solenoid magnets, TOF-c10 (Cnntinued) : . . . . . . . . 3-38

3-14. Solenvid magnets, TDF-010 (Continued) . . . . . . . . . 3-39

3-15. Service stack, TDF-011. . . . . . . . . . . . 3-41

3-16. Maximum cryostable current density for S-0 solenoid. . . . . . . . . . . . . 3-45

3-17. Maximum cryostable current density for S-1 solenoid. . . . . . . . . . . 3-46

3-18. Coil configuration and final temperatures

following quench for four sets of current leads . . . . . 3-49

3-19. Conductor yield strength vs electrical conductivity . . . - 3-52

3-20. Comparison of coolants and cooling configurations . . . . 3-55

3-21. Baseline concept for the TDF choke coil . . . . . . . . 3-57

3-22. Operating efficiency as a function of magnet geometry . . . 3-60

3-23. Maximum efficiency for any given magnet volune . . . . . . . 3-62

3-24. Comparison of internaliy and externally cooled resistive coils . . . . . . . . . . . . . . 3-6B

3-25. TDF internally cooled conductor concept, TDF-014 . . . . 3-71

3-26. Power required for choke coil to produce $15 \mathrm{~T}$ for varying background fields . . . . . . . . . . . 3-72

3-27. Alternate solenoid options . . . . . . . . . . . 3-73

3-28. Coil winding support alternatives . . . . . . . . . . . 3-78

3-29. TDF arrangement . . . . . . . . . . . . . . . . 3-81

3-30. Anchor cell magnet conductor . . . . . . . . . . . . 3-82

3-31. Anchor cell winding pack--typical . . . . . . . . . . . 3-84

3-32. Typical coil-case cross section . . . . . . . . . . . 3-87

3-33. Anchor cell coil set . . . . . . . . . . . . . . . 3-89

3-34. Cold mass support concept . . . . . . . . . . . . . . 3-9l

3-35. Anchor cell vacuum vessel . . . . . . . . . . . . . . 3-92

3-36. Section of anchor cell through the mouths of coils $\mathrm{T}-1$ and $M-2+.+.+.+.+.+.+3-93$

3-37. Section of anchor cell through the mouth of coil $\mathrm{M}-3$ 
3-38. Inner shell of anchor cell vacuum vessel . . . . . . . . . 3-96

3-39. Anchor pulmping beam dump . . . . . . . . . . . . . . 3-97

3-40. Anchor cell shielding detail. - . - . . . . . . . 3-9c ,

3-41. Anchor cell sections at $(a) z=9.5 \mathrm{~m}$ and

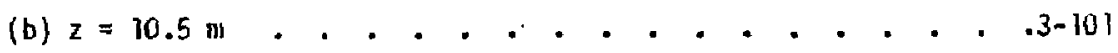

3-42. Anchor cell magnetic field on-axis... . . . . . . . . 3-103

3-43. Operating current vs peak field. . . . . . . . . . . 3-105

3-44. Notation for coil electromagnetic forces. . . . . . . . 3-709

3-45. Section through anchor coil at $z=9.75 \mathrm{~m}$. . . . . . . . $3-117$

3-46. Resistance change as a function of radial

location of conductor . . . . . . . . . . . . . 3-122

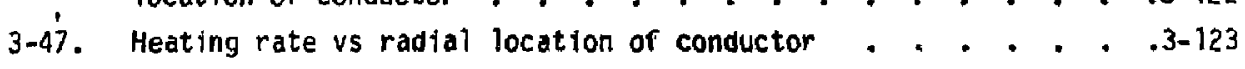

3-48. The internally-cooled choke coil concept at

$2800 \mathrm{~A} / \mathrm{cm}^{2}$ operated within design constraints . . . . . 3-125

3-49. Neutron-streaming considerations . . . . . . . . . . .3-134

3-50. Central cell hearn-auct geometry . . . . . . . . . . . 3-137

3-51. Central cell neutral-beam and

beam-dump configuration . . . . . . . . . . . . . . 3-138

3-52. Arrangement of beamline components . . . . . . . . . . .3-140

3-53. Beamilne layout . . . . . . . . . . . . . . . . . . . . . . . . . .

3-54. Extraction grid with slits . . . . . . . . . . . . .3-144

3-55. Extraction grid with circular apertures . . . . . . . . 3-146

3-56. Beam focusing . . . . . . . . . . . . . . . . . 3-147

3-57. Baseline neutralizer tube . . . . . . . . . . . . . 3-150

3-58. Heutralizer geometry . . . . . . . . . . . . . .3-153

3-59. Ion dump arrangement . . . . . . . . . . . . . . 3-156

3-60. Magnet aimensions . . . . . . . . . . . . . . . .3-159

3-61. Drift-auct geometry . . . . . . . . . . . . . . .3-160

3-62. Drift-duct location . . . . . . . . . . . . . . 3-166

3-63. Duct geometry . . . . . . . . . . . . . . . . . .3-168

3-64. Physical model . . . . . . . . . . . . . . . . . . . . . . . . . .

3-65. Dependence of $T_{D}$ on $f \cdot c^{-} \cdot e^{-} \cdot . \cdot . \cdot .3-172$

3-66. Duct-dump relationship . . . . . . . . . . . . .3-173

3-67. Mosel illustration . . . . . . . . . . . . . . . .3-174

3-68. Dependence of transmission on conductance . . . . . . . . 3-176 
3-69. Uetail of duct wall . . . . . . . . . . . . 3-179

3-70. Drift-auct i inal design . . . . . . . . . . . . . . . . . . . . . . . .

71. Variation of sputtering yield with angle of incidence for $\mathrm{H}^{+}, \mathrm{O}^{+}$on $\mathrm{Mo} . . .4 . . . . .3-183$

3-72. Beams intersecting plasma . . . . . . . . . . . . . 3-785

3-73. Beam absorption in plasma vs plasma radius . . . . . . . . 3-186

3-74. Beani vs dump geometry in $x-2$ plane. . . . . . . . . . . 3-187

3-75. Beam ys dump geometry in $Y-Z$ plane . . . . . . . . . . . 3-188

3-76. Power-density distribution on dunip face (one quadrant) . . . . . . . . . . . . 3-190

3-77. Plotted quaurant location. . . . . . . . . . . . . 3-191

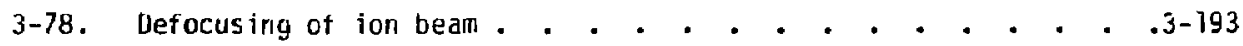

3-79. Defocusing model . . . . . . . . . . . . . . . . . . . . . . . . .

3-80. Power-density distribution on ion dump surface after defocusing . . . . . . . . . . . 3-196

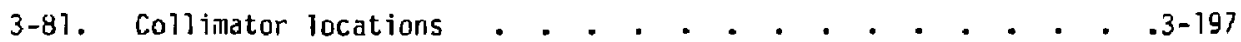

3-82. Rear collimator . . . . . . . . . . . . . . . . . . . . . . . . . .

3-83. Front collimator . . . . . . . . . . . . . . 3-200

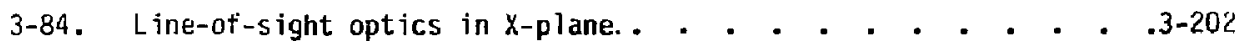

3-85. Diffusion model . . . . . . • . . • . . • . • . . 3-203

3-86. Vacuum-vessel sectioning . . . . . . . . . . . . . 3-205

3-87. Cryopanel structure, showing TOF/NBIS and cryopanel detail . . . . . . . . . . . . . . . . 207

3-88. Cryopanel structure . . . . . . . . . . . . . . . 3-208

3-89. Liquid-nitrogen flow system (each panel) . . . . . . . . 3-215

3-90. Liquid-helium flow system (each panel) . . . . . . . . . 3-216

3-91. Magnetic field identification . . . . . . . . . . . . . . . . . . . . . . . . . . . .

3-92. Superconducting shield design . . . . . . . . . . . . . - •222

3-93. Electrical and fluid connectors to ion source . . . . . . . 3-231

3-94. Vacuum-vessel disassemzly . . . . . . . . . . . . . . 3-234

3-95. Servicing a source . . . . . . . . . . . . . . . . 3-237

3-96. Renoving cylindrical section for servicing . . . . . . . . 3-238

3-97. Anchor cell NBI locations . . . . . . . . . . . . . . 3-241

3-58. Neutral-beam plasma targets . . . . . . . . . . . . . 3-244

3-99. Anchar cell vacuum vessel . . . . . . . . . . . . . . . . . . . . . . . . 
3-iu0. Trafisil rin pung ing-bealn geometry

3-101. Transilivi. bean plasma power aensity

for two configurations. . . . . . . . . . . . . . 3-249

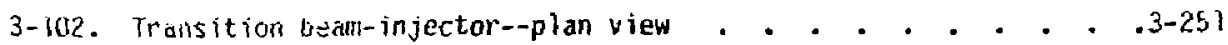

3-103. Träsitiran beari-injector-elevation view. . . . . . . . 3-252

3-104. Wansiturin beam-injector-eno view showirns somections. . . . . . . . . . . . . . . 3-253

3-105. Tramsiticin becin-power fractions. . . . . . . . . . 3-255

3-106. Irasicicin beam-dump patterns . . . . . . . . . . . . 3-257

3-70\%. Magnetic tielas around transiticn pumping beári . . . . . . . . . . . . . . . . 3-258

3-308. Anchar cell veall-injector-oplan view . . . . . . . . . . 3-25y

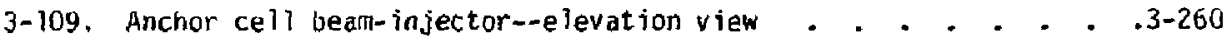

3-110. Anchor cell beam-injector--end view showing connections. . . . . . . . . . . . . 3-26i

3-111. Anchor cell fuiming-beam geometry . . . . . . . . . . 3-263

3-112. Anchor cell pumping-beam access. . . . . . . . . . . 3-264

3-113. Anchor cell pumping-beam power fractions . . . . . . . . 3-266

3-114. Magnetic fields around anchor purming injector . . . . . . 3-269

3-115. Anchor cell pumping beam-dump patterns . . . . . . . . . 3-270

3-176. Anchor cell slosining beam plasma patterns . . . . . . . 3-271

3-117. Anchor cell sloshing beam access . . . . . . . . . . 3-273

3-718. Anchor cell sloshing beam power fractions . . . . . . . . 3-276

3-119. Anchor cell sloshing beam-dump patterns . . . . . . . . 3-277

3-120. Magnetic fields around anchor cell pumping beam . . . . . . 3-279

3-127. Beam access constrictions . . . . . . . . . . . . . . 3-280

3-122. Beam acceptance angles. . . . . . . . . . . . . . . 3-281

3-123. (a) Beam power loss to beam scraping

(b) Beam cost increases slowly with number of sources . . . .3-284

3-124. Ion-dump characteristics . . . . . . . . . . . . . .3-288

3-125. Direct recovery configuration . . . . . . . . . . . . . . . . . . . . . . . .

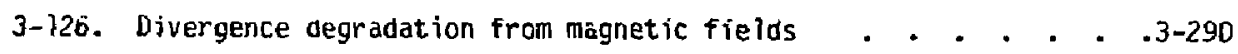

3-127. ECRH/anchor cell interface . . . . . . . . . . . . . 3-294

3-128. Cross section of anchor cell vacuum vessel at ECRH launcher . . . . . . . . . . . . . . . 3-296 
3-129. ECRH launcher detail . . . . . . . . . . . . . . 3-298

3-130. ECRH trangmissior system . . . . . . . . . . . • . . 3-299

3-131. ECRH system bleck diagram . . . . . . . . . . . . . . 3-301

3-132. ECRH loss summary . . . . . . . . . . . . . . . . 3-303

3-133. Flow diagram of deuterium gas system . . . . . . . . . . 3-311

3-134. Schematic sectional view of one of four startup plasma guns. . . . . . . . . . . . . . . . . . . . . . . . . .

3-135. PIasma-gun supply system diagram . . . . . . . . . . . 3-316

3-136. Sectional view, showing the location of the TDF startup plasma gun assemblies (two guns on each end cover) . . . . .3-317

3-137. Two-channel pellet injector flow diagram (one of two) . • . 3-323

3-138. Outline of centrifugal pellet injector, showing principal features and interface connections . . . . 3-325

3-139. Sectional view of TDF reactor, showing the location of one pellet injector and its mechanical interface . . . . 3-327

3-140. Dual isolation valve assembly and the pellet drift-tube extension . . . . . . . . . . . . 3-328

3-141. Test module seal . . • . . • . . • . . • . . . . 3-331

3-142. First-wall configuration . . . . . . . . . . . . . . 3-338

3-143. TUF magnet modeis for neutronics analysis . . . . . . . 3-347

3-144. Nuclear heating in the TDF central cell bulk shield . . . . 3-349

3-145. First wall neutronics modeling geometry . . . . . . . . . 3-352

3-146. Bulk shield neutronics modeling geometry . . . . . . . . . 3-359

3-147. Energy spectrum of neutrons and gamma photons streaming into the neutral beam duct . . . . . . . 3-366

3-148. Angular distributions of neutrors and gama photons streaming into the neutral beam duct . . . . . . . . . . . . .

3-149. Geometrical model for the neutral beam duct in TDF . . . . 3-368

3-150. Magnet shielding requirements for different neutron wall loadings . . . . . . . . . . . . . . . 3-375

3-151. Shield configuration for the transition ana anchor coils. . . . . . . . . . . . . . . . . . . . . . . . .

3-152. TUF test configuration . . . . . . . . . . . . . . . . . . . . . . . . .

3-153. Detailed TOF test configuration . . . . . . . . . . . . . . . . . . . . . .

3-154. Neutron fluence requirements and capabilities per run . . . .3-393 
3-155. Total integrated neutron fluence requirements and capabilities . . . . . . . . . . 3-400

3-156. Prototype of the TDF continuous cryopumping system tested at LLNL . . . . . . . . . .3-406

3-157. Regenerable cryopumping panel concept for TOF . . . . . . .3-407

3-158. Une of 12 six-panel degassing assemblies. . . . . . . .3-408

3-159. Helium-appendage pumping installation in the end cells . . 3-409

3-160. Details of the helium-argon cryotrapping panel . . . . . .3-410

3-161. Plot of tritium inventory us excess capacity for the TOF end-cell pulliping system . . . . . . . . . .3-413

3-16ї. Baseline panel design . . . . . . . . . . . . . . . 3-420

3-163. Dump panel assembiy. . . . . . . . . . . . . .3-421

3-16.4. Maximum heat flux on plasma end dump surface at various dump locations. . . . . . . . . . . . .3-422

3-165. Basic end dump panel . . . . . . . . . . . . . . .3-423

3-166. Sputtering rate enhancement vs arigle of incidence from normal . . . . . . . . . . . . . . . . . . . .

3-167. Calculated power density normal to the beam after passing through the plasma . . . . . . . . . . . . 3-427

3-168. Calculated power density normal to the beam for four sources on the dump . . . . . . . . . . . .3-428

3-169. TOF source startup sequence for central cell neutral-beam injectors. . . . . . . . . . . . . . .3-430

3-170. Sputtering thickness for 1 full-power year for various materials at increasing heat flux . . . . . . . . $3-432$

3-171. Maximum temperatures of the beam dump front surface . . . . .3-433

3-172. Water pressure requirements as a function

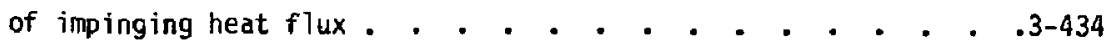

3-173. Panel coil geometry. . . . . . . . . . . . . .3-436

3-174. TDF end cell configuration . . . . . . . . . . . 3-437

3-175. Tritium giffusion model . . . . . . . . . . . . . .3-438

3-176. Neutral-beam deposition in metal surface. . . . . . . .3-439

3-177. Internally finned, water-cooled dump . . . . . . . . . .3-442

3-178. The central cell neutral-beam energy overlap on a normal dump surface with no plasma trapping . . . . . . 3-444

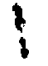

xxiv 
3-179. Saurce startup sequence for the centrat ce11

neutral-beam injectors. . . . . . . . . . . . . 3-445

3-180. TDF plasma trapping fraction increase at startup . . . . . 3-446

3-181. The heat flux scenario for the reactor startup phase study of the neutral ournp at various maximum flux locations . . . 3-448

3-182. The thernal analyzer front plate noddis.tion used to predict therma 1 gradients in the front plate of the neutral-beam dump during system startup . . . . . . . . .3-449

3-183. The begin-1ife temperature profile of the beam aump's front surface during reactor startup at the peak heat flux location of source $1 . .+. .+. .3-450$

3-184. The begin-life tenperature profile of the beam dump's front surface during reactor startup at the peak heat fiux at the intersection of sources 3 and 4. . . . . . 3-451

3-185. The eld-1ife temperature profile of the beam dump's front surface during reactor startup at the peak heat flux location of source 1. . . . . . . . . . . . 3-452

3-186. The end-7ife temperature profile of the beam oump's front surface during reactor startup at the peak heat flux at the intersection of sources 3 and 4. . $^{\circ}$. . . . . 3-453

3-187. The begin-1ife maximum temperature of the bean dump's front surface during reactor startup . . . . . . . . . 3-454

3-188. The end-iffe maximum temperature of the beam dump's ouring reactor startup . . . . . . . . . . . . . . . 3-455

3-189. Peak heat fluxes on central ceti beam oumps . . . . . . . . 3-457

3-190. Pumping power for TOF central cell beam dumps . . . . . . .3-458

3-191. Effect of misalignment between iseam and plasma. . . . . . 3-460

3-192. Effect of plasma loss on the beam dump surface. . . . . . .3-461

3-193. Transition region beam dump locations. . . . . . . . . 3-462

3-194. Surface heat load on the anchor pumping beam dump . . . . . 3-465

3-195. Surface heat load on the anchor sloshing beam dump . . . . 3-466

3-196. Surface heat load on the transition pumping beam dump . . . 3-467

3-197. End cell plan layout used in cost trade study . . . . . . 3-46

3-198. Summary of eni cell cost study . . . . . . . . . . . 3-470

3-199. Vacuum vessel suríace area . . . . . . . . . . . . . 3-471 
3-200. Eno . - 11 vacuuni vessel thick.lesses ased for trade study relative volumes

3-201. End aump surface heat loads based on magnetic field strength . . . . . . . . . . . . . .3-474

$3-202$. First wall of the central cell . . . . . . . . . .3-476

3-203. Tritium processing and treatment systems for TDF . . . . . 3-507

3-204. Fuel cleanup system for $\mathrm{TDF}$. . . . . . . . . . .3-509

3-205. I sotope separation system for TUF . . . . . . . . . . . 3-511

3-206. Contaminated air aistribution system for TOF . . . . . .3-515

3-207. TUF tritiated-water recovery system . . . . . . . . . 3-517

3-208. Tritium concentrations remaining in the reactor

wu?loug as a function of time and of cieanup rates

for an accidental release of $100 \mathrm{~g}$ of tritium . . . . . . 3-522

3-209. Tritiun concentration in tritium processing building

as a function of time ais of cleanup rate for an

accidental release of $300 \mathrm{~g}$ of tritium . . . . . . . . . . . . . 25

3-210. Tritium concentration in hot cell as a function

of time and of cleanup rate for a $100 \mathrm{Ci} / \mathrm{d}$ source term . . . .3-526

3-211. Plot plan for TOF facilities . . . . . . . . . . 3-544

3-212. Heat transport system . . . . . . . . . . . 3-548

3-213. Emergency-cooling water system . . . . . . . . . 3-549

3-214. Activity as a function of time after reactor shutdown . . . 3-552

3-215. Decay heat vs time after reactor shutoown . . . . . . . . 3-553

3-216. Processing of activated structure wastes. . . . . . . . . 3-554

3-217. Raoioactive waste handling system . . . . . . . . . 3-557

3-218. Interface of facility systems . . . . . . . . . . . 3-559

3-219. Reactor and support buildings (plan below el. $130 \mathrm{~m}$ ) . . . . 3-560

3-220. Reactor and support buildings (plan below el. $113 \mathrm{~m}$ ) . . . . 3-561

3-221. Reactor and support buildings (plan below el. $106 \mathrm{~m}$ ). . . . 3-562

3-222. Reactor and support buildings ( $p$ lan below el. $98 \mathrm{~m}$ ) . . . . 3-563

3-223. Reactor building (el. $100 \mathrm{~m}$ ) in mare detai\}

(Section A-A from Figure 3-219) . . . . . . . . . . . 3-564

3-224. Reactor complex (Section B-B from Figure 3-219) . . . . . 3-565 
3-225. Cryogenics building of reactor complex (Section C-C from Figure 3-219) . . . . . . . . . . .3-566

3-226. Tritium and hot-cell bulldings (Section D-D from Figure 3-219) . ... . . . . . . . .3-567

3-227. High-v01tage side of heavy-duty substation with single phase transformers. . . . . . . . . . . .3-581

3-228. Heavy-duty substation 13.8-kV power-distribution system . . . .3-582

3-229. Motor control and load control centers for the superconductor coil power supplies . . . . . . . . . .3-585

3-230. Light-duty substation and essential power-distribution system . . . . . . . . . . . $3-586$

3-231. No-break control power for the superconductor coil protection and fuel reprocessing systems . . . . . .3-588

3-232. The supervisory control and diagnostics system (SCDS) . . . .3-593

3-233. The plasma diagnostics data processing and display system : . . . . . . . . . . . . . .3-596

3-234. The local control and instrumentation system (LCIS) subsystem . . . . . . . . . . . . . . . .3-601

3-235. The safety interlock system (SIS) . . . . . . . .. . . 3-604

3-236. A typical primary power supply for the superconducting coils consisting of a silicon-contralled rectifier with low voltage and high current. . . . . . . . . . . 3-619

3-237. The magnet power conversion system for the series-connected chioke coils, $M-1$. . . . . . . . . . . . . . . 3-621

3-238. The magnet power supply and coll protection system for the M-2 and M-3 pair of superconducting coils . . . . . . . . 3-\$222

3-239. A conceptual plan view of the power conversion and magnet protection equipment . . . . . . . . . . . .3-624

3-240. Section A-A elevation view of the power conversion and coll protection equipment, from Figure 3-239. . . . . . 3-625

3-241. The motor and load control centers for the superconducting coil power supplies . . . . . . . . . . . . . . 3-627

3-242. The contact maintenance boundary and shield thicknesses . . 3-631

3-243. An estimate of first-time replacements for selecteo components 
3-2.44. The choke coil replacenents scheduled for the device's lifetinic . . . . . . . . . . . . . . . 3-639

3-245. Test module removal . . . . . . . . . . . . 3-640

3-246. Choke coil and beam dump removal . . . . . . . . . . . 3-641

3-247. Beamine removal: typical for anchor sloshing, transition pumping, or anchor pumping . . . . . . . . 3-643

3-248. Central cell beamline removal . . . . . . . . . . . 3-644

3-249. Removal of cryopanel modular assemblies . . . . . . . . 3-646

3-250. Particle dump panel removal . . . . . . . . . . . . 3-647

3-251. Helium pump module renoval . . . . . . . . . . . . 3-648

3-252. Magnet vacuum vessel assembly removal . . . . . . . . . . 3-650

4-1. Major gaseous tritium leakage pathways . . . . . . . . . 4-12

4-2. Tritium-release pathways to environs . . . . . . . . . . 4-13

4-3. Maximum-allowable tritium-release rate from plant butldings under normal conditions . . . . . . . . . . . . . 4-15

4-4. Maximum-allowable tritium-release in plant bulldings from an accidental release . . . . . . . . . . . . . . 4-18

4-5. Single confinement compared to double confinement . . . . . 4-22 


\section{LIST OF TABLES}

1-1. Characteristic times for fuel power cycle tests

on blankets and auxiliary equipment. . . . . . . . . 1-4

1-2. TDF physics parameters . . . . . . . . . . . . . . 1-9

1-3. TDF engineering parameters . . . . . . . . . . . . . 1-13

2-1. Tandem mirror parameters for the central cell based on the stream-stabilized base case . . . . . . . . . 2-4

2-2. Tandem mirror parameters for the TDF end cells based on the stream-stabilized base case . . . . . . . . . 2-5

2-3. TDF startup scenario . . . . . . . . . . . . . . . 2-11

2-4. Heating, fueling, and pump system listing . . . . . . . . 2-14

2-5. Design levels for the tandem mirrors of the TDF central cell, without a stream plasma . . . . . . . . . 2-29

2-6. Design levels for the tanden mirrors of the TDF end cells, without a stream plasma . . . . . . . . . 2-30

B-1. Definition of the parameters used in Table B-2 . . . . . 2-39

$B-2$. Values of the parameters used in a TDF startup scenario - . 2-40

D-1. Beam-plasma first-wall parameters for the TDF charge-exchange wall flux computation . . . . . . . . 2-49

D-2. Particle and power balances for the TDF central cell beams, determined from four beams at one end of the device. . . . . . . . . . . 2-51

E-1. Pellet injector requirements for the central cell . . . . 2-62

F-1. Neutral beam parameters for the central cell . . . . . . . 2-68

F-2. General halo parameters for zone 1 . . . . . . . . . . 2-83

F-3. Particle and power balance parameters for zone 1 . . . . . 2-84

3-1. TDF magnet field requirements . . . . . . . . . . . . 3-13

3-2. Design criteria that affect the magnet configuration . . . . 3-13

3-3. Peak fields in each conductor bundle. . . . . . . . . . 3-17

3-4. Energy stored in each coil . . . . . . . . . . . . . 3-19

3-5. Solenoid magnet requirements . . . . . . . + . . . . 3-25

3-6. Characteristics of LHeI - and LHeII-cooled conductors . . . . 3-28 
3-7. Cost trade stuay of LHeI vs LHel I for central cell solenoids. . . . . . . . . . . . . . . . . $3-29$

3-8. Cryoplant cost as a function of neutron heat deposition in the LHeI-cooled central cell solenoids . . . . 3-31

3-9. Major charcteristics of the solenoid magnets . . . . . . . 3-34

3-10. Input data for single lead-set quench analys is . . . . . 3-50

3-11. Anchor cell coil winding parameters . . . . . . . . . . 3-85

3-12. Dimensions of coil-case cross section . . . . . . . . . 3-88

3-13 Peak fields in anchor cell coils . . . . . . . . . . 3-104

3-14. Out-of-plane electromagnetic running loads on anchor set . . 3-107

3-15. In-plane electromagnetic running loads on anchor set . . . 3-108

3-16. Force sumary--anchor set . . . . . . . . . . . 3-111

3-17. Helium case sizing for magnetic running load . . . . . . . 3-112

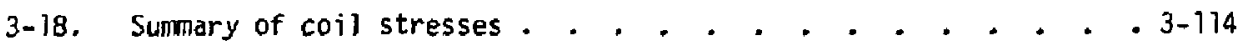

3-19. Center of gravity and support locations . . . . . . . . 3-115

3-20. Operating parameters at the inner and outer coil layers . . 3-127

3-21. Central cel1 NBIS requirements and design capabilities . . . 3-131

3-22. TOF standard ion source . . . . . . . . . . . . . 3-148

3-23. Neutralizer pararieters . . . . . . . . . . . . 3-155

3-24. Ion species, their relative abundance and Larmor radius in $B=0.128 T$ for $D$ and $B=0.157 T$ for $T . .3-158$

3-25. Reflection magnet parameter for one beamline (four beams) . . . . . . . . . . . . . . . . . 3-158

3-26. Drift-duct parameters . . . . . . . . . . . 3-177

3-27. Gas sources and magnitudes . . . . . . . . . . . . . 3-209

3-28. Getter and cryopump beamline comparison . . . . . . . 3-2io

3.29. Cryopanel area . . . . . . . . . . . . . . . . 3-212

3-30. Cryopanel parameters for each NBIS unit . . . . . . . . . 3-214

3-31. TART results . . . . . . . . . . . . . . . . . 3-224

3-32. Results of neutron streaming and shielding caicilations for the central cel] neutral beamiines . . . . . . . . . 3-224

3-33. Service requirements for eight beamlines . . . . . . . 3-230

3-34. Power supply requirements . . . . . . . . . . . . . 3-230

3-35. Coolant requirements . . . . . . . . . . . . . . 3-232

3-36. Summary of anchor cell neutral-beam requirements . . . . . 3-243 
3-37. Sumary of transition beám paraneters . . . . . . . . . 3-247

3-38. Characteristics of a transition neutral-beam ion source . . . . . . . . . . . . . . . 3-250

3-39. Summary of anchor cell pumping beam parameters . . . . . 3-265

3-40. Characteristics of the anchor cell pumping beam ion source. . . . . . . . . . . . . . 3-267

3-41. Summary of anchor cell sloshing peam parameters . . . . . 3-274

3-42. Characteristics of anchor cell sloshing beam ion source . . 3-275

3-43. Surmary of anchor cell microwave requirements . . . . . - 3-295

3-44. Sunmary of system parameters (based on end values) . . . . 3-302

3-45. Supply parameters for gyrotron oscillator (basen or a 60-GHz tube developed by Varian) . . . . . . . . . 3-302

3-46. TDF startup scenario . . . . . . . . . . . . . . 3-308

3-47. Gas-injection requirements/design data . . . . . . . . 3-310

3-48. Startup plasma-gun requirements . . . . . . . . . . . 3-313

3-49. Plasma-gun design and interface data. . . . . . . . . . 3-318

3-50. Pellet-injector design criteria . . . . . . . . . . 3-320

3-51. Pellet-injector requirements . . . . . . . . . . 3-321

3-52. Centrifugal injector data . . . . . . . . . . . . 3-324

3-53. Vacuum-vessel thicknesses for the TOF external vacuum vesse1 . . . . . . . . . . . . . 3-336

3-54. Mechanical properties of $304 \mathrm{~L}$ stainiess steel at elevated temperatures. . . . . . . . . . . 3-337

3-55. Flux-degradation results . . . . . . . . . . . . . 3-353

3-56. Raoiation damage and heating limits (bulk only) . . . . . . 3-357

3-57. Tof buik shield performance (neutronics) . . . . . . . 3-362

3-58. Totâl magnet nuclear input by zone (bulk only) . . . . . . 3-363

3-59. Kesults for the S-I superconducting magnets . . . + . . 3-371

3-60. Results for the superconductive shiela . . . . . . . . 3-372

3-61. Categories of fusion test requirements . . . . . . . . . 3-384

3-62. Generic test categorization . . . . . . . . . . . . . 3-387

3-63. MARS blanket test categorization . . . . . . . . . . 3-38B

3-64. Fusion Breeder Program blanket test categorization . . . . . 3-389

3-65. Fusion neutron facilities . . . . . . . . . . . . 3-392

3-66. End cell vacuum parameters . . . . . . . . . . . . 3-405

3-67. Summary of beam dump heat flux, size, and life . . . . . 3-463

3-68. End dump parameters and costs . . . . . . . . . . . 3-475 
3-69. First wal' parameters for circumferential coolant flow . . . 3-479

3-70. Projected range of deuterium ions in beam-dump materials . . 3-482

3-71. Plasma end-dump systen requirements . . . . . . . . . 3-485

3-72. Particles incident on end dump. . . . . . . . . . . 3-488

3-73. Atomic number densities and sputter yields . . . . . . . 3-489

3-74. Mechanical properties of candidate materials at $200{ }^{\circ} \mathrm{F}$. . 3-491

3-75. Mechanical properties of candidate materials at $300^{\circ} \mathrm{F}$. . 3-492

3-76. Mechanical properties of candidate materials at $400{ }^{\circ} \mathrm{F}$. . 3-493

3-77. Mechanical properties of candidate materials at $500{ }^{\circ} \mathrm{F} . .3-494$

3-78. Mechanical properties of candidate materials at $600{ }^{\circ} \mathrm{F} . .3-495$

3-79. Panel-coil structural design criteria . . . . . . . . . 3-497

3-80. Stress categories and maximum allowable stress intensities . . 3-498

3-81. Plasma end dump heat flux vs panel thickness . . . . . . 3-499

3-82. Fuel and plasma-stability requirements for a 2-MW full-power operation of the fusion device . . . . . 3-504

3-83. Reactor fueling rates and vacuum-pump exhaust rates for fuli-power (20 MW) operation . . . . . . . 3-505

3-84. Estimated contaminant-gas concentrations in the vacuum-pump exhaust for the plasma vesse] of TDF . . . . . 3-508

3-85. The approximate dimensions of the ISS distillation columns of TDF . . . . . . . . . . . . . . 3-512

3-86. Estimated tritium inventories at various locations in TDF . . . . . . . . . . . . . . . . . 3-520

3-87. Facility characteristics and assumed conditions for serious accident cases involving release of tritium inventories to their respective spaces . . . . . . . . 3-521

3-88. Responses of facility cleanup systems to accidents and est imates of tritium losses to the atmosphere . . . . 3-5.8

3-89. Major electrical power loads . . . . . . . . . . . 3-531

3-90. Characteristics of hypothetical site for TDF . . . . . . 3-536

3-91. Safety factors for assumed various loading conditions . . . 3-539

3-92. Estimate of facility power loads . . . . . . . . . . . 3-541

3-93. Capacity requirements for ac power system . . . . . . . 3-542

3-94. Major plant cooling loads . . . . . . . . . . . 3-546 
3-95. Amount of characteristics of radjoactive waste from tiree reactor types . . . . . . . . . . . 3-551

3-96. Power system nomenclature . . . . . . . . . . . . 3-583

3-97. Estimated cryogen requirements . . . . . . . . . . 3-607

3-98. Anchor cell region cryogen requirements as provided by the FEOC cryoplant study . . . . . . . . . . . 3-609

3-99. Cryogenic requirements as determined from heat load estimates for solenoid coils S-0 and S-1 . . . . . . . 3-610

3-100. The TDF's coil power conversion and protestion requirements data . . . . . . . . . . . . . . 3-616

3-101. Magnet power supply design data . . . . . . . . . . . 3-618

3-102. Uesign data for the magnet protection system . . . . . . . 3-623

3-103. Preliminary list of special purpose remote handling equipment. . . . . . . . . . . . . . . . . . 3-633

3-104. Expected lifetimes of components in the central cell, anchor cells, and end cells................. 3-636

4-1. Sources of safety hazards in TDF design . . . . . . . . . 4-3

4-2. Safety guidelines for TOF operating conditions . . . . . . 4-4

4-3. Dose-limit guidelines for TDF . . . . . . . . . . . . 4-5

4-4. Characteristics of TUF barriers for conf inement of radioactive materials . . . . . . . . . . . . . 4-8

4-5. Design steps for reducing safety-related hazards. . . . . . 4-25

5-1. Tof capital cost summary, as of mid-1982 . . . . . . . . 5-2

5-2. TDF direct cost summary, as of mid-1982 . . . . . . . . . 5-3

5-3. TDF capital cost accounts . . . . . . . . . . . . . 5-5

5-4. Total direct cost of TUF, compatible with FEDC . . . . . . 5-9

5-5. Total cost developed by the FEDC . . . . . . . . . . . 5-9

5-6. Estimated capital cost . . . . . . . . . . . . . . 5-10

5-7. Direct cost elements, as of mic-7982 . . . . . . . . . 5-11 


\subsection{INTRODUCTION}

The scientific feasibility demonstrations expected in several currentgeneration fusion experiments reflect a maturity in the understanding of plasma physics and signal the urgency to pursue further the technology of fusion. Fusion technology has progressed remarkably to be able to produce, heat, and contain plasmas in the breakeven experiments now in hand, but the nuclear technologies needed for engineering breakeven devices are in their infancy. If an Engineering Test Reactor (ETR) designed for engineering breakeven (electrical power output equal to the required recirculating power) is to be undertaken with acceptable risk, arid if it is to be operated efficiently (to maximize the benefit/cost ratio through minimal unplanned outages for maintenance) these technologies must be developed as early as possible.

What sets off the technologies for the coming decade from those of the past is the introduction of tritium and the production of neutrons, and all that this implies for fusion systems. The impact should not be viewed solely in terms of blankets and tritium systems required for deuterium-tritium (D-T) operation, because the impact on component technologies for plasma production and on the entire configuration of the device is very significant. For these reasons one intuitively believes the experience gained in operating D-T fusion devices is much preferred to attempts to systematically solve nuclear technology problems with complementary facilities, including fission reactor tests of blankets and materials. While such auxiliary facilities have a role in the program, they cannot entirely substitute for an operating $D-T$ device whose purpose is to guide the development of nuclear engineering for fusion and to be a test and demonstration bed for these technologies.

In charting a course leading to the construction of an ETR, one must determine the risks involved and have a clear set of criteria for success not related to physics performance alone. Failure might be defined by an availability too low to recover engineering data needed for the commerical demonstration to follow, or by a premature closing of the facility for reasons of safety or inability to repair the device (at reasonable cost) after a failure. In a broad sense then, success could be defined by the efficient operation of an ETR to generate this data base. 
The Technology Dèmonstration Facility (TDF), first proposed by Fowler and Logan', is base: on a tandem mirror operating in the Kelley mode (as explained shortly). In TDF one can produce a high flux of neutrons (>l $\mathrm{MW} / \mathrm{m}^{2}$ ), operate with steady state at modest availability, and therefore reach interesting levels of fluence for blanket and material testing. We would expect that the availability, initially low (5-20\%), would reach 40-50\% after a number of years of operation. Our objective is to gain the experience necessary to reduce the risks inherent in constructing an ETR from the data base that will be avaliable in this decade. This early experience should shorten the time needed to construct and operate ETR, and hopefully allow an early start on the device.

\subsection{MISSION}

To accomplish the general objectives outlined above we have adipted the Following mission for TDF:

- Demoistrate the steady-state operation of plasma heating systems, vacuum pumping and tritium handling systems, and superconqueting magnets in a high flux neutron environment.

- Provide a steady-state neutron environment sufficient to test blankets that are designed to address power/fuel cycle issues for fusion.

- Develop and demonstrate instrumentation, control, and mairitenance methods to show the safety and viability of operating fusion power devices.

The companapts of ThF far plasma production and containment are at the MFTF-B level of difficulty. Magnets, neutral beams, and electron-cyclotron resonant heating (ECRH) systems differ little in their parameters from those in MFTF-B. Even the steady-state operation requirement is only a slight extension of the 30-s capability of MFIF-B, except for cryopanels that must be continuously purged. It is the D-T operation, with attendant shielding, trịtium handling, design constraints to accomodate maintenance, and required reliability that will extend the engineering data base on the confinement and production technologies. 
Our major feature in this device however is the high flux, modest fluence capability of the machine, which will enable significant testing of power and fuel cycle components and systems. 8 lankets can be operated at a first-wall flux $>1 \mathrm{MW} / \mathrm{m}^{2}$ for periods of time sufficient to discover failure modes that might occur well beyond any initial (first-operation type) tailure but short of its expected operating life.

To be more specific on the required run time and fluence, we can classify the power/fuel system tests according to characteristic times. For example, neutronics tests require only seconds of exposure at a flux well below $1 \mathrm{MW} / \mathrm{m}^{2}$ to gather important data. For our purposes we defirie three characteristic times for blanket and other power/fuel cucle components: (1) the time to reach thermal equilibrium in the blanket and heat exchanger system, (2) the time to reach an equilibrium tritium distribution in the blanket and recovery system (and other parts of the fusion device), and (3) the time required to discover early failure modes in the system. These failure effects, such as corrosion in lead-lithium blankets and the subsequent transport of activated corrosion products, radiation induced changes to material properties (density, porosity, thermal conductivity, resistivity), and compatibility of materials, are those that could lead to premature fajlure of components and/or systems. Radiation-induced sintering of ceramic tritium breeding materials, for example, is of particular concern, since it could drastically change the tritium holdup characteristics and lead to an excessive inventory of tritium.

One can roughly estimate these characteristic times from available basic materials data or wesigns of blankets, as is done in Table $1-1$. Liquid metal blankets reach thermal equilibrium in $20.1 \mathrm{hr}$, while solid-breeder blankets require some what longer $(<\mathrm{hr})$. Tritium extraction at equilibrium requires only 10-20 minutes for lead-iithium blankets because tritium solubility is extremely low there. For blankets using a pure lithium breeder/coolant this time is tens of hours. Blankets with ceranic breeding materials could require up to a few hundred hours for recovery at equilibrium.

Finaliy, the premature fajlure effects might require from $10^{3}$ to $10^{4}$ hours of testing to discover problems of this type. In TOF, we would expect to be able to do this, and it is this capability which makes short tandem mirrors like TDF or TASKA ${ }^{2}$, which is a design study carried out at 
Table 1-1. Characteristic times for fuel/power cycle tests ${ }^{a}$ on blankets and auxiliary equipmer:*

\begin{tabular}{llll}
\hline Purpose & Uninterrupted test & Total test & Issues being \\
of test & time and subject & time needed & addressed
\end{tabular}

$\begin{array}{lll}\text { Thernal/ } & 21 / 10 \mathrm{hr} \text { (1iquid } & \text { Tens of } \\ \text { hyoraulic metal b]ankets) } & \text { well-instrumented } \\ \text { behavior } & 21 / 2 \mathrm{hr} \text { (ceramic } & \text { tests } \\ & \text { breeding blankets) } & \end{array}$

Design confirmation--or:

heat transfer/removal, structural effects, MHD pressure drops, offnormal (LOCF, LOCA) respanse.

\begin{tabular}{|c|c|c|c|}
\hline $\begin{array}{l}\text { Tritium } \\
\text { Breeding/ } \\
\text { Recovery }\end{array}$ & $\begin{array}{l}21 / 10 \mathrm{hr} \text { (lead lithium) } \\
210 \mathrm{hr} \text { (pure 1ithium) } \\
2100 \mathrm{hr} \text { (ceramic breeders) }\end{array}$ & $\begin{array}{l}\text { Tens of } \\
\text { wel1-instrumented } \\
\text { tests }\end{array}$ & $\begin{array}{l}\text { Design confirmation--or: } \\
\text { production/recovery rates, } \\
\text { tritium accountability, } \\
\text { permeation rates. }\end{array}$ \\
\hline $\begin{array}{l}\text { Early } \\
\text { failure } \\
\text { modes }\end{array}$ & $1=100 \mathrm{hr}$ & $1,000-30,000 \mathrm{hr}$ & $\begin{array}{l}\text { Corrosion, sintering, } \\
\text { mass transport, materials } \\
\text { compatibility, weld } \\
\text { performance, safety systems, } \\
\text { any problems that lead to } \\
\text { early system failures. }\end{array}$ \\
\hline $\begin{array}{l}\text { End of } \\
\text { life }\end{array}$ & $2100 \mathrm{hr}$ & $10,000-100,000 \mathrm{hr}$ & $\begin{array}{l}\text { Lifetime denonstration--or: } \\
\text { radiation damage to } \\
\text { structures, fatigue, } \\
\text { embrittlement, swelling, } \\
\text { all issues in the design } \\
\text { which } 1 \mathrm{imit} \text { the design-life } \\
\text { to } 210 \mathrm{~m} / \mathrm{yr}^{\prime} / \mathrm{m}^{2} \text {. }\end{array}$ \\
\hline
\end{tabular}

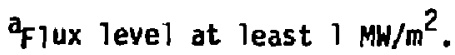


Karlsruhe, FRG, with the assistance of the University of Wisconsin, unique among facilities that cost approximately one billion dollars. (1982 dollars, with contingency and all indirect costs).

other characteristic test times could be defined. To qualify a blanket design for a next-generation device, rather than simply confirm a design, could require a few years' testing in IDF. End of life testing for radiation damage to structural materials requires an integrated fluence of 5-10 $\mathrm{MW} \cdot \mathrm{yr} / \mathrm{m}^{2}$ and is not possible in TDF.

A final part of the mission concerns the safe operation and maintenance of the device and the role of this facility in generating a data base that would be required to site and construct an ETR. One would expect to develop the necessary instrumentation and control for safe operation; establish operations and maintenance procedures based on experience in running the facility; and demonstrate the adequacy of systems for emergency events such as loss of flow or coalant, tritium release to secondary containment, and other such off-normal events. Experience gained here would be invaluable in establishing the safety of fusion power plants.

\subsection{PHYSICAL DESCRIPTION}

By limiting the central cell to the shortest length consistent with a minimal test area in the center of the machine, TDF can be comparable in size to present generation tandem mirrors. The magnet set is about as long as the Tandem Mirror Experiment-Upgrade (TMX-U) set, while the overall lengtii is a little less than the length of the Mirror Fusion Test Facility (MFTF-B). High gas throughout for stream stabilization of the plasma results in a requirement for a large cyropanel surface area (similar to MFTF-B) at each end of the machine. TDF is, consequently, much longer than TMX-U even though the magnet lengths are about the same. Figure 1-1 shows the general layout of the magnets, vessel, beams, fueling and vacuum systems, and shielded central cell.

Testing space for blankets is in the 8-m-long central cell, with provision for two modules that are $1 \mathrm{~m}$ wide. An uncollided neutron flux of $1.4 \mathrm{MW} / \mathrm{m}^{2}$ at a $25-\mathrm{cm}$ radius is generated by the fusion reactions in the 10-cm radius plasma. A total of $8 \mathrm{~m}^{2}$ of test surface at this flux is available, about half of it readily accessible between solenoids. This central portion of the machine is shown in $\mathrm{F}: \mathrm{g}, 1-2$. 


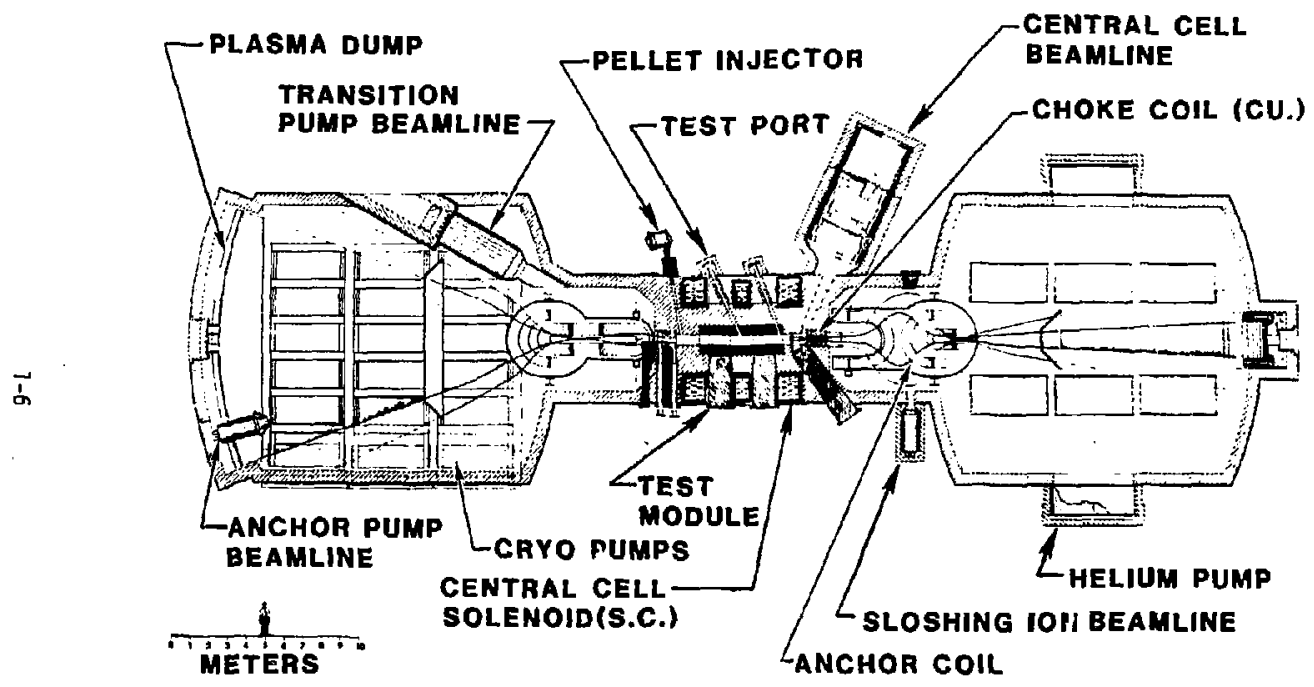

Figure 1-1. Cross section of TDF machine. Some components are shown rotated out of plane for clarity, ana only one of the eight central cell beamlines is shown. (See Figs, $1-5$ and 1-6 for the complete configuration). 


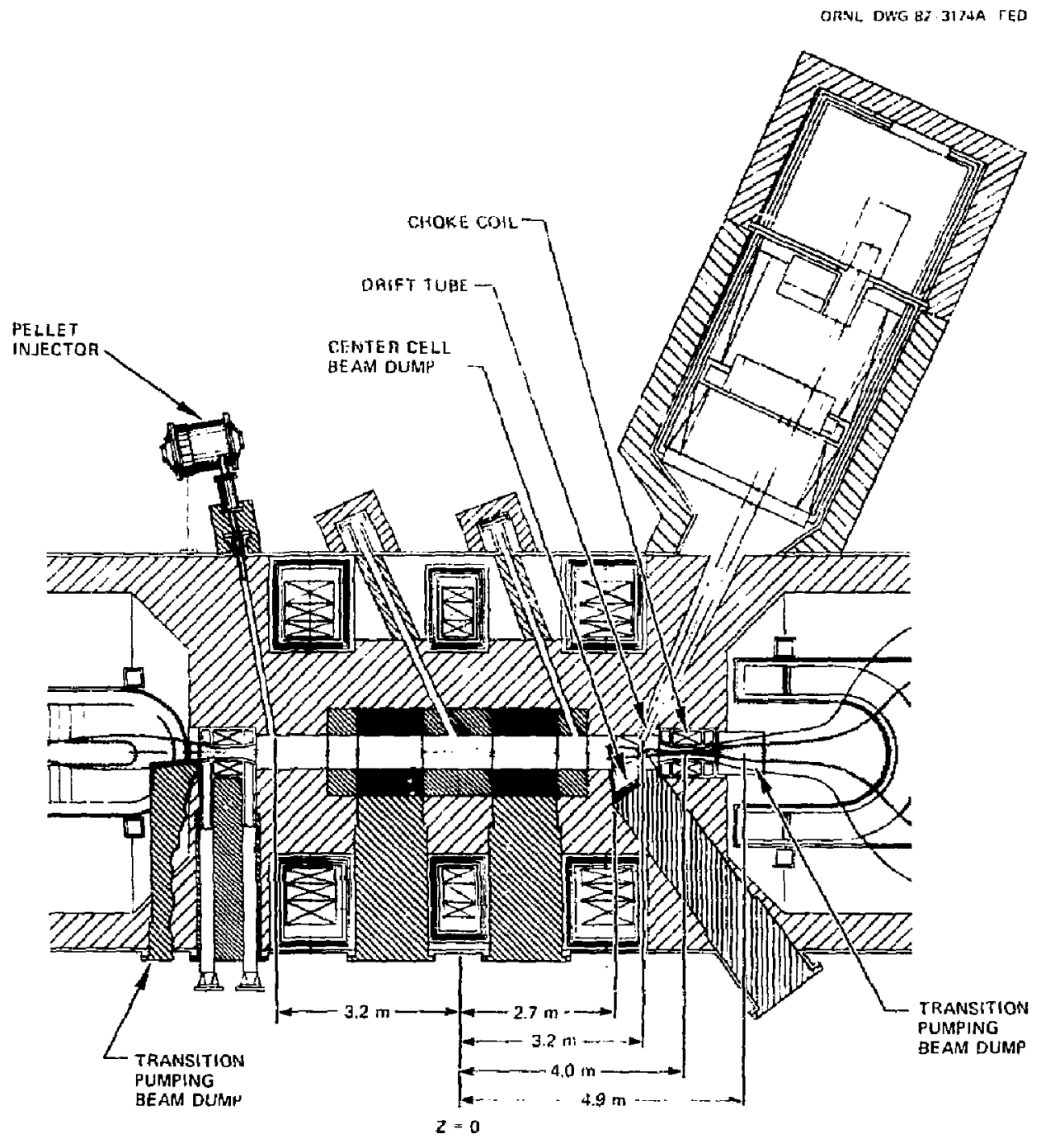

Figure: 1-2. Central portion of TDF, showing the two blanket module test lacations, scheme for removing choke coils, beamijne penetration through loing, and beam aump access for repair. Also shown is the access to the $h$.. flux test area under the superconducting coils, which allows for inserting material samples for raiaation aamage stuoies. 
Maintaining the high temperature and density of the central cell plasma requires the absuption of $52 \mathrm{MW}$ of $80-\mathrm{kV}$ beam power. Shielding of these beamlines and rentrdl cell to permit contact maintenance, access for maint ellunce of short life components, and accessibility and rapid change for test modules wert: important requirements to be met in designing the machine.

The end cells are $16 \mathrm{~m}$ in diameter and nearly $20 \mathrm{~m}$ long. These end cells are well isolated gemetrically from the neutron "line source," which is the 8-nillong plasina column ill the central cell. Only modest shadow shielding of the transition and anchor (superconducting) coils is required, because the copper choke coils at the ends of the central solenoid provide considerable axial shielding.

\subsection{PHYSICS DESCRIPTION}

Fusion reactions in TDF occur in the $4.5 \mathrm{~T}$ solenoid over the $8-\mathrm{m}-10 \mathrm{ng}$ region between the 15-T copper choke coils. This beam driven hot plasma ( $37 \mathrm{keV}$ ion energy) is magnetically confined with a lifetime of $211 \mathrm{~ms}$ (see Table 1-2 for these and other key physical paramenters). At a density of $6 \times 10^{14}$ the fusion power density of neutrons is $270 \mathrm{~W} / \mathrm{cm}^{3}$ averaged over the cross section. Microstability of this population is provided by a warm backgraund plasma ( $5 \mathrm{keV}$ ) with a density of $5 \times 10^{13}$ and a lifetime less than $1 \mathrm{~ms}$.

The warm plasma is electrostatically confined by a potential in the anchor which rises $4 \mathrm{keV}$ above the 13-kV central cell potential. These poorly confined ions produce a large flow of streaming plasma through each anchor and guarantee their microstability according to experimental results taken from the $2 X I I B$ and TMX mirror experiments. 2,3

Density, field, and potential profiles in TDF are given in Fig. 1-3. Notice that there is a substantial density drop between the choke coil and the anchor, and that trapped particle modes set a limit to this density drop. Further, the potential profile must be such that passing ions penetrate further into the anchor than do the electrons before being turned back (electrostatically) to the central cell. This axial difference in orbits leads to an orbit-averaged correction to the simple MHO trapped particle theory (somewhat analogous axially to the more familiar Larmor-orbit 
Table 1-2. TDF physics parameters.

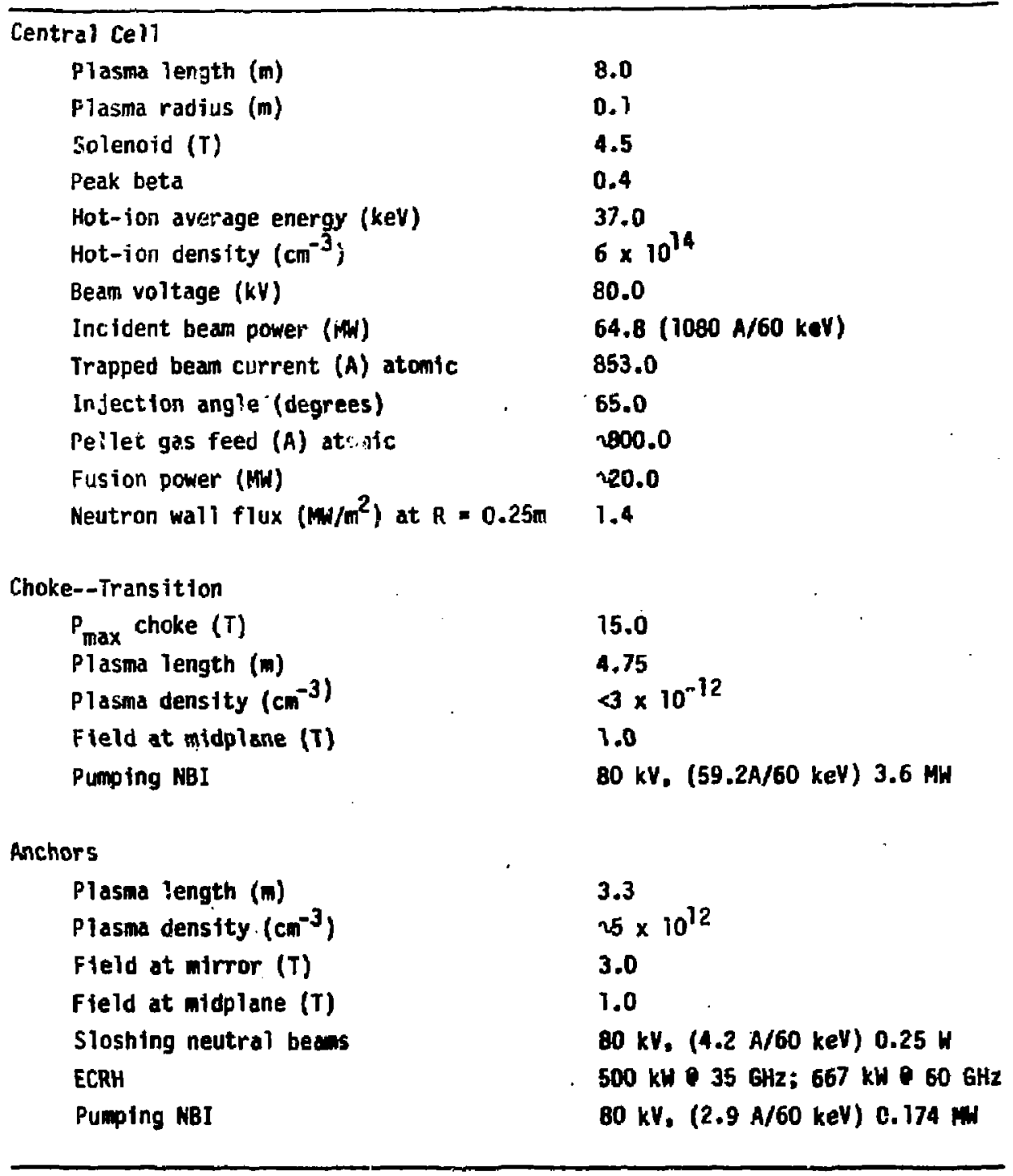




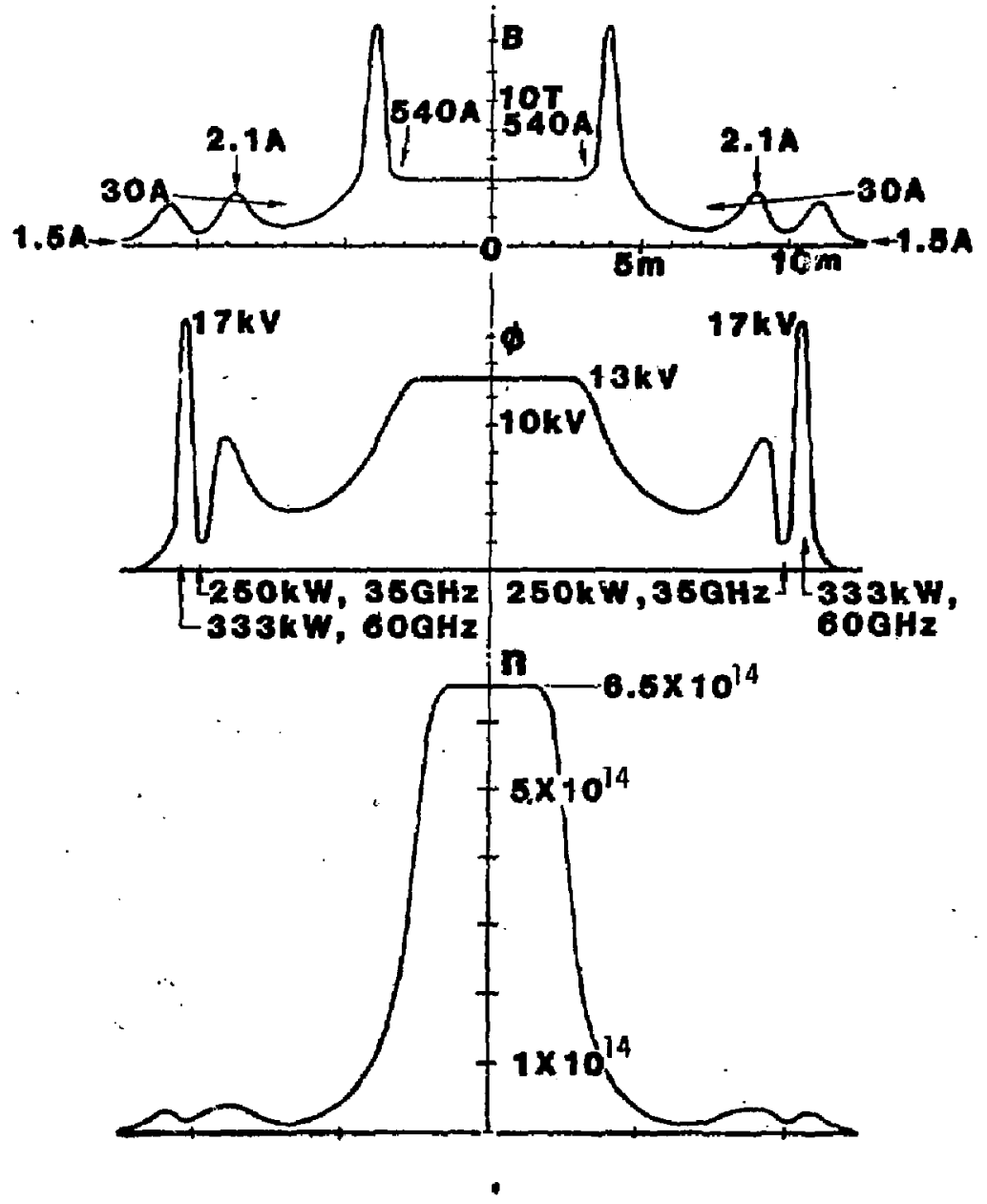

Figure 1-3. Proflies of magnetic field, potential, and density along the machine axis. The central cell population is nearly all magnetically trapped, and thase fons that escape are confined by the $40 \mathrm{kV}$ confining potential. 
corrections to MHo theory). This correction converts an otherwise unstable mode to a stable (negative energy) drift wave and provides stability criteria involving densi's ratios an sertain anchor and transition lengths.

To provide the proper axial potentials that result in the desired orbits, we rely on the thermal barrier construct of $[M X-U$. The potential dip of the barrier reflects the passing electrons, while the tilted potential initiateo by sloshing ions (and emphasized by ECRH) allows pascing ions to turn beyond the barrier. Trapped ion modes should thus be stabilized. Again, we do not rely on the microstability of the thermal barrier, as we do in TMX-U ano MFTF-B, rather we inject $800 \mathrm{~A}$ of gas in addition to the $850 \mathrm{~A}$ of beam (trapped in the central cell) to provide the stream stabilized flow necessary for stability. Recent data ${ }^{4}$ on TMX-U show that such a riow is not necessary when the ion sloshirg distribution is set up, Consequently, we now know that TDF is designed ton conservatively, that the gas flow could be reduced, the electron temperature increased above $2 \mathrm{keV}$, and the drag time on eiectrons increased. The higher $Q$ that would obtain means that less beam current would be required.

To maintain the thermal barrier we require ECRH at 35 and $60 \mathrm{GHz}$, totaling about 1.2 MW. These systems would be very much like the present MFTF-B systems. Charge exchange pumping with neutral beams is required in both the anchor thermal barrier and in the transition to maintain the potential at values below the central cell potential. The pump beam current (30 A each end) is somewhat less than that arailable in TMX-U.

In summary, TDF is based on rather conservative physics. The millisecond containment times needed for Kelley mode operation ${ }^{3}$ are very short, and most of the findamentals have been demonstra:ed experimentally. (Kelley mode refers to the two-conponent ito distribution in the centra\} ceit, with the majority species being the iot, magnetically trapped ions. This differs from the "two-component" or "wetwood burner" operation proposed in various Tokamaks, which have the hot component as the minority species. The mode is named after Or. G. Keliey, formerly at ORNL, for his work on different modes of operation in mirror devices.)

From $T M K-U$ we should verify that thermal barriers can be constructed, and from TMK-S ( $S$ for symmetric, denoting planned modifications to add a throttle coil) and TARA (an axicell tandem at MIT), we should discover whatever 
constrajnts the trapped particle modes will impose. These latter two issues should be resulved very soon in the program and are the only physics areas where experinental verification is not in hand.

\subsection{ENGINEERING DESCRIPTION}

The engineering philosophy used in the TOF study was tu provide a high reliability device for engineering demonstration and testing. We chose to use technology that was either in-hand or scheduled to be demonstrated in programs presently in place. This approach, coupled with the conservative physics assumptions that were used, should produce a machine with a high probability of fulfilling its mission in a timely manner.

The goal of the design effort was to describe each of the required subsystems in sufficient detail to allow one to do credible cost estimating and establish a configuration that meets interface requirements and points out critical technology issues.

The results of the 10 man-year design study ${ }^{4}$ can be sumarized by saying that the TDF produces $20 \mathrm{MH}$ of fusion power, developing an uncollided neutron flux of $1.4 \mathrm{~mW} \cdot \mathrm{m}^{-2}$ on an area of $28 \mathrm{~m}^{2}$, while consuming about $0.1 \mathrm{gm}$ of tritium per hour and 250 W of line power, for a cost of about $\$ 1000$ million. A summary of the major engineering parameters are given in Table 1-3. Major participants in the study were LLNL, TRQ, GD Convair, and the FEDC at Dak Ridge. Fdditional participation from Argonne, MIT, LASL, UCLA, SAI, Bechtel, and the University of Wisconsin was invaluable.

To describe TDF we begin with the central-cell region where virtually all of the neutrons are produced. The axial magnetic field of $4.5 \mathrm{~T}$ is produced by three Nb-Ti superconducting magnets spaced to allow the insertion of test modules up to $1 \mathrm{~m}$ in axial dimension. The ends of the central cell provide access for the central-cell beams that drive the fusion reactions. The main design considerations in this area are shielding for the solenoid magnets in the area adjacent to the beam ducts and the desire to keep the 15-T resistive choke coil close to the solenoids for field reinforcement. A detailed trade study between shielding the beam and magnet requirements in this area largely set the parameters for the rest of the design. The choke coil presents a novel design problem for fusion reactors because it must withstand a high 
Table 1-3. TDF engineering parameters.

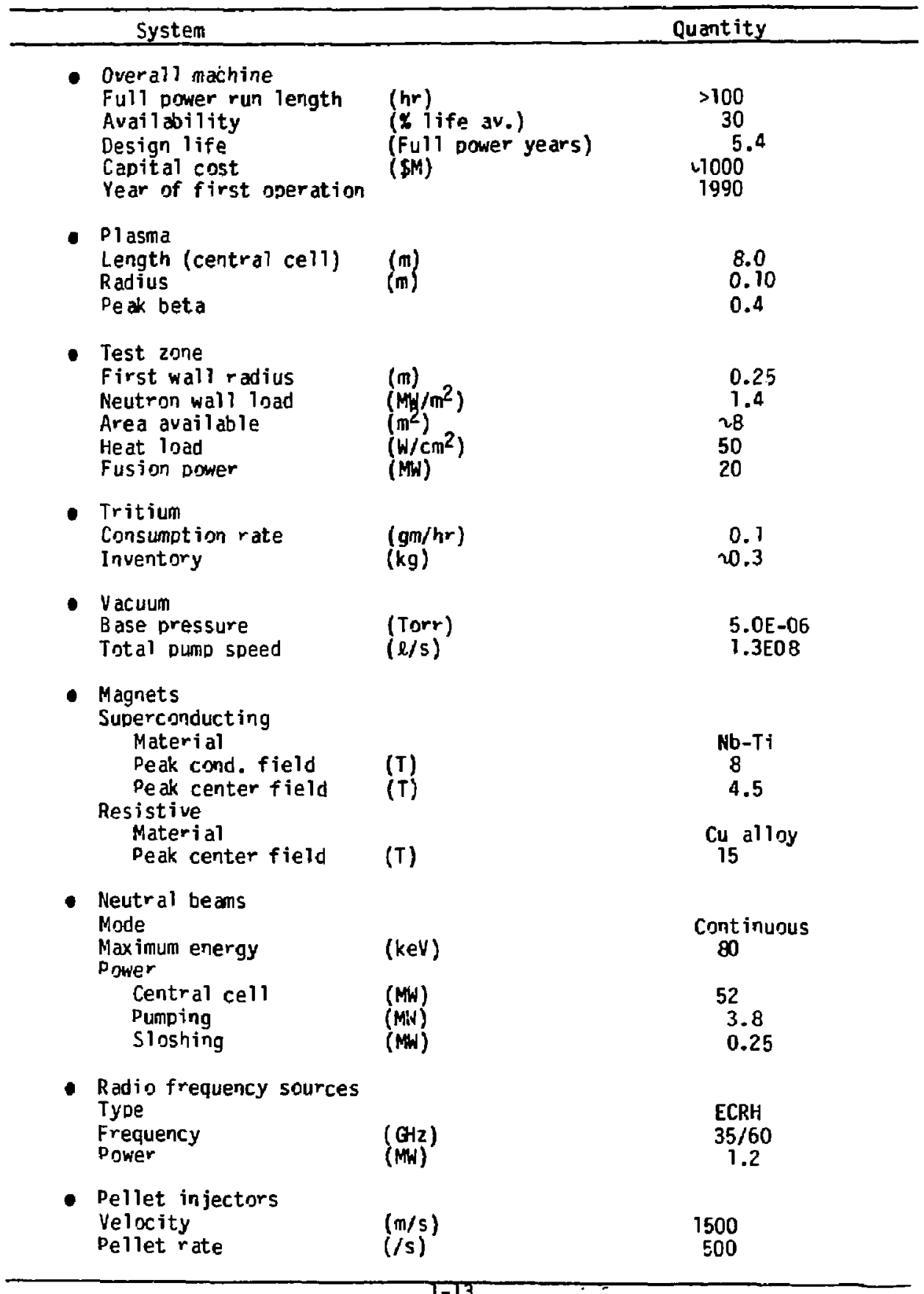


radiation load and has high internal heat loads and magnetic stresses. The preferred design uses internally water-cooled copper alloy conductors chosen for high strength and acceptable resistivity. The useful lifetimes of these coils is limited not by physical danage but by the radiation-induced increase in resistivity that eventually causes heat generation in excess of the cooling capacity of the conductor.

The principal testing area is the wall of the central cell in the area between the central cell beam ducts (Figs. 1-1 and 1-2). There is direct access to two full-diameter test zones between the solenoic magnets, each capable of accomadating a 1-m-1ong test module. Figure 1-4 shows how TDF test modules would be inserted in this test area. Access to the areas inside the coil boundaries are also conveniently avajlable to smaller test specimens, either as long-term resident samples for fluence testing or short-term samples inserted in "rabbit tubes." The major test capability offered by TDF is the ability to test full-size prototype blanket modules to qualify them for use in experimental power reactors. With steady-state run lengths of $>100$ hours, experimental components can be brought to thermal and tritium breeding equilibrium, and energy extraction may be demonstrated.

To generate the high-wall fiux in TDF, despite having a modest plasma $Q$, we must provide 52 MW of neutral beans in the central cell. Figures 1-5 and 1-6 show how four beamlines are arranged at each end of the machine, with four sources in each beamline. The beams are designed to operate in a relatively high radiation environment for several hundreds of hours. The beamlines are located in an area where the background magnetic fields are as high as $2000 \mathrm{G}$. Superconducting magnet ic shielding is employed to get the local fields down to the few gauss level needed for neutral beam source operations.

Each $80-k V$ source has a $70 \mathrm{~A}$ extraction current, of which $37 \mathrm{~A}$ is incident on the plasma. Only 24 sources are needed to provide the incident $1080 \mathrm{~A}(65 \mathrm{MW})$ necessary to trap $52 \mathrm{MH}$ of power. For redundancy and reliability an extra source is provided in each beamline. Half of the beamlines use deuterium gas while the other half use tritium.

Because the TDF plasma is small in diameter, about $15 \%$ of the incident beam power passes through to beam dumps. These dumps have been designed for a peak power density of $1000 \mathrm{w} / \mathrm{cm}^{2}$. The dump, like the choke coil, is not a lifetime element and both are built into shielded modules designed for convenient removal and replacement. 


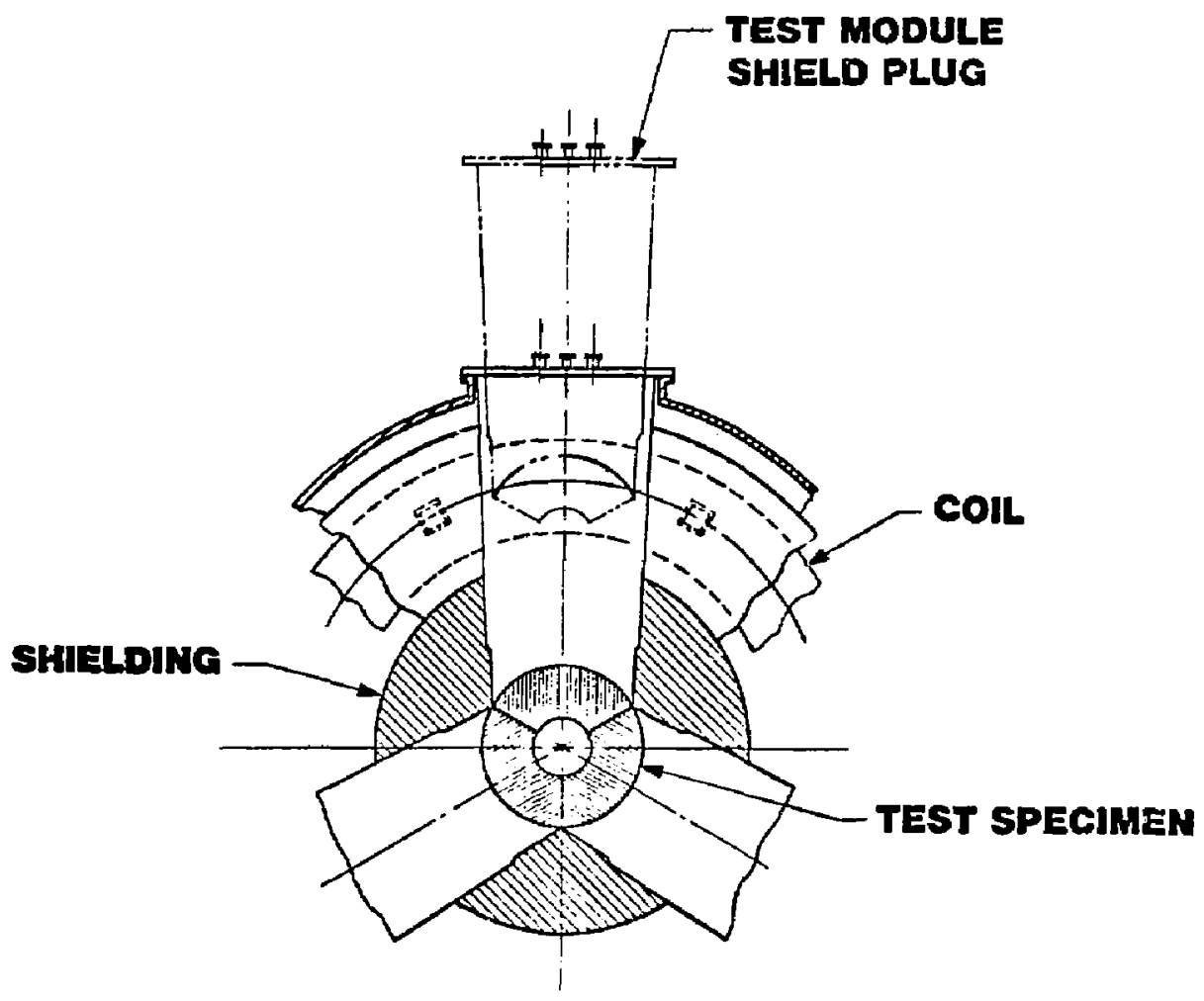

Figure 1-4. Details of the blanket test ared showing how moaules could be redily removed. 
<smiles></smiles> 


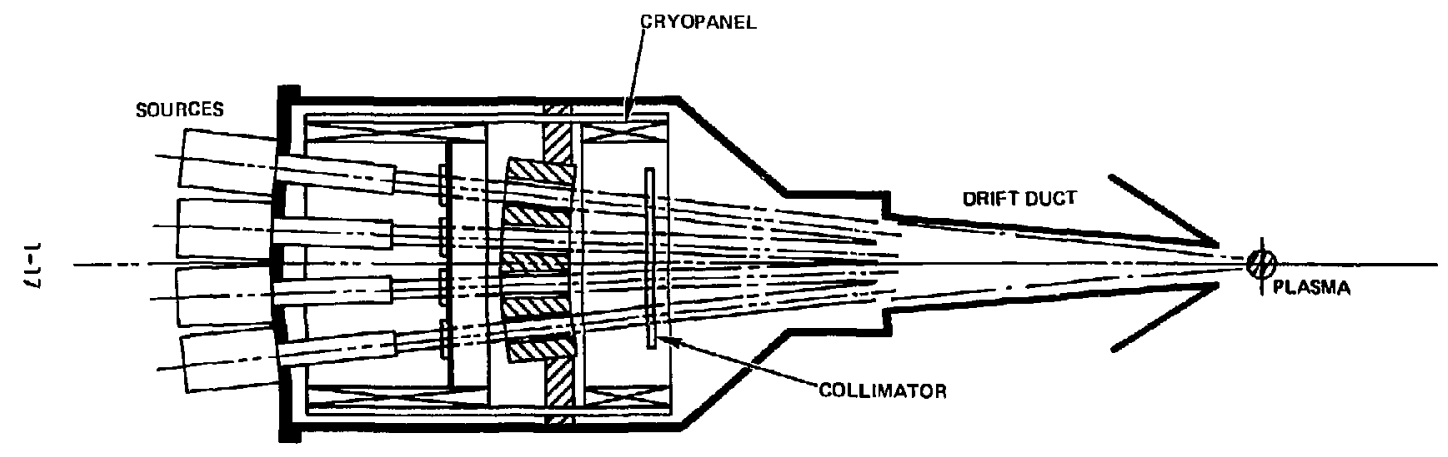

Figure 1-6. An indiviaual beamline with four sources, one of them a spare to improve reliability. The duct design and source optics are chosen to minimize neutron streaming without incurring beam blockage by high gas pressure in the duct. 
Beyond the central cell are the transition and anchor regions, which stabilize the central-cell plasma. The fusion power in this region is small, and only minimal shielding is required. The magnets are $\mathrm{C}$-shaped with a $\mathrm{Nb}-\mathrm{Ti}$ conductor and are housed in an independent vacuum vessel. Three neutral beam systems deliver particles to this area: two are pump beams that enter at shallow angles; the other provides sloshing ions normal to the axis. The relatively complex magnet geometry couplei with rigorous beam geometrical requirements control the design in this area.

The end cell is the outboard part of the system, and its principal functions are to house the primary vacuum pumping and provide a dump for the plasma-leakage power. Vacuum pumping is provided by cycling cryopumps located above and below the plasma leakage fan. Pumps of the type called for here have been developed and tested by Batzer. 5 There is redundant pumping capacity so that some of the pumps are always off-line being degassed. The internal tritium inventory can be controlled by the amount of excess capacity specified. The plasma dump is lecated at the extreme end of the machine, and its power-density limit largely sets the size of the end vessel. In this case, we limit the plasma power density at the dump to $250 \mathrm{w} / \mathrm{cm}^{2}$. This dump, like all those in the TDF, is water-cooled. The plasma startup guns are housed behind movable shielding at the end wall, and they inject a low-energy plasma along the field lines during startup.

The TDF is designed for maximum contact maintenance. Specialized tooling is provided for scheduled maintenance tasks, and general-purpose tooling is used for random failure repair. Therefore, contact operations can be performed in the vault during regular work schedules after a short cooldown with the magnets de-energized and the tritium removed. In this way, all the major connections $c$ an be made and broken in a contact mode, but components are removed with remote tooling because of the high flux of gamma rays coming from unshielded machine ports. Because the components are designed for high reliability and most maintenance tasks can be performed in this efficient contact mode, we are confident that the TOF will be a high-availability device with a specified $50 \%$ in the last half of its $15-\mathrm{yr}$ life. 


\subsection{CONCLUSIONS}

Early experience on nuclear technology can be gained in TDF. Its main feature is the ability to test blanket modules at a high wall flux for more than 100 hours per run. By using conservative physics and technology, the machine can be quickly built and made operational by the end of this decade. With the expected availability, the total first wall fluence in the machine will approach $5 \mathrm{mw} / \mathrm{yr} / \mathrm{m}^{2}$, so that a number of blanket modules could be tested for 1,000-10,000 hours to determine if there are any early-1ife railure modes. With the engineering data base obtained in TOF, one could proceed confidentiy with an ETR during the 1990s. 
1. T. K. Fowler and B. G. Logan, Tandem Mirror Technology Demonstration Facility, Lawrence Livermore National Laboratory, Livermore, CA, UCID-19193 (September 1981).

2. D. L. Corre11, et al., "Production of Large-Radius, High-Beta Conf ined Mirror Plasmas," Nucl. Fusion 20, 655 (1980).

3. R. P. Orake, et al., "The Effect of End-Cell Stability on the Confinement of the Center Cell Plasma in TMX," Nucl. Fusion 21, 359 (1981).

4. T. C. Simonen, et al, TMX-Upgrade (TMX-U) Operation in the Sloshing-Ion Mode, Lawrence Livermore National Laboratory, Livermore, CA, UCID-19568 (September 1982).

5. T. H. Batzer and W. R. Cal1, A Continuous Cryopump for Steady-State Mirror Fusion Reactors, Lawrence Livermore National Laboratory, Livermore, CA, UCRL-87732 (September 1982). (Also given at the 29th National Vacuum Symposium, Baltimore, MD, November 16-19, 1982). 


\subsection{INTROUUCTION}

The physics base for TOF is described by Fowler and Logan.' T.ie basic premise is that a tandem mirror machine can be designed to yield a fusion reactor relevant neutron wall loading of $r_{n}>1 \mathrm{MW} / \mathrm{m}^{2}$, using generally conservative or established physics. The design is compatible with a relatively law cost, particularly if fusion $Q>1$ is not also required. The D jasma parameters that are needed in the central cell (of the tandem contiguration) to yield the desired high D-T neutron wall loading are consistent with performance calculated for a neutral-beam driven, stream-stabilizec tandem mirror design operated in a two-component "Kelley"-like mode with modest electron thermal conduction barriers and electrostatic ion plugs in the end minimum- $B$ anchor cells. The physics requirements for stream-stabilized minimum- $B$ or anchor operation have been established in experiments on $2{ }^{2} \perp \mathrm{IB}^{2}$ and TMX. ${ }^{3}$ The physics of tandem electrostatic ion plugging was established on experiments on TMX, ${ }^{3}$ TMX-U, $^{4}$ Phaedrus ${ }^{5}$, and Garma- $6^{6}$. The general physics of various combinations of electron-cyclotron resonant heating (ECRH), ion-cyclotron resonant heating (ICRH), ano sloshing ion population buildup are currently under study on TMK-U, ${ }^{4}$ Phaedrus, ${ }^{5}$ Gamma-6, ${ }^{6}$ and STM- $1 .{ }^{7}$ The new physics requirements for neutral beam ion pumping and selective use of ECRH for barrier formation are to be tested in TMX-U $U^{8}$ and MFTF-B. ${ }^{3}$ Further supportive studies are expected from the TARA, ${ }^{10}$ Gamma-10, ${ }^{11}$ and AMBAL-1 ${ }^{12}$ experiments.

Placing the major emphasis for TDF on attaining an uncollided $14 \mathrm{MeV}$ neutron wall loaging $\Gamma_{n}>1 \mathrm{MW} / \mathrm{m}^{2}$ and not simultaneously requiring a fusion $Q>1$ (where $Q \equiv$ fusion power/input power) eases the physics reguirements for TDF. This allows lis to ease the high voltage technology requirements for the neutral beams and create a relatively short minimum-cost aesign. Prior work on advanced mirror machine designs for a high-wall loauing application include the 1974 FERF $^{13}$ single yin-yang cell study and the 1982 TASKA tanaem mirror study. ${ }^{14}$ The TASKA study, done by the joint Kernforschungszentrum Institute for Technical Physics (KFK) in Karlsrihe, Germany and the University of Wisconsin-Madison groups, consiaerea a tandem mirror design with an ICRH, U-T neutral-beam fuelea high mirror field central cell, use of neutral beam pumping to yieló an electron thermal barrier in the transition sections, ana use of ECRH and neutral beam injection in the 
yin-yang anchor for magnetohyorodynamic (MHD) stabjijty and electrostatic ion plugging. The superconducting magnet set for TASKA included an axisymmetric hybrid choke mirror coil with a normal copper insert producing a $20 \mathrm{~T}$ field. Our TOF design uses a magnet configuration similar to the TASKA design, but with $0-T$ neutral beam injection as the main heating source, and pellet injection for fueling in the central cell. Pellet fueling is required for warm plasma stabilization of the central cell and a! lows conservative siream-stabilization of the anchors. The design also includes neutral beam pumping and the use of ECRH in each anchor, to yielo an asymetric electron thermal barrier ano ion plug feature. This aesign refinement is required to ensure stability against possible trapped ion mode instabilities. 15 The steaay state operating feature of the tandem mirror aesign is very important to the Lility of high wall loadings for blanket module engineering tests, since this aljows the higher integrated wall damage exposures relevant to reactor materials testing, as well as tritium breeding and a test of thermal hydraulics under equilibrium conditions.

\subsection{TDF EQQUILIBRIUM PARAMETERS}

\subsubsection{Orerview}

The primary goal for TDF is to build up and maintain the plasma conditions that are needed to yield the desired D-T neutron wall loading $\Gamma_{n}$ in the central cell with the minimum technology requirements on the supportive enc cells and at the lowest overall cost.

To establish the general machine parameters, we first consigered the line source neutron yield and first wall radius requirements needed to yielo the desireo $\Gamma_{n}$ (jiscussed in Section 2.6.1). These requirements set lower limits on the needed high central cell magnetic field, high plasma density, and reacting ion temperature; this in turn constrains the plasma parameters needed for the supportive end cells for a given magnetic configuration. For a practical selected machine input configuration we then calculated the detailed equilibrium plasma requirements and the associated machine requirements with an aciaptation of the MARS reactor code (discussed in Section 2.2.2). We then usea the initial machine magnetic profile and the calculatea first 
approximation $\beta$ ( $\left.\beta \equiv 8 \pi \Sigma n k T / B^{2}\right)$ to calculate the $\beta$-weighted integrals of normal and geodesic curvature. Finally, we iterated the machine magnetic profile unti? it was line average MAC-stable and hao an acceptabie minimum level of field curvature distortion to arive radial diffusion.

Tables 2-1 and 2-2 list the base case calculated machine requirements and the resultant point-equilibrium plasma characteristics for stream-stabiized operation of the final, iterated magnetic field configuration.

The code input parameters we used included:

Machine Plasma and Magnetic Geometry

- A minimum practical hot plasma radius $r_{p}-10 \mathrm{~cm}$ in the central cell. This design choice was based largely on the plasma target size needed to efficiently trap the $\sim 0.4 \mathrm{deg}$ (half-angle) $1 / \mathrm{e}$ divergence expected for $80-k V$ neutral beams (e.g., for a neutral beam at a oistance of $\sim 10 \mathrm{~m}$ the $1 /$ e fall-off in neutral-beam footprint current density wiath uccurs at a plasma radius of $\sim 7$ $\mathrm{cm})$. We selected a relatively minimum $r_{p}=10 \mathrm{~cm}$ for the base case as a compromise of efficiency and power, since the requireo neutral beam current scales proportional to $r_{p}^{2}$, ana $\Gamma_{n}$ scales proportional to $r_{p}$ (approximately).

- A maximum magnetic field in the choke coil = $15 \mathrm{~T}$. This was a practical choice baseo upon use of a normal copper coil with an $8.75-\mathrm{cm}$ throat radius. This radius is needed to clear the magnetically mapped hot plasma column, including a halo of one 3.5-MeV alpha particle gyrodiameter (at B - $15 \mathrm{~T}$ ).

- A central cell field of $\approx 4.5 \mathrm{~T}$. This is the maximum practical solenoid field preserving a high mirror ratio $\left(R_{\text {vac }}-3.33\right)$ with the 15-T choke coil, which is necessary for gooo confinement of the central cell hot ions.

- A minimum central cell wall radius $r_{w} \sim 25 \mathrm{~cm}$ to clear both the hot plasma and a predicted halo plasma, scaling proportional to $\left(r_{p}+2 r_{\alpha}\right)$, where $r_{\alpha} \equiv$ alpha gyroratio.

- A minimum central cell length of $-8 \mathrm{~m}$ to allow an adequate area for full-scale materials and blanket testing.

- Transition and anchor magnetic fields, chosen as neeoed for several purposes: (1) to clear the magnetic mapping of the hot plasma and halo, (2) to provide reasonable ECRH parameters, (3) to create 
Table 2-1. Tandem mirror parameters for the central cell based on the stream-stabilizea base case.

\begin{tabular}{|c|c|}
\hline Parameter & Value \\
\hline Plasma length (m) & 8 \\
\hline Plasma radius, $r_{p}(m)$ & 0.1 \\
\hline Solenoid field, $B_{c}(T)$ & 4.5 \\
\hline Peak beta, $\hat{\beta}_{c}$ & 0.4 \\
\hline E lectron temperature, $\mathrm{T}_{\mathrm{ec}}(\mathrm{keV})$ & 2.1 \\
\hline $\begin{array}{l}\text { Hot ion average energy, } \bar{W}_{i h}(\mathrm{keV}) \\
\text { Ratio of plasma radius to mean hot ion }\end{array}$ & 37 \\
\hline gyroracius, $r_{p} / a_{i}$ & 10 \\
\hline Hot ion lifetime, $\tau$ (ms) & 11.5 \\
\hline Hot ion density, $n_{i h}\left(\mathrm{~cm}^{-3}\right)$ & $6 \times 10^{14}$ \\
\hline harm ion temperature, $T_{i w_{-}}(\mathrm{keV})$ & 5.3 \\
\hline Warm ion density, $n_{i w}\left(\mathrm{~cm}^{-3}\right)$ & $5 \times 10^{13}$ \\
\hline Beam voltage $(\mathrm{kv})$ & 80 \\
\hline Incjdent beam power (MW) & $64.8(1080 \mathrm{~A} / 60 \mathrm{keV})^{\mathrm{a}}$ \\
\hline Trapped beam atom current $(A)$ & 853 \\
\hline Injection angle (aeg) & 65 \\
\hline Beam particle trapping fraction, $f_{t}$ & 0.79 \\
\hline Pellets gas feed $(A)$ & 800 \\
\hline Fusion power (MW) & 20 \\
\hline Ratio of fusion power yield to input power, $Q$ & 0.39 \\
\hline Neutron wali flux at $r_{w}=0.25 \mathrm{~m}, \Gamma_{n}\left(\mathrm{MH} / \mathrm{m}^{2}\right)$ & 1.4 \\
\hline Central cel 1 potential, $\phi_{e}(k V)$ & 13 \\
\hline
\end{tabular}

a The ion source beam output is taken as a $80 \%: 15 \%: 5 \%$ species mix for either $\mathrm{D}^{+}: \mathrm{O}_{2}^{+}: \mathrm{O}_{3}^{+}$, or $\mathrm{T}^{+}: \mathrm{T}_{2}^{+}: \mathrm{T}_{3}^{+}$, yielding a $55 \%: 29 \%: 16 \% \mathrm{D}^{+}$(or $\mathrm{T}^{+}$) energy mix at 80:40:27 keV for the neutral beam composition trappea in the central cell. 
Table 2-2. Tanoem mirror parameters for the TDF eno cells based on the stream-stabilized base case.

\begin{tabular}{|c|c|}
\hline Parameter & Value \\
\hline \multicolumn{2}{|l|}{ Choke and transition coils } \\
\hline Choke maximum field strength $(T)$ & 15 \\
\hline Plasma average length $(m)$ & 4.75 \\
\hline Plasma radius $(\mathrm{m})$ & 0.20 \\
\hline Plasma density $\left(\mathrm{cm}^{-3}\right)$ & $<3 \times 10^{12}$ \\
\hline Magnetic field at midplane (T) & 1 \\
\hline Peak beta, $B_{t}$ & .01 \\
\hline Total pumping $N B 1^{*}, * \star$ & $80 \mathrm{kV},(59.2 \mathrm{~A} / 60 \mathrm{keV}) 3.6 \mathrm{MW}$ \\
\hline Injection angle (deg)/ magnetic fiela (T) & $30 / 1$ to 3 \\
\hline \multicolumn{2}{|l|}{ Anchor coils } \\
\hline Plasma length (m) & 3.3 \\
\hline Plasnla average radius $(m)$ & 0.21 \\
\hline Plasma density $\left(\mathrm{crar}^{-3}\right)$ & $5 \times 10^{12}$ \\
\hline Magnetic fielo at mirror (T) & 3 \\
\hline Magnetic field at mioplane (T) & 1 \\
\hline Barrier potential, $\phi_{b}(k V)$ & 12.2 \\
\hline Sloshing neutrai beams & $80 \mathrm{kV},(4.2 \mathrm{~A} / 60 \mathrm{keV}) 0.25 \mathrm{MH}$ \\
\hline Injection angle (deg)/magnetic field $(T)$ & $90 / 2.8$ (at point a') \\
\hline Beani trapping fraction & 0.28 \\
\hline \multicolumn{2}{|l|}{ Electron-cyciotron resonant } \\
\hline \multirow[t]{2}{*}{ heating ${ }^{\star \star}\left(\mathrm{KW}\right.$ at $\left.\mathrm{GH}_{\mathrm{Z}}\right)$} & 500 at 35 (at point b) \\
\hline & 667 at 60 (at point a) \\
\hline Peak beta, $\hat{\beta}_{b}$ & 0.5 \\
\hline Hot electron average energy, $w_{e, h}(\mathrm{keV})$ & 396 \\
\hline Warm electron temperatı re, $T_{e, W}(k e V)$ & 6.5 \\
\hline Maximum plug potential, $\phi_{e}+\phi_{c}(k v)$ & 17 \\
\hline Totaj pumping NBI ${ }^{*}{ }^{* *}$ e & $80 \mathrm{kV},(2.9 \mathrm{~A} / 60 \mathrm{keV}) .174 \mathrm{MH}$ \\
\hline Injection angle (deg)/magnetjc field (T) & $15 / 1$ to 2.5 \\
\hline
\end{tabular}

Note: See Fig. 2.l for definitions of points $a, b, a^{\prime}$ and $\phi_{e}, \phi_{c}$ and $\phi_{b}$. *An ion source output species mix of about 90,7 , and $3 \%$ is desirable for $0^{+}: 0_{2}^{+}: 0_{3}^{+}$. **Total inciuent power for both ends. 
sufficiently high mirror ratios $(-3$ in the anchor) to reuuce neutral-beam requirements, and (4) to meet high $B$ plasma stability requirements.

Plasma input for Parameter Set

- Limit injection energy of neutral beams to $80 \mathrm{kV}$. This was so we coulo use existing MFTF-B neutral beam development, maintain good beamline jon to reutral conversion efficiency, and take advantage of the tact that $U-T$ fusion reactivity $\langle\sigma v\rangle_{U-T}$ is nearly flat for mean ion energies of $240 \mathrm{keV}$. Calculations are generaily baseo or an ion source species mix of $80 \% \mathrm{D}^{+}, 15 \% \mathrm{~V}_{2}^{+}$, and $5 \%$ $D_{3}^{+}\left(\right.$or $\mathrm{T}^{+}: \mathrm{T}_{2}^{+}: \mathrm{T}_{3}^{+}$) for central cell neutral-beam injection.

- A limited peak $\beta_{\mathrm{c}} \leq 0.4$ in the central cell (based on theory, Fokker-Planck calculations and previous experiments). 16

- A limited peak $B_{A} \leqslant 0.5$ in the yin-yang anchor cell (based on theory and previous experiments). 16

- Use of $D_{2}$ pellet injection in the central cell to provide fueling for stream stabilization of the anchor cell (aescribea in Section 2.4.2) and warm plasma stabilization of drift-cyclotron-ioss-cone (OCLC) modes in the central cell (as described in Section 2.4.3).

- Use of pumping neutral beams and ECRH to (1) form potential plugs at the outboard sides of the anchors and (2) form electron barriers at each anchor midp? to separate electrons in the central cell from those in the plug regions.

- Volume averaged calculations performed with an assumeo cubic density profile of $n \propto n_{0}\left[1-(r / a)^{3}\right]$ where $a \equiv r_{p}$ and uniform radial temperature profiles. Preaictions for the outer cool "halo" plasma shield are discussed in Section 2.3.4.

a Neutral-bealn injection at 65 deg in the centra! cell to limit $\beta_{\perp} / \beta_{\|}<2.5$ for Alfven Ion Cyciotron (AIC) stability. Injection off the midplane at $90 \mathrm{aeg}$ in the anchor cell is used to develop two slushing ion density peaks that will suppress the AIC or UCLC instability as aiscussed in Section 2.4.2.

The hot $D-T$ ion reacting component ( $\sim 92 \%$ fraction-of-density) is .:agneticaily confined in the 4.5-T central cell by the 15-T choke coils. The injected 80-keV, 40-keV, and 27-keV hot ions tend to drag down in energy on 
the 000 (few $\mathrm{keV}$ ) central cell electrons until they are scattered into the loss cone and out over the choke coil, where almost all are airectly lost out an ena of the machine in a single transit time. The phase space of the minority, warm ions, is split into a magnetically-containea group in the central cell and an electrostatically-contained group in the full machine length between the potential end plugs in the anchor cells. The warm plasma density in the central cell is arranged through pellet fueling sufficient for a hot-plus-warm combined stable, nearly monotonic, ion distribution function and through sufficient loss flow of hot plus warm ions from the central cell for stream stabilization of the anchor cells. The row fraction of the local density from the central cell loss flow of warm ions that pass through the transition and reflect on the anchor plug potential is sufficient to couple the central cell piasma to the MHD-stable anchors. This is predicteo to stabilize any trappeo mode instabilities.

Producing a plug potential in the anchor cells higher than the ambipolar potential of the central cell by a potential difference of the plug from the central cell $\Delta \phi$ with a common electron temperature $T_{e}$ would normally require a higher plug plasma density than the central cell has, since $\Delta \phi \propto$ $\mathrm{T}_{e}$ in $n / n_{c}$. However, TOF will increase $\Delta \phi$ by including a modest thermal barrier in the inboard side of the anchor cell. This will allow us to separate the electrons into oifferent temperature regions, with a much higher $T_{\mathrm{e}}$ in the plugs.

He form the barrier using a combination of three methoos: (1) ion pumping in the transition and anchor regions to locally enhance ion losses (into the central cell if possible), (2) local ECRH at the anchor mioplane to produce a very hot magneticaliy-contained sloshing e?ectron group with a density minimum to yieid a barrier potential minimum, and (3) local ECRH of a hot electron group at the outboard sloshing-ion density peak to produce a nigher $T_{\mathrm{e}}$ at the ambipolar maximum potential plug. Barrier physics is relatively new and has yet to be tested on $T M X-U$ and MFTF-B experiments, but we feel that the requirements for a "barrier minimum to plug peak potential" difference of $\sim 77 \mathrm{kV}$ is sufficiently conservative for the $80-\mathrm{kV}$ neutral-beam injection pump system to be a low-risk element for this study.

The sloshing-ion populations developed by 65 deg injection in the central cell and 90 deg injection off midplane in the anchors are designed to stabilize the AIC instability. Even without pellet injection, these sioshing 
ion distributions will also tend to trap warm plasma for DCLC stable populations in these regions. An equilibrium case without the extra stream stabilization pellet fueling is therefore discussed in Section 2.5 .

Figure 2-1 shows the base case axial magnetic fielo, plasina potential, and total plasma densities.

\subsubsection{Code Base for Calculations}

The parameters shown in Tables 2-1 and 2-2 are calculated using a coce developed for calculating operating points for thermal-barrier, tandem-mirror reactors. The majority of the code equations are detailes in Ref. 17, Section IV. In general, the equations are statements of the particle ard energy balance of central-cell ions and electrons, hot ard warm plug electrons, ano sloshing ions. There are energy exchanges between these oifferent species, and particle sinks of one species can serve as particle sources for another. The relationships used to calculate neutral-bean anci ECRH powers have changed since the report was published, reflect ing improvements in modeling. These new scaling laws are described in Appendix $A$. The formuli for the warm plasma density that passes into the eno-plug region has also been improved on, using results based on work similar to that presented in Ref. 18. The configuration for TOF includes a thermal barrier, potential minimum, and a plug potential peak in the yin-yang anchor region. The transition region is pumped by a neutral beain, and the entire configuration is stable to the recently preoicted "trapped particle modes." 19

TUF was originally designed to operate in the Kelley mude; no thermal barriers were necessary, ano we colild attain a $Q$ value of about 0.5 . This was essentially the way TWX had been run. Theretore, it would require no new pliysics aevelopment and was quite attractive. However, the new trappea particle stability criterion required us to use a thermal barrier so that electrons coulo have their turning points inboard of the ion-contining peak. The presence of sloshing ions and hot electrons was then necessary to racilitate making the potential depression of the barrier. However, with the stream stabilization assumed in the original 1DF, the sloshing ions need not de as robust as requireo when the stream current is absent; this is a conservative aspect of the TDF operating mode. 

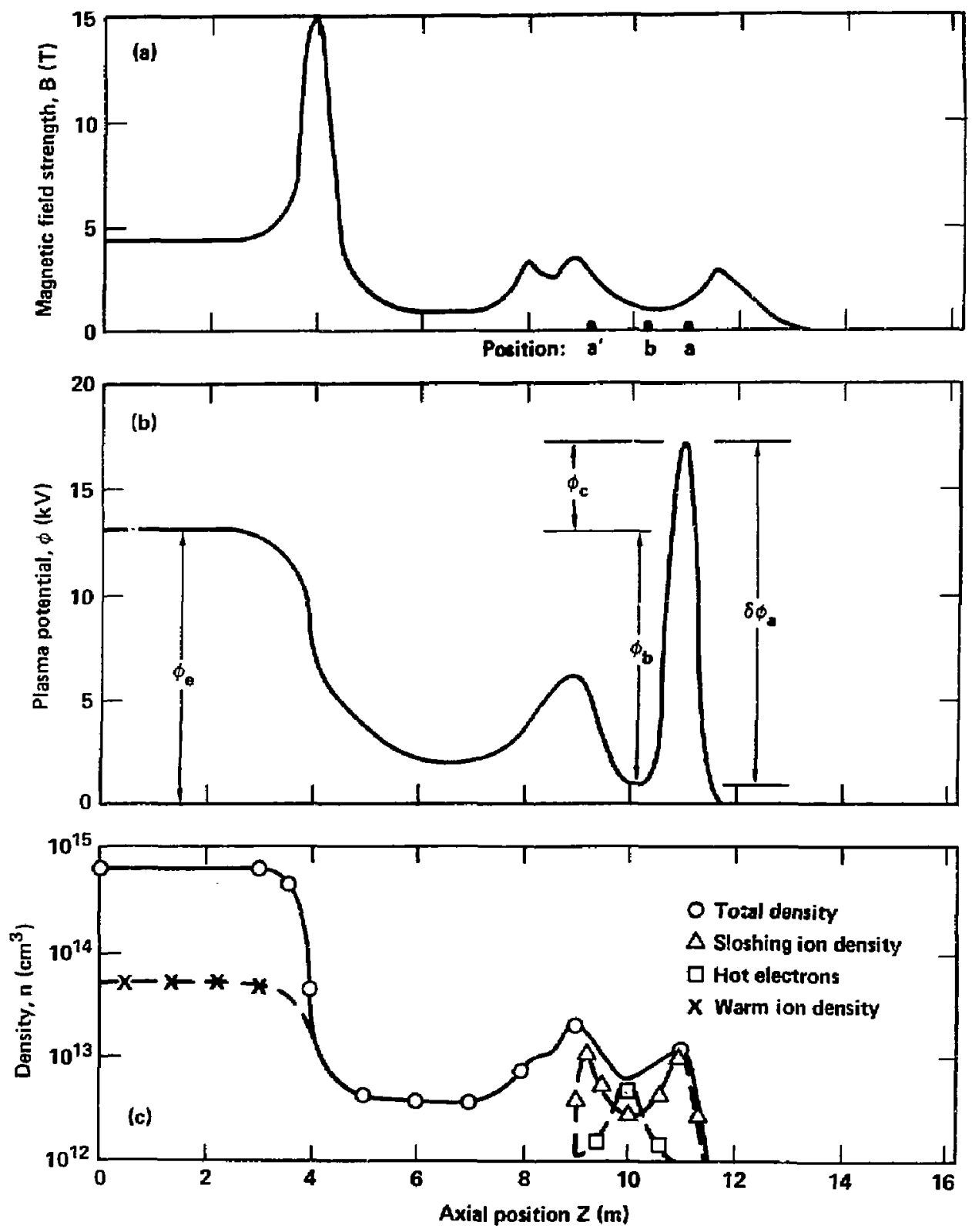

Figure 2-l. Base case axial magnetic field, plasma potential, and density profiles.

$2-9$ 
The cooe had to be modified in the central cell to include two species of ions: a warm stabilizing plasma and a hot mirror-confined plasma prouuced by neutral-beam injection. The confinement and energetics of these hot ions are aescribed by the Logan-Rensink model, 20 which fits a number of Fokker-Planck cases.

The formulas useo to compute the machine input powers requireo to sustain the JUF plasina are presenteo in Appendix $A$.

\subsubsection{Startup Scenario}

The startup scenario for TDF is only in a scoping phase. It is aifficult to define startup of any tandem mirror, particularly one with thermal barriers, since there are several helom and ECRH sources which nust be phased p'operly relative to each otner. However, we have outilined two approaches for cefining startup: (1) qualitatively stating the intermediate steps needeo to go from the initial stream-gun-produced plasma to the moderate beta-neutron-producing plasma and (2) using the equilibrium code to compute sustaining powers to achieve plausible, intermediate operating points that might simulate a start-up sequence. We will describe both of these methodologies briefly.

Table 2-3 shows the steps needed to start up the TDF plasma according to the first method. After about 100 hours of glow discharge cleaning to remove oxygen and carbon impurity from the walls of the machine, we begin an initial phase lasting about $10 \mathrm{~ms}$. The purpose of this phase is to produce a target plasma for beams and ECRH in both the central and eno cells. This would be

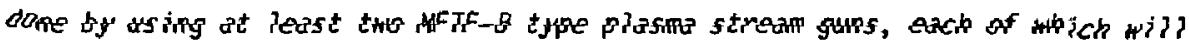
inject around $226 \mathrm{~A}$ of plasma.

The next 20 to 100 ms make up the "tandem $\phi$ " phase and involve applying about $100 \mathrm{kH}$ of $35 \mathrm{GHz} \mathrm{ECRH}$ in the end cells, while aading about $120 \mathrm{~A}$ of $\mathrm{O}_{2}$ gas in each anchor and $170 \mathrm{~A}_{\text {of }} \mathrm{O}_{2}$ gas in the central cell. The sloshing ion neutral beams are turned on ouring this perioo. The combination of the stream plasma leaving the central cell and the sloshing ions should proauce microstability. This phase is intenced to produce an anchor plasma of nigher censity (factor of 2 higher than initial phase), an electron temperature on the oroer of $1 \mathrm{keV}$, and anchors which are microstable. 


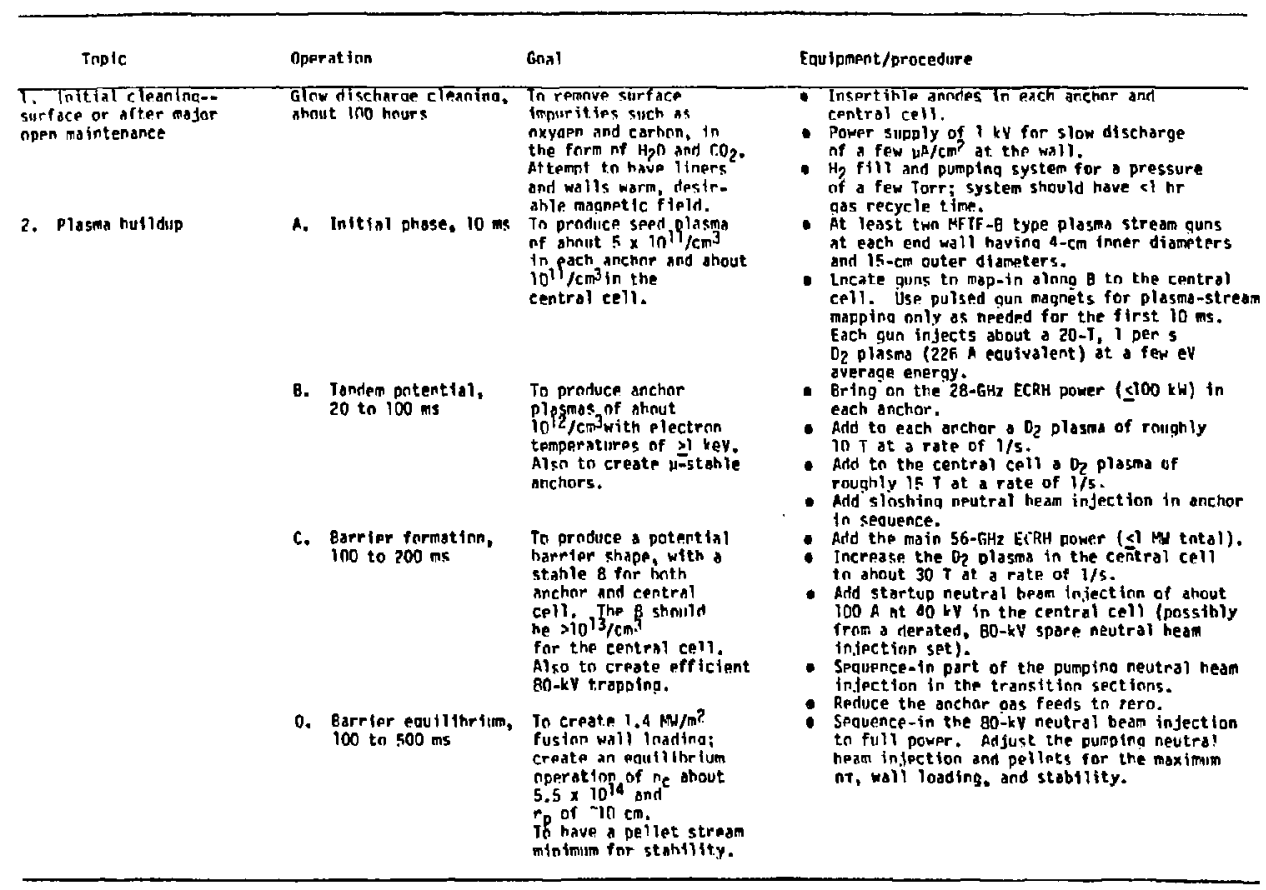


The next phase, which lasts about 100 to $200 \mathrm{~ms}$, is the barrier formation phase. In this period the main $60-\mathrm{GHz}$ tubes are turned on with a total power level not exceeding $1 \mathrm{MH}$. The central cell is feo by gas at a source strength of about $350 \mathrm{~A}$, and the central cell startup neutra? beams (40 keV) are turned on, delivering about $4 \mathrm{MW}$ of power. Since the central cell warm aensity (fueled from the $\mathrm{D}_{2}$ gas) is increasing during this period, the transition region pump beams should be started. These beams keep the source strength of warm ions trapped in the transition region and barrier small enough to let the potential wells form in these regions. During this phase, the gas feeds in the anchor are turnea off. This is a crjtical phase, since it produces the thernal barrier and sloshing ions, both of which are necessary for plasma stability and high performance. Also during this phase, a high-oensity target plasma is provided for the 80-keV central cell beams.

In the final phase, which lasts 100 to $500 \mathrm{~ms}$, we bring the $80-\mathrm{keV}$ central cell neutral beams to full power, start to refuel the warm ions with frozen $\mathrm{D}_{2}$ pellets (insteac of gas), and adjust the barrier and transition region sustaining powers to achieve the equilibrium-stable plasma parameters shown in Tables 2-1 and 2-2.

The secono approach to defining startup requirements is to run cases with the plasma performance code at plausible, intermeaiate density and temperature operating points and then calculate the sustaining powers required at these points. The calculations assume operation with thermal barriers, and lience really can only model startup during the last few hundred ms, while the barriers are being produced and afterwaros. The goal is to find a start-up pathway that does not require powers greater than for the operating point. From an initial low-density (low beta) barrier and central-cell plasma, we buila up the barrier beta by increasing the hot electron energy through ECRH. Increasing barrier beta by energy rather by density helps lower the required ECRH power. This approach begins to build the Darrier density (at a reduced electron energy) when the barrier beta is around one-half of the desired equilibrium value. Last, we begin to bring up central-cell beta to the desireo value, while increasing the barrier beta from one-half of its final value to the full value.

To start up the anchor/barrier region independently from the central ce11, we must control the densities in these regions separateiy. In the 
current physics model, these densities are interrelated by $g$ and $G$, oefined by the ion-density ratios:

$$
g=\frac{\text { passing from central cell }+ \text { trapped in barrier }}{\text { passing from centralcell }} \text {, }
$$

ano

$G=\frac{\text { passir.g from central cell }+ \text { trapped }+ \text { sloshing }}{\text { passing from central cell }}$.

$B y$ increasing $g$ and $G$ in the barrier, we can increase the barrier density while keeping the central cell density fixed. By decreasing $g$ and $G$ after the barrier has been built up, the central cell density can be increased at fixed-barrier aensity by increasing central cell beta. These calculations give us the sustaining powers required and plasma temperatures needed for a given set of betas and of $\mathrm{g}$ values. The fueling requirements must then be computed, given: 1) the timescale for the startup, (2) the set of g values prescribed, and (3) the loss rates of the inaividual species. See Appenaix $B$ for an example.

In sumnary, the basic elements of TDF startup should not be orastically different than for MFTF-B, where we have given much thought to the problem and where some of the ideas expressed here originated. The quantitative approach is potentially fruitful. The first attempt at a scenario has yielded reasonable startup powers for central cell beams and charge-exchange pumping beams, as well as stabilizing stream currents. There remains a minor problem with the sloshing beams and ECRH powers--the startup power presently exceeas the equilibrium power. A revised scenario may renove this problem and will be considered in future work.

\subsection{PHYSICS REQUIREMENTS FUR STREAM-STABILIZED OPERATION}

This section briefiy aetails the physics requirements for heating, fueling, and ion pumping for the TOF system. They are summarized in Table 2-4. The magnetic field geometry and associated physics requirements for stability are detailed in Section 2.4. 
Tahle 2-4. Hestina, fueling, and pump system listino. Under total, I T-1/s $0_{2}=11.28$ A (atonic).

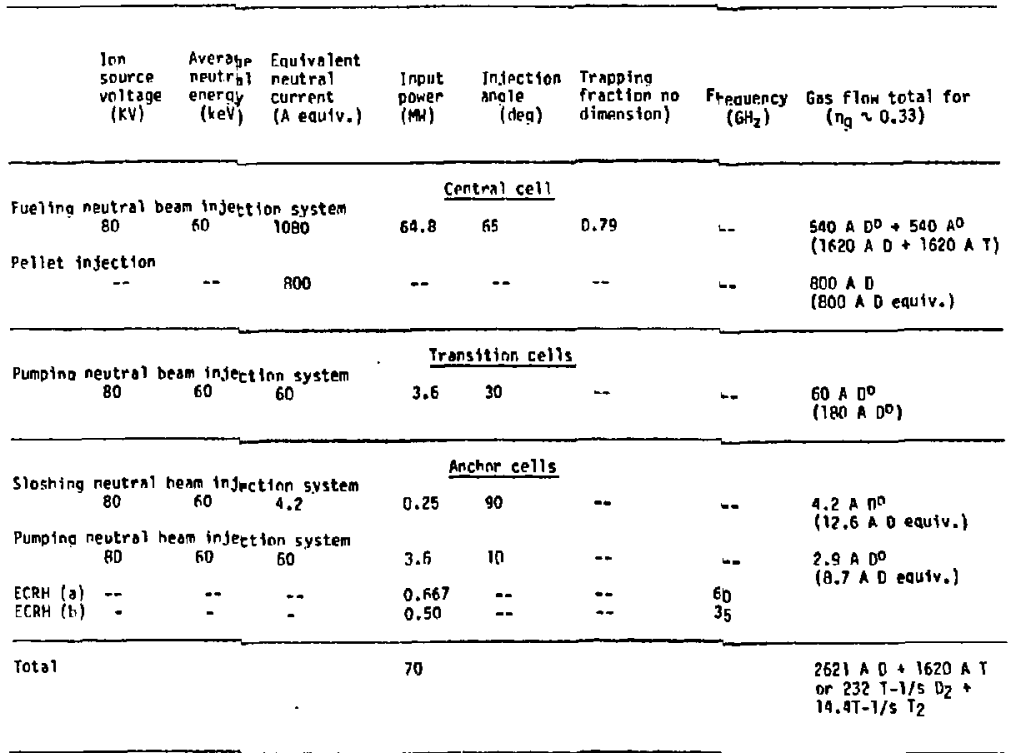

$n_{0} \equiv$ qas effictoncy of the neutral heam.

(a) Point $a$ is def ined as the nuthoard sinshing inn rensity peak in the arch ${ }_{\mathrm{pr}}$

(b) Paint b is defined is the atdplane sloshing ton denstity minimum in the ahehor. (See flạ. 2-1.) 
The TDF central cell is operated as a high $B$, short prototype sertion of a $0-T$ reactor intended to yield a steady-state uncollided neutron wall loading $\Gamma_{n} \sim 1.4 \mathrm{MH} / \mathrm{M}^{2}$. The hot reacting $D-T$ ions are the majority plasma species in the central cell, with on $1 y$ the minimum $8 \%$ warm plasma added as needed to stabjlize the microinstabilities. The effective $n \tau$ calculated from the Logan-Rensink mode ${ }^{20}$ for the hot jons is limited to $\sim 6.6 \times 10^{12} \mathrm{~cm}^{3} / \mathrm{s}$ because of the fairly rapid energy drag down on the 2.1-keV electrons and warm stabilizing ions; the partially cooled hot ion is scattered (by $\sim 15$ to $30 \mathrm{keV}$ ) into the loss cone and out over the 15-T choke coil. Establishing the high density D-T plasma of interest with such a low $n \tau$ requires intensive heating and fueling with the $70-\mathrm{MW}, 80-\mathrm{kV} \mathrm{D}^{0}$ and $T^{0}$ neutral-beam injection (NBI) system.

Central Cel1 Neutral-Beam Injector Considerations. We selected extraction at $80 \mathrm{kV}$ for the $D^{\circ}$ and $T^{\circ}$ NBI to take advantage of an existing $80-\mathrm{kV}$ ion source technology developed for MFTF-B. This choice is also reasonably opt imum for the high neutron yield needed to support $\Gamma_{n}$. The neutron yield is limited both by a basic $\beta_{C}$ (or nkT) limitation for a fixed magnetic field and the fact that the $\langle\sigma \mathrm{v}\rangle \mathrm{D}-\mathrm{T}$ reactivity becomes relatively flat $\left(\sim 8 \times 10^{-16} \mathrm{~cm}^{3} / \mathrm{s}\right)$ for mean ion energies $\bar{W}$ over $40 \mathrm{keV}$ (but less than $200 \mathrm{keV}$ ). Fokker-Planck calculations for the stream-stabilized D-T system yield average ion energies $\bar{W}$ of about $37 \mathrm{keV}$ for the 80-kV NBI system.

The NBI current requirements 1 isted in Table 2-4 are based on Mars code calculations of the form:

$I^{0} \sim 0.45 n^{2} \mathrm{fla} \mathrm{a}^{2} L /\left(\mathrm{f}_{t} \mathrm{n \tau}\right)$

where the factor 0.45 comes from integrating over the radial cubic density profile $^{n}=\hat{n}_{\left[1-(r / a)^{3}\right]}$ assumed for a plasma column with a radius of a, cell effective length $L$ is $7.2 \mathrm{~m}$ from Fokker-Planck calculations, the particle trapping fracion is $f_{t}$, and $n \tau$ is calculated from the Logan-Rensink 20 Fokker-P lanck model. 
The NBI system is arranged to inject at an angle of 65 deg just inside the choke coils. The limitation to an angle $\leq 75$ deg is based on Fokker-Planck requirements that the jon anisotropy must be reduced sufficiently for $B_{1} / B_{\| l}<2.5$ to be stable against the AIC instability. We selected 65 deg to allow better bean duct access between the choke coils and the first solenoid (with a minimum duct-to-first-solenoid clearance of $-45 \mathrm{~cm}$ needed for the neutron and associated gamma ray flux $\mathrm{N}$, $\gamma$ shielding for the superconducting solenoid), though this lower angle increased the NBI requirements. This NBI increase occurs because the effective mirror ratio $R_{\text {eff }}$ is proportional to $(\sin \theta)^{2}$ and $n \tau \alpha$ $\log _{10} R_{\text {eff }}$.

Appendix $C$ details effects of the ion source output species mix and trapping of various $0^{\circ}$ and $T^{\circ}$ energy components with an average trapping fraction 0.79 . For discussion here, we assume an ion source output mix of $80 \% \mathrm{D}^{+}, 15 \% \mathrm{D}_{2}^{+}$, and $5 \% \mathrm{D}_{3}^{+}$(or equivalent $\mathrm{T}^{+}: \mathrm{T}_{2}^{+}: \mathrm{T}_{3}^{+}$species) prior to dissociation to $D^{\circ}$ components at $80 \mathrm{KeV}, 40 \mathrm{keV}$, and $26 \mathrm{keV}$. This yields an average input $0^{\circ}$ energy of $\approx 60 \mathrm{keV}$ for an 80-kV NBI system. Trapping is calculated separately for each species, using a one-dimensional Gaussian neutral beam with a divergence of 0.4 deg, incident on an axisymmetric plasma column with a cubic density profile and a radius of $10 \mathrm{~cm}$. The trapping fraction is calculated using appropriate average energy-ion and electron-ionization cross sections, corrected for a limited number of generations of charge-exchange eyents prior to final ionization. The $f_{t}$ value quoted is the minimum trapping expected; it is made up of $55 \%$ $80-\mathrm{KeV}, 39 \% 40-\mathrm{KeV}$, and $16 \% 26-\mathrm{KeV} \mathrm{D}^{+}$input.

In Appendix $D$ we discuss a more detailed Monte Carlo calculation that can correctly handle the relatively forward-peaked, velocity-dependent charge-exchange "scattering" in the very limited geometry where the approximate plane parallel incident neutral beam is about the same width as the trapping p?asma colum diameter. This scattering occurs in each of several possible generations of charge-exchange events, since charge-exchange cross sections are typically higher than those used for ionization. Details of pellet injection and preliminary pellet range calculations are described in Appendix $E$. 
Anchor Sloshing NBI Considerations. The NBI heating and fuel ing requirements for the anchor cells are relatively modest because of lower cell densities and smaller volumes. However, we need a sloshing double-peaked ion density distribution, together with local ECRH, to facilitate formation of a local minimum-potential electron barrier at the anchor midplane and a maximum-potential peak at the outboard sloshing ion density peak. The use of $B 0-k Y$ ABI appears satisfactory for the predicted modest relative central cell ion plugging potential $\phi_{C} \sim 4 \mathrm{kV}$ and the 1imited barrier to plug peak potential, which nas a difference of $216.2 \mathrm{kV}$. The high anchor $\beta$ required for central cell MHO stabilization is produced largely by ECRH-heated electrons. It is desirable to use only a deuterium neutral beam here, to avoid high D-T neuticon production locally, since this would require extra $N$, $\gamma$ shielding on the yin-yang superconducting coils. Significant $D-D$ neutron production will occur but the heating and damage is calculated to be low enough that it will not require extra shielding.

Injection is at $90 \mathrm{deg}$, but it is off the midplane at the inboard $2 \mathrm{~T}$ mirror region to achieve the needed sloshing density distribution. NB1 and neutral beam dump access is very limited, though some variation in injection point and angle is available. Injection is at point a (inboard sloshingdensity-peak conjugate to the plug-density-peak) to reduce cold electron loading for plug ECRH at point a. Trapping of the anchor sloshing-NBI is similar to that just described for the central cell, except that the trapping chord geometry is generally along the major axis of the mapped hot-plasma ellipse (even then the $f$ nd) and hence $f_{t}$ is relatively low).

\subsubsection{Pellet Fueling for Stream Stabilization}

Since we decided to use established physics for TDF, we had to allow for additional fueling for stream stabilization of the anchors in the base case. In Section 2.4.3 we discuss the relevant streaming current density required in the anchor midplane. We have planned deuterium pellet injection into the central cell to fill in the loss cone and yield the end-joss currents needed for stream stabilization.

We considered several types of warm plasma sources, including gas-box fueling and $D_{2}$ pellet injection ( $D_{2}$ is sufficient, since almost all of the central cell $0-T$ yield is from hot-ion hot-ion collisions). Pellet injection appears the most desirable approach for higher gas efficiency ( 100\%), input 
fueling throughout the pellet range in the hot core (instead of mostly at the surface), and much lower charge-exchange losses. These losses are estimated from calculations 21 indicating that hot electrons play the dominant role both in evaporating (or ablating) the pellet and ionizing the atoms.

Section 3.4 discusses the details of the centrifugal type of extruded $D_{2}$ pellet irjector. The physics constraints with pellet injection include:

- A limited maximum size of the $D_{2}$ pellet. This is because the number of atoms $\Delta n$ in a single pellet needs to be much less than the total number of hot ions $N$ in the central cell to 1 imit the perturbation of the cold plasma input on the equilibrium hot plasma. For $\Delta n / N<10 \%$ the required diameter of a $D_{2}$ pellet is $\$ 0.6 \mathrm{~mm}$.

- A repetitive pellet injector rate of at least $0.64 \mathrm{kHz}$, to furnish the necessary average stream current of up to $\sim 800 \mathrm{~A}$.

\subsubsection{Neutral Beam Pumping and ECRH for Barrier Formation}

Our initial design involved only basic tandem mirror physics, using an axisymmetric, high-density, simple two-coil mirror plug at each end of the central cell and outboard yin-yang anchors for MHD stabilization. This design would not have required neutral beam pumping or ECRH. However, the possibiłity of the trapped particle modes described by Rasenbluth et al. 19 led to our current more complicated end barrier and plug design. It uses a single axisymmetric 15-T choke, or throttle, mirror coil at each end of the central cell to magnetically contain the main group of high-energy ions undergoing fusion and a relatively modest barrier and outboard potential plug in each anchor cell to electrostatically contain a minority warm plasma component. This warm component is needed to suppress loss cone instabilities. The design also offers enough warm plasma connection between the anchors and the central cell to avoid trapped-particle instability modes.

Before settling on this design, we considered using an axicell pair of 15-T coils. However, the NBI power and neutron shielding requirements appeared excessive for the minor gain in performance, so the baseline design was simplified to a single choke coil.

In our current design the transition cell contains the major bad curvature region, so a low $B$ weighting of the various magnetic field 
curvature integrals is essential there and is discussed in Section 2.4.1. To provide this low $B$ we needed some form of ion pumping in the transition region to reduce the local ion density (and thus the $B$ ) to the minimum passing ion density requirements for MHD connection and anchor stream stabilization. This is discussed in Section 2.4.3. We have used charge-exchange neutral-beam pumping, though we are considering other techniques to reduce power.

The requirement for a pumping neutral beam is that the ion resulting from neutral beam charge-exchange on the plasma ion is located in the loss-cone region of phase space. Ideally the resultant ion would pass into the central cell for fueling and heating, but this is difficult to arrange with the high mirror ratio (about 15) for the transition to choke coil. This is particularly true for the fractional energy species components in the pumping neutral beam. So most of the transition neutral-beam pumping power is lost through reflection of the charge-exchange ion on the choke coil field and passage back through the anchors and out along field lines to the end walls.

The phase space plot for the $Z \approx 6.5 \mathrm{~m}$ position of the transition section is shown in Fig. 2-2, with the 80-kV pumping neutral beam oriented at $30 \mathrm{deg}$. Neutral-beam injection angles of $<76$ deg would be required for pumping over the 15-T choke coil field. The on-axis phase space plot shows that using $80 \mathrm{kV}$ for the pump neutral beam allows both the full- and halfenergy beam components to pump and permits only fractional trapping of the third-energy component at some beam-plasma intersections. The use of $80 \mathrm{kV}$ rather than the minimum of $\sim 30 \mathrm{kV}$ needed for full energy component pumping, and the pump neutral beam requirement for $290 \% D^{+}$purity from the special ion source $\left(<7 \% \mathrm{D}_{2}^{+}\right.$and $\left.<3 \% \mathrm{D}_{3}^{+}\right)$are conservative steps we took to ensure efficient pumping along the 3-D intersection of the pump beam with the elliptical plasma column. The total incident pumping beam current requirement is based on code calculations described in Section 2.2.2 and shown in Table 2-4. The calculated potential depression (shown in Fig. 2-1) in the transition to $\Phi \sim 5 \mathrm{kV}$ represents a substantial $\sim 8 \mathrm{kV}$ barrier for the $2.1 \mathrm{keV}$ central-cell electrons.

The anchor cell must furnish both the $B$ weighted MHD stabilizing term for 1 ine curvature integrals and the electrostatic plugjing function with $\Phi>13 \mathrm{kV}$ to contain the warm ion loss cone stabilization population in 


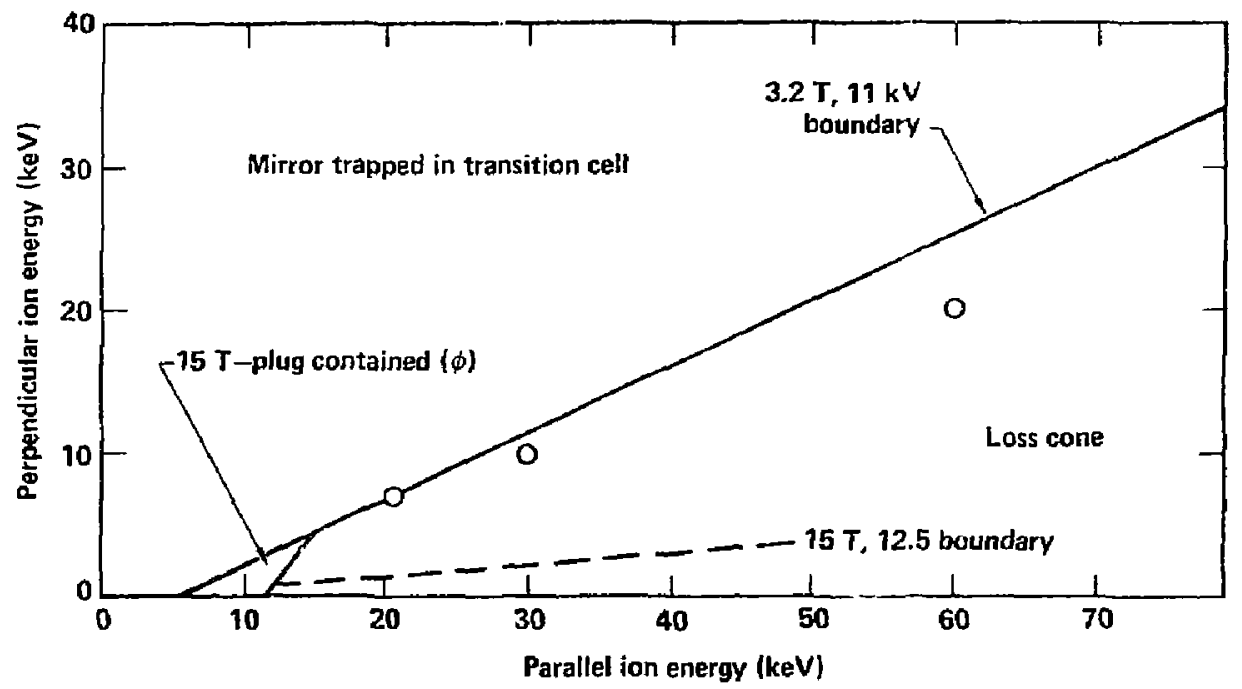

Figure 2-2. Phase space plot for the $Z \sim 6.5 \mathrm{~m}$ position of the transition section. The positions of the $30-$ deg pumping neutral beam components are shown by the circles. 
the central cell. For maximum stabilization of the trapped particle modes in the central cell, the anchor should also have an asymetric axial potential distribution, allowing the stabilizing warm ions to penetrace as far as possible in the anchor cell before reflection. This second requirement is satisfied by forming a barrier minimum potential near the anchor midplane and a plug potential maximum on the outboard side of the cell. This dual potential function requires production of a sloshing plasma to furnish a double-humped ion density population with an ion density peak for the outboard potential maximum and a central density minimum for the anchor midplane minimum potential barrier. An anchor pump neutral beam prevents the barrier ion density minimum from trapping passing ions and filling up. The s-corrected phase space plot for the anchor midplane is shown in Fig. 2-3. With an end fan injection angle $<10 \mathrm{deg}$, the full- and half-energy pump neutral beam components would be pumped through the transition section into the central cell.

The anchor pump beam requirements are also shown in Table 2-7. Again we are conservative, pumping with an $80-k V$ NBI system, orienting the injection angle $<16 \mathrm{deg}$, and requiring a high-purity ( $>90 \%$ ) $\mathrm{D}^{+}$fraction from the neutral beam ion source. This ensures efficient pumping all along the neutral beam/anchor plasma intersection path. It also has the advantage of allowing us to use comon $80-k V$ neutral beam components thrcughout.

To form the deep anchor barrier minimum ( $12-k V$ barrier) and $77 \mathrm{kV}$-anchor plug potential peak for the base case requires the addition of ECRH in local regions. ECRH power near the anchor midplane helps form a deep barrier minimum when electron energies are driven high enough for magnetic containment of the electrons. The vacuum field of $1 \mathrm{~T}$ will be $B(\beta \sim 0.5)$ depressed to $\bar{B} \sim 0.84 \mathrm{~T}$, since $B=B_{\text {vac }}(1-\bar{B})^{1 / 2}$ and the volume averaged $\bar{B}=0.3$. ECRH is planned at $35 \mathrm{GHz}$ for equilibrium, first and second harmonic, off-axis, near-midplane heating of the anchor. ECRH at 28 $\mathrm{GHz}$ would also be useful for startup.

The second ECRH region is at the sloshing ion density peak below 2.0 T on the outboard side of the anchor. The goal here is to moderately heat electrons that are electrostatically trapped by the ion density peak and thereby raise the peak's ambipolar potential proportional to $T_{e}$. In this case the fundamental frequency appears most appropriate so we use $60 \mathrm{GHz}$ 


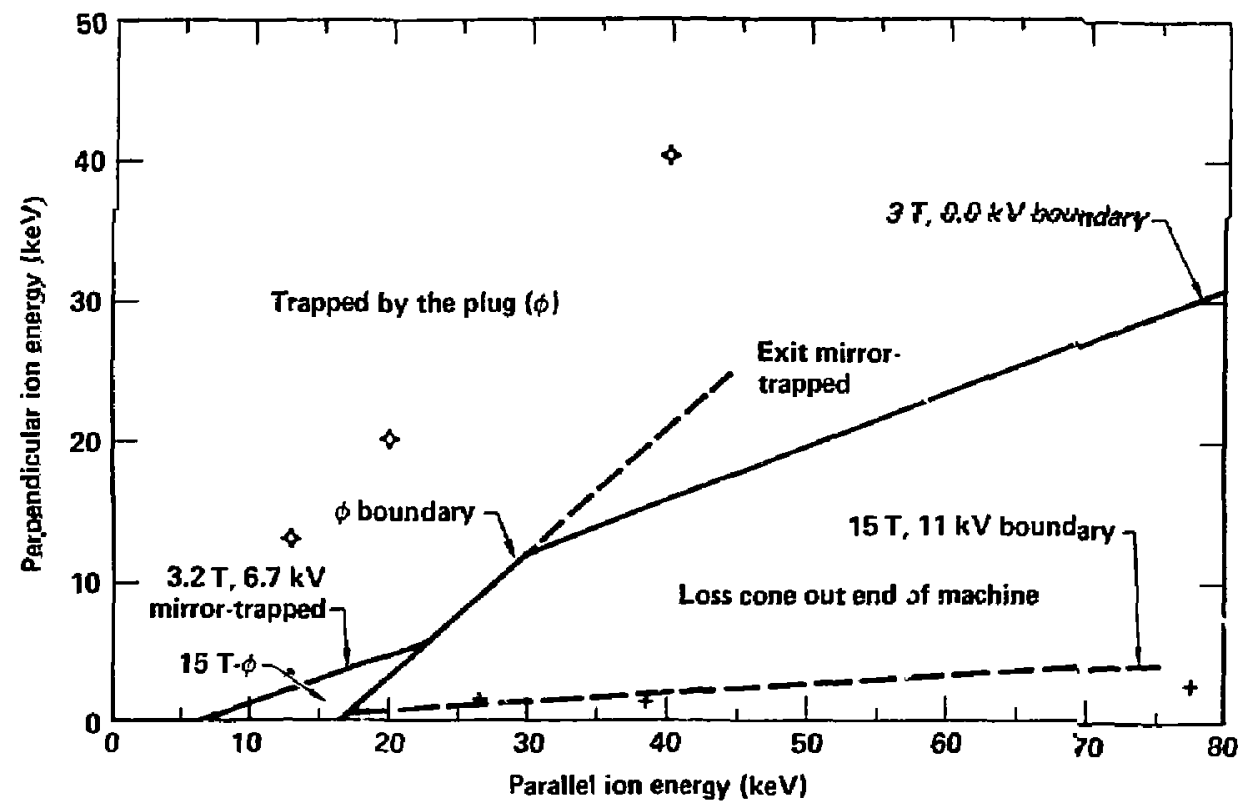

Figure 2-3. The B-corrected phase space plot for the anchor midplane. The stars show the approximate location of the sloshing neutral beam input components produced by $90-$ deg injection off the midplane at the 2-T a' field point; the crosses show the approximate locations of the 10-deg pumping neutral beam. 
gyrotrons. All the equilibrium ECRH power requirements are given in

Table 2-4. The neutral beam pumping and ECRH are approximate incident powers with expected losses taken into account.

\subsubsection{Yacuum and Halo Requirements}

The central cell vacuum requirements for TDF are based upon the requirement that the hot ion charge-exchange loss $t$ ime ${ }^{T_{c x}}$ be much longer than the predicted Logan-Rensink Fokker-Planck mode $1^{20}$ scattering loss time over the 15-T choke coil. The 11.5 -ms hot ion central cell loss time (from the $n t$ shown in Table $2-1$ ) then requires $\left(n_{0}<\sigma V>\right)^{-1}>11.5 \times 10^{-3}$ or $n_{0}<10^{9}$ for $<0 V_{c x}>-9 \times 10^{-8} \mathrm{~cm}^{3} / \mathrm{s}$. For an expected operating wall base pressure of $10^{-5}$ Torr this model requires a neutral gas attenuation factor of $>350$. Similarly, the transition and anchor regions require that the total input trapping rate of penetrating cold gas (by ionizing or charge-exchanging on the extant warm or hot plasma) be much less than the effective pumping rate to prevent filling in the low density barrier regions. Again the result is a requirement that $n_{0}<10^{9}$ and the halo produce an attenuation factor $>10^{2}$.

The $2 X I I B$, TMX, and TMX-U $U^{22}$ experiments have all shown formation of an outer c00l plasma "halo" that pumps most of the cool gas from the walls or beam dumps to the end walls. This attenuates the neutral energy gas flux, which penetrates into the hot plasma interior. The attenuation would require a halo plasma layer $\int \mathrm{n}_{0} d l>4 \times 10^{13} / \mathrm{cm}^{2}$, with $\mathrm{T}_{e}>40 \mathrm{eV}$ so that the electrons could efficientiy ionize the Frank-Condon neutrais of about $4 \mathrm{eV}$, which result from short mean-free-path dissociation (or dissociative ionization) of $D_{2}$ or $T_{2}$ molecules recyciling from the wall. Appendix $F$ describes the physics model of the halo predicted for the TDF case with a main halo thickness in the central cell of $7 \mathrm{~cm}$. This is about equal to a 3.5-MeV alpha fusion reaction product orbit diameter (all $\mathrm{W}$ 1) at the 15-T choke coil field limit when mapped back to the central cell. The attenuation we require agrees well with the expected halo, though it suggests that even higher attenuation and a more efficient halo plasma would offer a longer hot ion lifetime and less gas throughput. However, further increase in halo 
$\int \mathrm{n}_{\mathrm{el}}$ is limited, because it would also become a significant attentuation loss for the incident neutral beams and increase the resultant wall heat. loading.

\subsection{DESIGN SELECTION FOR STABILITY}

The TDF design involves a combination of magnetic and electrostatic features in the end cells of a tandem mirror configuration. This section briefly describes the associated vacuum-magnetic field design needed for flute and ballooning stability. We also discuss the expected axial pressure profile, the warm plasma fraction or stream current needed to stabilize the DCLC instability, and the pressure anisotropy limit required to stabilize the Alfen-Ion-Cyclotron (AIC) instability.

\subsubsection{The Magnetic Field Arrangement Used to Provide Stability from Flute} and Ballooning

We used computer studies by R. Bulmer, L. Pedrotti, and R. Wong ${ }^{23}$ to help design the TDF vacuum-magnetic field such as to reduce parallel currents and associated quadrupole flux surface distortions to a minimum level acceptable for the TDF base case $n \tau$. The MHD plasma equilibrium and stability were evaluated using the approach of Pearlstein et al. 24

Figure 2-4 shows the marginal flute and ballooning stability boundaries, calculated by $R$. Wong with the TEBASCO code under base case conditions. Note that the design point for a peak anchor $\hat{\beta}_{a}=0.5$ and central-cell peak $\hat{B}_{c}=0.4$ is comfortably stable. This good design stability margin is aided by the fact that only the low- $\beta$ warm ion component in the central cell is contained by the anchor plugs and recirculates through the transition section (for a transition $\beta_{t} \leq 0.012$ ), while the main high- $\beta_{c}$ component in the contral cell is generally lost when scattered over the 15-T choke coil field, and its single-pass density makes a negligible contribution to the $B_{t}$ in the unfavorable curvature transition section.

The design flexibility in the relatively simple transition magnet section between the choke coil and the anchor allows reducing parallel currents to an acceptably low value, but not $J_{\|} \sim 0$ as attained in MFTF-B under 


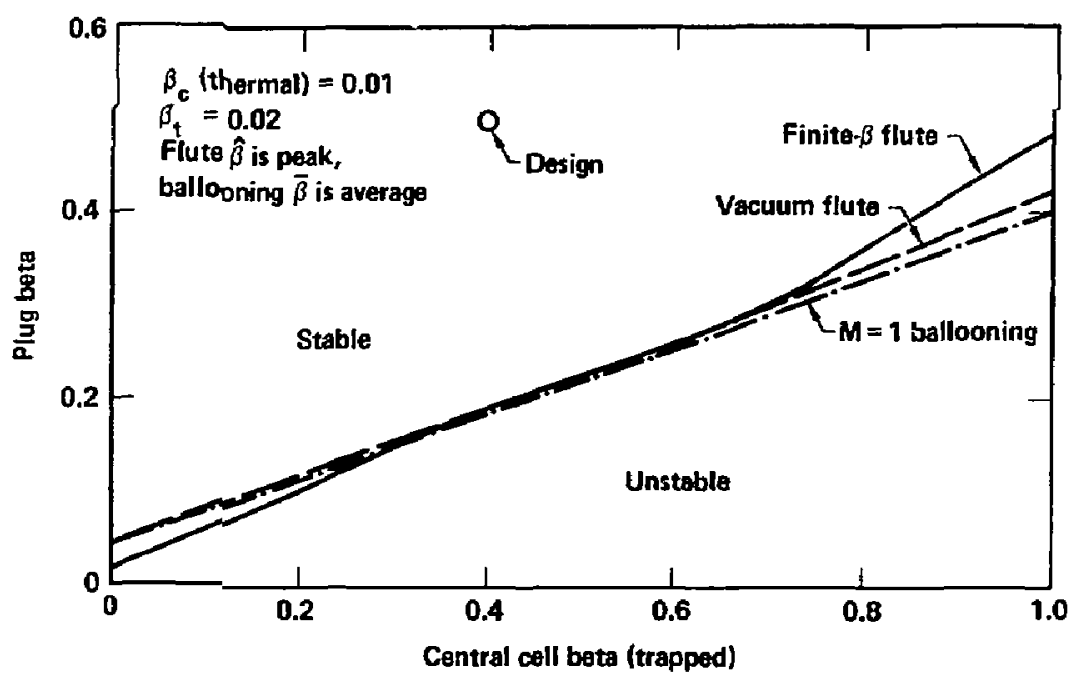

Figure 2-4. Marginal flute and ballooning stability boundaries calculated by $R$. Wong with the TEBASCO code under base case conditions. 
selected conditions with a more elaborate system of transition magnets. However, the remnant quadrupole moment induced radial losses should be minor compared to axial losses.

\subsubsection{Neutral-Beam Orientation Necessary To Avoid Alfven Ion Cyclotron} Instabilities

The electrostatic AIC instability is produced by strongly anisotropic ion velocity distributions. AIC was an important limiting factor for TMX ${ }^{3}$; we supressed it in TMX-U $U^{4}$ using angled NBI to produce a sloshing-ion distribution.

We have taken this same approach for TDF. The design uses 65 deg injection (90 deg would be normal to the magnetic axis) in the central cell to attain a Fokker-Planck value of $\overline{\left\langle\beta_{1} / \beta_{11}\right\rangle} \times 2.5$ for a hot sloshing-ion distribution predicted to be stable for the finite plasma dianeter of the central cell (calculations by $M$. Rensink ${ }^{25}$ ). The NBI for the anchor cell is arranged for $90 \mathrm{deg}$ injection, but off the midplane to attain a similarly stable sloshing-ion distribution.

\subsubsection{Stream Stabilization of Drift-Cyclotron-Loss-Cone Modes}

Loss-cone ion distributions tend to drive several instabilities, such as those of the axial-loss cone (ALC) and drift-cyclotron-loss cone (DCLC). The OCLC mode appears to have the more stringent instability limit for TDF conditions. The base case TOF design satisfies the DCLC quasi-linear theoretical requirements ${ }^{26}$ for the ratio of the warm-ion density (in the lass cone) to the hot ion density $n_{w} / n_{h} \sim 0.08$ in the central cell with $r_{p} / a_{i} \sim 11$. Fokker-planck runs also show that this results in an almost monotonic ion energy distribution function. In the anchor, we predict that DCLC stabilization requires a stream-stabilizing plasma flow of about $1600 \mathrm{~A}$. This flow will come from total central cell losses, including about 800 A from $\mathrm{D}_{2}$ pellet fueling. The stream current density required at the midplane of the anchor is 27

$$
j_{S} \approx \frac{1.9 \times 10^{-11} n\left(\mathrm{~cm}^{-3}\right)(\Delta \Phi / 4.5)^{3 / 2}(\mathrm{kV})\left(\mathrm{a}_{\mathrm{j}} / \mathrm{r}_{\mathrm{p}}\right)^{4 / 3}}{M^{1 / 2}(\text { AMU) E (keV)(R-1) }},
$$


where $j_{s}$ is in $A / \mathrm{cm}^{2}, R$ is the effective mirror ratio, $r_{p}$ is the plasma radius, $a_{j}$ is the ion gyroradius, and $\Delta \Phi$ is the approximate average difference in ambipolar potential between the anchor plug and the outboard mirror.

\subsubsection{Tandem Mirror Magnetic and Barrier/Plug Combination}

\section{Used to Avoid Trapped Particle Modes}

The magnetic design and operation of the tandem mirror depend upon line-averaged $\beta$-weighted field curvature integrals for stability. The approximately neutral stability in the central cell and certain bad curvature regions, such as the transition, will be stabilized by their connection to the good curvature high- $B$ anchor cells. Recent studies by $R$-senbluth et al. 19 have shown the possibility of trapped-particle instability modes isolated from the MHD anchors. To avoid these modes, Pearlstein et $=1.15$ note that the main ion potential plug at the outboard side of the anchor nust be arranged so that a sufficient population of the contained warm ions (about 10\%) passes through the machine, including the good curvature anchors, before recirculation. This will satisfy:

$\omega_{\star i} / Y_{c}>\frac{4\left(1+A_{r}\right)}{A_{r}^{2}}$,

where the Rosenbluth factor

$$
\begin{aligned}
& A_{r}=\left(r_{p} / a_{j}\right)^{2} \frac{B_{c}}{B_{a}}\left(\frac{g_{b} n_{p}}{n_{c}}\right) \times\left(\frac{2 L_{a}}{L_{c}}\right), \\
& w_{\star} i \quad \text { is the diamagnetic drift frequency, } \\
& r_{c} \text { is the MHO charac eristic instatility growth rate, } \\
& r_{p} / a_{i} \quad \text { is the ratio of the plasma radius to the ion larmor } \\
& \text { radius in the central cell, } \\
& \left(g_{b} n_{p} / n_{c}\right) \text { is the ratio of total density on the transition to } \\
& \text { the central c } 11 \text { density, } \\
& L_{a} / L_{c} \quad \text { is the ratio of anchor stabilizing length to the } \\
& \text { central cell length. }
\end{aligned}
$$


We meet the need for a warm ion turning point using all of the good curvature in the anchor by running the axisymetric choke coil only as a magnetic barrier for the central cell hot ion plasma components and forming an asymmetric anchor potential with a minimum potential electron barrier at the anchor midplane and the maximum potential warm ion plug at the anchor sloshing-ion outboard density peak. Figure 2-1 shows the machine magnetic and electrostatic potential axial distribution, and defines the input beam and ECRH locations at points $a, b$, and $a$ '.

\subsection{EFFECT OF FULL THERMAL BARRIER OPERATION}

OCLC requirements for the anchar cells determine the streaming current for the base case. However, the sloshing-ion distribution produced by off-midplane NBI into the anchor for AIC stability will also trap and fill in a warm plasma. This plasma wiil tend to build up a monotonic ion distribution similar to that of a central cell of a simple tandem, though the anchor pump beam will prevent the loss cone from being completely filled. The resultant distribution should markedly reduce the stream current density required for stability.

We are continuing to make fokker-Planck calculations of particle balance and streaming requirements for this case. Here we explore the impact of using only an effective streaming flow (hot ion loss) of about $800 \mathrm{~A}$ from the central cell, derived from the code of Section 2.2.2, with a no-pellet injection case. In the initial calculations we found that the lower strean flow allowed bo.h $T_{e}$ and $T_{i c}$ to increase but required a density decrease for $B_{C} \leqslant 0.4$. However, since $\left.<O v\right\rangle_{D-T}$ is relatively flat for average ion energies $W>40 \mathrm{keV}$, we reduced the neutral beam voltage and increased the density to maintain the same average $\beta$ and not ion energy in the central cell (just below $40 \mathrm{keV}$ ), but over a 1 arger $15-\mathrm{cm}$ radius plasma. This requires higher neutral-beam input current but about the same power to obtain a predicted higher wa?l loading of $\Gamma_{n} \sim 2.1 \mathrm{MN} / \mathrm{m}^{2}$. The parameter set for this neutral-beam, high-voltage-adjusted, larger-plasma-diameter, no-pellet injection case is shown in Tables 2-5 and 2-6. We expect further optimization for this case. 
Table 2-5. Design levels for the tandem mirrors of the TDF central cell, without a stream plasma.

\begin{tabular}{|c|c|}
\hline Parameter & \\
\hline Plasma length (m) & 8 \\
\hline Plasma radius (m) & 0.15 \\
\hline Solenoid field (T) & 4.5 \\
\hline Peak beía, $\hat{\beta}_{c}$ & 0.4 \\
\hline Electron temperature, $\mathrm{T}_{\mathrm{ec}}(\mathrm{keV})$ & 4.1 \\
\hline Hot ion average energy, $w_{i h}(k e V)$ & 37 \\
\hline Hot ion lifetime, $T$ (ms) & 200 \\
\hline Hot ion density, $n_{i h}\left(\mathrm{~cm}^{3}\right)$ & $5.25 \times 10^{14}$ \\
\hline Warm ion temperature, $T_{i w}(\mathrm{keV})$ & 10 \\
\hline Warm ion density, $n_{i w}\left(\mathrm{~cm}^{-3}\right)$ & $5.25 \times 10^{13}$ \\
\hline Warm ion density times lifetime, $n \tau\left(\mathrm{cm}^{-3} \mathrm{~s}\right)$ & $5.14 \times 10^{12}$ \\
\hline Beam voltage $(\mathrm{kV})$ & 55 \\
\hline Trapped beam power (MW) & 51 (1244 A/41 keV) \\
\hline Injection angle (deg) & 65 \\
\hline Beam trapping fraction, $f_{t}$ & 0.94 \\
\hline Fusion power (MW) & 35.6 \\
\hline \multicolumn{2}{|l|}{ Neutral wall flux at } \\
\hline $\mathrm{R}=0.27 \mathrm{~m}, \Gamma_{\mathrm{n}}\left(\mathrm{MW} / \mathrm{m}^{2}\right)$ & 2.1 \\
\hline Central cell potential, $\phi_{e}(k V)$ & 25 \\
\hline
\end{tabular}


Table 2-6. Design levels for the tandem mirrors of the TDF end cells, without a stresm plasma.

\begin{tabular}{|c|c|}
\hline Parameter & Value \\
\hline \multicolumn{2}{|l|}{ Choke coil } \\
\hline Maximum magnetic field strength $(\mathrm{T})$ & 15 \\
\hline Plasma length (m) & 4.65 \\
\hline Plasma radius (m) & 0.45 \\
\hline Plasma density $\left(\mathrm{cm}^{-3}\right)$ & $4.25 \times 10^{12}$ \\
\hline Magnetic field at midplane $(T)$ & 1 \\
\hline Pumping $\mathrm{NBI}^{\star}$ & $80 \mathrm{kV},(92 \mathrm{~A} / 68 \mathrm{keV}) 6.3 \mathrm{~kW}$ \\
\hline Injection angle (deg) & 30 \\
\hline Barrier potential, $\phi_{b}(k V)$ & 20 \\
\hline \multicolumn{2}{|l|}{ Anchor coj1s } \\
\hline Plasma length (m) & 3.3 \\
\hline Plasma radius (m) & 0.28 \\
\hline Plasma density $\left(\mathrm{cm}^{-3}\right)$ & $8 \times 10^{12}$ \\
\hline Magnetic field at mirror (T) & 3 \\
\hline Magnetic field at midplane (T) & 1 \\
\hline Anchor cell potential, at a' (kV) & 20 \\
\hline Sloshing neutra? beams ${ }^{*}$ & $80 \mathrm{kV},(11.3 \mathrm{~A}) 0.68 \mathrm{~mW}$ \\
\hline Beam trapping fraction & 0.29 \\
\hline ECRH (total absorbed) (MW at $G H z$ ) & 1 at $35,0.560$ at 60 \\
\hline Hot electron temperature (keV) & 466 \\
\hline Warm electron temperature $(k \in V)$ & 21.2 \\
\hline Confining potential, $\phi_{e}(k V)$ & 12 \\
\hline Pumping NBI ${ }^{\star}$ & $40 \mathrm{kV},(14.8 \mathrm{~A} / 34 \mathrm{keV}) 500 \mathrm{~kW}$ \\
\hline
\end{tabular}

\footnotetext{
* Total powers, incident to both ends. Pumping beam requirements are for a 90\%:7\%:3\% jon source output species mix.
} 


\subsection{FUSION REACTOR CONOITION WALL LOADING}

In designiry TDF our major goal was to produce fusion reactor-like wall loading for engineering materials testing. The primary feature of this wall loading is the 14.7 - MeV neutron flux $\Gamma_{n} \geq 1.4 \mathrm{Mw} / \mathrm{m}^{2}$. A secondary feature is the front surface heat loading from exposure to the hot and halo plasmas, expected to average $<50 \mathrm{~W} / \mathrm{cm}^{2}$, but with locally much higher heat loads near $N B I$ as discussed in Appendix $D$.

\subsubsection{D-T Neutron Yield and Wall Loading}

The machine parameters 3 isted in Table 2-1 include a calculation of the D-T neutron yield and wall loading for the axisymetric central cell. The neutron wall loading has the form

$\Gamma_{n}\left(\frac{M W}{m^{2}}\right)=\frac{0.45 n^{2}<\sigma V>{ }_{D T} \pi r_{p}^{2}}{4 \times 2 \pi r_{W}} \times \frac{14.1 \mathrm{MeV} \times 10^{4}}{6.25 \times 10^{18}}$,

where

$n$ is total peak density in $\mathrm{cm}^{3}\left(n_{D}=n_{T}=n / 2\right)$, $<O V\rangle_{D T}$ is the calculated $D-T$ reactivity in $\mathrm{cm}^{3} / \mathrm{s}$, $r_{p}$ is plasma radius in $\mathrm{cm}$, $r_{W}$ is the wall radius in $\mathrm{cm}$.

The factor 0.45 comes from integrating the assumed cubic density profile $\left\{\alpha\left[\left(1-\left(r / r_{p}\right)^{3}\right]^{2}\right\}\right.$ over the plasma radius, and the final factor converts the reaction rate loading $/ \mathrm{cm}^{2}$ to $\mathrm{WW} / \mathrm{m}^{2}$. We based the initial calculation for $\langle 0 V\rangle_{D-T}$ on a simplified hot ion distribution function generated by considering the trapping and electron drag-dominated equilibrium for each of the three neutral beam input energy components separately to form a $50 \% D^{+}$and a 50\% $T$ hot reacting plasma with an average energy of $\bar{W} \sim 39 \mathrm{keV}$. Calculations of $\langle 0 V\rangle_{D-T}$ for this case made with the SIGMAV code developed by DeVoto and Slaughter ${ }^{28}$ yielded a hot-plasma reactivity of $<\sigma \mathrm{V}\rangle_{\mathrm{D}-\mathrm{T}} \sim 7.8 \times 10^{-16} \mathrm{~cm}^{2} / \mathrm{s}$. We found the hot ion $\mathrm{D}-\mathrm{T}$ fusion 
reaction with the $8 \%$ warm DCLC stabilizing deuterium ion background to be negligible. Therefore, for use with the total (hot and warm) plasma density $n$, the corrected average reactivity is $\langle\sigma /\rangle_{D-T} \sim 6.8 \times 10^{-16} \mathrm{~cm}^{2} / \mathrm{s}$ for a mean total plasma density average ion energy of $W \sim 36 \mathrm{keV}$.

This parlicular D-T reactivity for the hot plasma with $W \sim 39 \mathrm{keV}$ is in excellent agreement with previous calculations by Marx et al. ${ }^{29}$ for a simple mirror contained plasma with a mirror ration $R=5$. However, more recent Fokker-Planck calculations of $W$ for the TDF case show an average energy $W$ dependence on the warm-jon net confining potential $\phi_{C}$. For $\phi_{C}=4 \mathrm{kV}$, Rensink's Fokker-Planck calculations yield $W=29 \mathrm{keV}$, with a slightly lower $\Phi_{c}$ required for a self-consistent $f i t$ of ion losses to the stream current we used in the base case parameter set of Table 2-1. The calculations could be iterateo further; however, the resultant value of $I_{n}$ turns out to be about $1.4 \mathrm{~mW} / \mathrm{m}^{2}$ in either case; this is because a lower $W$ allows a higher $n$ (since $B_{C}-0.4$ is regarded as fixed) and $\langle 0 V\rangle_{D-T}$ is relatively insensitive to energy, near or above $40 \mathrm{keV}$. The base case line source neutron yield calculated for $D-T, D-D$, and $T-T$ reactions is shown as a function of $Z$ in Fig. 2-5.

\subsubsection{First Wall Thermal Loading}

The first wall in the central cell is also subject to substantial thermal radiation from its exposure to the hot reacting plasma. The front first-surface loading includes:

- An energetic neutral-particle flux from hot plasma ions undergoing charge-exchange on either of the attenuated neutral beams, on superthermal neutrals penetrating through the outer halo plasma, or on incompletely stripped impurities. We estimate the average heat loading to be from 10 to $30 \mathrm{~W} / \mathrm{cm}^{2}$ for expected vacuum conditions, with a strong dependence on wall positions near the NBI system and on halo attentuation of wail-recycled gas.

- Vacuum ultraviolet-line radiation estimated at an average of a few $\mathrm{W} / \mathrm{cm}^{2}$ at the wall. This radiation is caused mostly from the excitation of moderate- to high-z impurity ions that are partially stripped. Thus, such loading is strongly dependent on the 


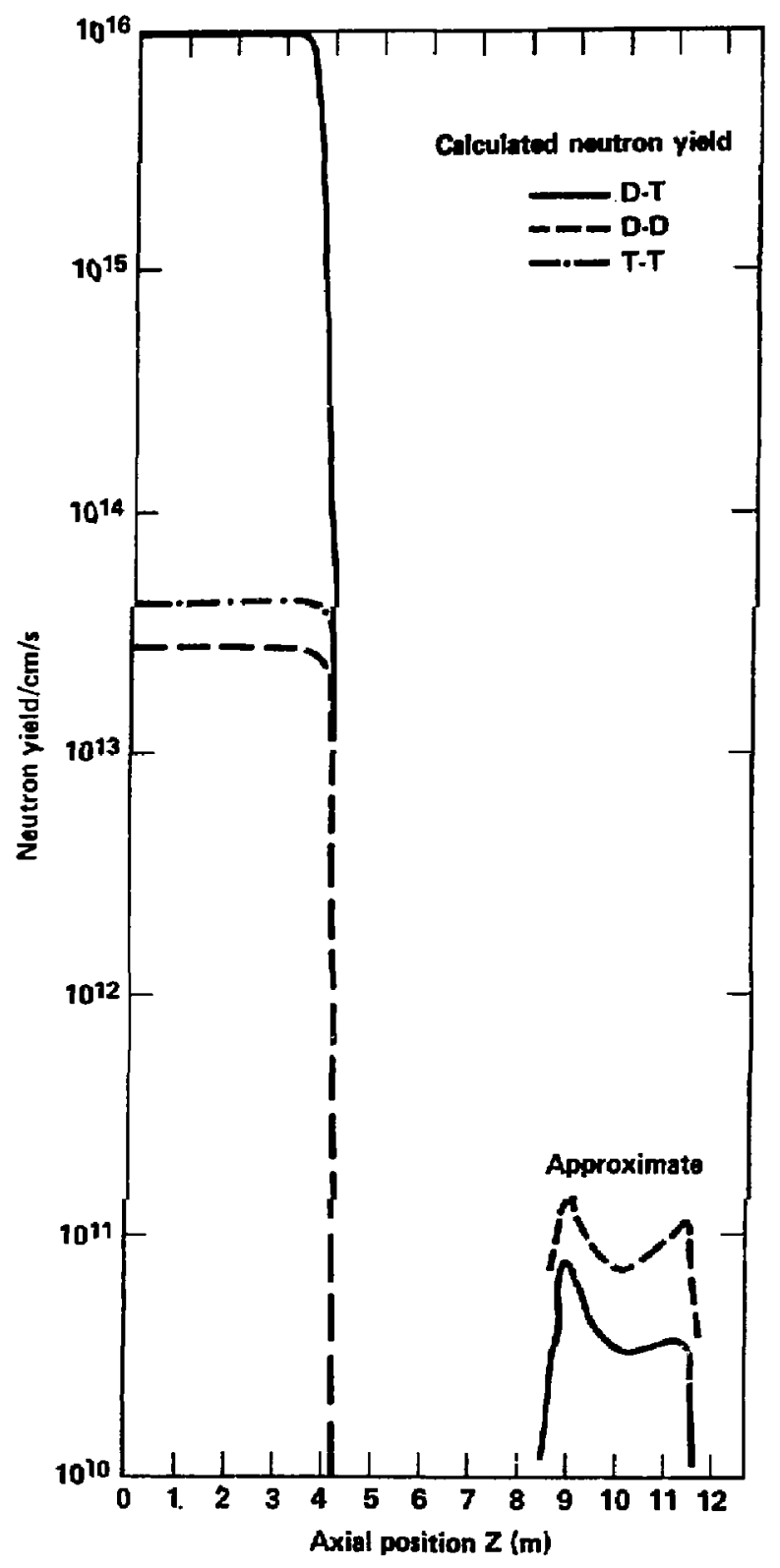

Figure 2-5. The line source neutron yield calculated for $D-T, D-D$, and $T-T$ reactions, as a function of $Z$. 
fractional impurity density, charge q-state, and $T_{e^{*}}$ The effective charge q-state is modified from expected coronal equilitrium by NBI and by any charge-exchange source for electron pickup. The oxygen impurity accumulation expected in TDF is discussed in Appendix G.

- Electron-cyclotron emission (ECE) from the hot electrons gyrating in the high magnetic field. Losses with expected self-absorbing and wall reflection are estimated to be a few $\mathrm{W} / \mathrm{cm}^{2}$. Power is proportional to $n_{e} T_{e} B^{2}$.

- Ordinary hot plasma electron bremstrahlung is predicted to be <] W/cmi proportional to $n_{i} n_{e} Z_{j}^{2}\left(T_{e}\right)^{1 / 2}$.

- Local high-power fluxes caused by forward-peaked neutral-beam charge-exchange neutral-beam "scattering" in regions near the remnant neutral heam dump and near ECRH horns (or refracted or reflected paths). Loading will depend on NBI design, divergence, and the plasma $f_{n} \cdot d l$. Total loads are likely to be several hundred $\mathrm{W} / \mathrm{cm}^{2}$ near neutral beam dumps. This topic is discussed further in Appendix $D$. The results there indicate an unexpectedly high local heat load requiring a larger $(75 \mathrm{~cm})$ radius first wall in the $\mathrm{NBI}$ input region.

APPENDIX A, COOE FORMULATION FOR THE TOF CASE

We used the MARS base code. 17 Its expression for the transition region charge-exchange pumping power is derived from the trapping current given by Futch and Lodestro. 30 In the form the MARS code uses:

$$
\begin{aligned}
& P_{\text {pump }}(M W)=4.49 \times 10^{-33} v_{t} \bar{n}_{p}^{2}(t) \frac{\left[1+0.0441 R_{t}\right]^{3.33} g_{p} f_{\text {ion }}}{P_{c x} T_{c}^{3 / 2}\left(g_{t}-1\right)} \\
& (x) \frac{g_{t}^{3.33}}{\left(g_{t}-1\right)^{2.33}} \frac{{ }_{A}}{A} E_{p u m p} \frac{\left(L_{t}+0.78 L_{g}\right)}{L_{t}},
\end{aligned}
$$


where

$v_{t}=$ the volume of the transition region $\left(\mathrm{cm}^{3}\right)$,

$n_{p}(t)=$ the passing ion density at the bottom of the transition region $\left(\mathrm{cm}^{-3}\right)$, with the bar above $n_{p}^{2}$ representing a

radial profile average using cubic profiles,

$\mathbf{R}_{t} \quad=$ the plasma mirror ratio between the barrier field and transition midplane field,

$f_{\text {ion }}=$ the factor 71.2 used to model the fact that some of the charge-exchange neutrals will be reionized before leaving the plasma,

$p_{c x}=$ the probability that an interaction between a beam particle and the background plasma is a charge-exchange event,

$T_{c}=$ the central celi warm ion temperature (kev'),

$9 \quad=$ the ratio of the traprad-plus-passing density to the passing density, with subscripi t the transition value and subscript $p$ the pump-beam location value $\left(g_{p}=1.16 g_{t}\right)$,

$A=$ atomic mass of the background plasma $\{a m u$,

$E_{\text {pump }}=$ the pump beam injection energy (kev),

$\mathrm{L}_{t} \quad=$ the transition region length $(\mathrm{cm})$,

$\mathrm{L}_{\mathrm{a}} \quad=$ the anchor region length $(\mathrm{cm})$.

The expression for the power required to sustain the sloshing ions at the thermal barrier may be written:

$P_{n b, a}(M W)=4.1 \times 10^{-32} \frac{\left(G_{b}-g_{b}\right)^{2} n_{p}^{2}(b) E_{i n j, a}, V_{b}}{A_{s} E_{i n j, a}^{3 / 2} \log _{10}\left(R_{e f f, s}\right)} \frac{B_{b}}{B_{a}}$,

sinere:

$G_{b}=$ the ratio of total ion density (passing + trapped + sloshing) to passing ion density,

$g_{b}=$ the same as $g_{p}$ and $g_{t}$ in $E q .(A-1)$ but at the barrier midplane, $n_{p}(b)=$ the passing ion density at the barrier midplane $\left(\mathrm{cm}^{-3}\right)$, $E_{i n j, a^{\prime}}=$ sloshing ion beam energy at the injection point (keV), 
$v_{b}=$ thermal barrier volume $\left(\mathrm{cm}^{3}\right)$,

$B_{b, a}=$ plasma magnetic field at the barrier (point $b$ ) and potential peak (point a),

A $\quad=$ atomic mass of sloshing ions (amu),

$E_{i n j, a}=$ sloshing ion energy at the potential peak (keV),

$R_{e f f, s}=$ the effective sloshing ion mirror ratio, including the effects of expelling potential.

The formula used to compute the power required to sustain the hot electrons is:

$P_{E C R H, b}($ NWW $)=1.52 \times 10^{-31}\left(1-F_{e c}\right) \frac{\bar{n}_{p}^{2}(b) E_{e h}^{-1 / 2}}{\log _{10}{ }^{R} \text { beff }}+P_{\text {sync }}$,

where the previously undefined symbols are:

$F_{\text {ec }} \quad=$ the cold electron fraction at the barrier,

$E_{\text {eh }} \quad=$ hot electron energy, (kev),

$R_{\text {beff }}=$ the effective hot electron mirror ratio,

$P_{\text {sync }}=$ the synchrotron radiation power, given by:

$P_{\text {sync }}($ MN $)=1.21 \times 10^{-19} B_{b, v a c}^{2} \bar{n}_{p}(b) v_{b}\left(\frac{E_{e h}}{10^{3}}+\frac{E_{\text {eh }}}{10^{3}}\right)^{2}$.

The power formula for the warm electrons which exist at the potential peak (point a) is:

$P_{E C R H, a}(M W)=5.85 \times 10^{-31} \frac{\overline{n_{e w}^{2}}(a) V_{b}\left(T_{e w}-T_{e c}\right)}{r_{e w}^{3 / 2}\left[\exp \left(\delta \phi_{a} / T_{e w}\right)-1\right]}\left(1+\frac{B_{b}}{E_{a}}\right)^{-1}$,

where

$n_{\text {ew }}(a)=$ warm electron density at point a $\left(\mathrm{cm}^{-3}\right)$,

$T_{\text {ew }}=$ warm electron temperature (keV),

$\mathrm{T}_{\text {ec }}=$ central cell electron temperature (keV),

$\delta \phi_{\mathrm{a}} \quad=$ the potential difference between the bottom of the thermal barrier and the potential peak. 
The power required to sustain the hot ion population in the central cell is the largest single requirement and is given by

$$
P_{n b, c c}(M W)=1.6 \times 10^{-22} \frac{\overline{n_{H,} c^{n} c c} E_{i n j, c} V_{c c}}{\left(n_{\tau}\right)_{H, c c}},
$$

where

$$
\begin{aligned}
n_{H}= & \text { hot ion density in the central cell }\left(\mathrm{cm}^{-3}\right), \\
n_{\mathrm{CC}}= & \text { total plasma density in the central cell }\left(\mathrm{cm}^{-3}\right), \\
E_{i n j_{3} \mathrm{C}}= & \text { average beam injection energy in the central cell (keV), } \\
V_{\mathrm{CC}}= & \text { central cell volume }\left(\mathrm{cm}^{3}\right), \\
\left(n_{\tau}\right)_{\mathrm{H}, \mathrm{CC}}= & \text { hot ion conf inement parameter }\left(\mathrm{s} / \mathrm{cm}^{3}\right), \text { computed from } \\
& \text { the Logan-Rensink model. } 20
\end{aligned}
$$


We have considered an example case in which the initial cordition places both the central and anchor cell betas at $1 / 10$ of their equilibrium values. Since the ratios are roughly equal, we set $g$ for the central cell and $G$ for the anchor cell at their equilibrium values for the initial conoitions. Based on pressure balance in the barrier, the initial, computed, hot electron density and temperature are similar to that in Elmo bumpy torus (EBT) operation, and those techniques might apply here.

Tables $B-1$ and $B-2$ show the set of cases run with the equilibrium code to model stariup. Notice that they do not mention time. The time scales are determined once the source strengths ar'e specified (or vice versa) to give the quoted $B$ and $g$ values.

Table B-1 defines the parameters of Table B-2. The first three steps increase barrier beta, $\beta_{b}$. This keeps the central cell and barrier densities approximately fixed (by keeping $g$ and $G$ fixed), while the added ECRH power at $b$ increases the hot electron energy $E_{\text {eh }}$ to almost $1 \mathrm{MeV}$. We need the slight increase in $g$ to keep $E_{\text {eh }}$ below $1 \mathrm{MeV}$. At this point the absoribed power needed to sustain the hot ions in the central cell $\left(P_{C C}\right)$ is quite modest, as is the power required to pump out the barrier and transition region $\left(P_{\text {pump }}\right)$. This is because the central cell density is stiji quite low, and both of these powers are proportional to the square of this density. The streaming current requirement $j_{s t}$ is aiso about an order of magnitude below the steady-state value. Notice that in this initial phase, the trapping fraction of the sloshing beam is quite low.

In the next three steps, which are virtually the same, we increase the density in the barrier by raising $g$ and $G$ at fixed barrier $B$. This lowers the hot electron energy and the warm elactron temperature and dramatically increases the ECRH powers required, but not above the steady-state powers. The central cell beam power and pumping power remain at modest values.

The last four steps show the sequence of events that occurs when the central cell $B$ is ramped up along with the barrier $B$ to the operating points. Comparing this with the data of Tables 2-1 and 2-2 shows that the first powers are incident for both ends, whereas these are absorbed for both enrs. Also note that since internally the code assumes no half - and third-energy beam components, the pump beam power is lower than that presented 
Table B-1. Definition of the parameters used in Table B-2.

Symbo 1

Definition

9

Ratio of the sum of the lucal trapped + passing ion densities to the passing ion density. It applies in the anchor and transition regions.

G Ratio of the sum of the local trapped + sloshing + passing ion densities to the passing ion density. It applies in the anchor region.

$B_{c} \quad$ Beta value in the central cell.

$B_{b} \quad$ Beta value in the anchor/barrier region.

$T_{\text {ew }}$

Tec Warm electron temperature at the potential peak (keV). Central cell electron temperature (kev).

Eeh Hot electron energy at the barrier midplane (keV). Warm plasma stream current required for stability (A). Neutral beam power in the central cell (MW).

$P_{\text {pump }}$ Plosh $P_{E C R H, a}$ The ECRH power required at the potential peak, point a $(k W)$. $P_{E C R H, b}$ The ECRH power required at the thermal barrier midplane, point $b(\mathrm{~kW})$.

* All powers are absorbed in plasma; end cell powers are for both ends. 
Tahle R-2, Values of the parameters used in o ThF-startup scenario,

\begin{tabular}{|c|c|c|c|c|c|c|c|c|c|c|c|c|c|}
\hline \multirow[b]{2}{*}{$\begin{array}{l}\text { Sequence } \\
\text { of steps }\end{array}$} & \multicolumn{11}{|c|}{ Farmeter values } & \multirow[b]{2}{*}{$\begin{array}{l}P_{E R C H, a} \\
\text { (HH) }\end{array}$} & \multirow[b]{2}{*}{$\begin{array}{l}P_{E R C H, b} \\
(K H)\end{array}$} \\
\hline & 9 & $\mathbf{G}$ & $B_{c}$ & $\mathrm{~A}_{\mathrm{h}}$ & $T_{(k e v)}^{T}$ & ${ }_{(k e v)}^{T}$ & $\begin{array}{l}\mathbf{E}_{\text {eh }} \\
\{k \in V)\end{array}$ & (A) & $\begin{array}{l}P_{\text {cc }} \\
\text { (H) }\end{array}$ & Ppump $_{(\text {pu) }}$ & $\begin{array}{l}P_{\text {slnsh }} \\
(k H)\end{array}$ & & \\
\hline \multicolumn{14}{|l|}{ Phase 1} \\
\hline 1 & 3 & 6 & 0.04 & 0.05 & 4.2 & 1.1 & 205 & 105 & 1.7 & 39 & $8(0.04)=$ & 9 & is \\
\hline 2. & 3 & 6 & 0.04 & 0.1 & 4,11 & 1,2 & 243 & 104 & 1.7 & 39 & $5.4(0.04)$ & 8.8 & 20.6 \\
\hline 3. & 3.8 & 7.6 & 0.04 & 0.25 & 3.7 & 1.1 & $96 \mathrm{~B}$ & 116 & 1.8 & 37 & $3.5(0.05)$ & 14 & 73 \\
\hline \multicolumn{14}{|l|}{ Phase II } \\
\hline 4. & 9 & 19 & 0.04 & 0.25 & 3.45 & 0.87 & 383 & 237 & 2.7 & 56 & $17.5(0.12)$ & 100 & 161 \\
\hline 5. & 9.75 & 18.5 & 0,04 & 0.25 & 3.43 & 0.86 & 351 & 253 & 2.8 & 59 & $20.4(0.13)$ & 121 & 180 \\
\hline h. & 10 & 20 & 0.04 & 0.25 & 3.54 & 0.85 & 349 & 261 & 7.9 & 61.5 & $21(0.13)$ & 125 & 182 \\
\hline \multicolumn{14}{|l|}{ Phase 11I } \\
\hline$?$ & 9.23 & 18.5 & 0.13 & 0.3125 & 3.95 & 1.7 & 151 & 966 & 15.6 & 536 & $152(0.33)$ & 734 & 789 \\
\hline 8. & 5.45 & 10.9 & 0.22 & 0.375 & 4.5 & 5.61 & 709 & $115 n$ & 26.2 & 907 & $126(0.31)$ & 501 & 526 \\
\hline 9. & 3.9 & 7.8 & n.31 & 0.4375 & 4.8 & 1.96 & 279 & $12 \mathrm{~F} 4$ &, 36.5 & 1650 & $100(0.3)$ & 379 & 378 \\
\hline 10. & 3 & 6 & 0.4 & 0.5 & 6.6 & 2.2 & 200 & 1536 & 50.1 & 2440 & $74(0.28)$ & 296 & 227 \\
\hline
\end{tabular}

Nate: The aad is met for the laraest power reautrement $\left(P_{c c}+P_{\text {pump }}\right.$ ). He need to accomplish further work where work where Phase $1 \mathrm{i}$ and Phase 111 meet. The $q$ = $q\left(R_{c}, R_{h}\right)$ noods to he optimised,

-The numbers in porentheses are the sloshing-hejm-trapping fractinns. 
in Table 2-2. To account for the 90\% full-, $7 \%$ half-, and $3 \%$ third-energy components for the special pump beams, we need to multiply the $P_{\text {plinip }}$ column by $3 / 2$; also, the ECRH powers must be multiplied by 2 to account for a conservative 50-50, extraordinary-ordinary mode $\mathrm{mlx}$ that comes out of the gyrotron and is divided by the $90 x$ deposition fraction. When the central cell $B$ is increased, both the central cell beams and transition pumping beams, which comprise the largest power requirement, are monotonically increasing during the buildup. This is consistent with our goal that startup should not take more power than the final state.

However, the sloshing beam power and the ECRH power pose a problem because they grow 1 arger than for the operating point by a factor of between 2.5 and 3.5. This occurs once the barrier density has built up, the electron energy has been reduced, and the central cell and barrier betas have been increased. It suggests that the central cell beta we chose for a given barrier beta was too large for our $g$ and $G$ values. Comparing cases 6 and 7 we see that the $g$ and $G$ values are virtualiy the same, but the ratio of $B_{c}$ to $B_{b}$ is larger by a factor of 2.6. (The barrier density in case 7 is also a factor of 2.6 larger.) The higher density suggests a lower electron energy; both translate to higher ECRH power.

A possible solution would be to raise $B_{c}$ more slowly, keeping $B_{c} / \beta_{b}$ fixed at fixed $G$ and $g$, until barrier beta reaches its equi\}jbrium value. Then we could raise $B_{c}$ while lowering $g$ and $G$ and keeping $B_{c} g$ and $B_{c} G$ constant. This would maintain the barrier parameters, and hence the sustaining powers, somewhat constant. These ideas should be tested further. 


\section{The Ion Source Species Mixture}

The arc plasma in an jon source that uses a molecular gas typically involves several ion states, with the specific ion species mix depending on the arc conditions and wali effects. For a posicive ion source invoiving $D_{2}$ or $T_{2}$ gas, the main full energy $w \equiv \mathrm{eV}_{0}$ extracted ion species are $0^{+}, D_{2}^{+}$, and $D_{3}^{+}$. Then total ion current $=I^{+}\left(f_{1}+f_{2}+f_{3}\right)$, where $f_{1} \equiv$ the fraction of $D^{+}, f_{2} \equiv$ the fraction of $D_{2}^{+}, f_{3} \equiv$ the beam fraction of $\mathrm{D}_{3}^{+}$. This is true when we ignore possible impurities and negative ions.

In the gas-filled charge-exchange neutralization celT, the $D^{*}$ fraction at the full energy can undergo electron pickup with the cross section $\sigma_{10}(w)$, and the resultant $0^{\circ}$ can undergo electron loss with the cross section $\sigma_{0 r}(w)$. The equilibrium neutral fraction is the product of the full enGrgy neutral fraction $F_{\infty}^{0} \equiv \sigma_{10}(w) /\left[\sigma_{10}(w)+\sigma_{01}(w)\right]$

and a gàs-ce 11 beam attenuator or thickness parameter $f_{\pi}(w) \equiv$ $1-\exp \left(-\sigma_{T} J n d l\right)$, where the total cross section $\sigma_{T}(w)=$ ${ }^{\sigma}{ }_{10}(w)+\sigma_{0}(w)$. This assumes we neglect small negative ion fractioris. The molecular ions must first undergo one or more dissociative collisians before forming a beam of half-energy or one-third-energy $D^{0}$ or $\mathrm{D}^{+}$compqnenis; each of which have a similar equilibrium neutral fraction at the appropriate energy. For relatively thick neutralizer cells in this case, we assume that the molecular ions completely dissociate into atomic fragments with relatively short mfp. Then each of the resultant fractional energy neutral beams have the same simple parameter for cell thickness and the same form:

$I^{0}(w)=I^{+} \times f_{j} \times F_{\omega}^{0}(w) \times f_{\pi}(w)$,

$I^{0}(n ; 2)=1^{+} \times f_{2} \times 2 F_{\infty}^{0}(w / 2) \times f_{\pi}(w / 2)$,

$I^{0}(w / 3)=I^{+} \times f_{3} \times 3 F_{\infty}^{0}(w / 3) \times f_{\pi}^{\pi}(w / 3)$. 
The average neutral beam energy $w=\sum p_{i} / \Sigma I_{i}^{0}$, with $p_{i}=I_{i} W_{i}$

for $i=1$ to 3 , or

$$
W=w_{0}\left[\frac{f_{1} F_{\infty}^{0}(w) f_{\pi}(w)+f_{2} F_{\infty}^{0}(w / 2) f_{\pi}(w / 2)+f_{3} F_{\infty}^{0}(w / 3) f_{\pi}(w / 3)}{f_{1} F_{\infty}^{0}(w) f_{\pi}(w)+2 f_{2} F_{\infty}^{0}(w / 2) f_{\pi}(w / 2)+3 f_{3} F_{\infty}^{0}(w / 3) f_{\pi}(w / 3)}\right] \text {. }
$$

It is common to operate the gas cell thickness fndl so that $f_{\pi}(w)=0.95$; this limits the neutralizer gas flow and minimizes scattering, which results in Ind $\sim 3 / \sigma_{T}(w)$. Evaluating the various cross sections for a typical 80-kV ion source mix of 80:15:5 for $D^{+}: D_{2}^{+}: D_{3}^{+}$, we get an average neutral beam energy of just over $60 \mathrm{keV}$, with full energy, half energy, and one-third energy $D^{\circ}$ currents of 55:29:16 respectively. Figure $\mathrm{C}-1$ shows curves for $\bar{w}$ for typica? species mix combinations of $D^{\circ}$ or $T^{\circ}$ beams.

The Neutral Beam Trapping Fraction

The factors involved in calculating the neutral beam trapping fraction $f_{t}$ are (1) the input neutral beam optics, ilucluding minimum divergence; (2) the chord length and interaction geometry of the plasma with the target beams; and (3) the appropriate relative velocity-dependent ionization cross sections $\sigma_{i j}$ and $\sigma_{e j}$ (plus a correction for one or more generations of $\sigma_{c x}$ events prior to final reionization).

We take the neutral beam profile to be a perfectly focused nearly plane parallel beam. It has a focal length of about $10 \mathrm{~m}$, whth an intrinsic beam of $1 / \mathrm{e}$ at a half-angle divergence of $\theta_{0} \simeq 3.5 \mathrm{deg} /\left(v_{0}\right)^{1 / 2}$ or 0.4 deg for an $80-k V$ NBI system. Fractional energy components have a slightly higher divergence, which we ignored. For the relatively long TDF plasma column target, we will consider only a centered-on-axis, 1-D beam focus (transverse to the axis) and a near-Gaussian minimum divergence. The divergence-limited, neutral beam current density then has the form

$$
j=j_{0} \exp \left[-\left(\theta ; \theta_{0}\right)^{2}\right]
$$




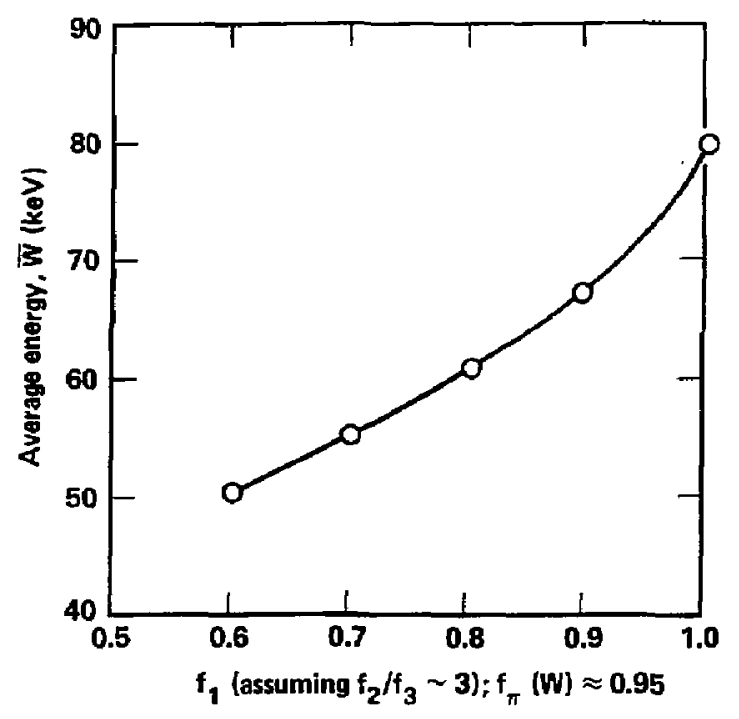

Figure $C-1$. Average $D^{0}$ energy for an $80-k V$ NBI system with a $D_{2}$ gas $f i l$ ] in the charge-exchange cell. 
For a large focal distance $L$ and for small angles, $\theta=y / L$, where $y$ is the transverse dimension in the plasia colum and $\theta_{D}($ Rad $)=$ $0.4 / 57.3 \sim 0.007$. For the case of $\mathrm{L} \sim 10 \mathrm{~m}$ and with $\mathrm{y}$ in $\mathrm{cm}$,

$j=j_{0} \exp \left[-(y / 7)^{2}\right]$

This simple Gaussian approximation to the beam makes it clear that a plasma with a radius of $10 \mathrm{~cm}$ will have $f_{t}<0.95$, simply because $t r$ "Gaussian wings of the beam are missing the plasma column. The compara y-sized beam and plasma target dimensions require that the beam attenuation calculation include the geometry of the beam current density profile, the $F^{\circ}$ asma target density profile, the changing beam chord length, and corrections for forward-angle-weighted charge-exchange in a relatively small geometry. Neglecting this charge-exchange correction, $f_{t}$ for an axisymetric plasma has the form

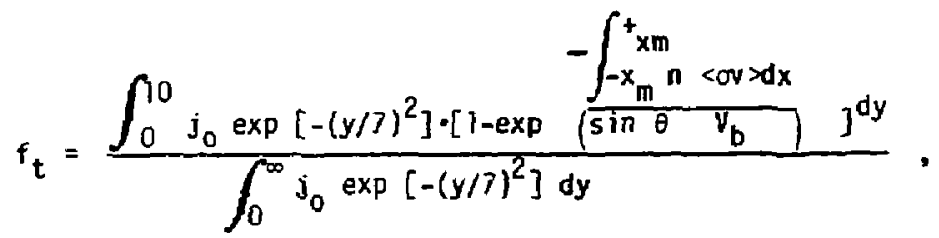

where

$$
\begin{aligned}
& \text { a } \quad \equiv \text { plasma radius, } \\
& \text { cov }\rangle_{i} \equiv\left(\left\langle\sigma_{i j} v_{i R}\right\rangle+\left\langle\sigma_{i e} v_{e}\right\rangle\right) \text {, } \\
& v_{b} \quad=\text { beam component velocity; } v_{i R} \text {, equals relative } \\
& \text { ion-reutral collision velocity, } \\
& \theta \quad=\text { angle of injection relative to machine axis, } \\
& x_{m}(y)=\left(a^{2}-y^{2}\right) 1 / 2 \text {, } \\
& n(y) \quad=n_{0}\left[1-\left(\frac{x^{2}+y^{2}}{a^{2}}\right)^{3 / 2}\right] \text {, } \\
& \int n \cdot d x=2 n_{0}\left\{\frac{3 \sqrt{a^{2}-y^{2}}}{a}-\frac{3 y^{2}}{8 a^{2}}\left[\sqrt{a^{2}-y^{2}}+\frac{\left.y^{2} \ln \left[\sqrt{a^{2}-y^{2}}+a\right) / a\right]}{a}\right]\right\} \text {. }
\end{aligned}
$$


Adding a correction for one or more generations of charge-exchange events prior to a final reionization definitely complicates the analytical calculation of the total $f_{t}$ for this very finite geometry. A more detailed Monte Carlo code calculation for this problem appears in Appendix $D$. 
APPENDIX D, A MONTE CARLO STUDY OF CHARGE-EXCHANGE FIRST WALL POWER DENSITIES FOR THE TOF CENTRAL CELL EEAMS

\section{Overview}

Sets of four central cell neutral beams at each end of TDF provide a total of $31.43 \mathrm{MW}$ of injected power. A fraction of these incident beans are ionized and trapped in the plasma. The remaining neutrals either penetrate with no interaction and are absorbed by the beam dumps or undergo charge-exchange with the plasma ions. The secondary neutral atoms formed in the charge-exchange process either escape and impact the first wall or undergo ionization or further charge-exchange interactions with the plasma. Therefore, considering particle and energy balance, a fraction of the incident beam power is absorbed in the plasma, a fraction escapes as shine-through to the beam dumps and the remainder appears as charge-exchange neutral bombardment of the surrounding first wall. Depending on the beam and plasma parameters, the charge-exchange neutral power to the first wall can be a significant fraction of the incident beam power. Thus, it is important to accurately predict the power density distribution of the charge-exchange flux over the first wall surface.

In general, modeling the steady-state neutral-beam/plasma/wa!1 system in three spatial dimensions is a very complex process. Various analytical and numerical investigations have incorporated simplifying assumptions regarding system parameters. 31,32 However, we must use Monte Carlo methods 33,34 if we wish to formally investigate charge-exchange phenomena in a threedimensional system and retain those phase-space anisotropies inherent in the interaction of a neutral beam with mirror-confined plasmas. Accordingly, we adapted a Monte Carlo neutral beam interaction code, MCNEUT, and employed it to model charge-exchange production and transport for the TDF central cell beams. In addition to computing general particle and power balances for neutral beam interaction, we mapped charge-exchange power density distributions over the first wall surface in both the axial and azimuthal directions. 


\section{Monte Carlo Modeling of the TDF Beam-Plasma Interaction}

MCNEUT was originally written at LLNL to moder the energy spectra and spatial origination positions of charge-exchange fluxes in the $2 X I I B$ minimum- $B$ mirror device. 35 The authors configured the code for single-species beams on single-species plasmas and modified it later to accommodate multi-species beams on multi-species plasmas and to allow for more than one incident beam direction.

The code follows many neutral particle histories in three dimensions from their emission through a target plasma, which is represented by a bi-Maxwellian distribution function in steady-state. We simulate ionization and charge exchange events by Monte Carlo techniques and then follow descendent generations of neutrals until each particle is either ionized and integrated into the background plasma or escapes and bombards the first wall.

For the TOF central cell neutral beams, we modeled the plasma as a cylinder $5 \mathrm{~m}$ long with a $0.10 \mathrm{~m}$ radius. We configured four $80-k e V$ neutral beams spaced 50 deg apart to subtend an angle of 65 deg relative to the z-axis. The intersection was at the midpoint of the 5-m-long cylindrical plasma, $2.5 \mathrm{~m}$ from one end. For convenience, this intersection point is designated as $z^{\prime}=0$ for defining the axial location of charge-exchange flux distributions, different from the $z=0$ datum coordinate at the center of the machine. We portioned the target plasma radially into 32 annular zones, assuming constant plasma properties in each. Escaping charge-exchange neutrals were scored on a cylindrical first wall detector grid with 32 azimuthal bins in the $\theta$ direction and 20 axial bins in the $z^{\prime}$ direction; the bin widths were $\Delta \theta=10 \mathrm{deg}$ and $\Delta z^{\prime}=5 \mathrm{~cm}$, respectively.

Table D-1 lists the input parameters we employed in the code. Note that the plasma density profile is Gaussian, whereas the TDF density profile is cubic with a plasma radius of $10 \mathrm{~cm}$. Accordingly, we chose the scale length of the Gaussian profile such that, when it was normalized to the same peak (on-axis) number density and some radial cutoff, both profiles yielded the same plasma number density per unit length in the z-direction. One other feature should be noted: the incident beams are modeled as right-circular cones emanating from point sources with a divergence half-angle defining the standard deviation of a Gaussian beam profile. We chose the distance from 
Table $0-1$. Beam-plasma first-wall parameters for the TOF charge-exchange wall flux computation.

\begin{tabular}{|c|c|}
\hline Parameter & Value \\
\hline Number of central cell beans at each end & 4 \\
\hline Injection angle (deg) & 65 \\
\hline Angle between beams (deg) & 50 \\
\hline Beam energy at full energy component (kev) & 80 \\
\hline Beam fractions at $f u l l, 1 / 2,1 / 3$ energy components $(x)$ & $55,29,16$ \\
\hline Beam species & D-T \\
\hline Injected particle current per beam (A) & 131 \\
\hline Total injected current at each end (A) & 525 \\
\hline Beam profile & Gaussian \\
\hline Beam source--plasma distance $(m)$ & 5.55 \\
\hline Beam half-angular divergence (deg) & 0.4 \\
\hline Cylindrical plasma length (m) & 5 \\
\hline Beam intersection point from one end (m) & 2.5 \\
\hline Plasma radius $(\mathrm{cm})$ & 10.0 \\
\hline Plasma profile & Gaussian \\
\hline Plasma radial scale length $\left(c_{m}\right)$ & 10.0 \\
\hline Prasma density $\left(\mathrm{cm}^{-3}\right)$ & $6.5 \times 10^{14}$ \\
\hline Temperature perpendicular to the magnetic field (keV) & 34 \\
\hline Temperature parallel to the magnetic fjeld (keV) & 4 \\
\hline Electron temperature (keV) & 2.1 \\
\hline On-axis magnetic field strength $(T)$ & 4.5 \\
\hline Number of annular plasma zones & 32 \\
\hline Plasma radial zone width $(\mathrm{cm}\rangle$ & 0.31 \\
\hline Cylindrical radius of first wall (cIn) & 25 \\
\hline Dimensions of first wall detector grid & $32 \times 20$ \\
\hline Grid azimuthal bin width (deg) & 10 \\
\hline Grid axial bin width $(\mathrm{cm})$ & 5 \\
\hline Number of neutral histories & 40,000 \\
\hline
\end{tabular}


each beam point source to the $z^{\prime}=0$ point on-axis such that, with a divergence half-angle equivalent to the expected beam divergence of 20.4 deg, less than $7 \%$ of the beam footprint fell outside the plasma diameter.

Results

Table D-2 summarizes overall particle and power balances as determined by MCNEUT for the input shown in Table 0-1. Note that these results are for the four central celi beams at one end of the machine. Note carefully the definitions of trapping fraction, total trapped current and net trapped current. The Monte Carlo statistics for these integral quantities are $<0.6 \%$.

A.3so note that the ratio of the net trapped current to incident current does not equal the ratio of the net trapped pnis to the incident power. This is because an incident beam particle of fixed energy can charge-exchange with plasma ions that have a distribution of energies. Since some of these secondary neutrals can charge-exchange or escape before becoming ionized, this will, in general, result in a plasma energy trapping that does not scale directly with the particle trapping. Finally note that, because of the model we used for the neutral beams (i.e., conical Gaussian distributions emitted from a point source), the particle and energy balances do not necessarily coincide with those calculated earlier in this physics section. There, we assumed rectangular Gaussian beam footprints of 2$] \times 0.14 \mathrm{~m}$, whereas the Monte Carlo model employs circular Gaussian beam footprints with diameters of 20. 14.

Besides the integral quantities in Table 1, we obtained charge-exchange power density distributions at the $32 \times 20$ detector grid on the first wall surface. Figure $0-1$ is a polar plot of the charge-exchange power density distribution in the $x y$ plane for one of the four beams at a $z^{\prime}$ value of -0.025 $\mathrm{m}$; in other words, this is the azimuthal variation of charge-exchange power density over the first wall at an axial position displaced $2.5 \mathrm{~cm}$ from the beam intersection point at $z^{\prime}=0$. The direction of the incident beam on this plot is at $\theta=90 \mathrm{deg}$. Also, because the beam injection angle is 65 deg relative to the machine $Z$ axis, this incident beam on this plot is inclined 25 deg out of the $x y$ plane of the figure. Figure $0-2$ is the corresponding polar plot when all four beams are included. It shows the four individual distributions and their composite distribution. The maximum power density is $22369 \mathrm{~W} / \mathrm{cm}^{-2}$ at $\theta=185 \mathrm{deg}$. 
Table D-2. Particle and power balances for the TDF cenitral-cell beams, determined from four beams at one end of the device.

\begin{tabular}{|c|c|}
\hline Measurement & Result \\
\hline \multicolumn{2}{|l|}{ Particle balance $\left(s^{-1}\right)$} \\
\hline Incident current & $3.27 \times 10^{21}$ \\
\hline Trapping fraction & 0.99 \\
\hline Total trapped current & $3.24 \times 10^{21}$ \\
\hline Net trapped current ${ }^{c}$ & $2.66 \times 10^{21}$ \\
\hline \multicolumn{2}{|l|}{ Power balance (MW) } \\
\hline Incident power & 31.4 \\
\hline Shine-through power to dumps & 0.33 \\
\hline Charge-exchange power to walls & 4.0 \\
\hline Net trapped power & 27.0 \\
\hline $\begin{array}{l}\text { Fraction of incident partic } \\
\text { undergo at least one interactio } \\
\text { exchange or ionization) with th } \\
b \text { Number of particles/s that } \\
\text { least one interaction (charge-e } \\
\text { ionization) with the plasma. } \\
c \text { Number of particles/s that } \\
\text { and become trapped in the plasm } \\
\text { equivalent to a fueling rate. }\end{array}$ & $\begin{array}{l}\text { les thai } \\
\text { (charge- } \\
\text { plasma. } \\
\text { indergo at } \\
\text { change or } \\
\text { ire ionized } \\
\text { This is }\end{array}$ \\
\hline
\end{tabular}




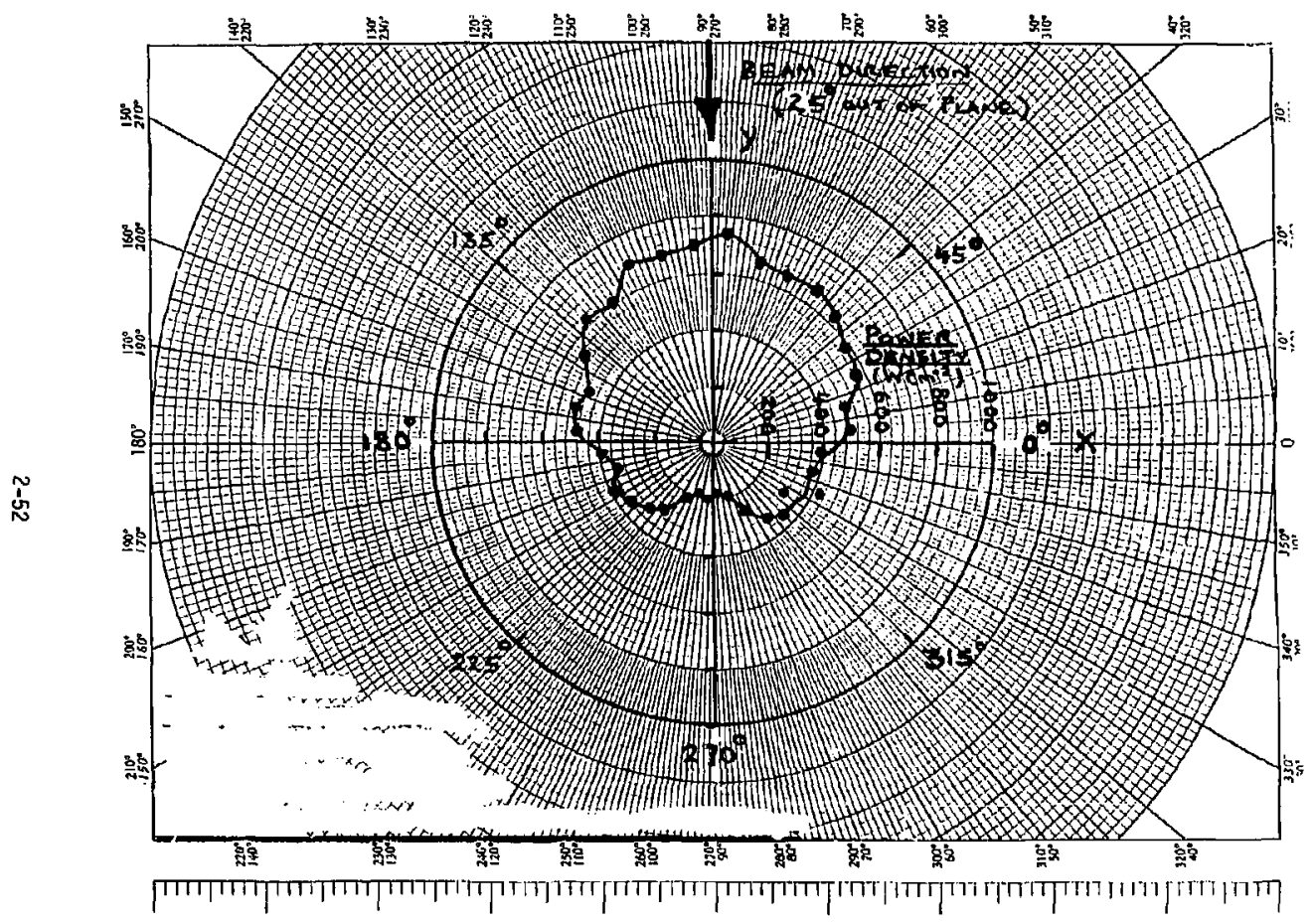

Figure $D-1$. Polar plot of charge-exchange wall flux in the $x-y$ plane for one of four $D-T$ beams on D-T plasma. The beam direction is 25 deg out of plane and $Z=-0.025 \mathrm{~m}$. 


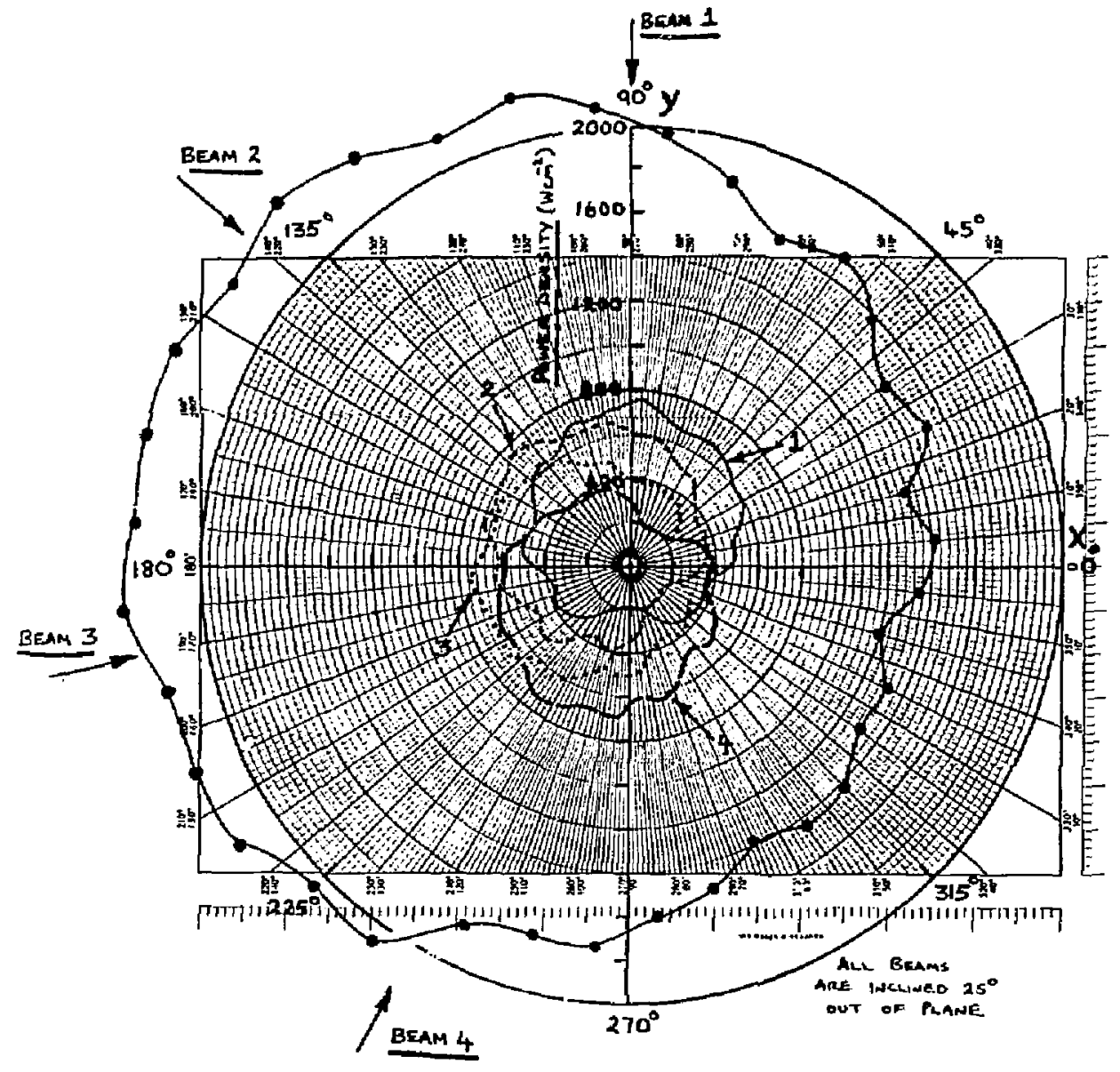

Figure D-2. Composite polar plot of charge-exchange wall flux in the $x-y$ plane for all four $D-T$ beams on D-T plasma. The beams are 25 deg out of plane and $z=-0.025 \mathrm{~m}$. 
From the code output, it is possible to construct polar plots similar to Fig. D-2 for each of the 20 axial grid positions along the first wall surface. However, it is mare instructive to pre-process this data and select only the maximum, minimum, and average charge-exchange power densities at each axial position and then plot these as functions of the corresponding $z$ ' coordinate. Accordingly, these data are shown in Fig. D-3. Peak values of the maximum, average, and minimum power density distributions are 2380 , 1875, and $1270 \mathrm{~W} / \mathrm{cm}^{-2}$ at the axial positions of $z^{\prime}=-2.5,-1.5$ and $-0.5 \mathrm{~cm}$, respectively.

\section{Discussion of Results}

One of the major features of the charge-exchange wall flux for the single beam incident on a thick target such as the central cell of TDF is the strongly backward-peaked distribution; in other words, maximum charge-exchange power densities occur at $2180 \mathrm{deg}$ relative to the incident beam direction. It is also interesting to note that the distribution is quite symmetric about the $y$-axis. This is to be expected in view of the incident beam direction. The slight deviations from true symmetry are, of course, attributable to Monte Carlo statistics ( $26 \%$ here).

The strong backward peaking of the single beam distribution in the composite has been somewhat ameliorated by the superposition of four beams 50 deg apart. However, the composite wall flux is still dominant in the general backward direction. Note that when the machine passes through startup, neutral fluxes progress from being strongly forward-peaked at low plasma densities to backward-peaked at full plasma conditions.

This backward peaking should be contrasted with the forward peaking observed $^{34}$ in the Mirror Advanced Reactor Study (MARS) for the pump beam charge-exchange distributions. ${ }^{36}$ Generally, for a single charge-exchange interaction, the product neutra] is emitted in the forward direction because the charge-exchange cross section is large for particle pairs with small relative velocities. 34 However, those forward-peaked neutrals directed into the bulk of a dense plasma have a strong chance of undergoing a further interaction--either charge-exchange or ionization. In addition, the incident neutral beam will be attenuated exponentially in the plasma; thus, the primary 


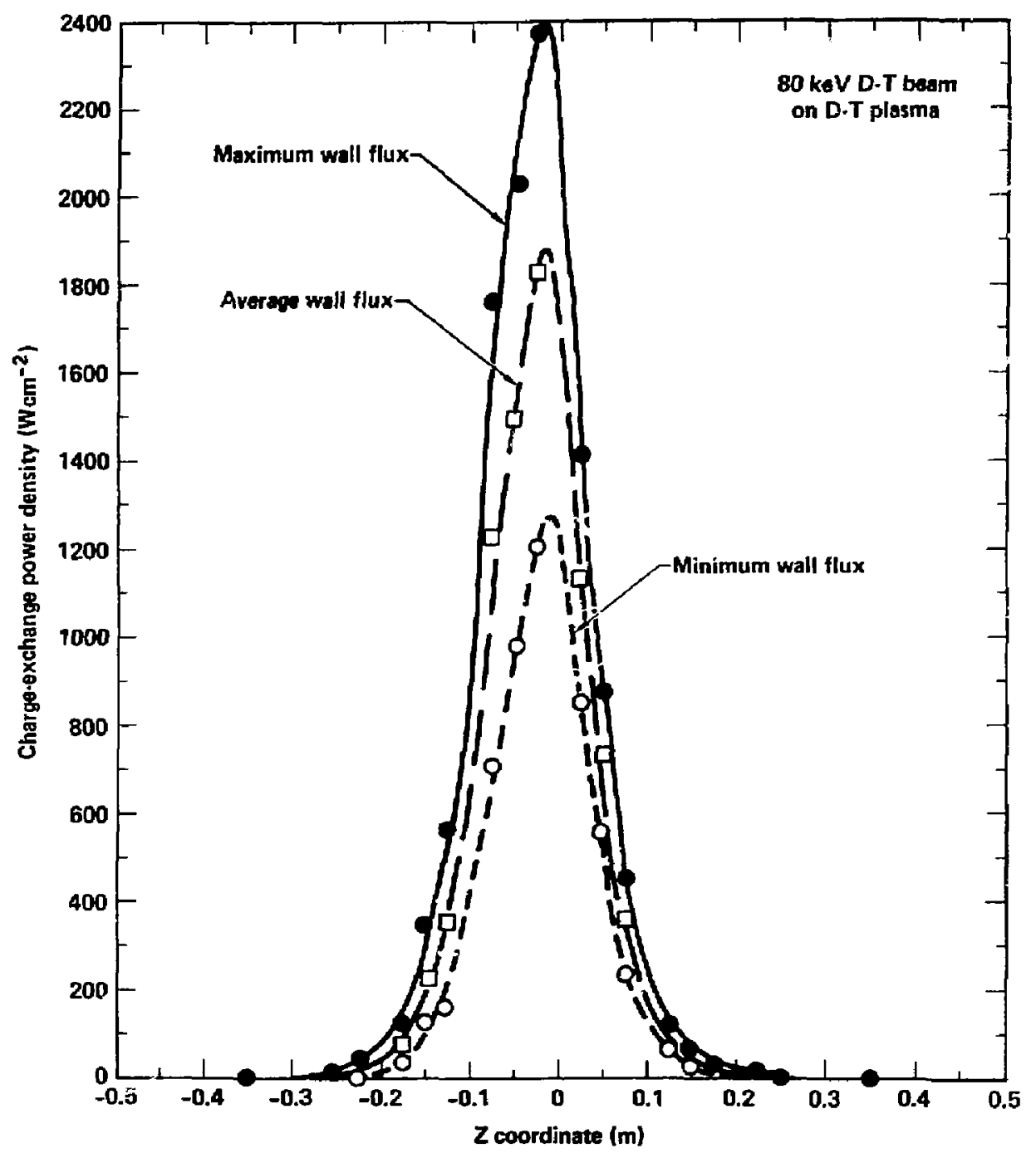

Figure D-3. Plot of an 80-keV beam on D-T plasma. 
charge-exchange production rate decreases along the chord length of the incident beam through the plasma. Therefore, the polar charge-exchange wall distribution is generally forward-peaked for a high energy neutral beam, a low plasma line density, or both. Conversely, for lower energy beam/higher plasma line density combinations, the wall distribution tends toward a maximum in the backward direction. As the beam trapping fraction approaches 100\%, strong backward peaking would be expected. This would then account for the backward peaking of the TDF charge-exchange wall distributions compared with the forward peaking in the MARS reactur. The MARS plasma density is only $1 \times 10^{14} \mathrm{~cm}^{-3}$, compared with $6.5 \times 10^{14} \mathrm{~cm}^{-3}$ for TDF. In addition, the MARS neutral beams are $97 \mathrm{keV}$ (negative ion) witr no half-or third-energy components, ${ }^{36}$ whereas the TOF beams are $80 \mathrm{keV}$ (positive ions) with appreciable half - and third-energy components (29\% and $16 \%$ respectively). Therefore, the bulk of the TDF charge-exchange neutrals are produced on the beam side of the plasma, and forward-directed products are strongly reabsorbed.

A significant feature of the axial plots of maximum, minimum, and average charge-exchange power densities is the tendency of the distributions toward peaks. Again, in comparison with the MARS reactar, TDF has considerably less total charge-exchange power loss to the walls $14.077 \mathrm{MW}$ from each end compared with 19.28 MW for MARS). However, in MARS this power is distributed over a greater axial extent in the z-direction, resulting in lower peak values of the maximum wall flux ( $7705 \mathrm{~W} / \mathrm{cm}^{-2}$ compared with $2380 \mathrm{~W} / \mathrm{cm}^{-2}$ for TDF). Again, this can be explained by the different beam/plasma conditions already discussed and by noting that the temperatures perpendicular and parallel to the magnetic field $\left(T_{1}\right.$ and $T_{11}$ ) in MARS are 58 and $59.5 \mathrm{keV}$, respectively, compared with 34 and $4 \mathrm{keV}$ in TDF. Clearly, there is a high probability of producing a charge-exchange neutral in ToF with a direction closely normal to the z-axis. These factors all contribute to the sharpness and the very high peak values of the TDF axial distributions. Fu:ther details on charge-exchange wall fluxes in the HARS reactor can be found in Ref. 34 .

The combination of these high-peak charge-exchange power densities and predominantly backward-directed emissions is rather unfortunate for TOF. At least in the case of forward-directed distributions, maximum charge-exchange flux densities go toward the beam dump surfaces. Clearly, heat loads of nearly $2400 \mathrm{~W} / \mathrm{cm}^{-2}$ are too large for even near-term high-heat flux technologies. Beam dumps based on the "hypervaporation" principle are 
currently being incorporated on the JET tokamak for heat loads of $27-15 \mathrm{~kW} / \mathrm{cm}^{-2}$ under quasi steady-state ( $\left.20 \mathrm{~s}\right)$ conditions. 37 However, such dumps have rather bulky configurations and could not be integrated in the first wall area of TOF, where space is at a premium. In practice, heat loads to water-cooled first wall surfaces should be limited to no more than $500 \mathrm{~W} / \mathrm{cm}^{-2}$. In the STARF IRE tokamak reactor design for example, maximum surface heat loads to the water-cooled limiter were restricted to $2240 \mathrm{~W} / \mathrm{cm}^{-2}, 38$

An alternative to reconfiguring beam parameters or geometries to reduce the peak heat loads to acceptable levels is to simply increase the radius of the first wall from its original value of $25 \mathrm{~cm}$. This would be necessary only in the vicinity of the high charge-exchange power densities and therefore would not be detrimental to neutron wall loading levels in the bulk of the central cell.

Accordingly, we re-ran the beam/plasma parameter set with two new values for the first wall radius: 50 and $75 \mathrm{~cm}$. Figure $0-4$ is a plot of the resulting maximum charge-exchange heat loads at each axial position as a function of the $z^{\prime}$ coordirate. For comparison we show the axial distribution at the original wall radius of $25 \mathrm{~cm}$. Note that the peak values have been reduced considerably from $2400 \mathrm{~W} / \mathrm{cm}^{-2}$. In fact, these reductions, caused by the distributed nature of the charge-exchange emission, are greater than the $1 / r$ scaling expected from a line source.

One other interesting feature of $\mathrm{Fig} . \mathrm{O}-4$ is that the wall fluxes at the wings of the distributions are actualiy greater for the larger wall radii. This can be explained by considering charge-exchange neutrals produced by neutral beam interactions in a given $x y$ plane and emitted at large angles relative to this plane. If these neutrals are to contribute to the charge-exchange flux at a given $2^{\prime}$ coordinate in the wings of the distribution, they must travel thruugh a larger plasma chord length to bombard the wall at the smaller radii than when bombarding the larger wall radi $i$ at the same $z$ ' coordinate. Thus, for coordinates sufficiently removed from $z^{\prime}=0$, lower attenuation within the plasma compensates for the apparent loss in intensity caused by the increased wall radius. In the wings it is obviously preferable to keep the wall radius relatively small. 


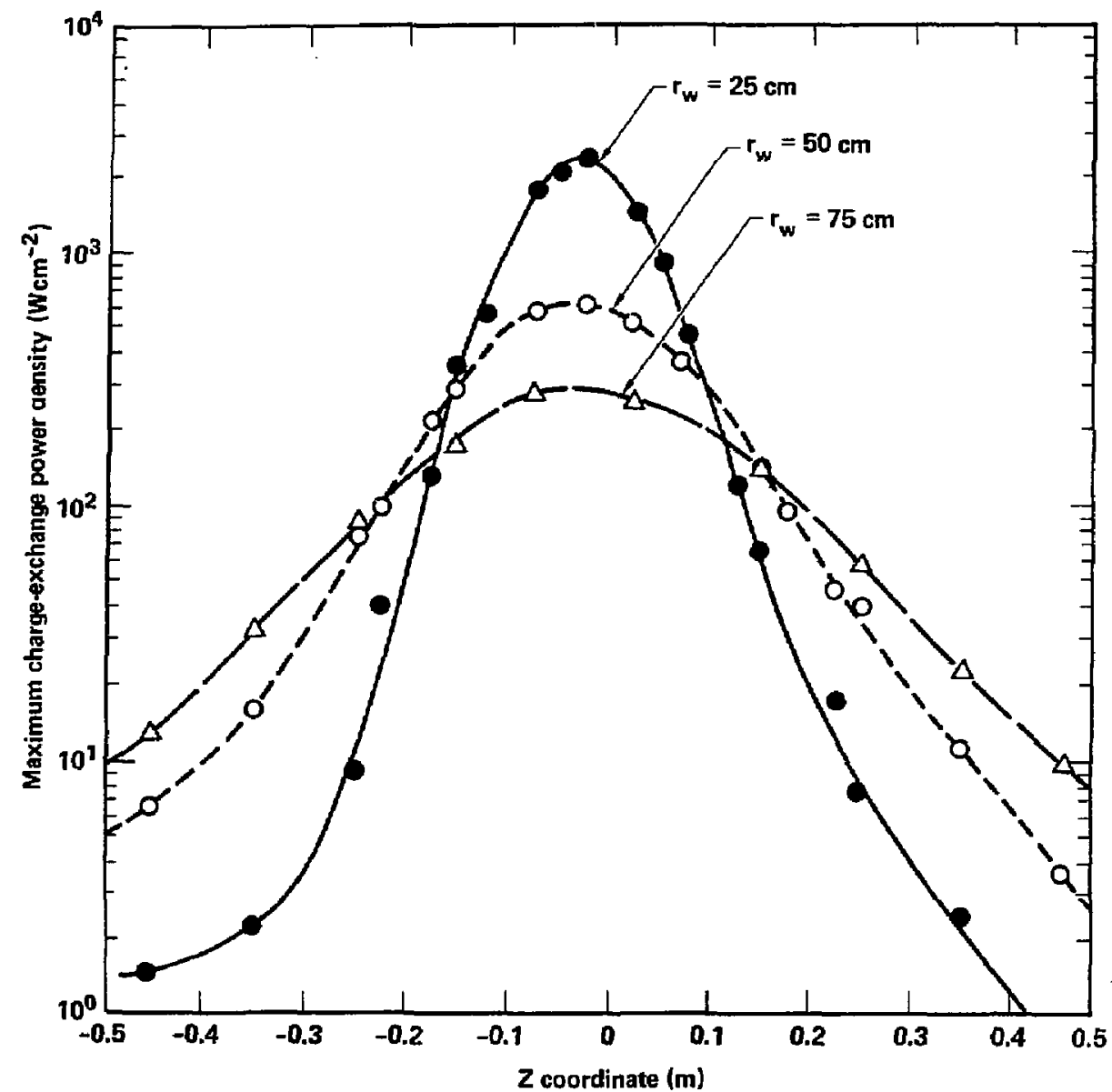

Figure 0-4. U-T beams on D-T plasma. The maximum charge-exchange wall fluxes with $\Gamma_{w}$ as a parameter. The beam injection point is here defined as $Z=0.0$. 


\section{Conclusions}

In conclusion, Monte Carlo calculations have shown that

- Peak charge-exchange power densities from the TDF central cell neutral beams are about $2400 \mathrm{~W} / \mathrm{cm}^{-2}$ for a first wall radius of $25 \mathrm{~cm}$.

- The heat loads have a predominantly backward-peaked distribution.

- The total charge-exchange power loss from the plasma is $4.077 \mathrm{MW}$ at each end of the device.

- One effective method of reducing the peak power densities to the first wall is to increase the wall radius from $25 \mathrm{~cm}$ to at least $75 \mathrm{~cm}$ in the vicinity of $z^{\prime}=0$ and taper it back to $25 \mathrm{~cm}$ at axial coordinates where heat lozds have fallen to acceptable levels.

- Such a revised design would keep maximum charge-exchange heat loads to less than $300 \mathrm{~W} / \mathrm{cm}^{-2}$ and could be accommodated by fairly converitional water-cooled first wall designs. 
APPENDIX E, PELLET FUELING CALCULATIONS

The requirements for warm ion flow stabilization are given in Table $E-1$. To satisfy them, we must inject $800 \mathrm{~A}$ of deuterium deep enough that warm ions exist over more than half of the plasma volume. Deuterium gas injectors can provide warm ions near the plasma's surface only. Frozen pellet injectors, such as those currently under development, have the potential of meeting the plasma penetration requirement of $>0.35 \mathrm{a}$, where a is the plasma radius.

Large pellets will penetrate deeper than small pellets, but, as the table indicates, the pellet size is limited to $10 \%$ of the ion population in the central cell. The frozen deuterium pellet can therefore be only about $0.6 \mathrm{~mm}$. We can also increase depth of penetration by injecting into the neck of the plasma near the throat of the central cell, where the plasma radius is only $5.5 \mathrm{~cm}$. However, coil restraints limit the injection point to the thick part of the neck, where the plasma radius is about $8 \mathrm{~cm}$. Since these two avenues posed problems, we explored the effect of increased pellet velocity, performing the andlys is described below.

We used a plasma physics transport code (Ref. 21) to investigate the ablation of frozen pellets of several sizes injected into the plasma at several velocities. Figures $E-1$ and $E-2$ are typjcal results that show the effect of pellet ablation on the plasma density for an acceptable and unacceptable size pellet. The densities were taken immediately after the complete pellet ablation. The perturbation disappears before the next pellet arrives a few ms later.

Figure E-3 shows some normalized parameters as functions of the plasma depth. At the plasma surface wilere $r=a$, tite parameters have a zero reference. In the center where $r=0$, they are maximum. The plasma has a cubic density distribution that determines the cumulative plasma mass distribution from the outside of the plasma to the center. Note that half of the volume lies between the outside of the plasma and $r=0.7 \mathrm{a}, 1 . e$. , the pellets must penetrate $30 \%$ of the plasma radius to directly influence $50 \%$ of the plasma volume. It also shows that the pellets need to penetrate $43 \%$ of the plasma radius to directly influence half of the plasma mass.

Figure E-A shows the needed pellet velocity in relation to the cumulative plasma volume and plasma mass of $\mathrm{Fig} . \mathrm{E}-3$. The three velocity curves correspond to the expected variation in pellet mass $( \pm 10 \%)$. It is evident 
that the lightest pellet with a velocity of $1500 \mathrm{~m} / \mathrm{s}$ will directly supply $60 \%$ of the plasma volume and $43 \%$ of the plasma mass. This more than meets the minimum requirements. It is also apparent that a pellet velocity of $2000 \mathrm{~m} / \mathrm{s}$ will directly supply more than $66 \%$ of the plasma volume.

Pellet injectors currently under development reach about $1200 \mathrm{~m} / \mathrm{s}$ and have the potential of achieving injection velocities of $2000 \mathrm{~m} / \mathrm{s}$. 
Table E-1. Pellet injector requirements for the central cell.

\begin{tabular}{|c|c|}
\hline Continuous operation fusion power(MN) & 20 \\
\hline Hot ion confinement time, $\mathrm{T}$ (ms) & 14 \\
\hline Plasma length (m) & $\sim 8$ \\
\hline \multicolumn{2}{|l|}{ Plasma radius, $r_{p}(\mathrm{~cm})$} \\
\hline$Z=0$ (at midfield of central cel1) & 10 \\
\hline $2= \pm 2.0 \mathrm{~m}$ & 10 \\
\hline$z= \pm 3.6 \mathrm{~m}$ & 8.5 \\
\hline$Z= \pm 4.0 \mathrm{~m}$ & 5.5 \\
\hline Peak ion density, $n_{0}\left(\mathrm{~cm}^{-3}\right) *$ & $6.5 \times 10^{14}$ \\
\hline \multicolumn{2}{|l|}{ Flat temperature distribution } \\
\hline Mean hot ion energy, $w_{i h}$ (keV) & 37 \\
\hline Electron temperature, $\mathrm{T}_{\text {ec }}(\mathrm{kev})$ & 2.1 \\
\hline Helium generation rate $\left(s^{-1}\right)$ & $7 \times 10^{18}$ with $1.14 \mathrm{~A}$ at $0.171 \mathrm{~g} / \mathrm{h}$ \\
\hline Fractional burnup $\left(T_{2}\right)$ & $7 \times 10^{-4}$ of $T_{2}$ to neutral-beam \\
\hline \multicolumn{2}{|l|}{ injectors } \\
\hline $\begin{array}{l}\text { Tritium fueling rate to neutral- } \\
\text { beam injectors }\left(s^{-1}\right)\end{array}$ & $1.01 \times 10^{22}$ with $1620 \mathrm{~A}$ at $182 \mathrm{~g} / \mathrm{h}$ \\
\hline $\begin{array}{l}\text { Deuterium fueling rate to } \\
\text { neutral beam injectors }\left(\mathrm{s}^{-1}\right)\end{array}$ & $1.01 \times 10^{22}$ with $1620 \mathrm{~A}$ at $120 \mathrm{~g} / \mathrm{h}$ \\
\hline $\begin{array}{l}\text { Deuterium fueling rate } \pm 0 \\
\text { pellet injectors }\left(\mathrm{s}^{-1}\right)\end{array}$ & $0.5 \times 10^{22}$ with $800 \mathrm{~A}$ at $58 \mathrm{~g} / \mathrm{h}$ \\
\hline $\begin{array}{l}\text { Fraction of plasma ions allowed } \\
\text { in a pellet }(\%)\end{array}$ & $\leq 10 \%$ \\
\hline
\end{tabular}

Pellet penetration of the plasma radius (cm) $\geq 0.35 a$

*Density distribution $n(r)=n_{0}\left[1-(r / a)^{3}\right\rceil$, where $a=r_{p}$. 


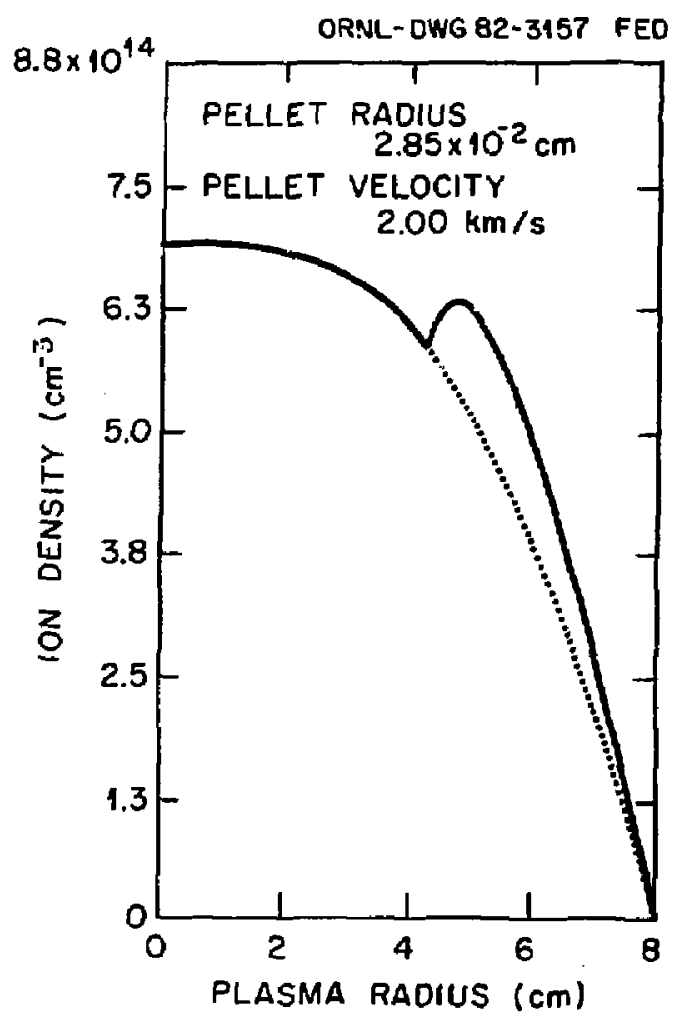

Figure E-1. Pellet deposition in the TDF plasma where the plasma radius is $8 \mathrm{~cm}$ and the pellet diameter is $0.57 \mathrm{~mm}$. 


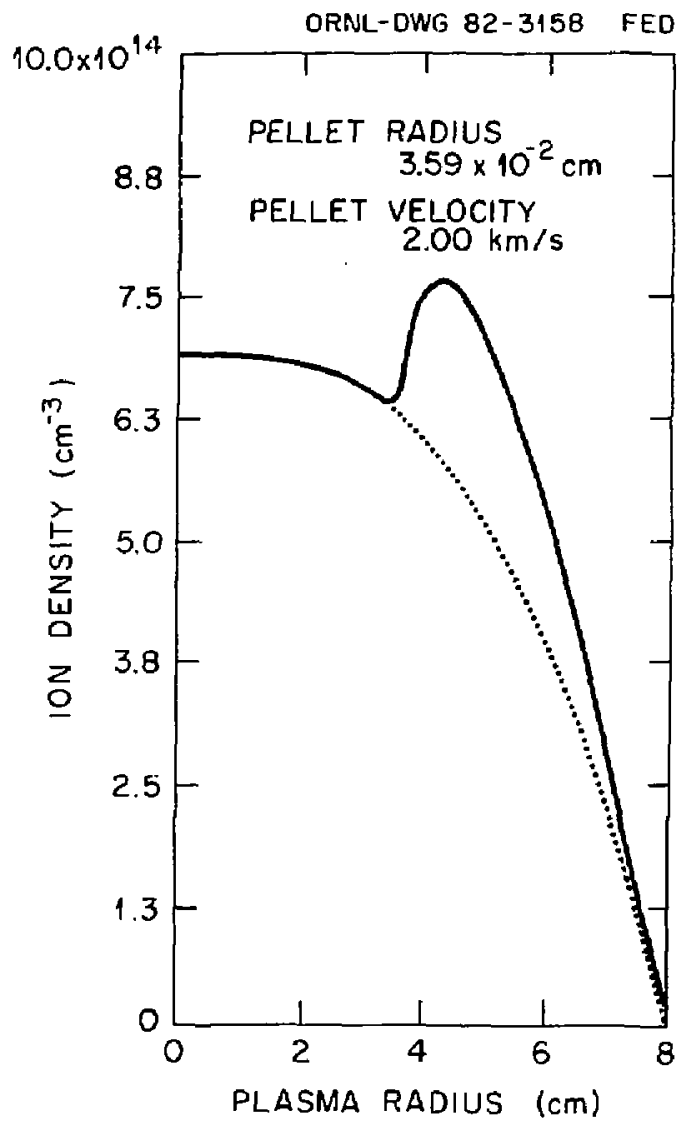

Figure E-2. Pellet deposition in the TDF plasma where the plasma radius is $8 \mathrm{~cm}$ and the pellet diameter is $0.72 \mathrm{~mm}$. 


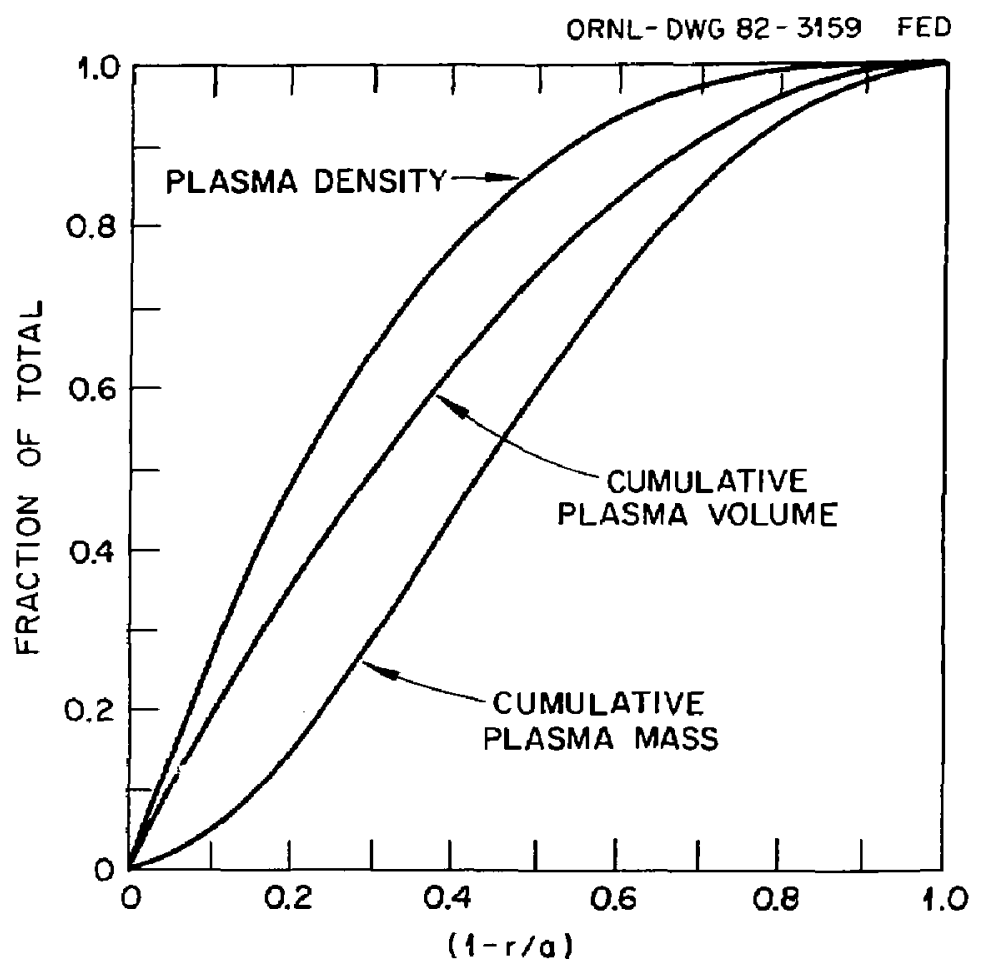

Figure E-3. The TDF normalized plasma volume and mass parameters as a function of $q(1-r / a)$. 


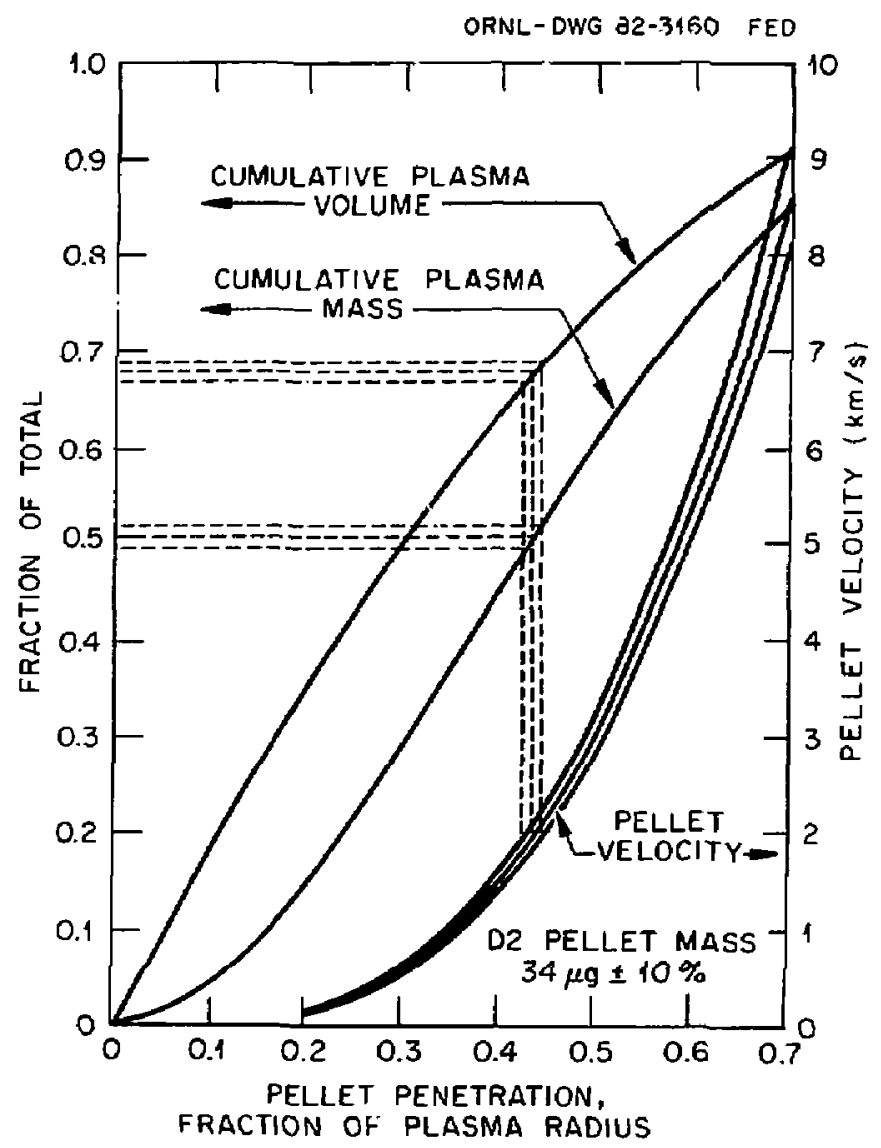

Figure E-4. The pellet velocity and plasma penetration, superimposed on normalized plasma parameters. 
APPENDIX $F$, VACUUM AND HALO REQUIREMENTS

A plasma halo fills the region between the main central cell plasma and the first wall. It shields the main plasma from impurities and cold gas that arise from wall outgassing, beam dump sputtering, and other such processes.

The halo model we used consists of two zones. The first extends from the main plasma at radius $r_{c}$ to the last magnetic field line $r_{h}$ to clear the high-field choke coils at the ends of the central cell. The second extends from $r_{h}$ to the first-wall radius $r_{w^{*}}$. Within each zane we assumed constant, equal density and temperature for both electrons and ions. Essentially all shielding of the main plasma occurs in zone 1 , so shielding criteria set the zone 1 parameters. Zone 2 must have a low density and temperature to keep sputtering at the end wall to a minimum.

Here we will discuss the required attenuation of cold gas and sputtered beam-dump neutials, particle and power balance, and TOF halo parameters. Units are CGS, with energies in electron volts and powers in watts unless otherwise stated.

\section{Beam Dump Sputtering}

Table F-1 contains our neutral-beam and beam-dump parameters. The sputtering yield is based on work by Bohdansky. 39,40 For normal incidence ( 0 cieg), the sputtering field of molybdenum (Ho) atoms per incident deuterium ion peaks at about $10^{-2}$ for a deuterium energy of about $2 \mathrm{keV}$; it drops to $10^{-3}$ for a deuterium energy of about $60 \mathrm{keV}$, as shown in Fig. F-1.39

Only Mo is considered here; other possible beam-dump materials for TDF give similar yields. Extrapolating from the $H$ ano $D$ data, $T$ will give sputtering yields perhaps a factor of 2 above those of $D$. Accounting for the half- and third-beam energy components, the sputtering yield for normal incidence is approximately $Y_{0}=Y_{D}+Y_{T}=3 / 210.8 \times 10^{-3}+0.15 \times 2$ $\left.\times 10^{-3}+0.05 \times 3 \times 10^{-3}\right)=1.9 \times 10^{3}$ atoms/incident particle.

The dependence of the sputtering yield on the incident beam angle is ${ }^{39}$

$$
Y(\phi)=Y(0) \cos ^{-f} \phi \text {. }
$$

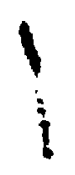


Table F-1. Neutral-beam parameters for the central cell.

Neutral beams

Total beam current (A)

1080

Total power (MW)

64.8

Average beam energy, W (kev)

60

Beam energy mix, for ion sources $80: 15: 5$

$\operatorname{mix}$ of $D^{+}: D_{2}^{+}: D_{3}^{+}(\mathrm{keV})$

$55 \%$

80

$29 \%$

40

$16 \%$

27

Beam angle (deg)

65

Trappíng fraction, $\boldsymbol{f}_{t}$

0.79

Beam-dumps

Surface material

Mo Tybdenum

Angle of dump to beam-off normal (deg)

69

Surface binding energy to deuterium (eV)

86 


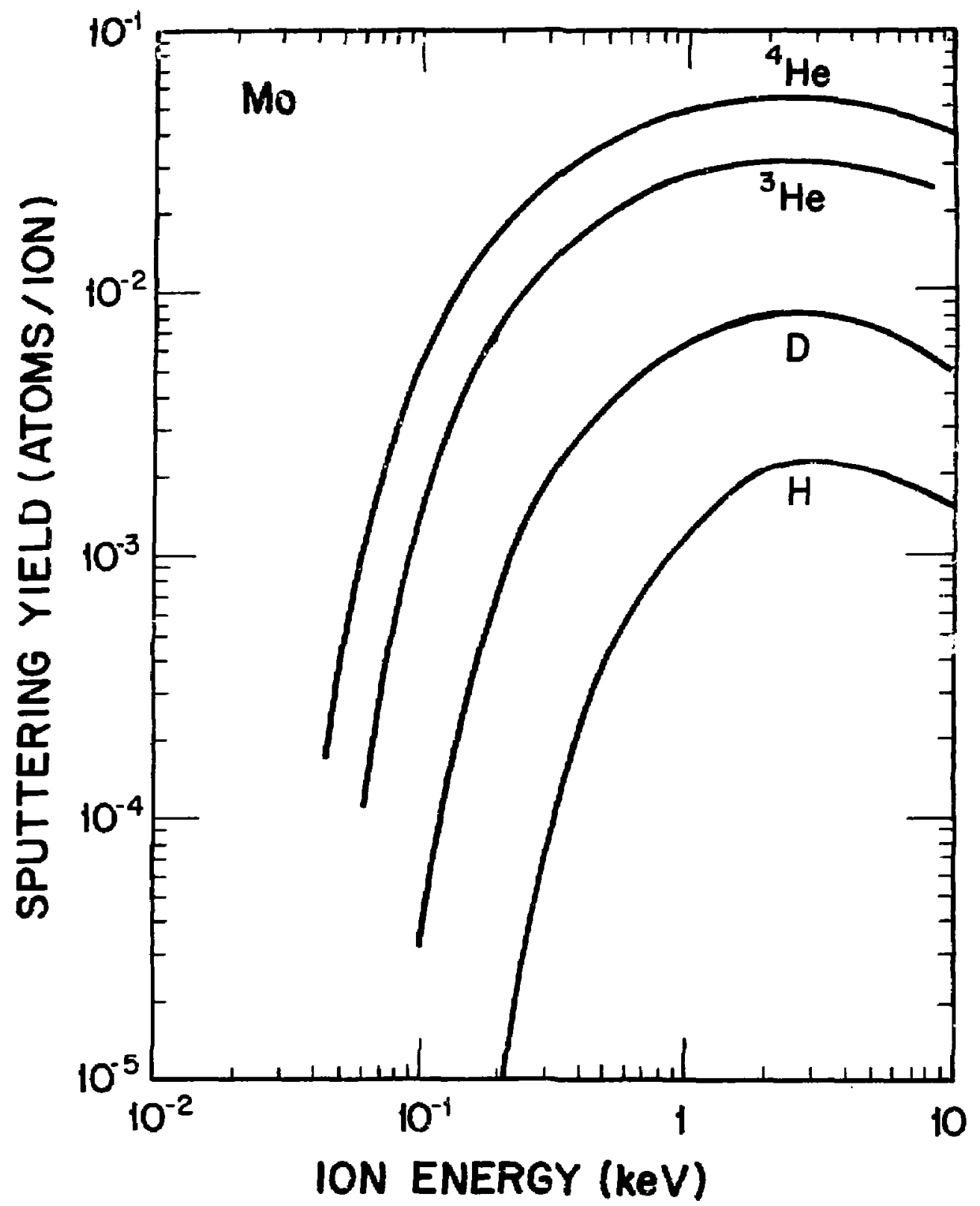

Figure $F-1$. Sputtering yield vs ion energy for molybdenum (Mo). The incident particle species are called out. 
where $f$ ranges from 1 to 2. For very iow- or high-energy beam particles $f$ is 1 , and $80 \mathrm{keV}$ is on the borderline of the high-energy regime, so $f=1$ is used here. The dependence of sputtering yield on energy is peaked at about $E=2 E_{b} / 3$, where $E_{b}$ is the surface binding energy and is proportional to $E^{-2} \cdot 39^{D}$ For very high-energy incident particles the sputtered atoms have a $\cos \theta \cdot \cos \zeta$ distribution, where $\theta$ and $\zeta$ are $0^{\circ}$ for norma 1 incidence.

Combining these dependencies, the approximate sputtering yield is

$Y(E, \theta, \zeta, \phi)=Y_{0}\left(\frac{\cos \theta \cos \zeta}{\cos \phi}\right)\left(\frac{2 E_{b}}{3 E}\right)^{2}$.

A very rough sketch of beam dump geometry is shown in Fig. F-2. Assuming that the neutral beam is strongly peaked at its center, the sputtered atom . must have $\theta>42 \mathrm{deg}$ and $\zeta<11 \mathrm{deg}$ to reach the halo. The probability of the sputtered atom penetrating the halo is $e^{-\lambda}$, where $\lambda$ is the ratio of path length to mean free path. This ratio is given by

$\lambda=7.15 \times 10^{-7}\left(\frac{\mathrm{nl}\langle\mathrm{\sigma ov}\rangle_{M_{0}} \mu_{M_{0}}}{E^{1 / 2}}\right)^{1 / 2}$,

where $n$ is the density of hot ions in the main plasma, $\ell$ is the path length, and $H_{m_{0}}$ is the ratio of Mo mass to proton mass. Although \& actually depends on $\theta$, it is taken as a constant here. The ionization rate is

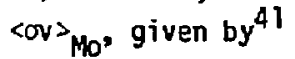

$\left\langle\right.$ sov ${ }_{M_{0}}=\left[\frac{10^{-5}\left(T_{e} / I_{M_{0}}\right)^{1 / 2}}{I_{M_{0}}^{3 / 2}\left(6+T_{e} / I_{M_{0}}\right)}\right] \exp \left(-I_{M_{0}} / T_{e}\right)$,

where $T_{e}$ is the halo electron temperature, $I_{M_{0}}$, which equals $7.3 \mathrm{eV}$, is the ionization potential for Mo, and the reaction rate has been averaged over Maxwellian electrons. 


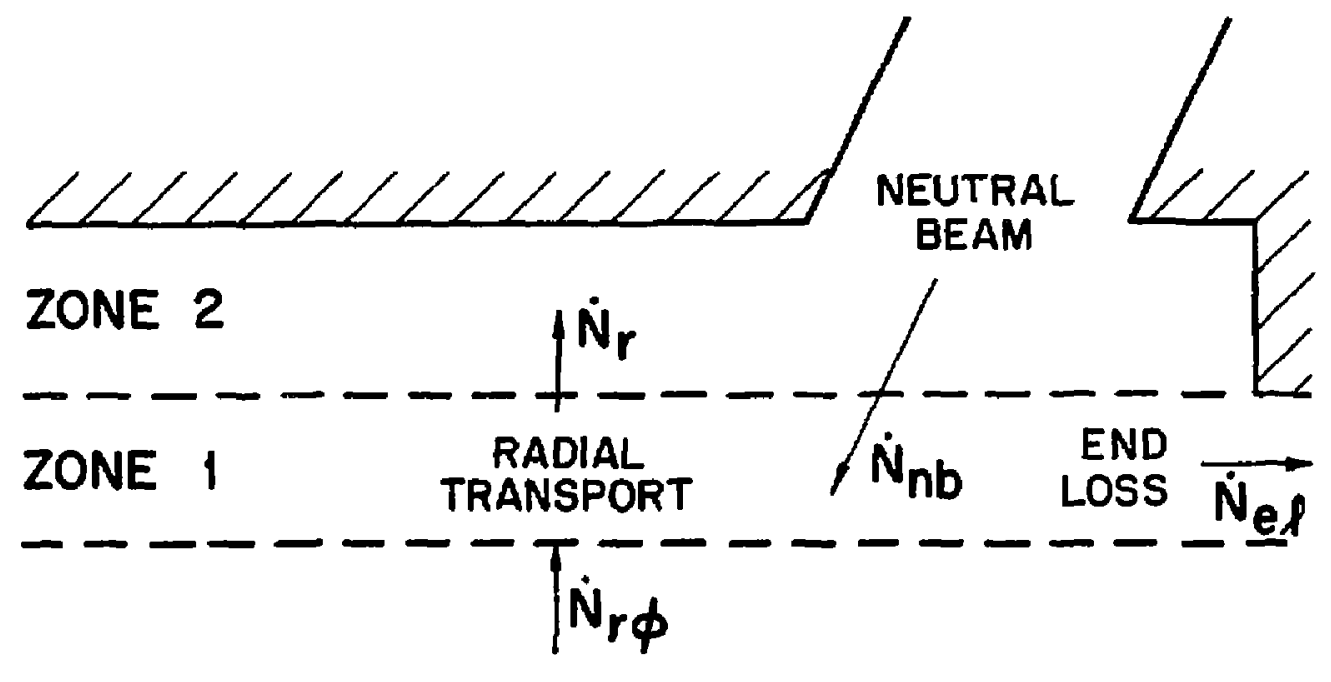

CENTRAL CELL PLASMA

ZONE 1

N. NEUTRAL

- - - - - - - - - - - - -

ZONE 2

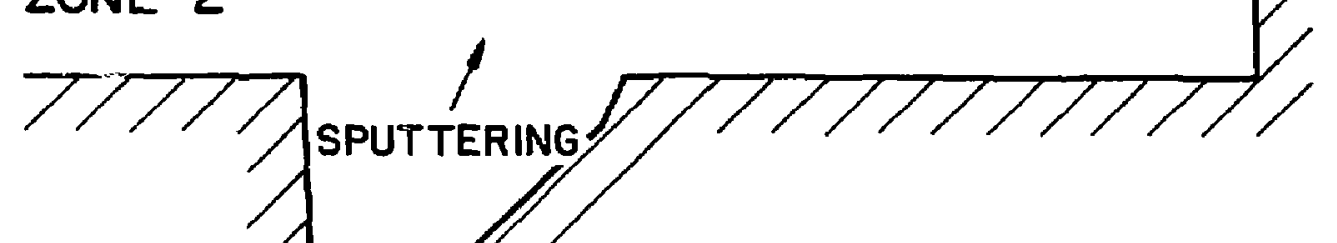

-. Figure F-2. Halo model geometry and particle balance terms. 
The number of sputtered Mo atoms per second that reach the main plasma is thus given by

$\dot{\mathrm{N}}_{M_{0}}^{S}=\frac{\dot{N}_{\mathrm{bd}} Y_{0}}{\cos \phi} \frac{E_{b}}{4} \int_{0^{0}}^{11^{0}} d \zeta \int_{42^{0}}^{90^{\circ}} d \theta \int_{E_{b}}^{E_{i n j}} d E \frac{\cos \theta \cos \zeta}{E^{2}} \exp (-\lambda) \simeq$

$$
\frac{2.8 \times 10^{8} \hat{n}_{b d}}{\left.(n \ell<\sigma V\rangle_{M o}\right)^{2}}\left(1+\frac{7.0 \times 10^{-6} \mathrm{nl}\langle\sigma V\rangle_{M 0}}{E_{i n j}^{T / 2}}\right) \exp \left(-\frac{7.0 \times 10^{-6} \mathrm{n \ell}\langle\sigma v\rangle_{\mathrm{Mo}}}{E_{i n j}^{T / 2}}\right),
$$

where $\dot{N}_{b d}$ is the particle source to the beam dump, the normalization factor is $9 \mathrm{E}_{\mathrm{b}} / 16$, and $\phi \simeq 69 \mathrm{deg}$.

To find the equilibrium density of Mo in the main plasma, we solve an Mo particle balance equation. Since $Z$ for Mo is high, Pastukhov conf inement 42,43 is very good--on the order of $(n \tau)$ Mo $_{\text {PAS }}^{\text {PA }} 10^{28} \mathrm{~cm}^{-3} \mathrm{~s}$. The minimum confinemer: iime is, therefore, set by classical radial transport, for which

$$
\begin{aligned}
& 0_{1}^{M_{0}} 2 J_{1} \rho^{2} 2 \frac{2.8 \times 10^{-6} \mu_{D T}^{1 / 2} z_{M_{0}}^{2} n_{c}}{M_{H_{H_{0}}} E_{h}{ }^{1 / 2} T_{M_{0}}}\left(\frac{102 \mu_{H_{0}}^{1 / 2}}{Z_{M_{0}} B_{c}} T_{M_{0}}^{1 / 2}\right)^{2} \\
& 2.3 \times 10^{-11} \frac{n_{c}}{E_{h}^{1 / 2}} 271 \mathrm{~cm}^{2} / \mathrm{s} .
\end{aligned}
$$

The radial loss time is approximately

$$
\tau_{M 0} 2 \frac{r_{c}^{2}}{20_{1}^{M_{0}}} \approx 0.70 \mathrm{~s}
$$


and the radial loss rate is

$\bar{N}_{M o}^{r} \sim f_{r p l} \frac{n_{M o}}{\tau_{M o}} v_{c} \imath 2.2 \times 10^{5} n_{M_{0}} s^{-1}$,

where $f_{r p l}$, which equals 0.6 , is a radial profile factor.

A density of impurities in the main plasma $n_{M_{0}}$ will reduce the fusion neutron production to a fraction of its $n_{M_{0}}=0$ value:

$\left(\frac{n_{c}-z_{M o} n_{M o}}{n_{c}}\right)^{2}=\left(1-7 \times 10^{-14} n_{M_{0}}\right)^{2}$.

Using particle balance, $\dot{N}_{M 0}^{r}=\dot{N}_{M 0}^{S}$, which implies $n_{M_{0}}=\dot{N}_{M_{0}}^{S} / 2.2 \times 10^{5}$, the fusion power reduction factor is: $\quad 1-\left(1-3.2 \times 10^{-19} \mathrm{H}_{M_{0}}^{5}\right)^{2}$ if all the sputtered Mo reaching the plasma edge penetrates and is distributed evenly in the main plasma.

Neutral gas. Neutral gas attenuation is modelled by proportionality Constants taken from Fig. $F-3$ for $T_{e}>10 \mathrm{eV}$ and scaled by <ov $\rangle_{e}$ for $\mathrm{T}_{e}<10 \mathrm{ev}$, where <ov $>_{\mathrm{e}}$ is the electron impact ionization rate.

For example, above $40 \mathrm{eV}$ (and assuming that the neutral density at the boundary between zones 1 and 2 is $n_{w}$ ) the neutral density a distance $\&$ into zone 1 is given by $n=n_{w} /\left(5.5 \times 10^{-2} n_{1} l\right)$, where $n_{1}$ is the constant density in zone 1 .

\section{Particle Balance}

Radial transport. We assume classical radial transport for lack of a detailed transport model. Only hot central cell ions are considered; the warm ions are neglected. The central cell ion radial transport coefficient is

$D_{r} \sim v_{\perp} \rho_{j}^{2} \simeq \frac{0.105 n_{h c}}{E_{h c}^{1 / 2} B_{c}^{2}} \sin ^{2} \theta_{i n b}$,

where $n_{n c}$ is the central cell hot ion density, $E_{h c}$ is the hot ion energy, $B_{C}$ is the central cell magnetic field, and $\theta_{i n b}$ is the neutral bean injection angle. 

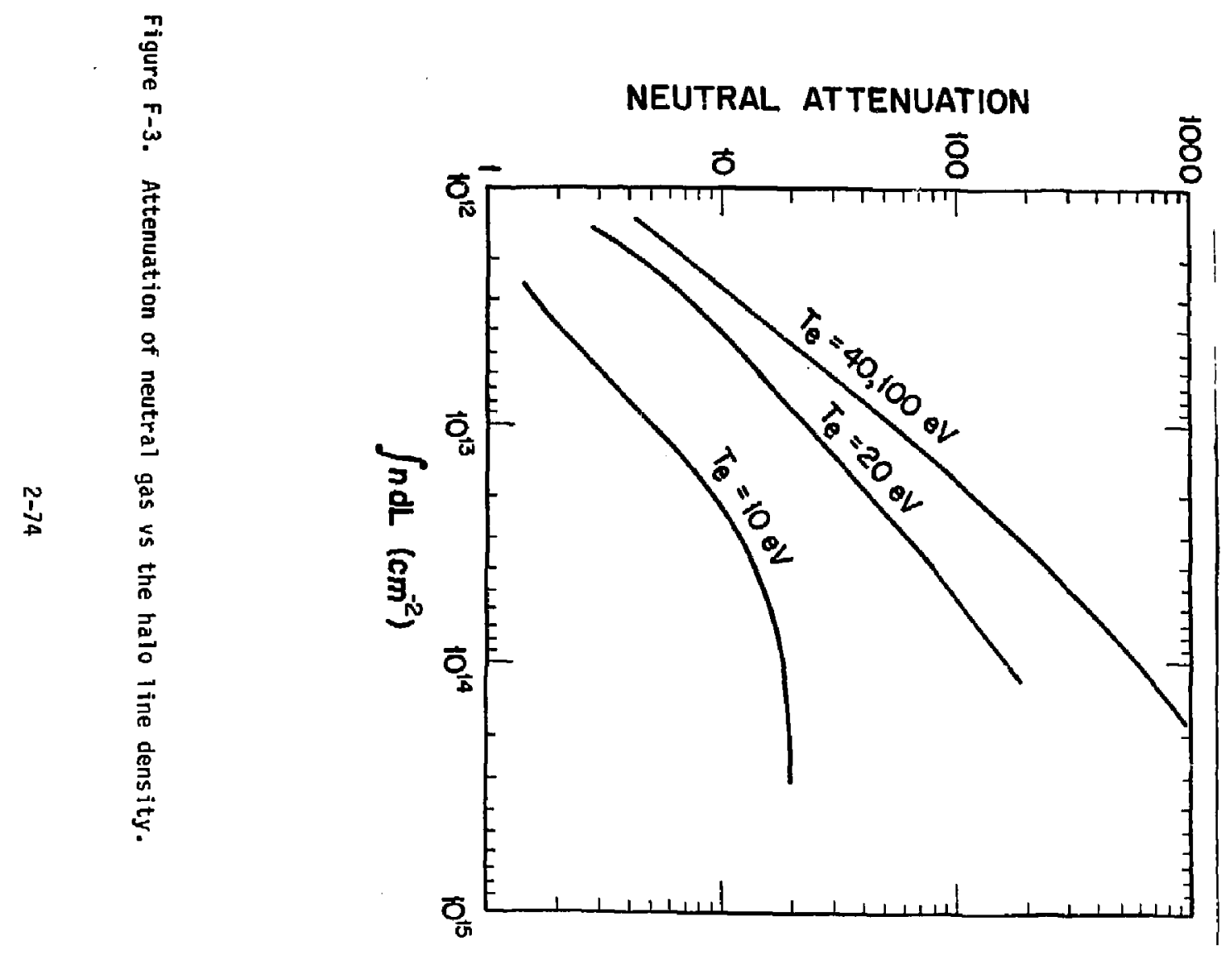
The radial loss time is approximateiy

$\tau_{r} \sim \frac{r_{c}^{2}}{2 D_{r}}$

so the radial loss rate from the central cell is

$\dot{N}_{r o}{ }^{2} f_{r p l} \frac{n_{c}}{\tau_{r}} v_{c}$.

For the TOF reference case, this is, $N_{\text {ro }} 22.4 \times 10^{20} \mathrm{~s}^{-1}$. Similariy, the loss rate from zone 1 to zone 2 is given by

$D_{r} 2 \frac{0.053 n_{1}}{T_{1}^{1 / 2} B_{C}^{2}}$

where $n_{1}$ and $T_{1}$ are the density and temperature in zone 1 . The

factor-of-two difference between the coefficients of EqS. $(F-10)$ and $(F-13)$ is because of isotropic thermal distribution in zone 1 .

Neutral beam ionization. The neutral beam trapping fraction for a region with density $n$, total path length $\ell$, ionization rate $\langle 0 v\rangle_{i n b}$, and charge-exchange rate $\langle\sigma v\rangle_{\mathrm{cxnb}}$ is

$f_{n b} 21-e^{-\lambda}$,

where

$\lambda_{n b}=7.15 \times 10^{-7}\left[\frac{\left.n \ell(<0 v\rangle_{i n b}+<0 v{ }_{c \times n b}\right)}{E_{i n b}^{1 / 2}} \frac{m_{i}}{m_{h}}\right]^{1 / 2}$

is the ratio of path length to the mean free path,

$E_{i n b}$ is the neutral beam energy,

$m_{i}$ is the ion mass,

$m_{H}$ is the proton mass,

$i$ is the path length on one side. 
For incident current $I_{n b}$ on an annular zone with trapping fraction $f_{t r}$ in the central circle, the total trapping rate is

$$
N_{n b} \simeq\left\{f_{n b}+f_{n b}\left[1-f_{n b}-f_{t r}\left(1-f_{n b}\right)\right]\right\} I_{n b} \text {. }
$$

The fraction of this current, which ionizes and fuels the halo zone, is

$$
\dot{N}_{n b i}=\frac{\langle\sigma v\rangle_{\text {inb }}}{\langle\sigma v\rangle_{\text {inb }}+\langle\sigma\rangle_{\text {cxnb }}} \dot{N}_{n b} .
$$

Ionization of background gas. As mentioned previously, the neutral gas density a distance $l$ into a halo zone from the outer perimeter is given by

$$
n_{g}=\frac{n_{w}}{c_{A} n \ell} \text {, }
$$

where $n_{W}$ is the density at the border, $c_{A}$ is a constant taken from Fig. $F-3$, and $n$ is the halo density. 44

The rate for ionization of gas is

$$
N_{i g}=d V n_{g} n\langle o v\rangle_{i g},
$$

where $\langle\sigma \gamma\rangle_{i g}$ is the ionization rate and the integral is over the halo volume. Equation $(F-18)$ is only a good approximation until $c_{A} n \ell \approx 1$, so the integral is broken into two parts. For zone 1,

$\dot{N}_{i g} 2 \pi L L_{c} \int_{r_{c}}^{r_{h}-\Delta r}\left[r d r \frac{\left.n_{w} n<\sigma v\right\rangle_{i g}}{c_{A} n\left(r_{h}-r\right)}\right]+2 \pi L_{c} \int_{r_{h}-\Delta r}^{r_{h}} r d r n_{w} n\langle\sigma v\rangle_{i g}=\frac{2 \pi L_{c}}{c_{A}} n_{w}\langle\sigma v\rangle_{i g} x$
$\left[r_{g} \ln \left(\frac{r_{h}-r_{c}}{\Delta r}\right)-\left(r_{h}-r_{c}-\Delta r\right)\right]+\pi L_{c} n_{w}^{n<\sigma v\rangle_{i g}}\left[2 r_{h} \Delta r-(\Delta r)^{2}\right]$, where $\Delta r=1 /\left(c_{A} n\right)$. For zone 2, a similar formula applies. of course, when $\Delta r$ is greater than the radial extent of the zone, $n_{g}=n_{w}$ throughout the zone. 
Reionization of charge-exchanged neutrals. Some fraction $f_{\text {ion }}$ of neutrals from charge-exchange events in the halo will reionize. To find the fraction we used the very rough model that follows. Our two major assumptions were:

(1) slab geometry suffices and (2) the main plasma is ignored because of its smaller volume.

The mean free path for reionization of a charge exchanged neutral is

$\lambda_{m f p} \approx\left[\frac{\left.7.15 \times 10^{-7} n<\sigma v\right\rangle_{i}\left(m_{i} / m_{H}\right)^{1 / 2}}{E^{1 / 2}}\right]^{-1}$,

where $\langle\sigma v\rangle_{i}$ is the ionization rate and $E$ is the neutral's energy. The very simple assumption used here is that the neutral escapes if its path length to escape the halo $\ell$ is longer than $\lambda_{\text {mfp }}$. Focusing on zone 1 and including the main plasma as part of zone $1, l=r_{h}-r_{c x}$ is greater than $\ell_{m f p}$ if a vector with direction $\vec{V} / v$ and magnitude $\lambda$ mfp lies on a zone of height $h$ on a sphere of radius $\lambda_{\mathrm{mfp}}$. The geometry is shown in Fig. F-4a. The ionization probability then, is, the area of this zone or zones divided by the total surface area of the sphere. The three possible cases are shown in Fig. F-4b. For case 1, $\lambda_{m f p}<r_{h}-r_{c x}$. and $f_{\text {ion }}=1$. For case 2, $r_{h}-r_{c x}<\lambda_{m f p}<r_{h}+r_{c x}$, and $f_{i \text { ion }}=0.5+\left(r_{h}-r_{c x}\right) / 2 \lambda_{\text {mfp }}$. For case $3, r_{h}+r_{c x}<\lambda_{m f p}$, and $f_{\text {ion }}=r_{h} / \lambda_{m f p}$

We can find charge-exchange on the neutral beam $\mathrm{N}_{\mathrm{nbcx}}$ and on the background gas $N_{c x g}$ by replacing $\langle\sigma\rangle_{\text {inb }}$ by $\langle 0 \%\rangle_{\mathrm{cxnb}}$ in the numerator of Eq. (F-17) and sov $\rangle_{i g}$ by $\langle 0 v\rangle_{c x g}$ in Eq. $(F-20)$. The source of halo ions caused by reionization of charge-exchanged neutrals is then

$\dot{N}_{i c x}=f_{\text {ion }}\left(\dot{\hat{N}}_{\text {nbcx }}+\dot{N}_{c x g}\right)$.

End loss. There are two end loss regimes, delineated by the ratio of ion-ion scattering time $\tau_{1}$ to the bounce time $\tau_{11}$. For $\tau_{\perp}<\tau_{\|}$, the halo is in the collisional regime, and the flow time to the ends dominates end 10s5, resulting in

$(n \tau)_{e \ell}=(n \tau)_{f l o w} \simeq \frac{R_{c} L_{c} / 2}{\sqrt{2 \pi T / m_{i}}}$, 
where $R_{C}$ is an appropriate mirror ratio and the $\pi$ comes from taking a directed Maxwellian velocity distribution. For $t<t$, the halo is in the collisionless reqime and

$$
(n \tau)_{e l}=(n \tau)_{s c a t} \approx n \tau_{i i} \text { ln } R_{c} \text {, }
$$

where the collisional scattering time is $\tau_{i j} \simeq 4.9 \times 10^{5}\left(T^{3 / 2}\right) / n$.

To choose the regime the computer code compares $(n \tau)_{f}$ low to $(n \tau)_{\text {Scat }}$, rather than $\tau_{\| l}$ to $\tau_{l}$ directly. This is for simplicity.

End loss is given by

$\dot{N}_{e \ell}=\frac{n^{2}}{(n \tau)_{e l}} v_{h}$.

Particle balance equation. Overall particle balance requires

$\dot{N}_{r o}+\dot{N}_{n b i}+\dot{N}_{i g}+\dot{N}_{i c x}=\dot{N}_{r}+\dot{N}_{e l}$,

where $N_{r o}$ is the gain and $M_{r}$ is the loss due to radial transport.

\section{Power Balance}

Radial transport. For zone 1, the power gain due to radial transport is

$P_{\text {ro }} \simeq \dot{N}_{\text {ro }} E_{\text {hc }} \simeq 1.4 \mathrm{MN}$

The lass is

$P_{r}=\dot{H}_{r 1} T_{1}$

For zone 2, the gain is

$P_{r o}=\dot{N}_{r 1} T_{1}$, 

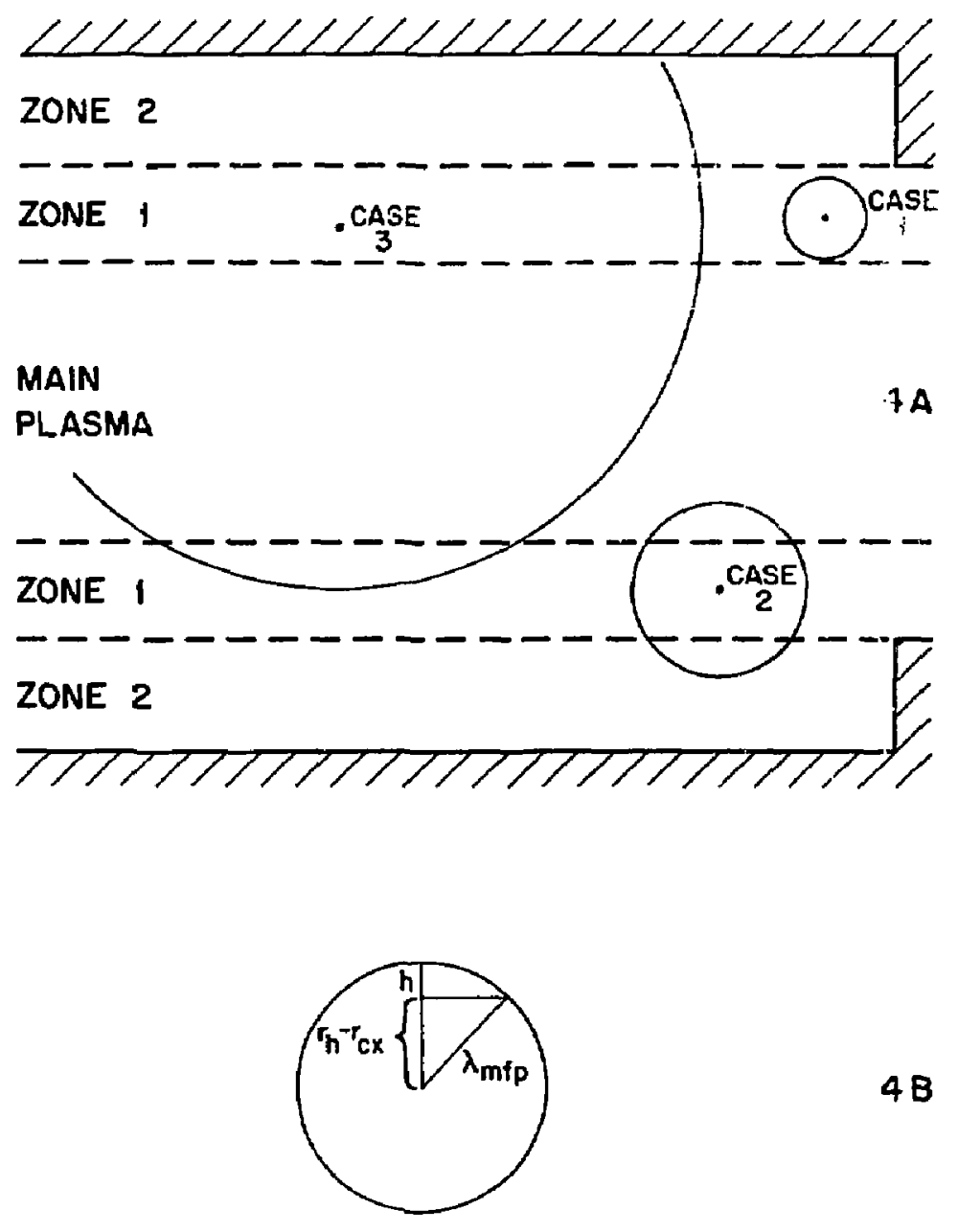

$4 B$

Figure $\mathrm{f}-4$. Our rough model for the reionization of charge-exchanged neutrals, showing (a) the model and (b) three possible special cases. 
and the loss is

$P_{r} \simeq \dot{N}_{r 2} T_{2}$

Alpha particle heating. Drag by halo electrons on alpha particles whose orbits intersect the halo will cause some heating to occur. The calculation for this is similar to that for reionization of charge-exchanged neutrals. If an alpha particle is born at radius $r$ in the main plasma and with angle $\theta$ with respect to the $z$ axis, two cases exist: if $0 \leq r \leq 2 \rho_{\alpha}-r_{c}$, where 20 is the maximum alpha particle Larmor radius, then

$f_{\alpha}(r)=\frac{r_{c}}{2 \rho_{\alpha}}$

If $\left(2 \rho_{\alpha}-r_{c}\right) \leq r \leq r_{c}$, then

$f_{\alpha}(r)=\frac{1}{2}\left(1-\frac{r_{c}-r}{2 \rho_{\alpha}}\right)$.

Since the electron temperature in the halo is low, we assume that any alpha particle whose orbit intersects the hato transfers all of its energy to the halo. This is not true if the halo density gets very low, since the slowing down time is proportional to $\mathrm{T}_{\mathrm{e}}^{3 / 2} / \mathrm{n}$.

For the radial density profile

$n(r)=n_{0}\left[1-\left(\frac{r}{r_{c}}\right)^{3}\right]$,

and the fraction of the alpha particle energy heating the halo is

$f_{\text {an }} 2 \int_{0}^{r_{c}}\left[1-\left(\frac{r}{r_{c}}\right)^{3}\right] f_{\alpha}(r) \frac{r d r}{r_{c}^{2}} \sim 0.13$.

The total power to the halo is thus

$P_{\alpha} \approx 0.2 P_{\text {fus }} f_{\text {ah }}=0.5 \mathrm{MH}$

for the reference case. 
Line radiation. Line radiation is neglected here.

Neutral beams. A halo zone causes a power drain of $\dot{N}_{n b} E_{i n b}$ on the neutral beam. However, if the charge-exchange time on background gas $\tau_{c x}$ is less than the drag time $\tau_{d r}$, this power will not significantly heat the halo zone. The pertinent times are

$\tau_{c x}^{2} \frac{1}{n_{n}<\sigma v>c x}$

and

$\tau_{d r} \approx \frac{\left(m / m_{i H}\right) T^{3 / 2}}{3.2 \times 10^{-8} n}$.

For $\tau_{c x} \ll \tau_{d r}$, which is the regime of both zones, charge-exchange on the background gas causes an energy drain of $1.5 \dot{N}_{n b} T$. The initial charge exchange events on the neutral beams also cause a small power drain, given by $1.5 \dot{N}_{n b c x} T$. Also, the power gain caused by beam particles slowing down before they charge-exchange is approximated here by $\dot{N}_{n b} E_{\text {inb }} \tau_{c x} / \tau_{d r}$. The total power change due to the neutral beans is thus

$P_{n b}=\dot{N}_{n b} E_{i n b} \frac{\tau_{c x}}{\tau_{d r}}-\left(\dot{N}_{n b}+\dot{N}_{n b c x}\right) \frac{3}{2} T$.

Charge-exchange on background gas. For a background gas temperature $T_{g}$ the power loss caused by charge-exchange is $P_{\text {cxg }}=3 / 2 \mathrm{~N}_{\text {cxg }}\left(T-T_{g}\right)$. We assume that the background consists mainly of Franck-Condon neutrals, with $\mathrm{T}_{\mathrm{g}} 23 \mathrm{eV}$.

Reionization of charge-exchanged neutrals. The reionization of charge-exchanged neutrals adds a power of $\mathrm{P}_{i c x}=3 / 2 \dot{N}_{i c x}{ }^{T}$. We assume that all reionization occurs in zone $?$.

End loss. End loss causes a power drain of $\mathrm{P}_{\mathrm{el}}=3 / 2 \dot{\mathrm{N}}_{\mathrm{e} \ell^{T}}$.

Power Balance Equation. Overall power balance is governed by $P_{r 0}+P_{\alpha}$ $+P_{\text {icx }}=P_{r}+P_{n b}+P_{c x g}+P_{e l}$. 
Halo Parameters for the Reference Case

We chose the reference case because it shielded the main plasma from sputtered beam dump atoms and cold gas while keeping neutral beam attenuation sma11. All of the shielding occurs in zone 1, whose parameters are given in Tables $F-2$ and F-3. Zone 2 has a very low density and temperature. We will discuss it only briefly and then concentrate on zone 1 .

The primary power inputs into zone 2 are radial transport from zone 1 and the $3-\mathrm{eV}$ ion from the ionization of Franck-Condon neutrals. Since only a small mirror ratio keeps these ions from hitting the wall at the ends of the central cell, the confinement time for an ion is very small--about $5 \times 10^{-4} \mathrm{~s}$ for a $3-\mathrm{eV}$ ion. The electrons are cols when they enter the plasma, and their thermal equilibration time is mich longer than the ion loss time, so they remain cold at about $0.025 \mathrm{ev}$. At this temperature, the ionization cross section is virtually zero, which impilos that very few events occur, and thus the halo dersity in zone 2 is negligible.

The ions entering zone 2 by radial transport are also quickly lost before they lose significant energy or transport very far radially. Thus, they form a tenuous boundary layer plasma at $189 \mathrm{eV}$ between zones 1 and 2. Although the question is too complex to address here, the details of the radiai transport are important for assessing the sputtering these ions cause at the end walls.

We considered these major factors in arriving at the zone 1 halo parameters:

(1) Sputtered beam dump atoms should be shielded from the main plasma.

(2) Neutral gas should be kept from the main plasma.

(3) Neutral beam attenuation should be as small as possible. Considerations (1) and (2) are at odds with (3).

The chief parameter controlling the system is the base pressure, which gives the neutral gas density $n_{w}$ at the first wall. The effect of varying $n_{w}$ on both density and temperature is shown in Fig. F-5. Since the ionization cross section is almost constant in this temperature range, the neutral beam attenuation has the same functional form as the density. We chose $n_{w}=2.9 \times 10^{12} \mathrm{~cm}^{-3}$ for the reference case since, at the minimum $\mathrm{n}$, it was considered tolerable to have fusion power degradation of $1.8 \%$ caused by Mo and the power loss of $0.2 \mathrm{MH}$ caused by charge-exchange of the main plasma on cold gas. He could have used a somewhat lower $n_{w}$, however, without greatly altering the halo parameters. 
Jabje F-2. General halo parameters for zone 1.

\begin{tabular}{ll}
\hline \multicolumn{1}{c}{ Parameter } & Value \\
\hline Density, $n\left(\mathrm{~cm}^{-3}\right)$ & $6.2 \times 10^{12}$ \\
Temperature, $T(\mathrm{e} !)$ & 189 \\
Inner radius, $\mathrm{F}_{\mathrm{c}}(\mathrm{cm})$ & 10 \\
Outer radius, $r_{h}(\mathrm{~cm})$ & 17
\end{tabular}

Surface heat to first wall from halo,

where radius $r_{w}=25 \mathrm{~cm}$

$\begin{array}{ll}\text { at } 260 \mathrm{keV}\left(\mathrm{W} / \mathrm{cm}^{2}\right. & 18.3 \\ \text { at } 189 \mathrm{eV}\left(\mathrm{W} / \mathrm{cm}^{2}\right) & 3.4 \\ \text { at } 37 \mathrm{keV}\left(\mathrm{W} / \mathrm{cm}^{2}\right) & 1.5 \\ \text { Total }\left(\mathrm{W} / \mathrm{cm}^{2}\right) & 23\end{array}$

Particle flux to first wall
at $260 \mathrm{keV}\left(\mathrm{cm}^{-2} / \mathrm{s}\right)$
$1.9 \times 10^{15}$
at $189 \mathrm{eV}\left(\mathrm{cm}^{-2} / \mathrm{s}\right)$
$0.1 \times 10^{17}$
at $37 \mathrm{keV}\left(\mathrm{cm}^{-2} / \mathrm{s}\right)$
$2.6 \times 10^{14}$

Beam-dump sputtering

Equilibrium Mo density in main plasma, $1.3 \times 10^{11}$

$\mathrm{n}_{\text {Mo }}\left(\mathrm{cm}^{-3}\right)$

Fusion power degradation ( $\mathrm{x}$ )

1.8

Neutral density
at main plasma edge $\left(\mathrm{cm}^{-3}\right)$
$1.1 \times 10^{10}$
at wa11, $n_{w}\left(\mathrm{~cm}^{-3}\right)$
$2.9 \times 10^{12}$
at wal1--base pressure (torr)
$8.8 \times 10^{-5}$

Charge-exchange of neutral bean ions
Charge exchange time, $\tau_{c x}$ (s)
$5.0 \times 10^{-4}$
Drag time, $\tau_{\mathrm{dr}}(\mathrm{s})$
$3.3 \times 10^{-2}$

Reionization of charge-exchanged neutrals

Mean free path $(\mathrm{cm})$

62

Reionization fraction

0.27 
Table F-3: Particle and power balance parameters for zone 1.

Product of density times lifetime $\left(\mathrm{cm}^{-3} \cdot \mathrm{s}\right)$

for end loss, $(\mathrm{n \tau})_{\mathrm{e}}\left(\mathrm{cm}^{-3}-\mathrm{s}\right)$

for transit flow loss, $(\mathrm{n} \tau)$ flow $\left(\mathrm{cm}^{-3} \cdot \mathrm{s}\right)$

for scattering loss, $(n \pi)_{\text {scatt }}\left(\mathrm{cm}^{-3} \cdot \mathrm{s}\right)$
$1.3 \times 10^{9}$

$1.2 \times 10^{9}$

$1.3 \times 10^{9}$

Particle gain

Radial transport, $\dot{N}_{\text {ro }}\left(\mathrm{s}^{-1}\right)$

Neutra)-beam ionization, $\dot{N}_{n b j}\left(s^{-1}\right)$

Neutral-gas ionization, $\dot{N}_{i g}\left(s^{-1}\right)$

Reionization of charge-exchanged neutrals, $N_{\text {icx }}\left(s^{-1}\right)$

$2.4 \times 10^{20}$

$1.1 \times 10^{20}$

$8.63 \times 10^{21}$

$5.33 \times 10^{21}$

Particle loss

Radial transport, $\dot{\mathrm{N}}_{\mathrm{r}}\left(\mathrm{s}^{-\mathrm{T}}\right)$

End loss, $\hat{N}_{\mathrm{el}}\left(\mathrm{s}^{-1}\right)$

$2.3 \times 10^{17}$

$1.43 \times 10^{22}$

\section{Power gain}

Radial transport, $P_{\text {ro }}(M W)$

1.4

Alpha particles, $P_{\alpha}$ (mis)

0.5

Reionization of charge-exchanged neutrals, $P_{\text {icx }}$ (MW)

0.24

Neutral beams, $P_{n b}$ (MW)

0.02

Power loss

Radial transport, $P_{r}$ (NiN)

$2.1 \times 10^{-5}$

Neutral-gas charge exchange, $P_{\text {cxg }}$ (WW)

End loss, $P_{\text {el }}$ (MW)

0.87

1.3

Total neutral beam attenuation in halo $(x)$

3.5 


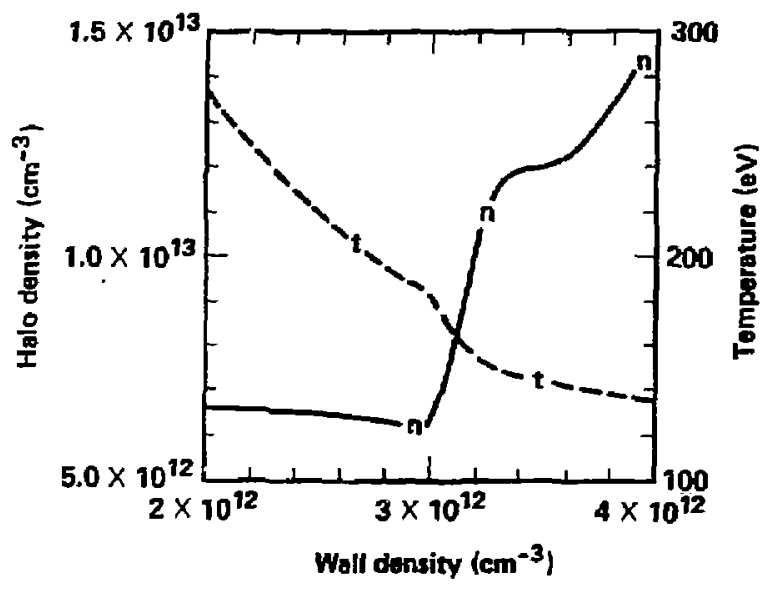

Figure F-5. Halo density and temperature vs neutral density at the first wall. 
The halo's particle balance is dominated by end loss and by ionization and charge-exchange of the cold neutral gas. For power balance, on the other hand, end loss is balanced by radial transport and alpha particle power, neither of which can be very accurately assessed. Figure F-6 shows the effect of increasing radial transport over classical transport. The neutral beam attenuation is $f_{n b}$ from $E q .(F-14)$; the total attenuation is given in Eq. $(F-16)$. T is essentially linear with the transport ratio. Thus, should some process increase transport over classical, the cost would be in increased neutral beam attenuation. Note that $n_{w}$ was not optimized here, which would heip siightiy.

The surface heat load of $23 \mathrm{~W} / \mathrm{cm}^{2}$ is somewhat higher than we anticipated. It has been spread over the whole first wall, since the bounce time for ions resulting from the neutral beams is less than the charge-exchange time.

In conclusion, the haln parameters are in the expected range. To maintain the present neutral ieam current to the main plasma requires an increase of about $3.5 \%$ in total current, to compensate for halo attenuation. No serious problems have been identified, but if zone 2 begins with a thin boundary layer of 189-eV ions, some sputtering of the end wall may occur. 


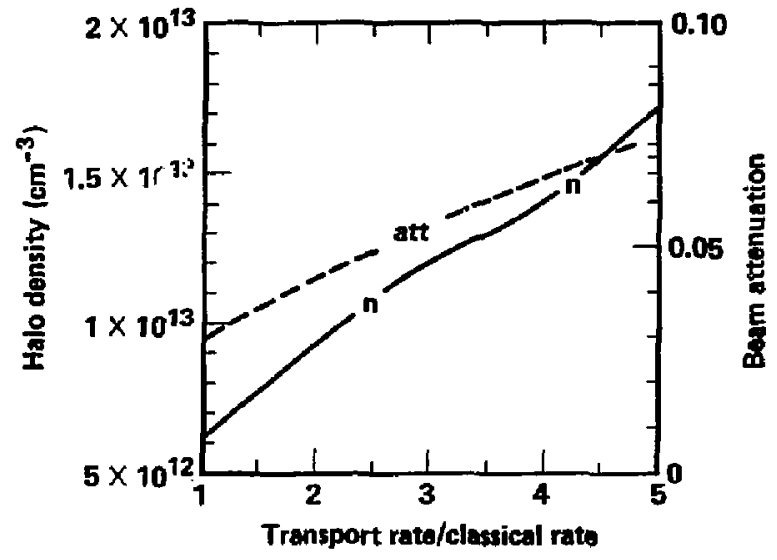

Figure $F-6$. Halo density and beam attenuation vs relative transport rate from the central cell. 
The major sources of impurities in TDF are expected to be i) Thermal oxygen and carbon desorbed from the wall and heavier metals such as iron sputtered from the walls and 2) Energetic impurities such as oxygen injected with the neutral beams $(20.1 \%$ oxygen now expected for steady-state NBI, unless purified by magnetic analysis). The thermal components from surfaces are predicted t;o have a very short mean-free-path for ionization and therefore to be highly a'tenuated by the halo plasma and pumped out to the end chamber walls. The energetic impurities, however, are predicted to penetrate, be trapped and stripped to a high charge state, with a relatively prolonged lifetime due to their high charge state and end plug potential confinement. The brief calculations for the main source of energetic impurities follows.

1. Total impurity input.. The total incident neutral beam current for $T D F \simeq 1150 \mathrm{~A}$ (atomic equivaient from central cell beams, sloshing ion beams, and pump beams). If this includes $0.7 \%$ oxi.jan the total input is $21.15 \mathrm{~A}$ oxygen or $20.6 \mathrm{~A}$ /end.

2. Trapping and equilibrium charge state. In general the cross section for ionization of impurities is $\geqq$ that for deucerium so a value $f_{t}$ (oxygen) $\geq f_{t}$ (D-T beams) is predicted. Here we assume a trapping fraction $f_{t}$ (oxygen) $\tau$ ) as the warst case. For low $q$ levels, the lifetime for ionization $\tau_{\text {ioniz }} \ll \tau_{\text {scatt }}$ or $\tau_{c x}$ for typical plasmas, so the impurity is rapidly stripped to a higher and higher charge state $q$, until the electron loss rate (ionization $q \rightarrow q+1$ ) is equal

to the electron pickup rate (charge-exchange $q \rightarrow q-1$ ), i.e., $\tau_{\text {ioniz }(q) \approx \tau_{c x}(q)}$. Hulse et al. in Ref. 45, shows $\bar{q} \ 6$ for oxygen in a plasma with $T_{e} \sim 2.1 \mathrm{keV}$ and a ratio of neutral to electron densities $n_{0} / n_{e} \sim 10^{-4}$ as expected with the TDF strong neutral injection and leakage of background super thermal or Frank Condon neutrals through the halo.

3. Impurity lifetime $\tau$. ine initial stripping process steps to $q \sim 5$ are so fast as to leave the maynetic moment or total kinetic energy unchanged. The step $q+5$ to $\sigma$ (or 7) is slower so some $0^{5}$ loss occurs by scattering out the loss cone, before the oxygen has cooled (from $\mathrm{dW} / \mathrm{dx}$ in the central cell to the bulk 2.1-keV electrons) such at $W \leq 20 \mathrm{keV}$, since $\phi_{c} \sim 4 \mathrm{keV}$. Rough calculations indicate at least $50 \%$ of the impurity ions are lost during 
the magnetically confined cooling phase, but here as a worst case we assume $100 \%$ trapped as $\vec{q} \sim 6$. Once cooled the $0^{6}$ ions are electrostaticaliy confined between the anchor plugs and tend to accumulate in either the transition or anchor barrier regimes. The lifetime for Pastakov electrostatic confinement has the form ${ }^{42}$ :

$\tau_{p}=\tau_{q D} q(R) \frac{q e \phi}{T_{i}} \exp \left(q e \phi / T_{i}\right)$

where $\tau_{q D}=\frac{\tau_{D D}(M / 2)^{3 / 2}}{q^{2}}$

and $q(R)$ is a function of the mirror ratio $R$ but of an order of unity.

4. Impurity density buildup. The maximum total input current of oxygen in Section $I$, the minimum warm plasma input of $800 \mathrm{~A} \mathrm{D}^{+}$from pellet fueling, and the assumption of similar Pastakov confinement for both warm ion groups then allows calculation of the equilibrium ratio of $n\left(0^{6+}\right) / n\left(0^{+}\right)$, since in equilibrium $n \approx\left(I f_{t} \tau\right) /$ Nolume. Therefore here the maximum impurity fractiorı

$f_{0} \equiv n\left(0^{6+}\right) / n\left(0^{+}\right) \approx \frac{1.2 \times 1 \times(16 / 2)^{1 / 2}}{800 \times 1 \times 6}\left(\frac{e \phi}{T_{i}}\right) \exp \left[\frac{e \phi}{T_{i}}(5)\right] \approx 0.1$ for $e \phi_{i} / T_{i} \imath 1$.

This would be prohibitive for radiation and more importantly scattering losses of $\mathrm{D}^{+}$and hence pumping requirements. Since they are proportional $^{46}$ to $\left(1+f_{0} q^{2}\right)$ or here $\left(1+0.1(6)^{2}\right) \approx 4.6$. However, more detailed calculations includiny oxygen scattering losses in the central cell and transition anchor regions (since there is essentially no cooling of oxygen Yushmanov trapped in the transition anchor regions, because the electron temperatures are so high), reduce the net oxygen trapped current by a factor of 210 to only $20.1 \mathrm{~A}$ with a more precise predicted result of $f \sim 1 \%$, which still yields $\left(1+f q^{2}\right)=1.36$. This would still result in a $36 \%$ reduction in $D^{+}$confinement $n \tau$ and a $36 \%$ increase in pumping current requirements.

Therefore further work is indicated to (1) refine the above calculations, (2) consider use of higher purity neutral beams with impurity content $\ll 0.1 \%$ (e.g. magneticaliy separate out heavy impurities from D-T), (3) study ICRH or other methods for impurity pumping for removal, or (4) consider quasisteady-state operation with remote dumps of $\phi_{c}$ to empty out impurities. 


\section{REFERENCES--SECTION 2}

1. T. K. Fowler and B. G. Logan, Tandem Mirror Technology Demonstration Facility, Lawrence Livermore National Laboratory, Livermore, CA, UCID-19193 (1981).

2. D. Correll et. a\}., Lawrence Livermore National Laboratory, Livermore, CA, UCRL-50051-78-2 (1978).

3. T. C. Simonen and TMK group, Summary of Results from the Tandem Alirror Experiment (TMX), Lawrence Livermore National Laboratory, Livermore, CA, UCRL-53120 (1981).

4. F. H. Coensgen et. al., TMX Upgrade Major Project Proposal, Lawrence Livermore National Laboratory, Livermore, CA, LLL-Prop-172 (1980).

5. R. J. Breun et. al., "Initial Results from the Tanden Mirror Phaedrus," in Proc. 8th IAEA Conf. Plasma Phys. and Cont. Nucl. Fusion Res. (Nuclear Fusion Supplement, Vienna, 1981) Vo1. 1, pp. 105-112.

6. S. Miyoshi-et. a1., "Plasma Confinement in Central Mirror of Gamma 6 (Tandem Mirror)," in Proc. 8th IAEA Conf. Plasma Phys. and Cont. Nucl. Fusion Res., (Nuclear Fusion Supplement, Vienna, 1981), Vo1. 1, pp. 113-118.

7. N. H. Lazer, "Electron and Ion Cyclotron Heating in a Symmetric Tandem Mirror STM-1, Bull. Am. Phys. Soc., 27, 961 (1982).

8. D. Grubb, et. al., "Measurements of Particle and Energy Confinement in Initial TMX-U Sloshing-Ion End Experiments," Bu1l. Am. Phys. Soc., 27, 997 (1982).

9. Physics Basis for an Axicell Design for the End Plugs of MFTF-B, D. E. Baldwin and B. G. Logan (editors), Lawrence Livermore National Laboratory, Livermore, CA, UCI0 19359 (1982).

10. R. E. Klinkowstein et. al., "The TARA Tandem Mirror Experiment," Bull. Am. Phys. Soc., 27, 967 (1982).

11. T. Kawaki, University of Tsukuba, Tsukuba, Japan, private communication (November 198c)...

12. G. I. Dimov, Institute of Nuclear Physics, Novosibirsk, USSR, private communication (November 1982).

13. T. H. Batzer et. al., Conceptual Design of a Mirror Reactor for Fusion Engineering Research Facility (FERF), Lawrence Livermore Nationa1 Laboratory, Livermore, CA, UCRL-51617 (1979) 
14. B. Badger et, a1., TASKA, Tandem Spiegelmaschine Karlsruhe, A Tandem Mirror Fusion Engineering Facility: A Preliminary Report, University of Wisconsin, Madision, WI, UWFOM-500 (1982).

15. L. D. Pearlstein et. al., "Stabilization of Tandem Mirror Trapped Particle Modes by Incomplete Cancellation of Trapped Particle Orifts," in Sherwood Meeting, Annual Contr. Fusion Theory Conference, 1982 (Santa Fe, NM, 1982).

16. B. G. Logan, Lawrence Livermore National Laboratory, Livermore, CA, private communication (1981).

17. G. A. Carlson et. al., Comparative End-Plug Study for Tandem-Mirror Reactors, Lawrence Livermore National-Laboratory, Livermore, CA, UCID 19271 (1981).

18. B. M. Boghosian, R. B. Campbell, and J. M. Gilmore, A Potential Solver for Sloshing-Ion Thermal Barriers, Lawrence Livermore National Laboratory, Livermore, CA, UCID 19215 (1981).

19. M. N. Rosenbluth, H. L. Berk, D. E. Baldwin, H. V. Wong, and T. M. Antonsen, "Fast-Growing Trapped Particle Modes in Tandem Mirrors," in Sherwood Meeting, Annual Contr. Fusion Theory Conference, 1982 (Santa Fe, NM, 1982) paper $32 A$.

20. B. G. Logan, A. A. Mirin, and M. E. Rensink, "An Analytical Model for Classical ion Confinement in Tandem-Mirror Plugs," Nucl. Fusion, 20, $1613(1980)$.

21. W. L. Houlberg, "Pellet Ablation and Plasma Penetration Studies," Oak Ridge National Laboratory, Oak Ridge, TN, private comunication (Harch 1982).

22. G. P. Grubb et. al., "Energy Confinement Studies in the Tanden Mirror Experiment (TMX): Power Flow," Phys. Fluids, 26 (7), 1987 (1983).

23. K. L. Wurıy, Läwrence Livermore National Laboratory, Livermore, CA, private communication (1982).

24. L. D. Pearlstein, T. B. Kaiser, and W. A. Newcomb, "Analytic Equilibrium with Quadrupole Symmetr! in the Paraxial Limit," Phys. Fluids, 24 (7), 1326 (1981).

25. M. E. Rensink, Lawrence Livermore National Laboratory, Livermore, CA, private comunication (1982). 
26. L. D. Pearlstein, G. R. Smith, and W. M. Nevins, in Physics Basis for MFTF-B (D. E. Baldwin, B. G. Logan, and T. C. Simonen, eds.), Lawrence Livermore National Laboratory, Livermore, CA, UCID 18496-part 2 (1980).

27. J. E. Osher, "Minimum Streaming Current Requirements for MFTF-B, Revised by Recent $R_{p} / a_{i}$ Scaling in $2 X I I B$, Lawrence Livermore National Laboratory, Livermore, CA, UCID-18060 (1979); also Ref. 3.

28. D. Slaughter, Fusion Reactivities for Several Ion Distributions, Lakrence Livermore National Laboratory, Livermore, CA, UCRL 87416 (1982).

29. K. D. Marx, et. a1, "Calculation of (OV) ${ }_{D T}$ for Anisotropic Mirror and Toroidal Distributions," Hucl. Fusion Lett., 16, 702 (1976).

30. A. H. Futch and L. L. LoDestro, Collisjonal Träping Rates for Ions in a Magnetic and Potential Well, Lawrence Livermore National Laboratory, Livermore, CA, UCRL-87249 (1982).

31. J. Hovingh and R. W. Moir, "Efficiency of Injection of Neutral Beams in Thermonuclear Reactors," Nucl. Fusion 14, 629 (1974).

32. G. A. Carison and G. W. Hamilton, "Wall. Bombardment Due to the Charge Exchange of. Injected Neutrals with a Fusion Plasma," Lawrence Livermore National Laboratory, Livermore, CA, UCRL-75306 (1974).

33. R. L. Miller, "Mante Carlo Simulation of Neutral Beam Injection for Mirror Fusion Reactors," Ph.D. Thes is (University of Illinois, 1979).

34. L. J. Perkins and T. B. Kaiser, Monte Carlo Studies of Charge-Exchange First Wall Heat Loads from Nettral-Beam Injection in Tandem Mirror Fusion Reactors," University of Wisconsin, Madison, W1, Fusion Engineering Program report to be published (1983).

35. T. B. Kaiser, MFE Quarterly Report, Lawrence Livermore National Laboratory, Livermore, CA (1978).

36. Mirror Advanced Reactor Study (MARS) - Interim Repart, Lawrence Livermore National Laboratory, Livermore, CA, UCRL-53333 (1983).

37. Joint European Jorus (JET) Annual Report, JET Joint Undertaking (1981).

38. C. C. Baker et a1.2 "STARF IRE - A Commercial Tokamak Fusion Power Plant Study," Argonne Natjona1 Laboratory, Argonne, 1L, ANL/FPP-80-1 (1980).

39. J. Bohdansky, J. Nucl. Mater. 93, 94, 44 (1980).

40. J. Roth, J. Bohdansky and W. Ottenberger, Data on Low Energy Light Ion Sputtering, Max-Planck-Institut fur Plasmaphysik, Garching, Germany, Report Ipp 9/26 (1979). 
41. D. L. Book, NRL Plasma Formulary Naval Research Laboratory, Washington, D. C. (1980).

42. V. P. Pastukhov, Nucl. Fusion 14, 3 (1974).

43. R. H. Cohen, M. E. Rensink, T. A. Cutter and A. A. Mirin, Nucl. Fusion 18, 1229 (1978).

44. T. C. Simonen, Lawrence Livermore National Laboratory, Livermore, CA, private communication $(6 / 25 / 82)$.

45. R. A. Hulse, D. E. Fost, and D. R. Mikkelson, "Charge-Exchange as a Combination Mechanism in High-Temperature Plasmas," J. Phys. B: Atom. Molec. Phys., 13, 3895 (1980).

46. P. Poulsen, Lawrence Livermore National Laboratory, Livermore, CA, private commication (1983). 

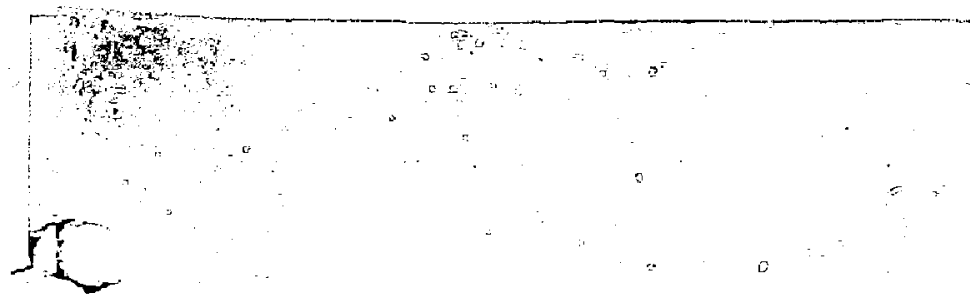

-

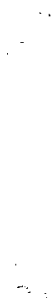

.

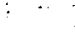

$=$

$\therefore$

SECTION 3.0. EMGIMEERIMG
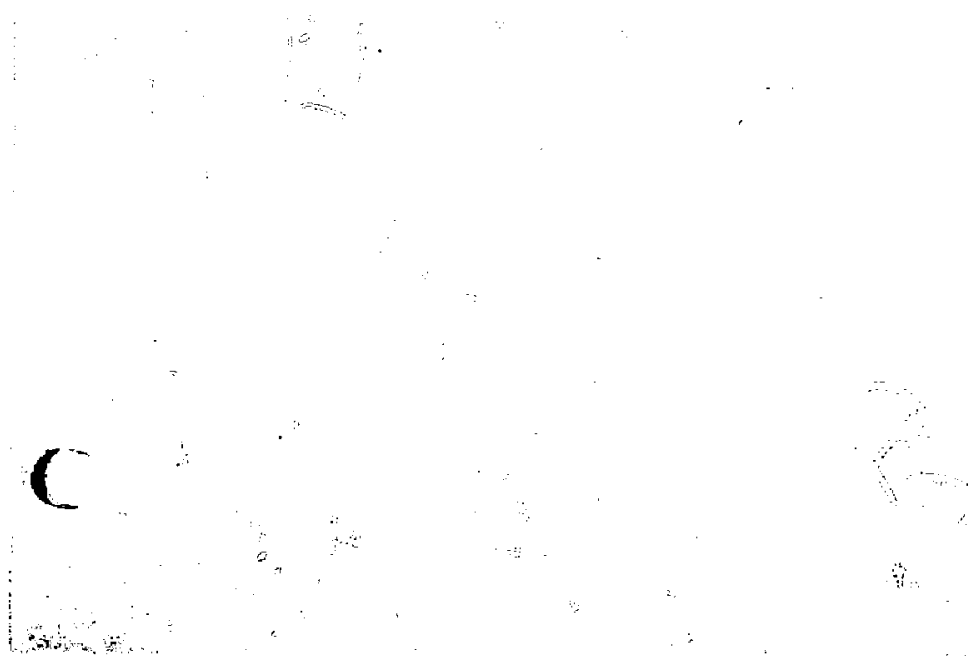

i: 


\subsubsection{Introduction and Summary}

A major component in the National Mirror Fusion Program Plan, TDF is a small, driven (power input greater than fusion power output) tandem mirror mach ine, which promises higher neutron flux $\left(1\right.$ to $\left.2 \mathrm{MW} / \mathrm{m}^{2}\right)$ and fluences at a lower cost than competing designs. Based on physics results from current machines, construction of TDF could be initiated in FY 7985 and completed in about 5 years. Data from this 59.4-m-long device will then be used in the final design of an economically attractive fusion reactor design.

This machine has been designed to meet two primary objectives:

- Provide nuclear fusion engineering data that demonstrates the engineering feasibility of steady-state operation, furnishes sufficient testing area under meaningful wall loads, and demonstrates the total fuel eycle operation, including tritium breeding and recovery.

- Demonstrate the construction, safe operation, and "maintainability" of a device that incorporates technologies usable in future fusion devices.

Figure 3-1, a cutaway view of the facility, shows the major systems and subsystems of TDF. In this drawing only one component from each system is shown, and for clarity these have been rotated into the plane of the drawing. Using this approach, we can portray the interfacing of the various components. For example, only one beamline is shown in the central cell at the right of the centerline; there are actuaily four beamlines symmetrically located on each side, and each of these is skewed to the plane of the drawing. Figure 3-2 shows the precise location of these beamlines.

Furthermore, the plasma and halo outlines depicted in Fig. 3-1 are actually oriented at $45 \mathrm{deg}$ to the plane of the drawing. Accordingly, aach of the components in the anchor and end cells is also displaced by that angle. The end view in Fig. 3-2 shows the proper orientation of these components. 


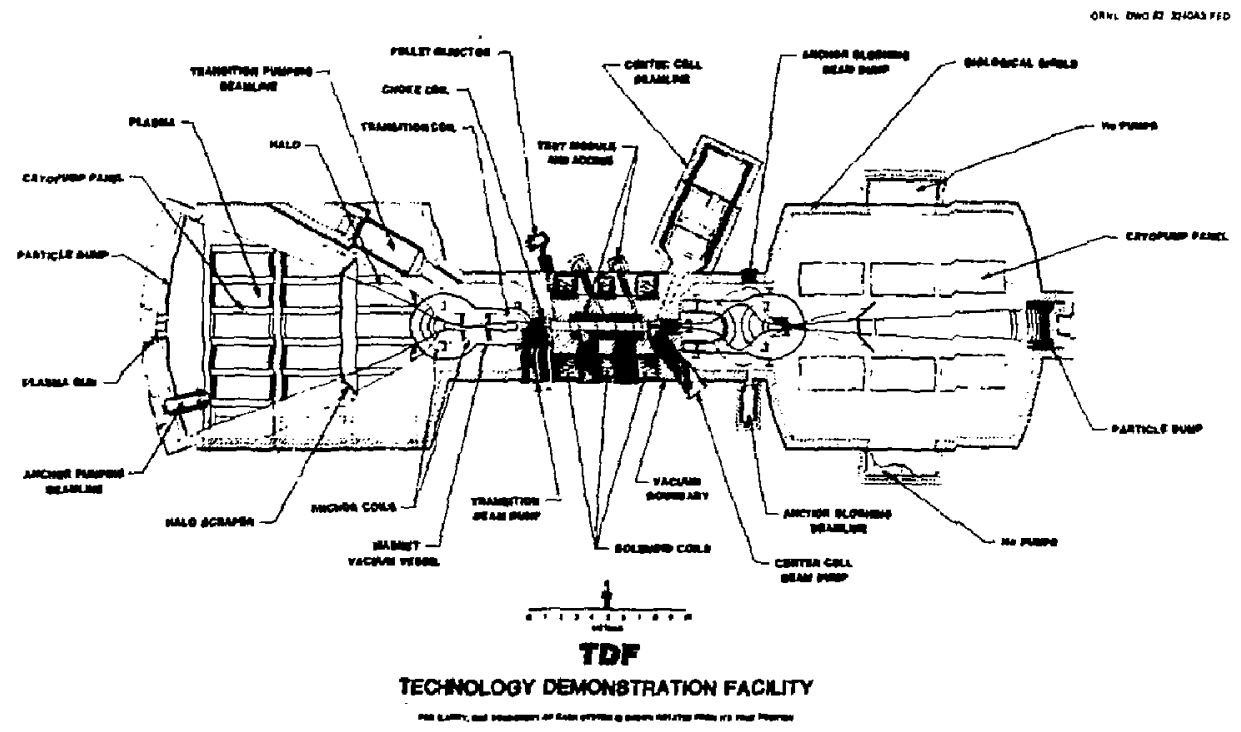

Fig. 3-1. The TDF conf iguration: an evolved design that meets the requirements of all system components. For clarity, one component of each system is shown rotated from its true position. 
TECHNOLOGY DEMONSTRATION FACHITY

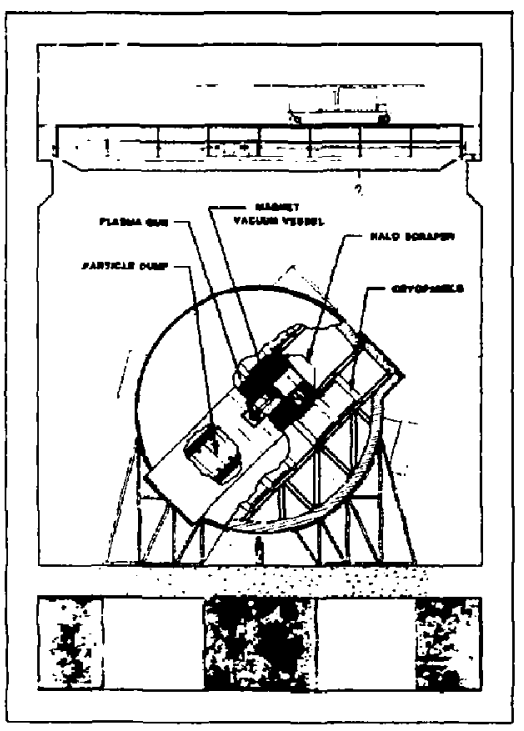

END VEW

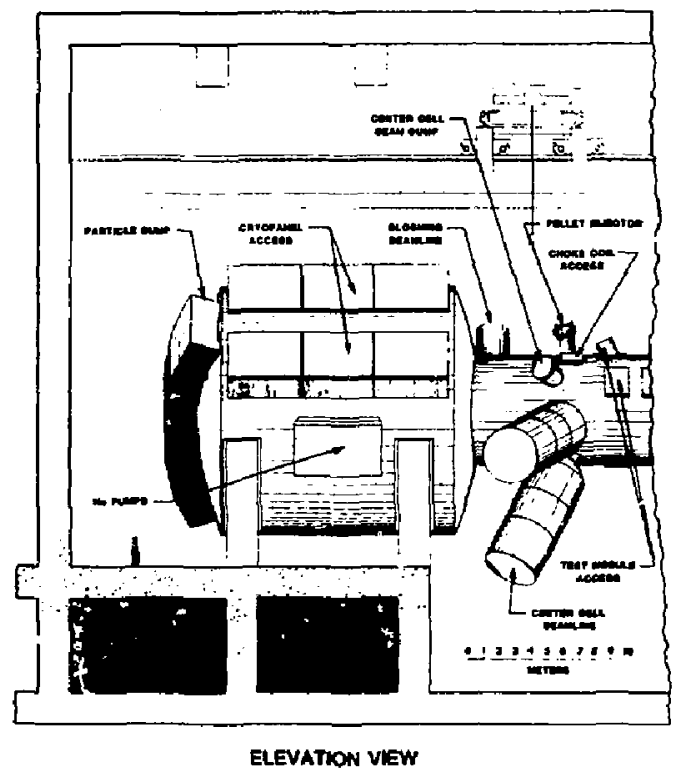

Fig. 3-2. Side elevation and end views of the nuclear engineering device, 
The TDF configuration has three main sections: the central ce11, the anchor cells, and the end cells. Figure 3-3 illustrates these three main areas and indicates the basic dimensions of the device.

The 8-m-long central cell is measured from the centerline of the choke coils. In adaition to these two high-field choke coils, the central cell also contains: the vacuum boundary, solenoid coils (SO and 51), nuciear shieiding $(5 / S)$, test mocules, beamlines, beam dumps, anc pellet injectors. The diameter of the central cell--7 m--was established primarily by the geometry of the two solenoio magnets.

The 6-m-long anchor cell is measured from the centerline of the choke coil to the intersection of the end-cell dome. This area includes the sloshing taamline, sloshing beam dump, transition beam dump, nuclear shield of heavy concrete, vacuum vessel, transition coil (TI), and the anchor coil set (M2 and M3). The diameter of the anchor cel1, measured to the outside of the vacuum boundary, is approximately $7 \mathrm{~m}$. This dimension allows for a reasonable clearance setween the plasma halo and the inner surface of the anchor-cell shielding.

The eno cell is approximately $16 \mathrm{~m}$ in diameter and 19-1/2 $\mathrm{m}$ in length, measuring to the shield plug covering the plasma guns. This outermost area contains the anchor pumping beamline, transition pumping beamline, particle dlimp, cryopump panels, nuclear shield of ordinary concrete, halo scraper, helium pumps, and plasma guns. Its final size is based on limiting the peak thermal load on the particle dump to $300 \mathrm{~W} / \mathrm{cm}^{2}$ and cantaining $576 \mathrm{~m}^{2}$ of cryopanel surface in each ce11. We have determined that by meeting the requirements for the peak thermal load we can provide sufficient volume for the cryopanels. He discuss these considerations in more detail in the following section on physical characteristics of the device.

\subsubsection{Design Approach and Requirements}

The overali layout of the TOF configuration was based on three considerations: modularity of each component, access to each component, ano integration of all systems to ensure total compatibility. At the same time these are the requirements that guarantee a configuration with "maintainabijity." 


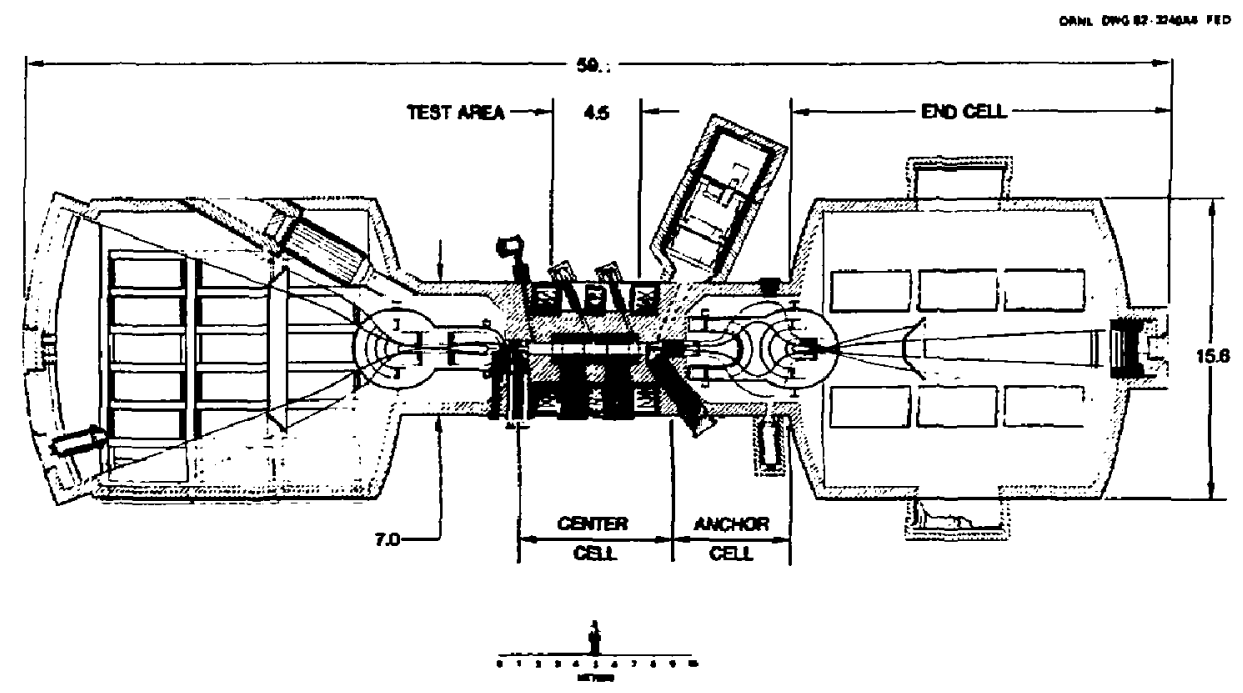

Fig, 3-3. The three areas of the machine: the central, anchor, and end cells. 
Moaular Design. Each component of TOF is modular, designed to be replaced individually. The module sizes depend on the particular component and the available access. For example, removal of the shield plug on the end-cell Lome provides reaay access to the small plasma gun. Hence, plasma-gun replacement involves removing only two small modules.

The sloshing beam aump located on the side of the anchor cell is also a module, conveniently mounted to the shield plug. This design allows us to replace the beam dump by handing only one relatively small component that incorporates the shield plug and the dump panel as well as the coolant lines. This same approach was used to design the larger central cell beam dumps for ease of access. Incorporating the sloshing beam aump into the shield plug was actually a necessity, however, because this dump panel is virtually inaccessible except by disassembling a portion of the central cell.

Not all components can be subdivided into small modules. The cryopanels are arranged so that each end cell has six assemblies, each containing six double-sided panels measuring $2 \times 4 \mathrm{~m}$. Therefore, replacing the cryopanels involves hanaling a moaule approximately $11 \mathrm{~m}$ long, $4 \mathrm{~m}$ wide, ana $2 \mathrm{~m}$ oeep.

The central cell neutrai-beam system is perhaps the best example of mocularity because it embodies both small and large modules. The ion sources are expected to require frequent replacement and are designed to be accessible and replaceable without disturbing the other components in the beamline. Keplacement of the larger internal components requires disassembly of larger modules (such as the rear superconducting magnetic shield, a portion of the nuclear shield, and finally, the total internal assembly of the beamlinel in one large module, which is then transported to a hot cell.

Access. Through such modularity we have provided for replacement access for each component. In the central cell the beamlines are arranged below the device midplane to permit overhead access for any replacement of the center components. The clustered beamline arrangement allows ready access to the 
choke coils, the central cell beam dumps, and the pellet injectors. Fortunately, access to the test modules is not affected by the arrangenent of the beami ine. Decause their injection angle is $65 \mathrm{deg}$.

In the anchor ce11, as we mentioned, access to the sloshing bean dump is through removal of the shield plug to which it is attached. Access to the sloshing beamline is possible by removal of the rear portion of the nuclear shield. The transition pumping beam, similar in cesign to the central cell dumps, is accessible through the bottom of the anchor cell.

Numerous access ports are provided in the end cely--six in each upper midplane, one for each cryopanel assembly, and shielcied covers for each set of helium pumps. The plasma guns, as well as the anchor pumping beamline, can be reachea through a small shield plug. The particle dump is accessible by removing the upper portion of the protruding shield that is mounted on the end-cell aome. Note that this access unaer the end cell was a configuration requirement; therefore, there is a $2-m$ clearance to the building floor. This dimension is sufficient to permit comfortable hands-on operations under the end cell; at the same time it can accommodate penetrations into the device and also any equipment that must be installed under the end cell.

Systems Integration. We integrated each of the systems and subsystem components by considering their functional requirements, the frequency of replacement, and their requirements for interfacing with adjacent components. This approach was particularly important in the central cell, where many components are competing for a limited amount of space. For example, the area along the plasma axis contains five components in close proximity: the four beamline drift tube openings and their corresponding beam dumps, the choke coil, the transition pumping beam oump, and the pellet injector. Figure 3-4 locates these components and gives their dimensions.

\subsubsection{Selected Physical Characteristics}

This discussion concentrates only on those physical characteristics pertinent to an overview. For additional information, refer to the section on the specific component. 


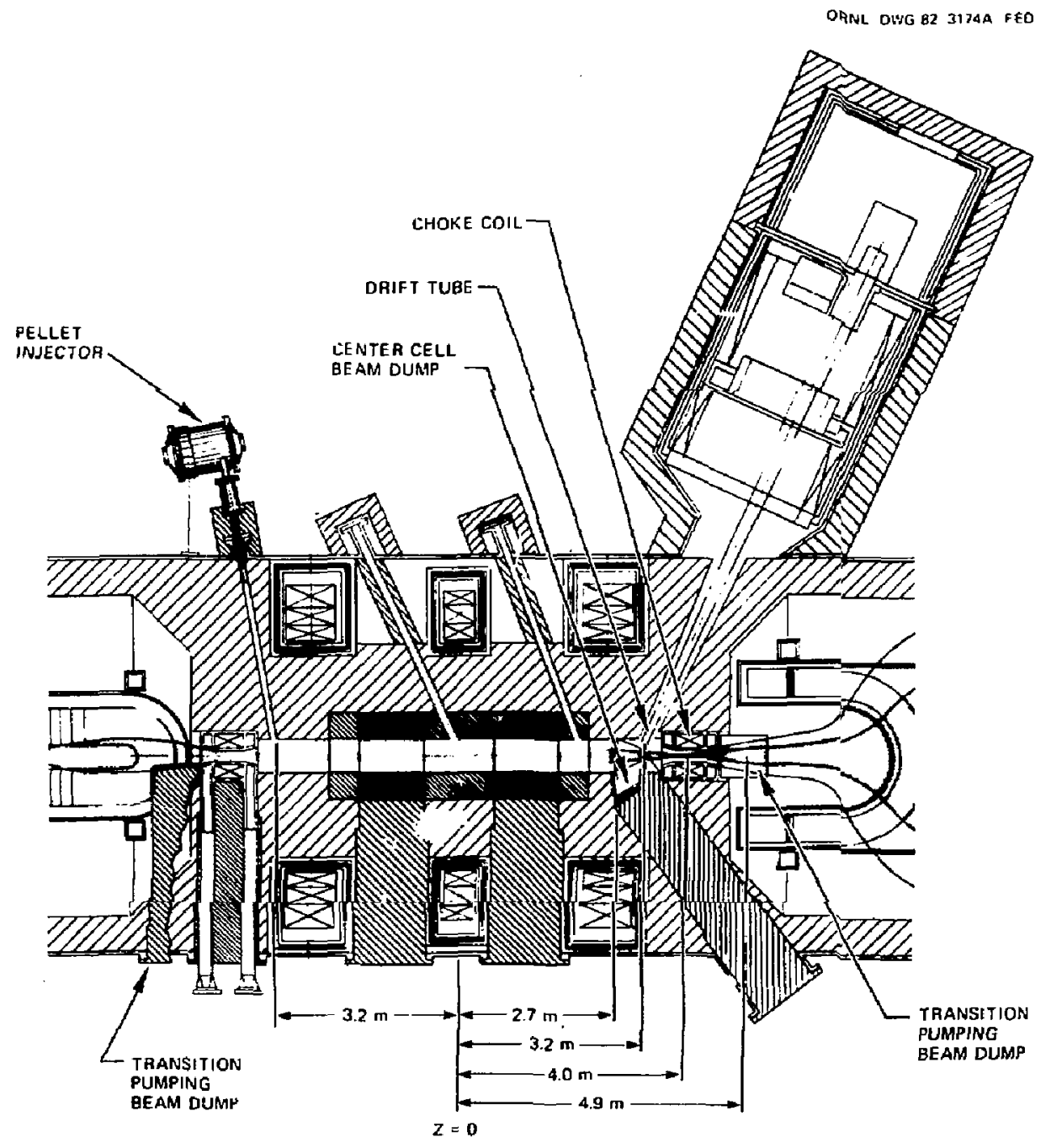

Fig. 3-4. Five components integrated into a limited portion of the central cell. The entire machine has been designed to permit easy access through its modular construction. 
Overall Device. We based our lluclear bulk shielding design for TDF on the requirement that calls for hands-on maintenance operations, 24 hours after shutdown, provided the snield remains intact. In the central cell, where the deuterium-tritium (D-T) fusions occur, the shield also provides protection to the superconducting coils to counter the effects of neutron heating, insulation damage, and increased copper resistivity. We determined that $115 \mathrm{~cm}$ of stainless steel plus $5 \mathrm{~cm}$ of lead cladding was sufficient to meet the biological activation limit $(2.5 \mathrm{mrem} / \mathrm{hr})$. Of this total, $90 \mathrm{~cm}$ of stainless steel placeo between the plasma and the magnet bore is aoequate to meet the requirements for coil protection.

The anchor cells and ena cells also have a biological shield. However, because these areas of the device receive essentially collimated neutrons streaming out of the central cell, the material and thickness requirements are less demanding. The anchar cell requires $60 \mathrm{~cm}$ of heavy (borated) concrete, whereas the end cell requires $60 \mathrm{~cm}$ of ordinary concrete.

The primary vacuum boundary is externaily located around the device, boti to simplify the vacuum joint interfaces for penetrations (i.e., those of the test modules and beam dumps) and to take advantage of the hands-on mode of operation for inspection and repair. The exception to this approach is at the neutral beamlines, where the vacuum boundary is located inside the beamline shielding.

We have provided for secondary vacuum containment around the superconducting magnets to avoid the additional downtime needed to thermally cycle these coils when the primary vacuum is lost during either routine changeouts of test modules or replacement of components (such as the choke coils or the beam dumps). Each of the solenoid coils has an individual vacuum vessel concentrically located around the structural case. Figure 3-1 shows this secondary vessel, as well as the transition and anchor coils that share a common vacuum containment. This common containment was requireu because of the coils' proximity. 
Central Cell. The central cell is a compact arrangement of many major systenis that comprise a tanden mirror device. The area allocated for engineering testing is of particular importance and is briefly discussed here. The test mooule region, as shown in Fig. $3-3$, is $4.5 \mathrm{~m}$ along the plasma axis, and with the test surface located at $R=0.25 \mathrm{~m}$, this yields a total available area of $7 \mathrm{~m}^{2}$. One-half of this area has ready access from between the solenoid coils; because the remainder is positioned in the plane of these coils, it is less accessible. Access to this latter test area requires removal of the test inodules between the coils. Therefore, we feel that any tests that require frequent changeout should be located between the coils and any long-term tests be located in front of the coils. This will minimize dountime.

The use of rabbit tubes into the less accessible test areas of the central cell provides an alternate means of introducing small test specimens without disturbing other test nodules. Figure 3-1 shows two such penetrations.

Anchor Cell. The anchor cell consists primarily of the sloshing beamline ano its beam dump, the transition pumping beam dump, and the magnet system in the cormon vacuum vessel. The anchor cell is sized to accomodate two requirements; its length was established by the support requirements for the magnet vessel and its diameter was based on maintaining a $0.5-\mathrm{m}$ clearance to the plasma halo. These sizing relationships, which represent a minimum volume for this cell, can be seen in Fig. 3-1.

One of our design objectives was to develop an overall configuration that minimized the building size for the reactor. This was partially achieved by designing a "foreshortened" ancior cell that does not allow for overhead access and remaral of the maxgot yessel, assembly. Reczuse this component requires removal through the end cell, it reduces the overall lerigth by approximately $5 \mathrm{~m}$. 
Eno Ce11. The end cell contains the largest component assembly that may require removal--the cryopanels. As previously mentioned, each assembly of six double-sidcd panels has an overall size of $11 \times 4 \times 2 \mathrm{~m}$. The orientation of the paneis (Fig. 3-2) is at 45 deg to the building floor and parallel to the plasma sheet. This skewed arrangement results from the orientation of the transition and anchor coils (also at 45 deg) because of the pool-boiling ciesign of these superconducting magnets. Each of the end and anchor cells has an orthogonal symetry relative to each other.

The center of the particle dump is located at $z=28 \mathrm{~m}$ and results from limiting the peak therma? load in this area to $<300 \mathrm{~W} / \mathrm{cm}^{2}$. This linear dimension, in conjunction with the aiameter required to contain the plasma eoge, is sufficient to contain the 36 cryopanels. 


\subsection{MAGNETS}

\subsubsection{0verview}

We have completed a reference magnet configuration for TUF. It consists of 11 coils, compatible with the design criteria shown in Tables 3-1 and 3-2. Three superconoucting solenoids in the central cell provide a 4.5-T field and allow test module clearance. Water-cooled choke coils provide a mirror peak fielu of $15 \mathrm{~T}$, while allowing neutral-beam access to the cential cell. The yin-yang pair flank a single transition coil, proviaing a 3:1 mirror ratio with a 3-T peak and allowing adequate clearance for neutral beams. The coils are designea to meet the coil engineering criteria of cooling, stability, and structural supports using state-of-the-art technology. The radiation shielding has been examined and is shown to be conservative.

Mignet Field Requirements. The field requirements are sumiarized in Table 3-i. Additional design criteria that virectly affect the configuration are included in Table 3-2. The resulting field profile is shown in fig. 3-5.

Magnet Configuration. The reference magnet configuration is shown in Fig. 3-6. It includes the coil geometry, cross section, position, and current aensity, as well as a description of the spacing between the SI and the $M I$ coils, which provices $35 \mathrm{~cm}$ of neutron shielding for the $\$ 1$ coil while allowing neutral-beam access to the central cell. The configuration is based on niobium-titanium conductor technology for all superconducting and water cooling for the choke coils.

External Field. Figure 3-7 is a plot of total field contours in the principal $v$ iew of the machine. The large area covered encompasses both the neutral-beam sources and the end region of the machine. Also shown is the flux tube, which corresponos in size to the $17-\mathrm{cm}$ radius of the plasma halo.

Conductor fields. The peak fields are shown in Table 3-3. 
Table 3-1. TDF magnet fielo requirements.

\begin{tabular}{ll}
\hline Central cell & \\
Plasma iength (m) & 8 \\
Plasma radius (cm) & 10 \\
Solenoid field (T) & 4.5 \\
Peak beta & 0.4 \\
Field variation ( $\mathrm{X})$ & $<5$ \\
Mirror region & \\
Field at mirrors (T) & 15 \\
Anchors & 2.87 \\
Plasma length (m) & 3.0 \\
Field at mirrors (T) & 1.0 \\
Field at miaplane (T) & 0.5 \\
Peak beta & \\
\hline
\end{tabular}

Table 3-2. Design criteria that affect the magnet configuration.

17-cm plasma haio clearance.

Circular flux lines in central cell and anchor.

Beam access to central cell, transition region, and anchor.

1.25-m test-cell clearance in central cell. 


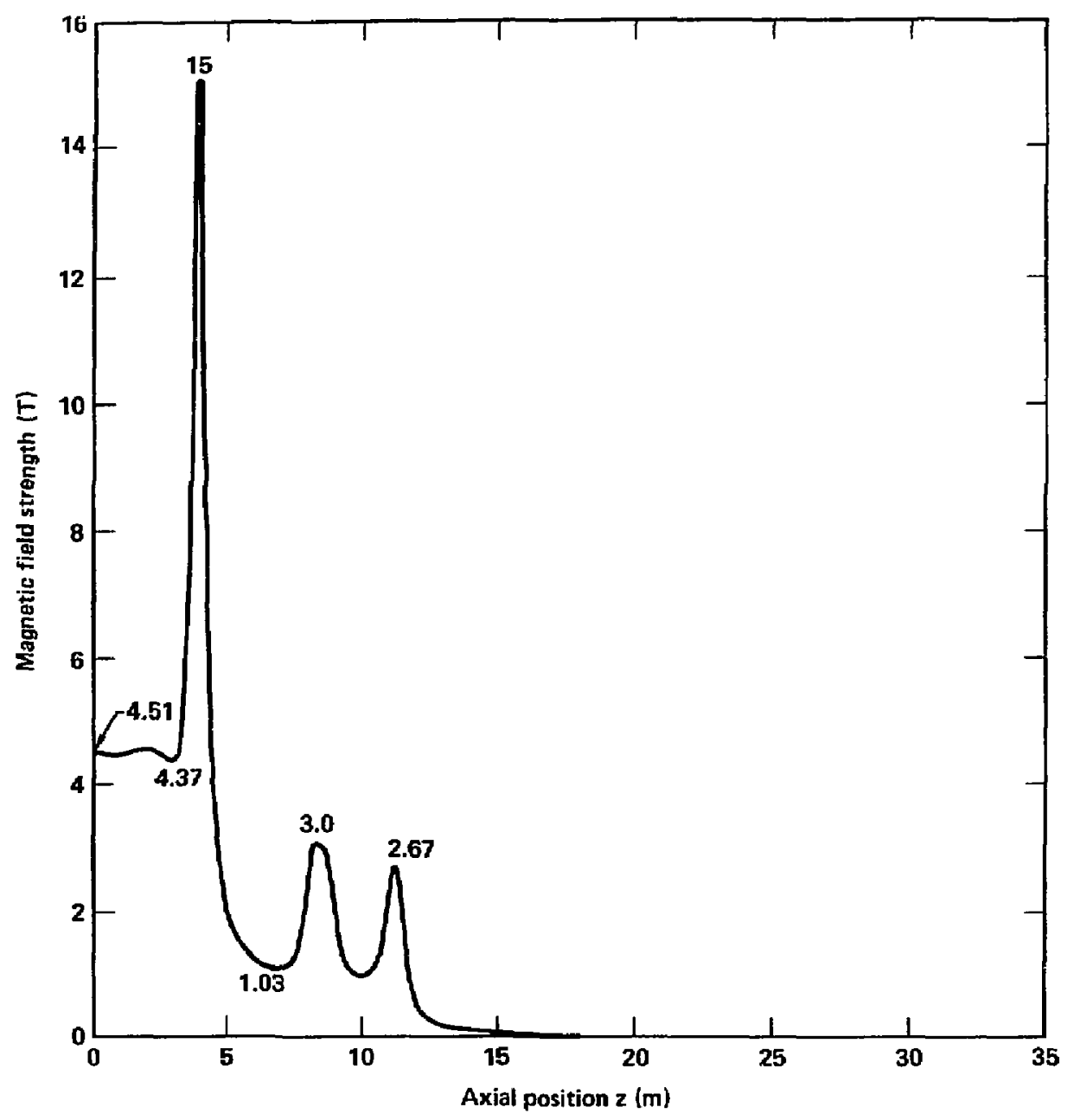

Figure 3-5. Center-line field profile. 


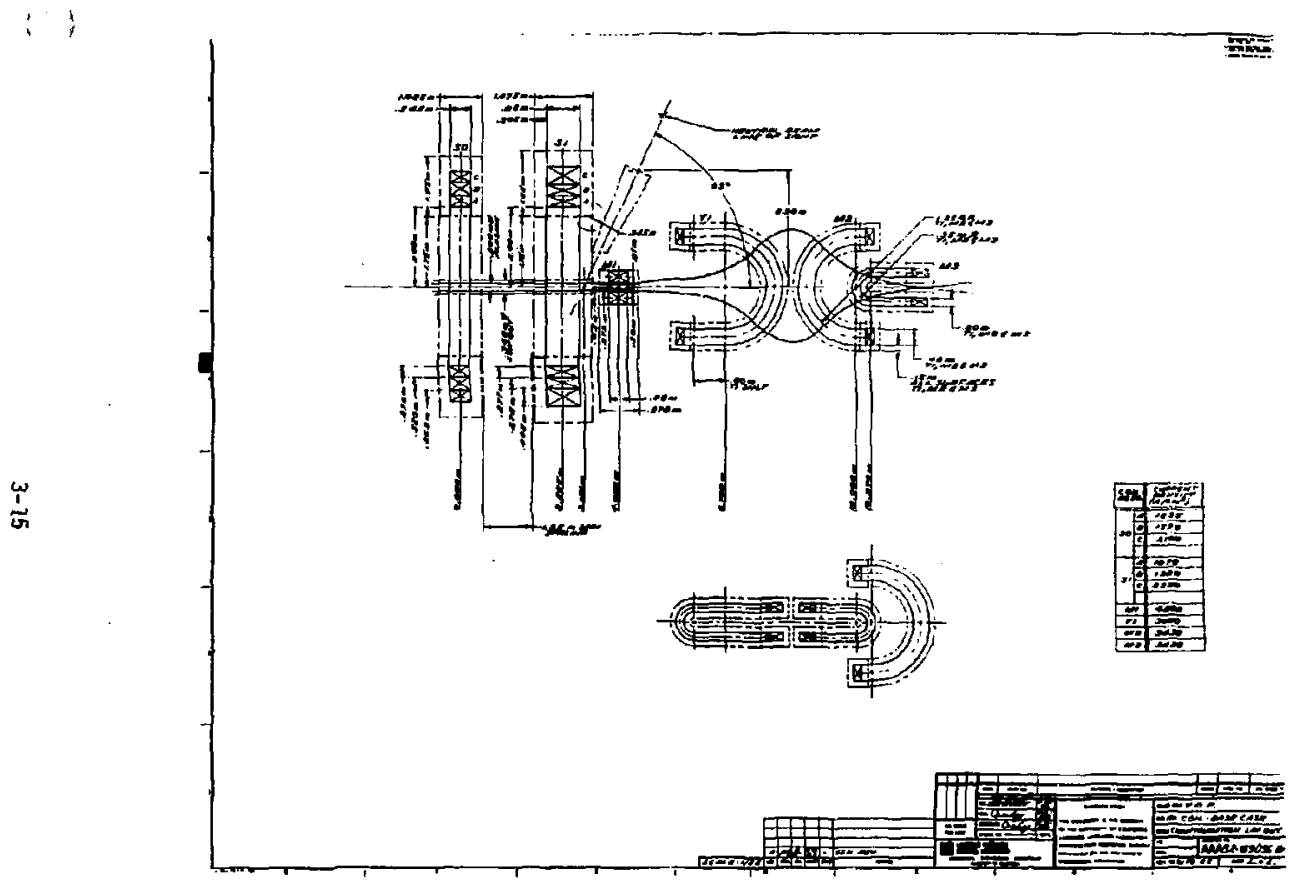

Figure 3-6. Heference magnet configuration. 


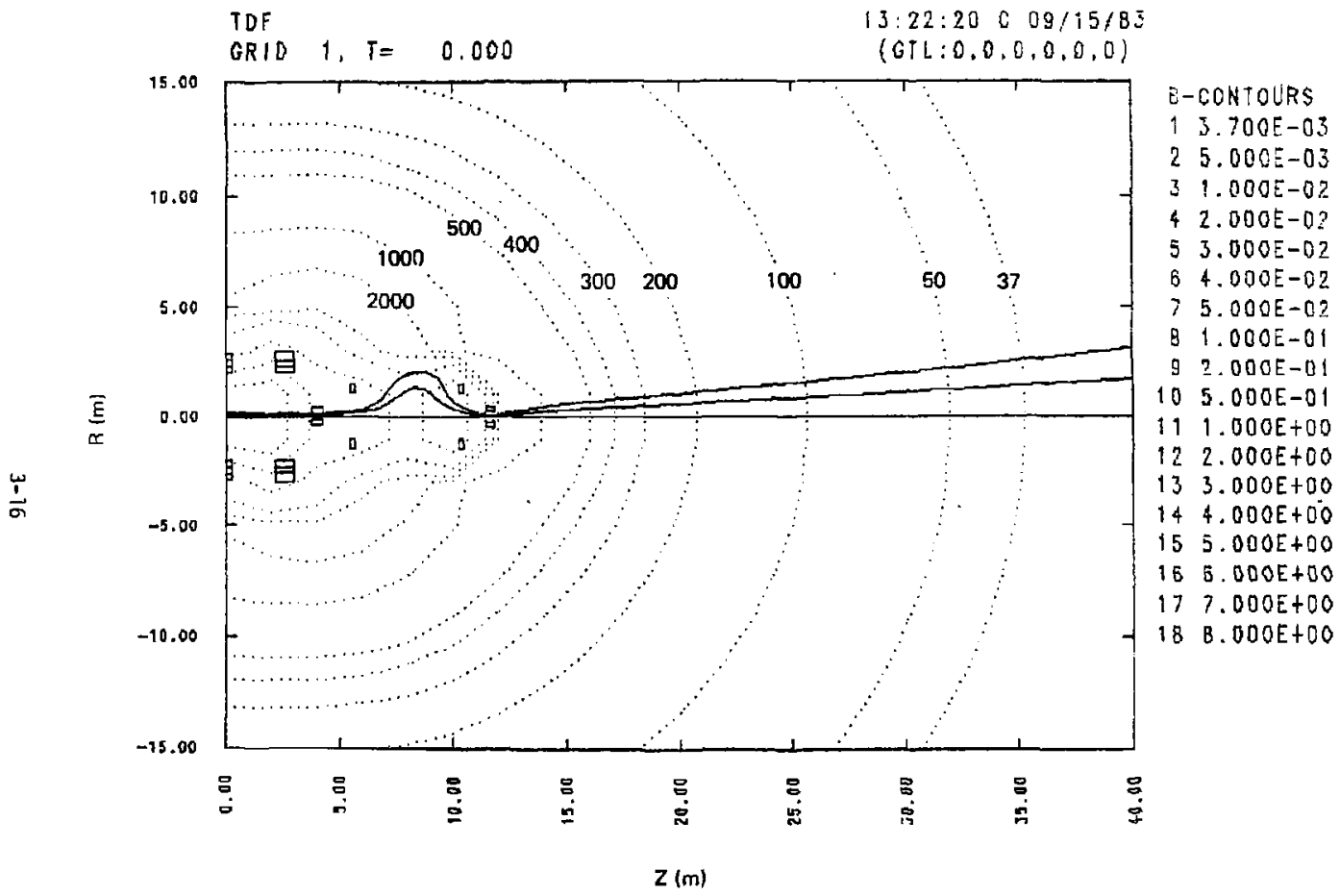

Figure 3-7. TDF external fields. 
Table 3-3. Peak fields in each conductor bundle.

\begin{tabular}{cc}
\hline Coi1 & Fieid (T) \\
\hline$S-0$ & 6.7 \\
$S-1$ & 7.6 \\
$M-1$ &.- \\
$T-1$ & 4.3 \\
$M-2$ & 4.7 \\
$M-3$ & 4.7 \\
\hline
\end{tabular}


Stored Energy. The energy stored in the magnetic field of each coil is shown in Table 3-4.

Ha lo Clearance. The coils were designed to allow the plasma halo clearance through the machine. The halo, which has a $17-\mathrm{cm}$ radius at the center line of the machine, clears all coils. It comes closest to the choke coil cryostat, as shown in Fig. 3-8.

Magnetic Forces. The magnetic forces calculated by the EFFI code are shown in Fig. 3-9. These nominal forces are calculated for the base case but do not represent the peak forces that must be analyzed as part of a detailed fault-case analysis.

\subsubsection{Sumfary Uescription of Central Cell and Choke Coil Magnet System}

This system consists of three superconoucting solenoids in the central cell with two resistive choke coils located symmetrically about it.

The layout of the magnet system is shown in Fig. 3-10. The design of the central cell is based on a reasonable extrapolation of existing technology, the magnets can be fabricated by the methods used to produce the solenoid magnets for MFTF-B.

The choke coils represent a design challenge because they produce a higher magnetic field than currentiy available coils and because their operational life is much longer than that of similar existing high field coils. The radiation environment in which the choke coils operate is aiso more severe than that for present technology coils. Developmental work is required to increase the operational life of the chcke coils and to ensure their integrity in the radiation environment.

Central cell Solenoids. The central cell superconducting solenoids produce a 4.5-T magnetic field with less than a $4.5 \%$ ripple, as required to confine the plasma. They have an inner diameter of $3.75 \mathrm{~m}$ and are separated by $1.25-\mathrm{m}$ gaps that allow access to the cell interjor for blanket module manipulation or for replacing portions of the shield. The current density in the windings is graded in three regions to achieve a more uniform stress distribution for the 
Table 3-4. Energy stored in each coil.

\begin{tabular}{cc}
\hline Coi\} & Energy $(M)$ \\
\hline$M-3$ & 12.47 \\
$M-2$ & 12.89 \\
$T-1$ & 13.73 \\
$M-1$ & 8.1 \\
$S-1$ & 700.0 \\
$S-0$ & 342.0 \\
\hline
\end{tabular}




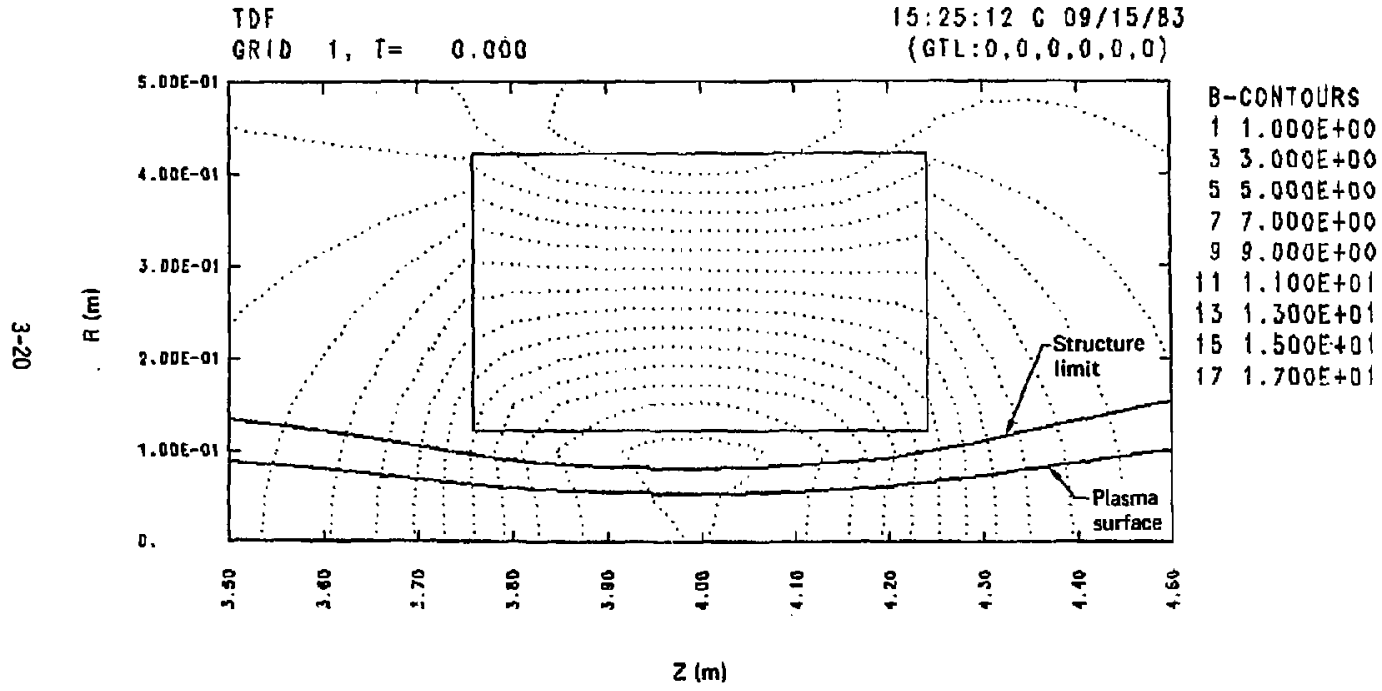

Figure 3-8. Flux bunole through the choke. 


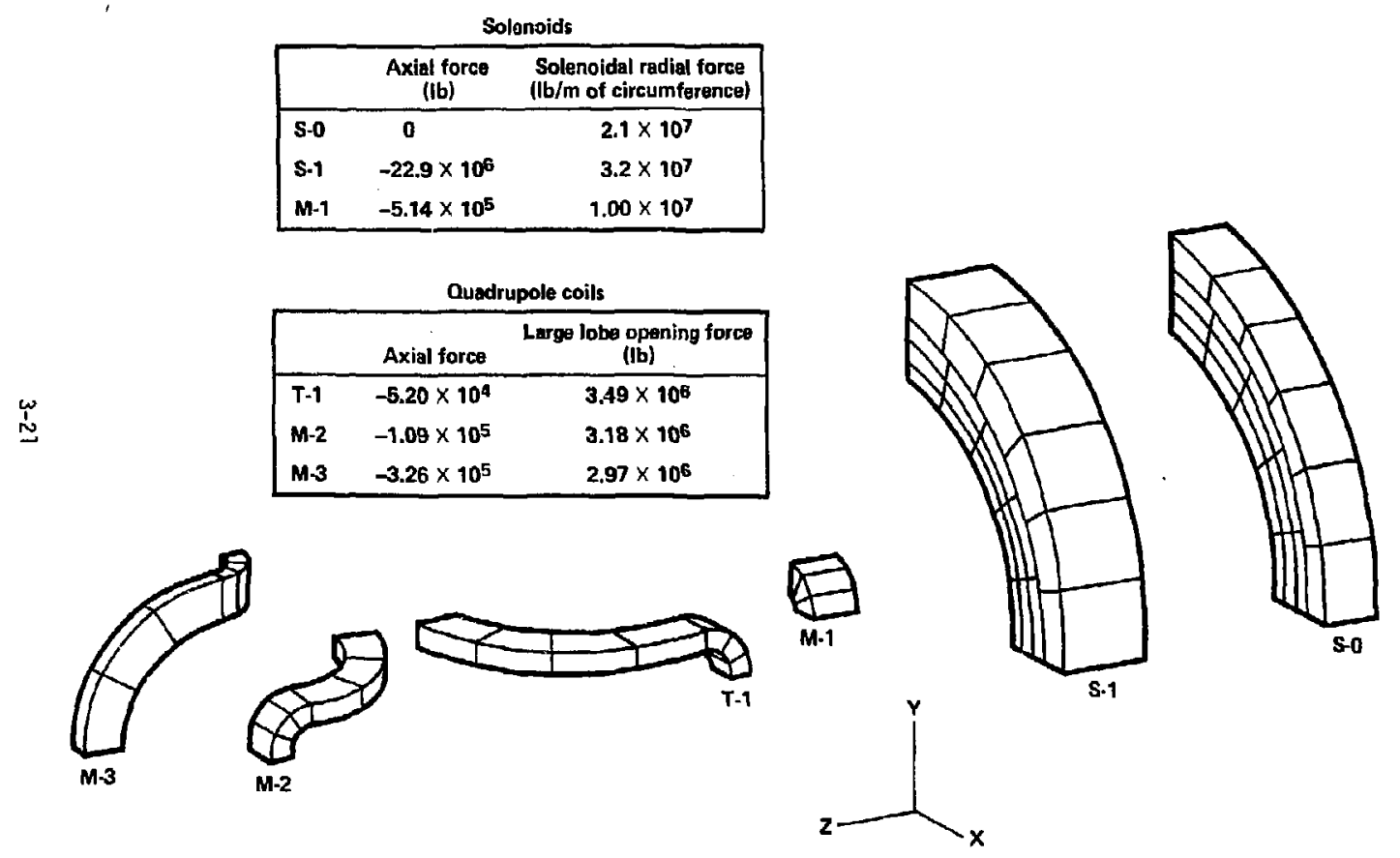

Figure 3-9. Easeline magnet system forces. 


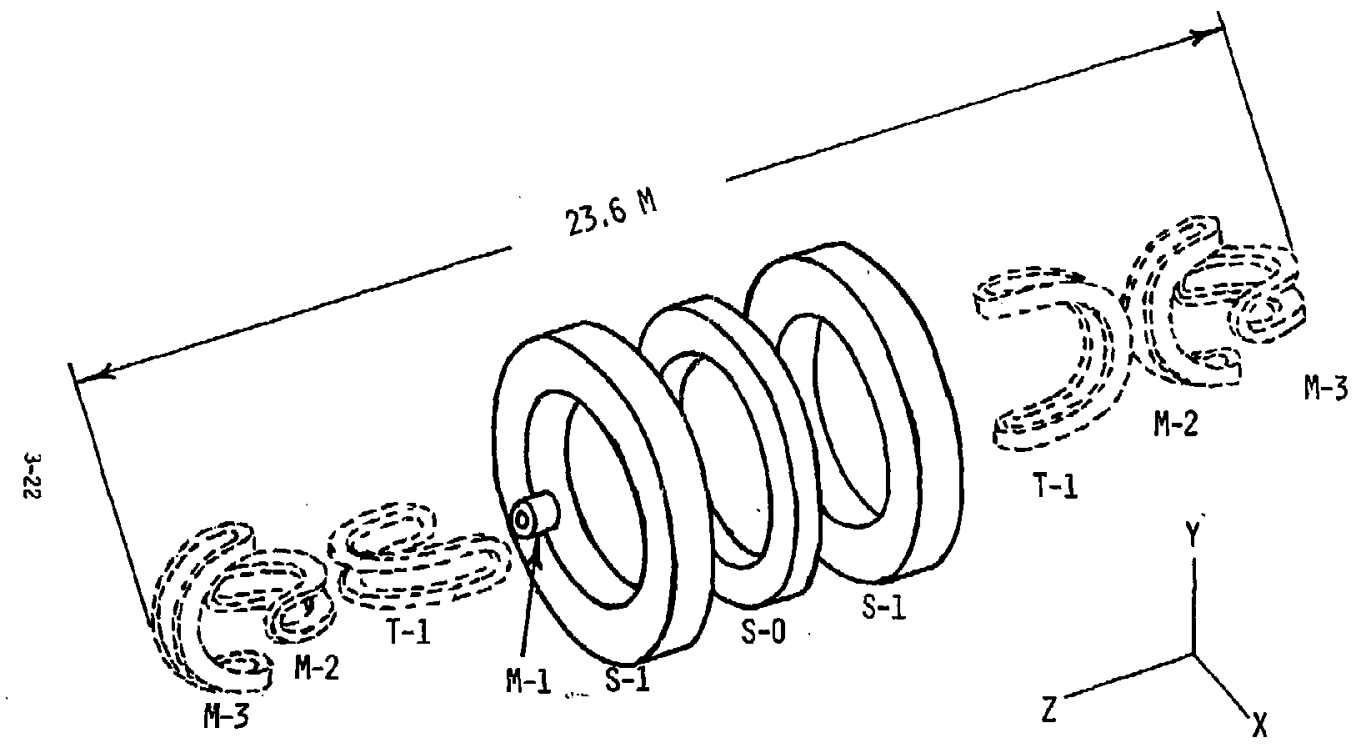

Figure 3-10. TOF magnet arrangement. 
most cost-effective design. The conductor consists of niobium-titanium superconductor joined to the copper stabilizer. The cryostable conductor is cooled with pool-boiling liquid helium at $4.2 \mathrm{~K}$.

Choke Coils. The choke coils are located $4 \mathrm{~m}$ along the axis on either side of the solenoid center 1 ine. Each coil produces a field of $12 \mathrm{~T}$, or a tatal field of $15 \mathrm{~T}$ when energized in conjunction with the central cell. We chose a resistive coil for this application because it uses current technology and satisfies magnetic field requirements within the space limitations. Our baseline configuration for this study uses a polyhelix-type coil with externally-cocled conductor; however, as a resuit of the trade-off studies described in this section, we recomend that an internaliy cooled conductor be considered for the finat design effort.

The deionized water used as a cuolant for the coil with the externally cooled conductor passes axially through the coil. The current density is $4800 \mathrm{~A} / \mathrm{cm}^{2}$. We chose to make the conductor of zirconium copper (AMZIRC), a material of relatively high yield strength and high conductivity--93\% International Annealed Copper Standard (IACS). Spinel $\left(\mathrm{Al}_{2} \mathrm{MgO}_{4}\right)$, was chosen as the insulator because it exhibits low swelling under radiation, is insensitive to chemical attack, and $c$ an be formed into the required shapes by sintering. The total power requirement for one coil is $26 \mathrm{Mw}$.

\subsubsection{Centrat Ceil Solenoids}

This section presents the proposed conceptual design for the central cell solenoids. Design considerations and requirentents, as well as trade-off studies, are presented as introductory topics. Engineering analyses come afterward.

Design Considerations and Requirements. The major design constraints on the magnets are determined on the basis of both plasma physics and space requirements for machine access, neutral-beam injection, and radiation shields. As a result of these constraints, the central magnetic field is $4.5 \mathrm{~T}$. The magnetic field ripple is limited to $4.5 \%$, the inner diameter of the magnet is $3.75 \mathrm{~m}$. The dimensional constraints must also allow sufficient space for support structures and the magnet cryostats. 
The magnets must also provide low-cost, reliable, ana safe operation. Therefore, it is desirable to base the magnet oesign oil such proven superconauctor technology such as copper-stabilized niobium-titanium superconauctor cooled at $4.2 \mathrm{~K}$ in the pool-boiling mode. Grading of the conductor reduces cost because of the effective use of the superconaucting material.

The central cell faces an intense neutron environment $\left(2 \mathrm{MW} / \mathrm{m}^{2}\right.$ of neutron wall loading) that can adversely affects material properties. For example, the electrical resistivity of an unsheilded stabilizer in this environment is increased and the mechanical and dielectric strengths of the insulator are reduced. In addition to these material properties effects, the critical parameters of the superconductor (critical current density, magnetic isela, and temperatuxe) are, in tinie, reuced. The neutron baat loads on the mignet winding also affect the cost of the cryogenic system. Therefore, the central magnets are provided with enough shielding to keep radiation damage ano neutron heating within acceptable limits for the full performance of the magnets over their design life of 5.4 full power years (FPY).

The radiation damage that increases the stabilizer resistivity is partially or wholly recoverable by annealling at room temperature. Damage to the insulators and superconductor is cumulative and nonrecoverable. Gama-ray irradiation affects organic iniulators. The radiation resistance of the structural materials is four orders of magnitude higher than that of the stabilizer, insulator, and superconductor. The structural materiais will out last any of these components.

The requirements and considerations were anajyzed, and a consistent set af requixements was defined and is summan ized in Takie 3-5. Reopsirements for the choke colls are also inclused in the tatle, but are not discussed in detail until Section 3.2.4. The most stringent constraints for the magnets include dimensional constraints, magnetic fields, and operational lives.

Engineering Trade-off Studies, Our engineering trade-off studies were focused primarily on the selection of the coolant. Cooling with HeI in the pool-boiling moce at $4.2 \mathrm{~K}$ was chosen for the baseline design befause it 
Table 3-5. Solenoid magnet requirements. The American Society of Mechanical Engineering (ASME) pressure-vessel design code is used both for the central solenoids and choke.

\begin{tabular}{|c|c|c|c|}
\hline Parameter & Central sol & enoids & Choke \\
\hline Coil & $5-0$ & S-1 & $M-1$ \\
\hline Central field (T) & $\begin{array}{l}4.5 \text { (combined } \\
\text { with } S-1)\end{array}$ & $\begin{array}{l}4.5 \text { (combined } \\
\text { with } 5-0 \text { ) }\end{array}$ & $\begin{array}{l}15.0 \text { (combined } \\
\text { with } S-0 \text { and } s-1 \text { ) }\end{array}$ \\
\hline Type of cooling & LHel & LHel & Wat: \\
\hline Conductor type & Super & Super & Anı zirc \\
\hline Operational life (FPY) & 5.4 & 5.4 & $i .0$ \\
\hline Clear distance to $\mathrm{S}-1(\mathrm{~m})$ & 1.25 minimum & $N / A$ & $N / A$ \\
\hline Inner winding radius (m) & 2.0 & 2.0 & 0.122 \\
\hline Clear bore radius (m) & $N / A$ & $N / A$ & 0.104 \\
\hline Z-axis location (m) & 0.0 & 2.585 & 4.0 \\
\hline
\end{tabular}


simplifies conauctor and coil engineering and uses simpler cryogenic equipnent. This option is also considered more reliable because it is basea on proven superconductor technology.

foditional trade- of $f$ studies show that neutron heat deposition in the winoing significantiy impacts the capital and operating costs of the cryoplant. Proper selection of materials and shield geometry limits neutron heat oeposition in the magnets, reduces neutron and gamma damage to the coil materials, and consigerably reduces the total cost of the cryoplant.

Choice of Coolant. Cooling with LHeI in the pool-boiling mode has been compared to cooing with LHell at $7.8 \mathrm{~K}$. Figure 3-? s sows the coil configurations for both cases.

Cooling with LHeIl allows a $30 \%$ reduction in magnet cross section because of the superior heat-transfer properties of Hell. As a resuit, both the weight and cost of the magnet can be reduced, Overall cryostat dimensions do not change appreciably, however, because more space is needed to accommodate an additional radiation shield conled with Hel.

Table 3-6 compares the characteristics of conductors cooled with LHeI to those of conouctors cooled with LHeII.

The LHeII-cooled conductor incorporates stainless steel reinforcement to support the hoop loads at a maximum stress of 40,000 psi. It also allows a sinaller winoing than the LHel conductor, but the conductor geometry is more complex and requires special manufacturing techniques and aoditional qualityassurance procedures.

Table 3-7 compares the costs of HeI and HeII central cell solenoids ano associated cryogenic equipment.

While cooling with LHeII does reduce magnet size and cost, several factors weigh against its use at this time. The use of LHeIl is not a developed technology, so developmental work would be required. Also, the service stack is more complex ror LHeII, and more refrigeration is necessary. Because these factors add to the total cost, we concluded that the use of LHeI is preferred for this machine.

Effect of Neutron Shielding on Cryoplant Cost. Neutron heat deposition in the windings directly affects the cost of the refrigeration equipment because a larger capacity refrigerator is needed to remove the heat. 
LHE I

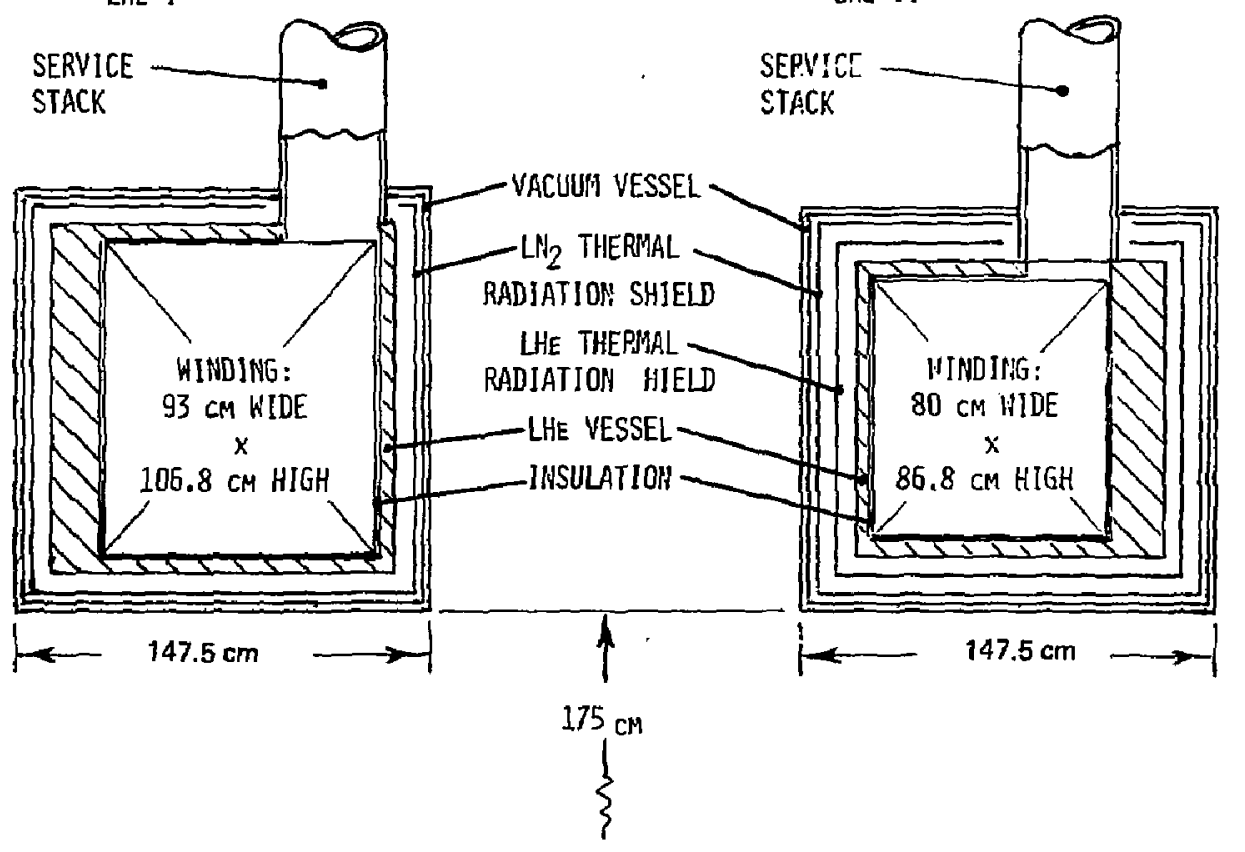

Figure 3-11. Coil configurations for Hel and HeII cooling schemes. 
Table 3-6. Characteristics of LHeI- and LHelI-cooled conductors.

\begin{tabular}{|c|c|c|}
\hline Coolant & LHEI & LHelI \\
\hline Superconauctor & niobium-titanium & niobium-titaniun \\
\hline Stabilizer & Half-hard OFHC copper & Half-hard UFHC \\
\hline \multicolumn{3}{|l|}{ copper } \\
\hline Reinforcing material & $N / A$ & Stainless steel \\
\hline \multicolumn{3}{|l|}{ 304LIV } \\
\hline Allowable stress (psi) & 28.000 & 40,000 \\
\hline Average current density $\left(A / \mathrm{cm}^{2}\right)$ & 2,100 & 2,750 \\
\hline Heat flux $\left(\mathrm{W} / \mathrm{cm}^{2}\right)$ & 0.25 & 1.00 \\
\hline
\end{tabular}


Table 3-7. Cost trade-off study of LHeI vs LHeII for central cell solenoids. Costs are in millions of dollars.

\begin{tabular}{|c|c|c|}
\hline Coolant & (Pool-boiling) & $\begin{array}{c}\text { LHeIl } \\
\text { (Superfluid) }\end{array}$ \\
\hline $\begin{array}{l}\text { Cost of coil, incluiting } \\
\text { liquid helium case }\end{array}$ & 9.20 & 6.85 \\
\hline $\begin{array}{l}\text { Cost of liquid helium thermal } \\
\text { radiation shield }\end{array}$ & $N / A$ & 0.009 \\
\hline $\begin{array}{l}\text { Cost of cryogenic plant } \\
\text { With LHeI } \\
\text { With LHeII }\end{array}$ & $\begin{array}{l}0.49 \\
N / A \\
\end{array}$ & $\begin{array}{l}0.31 \\
0.56 \\
\end{array}$ \\
\hline Total capital cost & 9.69 & 7.72 \\
\hline $\begin{array}{l}\text { Operating cost per lifetime } \\
\text { (5.4 FPY at } \$ 0.10 / \mathrm{kWh})\end{array}$ & 0.89 & 1.54 \\
\hline Total lifecycle costa & $10.5 B$ & 9.26 \\
\hline
\end{tabular}

aCost estimate does not include stack, Lit2 shield, vacium vesse1, cryogenic plumbing, or power and control system. 
Table 3-8 gives the total life cycle cost of the cryoplant per coil as a function of heat input per unit volume in the cald magnets.

As shown, cryoplant cost decraases rapidiy with lower neutron heating. Smaller heat input also means less radiation damage to the conductor and insulation. The magnets have a required operational life of 5.4 FPY. This requirement is satisfied by choosing a shield that limits the neutron heating below $0.06 \mathrm{mw} / \mathrm{cm}^{3}$, which gives a life cycle operating cost of approximately $\$ 1.8$ million per coil.

Selected Design Approach. This section presents the preferred design concept for the TOF central cell solenoids. The essential features of the design are cescribed, and key engineering analyses are discussed.

\section{Design Description.}

Cojl Configuration. The coils are layer-wound on thick stee 1 bobbins. Layer winding was chosen to keep layer tension constant, since the conquctors carry their own hoop forces, and to allow current density graoing by varying conductor size. (Section 3.4 .2 shows that a singie series-wound conductor is feasible.) This approach requires only one power supply for each magnet. The insulation chosen is polyimide fiberglass, and the nioblum-titaniun superconductor is stabilized with half-hara OFHC copper.

The conauctors are cryogenically stabilized to tolerate small mechanical disturbances during coil charging and to ensure full current recovery of a normal zone. The heat-transfer rate from a normal region in each of the three graues has a maximum value of $0.25 \mathrm{~W} / \mathrm{cm}^{2}$, which is consistent with present technology.

The coil's hoop forces are carried by the copper stabilizer. The axial coil loads have not been completely identified for all operating and faut conditions, but a large nominal axial force does exist on $5-1$ acting toward S-0. This large force of $23 \times 10^{6}$ lbs requires thick side plates on the inner sides of S-1; these loads are carried by cold intercoil struts to the inner and outer shells of $5-0$.

The design reflects the basic philosophy of using proven technology to provide an economical and reliable set of coils that meets overall requirements. Figures $3-12$ and $3-13$ show cross sections, and Table 3-9 shows major characteristics of the $S-0$ and $S-1$ solenoids. 
Table 3-8. Cryoplant cost as a function of neutron heat input in a typical central cell coil.

\begin{tabular}{|c|c|c|c|c|c|}
\hline Peak neutron heating $\left(\mathrm{mw} / \mathrm{cm}^{3}\right)$ & 3.0 & 1.4 & 0.5 & 0.03 & 0.006 \\
\hline Neutron heat load $(W)$ & 7884.1 & 3678.1 & 1312.8 & 78.7 & 15.7 \\
\hline other neat load $(H)$ & 217.5 & 217.5 & 277.5 & 217.5 & 217.5 \\
\hline Total heat load $(W)$ & 8101.6 & 3895.6 & 1530.3 & 296.2 & 233.2 \\
\hline Cryoplant power factor $\left(W_{e} / W\right)$ & 668.0 & 668.0 & 668.0 & 668.0 & 668.0 \\
\hline Cryoplant power requirement (MW) & 5.4 & 2.6 & 1.0 & 0.20 & 0.16 \\
\hline $\begin{array}{l}\text { Operating cost per year }(\$) \\
\text { at } \$ 0.1 / \mathrm{kWh}(\$ \mathrm{~m})\end{array}$ & 4.74 & 2.28 & 0.895 & 0.173 & 0.136 \\
\hline $\begin{array}{l}\text { Lifetime operating cost, } \\
5.4 \text { years total on time }(\$ M)\end{array}$ & 25.6 & 12.3 & 4.8 & 0.93 & 0.73 \\
\hline $\begin{array}{l}\text { Capital cost of cryoplant } \\
\text { at } 1.75 \mathrm{~kW}(\$ M)\end{array}$ & 14.2 & 6.8 & 2.7 & 0.52 & 0.41 \\
\hline Total Iife-cycle cost (\$M) & 39.8 & 19.1 & 7.5 & 1.45 & 1.14 \\
\hline
\end{tabular}




\section{KEY FEATURES}

- PEAK FIELD 6.7 T

- LHe I COOLED (POOL BOILING, 4.5K).

- THAEE WINDING SECTIONS USING GRADED CONDUCTORS

- $6 \mathrm{~cm}$ THICK side insulation FOR SPLICES AND LEADS

- conductors REACT OHN HOOP FORCES ( $2 B \mathrm{KSI}$, MAX.)

- APPROXIMATE WEIGH'T OF MAGNE'T COLD MASS IS 104 TONS

$\underset{\omega}{\omega}$

VACUUM VESSEL RADIATION SHIELD HELIUM VESSEL INSULATION

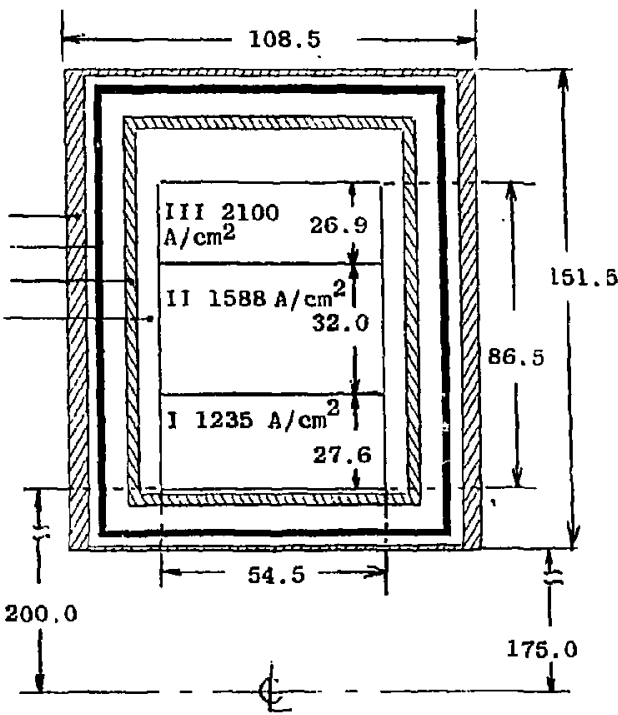

ALL DIMENSIONS IN CENTIME'TERS

Figure 3-12. Cross section and characteristics of the S-0 solenoia. 
- PEAK FIELD $7.6 \mathrm{~T}$

- Lhe I cooled (PoOl botling, 4.5K)

- three hiNdiNg sections USING GRADED CONDUCTORS

- 6 cin thick side INSULATION FOR SPLICES AND LEADS

- CONDUCTORS REACT OWN HOOP FORCES ( $28 \mathrm{KSI}$, YAX.)

- CASE SIDE EXTRA THICK $(12.7 \mathrm{~cm})$ TO REACT LARGE SIDE LOADS (NOMINALLY $5.0 \mathrm{~cm}$ )

- APPROXIMATE WEIGHT OF MAGNET COLD MASS IS 163 TONS

$\underset{w}{w}$

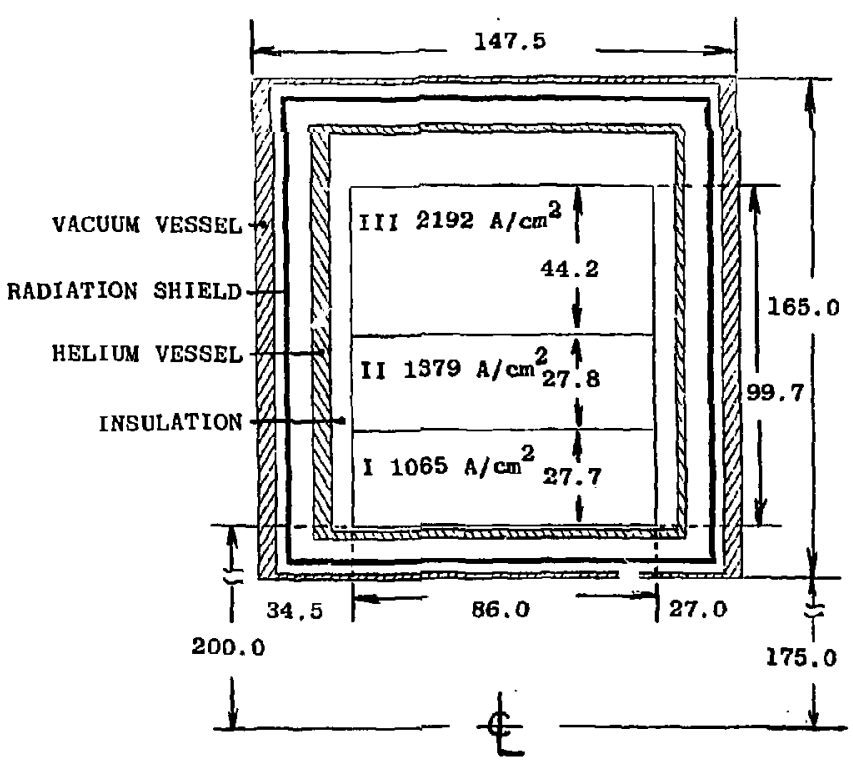

ALL DIMENSIONS IN CENTIMETERS

Figure 3-13. Cross section and characteristics of the 5-1 solenoid. 
Table 3-9. Major characteristics of the solenoid magnets.

\begin{tabular}{|c|c|c|c|}
\hline \multirow{2}{*}{$\begin{array}{l}\text { Parameter } \\
\mathrm{Coi} 1\end{array}$} & \multicolumn{2}{|c|}{ Central solenoids } & \multirow{2}{*}{ Choke } \\
\hline & $S-0$ & $5-1$ & \\
\hline Conductor material & \multicolumn{2}{|c|}{$\begin{array}{l}\text { NBTI superconductor with } \\
\text { half-hard OFHC copper stabilizer }\end{array}$} & AMZIRC \\
\hline Ampere turns $\left(10^{6} A\right)$ & 7.63 & 14.17 & 6.91 \\
\hline $\begin{array}{l}\text { Conductor current }(A) \\
\text { Current density }\left(A / \mathrm{cm}^{2}\right)\end{array}$ & 9,107 & 7,839 & 14,106 \\
\hline Grade I & 1,235 & 1,065 & 4,800 \\
\hline Grade II & 1,588 & 1,379 & -- \\
\hline Grade II I & 2,111 & 2,192 & - \\
\hline Peak field at conductor ( $T$ ) & 6.7 & 7.6 & 15.0 \\
\hline $\begin{array}{l}\text { Operating temperature max. } \\
\text { Conductor bundle }\end{array}$ & $4.2 K$ & $4.2 \mathrm{~K}$ & $120^{\circ} \mathrm{c}$ \\
\hline size (gl wide $x$ m high) & $0.545 \times 0.865$ & $0.860 \times 0.997$ & $0.48 \times 0.30$ \\
\hline Weight of winding (tons) & 56.5 & 106.6 & 2.0 \\
\hline
\end{tabular}


The characteristics of the choke $x_{i}:^{7} s$ are also included; a detailed discussion of choke coils is presented in section 3.2.4. The 5-0 solenoid conductor bundle is $0.545 \mathrm{~m}$ wide by $0.865 \mathrm{~m}$ high and produces $7.63 \times 10^{6}$ A-turns. The $S-1$ solenoid bundies are $0.86 \mathrm{~m}$ wide by $0.997 \mathrm{~m}$ high and each produces $14.17 \times 10^{6}$ A-turns. The winding cross section of $5-1$ is larger than that of S-0 because more ampere turns are required. Peak magnetic fields are $6.7 \mathrm{~T}$ for $\mathrm{S}-0$ and $7.6 \mathrm{~T}$ for $\mathrm{S}-1$. The winding consists of three regions of graded conductor. The current density in the inner two sections is limited by the conductor- allowable stress of $28 \mathrm{ksi}$, while that of the outer section is limited by the cryostability of the conductor.

Six-centimeter gaps on each side of the winding provide space to accormodate side insulation, splices, and leads. A plenum located radially outward from the winding stores additional helium for cooling and provides space for coliecting helium vapor formed by evaporation at the surface of the conductors. The winding is enclosed in a $304 \mathrm{LN}$ stainless steel vessel that also contains the helium coolant.

The vacuum vessel and associated $L N_{2}$-cooled radiation shield provide vacuum and radiation barriers that reduce liquid helium consumption. The helium vessel and vacuum vessel are made by welding prefabricated parts around the winding after the completion of winding operations. Approxinate weights of the winding of $S-0$ and $S-1$ are shown in Table 3-9.

The construction of the $S-1$ solenoid is similar to that of the S-O solenoid, except that the helium vessel incorporates a $12.7-\mathrm{cm}-$ thick lateral plate that supports the axial magnetic loads that act on the winding.

Detailed design features of $5-0$ and $5-1$ are shown in Figs. 3-12 and 3-13.

Conductor and Insulation. The conductor consists of a multistrand niobium-titanium/copper cable or monolith soldered onto the copper stabilizer. The conductor is graded for each winding region. The inner region of S-1 employs a conductor $2.00 \times 2.99 \mathrm{~cm}$. The size of the conductor decreases to $1.71 \times 2.68 \mathrm{~cm}$ and $1.35 \times 2.11 \mathrm{~cm}$ in the outer regions to accomodate higher current densities. 
The basic insulation concept uses "button-on-a-string" insulation for turn-to-turn insulation and "snow-fence" type insulation for layer-to-layer insulation. Turn-to-ground insulation is about $1 \mathrm{~cm}$ thick .

Conductor cooling. The conductor is cooled with pool-boiling Hel at $4.2 \mathrm{~K}$ and $1 \mathrm{~atm}$. Cryogenic stability is achieved for each graded zorie. The maximum current density in the niobium-titanium is always less than $75 \%$ of the short sample value at the maximum operating temperature of $4.4 \mathrm{~K}$. The analysis on which conductor current recovery is based is presented later in this section.

Coil casing. The coil casings designs are shown in Fig. 3-14. Each casing is made of 304LN stainless steel and consists of a bobbin and an outer ring. The outer ring is closeout-welded to the bobbin after completion of the winding operation. The casing supports the winding and contains the liquid helium used to cool the coil.

The casing of $5-0$ has an inner diameter of $3.88 \mathrm{~m}$, is $1.18 \mathrm{~m}$ high $x$ $0.77 \mathrm{~m}$ wide in overall cross section, and has 5-cm-thick walls. The casing of $\mathrm{S}-7$ has an inner diameter of $3.88 \mathrm{~m}$ and is $1.31 \mathrm{~m}$ high $\times 1.16 \mathrm{~m}$ wide in overall cross section. All walls but the lateral facing $5-0$ are $5 \mathrm{~cm}$ thick. This wall is thicker $(12.3 \mathrm{~cm})$ because it supports the axial load of $1.02 \times 10^{8} \mathrm{~N}\left(22.9 \times 10^{6} \mathrm{lb}\right)$ exerted by the windings of $5-1$. The 40,000-psi maximun bending stress on the wall results in a conservative safety factor of 2.5 .

The axial load on the thick wall is carried by six intercoil columns that transmit the load to the outer and inner rings of the S-0 casing. The load is then balanced by an equal and opposite load exerted by $S-1$ via a similar intercoil column arrangement. The supports are $0.5 \times 0.32 \mathrm{~m}(20 \times 12.5 \mathrm{in}$. $)$ in overall cross section and have a maximum compressive stiess of $287 \mathrm{MPa}$ $(41,600 \mathrm{psi})$ ano a safety factor of 2.5. The stress in the inner and outer rings of the casing of $S-0$ is very low because of the large area of the rings. The axial loa can be balanced in the unit flange of the machine without affecting $S-0$. The top of each coil casing has penetrations for the service-stack loads. 


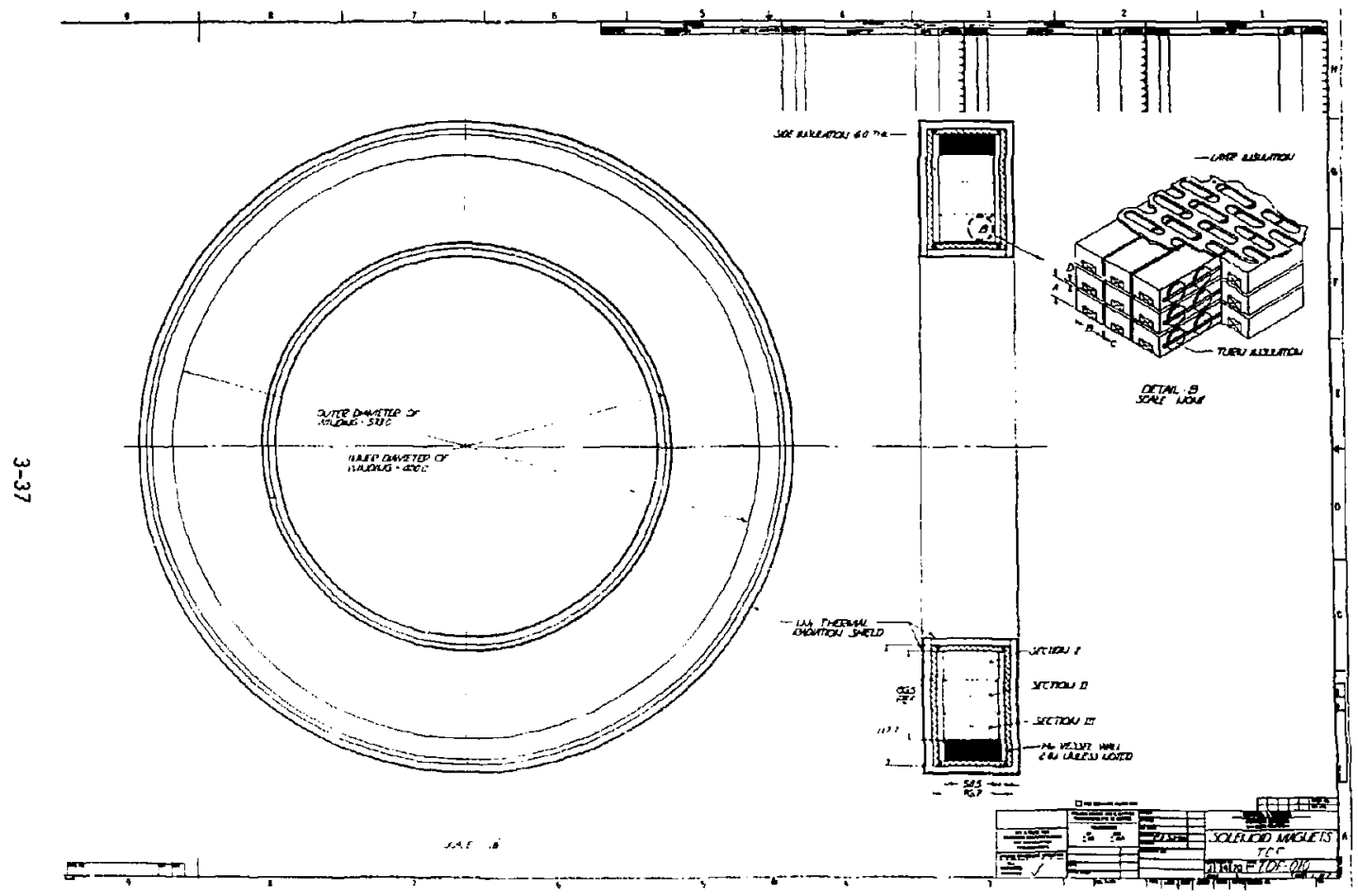

Figure 3-14. Solenoiu magnets, TDF-010. 


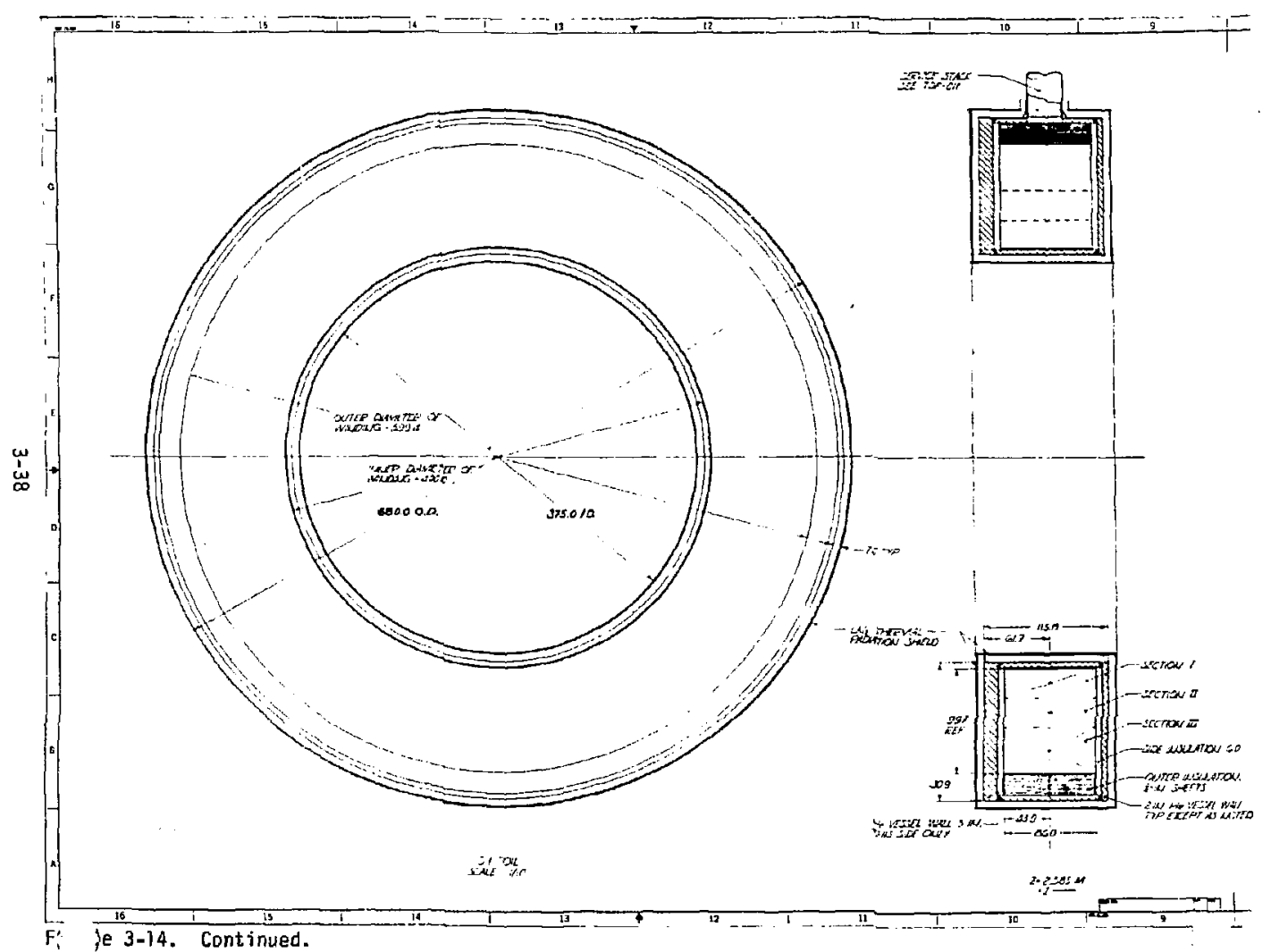


TOUACTOP AND WUSLATKON OATA

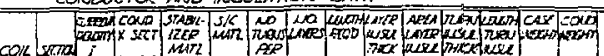

dent

AIRP

$7 x<$ maze

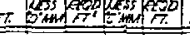

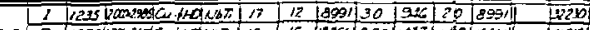

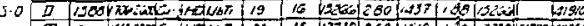

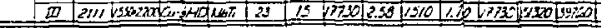

$\omega$

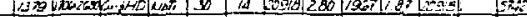

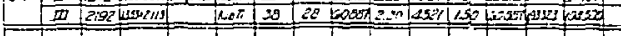

3

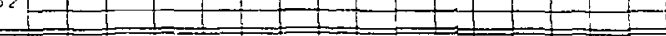

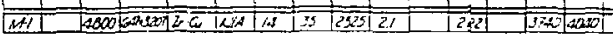

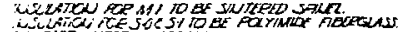

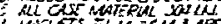

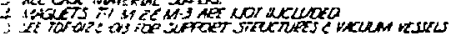

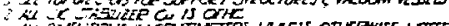

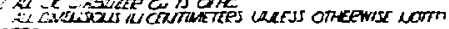
10TR .

Figure 3-14. Continued. 
Service Stack. A preliminary service-stack concept is presented in Fig. 3-15. This stack accomodater one set of power leads and accepts all the helium and nitrogen fluids needed to cool the coil and its radiation shield. It also has provision for instrumentation lines, liquid-level sensors, and emergency helium venting. Because the stack requires simultaneous penetrati! . of the vacuum vessel, the radiation shield, and the helium vessel, the use of bellows and coiled tubing is mandatory to allow for differential thermal contraction. The location of the magnet support struts can also affect relative movements in the stack and will have to be optimized in the final design. The fairly large p'enum chamber of the 5-0 and 5-1 solenoids collects the helium bubbles and allows them to migrate from the conductor to the service-stack opening. Since the conductors carry their own hoop forces, penetration of the heljum yesse]'s outer shell will not present a structural problem. The detail design of the service stack will be a straightforward design task.

\section{Engineering Analysis.}

Magnetics. The LLNL EFFI program was used to establish basic magnet concepts that satisfy physics requirements it a uniform current density. We optimized the magnets by designing conductors, chicking their stress loads and cryostability, and defining graded current density configurations that met the central field requirement. The EFF 1 program modeled these optimized magnets to verify resizing calculations and to determine the peak field (needed to determine conductor hoop stress and the type and anount of superconductar required).

The solenoids were optimized through an iterative process in which the winding pack is divided into an arbitrary yet reasonable number of grades (ttree or four). It was helpful to have a concept that had been analyzed for cryostability for various current densities because the size of the first grade could then be chosen by using an integral number of turns and layers. 


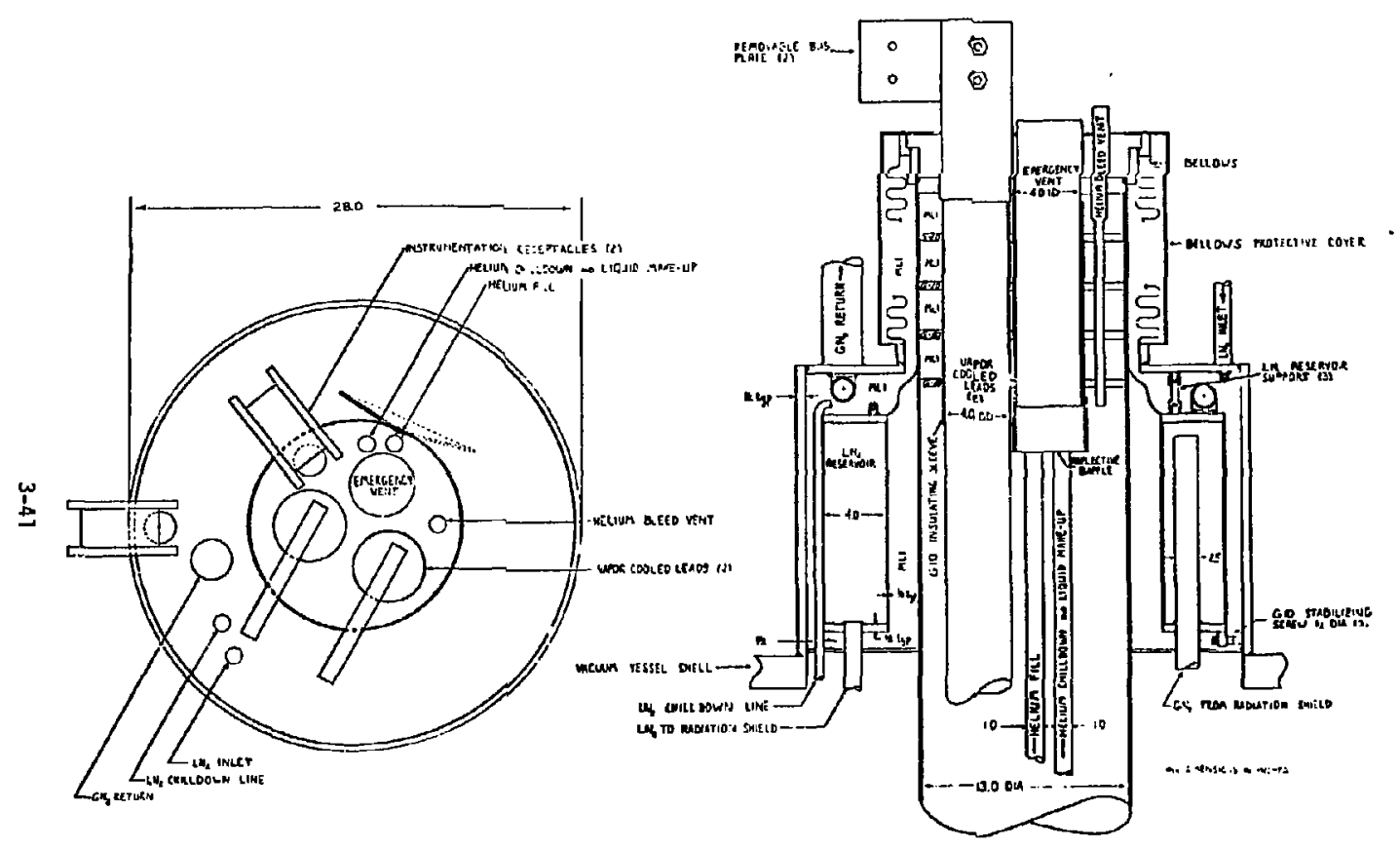

Figure 3-15. Service stack, TGir-011, 
We assume that the winding will be layer-wound to facilitate grading the current density. Knowing (or assuming) the field gradient wili allow us to find the peak field for the next graded section, which, in turn, will determine the amount of niobium-titanium superconductor and maximum allowable current density, provicied the maximum allowable stress is not exceeded.

Force and Stress Analysis. He performed stress analyses to size the conductors, windings, coil casings, and supports according to design criteria based on those used for MFTF-A, MFTF-B, and the Tandem Mirror Next Step (TMNS).

The STANSOL-II computer code. ${ }^{1}$ was used to evaluate radial and hoop stresses in the conductors. The conouctor pack is moteled as a thick-walled cylinder whose length is enual to the width of the conouctor. The effective radial modulus of the conductor and insulation is adjusted to $0.3 \times 10^{6}$ ps $i$ to account for gaps in the winding and for radiation-causeci swelling of the insulating material. This value is based on ata generated in the TMNS study. Adoitional design criteria include:

- Conductor: a yield-stress safety factor of 1.5 and the Von Mises equation used for combined stresses.

- Substructure/case: a yiela-stress safety factor of 1.5, ultimate strength safety factor of 3.0, and a primary bending illowable of 1.5 times the allowable tension.

Numerous STANSOL runs were made in different magnet locations and with varying substructure thicknesses. We found that the conductor stresses varied ittle from those of a self-supporting conductor and, because a selfsupporting conductor gives a simpler and more compact magnet, we decioed to size both $S-0$ and $S-1$ on the basis of the conductors carrying their own hoop stresses with no substructure support.

We used a conservative stress-analys is approach to size the thick side wall of $S-1$ and the intercoil support structure. The axial load of $22.9 \times 10^{6} 1 \mathrm{~b}$ was calculated using the EFFI program. The sideplate of the s- 1 coil was analyzed as an annular plate under uniform pressure as a result of the axial force, with only the top and bottom portion of the plate providing support. A maximum bending stress of 40,000 psi was found for the 12.3-cm-thick plate. The 304LN stainless steel plate material had a minimum yielo strength of 100,000 psi, which resulted in a safety factor of 2.5 . 
Conductor Cryostability. To swiport conductor grading iterations, we performed a cryostability analysis to determine the maximum cryostable current density as a function of field strength. To facilitate the magnetic field calcurations of Section 3.4.2, the stability analys is was done in terms of the overall current density, packing factor, and conductor unit cell size. In these terms, the stability criterion becomes:

$$
q>J^{2} \rho_{U}(t) \frac{A}{P} \frac{\lambda \phi}{1+\phi},
$$

where

$q=$ design heat flux $\left(W / \mathrm{cm}^{2}\right)$,

$\mathrm{J}=$ current density over conductor unit $\mathrm{cell}\left(\mathrm{A} / \mathrm{cm}^{2}\right)$,

$\rho_{0}(B)=$ stabilizer magnetoresistivity (ohm-cm),

$A=$ area of the conductor unit cell $\left(\mathrm{cm}^{2}\right)$,

$\mathrm{P} \quad=$ wetted perimeter of the conductor $(\mathrm{cm})$,

$\lambda \quad=$ packing factor (conductor area/unit cell area),

$\phi=$ copper-to-superconductor ratio in the conductor.

The conductor design determines the unit cell area and packing factor. The turn-to-turn ana layer-to-layer insulation are assumed to cover $50 \%$ of the conductor surface, which then determines the wetted perimeter. The copper-tosuperconauctor ratio was assumed to be approximately 10 to 15 to satisfy quench hot-spot constraints. (See "Quench Analysis" later in this section.)

The magnetoresistivity of the copper stabilizer is computed from:

$\frac{\rho_{0}(B)-\rho_{0}(0)}{\rho_{D}(0)}=4.37\left(\frac{B}{\rho_{0}(0)}\right)^{1.115}$,

where

$\rho_{D}(B) \quad=$ resistivity at field $B$ (ohm-en),

$\rho_{D}(0) \quad=$ degraded resistivity at zero field (ohm-cm),

(B) = field strength (I). 
Equation (3-3) is a power-law relation, similar to that of Kohler, ${ }^{2}$ that accounts for neutron-radiation-induced damage to the copper lattice that increases resistivity. ${ }^{3}$ The value of $\rho_{0}(0)$ is determined by assuming a $20 \%$ increase in the initial value of resistivity at $4.5 \mathrm{~K}$ and zero fiela:

$\rho_{D}(0)=1.20 \times \frac{1.73 \times 10^{-6} \Omega-\mathrm{CM}_{m}}{R R R}$,

where

$1.73 \times 10^{-6} \Omega-\mathrm{Cm}=$ resistivity of OFHC copper at room temperature, RRR = residual resistivity ratio (o $300 \mathrm{~K} / \mathrm{\rho} 4.5 \mathrm{~K}$ ).

The material selected for the central cell solenoio stablizer is half-haro copper with an RRR of approximately 90.

The design heat flux $q$ is limiteo by the conductor's ability to transfer heat to LHel by pool boiling. For an unconditional stability criterion, the design flux is the minimum film-boiling or recovery flux. This was determined through a series of tests measuring heat transfer to LHeI from conductors in bundles simulating actual winding packs. General Dynamics has established corrslations relating mininum iilm-boiling flux to the average gap oimension between conductors. ${ }^{4}$ Using these data, we set the design flux of the conouctor wetted surface at 0.25 $\mathrm{W} / \mathrm{cm}^{2}$.

Equation (3-1) was solved initially for several typical conductors and plotted as $J$ vs the field, $B$. These plots were used for the first sizing of the coils. After a trial in which the graded conductors were sized, Eq. (3-1) was again solved to give curves of $J$ vS $B$ to verify the conductor sizes chosen.

Figures 3-16 and 3-17 give these latter plots for the S-O and S-7 coils. Also indicated on the curves are the actual operating points for the three grades of conductor. The fact that the points fall well below the allowable curves emphasizes that the conductors are generally stréss-limited.

Radiation Effects. The magnet components most affected by radiation are the stabilizer, the insulator, and the superconductor. The following rajiation limits were developed for these components:

Stabilizer: $1.1 \times 10^{-4}$ displaced atoms (dpa) for copper stabilizer at. $8 \mathrm{~T}$ for 2 FPY. 


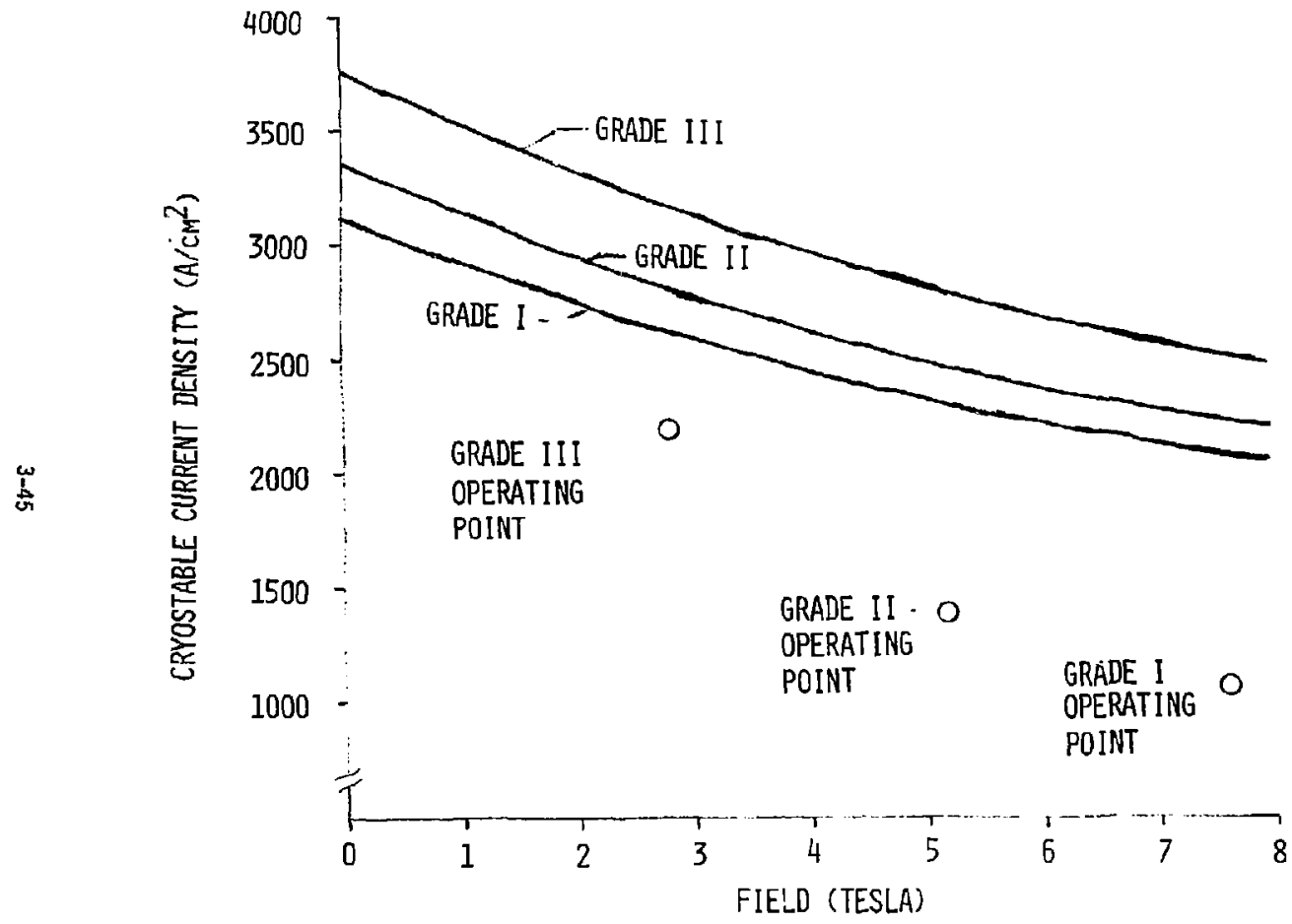

Figure 3-16. Maximum cryostable current density for S-O solenoid. 


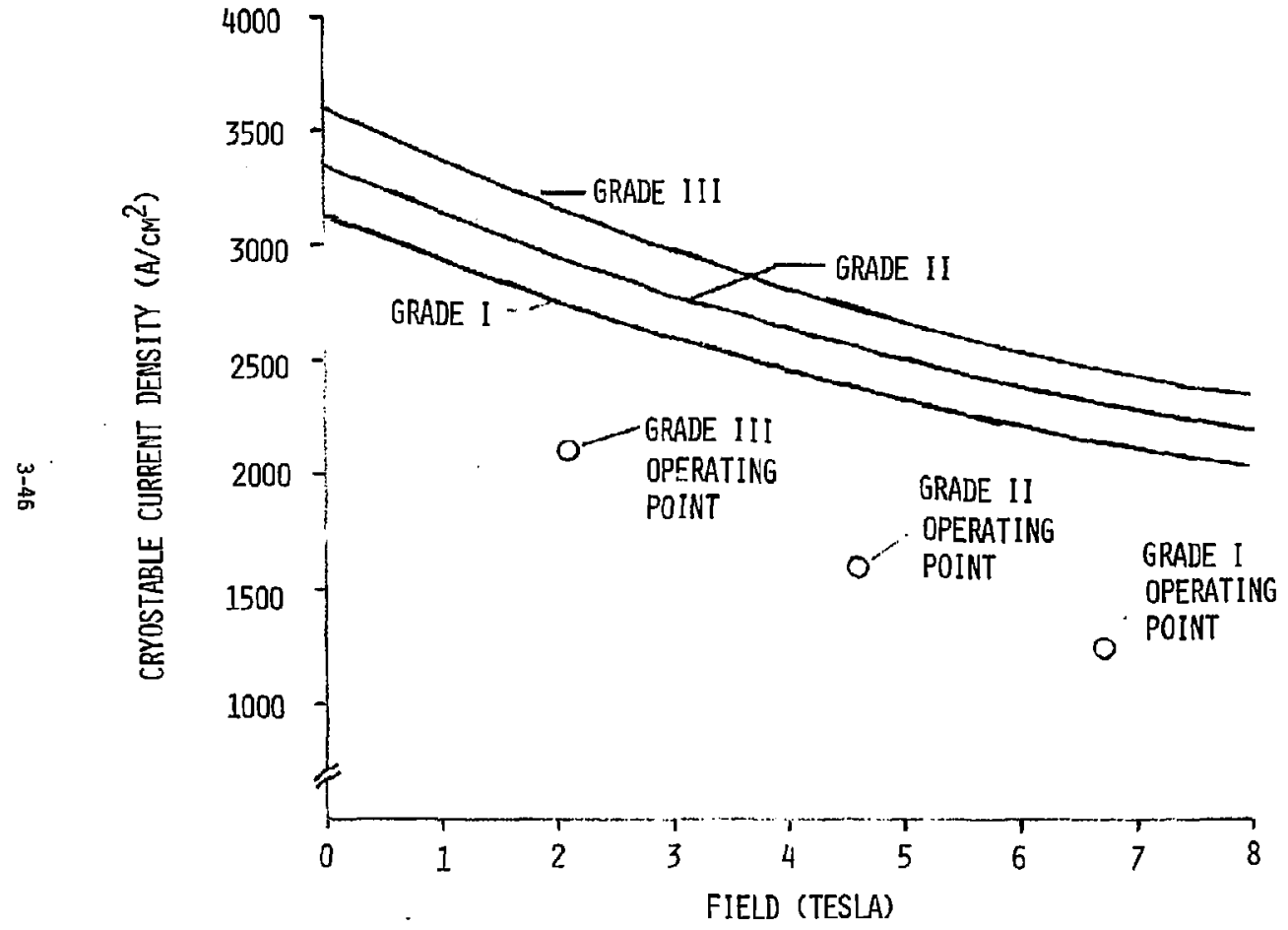

Figure 3-17. Maximum cryostable current density for S-I solenoia. 
- Insulator: $5 \times 10^{9}$ rads for polyimide-glass insulator over 5.4-FPY life.

- Superconductor: $10 \%$ decrease in critical current density over. 5.4-FPY life. This corresponds to a neutron fluence of $4 \times 10^{18}$ $\mathrm{n} / \mathrm{cm}^{2}$, at energies greater than $0.1 \mathrm{MeV}$.

For magnet cryostability analysis, the electrical resistivity of the copper stabilizer was allowed to increase $20 \%$ because of irradiation. This increase for half-hard OFHC copper at $8 \mathrm{~T}$ amounts * approximately $13 \times 10^{-9} \Omega \cdot \mathrm{cm}$.

ت. M. Williams et a $1 .{ }^{5}$ have experimentally det mined the increase in electrical resistivity $\left(\Sigma \rho_{i r p}\right)$ of $O F H C$ copper at $4 k$ for a fast fission neutron spectrum as follows:

$$
\left.\Delta \rho_{j y r}=400\left(1-\varepsilon^{-162} \mathrm{D}\right) \times 10^{-9} \Omega \cdot \mathrm{cm}\right)
$$

where $D=$ radiation damage in displaced atoms.

For $1.1 \times 10^{-4} \mathrm{dpa}$, this yields $\Delta 0_{i r r}=7.06 \times 10^{-\frac{c}{2}} .2 \cdot \mathrm{cm}$. In a subsequent room-temperature anneal, about $85 \%$ of this ra- ation-induced resistivity anneals out and $15 \%$ (about $10^{-9} \Omega \cdot-\mathrm{cm}$ ) remains as residual resistivity in the stabilizer. Thus, an allowance of $13 \times 10^{-9} \Omega \cdot-c m$ in the electrical resistivity of the copper stabilizer is adequate to accommadate six annealing cycles.

The polyimide-glass insulation retains better than $80 \%$ of its compressive strength up to a dose of $5 \times 10^{9}$ rads and is about an order of magnitude better than epoxy-glass insulation; its cost is about four to six times higher than that of epoxy-glass. Epoxy-glass insulation can still be used in relatively low-dose areas of the magnet.

The radiation limit for niobium-titanium superconductor is about the same as that for polyimide-glass insulator if the approximate relationship is $1 \mathrm{rad}=10^{9} \mathrm{n} / \mathrm{cm}^{2}$. Therefore, both constraints are satisfied.

Quench Analysis. Quench analyses were performed to determine the advantages of having multiple sets of current leads rather than a single set. The analyses were done with the $S-2$ coil, which has since been reconfigured as S-1 with three current densities instead of four. However, these data should be representative enough to compare the effect the number of leads has on the final temperature following a quench situation. 
Figure 3-18 represents the as-modeled coit configuration for the fourleao-set case and gives the final temperatures reached in various regions of the magriet following the dump of the magnet.

The data in Table $3-10$ were used in tire single-lead-set case and were input into the computer program, SUPEKQ, which relates voltage, temperature, current, aro time in tire event of a quench situation. SUPERQ is an iterative time-st $\lesssim p$ program that includes the enthalpy of both copper ana superconauctor, but does not incluae helium cooling effects. It represents a realistic case for the situation where a quench is initiated by a low helium level.

In the single-iead-set case, for an overall current density of 1532 $\mathrm{A} / \mathrm{cn}^{2}$ in the conductor pack, the maximum terıperature will reach $85 \mathrm{~K}$ using a aischarge voltage of $500 \mathrm{~V}$. Similar models were input into SUPERQ for the four-lead-set case to arrive at the final temperature of $67 k$ in the region of highest current density.

By comparing these final temperatures, we see that a single set of leads will be entirely adequate. The benefits of having a slightly lower final temperature does not outweigh the additional system complexity and risk of failure of the four-lead-set case.

\subsubsection{Choke Coils}

This section presents the selected conceptual design for the choke coils, gives the relevant engineering analysis, and discusses desigi considerations and requirements.

The design of the choke coils presents a technical challenge because of high field requirements, stringeni space limitations, and the severe radiation environment in which the coil is located. A number of alternatives were considered and examined before a design was selected; the most promising of these are presented at the enc of this section. These alternative designs merit further detailed analysis to determine which should be considered in the detailed jesign phase of TDF.

Design Considerations and Requirements. The selection of design parameters for the choke coil must be made in the context of overall machine design, as it is for most conponents. Systems plasma physics dictates a peak mirror field of I5 T. The coil system that produces this field should be small 


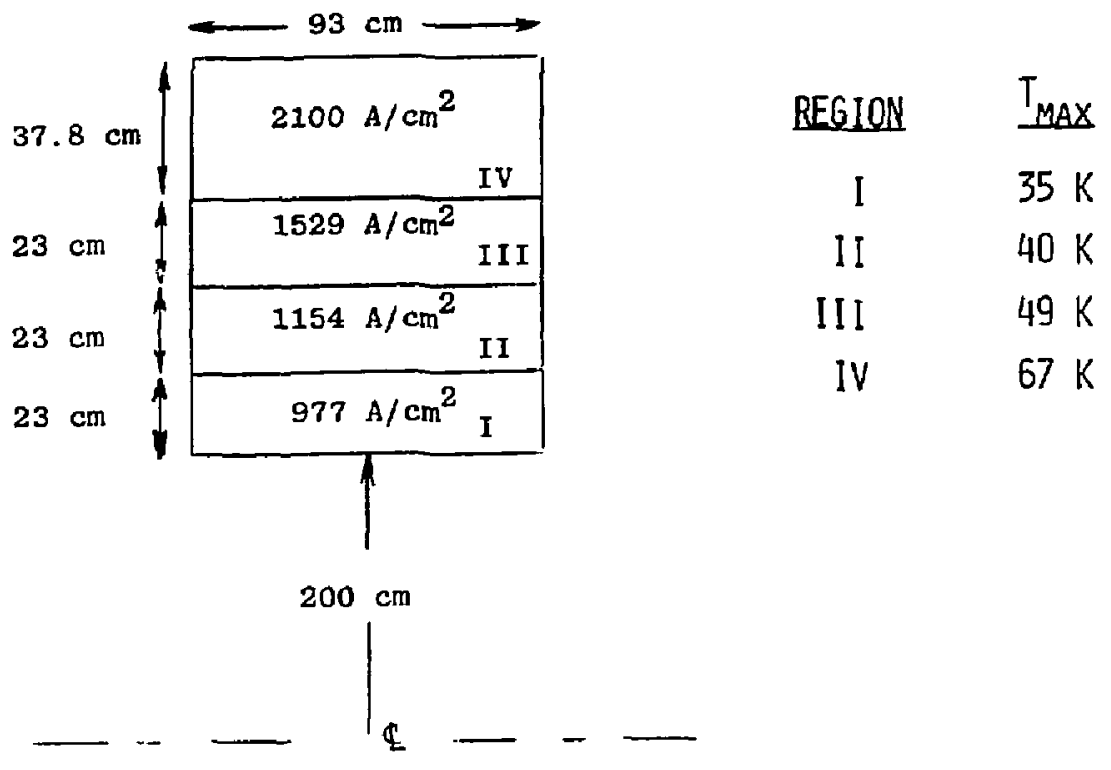

Figure 3-18. Coil configuration and final temperatures following quench for four sets of current leads. 
Table 3-10. Input aata for single-lead-set quench analys is.

\begin{tabular}{|c|c|c|c|}
\hline Operating current $(A)$ & 11272 & Deiay (s) & 10 \\
\hline Maximum discharge voltage (v) & 500 & Self field (T) & 7.9 \\
\hline Uverall current density $\left(A / \mathrm{cm}^{2}\right)$ & 1532 & Background fielo (T) & 0.0 \\
\hline Magnetic energy (MJ) & 570.15 & Field angie (deg) & 0.0 \\
\hline Critical temperature $(K)$ & 5.0 & $\mathrm{Cu} / \mathrm{SC}$ ratio & 11.2 \\
\hline Normal zone length $(\mathrm{cm})$ & 100 & Packing fraction & 0.815 \\
\hline frormal zone trigger voltage (V) & 0.001 & KRR & 80 \\
\hline
\end{tabular}


enough to permit latera? access for beam injection between the central cell anc choke coil. The length of the choke coil should be small to minimize overall machine costs. The choke coil shoulo also be radiation-resistant enough to operate for at least 1 FPY. Several approaches to achieve these goals have been examined. The resistive-coil approach was selecteo because it satisfies overall machine requirements and results in a simpler cesign.

Choke coil design requirements are summarized in Table 3-5. The onil utilizes AMZiRC copper for the conductor and is cooles with an axjal frow of deionized water. Each coil requires about $26 \mathrm{MW}$. Spinel was chosen as the coil insulator to increase operational time in the radiation environment.

Engineering Trade-Uff Studies. Uur engineering trade-off stwoies focused primarily on the choice of conductor and insulation and on the choice of ccolant and cooling configuration for the baseline design. Several alternatives that have potential for further development are presented in Section 3.2.4, "Alternative Design Approaches."

Choice of Conductor and Insulation. The conductor material for the resistive magnet must have high yiela strength to withstand hoop stresses and high electrical conductivity for low power gissipation. The best electrical conductors, in order, are silver, copper, gold, and aluminum. Precioitationstrenghthened copper alloys have high electrical conductivity, high yield strength, and a high recrystallization temperature. We consiuered and compareo several alloys of copper and one alloy of silver; Fig. 3-19 compares these al loys in terms of mechanical yield strength and electrical conductivity.

The compositions of the alloys are:

- Be-Cu (beryllium 0.4-0.75\%, cobait 2.4-2.7\%, balance copper),

- Cu-NI-Ti ( $5 \%$ nicke], 2.5\% titanium, balance copper),

- Glidcop AL-20 (0.4\% A] ${ }_{2} \mathrm{O}_{3}$, balance copper),

- Narloy Z (2.75-3.25\% silver, 0.3-0.5\% zirconium, balance copper),

- Corsil 900 (cadmium 10\%, Ag 90\%),

- LAC- $10.1 \%$ zirconium, $0.3 \%$ chromium, balance copper),

- MZC (0.03-0.06\% magnesium, 0.08 - 0.15\% zirconium, 0.4-0.8\% chromium, balance copper),

- AMZIRC (0.13-0.2\% zirconium, balance copper). 


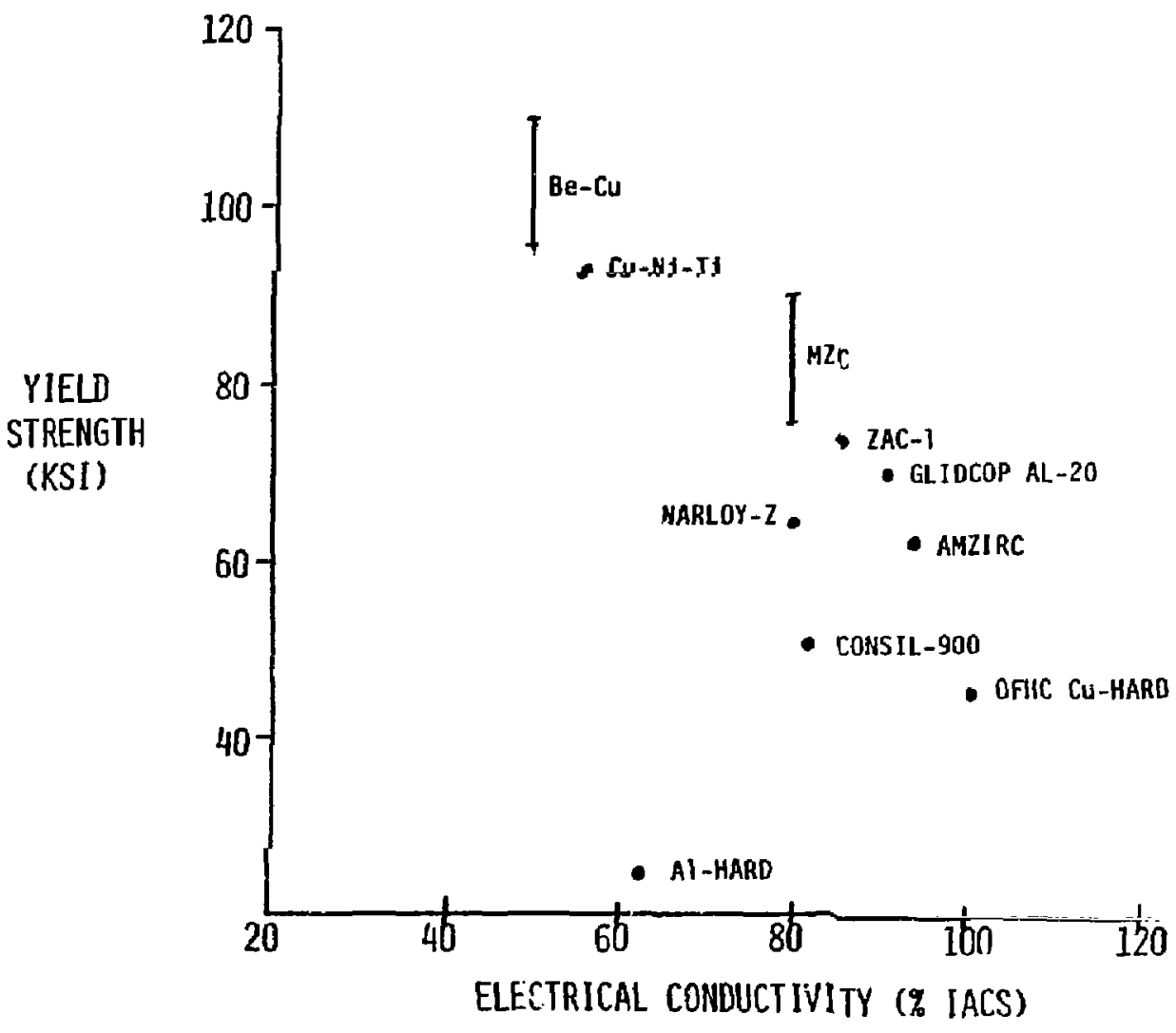

Figure 3-19. Conductor yield strength vs electrical conductivity. 
Consil 900 is a desirable conductor Decause of its low activation, but it does not have sufficient yield strength. Therefore, we selected AMZIRC for the conauctor material because it has both sufficient electrical conauctivity and yield strength.

Spinel $\left(\mathrm{Al}_{2} \mathrm{MgO}_{4}\right)$ has low swelling, no microcracking, and strengthens in a neutron raciation environment. A stress analys is shows that $3 \%$ volume swelling in spinel can be accommodated in the magnet structure without causing stress problems. This amount of swelling is expected in spinel after 2 FPY of magnet operation. Under the same conditions, alumina $\left(\mathrm{Al}_{2} \mathrm{O}_{3}\right)$ woula s,well 16\% by volume, develop microcracks, and deteriorate considerably in mechanical strength. On the basis of these observations, we selected spinel as the insulation material for the resistive magnet.

Choice of Coolant. Because the thermodynamic and physical properties of water make it an excellent neat-transfer medium, we chose it as the baseline cuolant for the resistive coil. In an externally cooled magnet, the water creates problems because of the ability of copper to dissolve in water ana to be oxidized by the oxygen within the water. (See Ref. 6 for complete details of problems caused by the use of water in externally cooled copper magnets.)

He investigated two alternate coolants that would eliminate the problems caused. We compared liquid nitrogen and gaseous helium to the water-cooleo baseline aesign, with the figure of merit being the total power $\left(1^{2} R+\right.$ pump and/or compressor work) required by the coil. The water-coolea baseline design required $25.9 \mathrm{MW}$ of power, based on an average conductor temperature of $100{ }^{\circ} \mathrm{C}$ and an average $20 \%$ increase in the resistivity of copper as a result of transmutations caused by neutron radiation.

Liquid-Nitrogen-Cooled Option. Assuming the magnet configuration remains the same as the baseline, the $I^{2} R$ power consumption will be reduced with $L N_{2}$ coolant by the fraction of copper resistivity that remains at $80 \mathrm{~K}$. For pure OFHC copper, the resistivity decreases linearly with temperature from room temperature to $\mathrm{LN}_{2}$ temperatures at a rate of about 6.9 $\therefore 10^{-9} \Omega \cdot \mathrm{cm} / \mathrm{K}$, so that $\rho_{300} / \rho_{80}$ is approximately 8 . This ratio is smaller in a neutron environment since the increase in resistivity causen by transmutations is independent of temperature. The increase in resistivity caused by neutron damage is equivalent to a $20 \%$ increase at room temperature 
and to a 760 s. increase at $L N_{2}$ temperatures. For damaged copper, then, $\rho_{300} / \rho_{80}$ is oximately $\frac{1.2}{2.6} \times\left(\frac{\rho_{3 \cup 0}}{\rho_{80}}\right){ }_{\text {undamaged }}=3.7$.

Thus, for an $L N_{2}$-cooled coil, the $l^{2} R$ power is reduced by a factor of 8 initialiy, but only by a factor of 3.7 at the ena of 1 FPY.

Un the other hand, refrigerator power is important. Refrigerators have efficiencies of less than 0.5 , so, at best, an $\mathrm{LN}_{2}$ refrigerator requires 7.t $W$ of input per watt of cooling.

The total powei consumed by an $L N_{2}$-cooleo magnet is $7.61^{2} \mathrm{R}+1^{2} \mathrm{~K}$, or over of times the joule heat dissipation. The benefit of the reouceo operating temperature is, for radiation-damaged copper, outweighed by the total power requirement, which is more than twice that of a water-cooled magnet. For this reason we rejected the $\mathrm{LN}_{2}$ option.

Gaseous Heliuni-Cooled Option. its main advantage as a coolant is that it is chemically inert. The hope was that the gas coulo be pumped through the coil at a velocity (and thus heat-transfer coefficient) high enough to keep the conductor cool without excessive pump power. A heat-transfer analysis shows that, to keep pumping power down in the range of $20 \mathrm{MH}$, the conductor temperature must exceed $125{ }^{\circ} \mathrm{C}$. At $125{ }^{\circ} \mathrm{C}$, the joule hrating increases to $35 \mathrm{MW}, 10 \mathrm{MW}$ higher than the baseline of $25 \mathrm{MiW}$. The total power consumption is around $55 \mathrm{MH}$, again more than double the water-cooled baseline requirement. Because the gaseous helium cooling concept is not as efficient as water cooling, it was also rejected.

Cooling Configurations. Figure 3-20 sumarizes the advantages ano disadvantages of the various coolants. We chose an externally cooled coil as the baseline design because it is more compact and economical than an internally cooled coil. Also, it has a current density of $4800 \mathrm{~A} / \mathrm{cm}^{2} \mathrm{vs}$ $2800 \mathrm{~A} / \mathrm{cm}^{2}$ for the internally cooled coil. Because the current must be lower in an internally cooled coil, the overall length would be $65 \mathrm{~cm}$ as opposed to $48 \mathrm{~cm}$ for the externally cooled coil. Another advantage of an externally cooled coil is the lower power requirement of $25.9 \mathrm{MWW}$ vs $37.0 \mathrm{MW}$ 

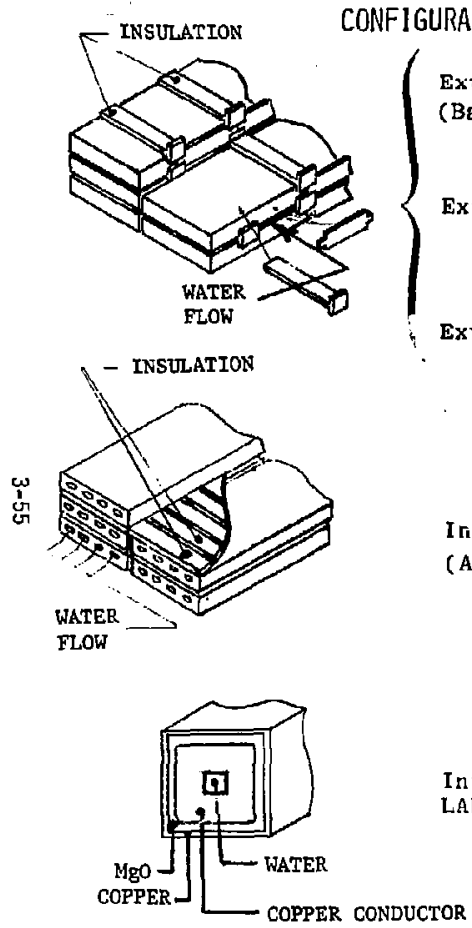

\section{COMMENT}

provides the highest Indicated allowable current density; icuever, long-torm electrical integrity is yitodionable.

Use of GHe cooling should lmprove electrical integrity but requires up to 2 times greater power.

Use of $\mathrm{LN}_{2}$ cooling should improve electrical integrity but requires nearly 3.2 times greater power consumption

Internaliy $\mathrm{H}_{2} \mathrm{O}$ cooled (Alternative)

Good long-term electrical integrity, power consumption not as low as externaly $\mathrm{H}_{2} \mathrm{O}$ cooled, but allowable current density is lower.

Good long-term electrical integrity; power consumption may be up to 2 times greater than externally cooled due to $h i g h$ leakage current; however, allowable current density too low.

Figure $3-20$. Comparison of coolants and cooling configurations. 
compared to internally the cooled coil. Axial cooling, too, is more straightforward than separate cooling of each of the 27 layers of winaing. Although we originally chose the externally cooled coil because of the listed advantages, this choice should be reconsidered as reliability data on the polyhelix configuration becomes available. The internally cooled option is analyzed in more detail in Sections 3.2.4 (A]ternative Design Approaches) and 3.2 .6 .

Selected Design Approach. The choke coil consists of a polyhelix wouno with a rectangular zircorium-copper conducior. The magnet is ceramic-illsulated ano axially cooled with flow of deionized water external to the conjuctor. Key engineering analysis and major design features follow.

\section{Design Uescription.}

Coil Configuration. The coil design incorporates features that promote long operational life in the raciation environment. The magnet carries $9.83 \times 10^{6} \mathrm{~A}$-turns and, in concert with the central cell magnets, proouces the 15-T mirror field. Table 3-5 gives the overall coil requirements, and Fig. 3-21 shows the arrangement of conductors and insulation.

Figure 3-9 gives more design details. The winding has an inner racius of $0.122 \mathrm{~m}$ ano is $0.48 \times 0.30 \mathrm{~m}$ in cross section. The coil has 35 layers, each with 14 turns. The insulation consists of thick spinel spacers placed between turns and between layers.

The spacers separate the conductors and form the cooling chanrel through which deionized water flows axially to remove $26 \mathrm{MW}$ of heat deposition. of this power, $20 \%$ is heat from $I^{2} R$ losses due to radiation-induced resistivity after 1 year of operation at full power. Less than 10\% of this powe: requirement is caused by neutron heat deposition.

The coil is placed irl a steel casing that incorporates inlet and outlet manifolds through which water is introduced and removed from the coil. The manifolas enclose and support the ceramic-insulated leaos. The casing is streamlined to avoid hot spots caused by plasma interception and to support the axial load of 257 cons exerted by the radial component of the magnetic fiela. 


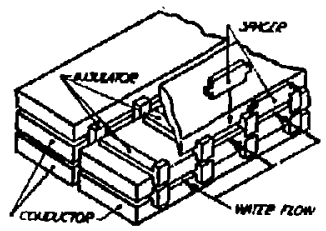

$w_{\substack{1 \\ j}}^{w}$

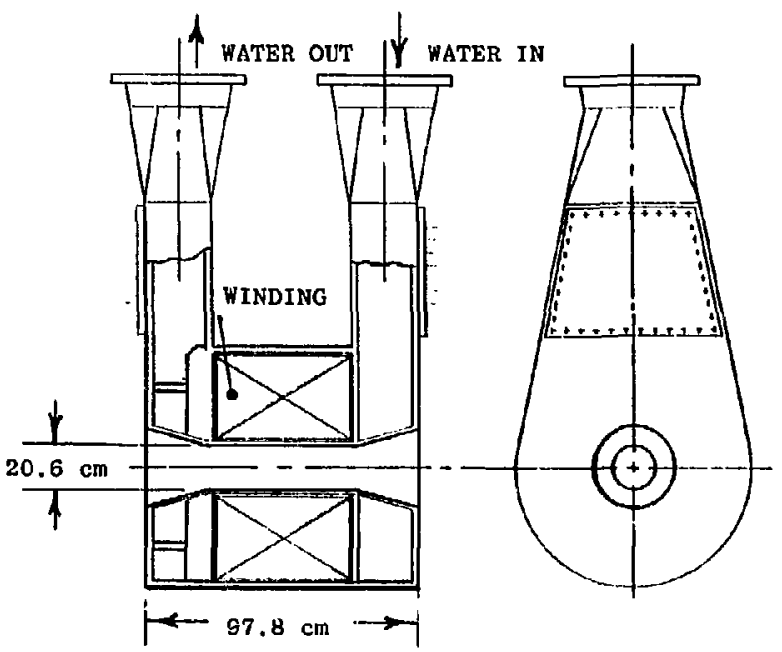

\section{CONFIGURATION}

- Axially coOled

- CONDUCTOR - ZIRCONIUM COPPER or MAGNESIUM-ZIRCONIUM-CHROMIUM COPPER

- SPINEL CERAMIC INSULATION

- 304L SS CASE

\section{KEY FEATURES}

- central field - 12.0T (15.0T w/Background)

- CURRENT DENSity - $4800 \mathrm{~A} / \mathrm{cm}^{2}$

- poner consumption $25.9 \mathrm{kH}$

- hater floh rate 2,450 GPH

- WEIGHT 6500 POUNnS

Figure 3-21. Baseline concept for the TDF choke coil. 
Conductor and Insulation. The conductor is $0.647 \times 3.207 \mathrm{~cm}$ in cross section and carries 14,106 A with a current density of $4800 \mathrm{~A} / \mathrm{cm}^{2}$. Because it is made of AMZIRC, the conductor is able to support the design circumferential stress of 18,300 psi with a safety margin of $100 \%$. Its initial conouctivity is $93 \%$ of that of OFHC copper. Heat from the additional $I^{2} R$ losses caused by radiation-induced resistivity is easily removed by axial cooling because of the large wetted surface of the conductors. The radiation-induced resistivity in AMZIRC copper is less than that of OFHC copper because of the initial content of alloying materials in the conductor. Inerefore, a slight increase in conductor cross section can compensate for the aduitional $I^{2} R$ losses.

The insulation is made of spinel spacers that maintain a 0.21-crn separation between conouctors and between layers. The spinel is highly resistant to raoiation and exhibits less than $3 \%$ swelling in operation. The swelling of the insulation is accormodated by a properly design coil assembly. We assume that the mechanical and electrical properties of spinel are superior to those of alumina and that radiation does not necessarily impose a limitation on the insulator's lifetime. Thin layers of soft material are interposed between the insulator and the conductor to eliminate point loais and prevent fracture of the insuiator.

Conductor cooling. The conductors are cooled with an external flow of oeionized water at a required flow of $12,5001 \mathrm{iters} / \mathrm{min}$. Introduced into the coil at $32.5^{\circ} \mathrm{C}$, the water flows at $2.2 \mathrm{~m} / \mathrm{s}$ between conductors. The maximum temperatures of the water and conductor are $60^{\circ}$ and $120^{\circ} \mathrm{C}$, respectively. A detailed analysis of the cooiing scheme is gi:en in Section 3.2.4 ("Selected Design Scheme").

Coil Casing and Support Structure. The general arrangement of the coil casing is shown in Figs. 3-20 and 3-21. Made of 1.25-cm-thick 316L stainless steel, the casing forms both a winding bobbin for the conouctors and a pressure vessel to contain the 175-psi cooling water maximum pressure. By means of manifolas at each end of the casing, the cooling water is forced to flow axially through the conductors. 
The "spoked-wheel" support at the ends of the coil reacts against the axial magnetic forces generated on the coil pack. While the water pressure generates low stress levels, giving a safety factor of 5 , the large axial force of $2.29 \times 10^{6} \mathrm{~N}(514,00016)$ requires closer consideration. The support was devised to allow the cooling water to freely enter and leave the coil pack from the manifolds and to resist the large axial force. The 12 main rectangular spokes (plus intermediate ones) are welded to the inner and outer case, and their loads are transmitted to the end of the manifold for pickup by a separate bucking ring anchored to the radiation shield.

The current leads run through the feed and exit water pipes, with a junction box at each end to make the transition to the conductors. We expect that the coil assembly and its water piping will have to be removed as a unit to replace the coil. The casing will be welded closed after winding the coil since it is not considered feasible to work on the conductor after the coil has been used. The small size of the entire coil makes this attractive. At this stage of the design, conservative hand calculations were performed to size the members. For the final design effort, more detailed analyses can be made to optimize the member sizes. .

\section{Engineering Analysis.}

Magnetics. Once the required central field is obtained, the next important consideration for the choke coil is to minimize power consumption. Although peak fields are of little concern when choosing a resistive conductor, the design of the $M-1$ choke coil was still a formidable task. Initially, there were two small resistive coils with current densities exceeding $9000 \mathrm{~A} / \mathrm{cm}^{2}$. Later these coils evolved into a slightly larger single coil with a current density of $4800 \mathrm{~A} / \mathrm{cm}^{2}$. Regardless of the values of current density, central field, inner base radius, conductor resistivity, and packing factor, the most efficient geometry for a coil of uniform current density can be determined by two dimensionless geometry factors, $\alpha$ and $\beta$. Figure 3-22, taken from Ref. 8, shows a plot of a vs $\beta$.

The most efficient geometry is at $\alpha=3.08$ and $\beta=1.86$. Moving off this point, the values of $\alpha$ and $\beta$ can vary widely for a given efficiency line. This results in magnets of varying volume. Further optimization is possible to obtain the least volume (and lowest cost) for the given field and 


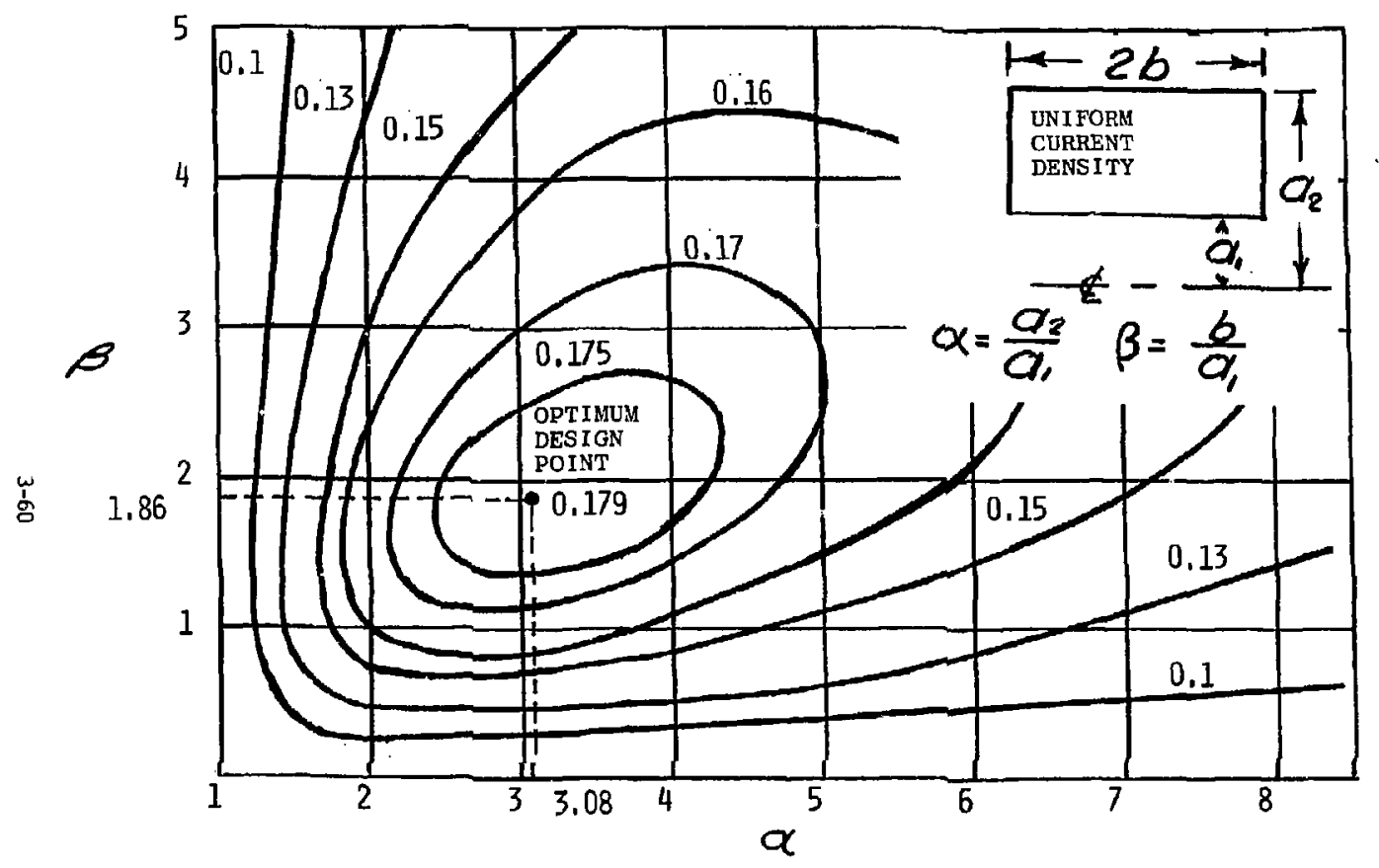

Ficmure 3-22. Operating efficiency is a function of magnet geumetry. 
power. Based on the work of Ref. 8, Genera] Dynamics created an aid to perform this operation, which is presented in Fig. 3-23. The figure shows the locus of the tangency points of constant volume tine with the efficiency curves; the 7 ine represents the most efficient magnet shape for any given volume. Conversely, if the volume is not known, the line can be followed towara the most efficient point $(\alpha=3.08, \beta=1.86)$ unti) a set of $\alpha$ and $B$ values is met that gives the required field. This will be the most efficient coil geometry possible. The above discussion is limited to coils of uniform current density.

work on the MARS study ${ }^{9}$ shows that the highest current gensities can be achieved by externally cooled conductors cooled by axially flowing water. This approach was used for the choke-coil baseline design. At a current aensity of $4800 \mathrm{~A} / \mathrm{cm}^{2}$, the conductor can be adequately cooled. The central field requirement of $12.0 \mathrm{~T}$ can be met, and $\alpha(3.46)$ and $\beta(1.97)$ are quite close to the maximum efficiency point.

Forces and Stresses. Stress analysis of the conductors is performed by modeling the coil with the STANSOL-II computer code. The stresses are well within allowable values for the AliZIRC conauctor.

The buckling analysis of the inner cylinder of the coil casing is based on the American Society of Mechanical Engineers (ASME) Boiler Code, Section III. For a 1.25-cm-thick inner wall of $316 \mathrm{~L}$ stainless steel, a safety factor greater than 5 for buckling is obtained, and the maximum stress is well below yield. The outer casing is placed in hoop tension by the relatively low water pressure of 175 psi maximum, and the stress level is well below yielo.

The analysis of the axial support is based on an axial force of $2.29 \times 10^{6} \mathrm{~N}(514,000$ lb) obtained from the EFFI computer program, and conservative hand calculations show the stresses are well below yield. A finite-element analysis of the final design should be made to optimize this part of the structure.

A check of the stress lesel in the proposed free-standing conductor shows a peak stress of just under 18,300 psi, we 11 within the range of present technology for conductor materials. 


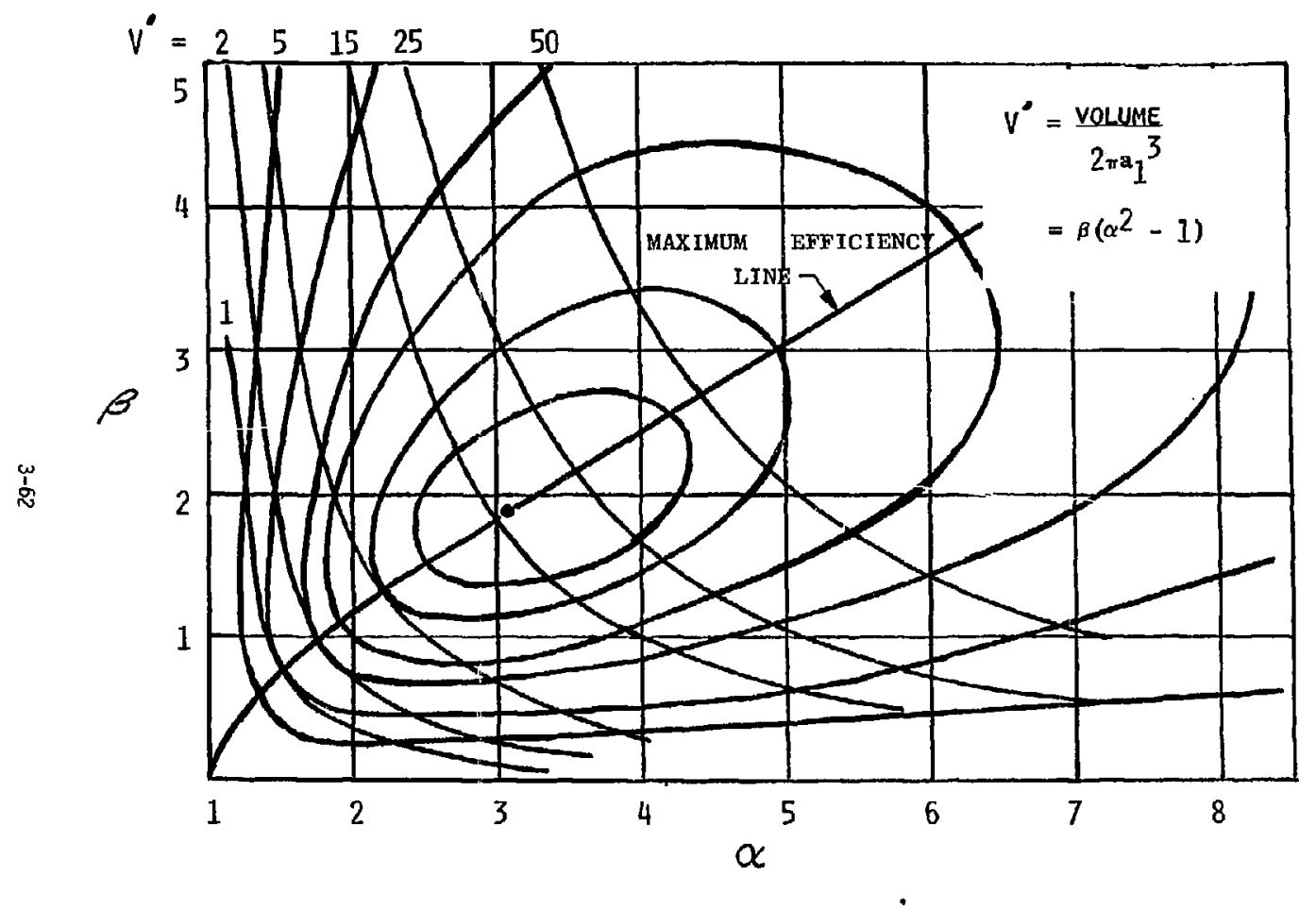

Figure 3-23. Maximum efficiency for any given magnet volume. 
Thermodynamics. We analyze the choke coils to determine coolant flow rates and to verify that there is enough heat-transfer capability to transfer the dissipated energy into the water.

First, we compute the total coolant load by adding the joule heating in the conductor to the heat deposited by neutron radiation:

$Q_{L}=\frac{J^{2} p V}{\lambda}+Q_{N}$

where

$Q_{L}=$ cooling load (W),

$J=$ current density in the coil $(w)$,

$\rho=$ average resistive of copper conductors $(\Omega \cdot \mathrm{cm})$,

$V=$ winding volume $\left(\mathrm{cm}^{3}\right)$,

$\lambda=$ packing factor,

$Q_{N}=$ integrated neutron heat deposition $(W)$.

Once the coolant load is determined, we find the required water flow rate by the following two energy balances:

$$
Q_{L}=\dot{m} c_{p} \Delta T_{w}=h A \Delta T_{c} \text {, }
$$

where

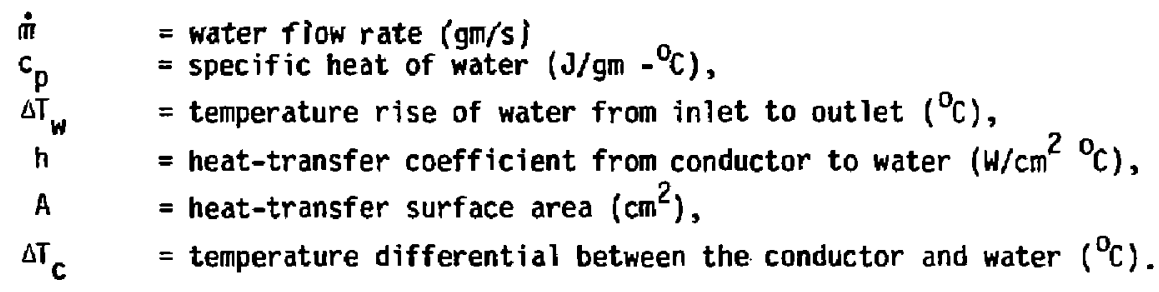

Two additional constraints must be satisfied: to avoid the possibility of boiling in a nonpressurized system, the conductor peak temperature is limited 
to $100^{\circ} \mathrm{C}$ maximum, and the heat-transfer coefficient is related to the water velocity within the coil by the colburn equation for flow inside a duct:

$h=0.23\left(0^{-0.2}\right) \times\left(0^{-0.8}\right)\left(y^{0.8} ;\right.$,

where

$D=$ flow passage hydraulic diameters $(\mathrm{cm})$,

$k=$ thermal conductivity of water $\left(\mathrm{W} / \mathrm{cm} /{ }^{\circ} \mathrm{C}\right)$,

$u=k$ inematic viscosity of water $\left(\mathrm{cm}^{2} / \mathrm{s}\right)$.

$v=$ water velocity $(\mathrm{cm} / \mathrm{s})$.

The final assumption: the inlet water temperature is $32.5^{\circ} \mathrm{C}$, a value expected to be typical of the temperature of water exiting a cooling tower.

The flow passage between layers is modeled as a gap between two parallel planes. The hydraulic diameter of the passage is twice the gap dimension. For this coil, the hydraulic diameter is $0.42 \mathrm{~cm}$. The spacers are assumed to block $60 \%$ of the passage on the average, so the flow area is computed to be $778 \mathrm{~cm}^{2}$, and the wetted area of the conductor is $3.85 \mathrm{~cm}^{2}$ ber centimeter length of conductor.

The neutron effects are most severe at the inner layer of the coil and drop off exponentially with radial distance. A volume-averaged value of resistivity degradation ( $20 \%$ increase in initial resistivity) was used to compute the average coil resistivity of $2.8 \times 10^{-6} \Omega \cdot \mathrm{cm}$. The volumetric neutron heat deposition of $2.6 \mathrm{~W} / \mathrm{cm}^{3}$ was used to compute the total neutron heat deposition of $0.6 \mathrm{MW}$. With these values, at $4800 \mathrm{~A} / \mathrm{cm}^{2}$ and a packing factor of 0.70 , the heat flux on the conductor wetted surface is $72.3 \mathrm{H} / \mathrm{cm}^{2}$, corresponding to a total heat dissipation rate of $22.7 \mathrm{MW}$. To 1 imit the water temperature to $60^{\circ} \mathrm{C}$ at the exit, the required mass flow rate is $209 \mathrm{~kg} / 5$, or 12,500 liters/min. This flow rate is equivalent to a water velocity of 2.2 $\mathrm{m} / \mathrm{s}$ with in the coil.

According to the colburn equation, the expected average heat-transfer coefficient at $2.2 \mathrm{~m} / \mathrm{s}$, with the $0.21-\mathrm{cm}-w i d e$ gap between layers, is about $1.0 \mathrm{~W} / \mathrm{cm}^{2} \mathrm{c}$. When multiplied by the total wetted surface area within the coil and equated to the required energy-removal rate, the maximum conductor temperature is computed at $120{ }^{\circ} \mathrm{C}$; this computation is based on an average 
water temperature of $46.3^{\circ} \mathrm{C}$. Altrough the maximum conductor temperature is $20^{\circ} \mathrm{C}$ higher than the goal of $100^{\circ} \mathrm{C}$, we foresee no serious threat to magnet performance. Because the conductor is designed to be self-supporting, spacer spacing could be relaxed to increase the wetted surface of the conductor and allow greater flow at the same velocity. Both effects combine to reauce conductor temperature to the desired operating point.

Failure Mechanisms. According to Ref. 6, the possible failure mechanisms in axially water-cooled magnets with organic insulators have been identified. Advanced design concepts provide potential solutions to these problems:

1. Radiolytic corrosion of copper.

2. Water absorption in compressed insuratat ion.

3. Water attack on copper insulator bond.

4. Water oxidation of mechanical joints.

5. Growth of capper-oxide whiskers in water.

The corrosion rate of copper in externally cooled cails is at a mihimum in deionized water that has a resistivity of $1 \times 10^{6} \Omega \cdot \mathrm{cm}$. The corrosicn rate is $7.7 \mathrm{mils} / \mathrm{yr}$ for 8 to $10 \times 10^{6} \Omega \cdot \mathrm{cm}$ resistivity water and 2.8 mils/yr for $0.005 \times 10^{6} \Omega \cdot \mathrm{cm}$ resistivity water, compared to only 0.5 $\mathrm{mil} / \mathrm{/r}$ for $1 \times 10^{6} \mathrm{\Omega} \cdot \mathrm{cm}$ resistivity water. From the standpoint of corrosion, $1 \times 10^{6} \Omega \cdot \mathrm{cm}$ is optimum for copper conductors. In the preserice of radiation, raoiolysis of water occurs. Radiation breaks $\mathrm{H}_{2} \mathrm{O}$ molecules down to $\mathrm{H}^{*}$ anc $\mathrm{OH}^{*}$ radicals that interact to produce other radicals as well as $\mathrm{H}_{2}, \mathrm{O}_{2}$, and $\mathrm{H}_{2} \mathrm{O}_{2}$. The oxygen concentration in the water is estimated at 8000 parts per bitTion (pp5). At this oxygen concentration and a water temperature of $60^{\circ} \mathrm{C}$, water resistivity cannot be as high as the $1 \times 10^{6} \Omega \cdot \mathrm{cm}$ "optimum." Radiolytic corrosion, which is oxidation of copper by $\mathrm{H}_{2} \mathrm{O}_{2}$, may erode $0.65 \mathrm{~mm}$ from copper in $2 \mathrm{FPY}$. Radiolytic corrosion can be suppressed by adding reducing agents, such as $H_{2}$ or hyorazine, and can be partially suppressed by an ion exchanger.

The choke coil is designed to use spinel insulation that is permeable to water. This cojl can be designed to aliminate mechanical joints where possible, or the mechanical joints can ba gold-plated. The coolant passages can also be gola-plated to reduce radiolytic corrosion and the growth of 
copper oxide whiskers. Further development witl be needed to verify whether these steps can eliminate failure mechanisms in water-cooled magnets in a radiation environment.

Radiation Effects. In the inner layers of the resistive magnet, the conductor, sees a very intense 14-Mev neutron environment. These neutrons affect both the mechanical and electrical properties of the conductor. The most significant changes in mechanical properties result from radiation-incuced void swelling, radiation creep, and radiation embrittlenent. Those changes in electrical properties are caused by both the transmutation of atoms and to the introduction of defects in the conductor lattice. ${ }^{7}$ The change in electrical resistivity that results when defects are introduced in the lattice (sometimes referred to as displaced-atom damage) saturates above any tenperature when the recombination rate of defects equals the generation rate of defects. In OFHC copper, the saturation values of aisplaceo-atom-induced resistivity is $\simeq 80 \times 10^{-9} \Omega \cdot \mathrm{cm}$ at $\approx 30^{\circ} \mathrm{C}$. The resistivity increase caused by transmutations does not saturate but increases linearly with neutron fluence. The primary transinutation products in copper are nickel, zinc, and cobalt. These products are assumed to be in solution in copper; thus, transmutations increase electrical resistivity and result in alloying of the copper conductor.

In small concentrations, nickel, zinc, and cobalt contribute $1.310^{-6} \Omega \cdot \mathrm{cm}, 0.310^{-6} \Omega \cdot \mathrm{cm}$, and $6.5 \times 10^{-6} \Omega \cdot \mathrm{cm}$ per atomic percent, respectively. For the choke-coil inner layer, the peak resistivity increase resulting from transmutations alone for a 2-FPy operation, is expected to be about $55 \%$ of its unirradiated value. As noted, the resistivity increase due to jefects (displaced atoms) would be about $5 \%$ of the unirradiated value. This is based on the assumptions that the introduction of transmutation products, or the production of hydrogen and helium caused by $(n, \rho)$ and $(n, \alpha)$ reactions, would not interfere with the basic recombination of defects process that leads to a saturation of defect density and thus to a saturation value for defect-inauced resistivity. If, indeed, the impurities interact with the defects, the saturation value would change and the defect-induced resistivity would assume a higher saturation value. The average change in the conductor's electrical resistivity is expected to be less than $20 \%$ over a 2-FPY operation. 
Alternative Design Approaches. At the outset of our conceptual design effort, we examined several choke coil approaches before selecting the baseline concept. Trade-off studies show that certain options have definite advantages inherent to the type of coil used. The externally cooled baseline option has lower power requirements than the internally cooled, and the coil can be accommodated in a smaller space. On the other hand, internally cooled coils are more reliable but have larger power and space requirements. Superconducting coils have very small power requirenents but are much more expensive. The alternatives discussed in detail below may have sufficieit potential for future TDF design studies.

Internally Cooled Resistive Coil. General Dynamics is developing high-field, internally cooled resistive coils applicable to mirror fusion reactors. The goal is to increase coil lifetime beyond the limits set by present technology. Thus, it is a prime candidate for TDF. In fact, recent developments indicate that this alternative is preferable to the selected baseline design because of increased coil reliability. The projected lifetime is substantially longer than that of an externally cooled coil because the turn-to-turn voltage is sustained by ory insulation rather than by water or by insulation in contact with water. The approach also avoids complications caused by copper-water chemistry, thereby reducing the chance of turn-to-turn shorts during operation. More details on coil-failure mechanisms are discussed in Rrf. 6.

Figure 3-24 compares an internally cooled conductor for TDF to the baseline externally cosled conductor coil. The internally cooled conductor is $l$ imited to a lower current density and therefore requires a higher cross-sectional area to establish the same central field. The power requirement for an internally cooled coil is also righer (roughly 0.6 vs 0.7 ), primarily because of the lower packing factor and ?arger build of the internally cooled coil. The larger build (3.76 times the volume) and more complex conductor will increase cost, but the expected increase in service life could make this alterrative attractive.

Major design features of the internaliy cooled concept include separate cooling and powering for each layer of the polyhelix. Although not necessary for adequate cooling, this approach does allow current grading (higher currents for the shorter, inner layers), which could lead to further coil 


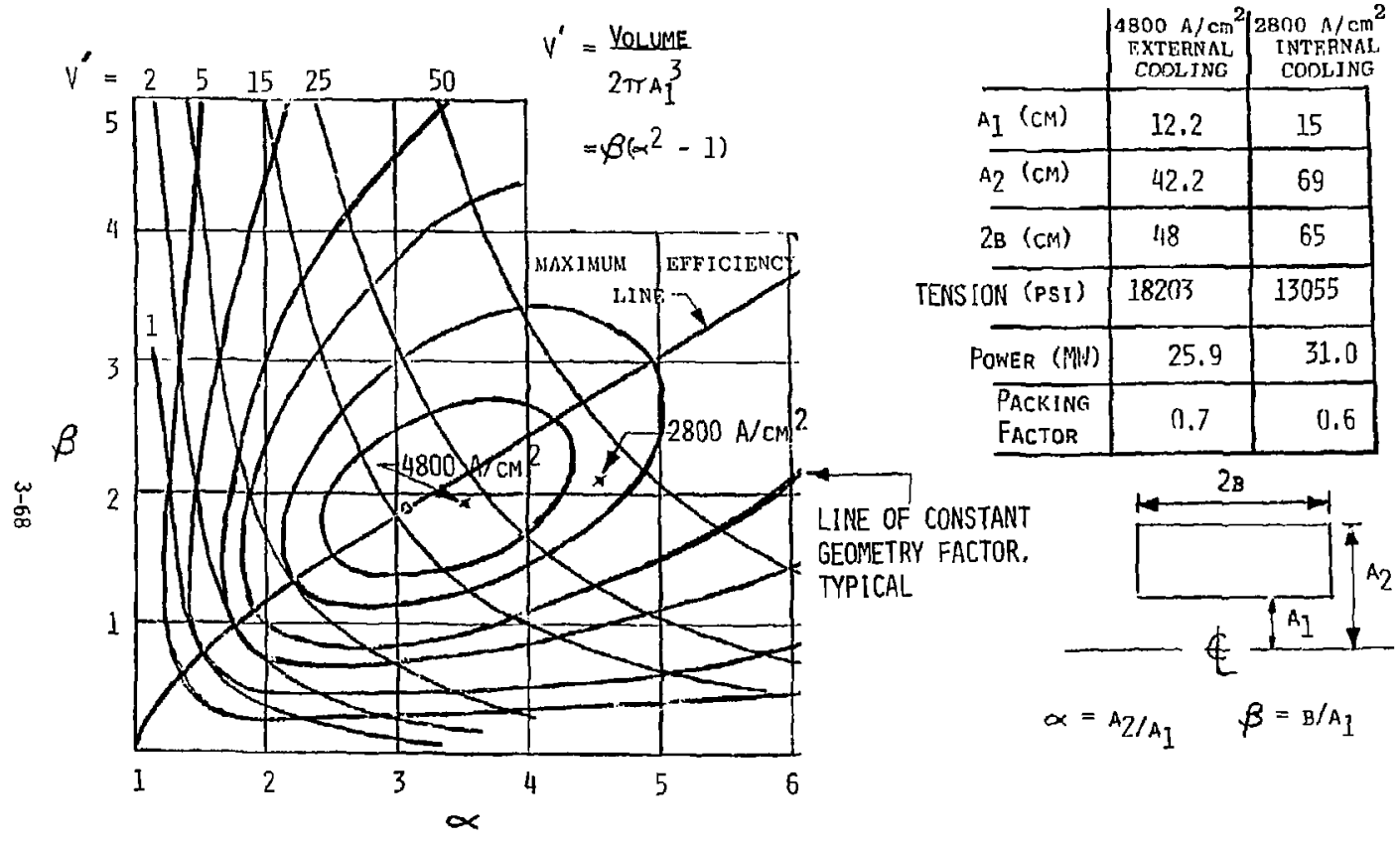

Figure 3-24. Comparison of internally and extenally cooled resistive coils. 
optimization. Getting 27 separate leads in and out of the coil is not a trivial task considering the huge side load $(514,000 \mathrm{lb})$ on the winding and the anchoring of each conductor to react the hoop forces.

The outstanding feature of this concept is the fact that the conductor is not encased in a metal sheath with the usual magnesium-oxide powder insulation, but by separate pieces of spinel. This feature raises the packing factor over that of a conventionally sheathed conductor (thus lowering the power required) and reduces the limitations on minimum bending radius-.-an important consideration for a coil whose inside radius is $15 \mathrm{~cm}$. Because the hoop stresses are low ( $13 \mathrm{ksi})$, the conductor can be made from a $z$ irconium-copper alioy that has 93\% IACS resistance and a high yield stress of $46 \mathrm{ksi}$ for $50 \%$ cold worked and aged. Half-hard OFHC copper was ruled out because of its low strength. Figure 3-25 gives additional design features.

The spinel insulation does not have to be thick since the separate power supply for each layer results in a low layer-to-layer voltage. One winding concept is to overwind a turn without the insulation in place, to relax the coil enough to insert the spinel strips, and then to start the next turn.

When the basic uncertainties of either the baseline or the alternative high-field coil are considered, the inevitable conclusion is that some developmental work should precede a final design effort. High-field resistive coils of this size have not been built before, and the high radiation environment compounds the problem. We feel that a low-cost development program can be scoped to yield the answers necessary for a reliable design.

For a detailed engineering analysis of internally cooled resistive coils see Section 3.2.6.

Background Superconducting Coil. The resistive coil of the baseline configuration receives a background field of about $3 T$ from the central cell coils. Without this background, the resistive coil would irequire more power. This need led us to consider an additional background superronducting coil to further reduce the power requirement. Although space constraints imposed by the need for an unobstructed neutral beam pathway limit the geometry of such a background coil, it should be considered during the final design because the power saving could be dramatic.

$\$$ 
Figure 3-26 shows the calculated ohmic power required by the Ml choke coil for varying background fields. The baseline case with a 3-T background fiela requires $25.9 \mathrm{NW}$ of power. If the background field can be raised from 3 to $6.2 \mathrm{~T}$, the ohmic power requirement will drop to $12.5 \mathrm{Mk}$. of course, the reduction in power will have to be balanced against the cost of an additional superconaucting magnet.

Figure 3-27a shows the approximate location of the alternative S-2 coil. Unfortunately, other constraint: prohibited placing $S-2$ directly over the $\mathrm{M}-1$ choke coil, its most efficient position. When General Dynamics made a preliminary optinlization of the coil, it became apparent that, with the relatively narrow builo, the peak field at the inner radius would exceed the $8 T$ allowable for a niobium-titanium conductor. The weight of the winding alone for this coil is approxintately 36 tons, and a cost trade-off against the chmic power saving of $13.4 \mathrm{kWH}(25.9 \mathrm{~kW}-12.5 \mathrm{HW})$ should be considered in the final design.

Superconducting Choke Coil. General Dynamics has considered carrying this one step further and eliminating the choke coil. This would require a superconductiag coil whose inner radius is large enough to accomodate the shielding required to protect the coil from radiation damage. Such a coil has been tentatively sizeo, assuming a plasma/halo radius of $20 \mathrm{~cm}$ and a shielding thickness of $90 \mathrm{~cm}$. Allowing $27 \mathrm{~cm}$ from the inner ragius to the outside of the vacuum vessel gives a winding inner radius of $137 \mathrm{~cm}$. The concept coil is shown in Fig. 3-27a. It would require LHe-II cooling and the development of a 16-T rated superconductor. Total weight would be approximately 834 tons.

With such a huge coil eliminating the $M-1$ choke coil, the next consideration is to elininate the $S-1$ coil as well and to move the new coil inboara. This configuration is also shown in Fig. 3-27b. Note that the effects of these alternatives on the physics have not been analyzed and that they are presented as options strictiy from the magnet point of view.

\subsubsection{Anchor Cell}

Uesign Requirements. The anchor set at each end of the machine consists of a transition coil (T-I) and the yin-yang anchor pair ( $M-2$ and $M-3$ ). The anchor-cell magnet set must produce a magnetic field on-axis, providing MHO 


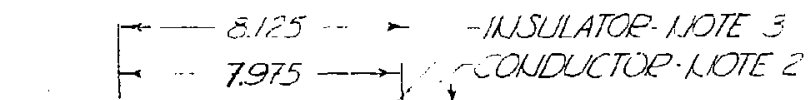
$1.2000-1$ 4 and SONOUCTOE CEOIS SECTIOU SCALE: FULL

LIOTFS:

' ALL DMIENSIONS W CENTMAFTERS"

$\therefore$ MATEFIAL - ANALIFL CU 1 HAPO

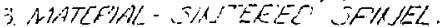

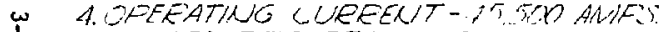

$\doteq$ S. THTEAL FIEIL EECQUKEO - IR.OT.

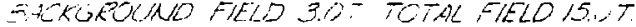
E.AX CONDUCTOE TAME $\angle 200^{\circ} \mathrm{C}$.

7. WATFF' WMET TFMAP $3: 2{ }^{\circ} \mathrm{C}$.

$\rightarrow$ WATEP OWITT TEMF \& EC"C

$\therefore$ WATFP FLOW EATE CIE NIS

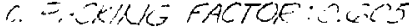

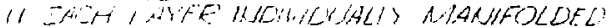

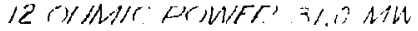

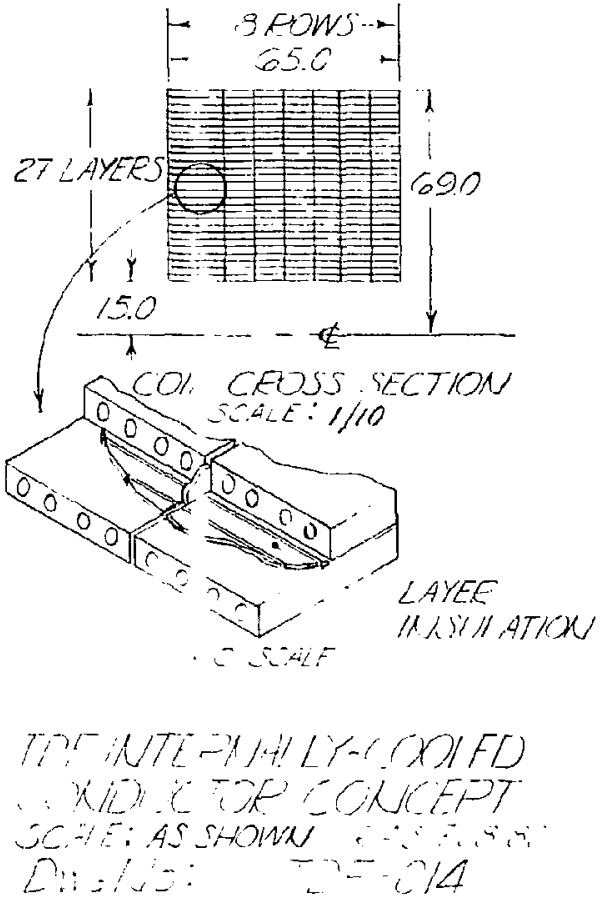

Figure 3-25. TUF internally cooled conductor concept, TOF-014. 


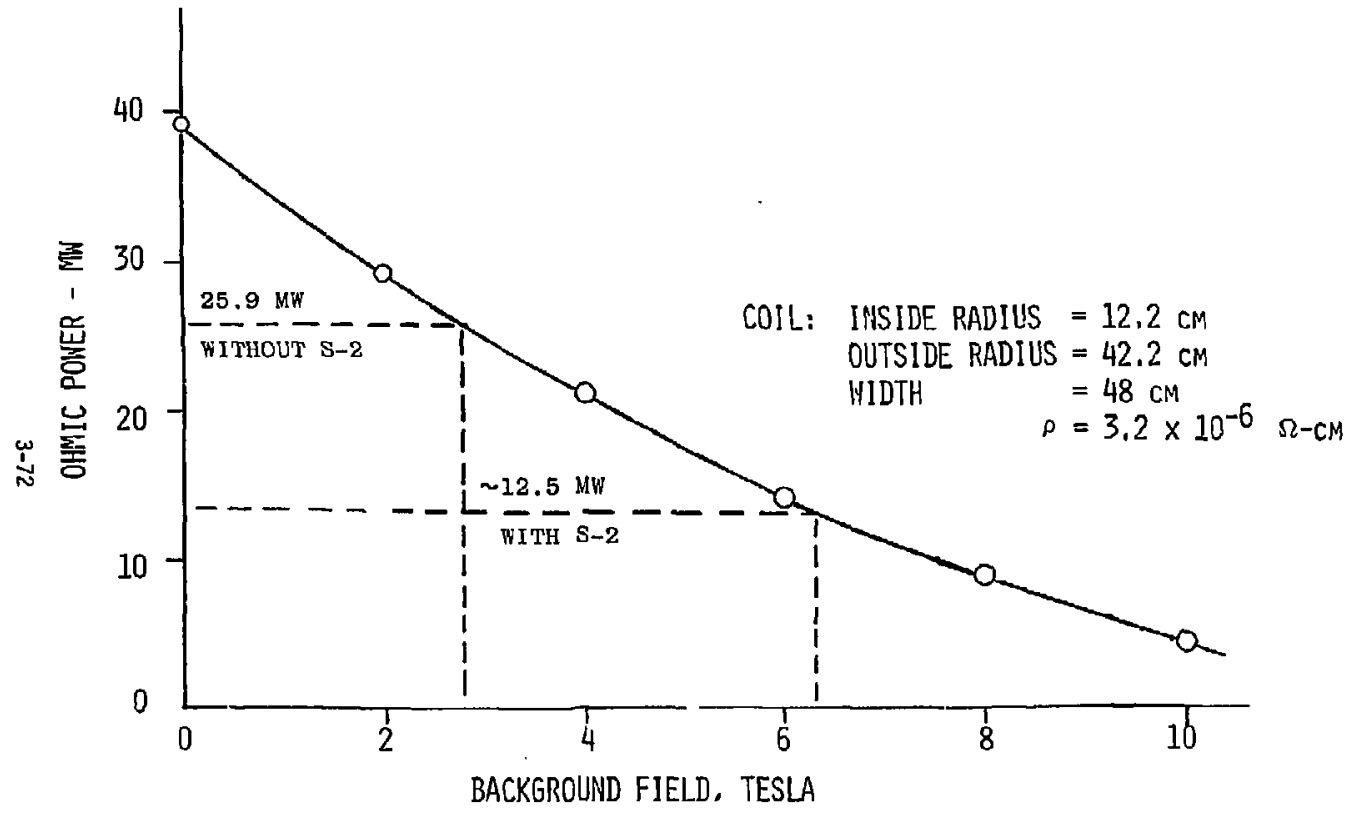

Figure 3-26. Power required for choke coil to produce $15 \mathrm{~T}$ for varying barkkground fielas. 


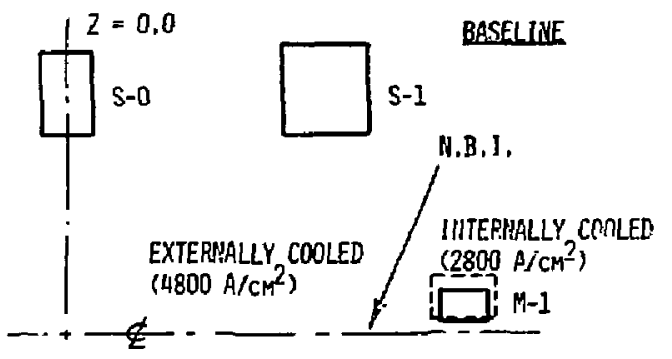

CONBENTS

EXTERNALLY COOLED COIL $\left(4800 \mathrm{H} / \mathrm{cm}^{2}\right.$ ) REQUIRES 25.9 Wh KHERE, IXTERSALLY COOLSD COIL (2800 A/ $/ \mathrm{Cm}^{2}$ ) REQLIIRES

31.1 MT G GEATER KIDTH OF

JNTERNALLY COOLED COIL WAY INCREASE LENTTTH OF BAACHISE.

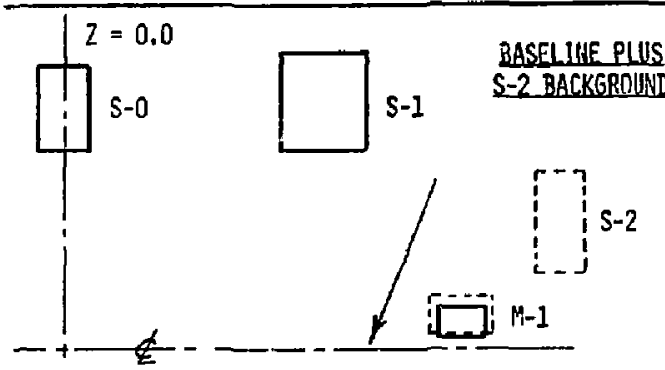

THF ADDITION OF S-2 BACKGOLND COIL (SUPERCONDUCTING) LOKEAS RFQUIRED FIFLD OF $m-1$ COIL, CURRENT DENSITY OF AN EXTERNALLY COOLED TYAE IS $3900 \mathrm{~A} / \mathrm{cm}^{2}$ AND THE POHER $17.1 \mathrm{MK}$.

CAPITAL COST OF S-2 IS APPROXIMIATELY

\$2.6M AND APPEANS ECONOMICALLY FEASTBLE.
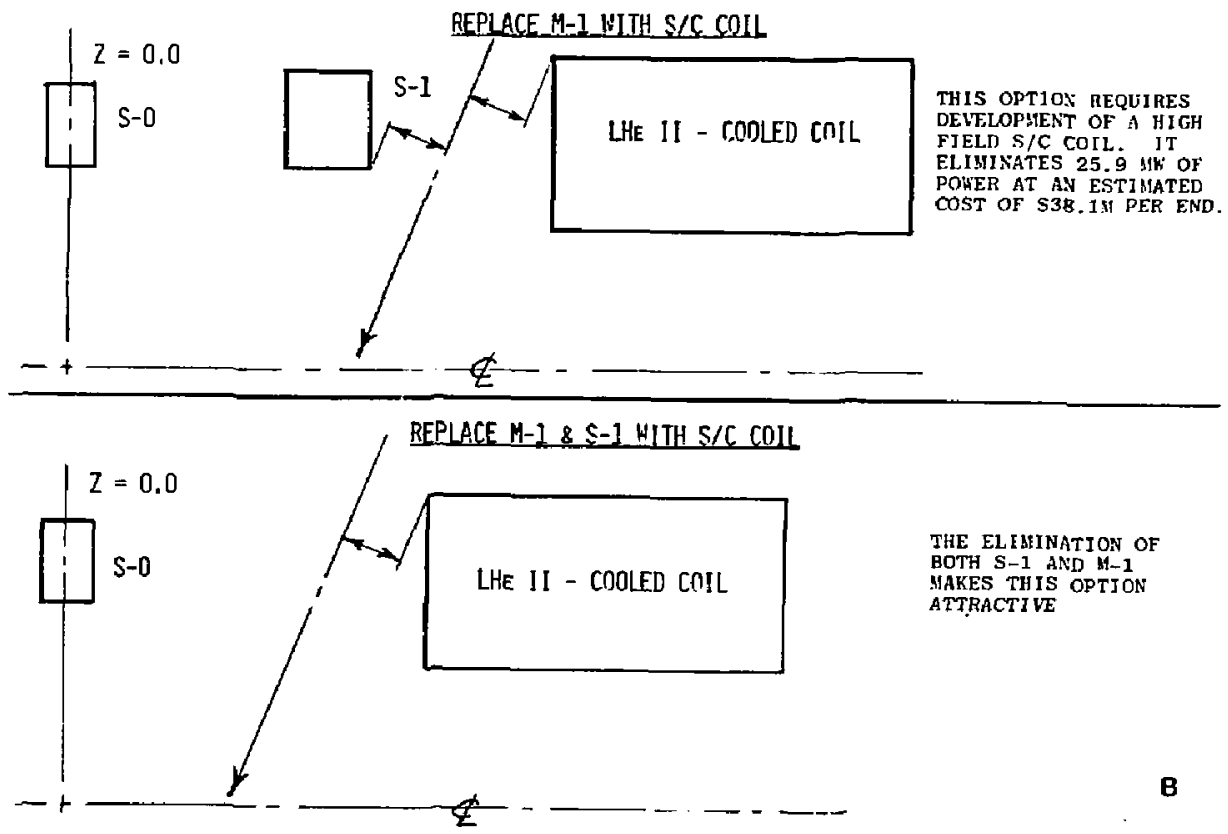

THE ELISINATION OF BOTH S-1 AND $\mathrm{H}-1$ MAKES THIS OPTJON ATTRACTI VE

Figure 3-27. Alternate solenoid options. 
stability for the plasma. Its configuration must also be compatible with neutral-beam access requirements and with a requirenient to clear the plasma halo. Finally, the magnet windings must be supported for magnetic forces, deaa weight, and seismic loass for the design life of the magnet.

Coil Configuration Requirements. MHD stability requirements dictate that the anchor cell winding configuration and coil currents produce a 3-T peak mirror field on-axis and a 3:1 mirror ratio.

To clear the plasma halo, the configuration of the anchor cell magnet set must provide a clear bore for the flux surface that is located at a radius of $17 \mathrm{~cm}$ in the central cell.

There must be space through which plasma heating can be introduced at appropriate points on the nachine axis by these methods:

- Anchor pumping beam directed up the mouth of coil M-3, at an angle of 15 deg to the machine axis, to strike the axis over the area between $z=9.2$ and $10 \mathrm{~m}$.

- Anchor sloshing beam cirected at 90 deg to the machine axis, through the mouth of coil $\mathrm{Ni}-2$, at a point $z=8.95 \mathrm{~m}$.

- Transition pumping beam, at an angle of 25 deg to the machine axis, aimed to strike the axis over the area between $z=4.7$ and $6.0 \mathrm{~m}$.

- Kf launchers directed at $z=9.5$ and $10.5 \mathrm{~m}$.

In addition to these quantitative requirements there is, for beam access, a challenging compromise betreen the space availability between magnet. structures and having enough beam footprint to achieve the desired beam power striking the plasma.

Design Life. TOF has a design life of 10 years, and the anchor cell coils have been designed for 1000 startup and shutdown cycles--a reasonable cyclic requirement for this design life ano the expected operating scenario. The anchor cell coits have been designed for a seismic load of $1 \mathrm{~g}$ laterally and vertically; this figure is based on an assumed ground acceleration of $0.25 \mathrm{~g}$ and on a dynamic amplification of 4 , found to be realistically canservative for such massive structures.

Design Criteria. These criteria have been used in the conceptual study:

- The winding should be cryostable.

- The maximum discharge voltage during a quench shou ta be $500 \mathrm{~V}$. 
Magnet Structural Design. The allowable stress for magnet structural sizing calculations is $207 \mathrm{MPa}$. This figure is based on an allowable alternating stress of $414 \mathrm{MPa}$, derived from crack growth and brittle fracture considerations, with an arbitrary conservatism factor of 2 to reflect that sizing is by hand calculations and that detailed analysis will probably produce nigher stress.

Vacuum Vessel Structure. Wall thickness is chosen in accordance with the ASME Boiler and Pressure Vesse 1 Code. The design pressure is 1 atm, and the design temperature is $93{ }^{\circ} \mathrm{C}$.

Engineering Trade-off Studies. Before proceeding with a point design, we conpleted several general design studies sn that we could evaluate alternative concepts.

Choice of Cooling. From a preliminary study of the relative cost of liquid-helium-cooled superconducting magnets vs water-cooled resistive coils we concluded that the resistive option would be cheaper than the superconducting option. The direct capital cost in 1982 dollars of two coil sets, one at each end of the machine, was estimated at $\$ 17.7$ million for the superconducting option and at $\$ 2.7 \mathrm{mi}$ ilion for the resistive option. The 10-year life-cycle costs were $\$ 17.7$ million (with the operating cost for the superconducting coils assumed to be negligible in this study) and $\$ 14.3$ million for the superconducting and resistive options, respectively.

A key assumption that strongly influenced the conclusion was that the superconducting coils would need significant shielding in the bore while the resistive coils, which could be insulated vith polyimide, would not. The need for general shielding in the bore of the aachor-cell magnet set meant that larger magnets were needed to provide the same clear bore and, consequently, that there was an increased MAT requirement to achieve the same field on-axis, both of which resulted in the greater capital cost. An evaluation of anchor cell shielding requirements contradicted the original shielding assumptions. Because of the relatively benign nature of the plasma within the anchor cell (regarding the neutron irradiation), neither option would require general shielding of the bore. The use of glass-epoxy insulation was found to be feasible, with perhaps some shadow shielding required to protect against streaning through the choke coil from the central cell. 
Motivated by the resuits of this neutronic study, we reworked the cost comparison, deleting the need for general shielding of the bore of the superconducting magnets. This reevaluation also incorporated relatively minor comments resulting from a General Dynamics and LLNL review of the original study. The reevaluation showed that the estimated capital cost of the supercoriducting option ( $\$ 1.2 \mathrm{million})$ is less than that of the resistive option (\$2.8 million) and that the life-cycle costs are much less. Although coil configuration changes affecting the actual cost have occurred since this study, the relative economy of superconducting coils for this application is still valid.

Choice of Conductor. The Grade-II yin-yang conductor for the MFTF-B Project was seiected. Hit seriours thought was given to alternatives since this conouctor has been successfulty manufactured, successfully wound in to quadrupole coils, and successfully operated at design conditioris. Also, the TDF anchor cell performance requirements are very similar to those for MFTF-B (a current density of $3430 \mathrm{~A} / \mathrm{cm}^{2}$ at $4.7 \mathrm{~T}$ on JUF, compared to $2.837 \mathrm{~A} / \mathrm{cm}^{2}$ at $5.28 \mathrm{~T}$ on MFTF-B). As discussed in Section 3.2.3 ("Engineering Analysis"), calculations confirm conductor cryostability for the TOF fiela ana current.

The size difference between the TDF quadrupole coils and those successfully fabricated and operateo on MFTF-B was of potential concern because it meant that the conductor would have to be wound inta a tighter radius of curvature on IDF than it was on MFTF-B. With the raqius of curvature being only half as large on TDF, the bending strain in the conauctor would be twice as great, which could damage the conductor. Alsa, it is more

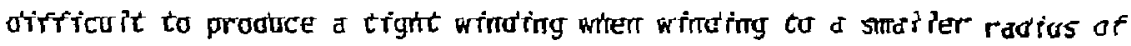
curvature. We have evaluated these potential concerns, however, and found them to be invalid. Although the bending strain during winding is larger, it is only 2.5\%, which is acceptable. Further, ouring practice winding of the MFTF-B " $A$ " coil, which has a minimum radius of curvature approximately equal to TDF, we encountered no practical difficulty, i.e., no solder joints broke, and the pack tightness was acceptable.

Vacuun Topology. The entire machine wi]l have a plasma vaciam vessel that could also provide the thermal guard vacuum for the anchor cell coils. However, the test samples are inside the plasma vacuum vessel, which would require that the superconducting magnets be warmed up and cooled down every time the plasma vacuum vesse] is opened for the installation of test modules. 
Because module installation will occur frequently, and the time required to cycle the superconducting magnets between cryogenic and room temperature will be long, the impact on operation wculd be most undesirable. Thus, we will place the anchor cell coils within a vacuum vessel independent of the plasma vacuum vessel so they can be kept cold when the plasma vessel is penetrated.

Consistent with the provision of a guard vacuum independent of the plasma vacuum vessel, two alternatives are possible. The three anchor cell coils $(T-1, M-2$, and $M-3)$ can each have their own guard vacuum vessel, or they can be placed within a common vessel. The advantage of individual vessels is that a single coil could be replaced or repaired without disturbing the other two coils in the set. A single vesse], on the other hand, simplifies support problems and reduces the space envelope; it also allows for the coil set to be assembled into the vacuum vessel and ther for the subassembly to be loaded into the machine as a unit, simplifying installation. Considering all these factors, the single-vessel approach was favored.

Winding Support. As a minimum, the helium case around the winding pack must be sized so that it is strong enough to act as the coil former during winding and to resist the winding magnetic running loads in plate bending. Sized in this manner, the case is not strong enough to resist integrated running loads whose major effects are lobe-opening forces, with equilibration of the major lobe-opening forces being the more difficult design problem. Equilibration of the major lobe-opening forces can be accomplished in several ways.

Consider coil T-1--because there are only two options for it, it is eisy to discuss. On MFTF- $B$, the " $A$ " coil will be supported against the major lobe-opening forces by a support yoke (deep beams across the major lobes connected by tension members at their ends). As an alternative, the case can be strengthened sufficiently so that it is free-standing against the lobeopening forces. These two options are illustrated in the upper sketch in Fig. 3-28. Because of its lower cost, the latter option was adopted for TDF. On TDF, the plasma halo fans out into the mouth of coil T-1 to such a degree that a support yoke is not practical. The deep beams have to be long enough for the tension members to clear the halo fan; this would result in such large bending moments in the deep beams that it is impractical to carry the load in this manner. 


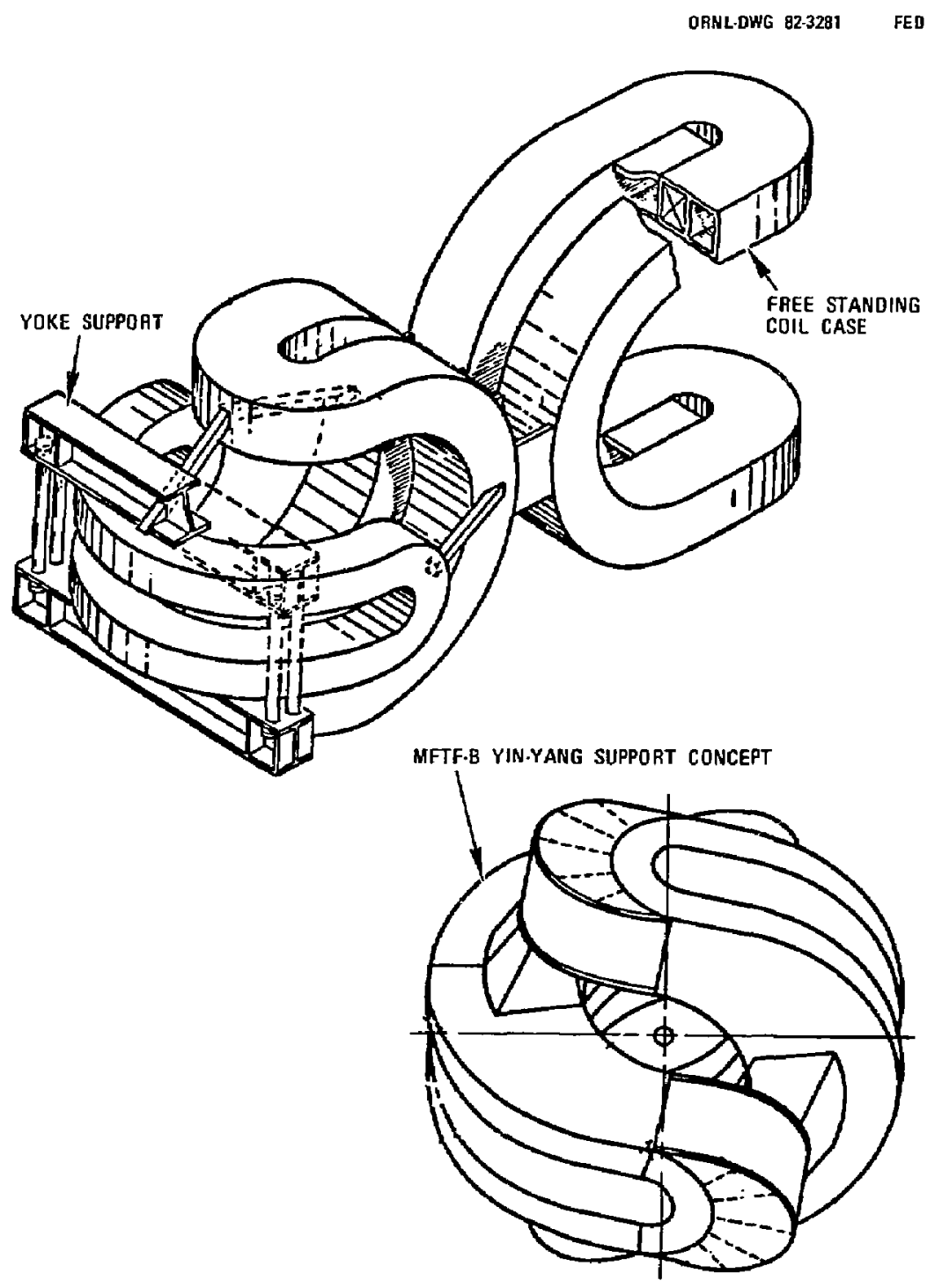

Figure 3-28. Coil winding support alternatives. 
Three options are available for coils $M-2$ and $M-3$ : the major lobe yokes or free-standing cases discussed above, as well as a third crcion not available for $T-1$. This is to connect coils $M-2$ and $M-3$ so that tney tend to support one another. This option was adopted for MFTF-B and is iliustrated in the lower portion of Fig. 3-28. Major lobe-support yclies were again rejecteo, as they were for coil $\mathrm{T}-1$, because the plasma halo fan $\mathrm{fil}$ ? $\mathrm{s}$ the mouths of cojls $M-2$ and $M-3$ to such an extent that it is deemed impractical to support the major lobe-opening force in this way. In an early aesign study for coil $\mathrm{M}-3,40 \%$ of the major lobe-opening force was carried by the yoke and $\mathrm{b} 0 \%$ by the coil case itself. The structure weight (and therefore cost) was found to be $20 \%$ less than the siructure weight that would have been requireo had the case been designed to be free-standing. In this study, however, the deep bean was made just long enough to clear the coil jtself. Had the ceep bearn been long enough for the tension members to clear the halo, the bending moment in the beam would have nearly triplea. It was judged, too, that resizing of the beam for this moment would result in a more expensive design than the free-standing case, leaving the support scheme useo on MFTF-b as the only alternative to free-standing coils. Although this design was not evaluated quantitatively, it was rejected in favor of free-standing coil cases for lobe spreading. The geometry of the MFTF-B yin-yang pair lenas itself to structural connections between the coils. A mooest extension of the case in the minor radius of the coils is sufficient to place the case in that region oirectly over the miopoint of the major radius of the other coil in the pair. For TUF, however, the axial separation of coils $\mathrm{M}-2$ and $\mathrm{M}-3$ in the yin-yang pair means that a much greater distance must be spanned to provide the structural connection between the minor lobes of each coil to the riliadle of the major lobe of the other coil in the pair. (Un MFTF-B the centers of the major radius of curvature of the two coils actually overlap by $140 \mathrm{~cm}$, while on TUF there is a 37-cm-wide axial spreading). A further consideration deals with design philosophy. A virtue of the free-standing approach is that coil forces and moments become statically determinate, providing greater assurance that they have been accurately computed and properly accounted for than in a design that requires accurate computation of the force-defiection characteristics of the coils for accurate computation of coil forces ano stresses. A design in which the coil cases are free-standing is also more tolerant of the sort of design change presently being incorporated into MFTF-B, i.e., axial separation of the coils, which will have a first-order effect on the structural design. 
Conclusions. Un the basis of the alternatives, we chose a baseline design for the anchar cell consisting of liquid helium-cooled $(4.5 \mathrm{~K})$ superconducting coils, with the same kind of niobium-titanium, copper-stabilized, Type-II yin-yang conductor being employed in MFTF-B. The coils will be suspenaed in a common vacuum tank, distinct from the main plasma vacuum vessel, that will permit installation of the anchor cell coil set as a subassembly and provide the thermal guaro vacuum needed to eliminate heating up and cooling down the superconducting coils every time the plasma vacuum vessel is penetrated to install test modules. The cold mass of the magnets will be supported from the common vacuum vessel, and external locations on the anchor cell will be provided for supporting the subassembly from the main machine structure. The individual coil cases are designed to be free-standing under the action of lobe-opening forces, with intercoil support structure needed only to transfer the resulting axial magnetic and inertial loads (dead weight and seismic load) from coil to coil.

Selected Design Approach. The baseline concept has just been discussed. Fjgure 3-29 7 llustrates the overall arrangement of TDF, showing the location and orientation of the anchor cells relative to other components. Notice that the two cells are rotated 90 deg about the machine axis relative to one another. For clarity, the anchor cells have been rotated so that their planes of symetry lie in and normal to the plane of Fig. 3-29; in practice the orientation will be such that the planes of symmetry are at 45 deg relative to the vertical ano horizontal. This orientation requces the tendency to trap helium bubbles under horizontal surfaces of the helium case.

Design Description. The baseline concept consists of three superconducting coils--T-T, M-2, and $M-3--e n c l o s e d$ within a single vacuum vessel. A detaileo description of these components follows.

Concuctor ana Insulation. The conductor and insulation are depicted in Fig. 3-30. The conductor consists of a square monolithic core with niobium-titanium stranos in copper, with a copper-to-superconductor ratio of 6:1. Adgitional stabilizer material is provided by a copper sheath surrounding the core and bonded to it by solder joints. Turn-to-turn and layer-to-layer insulation is provided by G-10CR sheets perforated to ensure adequate ventilation of helium bubbles from the winding. 


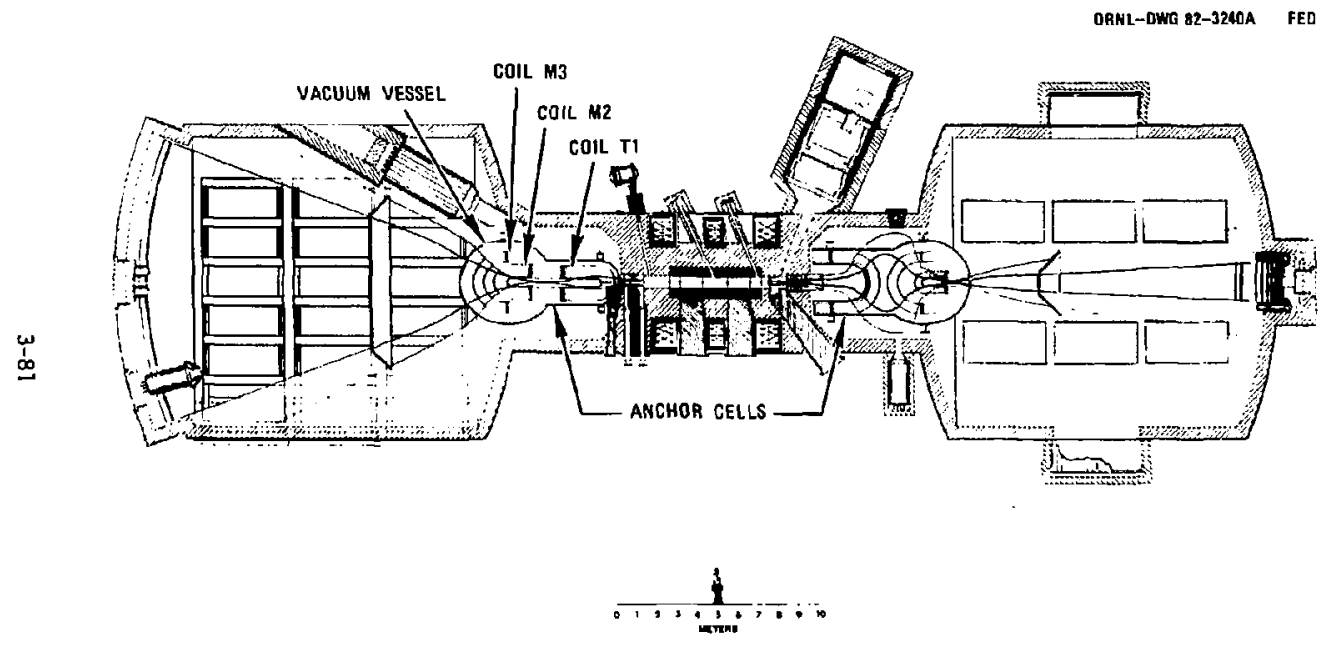

Figure 3-29. TDF arrangement. 


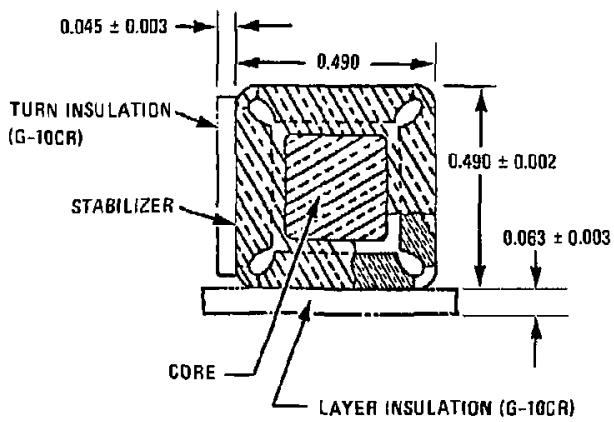

ALL. DIMENSIONS IN INCHES

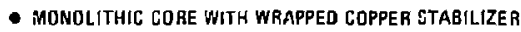

- NGTi SUPERCONOUCTOR CONTAINS $46.3 \pm 1,5 \%$ TITANIUM

- $\mathrm{Cu} / \mathrm{Sc}$ CORE $=6.00: 1$

- $\mathrm{Cu} / \mathrm{Se}$ OVERALL $=18.7: 1$

- bar core $=150$

- rra stabilizer $=220$

- 50/50 pb Sn Solden

- copper area 1.168 sq em

Figure 3-30. Anchor cell magnet conductor. 
The conductor shown in Fig. 3-30 is wound into the winding pack illustrated in Fig. 3-31. For all three ancior cell coiis, the winding is nominally $0.2 \times 0.4 \mathrm{~m}$ with 420 turns arranged in 28 layers, with 15 turns in each layer. A layer of 1.27-cm-thick G-70CR surrounds the winding and provides ground-wall insulation. The overall dimensions of the winding pack, incluoing ground-wall insulation, are $0.229 \times 0.419 \mathrm{~m}$.

Winding Configuraton. The winding configuration is described in Table 3-11. Each anchor cell coil is a quadrupole with major and minor radii of 1.25 and $0.35 \mathrm{~m}$, respectively. For coil $\mathrm{T}-1$, there is an $80-\mathrm{cm}$ straight leg between the minor and major lobes. The centers of major radius of curvature for the three coils are located at $6.6 \mathrm{~m}$ (coil $7-1$ ), $10 \mathrm{~m}$ (coil $M-2)$, and 10.37 ni (coil M-3), measured from the center of the central cel).

Support Structure. The support structure may be separated into four categories distinguished by the function performed.

1. Helium case. The winding cavity helium case is a rectangular, leaktight box weloed around the winoing pack, which serves as a coil foriner, onto which the winoing is wound; the structure, which also includes a helium vessel to contain the coil coolant, continuous $1 y$ supports the winoing pack against the in- and out-of-plane magnetic running loads (magnetic load integrated over the winoing crass section).

2. Helium-case support structure. The helium-case support structure is a heavy channel section that extenos the helium-case cross section parallel to the short dimension of the helium case. Its puruoses are to provide a composite box section that is strong enough to equilibrate the bending moments, shears, and axial tension produced by integration of the magnetic running load along the coil and, in particular, to provide enough benaing section to equilibrate the resulting critical stress, the bending moment produced by the major lobe-open ing forces.

3. Intercoil support structure. The intercail supports tie the coils together into a single unit and must be strong enough to transmit the intercoil forces, i.e., dead weight, seismic load, and axial magnetic load (the only magnetic load of a non-self-equilibrating character). 
ORNL-UWG $82-3484$

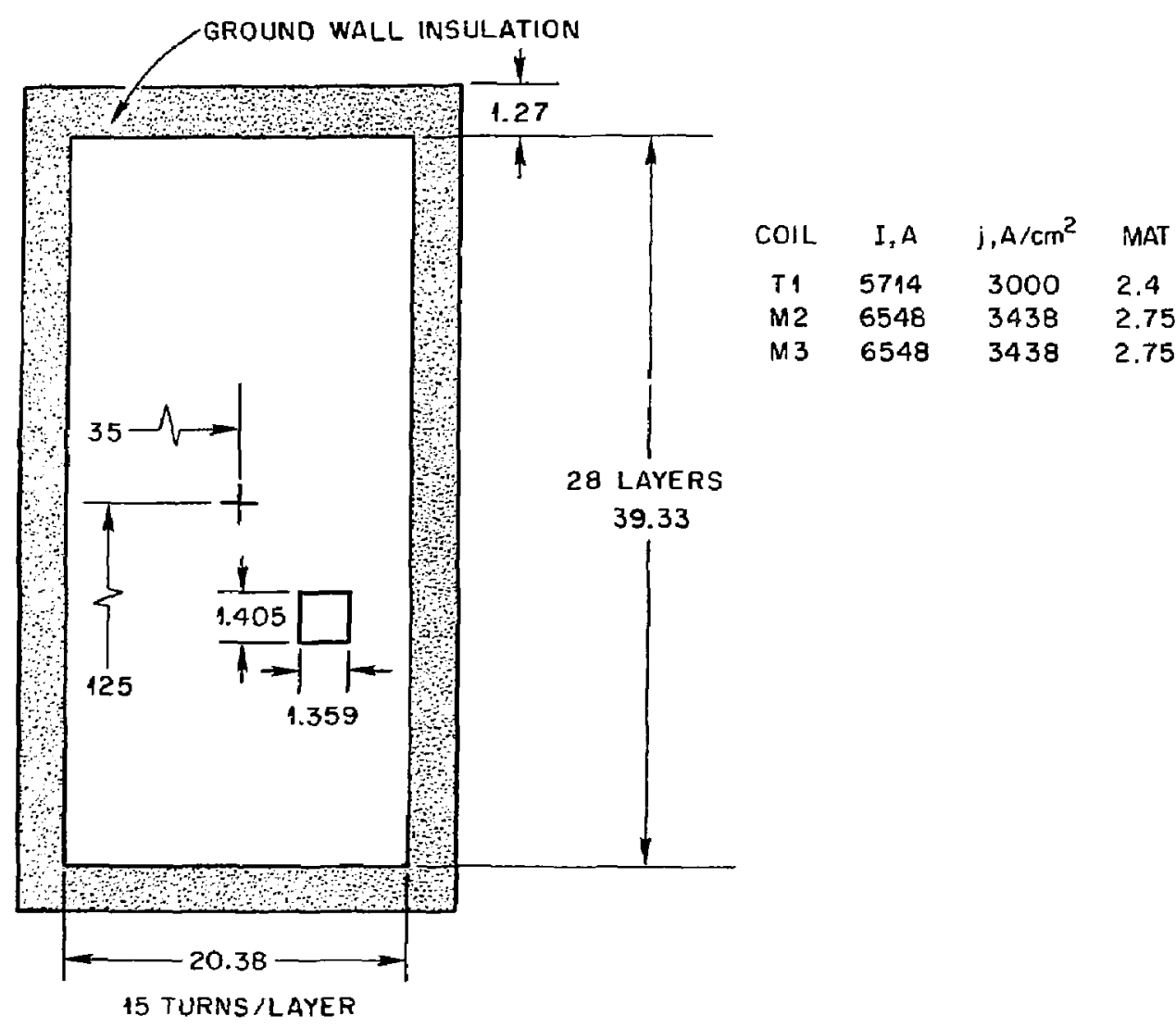

ALL DIMENSIONS IN $\mathrm{cm}$

Figure 3-31. Anchor cell winding pack--typical. 
Table 3-il. Anchor cell coil-winding parameters.

\begin{tabular}{|c|c|c|c|}
\hline & \multicolumn{3}{|c|}{ Coil } \\
\hline & $\overline{T 1}$ & M2 & $\overline{M 3}$ \\
\hline Major radius, $m$ & $\longleftarrow$ & $-1.25-$ & $\rightarrow$ \\
\hline Minor radius, m & $\leftarrow$ & $-0.35 \frac{7}{7}$ & $\rightarrow$ \\
\hline Straight, m & 0.8 & 0 & 0 \\
\hline Axial location, $m^{*}$ & 6.7 & 10 & 10.37 \\
\hline Winding pack, $m \times m$ & $\leftarrow$ & $0.204 \times 0.393$ & $\longrightarrow$ \\
\hline Mean turn length, m & 13.25 & 10.05 & 10.05 \\
\hline MAT & 2.40 & 2.75 & 2.75 \\
\hline $\mathrm{j}, \mathrm{A} / \mathrm{cm}^{2}$ & 2994 & 3430 & 3430 \\
\hline$I, A$ & 5714 & 6548 & 6548 \\
\hline
\end{tabular}


4. Cold-mass support structure. The cold-mass supports suspend the coils inside the anchur cell vacuum vessel and must be capabie of equilibrating the resulting loads acting on the coil set.

Figure 3-32 shows a typical coil case cross section to illustrate the composite box character of the case. Case dimensions for each coil are provided in Table 3-12. Figure 3-33 is an isometric of the complete anchor-cell set, showing the coil set at the left end of the machine.

The sices of the helium case against which the in- and out-of-plane magnetic running loads bear have thicknesses $t l$ and $t 2$ in $F i g .3-32$. These thicknesses vary from coil to coil, reflecting the fact that the magnetic running load varies from coil to coil. The two remaining sides of the rectangular box that constitutes the helium case provide the former onto which the coil is wouno; their thickness is chosen rather arbitrarily.

The helium-case support structure illustrated in Fig. 3-32 is an extension of the two short sides of the helium case, with a cross plate forming the top of the composite box. The $i$ and $t 3$ dimensions of the structure are chosen so that the resulting beam properties ensure adequate strength at critical locations along the coil center line. As shown in the figure, the helium-case support structure is oriented to produce the most effect on the cross sectional moment of inertia of the case in the direction of bending proouced by the major lobe-opening forces.

The case cross section is graded in each coil, with the basic section prevajling over most of the major lobe. The case cross section dimension $h$ is then increased linearly to a maximum value at critical coil sections--the junction between the major lobe and the straight section for coil T-I, ana the middle of the minor lobe for coils $M-2$ and $M-3$. The grading was cone to reduce cost. The maximum section does not have to be run all around the coil periphery; the resulting stresses are generally lower near the center of the major lobe, so the reduced section is adequate there. The basic section extends to $\pm 50 \mathrm{deg}$ of the center of the major lobe for coil T-1 ano to \pm 60 deg for coils $M-2$ and $M-3$.

Aoditional helium-case support structure is provided in the form of gussets in the mouth of coil $\mathrm{T}-\mathrm{l}$ joining the straight legs between lobes together across the mouth of the coil (see fig. 3-33). These gussets equilibrate the major lobe-opening forces in direct tension rather than permitting the maximum moment due to major lobe-opening forces to increase 


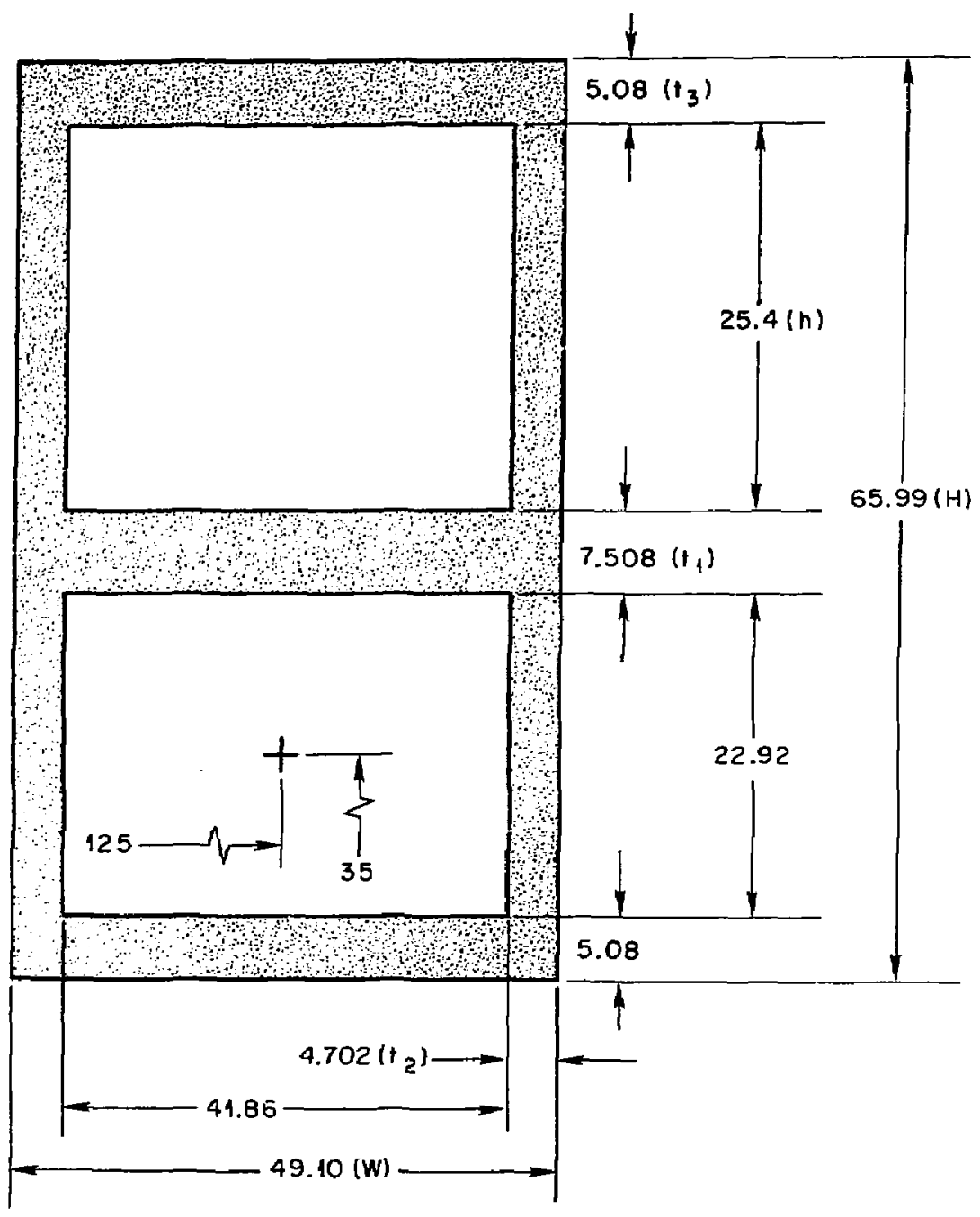

ALL DIMENSIONS IN cm

Figure 3-32. Typical coil case cross section. 
Table 3-12. Coil case cross section dimensions.

\begin{tabular}{lcccc}
\hline & $\begin{array}{c}\text { Coil T1 } \\
\text { Basic section }\end{array}$ & $\begin{array}{c}\text { Coil T1 } \\
\text { Max. section }\end{array}$ & $\begin{array}{c}\text { Coils M2 \& M3 } \\
\text { Basic section }\end{array}$ & $\begin{array}{c}\text { Coils M2 \& M3 } \\
\text { Max. section }\end{array}$ \\
\hline$t_{1}, \mathrm{~cm}$ & 7.51 & 7.51 & 7.89 & 7.89 \\
$\mathrm{t}_{2}, \mathrm{~cm}$ & 4.70 & 4.70 & 3.53 & 3.53 \\
$\mathrm{t}_{3}, \mathrm{~cm}$ & 5.08 & 7.62 & 5.08 & 10.16 \\
$\mathrm{~h}, \mathrm{~cm}$ & 25.4 & 38.1 & 25.4 & 53.34 \\
$\mathrm{H}, \mathrm{cm}$ & 49.10 & 49.10 & 47.93 & 47.93 \\
$\mathrm{H}, \mathrm{cm}$ & 65.99 & 81.23 & 66.37 & 99.39 \\
\hline
\end{tabular}




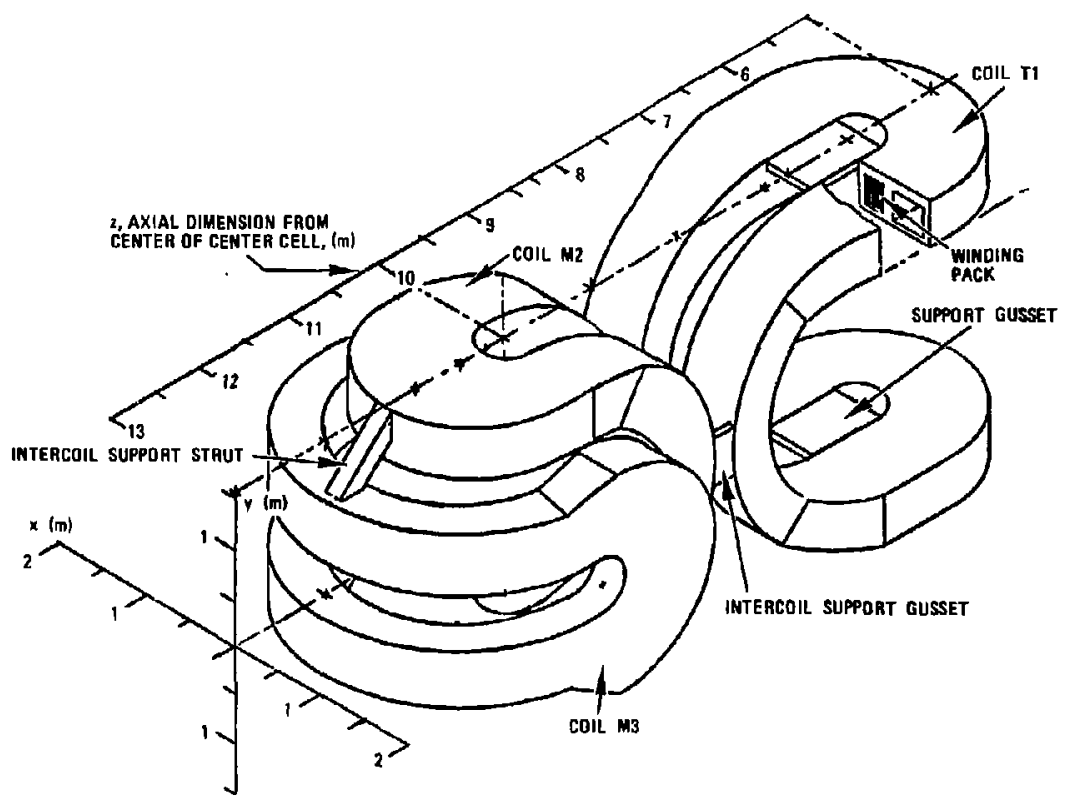

Figure 3-33. Anchor cell coil set. 
linearly along the $80-\mathrm{cm}$ straight leg. Analysis has shown that these gussets will not be in the way of the plasma halo. The plasma halo, while farining out in the nouth of coil $\mathrm{T}-1$, does so outboard of the straight legs.

The intercoil supports are depicted in Fig. 3-33. They consist of four gussets connecting coils $\mathrm{T}-1$ and $\mathrm{N}-2$ at the centers of their major lobes and of two beam supports connecting the minor lobes of cbil $\mathrm{M}-2$ to the centers of the major lobes of coil $\mathrm{M}-3$. The gussets are plates, each $25-\mathrm{cm}$ deep and $5-\mathrm{cm}$ thick; the beam supports are hollow box beams, 30-cm square with a $2.5-\mathrm{cm}$ wall thickness.

The cold-mass support concept is shown in Fig. 3-34. Struts from the coil cases to the reinforcing yokes on the vacuum vessel transmit the resulting loads on the coil set to the yokes.

Vacuum Vessel. The vacuum-vessel concept is depicted in the Fig. 3-35 isometric ano in the section views of Figs. 3-36 and 3-37. As aiscussed in Section 3.2.5 Vacuum Topology, a single vessel has been designed to enclose each anchor coil set. The major features of the vacuun vessel, discussed in more aetail below, are:

- Outer vacuum surface.

- Reinforcing yokes.

- Inner vacuum surface or bore.

- Rf penetrations.

The shape of the vacuum vessel has, through evolutions, become increasingly complex. A]though this complexity may not be reaojly grasped from Fig. 3-35, it is better understood when the current vessel is contrasted with an earlier configuration. The earlier configuration consisted of an outer cylindrical vessel with hemispherical heads completely surrounding the coil set and of an inner bore to permit the plasma to pass through the anchor cell. The diameter chosen for the cylinder was to be large enough to completely contain the plasma halo as it fans out in the mouths of coils T-l and $M-2$. The portion of the vessel that encloses the yin-yang is still pod-like in $\mathrm{Fig}$. 3-35, but the diameter has been reduced to more nearly conform to the coils. The result is that the bore of the vacuum vessel actually intersects the outer shell of the vesse], producing the slot (top of the vesse] in Fig. 3-35) that permits the halo to fan out beyond the outer shell througl the nouths of coils $T-1$ and $M-2$. In the present configuration, the portion of the vessel around coil Tl has been shaped to conform to the shape of the coil. 
OANL-DWG 82-3287 FED

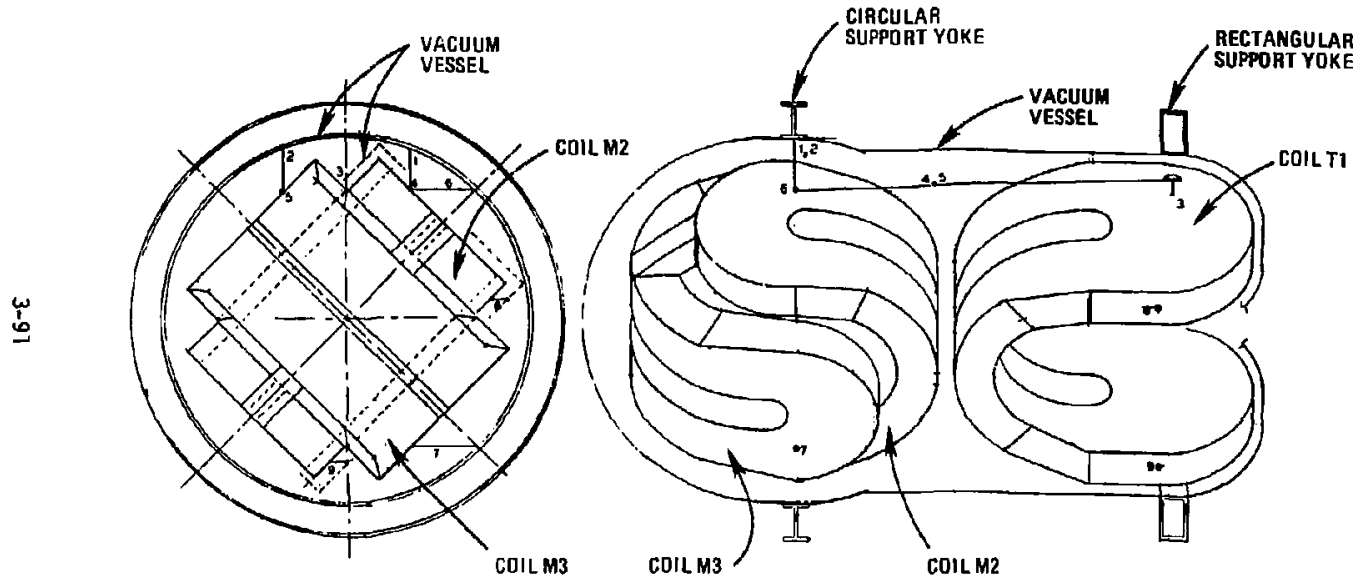

Figure 3-34. Cola mass support concept. 


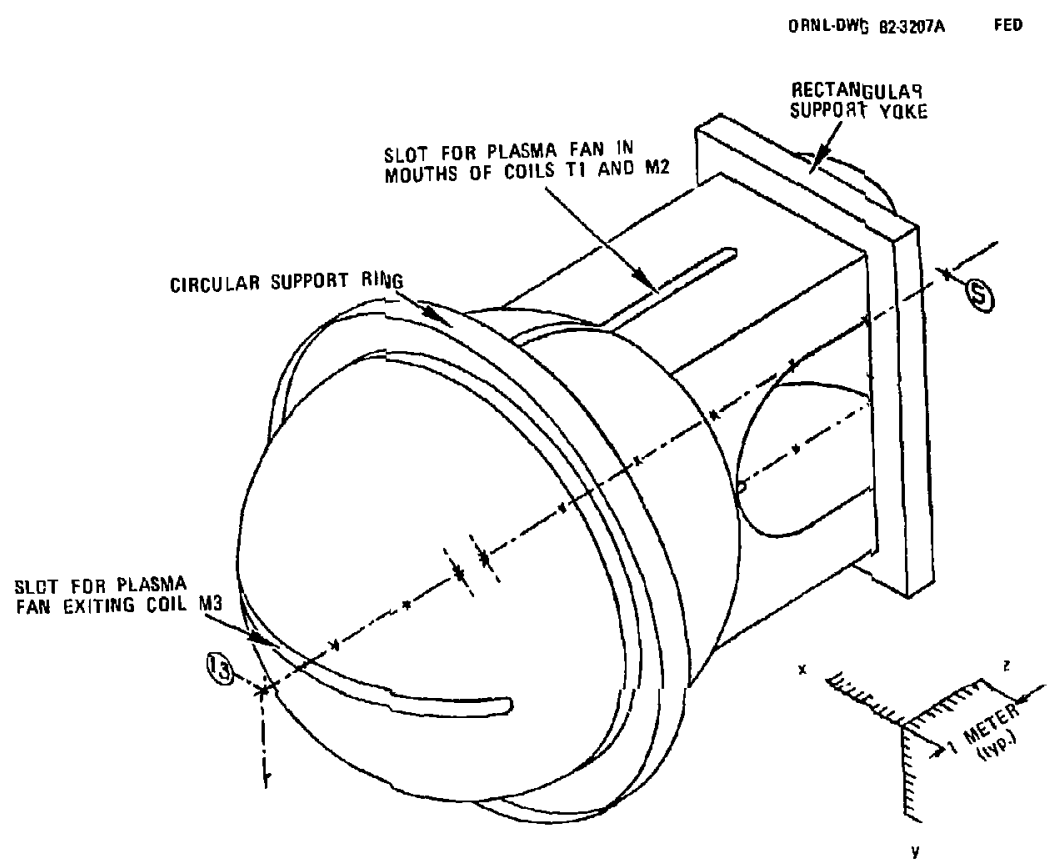

Figure 3-35. Anchor cell vacuum vessel. 


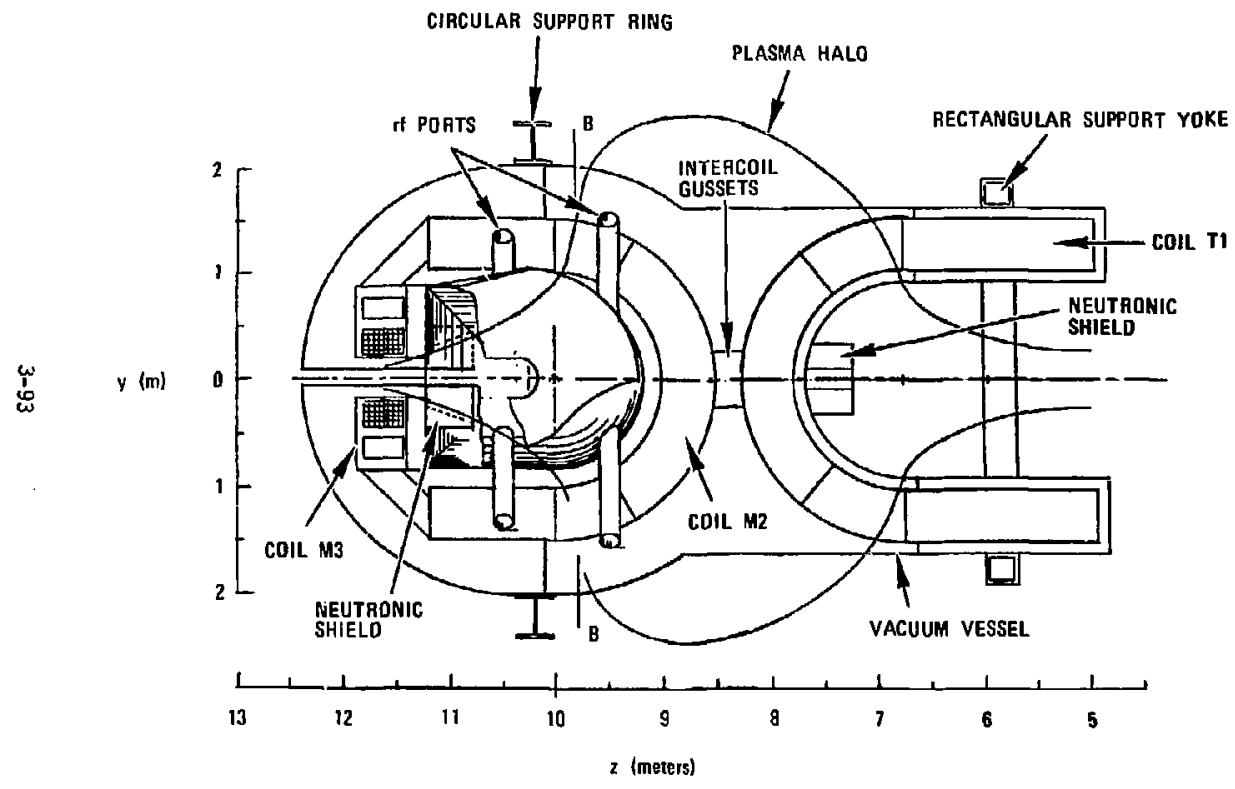

Figure 3-36. Section of anchor cell through the mouths of coils T-1 and M-2. 


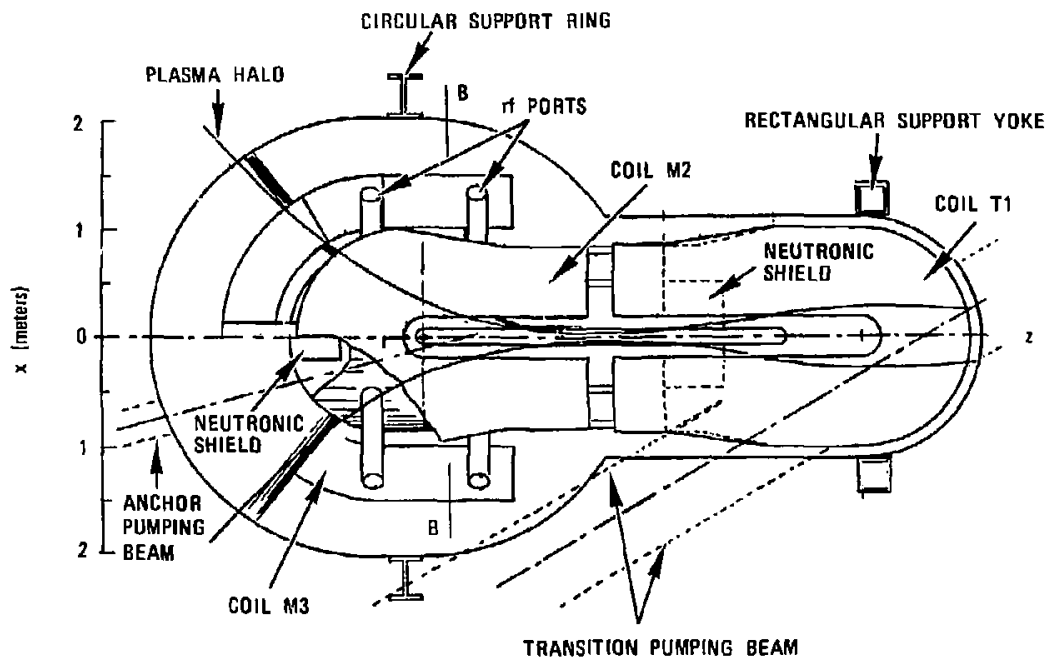

Figure 3-37. Section of anchor cell through the mouth of coll M-3. 
The changes made to the early pod-like configuration were primarily for two purposes: (1) to provide a larger annular gap between the vacuum vessel and the inside diameter of the shielding in order to ensure adequate cryopumping of the anchor cell and (2) to improve access for the transition pumping beam.

A sketch of the inner shell or bore of the vacuum vessel appears in Fig. 3-38. The bore shape was chosen to accommodate the plasma halo fan in the mouths of coils $T-1 / M-2$ ana in the mouth of coil $\mathrm{M}-3$.

A liquid llitrogen cold shield following the vacuum vessel wall is locateo on the coil side of the inner and outer vacuum vessel surfaces. In those areas where the vacuum vessel surface lies close to the coils, (e.g., most of the inner surface and where the oucer surface wraps the iegs of coit $i-i\rangle, a 10-\mathrm{cm}$ space is reserved for the yacuum vessel. This space is considered sufficient to accomnodate the vacuum vessel wall (including water-cooling channels), the liquid:nitrogen cola shield, multilayer insulation, and tolerance.

Atcess requirements for the neutral beams are listed in Section 3.2 .5 ("Coil Configuration Requirements"). The anchor cell pumping beam is aimeo up the moldth of coil M-3 at an angle of $30 \mathrm{deg}$ to the machine axis. Because of the arigle and location of injection, a beam aump must be located inside the yin-yang pair for the anchor pumping beam. The anchor pumping beam oump is illustrated in Fig. 3-39 ano is discussed in detail in the "Neutral Beam" chapter. The anchor sloshing bean is directed through the slot in the mouth of coil M-2. Unlike the anchor pumping beam, the sloshing beam requires is dump inside the coll set because it can pass out through the slot on the gther sice. Ithe contour of the vactum resse $i$ around coti $i-i$ provious ampie access for the transition pumping beam (see Fig. 3-37).

There are, in connection with the anchor pumping beam, local exceptions to the minimum vacuum vessel standoff distance mentioned above. To accomodate the anchor pumping beam dump and not interfere with the plasma halo, it is necessary to indent the vacuum vessel about $2.5 \mathrm{~cm}$ locally. The flat sides of the inrer surface of the vessel in the mouth of coil $\mathrm{M}-3$ also have cylinorical indentations (see view $A-A$ in Fig. 3-39) to enlarge the anchor pumping beam footprint on the plasm?. In these regions, it may be necessary to use a thin copper plate as a cold shielo that, although it has no active $L N_{2}$ cooling over its surface, is attachea along its edges to the actively cooled portions of the colo shield. This thin plate or "chill" would rely on conouction in 


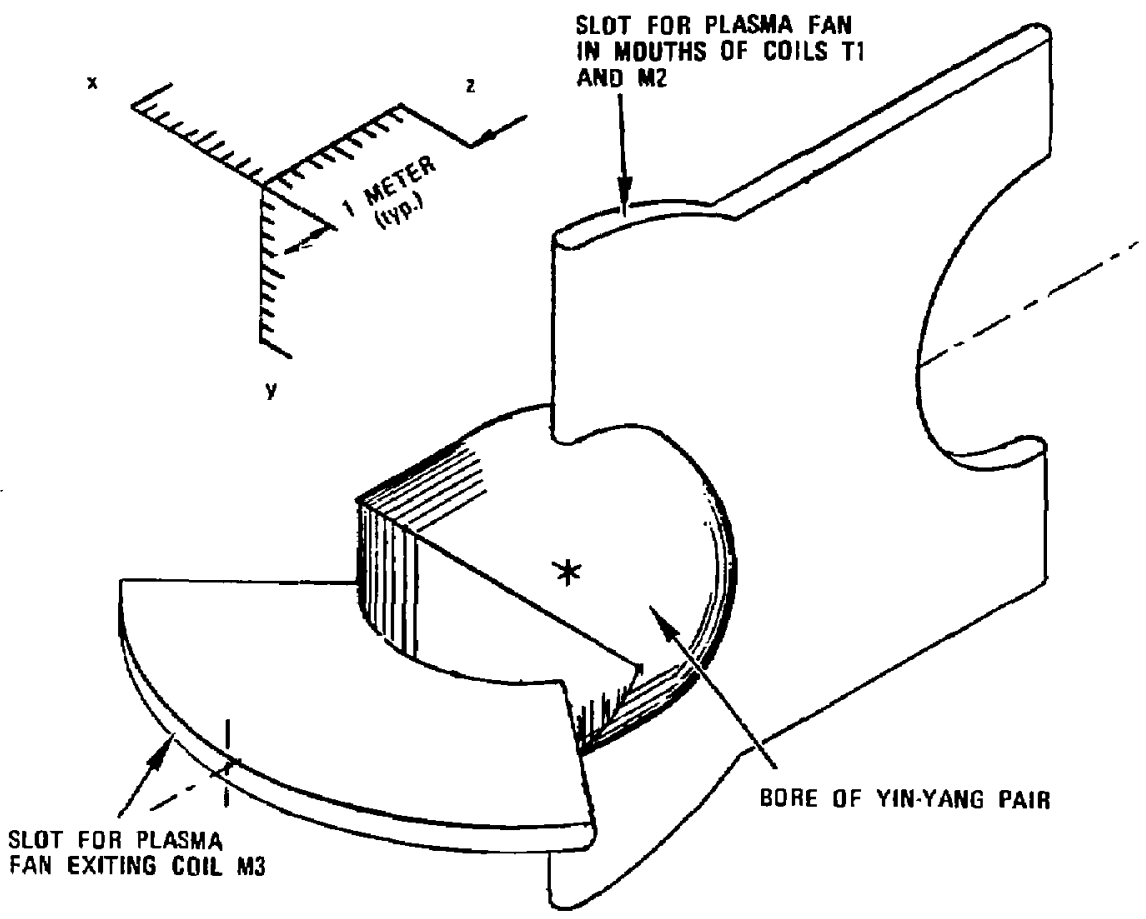

Figure 3-38. Inner shell of anchor cell vacium vessel. 
DAML-DW :23263

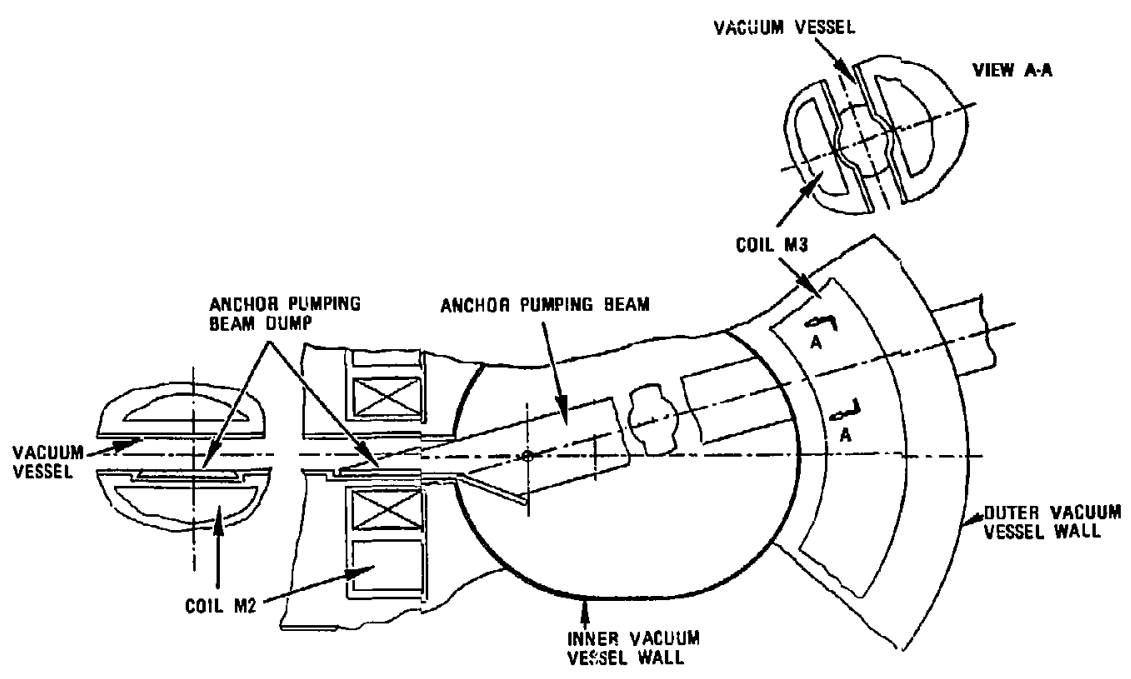

rigure 3-39. Anchor pumping beam dump. 
the plane of the chill to its actively cooled eages to provide a cold shielo in these very local regions where space does not permit use of the $\mathrm{LN}_{2}$ shield usea elsewhere.

The trajectory of the transition pumping beam is shown in Fig. 3-37; note that it physically interferes with the vacuum vessel and the case of coil $T-1$. An acceptable soluton to this problem is to notch the vessel and coil case locally to provide space for the beam. The spot where the trajectory interferes with the coil case (i.e., at the center of the major lobe) is a location of relatively low stress, and local notching is clearly feasible.

The entire inner surface of the vacuum vessel must be water-cooled. There is a uniform heat load of $1.5 \mathrm{~W} / \mathrm{cm}^{2}$ over the entire surface of the inner she]l, with locally higher loads in close proximity to the neutral beams. The heat load is $70 \mathrm{~W} / \mathrm{cm}^{2}$ where the anchor pumping beam passes through the mouth of coil $\mathrm{M}-3$ and $20 \mathrm{~W} / \mathrm{cm}^{2}$ where the anchor sloshing beam passes though the mouth of cail $\mathrm{M}-2$.

Local cooling will also be required where the transition pumping beam passes close to the outer shell of the vacuum vessel. The heat load at this location is $10 \mathrm{w} / \mathrm{cm}^{2}$. For this application, it is considered feasible to provide water-cooled surfaces designea to last for the life of the machine.

A shielding detail is shown in Fig. 3-40. Although general shielding of the anchor cell magnets is not required, shadow shields are necessary to shield local areas from neutron streaming from the central cell through the bore of plug coil M-1. The shielo is attached to the wall of the vacuum vessel anc must be water-cooled. Similar shielding is required in the same area of coj] M-3 (coj] M-2 is adequately shielaed by $\mathrm{J}-1$ in the region of neutron streaming from the central cell); Fig. 3-36 illustrates an unresolved problem in this regard. As shown in Fig. 3-36, the plasma halo clips the inside corner of the shielding on coil $\mathrm{M}-3$, violating the requirement for a 45-cm shadow-shield thickness in this area. This will require further study.

The shielding near the centers of the major lobes, depictea in fig. 3-40, is necessary on coils $\mathrm{T}-1$ and $\mathrm{M}-3$ to ensure that the following neutronic shielding criteria are met.

- Maximum integrated cose of $5 \times 10^{9}$ rads.

- Maxinum damage in copper of $1.1 \times 10^{-4} \mathrm{dpa}$ (producing a $7 \mathrm{n} \Omega-\mathrm{cm}$ increase in stabilizer resistivity).

- Maximum neutronic heating of $0.06 \mathrm{~mW} / \mathrm{cm}^{3}$. 


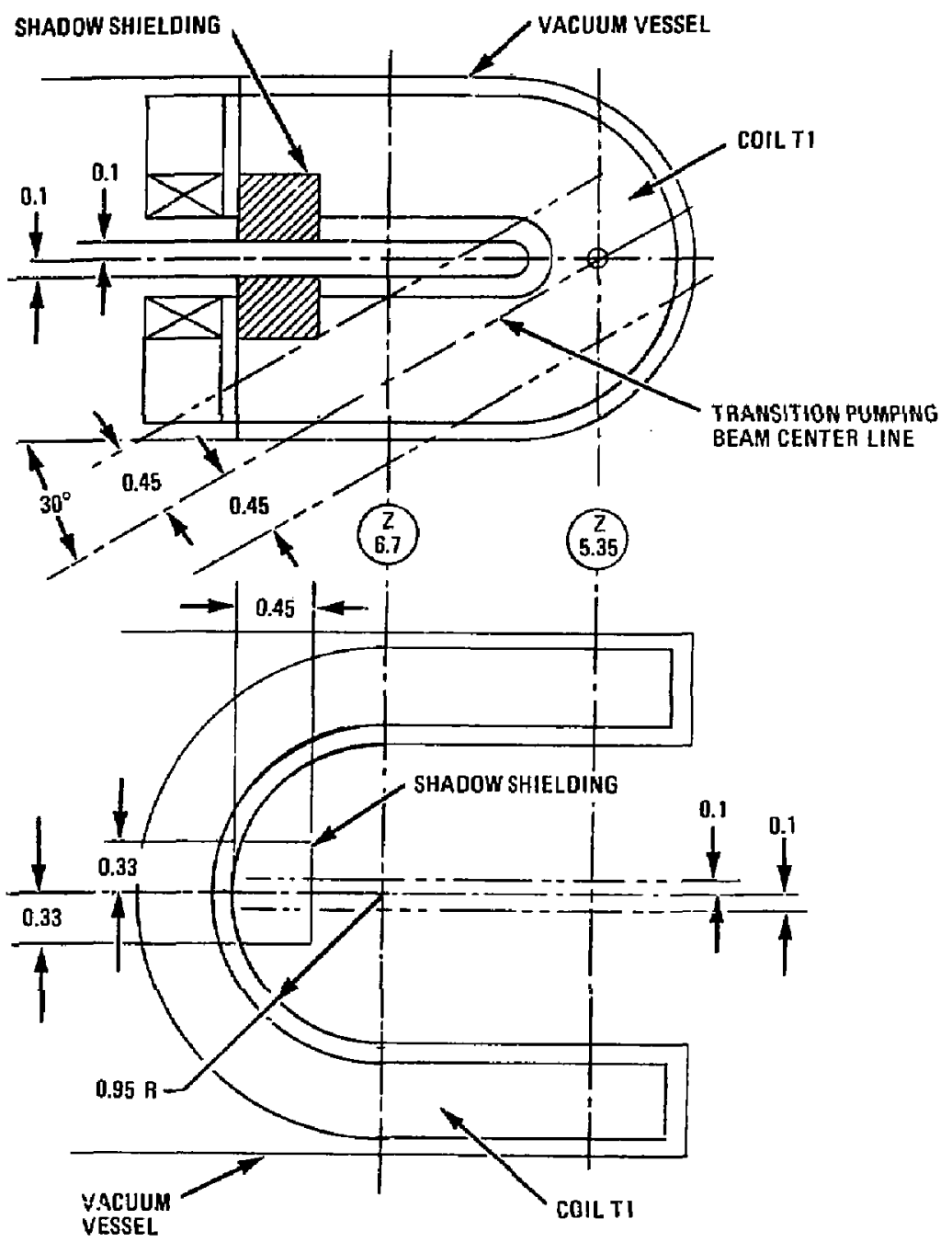

ALL DIMENSIONS IN $\mathrm{m}$

Figure 3-40. Anchor cell shielding detail. 
The outer shell of the vacuum vessel is reinforced by two support yokes (Fig. 3-35): a circular yoke of 1-beam cross section at $z=10.15 \mathrm{~m}$ (approximately the axial location of the center of gravity of yin-yang pair $M-2 / M-3)$ and a rectangular yoke of hollow rectangular section at $z=6 \mathrm{~m}$ (approximately the axial location of the c.g. of transition coil T-1). The yoke locations are dictated by beam access and halo clearance requirements. The reinforcing yokes provice support structure with bending strength sufficient for

- Supporting the cold mass insioe the vessel.

- Lifting ano handling a completed anchor-cell module consisting of the vacuum vessel and three magnets.

- Supporting a complete anchor-cell module from tha main plasma chamber.

A so shown in Figs, 3-36 ano 3-37 are the four 10-cm-oiam penetrations for $r f$. Two are aimea at each of the points $(z=9.5$ and $z=10.5 \mathrm{~m})$ on the plisma axis. Sections through the anchor cell at $z=9.5 \mathrm{~m}$ and $z=10.5 \mathrm{~m}$ are given in Fig. 3-41, which shows these penetrations. The physical interference between the pentetrations and the coil cases at $z=10.5 \mathrm{~m}$ will require minor locis ? notching of the coil cases.

One feature not shown in Fig. 3-41 is the stiffening requireo tor the flat-plate portions of the inner surface of the vessel, which are shaped to accommodate the fanning of the plasma in the mouths of the coils. To suppori the applied pressure load, these portions of the inner wall will require stiffening ribs. In those areas where the flat plates are immediately adjacent to the coil cases and space limitations preclude the use of stiffening ribs, standoffs made of a thermal insulating material to reduce heat leak would be used to provide suppori from the coil itself. Note that the pressure aifferential across these plates acts in a direction to push them against the coils.

\section{Engineering Analysis.}

Piagnetic Fielo Uistribution. Magnetic fiela calculations have been periormed with the EFFI computer program. The geometry of the conouctor used for this purpose was slightly different than that describea in Table 3-11 in that the nominal winding cross section of $0.2 \times 0.4 \mathrm{~m}$ was used, rather than 


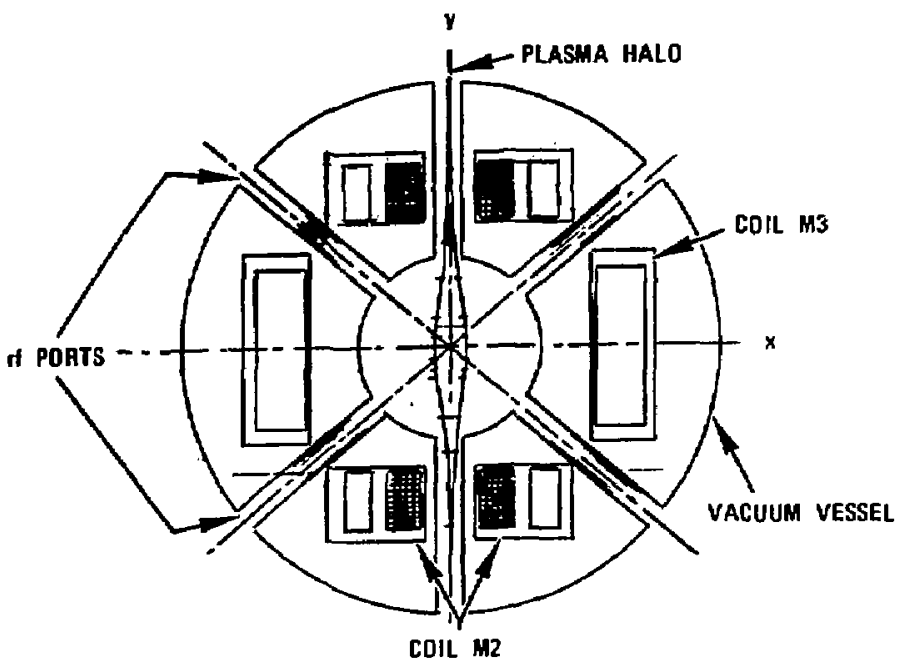

a) $z=9.5 \mathrm{~m}$

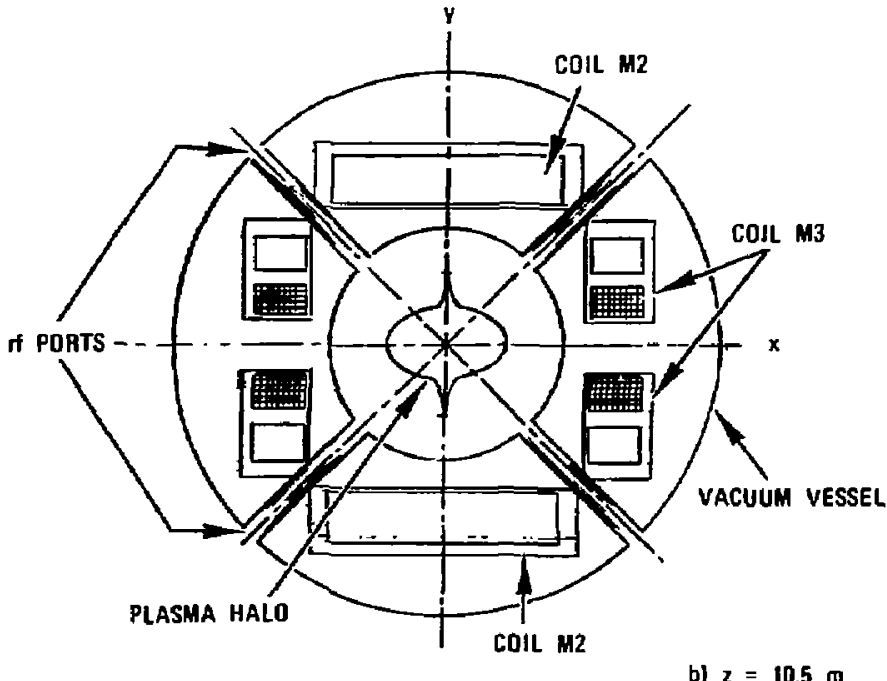

b) $z=10.5 \mathrm{~m}$

Figure 3-41. Anchor cel] sections at (a) $z=9.5 \mathrm{~m}$ and (b) $z=10.5 \mathrm{~m}$. 
the $0.204 \times 0.393 \mathrm{n}$ siated in Table $3-11$, with the correct center line configuration and with current density adjusted as necessary to ensure that the total MAT were correct. This was to permit magnet field and force calculations to proceea in parallel with conauctor choice and winaing layout. This oifference is very small and has a negligible effect on the results.

Figure 3-42 shows a plot of the magnet fieid on-axis for the anchor cell region. The desired field on-axis characteristics, i.e., a 3:1 mirror ratio and a 3-T peak mirror fielo are actually exceeded by the baseline magnet system; the mirror ratio is approxinately $3.7: 1$, and the peak mirror fiela is actually $3.45 \mathrm{~T}$. Therefore, $i \varepsilon$ is possible to reduce coil cost somewhat while still meeting the minimum field requirements. A potentially nore significant feature in the field distribution shown in Fig. 3-42 is the local field dip at the inboard yin-yang coil (near $z=8.5 \mathrm{~m}$ ). This aip does not improve performance, but is the result of an unnecessary feature of the configuration. It would be preferable if the field on-axis were to increase nonotonically from the transition minimum near $z=6.7 \mathrm{~m}$ to the first anchor peak near $8.7 \mathrm{~m}$. During the evolution of the baseline magnet systemis, an axial separation was intentionally introduced between coils $T-1$ and $M-2$ to allow space for coil structure. This separation was later proved to be larger than necessary to meet structural requirements; it also causes the local oip in fiela on-axis. The presence of the dip in field has been reviewed by TDF plasma physicists and found acceptable for performance.

When coil $\mathrm{T}-1$ is moved $20 \mathrm{~cm}$ closer to coil $\mathrm{M}-2--$ the amount of separation introduced unnecessarily--the dip can be eliminated. However, to avoid lengthy reanalys is of fields, forces, and coil structural requirements, the baseline was not reviseo to eliminate the dip as it would be in future TDF stuoies.

Fielo plots have also been generated at critical locations in the cross sections of the anchor cell coils to determine the peak field in the anchor cell magnets. The results of this evaluation are provided in rable 3-13.

Electromagnetic Analysis. Figure 3-43 presents a plot of operating current vs fielo for the conductor described in Section 3.2 .3 ("Selected Design Approach"). The values of operating clirrent were obtained from the experimentaliy aetermined critical current for this conductor by imposing an operating margin of 0.21 , where operating margin is defined to be equal to 1 minus operating current/critical current. 


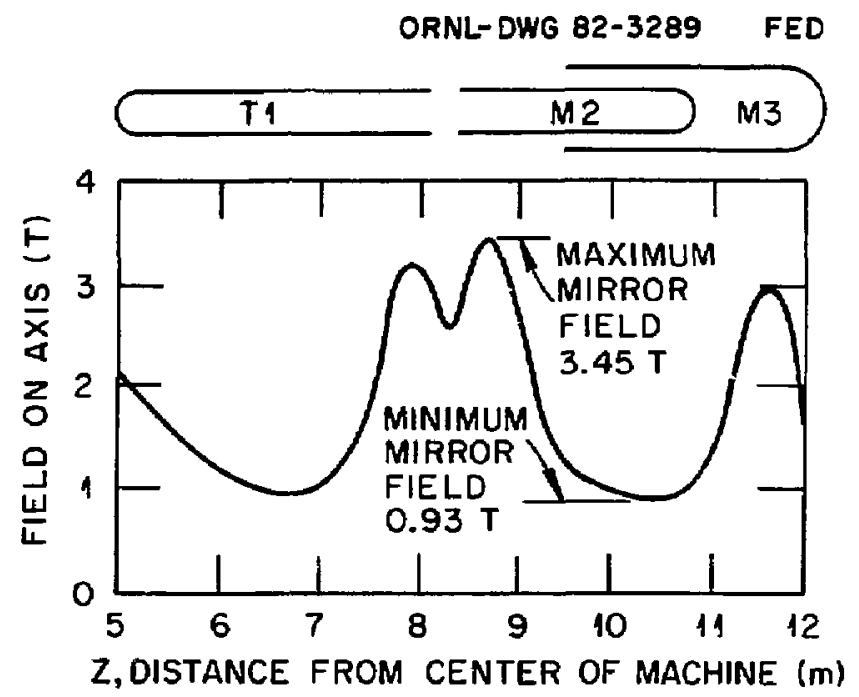

Figure 3-42. Anchor cell magnetic field on-axis. 
Table 3-13. Peak fielos in anchor cell cojls.

\begin{tabular}{lll}
\hline Coil & Peak field $(T)$ & Location \\
\hline$T-1$ & 4.3 & Center of ninor lobe \\
$M-2$ & 4.7 & Center of major lobe \\
$M-3$ & 4.7 & Center of minor lobe \\
\hline
\end{tabular}




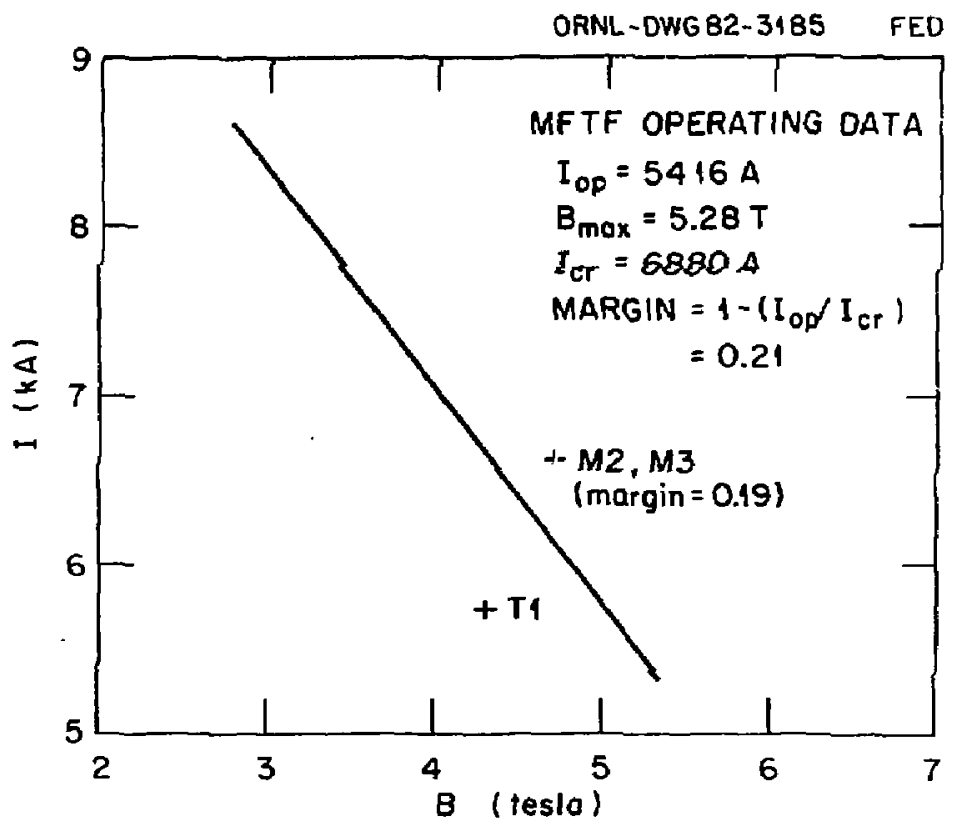

figure $3-43$, Operating current vs peak field. 
The margin of 0.21 was chosen because it exists in the MFTR-B application of this conductor. As seen in Fig. $3-43$, the conoition for coil T-1 is conservatively to the left of the curve. For coils $M-2$ and $M-3$, the operating point is slightly to the right (niargin of 0.19 , rather than 0.21 ), but it is acceptable for this conceptual design stuay.

A stability calculation performed for this conductor reflected an increase in resistivity caused by irraoiation. The stability margin wos found to be about $7 \%$, i.e., the critical heat flux for this conductor is $0.19 \mathrm{~W} / \mathrm{cm}^{2}$ and the heat flux required to dissipate the joule heating in a normal zone is $0.177 \mathrm{~W} / \mathrm{cri}^{2}$, neglecting heat flow away from the normal zone. No detailed dynamic stability calculations were performed. The $7 \%$ stability margin is inconsistent with the stability margin in previous applications of this conductor and is deemed to be unsatisfactory. For example, the final stability margin for this conductor in the MFTF-B "A" cell is $33 \%$. In future evolutions of TDF, design modifications (E.g., reduced current density) will have to be incorporated to ensure cryostability.

In an adiabatic quench analysis performed for the conductor, the dump resistance selected to limit the discharge voltage to $500 \mathrm{~V}$ resulted in a maximum hot-spot temperature rise of approximately $100 \mathrm{~K}$. It is judgea acceptable, however, on the basis that the resulting strain will only be $0.045 \%$, or considerably less than yield.

Coil Forces and Stresses. Electromagnetic forces on the coils were calculated using the EFFI computer code. The modeling of the conductor pack used in this calculation was the same as that usea to calculate fjejus (Section 3.2.3, "Engineering Analys is").

A summary of coil electromagnetic running loads is presented in Tables 3-14 and 3-15 for a one-quarter nodel of the coil set; running loads for the other three quadrants may be deduced by symmetry. A sketch of the quarter model is given in Fig. 3-44. Each coil lobe was divided into nine segments of equal arc length, or a $10 \mathrm{deg}$ angle for each segment, with forces given at the midpoint of each segment $(0=5,15, \ldots, 85$ deg relative to the beginning joint of each lobe). In-plane force is positive when directed radially citriard, and out-of-plane force is positive when it tends to cause lobe Etreacing. 
Table 3-14. Out-of-plane electromagnetic running loads on anchor set, $M N / m$.

\begin{tabular}{|c|c|c|c|c|c|c|}
\hline$\theta$, deg. & $\begin{array}{c}\text { Coil } \mathrm{M3} \\
\text { Major arc }\end{array}$ & $\begin{array}{cc}\text { Coil } & \text { M3 } \\
\text { Minor arc }\end{array}$ & $\begin{aligned} & \text { Coi1 } \text { M2 } \\
& \text { Minor arc }\end{aligned}$ & $\begin{array}{r}\text { Coil M2 } \\
\text { Major arc }\end{array}$ & $\begin{array}{c}\text { Coil Tl } \\
\text { Major are }\end{array}$ & $\begin{aligned} & \text { Coil } \text { Tl } \\
& \text { Minor are }\end{aligned}$ \\
\hline 5 & 2.4701 & 0,5920 & 1.0407 & 3.2085 & 2.9327 & 0.0561 \\
\hline 15 & 2.4770 & 0.6254 & 1.0252 & 2.7701 & 2.8208 & 0.4568 \\
\hline 25 & 2.4918 & 0,6918 & 0.9948 & 2.6420 & 2.6526 & 0.8724 \\
\hline 45 & 2.5568 & 0.8557 & 0.8950 & 2.7501 & 2.3582 & 1.6865 \\
\hline 55 & 2.6218 & 0.9322 & 0.8315 & 2.9115 & 2.2593 & 2.0441 \\
\hline 65 & 2.7333 & 0.9955 & 0.7657 & 3,1147 & 2.1774 & 2.3386 \\
\hline
\end{tabular}


Table 3-15. In-plane electromagnetic running loads on anchor set, MN/m.

\begin{tabular}{|c|c|c|c|c|c|c|}
\hline$\theta$, deg. & $\begin{array}{l}\text { Coil M3 } \\
\text { Major arc }\end{array}$ & $\begin{array}{l}\text { Coil MS } \\
\text { Minor are }\end{array}$ & $\begin{array}{l}\text { Coil M2 } \\
\text { Minor arc }\end{array}$ & $\begin{array}{l}\text { Coil M2 } \\
\text { Major arc }\end{array}$ & $\begin{array}{c}\text { Coil } \mathrm{Tl} \\
\text { Major arc }\end{array}$ & $\begin{array}{l}\text { Coil } \mathrm{Tl} \\
\text { Minor arc }\end{array}$ \\
\hline 5 & 0.9204 & 3.3528 & 4.1003 & 0.5495 & 1.2441 & 2.9112 \\
\hline 15 & 0.9171 & 3.6515 & 4.0817 & 0.7856 & 1.1480 & 3.1404 \\
\hline 25 & 0.9099 & 3.8649 & 4.0437 & 0.9000 & 1.0155 & 3.3073 \\
\hline 35 & 0.8976 & 4.0226 & 3.9846 & 0.9808 & 0.8977 & 3.4338 \\
\hline 45 & 0.8783 & 4.1399 & 3,8992 & 1.0587 & 0.8078 & 3.5313 \\
\hline 55 & 0.8474 & 4.2270 & 3.7841 & 1.1525 & 0.7366 & 3.6056 \\
\hline 65 & 0.7956 & 4.2884 & 3.6278 & 1.2722 & 0.6660 & 3.6599 \\
\hline 75 & 0.6990 & 4.3277 & 3.4159 & 1.4054 & 0.5699 & 3.6956 \\
\hline 85 & 0.4738 & 4.3470 & 3.1177 & 1.5016 & 0.3700 & 3.7134 \\
\hline
\end{tabular}




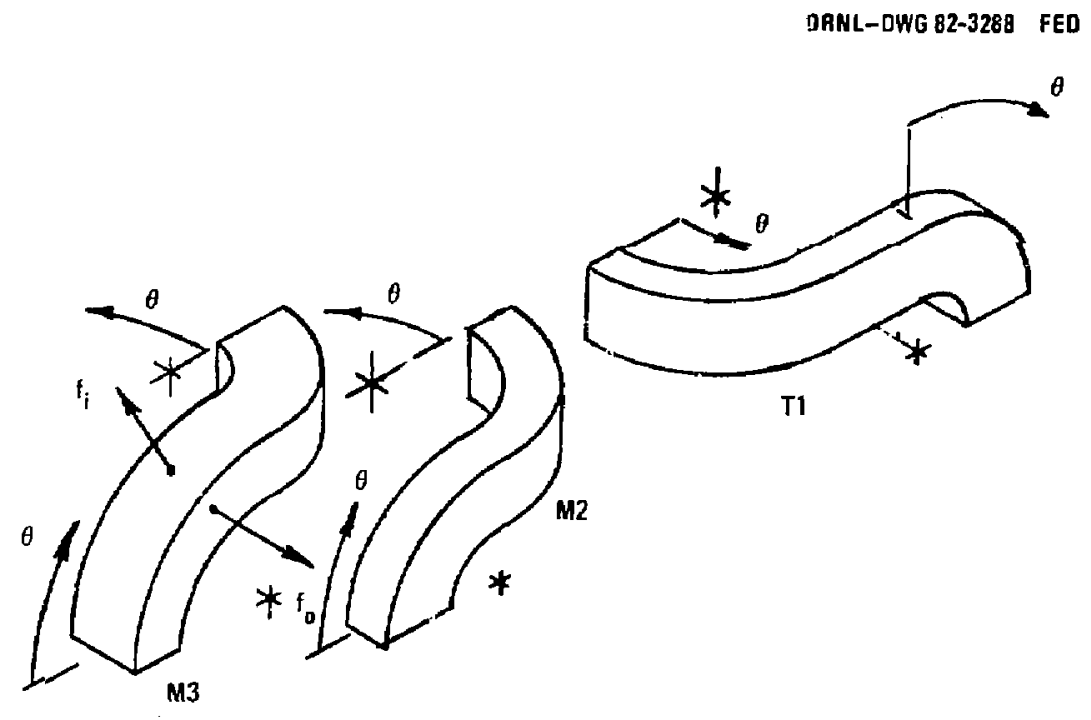

Figure 3-44. Notation for coil electromagnetic forces. 
A sumary of the resulting coil loads is given in Table 3-16. These loads on individual coils are caused by the coil dead weight, by the l-g lateral ano vertical seismic load, and by the resultant electromagnetic load. Because of the symmetry of the magnet configurations, integration of the running loads over the coil produces resultant load only in the axial direction.

Comparison with MFTF-B establishes the ability of indiviaual turns in the winding to transmit the electromagnetic running loads that accumulate from turn to turn ano layer to layer. The electromagnet ic running loads on individual turns are of about the same magnituoe on both TDF and MFTF-B (i.e., the product of conductor current times field is about the same); since the winding cavity dimensions (height and width) on TDF are about half as large as those on MFTF- $B$, the accumulated loads (and therefore the stresses due to this source of loading) are about half as large.

The sense of the in- and out-of-plane magnetic running loaos causes the winding pack to bear agajnst the two sides of the helium case with thicknesses tl and $t 2$, as shown in Fig, 3-32. The sidewalls are evaluated as being simply supported, with uniformly apploed load leading to the following design equation for the required thickness, in meters:

$t=(0.75 \mathrm{fL} / 207)^{1 / 2}$,

where $f$ is the running load in $M N / m$ and $L$ is the sidewall span in meters. The thicknesses $t 1$ and $t 2$ for this equation are determined with values for $f 1$ and $f 2$ (the maximum local running loads bearing on sidewalls $t$ l and $t 2$ ) selected fron Tables 3-14 and 3-15. The calculations are summarized in Table 3-17.

To shorten the fort involved in this study, the cases for coils $\mathrm{M}-2$ and M-3 have been made identical (see Table 3-12), with the thicknesses $t$ ) and t2 corresponoing to the coil M-2 dimensions in Table 3-17. Therefore, the structural limit of $207 \mathrm{MPa}$ will be slightly exceeded in wall tl of coil $\mathrm{M}-3$. kecal7, however, that the stress limit has an arbitrary factor of 2 to account for such simplifications.

As oiscussed in Section 3.2.3 ("Winding Support"), the coill case is designed to equilibrate lobe-opening forces in case bending. To accomplish this we sized by hand analysis, using beam theory. Force resultants (dxia) tension and bending moments about the two principal axes in the plane of the 
Tab le 3-16. Force summary--anchor set.

\begin{tabular}{|c|c|c|c|c|c|c|c|c|c|}
\hline \multirow[b]{2}{*}{ Force component } & \multicolumn{3}{|c|}{ Coil T1 } & \multicolumn{3}{|c|}{ SojJ ML } & \multicolumn{3}{|c|}{ Cois $\mathrm{M3}$} \\
\hline & $\begin{array}{l}\text { De ad } \\
\text { weight }\end{array}$ & Seismic & Maghetic & $\begin{array}{l}\text { Dead } \\
\text { weight }\end{array}$ & Seismic & Magnetic. & Wead & Seismic & Magnetic \\
\hline Axial (Z) & 0 & \pm 21.05 & -23.61 & 0 & \pm 17.74 & -49.49 & 0 & \pm 17.74 & -148.0 \\
\hline Horizontal (X) & 0 & \pm 21.05 & 0 & 0 & \pm 17.74 & 0 & 0 & \pm 17.74 & 0 \\
\hline Vertical (Y) & -21.05 & \pm 21.05 & 0 & -17.74 & \pm 17.74 & 0 & -17.74 & \pm 17.74 & 0 \\
\hline
\end{tabular}

A11 forces in $10^{3} \mathrm{~kg}$. 
Table 3-17 Helium case sizing for magnetic running roads.

\begin{tabular}{lllllll}
\hline Coi1 & f1 $(\mathrm{MN} / \mathrm{m})$ & L1 $(\mathrm{m})$ & t1 $(\mathrm{cm})$ & f2 $(\mathrm{MN} / \mathrm{m})$ & $\mathrm{L2}(\mathrm{m})$ & $\mathrm{t2}(\mathrm{cm})$ \\
\hline $\mathrm{T}-1$ & 3.7134 & 0.4186 & 7.508 & 2.6583 & 0.2292 & 4.701 \\
$\mathrm{M}-2$ & 4.1003 & 0.4186 & 7.889 & 1.5016 & 0.2292 & 3.533 \\
$M-3$ & 4.3470 & 0.4186 & 8.123 & 1.0638 & 0.2292 & 2.974 \\
\hline
\end{tabular}


cro:s section) were computed at 10-deg increments aiong the coil. The combineo normal stress was then calculated at the most highly stressed point in the cross section, using the equation

$\sigma=M x / Z x+M y / Z y+P / A$.

Here $M x, 2 x(M y, Z y)$ are the resultant moment and the section moduius with respect to an axis through the centroid of the coil case cross section, parailel to the long and short dimensions, respectively, of the winding pack, and $A$ is the area of the coil case cross section. Table 3-18 is a summary of combineo stresses at critical locations; it gives coil parameters for coils T-1 and $M-2$ at two critical locations. Detailed stress analys is was not done for coil M-3: Comparison of the loads ior coil Mi-2 to those for M-3 reveals that making the coil cases icentica! for the two coils is conservative, but not unauly so.

The coil weights are provided in Table 3-16. Stiffening rings built into the vacuum vessel suppurt the coils. The calculated axial locations of the coils' centers of yravity are reported in Table 3-19, which also incluaes the axial locations of the cold-mass support points. Using the information provioed in Tables 3-16 and 3-19, we computed support loads for static equilibrium of the anchor set, caiculated resultant forces and moments for the intercoil supports, and, through analysis, verified that the stresses within the intercoil supports are less than allowable.

As discussed previolisly, the coil cases are designed to equilibrate the large lobe-opening forces in benaing. Because this marner of winoing support is inherertily more flexible than the alternate support schemes considered, we were concerned that deflections might be too large, i.e., might cause unacceptable distortion of the magnetic field. Therefore, to obtain a rough order-of-magnitude estimate of the coil deflections, we performed a rudinentary deflection arialysis on coil $\mathrm{M}-2$, which has the largest lobe spreading forces. This calculation resulted in $0.64-\mathrm{cm}$ spreaoing of the wajor lobes, which is judgeo acceptable.

Vacuun Vessel Size. One requirement of the vacuurn vessel is that the plasma bore be sized and shaped to clear the plasma halo (the flux surface, a $17-c$ rin ravius cylinder in the centrai cell). Figures $3-36$ and 3-37 show the 
Table 3-18. Sumary of coil stresses.

\begin{tabular}{|c|c|c|c|c|}
\hline \multirow[b]{2}{*}{ Location } & \multicolumn{2}{|c|}{ Coil $\mathrm{Tl}$} & \multicolumn{2}{|c|}{ Coil M2 } \\
\hline & $\begin{array}{l}50^{\circ} \text { from center of major } \\
\text { lobe; location of max. } \\
\text { stress in basic section }\end{array}$ & $\begin{array}{l}\text { Junction of major lobe } \\
\text { with straight section; } \\
\text { location of largest } \\
\text { stress resultants }\end{array}$ & $\begin{array}{l}60^{\circ} \text { fron center of } \\
\text { major lobe; location } \\
\text { of max. stress in } \\
\text { basic section }\end{array}$ & $\begin{array}{l}\text { Center of minor lobe; } \\
\text { location of largest } \\
\text { stress resultants }\end{array}$ \\
\hline$M_{x}, N-m$ & 1.5441 & 4.0059 & 2.2321 & $6.6081^{\circ}$ \\
\hline $2 x, m^{3}$ & 0.01712 & 0.02584 & 0.01641 & 0.04095 \\
\hline$M_{y}, M N-m$ & 1.4603 & 0.9338 & 0.6745 & 0 \\
\hline$z_{y}, m^{3}$ & 0.01326 & 0.01549 & 0,01423 & -..- \\
\hline$F: \mathbb{M N}$ & 1.6711 & 1.2503 & 1.6373 & 7.2276 \\
\hline$A, m^{2}$ & 0.1217 & 0,1397 & 0.1159 & 0.1572 \\
\hline$\sigma, \mathrm{MPa}$ & 214.4 & 224.1 & 197.2 & 207.5 \\
\hline
\end{tabular}


Table 3-19. Center of gravity and support locations.

\begin{tabular}{lc}
\hline Component & Axial Location, * $\mathrm{m}$ \\
\hline Coil Tl & 6.99 \\
Coil M2 & 9.67 \\
Coil M3 & 10.71 \\
Rectangular yoke & 6. \\
Circular yoke & 10.15 \\
\hline
\end{tabular}

* Measured relative to the center of the center cell 
projection of the plasma halo on the two planes of symmetry of the anchor cell. Figure 3-36 illustrates the need for the slots in the sides of the vacuum vessel to accomodate the halo as it fans out in the mouths of coils $\mathrm{T}-\mathrm{l}$ and $\mathrm{M}-2$. Figures $3-36$ and $3-37$ also show that the halo clears the coils in these two projections.

The same two figures illustrate potential problem areas where it is unclear if there is physical interference between the halo and the vacuum vessel or even between the halo and the coils themselves. Considerable effort was expenced to ensure that interferences do not exist.

For example, consider the halo tan in the mouth of cuil $\mathrm{M}-2$. There is cleariy no probiem from $z=8.5$ to $9.1 \mathrm{~m}$; even in the $x-z$ plane (Fig. 3-37), the halo fan is thinner than the width of the slot and diminishes in thickness with distance $y$ from the $x-z$ plane. In the region $z>9.1 \mathrm{~m}$, however, as shown in Fig. 3-37, the width of the plasma halo in the $x-z$ plane exceeds the wioth of the slot, and it is possible that the walls of the slot may interfere with the halo. The point $z=9.75 \mathrm{~m}$ (the $B$ section in Figs. $3-37$ and 3-38) presents a potential problem because the wioth of the halo in the $x-z$ plane not only exceeds the widtl of the slot in the vacuum vessel at that point, but a]so exceeds the coil-case-to-coil-case clear space in the mouth of coil $\mathrm{M}-2$.

Figure 3-45 shows a section through the plasma near $z=9.75 \mathrm{~m}$; as can be seen, the halo is well away from the inside surface of the vacuum vessel. By drawing similar sections through a number of axial positions, we have demonstrated conclusively that the vacuum vessel described in this report satisfies the requirement to pass the plasma halo.

The wall thickness for the outer surface of the vacuum vessel was chosen in accordance with Section VIII of the ASME Boiler and Pressure Vessel Code. sizing in accordance with paragraph $\mathrm{UG}-28$ of the Code resulted in a requireo wall thickness of $0.478 \mathrm{~cm}$ for the hemispherical portions of the outer shell ario of $0.956 \mathrm{~cm}$ for the cylindrical portion. Time did not permit detailed design of the flat portions of the outer shel1, which will be stiffened $f$ lat plates. For titic purpose of estimating weight and cost, we assumed that these portions have an equivalent uniform thickness of $2.54 \mathrm{~cm}$.

Preliminary sizing of the support yokes was accomplished by designing each yoke to carry the full dead weight of the coil set as oiametrically opposed concentrated forces. Although this method of sizing is probably conservative, the yoke section dimensions that resulted are aeemed to be reasonable. 


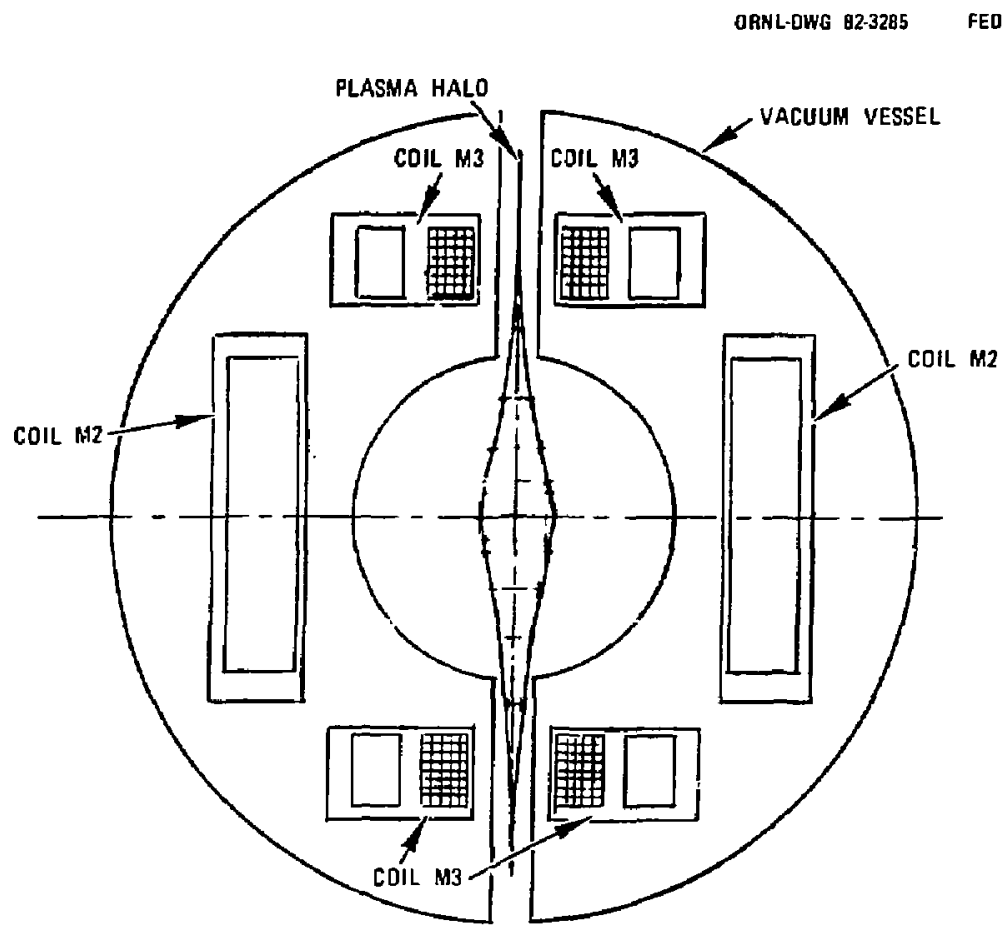

Figure 3-45. Section through anchor coil at $z=9.75 \mathrm{~m}$. 


\subsubsection{Internally Water-Cooled Resistive Coil}

A high-performance magnet that is internally cooled by water is an attractive alternative to a magnet that is externally cooled. Externally cooled magnets have problems with wet polymer insulation shrinking or swelling, embrittling, and extruding into the coolant passages. Also, copper whiskers can grow from one layer to another and create a short. ${ }^{6}$ Internally cooled coils have their own set of problems. Heat generation is proportional to the product of $J^{2}$ and $L$, where $J$ is current density and $L$ is length of coolant path. There is a limit to the amount of cooling poss:ble; therefore, there is a limit to the $J^{2} L$ product. Because internally cooled coils have longer coolant paths, they have a lower achievable current density. Another trait of internally cooled coils is the reduced wetted surface area (and coolant flow area), requiring larger water flow rates to improve hicat transfer. The net effect is to make pumping power more important than in an externally cooled coil. Higher flow rates are necessary, and the long coolant path must be broken down to little more than one coolant path per layer, at which point the coolant path is still longer than for externally cooled magnets. All this means that manifolding is more complex, copper operating temperatures are higher, and a pressurized system might be necessary.

The increased reliability of an internally cooled coil must be weighed against the penalties of more difficult thermodynamic cooling requirements. To quantify these requirements, a thermal analysis of an internally cooled coil concept was performed. The maximum current density vs hydraulic length and operating parameters was determined for the proposed alternative baseline TOF choke coil. By analyzing the results, one can select an optimum conductor and operating parameters for minimum operating power.

Theory. The internally cooled coil can be modeled as flow through a pipe with frictional losses and heat generation within the pipe wall. In the steady state heat is generated by electrical resistance, and neutron heat deposition is carried away by the water. The problem is described by the following energy balances:

$Q=$ N $(C p)\left(T_{\text {out }}-T_{\text {in }}\right)=h(P w) L\left(T_{\text {cond }}-T_{a}\right)=\operatorname{Qnt} \frac{J^{2}(A U C) \rho L}{\lambda}$, 
where

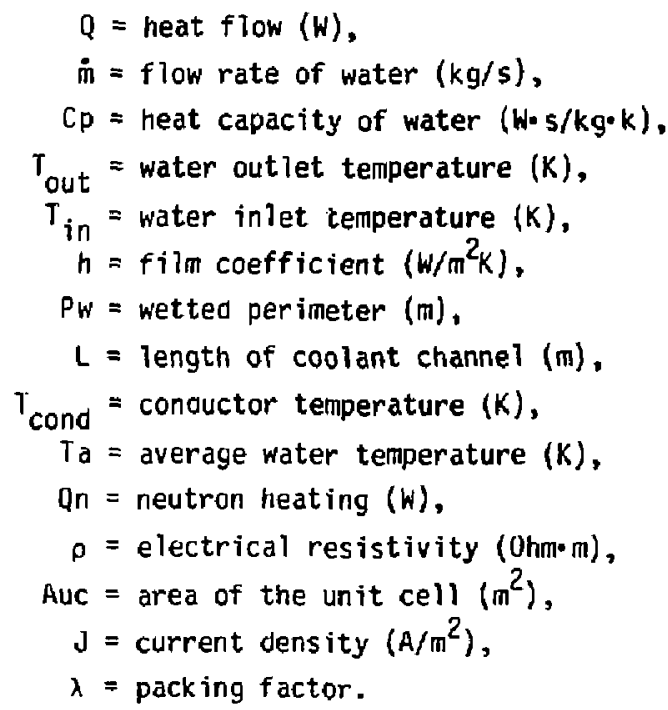

The figure of merit of a resistive coil is the total power it dissipates. This power is the sum of the electrical work needed to overcome resistance and the pump work required to cool the coil:

$W=\frac{J^{2} p \text { AuC } L}{\lambda}+w p$

$W p=\frac{m \Delta p}{\rho_{f}}$,

$\Delta p=f \frac{L}{U} \rho f \frac{v^{2}}{2}$

where

W'p = pump power (W),

$\Delta P \quad=$ pressure $\operatorname{arop}\left(\mathrm{N} / \mathrm{m}^{2}\right)$,

$o_{f} \quad=$ density of water $\left(\mathrm{kg} / \mathrm{m}^{3}\right)$,

$f \quad=$ friction factor

D = hydraulic diameter, $m$

$\checkmark \quad=$ velocity of water, $\mathrm{m} / \mathrm{s}$. 
The conouctor pack for TDF for a current density of $2800 \mathrm{~A} / \mathrm{cm}^{2}$ is shown in $F$ ig. 3-25. A metnod is being developed to determine the geometry of the optimum internally cooled conductor that will fit in a given conductor pack. The conouctor is shown in Fig. 3-25. The values for areas, packing factor, and length of different layers are also listed.

Various assumptions must be made to set values for several of the above variables. The inlet water tempercture is assumed to be coming from a cooling tower and estimated to be at $32.5^{\circ} \mathrm{C}\left(90^{\circ} \mathrm{F}\right)$.

The conductor temperature is assumed to vary linearly with length. Heat transfer is assumed to occur between this average conductor temperature and an average water temperature. The peak conductor teniperature was kept below $200{ }^{\circ} \mathrm{C}\left(392^{\circ} \mathrm{F}\right)$ to avoid recrystalization of the cold-worked capper and the subsequent loss of strength. Therefore, both a peak and an average conauctor temperature $\left(T_{C p}\right)$ were calculated. The peak conductor temperature is approximated by the following relationship:

$$
T_{\text {cono }}-T_{a}=T_{c p}-T_{\text {out }}
$$

Conductor resistivity is affected by temperature changes, transmutations, lattice deformations, and radial location. Total resistivity is calculated as follows:

$\rho=\rho$ temperature $+\rho$ transn!utations $+\rho$ lattice.

The temperature effect on resistivity is a function of the type of copper used and the amount of cold-working. The TDF internally cooled coil will use zirconium-copper, which has an IACS of 93\%. Through colo-working, the conductivity of zirconium-copper will degrade about $3 \%$, so a value of $90 \%$ is used in the equation that follows. (Pure, fully annealed copper has an IACS of $100 \%$ ). Note that the average conductor temperature is used to compute the temperature-dependent component of resistivity.

o temperature $=0.006818 \mathrm{~T}_{\text {cond }}+\frac{1.55}{0.90}\left(10^{-6} \Omega \cdot \mathrm{cm}\right)$ 
Transmutation effects for TOF were, except for changes caused by wall loaging and bore radius, assumed to be similar to those computed by the University of Wisconsin for the Mirror Advanced Reactor Study (MARS). The transmutation effects drop off as the location increases radially because of progressively thicker wall copper and water that acts as shielding. Figure 3-46 shows the change in resistivity per total resistivity as it changes with the radial location of the conductor.

The following are values for radial positioning corresponoing to the inner and outer layers, the miodle layer, and a layer between the inner and middle layers, in $\rho$ transmutations $\left(10^{6} 8 \cdot \mathrm{cm}\right)$.

- Inner layer: 1.1487.

- One-fourth: 0.4022 .

- Midde: 0.1081.

- Uuter: 0.0089 .

The increase in resistivity caused by lattice displacenents is $0.6 \times 10^{-6} \Omega \cdot \mathrm{cm}$, which is the saturation value of the change in resistivity vs dpa in the severe neutron radiation environment of TUF. This value has no radial or temperature dependence.

Neutron heating also varies in going from the inner to the outer layer. Fig. 3-47 shows the neutral heating rate as it changes with the radial location of the conouctor.

The following are vaiues for the inner, one-fourth, middle, ano outer layers in $\mathrm{W} / \mathrm{cm}^{3}$ :

- Inner: 10 ,

- une-fourth: 3,

- Middle: 2,

- Outer: 1.

The velocity of water was constrained to $12 \mathrm{~m} / \mathrm{s}$, based on an assessment of maximum practical velocities ano cavitation limits. Corrosion/erosion of a copper pipe flowing water at $12 \mathrm{~m} / \mathrm{s}$ continuously for one year is limicea to a depth of no more than $70 \times 10^{-6} \mathrm{~m}$. Further experimental work may allow this constraint to be relaxed.

Although aetai?ed configurations for the power buses are not available yet, the resistive heating calculation must include the bus length and cross section. The buses were taken into account by adding an effective length to the length of conductor. An arbitrary value of two meters was chosen. 


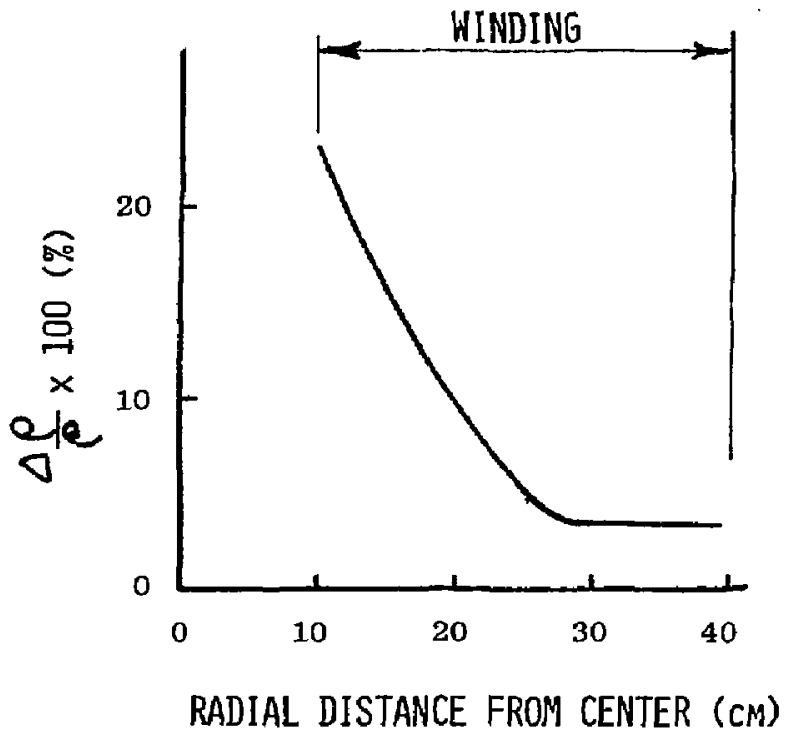

Figure 3-46. Resistance change as a function of radial location of conductor. 


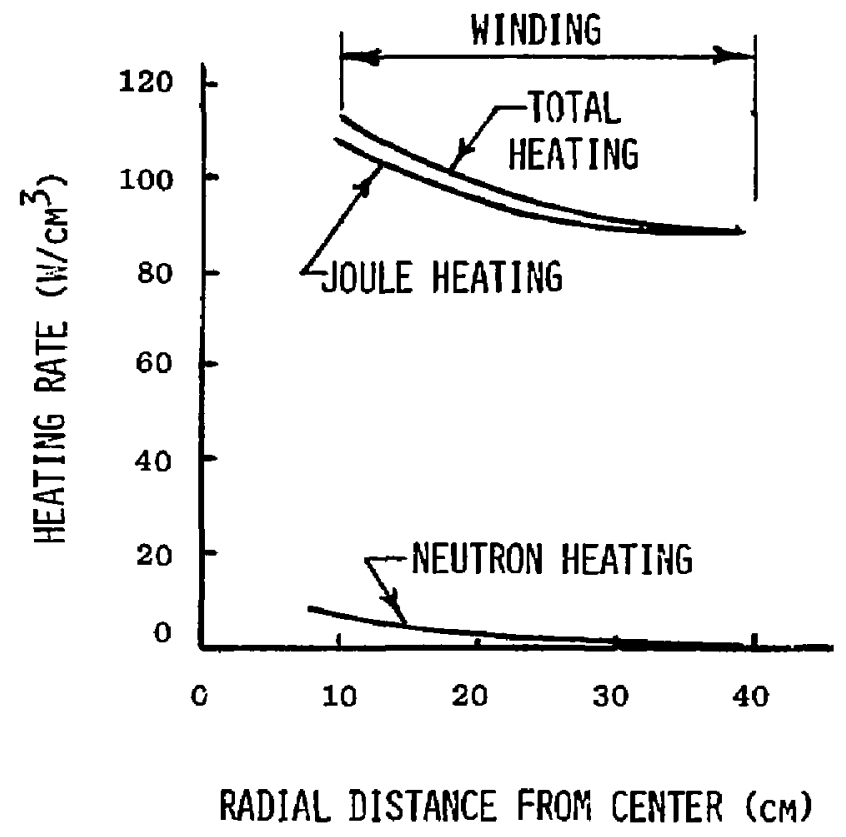

Figure $3-47$. Heating rate vs raoial location of conductor. 
To account for possible increased thermal resistance caused by the formation of a copper-oxide scale, a fouling coefficient was included in the computation of the film coefficient. The added resistance was assumed to be the result of conduction across a copper-oxide surface film. There is very little data on the thermal conouctivity of copper-oxide or on the thickness of oxide that may form. The thernal conductivity is assumed to be around 6 to $9 \mathrm{~W} / \mathrm{m}^{*} \mathrm{~K}$. Knowing the electroplating and other coating means can only achieve a thickness of one-half nil, we assumed that a worst-case thickness would be 0.5 mils. An equivalent filn coefficient $h_{\mathrm{cr}}$ was calculated.

$$
\begin{aligned}
h_{c r} & =\frac{6 \frac{\mathrm{w}}{\mathrm{m}^{*} \mathrm{~K}}}{0.0005 \text { in } \frac{\mathrm{l}}{12} \frac{\mathrm{ft}}{\mathrm{in}} \cdot \frac{1}{3.281} \frac{\mathrm{m}}{\mathrm{ft}}} \\
& =470 \times 10^{3} \frac{\mathrm{W}}{\mathrm{m}^{2} \cdot \mathrm{k}} .
\end{aligned}
$$

To understand how important this term is, we can compare it to the film coefficient for the coolant water, which is approximately $30 \times 10^{3} \mathrm{w} / \mathrm{m}^{2}+k$ at the TUF service conditions. So, the water film coefficient is $1 / 16$ of the fouling coefficient. The overall heat-transfer coefficient $U$ is as follows:

$$
u=\frac{1}{\frac{1}{h_{\text {water }}}+\frac{1}{h_{c r}}} .
$$

Typical values of $U$ are $30 \times 10^{3} \mathrm{~W} / \mathrm{m}^{2} \cdot \mathrm{K}$.

Results ano Discussion. Power includes joule heating and pump work. The inner layer operates at a power of less than $1 \mathrm{MW}$, and the outer layer operates at a power of $2 \mathrm{MW}$. The outer layer takes more power than the inner layer because of its greater length, which more than makes up for its lower resistivity. Total power for all layers is $31 \mathrm{Mw}$. The operating window is constrained from the right by the $12-\mathrm{m} / \mathrm{s}$ maximum velocity and from the left by the $200{ }^{\circ} \mathrm{C}$ peac conductor temperature. The reduction in the operating window is shown by the bands an each of the lines that reflect margins required to account for uncertainties in $f i l m$ coefficient, resistivity, current density, and mass flow rate. A $10 \%$ uncertainty in film coefficient is 
al lowed for by constraining the peak conductor temperature to anly $180^{\circ} \mathrm{C}$. A $10 \%$ uncertainty in resistivity ano $2 \%$ uncertainty in current density result in the inner and outer layer power curves shifting up $14 \%$. A 5\% uncertainty in flow requires constraining the velocity to $95 \%$ of $12 \mathrm{~m} / \mathrm{s}$.

Given the velocity and conductor temperature constraints and the bands of uncertainty in resistivity, current censity, velocity, and film coefficient, the TuF internally cooled coil is able tu operate at a current density of $2800 \mathrm{~A} / \mathrm{cm}^{2}$ (see Fig. 3-48). The fact that the inner layer has a wider operating window than the outer layer indicates that it could be optinized. Examining Eq. (3-10) of this section we can see that the $J_{2} L$ product can be higher ana still allow an adequate operating window, i.e., reasonable water outlet and conductor temperatures. Physically, this means that the inner layer can either share its cooiant with the second liyer (increase $L$ ) or it can be operated at higher current densities (increase J). Even though raising the current aensity will increase the power needed for the first layer, less total power will be needed because the magnet can be made smaller. An added benefit is the lower capital cost of a smaller mignet. On the other hand, if the coolant path of the inner layer is joined with she coolant path of the second and possibly even the third layer, less piping and pumps are needed.

If the curves for the inner ano outer layers are extrapolated out to lower and higher velocities, the total power can be seen to first decrease and then increase with increasing velocity, wit minimum power occuring over the TUF operating window of velocities between 8 to $12 \mathrm{~m} / \mathrm{s}$. The reason the curve has a minimum point is that pump work beconles a more important part of the total work at higher velocities. At lower velocities, the conauctor operates hotter and, therefore, has a higher resistive heating.

The operating conditions at the "sur corners" of the window are shown in Tabie 3-20.

Two values of interest are total resistivity "p" and the transmutation portion of resistivity " $p$ trans". The change in total resistivity from 3.4 to 2.3 (or 3.6 to 2.5) results fror the change in transmutation.

Another parameter of interest is the pressure arop, which ranges from 12 psi at the inner layer (for maximum conductor temperature) to $390 \mathrm{psi}$ at the outer layer (for maximum coolant velocity). As discussed previously, the inner layer would be optimized by increasing either current density or length, so the value of 12 psi would be changed. A pressurjzed system is required to al', w for pressure drops of 400 psi. 


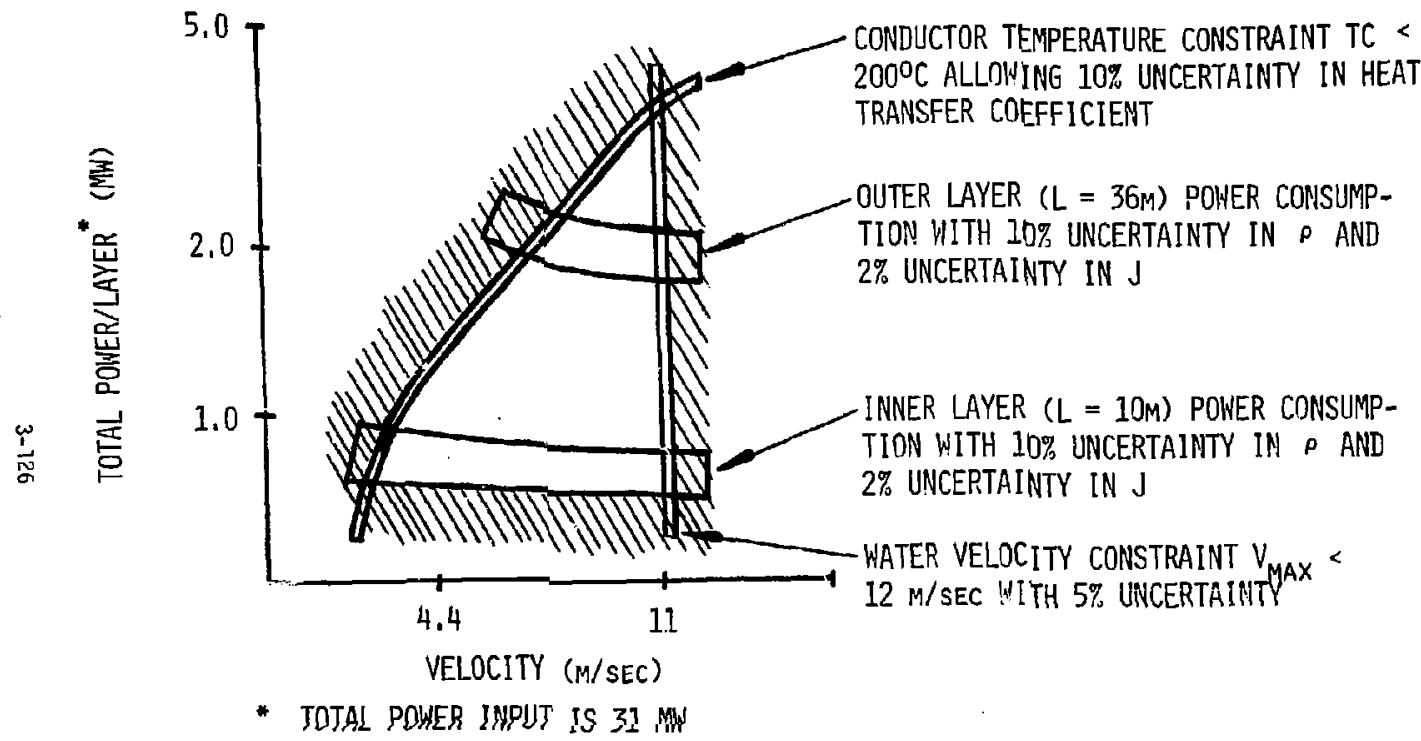

Figure 3-48. The internally-cooleo choke coil concept at $2800 \mathrm{~A} / \mathrm{cm}^{2}$ operated within design constraints. 
Table 3-20. Operating parameters at the inner and outer coil layers.

\begin{tabular}{|c|c|c|c|c|}
\hline Parameters & $\begin{array}{c}\text { Inner layer } \\
\text { maximum } \\
\text { velocity }\end{array}$ & $\begin{array}{l}\text { Inner layer } \\
\text { conductor } \\
\text { temperature } \\
\text { maximum }\end{array}$ & $\begin{array}{l}\text { Outer lajer } \\
\text { maximum } \\
\text { velocity }\end{array}$ & $\begin{array}{l}\text { Duter layer } \\
\text { conductor } \\
\text { temperature } \\
\text { maximum }\end{array}$ \\
\hline$L(m)$ & 10 & 10 & 36 & 36 \\
\hline$W(M H)$ & 0.7 & 0.8 & 1.8 & 1.9 \\
\hline$w_{p}(k H)$ & 4 & 0.1 & 13.7 & 2.6 \\
\hline$Q(\mathrm{MH})$ & 0.9 & 0.9 & 1.8 & 2.0 \\
\hline QN (NW) & 0.2 & 0.2 & 0.06 & 0.06 \\
\hline$V(m / s)$ & 12 & 3 & 12 & 6 \\
\hline$U\left(w / h \cdot c m^{2} \cdot k\right)$ & 3.8 & 2.2 & 4.4 & 3.5 \\
\hline$\varphi_{\mathrm{p}}(\mathrm{Pa} / \mathrm{ps} \mathrm{i})$ & $758,500 / 110$ & $82,700 / 12$ & $2,689,000 / 390$ & $896,000 / 130$ \\
\hline$\rho(\mu \cdot \Omega \cdot \mathrm{Cm})$ & 3.4 & 3.6 & 2.3 & 2.5 \\
\hline$\rho_{\text {trans }}(\mu \cdot \Omega \cdot \mathrm{cm})$ & 1.1 & 1.1 & 0.0089 & 0.0089 \\
\hline $\mathrm{T}_{\text {out }}\left({ }^{\mathrm{O}} \mathrm{C} /{ }^{\mathrm{O}} \mathrm{F}\right)$ & $75 / 167$ & $180 / 356$ & $120 / 248$ & $200 / 392$ \\
\hline${ }_{c}\left({ }^{0} \mathrm{C} / \gamma^{0} \mathrm{~F}\right)$ & $70 / 158$ & $135 / 275$ & $85 / 185$ & $125 / 257$ \\
\hline$T_{C p}\left({ }^{0} \mathrm{C} /{ }^{0} \mathrm{~F}\right)$ & $90 / 194$ & $210 / 410$ & $125 / 257$ & $210 / 410$ \\
\hline
\end{tabular}

Lastiy, the temperatures of the water outlet and the average and peak conductor are of interest. The hottest water outlet temperature is $200{ }^{\circ} \mathrm{C}$ at the outer layer for the peak conductor temperature of $210^{\circ} \mathrm{C}$. Actually, the peak conductor temperature would only be allowed to reach $200{ }^{\circ} \mathrm{C}$. So, the water outlet temperature would be less than that. To avoid boiling and vapor formation at the magnet outlet, a water temperature of $200^{\circ} \mathrm{C}$ requires an out let pressure of 230 pounds/square inch absolute (psia). Combined with the 390-psia drop in the system, TDF will require a peak operating pressure of 620 psia. 


\subsection{NEUTRAL BEAMS}

\subsubsection{0verview}

The neutral-beam injection systems (NBIS) perform several key functions necessary for the successful and efficient operation of TDF. The oiversity of the systems is indicated by the fact that eicn beam subsystem has a markedy different task. One set of beams (central cell NBIS) is needed to fuel, heat, and maintain the central cell plasma at a lever that ensures the desired neutron flux at the test mooules. Several beams are needed to "pump out" unwanted, trapped, low-energy ions both in the transition regicns a J anchor cells (transition-region and anchor-cell pump beams). These unwanted ions would otherwise modify the axial potential distribution in a way that would a] low increased axial particle and energy loss. Ion pumping is accomplished by choosing a neutral-beam injection angle and energy such that the incident neutral particle, after charge-exchange with the unwanted ion, can overcome potantial and magnetic mirror barriers and leave the system. A fourth set of beams is used to heat and maintain the proper trapped ion population in the anchor cells (anchor sloshing beans).

Because of the different tasks assigned to the central and end cell beams, the problems associated with their design are considerably oifferent. The central cell NBIS design is dominated by the high power requirements of $70 \mathrm{MiN}$ absorbed in the plasma. This means that primary attention had to be directed toward efficient beam generation and transport, both to increase the Q-value of the reactor and to reduce requirements on power-absorbing surfaces. In the design reported here, an injector efficiency (trapped power/source power) of $41 \%$ was achieved. The neutral beams are grouped in eight beamlines, each containing four sources. Four beamlines, two of which inject tritium and two deterium, are arranged in a semicircle at each end of the central cell. This geometry allows good interfacing with and the good servicing capability of the choke coils and the shine-through beam dumps.

In contrast, the end ce!l beam system design, particularly that of the pump beams, is dominated by the topology of the various end cell components, and the power requirements are rather modest. For example, the injection angle of the transition pump beam cannot be made arbitrarily small because of interference with the anchor cell coils. All end cell beam sources can only be positioned at sizable distances from the interaction point. This, together 
with the narrow gap allowable in the vacuun chamber surrounding the transition and anchor cell magnets, means that the beams have to be well focused and collimated to prevent excessive power deposition on the magnet vacuum wall surfaces.

While some beamline components are not expected to need servicing during the lifetime of the reactor, others, like the ion sources, as well as the ion and shine-through dumps, will need more frequent repairs or exchanges. Since most of the components will either be activated directly by neutron irradiation or contain dissolved tritium like the dump surfaces, much of the servicing will have to be performed remotely. To facilitate remote hanoling and servicing, the beamlines are modular. For example, a complete beamline can be removed for servicing or just a single source exchanged, since it will have the shortest lifetime of al] the reactor components.

Another aesign rationale is to minimize development cost. Thus, we use current technology, such as present-day parameters for ion-source current density ano beam divergence and maximum power dissipation for particle dump surfaces. Standard configurations like the magnetic separation of remnant ions and neutrals are also used even though such schemes might be more space-consuming than unproven concepts.

Some aspects must rely on, or could benefit from, advancements in neutral-beam technology. Consistent with constant wave (steady-state) operation of the reactor, it will be necessary to develop continuous beam ion sources. The primary source problem, component lifet ime, should be solved within the next two years, either with improvement of the Oak Ridge hollow cathode of with development of the TRW rf surces. Extraction grid cooling and mechanical stability must also be evaluated and improved to ensure proper curvature for bear focusing under continuous beam conditions. On the other hand, radiation hardening of the sources is not absolutely necessary, since carefully aesigned shielding can be installed between the plasma and the source insulators.

There are several areas of the system where further technological developmert is desirable but unnecessary. As indicated above, improved beam divergence would facilitate beam injection in the end cells and increase the beam fraction deposited in the central cell plasma. It would als's allow plasma-source distance to be increased, facilitating the magnetic shielding problem. Changing the species $m j x$ in the pump beams to decrease the 
number of molecular ions woula improve beam pumping efficjency. Finally, it would be desirable to improve the particle beam dumps, both to increase the allowable power density ano to find materials or combinations of materials to decrease the aiffusion of tritium into the cooling water.

\subsubsection{Central Cell Neutral-Beam Requirements}

The basic parameters are the desired neutron wall loading and the total available test module surface area. These requirements, i.e. neutron flux and plasnla radius and length, together with hot-ion confinement time and ion temperature, determine the neutral-beam voltage and the current that needs to be trapped to maintain the desired fusion reaction rates. Plasma density electron temperatures, as well as plasma and beam density profiles, determine the fraction of the incicent beam trapped by the plasma; that is, they determine the basic neutral-beamline parameters of a $64.9 \mathrm{MW}$ beam incident on the plasma. The neutral-beam injection angle represents a compromise between the ion lifetime and a geometrical factor determined by the magnetic-filled coil configuration.

Table 3-2l shows the central cell NBIS requirements for a $1.4-\mathrm{MH} / \mathrm{cm}$ neutron load at $R=0.25 \mathrm{~m}$ and a plasma of $8 \mathrm{~m}$ length, confined to a radius of 0.1 in by a 4,5-T magnetic field. The required beam voltage of $80 \mathrm{kV}$ is we 1 within the range of present-day technology and ensures a reasonably large charge-exchange cross section for the primary positive ions in the neutralizer cell. The large trapped beam currents mean that a sizable number of sources have to be arranged around the device without interference with other components.

A redundancy of at least $20 \%$ is required because ion source lifet ime is estimated, at most, to be of the order of the duration of an average blanket module test.

The environment in which the system must operate gives another set of requirements. The residual neutron flux will determine the insulator lifetime of the ion source. Even more important is the heat deposited by the neutrons in the approximately $200-\mathrm{m}^{2}$ cryopanels of all beamines because, for each watt that must be removed from the cryogen, 600 to $1000 \mathrm{~W}$ must be expenced in the cryoplant. 
Table 3-21. Central cell NBIS requirements and design capabilities.

\begin{tabular}{|c|c|c|}
\hline Item & Requirements & capabilities \\
\hline Incident power (MW) & 4.9 & 76 \\
\hline Beam voltage $(\mathrm{kV})$ & 80 & 80 \\
\hline Trapped current $(A)$ & 853 & 1212 \\
\hline Trapping fraction & 0.79 & 0.79 \\
\hline Injection angle (deg) & $\geqq 65$ & 65 \\
\hline Species & $50 \% 0 ; 50 \% \mathrm{~T}$ & $50 \%$ D; 50\% T \\
\hline Species mix $(x)$ & $80 / 15 / 5$ & $80 / 15 / 5$ \\
\hline Redundancy $(x)$ & $\begin{array}{l}>20 \\
\text { (One spare scurce per three operat } \\
\text { sources or one spare beamline per } \\
\text { three operating beamlines) }\end{array}$ & 33 \\
\hline Injection points & $1 / 2$ power at each end of $8-\mathrm{m}-$ long & plasma columr \\
\hline $\begin{array}{l}\text { Lifet ime of source insulators } \\
\text { due to neutron load (FPY) } \\
\text { l0-cm-wide }\end{array}$ & 0.5 & $\begin{array}{l}\text { I (polyimide) } \\
\text { I (Macor with } \\
\text { shield) }\end{array}$ \\
\hline $\begin{array}{l}\text { Cryopanel męutron heat } \\
\text { load (mW/cm })\end{array}$ & $<10$ & 1 \\
\hline $\begin{array}{l}\text { Maximum } B-f \text { ield at } \\
\text { source }(G)\end{array}$ & $\leqq 2$ & 1 \\
\hline $\begin{array}{l}\text { Maximum B-field at } \\
\text { neutralizer (G) }\end{array}$ & $\leqq 10$ & 10 \\
\hline
\end{tabular}


The specified limits for remnant magnetic fields at the ion source and neutralizer ensure that the ion beam, and therefore the neutral-beam aivergence, remains within specified limits.

\subsubsection{Central Ce1\} Beams}

Approach. The central cell NBIS requirements are well defined and can be met by using mostiy known technologies. Implementing them is not strajghtforward, however, partly because of cost and because component interface and serviceability have to be considered, but primarily because several components must be evaluated intensively since their intended use goes beyond known parameter ranges for fusion reactor concepts. The reasons $c$ an be traced to three new operational conditions: (1) high total incident beam power, (2) steady-state operation, and (3) the neutron flux and magnetic field environment.

Beam Duct. The requirement for a very high power injection and limited access area results in a beam-current density that is very high because of a partial overlap of adjacent beams. This, together with the necessarily long ducts, leads to a substantial beam loss in the ducts that must be made up with increased source current. Particles lost from the beam increase the pressure of the ambient background gas further, increasing beam loss; in principle, complete blockage of the bearm can occur. Because the plasma is a strong neutron source, the beam duct also constitutes a source of streaming neutrons; thus, an improper duct oesign can result in excessive neutron loading of critical components. The beamlines also compete directly with the adjacent fiela coils for critical space.

Beam Dumps. The high beam power density requires that special consideration be given to both the neutral-beam and ion-beam dumps, which will have to operate at a power dissipation rate close to the state of the art. Careful aesign is requirea to achieve reasonatle lifetimes in the presence of sputtering ano blistering. In addition, gas emanating from the dumps needs to be pumped efficiently to prevent beam losses resulting from increased background pressure both in the beam duct and in the beamline area. Finally, the shine-through neutral-beam dusips need to allow easy servicing while not interfering with field coils and blanket modules. 
Ion Source. Ion sources have reached a state of development that al lows them to geliver the required current densities for several seconds, so more development is needed for steady-state operation. In addition, the IDF plasma is an intense neutron source. Because the beam ducts provide an excellent path for neutron streaming, neutron oamage to key components, especially to the source insulators, has to be evaluated carefully.

Cryopanels. The tritium and deuterium gas emanating from the neutralizer and ion Gumips has to be pumped steadily by cryopaneis. Therefore, an operational node must be developed to al Tow routing outgassing. Another concern is neutrcn heat deposition in the cryopanels. To avoid this, the panels mist be iucdted so as mot to intercept streaming neutroms.

Magnetic Shielos. To 7 imit divergence growth of the neutral beam, the stray magnetic field at the ion source and neutralizer must be reduced to trie values indicated in Table 3-21. Two types of shields are possible, inactivg (magnetic material) and active (superconductor). Both types were evaluated in terms of weight, effectiveness, and engineering feasibility.

Central cell NBIS components that fulfill the requirements in Table 3-2, 1 can be arranged several ways. To show that the design reported here has the required parameters and is optimized, we discuss the rationale used to choose one particuiar design.

An axial and aximuthal cross section of the NBIS configuration is shown schematically in Fig. 3-49. The 8-m-long plasma is surrounded by solia material of consiơerab́le thicḱness, consisting or neutron snieias, test blankets, and magnetic-field-producing coils. Ducts are inserted through this structure to allow neutral beams to pass from the source into the plasma. The beam axis is inclined at 65 deg with respect to the plasnia axis. Because the plasma raoius is small compared to the source dimensions, it is obvious from Fig. $3-49$ a that, in the plane perpendicular to the plasma column, the neutrai beam should be focused geometricaliy onto the plasma column and should have a minimum source divergence in that direction.

In contrast, the optimum focusing conditions in the plane containing the plasma axis ( $F$ ig. 3-49b) is not self-evident, and any arrangement seems feasible from the view of the beam-klasma footprint. Un the other hand, the specific TDF environment of high neutron flux from the plasma column imposes substantial requirements on the neutron shieiding: 


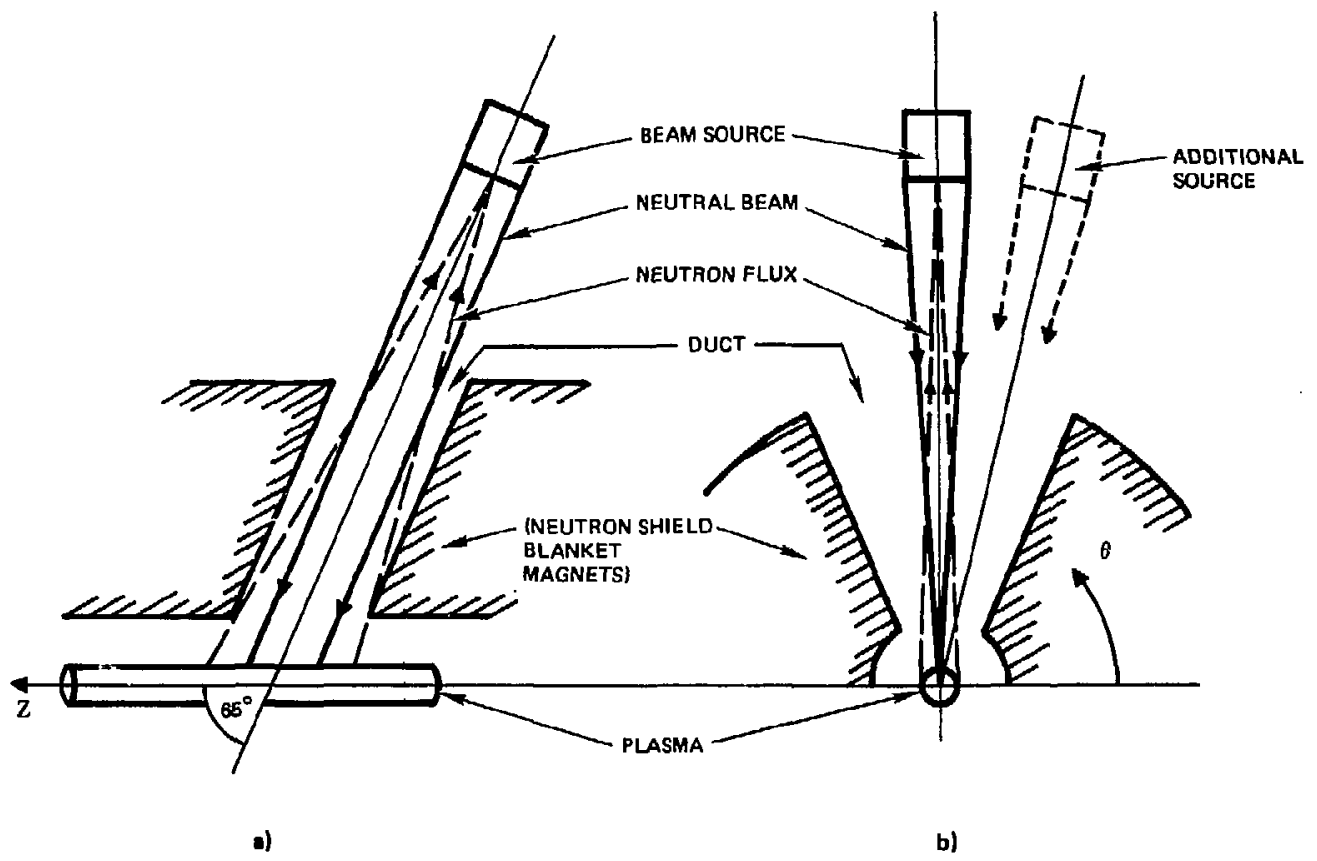

Figure 3-49. Neutron-streaming considerations. 
- The neutron flux on the ion source insulators shoula be low enough to ensure a reasonable insulator lifetime.

- The power deposited by neutrons in the cryopanels surrounding the beamline should remain at a tolerable level.

The most effective way. to limit the neutron loading of the source components is to restrict the auct width close to the plasma surface in 2 , i.e., parallel to the plasma axis, thereby minimizing the plasma seen by the neutron-sensitive components. This minimum width, on the other hand, is oetermined by the size of the neutral beam, which, in turn, is given by the beam divergence and the focusing in that plane. It follows, therefore, that the neutral beam should be focused onto the plasma and should have a minimum beam divergence in the $Z$-direction, as we 7 as in the $\theta$-direction.

Although a narrow beam dust seems optimal from the view of neutronics consicerations, other aspects influence duct aesign. The most prominent of these is the possibility of beam blockage. The gas density inside the ouct is given by the balance of pumping speed, which is determined by the auct geometry and external pressure, and by the gas entering the duct; this gas comes primarily from the walls and resuits from outgassing. This outgassing can be enhanced by wall heating from neutral-beam particles. The shine-through beam dumps are another gas source, where that fraction of the neutral beam not absorbed in the plasma is captured and converted into thermal particles. The neutral-beam particles jonized by the gas within the duct are removed along the magnetic field lines onto the duct wall. This beam loss must be made up by increasing the source current because the current incidient on the plasma should remain the same. This, of course, will increase capital and operating costs.

In addition to fractional beam loss, which will always be present to some degree, an improper duct design can lead to beam blockage. The particles lost from the beam by ionization will recombine at the auct surface, increase the gas density, and lead to a higher beam loss that, in turn, can cause gas density to increase exponentialiy in time until complete beam blockage occurs. This problem is of particular concern for TOF because of the requirement for constant wave operation and beccuse of the large beam currents and current aensities involveo.

To prevent beam blockage and ensure minimum beam loss in the auct, conductance to the cryopanels pumps located above the auct should be 
maximized. This can be accomplished in two ways: (1) In the plane containing the Z-axis, the source end of the duct should be widened while keeping the plasma end narrow to limit the neutron flux and (2) In the $\theta-p$ lane the di.t can be wide from the view of neutronics because the angle that determ: ies the neutron flux onto the source components is given by the plasma radius, not. by the duct width. It is also evident that a wide duct in $\theta$ allows the side-by-sice grouping of several indiviaual beams.

The result is that the beams should be focused onto the plasma and shoulu have minimum aivergence both parallel and perpendicular to the plasma axis. The beam duct should also be $y$-shaped in both planes, with a wide opening at the source eno, as inoicated in Fig. 3-50. The upper width of the ouct should be large enough for good puming speed, but narrow enough so that the cryopanels can, for neutronics reasons, be positioned outside the line of sight from the plasma.

Figure 3-50a shows the bear ducts between the superconducting $s-1$ cail and the normal conducting chake coil. Sufficient space has to be provided for the coolant manifold of the choke coil and the vacuum cryostat of the S-l coil. The vacuum cryostat must be provided with enough neutron shielding to keep the resistivity increase of the copper statilizer within prescribed limits. To provide sufficient space while preserving the plasma colum length at $8 \mathrm{~m}$, the three-coil neutral cell concept was adopted.

As detailed in Section 3.3.1, the total beam current required to be incident on the plasma could best be achieved by placing at each central-cell end four beanilines containing four sources each. This arrangement also meets requirements for at least $20 \%$ redundancy. As shown in Fig. 3-51, these beamlines were not placed symmetrically arounc the plasma, but were grouped in one-half plane for the following reasons:

- The average path length between the beam dumps and the bean duct opening is increased, decreasing the probability of gas entering the ducts from the dumps ana increasing the effectiveness of halo pumping.

- Since one-half plane is free of beami ine vacuum vessels, enough space is available for access to both the beam dump and choke coil. This way both can be moaular, as indicated for the beam dump in Fig. 3-50a. The accesses for the toam aump and choke coil modules are azimuthally offset from each other. 


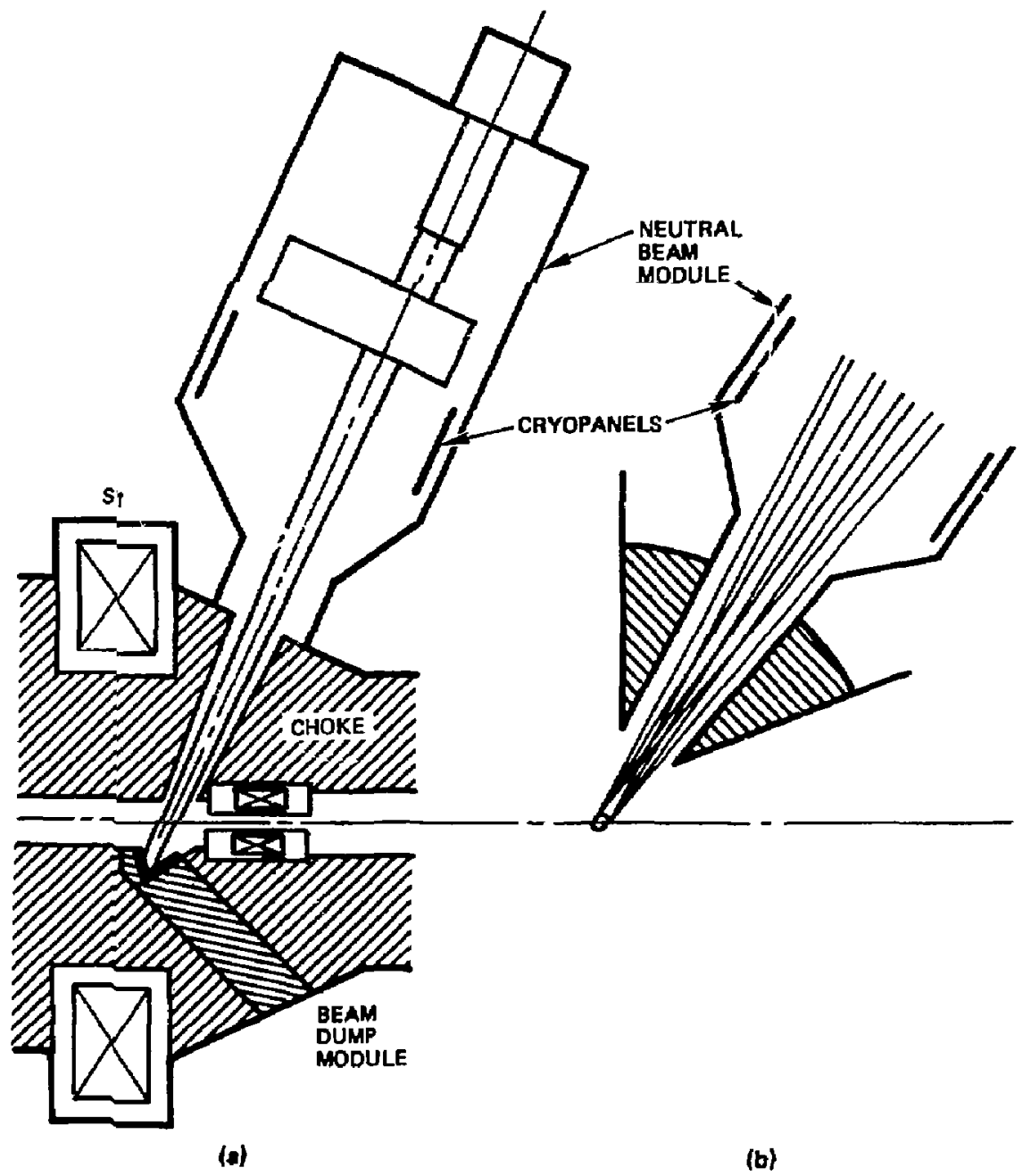

Figure 3-50. Central cell beam-duct geometry. 


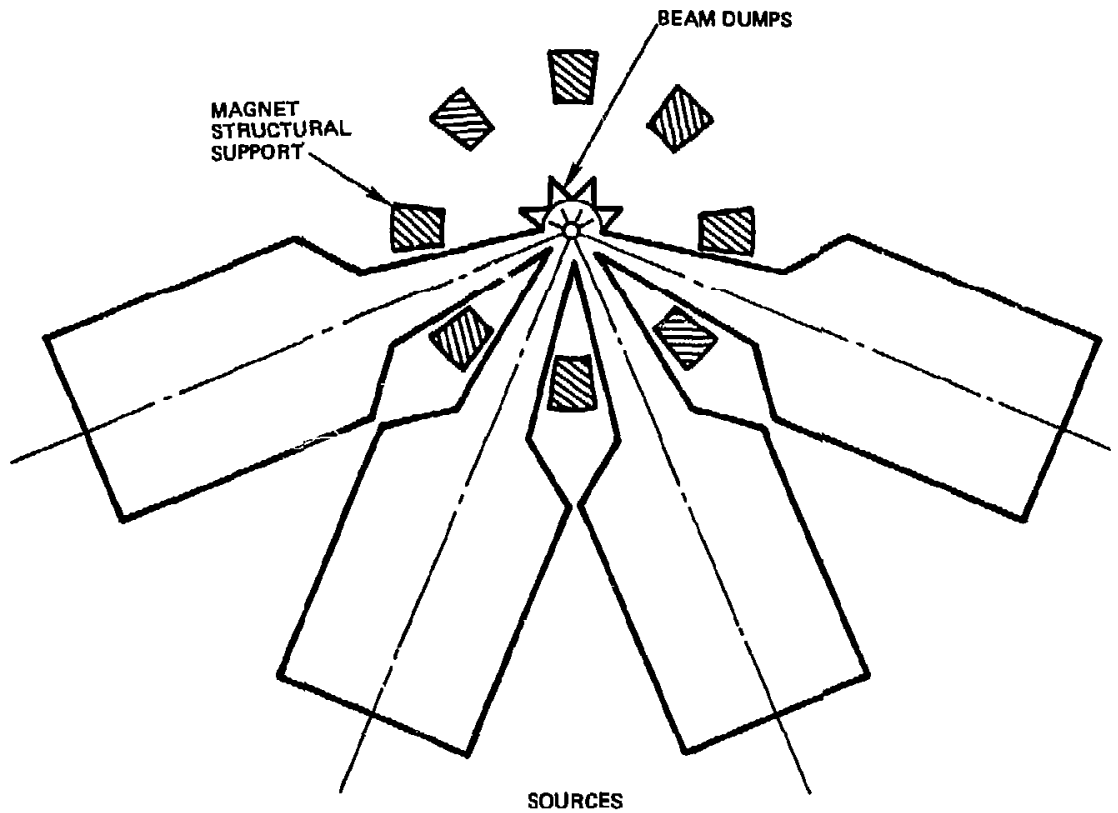

Figure 3-51. Central cell neutral-beam and beam-dump configuration. 
The grouping of components within one beamline is orawn schematically in Fig. 3-52. The concepts used are standard. A reflection magnet separates the mixture of neutral-beam particles and ions emanating from the neutralizer. This means of separation, compared to the straight line electrostatic separator with energy recovery, was chosen because it represents a well-aeveloped concept. A1so, the baffle, together with the reflection magnet, conveniently separates the vessel into high-; intermediate-, and low-pressure sections. The magnet and additional shield naterial serve as a neutron absorber, providing additional neutron protection for the rear cryopariels and the ion source components. Continuous cryopunping is effected by cyc]jicaljy autgassing the sryphane] sections (see Section 3.3.6).

The ion sources are expected to have a shorter lifetine than other TOF system curinonents; therefore, gate vaives between the neutralizer and sources allow eas.y source replacement. In contrast, no gate valves should be needed in the begm duct to separate the main system from the beamline components. Gate valves of the required size are cumbersome, bulky, and costly. Also, the lifetimes of beamline components, other than the ion sources, can be expected to be long enough that servicing and maintenance can be scheduled to coincide with TUF servicing or other repair work. The whole beamline assembly can be removed from the vacuum envelope, facilitating beamline servicing.

\section{Component Design}

Ion Source. Ane of the key paraffetars of the ThF dexice is the tota? neutral-beam power of $64.9 \mathrm{MW}$ incident on the central cell plasma. A total current of $853 \mathrm{~A}$ is specified to be trapped in the plasina, with beam energy set at $80 \mathrm{kV}$.

Assuming that the neutralizer efficiency, team losses, deposition efficiency, and plasma deposition efficiency are known, the total ion beam source current required can be calculated from the required trapped neutral-beam current.

$$
I_{s, \text { tot }}=\frac{I_{p, \text { tot }}}{(1-\ell)^{n} \sum_{k=1}^{3} k^{n}{ }_{P k}{ }_{N k} f_{k}} \text {. }
$$




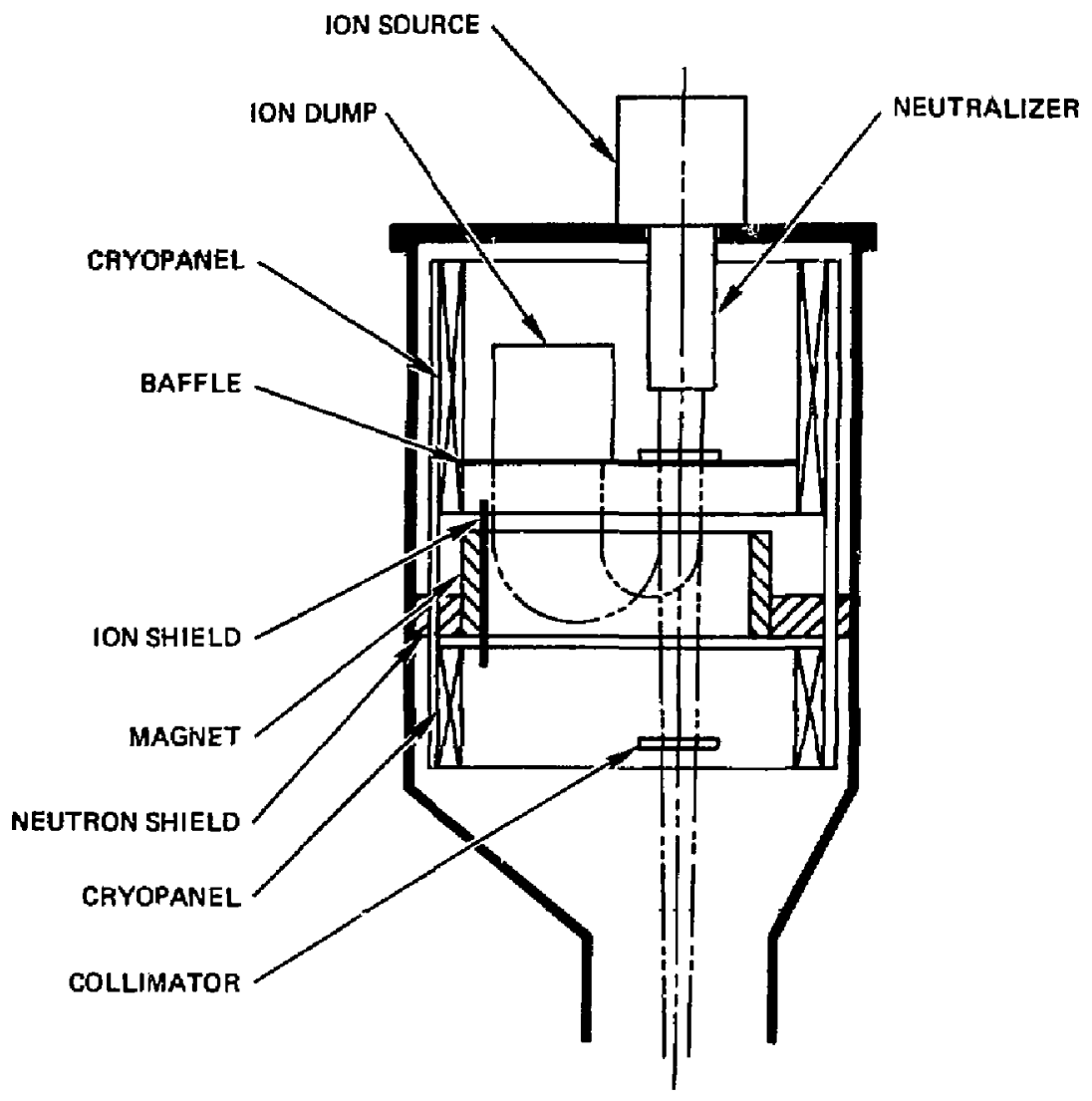

Figure 3-52. Arrangement of beamline components. 
The following notation is used:

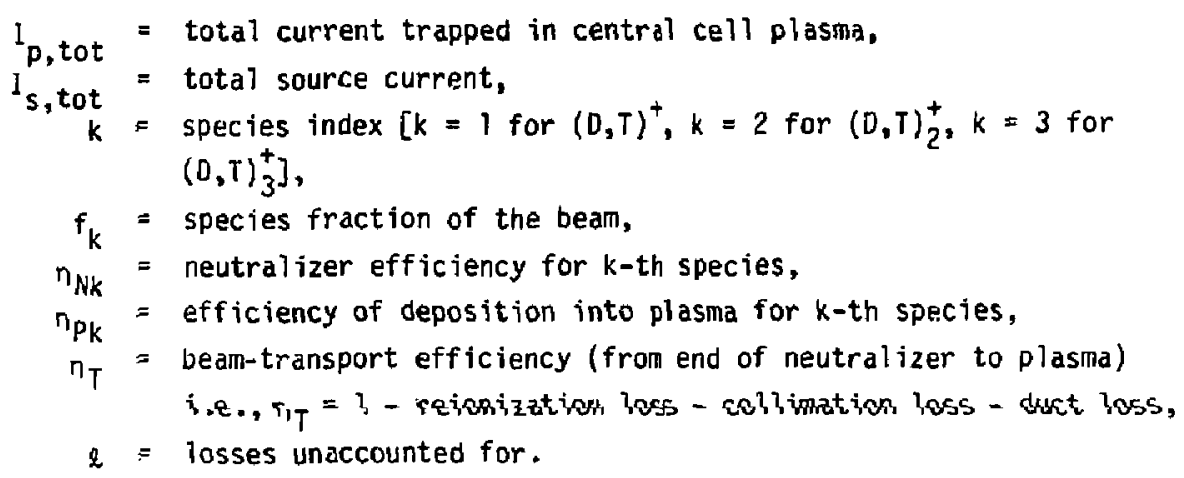

with

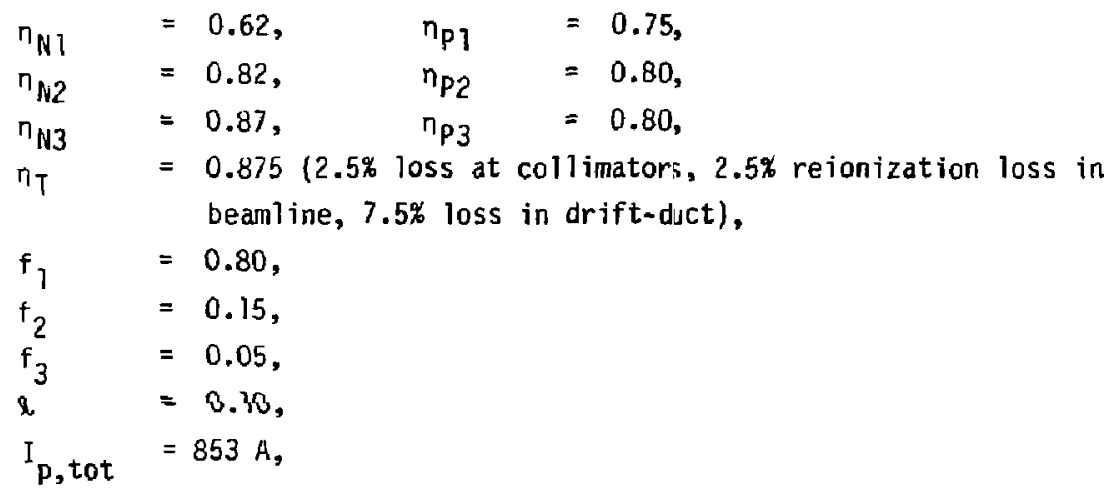

then the total ion source current required is $I_{s, \text { tot }}=1618 \mathrm{~A}$.

TDF ion sources have to operate in continuous mode. Since the long pulse ion source at Oak Ridge National Laboratory (ORN.) ${ }^{10,11}$ is presently closest. to this requirement, it is the chosen baseline source. This source produces an jon beam of up to $200 \mathrm{keV}, 100 \mathrm{~A}$ with 10 - to 30 -s pulse lengths. It has been determined ${ }^{12}$ that, by reducing the number of grids from four to three ana with minur design, such a source could be used in the $\mathrm{Cw}$ mode while extracting up to $100-A$ beams at $80 \mathrm{keV}$. For TDF use, the current for an individual source will be set conservatively at $I_{s}=70 \mathrm{~A}$. This means that 
24 sources are neeaed to satisfy the $I_{s, \text { tot }}=1618$ A requirement. Because of the expecteo short source lifetime, a source redundancy of at least 25\% is required to avoid blanket test interruptions by source fajlure. To provide redundancy and allow a convenient source grouping, the total number of sources was set at 32. For engineering, fabrication, and maintenance, it is oesirable to group several sources together and to combine all of their associated beamline components within one common vacuum envelope. These sources then share the same cryopanels, drift auct, reflecting magnet, shields, and other components or subsystems, thereby substantially easing construction and maintenance.

As shown in Fig. 3-53, four sources are sharing one beamline. Four of these beamlines are positioned at each end of central cell. This allows redundancy to be arranged in two ways: (1) one source in all beamlines is spare; (2) in three beamlines all four sources operate, while one complete beam! ine is unused.

As discussed in Section 3.3.3, the beams of each beamline have to de focused onto the same point of the plasma for optimum power deposition into the plasma and for neutronics reasons. Because this results in a beam overlap, specific considerations must be made for beam-duct blockage (Sectic. 3.3.4) and for shine-through dumps on the other side of the plasma (Section 3.3.5). The issue here is whether the divergence of the beams is low enough and whether the source grios can be properly shaped to ensure the required fccusing.

That the accelerator grid structure of the source greatly affects the optical quality of the ion beam grid design is well known. In one design, the grids are constructed with elongated apertures (slits). The advantage of this design is that the source can be easily focused by bending the grids independently in two directions: (1) parallel and (2) perpendicular to the grids. Such grid structures can usually be finely tuned during source testing. The difficulty, however, is that individual beamlets are somewhat defocused in the $Y$-direction, although the source as a whole is well focused. The defocusing, weak focusing, or divergence as it is sometimes called results from plasma meniscus at the extraction grids. This meniscus is rather shallow and has slow depth variation parallel to the slit, causing increased beamlet djvergence in that direction, as illustrated in Fig. 3-54. The beam 


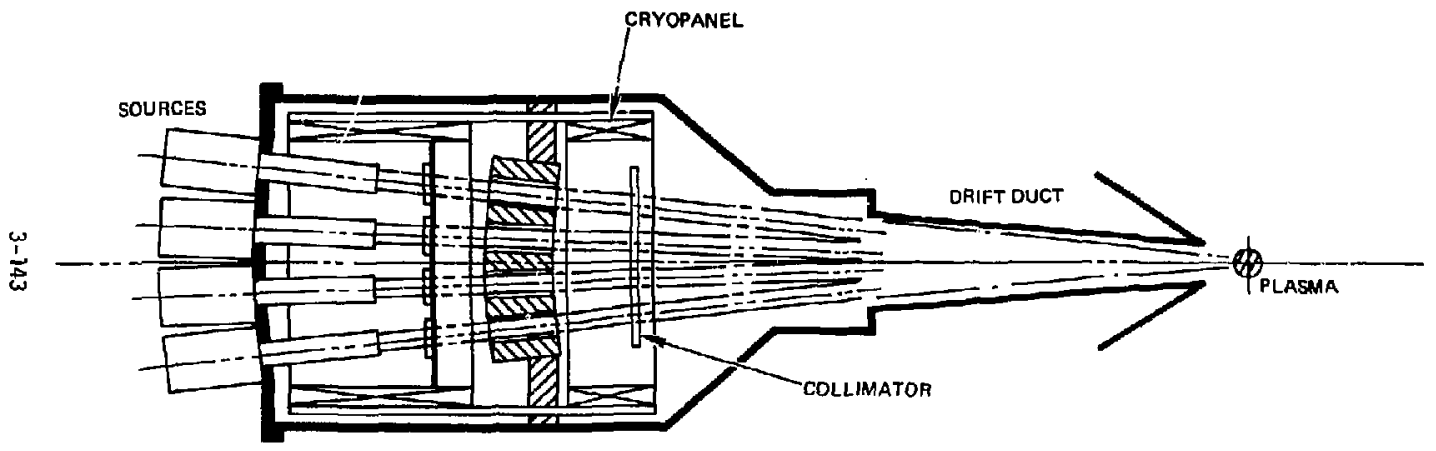

Figure 3-53. Beamline layout. 

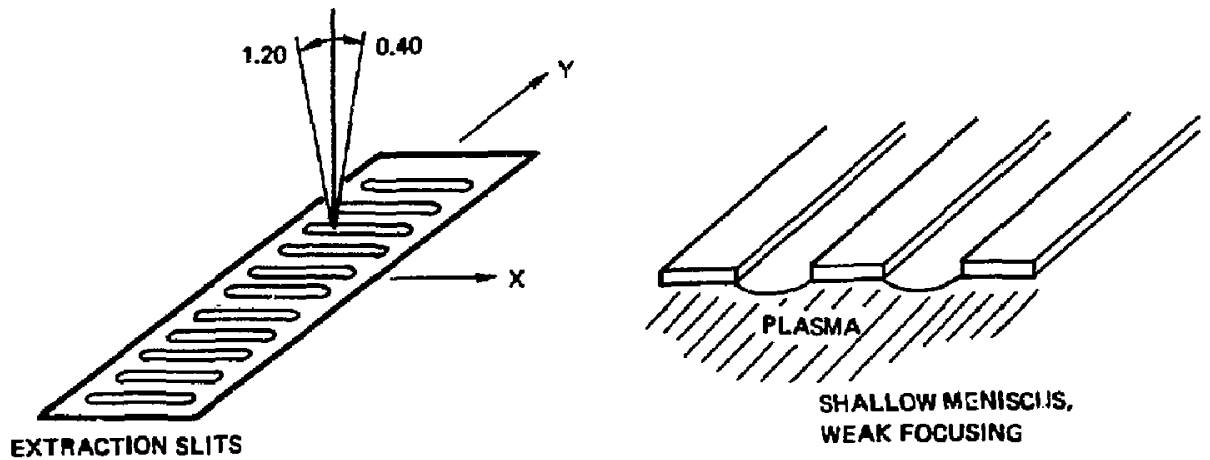

Figure 3-54. Extraction grid with sljts. Entries constitute present technological 1 imits. 
civergence in the $X$-direction is given by the thermal ion energy distribution of the source plasma. The second grid design uses circular apertures. Not only is the plasma meniscus deeper at the extraction grid, enabling better focusing, but the resulting beamlet divergence is isotropic (see Fig. 3-55).

The circular aperture grids, however, have disadvantages. The grid structure is somewhat rigid and therefore harder to aujust. These grids have lower optical transparency than grids with slits; consequently, their overall area has to be increased to accommodate the same current. In addition, water cooling channels are more difficult to construct.

For spatial and neutronics reasons (Section 3.3.7), the beam ouct has to be narrow in the direction of the plasma axis at the plasma end. This auct has poor optical transmission for a divergent beam, since a large fraction will be intercepted by the duct wa1?. In addition, the plasma radius is sma11. This means that a source with the sinallest possible divergence in both $X$ - and $Y$-direction is required. Therefore, a source with circular aperture grids was selected as a design standard. The divergence 1 imit was conservatively set at $0.5 \mathrm{deg}$, rather than $0.4 \mathrm{deg}$, the technological 1 imit. Assuming $f_{x}=950 \mathrm{~cm}$ source distance fram plasma and $v=0.5^{\circ}$ source divergence, the beam/plasma footprint will be $8.3 \times 8.3 \mathrm{~cm}$ (Fig. 3-56). The parameters of the TDF standard ion source are assembled in Table 3-22.

The ion source has the shortest lifetime of all beamline cconponents. It is thus necessary to redesign the ORNL source to facilitate easy access and replacement without impairing the vacuum integrity of NBIS. For this purpose, an isolation valve will be installea at the interface of each source with the back wall of the NBIS vacuum vessel. Such an arrangement will enable independent servicing of each source.

When new or overhauled sources are installed on a NBIS unit, fine adjustments will have to be made to direct the beam precisely at the plasina column. The entire source will not have to be moved, as is of ten done; rather, the orientation of the accelerator grid will be adjusted. The Oak Rigge source has such an arrangement, where the acceleration grid structure is suspended in a gimba? that is controlled remotely by stepping motors (not shown). During the adjustment cycle, the beam is fired from the source in short puises and its profile/position is monitored on shine-through beam durnps using an infra red camera. This method may be also used to check the quality 

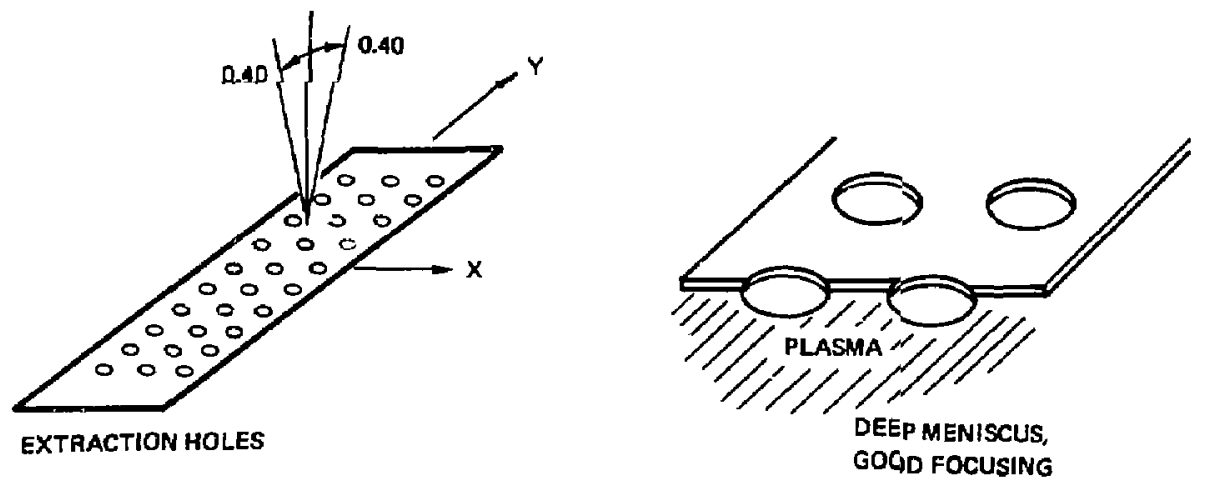

Figure 3-55. Extraction grid with circular apertires. Entries constitute present technological limits. 

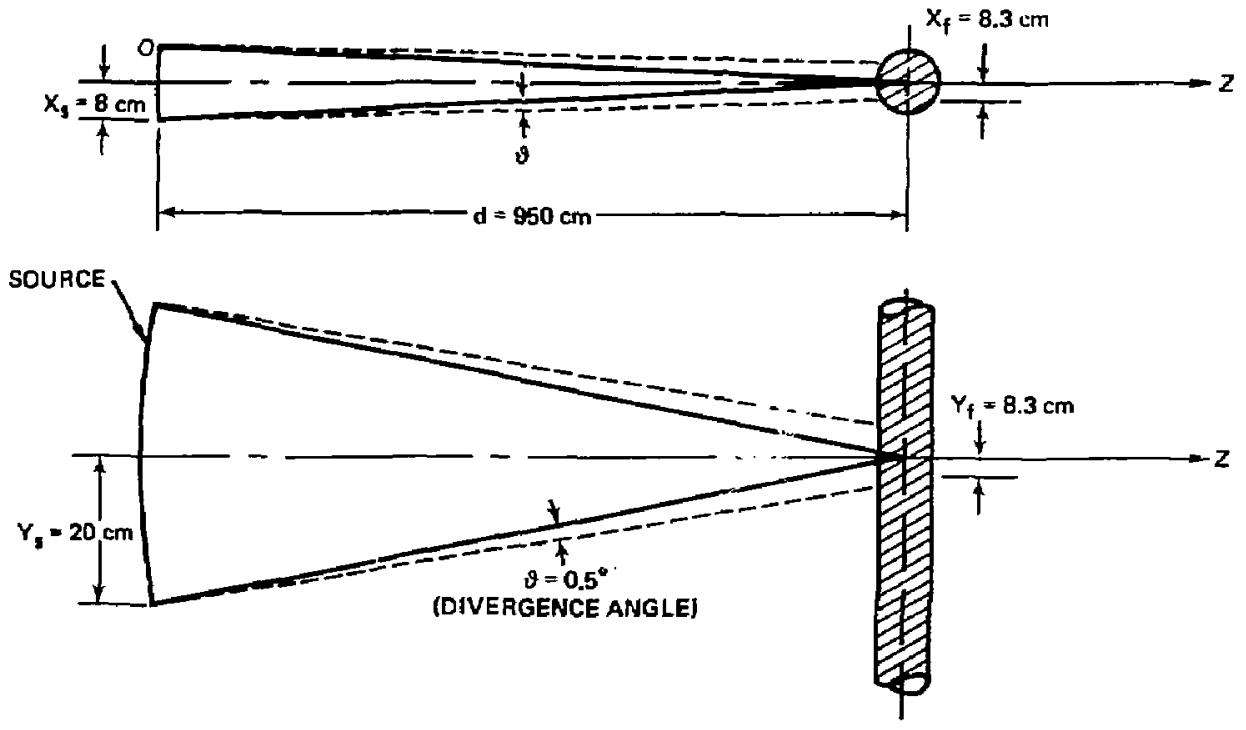

rigure 3-56. Bearn focusing. 
Table 3-22. TDF standard ion source.

\begin{tabular}{|c|c|c|}
\hline Parameter & Symbol & Value \\
\hline Extractea current $\left(A^{+}\right)$ & $\stackrel{+}{i_{s}}$ & 70 \\
\hline Particle energy (keV) & Eo & 80 \\
\hline Species mix, $0^{+} / 0_{2}^{+} / 0_{3}^{+}(x)$ & -- & $80 / 15 / 5$ \\
\hline Power delivered (MW) & $P_{S}$ & 5.6 \\
\hline Number of grids & -- & 3 \\
\hline Grid size $(\mathrm{cm})$ & $2 x_{0} \times 2 y_{0}$ & $40 \times 16$ \\
\hline Apperture diameter (cm) & $a_{a}$ & 0.2 \\
\hline Beam divergence (aeg) & $\theta$ & 0.5 \\
\hline Focal length $(\mathrm{cm})$ & $f_{x}, f_{y}$ & 950 \\
\hline Pressure (Torr) & $P_{S}^{n}$ & $5 \times 10^{-3}$ \\
\hline Grid transparency $(8)$ & $\mathrm{t}_{g}$ & 45 \\
\hline Current aensity $\left(\mathrm{Acm}^{-2}\right)$ & $j_{5}$ & 0.25 \\
\hline
\end{tabular}


of source focusing. While focusing is usually nonadjustable in situ, some aajustment is possible by shiming the grid structure at assembly time.

Ion sources contajn a number of electrical insulation components, usually made of ceramics, Kapton, Mylar, Polyimide, or epoxy and vacuum seals using elastomer 0-rings. All of these components will be exposed, to some degree, to radiation effects that might jegrade them and render them ineffective. Thus they must be made of materials that can survive the radiation environmer:. Special shielding around the interface flange and within the source itself will be used to reduce the requirements to reasonable levels.

Neutralizer. The ion bean emerging fron the acceleration grid structure of the ion source is transformed into a neutral beam by charge-exchange collisions of ions with neutral gas atoms. To keep this gas target density high ano the gas load onto the beamline cryopanels low, the gas is contained in a low-conductance rectangular duct tightly surrounding the ion bearn. The length of this neutralizer (Fig. 3-57) is chosen to privide an optimum target thickness $\pi$.

A typical ion source has a gas efficiency of 50\%. The unused gas streams along with the ion beam through the grid structure into the neutralizer, where it becomes part of the gas target. Depending on neutraljzer ouct conductance ario the required target density, additional gas might have to be introduced into the neutralizer. Since the neutralizer is made as narrow as possibie and since the ion beam has a finite aivergence, a certain fraction of the beam will be intercepted by the walls. Water cooling, therefore, is required.

The neutralizer length is optimized for the major consituent of the bean $\mathrm{U}^{+}(80 \mathrm{keV})$ and $\mathrm{T}^{+}(80 \mathrm{keV})$. The neutralizer friciency for this species can be obtained from:

$B_{N 1}=\frac{D^{o}(L)}{D^{+}(0)}=\frac{\sigma_{10}}{\sigma_{10}+\sigma_{01}}\left[1-\exp \left(-\left(\sigma_{10}+\sigma_{01}\right) \int_{\sigma}^{L} n(z) d z\right)\right]$,

where $D^{+}(0)$ is the count of ions entering the ouct, $D^{\circ}(L)$ is the count of neutra]s leaving the duct, $\sigma_{10}=2.0 \times 10^{-16} \mathrm{~cm}^{2}$ and $\sigma_{01} \approx 1.4 \times 10^{-16} \mathrm{~cm}^{2}$ are the neutralization and ionization cross sections, respectively, and $n(z)$ is 


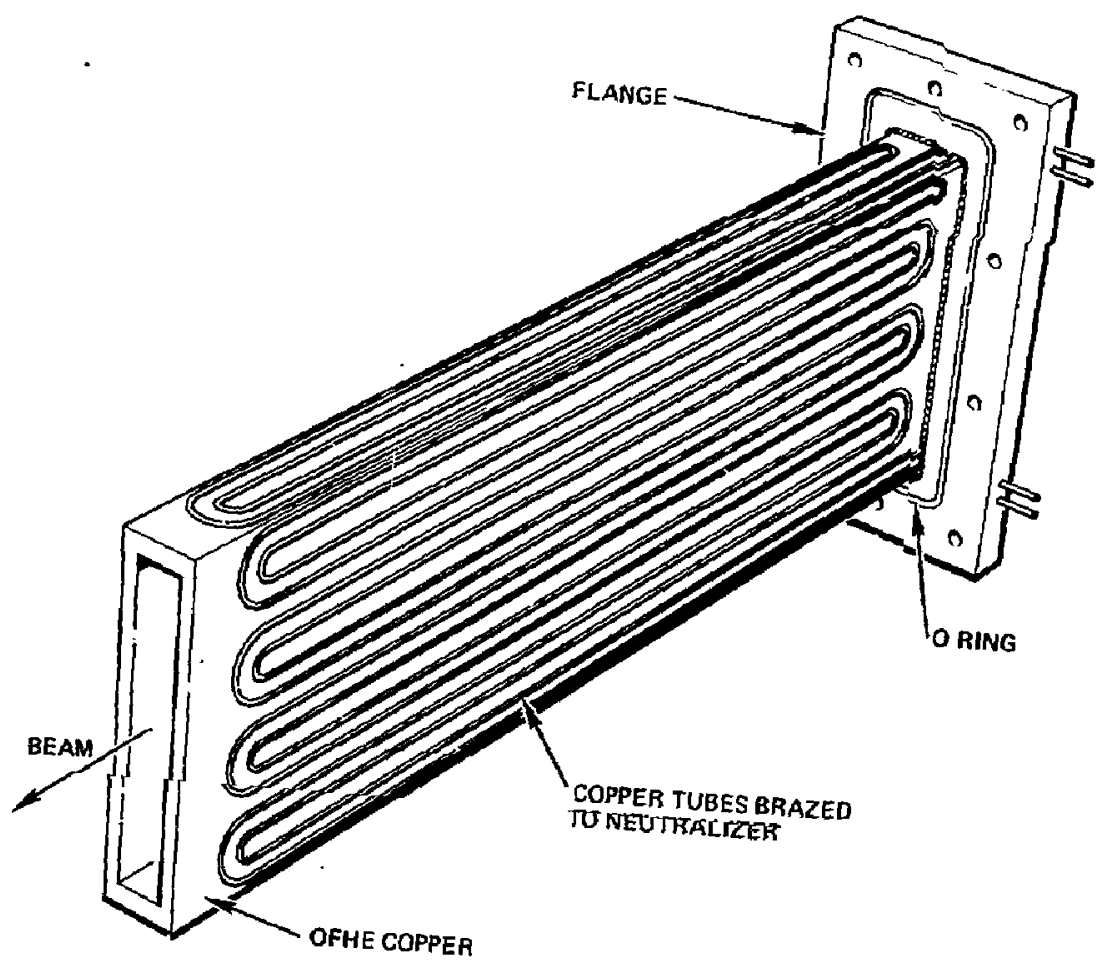

Figure 3-57. Baseline neutralizer tube. 
the gas density distribution along the duct. Duct length is determined by requiring a high percentage (here $95 \%$ ) of equilibrium neutralization under a law excess gas condition. For a duct of nearly uniform cross section, Eq. $(3-16)$ becomes

$0.95=1-\operatorname{xp}\left(-\left(\sigma_{10}+\sigma_{01}\right) \frac{\text { nentrance }_{\text {exit }}-n_{\text {exit }}}{2}\right)$.

The target derisity is defined as

$\pi=\frac{n_{\text {entrance }}-n_{\text {exit }}}{2} L$.

Thus, Eq. (3-17) yielas

$\pi=-\frac{\ln (0.05 \mathrm{n})}{\sigma_{10}+\sigma_{01}}=8.81 \times 10^{15} \mathrm{~cm}^{2}$.

From the cryopanel pumping speed and the volume and conductances outside the neutralizer, one can estimate the pressure at the neutralizer exit to be $P_{\text {exit }}=10^{-4}$ Torr. The entrance pressure, $P_{\text {entrance, }}$ can be obtained from the following considerations. The gas pressure in the source is $P_{S} \simeq$ $5 \times 10^{-3}$ Torr so gas will flow into the neutralizer. However, the acceleration grid system constitutes an impedance to the flow. Assume a grid aperture diameter $d_{a}=2 \mathrm{~mm}$. The sequence of apertures on each grid wiil appear to gas molecules as a tube whose total length is equal to the total gria thickness plus total grid spacing, here about $1.26 \mathrm{~cm}$. Thus the conductance of a single aperture is

$C_{a}=3.81 \sqrt{\frac{T}{M}} \frac{u^{3}}{l_{a}+.17 D}=0.237 \ell / s$,

where $T$ was taken to be $400 \mathrm{~K}$. But, a grid of dimensions $2 x_{0}=14$, $2 y_{0}=40$ and transparency $T_{G}=0.45$ has a total of

$16 \times \frac{0.45 y_{0} x_{0}}{\pi a_{a}^{2}}=8027$ apertures.

which yields a total grid conductance of $C_{G}=1900 \mathrm{\ell} / \mathrm{s}$. 
Denoting a flow rate by $Q$, the $C_{G}$ relates to pressure as

$C_{G}=\frac{Q}{P_{S}-P_{\text {entrance }}}$.

On the other hano, the duct conductance is given as

$C_{N}=\frac{Q}{P_{\text {entrance }}-P_{\text {exit }}}$.

So,

Pentrance $=\frac{C_{G} P_{S}+C_{N} P_{\text {exit }}}{C_{N}+C_{G}}$.

If we assume a pressure in Torr and conductance in $\mathrm{cm}^{3} \mathrm{~s}^{-1}$, a substitution to (Eq. 3-18) yielos

$\frac{2 \pi}{L}=\left(\frac{C_{G} P_{S}+C_{N} P_{\text {exit }}}{C_{N}+C_{G}}-P_{\text {exit }}\right) \times 3.53 \times 10^{16}$,

since, for a rectangular duct,

$C_{N}=9.71 \times 10^{3} \sqrt{\frac{T}{M}} \frac{a^{2} b^{2}}{(a+b) L}$,

where $a$ and $b$ are dimensions of the duct cross section. Equation (3-25) can be converted into a puadratic equation for 1 :

$C_{G}\left(P_{S}-P_{\text {exit }}\right) L^{2}-B C_{G} L-\alpha \beta=0$,

where $\alpha=C_{N} L$ and $\beta=2 \pi / 3.56 \times 10^{16}=623$. Using $a=40 \mathrm{~cm}$,

$b=16 \mathrm{~cm}$ resuits, the $\mathrm{L} \simeq 150 \mathrm{~cm}$.

The final design features a duct that is slightly tapered in the direction

of the beam, as shown in Fig. 3-58, with conductance $C_{N}=4734 \mathrm{l} / \mathrm{s}$ and optical transmission factor $T_{N}=0.996,007$. This yields about 19-kW power dissipation there. 


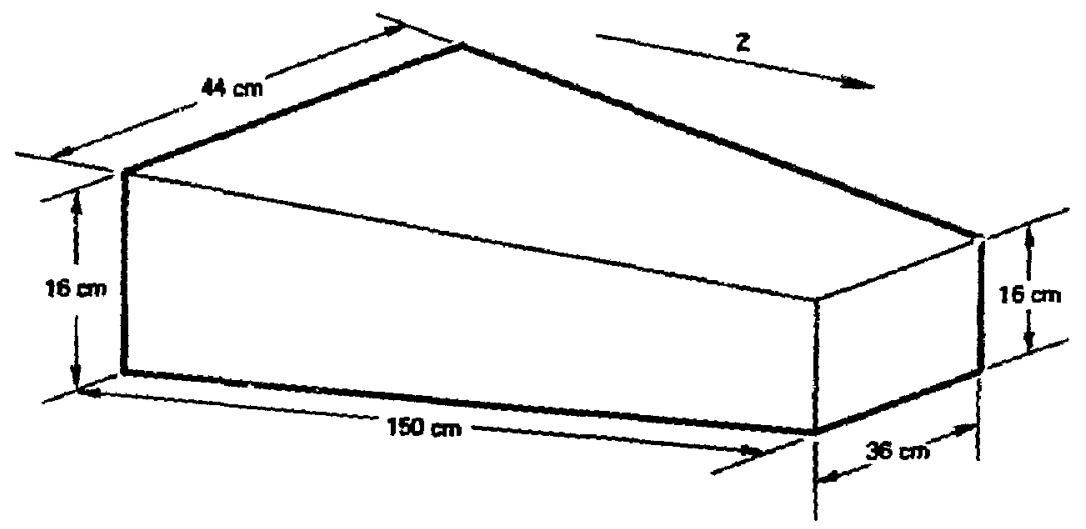

Figure 3-58. Neutralizer geometry. 
Gas streaming from neutralizer can now be obtained as

$Q=\frac{C_{G} C_{N}}{C_{G}+C_{N}}\left(P_{S}-P_{\text {exit }}\right)=\Sigma_{10} T-2 / 5$.

The final neutra]izer parameters are shown in Table 3-23.

Reflection Magnet. The ions contained in the neutral beam that exits the neutralizer carry a sizable fraction of the total power delivered by the jon source. In the case of the TDF cenitral cell injector, about 2 MH of the 5.6 $\mathrm{MW}$ delivered by a single ion source are carried by the unneutralized ions. It is necessary, therefore, to oissipate this power in a well-aefined manner onto a power absorbing surface. In addition, care has to be taken to pump the gas emanating from these ion aumps efficiently because gas in the path of the neutral beam can lead to reionization and subsequent beam loss.

As shown in Fig. 3-59, the reflection magnet chosen to separate the ions ano neutrals is similar in configuration to the Doublett III (Ref. 13) beamline magnet. This arrangement has two advantages: (1) the design is basea on a proven concept, and (2) beam loss caused by background gas is minimized. The magnet, together with the baffle, separates the beamline vesse 1 into three compartments. The high-pressure section, in which the gas emanating from the neutralizer and jon dumps is dominant, contains only a short neutral-beam path. The transition section between the reflector magnet and the beam duct is kept at low pressure.

The three major beamline components--source, reflection magnet, and ion dump, together with some characteristic particle orbits--are shown in Fig. 3-59. The minimum separation $\Delta W$ of source and ion dump is given by the Larmor radius $\rho_{1}$ of the lightest, least energetic ion and the incident beam wioth $\mathrm{H}_{\mathrm{s}}$ as

$\Delta W=2 \rho_{1}-W_{S}$

To collect the most energetic ion of Larmor radius $\rho_{2}$ on the ion dump, the magnet width $W_{m}$ has to be at least

$w_{m}=z_{2}+w_{s}$ 
Tabie 3-23. Neutralizer parameters.

\begin{tabular}{|c|c|}
\hline Parameter & Vaiue \\
\hline Entrance size, $a_{1} \times b_{1}(c m \times c m)$ & $i 5 \times 44$ \\
\hline Exit size, $a_{2} \times b_{2}(\mathrm{~cm} \times \mathrm{cm})$ & $16 \times 36$ \\
\hline Length, $L(\mathrm{~cm})$ & 150 \\
\hline Conouctance, $C_{N}$ (1iters $/ s$ ) & 4734 \\
\hline $\begin{array}{l}\text { Entrance pressure, } P_{\text {entrance }} \text { (Torr) } \\
\text { Exit pressure, } P_{\text {exit }} \text { (Torr) }\end{array}$ & $\begin{array}{l}1.5 \times 10^{-3} \\
10^{-4}\end{array}$ \\
\hline Gas throughput, $Q$ (Torr-liters/s) & 10 \\
\hline Target tinickness, $\pi\left(\mathrm{cm}^{-2}\right)$ & $7.68 \times 10^{15}$ \\
\hline Uptical transmission factor, $T_{N}$ & 0.996607 \\
\hline Beam power dissipated $(\mathrm{kW})$ & 19 \\
\hline
\end{tabular}




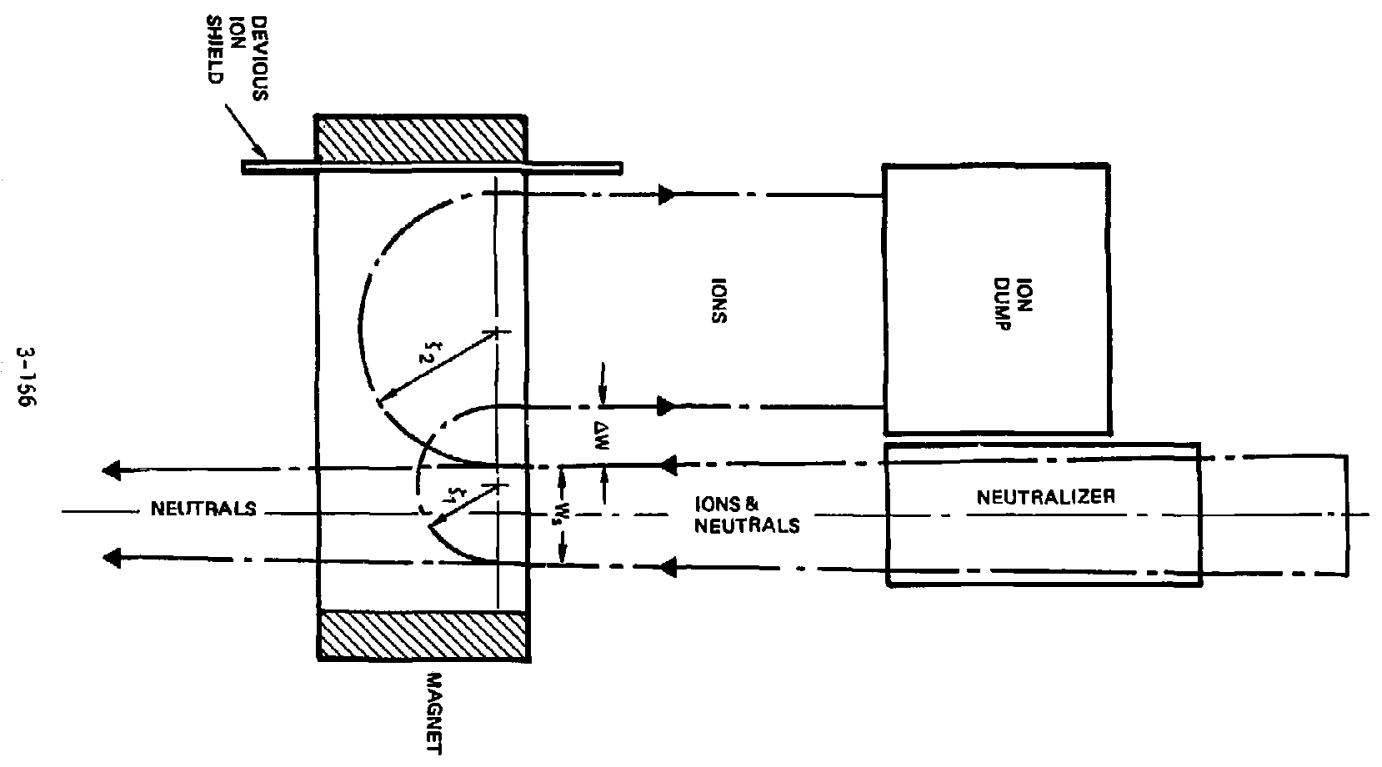

Figure 3-59. Ion-dump arrangement. 
This shows that the field should be high to keep the magnet size and weight low. On the other hand, the field cannot be made arbitrarily large since $\Delta W$ has to remain finite. For source width $W_{S}=32 \mathrm{~cm}$ and a source-to-neutralizer distance of $\Delta \mathrm{W}=20 \mathrm{~cm}$, it fol lows from $\mathrm{Eq}$. (3-28) that $p_{1}$ should be $26 \mathrm{~cm}$. Because the 1 ightest, least energetic ion is $\mathrm{D}^{+}$at $27 \mathrm{kV}$ or $\mathrm{T}^{+}$at $27 \mathrm{kV}$, a magnet field strength of $0.128 \mathrm{~T}$ for the deuterium beams and $0.157 \mathrm{~T}$ for the tritium beams is required. The needed magnet widih $W_{m}$ ciepends on the heaviest, most energetic ion that is still to be directed onto the ion dump (Eq. 3-29). Table 3-24 shows that the relative abundance of the molecular ions and, therefore, the energy carried by them is relatively low. Limiting the ion dump collection to $(D, T)_{1}^{+}(80 \mathrm{kV})$ rather than $(0, T)_{3}^{+}(80 \mathrm{kV}) \mathrm{can}$ save magnet length and therefore weight by about the square rcot of 3 .

Figure 3-60 shows the magnet dimensions adopted. Mast of the $(D, T)_{1}^{+},(80 \mathrm{kV})$ ions and a fraction of the $(\mathrm{D}, \mathrm{T})_{2}^{+}(80 \mathrm{kV})$ ions will be directed toward the inside of the magnet gap, where they are collected on a low power density dump consisting of water-cooled copper sheets (devious ion shield). With $70 \mathrm{~cm}$, the magnet depth is smaller than the Larmor radius of the $(0, T)_{3}^{+}(10 \mathrm{kV})$ ions. The shield, therefore, has to be extended beyond the magnet gap. It should be noted that a step-function field distribution was assumed. The actual magnet is $10 \mathrm{~cm}$ wider to accommodate the fringing field. Four beams are located adjacent to each other in one beamline. This makes it possible to construct the reflection magnet as one unit, shown in Fig. 3-60. With the gap dimensions shown, the magnet assembly weighs 9 tons.

The power consumed in the magnet power beamline can be estimated from

$P=\rho j g L B_{0} / \eta \mu \Delta \cdot\left(\mu_{0}\right) \cdot$

Here, $\rho$ is the resistivity of copper $\left(1.7 \times \omega^{-6} \Omega \mathrm{cm}\right), j$ is the usable current density in the conductor (assumed to be $250 \mathrm{~A} / \mathrm{cm}^{2}$ ), $g$ is the gap width, $L$ is the conductor length, and $n$ is the conductor filling factor (assumed to be 0.6). The magnet parameters are assembled in Table 3-25.

Drift-Duct. Neutral beams are injected into plasma devices through drift-ducts that are usually long and narrow for geometrical and neutron shielding considerations (Fig. 3-61). The major problem encountered with 
Table 3-24. Ion species, their relative abundance and Larmor radius in $B=0.128 \mathrm{~T}$ for $D$ and $B=0.157 \mathrm{~T}$ for $T$.

\begin{tabular}{lcc}
\hline $\begin{array}{l}\text { Species } \\
\text { Dor T }\end{array}$ & $\begin{array}{c}\text { Atonic } \\
\text { current (A) }\end{array}$ & $\begin{array}{c}\text { Larmor } \\
\text { radius (cm) }\end{array}$ \\
\hline$(D, T)_{j}^{+}(27 \mathrm{kV})$ & 1.13 & 26 \\
$(\mathrm{D}, \mathrm{T})_{1}^{+}(53 \mathrm{kV})$ & 1.03 & 37 \\
$(\mathrm{D}, \mathrm{T})_{1}^{+}(80 \mathrm{kV})$ & 20.90 & 45 \\
$(\mathrm{D}, \mathrm{T})_{2}^{+}(53 \mathrm{kV})$ & 0.14 & 52 \\
$(D, T)_{2}^{+}(80 \mathrm{kV})$ & 1.56 & 64 \\
$(D, T)_{3}^{+}(80 \mathrm{kV})$ & 0.66 & 78 \\
\hline
\end{tabular}

Table 3-25. Reflection-magnet parameter for one beamline (four beams).

\begin{tabular}{cccc}
\hline Species & Field $(T)$ & Weight (tons) & Power (kW) \\
\hline$D$ & 0.128 & 9 & 35 \\
$T$ & 0.157 & 9 & 43 \\
\hline
\end{tabular}




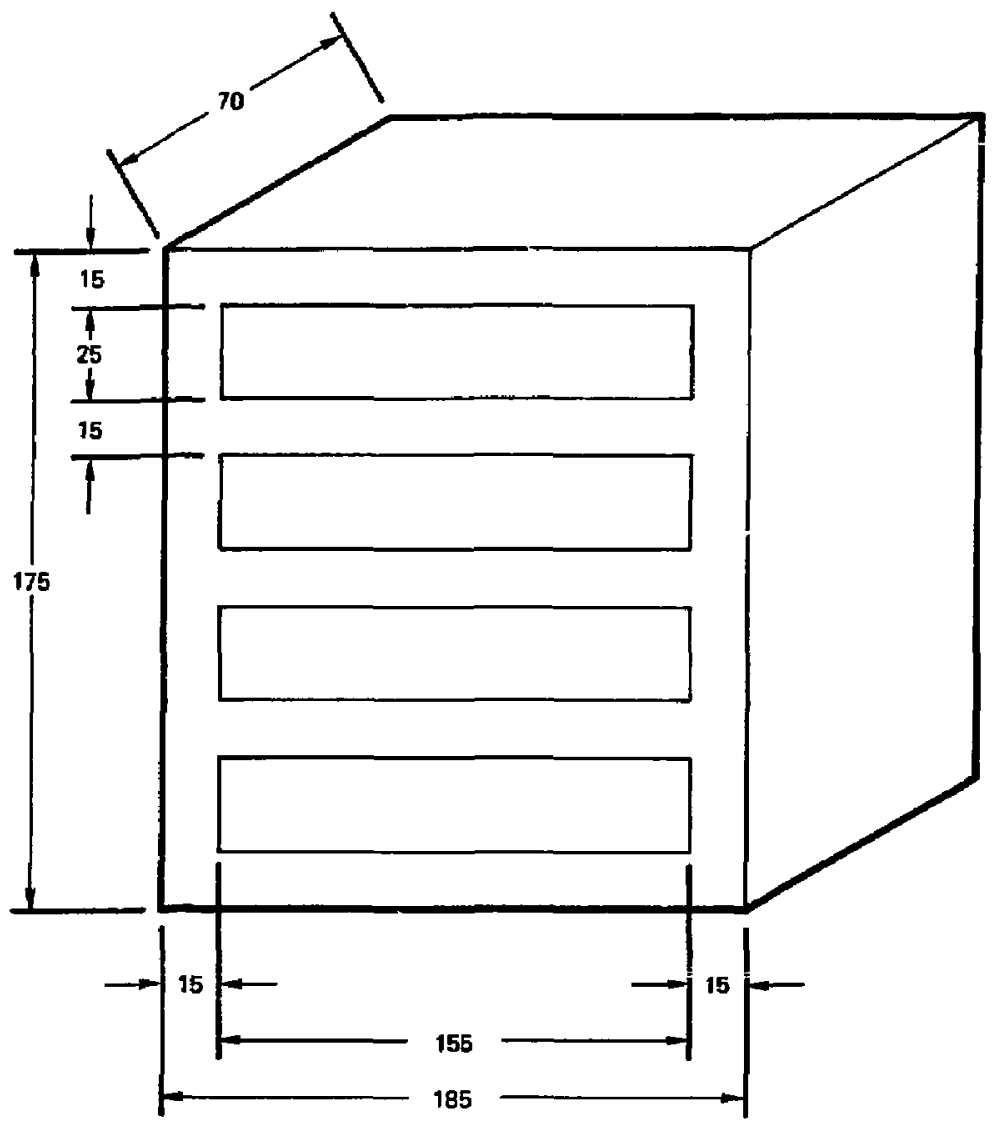

Figure 3-60. Magnet dimensions. 


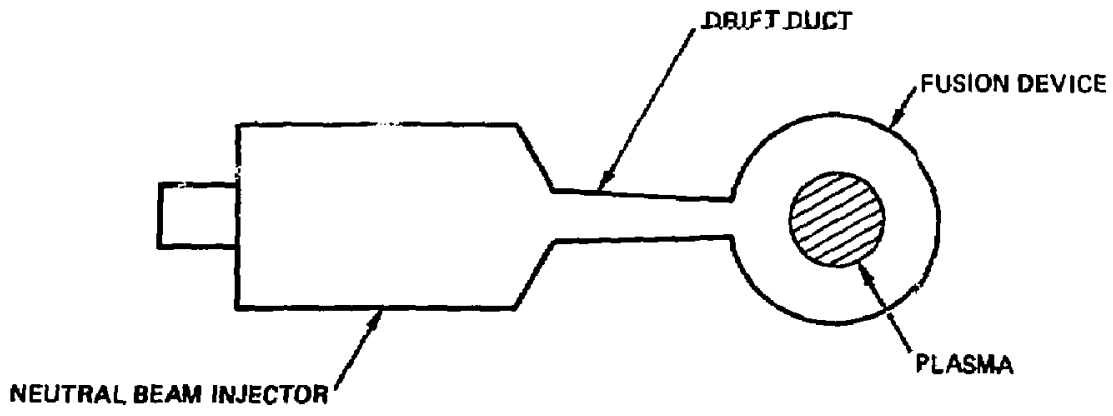

Figure 3-6.. Drift-duct geometry. 
these ducts is that some of the beam particles undergo ionizing collisions with thermal gas within the duct and are reflecter by stray magnetic fields onto the auct wal]. This phenomenon was responsible for significant power loss from the neutral bearr in devices such as PLT, ${ }^{14}$ PDX, ${ }^{15}$ and others. 16 Early analysis of drift-duct physics was done by Riviera and Sheffiela ${ }^{16}$; later it was moaified by Hemsworth. 17

The importance of the development of a good neutral-beam transport mode 1 for drift-ducts can be seen from the following considerations. The ions generated via collision from beam particles and transported to the wall will recombine at the surface. This increases the gas pressure in the duct which, in turn, increases the rate of heam particle loss oue to ionization. If a stable equilibrium is not reached, the situation can lead to a complete beam blockage.

Urift-ouct designs can be examined with Monte Carlo techniques. However, these methods are usually tailored to a particular design and are, therefore, not versatile; only simple cases can be treated effectively. Finally, Monte Carlo analysis is not very cost-effective. Therefore, we focused on an analytical methoo. Notice below that a reasonably accurate analytical method can, in fact, be constructed. This model vias initially createo from very general considerations and later refined for TDF.

A number of effects that contribute to beam blockage have been identified. They are of varying importance, depending on fusion device configuration:

Classical Effect. In magnetic confinement devices, the neutral beam is ionized by collisions with background gas, and the ions are removed to the wall by the magnetic field. Thus, it follows that the beam propagating along the $Z$-axis is attenuated accoroing to

$P=P_{0} e^{-\int_{0}^{L} \sigma n(z) a z}$

where $P_{0}$ is beam power at $z=0, P$ is beam power at $z=L, 0$ is the reionization cross section, and $n(z)$ is the background (thermal) gas density oistribution. The difficulty encountered in developing a beam transport model 
is that the density oistribution is unknokn, unless experimentally obtained. One usually takes $n(z)$ to be constant and casigns it some experimentally obtained value. While this procedure makes it possible for one to estimate beam loss for new aesigns by extrapolating from existing designs, results obtained by this method are crucie at best because $n(z)$ is never constant, usually not even linear and, in fact, strongly a function of duct shape.

The classical effect is the most important one in the TUF configuration and, therefore, forms the basis of the model to be developeo.

Culham Effect. In the first neutral-beam injection experiment at Culham in 1973, it was found that the beam loss far exceeded original estimates. 14 The unexpectedly high drift-duct pressure was attributed to the interaction of collisionally ionized particles with the arift-auct wall. It is believed that the bombarding particles "knock off" additional gas molecules from the surface. This gas contributes to local pressure and is responsible for increased losses. Knock-off yields (released particles/incident particles) are generally unknown and their contribution is, therefore, difficult to estimate. However, for continuously operating beamlines, it is clear that the yield becomes unity after some time since no adsorbed gases are left at the surface.

Princeton Large Tok amak Effect. During the testing phase of PLT neutral beamlines, a slow rise $(300 \mathrm{~ms})$ in drift-auct pressure was responsible for excessive neutral-beam loss. This increase in pressure was attributed to a gas source within the duct itself: diffusion of gas previously absorbed inside the duct wall material. It is well known that, unless special metailurgical methods are used during the manufacturing phase, most metals will contain appreciable amounts of gases. ${ }^{18}$ As the duct wall is heated by particle bombariment, these gases diffuse to the surface and are desorbed from it. A complete diffusion model for this case was devised by Stewart, et al. 19 Because it also requires some inputs that must be obtained experimentally, it is of rather questionable value for any new design, except perhaps as a basis for extrapolation.

Because of the gradual surface clearing and wall outgassing of the continuously operating TDF beamlines, both the Culham and PLT effects will become of minor importance after some operating time. For this analysis, 
"knock off" and desorption will be represented by a single constant, $r_{\text {wa }}$ $(x, y, z)$, that is the wall outgassing rate in molecules per second at the point $(x, y, z)$.

Beam-Divergence Effect. In most practical beamline configurations it is impossiole to design beam collimators (scrapers) so that no point on the drift-duct wall "can see" the source (Section 3.3.5, "Magnetic Shie)ding Hequirements for Low Divergence"). An optical model for the source assumes that each point of the source is an emitter of beamlets directed toward some common point (focus). Each of these beamlets is divergent and has a Gaussian power distribution profile. Clearly, any point of duct wall in direct line of sight with the source is illuminated by it. The far end of the ouct represents another aperture to the beam. It is necessary to choose the dimensions of the far end duct opening so that the highest possible optical (ignoring reionization) transmission is achieved. Even in such an optimal case, the fraction of beam lost because of opical inefficiency may constitute a significant gas load on the drift-duct, causing a local increase in pressure there and, consequently, increased beam blockage caused by reionization (enchanced classical effect).

In the following inodel, the beam-divergence effect is taken into account as follows. Consider a beam passing through a set of two rectangular apertures, each representing one of the drift-duct end openings. Let $f_{r}$ be the power fraction of the beam that passes through a rectangular aperture of size $2 x^{\prime}$ by $2 y^{\prime}$ (Ref. 20). From preliminary studies, duct openings were sized at approximately $100 \times 120 \mathrm{~cm}$ and $50 \times 70 \mathrm{~cm}$, 1ocated at $z=625$ and $850 \mathrm{~cm}$, respectively. The fraction of the beam lost within the ouct is calculated apapproximately as

$f($ lost $)=f($ entrance eno $)-f($ exit end $)$.

Using previously defined source parameters, namely $\theta_{x}=\theta_{y}=0.5 \mathrm{aeg}, f_{x}=$ $f_{y}=950 \mathrm{~cm}$ and $x_{0}=8 \mathrm{~cm}, y_{0}=20 \mathrm{cmin}$ results in $\mathrm{f}($ lost $)=2.5 \%$, which is significant. In fact, for a $240-A$ beam, it represents a 6-A 10ss. This number is reauced somewhat if a collimator is placed just before the ouct entrance, but that is a minor point. 
Gas-Plume Effect. From experiments involving neutralizers in various beamlines, we know that thermal gas is ejected from the neutralizer eno with approximately Lambertjan spatial distribution. Because of the rejatjvely large mean free path for thermal gas within the beamline, the path of ejected gases can be traced using ray optics. It is easy to see that some of this gas impinges on the orift-duct walls. This case is analogous to the beam-divergence effect above, except here the neutralizer is taken as a cosine law "source."

for the duct dimensions above, the fraction of the beam lost is $1.23 \%$. Since the thrermal particles ejected by the neutralizer are at about a one-toone ratio to beam particles, the $1.23 \%$ is equivalent ta about 0.9 of the particle current. Ignoring this is hard to justify, but one can show that placing a collimator just before the drift-duct entrance can greatly reduce the flow of thermal particles onto the duct wall. In fact, if a $16-x 30-\mathrm{cm}$ collimator is placed $150 \mathrm{~cm}$ before the duct, $f(l o s s)$ becomes virtually zero. Gas-plume effect will, therefore, be ignored in the analys is.

Plasma Generation. As noted earlier, ions and electrons are generated inside the duct as a result of reionization. In the presence of a magnetic field, they are deflected from the beam so that their average velocity relative to the beam is non-zero. Since the lifetime of these particles within the duct (before they recombine at the wall) can be significant, they can interact with the beam. Although it is clear that the net plasma density within the duct is much less than that of the background gas, the charge-exchange $[(1,0) \rightarrow(0,1)]$ collision has a cross section that is one or two orders of magnitude larger than the reionization process. Uepending on the size of the beam(s) and the strength and direction of the magnetic field, this may result in appreciable net beam attenuation.

The amount of plasma in the duct can be estimated from particle balance considerations. The parallel velocity of reionized particles is approximately

$V_{11}=\sqrt{\frac{K T}{V T}} \cos \Psi$,

wriere $K T$ is particle energy, $M$ is mass, and $\psi$ (which equais $45 \mathrm{deg}$ ) is the argle between the beam and magnetic field. Because, in a magnetic fieid of sorel tesla, the Larmer radius of $80 . \mathrm{keV}$ jons is just a few centimeters 
(small in comparison to the size of the duct), the ions will essentially follow the magnetic lines of force to the wall. Denoting $a=45 \mathrm{~cm}$, the average aistance from the collision site to the wali, the average ion lifetime in the duct is

$\overline{\mathrm{t}}=\frac{\overline{\mathrm{a}}}{v_{11} \sin \Psi}=\frac{2 \overline{\mathrm{a}}}{\sqrt{\frac{K T}{\mathrm{H}} \sin 2 \Psi}}$.

Assuming about a $5 \%$ loss of the original approximately $245-A$ beam within the duct and charge-exchange cross section, $\alpha=3 \times 10^{-14} \mathrm{~cm}^{2}$ results in about a $0.1 \%$ additional attenuation because of plasma. This is much too low ano can be ignored.

Gas Load from Neutral-Beam Dumps. Hecause of the geometrical configuration of the neutral beamlines at the end of the central cell, the shine-through beam dumps are located close to beamline drift-oucts. A sizable fraction of the neutral beam incident on the plasma is not absorbed and emanates from the shine-through dumps as gas. This gas can enter the bean ou: $t$ and must be taken into account in the overall gas balance of the ducts.

The expected particle shine-through current for one injector is about $44 \mathrm{~A}$. Al the dump, this will turn into $22 \mathrm{~A}$ of molecular current. If 5 to $20 \%$ of this gas is drawn into the duct, 1 to $4.5 \mathrm{~A}$ additional thermal gas has to be pumped out. This effect is substantial and must be included in the analysis.

From the overail injector geometry, it has been detemined that four neutral beams will share one duct. The location and orientation of the duct with respect to the reactor coils is shown in $F i g .3-62$. As described in Section 3.2, the far end (plasma end) of the duct should be made as narrow as possible to minimize neutron streaming. A small plasma end aperture will also limit the amount of dump gas intake into the duct. On the other hand, the optical transmission of the duct will be reduced ano the effect of beam loss by the apertures will be enhanced. A trade-off study is therefore required.

Sinilar considerations have to be given to other geometrical features of the auct. For example, a minimum amount of shielding is needed between the duct wall and the edge of coil S-1. Because the leation of the duct and coil 


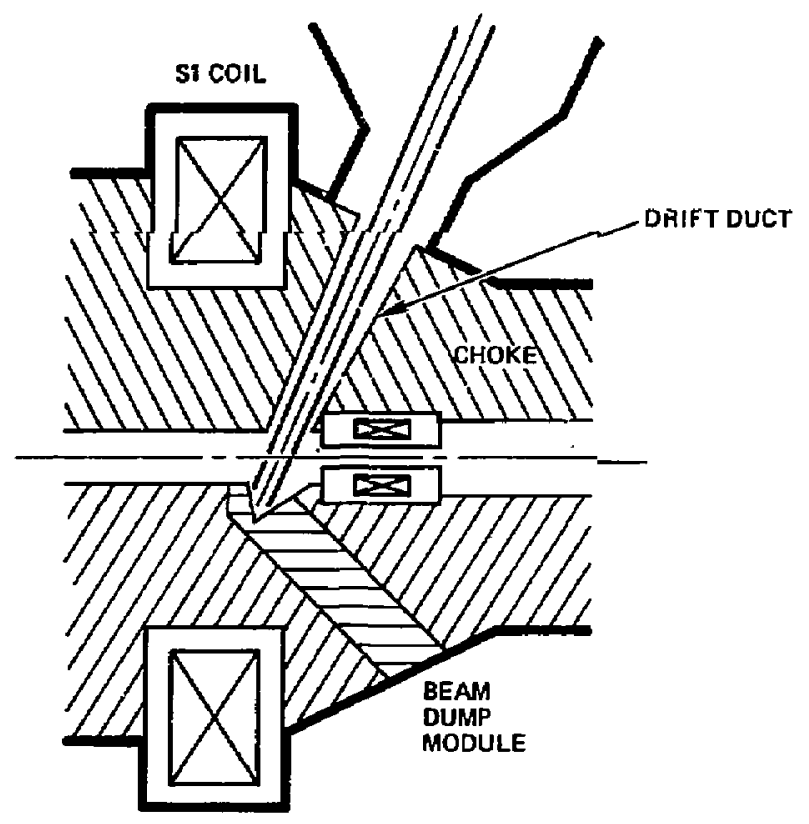

Figure 3-62. Drift-duct location. 
is fixed, shield thickness can be increased (desirable to limit neutron damage to the superconouctor) on $7 y$ at the expense of reduced duct conductance (and higher beami loss) and vice versa.

Those geometrical features of the duct that will be used $i_{i}$ analys is are defined in Fig. 3-63.

A number of assumptions will simplify analysis. Some of them are not absolutely necessary because the model could be extended to even more general cases, but minor increases in accuracy are not always worth the trouble. The assumptions are:

- The density gradient is always pciallel to the duct central (2) axis, i.e., there is no density variation in the plane perpendicular to the cuural axis.

- The gas inside the duct is at constant temperature everywhere (hence, $T_{\text {gas }}=T_{\text {duct } \text { wall) }}$.

- An infinite pumping speed exists at the flared end of the duct.

- There is no pump at the narrow (far) enc of the auct $(z=1$ ).

- The wall degassing rate is uniform and constant in time (steady-state).

- The attenuation due to plasma is negligible.

- The gas load due to the neutralizer gas-plume effect is negligible.

- A fraction of the gas generated at the beam dumps will be orawn into the duct.

- A strong magnetic fiela is present within the orift duct (in sase of TDF $|B|=20$ to $50 \mathrm{~kg}$.

Theory. Once all the beam loss mechanisms and gas sources have been iaentified, flow equations can be used to determine the density distribution inside the duct. The particle balance is depicted in Fig. 3-64.

Gas generated in a cross sectional duct segment of thickness $\Delta z$ located at an axial distance $z$ is

$k_{g}(z)=$ beam loss + outgassing + other sources. 

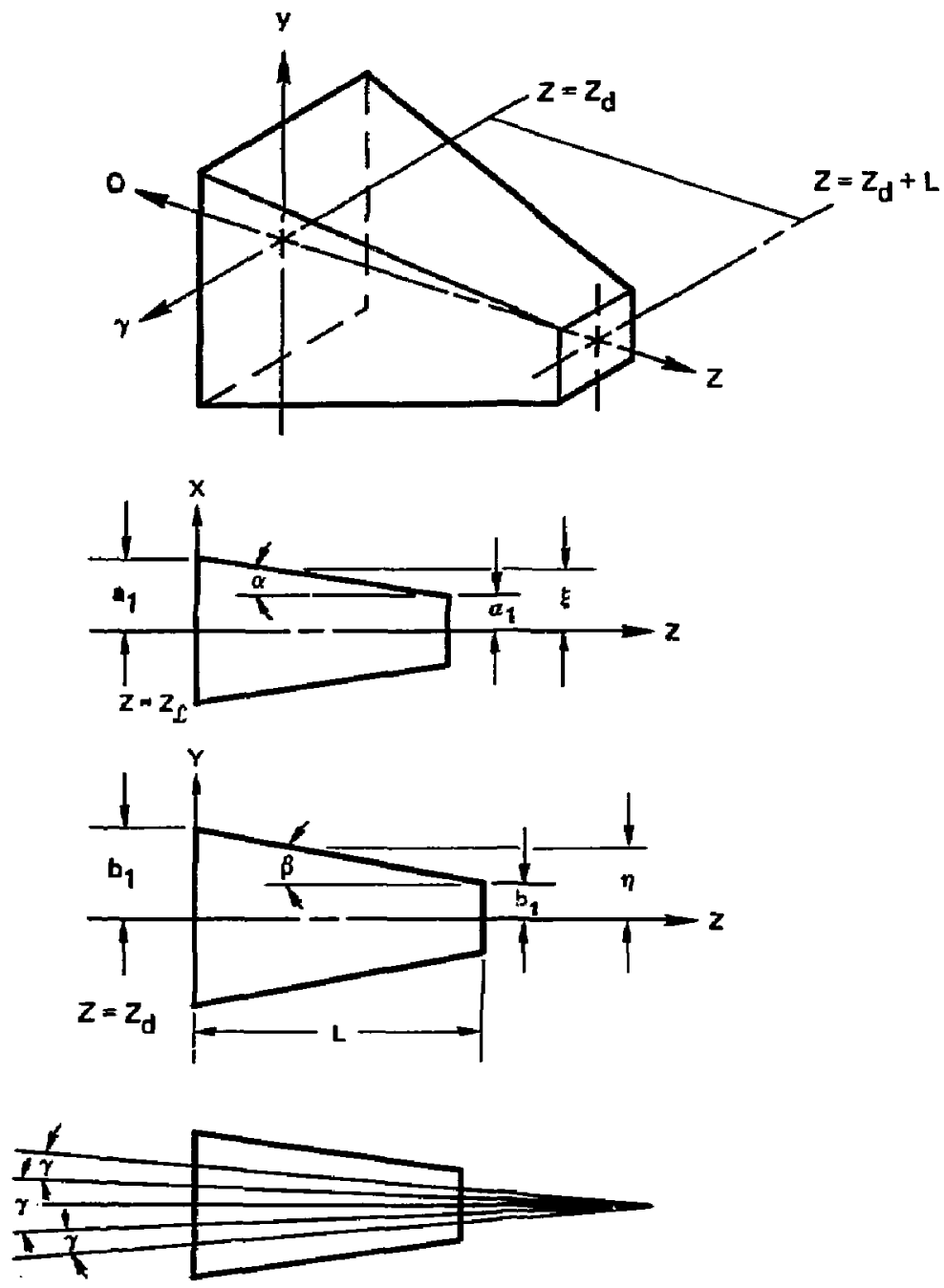

Figure 3-63. Duct geometry. 


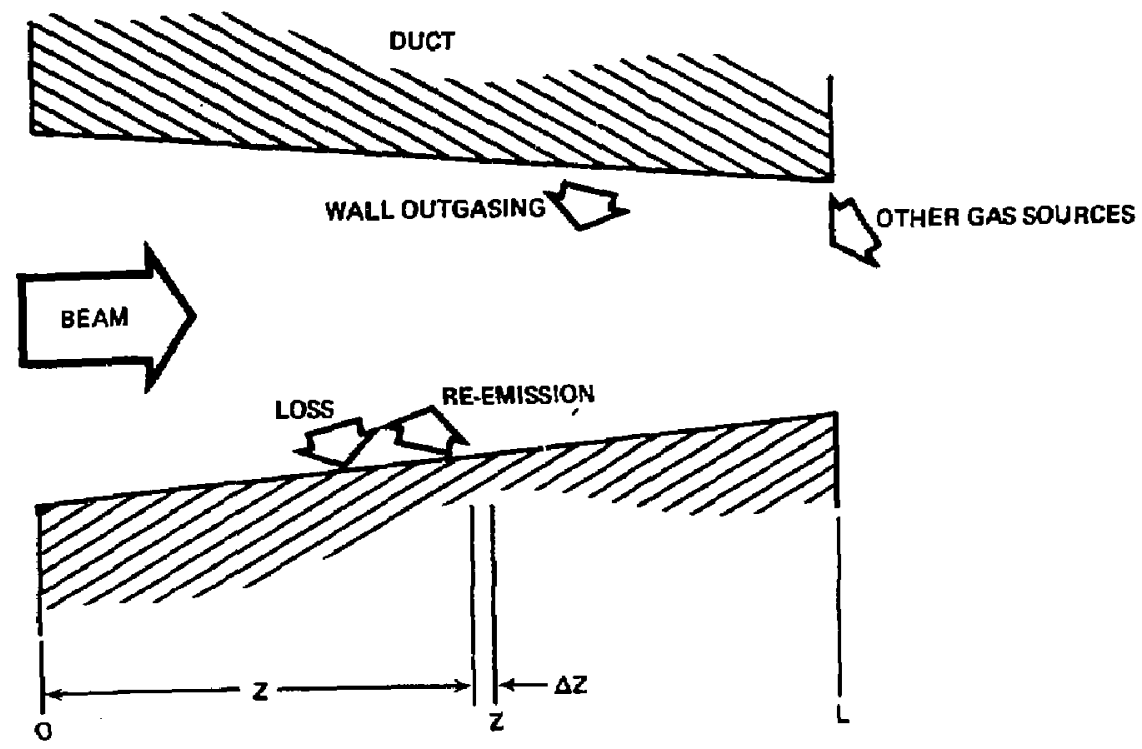

=igure 3-64. Physical model. 
$R_{f}(z)=\int_{z}^{L} k_{g}(\zeta) \alpha$.

The corresponoing gas aensity can be written as

$n(z)=\frac{R(z)}{C_{\Delta}(z)}+n(z-\Delta z)$,

where $C_{\Delta}(z)$ is the conductance of a duct element of length $\Delta z$ with a cross section corresponding to the point $z$. Since the beam loss is related to the gas density, one can write the axial censity gradient as

$\frac{d n}{d z}=\frac{R_{B} a}{2} \frac{e^{-\int_{0}^{z^{\prime}} Q(\zeta) d \xi}-1\left(1-f K_{s}\right) e^{-\int_{0}^{L} Q(\zeta) d \zeta}+\int_{z^{\prime}}^{L} r_{w}(\zeta) d \zeta}{g .71 \times 10^{3} \sqrt{\frac{T}{M}} \frac{B \xi^{2} n^{2}}{\xi+n}}$

where $Q(\zeta)=\sigma n(\zeta)+\lambda(\zeta), r_{w}(\zeta)=2(\zeta+n) r_{w a}$, and $\lambda(\zeta)$ is a view factor defined as the fraction the beam lost at cross section $z$ because of direct source illumination. The other symbols are identified in Fig. 3-54.

Analytic solutions for Eq. (3-38) are difficult to find. If the equation is rawritten in different form, however, it becomes suitable for application of numerical methods. One may proceed as follows: subaivide the duct of length $L$ into 1 segments, assume initially uniform density (background density), and obtain the density profile for the entire duct by repeated application of Eq. (3-33), working step by step from $Z=0$ to $Z=L$. This newly obtained profile is now used as a starting point for recalculating the density profile, and the process is repeateo. The iteration converges rather rapidly for most configurations; 20 to 30 steps are usually enough to stabilize the output to six significant digits. 
Once the density profile $n(z)$ has been obtained, the total transmission factor $T_{D}$ for the duct is easily computed as

$$
T_{D}=\frac{\text { Power in }}{\text { Power out }}=e^{L} \sigma n(z) d z
$$

The coefficients $f$ and $k_{s}$ used in Eq. (3-28) remain to be determined. The shine-through quotient, defined as

$k_{s}=1-\frac{\text { Power absorbed in plasma }}{\text { Power incident on plasma }}$,

is usually known ahead of time from plasma heating calculations. On the other hand, the fraction ( $f$ ) of dump-generated gas drawn into an adjacent drift-duct has to be obtained from some particle-transport model for the dump-duct region. Ideally, one would assume that gas particles are emitted from the dump with a cosine distribution and then calculate the relevant view factors. From the functional dependence of $T_{D}$ on $f$, this complicated computation can be considered superfluous (Fig. 3-65).

Since $T_{D}$ depends only weakly on $f$, it will suffice to obtain $f$ by a simpler, but rather crude method of comparing conductances. Consider the configuration in Fig. 3-66, adopted from Drawing No. 1076.

For computation, it can be translated into the following model: the dump is located between two ducts connected and to end, each having a pump on the far end (the plasma is assumed to act as an infinite pump), as shown in Fig. 3-67.

The size and shape of the "plasma duct" is inferred from the following considerations: the smaller aperture is the same size as the plasma halo (assumed to act as an infinite pump), and the larger aperture is equivalent to the distance between two adjacent beam dumps. Assuming $\theta_{1}=6 \mathrm{deg}$, $\theta_{2}=18 \mathrm{deg}, a_{0}=60 \mathrm{~cm}, d_{0}=45 \mathrm{~cm}, \mathrm{~L}=300 \mathrm{~cm}, \mathrm{R}=70 \mathrm{~cm}, r=25 \mathrm{~cm}$, and duct width (both ducts) $2 \mathrm{~b} \simeq 60 \mathrm{~cm}$, we obtain conductances for the drift duct and plasma duct, respectively: $C_{D}=25,0001 \mathrm{iters} / \mathrm{s}$ and $C_{p}=50,000$ liters $/ \mathrm{s}$. He expect, however, that $C_{D}$ has been over estimated 


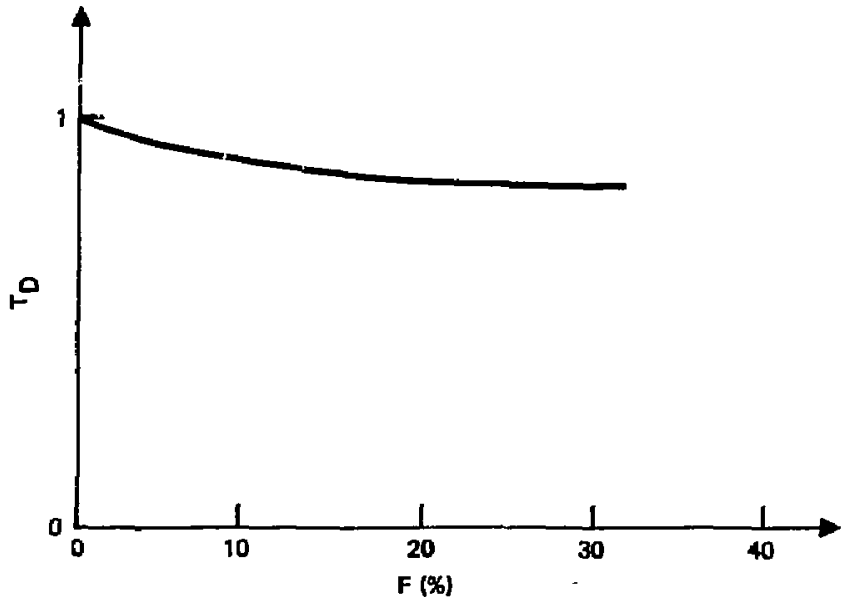

Figure 3-65. Jependence of $T_{0}$ on $f$. 


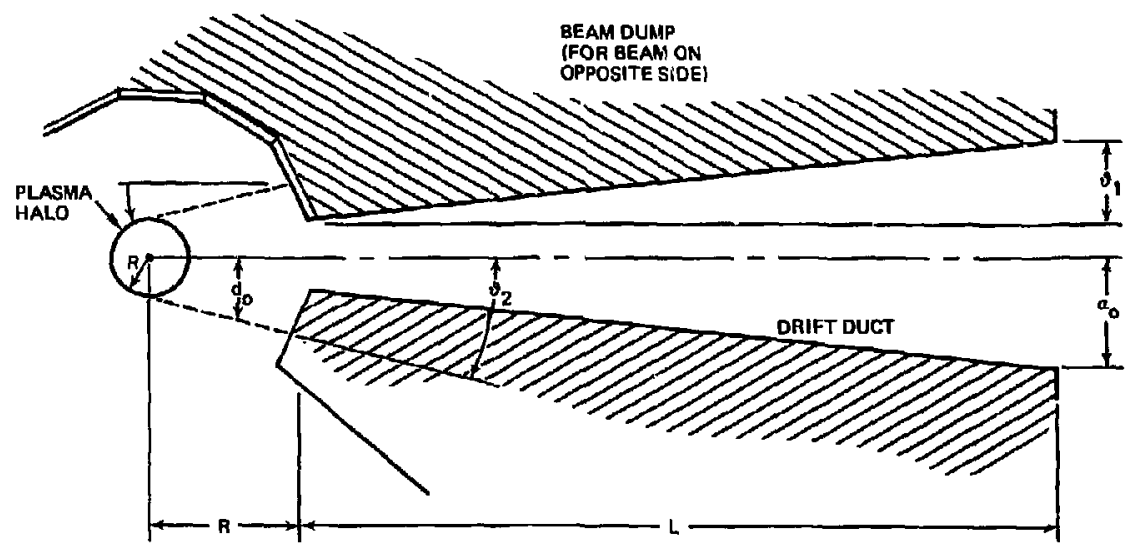

Figure 3-65. Duct-dump relationship. 


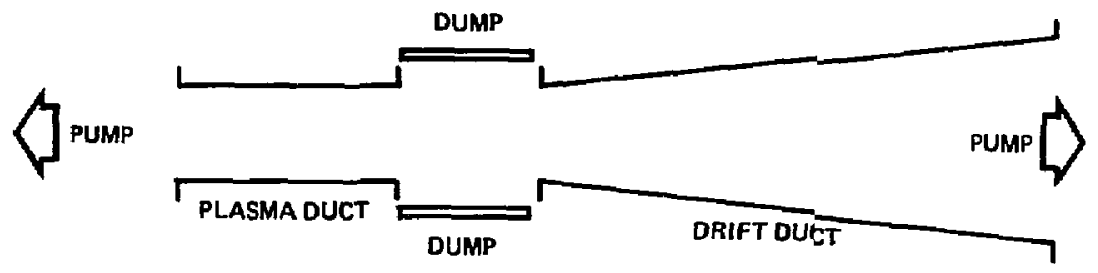

Figure 3-67. Model illustration. 
by about a factor of 3 because the duct intake is not in direct view of the adjacent dump. Using adjusted value $C_{D}=8000$ ijters/s,

$$
f=\frac{C_{D}}{C_{D}+C_{p}} \simeq 14 \% \text { (max) } .
$$

These calculations can be used to determine the beam transmission factor $T_{0}$ for any given duct configuration. For the initial duct design, we can discuss the functional dependence of $T_{D}$ on various parameters, particularly duct conductance, which is shown schematically in Fig. 3-68. Notice that appreciable beam loss can occur for small conductances and that complete beam blockage results below some minimum conductance. After a regime in which transmission increases rapidly with conductance, the curve levels off for $T_{C} \geq 0.95$. This means that a further increase in duct conductance has little effect on transmission.

As discussed in Section 3.3.3, tine drift-duct cross section and, therefore, the conductance, cannot be made arbitrarily large. (1) The neutron flux on some beamline components should be minimized to limit heat transfer to cryopanels and to increase source insulator lifetime. (2) A limited space for ducts is available between the magnet coils and their neutron shields. This space and the curve describing the transmission dependence on conductance show a trade between a narrow, low-conductance duct that limits neutron flux but causes appreciable beam loss and a wide duct. The duct design should result in a conductanre represented by this design region so that small errors in design, assessment of gas inputs, etc. do not appreciably alter the design transmission coefficient. The parameters of the beam duct finally accepted for TOF are shown in Table 3-26.

The duct walls under particle bombardment are water-cooled to keep them at constant temperature. The wall material will be further treated with vacuum metallurgy methods to lower the content of absorbed gases and other impurities. Order-of-magnitude improvements over untreated material are readily achievable. ${ }^{18}$ Quoted results for 304 stainless stee 1 are approximately $1 \mathrm{ppm}$ of $\mathrm{H}_{2}$ and $20 \mathrm{ppm}$, for carbon, sulphur, and phosphorus. Using these two assumptions, it is possible to estimate maximum steady-state 


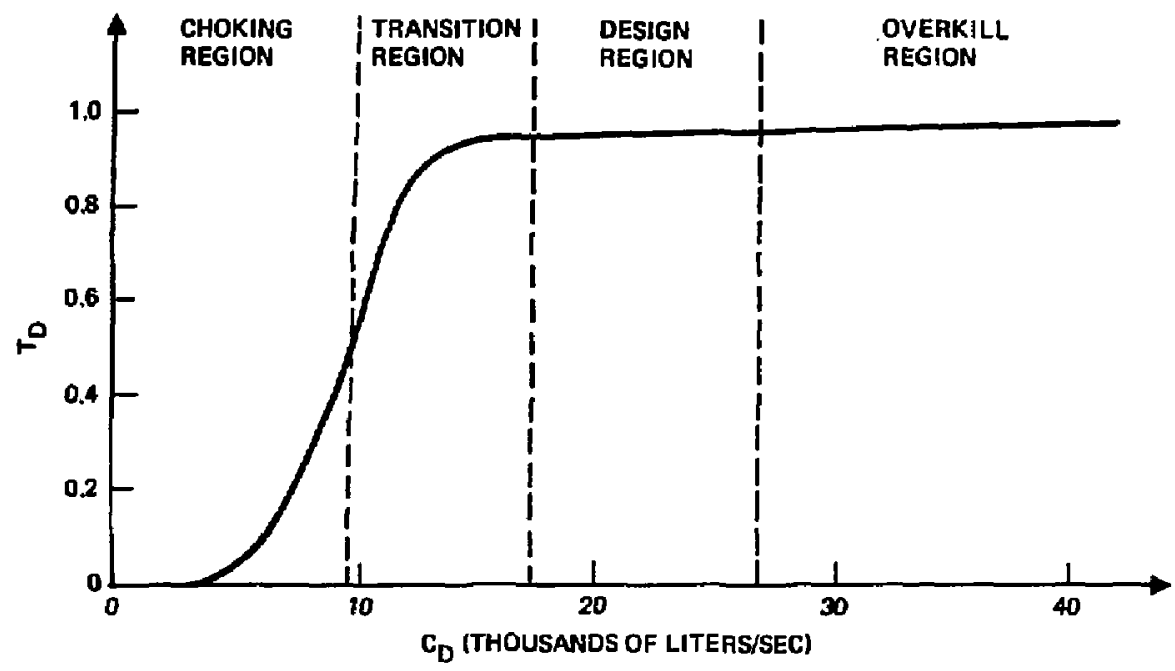

Figure 3-68. Dependence of transmission on conouctance. 
Table 3.26. Drift-duct paramete:s.

Initial parameters

Neutral-beam current entering duct, $I_{0}$ (A) 56.35

Power of neutral beam entering auct, $P_{0}(\mathrm{~kW}) \quad 3436$

Number of beams

4

Angular separation of beams, $T$ (deg) 3.31

Source dimensions $(\mathrm{cm})$

$2 x_{0}$

$2 \mathrm{y}_{0}$

16

Source divergence, $\theta$ (deg)

40

Source focal length, $f_{s}(\mathrm{~cm})$

0.5

Front collimator dimensions (cm)

${ }_{b}^{a}$

$\mathrm{b}_{\mathrm{c}}$

Location of duct entrance, $Z_{d}(\mathrm{~cm})$

Duct wall outgassing rate, $r_{\text {wa }}\left(\mathrm{s}^{-1} \mathrm{~cm}^{-2}\right)$

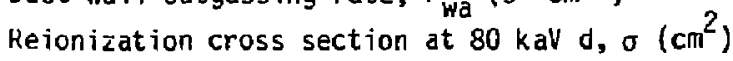

950

Magnetic field in thermal duct, $\mathrm{B} B \mathrm{I}$ ( $k G$ )

8.5

Temperature of thermal gas, $T$ (deg)

15

Shine-through factor, $k_{s}$

630

$1 \times 10^{13}$

$1 \times 10^{14}$

220

400

0.8

\section{Design parameters (final oesign)}

Neutral-beam current leaving duct, $I_{1}(A)$

53.2

Power of neutral beam leaving duct, $P_{1}(\mathrm{~kW})$

3251

Duct transmission factor, $T_{U}$ (four beams)

$\geq 0.927$

Pawer dissipated in duct, $P_{L D}(k W)$

740

Duct dimensions $(\mathrm{cm})$

$a_{0}$

60

$b_{0}$

35

$a_{1}$

35

${ }^{b}$

19

L

225

Duct dimensions (deg)

$\alpha$

6.34

Bo

4.07 
wall degassing. The design of the ouct wall is shown in Fig. 3-69. Because such an arrangement will limit wall outgassing, it may be assumed to be cunstant throughout the entire duct.

0 ther considerations are sputtering and heat loav. Bombardment of the auct wall by energetic particles will cause wall face erosion. The thickness of the cooled shield should be adjusted so that it remains a lifetime component. The total heat generated within the duct is close to $800 \mathrm{kw}$. This heat load is not uniformiy distributed, but varies along the duct and depends on the direction of the magnetic field, Cooling lines have to be carefully laid out to prevent local overheating.

List of Parameters. Table 3-26 shows requirements and final design parameters. They are related to Figs. 3-63 and 3-70.

Power Absorbing Surfaces. Of the $5.6 \mathrm{NW}$ of ion bean power delivered by an ion source, $2.3 \mathrm{MW}$ will be absorbed as neutral-beam particles in the plasma. The rest of the ion- and neutral-beam power will be intercepted and must be dissipated on various surfaces. While the power density on parts like the neutralizer ano beam duct walls is not especially high and can be handled with conventional water-cooling, other surfaces like the eages of the beamedge-aefining collimator are subjected to high power density and require special attention. The probiem is particularly severe for the ion oumps, which must handle about $2 \mathrm{MW}$ per ion source, and for the shine-through neutral-beam dumps, which receive that part of the neutral beam not absorbed by the plasma. While the total power received per single beam is less than $1 \mathrm{MW}$, the power density is high because of the partial spatial overlap ci the four beams contained in one beanline.

Three main issues must be addressed:

- Maximum power oensity that can be acsorbed safely.

- Lifetime limited by sputtering and erosion.

- Tritium diffusion into the cooling water.

Beam-Dump Lifetime. Beam-dump lifetime is limited by erosion of the front surface produced by sputtering. Beam dumps intended to dissipate high-power heat fiux usually have thin front walls to facilitate efficient heat transfer. Since the dump coolant is highly pressurized, only limited erosion of the front wall can be tolerated, usually $10 \%$, but no more than $30 \%$ 


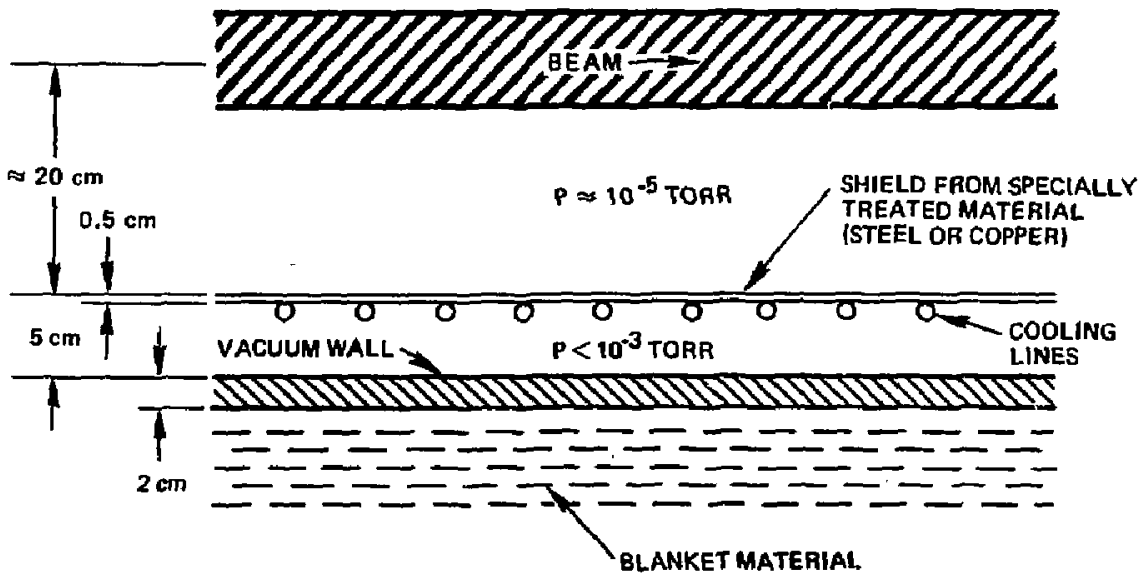

Figure 3-69. Detajl of duct wall. 


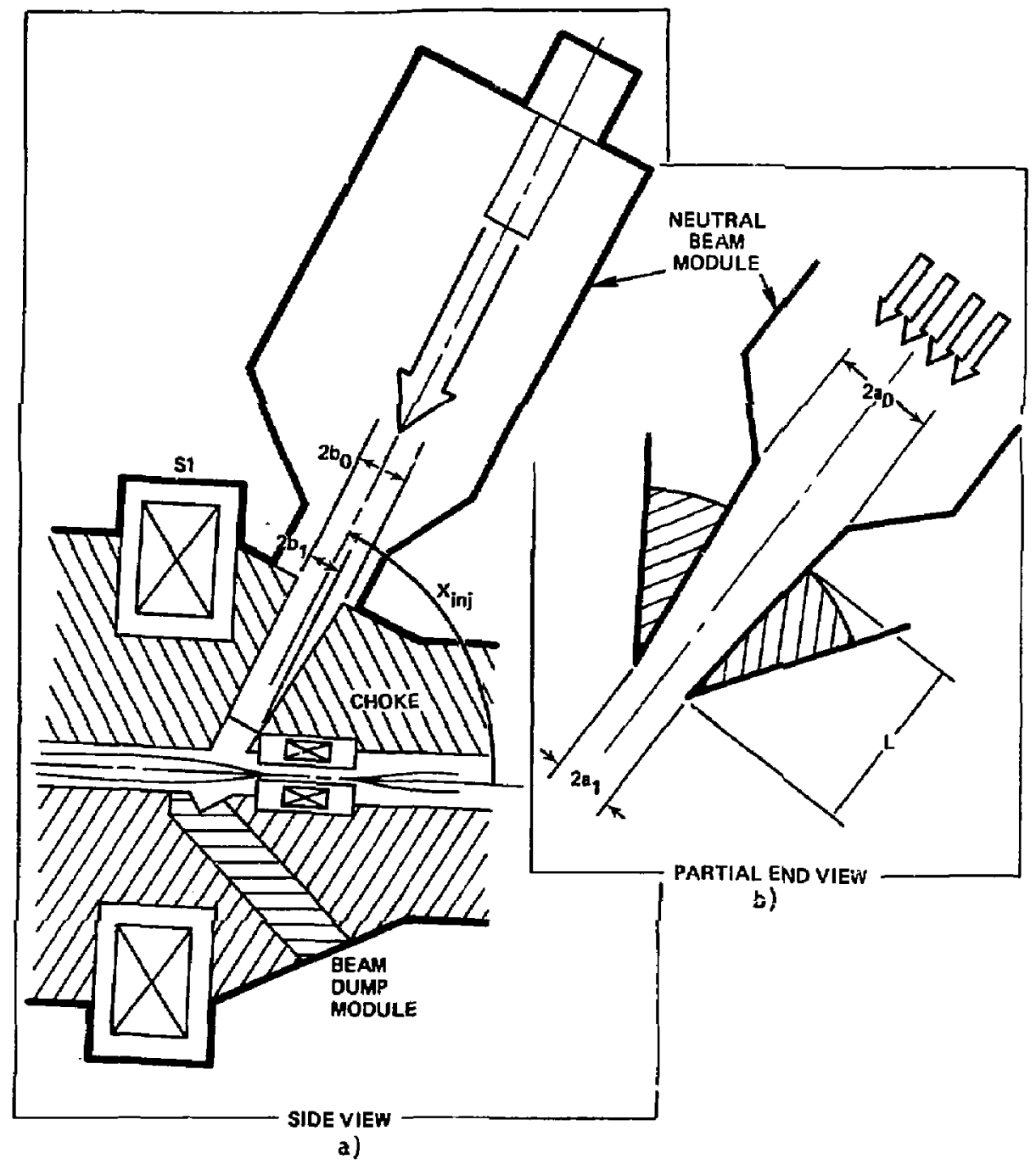

Figure 3-70. Urift-duct final design. 
nf tine initial wall thickness. Beam-dump lifetime can be estimated easily if a erosion rates are known.

$I^{f} \gamma$ is a sputtering yield (i.e., the average number of particles removej from the first wall for every particle arriving) and $j_{\text {inc }}$ is the incident current density, the rate of arriving particles per unit area is then

$R_{a}=\frac{j_{\text {inc }}}{e}\left[s^{-1} \mathrm{Cin}^{-2}\right]$,

where $\mathrm{e}$ is the elementary charge. The wall material particle-removal rate is then given as

$R_{w}=\frac{j_{\text {inc }}{ }^{T}}{e}\left[s^{-1} \mathrm{~cm}^{-2}\right]$.

The number of particles per unit volume of wall material is $\rho N / M$, where $\rho$ is oensity, $N$ is the Avagadros number, and $M$ is atonic mass. Then the erosion rate can be expressed as

$r_{w}=\frac{R_{w}}{\rho N / M}=\frac{j_{i n c^{T}} M}{e^{N} p}\left[s^{-1} \mathrm{~cm}\right]$.

Assuming the front wall has an initial tnickness $j$ of shich maximal fraction $f$ may be eroded, the lifetime of the oump can be written

$T_{\text {life }}=\frac{f a}{r_{w}}=\frac{f d \sigma N \rho}{T j_{\text {jnc }}^{M}}[s]$.

So far normal incidence is assumed.

The sputtering yield is a strong function of incident angle. Particles entering the dunp material at large angles with respect to the surface normally aeposit most of their energy ano transfer most of their momeritum to substrate atoms near the surface, thereby increasing sputtering yield. To account for this effect, an angle-dependent correction factor $A(\alpha)$ is intraduceo.

The expression for lifetime then becomes

$T_{\text {life, } \alpha}=A(\alpha) T_{\text {life, }} 90 \mathrm{deg}$. 
As seen from $F i g .3-71, A(\alpha) \approx(\cos \alpha)^{-1}$.

If $E_{a v}$ is the average energy of arriving particles and $P_{\max }(\alpha)$ the maximum power density to be dissipated by the dump, then

$j_{\text {inc }}=\frac{P_{\text {max }}(\alpha)}{E_{a v}}$.

Thus

$T_{1 \text { ife }}(h r s)=\frac{A(\alpha) \text { fdeN } p E_{a v}}{3600 \mathrm{~T} P_{\max } M}$.

Note that $P_{\max }(\alpha)=P_{\max } \cos \alpha$. Ir view of the cosine dependence for $A(\alpha)$, it is clear that there is nothing to choose in terms of $\alpha$ to prolong $T_{\text {Tife; }}$ i.e. $T_{\text {ife }}=$ const( $\alpha$ ).

It turns out that the most reasonable choice for front wall material is tungsten, Assuming $d=2 \mathrm{~mm}, \alpha=60 \mathrm{deg}, f=0.1, p=2 \mathrm{~kW} / \mathrm{cm}^{2}$, $E_{\partial V}=80 \mathrm{keV}, M=183, \rho=16.6 \mathrm{~g} / \mathrm{cm}^{3}$, and $\gamma=4 \times 10^{-4}$, the resulting dump lifetime is 2425 hours, i.e., 101 full power days. This is the equivalent of about one calendar year. Such a lifet ime is quite rerisonable.

Because tungsten is a high-Z material, the total sputtering rate into the plasma becomes of great cancern. Assuming a total neuträl-beam current onto the plasma per machine, $I_{0}=1280 \mathrm{~A}$, and roughly a $25 \%$ shine-through factor, we have a total sputtering rate per machine and

$S_{\text {total }}=\frac{I_{0} K S}{e} T=9 \times 10^{17}\left[s^{-1}\right]$.

This is considered to be well below the dangerous thresnold; hence, using tungsten will not increase Bremstrahiung radiation. 


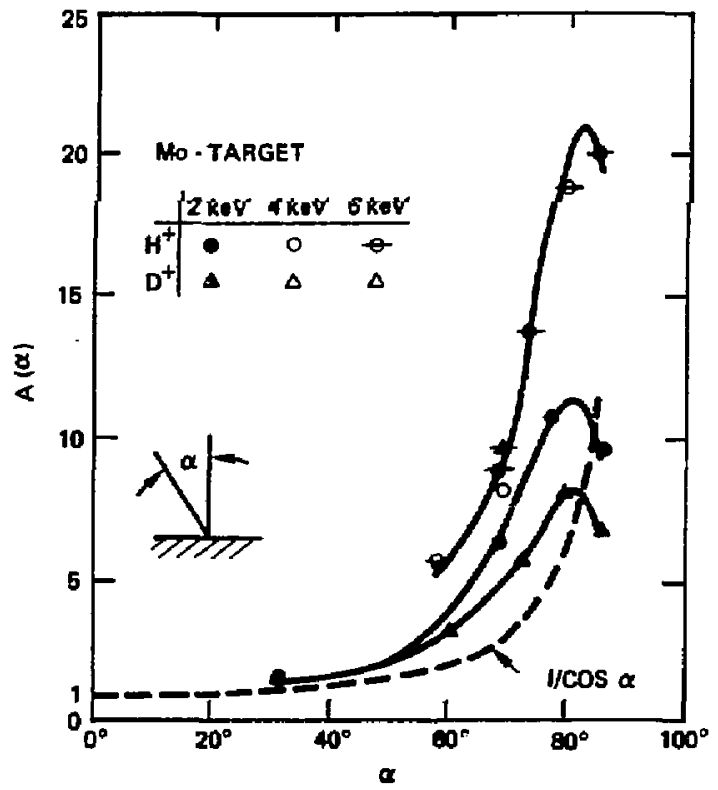

Figure 3.7?. Variation of sputtering yield with angle of inciden:e for $\mathrm{H}^{+}$, $\mathrm{O}^{+}$on Mo. The dashed line indicates a $\cos ^{-1}$ dependence. 
Power Density. The purpose of this section is to determine the feasibility of ion and shine-through dumps. The input is the set of source parameters (Section 3.3.1), and the output is the dump inclination angle required to limit power density at the surface to less than $2 \mathrm{~kW} / \mathrm{cm}^{2}$. Because this alone is not sufficient for the ion pump, other methods like beam defocusing are considered.

The shine-through dump is easiest to analyze. In the model used, four beams are directed onto the plasma, and the shine-through beams over ap on a single, common dump, here just a flat plate (Fig. 3-72). This configuration is used for each neutral beamline. The power density distribution for eacin of the four beams is obtained by using a formula developed by pittinger. 20 Beam absorption in the plasma varies as a function of plasma, adius. Dependence on $x$, the beam local coordinate, is shown in Fig. 3-73.

Define a shine-through coefficient

$K_{s}(x, y)=1-$ absorption $(x, y)$.

Then the shine-through power distribution for each beam is given by

$P_{S T}(x, y, z)=P_{N}(x, y, z) K_{S}(x, y)$.

where $P_{N}$ is the power density of the incident neutral beam, Now there are four beams incident on a dump located at distance $d_{0}$ from the plasina and inclined at angle $\alpha$ with respect to the plasma axis (see Figs. 3-74 and 3-75).

For a beam with angular displacement $\delta$,

$$
\begin{aligned}
& x=(\xi-d \tan \delta) \cos \delta, \\
& y=\pi \cos \alpha, \\
& z=z_{f}+\frac{d}{\cos \psi},
\end{aligned}
$$




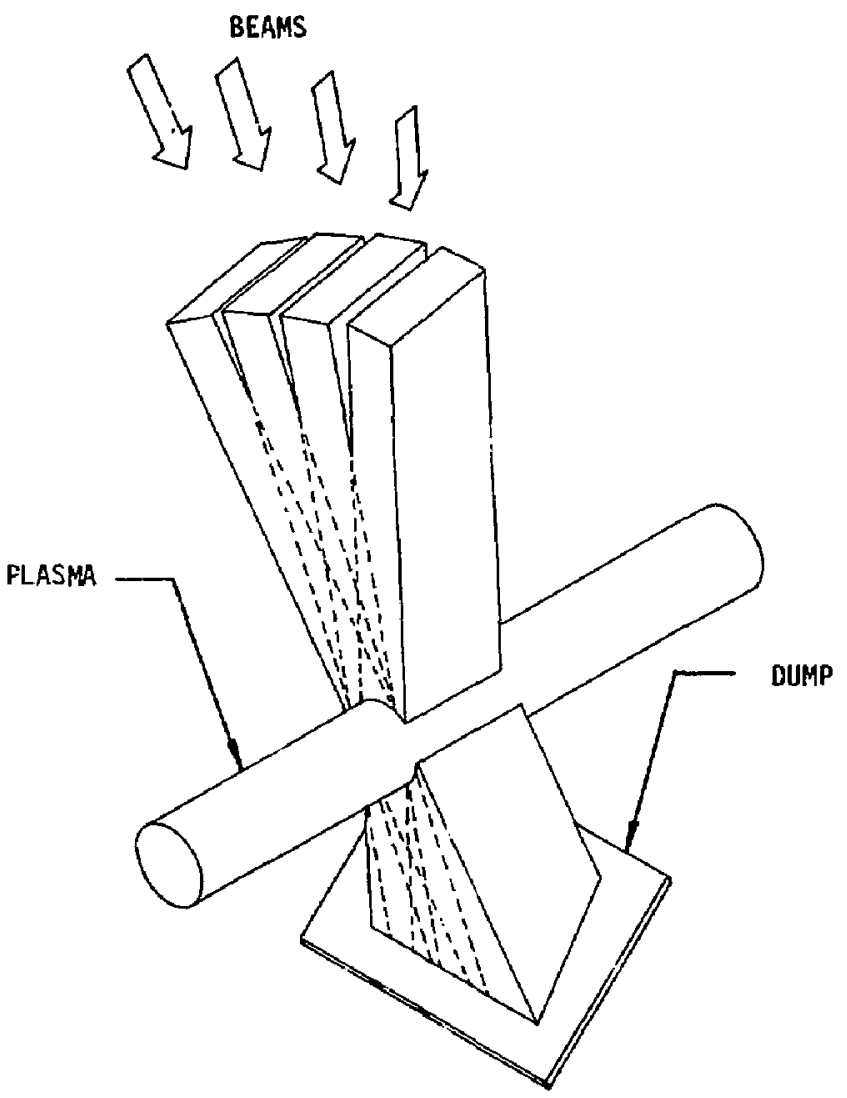

Figure 3-72. Beams intersecting plasma. 


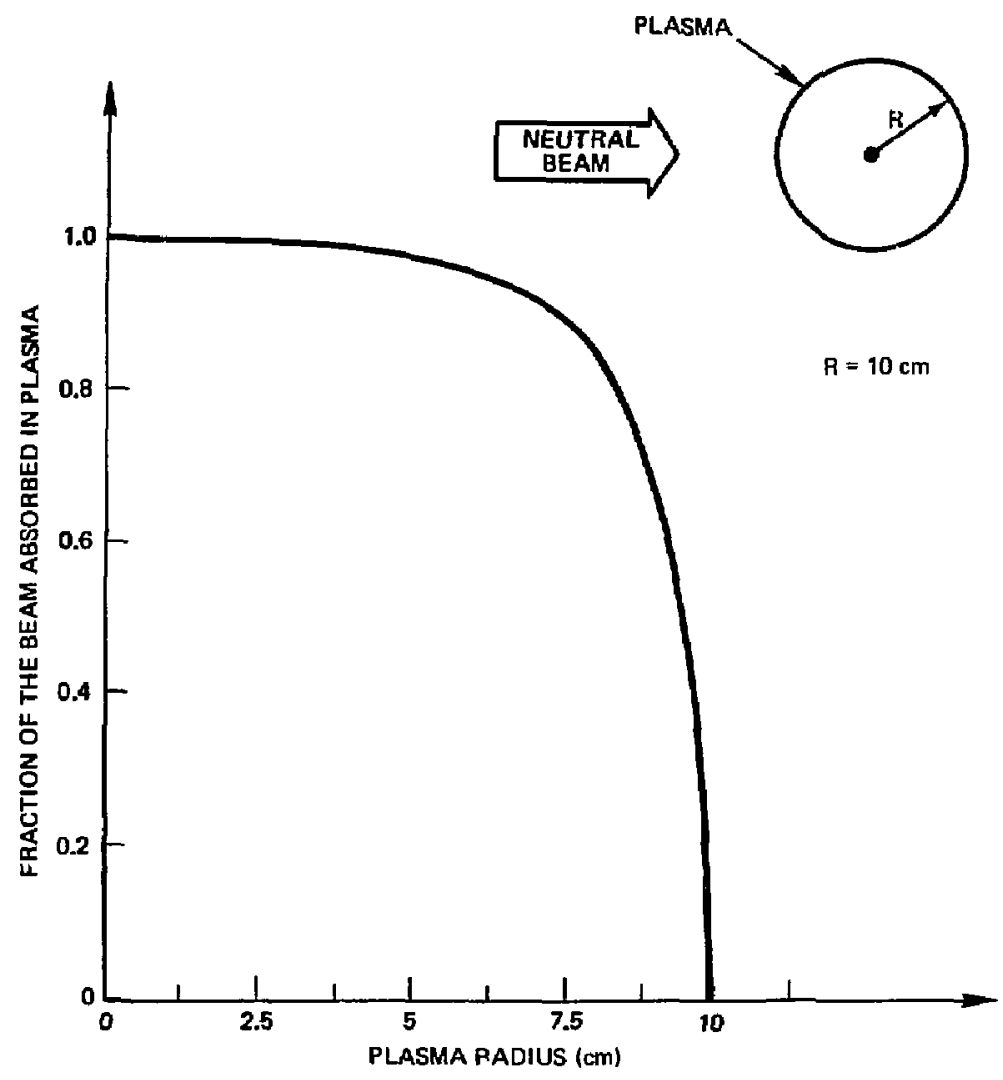

Figure 3-73. Beam absorption in plasma vs plasma radius ( $\equiv$ local coordinate $X$ ). 


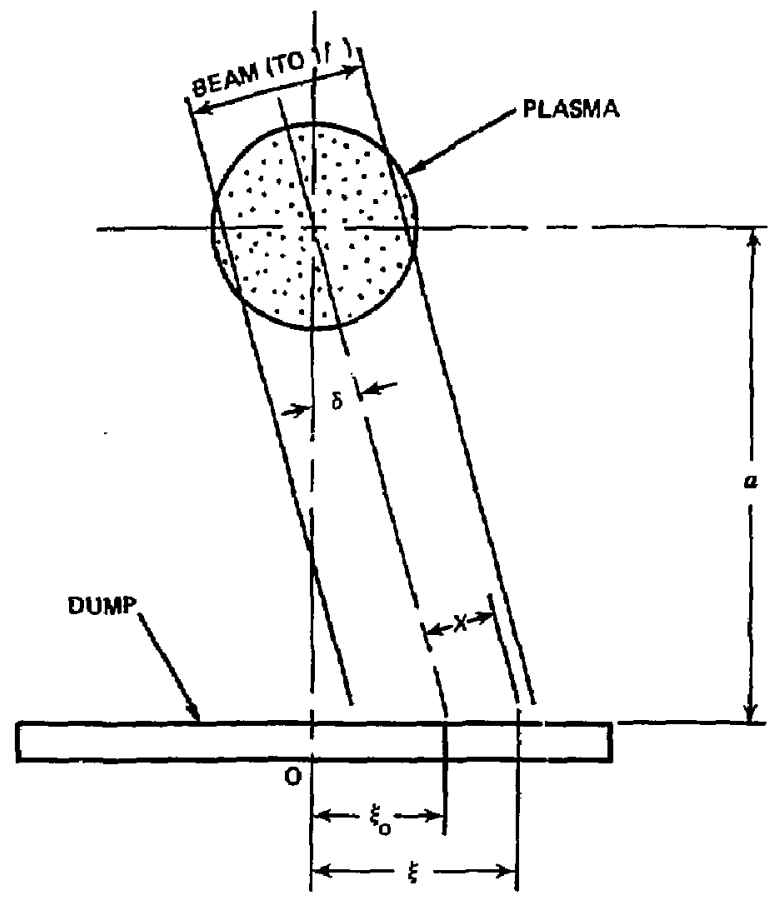

Figure 3-74. Beam vs dump geometry in $x-2$ plane. 


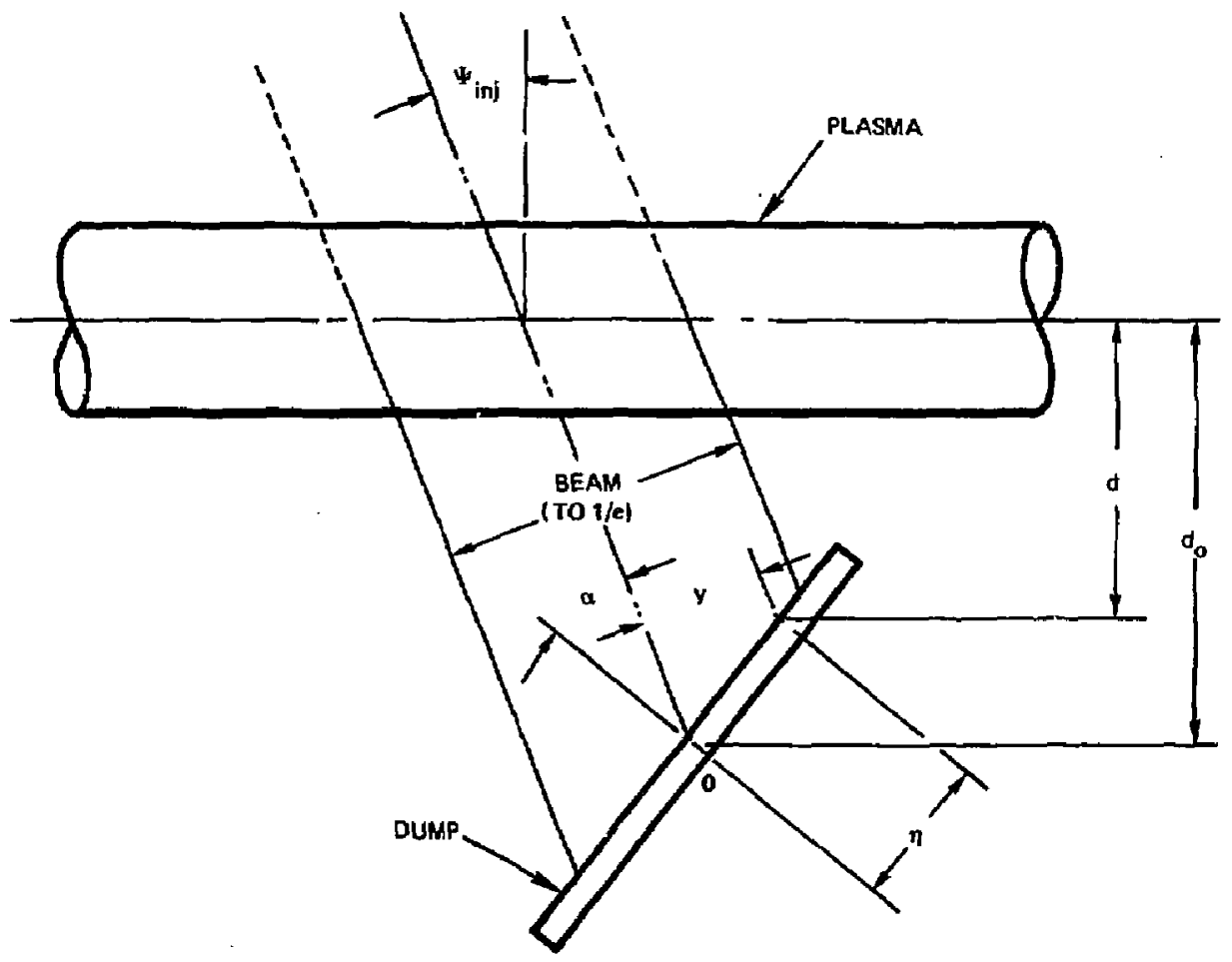

Figure 3-75. Beam vs dump geometry in $Y-2$ plane. 
and

$a=a_{0}+y \sin (\alpha+v)$.

Then, in the $(5, n)$ coorainate system of the oump, the power density distribution is

$P_{\mathrm{NU}}\left(\xi,,^{n}\right)=\left(\frac{\cot ^{2} \delta+\cot ^{2} \alpha}{\left.\cot ^{2} \delta+\cot ^{2} \alpha+\right]}\right)^{1 / 2} \sum_{\text {beams }}^{4} P_{S T}(x, y, z)$.

For small $\delta$ 's, this can be simplifieo to

$P_{\text {MD }}(u, b)=\cos a \sum_{\text {beams }}^{4} P_{S T}(x, y, z)$.

A computer plot of this result for the TDF beamline is shown in Fig. 3-76. It relates to the dump quadrant shown in $\mathrm{Fig.} \mathrm{3-77,} \mathrm{which} \mathrm{represents} \mathrm{the} \mathrm{case}$ with the highest power density. The incidence angle is $\alpha=75 \mathrm{deg}$.

The power density everywhere is less than $2 \mathrm{~kW} / \mathrm{cm}^{2}$ and, except for a few hot spots, is less than $1 \mathrm{~kW} / \mathrm{cm}^{2}$ throughout most of the surface. Note that the incidence angle a that was previously assumed to be 60 deg must be increas: 1 to at least $75 \mathrm{deg}$, shortening dump lifetime from 110 to 52 full power days. To prolong dump life, it is necessary to increase either the front wall thickness or the allowable erosion.

The situation for the ion dump is somewhat oifferent. The ion beam is first reflecteo by a magnet before it impinges onto the oump. Each of the four beams within a beamline has its own ion dump. For this analys is, it is sufficient to assume initially that the reflection does not affect any beam parameter except perhaps oirection and lateral displacement, Hence, it is possible to assume that the dump is placed a oistance $z_{i d}$ from the source and that the power aensity distribution is calculated using

$P_{i a}(x, y)=P_{i, t o t}\left(x, y, z_{i 0}\right) \cos \alpha$,

where $P_{i, t a t}$ is the incident ion power-density distribution ano $\alpha$ is the inciderse angle. To bring $P_{i d}$ under the $2-\mathrm{kw} / \mathrm{cm}^{2}$ 1imit everywhere 


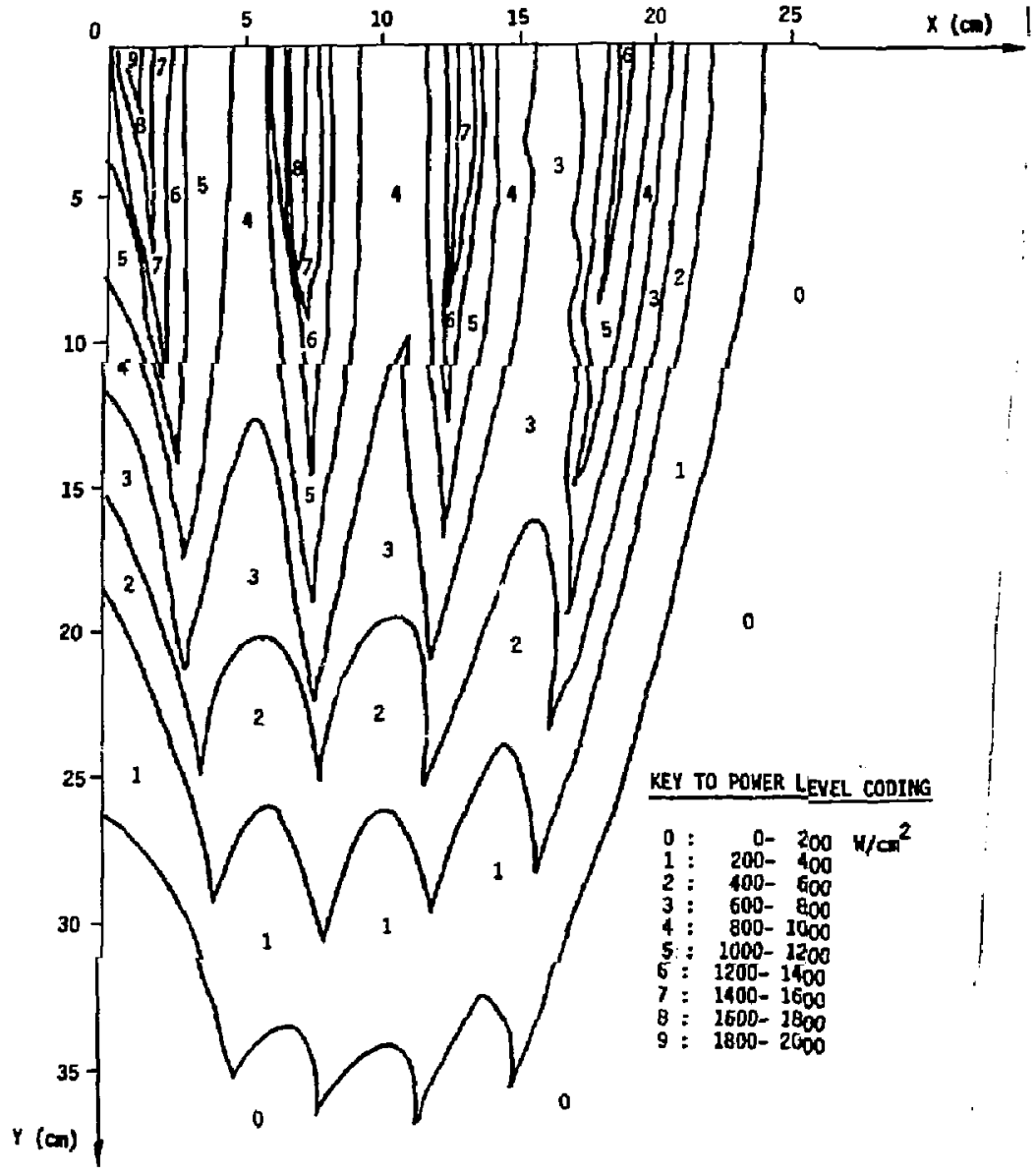

Figure 3-76. Power-density distribution on dump face (one quadrant). 


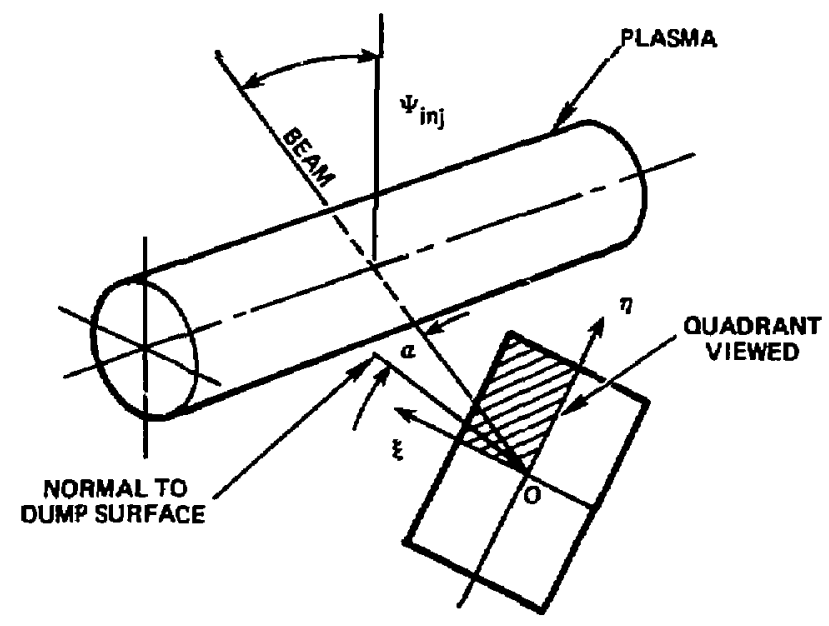

Figure 3-77. Piotteo quadrant location. 
requires that $\alpha$ be rather large, about 85 deg. That, however, is not acceptable because of the orastically shortened dump lifetime. The problem can be resolved, however, by taking advantage of the reflection magnet. Magnetic defocusing of the ion beam would yield reduced power densities in the dump area, and such an approach is indeed suitable for the NBIs. The defocusing model presented uses strictly geometrical optics and ignores the fringing field of the magnet.

Consider a magnet with pole pieces slanted at angle $\Omega$ where the ion beam exits (Fig. 3-78).

Let $w$ be the displacement from the center of a Gaussian beam where power density drops to $1 /$ e of its peak value. Mark the center of the beam as a ray $C$ or $C^{\prime}$, rays displaced from $C$ by $w$, and by $y$ as $A, A^{\prime}$ and $B^{\prime}\left(B^{\prime}\right)$, respectively. The sloped pole pieces are designed so that the reflected ray $A$ ' is unaffected by oefocusing (Fig. 3-79).

The task is to calculate the power density at the pump located a oistance $z_{i a}$ from the source (measured along ray $C^{\prime}$ ). Since the separation of the dump and the magnet is $z_{m d}$ and that of the source ana the magnet is $z_{m}$ then, for small $\Omega$,

$z_{i c}=z_{m}+z_{m d}+\pi r_{L}\left(D^{+}\right)$,

where $r_{L}\left(D^{+}\right)$is the Larmor radius of $D^{+}$specie. Because of the large focal length of the source, the convergence of rays $A, B$, and $C$ may be ignoreo. To locate a given ray on the dump it is necessary to find $\phi$. First,

$\tan \Omega=\frac{u_{0}}{2 r_{L}+y-q}$

where

$q=r(1+\cos \rho)$,

$u=r_{L} \sin \Phi$. 


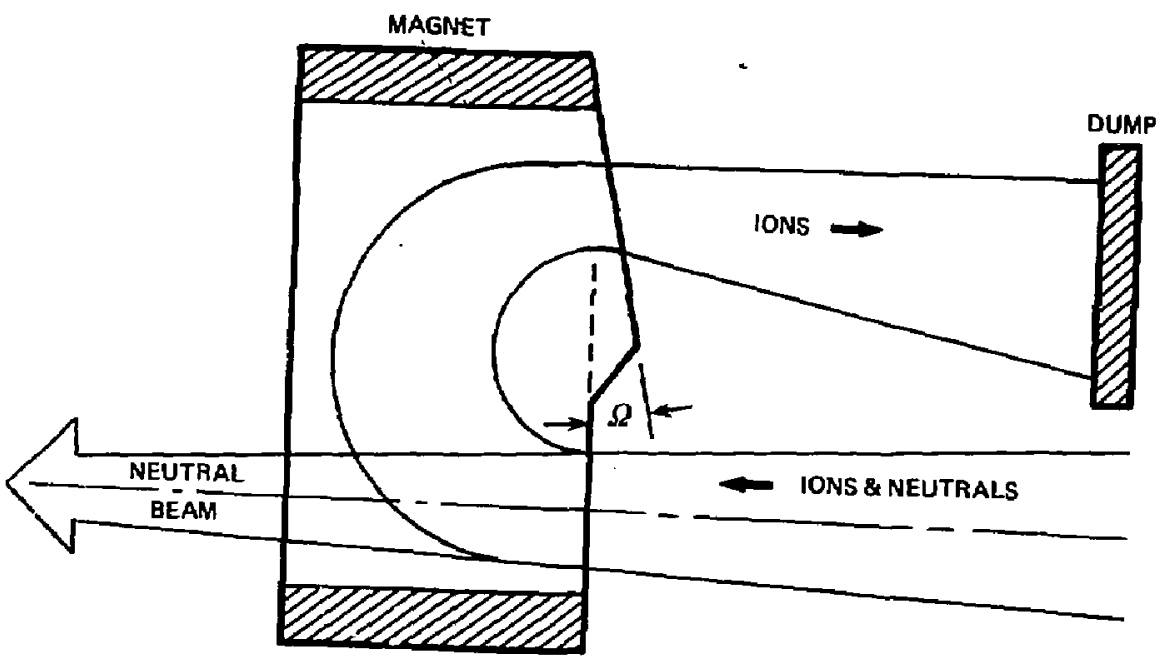

Figure 3-78. Defocusing of ion beam. 


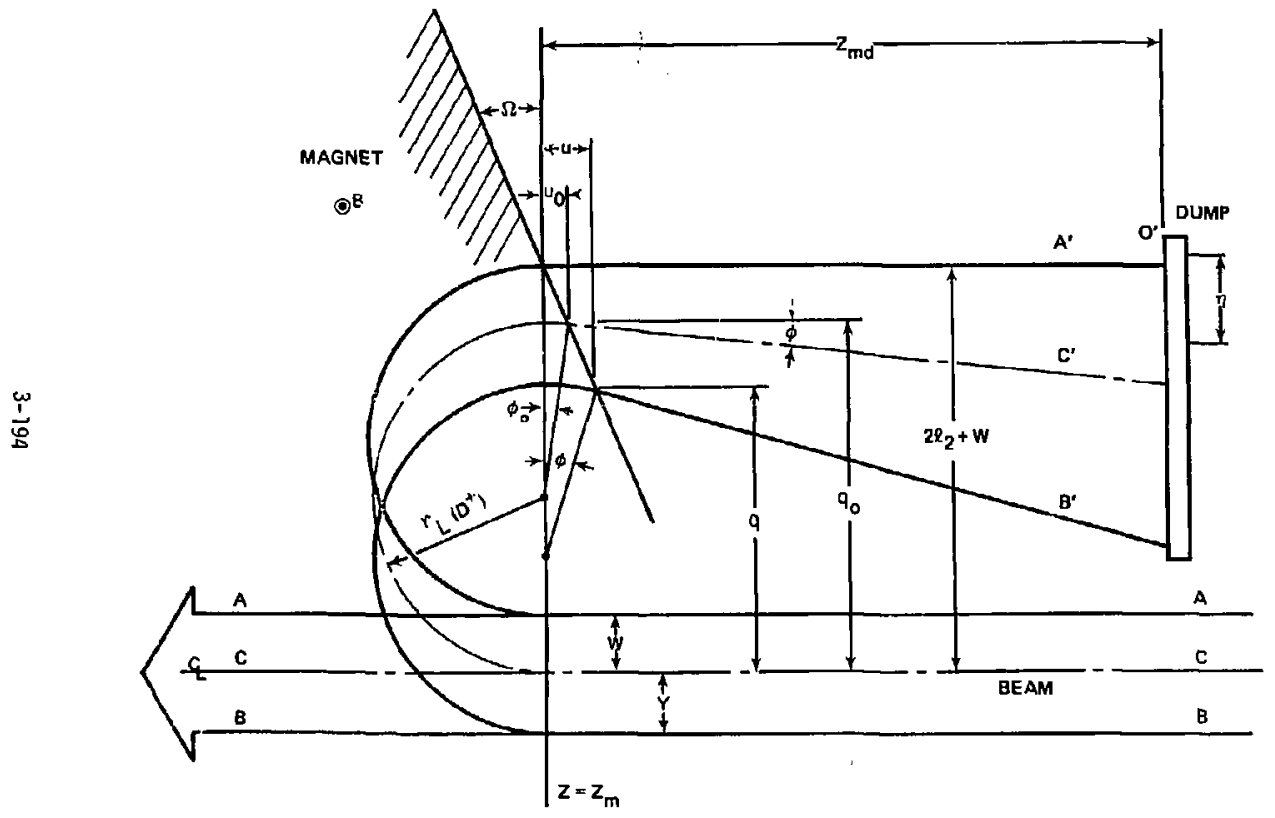

Figure 3-79. Defocusing model. 
Substituting these into the equation

$r_{L}^{2}=u_{o}^{2}+r_{L}^{2} \cos ^{2} o$

yields the quadratic equation

$K^{2}-2 K S^{2}\left(1+\frac{y}{r_{L}}\right)+s^{2}\left(1+\frac{y}{r_{L}}\right)+s^{2}-1=0$,

where $K=\cos \Phi_{0}$ and $S=\sin \Omega$. Solving for $K$ results in

$k=1+\frac{y}{r_{L}}\left[s^{2} \pm \sqrt{s^{4}-s^{2}+\left(1-s^{2}\right)\left(\frac{r_{L}}{r_{L}+y}\right)^{2}}\right]$.

Let $(\xi, n)$ be a coordinate system of the ion dump with origin on ray $A^{\prime}$.

Then

$$
\begin{aligned}
P_{i d}\left(\xi,,^{n}\right) & =P_{i, \text { tot }}\left(x, w-y+z_{m d} \sin \rho\right) \cos \alpha \\
& =P_{i, \text { tot }}\left(x, w-y+z_{m d} \sqrt{1-k^{2}}\right) \cos \alpha,
\end{aligned}
$$

where $\alpha$ is again beam incjdence angle onto the surface. A plot of $P_{i a_{2}}(\xi, n)$ for $\alpha=$ TBD is shown in Fig. 3-80. Note that everywhere $P_{i a} \leq 2 \mathrm{kw} / \mathrm{cm}^{2}$ as required.

Collimators. There are two sets of collimators in each beamline--front and rear (their locations with respect to the plasma, shown in Fig. 3-81).

Collimators clip the edges (wings) of the beam that would otherwise impinge on the beam duct, causing excessive heating and increasing the gas load. In essence, each collimator is an aperture with well-cooled eages that trim the beam to the desired profile. A major constraint in collimator design is the incident power density to which the edges may be exposed. This number is usually under $2 \mathrm{kw} / \mathrm{cm}^{2}$.

The rear collimator clips the beam before it enters the magnet yoke. It is estimated that the collimator edges will be exposed to $1000 \mathrm{w}^{\prime} \mathrm{Cm}^{2}$ power dersity with an aperture size of $15 \times 35 \mathrm{~cm}$ (Fig. 3-82). 


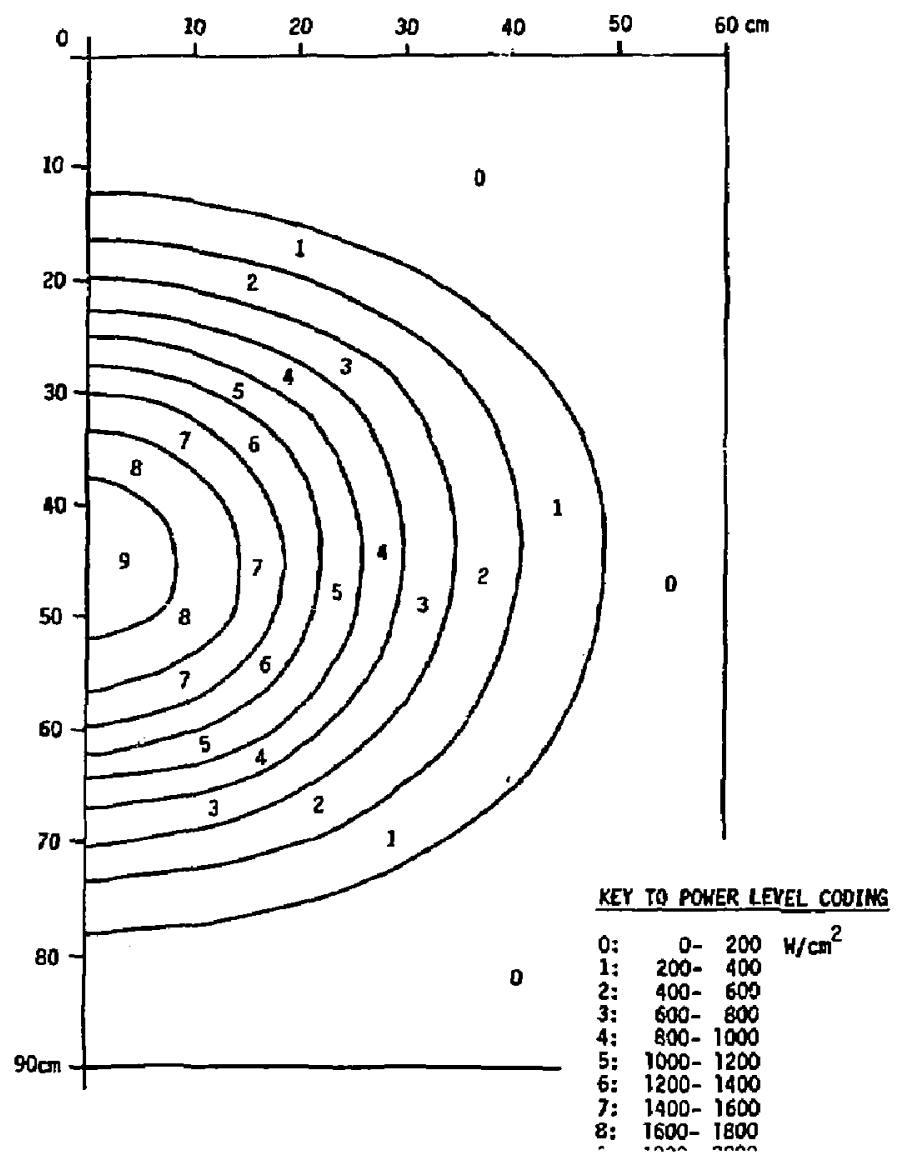

Figure 3-80. Power-density distribution on ion-dump surface after defocusing (one-half). 


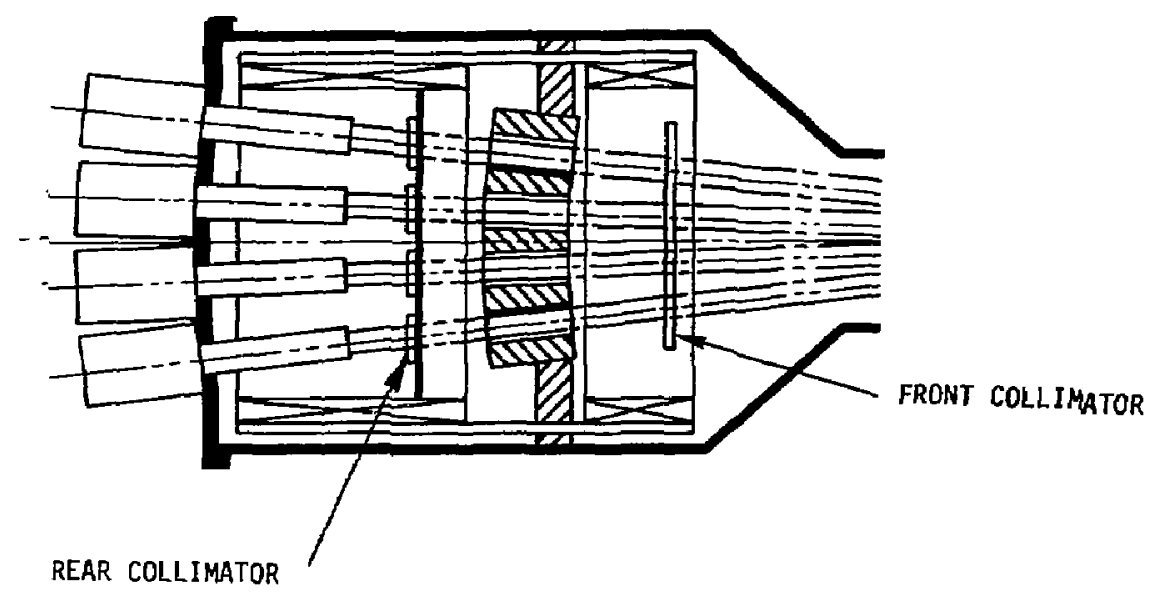

gure 3-81. Collimator locations. 


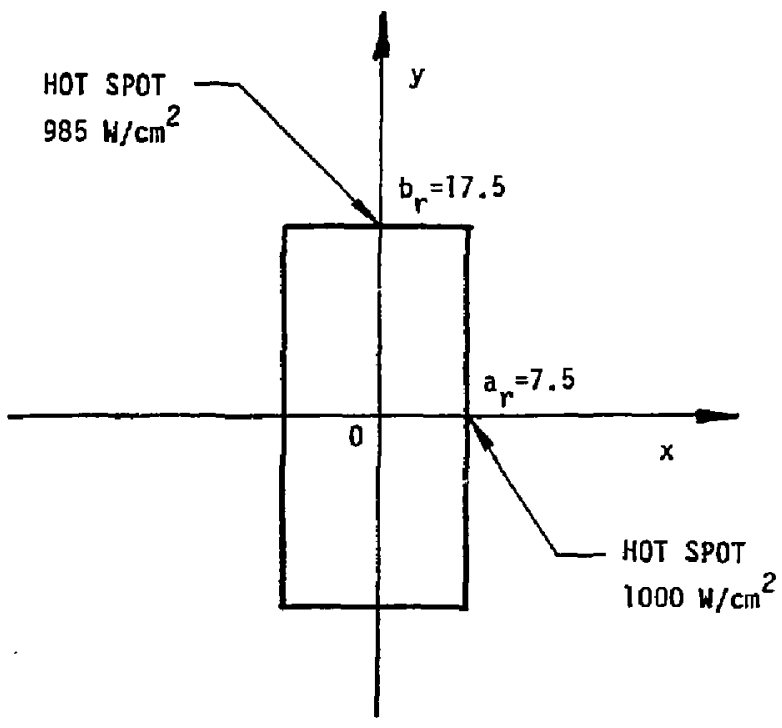

Figure 3-82. Rear collimator. 
The transmission factor is defined as the fraction of the incident power passing through the coilimator. It can be calculateo from Ref. 8 ,

$$
\begin{aligned}
& T_{R C}=\frac{z_{r c^{2}}^{2}}{{ }_{X_{\text {XYO }}}^{2}}\left(A \operatorname{erf}(A)+\frac{1}{\sqrt{\pi}} e^{-A^{2}}-B \operatorname{erf}(B)-\frac{1}{\sqrt{\pi}} e^{-B^{2}}\right)^{2} \\
& \times\left(C \operatorname{erf}(C)+\frac{1}{\sqrt{\pi}} e^{-C^{2}}-0 \operatorname{erf}(D)-\frac{1}{\sqrt{\pi}} e^{-0^{2}}\right),
\end{aligned}
$$

where

$$
\begin{array}{ll}
A=\frac{g X_{0}+a_{r}}{2 \theta}, & 0=\frac{h y_{0}-b_{r}}{Z \theta}, \\
B=\frac{g x_{0}+a_{r}}{Z \theta}, & g=\frac{Z}{f y}, \\
C=\frac{h \gamma_{0}+b_{r}}{Z \theta}, & h=\frac{z}{f y} .
\end{array}
$$

The power lost at the rear collimator is given by

$P_{L r c} \simeq P_{S}\left(T_{N}-T_{r}\right)=5600 \times 5.197 \times 10^{-3}=29.15 \mathrm{~kW}$.

The gas generated is equivalent to $3.21 \times 10^{-5}$ Torr-hiters/s.

The parameters that influence the design of the front collimator are different from those that influence the rear collimator. Ideally, the collimator would pass only that fraction of the beam that is airecteo onto the plasma and block everything else. However, because the aperture is located an appreciable distance from the plasma (as a result of beamline geometry) and source size is finite, it is apparent from Fig. 3-83 that this ideai situation cannot be achieved.

It turns out that attempts to reduce $x_{\text {pmax }}$ (Fig. 3-83) rest.]t in a loss of beam reaching the plasma. A careful trade-off between these two effects 


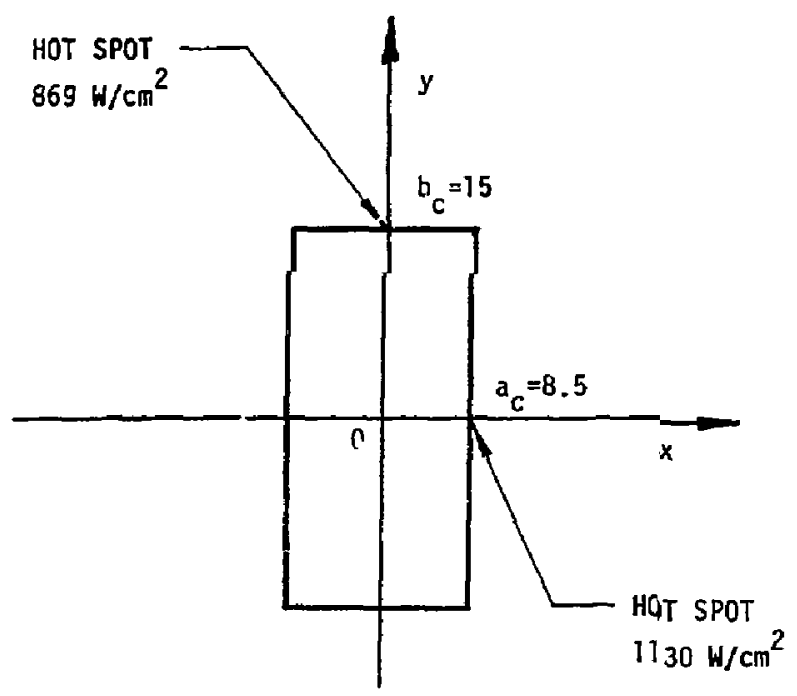

Figure 3-83. Front collimator. 
was required, and the final design for the front collimator yielded an aperture size of $17 \times 30 \mathrm{~cm}$ (Fig. 3-84).

The transmission factor (Eq. 3-64) associated with these dimensions is $T_{f c}=0.977702$. Consequently, the power lost at the collimator is

$P_{L r c}=P_{N_{t o t}}\left(T_{r c}-T_{f c}\right)=41 \mathrm{kw}$,

while the equivalent gas load is $4.26 \times$ Torr-liters/s.

Another collimator is located in the beamine, namely the ion colimator that trims the reflected ion beam incident on the ion dump. Its sole purpose is to keep any of the beam from falling outside the aump area. The design of this collimator is not critical. If ion beam defocusing is ignored, its size is $17 \times 29 \mathrm{~cm}$; with defocusing, it will increase to about $17 \times 40 \mathrm{~cm}$.

Tritium Uiffusion. In Section 3.3.1 we showed that four of the eight beamlines inject tritium. Energetic tritons will be implantea in both the ion and shine-through dumps. The aiffusion of the implants into cooling water ara their accumulation could becone a significant radiological hazard. Tritium recovery from water is a rather costly process, but it inay be requirea. In this section we attempt to determine the magnitude of the problem.

The following moael was used in the diffusion analysis. The beam oump is represented by a flat wall of thickness $t$ (Fig. 3-85), with a vacuum on one side and a pressurized coolant $\left(\mathrm{H}_{2} \mathrm{O}\right)$ on the other. A beam (ion or neutral) of current density $j_{\text {total }}$ impinges on the vacuum side of the dump at an incidence angle of $\alpha$.

Although the situation for both the ion and shine-through cumps is postulated to be essentially the same for the purposes of this analysis, the ion ouinp has a larger area (it absorbs twice the power) than the shine-through oump. As a result, only the shine-through dump needs to be treated.

Diffusion is a temperature-dependent process. Starting from the power density distribution plot in Fig. 3-80, it is possible to determine the temperature profile in the wall.

$T(\xi, \pi, \zeta)=T_{c}+\frac{\zeta}{\lambda} P(\xi, n)$

where $(\xi, n, \zeta)$ is the coordinate system of the oump, $\lambda$ is the thermal 


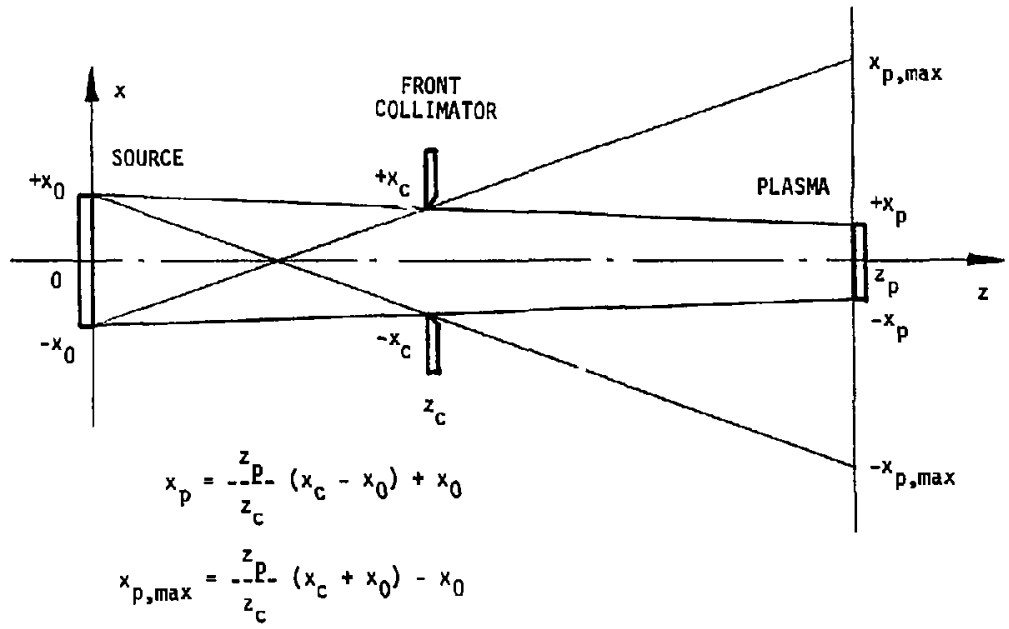

Figure 3-84. Line-of-sight optics in X-plane. Y-plane optics analogical. 


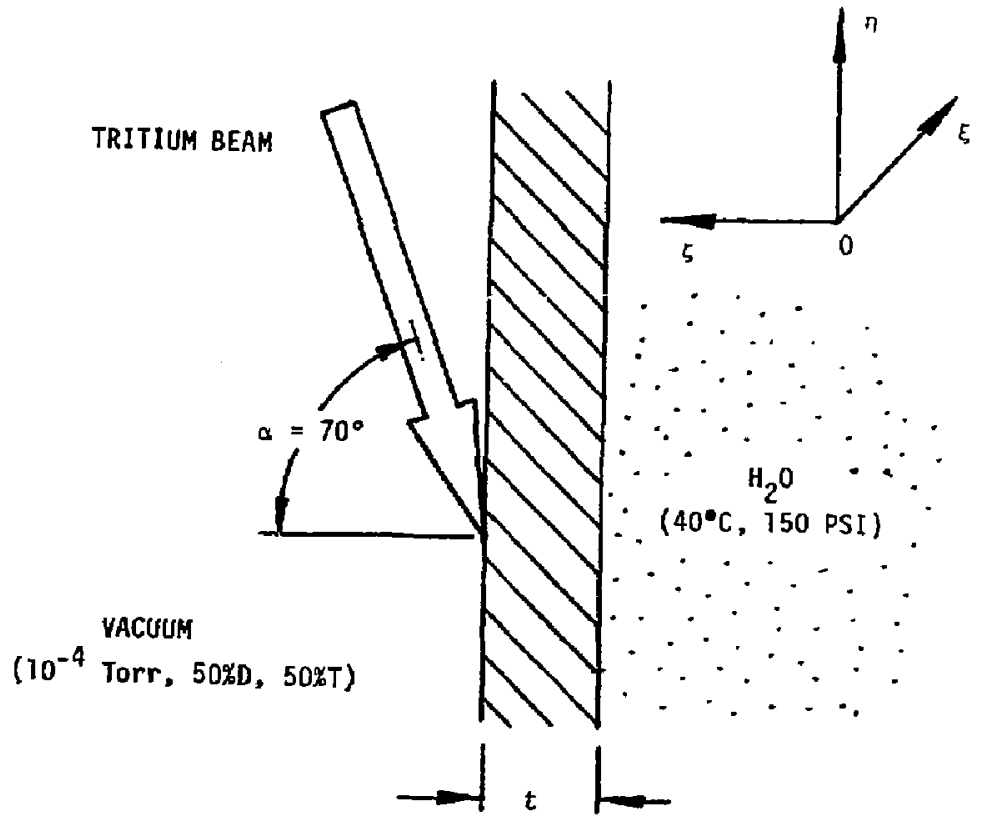

Figure 3-85. Diffusion model. 
conductivity of the wall material, and $T_{c}$ is the wall temperature at the coolant side.

The current density distribution is given by

$j_{\text {tota } 1}(\zeta, n)=\frac{P(\xi, n)}{E_{a v}} \approx 1.587 \times 10^{-5} P(\xi, n)\left[\mathrm{A} \mathrm{cm}^{-2}\right]$,

and the energy spectrum of the beam particles used is $D^{+}(61 \%$ at $80 \mathrm{keV})$, $\mathrm{D}_{2}^{+}(25 \%$ at $40 \mathrm{keV})$ and $\mathrm{D}_{3}^{+}(14 \%$ at $27 \mathrm{keV})$. Finally, it was assumed

that the plate material is AMIRC (98\% copper) and that temperature $T_{c}$ is $\sim 150^{\circ} \mathrm{C}$.

These parameters were used as input for a aiffusion computer coue ${ }^{21}$; on the basis of these data, the tritium leakage rate into the cooling water was found to be approximately $4.8 \mathrm{mg} /$ day per shine-through dump. Because there are 4 shine-through and 16 ion aumps for tritiun beams and the ion dumps absorb twice the power, the total tritium leakage rate for the central cell beam injector dumps is $57.6 \mathrm{mg} /$ day, or $21 \mathrm{~g} / \mathrm{FPY}$. This figure is significant enough to require continuous tritium recovery from the cooling water.

\section{Vacuum System}

Approach. To limit beam losses resulting from reionization and scattering, the background gas density along the beam path must be kept at the lowest piessure attainable. This situation is complicated by the presence of several gas sources within the vacuum envelope of the injector, namely beam dumps, collimators, the neutralizer, and the arift duct. Most conventional neutral-beam injectors feature vacuum pumps (cryoparlels) in close proximity to the beamline axis. One advantage of this approach is high conouctance between the gas sources and pumps. As seen from Fig. 3-86, cylindrical cryopanels (front ano rear) completely envelop the beams, as well as all of the dumps. Cryopanel size is linited by the maximum size of the vacuum chanber; consequently pressure distribution inside the chamber is influenced by constraints on the external geometry.

The neutralizer represents by far the largest gas source. To intercept a large fraction of streaming thermal gas, a baffle is positioned some distance from the neutralizer end. Because of the gas buildup, it is advantageous to 


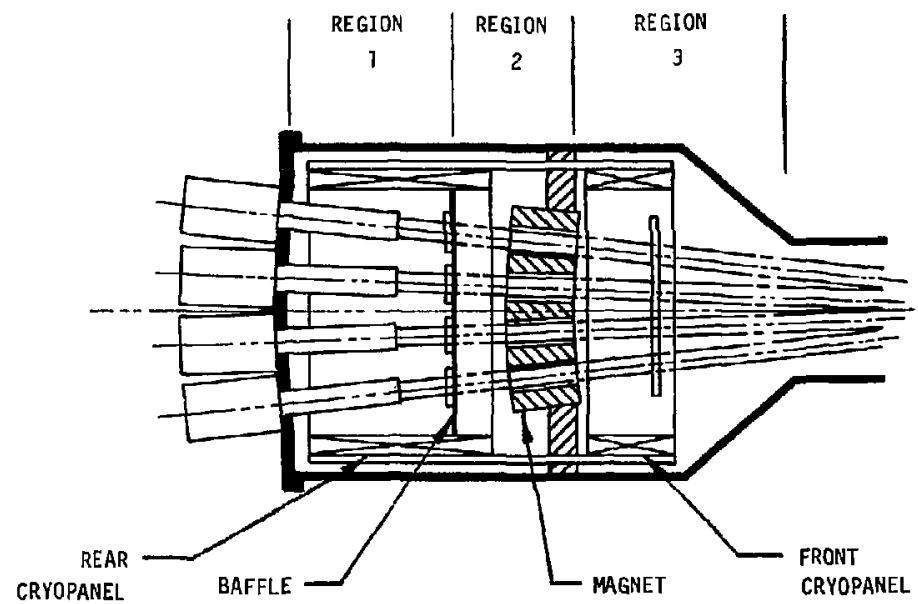

Figure 3-86. Vacuum-vessel sectioning. 
exteno this baffle radially all the way to the cryopanels, which effectively seals off this high-pressure section (Region 1) from the rest of the vacuum chamber. However, apertures in the baffle allow beam passage; therefore, some downstream leakage of gases cannot be prevented. The distance between the neutralizer end ano the baffle is a result of a trade-off between charge-exchange loss and gas sxreaming, both of which have to be minimized.

The reflection magnet, a large, bulky unit, constitutes a natural barrier within the beamline, subaividing the remaining vacuum chamber space into halves, Regions 2 and 3 (Fig. 3-86). The powerful ion beam is disposed of by directing it onto beam dumps in Region 1, causing yet higher pressure there. That leaves only small gas sources, such as collimators, ion shields, etc., in Regions 2 and 3 , which is a good prerequisite for higher vacuum. A complete list of gas sources and their magnitudes is given in Table 3-27.

Cryopanels. Pumping the beamline from air on startup is accomplished by a secondary vacuum system (a torepump). To remove excess gas from the chaniber and keep the pressure there at a suitable level, another means of puming must be provided. Only two candigates are suitable for this task: getter pumps and cryopanels. The gettering system is an attractive option because it does not liberate gases, it is insensitive to minor thermal variations, and it is totally immune to neutron bombardment. However, the deployment of gettering surfaces requires approximately 1.5 times the NBIS space required by cryopanels; as a result, cryopaneis were selected. A comprehensive comparison of getter pumps ano cryopanels is given in Table 3-28. As previously described, the central cell cryopaneis are cylindrical and envelop most of the gas sources. Each cryopanel has the geometry shown in Fig. 3-87. Hydrogen gas pumping occurs on the surface of the liquid helium panels. These panels are surrounded by liquid-nitrogen-cooled shields and louvers for protection against thermal radiation and to cool incoming gas molecules. Each of the front ano rear cryopanels is designed for continuous operation. Because of the need to occasionally liberate the gas frost from the liquid helium panels (aegassing process), the cylirider has been subdivided into six angular sections, five pumping and one degassing at any given time (see Fig. 3-88). The aegassing cycle proceeds as follows: Louvers on each segment to be degassed rotate on their supporting hinges until they close the communication path between the liquid helium panels and gas sources. Valve 1 


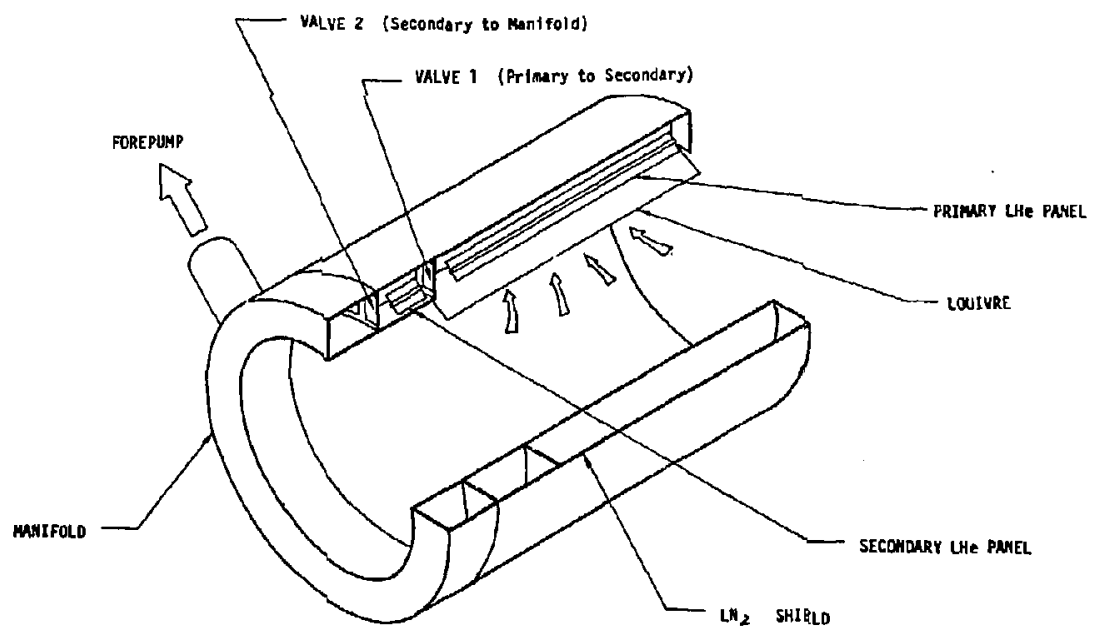

TDF - MBIS, CAYOPAMEL DETAIL

(MOT TO SCALE)

Figure 3-87. Cryopanel structure, showing TOF/NBIS ano cryopanel oetail (not to scale). 


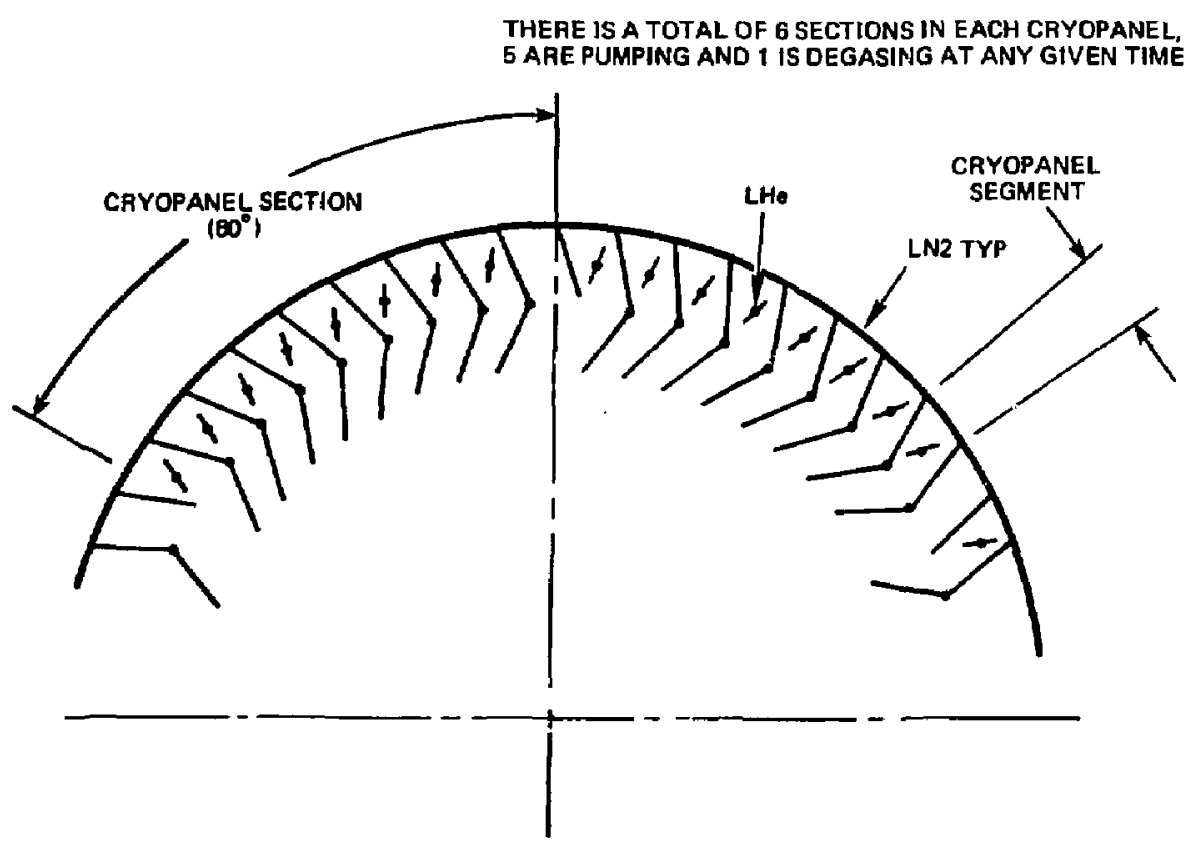

Figure 3-88. Cryopanel structure. 
Table 3-27. Gas sources and magnitudes.

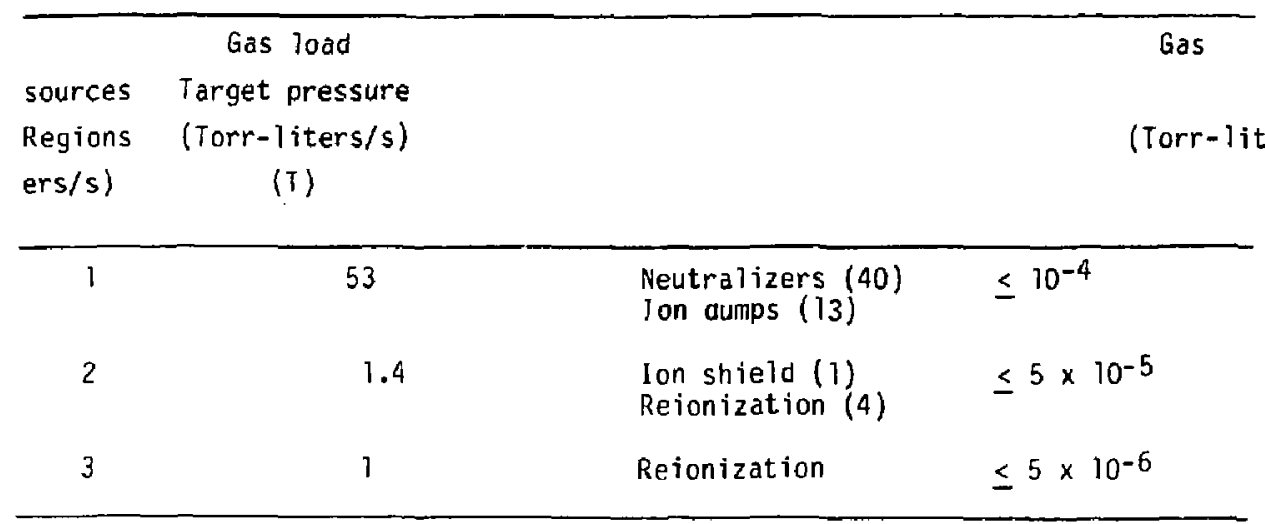


Table 3-28. Getter and cryopump beamine comparison.

\begin{tabular}{|c|c|c|c|c|c|c|c|c|c|c|}
\hline \multirow[b]{2}{*}{ Type } & \multirow[b]{2}{*}{$\begin{array}{l}\text { Minimumi } \\
\text { Degas Time } \\
\text { (Minutes) }\end{array}$} & \multirow[b]{2}{*}{$\begin{array}{l}\text { Degas } \\
\text { Fraction }\end{array}$} & \multirow[b]{2}{*}{$\begin{array}{l}\text { Ninimum } \\
\text { Cycle Time } \\
\text { (Minutes) }\end{array}$} & \multicolumn{2}{|c|}{$\begin{array}{l}\text { Power Heeded } \\
\text { Per Deaml ine }\end{array}$} & \multicolumn{2}{|c|}{$\begin{array}{l}\text { Operational } \\
\text { Temperature }\end{array}$} & \multirow[b]{2}{*}{ Configuration } & \multirow{2}{*}{$\begin{array}{l}\text { Pumping } \\
\text { Speed } \\
\text { of Panel 's } \\
\text { Projected } \\
\text { Area } \\
\text { L/S-Cm2 }\end{array}$} & \multirow[b]{2}{*}{ Panel Life } \\
\hline & & & & $\begin{array}{l}\text { During } \\
\text { Outgas } \\
\text { (m) }\end{array}$ & $\begin{array}{l}\text { Steady } \\
\text { State } \\
\text { (mi) }\end{array}$ & Outgas & Pumping & & & \\
\hline $\begin{array}{l}\text { SAES ST707 } \\
\text { Alloy Getter }\end{array}$ & 35 & 0.2 & 210 & 0.02 & 0.0005 & $500^{\circ} \mathrm{C}$ & $25^{\circ} \mathrm{C}$ & $\begin{array}{l}\text { WPI250/2 wafer } \\
\text { pump modules } \\
\text { arranged in } \\
\text { panel form }\end{array}$ & 4.1 & $\begin{array}{l}30 \text { up to air } \\
\text { exposures } \\
\text { allowed. } \\
1 \text { yr. life } \\
\text { when exposed } \\
\text { to } 1 \mathrm{TL} / \mathrm{s} \mathrm{o}^{2} \\
\text { or } 0.2 \mathrm{~T} / \mathrm{s}^{2} \\
\mathrm{~N}_{2}\end{array}$ \\
\hline $\begin{array}{l}\text { Cryogenic } \\
\text { LHe Panel } \\
\text { (LLML Type) }\end{array}$ & 7 & 0.2 & 42 & - & - & $18^{\circ} \mathrm{K}$ & $4.2^{\circ} \mathrm{K}$ & $\begin{array}{l}2 \text { louvered LN } \\
\text { cooled shroud } \\
\text { surraunding } \\
\text { LHe panels }\end{array}$ & 6 & Machíne \\
\hline
\end{tabular}


opens, and liquid helium is diverted from the primary panels into secondary liquio helium panels (Fig. 3-8\%). The resulting warmup of the primary panels causes gas previously trapped there to be transferred onto secondary panels. Unce the transfer is accomplished, vaive 1 closes, valve 2 opens, and liquid helium is diverted back from the secondary to the primary panels. The louvers can now be opened again, and pumping resumes. As the secondary pane 1 warms $u p$, the gas is discharged into a manifola and proceeds to the forepump. One meter of the type of cryopanel under consiceration has a pumping speed of $6 x$ $10^{4}$ liters/s. So from the gas load and pressure identification in Table 3-27 for regions 1,2 , and 3 , we obtain the following requirements on cryopane 1 size:

- Region 1: $8.3 \mathrm{~m}^{2}$ (actively pumping).

- Region 2: $3.3 \mathrm{~m}^{2}$ (actively pumping).

- Region 2: $8.7 \mathrm{~m}^{2}$ (actively pumping).

Gas-Pressure Distribution. Eackground pressure in the three vacuum regions was determinea from a set of linear equations relating to gas loads, conductances, pumping speeds and pressures at various points in the vacuum chamber. Each region was divided into naturally occurring sections, each of which was treated as a separate entity capable of comnuicating with neighboring sections (Fig. 3-86).

The model given below is based on gas flow balance. In steady-state the gas generated in any section equals a net gas outflow from that section. The inflow or outflow is governed by conductance and pressure difference with respect to neighboring sections or a cryopanel. Let $q_{1}, \ldots \ldots \ldots Q_{1}$ be the magnitudes of actual gas sources (dumps, etc.) in Sections 1 through 11 , respectively. Then

$Q_{n}=\sum_{k \neq m}^{11} c_{n_{k}}\left(P_{n}-P_{k}\right)+s_{n} p_{n}$,

where $C_{n k}$ denotes a conductance from the $n$-th section to the $k$ - $t$ h section, $P_{k}$ is the gas pressure in the $k-t h$ section and $S_{n}$ is the effective pumping speed of the cryopanels (if any) in the $n$-th section.

Simultaneous solution of this set yields the gas pressures in various sections. An overview of the situation is given in Table 3-:9. 
Table 3-29. Cryopanet area.

\begin{tabular}{lcll}
\hline Region & $\begin{array}{l}\text { Total gas 1oad } \\
\text { (Torr-jiter/s) }\end{array}$ & $\begin{array}{l}\text { Cryopanel area } \\
\left(\mathrm{m}^{2}\right)\end{array}$ & $\begin{array}{l}\text { Resulting } \\
\text { pressure } \\
\text { (Torr) }\end{array}$ \\
\hline 1 & 53 & 8.3 & $6 \times 10^{-5}$ \\
2 & 1.5 & 3.3 & $2.5 \times 10^{-5}$ \\
3 & 1 & 8.3 & $5 \times 10^{-6}$ \\
\hline
\end{tabular}


Cryogenic System. Energy removed from gas molecules, thermal radiation, and neutron heating constitutes a thermal load that must be continuously removed by the cryogens. Because large flow cross sections and generous fluid inventory are required for free convective flow, a forced-flow liquid helium and $\mathrm{LN}_{2}$ system was selected. Heat is deposited in both the $\mathrm{LN}_{2}$ and liquid helium.

Assuming an average neutron heating of $P_{N}<5 \times 10^{-4} \mathrm{~W} / \mathrm{cm}^{3}$ for aluminum, this yieids less than 150 and $50 \mathrm{~W}$ heat input, respectively, for the $L N_{2}$ and liquid helium cryopanels, with a total structural volume of $3 \times 10^{5}$ and $1 \times 10^{5} \mathrm{~cm}^{3}$. Thermal radiation from the beamline vacuum enclosure can be greatly reciuced by proper shielding; it is estimated at $350 \mathrm{~W}$. The radiation from "hot" beamline components (neutralizers, aumps, collimator) cannot be shieldea as effectively, and it represents an additional $1150 \mathrm{w}$. The bulk of the thermal radiation is oeposited into $\mathrm{LN}_{2}$ panels, with only about $8 \mathrm{~W}$ being deposited into the LHe system. There is virtualiy no gas condensation on the $\mathrm{LN}_{2}$ panels, while it accounts for about $6 \mathrm{w}$ heat input at the LHe panels. A detailed account of cryopanel parameters is given in Table 3-30.

Helium and nitrogen flow paths are similar, as can be seen from Figs, 3-89 and 3-90.

Magnetic Shield. Proper operation of the neutra! beamlines requires that the area between the points of initial ion generation (i.e., the discharge) ana the final point of separation of neutral-beam particles and residual ions (the reflection magnet) be practically free of external magnetic fields. In the arc chamber a magnetic field would influence primarily electron orbits, which can result in unwanied discharge characteristics and nonuniform plasma densities. It can also adversely affect the ion velocity distribution function at the extraction points. In the neutralizer, the straight-line ion trajectory must be maintained before neutralization. A deflection by perpendicular magnetic fields will result in beam defocusing and increased beam divergence. The beam exiting the neutralizer consists of an equilibrium mixture of ions and neutrals. A magnetic deflection of the ions in the space between the neutralizer and reflection magnet can, therefore, result in (1) beam loss as a result of the ionization of neutrals and (2) uncontrolled 
Table 3-30. Cryopanel parameters for each NBIS unit.

\begin{tabular}{|c|c|c|c|}
\hline Parameter & Region 1 & Pegion 2 & Region 3 \\
\hline Effective pumping area $\left(m^{2}\right)$ & 8.3 & 3.3 & 8.7 \\
\hline Net capture probability & 0.2 & 0.2 & 0.2 \\
\hline Neutron heating $\mathrm{LN}_{2} / \mathrm{LHe}(\mathrm{W})$ & $41 / 40$ & $16 / 15.5$ & $43 / 42$ \\
\hline Thermal radiation heating $L N_{2} /$ LHe $(w)$ & $1289 / 3.3$ & $5.6 / 7.3$ & $145 / 3.4$ \\
\hline $\begin{array}{l}\text { Gas condensation neat impact } \\
\text { Live } / \text { Le }(W)\end{array}$ & $0 / 2.4$ & $0 / 1.0$ & $0 / 2.4$ \\
\hline Total heat input $\mathrm{LN}_{2} /$ LHe $(W)$ & $1330 / 45.7$ & $72 / 17.8$ & $188 / 48$ \\
\hline LN $\mathrm{N}_{2}$ flow rate (ljters/hr) & 29.3 & 3.3 & 4.2 \\
\hline Lhe flow rate (liters/hr) & 62.8 & 24.5 & 65.9 \\
\hline $\begin{array}{l}\text { Pressure arop through } L_{2} \\
\text { circuit (psi at } 77 \mathrm{~K} \text { ) }\end{array}$ & 5 & 3 & 5 \\
\hline $\begin{array}{l}\text { Pressure drop through Lhe } \\
\text { circuit (psi at } 3.8 \mathrm{~K} \text { ) }\end{array}$ & 0.4 & 0.2 & 0.4 \\
\hline
\end{tabular}




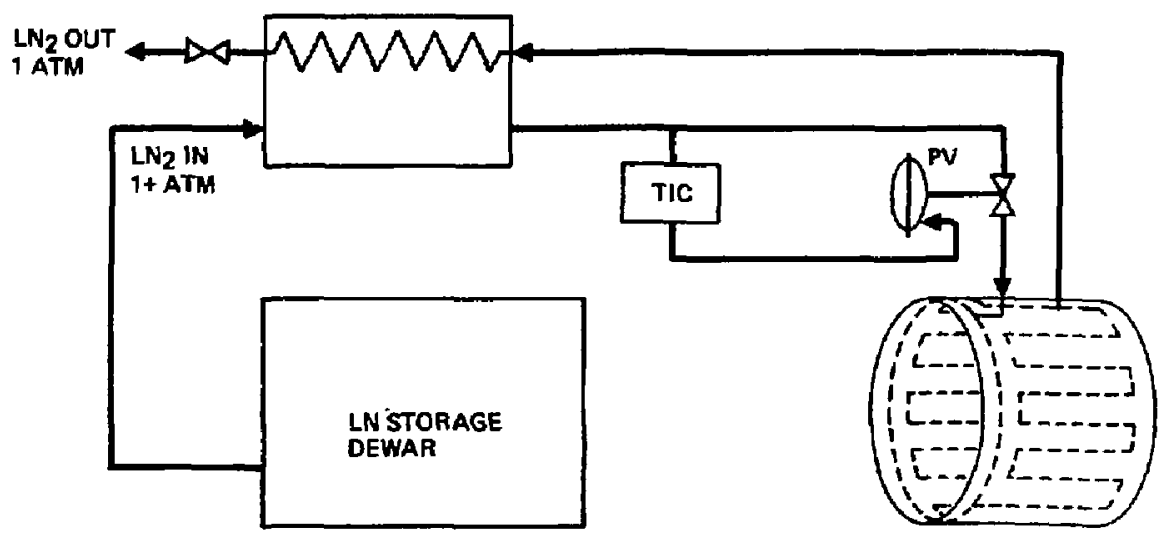

Figure 3-89. Liquid-nitrogen flow system (each pane1). 


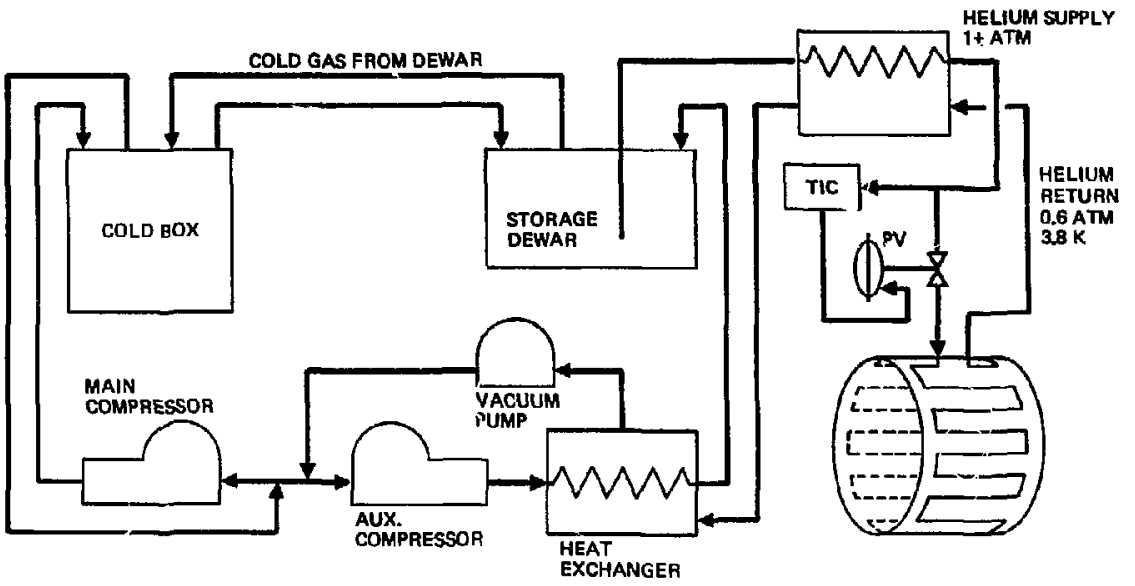

Figure 3-90. Liquid-helium flow system (each pane1). 
modification of the ion aistribution at the ion dumps. For these reasons, the maximum fields allowed are $2 \mathrm{G}$ in the arc chamber and $10 \mathrm{G}$ at the entrance of the reflection magnet.

The majn source of magnetic fields at the beamline is, of course, the stray field of the main system solenoids, but fields can also be produced by high-current electrical leads located close to the beams. The latter is of particular concern for arcs with high-current filaments, which ao not have to be considered here since the design is based on either the hollow-cathode arc or an $r$ t aischarge.

The vacuum magnetic fields, which are due primarily to the central cell solenoias, are indicated in Fig. 3-9l for two prominent beamline prints, the last gria of the source and the entrance of the reflection magnet. The fields are of the same magnitude as those considered for MFTF, but are essentially parallel to the beamline axis, compounding the shielding problem. To facilitate shieloing, it woulo be highly desirable to place the beam sources farther from the magnets because the field decays as $R^{-3}$; however, beam aivergence limits plasma source oistance to the 9.5-m design value.

Because IDF is steady state, not pulsea, shielding with ferromagnetic material seems to be inoicated. A oetailed analysis shows that other shielos might be preferable. First, the option of shielding the entire beamline vessel would require a $40-\mathrm{cm}$-thick iron wall weighing 200 tons. Also, it would not be very effective because the field inside a cylindrical shielo immersed in an axially directed external field decays as exp- (2.42/R). This means that a $1.8-k G$ field in a 1.5 -rii-radius shield would be reduced to only $70 \mathrm{G}$ at à Gistance of $2 \mathrm{~m}$.

The second option is to shield the individual source-neutralizer combinations in a way similar to the multilayered shields proposea in Ref. 2 ? or to the shields of the Doublet III beamlines. It was shown that such shielas, in an environmental magnetic field of comparable magnituae, can indeed reduce the field to the required value. ${ }^{23}$ The space between the neutralizer and the reflection magnet would have to be left unshielded to effectively pump the gas emanating from the neutralizer. As meritioneo earlier, this field is undesirable because it can cause beam loss and an unoiesirable power oistribution on the ion aumps. Using the stray field as an ion deflector as proposed in Ref. 23 cannot be done in the TOF case because 


\begin{tabular}{|c|c|c|c|c|}
\hline LOCATION & $\begin{array}{c}B_{\mathrm{f}} \\
(\mathrm{KG})\end{array}$ & $\begin{array}{c}\mathrm{B}_{\mathbf{2}} \\
(\mathrm{KG})\end{array}$ & $\begin{array}{c}6 \\
(\mathrm{KG})\end{array}$ & $\begin{array}{c}\hat{\theta} \\
(\mathrm{O})\end{array}$ \\
\hline$P_{1}$ & .797 & .086 & .802 & 83 \\
\hline$P_{2}$ & 1.799 & .433 & 1.860 & 76 \\
\hline
\end{tabular}
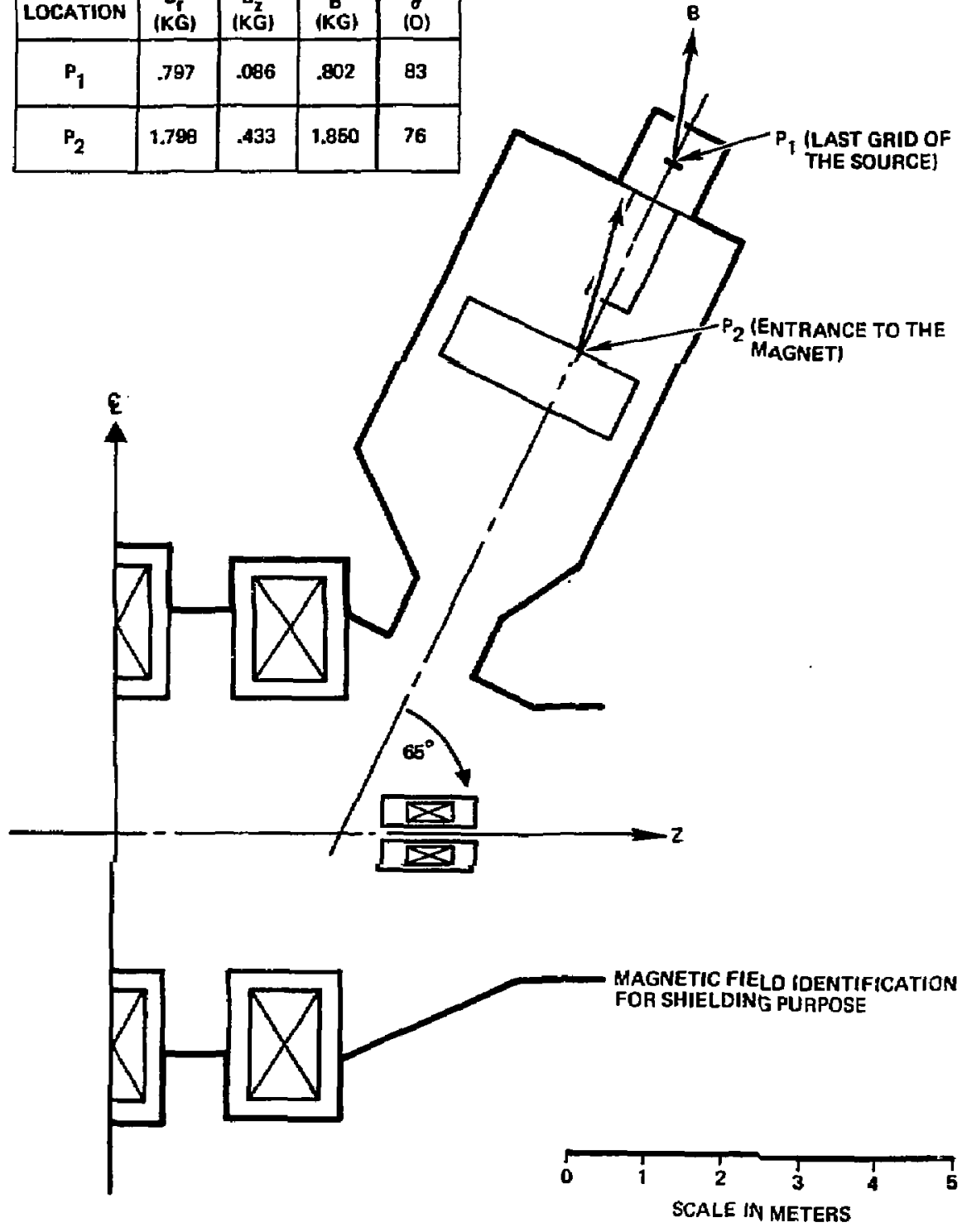

Figure 3-91. Magnetic field identification. 
the field is longitudinal rather than transversal. Also, inaiviaual shields for each beam would weigh 3 tons, which, together with the refraction magnet, means that there would be 20 tons of magnetic material in a beamline.

A lightweight shield could be constructed to eliminate the harmful perpenaicular field in the neutralizer to the reflection field region. However, the resulting fringe field in the source region wo'ld be intolerably high.

One potential shielaing option has not been considered here because extensive computer calculations would be required to evaluate it.

The beamline vacuum vessel is to be surrounded by neutron shielding material. For neutronics reasons, this material can be replaced by soft iron of sufficient thickness. Itis iron cyinoler woolor reouce the fie io ins ion to a level that could easily be reauced further by small, individual, magnetic shielas.

Because the use of ferromagnetic materials as shields does not seem a reasonable solution primarily because of weight problems, a superconoucting magnetic shield was adopted as a baseline design for the central cell neutral beamlines. Notice, apart from the difference in weight, that the superconaucting shields can be more effective since, unlike iron shielos, they do not pull field lines from a large area toward the shieid, thereby increasing flux that has to be eliminated. Rather, they "push" the vacuum field lines away from the region to be shielded.

Although superconducting shields have been used in a trial-and-error fashion for a long time, only recently has work been done to optimize the them. 24 Such shields have been buift for a variety of applications. They have produced regions containing fields of only $10^{-10} \mathrm{~T}$, used to calibrate sensitive magnetrometers. Long, narrow shielas have been constructed at CERN and SLAC for field-free propagation of particles in high-energy experiments. Finally, a superconducting shield for a Baseball II beamline, measuring 36 in. in length and $16 \mathrm{in}$. in oiameter, excluded an external field of $1.5 \mathrm{kG} .{ }^{24}$ Superconducting shields are ideal if a cryogenic environment has already been provided, like the cryopanels surrounding the beamlines of the TDF central cell.

Type I superconductors are ideal magnetic shield materials because they totally exclude fields to within a surface depth of $10^{-5} \mathrm{~cm}$. Their arawback 
is that the critical field is low, $1.5 \mathrm{~kg}$ for niobium, beyond which complete field penetration occurs.

In contrast, Type II superconductors, whose critical field can be as much as a factor 30 higher, are not totally diamagnetic. An external magnetic field induces a current in a surface layer whose thickness increases with increasing field so that a critical current $d_{c}$ is not exceeded at any point. The thickness of this layer and, therefore, the fieid penetration depth is given by

$\sigma=\frac{10}{4 \pi} \frac{H}{J \mathrm{c}}(\mathrm{cm})$,

where the external field $H$ is given in gauss and the critical current is given in amps per square centimeter. For a typical Type II superconductor, $J_{c}=$ $10^{5} \mathrm{~A} / \mathrm{cm}^{2}$, which means that, for example for $H=2 \mathrm{kG}, d=0.016 \mathrm{~cm}$ or about 6 thousandths of an inch.

The critical current $J_{c}$ of Type II superconductor is temperaturedependent. Thus, if local heating occurs (for example because of rapid flux change), $J_{c}$ is reduced, allowing additional flux penetration that can, in turn, cause additional heating. If equilibrium is not reached, flux jumping occurs. This stability probiem occurs particulariy in large cross-section superconductors, like the sheets from which cylindrical superconducting shielos would be constructed. Stabilization can be effected by keeping the superconductor in close thermal contact with a cold base material.

Because thin cross-section wire is not as sensitive as the flux jumping just described, effective magnetic shields have been constructe in the form of solenoids. The current induced in the wire will be such that the magnetic field energy inside the solenoid is minimal. Since the current is constant throughout the solenoid, fieids in some parts of the volume will be overcompensated ana in others undercompensated if the vacuum field was not spatially constant. In principle, this problem can be eliminated by proper spatial oistribution of the solelioid windings.

The shielding property of the superconductor should be constant in time. The current decay that results from field creeping is estimated to change the ins ide field by one part in $10^{4}$ in seven years. 
As shown in Fig. 3-92, the superconducting shield of the central cell NEIS is constructed in two parts, one inside and one outside the vacuum chaniber. The niobium-titanium superconducting material is laminated onto an aluminum cylinder that provides both mechanical support and cooling of the niobium-titanium. As mentioned, close thermal contact of the niobium-titanium sheet is recessary for the thermal stability that prevents flux jumping. The aluminum cylinder is cooled to $4.2 \mathrm{~K}$ by liquid-helium-carrying coils. It is surroundec on either side by $L N_{2}$ shielas and, on the side of a room-temperature wall, by an additional thermal radiation shield. The external shielo section, which is movable for ion source servicing, is terminatea by a flat, washer-shaped disk that allows the external shield structure to de stortened canstuterdib. The forle provides accers to the beamlines of service lines. The shielding properties of the siperconducting shield, featuring a shortened external structure with the disk, have not been evaluateo in aetail because the appropriate computer model ij not currently available. Chargin and Anderson ${ }^{25}$ show that such a termination to a superconducting cylindrical shield can be useo effectively zo shorten the cylincier. The alternate choice of a 4-m-long external shield in the form of a constant-0iameter cylinder would reouce the magnetic fielis at the point of the source grids to $2 \mathrm{G}$.

Neutron Shield. While neutronics calculations at results are covered in a separate section, it is important to unoerstand some neutronics considerations that influence the beamiine design.

The first walf is oesigned for a neutron flux of $2.0 \mathrm{MW} / \mathrm{m}^{2}$ and, because the operation is steady state, the lifetime and hezt oeposition due to gamma rays, neutrons, and secondary radiation will be ir portant considerations. Beamline-related problems include (1) heat depos.tion in the cryopanels and superconaucting shield and (2) radiation damage of the ion-source insulator. In general, heat deposition inco the cryogen is important to system capital cost since the heating influences cryoplant s ze. Operating costs also increase because 600 to $1000 \mathrm{~W}$ must be expenced in the plant for each watt cepssited in the cryogen. The other critic:l area, related to damage of the 


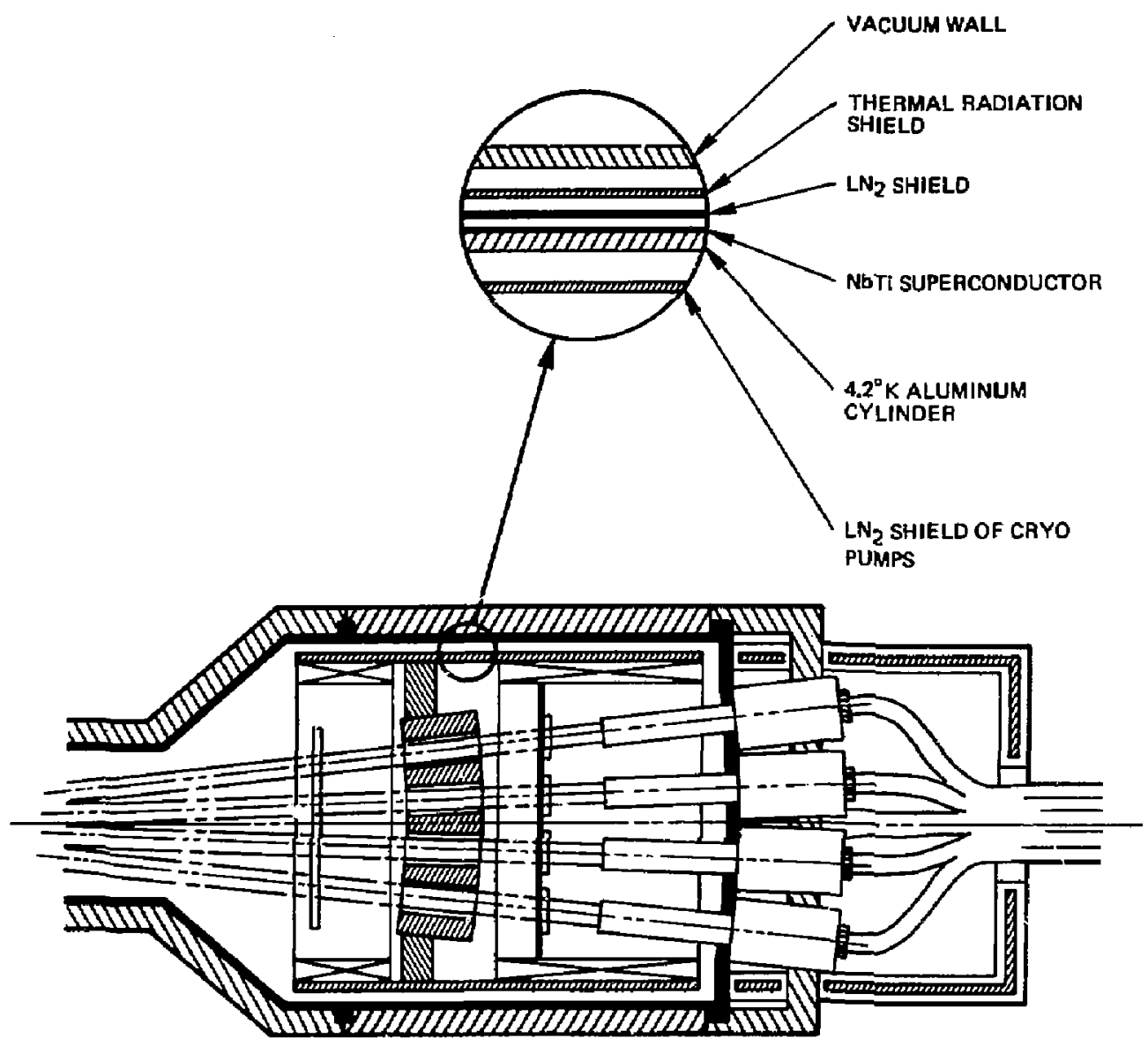

Figure 3-92. Superconducting shield design. 
ion source insulation, is important because it has a strong influence on the lifetime of this component. The radiation limits used to govern the design

$\begin{array}{lll}\text { were: } & \text { Shield heat load } & 10 \mathrm{MH} / \mathrm{cm}^{3} \\ & \text { Dose in polyimide insulator } & 5 \times 10^{9} \mathrm{rad} \\ & \text { Dose in Macor insulator } & 6 \times 10^{8} \mathrm{rad}\end{array}$

Neutron damage to beamline components is an inherent problem because the beam ouct can readily transport neutrons from the plasma to the components ejther by direct line-of-sight propagation or by scattering neutrons from the wall surface. Scoping calculations were performed at TRW, using the TART NPP code to study the influence of duct shape on outside neutron flux. To obtain the maximum number of sources per beamline, sources are arranged azimuthally adjacent to each other, separated by an angle of $3.3 \mathrm{deg}$. The deposition rate onto an aluminum sampler at the duct exit is determined as a function of auct wiath (Table 3-31). Increasing the number of beams per duct, i.e., increasing the duct width in $\theta$, did not increase the energy deposition. This result was anticipated because the plasma diameter is smaller than the duct opening. In addition, doubling the duct width increased the energy deposition by almost a factor of 2 . From these results, a preliminary duct shape was selected. The ouct is $V$-shaped in $\theta$, and the beams are stacked side by side in this plane. In the plane containing the plasma axis, and at the plasma end, the duct is made as narrow as neutral-beam transport considerations allow to 1 imit neutron and gas streaming into the duct. At the source end, the duct is widened to enhance pumping but left narrow enough to keep the first cryopanels in the shadow of the neutron shiela surrounding the duct.

As oetailed in the neutronics chapter of this repurt, the radiation flux and neutron heating of the various components was calculated for the detailed neutral beamline configuration. The results are given in Table 3-32. Both the heating of cryogenic panels and the expected lifetime of source insulation are found to be within design requirements.

\section{Systems Design}

Instrunientation, Control, and Data Acquisjtion. Monitoring and control of neutral-beam injector operations are performed by the instrumentation, control, and data-acqujsition system (ICDAS). Functionally, 
Table 3-31. TART results.

\begin{tabular}{lcccccc}
\hline $\begin{array}{c}\text { Beams/ } \\
\text { duct }\end{array}$ & $\begin{array}{l}\text { Duct width } \\
\text { in Z (cm) }\end{array}$ & $\begin{array}{c}\text { Neutron } \\
\text { edep }\end{array}$ & $\begin{array}{l}\text { Neutron } \\
\text { flux }\end{array}$ & $\begin{array}{c}T \\
\text { edep }\end{array}$ & $\begin{array}{c}\text { Tlux } \\
\text { flux }\end{array}$ & $\begin{array}{l}\text { Total } \\
\text { edep }\end{array}$ \\
\hline 2 & 60 & $1.25-2$ & $9.14+15$ & $4.83-3$ & $4.87+15$ & $1.73-2$ \\
3 & 60 & $1.16-2$ & $1.01+16$ & $6.46-3$ & $5.36+15$ & $1.80-2$ \\
4 & 60 & $1.08-2$ & $9.41+15$ & $5.69-3$ & $4.75+15$ & $1.65-2$ \\
5 & 60 & $1.16-2$ & $1.06+16$ & $5.35-3$ & $4.91+15$ & 1.72 .7 \\
4 & 120 & $2.18-2$ & $1.99+16$ & $1.09-2$ & $9.70+15$ & $3.27-2$ \\
\hline
\end{tabular}

Table 3-32. Results of neutron streaming and shjelding calculations for the central ce $]$ neutral beamlines.

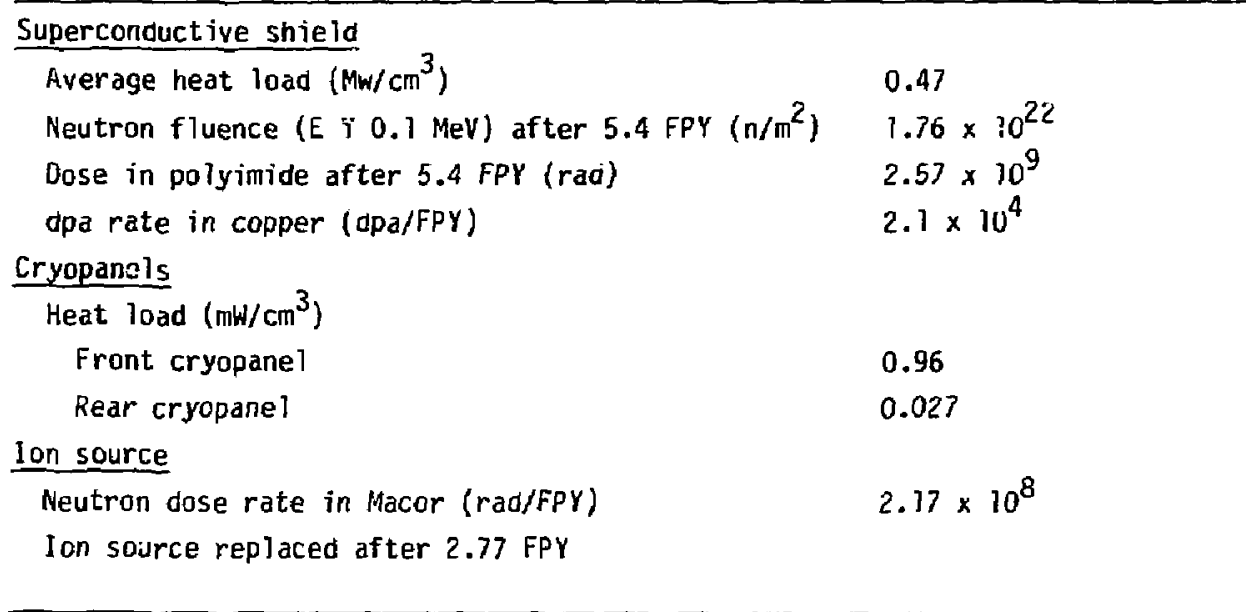


the system is composed of three interrelated subsystems: data acquisition, control, ana interlock.

1. The data-acquisition subsystem consists of sensors, sensor-signal processing electronics, and aisplays capable of monitoring routine injector operating parameters and presenting them to the operator in a meaningfu? manner. The provision to acquire aiagnostic data during the operation is also includea.

2. The control subsystem provides for adjustment of beamline operating conations. Control inputs allow ion source voltages to be adjusted, event timing to be specific.1, and mechanical devices to be positioned. The effect of a control input on the system may be ascertained via the data-acquisition system.

3. The interlock subsystem is an independent portion of the ICADS. Its role is to ensure complete safety for operating personnel and to protect beamline and reactor equipment in the event of improper injector operation or a hardware malfunction within injector subsystems.

The following sections describe the requirements placed on the ICDAS and design concepts for a $Q Q$ system capabie of satisfying them.

General. A computer-driven instrumentation, control, and aata-acquisition system will be used for this NBIS. The system, configureo like that of the TOF reactor ano compatible with it, will use CAMAC hardware interfaced to a computer.

To understand the trade-offs involved in the design of the ICACS, it is worthwhile to review the CAMAC standard. The CAMAC system was developed to stanaardize protocol for the transmission of data and control signais between instrumentation modules and a digital controller. It imposes mechanical requirements on compatible hardware, thereby allowing flexible data-acquisition and control systems to be configured.

The basic element of the CAMAC system is the module that provides the interface between the external sensors and controls and the system. Physicaily, as many as three modules reside in a crate, the standara mounting structure, which includes power supplies and intermodule connectors and wiring. The modules communicace with the crate-contained modular controller via dataway, a standard interface bus. The crate contraller provides the capability for addressing individual modules and for transferring data and 
control signals to and from them. Physically, the crate controller resides at two locations, or slots, in the crate. In the most common CAMAC-configureo system, the controller provides no intelligence, but simply interfaces the dataway to an external TDF computer via a data highway. This is the configuration of the TDF instrumentation and control system, an the eventua? form of the neutral-beamline ICOAS.

Data Acquisition and Display. The data-acquisition subsystem is required to sense, process, and display a limited set of process-related and diagnostic data.

Each beamline will be instrumented with sensors and monitors to perform. at minimum, the measurements required on MFTF-B. These include sensors that proviae inputs to the interlock electronics ‘e.g., vaive status], that measure support subsystem performance (e.g., gas pressures), and that Arovide beam diagnostic data (e.g., cooling water temperature rises and flow rates).

Special displays will be provided for the power-supply monitors and the beam-energy aiagnostic measurements. Uscillographs are to provioe displays of ion-source power-supply waveforms. Panel aisplays will be usea to read out static supply voltages. Similarly, energy deposition on all iristrumented surfaces (collimators, shine-tirough, and ion dumps, neutralizers, etc.) will be displayed on panel meters. It was suggested that displays be interfaced to the ICDAS via CAMAC, a concept that will be followed in the praposed design.

Control. The NBIS control system is required to provide two general types of control inputs. The first, referred to as event timing, responos to a TDF facilities clock and produces properiy sequencea triggering signals to beamline components. These produce the staged operation of valves and power supplies necessary to produce the ion beam. The same timing circuitry produces triggering signals to initiate data acquisition in the ICDAS. The second category of control inputs allows the beamline to be operated manually, that is, indepenciently of the computer. Manual controls provide for aajustments of power-supply voltages, the actuation of valves and motors, the resetting of fault shutdowns, etc.

During the early stages of operation, the beamlines must be capable of complete manual operation for checkout, debugging, and source conditioning. 
Beamline manudl control inputs are provided by control-panel-mounted switches. Thumb-wheel and rotary selector switches, interfaced directly to the power-supply control electronics, program output voltages and currents. Similarly two- and-three-position toggle switches apply power to control relays to activate valves and positioning motors.

The sequencing or staging of beamline operations is performed by CAMACstandard timing-pulse generator modules. After initiation of counting by a start-pulse input from the TDF ICS, time-successive, independent outputs are ayailable from the comparators. They, in turn, initjate the typical control ano monitoring activities. Flexibility in sequencing is obtained by dilowing manual control of the comparison register's inputs, which is accomplished by control-panel-mounted thumb-wheel switches. These switches are interfaced to the dataway in a manner analogous to that used for the displays: all switches are bused together and provide a set of common inputs to a single BOC-to-binary converter, and the output feeds a digital-input gate mooule. The thumb-whee] switches are scanned unaer microprocessor control, and the selected data are applied to the dataway. The switch-read operation is followed by a CAMAC write, whereby individual comparators are loaded with a programmed set-point time interval. Because of the expandable nature of the CAMAC-standard architecture, inclusion of these additional control provisions is easily obtained should it become necessary.

Interlocks. The function of the interlock subsystem is to ensure that the NBIS is not allowed to operate in a mode hazarcous to operating persornel or damaging to beamline components. Because of the high potential or serious damage that improper injector operation may produce, the interlocks themselves are required to be failsafe. Successful beaml ine inter lock subsystem design requires a careful assessment of potential failures or improper operating modes, the selection of parameters inaicative of these, the choice of sensors capable of adequately metering these key paraneters, ano finally a reliable hardware mechanization of the interlock function.

The interlack system is required to operate independently of the computer, to be as failure free as feasible, and to be so configured as to preclude operation of the NBIS or any of its subsystems when doing so would present a hazard or result in damage to the apparatus. Each beamline is required to 
coritain its own interlock system. It is reasonable to assume that other beamline subsystems will be similarly instrumented if a fault internal to the subsystem enciangers the safety of operating personnel or of the equipment itself.

The sensors monitoring critical parameters would be combined with inputs derived from other NBIS and TDF interlock sensors to create the overall commands to inhibit system operation if a malfunction is detected.

The most fundamental interlock function is to prevent the operation of the sources if the proper beamline operating conditions are not met. For example, the beatn may not be operated if the source isolation valve is closed, the cooling water is not flowing, the system pressure is not sufficiently low, the bending magnet is not providing the correct field, etc. In general, any single failure to satisfy the interlock conditions will inhibit the beam.

Even in the absence of beam, aamage may be done to beamline components because of improper conditions within the beamine. For example, to avoid boiling off liquid helfum, it is necessary to ensure that the liquid nitrogen has cooleo the cryopanels sufficiently before the helium is introduced. It is clear that some interlock functions must be staged; that is, certain beamline operations must be performed in a specified order, and the interlocks must be inspected corresponoingly.

Personnel safety is the critical interlock function. The presence of high voltage, high fluxes of neutrons, and $x$ rays requires extreme precautions. All areas containing hazardous equipment will be required to have limited access. Further, the equipment contained therein will be interlocked to preclude jeopardy to service personnel. A viable safety-motivated interlock is to equip each restricted access door with a key-operated lock and further require that the key be inserted into an interlock in the cuntrol room before energizing the beam. This will enstre that all personnel are in a safe area ouring NBIS operation.

In addition to inputs derived from beamline components, other TDF systems provide interlock signals; for example, a plasma must be establishes in the reactor before the bearn is injected to avoid oamage to the shine-through dumps. The injector power supply subsystem contains its own independent interlock system that provides a single input to the beamline interlock system. 
The electronics comprising the interlock system will be composed of highreliability components. The circuitry will be hardwired and configured to provide failsafe operations through selective redundancy. That is, single point failures (or reasonable combinations of failures) will always proouce a beam "of $f$ " condition. Both a seif-check capability and status indicators will be provided; the status indicators will show the conoition of all critical beamline components directly. The assertion of interlocks will be accomplished by audible ano visual alarms to notify operators of a malfunction.

Service Requirements. Each NBIS unit requires a multituoe of puwer, coolant, and cryogen supplies to functian properly. These services have to be made dvailable at the place of veamline deployment. An overall sumary is given in Tabie 3.33.

Electric Power. There are two kinds of power-consulning devices within the beanline: the sources and the reflection magnet. While the magnet is serviced by a simple, low-voltage, medium-current line, each source requires several high-voltage and high-current lines. Table $3-34$ gives a detailed account of the power supply needs and their parameters. Note that the data are given for only one of four sources. Electric and $I \& C$ cables for each source will be harnessea together and terminate preferabiy at a single multiple connector (Fig. 3-93). Such an arrangement would facilitate quick replacement of the source.

Coolant. It has been calculated that on iy about $3.44 \mathrm{MW}$ of the 5.6-MW beam at each source will pass through a front collimator. The difference, 2.16 MW, is uitimately dissipated within the vacuum enveiope of the injector. If all four sources operate at the same tine, some $8.7 \mathrm{Mk}$ will have to be removed from the beamline. Assuning that the coolant will be water ano aliowing an average temperature rise of $T=30{ }^{\circ} \mathrm{C}$ yields a floi rate $Q=64.4$ iiters/s, i.e., 232 milliliters/hr. The coolant velocity in supply 1 ines show 1 a not exceed $200 \mathrm{~cm} / \mathrm{s}$; hence, the total required flow cross section is about $322 \mathrm{~cm}^{2}$. Roughiy $90 \%$ of the heat is generated at the four ion dumps. The remaining $10 \%$ is created at the negative ion dump, devious ion shield, collinators, ana sounce ano is summarized in Table 3-35. Note that ali of the coolant is subject to tritium recovery. 
Table 3-33. Service requirements for eight beamlines.

\begin{tabular}{|c|c|c|}
\hline $\begin{array}{l}\text { Uperation at } \\
\text { maximum power } \\
\text { Service }\end{array}$ & $\begin{array}{l}\text { Norma) operation } \\
\text { scenario } \\
\text { (32 sources) }\end{array}$ & (24 sources) \\
\hline Electric power (MW) & 179.2 & 134.4 \\
\hline Cooling lliters $/ 5 \mathrm{H}_{2} \mathrm{O}$ & 640 & 480 \\
\hline \multicolumn{3}{|l|}{ Cryogens (itters/s) } \\
\hline $\mathrm{LN}_{2}$ & 1.088 & 0.816 \\
\hline LHe & 0.452 & 0.339 \\
\hline
\end{tabular}

Table 3-34. Power-supply requirements.

\begin{tabular}{|c|c|c|c|c|c|}
\hline Parameters & Arc & $\begin{array}{l}\text { Acceleration } \\
\text { grid }\end{array}$ & $\begin{array}{l}\text { Gradient } \\
\text { grid }\end{array}$ & $\begin{array}{l}\text { Supfiressor } \\
\text { griq }\end{array}$ & Magnet \\
\hline Voltage & $\begin{array}{l}100 \mathrm{~V} \\
(\mathrm{fu} ! l \\
\text { load) }\end{array}$ & 20 to $80 \mathrm{kV}$ & $\begin{array}{l}0 \text { to } 60 \mathrm{kV} \\
\text { to ground } \\
-2 \text { to }-30 \mathrm{kV} \\
\text { to acceler- } \\
\text { ation grid }\end{array}$ & 1 to $5 \mathrm{kV}$ & $50 V$ \\
\hline $\begin{array}{l}\text { Current } \\
\text { Regulation }\end{array}$ & $\begin{array}{l}4000 \mathrm{~A} \\
\pm 3 \%\end{array}$ & $80 \mathrm{~A}$ & $\begin{array}{l} \pm 3 \mathrm{~A} \\
\pm 1 \% \text { to } \\
\text { acceler- } \\
\text { ation grid }\end{array}$ & 0 to $16 \mathrm{~A}$ & $300 \mathrm{~A}$ \\
\hline Tracking & & & $\begin{array}{l} \pm 5 \% \text { to } \\
\text { acceler- } \\
\text { ation grid }\end{array}$ & & \\
\hline
\end{tabular}




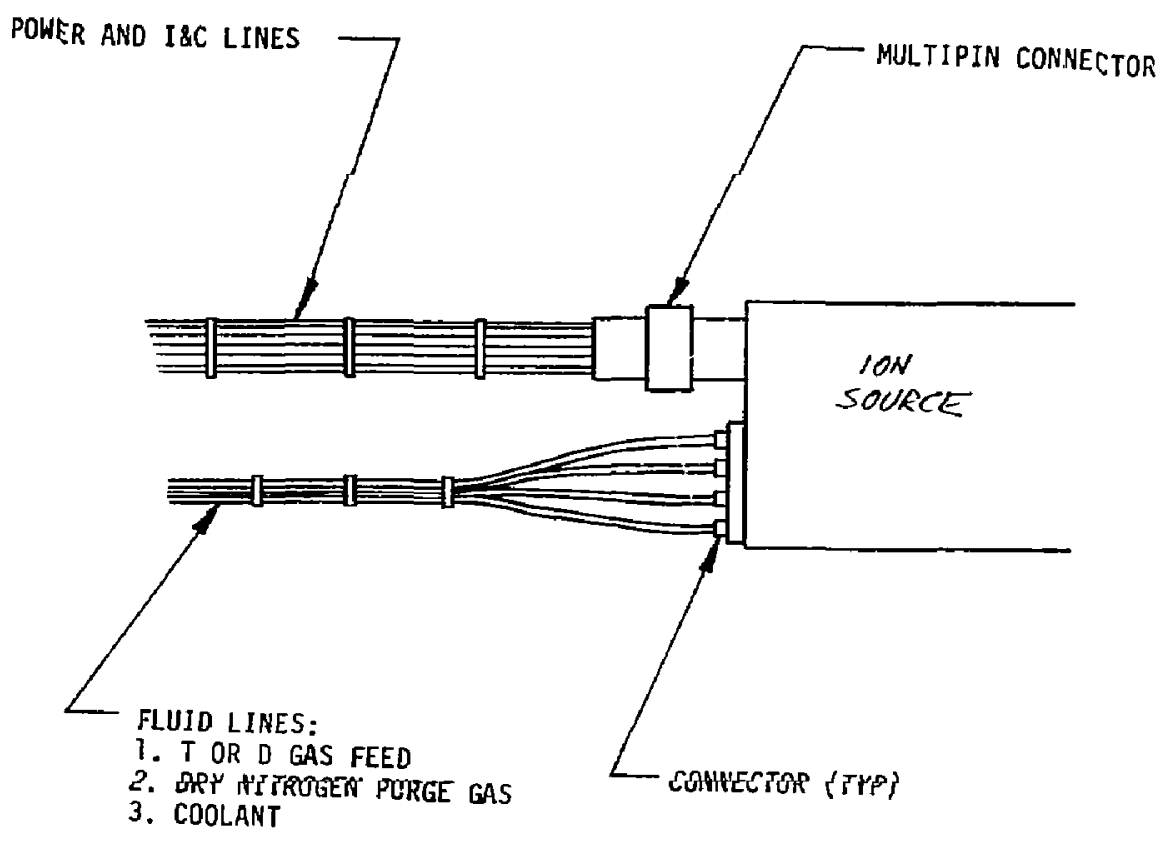

Eigure 3-93. Electrical and fluid connectors to ion source. 
Table 3-35. Cootant requirements.

\begin{tabular}{|c|c|c|c|c|}
\hline Comporient & $\begin{array}{l}\text { Power to be } \\
\text { removed } \\
\left(k H^{\prime}\right)\end{array}$ & & $\begin{array}{l}\text { water flow rate } \\
\text { at } \Delta T=30^{\circ} \mathrm{C} \\
\text { (liters/s) }\end{array}$ & $\begin{array}{l}\text { Supply line } \\
\text { identif icatio } \\
\text { (cm) }\end{array}$ \\
\hline \multicolumn{2}{|c|}{ Positive ion dump (each) } & 1948 & 15.5 & 10 \\
\hline \multicolumn{2}{|c|}{ Negative ion dump $(a 114)$} & 260 & 2.1 & 4 \\
\hline \multicolumn{2}{|c|}{ Uevious ion shielo $(a 114)$} & 128.5 & 1.0 & 3 \\
\hline \multicolumn{2}{|c|}{ Front collimator (al1 4) } & 167 & 1.3 & 3 \\
\hline \multicolumn{2}{|c|}{ Rear collimator (all 4) } & 76.6 & 0.61 & 2 \\
\hline \multicolumn{2}{|c|}{ Ref lecting magnet } & 15 & 0.12 & 1 \\
\hline \multicolumn{2}{|c|}{ Neutralizer (all 4) } & 76 & 0.61 & 2 \\
\hline \multicolumn{2}{|c|}{ Source (each) } & 100 & 0.80 & 3 \\
\hline \multicolumn{2}{|c|}{ Orift auct } & 760 & 6.0 & 7 \\
\hline \multicolumn{2}{|c|}{ Total (whole beamline) } & 9675 & 77.0 & \\
\hline
\end{tabular}


Cryogen Supply. In Section 3.3.3, we concluded that the cryopump at the $20 \mathrm{~K}$ system for each beamline requires 0.102 liters/s of liquid nitrogen a' $77 \mathrm{~K}$ and 0.0425 liters/s of liquid helium at $4.2 \mathrm{~K}$. Magnetic shield needs are 0.034 liters/s or liquid nitrogen and 0.014 liters/s of liquid helium per beamline. Separate lines are required for the front cryopanel, rear cryopanel, and internal and external magnetic shields. Each line must have a flexible section terminated by a connector.

Gas feed. Each source has two gas lines: fuel and purge. An equivalent of $70 \mathrm{~A}$ of the fuel is converted into beam while about $80 \mathrm{~A}$ is ejected from the source as neutral gas. The total particle current of $150 \mathrm{~A}$ to be supplied by the gas feed is equivalent to

$Q=\frac{I_{S}}{e N_{L}}=35 \mathrm{cmil}^{3} / \mathrm{s}$ at standard pressure.

This does not constitute a problem for deuteriun injectors; where tritium is hancieo, however, special precautions have to be taken to prevent an accidental release to the surrounaing atmosphere. These precautions apply especially to cut-off valves and connectors.

Dry nitrogen will be universally used in TDF as a purge gas. Each source must be capable of being purges independently.

\footnotetext{
Vacuum Forepump. Each beamline has a separate transfer pump external to the injector.
}

Installation ana Servicing. NBIS units are to be deployed in locations that are not always directly accessible by overhead crane. Mareover, it may not te possible to replace some short life components (i.e., sources or dumps) without disturbing at least some beamline subsystems. This section juentifies the access problem.

Installation. The NBIS vacuum vessel can be disassembled into two major jarts $(F i g .3-94)$ : the nose section that leads to the arift auct, and the cylinorical section that contains all the other beamline components. 


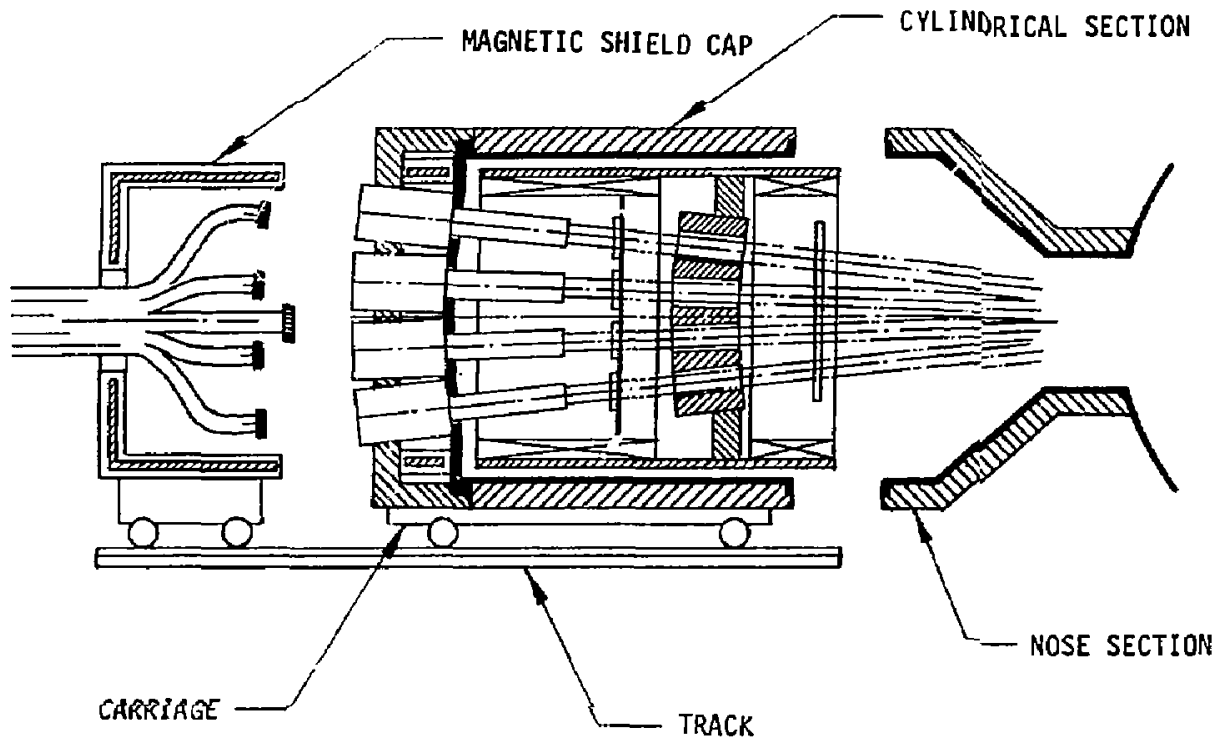

Figure 3-94. Vacuum-vessel disassembly. 
The nose section is basically an empty shell that is permanently attached ectly to the T: vacuum vessel at the narrower opening. External stiffeners und reinforcement bars may be added to improve rigidity and stability. A carriage on short tracks with be used to support the cylindrical section of NBIS, which represents $95 \%$ of the unit's bulk weight. During installation for servicing), the cylindrical section would be dropped onto the carriage from an overhead crane and would then be moved forward to couple with the nose section. Except for the interface flange, there are no electrical or mechanical connections between the nose and the cylindrical section. Boits in that flange are attended remotely, manualiy, or preferably without moving any biological shielding material. The magnetic shield cap with all cabius and connectors is also delivered by crane and rests an the tracks, Mauement af the carrianes is controlled by electromechanical actuators mounted between the tracks.

Servicing. Mechanical design and configuration details for the NBIS units are consistent with the following servicing philosophy. Short-life and high-failure-rate components (e.g., ion sources and dumps) are to be replaced with the beamline in situ, causing minimal disturbance to the main assembly. These components will then be transferred to a cell for servicing, disassembiy, or scrapping. On the other hand, access to other beamline components can be gained only by major disassembly, which inciudes removal and transport of the cylindrical section to the hot cell. Each scenario will be discussed in detail.

In Section 3.3.3, "Neutron Shield," we determined that the ion sources penetrate the rear of the binlogical shield. Since earh source bas an individual shield, there is no radiation hazard in the area of the service connectors as long as all the sources are in plice. Consequently, all the connectors and source fasteners may be attended manually. Once the source isolation valve is loose and the fasteners are removed, the source can be removed by a remotely operated manipulator. A dummy shield can be placed into the empty space temporarily to seal off the radiation.

The sequence will be as follows:

1. Power disconnected (Fig. 3-95a).

2. Magnetic shield moved back (Fig. 3-95b).

3. Faulty source disconnected. 
4. Faulty source-isolation valve shut.

5. Faulty source fasteners removed.

6. Faui y source removed (Fig. 3-95C).

7. Durmy stivela or a new source put in place.

8. Steps 1 through 5 reversed.

Note that the magnetic shieid cap was not neartivaif altimigi ia. movea. It turns out that ai, identical sequence is requil. I lo seivise lie iul dumps. However, their projected lifetime is substantial ionger thin thaci of sourses, so their attendance is of lesser importance.

The lifetime of most other beamline components is compar zble to or esceecis the life of the TOF device, however, accidental fajlures annot be exciuded $i$. a system as complex as the NBI5. Repairs connected wiil such failures nay require an extensive effort. As a result of this and the configuration of the magnetic shielding, it is preferable not to attempt detail disassembiy an site. This would create a radiation hazard in an area whice there is manipulator support. A more favorable approach is to transfer the entire cylindrical section to a hot cell, where it can receive proper attention. Although this process requires major disasscmbly, the actual number of joints and connections is quite limited and the whole task can be perforried in a shurt time. The detailed sequence is as follows:

1. TDF magnet coils detached.

2. TDF and WBIS cryopanels brought up to room temperature.

3. TOF brought up to air (ary nitrogen fill).

4. NBIS pcier disconnected ( $F$ ig. 3-96a).

5. Magnetic shield cap moved back, and shield around flange removeo (Fig. 3-96a).

6. Flange fasteners removed, cables and hoses disconnected, cylindrical sectian moveo back (Fig. 3-96a).

7. Cylindrical section transferred by overhead crane to hot cell for detail subassembiy.

8. Shielding cap placed in lieu of cylindrical section to seal off radiation leakage from inside TOF. 


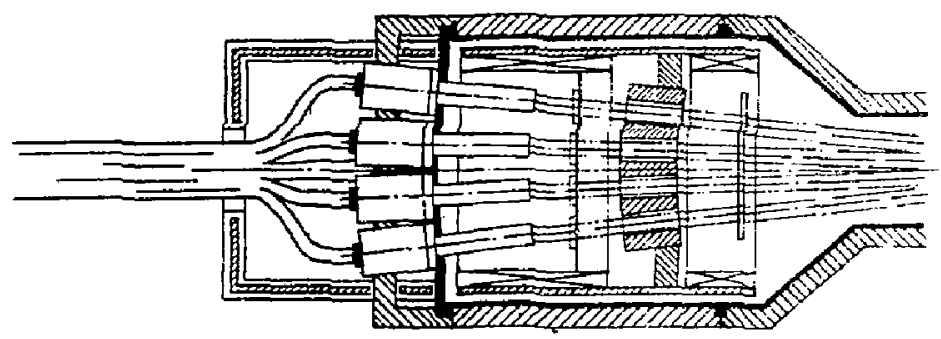

a)

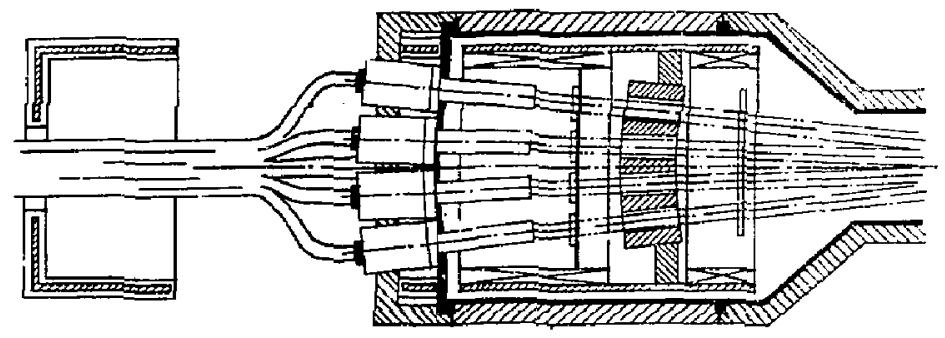

b)

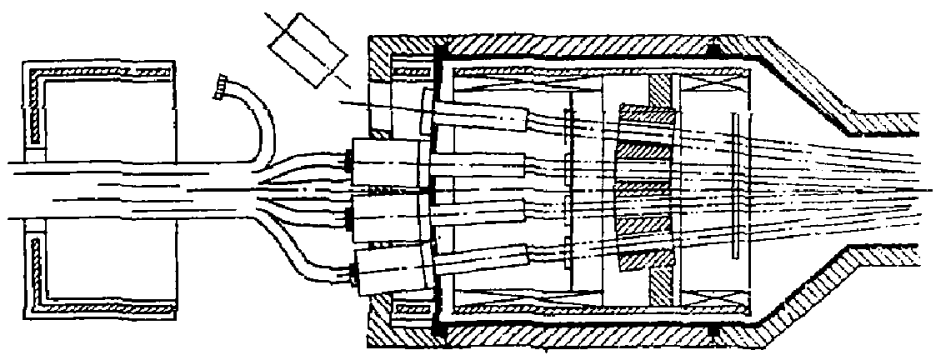

c)

Figure 3-95. Servicing a source. 


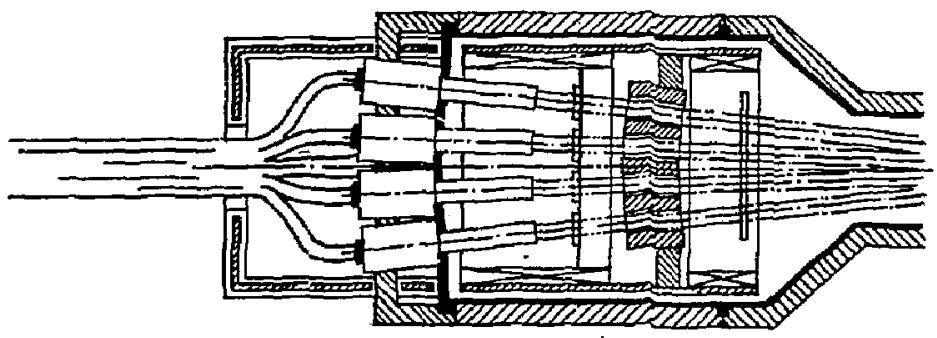

a)

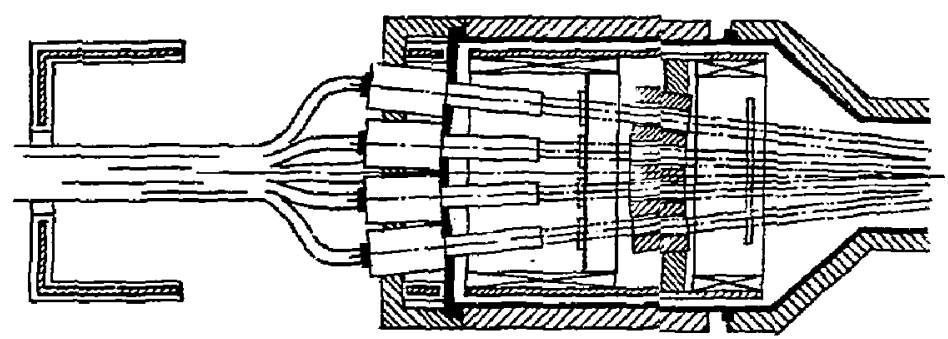

b)
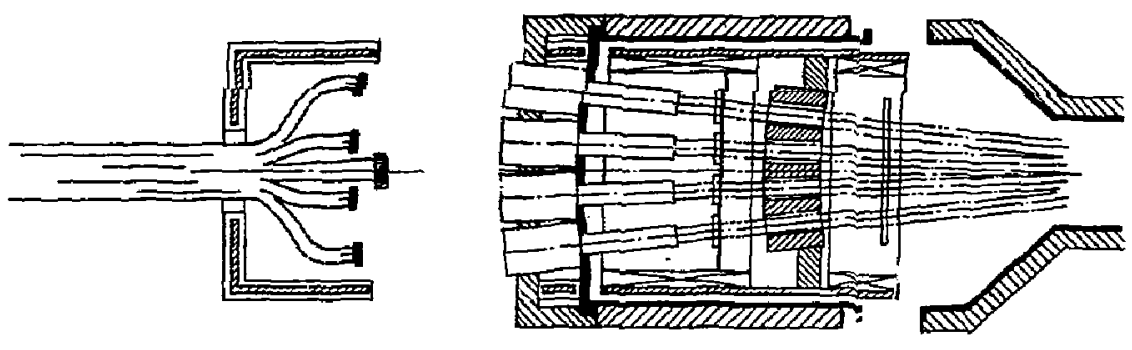

c)

Figure 3-96. Removing cylindrical section for servicing. 
Lentral Ceil NBIS, Key Techrica! Issues

Ion Source. Ion source developnerit is at the state where fuli-power operation at the requireo currerit densizies is possible for several seconds.

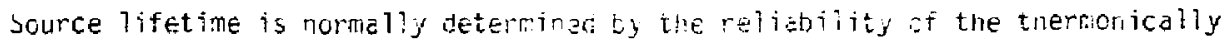
emitting cathooe. A riew oeviapnert drag electroceles rf gischarge lias consigerable promise in terms of iorg life and reliacility. The secono problem is extraction grid erosirn, which car inflience lon rce pertormance

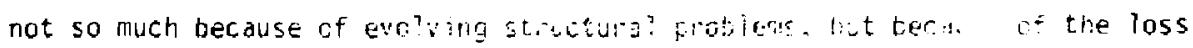
of optimuri surface siaje for ioad tocusilg.

Present ion sources can achieve the beam aivergence of 9.1 ssumed in the central cell beamline gesigri. While this divergence ic staticien: to ootain the required power oeposition in the plasia uthru erceeding the maximum design power density on the shine-through team dumps, sources wit srialler oivergence are needed to increase tho chitunt of bear intercepted $t$ the plasma and to allow the sources to be placed a larce distance away from $t$ plasma. This would reduce the demards on radiaticn anc nagnetic snieloing o the sources and neutralizer.

Power-Absorbing Surfaces. Soth the ion cunins (which absorb the energy of the residual ions) and the shine-through dunips (which receive the neutrai beam not trapped in the plasma) operate at a poiter deposition rate just below the state of the art. Currently, this can only be achieveo by incluoing the aump surface with respect to the particle trajectory. Unitortunctely, this carries the penaity of a nigher sputtering rate end, therefore, a shorter oump lifet ime.

From Monte Carlo calculations, it was found that $80-k V$ tritilim neutrals or ions are deposited an average of $3000 \mathrm{~A}$ below the beam-dump surface. Since the transport of hyorogen and its isotopes in metallic lattices proceeds by interstitial diffusion, the transport rate can be high particularly for the elevated beam-dump operational temperature. First calculations show that the tritium deposition into the cooling waters of the beam dumps is high enough to make tritium processing of the cooling vater necessary. Dunip designs should be developed to limit tritium aiffusion; for example, use leninates cf several materials, some of which have stïali tritium solubility. 
Magnetic Shielding. To limit divergence growth of the neutral beam and ion source, the neutratizer and the space between the neutralizer and the reflection magnet must be shieldea from stray magnetic fields. Shielding this volume is difficult because the local magnetic fields have a magnitude of 0.6 to $2 \mathrm{~T}$ anc are almost colinear with the neutrai beam axis. However, effective shielding can be achieved with either ferromagnetic or superconducting material. Shielaing with ferromagnetic material has the advantage of being free of maintenance requirements. Its disadvantage is weight, which amounts to 200 tons if the entire beamline is to be shiejded and to 12 tons if individual components are to be shielded. One option is to use an external soft iron cylinder around the beamline to reduce the interior field to $100 \mathrm{G}$; final field reduction can be achieved with individual component shields. A more attractive approach is to use an active superconouctor (niobium-titanium) cylinaer. Although such shields have been built and tested on a small scale, their effectiveness at the size required by the TDF/NBIS has not been confirmed.

\subsubsection{End Cell Beam Requirements}

This portion of the report addresses the three neutral-beam injectors required for the anchor cel1. The injectors are designated by function as (1) transition pumping (TP), (2) anchor cell pumping (ACP), and (3) anchor cell sloshing (ACS). Figure 3-97 shows the layout of the injectors in the two principal planes of the anchor and end cell regions. The purposes of this section are (1) to document the results of the FY 1982 preconceptual design effort on TLF and (2) to describe the key issues encounterea.

Uuring the preconceptual design effort, there was considerable interchange between physicists and engineers to solve anchor cell access problems. This was the key to our success.

The aesign descriptions are not detailed because this was a preconceptual design effort. Performance allucations ano assessments have been carrjeo out at the major component level. Particulars of the detailed design are left to subsequent design efforts, where hardware cesign support is includeo. In general, specified component characteristics are within the state of the art. 


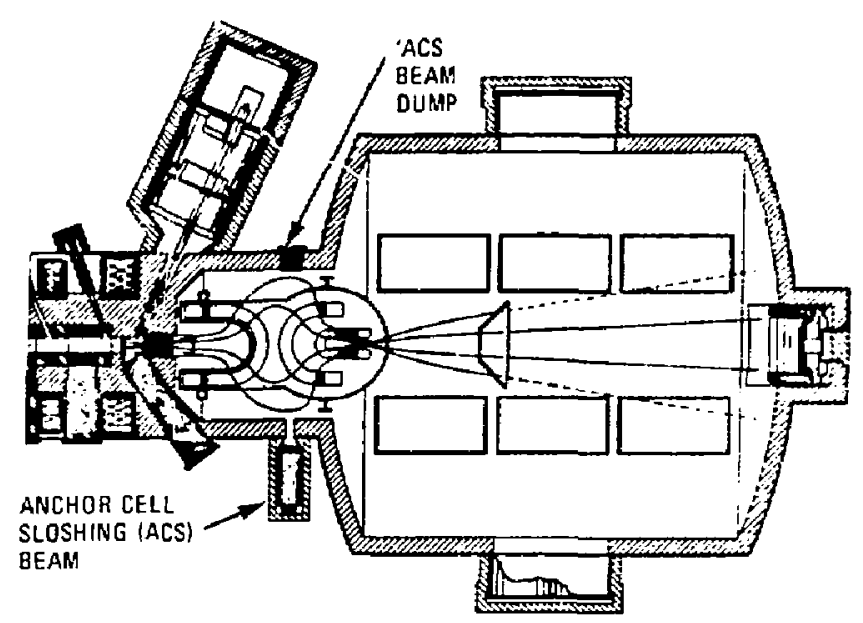

\section{$X-Z$ PLANE VIEW}

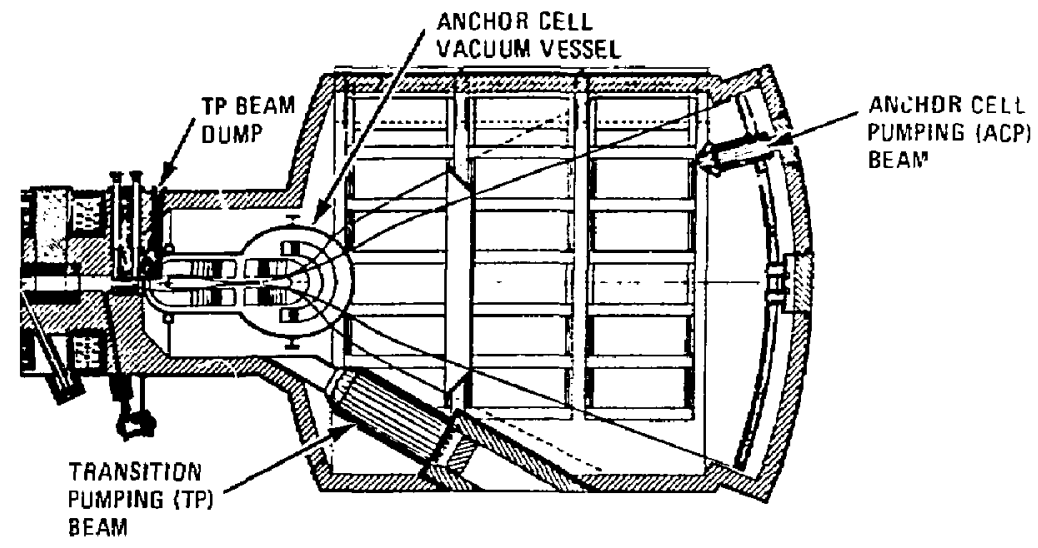

Y-Z PLANE VIEW

Figure 3-97. Anchor-cell NBI locations. 
A summary of salient requirements allocated to the anchor cell neutral-beam system is given in Table 3-36. In addition, geonetric constraints are in:posed by the device and its major components. As evident in the discussion that follows, the yin-yang coil pair used in the anchor cell imposes consicierable constraints. Before we interpret the requirements, however, something should be said about the coordinate system used.

Figure 3-97 shows one end of TOF in plan view. The plasma axis (going awoy from the central cell) is designated the $Z$ axis. Coil M-3 has its major radius open in the $Y-Z$ plane; hence, the aforementioned plan view is in the $\gamma-2$ plane, with the $X$ coordinate out of the plane of the paper. The opposite end is rotated $90 \mathrm{deg}$, so references to beams in specific planes, etc., have to be rotated/translated properly.

Note that all three injector requirements are specified in Tabie 3-36. Injection angles are given with respect to the plasma axis ( 2 axis). The injection plane is specified to ensure that the injected beam meets the assumed trapping fractions. This dependence of trapping fraction on the plane of injection comes aboul because the plasma is assymmetrical. The beam intercepts are specified in terms of the magnet field along the $Z$ axis. Figure 3-98 shows the translation of these intercepts into 2-axis positions. It also shows the interrelationship of the beam footprints. The species mix is specified to ensure consistency in modeling both the physics and neutral-beam performance. Species mixes of this quality are achievable in current sources. The trapping fraction is not a requirement on the beamine design directly, but does allow calculation of required beam-dump power-handling capability.

Specifically, 70\% of the injected current goes to the beam aump in the case of the anchor sloshing beam, and only $40 \%$ of the transition neutral-bean current goes to its dump. In the path traversed by the anchor pumping beam (upstream in the end cell particle fan), there is significant charge exchange; hence, an additional loss factor of $30 \%$ must be applied. The pumping beam is made large enough so the power intercepting the plasma is of the specified amount (1.45 A $\times 80 \mathrm{keV}$ or $116 \mathrm{~kW} /$ anchor). There are no serious nuclear shielding problems in the anchor cell nor the end cell. This neutron flux on the wall is about $3 \times 10^{10} \mathrm{n} / \mathrm{cm}^{2} \mathrm{~s}$ in the anchor cell requires that the sloshing beamline be properiy shielded. No significant cryopanel heating or 
Table 3-36. Sumary of anchor cell neutral-beam requirements. (Note that all have a beam energy of $80 \mathrm{keV}$, a species mix F/H/T (X) of $80 / 15 / 5$, a neutron fiux of $310^{10}$, and a pulse length capability of $\left.\$ .5.\right\}$.

\begin{tabular}{|c|c|c|c|}
\hline \multirow[b]{2}{*}{ Parameter } & \multicolumn{2}{|c|}{ Anchor } & \multirow{2}{*}{$\begin{array}{l}\text { Transition } \\
\text { pumping }\end{array}$} \\
\hline & Pumping & Slosning & \\
\hline Injection angle (deg) & $\leq 15$ & 90 & 30 \\
\hline Injection plane & $Y-Z$ & $x-z$ & $\gamma-i$ \\
\hline Magnet ic field at intercept (T) & $1.0-2.5$ & 2.85 & $3.0-1.0$ \\
\hline Injected current ${ }^{a}(A)$ & 2.9 & 4.2 & 60 \\
\hline Assumea trapping fraction ${ }^{b}(\%)$ & $30^{\mathrm{c}}$ & 30 & 60 \\
\hline Magnetic flux (G) & $68 /<1$ & $800 / 600$ & $300 / 40$ \\
\hline (axial/trans. at source) & & & \\
\hline
\end{tabular}

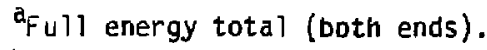

Wat a requirement.

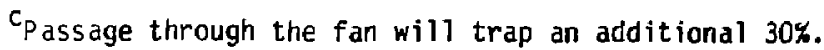




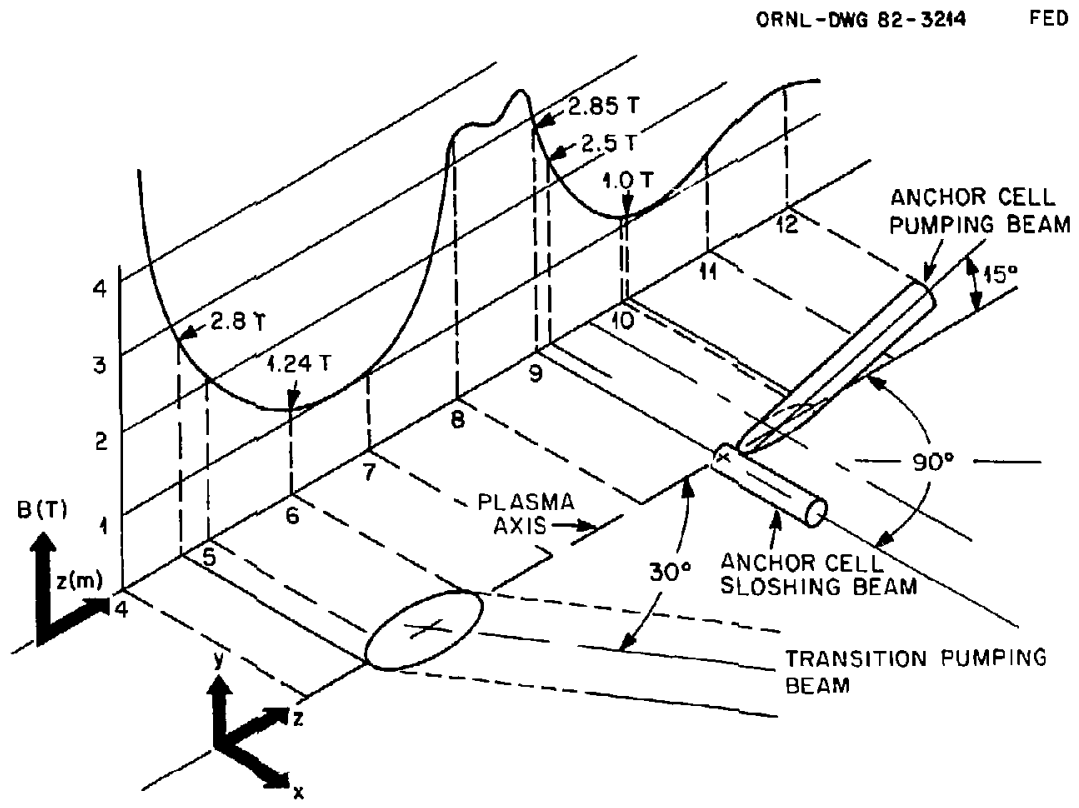

Figure 3-98. Neutral-beam plasma targets. 
radiation damage is expected. Finally, the magnetic flux was specifiad in a simplistic manner to indicate grossly the size of the magnetic shielding problen. Detailec plots of the field around the injector will be given in the aiscussion section that folrows.

\subsubsection{End Cell Beam Discussion}

Aijitcach. Figure 3-99 shows the penetrations of the three neutral neams into the anchor celi. (Note: "anchor cell" is used loosely here to cover both the anchor cell that is the yin-yang coil pair $M-2 / M-3$ and the transition coil T-1.) Each of these neutral-beam injectors will be described in detaii, followea by a discussion of key issues encountereo in the preconceptual Gesign. All the beamline performance estimates and major component sizings were done with the aid of a computer model developed on the Engineering iest Facility Program.

\section{Component Design}

\section{Transition Pumping Neutral-Beam Injector. Tab?e 3-37 sumiarizes} parameters of the transition neutral-beam injector. Six narrow beams form a $f$ an that covers the $Z$-axis intercept from 4.7 to $6.0 \mathrm{~m}$. This interface is shown in Fig. 3-99 and detailed in Fig. 3-100. The heat flux distribution of the six-beall fan projected on the 2 axis is shown in Fig. 3-10\%. Part of the rationale for this six-bean configuration is suggesteo by comparing it with the two-beam fan composite. Either option injects the same power over the $i=4 . \therefore$ to $6.0-\pi$ region, but the beam flux outsiae this desired region is much higher for the two-beam fan. This spillo:sr heats the choke coil, which is undesirable.

Figure 3-100 shows the six sources configured in a line array spaceo $25 \mathrm{~cm}$ apart, and Table 3-38 gives the parameters for these ion sources. Niore details of the beam design Jayout are shown in Figs. 3-102 through 3-104. Note that the high-failure-rate items (ion sources and ion beam dumps) are accessible from the back. The particulars of the beamline are summarized in Table 3-37. Figure 3-97 shows the injector pasitioned with in the end cell. Access for maintenance is through the back panel, where all connections are 


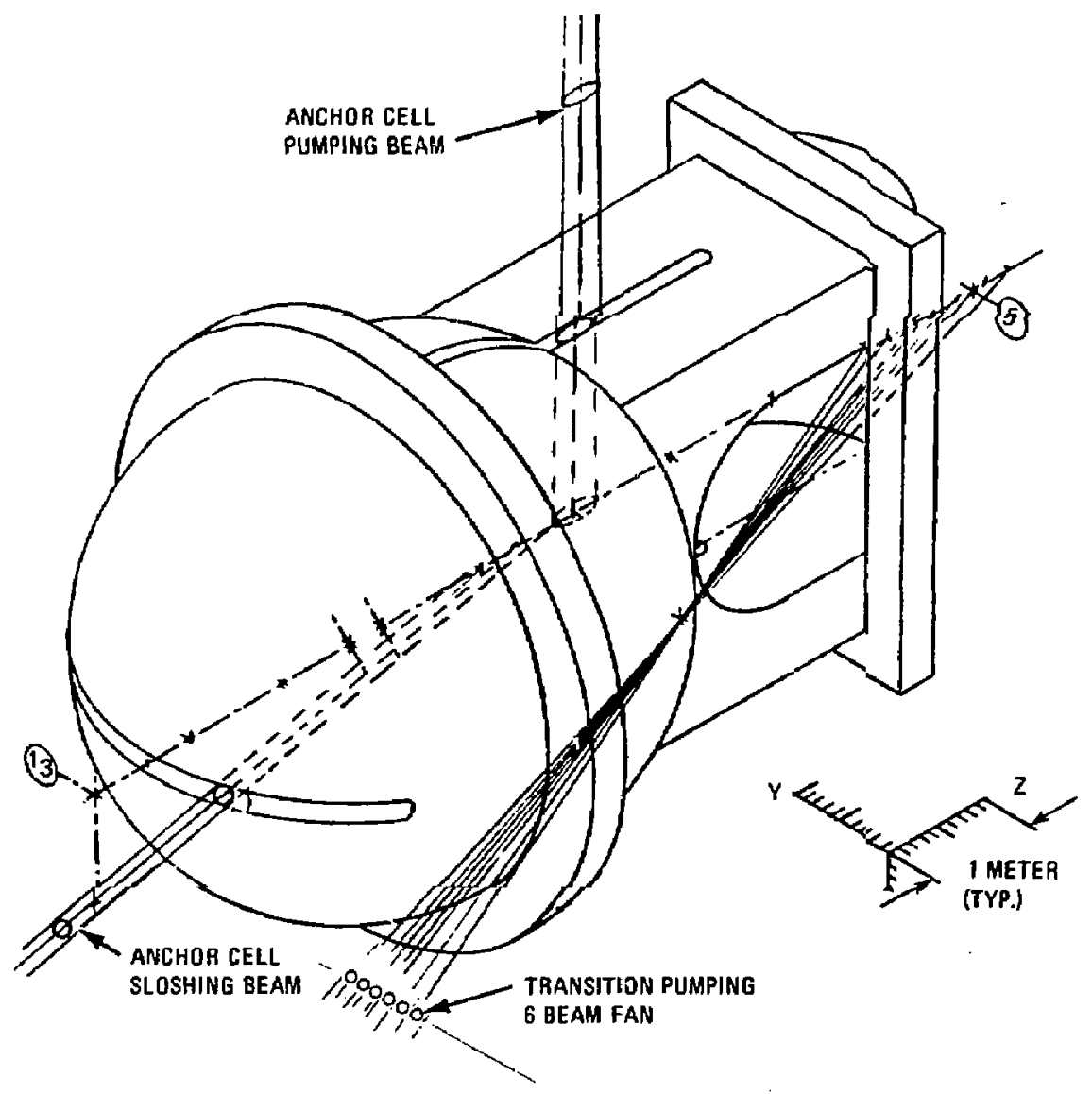

Figure 3-99. Anchor cell vacuum vessel. 
Table 3-37. Summary of transition beam parameters.

\begin{tabular}{|c|c|c|c|}
\hline Energy (kev) & 80 & Source current (A) & 14.34 \\
\hline Power injected (MW) & 3.793 & Source separation $(\mathrm{cm})$ & 25 \\
\hline$F_{d} 11$ energy & 2.880 & & \\
\hline Half energy & 0.674 & Cryopump area $\left(m^{2}\right)$ & \\
\hline \multirow[t]{2}{*}{ One-third energy } & 0.238 & Gas cell chairber & 12 \\
\hline & & Urift cell chamber & 7 \\
\hline \multirow[t]{2}{*}{ Prime power (MW) } & 7.311 & & \\
\hline & & Beamline diameter (m) & 2 \\
\hline \multirow[t]{2}{*}{ Efficiency $\{\%\rangle$} & 51.9 & & \\
\hline & & Beamline transition & \\
\hline$\left.F_{4}\right] 1$ energy current $(A)$ & 36 & section length (m) & 0.7 \\
\hline Nutiber of sources & 6 & $\begin{array}{l}\text { Drift-duct cross section } \\
\text { width/height }(c, m)\end{array}$ & $80 / 55$ \\
\hline \multirow[t]{2}{*}{ Divergence, $1 / E$ (deg) } & $0.25 \times 0.5$ & & \\
\hline & & Drift-duct length $(\mathrm{cm})$ & 200 \\
\hline \multirow[t]{2}{*}{ Beam crossover distance (cm) } & 835 & & \\
\hline & & Cost (\$N) & 6.6 \\
\hline
\end{tabular}




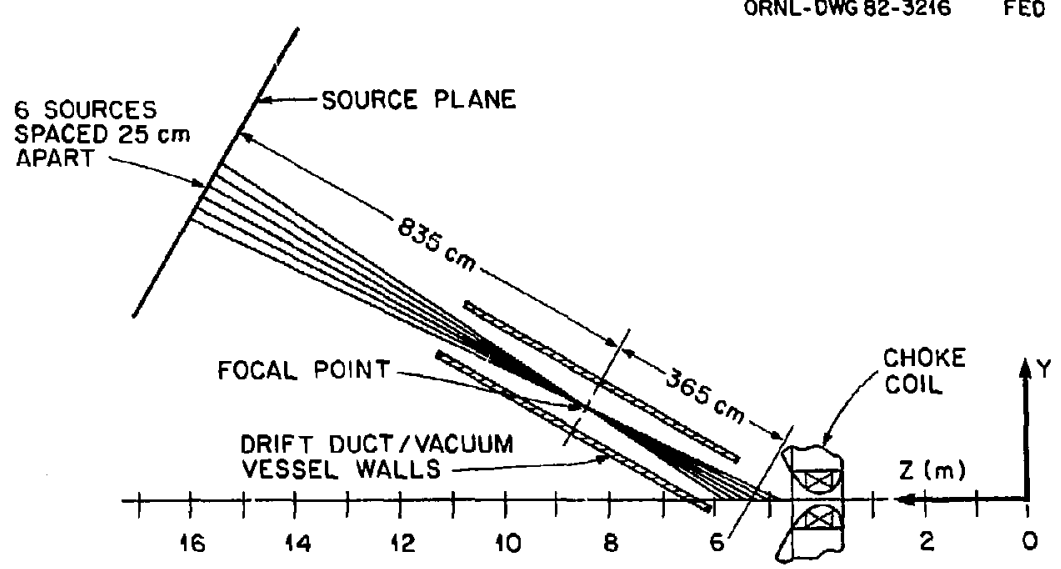

Figure 3-100. Transition-punping-beam geometry. 

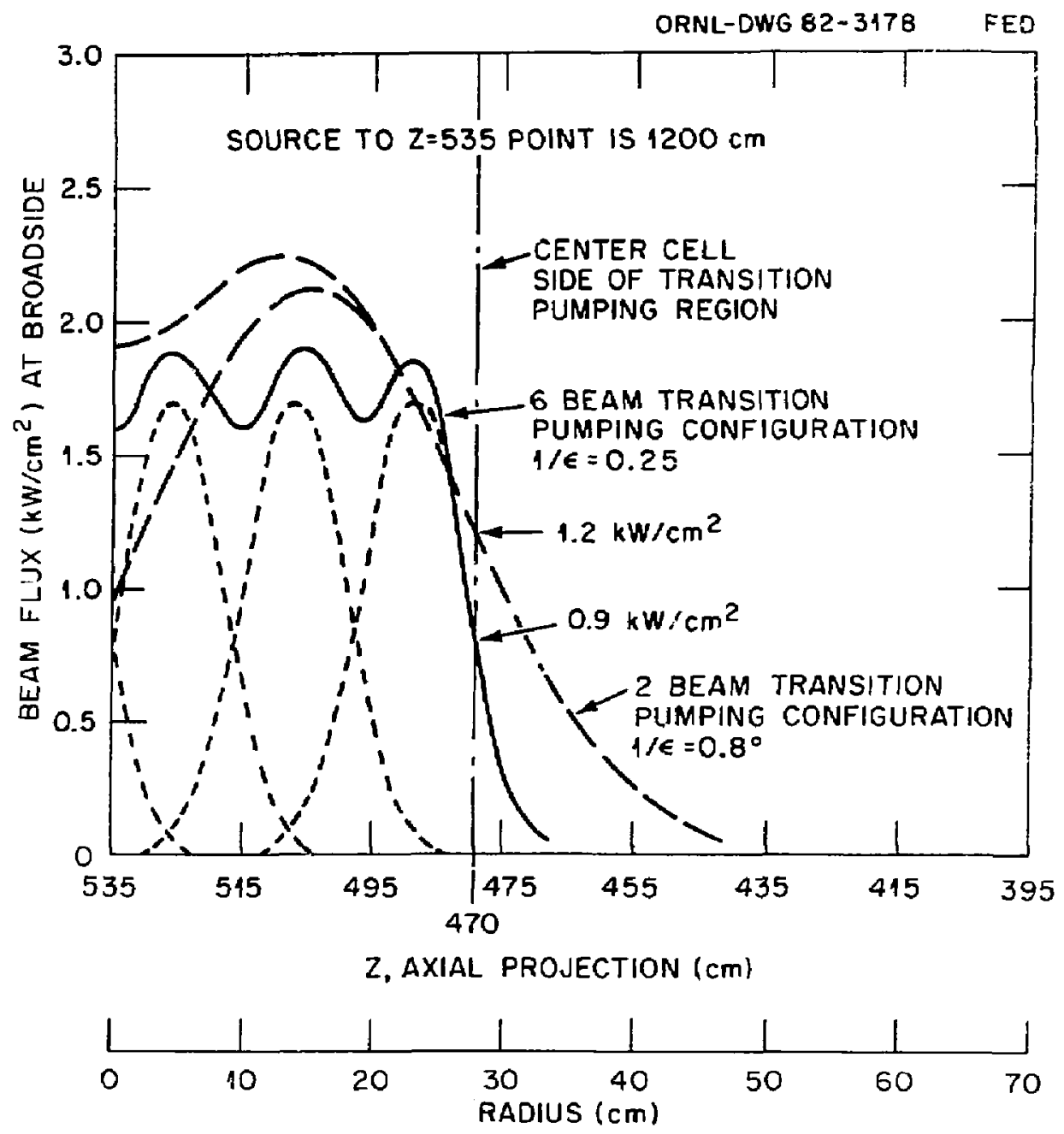

:igure 3-101. Transition beam plasma power density for two configurations. 
Table 3-38. Characteristics of a transition neutral-beam ion source.

\begin{tabular}{|c|c|}
\hline Characteristic & Performance \\
\hline \multicolumn{2}{|l|}{ Discharge } \\
\hline Current density $\left(A / \mathrm{Cm}^{2}\right)$ & 0.3 \\
\hline Species mix $(x)$ & $80 / 15 / 5$ \\
\hline Gas efficiency $(x)$ & 50 \\
\hline Gas flow/source (Torr-liters/s) & 3 \\
\hline Maximum stray B (G) & 4 \\
\hline \multicolumn{2}{|l|}{ Extracted beam } \\
\hline Aperture grid & Rectangular \\
\hline Grid transmission efficiency $(x)$ & 60 \\
\hline Beam oivergence (deg) & $0.25 \times 0.5$ \\
\hline Area $\left(\mathrm{cm}^{2}\right)$ & 80 \\
\hline Ion beam current, a11 energies (A) & 14.4 \\
\hline Full energy (keV) & 80 \\
\hline \multicolumn{2}{|l|}{ Grouping } \\
\hline Sources/beaml ine & 6 \\
\hline Beamlines/eno & 1 \\
\hline Spare sources & 1 \\
\hline Total number of sources & 12 \\
\hline
\end{tabular}




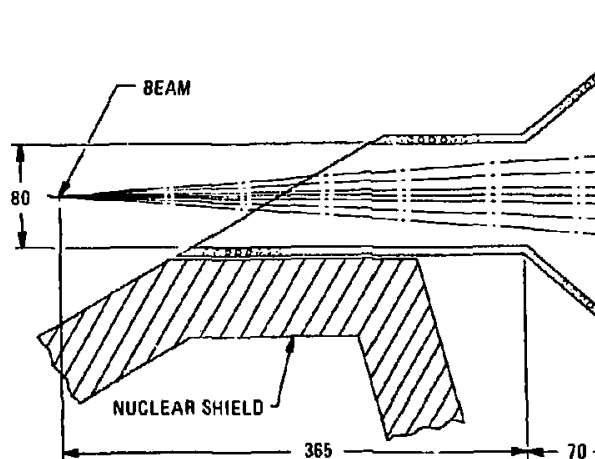

ALL DIMENSIONS IN $\mathrm{cm}$

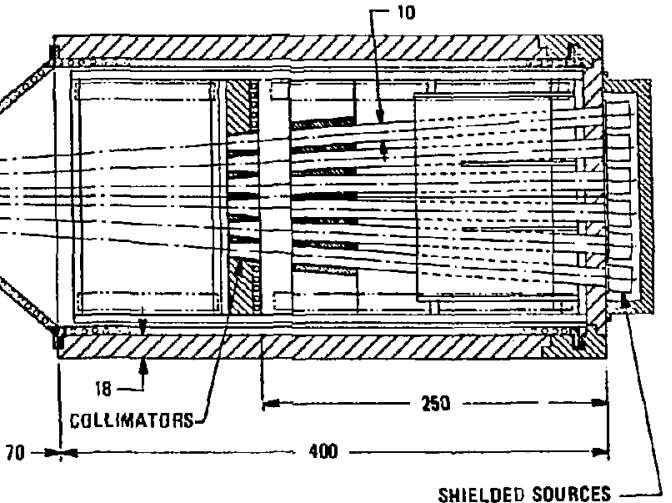

PLAN

Figure 3-102. Transition beam injector-plan view. 


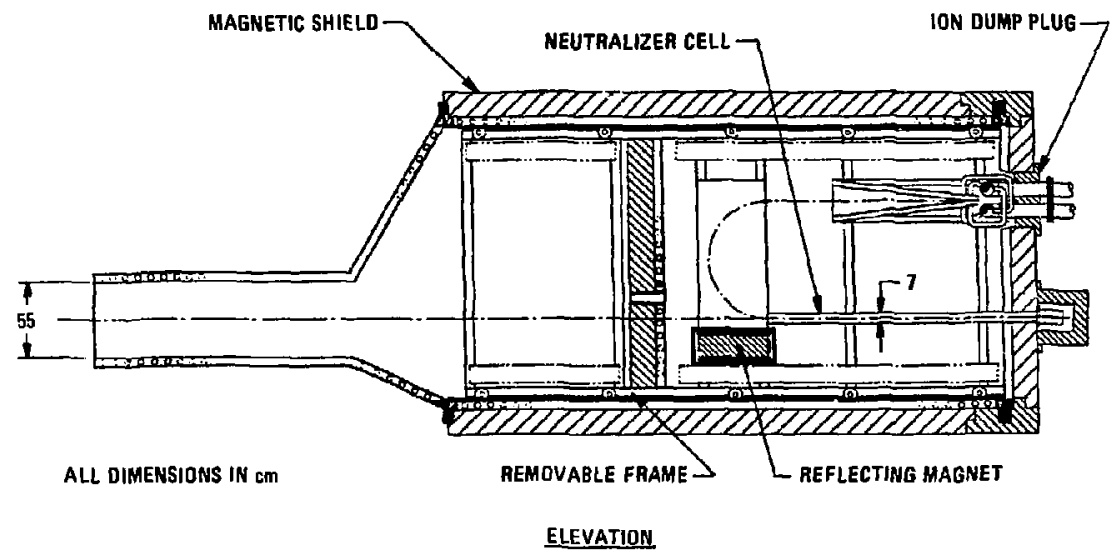

Figure 3-103. Transition beam injector--elevation view. 


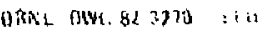

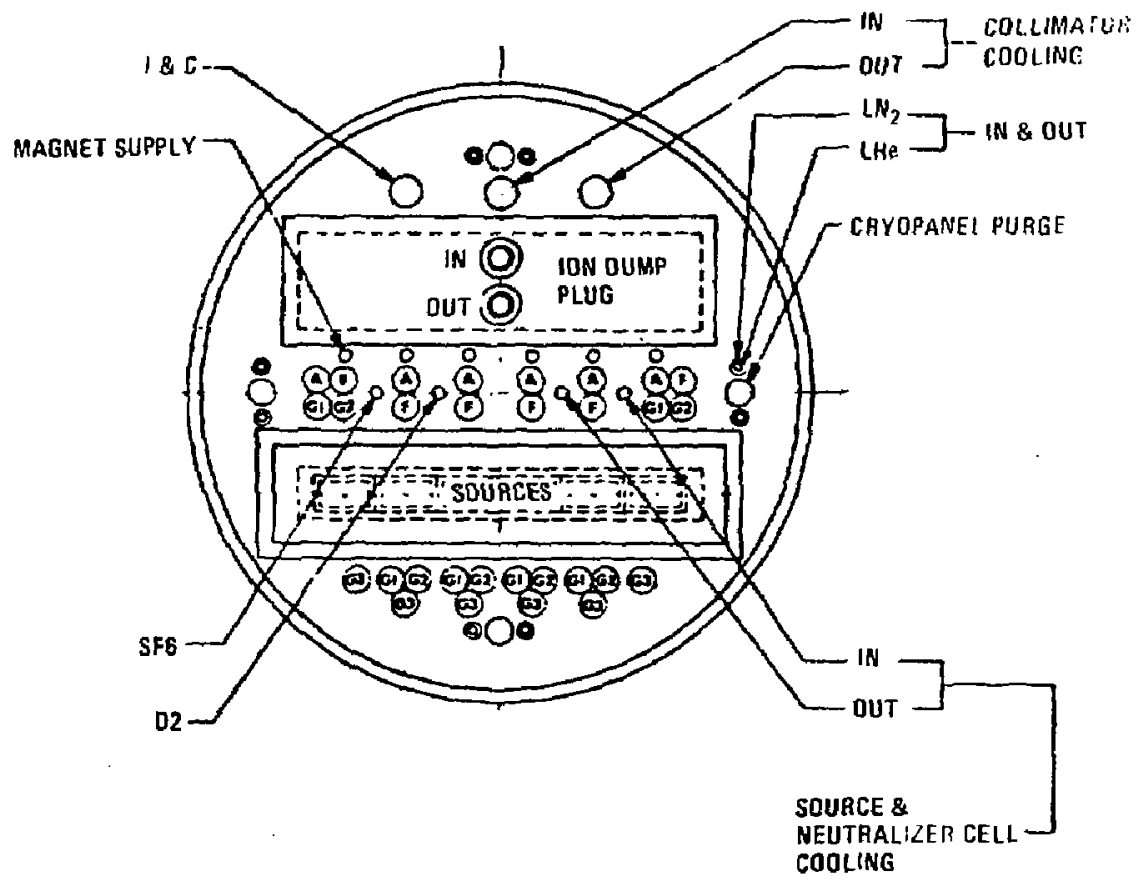

ELEVRTION

BACK END VIEW

Figure 3-104. Transition beam injector--end view showing connections. 
made (see $\vec{f}$ ig. $3-154$ !. The irjectar assembly is mounted on roller bearing surfaces and can be removed in total from the back. The injector is cylindrical (2-in tian ana 4-m length) and encased in an 13-cm-thick ingot iron magrietic shiela. Cryopumps are cylindrical, similar to the Dovolet 111 beanline. A modification to the cryopanel oesign will allow the $\mathrm{LN}_{2}$ cheyrons the clusing motion needed to regenerate portions of the cryopump while the beamine is operating. Such a design has been developed on the flat cryopanels used in the end cell. As seen from Figs. 3-102 and 3-103, two chambers are to be pumpedi--one in the gas cell area and one in the orift chamber. The pressures in the chambers are estimatea to be $1.1 \times 10^{-5}$ ano $4.8 \times 10^{-6}$ Torr, respectively. A power balance analys is that reconciles the power and efficiency numbers in Table 3-36 is portrayed is $\mathrm{Fig}$. 3-105. Each unneutralized full energy bean striking the ion dump has a power of $317 \mathrm{~kW}$. The peak power density is taken from

$i=\frac{I_{t}}{16 R X R Y}\left[\left(\operatorname{erf} \frac{R X-Y}{2 \theta}+\operatorname{erf} \frac{R X-Y}{2 \theta}\right)\right]$

and the peak at $y=x=0$

$\left.x\left(\operatorname{erf} \frac{k Y+Y}{z \phi}+\operatorname{erf} \frac{R Y-Y}{z \phi}\right)\right]$

and

$\underset{\text { peak }}{i_{\text {where }}}=\frac{I_{t}}{4 R X R Y}\left(\operatorname{erf} \frac{R X}{2 \theta} \operatorname{erf} \frac{R Y}{Z \phi}\right)=7.2 \mathrm{kW/ \textrm {cm } ^ { 2 }}$,

$$
\begin{aligned}
R X & =X O(1-1 / F), \\
R Y & =\gamma(1-1 / F), \\
\theta & =\text { divergence in the } x \text { direction, } \\
\phi & =\text { divergence in the } Y \text { direction, } \\
1 & =\text { distance to the dump, } \\
I_{t} & =\text { beam power, } \\
\bar{r} & =\text { focal length. }
\end{aligned}
$$




\section{FROM ACCELERATQR}

- $807 \times 6$ @

- $152 \times 6 @ E / 2$

- $5 i \times 6 @ E / 3$
NEUT RALIZER

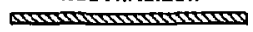

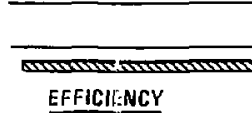

- 0.01@E

- 0.75 @ $\mathrm{E} / 2$

- 0.79@E/3
TO PLASMA

- 2,880 kW @ E

- $675 \mathrm{~kW} @ \mathrm{E} / 2$

- $233 \mathrm{~kW} E / 3$

Figure 3-105. Transition beam power fractions. 
For the ion durnp configuration shown in Figs. 3-102 and 3-103, the peak power density is reduced to $1 \mathrm{~kW} /(\mathrm{cm})^{2}$ by adjusting the dump angle to about 6 deg. The source spacing is such that there is no significant overlap of the ion beams on the ion dump. Figure 3-106 shows the neutral beam pattern on the beam dump. There is substantial overlap in this case, but the power densities are lower because the source to beam-dump distance (1) is much longer, and the plasma traps $60 \%$ of the power.

The stray magnetic field in the vicinity of the transition pumping beam injector is shown in Fig. 3-107. The low divergence source requires that the stray magnetic field inside the injector around the ion source and neutralizer be less than $4 \mathrm{G}$. The shieloing required in the transverse direction is $-100 / 4$, or a factor of 25 . The field strength in the axial direction is an ot uer of magnitude higher. Because the shielding factor is so modest, the shield thickness is set by the flux censity saturation limit of the shield material (ingot iron). Making this assumption, the thickness is given by

$t=\frac{D}{\left(\frac{B_{S A T}}{B_{E X T}}-1\right)}=18.2 \mathrm{~cm}$,

where BSAT is the saturation limit of the

$S_{t}=\frac{\mu t}{D}+1=911$ transwerse field.

and

$S_{A}=q S_{t}=6.38$ axial field,

where $\mu$ is the permeability $\left(10^{4}\right)$ ano $q(0.7)$.

Anchor Cell Pump Beam. The anchor cell pump beam and the anciror cell sloshing-beam injectors are smait ( $1 \mathrm{~m}$ diam $x 2 \mathrm{~m}$ long) source injectors that supply about 200 to $300 \mathrm{~kW}$ of injected power. Figures 3-108 through 3-110 show details of the injector design layouts. Two ion sources are built into the system, one for operation and one for a spare.

There is a $30 \%$ trapping fraction of the pumping beam as it traverses the particle fan of the end cell on its way to the plasma target inside the yin- 


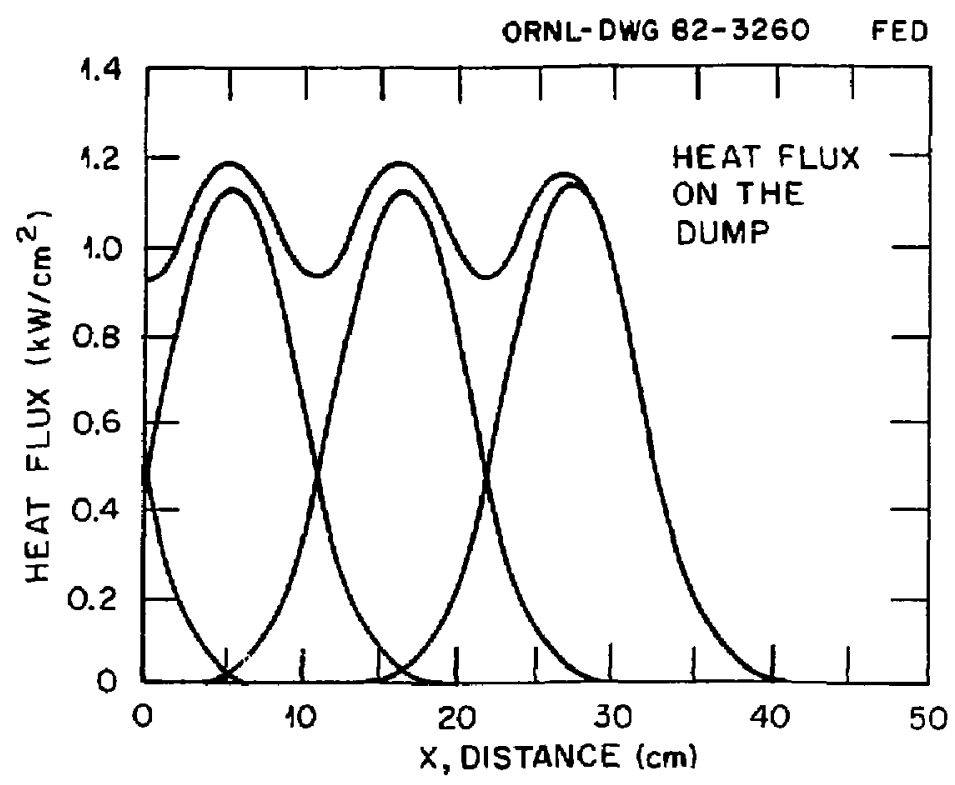

Figure 3-106. Transition beam oump patterns. 

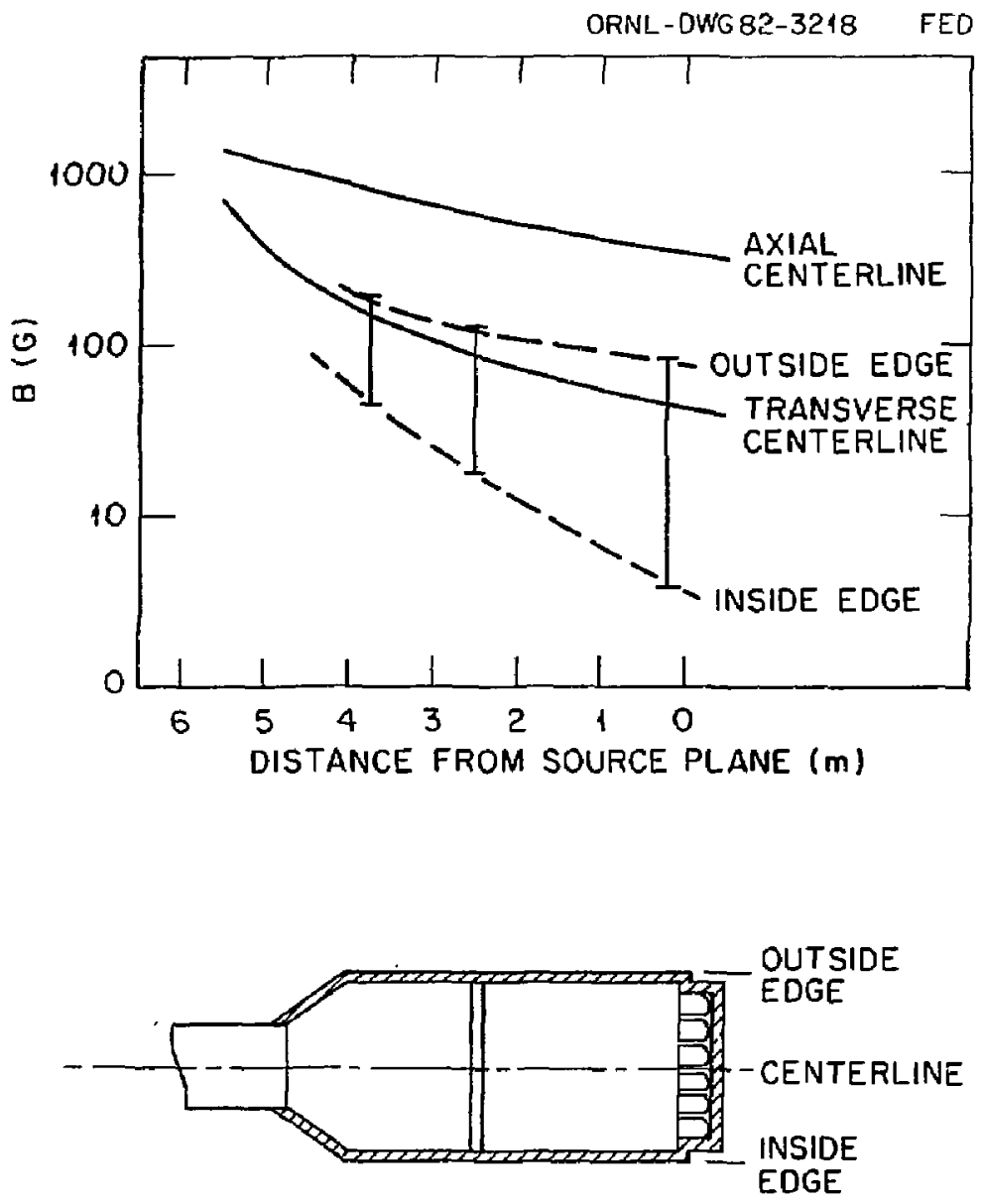

TRANSITION PUMPING BEAMLINE

Figure 3-107. Magnetic fieles around transition pumping beam. 


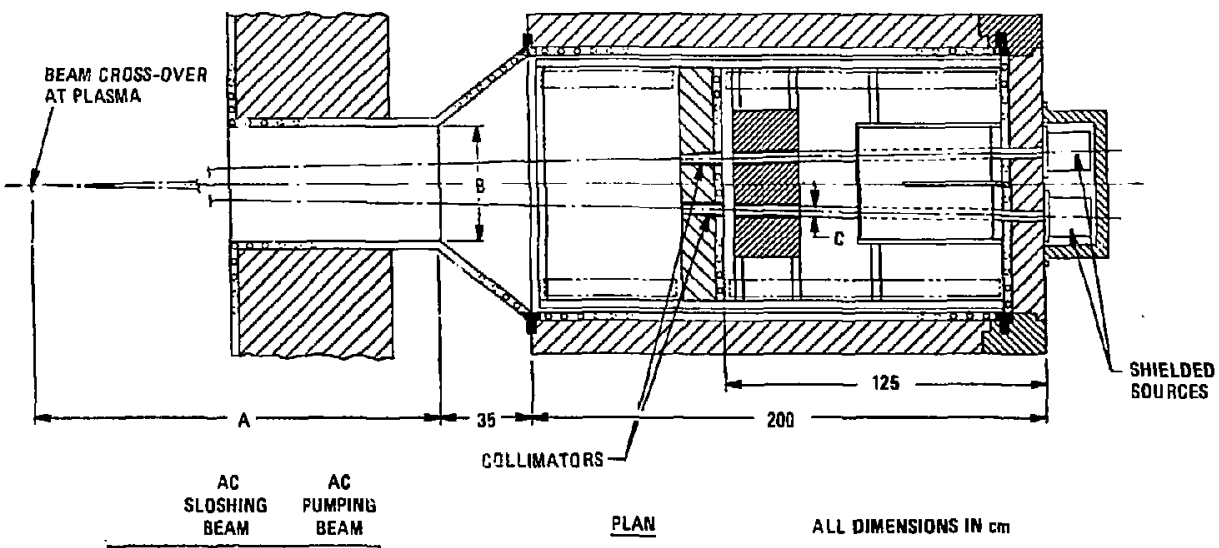

Figure 3-108. Anchor cell beani injector--plan view. 


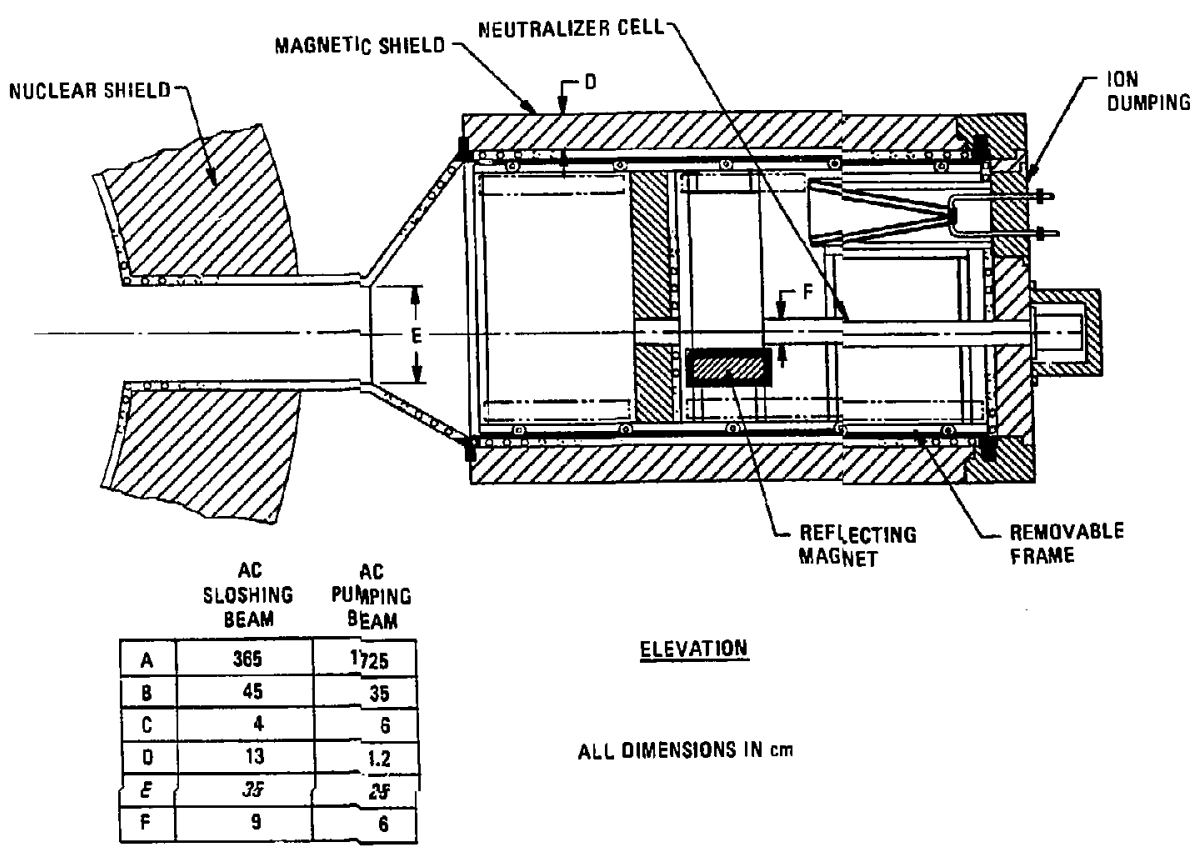

Figure 3-109. Anchar cell beam injector--elevation view. 


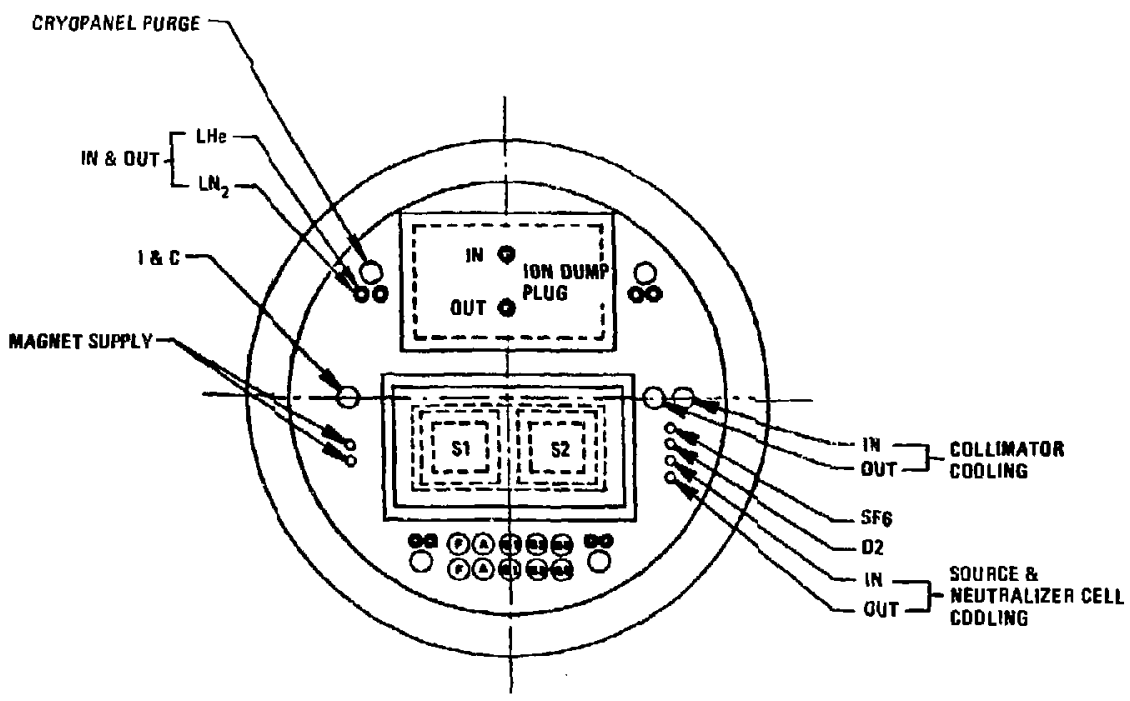

Figure 3-Iro. Anchor cell beam injector--end view showing connections. 
yang coil pair (see Fig. 3-97). Because of the great source-to-plasma distance, the absolute beam extent (distance $x$ beam angle) is large (Fig. 3-111). To get power through the constricted penetrations of the vacuum vesse1, the angular extent of the beam must be limited. In theory, this smal1 angular extent $c$ an be achieved by collimation of a modest divergence beam, use of a low-divergence beam, or a combination of both. Colitimation wastes power. Low-divirgence ion sources are unter development, with the present state of the art at $0.25 \times 0.9 \mathrm{deg}$ (built and tested); a near-future (FY 1983) development has a goal of $0.25 \times 0.25 \mathrm{deg}$. The anchor pumping beam has been sized on the basis of low-divergence $(0.25 \times 0.25 \mathrm{aeg})$ optics. If this divergence is not attained successfully, the source size will de increased in power and the colimation necessary to achieve the desired absolute beam extent with sufficient power will be appliea. This backup solution is practical because only a few hundreds of $k$ jlowatts of power is required.

The 0.25-deg-2/e beam contour, when projected onto the plasma at a oistance of $1830 \mathrm{~cm}$ and a grazing angle of $15 \mathrm{deg}$, spreads out from $Z=$ $9.6 \mathrm{~m} \pm 0.66$, depositing $86 \%$ of its power in this region $(Z=8.94$ to $Z=$ 10.26), thus meeting the pumping beam requirement. At $Z=8.95$, the sloshing beam is injected; hence, there is a slight overlap in this region.

Figure 3-172 shows the details of the anchor cell penetration of the pumping beam. Table 3-39 sumarizes the pumping bean parameters. The figure shows the beam dump located inside the yin-yang coil pair. Although the beam dump is expected to be a long-life iten, access for maintenance through the particle fan exit is planned. This opening, which is $-20 \mathrm{~cm}$ in height ( $x$-direction), was not sufficient for pumping beam ingress and was, therefore, enlarged to $238 \mathrm{~cm}$, as shown in Fig. 3-112. It scrapes the beam at about the $2.5 / e$ contour, thus passing $>90 \%$ of the beam power. The figure also shows the contour limited in the orthogonal direction $(Y \cos 15 \mathrm{deg})$. This is effected by collimation back in the injector.

The anchor pumping beam power fractions from a single operating source are given in Table 3-39. A pictorial presentation of the power balance for this injector is in Fig. 3-113. Part of the $194 \mathrm{~kW}$ of full energy is trapped in the fan, leaving $136 \mathrm{~kW}$ at the plasma. This corresponds to $1.7 \mathrm{~A}$, compareo to the required value of $1.45 \mathrm{~A}$. For the full-energy ion beam, the power density on the aump shown in the design layout is $1.58 \mathrm{~kW} \times$ in 10 deg $=$ $0.275 \mathrm{~kW} /(\mathrm{cm})^{2}$. Table 3-40 summarizes the characteristics of the ion source. 


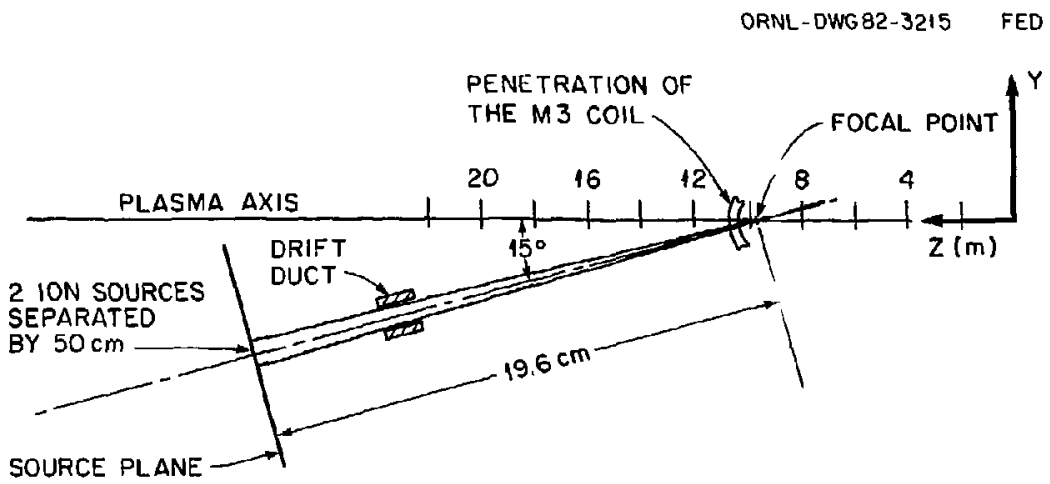

Figure 3-111. Anchor cell pumping beam geometry. 


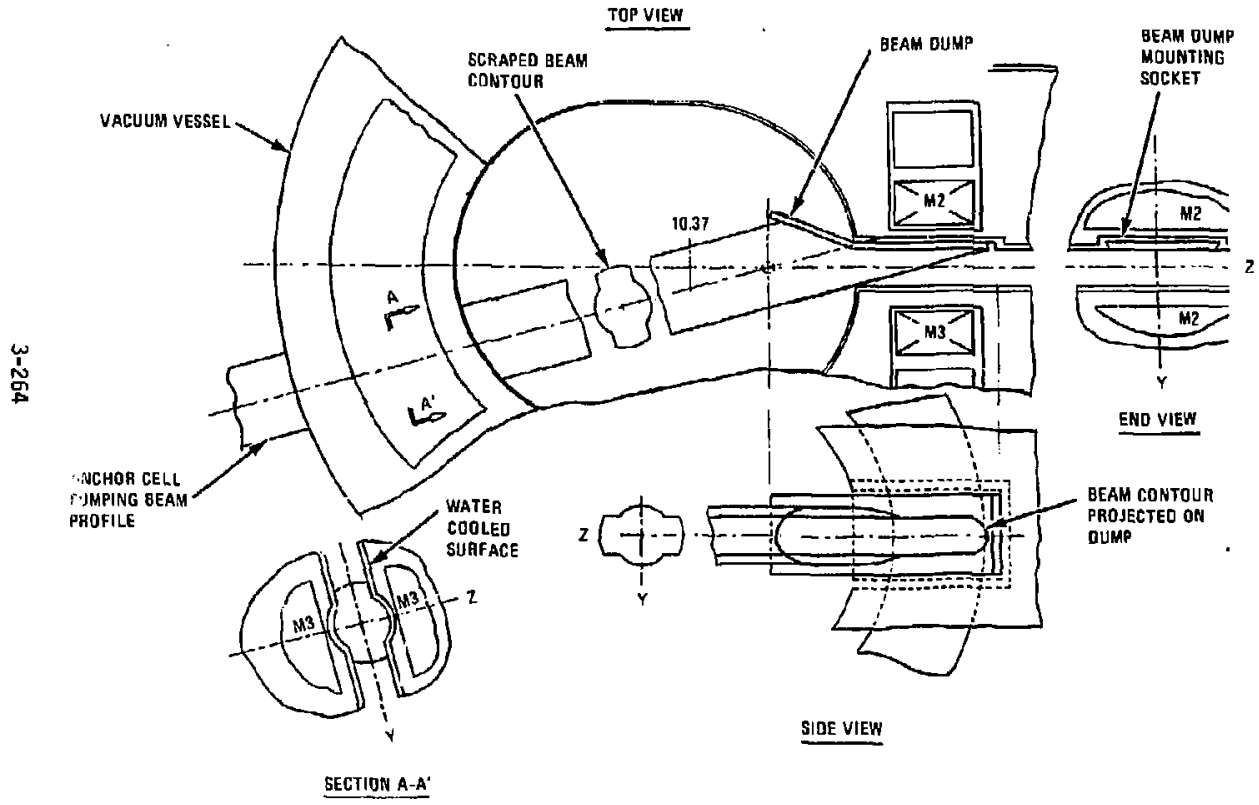

Figure 3-112. Anchor cell pumping-beam access. 
Table 3-39. Sumary of ancho cell pumping beam parameters.

\begin{tabular}{|c|c|c|c|}
\hline Parameter & Capability & Parameter & Capability \\
\hline Energy (keV) & 80 & Source separation $(\mathrm{cm})$ & 25 \\
\hline Power injected ( $\left(\mathrm{i} / \mathrm{W}^{\prime}\right)$ & 0.256 & Cryopump area $\left(m^{2}\right)$ & \\
\hline Full energy & 0.794 & Gas-cell chamber & 1 \\
\hline Haif energy & 0.046 & Drift-cel1 chamber & 0.25 \\
\hline \multirow[t]{2}{*}{ One-third energy } & 0.016 & & \\
\hline & & Beamline length (m) & 2 \\
\hline \multirow[t]{2}{*}{ Primle power (MW) } & 0.412 & & \\
\hline & & Length of beamline & \\
\hline Efficiency $(x)$ & 51.9 & transition section (m) & 0.35 \\
\hline \multirow[t]{2}{*}{ Number of sources. } & 2 & Drift-duct cross section & \\
\hline & & width/height $(\mathrm{cm} / \mathrm{cm})$ & $35 / 25$ \\
\hline \multirow[t]{2}{*}{ Divergence, l/E (deg) } & $0.25 \times 0.25$ & & \\
\hline & & Drift-duct length $(\mathrm{cm})$ & 150 \\
\hline \multicolumn{4}{|l|}{ Beam crossover } \\
\hline distance $(\mathrm{cm})$ & 1960 & Cost (SM) & 0.75 \\
\hline Source current (A) & 5.8 & & \\
\hline
\end{tabular}




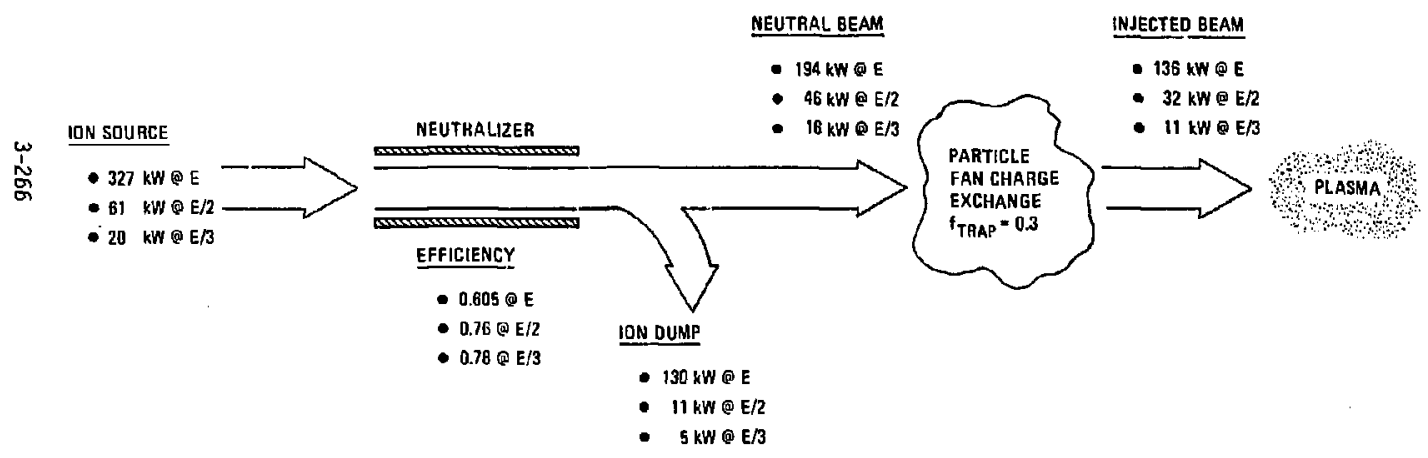

Figure 3-113. Anchor cell pumping beam power fractions. 
Table 3-40. Characteristics of the anchor-celf pumping beam ion source.

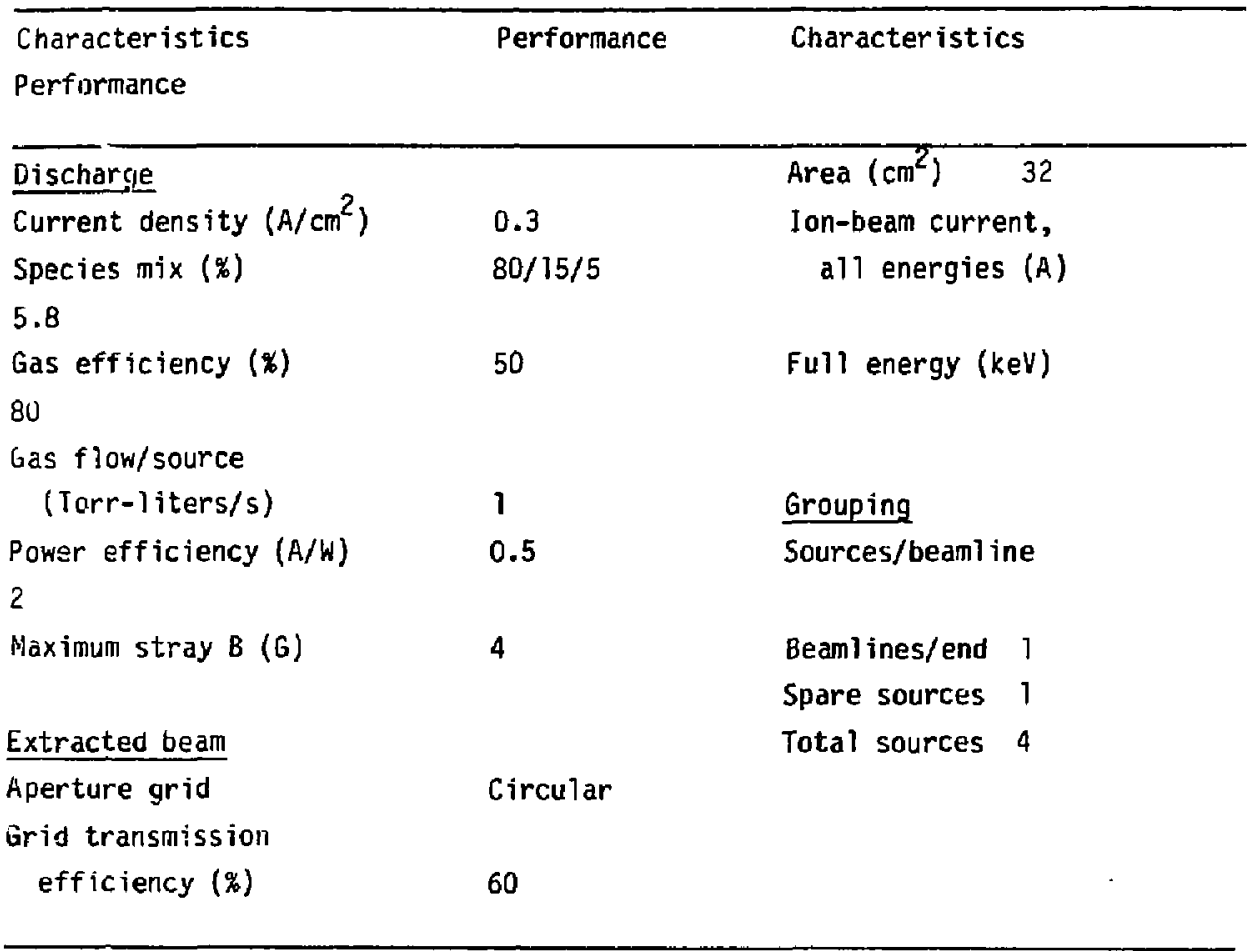


Table 3-39 shows the cryopump load to be small; $1 \mathrm{~m}^{2}$ handles the gas in the first chamber at $\sim 10^{-5}$ Torr. In the second chamber, a significant portion of the gas is conducted through the short arift duct into the end cell, which is continuously pumped by large cryopanels. The remainoer is handled by $0.25 \mathrm{~m}^{2}$ of panel, operating in a chamber pressure of $-5 \times 10^{-6}$.

One difference between the anchor pumping and sloshing beams is the particle deflector, which resides around the drift ouct and transition section on the pumping beam injector. A particle flux of about $300 \mathrm{~W} /(\mathrm{cm})^{2}$ impinges on the injector walls. The magnet flux that guides these particles is almost totally along the axis of the beamline (see Fig. 3-114). For the drift-duct dimensions planned, the power interceptea by the injector duct would be $-250 \mathrm{~kW}$ without a diverter. The diverter modifies the magnetic field to introduce a perpendicular component ( $X$ direction) that bends the particle flux around the injector. A field of $\sim 100 \mathrm{G}$ should suffice. Heat-transfer surfaces line the drift-duct and transition section outside surfaces of the injector to provide a particle dump.

The ion source and gas cell of the beamline must be shieldea from the magnetic fields of the diverter and TOF stray fields. Assuming a fiela magnitude of $140 \mathrm{G}$, the shield thickness is $1.2 \mathrm{~cm}$ and the shielding factor about 120.

Figure 3-175 shows the beam-dump patterns for a surface broadside to the beam. The two sources have essentially the same pattern, separated by only $0.5 \mathrm{~cm}$. The beam dump is only required to handle one beam at a time. Each beam has a peak power density at broadside of $\sim 360 \mathrm{kH} /(\mathrm{cm})^{2}--$ a very mooest load, eve:، at broadside. Figure $3-112$ shows the surfaces of the dump at 35 and $15 \mathrm{deg}$. The surfaces were sized and set at angles to cover areas where the flux is greater than $\sim 10 \mathrm{~W} /(\mathrm{cm})^{2}$. This dump is extracted by slioing the assembly out of the "Jove-tailed" mounting socket (see Fig. 3-112, end view), rotating it $90 \mathrm{deg}$, and extracting it through the particle-fan egress.

\section{Anchor Cell Sloshing Beam. Except for ion source size and} divergence, this injector is of the same basic type as the anchor cell pumping beam. The beam is injected into the plasma fan through its broad dimension at $Z=8.95 \mathrm{~m}$ (Fig. 3-116), which accommodates a 90-oeg injection angle. Arl 

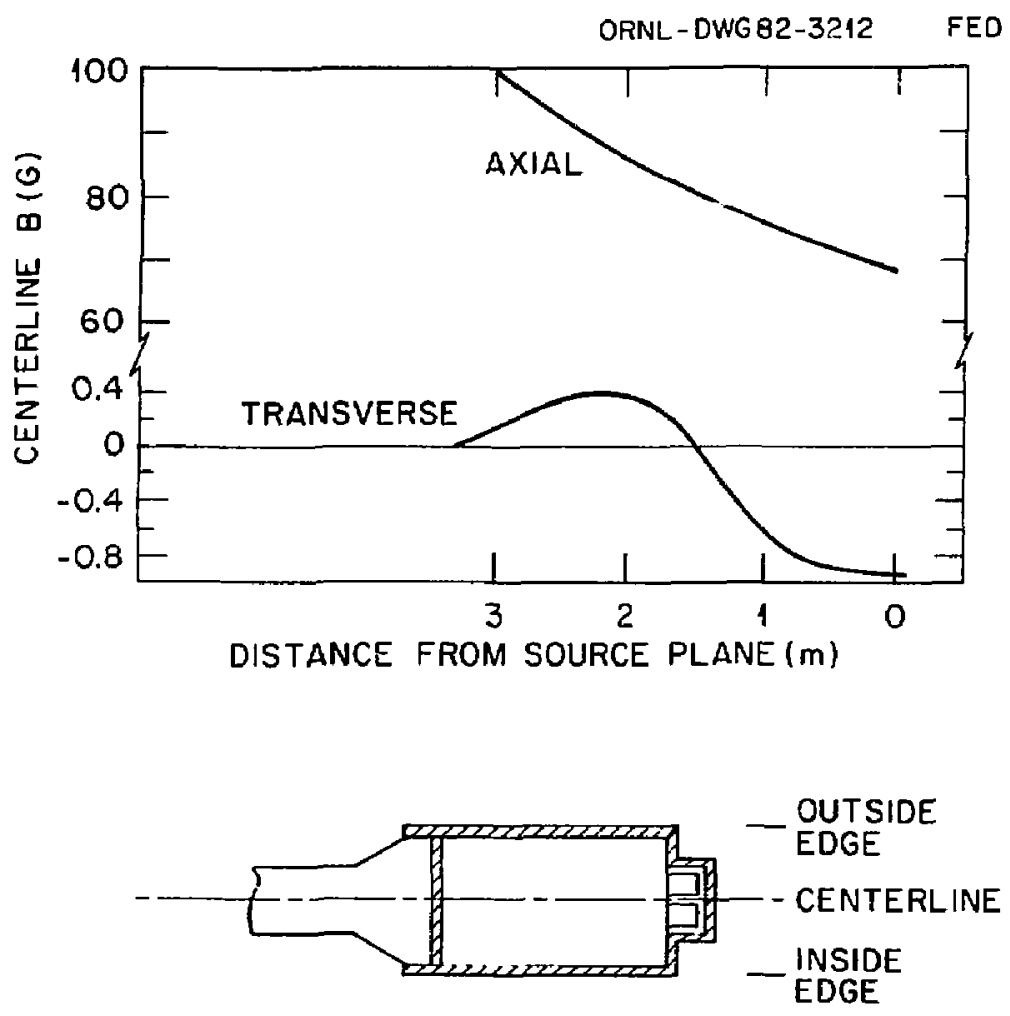

ANCHOR PUMPING BEAMLINE

Figure 3-114. Magnetic fields around anchor-pumping injector. 


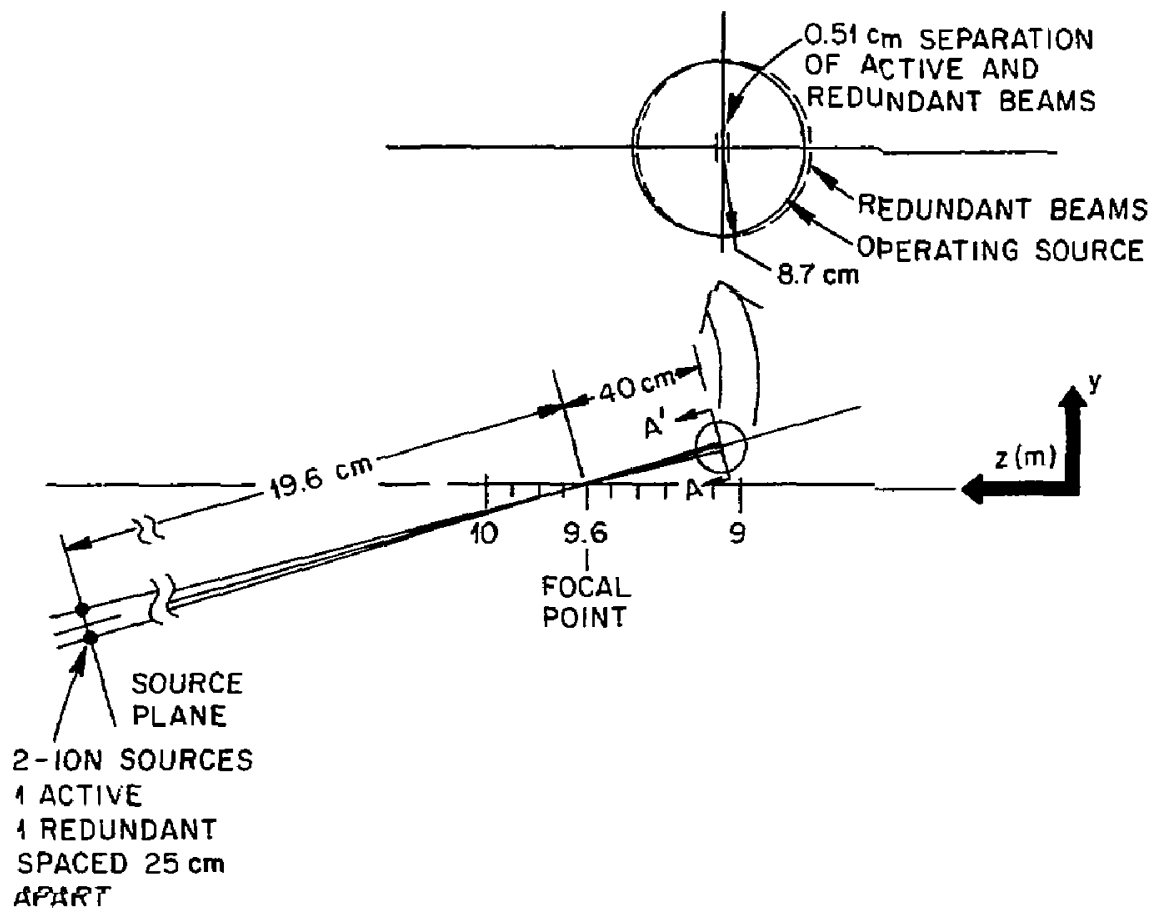

Figure 3-115. Anchor cell pumping beam-dump patterns. 
ORNL-DWG B2-3209 FED
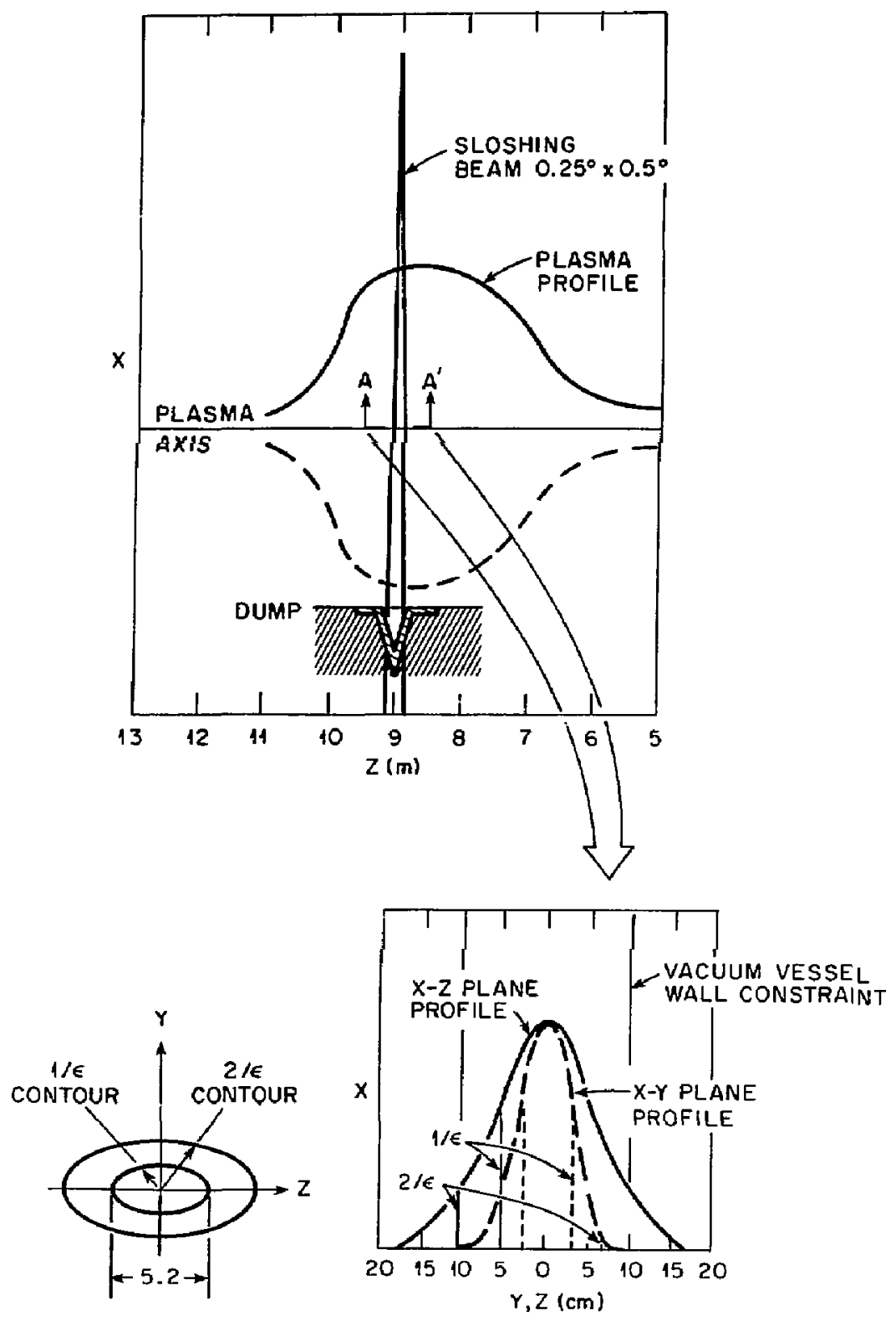

Z-Y PLANE

Figure 3-116. Anchor cell sloshing-beam plasma patterns. 
external beam dump is positioned on the anchor cell wall. Figure 3-117 shows the passage of this beam through the small radius slot of the $M-2$ coil to the dump. At the coil slot egress to the dump, the vacuum vessel wall is at the $3 / \mathrm{e}$ beam contour in the $Y$ direction for a $0.25-\mathrm{deg}$ beam divergence. This spacing assures the wall loading will be small--below $70 \mathrm{~W} /(\mathrm{cm})^{2}$. It is also implicit that this passage have low transmission losses (neglecting plasma trapping, of course). In the orthogonal plane, the divergence is 0.5 deg. At the plasma axis, the $2 /$ e extent along the axis is $\pm 10 \mathrm{~cm}$. Hence, about $86 \%$ of the sloshing beam power is injected into the region between $Z=9.05 \mathrm{~m}$ and $8.85 \mathrm{~m}$. The exploded view of the beam pass in Fig. 3-117 shows the plasma fan drawn to scale. The plasma width matches the $1 / e$ contour at the axis. Ideally, the divergence (1/e) stould be smaller to improve the trapping fraction. However, 1/e values of less than 0.25 deg represent a high deve lopment risk.

The design layout for this injector is shown in Figs. 3-108 through 3-110. As with the other injectors, access for maintenance and connections is through its back. The injector is enclosed in a nuclear shield. A neutron wall loading (at the anchor cell wall) of $3 \times 10^{8} \mathrm{n} /(\mathrm{cm})^{2}-\mathrm{s}$ is estimated. This is not a significant level in terms of cryopanel heating or radiation damage, however, the shield ( $25 \mathrm{~cm}$ in thickness) is required to meet aose levels 24 hours after shut down.

Table 3-41 gives a sumary of anchor cell sloshing beam parameters. The ful1-energy-power fraction is $210 \mathrm{~kW}$, corresponding to a current of $2.6 \mathrm{~A}$ where $2.1 \mathrm{~A}$ is required. This power level is available from a single source. As for the pumping beam, there is a spare source. The characteristic; of the ion source are summarized in Table 3-42. A power balance is given in Fig. 3-118. In this case, the ion-oump power density is slightly lower because the divergence is double in one dimension, even though the injected power is slightly higher. The power density is $863 \mathrm{~W} /(\mathrm{cm})^{2}$ at broagside, or $150 \mathrm{~W} /(\mathrm{cm})^{2}$ on the ion dump surface.

Figure $3-179$ shows the redundant bean crossover with the operating beam at the plasma. This crossover results in a $23-\mathrm{cm}$ separation of the beam patterns on the dump surface some $2.7 \mathrm{~m}$ away. This dump is positioned approximately broaasice to the beam. The power density is $1.95 \mathrm{~kW} /(\mathrm{cm})^{2}$ at broadside. (A 30-deg dump surface would reduce this density to a tolerable leve1.) 


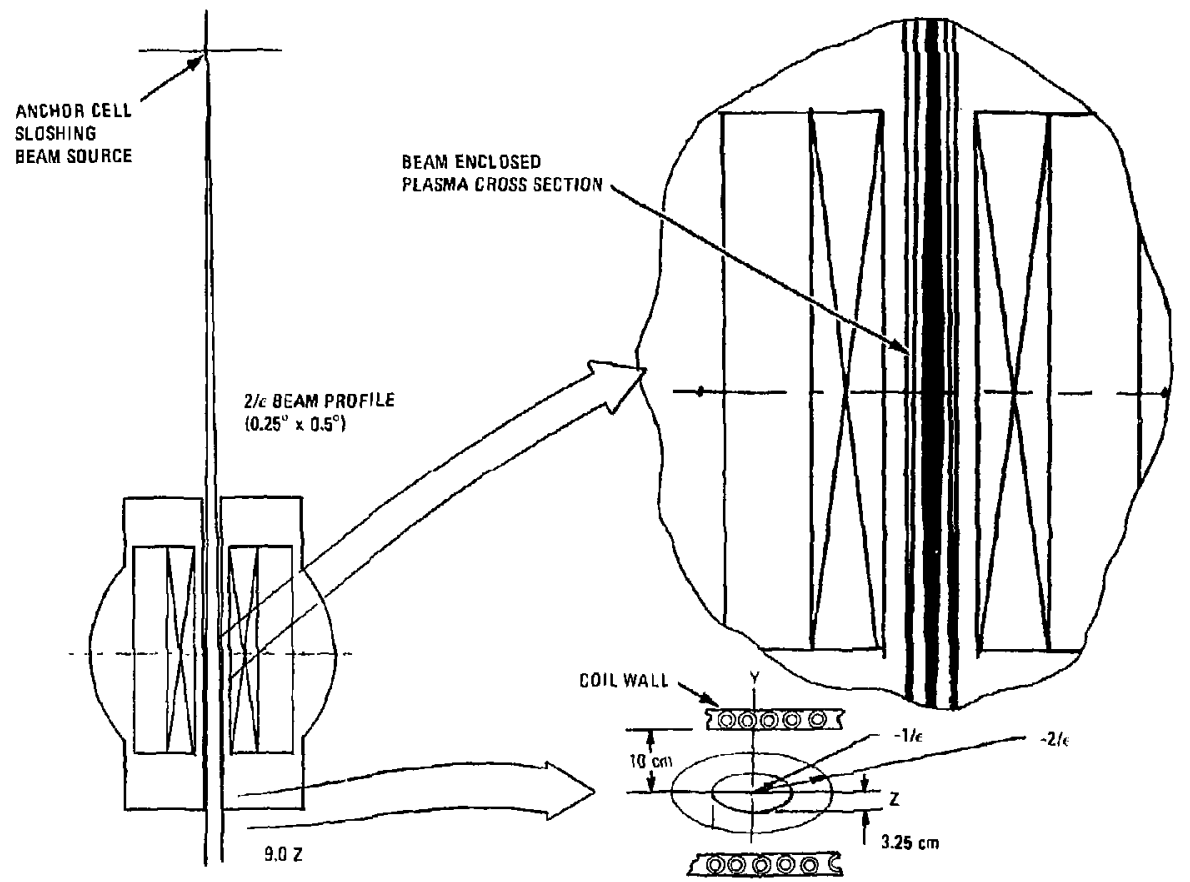

Figure 3-117. Anchor cell sloshing beam access. 
Table 3-41. Sumary of anchor cell sloshing beam parameters.

\begin{tabular}{|c|c|c|c|}
\hline Parameter & Capability & Parameter & Capability \\
\hline Energy (keV) & 80 & Source separation (cm) & 25 \\
\hline Power injected (MW) & 0.277 & Cryopump area $\left(m^{2}\right)$ & \\
\hline Full energy & 0.210 & Gas-cell chamber & 1.8 \\
\hline Half energy & 0.049 & Drift-cell chamber & 0.25 \\
\hline \multirow[t]{2}{*}{ Une-third energy } & 0.017 & & \\
\hline & & Beamline (m) & \\
\hline \multirow[t]{2}{*}{ Prime power (MW) } & 0.537 & oiameter & 7 \\
\hline & & Length & 2 \\
\hline Efficiency $(x)$ & 51.5 & Length of tran- & \\
\hline & & sition section & 0.35 \\
\hline \multicolumn{4}{|l|}{ Fuil energy } \\
\hline \multirow[t]{2}{*}{ current (A) } & 2.63 & Brift-duct & \\
\hline & & cross section, & \\
\hline Number of sources & 2 & width/height $(\mathrm{cm} / \mathrm{cm})$ & $45 / 35$ \\
\hline Divergence, W/E (deg) & $0.25 \times 0.5$ & Drift-duct length $(\mathrm{cm})$ & 35 \\
\hline Beam-crossing & & Cost $(\$ M)$ & 0.84 \\
\hline distance $(\mathrm{cm})$ & 600 & & \\
\hline Source current $(A)$ & 6.3 & & \\
\hline
\end{tabular}


Table 3-42. Characteristics of anchor cell sloshing-beam ion source.

\begin{tabular}{|c|c|c|c|}
\hline Characteristics & Performance & Characteristics & Performance \\
\hline Uischarge & & Beam divergence (deg) & $0.25 \times 0.5$ \\
\hline Current density $\left(\mathrm{A} / \mathrm{cm}^{2}\right)$ & 0.3 & Area $\left(\mathrm{cm}^{2}\right)$ & 35 \\
\hline Species mix $(\%)$ & $80 / 15 / 5$ & $\begin{array}{l}\text { Ion beam current } \\
\text { (a11 energies) (A) }\end{array}$ & 6.3 \\
\hline Gas efficiency ( $\%$ ) & 50 & Full energy (keV) & 80 \\
\hline \multicolumn{4}{|l|}{ Gas flow/source } \\
\hline (Torr-liters/s) & 1.3 & Grouping & \\
\hline Power efficiency $(A / W)$ & 0.3 & Sources/beam? ine & 2 \\
\hline \multirow[t]{2}{*}{ Maximum stray B (G) } & 4 & Beaml ines/end & 1 \\
\hline & & Spare sources & 1 \\
\hline Extracted beam & & Total sources & 4 \\
\hline \multicolumn{4}{|l|}{ Grid transtrission } \\
\hline efficiency $(\%)$ & 60 & & \\
\hline
\end{tabular}




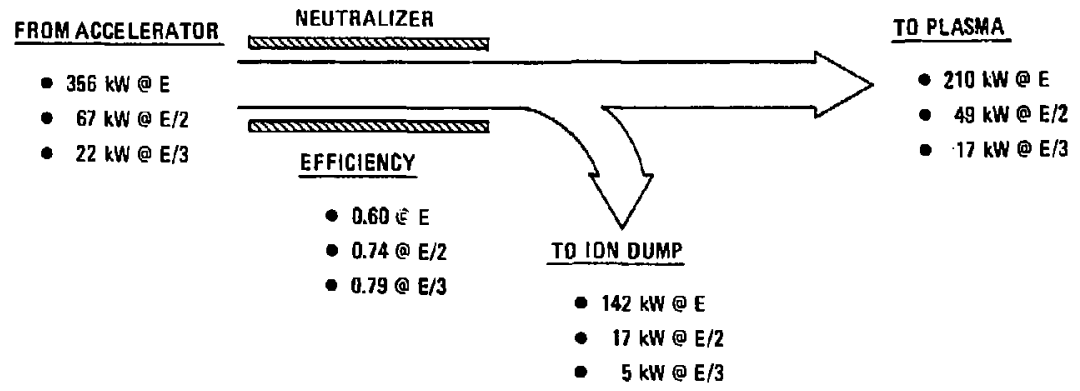

Figure 3-118. Anchor cell sloshing beam power fractions. 


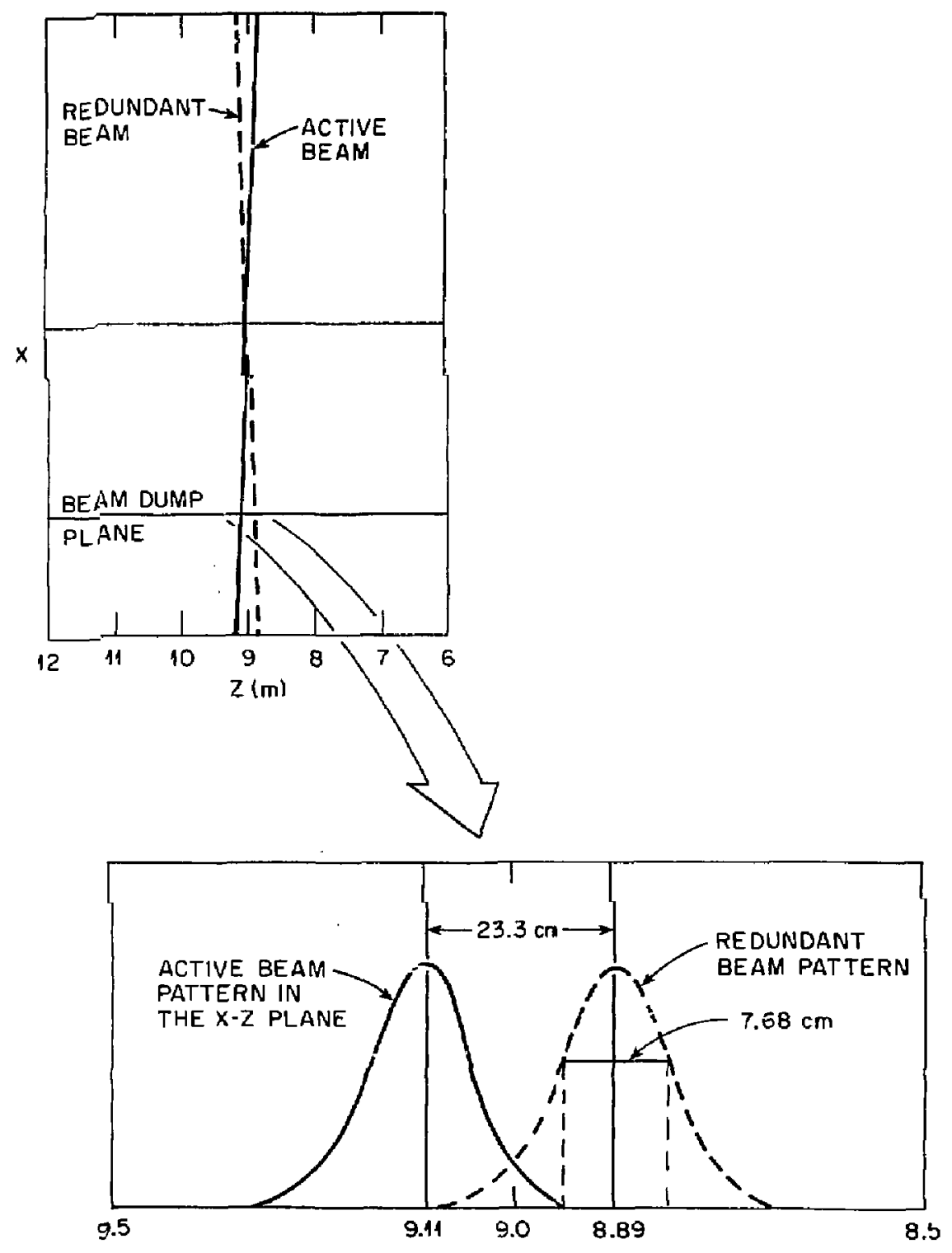

Figure 3-119. Anchor cell sloshing beam dump patterns. 
Cryopumping demands are small in this injector. One square meter of pump is sufficient to handle the gas load in the first chamber at a pressure of $7 \times 10^{-6}$ Torr. In the orift chamber, the duct conductance ano a $0.25-\mathrm{m}^{2}$ panel share the load at $6 \times 10^{-6}$ Torr. Both cryopumps are $>21 \%$ oversized to allow continuous operation with five sections pumping and one section regenerating.

Figure 3-120 shows the axial and transverse magnetic fielo strengths. because the injectors are close to the coils in the anchor cell, the flux density is highest for the sloshing beam. To avoid saturation, the shield should be $>13 \mathrm{~cm}$ thjck, which gives a shielding factor of $\sim 1300$, compared to the $350: 1$ (1400 G/4 G) required. Although this shield could be made smaller if the sloshing injector were moved back, doing so would increa: ? the extent of the absolute beam width and result in higher vacuum-vessel wall loading in the beam passage through $M-z$. The magnetic shield, which weighs 5.7 tons, provides nuclear shielding as well. The concrete nuclear shield could be reduced somewhat.

\section{Key Technical Issues}

Anchor Cell Beam Access Requires Low Divergence and Collimation. Along with functional and performance requirements for anchor-cell beam injection, there are constraints. These constraints impose limitations on the solutions to beam access. Specifically, the salient constraints are (1) coil geometry, (2) TDF cevice stray magnetic tields, (3) plasma shape, (4) source position. limited by device geometry, and (5) technology development. Figure 3-121 shows a plan view of the anchor cell (both the yin-yang coil pair and transition coil). Note that the coil geometry blocks the egress of the anchor pumping bean for the required $15-d e g$ injection angle and $Z$-axis intercept. Figure 3-122 shows the 1 imited ingress of the $M-3$ coils' vacuum-vesse 1 opening. The sloshing beam requires injections at $Z=9 \mathrm{~m}$ and normal to the axis. The enlarged view (Fig. 3-121) near this injection point shows the opening set by the M-2 coil spacing and its vacuum vessel. For the transition pumping bearn, there are at least three possible interference points between the drift-ouct pass and the device. In addition, the proximity of the choke 

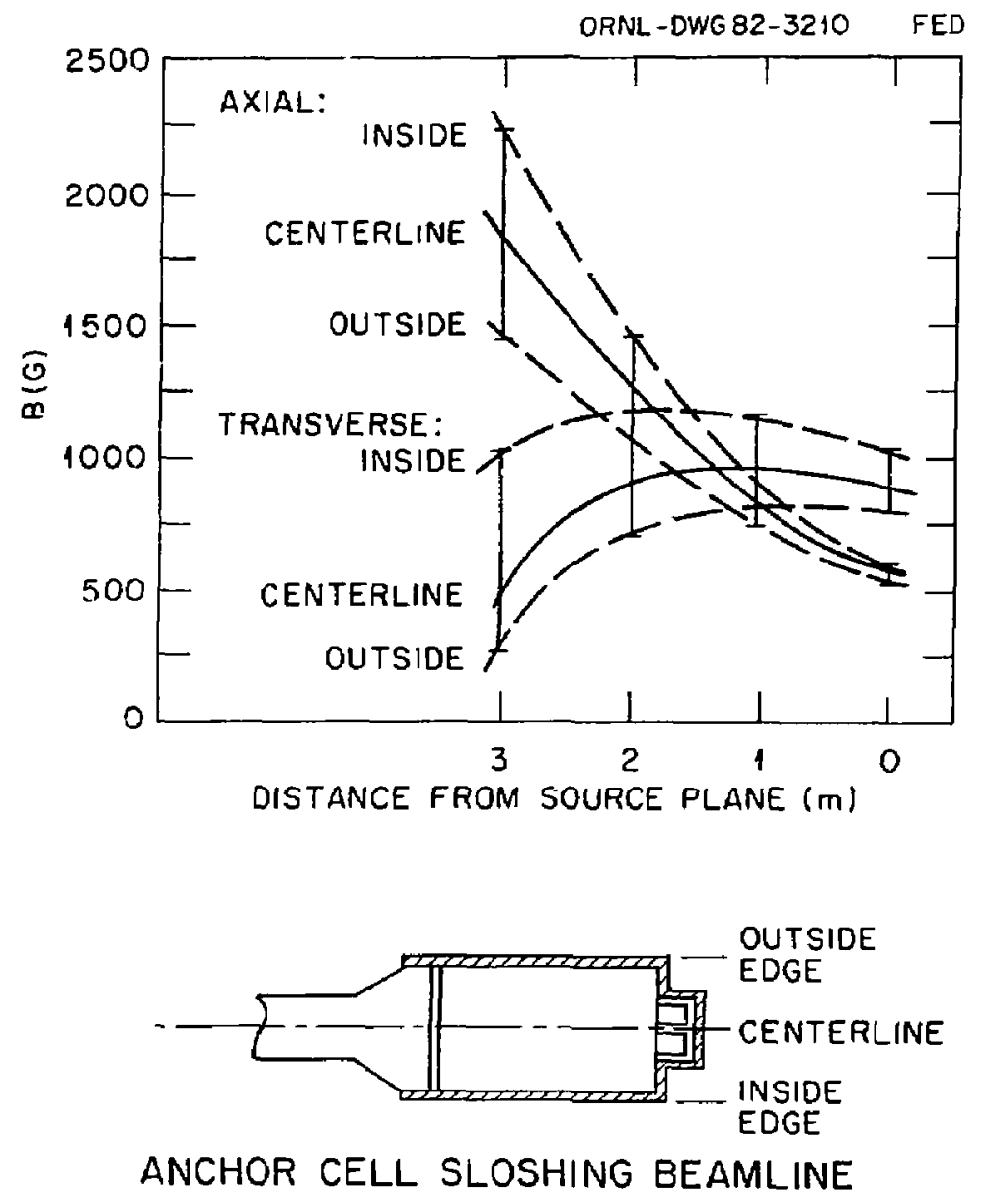

Figure 3-120. Magnetic fields around anchor cell pumping beam. 


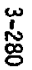

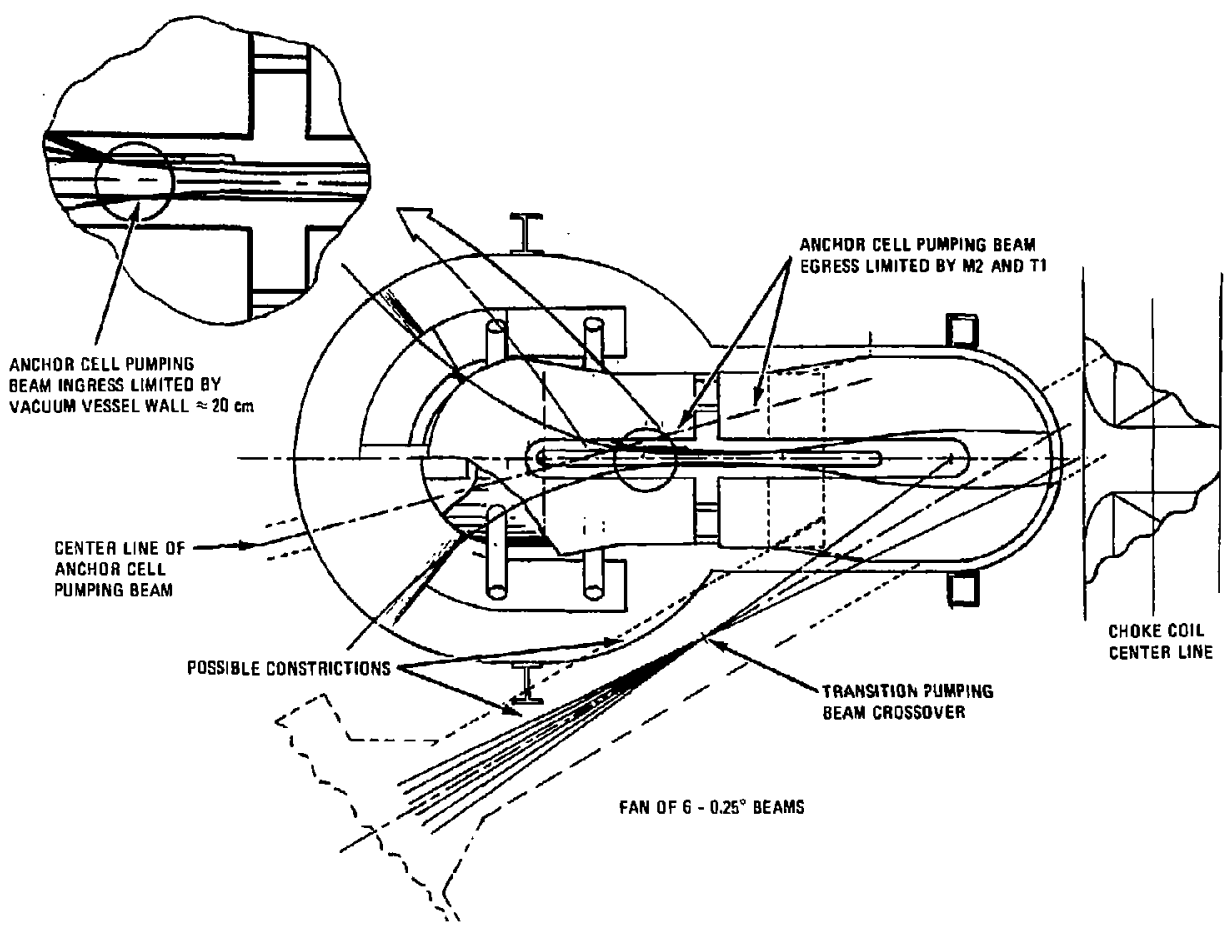

Figure 3-121. Beam access constrictions. 
ORNL OWG 82-3201 ILU

$\omega$
1
0
0

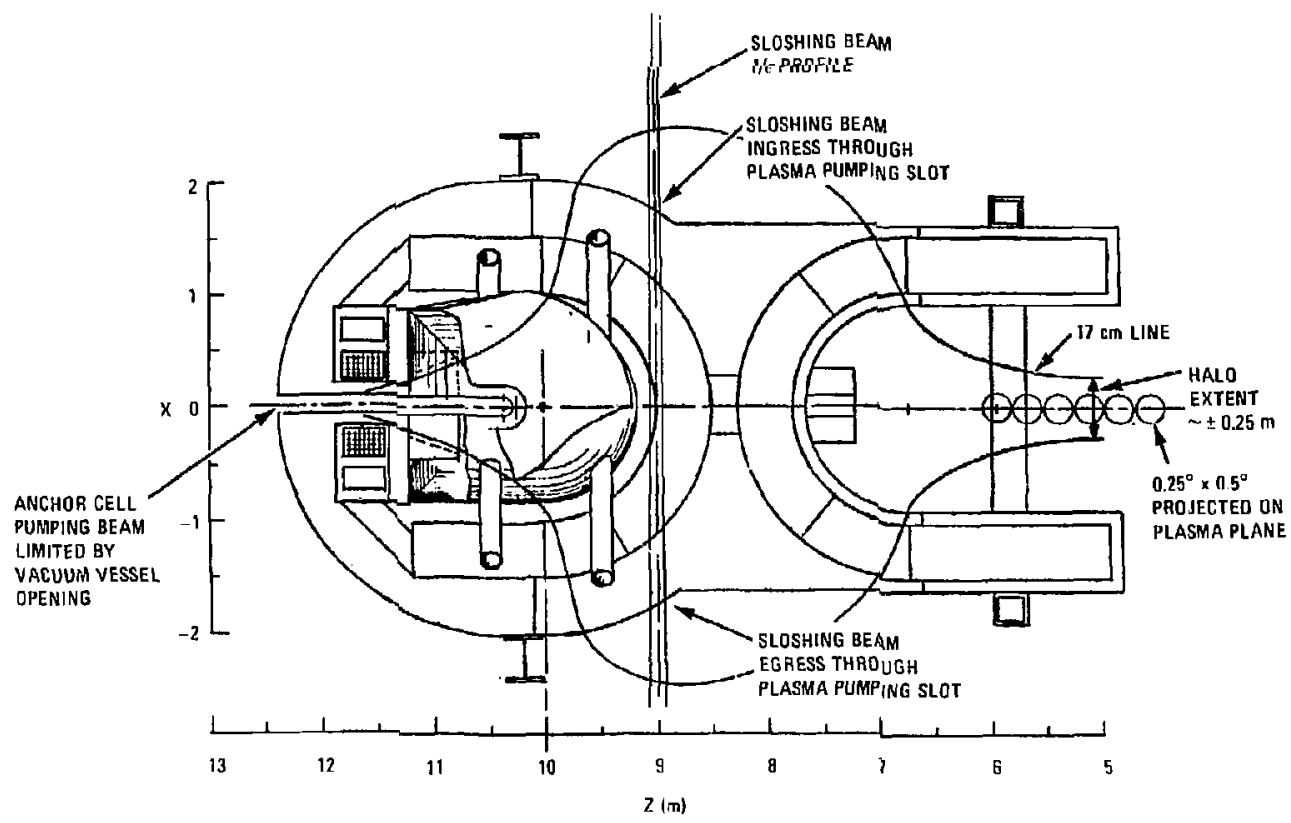

Figure 3-122. beam acceptance angles. 
coil makes it desirable to limit this beam's angular extent. Probably the most serious of the transition bean constraints is interference with the circular "I" beam providing structural support and a mounting surface.

Solutions Consigered. During the preconceptual design study, a number of access solutions were investigated. First, anchor cell vacuum-vessel penetrations for the sloshing beam were considered. The two possible entries were (1) through $M-2$ and (2) between $M-2$ and $M-3$. Because the trapping fraction of the plasma is important to efficiency, the sloshing beam had to be positioned to pass through the broad dimension of the plasma, which entailed shooting it through the small radius of $\mathrm{M}-2$. (Note that this solution also provides beam egress to an external beam dump.) The alternate solution, with the penetration passing between $M-2$ and $M-3$ at $\sim 45$ deg, yielded only a 5\% trapping fraction.

In the case of pumping beams, both anchor and transition, the choice of vacuun-yessel penetration (injection angle and axial position) could be traded off with beam energy. For the anchor pumping beam, penetration through the exit port for the particle fan was deemed best. Positioning the neutral-beam injector in the plane of the far assured a good trapping fraction. The 15-deg injection was chosen to limit the overlap with the sloshing beam. At this angle, the 80-keV energy was appropriate. Even at the 15-deg angle, beam width must be restricted to avoid interaction with the sloshing beam. (See Fig. 3-98.) The ingress through the $M-3$ small radius opening was only about $20-\mathrm{cm}$ high. This also imposed restrictions on the beam width of the anchor pumping beam, but in the orthogonal plane. Hence, to meet access constraints on the anchor pumping beam, the beam must be nearly symmetrical and have a narrow beam width. Further, as noted earlier, a beam egress was impossible; therefore, the dump must be located inside the yin-yang pair. The 15-deg injection angle and $Z$ intercept region (i.e., between $Z=10.02$ and 9.2), along with the beam positioned in the $Y-Z$ plane, dictates this dump location.

Three solutions were considered for the transition beam, each involving different beam wioths. Initially, two sources were ronsidered, each using aivergence $(-0.8 \mathrm{deg})$ sufficient to cover the $Z=4.7$ to 6.0 intercept at the required injection angle of $30 \mathrm{geg}$. Here the heams were positioned in the $Y-Z$ plane (the plane of Fig. 3-121's viẹw) because it provided the greatest access in the orthoganal plane $(x-Z)$. Also, the plasma being nearly 
symmetrical over the intercept imposed no preferences (as in the sloshing beam ase) from the standpoint of trapping fraction. A second approach used a single beam with $\sim 1.5$-deg divergence, the advantage being that a second source could provide $100 \%$ redundancy. The beam crossover point in this case had to be at the plasma; this, in conjunction with the relatively wide divergence, required a wider drift-duct passage and, hence, greater interference with the vacutm vesse] and its support ring. The third approach--a six-beam fan of smal1-beam-width beams--is shown in Fig. 3-12i. Here the choice of beam crossover was based on naking the orift-duct channe 1 as narrow as possible. Consideration was given to noving this crossover back near the support ring, which would necessitate closer source spacing (less than $25 \mathrm{cml})$. This solution of a six-beam fan provided a better energy ueposition pattern and reduced the loaaing on the choke coil by about 2:1 from the two-source solution (see Fig. 3-101). In the orthogonal plane (Fig. 3-123) the useful divergence is limited slightly in the vicinity of the T-l coil pass and by the height of the plasma as it comes out of the choke coil. At a nominal source-to-plasma distance of $1200 \mathrm{~cm}$, a 0.5 -deg divergence is $20-\mathrm{cm}$ high ( $x$ direction), which matches the plasma diameter. Increasing the divergence significantly beyond $0.5 \mathrm{deg}$ would have a degrading effect on the trapping fraction, result in a larger transition beam-power requirement (a)ready at $-3 \mathrm{MW}$ ), and increase the size of the beam dump or the load on the choke coil. The size of the beam dump is constrained by the access between the T-1 coil yoke and the shield wall of the central cell. Figure 3-123b shows a cost comparison of the approaches, which indicate anly a $70 \%$ variation in cost.

Divergence Needs. This discussion of beam access indicates, in genera1, that the solutions required smail beam widths. Small beam widths are achieved by using sources with low aivergence augmented by collimation and by positioning them close to the plasma. As noted, source position is constrained by device geometry and the difficulties of shielding against the environment imposed by the device. The two major environmental hazards are stray magnetic fields and the particle fan in the end cell in which the pumping beams reside. The nuclear environment in the anchor cell is not severe. Although neutron flux levels are at least two or three orders of 


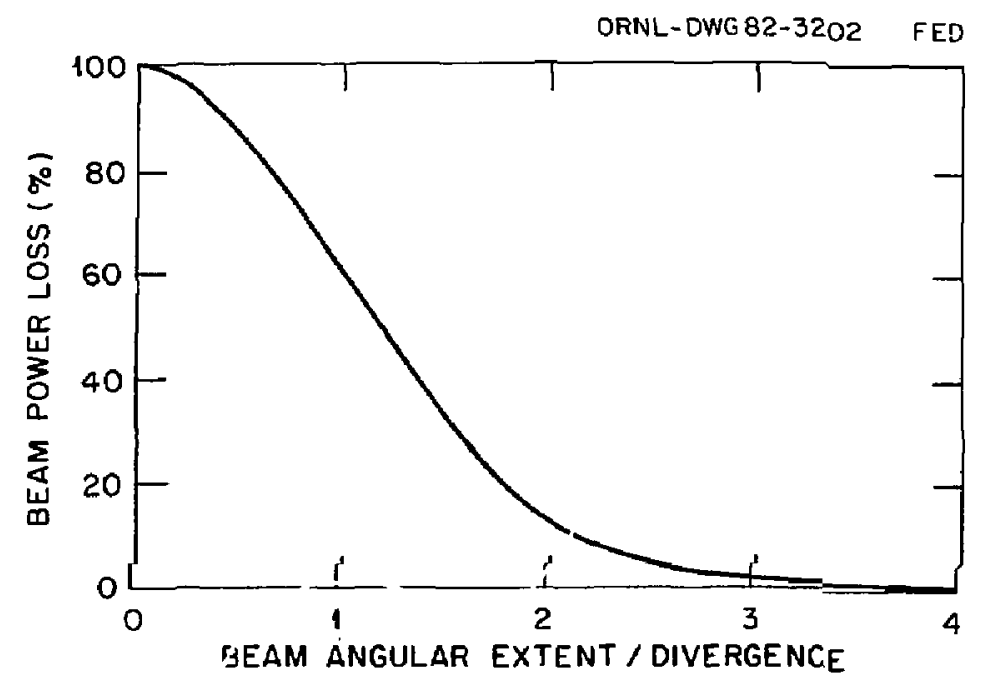

A

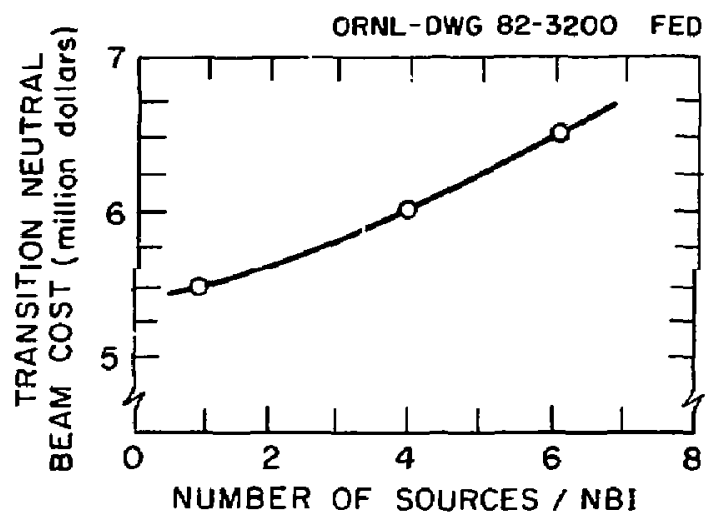

B

Figure 3-123. (a) Beam power loss to to beam scraping. (b) Beam cost increases slowly with number of sources. 
magnitude below those of the central cell, shielding is required. Maintenance .s inhitited by activation. Radiation oamage of beamline components or nuclear heating are not major concerns.

For the sloshing beam, the major concern is the stray magnetic field. This problem is mitigated by moving the beamline back, which increases beam extent at the coil constricicion. Figure 3-120 shows the field strength to be about $1 \mathrm{kG}$ for the source $6 \mathrm{~m}$ from the plasma. At this position, to clear the vacuum vessel walls of the anchor cell, the angular extent of the beam must be 1 imited to about $1.4 \mathrm{deg}(20 \mathrm{~cm} / 820 \mathrm{~cm}$ ) in the $Y$ direction (see Fig. 3-117). This angular extent is satisfied by a $0.25-d e g$ divergence. Magnetic shielding to achieve this beam width is essentjal. For example, with the $0.25-$ deg divergence source, the residue magnetir transwerse field must he limited to less than 76 .

The angular extent desired can be achieved by using a low-divergence (goal of $0.25 \mathrm{deg}-1 / \mathrm{e}$ ) source and collimation. Figure $3-123$ a shows the ilupact of scraping the beam with a collimator to achieve small beam wisths. For example, to limit the angutar extent of a $0.5-b y-0.5-$ deg bearn to 0.25 by 0.25 , about $85 \%$ of the power is lost or, equivalently, the beam] ine must be made approximately seven times larger in power. The pumping beam would grow froin 0.256 to $1.8 \mathrm{MW}$, with the cost increasing from $\$ 2.0$ to $\$ 3.0 \mathrm{million}$.

The anchor puriping beam for the TOF is scraped by the ingress to the vacuum vessel enclosing $M-3$ (see Figs. 3-99, 3-112, 3-121, and 3-123). The source is positioned $16.2 \mathrm{~m}$ from this vacuum-vessel opening (see Fig. 3-97). With this opening enlarged to $28 \mathrm{~cm}$ for beam passage, an anguiar extent of

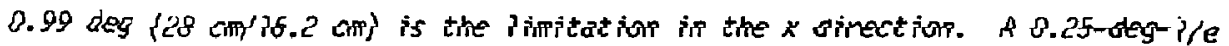
divergence beam would have its $2 /$ e contour coincide with this limitation, and only $14 \%$ of the beam power would be last (see Fig. 3-123a) if the beam were symmetrisal and scraped an equal amount in both planes. Scraping in the orthogonal $p$ lane $(Y-Z)$ is done back at the beamline because the particle fan requires a slot-shaped opening in the vacuum vessel. As noted previausly, this co:limation is required to keep the anchor pumping and sloshing beams from overlapping in the plasma. While the pumping beam could be moved further upstreani in the particle fan to reduce the beam extent, maintenance would become more difficult and fan particle flux would increase, creating high 
loading on the neutral-beam enclosure walls. Figure 3-117 shows that the magnetic field would be no problem if it were necessary to move the pumping beam in closer.

Finally, the transition beam was required to have a divergence of 0.25 by $0.5 \mathrm{deg}$, based on the $1200-\mathrm{cm}$ source-to-plasma distance and the plasma target oimensions. The magnetic field profile for this neutral-beam location is shown in Fig. 3-107. From the standpoint of magnetic fielu, the beamline could be moved up slightly, but not enough to make divergence requirements significantly easier. Maintenance would be slightly more difficult, and particle fan loading would increase slightly but is not a consideration.

Status of Low-Uivergence Source Development. A recent source was built with a measured divergence of 0.25 by $0.9 \mathrm{deg}$, and a design for an ion source with a divergence of 0.25 by $0.25 \mathrm{deg}$ is under development. Sources with divergences of 0.5 by $0.8 \mathrm{deg}$ are more common, however. Developers are finding parameters such as cathode construction and the uniformity of ion-source plasma density important in achieving low divergence. Small sources with reasonable aspect ratios (height to width) $\sim 2: 1$ are thought to offer lower development risk. Of the three source needs discussed, the anchor puning beam is the most critical for divergence. It should be as close to a 0.25 -by-0.25-deg divergence capability as possible. The other two sources specified at 0.25 by $0.5 \mathrm{deg}$ could be relaxeo somewhat in the wide dimension. As stateo at the outset, the approach proposed is a combination of low-divergence optics in conjunction with collimation. If the oivergence desireo is not achieved fully, source size could be increased and a higher percentage of the beam could be scraped to achieve the equivalence of low divergence.

Direct Recovery Development Risk Is Worthwhile. In the foregoing discussion the ion dump for the transition pumping beam had to be designed to handle a heat flux of $7.23 \mathrm{~kW} /(\mathrm{cm})^{2}$. This was accommodated by setting the beam-dump surface at 6 deg with respect to the beam, thereby reducing the incident flux to $750 \mathrm{~W} /(\mathrm{cm})^{2}$. At this angle, however, the sputtering rate is 25 times higher than at broadside for the same heat flux. Dump life is est imated at $1 / 3 \mathrm{FPY}$. For all six beams, the full-energy power dissipated in 
the ion dump is $-1.9 \mathrm{NH}$. A direct-recovery device would lower the heat fiux to the ion dump by a factor of 10:1; it would also increase overall beamline efficiency from 51 to $61 \%$. In doing this, the power dissipated at the aump is about $1.2 \mathrm{MH}$ less, which allows the thickness of the beam dump to increase. The particle rate is expected to be about the same for the recovery and no-recovery cases; however, the energy is 10:1 less, which impacts the sputtering rate. Figure 3-124 shows the trade-off of bean-oump angle and expected life for both the recovery and no-recovery cases. Clearly, direct energy recovery, although it requires further development, is an improvement. A proof-of-principle experiment was run, and a report was written. ${ }^{26}$ Figure 3-125 shows a schematic of the experiment for a 20-A-35-keV hydrogen beam. Recovery efficiencies of $>80 \%$ were measured.

Magnetic Shielding Requirements for Low Divergence. Low divergence is critical to beam access in the anchor cell. Source divergence in the 0.25 -to 0.5 -deg range is required. On the basis of simple theory, we know that the resiaue magnetic flux inside the shield injector enclosure both steers and broaders the beam. A charged particle moving through a $B$ field perpendicular to its velocity vector traces a circular path with a radius dependent on both particle energy and $B$ field flux density. The circular path in the neutralizer is terminated when there is a charge exchange and the ion is neutralized and no longer under the influence of the field. This somewhat random event leads to a scattering of the normally low divergence beam with a nonzero mean. Although the steering error (bias) is easily compensated for, the divergence degradation nust be tolerated. A quantitative modeling of this distortion was done by J. Conrad. ${ }^{27}$ Figure 3-126 shows the dependence on beam energy ana flux density to contain the beam distortion to within $20 \%$. The residue fields perpendicular to the beam must be less than $4 \mathrm{G}$ for the s0-keV beam energy.

Drift-Duct Design Optimization. For multiple source beamines, drift-auct optical efficiency depends on geometry and beam aivergence. The orift-auct cross section of a continuous duct is made smallest if beams from the sources cross over in the center of the drift-auct length. This minimum cross section duct reduces neutron and plasma impurity streaming into the beamline and 


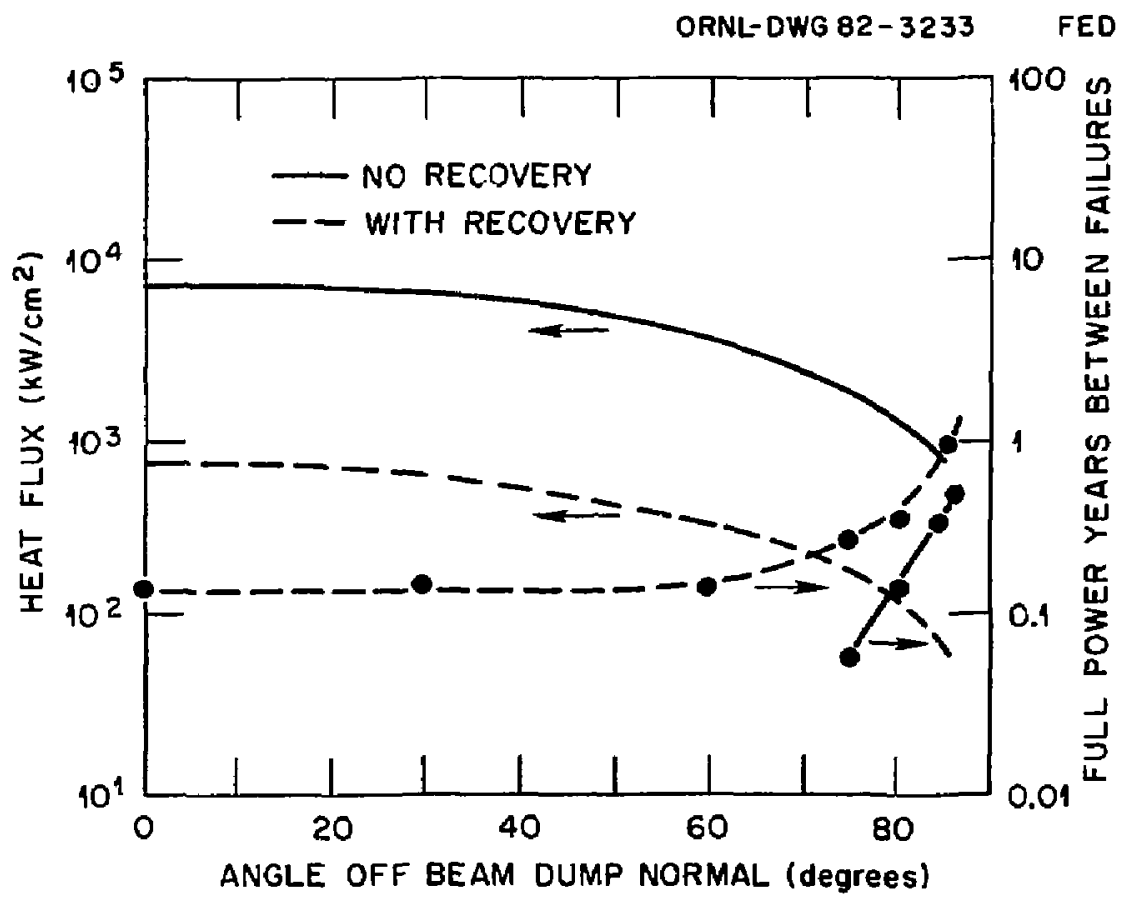

Figure 3-124. Ion-dump characteristics. 

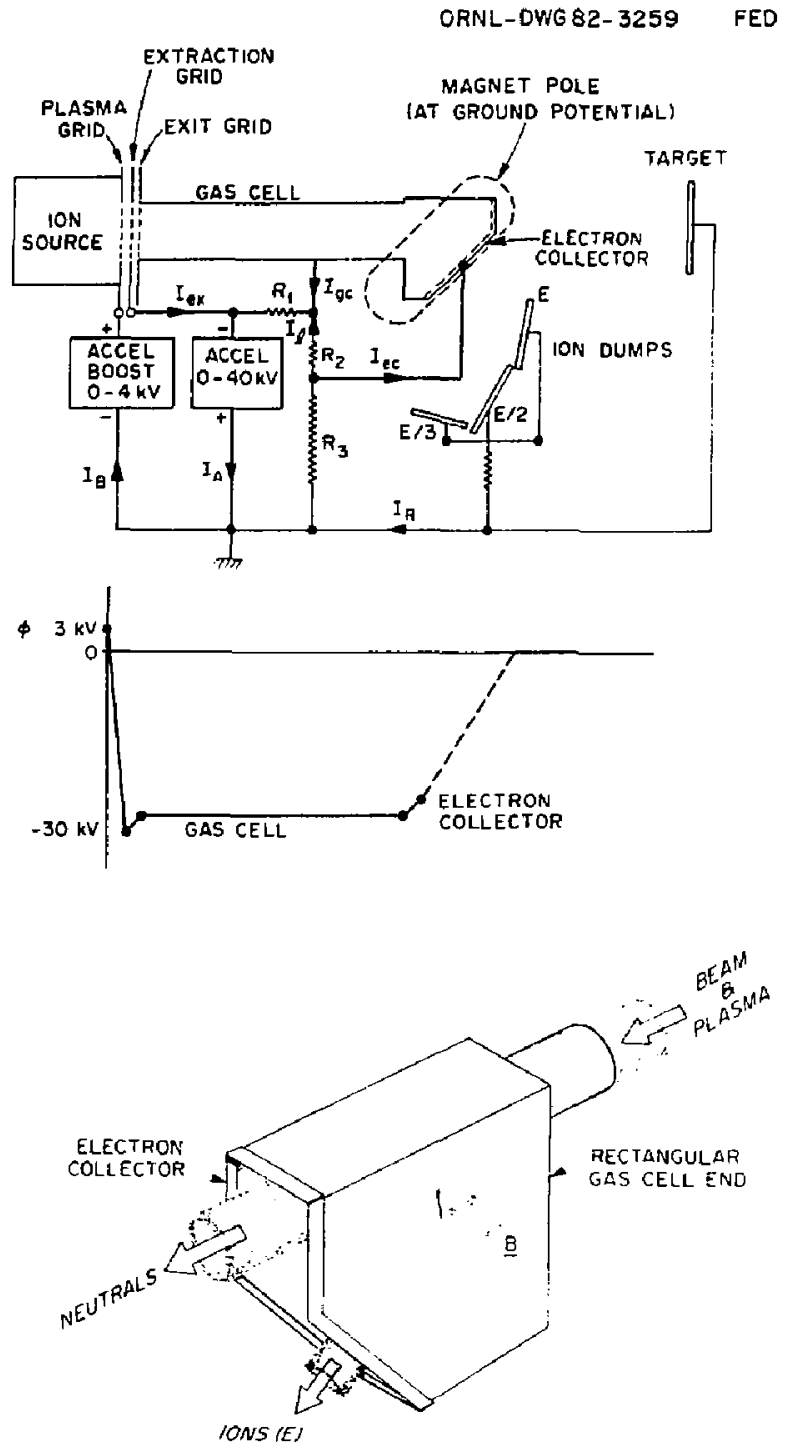

Figure 3-125. Direct-recovery configuration. 


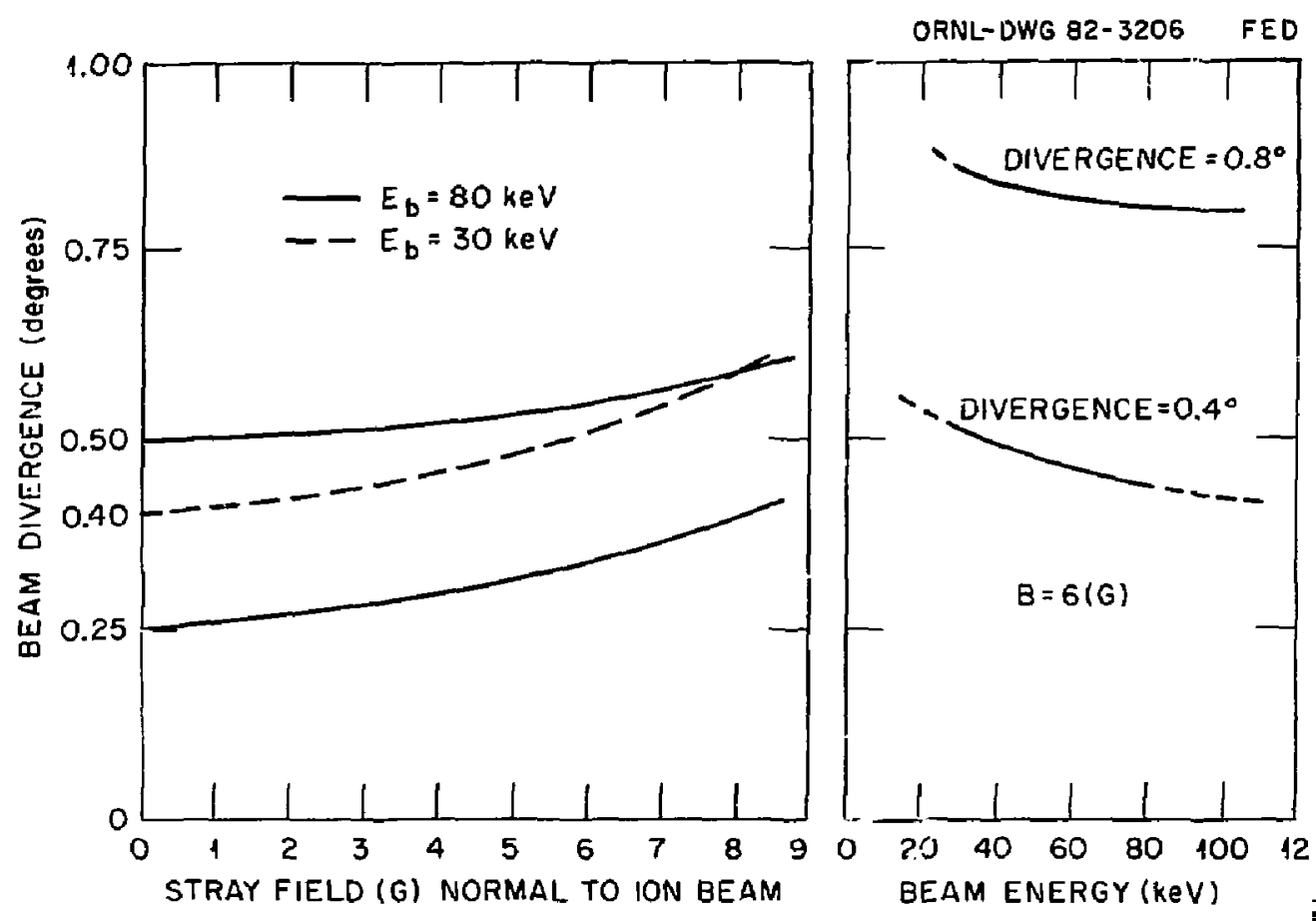

Figure 3-126. Divergence degradation from magnetic fields. 
minimizes TUF vacuum-vessel penetrations. For the anchor cell, the drift duct is not continuous and is composed of the beamline orift duct and the anchor cell vacuum-vessel penetrations. Each of the three neutral-beam injector interfaces is different. In the case of the six-source transition beam, the width of the beam fan is restricted as it passes the vacuum vessel even though no formal duct is constituted by the vacuum-vessel penetration. Here the beam crossover is at the center of this restricted passage. For the anchor cell pumping and sloshing injectors, the beam crossover is at the plasma. The rationale here was that redundant sources must iliuminate the same plasma target, which is only possible with the crossover at the target, not upsiream in the drift duct. Further, the main constriction for the pumping beam is the penetration of the vacuum vessei, which is $7600 \mathrm{~cm}$ from the source but only $300 \mathrm{~cm}$ from the crossover at the plasma. The crossover of operating and reaunaant source beams was not placeo in the center of this constriction because the illuminated region of the plasma would be source-beam dependent and the beam dump extent would be enlarged. Because the two source beams are in the plane of the particle fan, which aiready requires a slot-shaped egress, little impact was incurred by shifting the crossover. The sloshing beams ' sources are also aligned with a slot-shaped penetration in the vacuum vessel, in this case that of the plasma pumping channel. Moving the source beam crossover wollo have little impact on the sloshing beam injector's drift auct, which is a snall portion of the total drift-duct extent (i.e., injector drift duct plus vaculum- vessel penetration). With the crossover at the plasma, both beams illumirate the plasma target and the bean-dump extent is smaller than it would have been with the crossover at the drift-duct center (see Fig. 3-121).

Neutra]-Beam Injector Sizing for Acceptable Avajlability. A steady-state device such as the TOF inherent7y requires high availability subsystems. Total duplication of all subsystems is prohibitively expensive. Selected reaundancy can meet the availability requirement in a cost-effective manner. The first ground rule is that the injector itself (e.g., structure, shielas, vacuum bounciary, power supplies, auxiliary/support equipment, etc.,) has a long mean time between failures (MTBF). Second, higher failure rate components can be oversized and/or duplicated. Finally, complete loss of any 
function is a serial failure; that is, the TDF would be down. Partial failures can be sustained in most cases. Degradation is graceful in that small reductions in plasma heating power in a given region do not have a catastrophic effect. Some preliminary investigations (failure-effects analysis, in a sense) support the latter conclusion. For example, the transition pumping funciion was found to be quite forgiving of small changes in power and illuminated region.

No quantitative allocation of availability was assigned to the anchor cell neutral-beam injectors. The approach taken was to oversize the sources by $\approx 20 \%$ in all injectors. Because the power requirements of the anchor pumping and sloshing beams are so small, the provision of redundant sources would have little impact. Power supplies would be switched between sources. For the transition pumping beam, the six-beam fan has inherent redunoancy. With five of six sources running, the impact on performance should be acceptable. Finally, the cryopumps are oversized by at least $20 \%$ so that five of six sections can provide full operation while the sixth panel/section is regenerated. 


\section{A. MICROWAVE SYSTEMS}

\section{.4.1. Overview}

The two microwave systems used as a thermal barrier in the TOF differ only in power level and frequency. Figure 3-127 shows the position of the microwave ports (two sets per system) in the anchor cel1. Both input and output ports have been provided since only 50 to $75 \%$ of the power is trapped (single-pass absorption) by the plasma. Launchers, fitted to receptacles that are inserted into the input ports, are supplied by circular waveguide. All remaining components are located autside the reactor hall in a "hands-on" area. In the discussion that follows three issues are broached, namely (1) component avajlability, (2) window survivability, and (3) power control.

\subsubsection{Requirements}

Table 3-43 summarizes requirements for the two microwave systems, one at $35 \mathrm{GHz}$, the other at $60 \mathrm{GHz}$. A total power (for both ends of the device) of $500 \mathrm{~kW}$ at $35 \mathrm{GHz}$ and $676 \mathrm{~kW}$ at $60 \mathrm{GHz}$ is required. Injection is at $9.5 \mathrm{~m}$ and $10.5 \mathrm{~m}$ along the $z$-axis. Mode control is required, with the e-vector oriented perpendicular to the magnetic flux. The neutron flux must be specified to determine the suitability of $\mathrm{rf}$ window material and the need for nuclear shielding. Cyclotron resonance can occur inside the waveguide. If the window (vacuum break) is too far from the plasma edge, where the magnetic field supports resonance at the transmitted frenuency, breakdown can occur. Magnetic flux data at the 9.5 - and $10.5-\mathrm{m}$ injection points indicate that resonance is possible behind the launcher. This necessitates positioning the window close to or within the launcher.

\subsubsection{Design Description}

Section cuts of the design layout shown in Fig. 3-128 were made at the microwave inje:tion points. Each 10-cm-diam input part enters the anchor cel1 at $\sim 45$ deg with respect to either the $x$ or $y$ axis and is fitted with $a$ mounting flange at the outside of the vacuum vessel. A launcher receptacle 


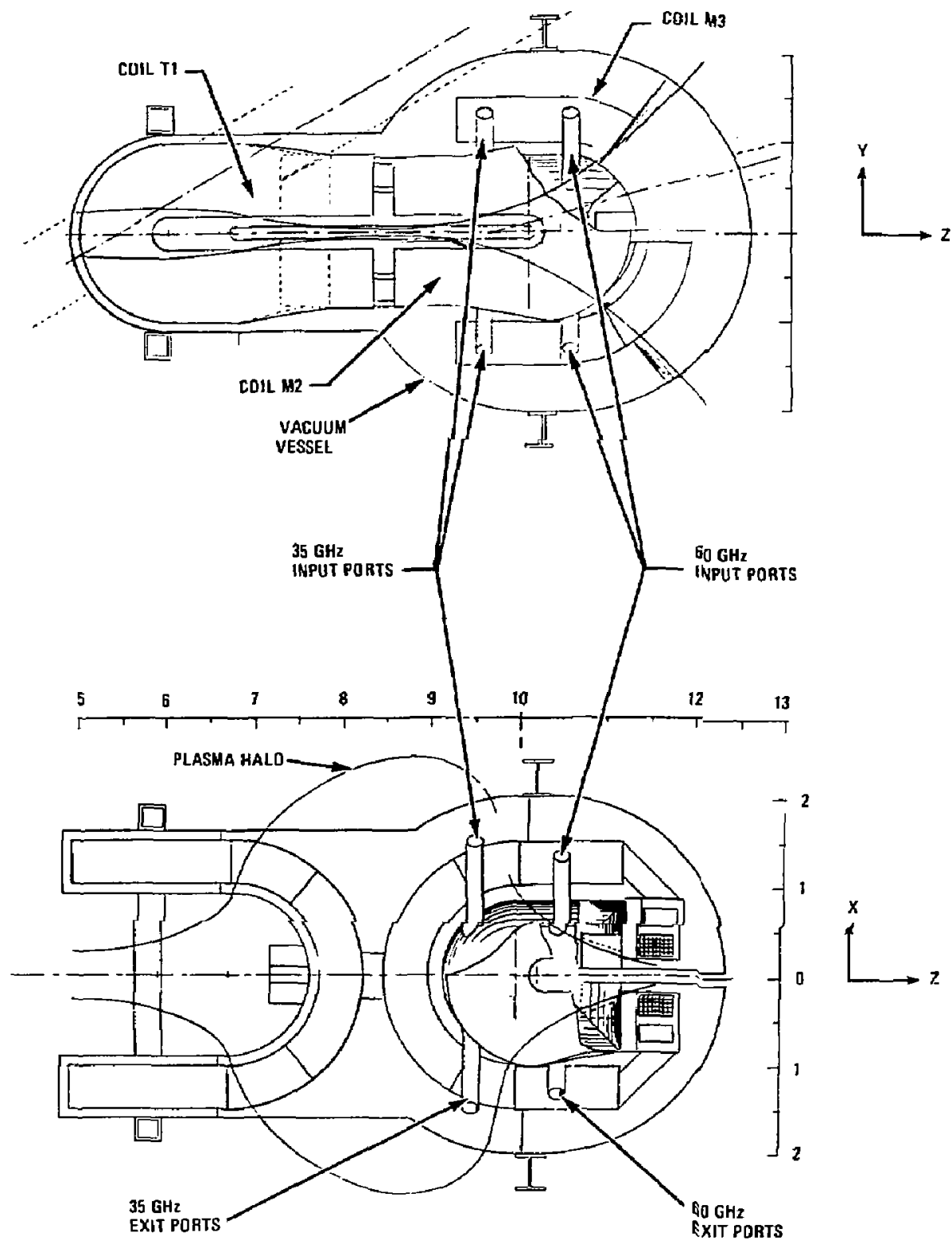

Figure 3-127. ECRH/anchor cell interface. 
Table 3-43. Summary of anchor cell microwave requirements.

\begin{tabular}{lll}
\hline Parameter & System 1 & System 2 \\
\hline Frequency (GHz) & 35 & 60 \\
Power (KW) & $500^{\mathrm{a}}$ & $676^{\mathrm{a}}$ \\
Position in Z (m) & 9.5 & 10.5 \\
Mode & $--\mathrm{b}$ &.$- \mathrm{b}$ \\
Pulse length (s) & 5.5 & 5.5 \\
Absorption ( & -50 & -50 \\
Neutron flux (W/cm $\left.{ }^{2}-\mathrm{s}\right)$ & $-3 \times 10^{8}$ & $-3 \times 10^{8}$ \\
Magnetic flux (T), & $-2.6 / 0.3$ & $-0.3 / 2.8$ \\
$X-Z / Y-Z$ at launcher & & \\
\end{tabular}

Total (both ends).

Extraordinary.

Csingle pass. 


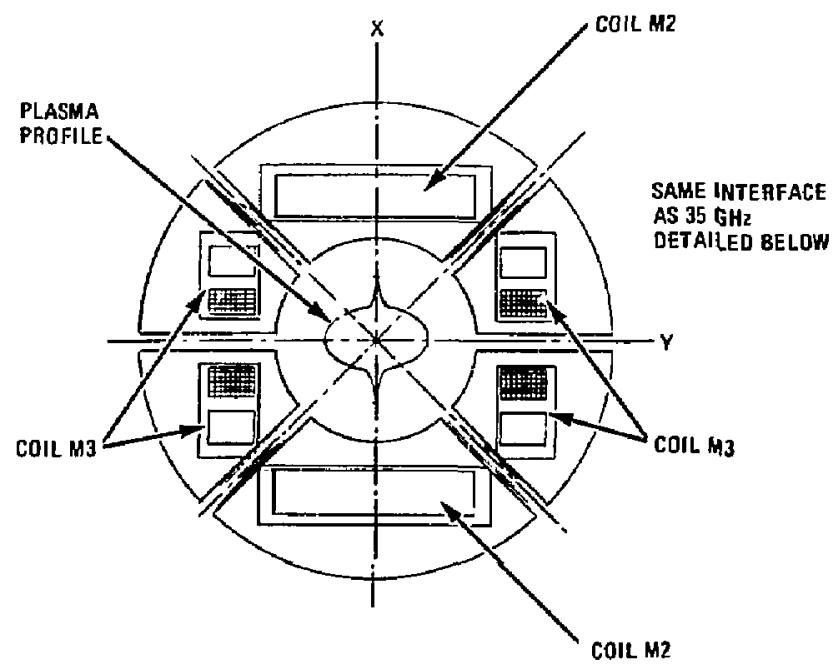

ANCHOR CELL SECTION

CUT AT $Z=10.5 \mathrm{~m}$

(60 $\mathbf{G H z})$

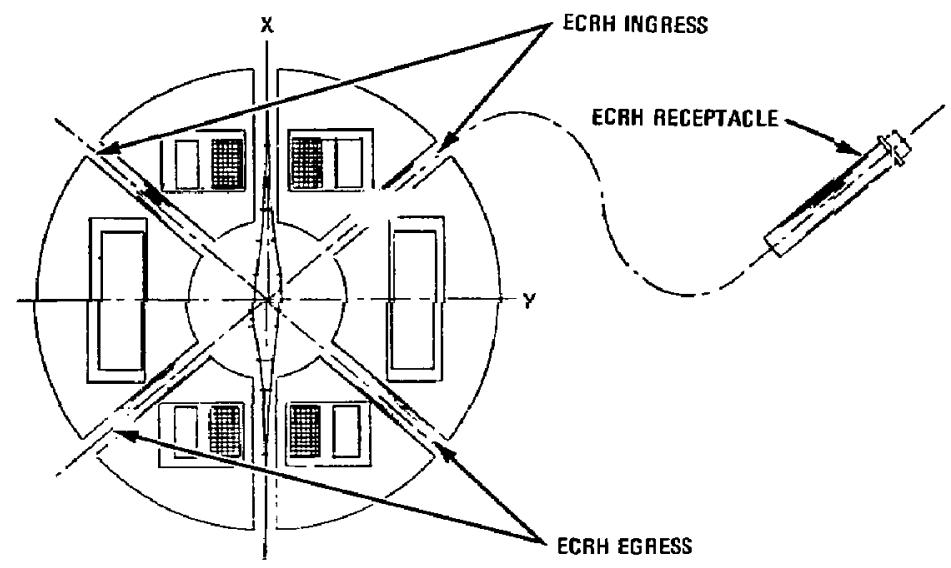

ANCHDA CELL SECTION

CUT AT Z=9.5 m

(35 $\mathrm{GH} 2)$

Figure 3-128. Cross section of anchor cell vacuum vessel at ECRH launcher. 
inserts into the port and is secured to the mounting flange by a ring. This arrangement facilitates maintenance and provides support for the launcher assembly.

Figure $3-129$ is an elaboration on the launcher assembly, which is composed of a short section of smooth, overmoded circular waveguide, a cooled BeO window, and mounting flanges to interface with the launcher receptacle and transmission lines. Floracarbon-100 (a trade name) cools the window. This window is tailared after the Varian window design for the gyrotron. The mode compatability of this window with the $H E_{11}$ mode is a component detailed design problem that, given a little development, is assumed to be tractable. A short section of corrugated circular waveguide is provided between the window and the transmission line disconnect, which is mounted to the ECRH receptacle. The entire transmission line is pressurized to about 1 atm of sulfur hexafloride $\left(S F_{6}\right)$ and cooled with a water pipe wrapped about the outside over the portion within the anchor cell.

The egress ports shown in $\mathrm{Fig}$. 3-128 act as dumps for the refracted beam of the launcher. After the untrapped power passes through the egress, either absorbing or reflecting surfaces are needed to prevent returned power. The objective of these "dumps" is to minimize reflections off the vacuum-vesse 1 wails (inside the plasm chamber) that might inject power at spurious locations. Their success depends on the vacuum-vessel geometry, magnetic fields, and plasma characteristic, the last two of which have an impact on the degree of beam refraction. A rule of thumb for estimating this impact is to compare the plasma to cyclotron frequency. If the ratio is less than say 0.2 to 0.1 , the refraction of the ECRH beam should be minimal. This appears to be the case for the anchor cell; therefore, the egress port is expected to be an effective dump. Application of a ray-tracing code is needed to confirm the degree of refraction.

Figure 3-130 shows details of the transmission system from the launcher to the high-power oscillator (gryatron), where undesirable modes are filtered out by the mode filter. The mode is then converted from the desired mode (probably $\mathrm{TE}_{02}$ or $\mathrm{TE}_{03}$ ) to the $\mathrm{HE}_{11}$, which is transmitted through the low-loss corrugated waveguide. A high-power switch and dummy load provide a local checkout capability, and three separate $S_{6}$ systems provide double protection against tritium release during an accident. The monitoring 


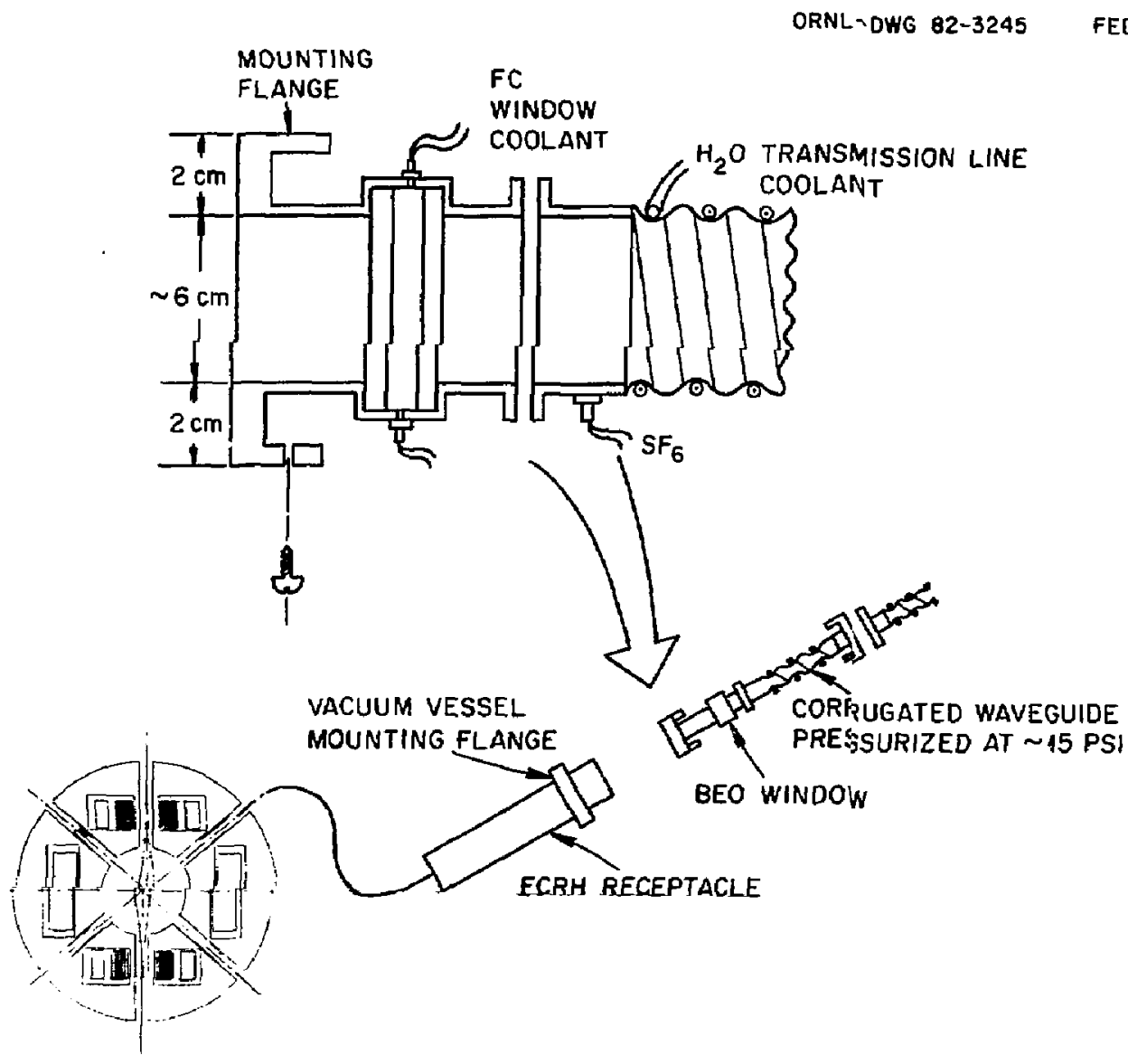

Figure 3-129. ECRH launcher detail. 


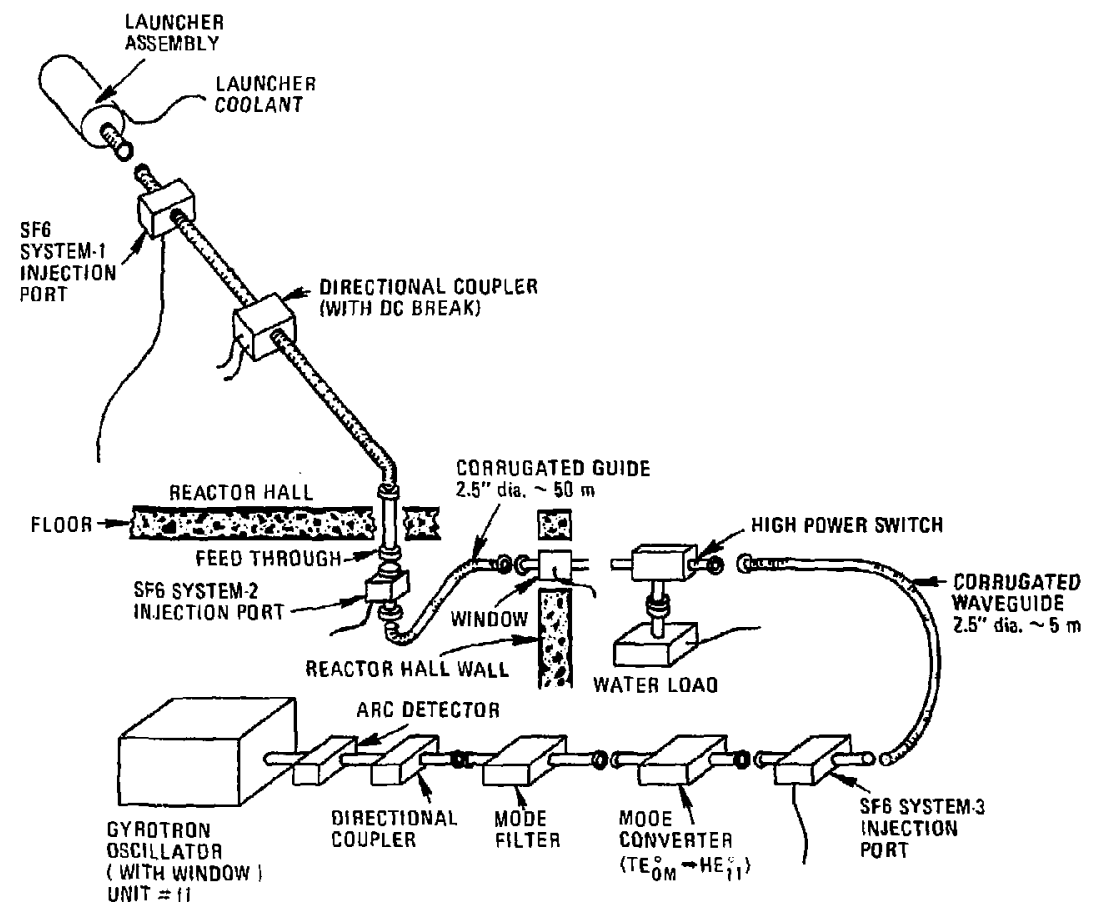

Figure 3-130. ECRH transmission system. 
provisions shown are, specifically, the arc detector and directional coup?er, which report conditions at the gyrotron output, and another coupler at the launcher end, which provides samples' forward and reverse power level to estimate injected power and launcher VSWR. The transmisson line is a 2.5-in.-i.d. corrugated waveguide.

The block diagram in Fig. 3-131 shows the equipment and support system interfaces required for one end of the TDF. Table 3-44 sumarizes the system parameters. This equipment provides $250 \mathrm{~kW}$ of $35-\mathrm{GHz}$ and $338 \mathrm{kH} \mathrm{of} 60-\mathrm{GHz}$ microwave power; the prime power comes in at $13.8 \mathrm{kV}$ for the high-voltage system. Table 3-45 gives the supply parameters for a single gryotron oscillator. Each gyrotron has its own modulator to provide individual contro1. The cooling requirements for the launcher and transmission line internal to the anchor cell are small (on the order of $10 \mathrm{~kW}$ ). A fiber-optics system is used to transmit, monitor, and comand signals between the processor and the transfators located at the control or monitoring sampler. Interface with the main instrumentation and control (I\&C) of TDF is by means of a fiber-optic buss. The monitoring and control processor has a distributed architecture, receiving and transmitting microlevel commands to this I\&C buss. Microscheduling is done in the processor and transmitted to the controllers. Raw monitoring data are smoothed and formatted for local diagnostics or transmission to the $I \& C$.

Figure 3-132 shows transmission-system loss estimates. Most of the larger contributors to this loss are associated with mode-control components. At present, there are few data on the mode mix at the gyrotron output. The only data taken were cold test data on the 60-GHz tube, indicating that $>90 \%$ of the output wauld be at the desired mode. The 0.3-dB loss corresponds to a 93\% mode purity from the tube, a total transmission loss of 1.7 , or $0.33 \mathrm{~dB}$. For the 35-GHz system, the tube output is assumed to be $200 \mathrm{~kW}$. At the launcher, the power would be $135 \mathrm{~kW}$, or $270 \mathrm{~kW}$ total compared to the $250 \mathrm{~kW}$ required. Similarly, the 60-GHz tube is assumed to output $275 \mathrm{~kW}$, which gives a power of $185 \mathrm{kH}$ per tube at the launcher, or $370 \mathrm{~kW}$ tatal compared to the $338 \mathrm{kH}$ needed. Gyrotron efficiency is expected to be approximately $45 \%$. Using this figure, the $0.33-d B$ transmission loss, and an assumed $5 \%$ loss in the modulator power supply gives an overall efficiency of $\sim 28 \%$. 


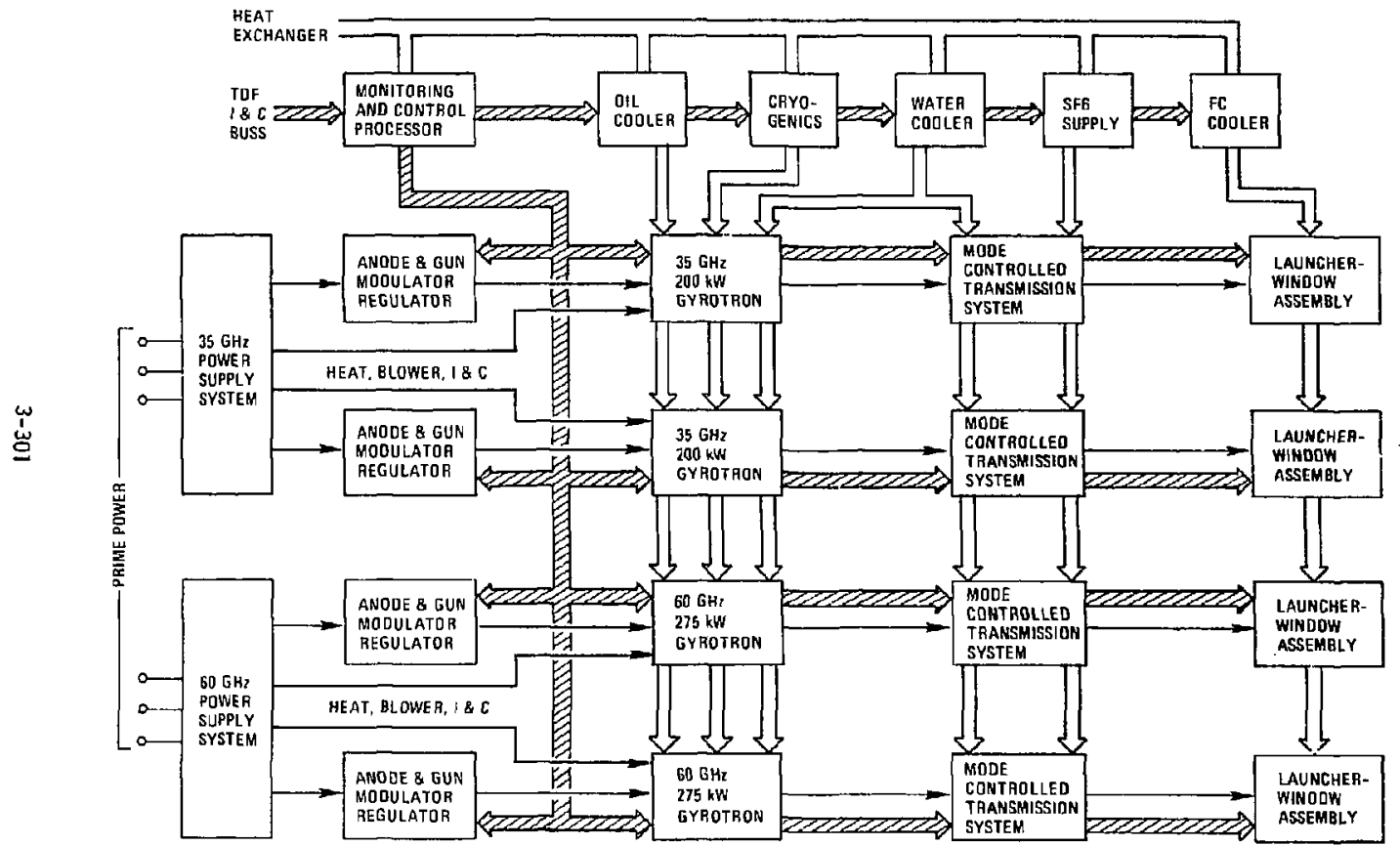

Figure 3-131. ECRH system block diagram. 
Table 3-44. Surmary of system parameters (based on end values).

\begin{tabular}{|c|c|c|}
\hline Parameter & System I & System 2 \\
\hline Frequency (GHz) & 35 & 36 \\
\hline \multicolumn{3}{|l|}{ Power (kW) } \\
\hline Generated & 400 & 500 \\
\hline Injected & 250 & 338 \\
\hline Prime power (kW) & 944 & 1285 \\
\hline Efficiency $(x)$ & 28.6 & 28.6 \\
\hline Gyrotrons & 2 & 2 \\
\hline Launchers & 2 & 2 \\
\hline
\end{tabular}

Table 3-45. Supply parameters for gyrotron oscillator (based on 60-GHz tube developed by varian).

\begin{tabular}{|c|c|c|c|}
\hline Beam voltage $(k V)$ & 80 & LHe $(\pi / h)$ & $\pi$ \\
\hline Beam current $(A)$ & 8 & $\mathrm{LN}_{2}(\pi / \mathrm{h})$ & 0.5 \\
\hline Gun-anode voltage (kV) & 20 & Water $(g p m)$ & TBS \\
\hline Heater voltage (V) & 12 & $0 i 1(\mathrm{spm})$ & TBS \\
\hline Heater current $(A)$ & 2 & & \\
\hline
\end{tabular}


ORNL-OWG 81-3342A FED

- All losses

IN dB's

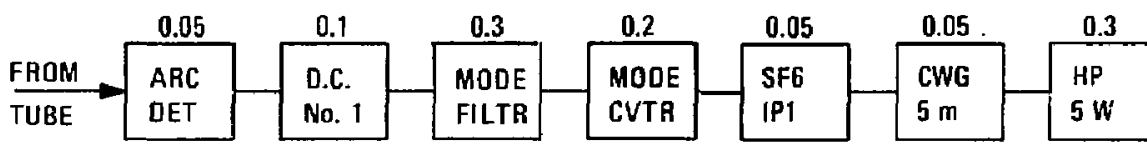

\begin{tabular}{|c|c|c|c|c|c|c|}
\hline 0.1 & 0.1 & 0.05 & 0.05 & 0.05 & 0.1 & 0.05 \\
\hline WNOW & CWG & SF6 & FEED & CWG & D.C. & CWG \\
\hline No. 1 & $50 \mathrm{~m}$ & IP2 & THRU & $10 \mathrm{~m}$ & No. 2 & \\
\hline
\end{tabular}

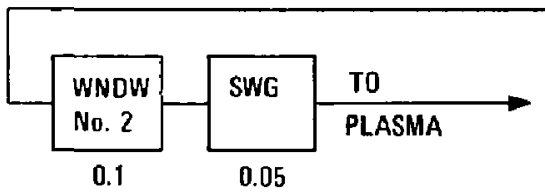

Figure 3-132. ECRH loss summary. 
Component Availability. The least available major component is the high-power gyrotron. Long-pulse (hundreds of miliiseconds) and $\mathrm{CW}$ versions of the $28-\mathrm{GHz}$ gyrotron are presently avajlable. At $35 \mathrm{GHz}$, medium- (tens of milliseconds) and short-pulse (less than 1 miljisecond) tubes are available from Thomson CSF. The Naval Research Laboratory (NRL) developed a $35-\mathrm{GHz}$, short-pulse, $70-\mathrm{kW}$ tube and is now working on a $35-\mathrm{GHz}$, I-MW version that provides hundreds of kilowatts but a shart pulse. A $35-\mathrm{GHz} \mathrm{cW}$ tube is clearly feasible but needs development. A scaling of frequency from the $28-\mathrm{GHz}$ version is a possibility. A scaling effort to produce a $56-6 \mathrm{~Hz}$ tube from the $60-\mathrm{GHz}$ design is currentiy underway.

Development of the $60-\mathrm{GHz} \mathrm{Cw}$ tube is proceeding nicely. One vencior, Varian, has operated a tube at $124 \mathrm{~kW}$ for up to one hour. This tube was demonstrated at we 11 over $200 \mathrm{~kW}$ (its required power output) for short pulses. Hughes, another vendor, has reached $280 \mathrm{~kW}$ at short-pulse durations and additional low-power or beam-only tests at tens of milliseconds. Short-pulse, high-frequency (>90 GHz) tubes have also been built and tested. The 60-6Hz, 275-kW cw tube is not available today, but should be obtainable by the time TUF requires it.

Other components have limited availability. Transmission systems in which the mode is controlled have been developed at GA Technologies (28 $6 \mathrm{~Hz}$ in the past, $60 \mathrm{GHz}$ currently) and are under development at Princeton (60 GHz) as well. The high-power switch needs development. Other fusion microwave systems have similar needs; therefore, the components should be available for TDF. The mode purity of these devices will be important in achieving the aforementioned transmission efficiency.

Window Survivability. In the design description the window was positioned facing the plasma. Normally this position would be of concern because of neutron /radiation damage ano the possible deposition of impurities from the TUF. The radiation damage threshola is $\sim 10^{20} \mathrm{n} / \mathrm{cm}^{2}$. With a neutron flux at the wall of $<10^{10} \mathrm{n} /(\mathrm{cm})^{2}-\mathrm{s}, 10^{10} \mathrm{~s}$ or -300 FPY would be neeaed to reach a oamage level--hardly a concern. The planned window will be recessed about $5 \mathrm{~cm}$ to reduce the deposition of impurities. While it would be desirable to move the window back further in the guide, there is concern about forming a plasma in the guide. Because of the high magnetic fields in the 
vicinity of the launcher, it is possible to have cyclotron resonance at the triving frequency ( 35 or $60 \mathrm{binz}$ ) if the vacuum break is positioned outboard rom the resonance surface.

Power Control. Mode control, which is expensive in terms of transmission system loss, cost, and complexity, is needed to control power deposition in the tramission system. A mixed mode system can have a transmission loss of less than $10 \%$ compared to the $33 \%$ estimated for the Tof microwave system. However, unknown ana/or spurious modes can reduce power-handling capability on the transmission system and cause power deposition in unwanted portions of the plasma. The result is lower effective heating in desired portions of the plasma. Overall, however, the mode-controlled system may rave higher efficiency.

Power absorbed by the plasma vs that of the cavity (plasma chamber) vialis and its opening is another part of the power-deposition control problem. This problem has been modeled using a power-balance approach. Power transmitteo to the plasma is:

$$
\begin{aligned}
& T_{\text {plasmá }}=1 \text { - losses } \\
& \text { Losses }=Q_{\text {loaded }} / Q_{\text {unloaded }} . \\
& Q_{\text {loaded cavity }}^{-1}=\text { power in/w - energy stored } \\
& =1 / Q_{\text {plasma }}+1 / Q_{\text {apertures }}+1 / Q_{\text {cavity walls }} .
\end{aligned}
$$

where

$$
\begin{aligned}
& Q_{\text {aperture }}=\frac{8 \pi \text { volume of cavity }}{\text { Wavelength } \times \text { area of aperture }}, \\
& Q_{\text {unloaded }}^{-1}=1 / Q_{\text {aperture }}+1 / Q_{\text {cevity walls }}, \\
& Q_{\text {plasma }}=\frac{-4 \pi \text { length of cavity in wavelengths }}{\ell n[1-\text { single-pass absorption }]},
\end{aligned}
$$


$Q_{\text {cavity walls }}=3$-cavity volume/28-cavity wall surface area,

$\delta=$ skin depth $=1 / \sqrt{\pi} f_{\mu \sigma,}$

where $\mu$ is permeability, $f$ is frequency, and $\sigma$ is conductivity.

The power transmitted to the plasma was estimated at about $72 \%$, based on a single-pass absorption of 0.5 . This means that $28 \%$ of the $370 \mathrm{~kW}$ of $60-\mathrm{GHz}$ and $270 \mathrm{~kW}$ of $35-\mathrm{GHz}$ power is either absorbed or reflected by the cavity. Hopeful1y, most of this $180 \mathrm{~kW}$ is carried away by the dump ports opposite the injection ports, but this depends on the amount of microwave beam refraction as it passes through the plasma. The more exact solution is to do a raytracing analysis for the plasma magnetic fields and cavity geometry. Another approach would be to evaluate the impact of microwave power reflected to other points in the plasma. It is possible that the impact is slight and that a more exact analysis of power flow is not required.

The power-balance approach is expedient but gross. The cavity geometry is very complex. The power injected into the plasma is beamed or focused not omnidirectiona 1, hence more power should be absorbed by the dump ports than by any port of a simple carity. Clearly more investigation is needed. 


\subsection{LOW-ENERGY DEUTERIUM INJECTION}

The systems defined in this section inject low-energy deuterium into the machine to provide (1) initial gas fill, (2) a plasma target for the anchor cell neutral-beam injectors during the startup scenario defined in Table 3-46, and (3) low-energy ion-flow stabilization during operation. All nascent high-energy ions, both deuterium and tritium, are supplied with neutral-beam injectors described in section 3.3 .

before startup (see Table 3-46), the vacuum vessel is pumped down arid backfilled to a few mitorr of hyorogen when performing glow-oischarge cleaning. Gas injectors, also known as gas puffers, perform this function. After glow-discharge cleaning is completed, plasma guns located on the end walls inject a low-energy plasma into the anchor cell to provide a target for the associated neutral-beam injectors. About 0.1 s after the initial startup phase, the gas injectors are phased out and pellet injectors are phaseo into operation. Frozen pellets are injected into the central cell to establish the stabilizing low-energy ion flow through the mirror-plug region needed for continuous stable operation. The requirements and design features of these three types of deuterium injéctors are described in detail below.

\subsubsection{Gas Injectors}

Summary. Deuterium gas injectors, also known as gas puffers, will inject into three cells: tine left anchor cell, the central cell, and the right anchor cell. During glow-discharge cleaning and startup, deuterium gas will be injected into all three cells.

Two independent gas injector systems will be provided, either of which can inoependently meet the requirements. The use of components proven in gas-puffing systems of present-day fusion experiments and the two-system redindancy ensure high system availability. The shelf life of the equipment is the driving factor that determines system reliability. 
Tab7e 3-46. TDF startup scenarjo.

\begin{tabular}{|c|c|c|c|}
\hline \multicolumn{2}{|r|}{ Topic } & \multicolumn{2}{|r|}{ Equipment } \\
\hline (1) & $\begin{array}{l}\text { Initial surface } \\
\text { cleaning (or } \\
\text { after major } \\
\text { open maintenance) }\end{array}$ & $\begin{array}{l}\text { Glow-discharge cleaning } \\
\text { about } 100 \text { hours }\end{array}$ & $\begin{array}{l}\text { Insertable anodes in each } \\
\text { anchor and in central cell. } \\
\text { - I-kV power supply for slow } \\
\text { aischarge of a few } \mu \mathrm{A} / \mathrm{cm} \mathrm{C}^{2} \\
\text { at wall. } \\
\text { - } \mathrm{H}_{2} \text { fil and pumping sys- } \\
\text { tem for a few miorr pres- } \\
\text { sure with sl-hr gas-recycle } \\
\text { times. }\end{array}$ \\
\hline \multirow[t]{4}{*}{ (2) } & Plasma buildup & $\begin{array}{l}\text { Initial phase } \\
\text { about } 10 \mathrm{~ms}\end{array}$ & $\begin{array}{l}\text { Two or more MFTF-B type 4- } \\
\text { cm-i.d./15-Cm o.d. piasma } \\
\text { stream guns at each end } \\
\text { wall. } \\
\text { Locate guns to map in along } \\
\text { B to C cell only as needed } \\
\text { during first } 10 \text { nis. Each } \\
\text { gun injected about } 20 \text { Torr- } \\
7 \text { iter/s } D_{2} \text { plasma (about } \\
226 \text { A equivalent) at a few } \\
\text { eV average energy. }\end{array}$ \\
\hline & & $\begin{array}{l}\text { Tandem } \phi \\
\text { about } 20 \mathrm{~ms}\end{array}$ & $\begin{array}{l}\text { Bring on } 28 \mathrm{GHz} \text { ECRH power } \\
\text { (s } 100 \mathrm{~kW} \text { ) in each anchor). } \\
\text { - Add about } 10 \text { Torr-1iter/s } \\
\mathrm{D}_{2} \text { for each anchor (rough } \\
\text { estimate). } \\
\text { - Adci about } 15 \text { Torr-1iter/s } \\
\text { D2 for the central ce } 1] \text {. } \\
\text { Sequence in s?oshing NBI in } \\
\text { anchor. }\end{array}$ \\
\hline & & $\begin{array}{l}\text { Barrier formation } \\
\text { about } 100 \text { mis }\end{array}$ & $\begin{array}{l}\text { - Add main 56-GHz ECKH power } \\
\text { ( } \leq 1 \mathrm{MW} \text { tota1). } \\
\text { - Iñcredse } \mathrm{D}_{2} \text { in centraf cel1 } \\
\text { to about } 30 \text { Torr-liter/s. } \\
\text { - Ado startup (about } 100 \mathrm{~A} \text { at } \\
40 \mathrm{kV} \text {; WBI in C cel1 possi- } \\
\text { bly from derated } 80-\mathrm{kV} \text { spare } \\
\text { NBI set. } \\
\text { - Sequence in part of pump NBI } \\
\text { in transition sections. } \\
\text { - Reduce anchor gas feeds to } \\
\text { zero. }\end{array}$ \\
\hline & & $\begin{array}{l}\text { Barrier equilibrium } \\
\text { operation } \\
\text { about } 100 \mathrm{~ms}\end{array}$ & $\begin{array}{l}\text { Sequence in } 80-k V \text { NBI to full } \\
\text { power; adjust pump NBI ana } \\
\text { pellets for max nT, wal1 } \\
\text { loading } \Gamma \text {, and stability. }\end{array}$ \\
\hline
\end{tabular}


Requirentents.

1. Deuterium gas injectors will be provided to control pressure in the vacuum vessel before and during the initial startup phase.

2. The central cell and end anchor cells will each be provided with two independent gas injectors.

3. Sufficient system redundancy witl be provided to erisure operation when one injection system is out of service.

4. Two operating modes will be provided, one for pulsed flow and one for modulated flow of the aeuterium gas.

5. Gas flow rates will be sufficient to backfill the vacuum vessel ano establish the desired deuterium vacuum pressure.

Uesign Description. The gas flow rate depenas on the size of the vacuum vessel, the capacity of the vacuum cryopumps, and the time required to backfill the vessel to the desired vacuum pressure. Flow rates given in Table 3-47 are ultraconservative inasmuch as they are more than the pellet injector and neutral-bean injector rates combinea ouring steaay-state operation. The high flow rates permit rapia backfilling of the vacuum vessel if necessary. These rates can be recuced greatly by adjusting the upstream pressure regulator, Pd, and the flow valve controller, FVC, shown in Fig. 3-133.

Referring to the deuterium gas flow-system diagram of Fig. 3-133, we see that there are three categories of gas injection system equipment: the gas supply, control valves and injectors, and the I\&C for measuring and controlling gas fiow. Because of interfacing, some fuel-recovery and fuel-processing equipment is also shown. $[A C, C C$, and RAC are mnemonics for left anchor cell, central celi, and right anchor cell, respectively. The deuterium gas supply system is shared with the deuterium neutral-beam injectors ano the pellet injectors. Several types of control valves are needed: remote-shutoff valves, (RM), pressure-regulator valves (PRV), modulated flow-control valves (FCV), and pulsed selector valves (PSV) that connect to the injector nozzles. The I\&C components consist of pressure regulators ( $P d$ ), flow-valve controllers ( $F V C$ ), programmable satellite computers, ano the manual/auto (M/A) transfer station. For the pulsed flow-control moae, the pressure regulators and flow-control valves are preset; then, the PSV are opened and ciosed as commanded by the programmable control. 
Table 3-47. Gas injection requirements/design data.

\begin{tabular}{ll}
\hline Gas injection systems & 2 \\
Central cell injection nozzles located & 2 \\
near peltet injection tubes & 4 \\
End cell injection nozzles & 2 \\
Left-end anchor cells & 2 \\
Kight-enc anchor cells & 360 \\
Total maximum gas flow rate $(\mathrm{g} / \mathrm{hr})$ & 160 \\
To central cell & 100 \\
To each end cell & $>99$ \\
Gas composition $\left(\boldsymbol{X} \mathrm{D}_{2}\right)$ & \\
Operating control modes & Pulsed \\
Mode 1 & Modulated \\
Moge 2 & \\
\hline
\end{tabular}




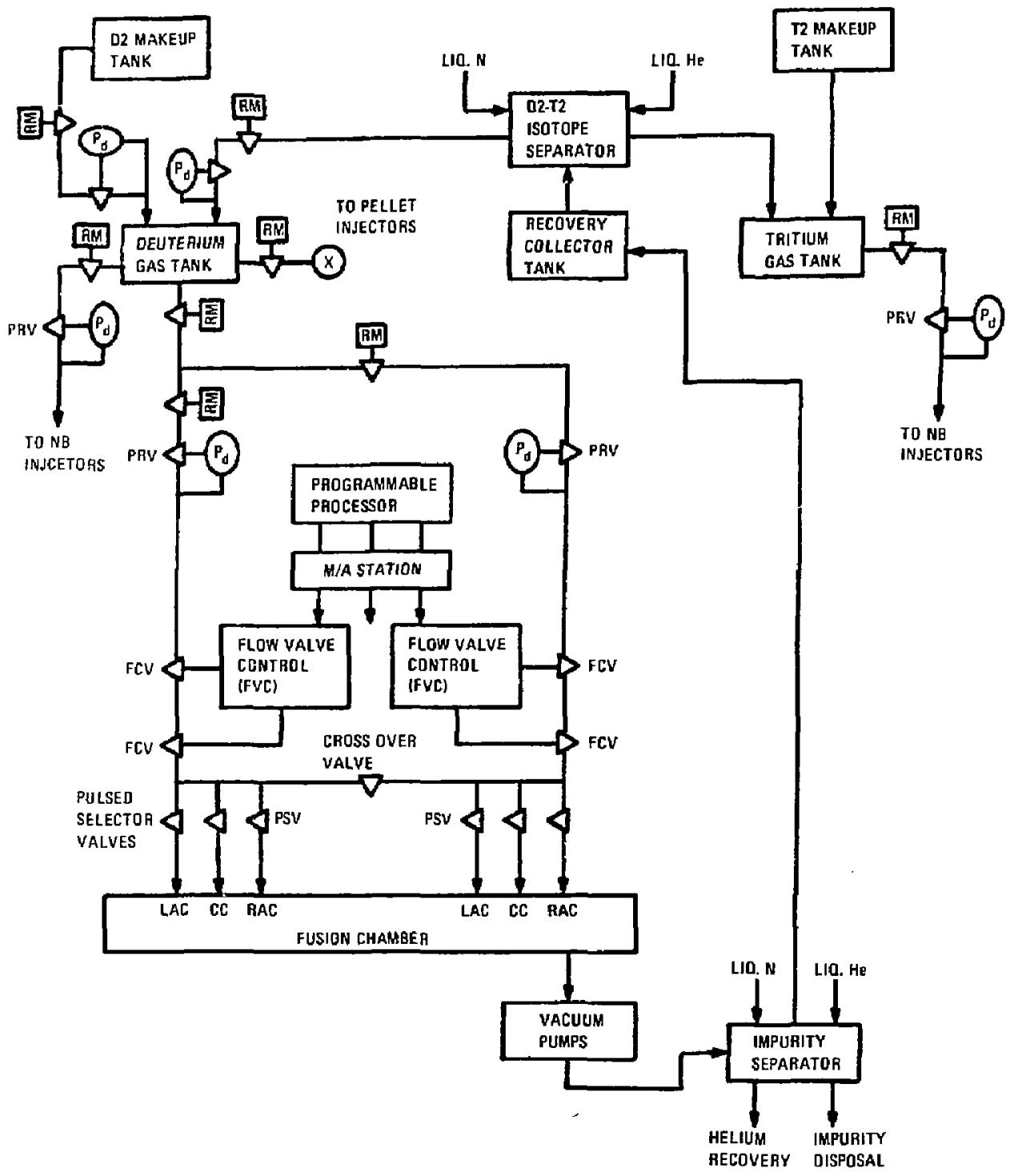

Fig. 3-133. Flow diagram of deuterium gas system. 
For the modulateo flow-control mode, the pressure regulators are preset, the desired PSV are openeo as needed, and the FCV are controlled to provide the desired modulated flow.

Key Issues. Gas-puffing systems are well developed for use with present-day experimental fusion machines. The pulsed selector valves and FCV should be close to the injector nozzles so that the entry of gas into the vacuum vessel is not aelayed significantly. Large-diameter lines between the PSV ano injectors reduce delay time, but may introduce radiation streaming problems unless the lines follow a longer contorted path that, in turn, increases delay time.

\subsubsection{Plasma Gun Injectors}

Summary. Two or more MFTF-b plasma stream guns will be located on each end wall for plasma field mapping and to provide a target plasma for the anchor neutral beams during the initial $10 \mathrm{~ms}$ of startup. The plasma gun assemblies will be modified so that they do not retract when dormant (not in use). A movable tungsten shield will close to provide nuclear radiation shielding when the guns are dormant. The plasma guns will be located about one meter off the axis of the machine, in the plane of the plasma fan at the end walls. Openings between the particle and oumps must be sufficiently large (about $1 / 2$ in in diameter) to accommodate the expanding plasma when it emerges from the guns.

Requirements. Except for additional nuclear radiation shielding, the detailed performance requirements for the plasma guns will be the same as those for MFTF-B. The general requirements for the plasma guns are given in Table 3-4B. If the guns are reliable enough, only two are needed on each end wall. Otherwise, aoditional plasma guns are needed as quick replacements for failed units. Most of the time, the plasma guns are dormant. Their sheif life under limited radiation exposure is the driving reliability factor.

Cooling for the radiation shielding must be provided on a continuous basis. The movable shield must not degrade the performance of the plasma gun when it is in the fully open position. Air-cooling of the gun assembly will 
Table 3-48. Startup plasma gun requirements .

- At least four startup plasma guns will be provided to create a low-energy plasma target for neutral-beam injection into the anchor cells.

- The plasma guns wijl use the design developed and tested for MFTF-B.

- At least two guns will be located on each end wall, about $1 \mathrm{~m}$ off the machine axis, ano oriented in the plane of the end particle fan.

- Enough redundancy will be provided for assure operation with one plasma gun out of operation.

- Each plasma gun will be independently replaceable.

- The plasma guns will be protected from radiation dosage and heat damage at all times.

- Movable radjation shielding must not interfere with plasma gun operation when in the open position.

- Movable shield open ana close time will be about one ininute.

- Performance will be the same as specified for the MFTF-B plasma guns. 
be provided even though the heat capacity of the components is large enough to absorb the energy dissipation of a single 10-ms shat without damage.

Design Description. Plasma gun design and interface data are given in Table 3-49. The plasma gun arc is the source of low-energy electrons that ionize deuterium gas injected before the pulsed line discharge. Plasma oensity in the gun is relatively high, but is reduced about two orders of magnitude at the anchor cell by means of magnetic expansion.

Figure 3-134 is a schematic diagram of the plasma gun, and Fig. 3-135 is a connection diagram to the power supplies and gas systems. The air-cooling system will be needed when the plasma guns are tested ard for initial fluxmapping operations. The pulsed arc is formed along the magnetic field lines betwetn the molybdenum cathode ano anode washer stack. An auxiliary pulsed magnet can be positioned to control plasma flux mapping. A pulsed piezoelectric valve is used to admit the deuterium gas to the plasma gun.

The arc contains roughily $2 \mathrm{kA}$ of low-energy electrons that produce about $250 \mathrm{~A}$ of plasma flow from the gun along the magnetic field lines. A relatively low-voltage $(-1-k V)$, pulse-forming network power supply provides a 2500-A pulse to the gun. Likewise, the magnet power supply consists of a 16.7- $\mu \mathrm{F}$ capacitor bank discharged to provide $1760 \mathrm{~A}$ to the auxiliary magnet that produces a 3-T magnetic fieid in the gun. Energy stored in the magnet power supply is about 2.5 times the energy stored in the arc power supply.

Figure 3-136 shows a sectional view of the shielded plasma gun housing assembly, including the rotatable muzzle shield and actuator. The heavy eccentric muzzle shield will require considerable drive torque for positioning, but its opening and closing time is about one minute so that a worm gear box can be used to multiply the torque of a fractional horsepower nictor. The fixed-position nuclear shielding is water-cooled. The movable shield may be cooled by radiation and conduction, or it may be water-cooled separately as described below.

Key Issues. Cooling of the movable radiation shield is the major aesign consideration because the peak heat load on the shield surface is about $300 \mathrm{~W} / \mathrm{cm}^{2}$. There are at least two possible solutions to the problem. One solution is to spring-load the drive shaft so that the backside of the 


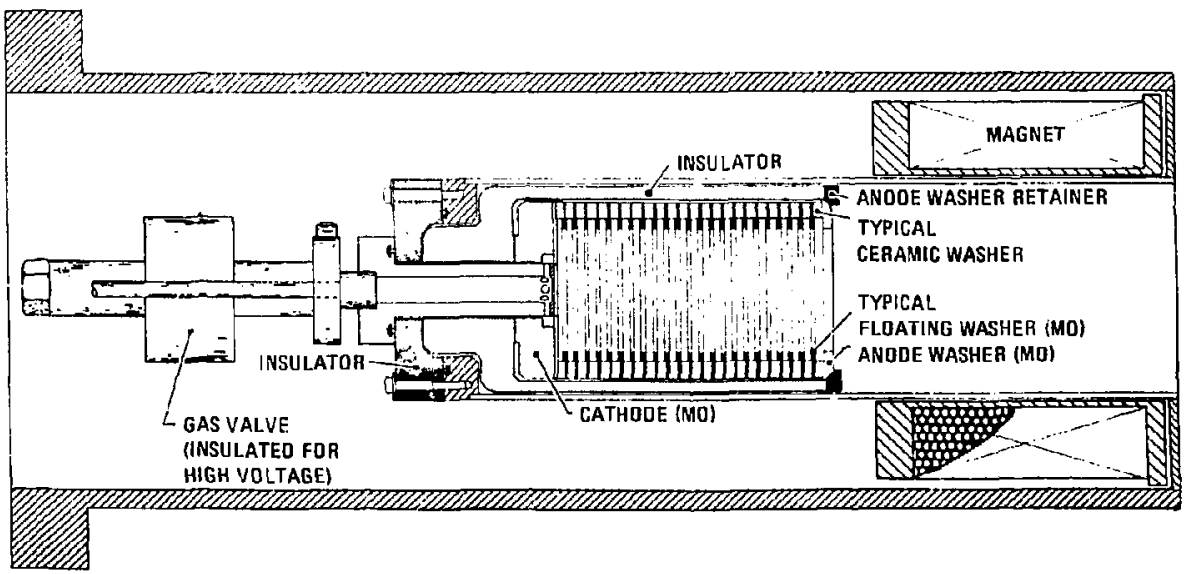

Fig. 3-134. Schematic sectional view of one of four startup plasma guns. 


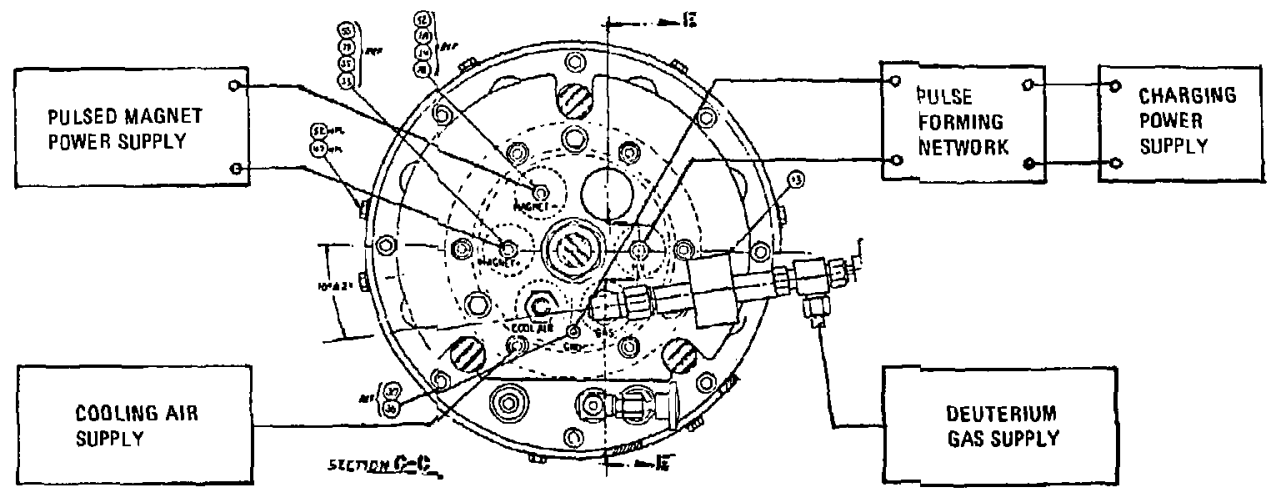

Fig. 3-135. Plasma-gun supply system diagram. 


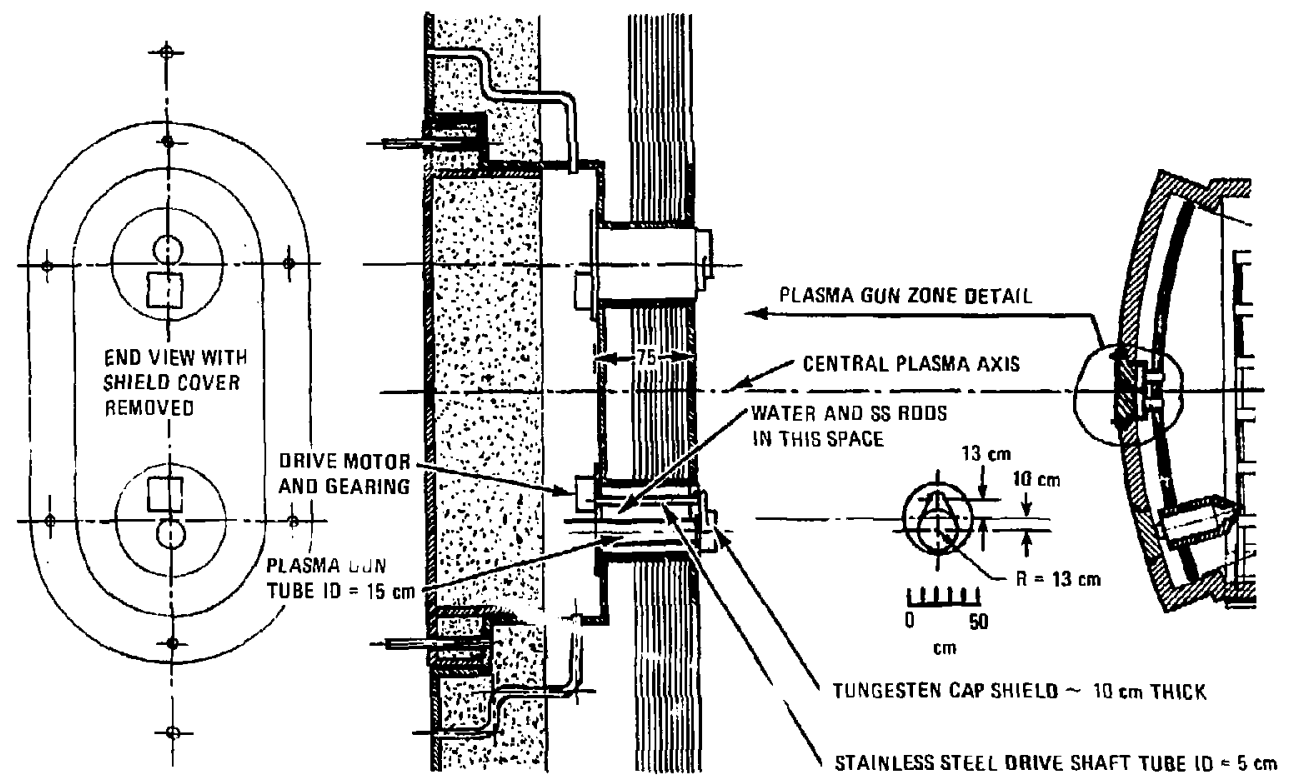

Fig. 3-i36. Sectional view, showing the location of the TDF startup plasma gun assemblies (two guns on each end cover). 
Table 3-49. Plasma-gun design and interface data.

\begin{tabular}{|c|c|c|c|}
\hline $\begin{array}{l}\text { Guns ( } 2 \text { on each } \\
\text { end-ce } 11 \text { cover) }\end{array}$ & 4 & $\begin{array}{l}\text { Cooling air flow } \\
\text { (Torr-liters/s) }\end{array}$ & TBD \\
\hline Location & $r= \pm m$ & $\begin{array}{l}\text { Magnet pulsed-power } \\
\text { supply }\end{array}$ & \\
\hline Current/gun (A) & 2000 & $\begin{array}{l}\text { Amps (kA) } \\
\text { Volts (kV) }\end{array}$ & $\begin{array}{l}2 \\
2.4\end{array}$ \\
\hline $\begin{array}{l}\text { Arc electron } \\
\text { temperature (eV) }\end{array}$ & 5 to 250 & Power, $10 \mathrm{~ms}$ (MW) & 4.8 \\
\hline $\begin{array}{l}\text { Plasma } \\
\text { Pulse length (ms) } \\
\text { Pulse flow (A) } \\
\text { Ion temperature (eV) } \\
\text { Density }\left(\mathrm{cm}^{-3}\right)\end{array}$ & $\begin{array}{l}10 \\
250 \\
10 \\
10^{13} \text { to } 10^{14}\end{array}$ & $\begin{array}{l}\text { Accel cathode pulsed } \\
\text { powe: supply } \\
\text { Amps, } 10 \mathrm{~ms}(\mathrm{kA}) \\
\text { iolts (kV) } \\
\text { Power, } 10 \mathrm{~ms}(\mathrm{MW}) \\
\text { Energy (kJ) }\end{array}$ & $\begin{array}{l}2.5 \\
1 \\
2 \\
20\end{array}$ \\
\hline $\begin{array}{l}\text { Deuterium gas flow } \\
\text { (Torr-liters/s) }\end{array}$ & 22 & & \\
\hline
\end{tabular}


tungsten is in firm contact with a thin graphite plate secured to the water-cooled stainless steel plate. The grapinite reduces the sliding friction and transmits heat from the tungsten to the stainless steel. Another solution is to provide water-coaling channels down the hol low drive shaft and water-cool the tungsten shield. Flexible hydraulic hoses connecting the drive shaft to the water supply lines must allow for $180 \mathrm{-deg}$ rotation of the shaft. In this case, the tungsten cap stield is secured to a water-cooled chromium-copper base plate that is secured to the drive shaft.

\subsubsection{Pellet Injectors}

Summary. To stabilize the plasma during continuous operation, small frozen oeuterium pellets are injected into the central cell plasma to provide the low-energy part of the plasma spectrum. As they penetrate into the plasma, the frozen pellets ablate. Upon heating, the pellet ablation gases interact with high-energy ions and become a source of low-energy deuterons that help stabilize the plasma.

For reasons given below, many small frozen deuterium pellets are injected into the plasma with a velocity $>1500 \mathrm{~m} / \mathrm{s}$ to provide a quasi-continuous flow of low-energy plasma that supplements the high-energy plasma flow through the central cell mirror plug.

Centrifugal pellet injectors now under development can be modified to meet the requirements defined below. Two dual-channel centrigual injectors are proposed, one at each end of the central cell. These injectors will provide adequate pellet penetration and not interfere with test-module installation and removal. Although injection into the small neck of the centrai cell piusma is desirable for enhanced pellet penetration, the plug cails and beam-dump obstructions make injection into the thick part of the plasma neck more desirable.

Requirements. A sumary of the peilet injector aesign criteria is given in Table 3-50. These criteria are the basis for the pellet-injector data requirements given in Table 3-51. To prevent large variations in plasnia aensity, the number of atoms contained in the pellet mass must be no more than $10 \%$ of the iun poptiation in the centrol cell. This 1 imits the maximum pellet 
Table 3-50. Pellet-injector design criteria.

- All stabilizing warm ion flows will be supplied with deuterium pellet injecturs.

- Pellets will be injected in the central cell region only, preferably near the ends of the cell, where the plasma penetration is greatest.

- Pellet mass will be limited to $10 \%$ of the ion population of the central cell.

- Pellets will have sufficient injection velocity to penetrate the plasma at least $30 \%$ of the plasma radius so that warm ions exist in the largest part of the plasma volume.

- Sufficient pellet redundancy will oe provided to assure operation with one injector out of service.

- Pellet injectors will be located so that they do not interfere with the removal and replacement of other components nor degrade their performance. 
Table 3-51. Pellet injector requirements.

\begin{tabular}{|c|c|c|c|}
\hline Pellet size (g) & 34 & $\begin{array}{l}\text { Magnetic field in vicinity } \\
\text { of pellet injector }(T)\end{array}$ & 0.2 \\
\hline Pellet uiameter (mm) & 0.6 & Pellet velocity $(\mathrm{m} / \mathrm{s})$ & $>1500$ \\
\hline Injection point, $Z(m)$ & \pm 3.35 & Pellet rate $\left(s^{-1}\right)$ & 500 \\
\hline $\begin{array}{l}\text { Injector distance to } \\
\text { plasma (m) }\end{array}$ & 5.4 & Reliability & $\geq 0.90$ \\
\hline $\begin{array}{l}\text { Scatter-cone half } \\
\text { angle (dag) }\end{array}$ & $<0.5$ & & \\
\hline
\end{tabular}


diameter to $0.6 \mathrm{~mm}$. The analys is in Section 2.3 .2 shows that a pellet velocity $>1500 \mathrm{~m} / \mathrm{s}$ will satisfy the minimum requirements. The velocity-penetration relationship is highly sensitive to the plasma electron temperature. Some velocity reserve is needed to accomodate the uncertainty in the plasma electron temperature. A pellet injector program is currently underway to achieve pel let velocities near $2000 \mathrm{~m} / \mathrm{s}$.

Design Description. Centrifugal injectors are needed to meet the high-injection pellet rate requirement. Centrifugal injectors now under oevelopment are potentially capable of meeting the requirements if high reliability ( $>95 \%$ ) is not a requirement. To improve system reliability, two channel pellet injectors (Fig. 3-137) are needed so that ane pellet injector can provide the required pellet rate if the other one fials. Nomaliy only one of the two pellet channels would be in operation, with a pellet rate of $250 / \mathrm{s}$. When one injector fajls, the second channel in the other injector will be activated so that one injector supplies 500 pellets/s.

The design data for the centrifugal peliet injectors are given in Tabie 3-52. Some variation in arbor speed is provided so that the pellet velocity and rate can be varied over a limited range. The top speed corresponds to $2000 \mathrm{~m} / \mathrm{s}$. A pellet collimating tube is needed to limit the maximum half-cone scatter angle to $0.5 \mathrm{deg}$. This technique has been demonstrated with Teflon tubing at lower pellet velocities,

There are six types of interface connections to the pellet injectors, four fluid tube lines, electrical cable, and mechanical interface connections to the supporting structures and isolation valves. When a pellet injector fails, the isolation valve is closed, the injector is purged to remove deuterium and tritium, the supply-line valves are closed sequentially, and the electrical power is turned off.

An outline drawing of the pellet injector is shown in Fig. 3-138. The injector weighs about $1000 \mathrm{~kg}$, excluding support structure. The isolation valve assembly consists of two rotary isolation valves, with provision for vacuum removal of leakage gas through the valves. The assembly also coritains sensors for measuring pellet rate and pellet velocity. 


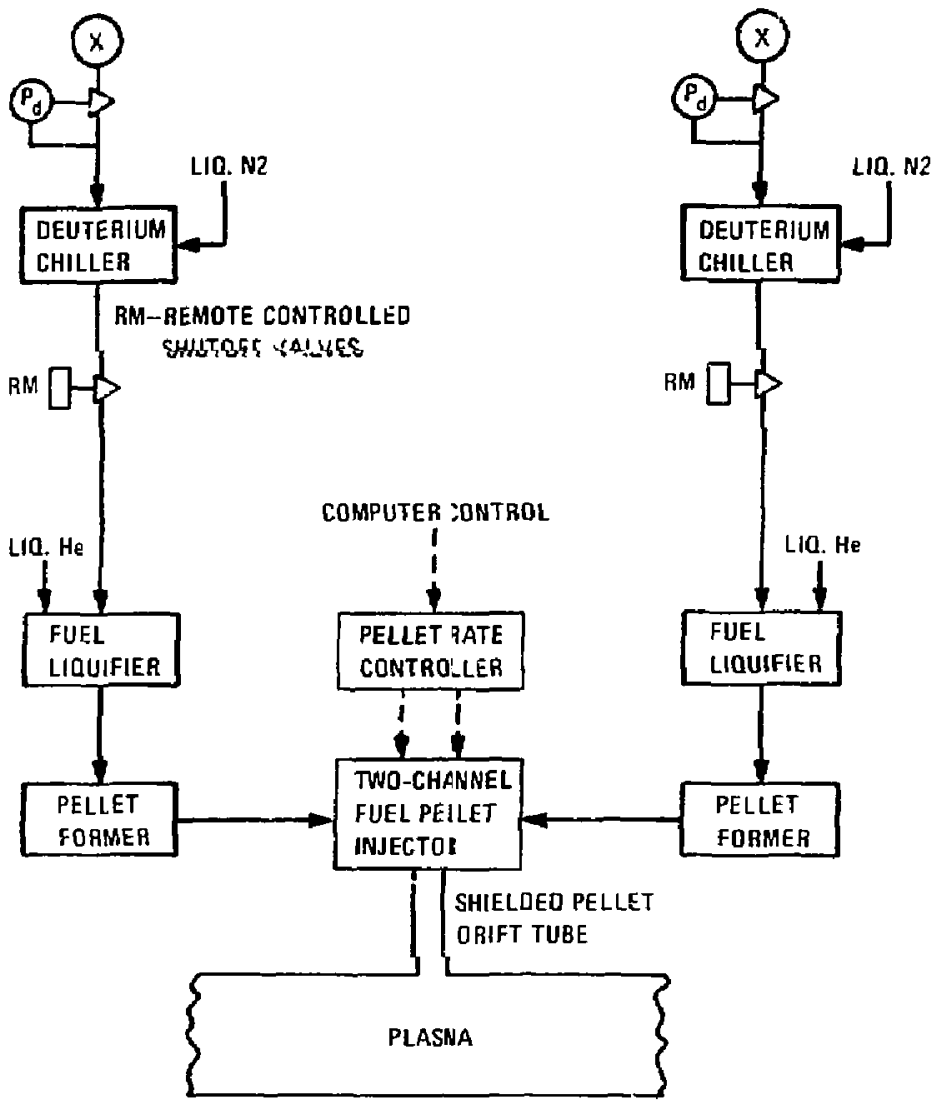

Figure 3-137. Two-channel pellet injector flaw diagram (ane of two). 
Table 3-52. Centrifugal injector data.

$\begin{array}{ll}\text { Two-channel injectors } & 2 \\ \text { Arbor speed (rpm) } & 27,000-36,000 \\ \text { Pellet velocity (an/s) } & 7,500-2,000 \\ \text { Pellet rate, max (s-1) } & 450-600 \\ \text { Scatter cone half angle with collimating tube (deg) } & <0.5 \\ \text { Interface connections/injector } & 6 \text { types } \\ \text { Liquid helium (g/hr) } & 360 \\ \text { Liquid nitrogen (g/hr) } & 1800 \\ \text { Deuterium (g/hr) } & 75 \\ \text { Elecum (Torr-liters/m) } & 500 \\ \text { Mechanical, start/run (w) } & 1500 / 750 \\ \text { Approximate weight (kg) } & ? \\ \text { Approximate space of cylindrical envelope (m) } & 1000 \\ \text { Jiameter } & \\ \text { Length } & 1.00 \\ \text { Auxiliaries/injector isolation valve } & 1.40 \\ \text { Contral instrumentation } & 2 \\ \text { Pellet velocity } & 6 \text { sehsors } \\ \text { Pellet rate } & 2 \text { channels } \\ \text { Pellet mass } & 2 \text { channels } \\ \end{array}$




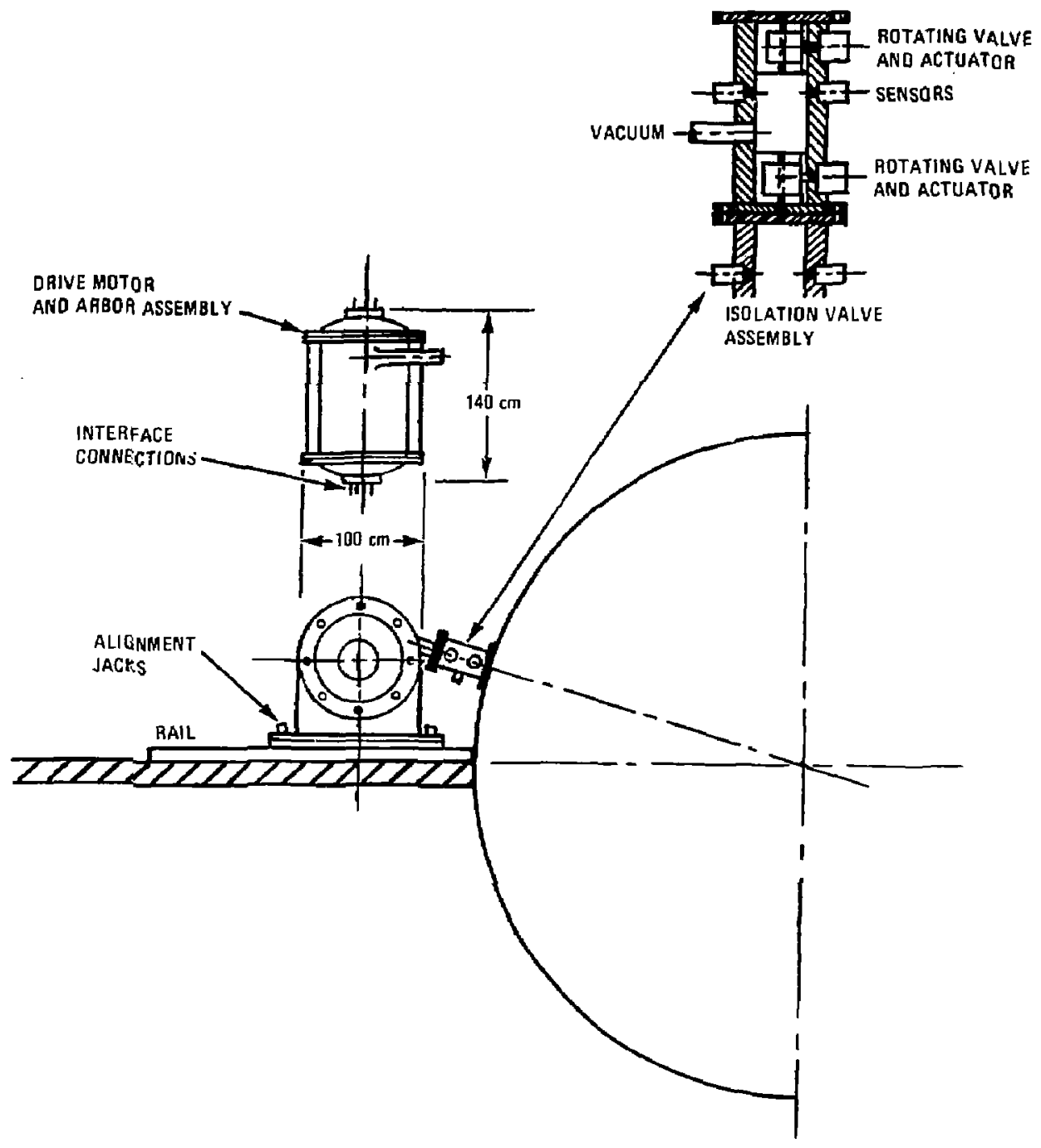

Figure 3-138. Outline of cenirifugal pellet injectar, showing principal features and interface connections. 
The fluid supply lines connect to the pellet former of the injector. The drive motor and its electrical connectors are located at the other eno. The ends of the injector should be readily accessible for equipment servicing and maintenance.

Key Issues. Pellet injectors have not yet been developed to meet the high-velocity, high-pellet-rate requirements. Continuous operation under these concitions could present reliability problems. Considerable reliability testing will be needed to assure high pellet injector availability.

The injectors are mounted in an awkward position, as shown in Fig, 3-139. The details of the supporting structure and alignment provisions have not been developed. The pellet injector tube, shown in $\mathrm{Fig}$. 3-140, must not interfere with either the plug coil or the beam-dump penetrations, nor must it allow excess nuclear radiation into the $5-1$ coils. To meet these requirements, the injector has to be mounted at an unusual angle relative to the machine. It cannot be readily supported from the floor of the building. These consigerations could present maintenance problems in obtaining ascess to and aligning the injectors. 


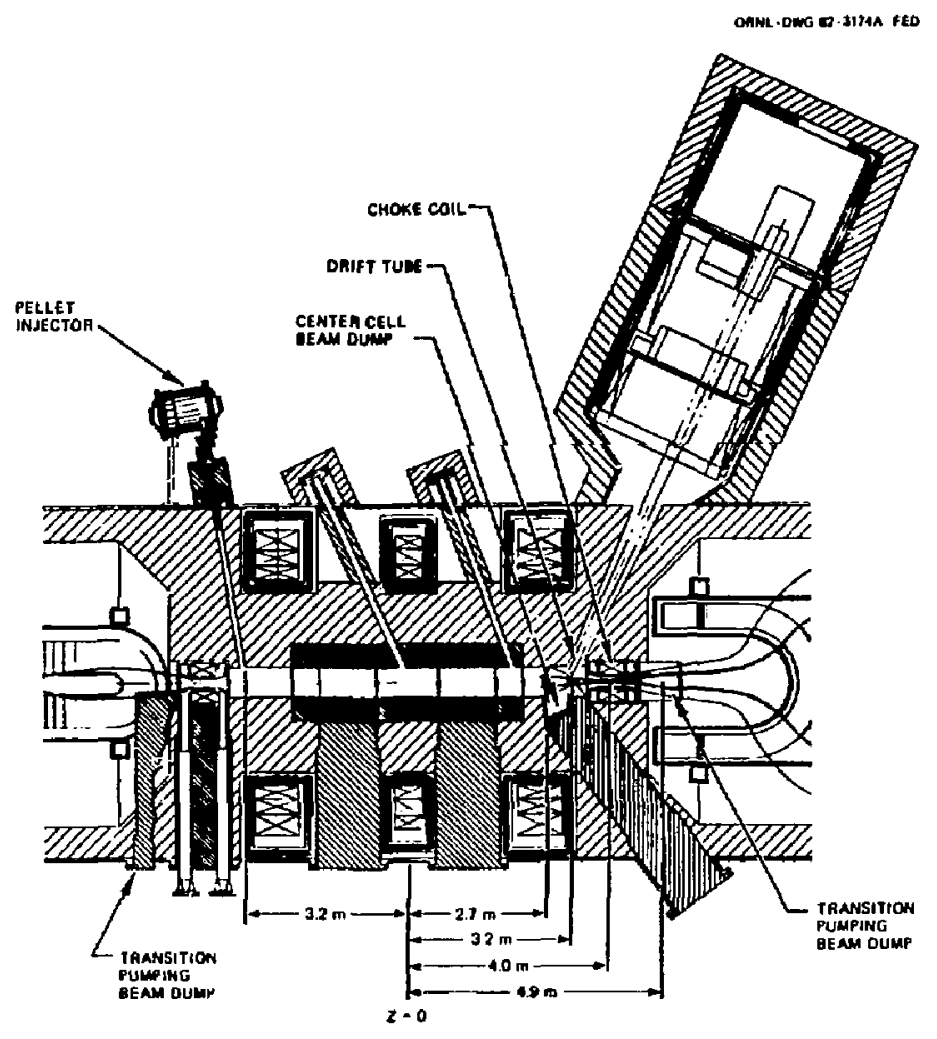

Figure 3-139. Sectional view of TOF reactor, showing the location of one ellet injector and its mechanical interface. 


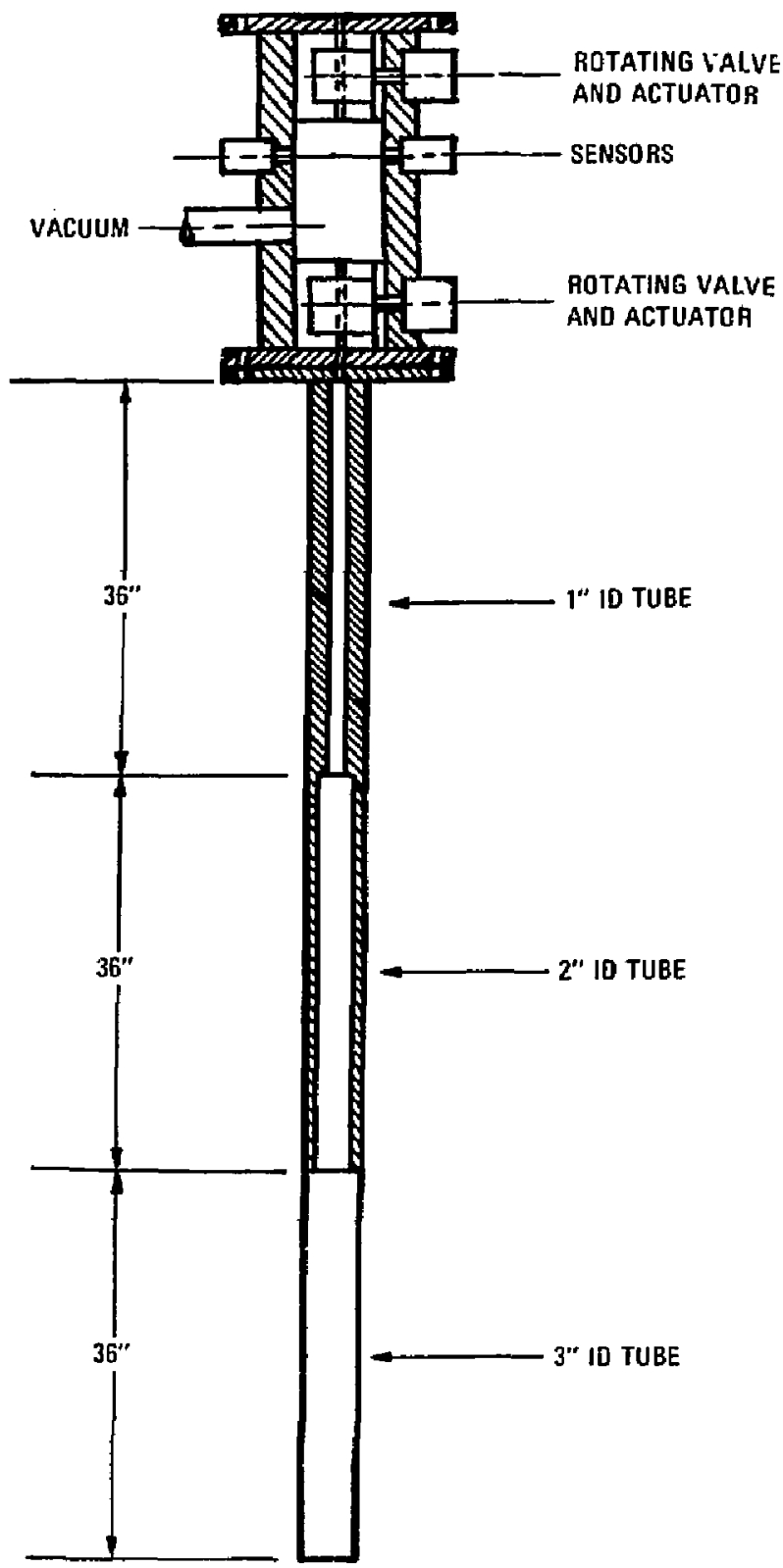

Figure 3-140. Dual isolation-valve assembly and the pellet drift-tube extension. 


\subsection{VACUUM VESSEL}

\subsubsection{Main Vacuum Vessel}

The main vacuum vessel is basically a skin that wraps around the outside of the entire machine. It is made up of a series of plates welcied together, with penetrations at various places for beamlines, test modules, and other services for the reactor vessei.

Together with the bulk shielding, the vacuum vessel performs several functions. First, it provides a vacuum contaiment for the plasma chamber and the supercorducting magnets. It also supports the magnet thermal shielding and aids in structural support of the magnet cold mass. As mentioned earlier, the vacuum sk in must provide access ports for component accessibjlity and feeathrough. Finally, it provides structural support for test-module installation and the neutral-bean dumps.

Essentially only two things are between the plasma and the outside: the snield and vacuum-vessel skin. Thus, there are two locations for the vacuum vessel--one inside the shield and one outside the shield. A trade study was performed at General Dynamics to determine which location would provice the optimum configuration. In sumary, the study concludes that "...the baseline vacuum vessel should be located external to the shield.

It was aecided that the central cell solenoid magnets shoula have their own vacuum vessels to eliminate warming the magnets every time maintenance or service is required on the TDF machine.

Because of the large intermagnetic forces that act to pull the solenoids inward, massive intercail support beans were needed to support the magnets. Since the beam must go from cold mass to colo mass, this would provide an undesirable heat leak. To eliminate the heat leak, the magnet vacuum vessel and $L N_{2}$ thermal shields were extended to cover the intercoit support bearsis as we 1$\}$.

When connected, the central cell neutral beaml ines will also be part of the main vacuum volume. Because they will be installed with their own vacuum vessel, an interface will be provided to seal the two vessels together. This interiace will have to provide structural support for the neutral beamlines as well. 
The other major interface connections to the vacuum vessel are the test modules. These modules are in six locations between the central cell solenoias and have, perhaps, two actual modules per penetration. This interface will have to provide all of the structural support for the modules, as well as for the vacuum. A seal design employing a separate vacuum ano structural connection is used to eliminate some problems that arise from this type of interface. A cross section of this configuration is shown in Fig. 3-141.

\subsubsection{Manufacturing}

A comprehensive mariufacturing plan will be required for the manufacturing, instailation, and test phase of the TDF vacuum vessels.

\section{Fabrication}

Magnets. The magnet vacuum vesse]s will be comprised of three weided subassemb lies.

1. On the first subassembly, the inner-radius-ring weided assembilies will be made up in four quarters from rolled 1/4-in. $\times 5-\mathrm{ft} \times 12-\mathrm{ft}, 316$ CRES plate, weided together to form the required diameter.

2. On the second subassembly, the outer-radius rings will be subassembled in a similar manner froun 1/2-in.-thick rolled plate.

3. On the third subassembly, the side subassemblies will be fabricateo from segments, approximately eight per side, plasma arc cut from 1-3/4-in.-thick plate stock for the S-0 magnet and from 2-in.-thici plate for S-1. The segments will be welded together to form a complete annulus.

The inner- and outer-radius rings will be welded to a side annulus plate to form a channel cross section. This welded subassernbly is made ready to receive the magnet cold mass.

End Cells. The end cell vacuum vessels will be fabricated in weided subassemblies limited by transportation and handling capabilities.

The entire end cell vacuum vessel will be built up "in plant" with jig and fixture shoring-type supports (tooling). Operations include cutting, 

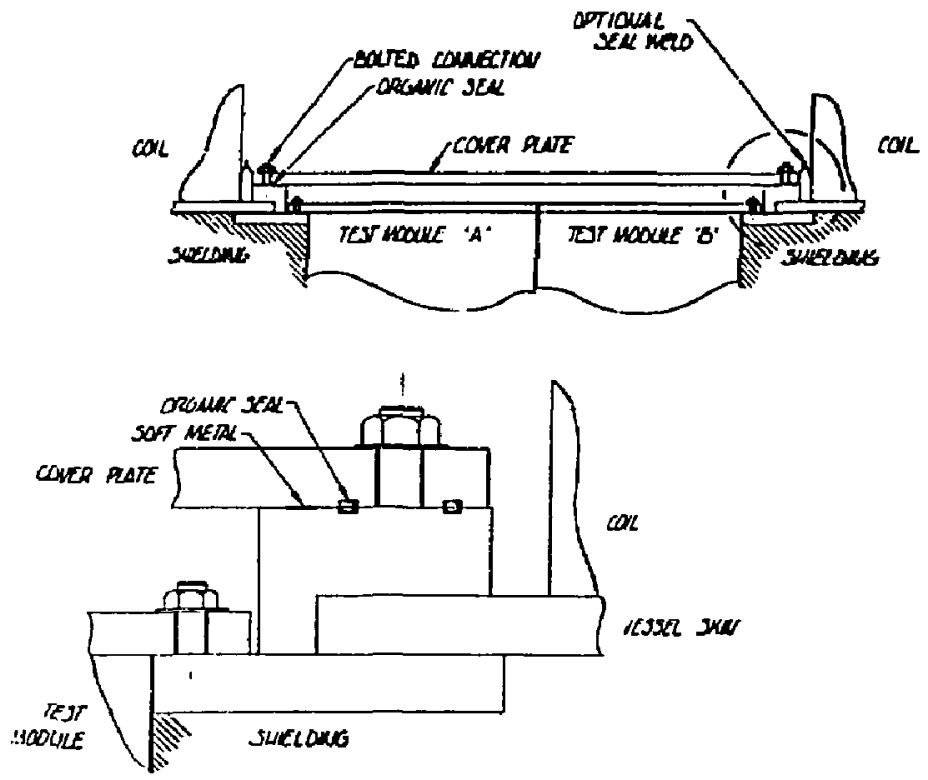

Figure 3-141. Test module sea1. 
shearing, rolling, bending, heat-treating, and grit blasting of the 1- to 2-in.-thick plate, After the subassemblies are completely weloed and inspected, the tack weld that held them in the completed assembly will be cut away and the subassemblies will be prepared for shipping. The planned size of these subassemblies is about $10 \times 15 \times 60 \mathrm{ft}$. Each subassembly is scheduled to weigh less than $90,0001 \mathrm{~b}$.

Central Cell and Anchar Cell. These vacuim vessels will be tabricated from rolled 3/8-in.-thick 316 CRES plate stock. Welded subassemblies wi]l be employed to alleviate handling and transportation problems. Circumferential rings will be welded in segments and fitted to the in-plant assembled vessels. After fit-up of the rings and inspection of the completed welos, the vessels will be dismantled and shipped to the installation site.

Weid-joint preparation for the vacuum vessels will include, as a minimum, a 60 -deg beveled edge for $100 \%$ weld penetration. The first weld pass from each side of the subassemblies will be performed with the automatic gas metal-arc weld (GMAW) process, or the gas tungsten-arc weld. The remaining passes would be accomplished with a high-rate deposit process, such as flux core metal arc. To minimize warpage and distortion, welding will be done on both sides of the material when possible.

Quality assurance procedures will be used throughout all the welding processes. X-ray and ultrasonic inspection of welds would be a part of these procedures.

A shipping and transportation plan will be part of the manufacturing plan for the vacuum vessel. The shipping plan will develop routes and envelopes of the vacuum vessel's subassemblies. Producibility engineers, in concert with structural and system engineers, will ensure adherence to the envelope parameters during the detail design phase.

\section{Installation}

Magnets: The magnet vacuum vessels are assembleo onto the completed magnet cold masses. The magnet, with its thermal shielo in place, is positioned inside the vacuum vessel's channel-shaped annulus subassembly and the support struts are located and attached. The opposite side plate is then 
welded in position with a close-out weld. Because of the inspection and test proceaures involved, it is desirable to make this close-out weld in an in-plant atmosphere, which will require special handling by shipping and transportation personnel. The completed $\mathrm{S}-1$ magnet is $25 \mathrm{ft}$ in diameter and will weigh 160 tons.

End Cells. The end cell vacuum-vessel subassemblies will be received on site, inspected, and stored in a prepareo marshalling area. After the centra] cell vacuum vessel ano magrets have been positioned ano secured and the end cell support structure is positioned, installation of the end cell vacuum vessel will begin. The subassemblies will be erected on shoring similar to that employed during the in-plant assembly. Manual tack welding will be used throughout erection of the subassambly. When all assemblies are in place, a complete first-pass weld will be accomplished manually, using gas-tungsten arc weld. The remaining passes will be accomplished with a flux-core, metal-arc weld process. All welding will be controlled procedurally and will be in accordance with ASME codes. Quality assurance will be maintained by 100\% weld inspection; x-ray ano dye penetrant procedure; will also be employed.

Central Cell and Anchor Cell. As-shipped weldeo subassemblies will be received at the erection site. They will be inspected ar.d stored in a marshalling yard until scheduled for use. Shoring, braces, and ScaD plates will hold the central cell assemblies in place during tack and first-pass welaing. The welds for the central cell vacuum vessel will be completed and inspected, ana any repair or rework will be accomplished. Also, vacuum bagging will be used for limited testing of all openings. When this testing is completed satisfactorily, the magnets can be installed.

When the magnets and central cell vacuum vessel have been satisfactorily located and mounted, anchor cell buildup will commence. Again, shoring, braces, and scab plates will be used to hold the subassemblies in place auring tack and first-pass welding, and all welding will be controlled proceaurally. The circunferential rings $w i j l$ be assembled onto the ressel skin and weided in place, and all welds will be inspected. 
The scab plates are to be ground off, any unnecessary or interfering shoring is to be removed, and the alignment and center line of the installation is to be established or verified by optical means.

Structural Analysis. The magnet vacuum vessel and the external vacuum vesse? are to be mace of 316 CRES with an operating temperature of about $100^{\circ} \mathrm{F}$. These vessels are to be designed to withstand 1 atm pressure. In addition, the external vacuum vessel has to resist loads due to the shielding material. These loads depend on how the shielding material is attached to the vacuum vesse1; however, for initial sizing, these procedures will be followed:

1. Design the shell thicknesses for external pressure, using the ASME Boiler Cocie as per NB 3133.3 and NB 3133.4 of Section III, Division I.

2. Aod a safety factor of 2 when sizing shell thickness even though the Boiler Code design procedure allows a sufficient margin of safety.

3. Undertake the detailed design of ring stiffeners and cicher shield-load attachment points at a later stage.

4. When the Boiler Code does not cover the loading situation, use conventional analysis procedures with a sufficient factor of safety.

Central Cell Magnet ( $S-0$ and $S-1$ ) Vacuum Vesse1. These magnet vessels are basically toroidal shells having a rectangular cross section under an externail pressure of $1 \mathrm{~atm}$. The Boiler Code does not address the problem of rectangular section toroids under external pressure. Hence, an approximate metiod was used to size these vesse15. The outer surface of the torus was treated as a cylindrical shell under external pressure and, hence, was designed according to the Boilar Code. Because of the nature of the simplifying assumption, a safety factor of at least 5 was deened advisable. A step-by-step procedure for designing a cylindrical shell under externa] pressure is outlined in NB 3133.3 of ASME Boiler Code, Section III. With this procedure, a thickness of 2 in. was found sufficient.

The magnet vesse? side plates were treated as bending annular plates under $1 \mathrm{~atm}$ pressure--a very conservative assumption. Two-inch-thick side plates were stressed to a maximum bending stress of 16,740 psi. For 316 CRES at $100^{\circ} \mathrm{F}$, the yield stress (as per the Boiler Code) is 30,000 psi. This 
resulteo in a safety factor of 1.79 using the allowable tension even though the boiler Code permits an allowable tension of 1.5 tines the tension aljowable when checking for failure under maximum bending stress. Hence, a 2 -in.-thick vacuum vessel was deemed sufficient to withstano 1 atm pressure.

Exterral Vacuum Vessel. The configuration of the external vaculim vessel changes from a cylinder to a spherical segment, as shown in Fig. 3-1. This arawing shows transition sections where the shell is approximately conical or spherical. As discussed previously, the Boiler Code pracedure was usea, a thicknesses were determineo using an additional safety factor of 2 . Table -53 lists vacuum-vesse $i$ thicknesses at various locations.

Materials. Because of its availability and cost relative to other, nore exotic materials, stainless steel was chosen as the baseline material for both the main and magnet vacuum vessels. Stainless steel is also nonmagnetic, a requirement of any material used in this application. See Tat le 3-54 for tne mecharicai properties of $304 \mathrm{~L}$ stainless steel at elevated temperatures.

\subsubsection{First Wall--Mechanical}

Design. Design of the first wall requireo a balance between the two main requirements, heat transfer and neutronics. Other requirements are structural rigidity and physical compatibility with the rest of the machine. Physical compatioility is a relatively minor requirement because we are concerned oniy witr: the central cell first wall.

The main task was to pruvide a surface over which a coolant fluia could flow, but which would not be destrayeo by the fusion environment in too short a lime period. To acconplish this, we used two concentric cylinaers that run the length of the central cell. The coolant water flows between the two cylinders and through a series of vanes positioned within the cylinders to maintain axial flow (see Fig. 3-142.)

An alternate configuration would be to use a series of parallel trace tuoes welued to the outside of the inner cylinder, which would also run the length of the central cell. 
Table 3-53. Vactum-vessel thicknesses for the TuF external vacuum vessel.

\begin{tabular}{ll} 
Location & $\begin{array}{c}\text { Thickness } \\
\text { (in.) }\end{array}$ \\
\hline Central cell & 0.75 \\
Anchor cell & 1.125 \\
Eno cell & 2.75 \\
End dame & 3.25 \\
\hline
\end{tabular}


Table 3-54. Mechanical propertjes of 304L stainless steel aỉ elevated temperatures.

\begin{tabular}{lllllll}
\hline $\begin{array}{l}\text { Temperature } \\
\text { (OF) }\end{array}$ & $\begin{array}{l}\text { Modulus of } \\
\text { elasticity } \\
\text { (Msi) }\end{array}$ & $\begin{array}{l}\text { Poisson's } \\
\text { ratio } \\
v^{*}\end{array}$ & $\begin{array}{l}\text { Coefficient } \\
\text { of thermal } \\
\text { expansion } \\
\text { (in./in./OF) }\end{array}$ & $\begin{array}{l}\text { Yielo } \\
\text { stress } \\
(\mathrm{ksi})\end{array}$ & $\begin{array}{l}\text { Tensile } \\
\text { utimate } \\
(\mathrm{ksi})\end{array}$ & $\begin{array}{l}10,000-\mathrm{hr} \\
\text { creep-rupture } \\
(\mathrm{ksi})\end{array}$ \\
\hline 200 & 28.0 & 0.3 & 8.4 & 60 & 120 & 90 \\
300 & 27.5 & 0.3 & 9.2 & 60 & 115 & 87 \\
400 & 27.0 & 0.3 & 9.6 & 56 & 110 & 83 \\
500 & 26.2 & 0.3 & 9.9 & 54 & 108 & 75 \\
600 & 25.5 & 0.3 & 10.3 & 50 & 106 & 71 \\
\hline
\end{tabular}

*Estimateo. 


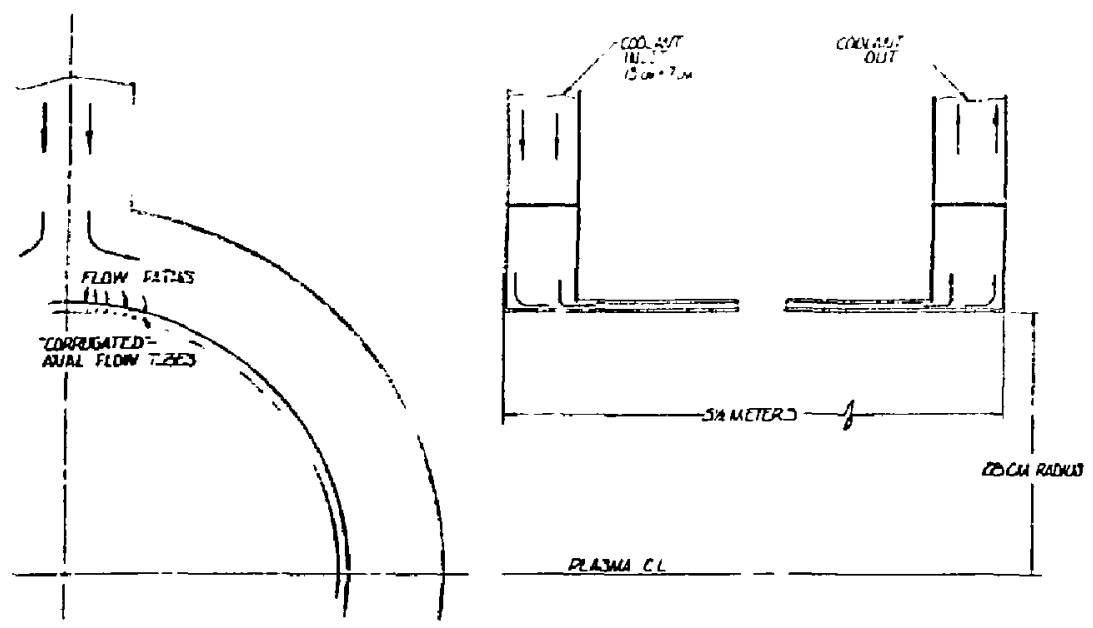

Figure 3-142. First-wa11 configuration. 
A thira configuration would provige separate first-wall sections for each test module and shield segment. Although this configuration would be basicaliy the same as the full length design, there would be many short wails insteac of one long one.

wall thicknesses were initially dictated by thermodynamics requirements, but their present thickness is a result of stress requirements. A center support is also needed to limit bending under the weight of the water. The length of the first wall is dictated by task definition.

keplacement or servicing of the central cell first wall can only be done by oisassembling the center cell shield sections should the full length design be chosen. While it woulo be easier to maintain the individual first-wall segments, manifolding and water piping would be more complex. From the stanopoint of maintenance, a modular or sectional first wall would be best. It would provide a concise piping supply and return lines and facilitate the removal of sections.

\section{Manuf scturing}

Eabrication. The detajls of the first wall will be made from stainiess steel, using such processes or operations as layout and rough cut, roll form, beno bn brake, and route or shear to final size.

Five welded subassemblies will be made from these details. The first-wall subassembly will be made by resistance welding 0.080 -in.-thick corrugated formed sheet to the rolled and welaeo inner bore. The outer channel skin will be rolled, formed in four sections, and resistance welced to the corrugated channel. The sections will be longitudinaliy welded tcgether. lhese welds will employ backup bars. The secona and third assemblies will be two annulus rings made up of an outer radius ring ana a side plate. The annulus assembly will have an "L"-shaped cross section, one left-handed and one right-handea. The fourth assenbly will be two annulus rings welded into eno-plate assemblies. The fifth assembly will be two stacks made from a tube and a built-up rectangular transition section.

Installation. The first assemblies to be installed will be the stacks, which will be inserted into openings and ciamped in place. The first-wall 
assembly will be fed into place from ejther end and located on station. The third and fourth assemblies will be fed in over the first-wall in-bore and located on station. The side-plate annulus ring will meet the first wall's outer skin. Then the stack will be weldea to the outer radius ring of the annulus assembly, and the side piate of the annulus assembly will be welded to the outer skin of the first-wall assembly. All welds will be in accordance with the ASME vessel cooe. The end plate annulus rings will then be welded into place as close-out of the first wall is accomplished.

Thermal Hyoraulics. The 6-m-long, 28-cm-radius central cell first wall nust accommoate a heat loading of $10 \mathrm{w} / \mathrm{cm}^{2}$. Assuming an inlet water temperature of $40^{\circ} \mathrm{C}$ and a temperature rise of $20^{\circ} \mathrm{C}$, a water flow rate of $180 \mathrm{gpri}$ is required to remove the heat. The 1.7-fps water velocity will cause a pressure orop of 0.3 psi through the channels, requiring $20 \mathrm{~W}$ of pumping power. A pressure of 10 psi will be adequate to suppress boiling. The maximum steel temperature reached at the front surface of the first wall is $144^{\circ} \mathrm{C}$, with a $54{ }^{\circ} \mathrm{C}$ conduction orop in the front plate.

Structural Analysis. The first wall, as shown in General Dynanics arawing TDF 016 , consists of a cylindrical $5.5-\mathrm{m}-1$ long shell with a support at mio-length ano a $28-\mathrm{cm}$ inner radius. The she $1 \mathrm{l}$ well is of corrugateo sandwich type construction, with the corrugation in the axial direction of the shell. The average operating temperature is about $250^{\circ} \mathrm{F}$, and the materiai chosen for first-wall construction is $304 \mathrm{~L}$ stainless steel. The coolant flow proauces an internal pressure of 100 isi insice the wall.

The Bailer Code does not have a design procedure for a sanawich type cylinurical shell with a pressure load inside the shell wall. Hence, the following faiture modes were assuned, with boiler cooe design procedures being followed when applicable.

i. Failure of the outer skin as a cylindrical sheil under internal pressure.

2. Faijure of a rectangular pressure vessel equivalent to the trapezoidal flow ARCA under internal pressure. 
3. Failure of a monocoque cylinder equivalent to the sandwich cylinder under external pressure.

4. Failure of the inner skin as a cylindrical shell under external pressure.

Analyses based on the Boiler Code for the four possible failure modes shown above indicate that modes 1 through 3 can be avoided if the outer and inner skins are each $0.125 \mathrm{in.} \mathrm{thick} \mathrm{and} \mathrm{have} \mathrm{a} \mathrm{corrugated} \mathrm{mietal} \mathrm{thickness} \mathrm{of}$ $0.051 \mathrm{in.,}$ while mode 4 can be avoided if the thickness of the inner skin is increased to $0.30 \mathrm{in}$. Even though moce 4 is the worst-case failure mode, this assumption would be conservative should the corrugation separate from the outer skin. Hence, we can cunclude that the outer skin can be $0.125 \mathrm{in}$. thick, while the inner skin should be 0.30 in thick.

First-Wall Material Properties. Because of its availabllity ano cost, steel was chosen as the base]ine naterial for the first wall. Stainless steel ineets the neutronics and thermooynamics requirements, so the use of exotic expensive material is not warranted. Table 3-54 shows the property values of the 3014L stainless steel usea in performing material-related stress and thermooynanic arlalyses.

\subsubsection{Structural Support}

The structural support for the TUF mirror machise is similiar to a briuge structure. It consists of a series of I-beans and support struts that carry the weight of the entire machine.

The overall structure of the machine can be broken into three aistinct sections: (1) the central cell, (2) the eno cell, and (3) the beamlines.

1. The support for the central cell magnet consists of two trusses that cover approximately 100 oeg of the magnat vessel bottom. Included in the magnet structure is the magnet cold-mass support and the intercoil magnetic support beam. The cola-mass support consists of four pairs of struts that support the magnet inside the vacuum vessel. These struts are aesigned to operate in tension, so the cross section can be low and long, providing a low heac isss. 
2. The end cell support is made up of one wide center-support truss that runs the length of the end cell and four tall, stabilizing towers that provide lateral stability. The main weight component taken up by the center section.

3. The beamline supports posed a more complex problem than either the central cell or end cell. The beamlines are situated at odd angles (at angles in two planes). To leave more floor space under the machine, it was deemed desirable to support the beamiines from abovi or at the eno cell come.

A support arch, which goes from the side walls up to the beamlines anc connects unaer the anchor cell, was designeo as the primary load-carrying support. In addition, some tension members that reach back to the anchor cell region will provide extra support by transferring a portion of the load to other parts of the machine.

The support for the anchor cell magnet set is in iwo parts: magnetic load and weight load. Most of the magnetic load is taken up by eight struts in tension. The weight load ano partial magnetic load is taken up by a beam-truss structure between the anchor magnet vacuum vessel and the inner wall of the shield.

A bucking ring, which welds to the inner wall of the shield, is added to react the magnetic forces acting on the choke coil.

\section{Manufacturing}

\section{Fabrication}

Magnets. The magnet supports will be fabricated from structural shapes and detail plates that are plasma arc cut from stainless steel. saddles and footprint pieces will be cut, sized, and saddle-rollea to shape. Assembly will be accomplished using standard shop aids (clamps, braces, and shoring) to position the details for welding. These supports could be assembled in plant or on site as costs and schedules demand. To hoid costs down and permit detajl batching, the six support assemblies will have a common set of details. 
Eno Cells. The support assemblies will be faoricated in much the manner as the central cell. Because of its size, however, the saicer-line support assembly will have to be final assemblea on site. As a cost-saving measure, the end cell supports may be made from structural steel in lieu of stainless steel if analys is of the magnetic fielo permits it.

A.l weloing will be to structural standards, and appropriate quality assurance proceoures will be applied.

\section{Instailation}

Magnets. The completed support assenbly will be positioned and affixed to mounting plates anchored in the facility floor. With the use of jacks and alignment optics, the supports wilt be shimmed in position ana weldea in place.

End Ceils. These supports will be installed like those for the magnets.

Structural Analysis for TDF End-Cell and Central-Cell Support: Weight Loaging. The end cell was conservatively assumed to be supporteo by its 15 vertical members. The certral cell was similarly assunied to be supported by its two vertical members. Remaining members were assumed to provide horizontal stability only and were not included in load-carrying capability.

The material for the structural members was designated as 316 siainless steel having an assunied yield of $30 \mathrm{ksi}$. Column dimensions were selected from. the AISC Manual of Steel Construction, using the 36-ksi yield-stress tables. An adaitional factor of safety was used to account for the higher yield stress of the tables.

Based on the stress analysis, the central columns for the end cell support should be W12 by 12-133; all other members should be W12 by 12-79. For the central cell supports, the columns should be 610 by $10-72$, and the other members should be w6 by 6-15.5. Sizing should be used as a preliminary guice only, not for manufacturing. 
Pre i iminary Stress Ana iys is or the Anchur-Cei i vacuun- iesse? Support

Structure. The vacuum vesse] was assumed to be supportea by its vertical members only. Nonvertical members were assumed to take lateral loaos, prov. . stability, and leave the vertical columns in compressive loading only.

The material for the structural members was designated as $304 \mathrm{~L}$ stainless steel. The environment was assumed to be $200^{\circ} \mathrm{F}$, giving a yjeld stress of $60 \mathrm{ksi}$.

The inboaro region is supported by five vertical members. All members are cesigriated as $4-\times 4-\times 3 / 8-i n$. square structural tubing. The four vertical support nembers of the outboara region are designated as $b-\times 5$ $i / 2-i n$. square structural tubing. Again, these are preliminary sizings only and are not intended for manufacturing. 


\subsection{SHIELDING}

\section{7,1. Shield--Mechanical}

The TDF central cell bulk shield is basically a hollow cylinoer with an inner radius of $85 \mathrm{~cm}$ and an outer radius of $175 \mathrm{~cm}$. The first $15 \mathrm{~cm}$ of the shieio is a water volume. The water is required for botin neutronics and heat transfer. The last $10 \mathrm{~cm}$ of the shield is toron carbide, a composite material. The remainder of the shield is steel.

The major factor to be considered in shield design is the sheer mass of the steel. Two methoas were thearized to design the shieid sections: (1) Shield sections could be built up of large steel plates weloeo together to form a framework "box" (egg crates); it was felt that this would assmilate a solid piece of steel fairly well. (2) Shield sections coulc be spuri-cast into solid cylingers; however, the size of the application may not al low this option.

Boron carbide is so hard it requires a oianond to cut and is, therefure, difficult to work with. Forturiately, the vendor can supply it to us in the section shapes and sizes we dictate so that no cutting is requireo. 28

maintenance ard replaceability of the silield should be a relatively smail task. Piaintenance is limited to the water channels at the inside radii. Snield sections such as the superconducting magnets should never have to be replaced, so no ease-of-removal features have been worked into the desiyn. Should removal be necessary, segments could be cut out in the reverse oroer of assembiy.

\section{Maniffacturing}

Fabrication. Shield fabrication will involve such processes as rolling, shearing, drilling, and plasma arc cutting. Details will be cut from 3/4-, 1-, $2-$, and $3-i n$-thick stainless steel material. Jo-size boron carbide plates will be procurea :rom a qualified subcontractor, and the to-size tungsten insert will be obtained from a tungsten producer.

All detai?s will be inspected and their conformance to the engineering design identifieu before they are delivered to the site by conmon carrier. 
installation. All shield details will be received at the site and stored. The central cell vacuura vessel will have been built up to the horizontal center line, and the outer shield ring will have been roll-formed in pieces approximately 4-1/2 by $8 \mathrm{ft}$. The plates will be laid in place in the bottom of the vacuum vesse 1 and structurally welded together up to the horizontal center line. Then the 3/4-in.-thick ring halves and ribbing will be positioned to form rectangular egg crating, and the details will be welded in place with high-deposit fillet welds. These egg crates will then be filled with boron carbide sheets. Segments cut from 5000-7b billets, with mating steps cut radially into each side, will be positioned in this bottom half of the shield structure. Tack welding will be performed between each segment to . nlo the segments in place. Concurrently, in another fabrication area, the vinter-jacket assembly is to have been built up from the inside wall out, using pressure vessel quality welas. This assembly is to be inspected and integrity tested before it is lowered into place on the bottom half of the 2-ft-thick strield wall.

When the water jacket and its associated plumbing are in place, the top half of the 2-ft-thick ring-well assembly will continue. Where wall openings are encountered, temporary shoring or frames will be used to permit the placement of blacks. The top block will be keystone-shaped to fit in the stepped sides of the segments.

The top half of the outer shield ring will have been subassembled in manageable sizes at a convenient site. The egg crating of these sized and roll-formed welded subassemblies will have teen welded in place, and the boron carbide plates will be located in the crating. The final operation witl be to place these assemblies on top of the existing shield structure and weld them in place.

When the shiela structure is complete, the rest of the vacuurn vessel will be instalied.

Thermoaynamics. The TDF central cell shield must be cooled to remove volumetric nuclear heating. Water was chosen as the cooling medium because of its good neutronics performance with steel. The 15 vol\% of water in the shiela was also dictated by neutronics performance, not cooling requirements. Therefore, it is necessary to determing if the shield will meet cooling requirements. An analysis of the shield configuration is shown in Fig. 3-143. 


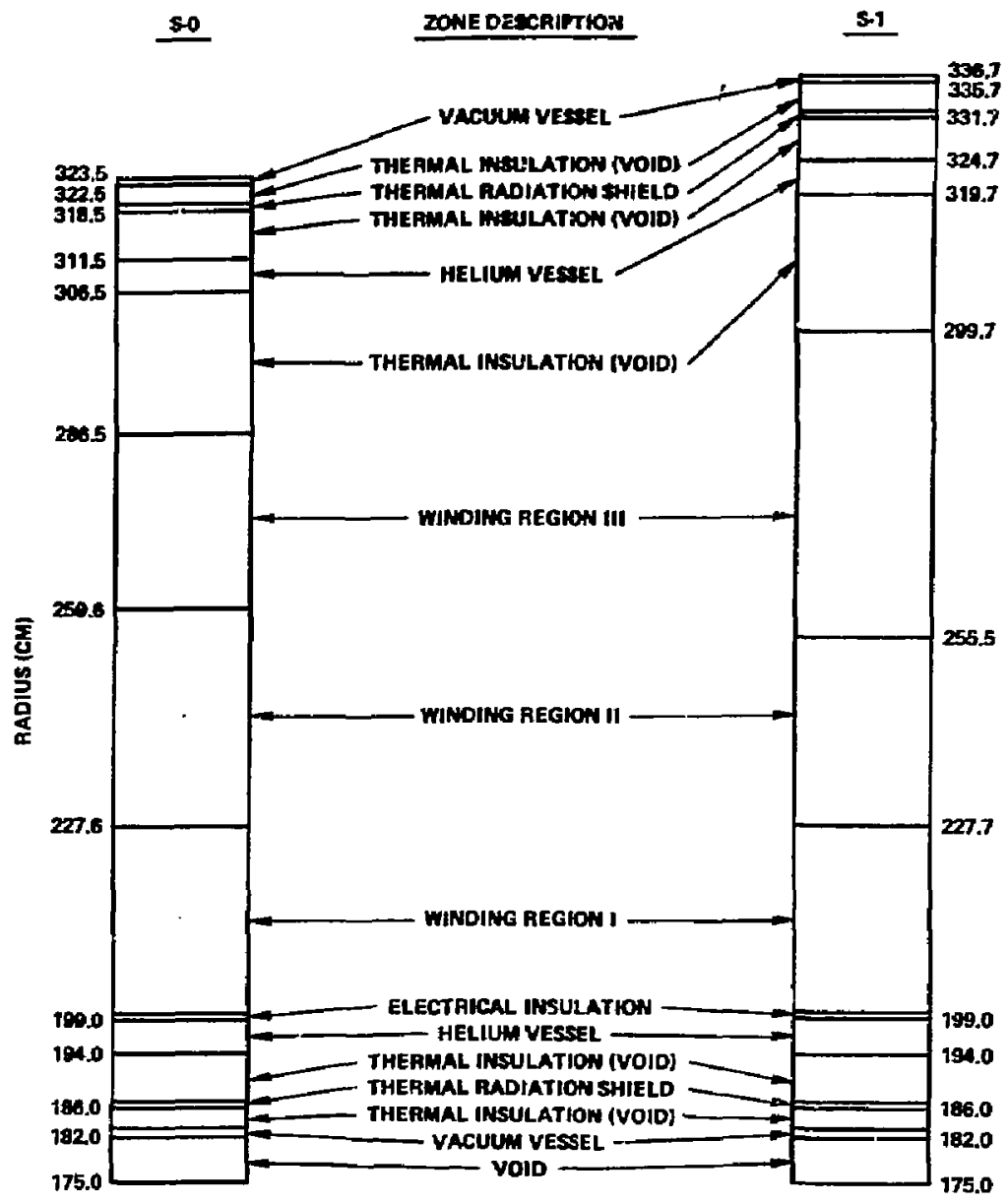

Figure 3-143. TOF magnet models for neutronics analys is. 
The water is assumed to be uniformly distributed and to flow circumferentially around the shield. The inner and outer surfaces of the shield are assumed to be insulated, i.e., no heat is transferred out of the shield except through the water. The nuclear heating profile used in the thermal analysis of the shield is shown in Fig. 3-144.

A tatal of $4.7 \mathrm{MW}$ of heat must be removed from the $128-\mathrm{cm}-1$ ong shield section. One-thousand gallons per minute of water at 150 psi will cool the shield and suppress boiling. The inlet temperature and temperature rise of the water are assumed to be 40 and $20^{\circ} \mathrm{c}$, respectively. The water velocity of 1.3 fps results in a small pressure drop through the shield. The predicted temperature reaches a maximum of $324^{\circ} \mathrm{C}$ on the inner stell and $320{ }^{\circ} \mathrm{C}$ on the outer shell.

\subsubsection{First Wall}

Summary. The primary function of the first wall in the central cell is to protect material test samples from the non-neutron environient associated with a mirror fusion plasma. To accomplish this, the first wall design cannot significantly attentuate or moderate the 14-MeV neutron flux of this facility. We used the neutron and gamma transport code ONEDANT to analyze several design options from a neutronics perspective. On the basis of these analyses, ve scoped a first-wall mechanical aesign. The design consists of a stainless steel, channeled structure that is cooled with water and modular so that segments can be easily removed from the central cell for maintenance or replacement access. Neutron flux effects, as measured by the reduction of material displacement per atom (dpa) potential, indicate a reduction of on $1 y$ about $25 \%$ with this design. We recomend redesigning the structure with a luminum.

Requirements. Nost material test samples are to be exposed to only the 14-MeV neutron flux associated with a D-T burning plasma. Consequently, the first wall must provide a barrier against the thermal radiation and neutral atom flux associated with this type of plasma. The wall must absorb all of the neutral flux and as much of the electromagnetic radiation as possible. Heat loads will necessarily be high, resulting in a neeo for adequate cooling 


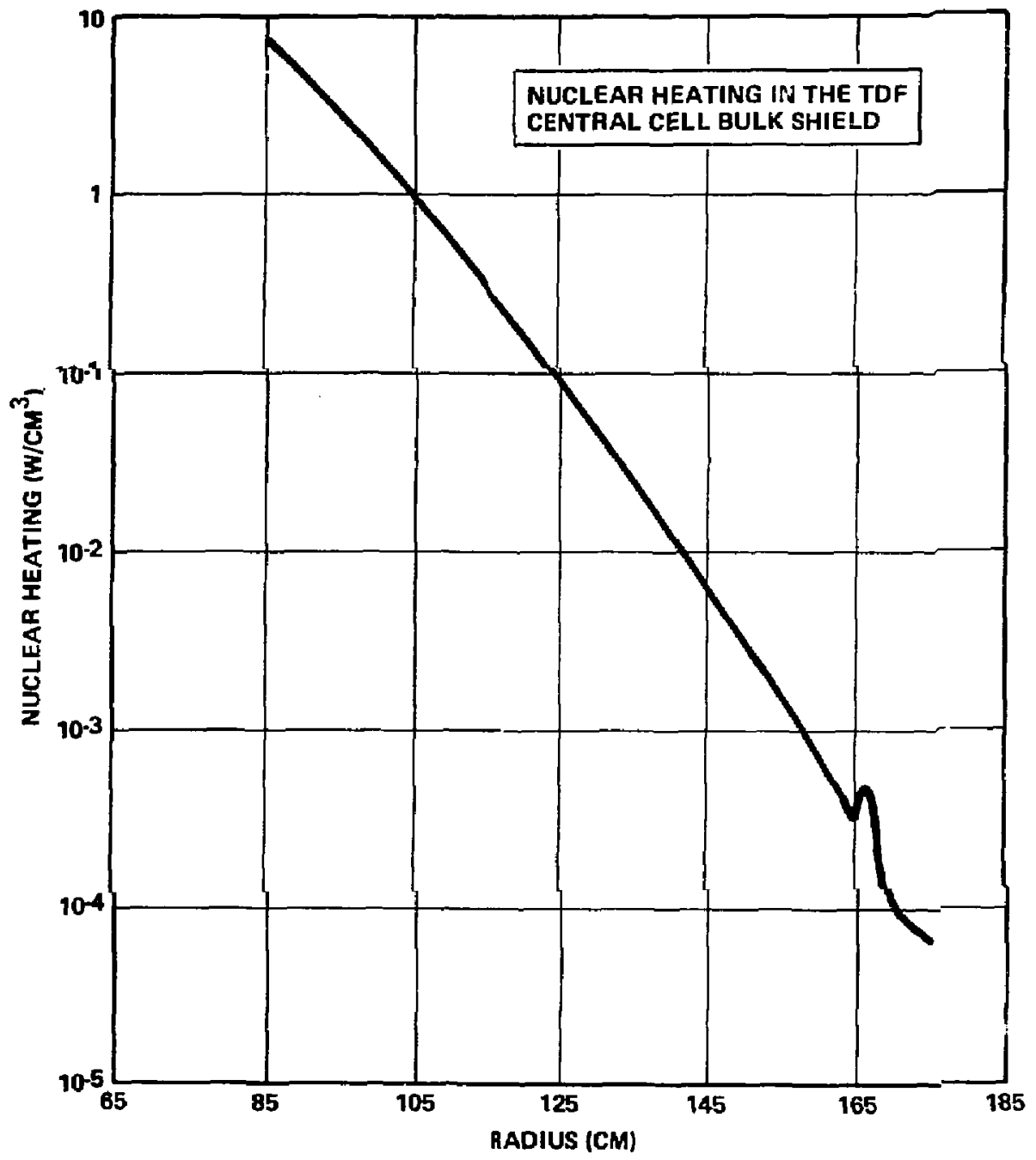

Figure 3-144. Nuclear heating in the IDF central cell bulk shield. 
and subsequently in requirements that the wall be leaktight and interfaced with a heat-exchanger system.

Neutral flux wall loadings above $10 \mathrm{~W} / \mathrm{cm}^{2}$ mean that first-wall material will be sputtered. A design adequate to ensure that the resulting material loss will not compromise the integrity of the first wall over the facility lifetime would aegrade the neutron flux at the material test samples. The alternative chosen was to require that the first wall be easily replaceable and removable through the blanket test ports.

0iscussion. The single most important application of FDF is for nuclear testing. Large test volumes and neutron wall loadings of $2 \mathrm{MW} / \mathrm{m}^{2}$ at a radius of $25 \mathrm{~cm}$ make it an ideal materials test platform. The reality of this engineered device, however, is that, in addition to the required 14-MeV neutron flux, other less desirable plasma components are also produced. In the absence of a first wall, test samples would be exposed to both a radiant heat load and a flux of neutral atoms. Both fluxes would significantly heat the test samples, ano the latter can give rise to sputtering and surface blistering. Although this is an appropriate environment for some sample testing (diverter and limiter surfaces of a tokamak and first walls of any fusion driver), most materials testing in this zone will be performed in the shielded, neutron-only conditions outboard of the first wall. The majority of unshielded materials testing has been reserved for the specialized blanket test facilities.

Clearly, the most important function of the first wall is to provide the shielding needea to ensure that test saniples are exposed only to neutrons. Specifically, it must provide this protection with a minimal impact on the 14-MeV neutron flux, both in terms of attenuation and energy moderation.

A second significant consideration for the first-wall design derives from the sputtering issue. Two distinct neutral fluxes are incident on the first wall. The first is a lower energy component that averages about $100 \mathrm{eV}$ and originates in the halo plasma. A higher energy flux, averaging about $30 \mathrm{keV}$, is traceable to charge-exchange events with neutral-beam-injection deuterons and tritons. This combined flux is responsible for a sputtering rate of about $0.13 \mathrm{~mm} / \mathrm{FPY}$ on the first wal]. The sputtered material is, in theory, trapped by a halo plasma and pumped out through the end cell. Should the halo not intercept the wall material, it will enter the lower portion of the plasma, where it will ionize. 
As a relatively high- $Z$ material, it will radiate away a great deal of plasma heat, resuiting in plasma control and yield prob?ems and in additional radiation shielding concerns. If the halo intercept theory does not prove out, the first wall can provide a suitable substrate upon which a sacrificial, low-Z armor could be placed. Sputtered armor that penetrated the halo plasma woula have a significantly lower radiation cooling effect than would the steel, assuming similar material sputtering rates.

Constraining the first-wall design to have a minimal impact on the 14-MeV neutron flux necessitatea a neutronics analysis of various mechanical designs that met all other requirements. ONEDANT, a discrete orainates neutron and galiuna transport code, was used for this purpose. A 30-energy-group neutron and 12-group gamma library complete with kerma and dpa response function aata was used for analysis. Modeling of the system geometry is depicted in Fig. 3-145; it consists of a one-dimensional radial slice through the TUF central cel1. Full-energy neutrons are uniformly produced in the 10-cm-radius piasma, stream through the 15-cri-radius void space, and are incident on a pair of zones of variable thickness that represent the first-wall structure arid coolant, respectively. A third variable-thickness region is a void that is followed by a thin sample zone of aluminum. This last zone is used as the first-wall neutronics effects reference. The variable used to measure the 14-Mev neutron flux degradation assaciated with a given first-wall oesign is the apa rate in the aluminum reference zone. Atomic oisplacement rates are indicative of both neutron flux strength and average neutron energy and, as such, provide an excellent diagnostic for determining relative flux-oegradation effects.

Preliminary mechanical design scoping and the ease-of-replacement requirement suggested that only standard structural materials need be considered. Both aluminum and stainless steel were investigated, with either water or nitrogen being considered as coolants. Clearly, flux degradation is proportional to the thickness of the structure and coolant regions, and a gas has a smalier effect than a liquid does (see Table 3-55). Of interest, however, was a quantification of this effect with thickness and material. Results obtained for several thickness combinations are tabulated below. Degradation results are reported as percent reductions in dpa with respect to the no-first-wall reference case. 


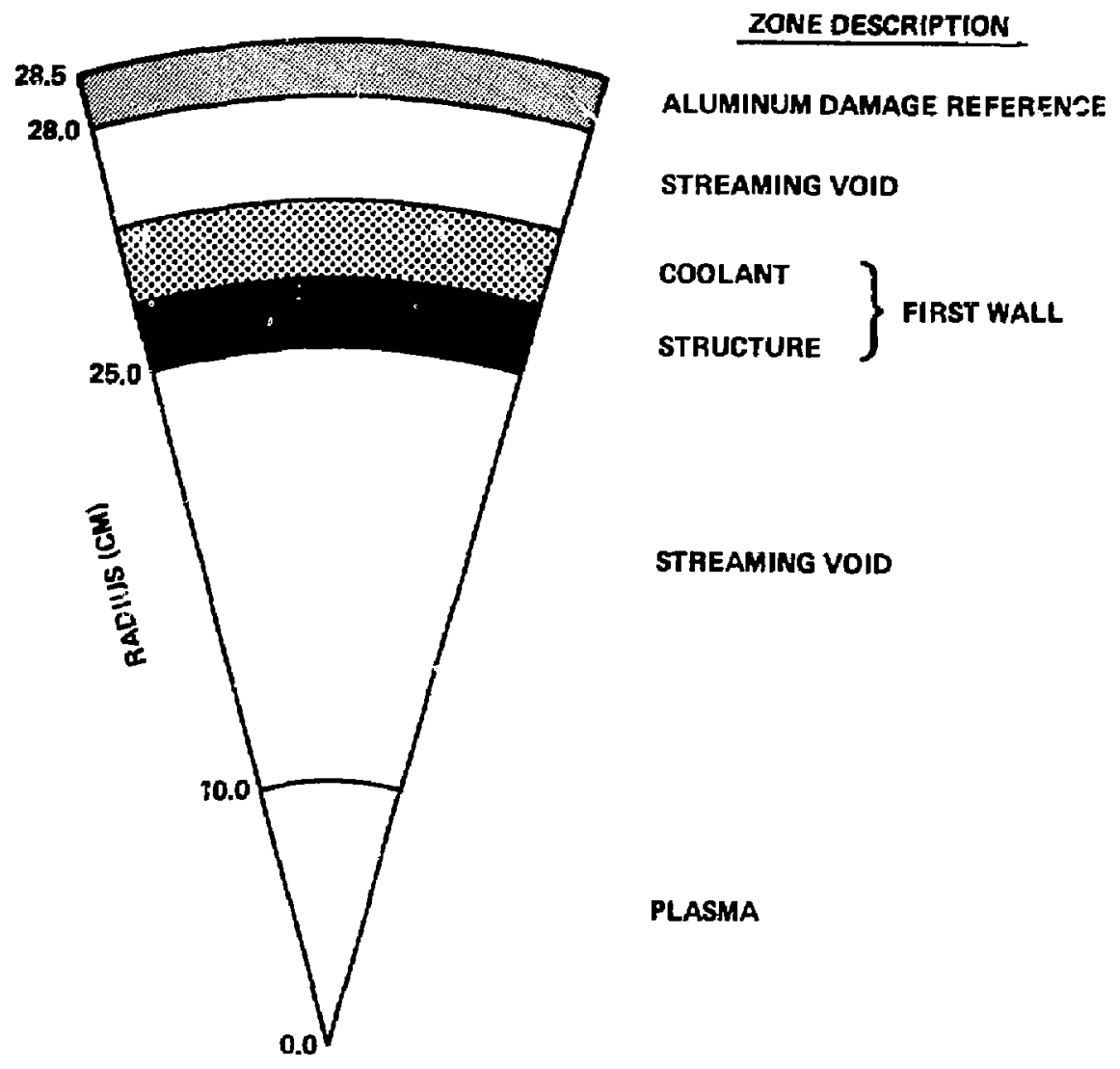

Figure 3-145. First wall neutronics modeling geometry. 
Table 3-55. Flux-degradation results.

\begin{tabular}{|c|c|c|c|c|}
\hline \multirow[b]{2}{*}{ Structure } & \multirow[b]{2}{*}{ Coolant } & \multicolumn{3}{|c|}{ Wall thickness $(\mathrm{cm})$} \\
\hline & & $\underline{0.4}$ & 0.635 & 1.0 \\
\hline \multirow[t]{2}{*}{ A luminum } & Water & 15 & 19 & 23 \\
\hline & Gas & 9 & 13 & 17 \\
\hline \multirow[t]{2}{*}{ Stainless steel } & water & 25 & 31 & 38 \\
\hline & Gas & 17 & 23 & 31 \\
\hline
\end{tabular}


These results indicate that aluminum is substantially more neutron transparent than an equal thickness of steel and, consequently, a more uesirable candidate. No mechanical reasons exist to mandate the use of steel over aluminum, particularly in light of the ease of replacement ana repair requireo of the design. Aluminum has the further advantage of having all short-lived activation products; as a result, it poses less of a waste management problem. On the basis of its superior neutron transparency properties and approximately equal mechanical factors, aluminum was preferred as the first-wall structural material. However, time aid not permit completion of the aluminum wall design. An interim design using stainless steel is included in the baseline.

As expecteo, the gas-coolant option provides for better neutron transparency than does a equal thickness of water cooling. A decision as to what cootant to use for the first wall requires compariscn of more than just neutrorics. Heat fluxes of $10 \mathrm{~W} / \mathrm{cm}^{2}$ from direct plasma heating and volumetric heat loads of about $25 \mathrm{w} / \mathrm{cm}^{3}$ from neutron energy deposition place a substantial requirement on first-wall cooling. Pressurized gas cooling would be possible, but the price of high velocities, large pumping powers, and substantial film arops. These factors combined put additional structural requirements on the oesign and, as such, mean lower neutronic transparency than the comparison at equal zone thickness would suggest. In addition, shorter first wall lifetimes resulting from temperature-enhanced radiation damage problems would increase first wall replacement and failure rates. It is clear, for the TDF application, that the small neutronics penalty associated with water cooling is far less severe than the consequences associated with the gas coolant concepts.

In summary, neutronics considerations (in light of the mechanical issues driving the choice of coolant) suggest that aluminum and water be used for the first wall materials. Every effort should be made to make both materials as thin as possible within the mechanical constraints associatea with the environment and the overall design. 


\subsubsection{Bulk Shielo}

Sumnary. The main function of the TDF central cell bulk shield is to protect the superconducting solenoid magnets ( $S-0$ and $S-1$ ) from bulk penetration of the neutron and gamana fluxes of the central cell. A detaileo one-dimensional neutronics ano photonics model is adequate for analyzing the radiation attenuation of the many shield designs investigated. Although three damage/heating requirenents were identified for bulk shield attenuation performance, peak magnet heat load became the driving requirement. If this most stringent requirement could be met with design, the other two damage criteria were satisfied with substantial margin. A two-region bulk shiela has been chosen for the TDF. Region 1 consists of an 80-cm-thick steel (Fe-1422) annulus cooled with 15 vol\% water. This is wrapped by region 2, a 10-cm-thick $\mathrm{B}_{4} \mathrm{C}$ (boron carbice) thermal neutron shield. Neutronics analysis showed that the neutron absorber in region 2 was more effective in reducing the magret heat load than an equal thickness of lead. This suggested that neutron heating (through capture and the emission of charged particles or prompt gamma) dominated gamma streaming from the bulk shield into the magnet, at least with respect to peak heating--the design requirement. All ihree design requirenents were satisfied with margins exceeding a factor of two even when the beam auct streaming contributions to $S-1$ were included. In addition, this design allows for approximately $60 \mathrm{~cm}$ (radially) of material test area behina the first wall.

Requirements. Only three major requirements were established for the neutronics/photonics analys is of the TDF butk shield. Brcause the shield function is to protect the superconducting magnets (considered lifetine components), all three requirements place limits on the allowable radiation a amage to magnet components. The most stringent requirement is the peak magnet heat load. Maximum neutron plus gamra heating of any liçuiohelium-cooled portion of the magnet should not exceed $60 \mu \mathrm{W} / \mathrm{cm}^{3}$ from all sources. This requirement is established to ensure that the nuclear heat load on the magnet does not cause excessively high cryogenic plant costs and power. It is significantly below the maximum heat loao allowed for inagnet cryostability. 
The second requirement is that the peak radiation dose to the polyimide electrical insulation of the magnet not exceed $5 \times 10^{9}$ rads over the 5.44-FPy 1ifetime of the facility. Dases must be kept below this limit to ensure that raoiation-induced property changes in the insulator do not lead to failure of the material's electrical isolation function.

The third requirement is that the peak dpa in the copper stabilizer of the superconducting winding pack not exceed $1.1 \times 10^{-4}$ before the first inagnet anneal at room temperature. This first anneal should occur after two FPY because only about $85 \%$ of the damage can be recovered. A shorter initial anneal period results in increased anneal frequencies later in the magnet life and in a corresponoing decrease in continuous machine run time. Sinre most long-tern blanket tests will be perforined with a mature machine (late in machine life), frequent anneal requirements will have a negative impact on the test program.

In addition to the bulk neutron and gamma streaming danage, a beam duct streaming component must also be considered. As will be discussed later, the neutral beam injector ducts provide penetrations into the bulk shield that effectively move neutron and gamma sources $f$ ar closer to the $S-1$ magnets than oo those that must pass through the bulk shield. Adequate tungsten shielding has been provided to protect against this beam auct penetration component, but the bulk shield damage requirements must be lower to account for bear duct streaming contributions. Thus, two sets of requirements have been established for the bulk shielding neutronics analysis. One set each for the S-0 and S-l magnets is risted in Table $3-56$.

Discussion. The superconducting central cell solenoios are lifetime components. Lead times for the corrstruction of these magnets and the cost of raplacing then once the machine has been assembled would preclude continued operation of the device. Consequently, every effort must be made to ensure their protection. The most obvious source of damage to the magnets is the intense neutron flux of the central cell plasma. A neutron wall loading of $2 \mathrm{MW} / \mathrm{m}^{2}$ at the first wall radius of $25 \mathrm{~cm}$ must be lowered about five orders of magnitude to reduce the magnet damage to acceptable levels. The shieiding needed for this reduction must fit between the material test samples at a radius of approximately $50 \mathrm{~cm}$ and the insioe bore of the magnet vacuum vesse? at a radius of $175 \mathrm{~cm}$. 
Table 3-56. Radiation damage and heating limits (bulk only).

\section{$\$-0$}

S-1

Peak magnet heat load $\left(\mu \mathrm{W} / \mathrm{cm}^{3}\right)$ 60 35

Peak aose in polyimide insulator ( rads/FPY $\times 10^{8}$ )

Peak dpa in copper stabilizer $\left(\times 10^{-4}\right)$

9

8.8

1.1

(Before first anneal--2 FPY) (opa/FPY $\times 10^{-5}$ )

5.5

4.7 
Neutronics and phatonics analyses were performed with the one-dimensional, discrete orainates coge ONEDANT. A coupled 30-group neutron and 12-group gamia library complete with kerma and dpa response functions was used for the transport calculations. Because both tatal nuclear heating data and peak heating data were requireo for each magnet, modeling was performed carefully so as to be as near to the actual mechanical design as possible. Cross sections of both the S-0 and S-1 magnets are shown in Fig. 3-143; the associated dimensions are radial locations with respect to the plasma center line. Unly the multi-layer insulation material of the chermal shield zone was not modeled. The MLI region consists of loly-density $\left(0.01 \mathrm{~g} / \mathrm{cmi}^{3}\right), 10 \mathrm{w}-\mathrm{z}$ material with minimal protection capability or damage concerns and, as such, was treateo as a void.

Figure 3-146 shows the remainder of the neutronics model from the plasma center line $(r=0.0)$ to the outside of the bulk shield (i.e., $r=17 \mathrm{~b} \mathrm{~cm}$, the inside bore radius of the S-O and S-1 magnets). Full-energy (14-MeV) fusian neutrons are uniformiy produced in the 10-cm-oiam plasma region (normalized to a $2-\mathrm{MW} / \mathrm{m}^{2}$ wall loading at $25 \mathrm{~cm}$ ), stream through a $15-\mathrm{cm}-\mathrm{ajam}$ void, and are incident on the first wall. The first wall was modeled as a $1-\mathrm{cm}$-thick water coolant sandwiched by two 0.25-cm-thick aluminum plates--a close approximation to the channeled first wall structure. The effect of changing to a stainless steel structure will not change the shield design. A material test sample region was assunied to exist to a radius of $50 \mathrm{~cm}$, but was taken as a void to investigate the worst-case shielaing requirements. The remaining region $(r=$ 50 to $175 \mathrm{~cm}$ ) was the bulk shield design flexibility region, where the various ofsign concepts were investigated for neutronics compatibility with the magnet danlage limits.

Several materials were consiaered in the neutronics analyses. Preliminary calculations of the nuclear heat-deposition rates mandated use of a water-cooled shield for heat removal. The water also provides an excelient neutron moderator. It quickiy reduces the energy of the fusion neutrons, thereby decreasing the magnet damage potential and increasing the capture probability in the shielo zone. Structural materials investigated included steel (Fe-1422 and 316 stäinless stee1), tungsten, lead, ana boron carbide. Several shield configurations were also tested. Homogeneous and heterogeneous designs, as well as graoed shield concepts, were scoped where different water 


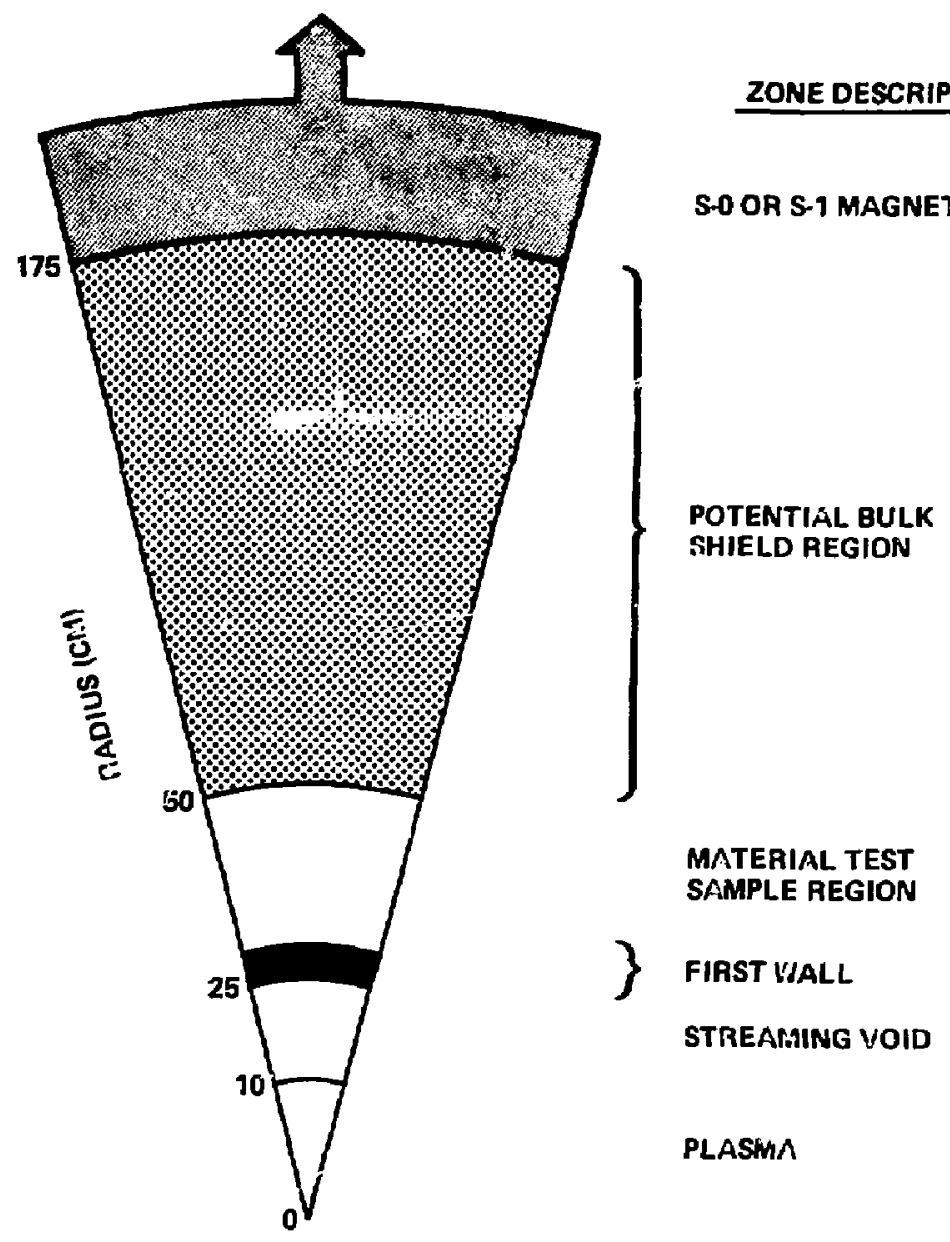

Figure 3-146. Bulk shield neutronics modeling geometry. 
fractions were used in various areas of the shielo. Shield thicknesses were also varien to determine shield attenuation factors that coula be used for extrapolatjons to higher or lower wall loadings.

Neutronics resuits showed that 15 vol\% of water in the shield was optimum for 316 stainless stee], Fe-1422, and tunysten. It provided enough moderation without aisplacing too much of the absorbing structural material. Although tungsten provided the thinnest shield, being about a factor of 2 thinner than the two steel options, tungsten costs and fabricability quickly elimijated it as a possible structural material when it became clear that there was adequate shiela space for the less effective, but significantly cheaper, steels. The 316/304 stainless steel option was then eliminated because of the higher activation levels associated with its nickel content. The steel composition chosen for fine-tuning neutronics analysis was $F \in-1422$, which consists of $82 \%$ iron, $14 \%$ manganese, $2 \%$ nickel, and $2 \%$ chromium.

The final step in the neutronics analyses was to determine the effectiveness of either a gamma shield or a thermal neutron shielo around the outside of the Fe-1422/water major shield. Neutrons captured in the bulk shield give rise to deeply penetrating garma rays that can pass through the remainder of the shield and contribute to nuclear heating. Lead is an excellent gamma shield; therefore, $10 \mathrm{~cm}$ of lead was placed just inside the magnet bore. A ieutronics analysis with the lead shield showed a reduction in magnet heat deposition; when a similar $10 \mathrm{~cm}$ of neutron absorber (boron carbide) was investigated, however, a greater reauction in magnet heating was chserveo. Thermal neutrons escaping the bulk shield are apparently captured in the magnet materials and, through charged-particle formation or capture galima production, result in more nuclear heating than the streaming garma radiation from the bulk shield.

On the basis of the neutronics analyses and some cost considerations, a water-cooled ano moderateo Fe- 1422 steel shield $(85 / 15$ vol\% composition of steel and water) followeo by a $10-\mathrm{cm}$-thick boron carbide thermal neutron shiela provides adequate shielding within the space available. The appropriate thickness of the steel/water zone was determined by a trade study. Neutronic analyses showed that a 70 -cm-thick steel/water shield (followed by boron carbide) marginally satisfied the design criteria. The same configuration with an 80-cm-thick steel/water zone satisfied all design requirements by at least a factor of 4 . In this trade study, the fabricated 
cost of the extra $10 \mathrm{~cm}$ of steel/water shield was compared with the lifetime operating cost of the cryosystem require to remove the additionai nuclear leating associated with the 70-cm-thick steel/water shield. Fabricated shield costs were about. $\$ 5.2$ million and $\$ 4.7$ million, respectively, for the 30 - and $70-\mathrm{cm}-$ thick cases (90 and $80 \mathrm{~cm}$ total thickness). The $\$ 0.5$ million difference compares with the $\$ 1.5$ million additional cryosystem operating cost. Ciearly, thicker shields are economically superior even in this conservative trade study. In addition, thicker shields provide more margin, which means higher possible wali loadings and fewer, less frequent magnet annealings. It is even possible, although marginal, that the polyimide insulator of the magnets could be replaced by the cheaper, but less radiation-resistant, 610 insulator.

The recommended bulk shield consists of an $80-\mathrm{cm}$-thick ( $r=85$ to $165 \mathrm{~cm}$ ) steel/water zone of 85 vol\% Fe-1422 and 15 vol\% water followed by a thermal neutron shield zone of 10 -cm-thick $(r=165$ to 175) boron carbide. Table 3-57 lists the neutronics performance of this shiela configuration for bulk contributions only (no neutron beam ouct aamage or heating included). All three damage linits have heen met with substantial margin. The energy multiplication factor is the ratio of total energy absorbed by all components ot the neutronics model (i.e., first wall, shield, and magnet) divideo by the fusion neutron energy input. This quantity reflects the magnitude of the urompt gamma production within the shield. The magnet energy deposition fraction is the fraction of total system energy that leaks from the shield ano is absorbed in the magnets in the form of nuclear heating.

Nuclear heat deposition in the magnets and bulk shield must be removea. Proper use of the neutronics analysis results provided information on the heat deposition in both the $S-0$ and $S-1$ magnets. The data are summarized in Table 3-58; again, they do not incluae beam duct contributions for the S-1 magnets, which will only add about $20 \%$ to the overail cooling requirements. Similarly, nuclear heat depositjon in the bulk shjeld was computed and is plotted in Fig. 3-144.

\subsubsection{Strecming and Shielding And ys is for the Neutral Beam Uuct}

Summary. A detatled three-dimensional neutronics and photonics calculation has been performed to analyze radiation streaming and shieloing in the neutral beam injector (NBI) system of TDF. A tungsten shield in the 35-cm-wide space 
Table 3-57. TDF bulk shield performance (neutronics).

Energy multiplication factor

Magnet energy deposition fraction $\left(x \quad 10^{5}\right)$

1.65

Superconaucting peak heating $\left(\mu \mathrm{w} / \mathrm{cm}^{3}\right)$

10

(aesign limit $=60$ )

Insulator peak-raoiation camage ( $\left.x 10^{-7} \mathrm{rads} / \mathrm{FPY}\right) \quad 2.4$

(aesign limit $=8.8 \times 10^{8}$ for polyimide ano $9.0 \times 10^{7}$ for $(610)$

Copper stabilizer peak aamage $\left(x 10^{6}\right.$ dpa/FPY

(design limit $=5.5 \times 10^{-5}$ )

Magnet nuclear neating (w)

(sum of 3 magneis) for liquid helium 
Table 3-58. Total magnet nuclear input by zone (bulk only).

\begin{tabular}{lcc}
\hline & \multicolumn{2}{c}{ Power deposition (W) } \\
\cline { 2 - 3 } & S-0 magnet & Each S-7 magnet \\
\hline Vacuum vessel & 24.3 & 29.6 \\
Kadiation shield & 7.81 & 9.53 \\
Helium vessel & 34.6 & 49.3 \\
Insulator & 0.82 & 1.21 \\
Grade I conducior & 17.6 & 26.0 \\
Grade II conductor & 0.62 & 0.88 \\
Grade III conquctor & 0.012 & 0.032 \\
& & 116.5 \\
\hline
\end{tabular}


available between the corner of the central cell solenoid and duct wal] provides adequate shielding ior the magnet. The peak raoiation aamage and nuclear heating in the magnet are well below specified design limits. Acceptable heat loaos were obtained in the aluminuti cryopanels. An extra 10-cm-thick tungsten shield in front of the steel source structure reduces the absorbed raoiation dose in the Macor insulator considerably, resuiting in an estimated lifetime of 2.77 FPY.

Requirements. Adequate shielding is needed to shield the central cell salenoids against radiction streaning into the neutral beam injection duct. Because of the limited space between the magnets and duct wall, the option of using the nore effective tungsten for shielding must be considerea. Arother requirement of the shield design for the duct is to eliminate excessive nuclear heating in the cryopanels. The raoiation effect on the Macor insulating eiectrode support structure in the ion source is of greatest concern. Because of the low radiation dose limit for Macor, adequate shielaing is essential to increase the life of the ion source.

Discussion. Fusion reactors must accommodate a variety of penetrations. The purpose ana size of these penetrations vary depending on the reactor type. ${ }^{28-30}$ Proper shielding is needed to protect the vital components in the penetration from excessive radiation damage caused by raciation streaming. In TDF, oifferent penetrations are used for pumping, heating, fueling, and ion sloshing beams. Because neutron source density in the anchor ana end celis is about six orders of magnitude lower than that in the central cell, only streaming in centra! cell penetrations is analyzed. The central cell penetrations in TUF have different sizes and injection angles and must fit between the central cell solenoids S-0 and S-1. Radiation streaming into these penetrations can lead to adverse radiation effects in the superconducting magnets. It is, therefore, essential to provide enough shielding between the duct wall ano the magnets to reduce the radiation effects in them to acceptable limits.

A major penetration in the central cell of TDF is the neutral beam injection duct, which is characterized by a large size. The duct opening at the first wall is 0.3 by $0.6 \mathrm{~m}$. Four such penetrations are at each ena of the central cell. Other central cell penetrations incluae pellet-fueling ana 
test-specimen-tube penetrations, which are smaller (about $7.5 \mathrm{~cm}$ diam) ano have less severe streaming problems than the aucts. Ising a simple neutronics nalysis basea on one-dimensional calculations, we estimated that a minimuni shieloing thickness of $15 \mathrm{~cm}$ is needed between these penetrations and the miagnets.

Evaluation of the ragiation flow into the ducts requires an accurate knowleoge of the neutron source and the geometrical configuration of the shielo, as well as the size of the ducts. The MCNP ${ }^{31}$ continuous, energy-coupled, neutron gamma Monte carlo code was used to madel the reactor geometry. The neutron source was sampled from the neutron linear source density distribution along the machine axis. The source density was nomarized to a central cell wall loaging of $2 \mathrm{~mW} / \mathrm{m}^{2}$ at a radius of $25 \mathrm{~cm}$. A trapping surface was located at the entrance surface to the duct. At this surface, all particles entering the ouct were counted according to energy and angle bins. Then the results were stored to serve as source distributions in later modeling of the duct itself. Cross-section data based on ENOF/B-V evaluation were used in the calculation.

The energy spectrum of neutrons and gamma photons streaming into the duct is shown in Fig. 3-147. The pronounced peak at 14.1 MeV in the neutron energy spectrum is due to the uncolliaed source neutrons ( $44 \%$ of the streaming neutrons) streaning directly into the duct. A total of $3.08 \times 10^{17}$ neutrons having an average energy of $6.91 \mathrm{MeV}$ and $5.63 \times 10^{16}$ gamma photons averaging $1.44 \mathrm{MeV}$ in energy stream inta the duct each second. The angular distributions of neutrons and gamma photons are shown in Fig. 3-148. The angle $\theta$ is measurea from the normal to the duct entrance surface. I $\hat{\imath}$ is clear that the angular distribution peaks at normal incidence. The 65 -cieg injection angle between the duct and plasma center lines implies that $\cos \theta=0.9$.

Therefore, a large fraction of streaming radiation will go directly towara the ion source at the end of the duct. In general, shielding requirements for the ion source are more severe for larger injection angles.

Figure 3-149 shows the geometrical model used to model the NBI systen in TUF. The model is an idealization and adaptation of the actual NBI system design. The duct opens from 0.3 by $0.6 \mathrm{~m}$ at the first wall to 0.7 by $1.18 \mathrm{~m}$ at a distance of $3.2 \mathrm{~m}$ from the plasma center line. A 1-cm-thick stainless steel duct lining was modeled in the calculation. Only the corner of centrai 


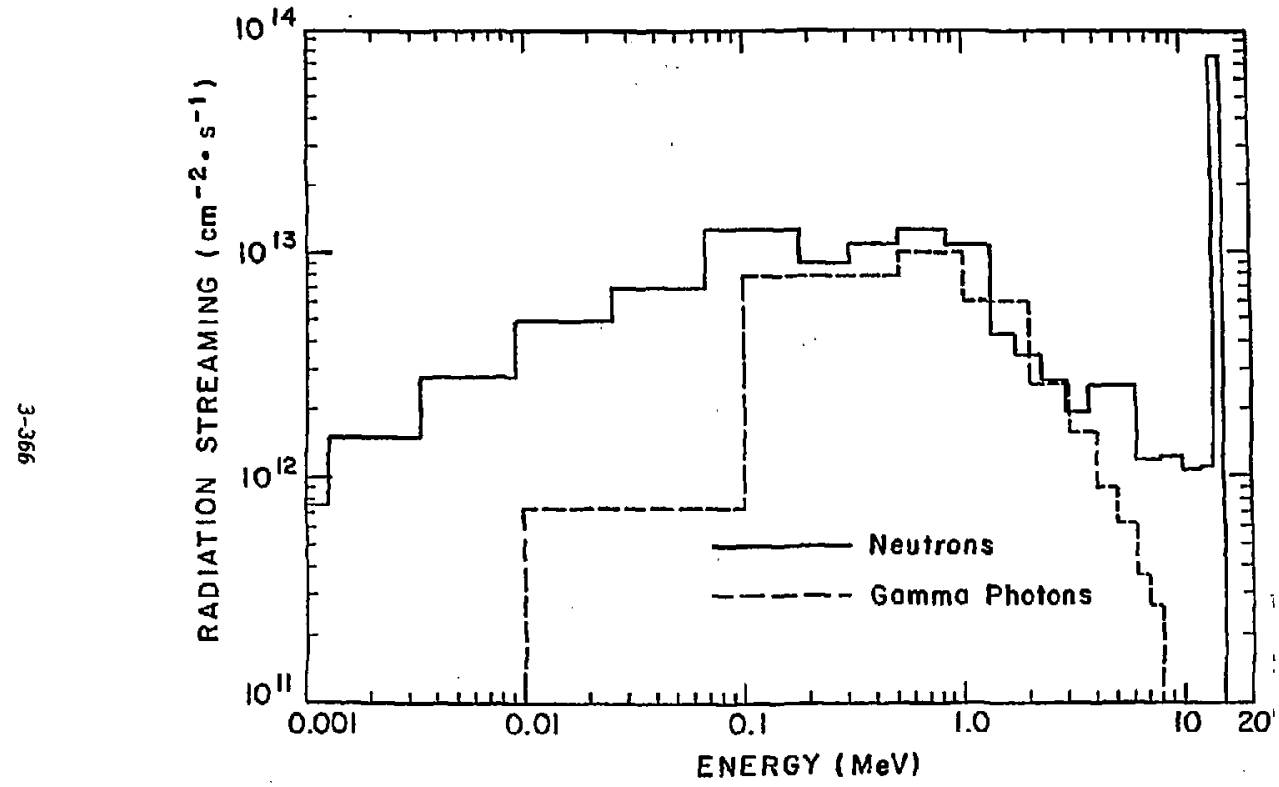

Figure 3-147. Energy spectrum of neutrons and gamma photons streaming into the neutral beam duct. 


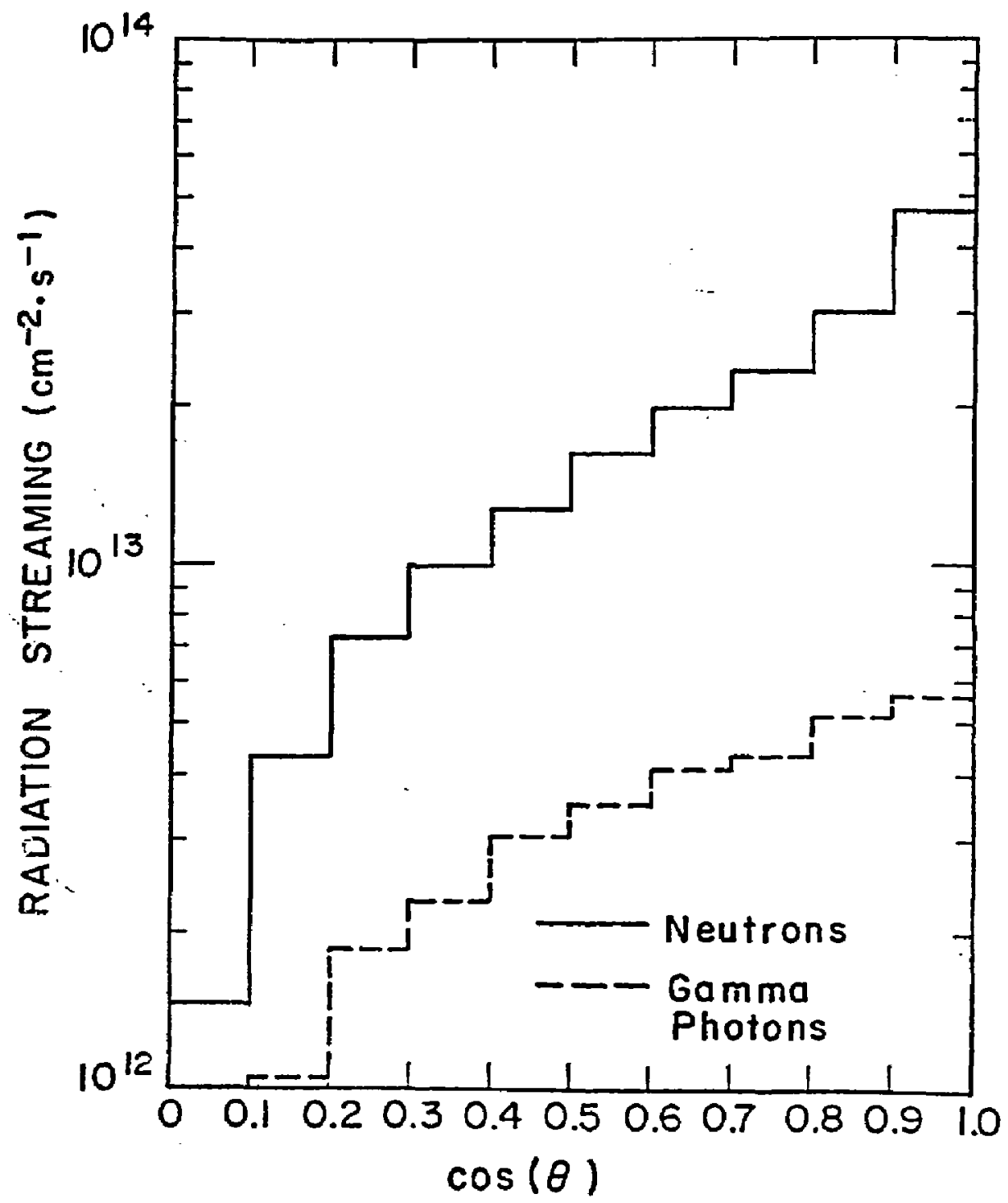

Figure 3-148. Angular distributions of neutrons and gamma photons streaming irito the neutral beam duct. 


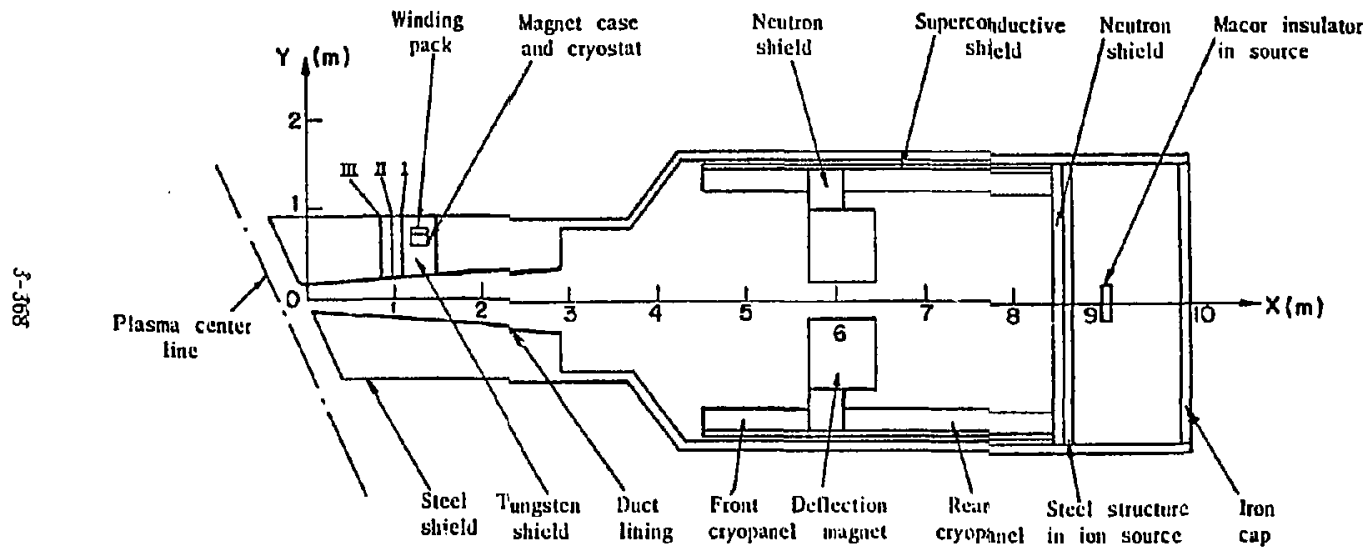

Figure 3-149. Geometrical model for the neutral beam auct in TOF. 
cell solenoin S-1, which has the largest radiation effects from streaming particles, was modeled. The magnet winding pack consists of 2 vol\% iobium-titanium, 23.88 vol\% copper, 54.12 vol\% 304 stainless stee 1, 10 vol\% liquid helium, and 10 vol\% polyimide insulation. The magnet case, cryostat, and vacuum vessel surrounding the winding pack have a total effective stainless steel thickness of $0.125 \mathrm{~m}$, which was modeled in the calculation. A space of only $0.35 \mathrm{~m}$ is available for shielding between the vacuurn vessel at the magnet corner ano the duct wall. The low-activation steel fe-1422 (14 wt\% manganese, 2 wt\% chromium, 2 wt\% nicke 7 , and 82 wt\% iron) was used in the shielo. The shiela consists of 56 yot\% Fe-1422, 26 vol\% $\mathrm{B}_{4} \mathrm{C}(0.87$ u.f.), 14 vol\% lead, and 4 vol\% water. The option of replacing $\mathrm{Fe}-1422$ by tungsten in the shielo zone at the magnet corner was considered to provide adequate magnet shieloing.

The deflection magnet and aluminum cryopanels were included in the model. The NBI systen uses a $0.05-\mathrm{m}$-thick superconductive shield that was also moaled in the calculation. Of greatest concern in the ion source was the effect of radiation on the insulating electrode support structure. Because of its fabricability, Macor machinable glass-ceramic is an attractive candidate insulation material for ABI systems. Its structure consists of a set of mica crystals embedded in a glass matrix. Because both glass and mica may unaergo radiolysis, an enhanced damage rate in Macor is possible. The Macor used in the ion source is concealed from the direct line of sight of the source lleutrons by an effective 0.1-m-thick stainless steel structure. Preliminary results showed that the ion source is not adequate to protect the Macor from excessive radiation effects. An extra $0.1-m-t h i c k$ tungsten shielc, with holes to allow for passage of the beams, is used in front of the stainless steel structure.

The NBI system model used in the three-dimensional Monte Carlo calculation employs neutron and gamma surface sources at the auct opening with energy spectra and angular distributions obtained from the previous streaming calculation. An angular source-biasing technique was used to get statistically adequate estimates for the quantities of interest. Completion of the MCNP run of 50,000 histories resulted in statistical uncertainties of less than $20 \%$. 
The shiezaing requirements for superconducting central cell solenoio s-? are determinea by a number of radiation limits. The apa in the copper stabilizer should not exceed $1.1 \times 10^{-4}$ before the first magnet anneal. Tnis corresponds to a $20 \%$ increase in resistivity. Annealing at room temperature results in only $80 \%$ recovery of radiation-induced defects. ${ }^{32}$ A minimum of two FPY is needed before the first magnet anneal, which results in a period of 1 FPY between the final two anneals for an estimated reactor i ifetime of 5.4 FPY. 33 Therefore, the dpa rate in copper should not exceed $5.5 \times 10^{-5} \mathrm{dpa} / \mathrm{FPY}$. Mechanical strength tests of irridiated magnet insulators show that polyimides are 5 to 10 times more radiation-resistani than comparably prepared epoxies. 34 polyimide is used as an electrical insulator in the TOF magnet coils. The design criterion used for polyimide is that the radiation dose not exceed $5 \times 10^{9}$ rad at the end of the machine's life. Irradiation of tne sliperconducting material (niobium-titanium) resuits in aegradation of the critical current density. In this work, we require that the peak neutron fiuence $\left(E>0.1 \mathrm{MeV}\right.$ ) not exceed $3 \times 10^{22} \mathrm{n} / \mathrm{m}^{2}$, which corresponds to a $10 \%$ decrease in critical current density. ${ }^{35}$ The 1 imit on the peak heat load in the magnet winding pack is con-sidered to be 0.06 $\mathrm{mW} / \mathrm{cm}^{3}$. Because only radiation effects in the magnet, as a result of streaming jnto the neutral beam injection duct, are considered in this calculation, the radiation limits required are a factor of 2 lower to allow for the extra contribution coming through the magnet bulk shieja.

Tabie 3-59 summarizes results for the Fe-1422 shielo case and three options in which tungsten replaces Fe-1422 along the ouct at the magnet corner. In Options $1, I I$, and III, the tungsten shield lengths along the ouct are, respectively, $0.4,0.5$, and $0.6 \mathrm{~m}$. The extra tungsten shielo is added to the side close to the first wall, as shown in Fig. 3-149, because most streaming neutrons will go into that part of the shield. The results show that option I satisfies the design criteria for polyimice dose and superconductor fluence, but only 0ption III meets a.1 design criteria. Therefore, we adopted Option III to shield the magnets against neutrons and gamma photons streaming into the duct.

The results for the superconductive shield are summarized in Table 3-60. A]though the results for dpa rate and heat load exceed the 1 imits used for the superconducting magnets, the superconductive shield can be annealed more 
Table 3-59. Results for the S-1 superconducting magnets.

Fe-1422

shielo

\begin{tabular}{lll}
\multicolumn{3}{c}{ Tungsten shield } \\
\hline Option I Option II
\end{tabular}

Peak opa rate in

$3.84 \times 10^{-4}$

$1.1 \times 10^{-4}$

$5.82 \times 10^{-5}$

$8.4 \times 70^{-6}$

copper stabilizer

(dpa/FPY)

Peak oose in

$3.56 \times 10^{9}$

$9.5 \times 10^{8}$

$5.7 \times 10^{8}$

$8.26 \times 10^{7}$

polyimide after

5.4 FPY (rad)

Peak magnet heat

load $\left(\mathrm{m} / \mathrm{w} / \mathrm{cm}^{3}\right)$

0.1

0.052

0.041

0.025

Peak neutron fiuence

$4.16 \times 10^{22}$

$1.13 \times 10^{22}$

$6 \times 10^{21}$

$8.7 \times 10^{20}$

( $E>0.1 \mathrm{MeV}$ ) in

superconductor after

$\bar{j} .4 \mathrm{FPY}\left(\mathrm{n} / \mathrm{m}^{2}\right)$ 
Table 3-60. Results for the superconductive shield.

\begin{tabular}{ll}
\hline Average heat load $\left(\mathrm{raw} / \mathrm{cm}^{3}\right)$ & 0.47 \\
$\begin{array}{l}\text { Neutron fluence }(E>0.1 \mathrm{MeV}) \\
\text { after } 5.4 \mathrm{FPY}\left(\mathrm{n} / \mathrm{m}^{2}\right)\end{array}$ & $1.76 \times 10^{22}$ \\
Dose in poly imide after $5.4 \mathrm{FPY}(\mathrm{rad})$ & $2.57 \times 10^{9}$ \\
dpa rate in copper (opa/FPY) & $2.1 \times 10^{-4}$ \\
\hline
\end{tabular}


frequently because of the small volume. Further, total nuclear heating is mall and does not require an excessive refrigeration system. The front and rear cryopanels have average heat loads of 0.96 ar.a $0.027 \mathrm{~mW} / \mathrm{cm}^{3}$, respectively, which are much lower than the design limit of $10 \mathrm{mw} / \mathrm{cm}^{3}$ useo in this study.

An experinental study of rajiation effects in Macor ${ }^{36}$ indicated slight aamage by 14-MeV neutrons at a fluence of $10^{22} \mathrm{n} / \mathrm{m}^{2}$, which was estimateo $f$ to correspono to a dose of $6 \times 10^{8} \mathrm{rad}$. Our calculations show that the aose rate in the Macor insulator is $2.17 \times 10^{8} \mathrm{rad} / \mathrm{FPY}$, implying that the ion source insulators will last $2.77 \mathrm{FPY}$ as far as radiation is concernea.

\subsubsection{Ena Cell Magnet Shielding}

Summary, Shadow shields have been designed to protect the transition and anchor coils against central cell neutrons streaming into the eia cell. Four shadow ghields are reeoed. Each shield is $0.45 \mathrm{~m}$ thick and $0.5 \mathrm{~m}$ wide. The neutron source in the ena cell is much smaller than the one in the central cel1. No radial shielding is required, and the stainless stee? in the magnet case and cryostat will provide adequate shielding against neutrons originating in the end cell plasma.

Requirements. Radiation damage to the transition and anchor coils can result from fusion neutrons generated in the end cell plasma, as well as from the central cell neutrons streaming into the end cell. A limited space is available for radial shielaing between the plasma halo and the magnets. This

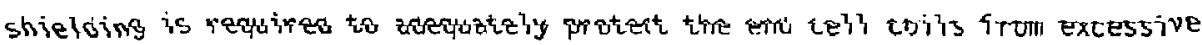
raujation effects.

Discussion. While the peak neutron-linear source density in the central cell of TUF is $1.39 \times 10^{16}(14.1 \mathrm{MeV}) \mathrm{n} / \mathrm{cm} * \mathrm{~s}$, the peak source density in the ena cell corresponds to only $2.8 \times 10^{77} \mathrm{D}-0(2.45 \mathrm{MeV}) \mathrm{n} / \mathrm{Cm} \cdot \mathrm{s}$ and $2.4 \mathrm{x}$ $\left.10^{10} \mathrm{D}-\mathrm{T}(14.1) \mathrm{MeV}\right) \mathrm{n} / \mathrm{cm}-\mathrm{s}$. The numbers are normalized to give a wall loading of $2 \mathrm{MW} / \mathrm{m}^{2}$ at a radius of $25 \mathrm{~cm}$ in the central cel1. To aetermine the shielding requirements for the transition (T-1) and anchor ( $M-2$ ana $M-3$ ) coils, one must consjder fusion neutrors criginating in both tre central and - end cells. 
The smallest distance between the coils and plasma center line is $25 \mathrm{~cm}$. These coils are surrounded by a 15-cm-thick case and cryostat, which have a total stainless steel thickness of $5 \mathrm{~cm}$.

The peak neutron wall loading at the irner surface of the coils, resulting from neutrons generated in: the end cell plasma, is $10^{-5} \mathrm{MW} / \mathrm{m}^{2}$. Based on a one-dintensional calculation, Fig. 3-149 gives the opa rate in copper stabilizer vs shiela thickness for different values of the wall loading. The shield has the same composition as that used in shielding the neutral beam injection penetration, namely 56 vol\% $\mathrm{Fe}-1422,26$ vol\% $\mathrm{B}_{4} \mathrm{C}, 14$ vol\% lead, and 4 vol\% water. The linit on the damage rate in copper stabilizer is $5.5 \times 10^{-5} \mathrm{dpa} / \mathrm{FPY}$, which is the same as that used for central cell coils. Previous results for shielding central cell coils revealed that the opd limit in copper is the design driver; therefore, the shielding requirements for the eno cell coils have been determined on the bas is of the dpa limit. The results in Fig. 3-149 show that a shield thickness of $3 \mathrm{~cm}$ is needed for a wall loadiing of $10^{-5} \mathrm{NiW} / \mathrm{m}^{2}$. Hence, the magnet case and cryostat will adequately shield against neutrons generated in the end cell plasma.

Almost all fusion neutrons are produced in the central cell. Because of this, central cell neutrons that stream into end cell may cause excessive damage to the end cell cails. Damage results when central cell neutrons pass through the $17.5-\mathrm{cm}-\mathrm{di}$ am opening of the choke coil at $z=4.5 \mathrm{~m}$, The neutron 1 inear-source density in the central cell orops rapioly at the choke coil. An effective central cell length, determineo by the points at which the source drops to half its peak value, is $6.8 \mathrm{~m}$. Large portions of the end cell coils will be concealed from the direct line of sight of central cell source neutrons.

[oj] parts that get a direct contribution from central cel] source neutrons are in the axial ranges 7.5 to $8.2 \mathrm{~m}, 8.6$ to $9.0 \mathrm{~m}$, and 11.3 to $11.8 \mathrm{~m}$. The peak neutron wall loading that results from central cell source neutrons streaming into the end cell is $0.009 \mathrm{MW} / \mathrm{m}^{2}$, which occurs at the surface of anchor coil M-2, perpenaicular to the plasma axis. This wall loading corresponds to a required shield thickness of $50 \mathrm{~cm}$, as shown in Fig. 3-150. If the shielding effect of the magnet case and cryostat are taken into account, only a 45 -cm-thick shield is needed. 


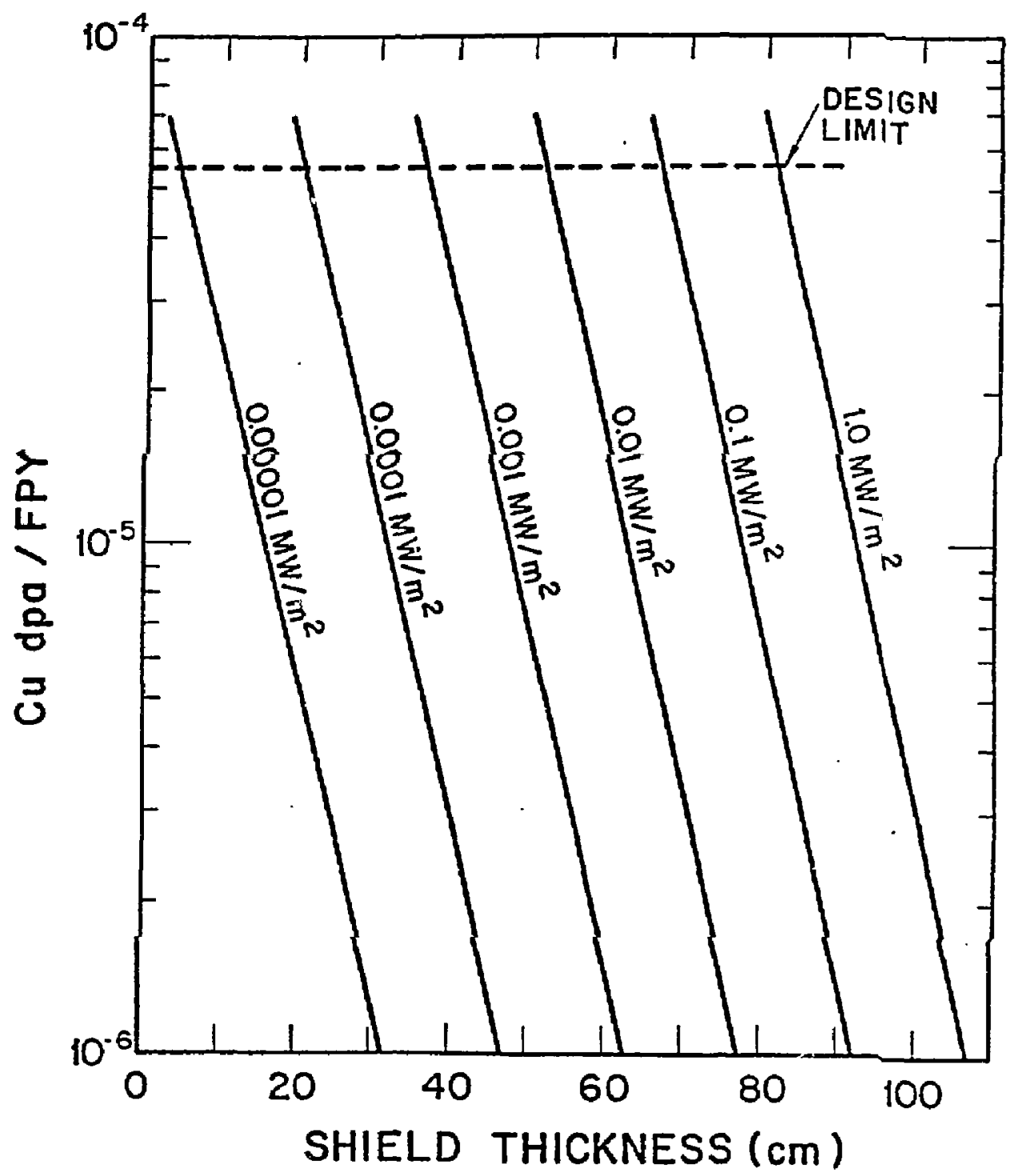

Figure 3-150. Magnet shielding requirements for different neutron wall loadings. 
Using the 17-cm flux lines to determine plasma halo size, we found that only a $5-\mathrm{cm}$ space is available between the plasma halo ana the $T-1$ and $M-2$ coils and that no shielding space is available for the $M-3$ coil. Hence, radial shielaing cannot be used because of the limited space. However, adequate shielding can be provicied with shadow shields, as shown in Fig. 3-151. The shadow shield used at the T-1 coil will adequately shield the parts of the $T-1$ and $M-2$ coils that have a direct contribution from central cell source neutrons. Although parts of the T-1 and M-2 coils with $y$ aimensions $>0.33 \mathrm{~m}$ and parts of coil $\mathrm{M}-3$ with $x$ dimensions $0.20 \mathrm{~m}$ are concealed from the direct line of sight of the central cell source neutrons, the shadow shielos have been extended, as shown in Fig. 3-750, to protect other parts of the coils ayainst seconaary scattered neutrons. The magnet case and cryostat will aoequately protect other parts of the coils, at larger uistances from the plasma axis, against scattered neutrons.

\subsubsection{Biological Shielding}

Requirements. Biological shielding is defined as the nuclear shielding needeo to protect working personnel ana the public, as opposed to magnet snieloing or other component shielding designed to control radiation damage and heating. Magnet and component shjelding requirements and designs are discussed fully in other subsections of this repart.

$B$ iological shielding requirements for the inside of the reactor room are dictated by the need for personnel access to the room $24 \mathrm{hr}$ after shutdown of the machine, including access to all unshielded equipment and up to the unbroken surfaces of the TDF biological shielding. Dose criteria include a general room level of $2.5 \mathrm{mrem} / \mathrm{hr}$, permissible local dose rates to the hands of $10 \mathrm{mrem} / \mathrm{hr}$, and permissible nighly localized streaming dose rates of $100 \mathrm{mrem} / \mathrm{hr}$. A further constraint is that the working staff not receive a cumulative whole-body exposure of more than 20 person-rem/yr.

Biological shielding for the builoing walls and roof shoula be jesignea to 1 imit the dose rate at accessible locations outside the building to $1 \mathrm{mrem} / \mathrm{hr}$ at any time during operation or shutdown. This criterion is set lower than the 2.5-nirem/hr maintenance dose rate inside the builaing because of the possibilicy that nonradiation workers (i.e., clerical help, vendors, visitors, etc.) will have access to the outside of the building. The annual oose at the site boundary should be limited to 10 mren. 

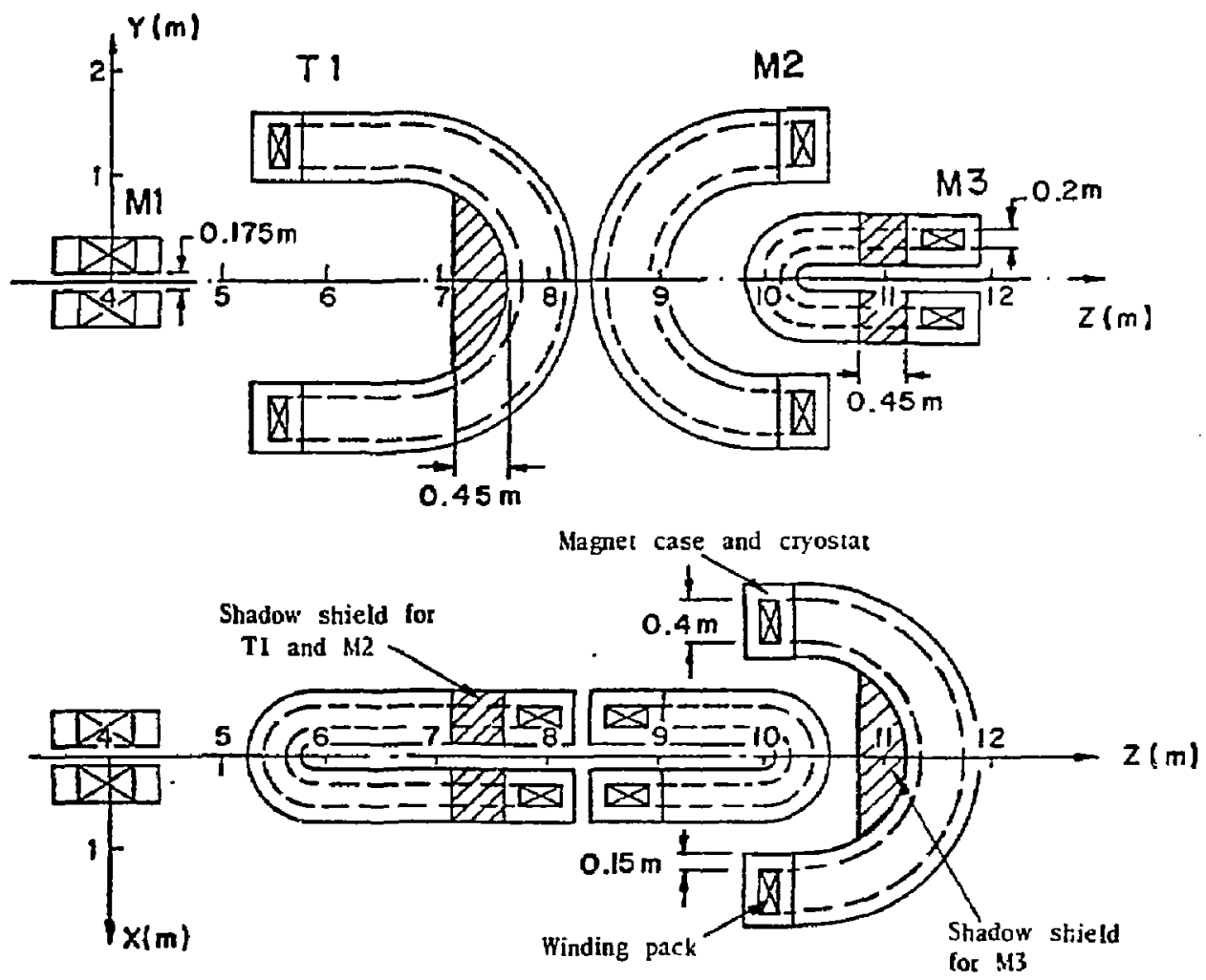

Figure 3-151. Shield configuration for the transition and anchor coils. 
The release of radioactive gases does not affect the biological shield design, but is considered elsewhere in this report in connection with tritium system design.

\section{Shield Oesign}

Reactor. Shutdown gamma radiation originates from inauced activation in the outer layers of biological shieloing and in unshielded equipment just outside the shield, such as the support structure, crane briage, etc. Earlier work on EIF and FED inaicated, should the outboard shielaing reduce the operating neutron leakage to a leve 1 of $22 \times 10^{6} \mathrm{n} / \mathrm{cm}^{2}-\mathrm{s}$, that activation of the shield and outlying components would be kept iow enough to result in a general shutdown dose rate of 2.5 mrem/hr $24 \mathrm{hr}$ after shutdown. Therefore, the conceptual design of the TOF biological shielding is based on reducing the operating external neutron leakage to $2 \times 10^{6} \mathrm{n} / \mathrm{cm}^{2}-\mathrm{s}$.

To limit nuclear heating in the solenoid coils, $0.9 \mathrm{~m}$ of shielding is incorporated between the plasma chamber and the solenoid coils of the central cel1. However, an overall shield thickness of at least $1.2 \mathrm{~m}$, assuming an optimized SS/H2C or SS/B4C configuration, is needed to reduce the leakage to $2 \times 10^{6} \mathrm{n} / \mathrm{cm}^{2}-5$. Therefore, additional biological shielding is incorporated between the solenoid coils to achieve this thickness. The solenoid coils are thick enough to aouble as biological shields in those locations.

The central cell beamlines are enclosed in biological shieloing consisting of $z \mathrm{ft}$ of heavy concrete. other penetrations into the centra] cell must have local shielding to prevent excessive streaming and activation.

Biological shielding requirements for the anchor cell are dictated by the intense neutron beam entering this cell from the central cell--2 $\times 10^{14} \mathrm{n} / \mathrm{s--}$ as well as a local $0-0$ source of $2 \times 10^{14} \mathrm{n} / \mathrm{s}$. With no shielaing, the surface current would be about $3 \times 10^{8} \mathrm{n} / \mathrm{cm}^{2}-5$. About $2 \mathrm{ft}$ of heavy concrete is needed around the anchor cell to reduce this current to $2 \times 10^{6} \mathrm{nicm}^{2}-\mathrm{s}$.

Because of its large size, the end cell experiences a much lower average neutron flux on its surfaces (except on the center Iine). Except for the addition of a required shadow shield over the particle dump region, biological 
shielding of 1 to $2 \mathrm{ft}$ of ordinary concrete is estimated to be adequate for the end celt.

Building. The concrete walls and roof of the building serve as biological shielding, as well as structure and containment. The wall shielding requirement is dictated either by (1) attenuation of the operating neutron dose rate of $2200 \mathrm{rem} / \mathrm{hr}$, or (2) attenuation of the activation gamma cose rate from a remotely removed portion of the central cell being transported to a hot cell without shielding. Roof shielaing is dictated by the skyshine neutron dose rate during operation for a detector outside the building on the ground level.

No calculations for TDF were performed in this phase of the study, but earlier results for FED indicated a 2-m-thick wall of ordinary concrete and a 1.3-m-thick roof, both preferably containing boron or having a low sodium content. Until more definitive information is generated for TOF, the walls are shown as being $2 \mathrm{~m}$ thick. 


\subsection{TEST FACILITIES}

\subsubsection{Summary}

A key issue in TDF design is the aevelopment of testing capabilities that ensure the fusion community receives maximum benefit from this machine. Consequently, TDF test facilities development and test planning have proceeded logically, beginning with the consigeration of general requirements for various types of fusion tests and, ultimately, with requirements for specific reactor blanket designs.

Five generic types or categories of tests are suitable for such a fusion device. They incluoe two basic types of fusion engineering tests: blanket module and materials tests. Although these terms are not mutually exclusive, we generally distinguish between integrated blanket module tests, inciuding materials interactions, thermal hyoraulics, etc., and isolated long-term materials tests. Integrated tests are generally distinguished by short- to meaium-term tests in easily accessible moaules, while isolated tests are oistinguished by moderate- to long-term tests of single specimens, possibly in remote areas of the machine. Short-term materials tests may generally be consioered in either category.

The specific flux and fluence requirements provided herein are appropriate to blanket concepts currently under consideration. To cevelop test capabilities and program plans appropriate to realistic future fusion reactors, we present a generic test categorization for specific blanket concepts and use two blankets--MARS and the Fusion Breeder Program (FBP) hybrid design--to illustrate how the specific requirements for various blankets can be systematically categorizeo and integrated into ToF testing plans.

After we have compared the blanket requirements with current ToF design capabilities, we aevelop the key design impacts of the testing requirements, including specific locations of the materials and blanket module test areas ano of the blanket test area configuration itself. The key factor in blanket module configuration is the vacuum seal location because short test times and rapio turnaround are important to the blanket test program. The result is an optional blanket port module, with an integral first-wall vacuum seal that 
allows single blanket modules to be replaced rapidly without breaking the integral machine vacuum. This port could be replaced by integrated blanket modules (90- to $360-$ deg circumferential extent) that have their own externa) seal and, if desired, optional first wall. We compare the TDF capabilities with the previously developed test requirements, as wel? as with the capabilities of other proposed machines, and show that the TOF concept can meet almost all of the desired blanket and materials test requirements.

Finally, we discuss the remaining key technical issues, including the development of specific test plans that are coordinated with the TOF machine operating plan ana with the individual blanket modules to be tested. ultimately, the blanket test modules and the materials test specimens ano configurations must be designed.

\subsubsection{Requirements}

These can be broken into two categories: blanket test and materials test requirements. Aithough the two are ciearly related, specific testing capabilities in the TOF configuration allow for two distinctly different test environments. They are (1) long-term testing in relatively inaccessible parts of the machine and (2) short-term testing within removable modules that can be replaced or reconfigured relatively quickly.

We generally refer to two separate sets of tests and test requirements relevant to TDF: long-tern materials tests (especially structural materials tests) suitable for long-term in-place testing and blanket module tests, including short-term materials and materials compatibility tests that can be performed within renlovable blanket modules.

Nuclear test requirements relevant to a wiae range of future-generation fusion reactors can be conveniently divided into five categories: neutronics, thermal hydraulics and mechanical effects, tritium breeding/axtraction, raoiation chemistry effects, and radiation damage testing. Each category has a distinct set of test environment requirements that can cover a range of future reactor concepts. The first faur categories generally cover blanket module testing, while the last category is basically for materials testing.

The basic blanket module test requirements are:

1. Neutronics tests require seconds at low flux. 
2. Thermal hydraulics tests require minutes, up to the order of an hour, at moderate flux levels of $0.5 \mathrm{~m} / \mathrm{h} / \mathrm{m}^{2}$ or greater.

3. Tritium breeding and extraction tests can require from 1 to $10 \mathrm{hr}$ for liquid breeders to about $300 \mathrm{hr}$ for solid breeders, at a wall loading of $1 \mathrm{MW} / \mathrm{m}^{2}$. Although essentially $100 \%$ availability would be required during this period, small outages could be tolerated, especially early during a run.

4. Radiation chemistry testing, including materials compatibility effects and other integrated blanket tests, would require the longest test periods of the blanket module tests. On the order of $1000 \mathrm{hr}$ would be needed to demonstrate integrated module operation and to obtain indications of radiation chemistry effects, while two or more years would be needed to qualify a blanket for a next-generation machine.

In aduition, for a hybrio blanket, fuel-cycle equilibrium testing would be requirea over a full cycle. This would be comparable to testing requirenients for category 4.

In category 4 , and in the following discussion on materials testing, we distinguish between moderate-length demonstration or confirmation testing ana long-term qualification testing. In the former we refer to tests that indicate whether a specific design functions as it was interidea to function (a demonstration) or if specific designs or materials previously selecteo for future machines are appropriate, within the time and fluence constraints required, to meet time lines for the design or construction of such a machine (confirmation). By qualification testing, we mean a long-term test or series of tests that verifies that a blanket concept or specific material actually meets or is expecteo to meet the ultimate ena-of-life conditions anticipated in the machine environment for which it is intended. This includes testing materials until actual end-of-life conditions are met or can be predicted reliably; it also includes testing modules until materials interactions, normal and abnormal conditions, overall reliability, and other factors are shown to meet module end-of-life criteria. In either case, qualification will generally iniply tests following, or concurrent with, aemonstration and confirmation testing, but of a longer and more rigorous nature. 
Some materjals test requirements follow:

- Testing of certain special materials, e.g., insulators, requires only low to moderate fluence (up to $0.1 \mathrm{MH}-\mathrm{yr} / \mathrm{m}^{2}$ ), and is not a limiting factor in setting machine requirements.

- At higher fluence levels (about $\left(M /-y r / m^{2}\right.$ ), a fusion spectrum can provide useful data for scoping studies of structural and other materials.

- Longer-tern materials testing, and materials qualification testing for a circa 2000 Engineering Test Reactor (ETR/DEMO) machine requires fluences of about $2 \mathrm{Mri}-\mathrm{yr} / \mathrm{m}^{2}$ at typical wall loadings of $1 \mathrm{mh} / \mathrm{m}^{2}$.

- Materials qualification for commercial prototype machine end-of-life conditions requires fluences of about 5-75 $\mathrm{Mk}-\mathrm{yr} / \mathrm{m}^{2}$ at wall loadings on the order of $3 \mathrm{Mw} / \mathrm{m}^{2}$.

Table 3-6i lists the five categories of blanket and materials tests and gives typical flux, test length, and fluence requirements for each. The categories are listed in orcer of increasing demand on test facility capabilities.

As this table indicates, the lowest level requirements cover neutronics testing, including tritium breeding verification, that can be performec over very short tinte periods at relatively low flux and fluence levels. Thermal hydraulic and mechanical tests require flux levels ideally within about a factor of 2 of the desired level of operation, i.e., approximately $1 \mathrm{kik} / \mathrm{s}^{2}$ for a $2-\mathrm{MW} / \mathrm{m}^{2}$ ETR or DEMO, ano $3 \mathrm{MW} / \mathrm{m}^{2}$ to possibly $5 \mathrm{MW} / \mathrm{m}^{2}$ for a commercial prototype reactor. For thermal hydraulic and mechanical tests, tens of minutes would be required to achieve thermal equilibrium, auring which time transient data can be obtainea, followeo by steady-state operation to obtain equilibrium condition information. Because of the minimal time and fluence requirenients of this and the neutronics categories, such tests wouid be especially suitea for the early stages of TDF operation when high long-term availability would not yet be attained. The following categories, all of which require longer term testing, could then be performed later in the machine life when higher availability would be expected.

The requirements for tritium breeding and exiraction tests depend on the specific tritium breeding material in the blanket. These tests, as distinct from those in category 1 , wi?l be carried out up to equilibrium concentrations 
Table 3-61. Categories of fusion test requirenents.

\begin{tabular}{|c|c|c|c|}
\hline Category & $\begin{array}{l}\text { 14-HeV neutron } \\
\text { val] loading } \\
\left(\mathrm{MH} / \mathrm{m}^{2}\right)\end{array}$ & $\begin{array}{l}\text { Test length } \\
\text { at } 1 \mathrm{MW} / \mathrm{m}^{2}\end{array}$ & $\begin{array}{c}\text { Fluence per run } \\
\left(\mathrm{MH}-\mathrm{yr} / \mathrm{m}^{2}\right)\end{array}$ \\
\hline $\begin{array}{l}\text { 1. Neutronics } \\
\text { Power deposition } \\
\text { Neutron calorimetry } \\
\text { Tritium breeding }\end{array}$ & $>0.01$ & 0.1 to $10 \mathrm{~s}$ & $10^{-9} \approx 10^{-7}$ \\
\hline $\begin{array}{l}\text { 2. Thermal--nydraulic } \\
\text { Thermal--mechanical }\end{array}$ & $>0.5$ & 0.1 to $1 \mathrm{hr}$ & $10^{-5} \times 10^{-3}$ \\
\hline $\begin{array}{l}\text { 3. Tritium breeding/extraction } \\
\text { Liquid metals } \\
\text { Solid breeders }\end{array}$ & $>0.5$ & $\begin{array}{l}\text { l to } 15 \mathrm{hr} \\
10 \mathrm{~s} \text { to } 100 \mathrm{~s} \\
\text { of hours }\end{array}$ & $10^{-4} \cdot 10^{-3}$ \\
\hline $\begin{array}{l}\text { 4. Radiation chemistry effects } \\
\text { Testing for corrosion and } \\
\text { material property changes } \\
\text { Qualification }\end{array}$ & $\begin{array}{l}21.0 \\
>1.0\end{array}$ & $\begin{array}{c}21,000-10,000 \mathrm{hr} \\
2 \approx 10 \mathrm{yr}\end{array}$ & $\begin{array}{l}0.5 \sim 1 \\
1 \approx 5\end{array}$ \\
\hline $\begin{array}{l}\text { 5. Radiation damage testing } \\
\text { for structural materials } \\
\text { ETR (confirmation) } \\
\text { Prototype (qual ification) }\end{array}$ & $\begin{array}{l}27.0 \\
224\end{array}$ & $\begin{array}{l}2 \sim 4 y r \\
2 \sim 10 y r a\end{array}$ & $\begin{array}{l}1-2 \\
5-10\end{array}$ \\
\hline
\end{tabular}

at 2 to $4 \mathrm{MW} / \mathrm{m}^{2}$. 
of tritium, while the category 1 tests will only need to praduce minimal levels of tritium sufficient to verify that tritium has inaeed been produced. Because solid breeding materials retain larger tritium concentrations, longer test times will be needed for these materials to reach equilibrium. Hence, Table 3-61 lists separate test times for liquid metals ( ${ }^{\left.83_{P b}-1{ }^{2} i \text { ana } L i\right)}$ ano solid breeders ( $2 \mathrm{~L}_{\mathrm{L}}$ and $\left.\mathrm{LAAlO}_{2}\right)$. In general, test times for liquid metals range from 1 to $15 \mathrm{hr}$ with a first wall flux of $1 \mathrm{MW} / \mathrm{m}^{2}$, while those for solid breeders range up to several nundred hours. Essentially 100\% availability would be required during these intervals because tritiun levels would begin to decrease as soon as the neutron source was removed. Although this woula present no particular problem early in the test interval, any arop in tritiun level as the test progresses and higher levels of tritium are attained would take progressively longer to nake up; this is especially true for liquids if extraction tests are being performed.

Tests of radiation chemistry and radiation-inducea material changes will entail durations on the order of $1000 \mathrm{hr}$ and up so that perfornance-degrading ano lifetime-limiting problems can be examined. liany radiation-inauced materials compatibility and corrosion problems can be examined in fission reactor environments. However, additional tests in a 14-MeV neutron environment will still be necessary to ensure that unforseen materials compatiblilty or corrosion problems do not occur in blankets in later fusion reactors; those tests carried out in fission reactor environments will still be useful, especially in screening out potentially unsuitable materials, thereby reducing the number of candidate materials that TUF will have to test. This 1,000- to 10,000-hr test category also includes radiation-inouced changes to material properties such as thermal conductivity, density, porosity and resistivity. Solid breeding blankets are especially sensitive to radiation-induced restructuring of ceramic tritium breeding materials, which will change tritium holdup characteristics and thereby affect the results of the tritium breeding/extraction tests. Radiation-induceu changes in resistivity can significantly degrade the performance of high-field copper magnets. Finally, this category includes long-terin blanket qualification testing, which is intended to examine those few module concepts and materials combinations that the shorter-term tests have inaicated as being prime 
canaidates for future-generation blanket modules. Possibly two years would be desirable for a late 1990s "next-generation" device, while even longer periods, up to $10 \mathrm{yr}$, woula be useful if TOF were used to test a blanket coricept for a prototype reactor.

Structural radiation lamage testing is divided into (1) materials confirmation tests for an ETR type machine and (2) materials qualification for a prototype machine. Because TUF would probably not operate long enough in advance of an ETR to be used to determine suitab]e materials choices, its most useful application would be to confirm those materials choices already made ana to indicate any potential problems as early as possible. For a prototype machine however, TOF would operate early enough to select materials and to pualify them as well. The former tests would reguire fluences on the orcier of $1 \mathrm{MW}-\mathrm{yr} / \mathrm{mI}^{2}$ to confirm the suitability of ETR blankets cperating at $2 \mathrm{MW}-y \mathrm{r} \mathrm{m}^{2}$, while the latter woulo require fluences of abaut $5 \mathrm{WW}-y \mathrm{r} / \mathrm{m}^{2}$ or greater to qualify prototype materials candidates to fluences of $10 \mathrm{MW}-\mathrm{yr} / \mathrm{nl}^{2}$ or greater.

\subsubsection{Jiscussion}

Design. 5sues. To more fuljy def ine TDF design requirements and develop an appropriate design, more specific testing requirements must be identified for fusion reactor designs now under consideration. To facilitate this, a generic categorization was developed to permit the needs of various fusion devices to be prestnted in a similar format useful in quantifying those factors important to the cesjon of JDF test facilities. This generic test categorization is shown in Table 3-62.

The categories of information included for each test (among the test categories already discussed) include the type of test and the parameters to be monitored, the duration, the number of times and different test conditioris, and the availability requirements. Together, these factors inoicate the total test time required for each category of test. Table 3-62 also includes examples of the kinds of information appropriate to each column.

$\mathrm{Pr} l$ iminary test needs for two blanket concepts--MARS and the FBP nybrid olenket-appear in Tables 3-63 and 3-64. We chose these two concepts to ...strate solle of the differences in TDF test requirenterits resulting from 
Table 3-62. Generic test categorization. Full power and $100 \%$ availability are assumed for the duration of the test unless specified orherwise.

\begin{tabular}{|c|c|c|c|c|c|c|c|}
\hline Test & $\begin{array}{l}\text { Desired } \\
\text { information }\end{array}$ & Duration & $\begin{array}{l}\text { Number } \\
\text { and } \\
\text { conditions }\end{array}$ & $\begin{array}{l}\text { Pariagiors } \\
\text { ronitored }\end{array}$ & $\begin{array}{l}\text { Avoil- } \\
\text { astility } \\
\text { require- } \\
\text { ments }\end{array}$ & $\begin{array}{l}\text { Total and } \\
\text { tine }\end{array}$ & Modules \\
\hline $\begin{array}{l}\text { Neutronics } \\
\text { Thermal } \\
\text { hydrauliss } \\
\text { Tritium } \\
\text { breeding }\end{array}$ & $\begin{array}{l}\text { Neutronics } \\
\text { MHD effects } \\
\text { Flows, tern- } \\
\text { peratures } \\
\text { Coefficients } \\
\text { Tritium breed- } \\
\text { ing ratio }\end{array}$ & Ahout 5 hours & $\begin{array}{l}3 \text { conflg }=- \\
\text { at ions with } \\
\text { several tests } \\
\text { per conf igur- } \\
\text { ation }\end{array}$ & $\begin{array}{l}\text { Temperature } \\
\text { Neutron flux } \\
\text { Coolant temperature. } \\
\text { flow, pressure pro- } \\
\text { flies } \\
\text { Tritium level }\end{array}$ & $\begin{array}{l}\text { 100x over } \\
5 \text { hours }\end{array}$ & $\begin{array}{l}\text { Ahout } 30 \\
\text { tests } \\
150 \text { hours }\end{array}$ & $\begin{array}{l}3 \text { (or 1, } \\
\text { configur- } \\
\text { able) }\end{array}$ \\
\hline $\begin{array}{l}\text { Fuel breed- } \\
\text { ing: one } \\
\text { full cycle }\end{array}$ & $\begin{array}{l}\text { Neutronics } \\
\text { Afterheat } \\
\text { levels } \\
\text { Irradiation } \\
\text { effects }\end{array}$ & $\begin{array}{l}3 \text { full-power } \\
\text { months } \\
\text { ( } 6 \text { at } 50 \% \\
\text { avallability) }\end{array}$ & Twa conditions & $\begin{array}{l}\text { As sbove, plus } \\
\text { Out-of-reactor } \\
\text { test } 5\end{array}$ & $\begin{array}{l}50 \% \text { over } \\
6 \text { months }\end{array}$ & $\begin{array}{l}2 \text { tests } \\
12 \text { months } \\
\text { total }\end{array}$ & $\begin{array}{l}1 \text { (config- } \\
\text { urable) }\end{array}$ \\
\hline $\begin{array}{l}\text { Radiation } \\
\text { effects/ } \\
\text { qualification }\end{array}$ & $\begin{array}{l}\text { Irradiation } \\
\text { damace to } \\
\text { threshold }\end{array}$ & $\begin{array}{l}2 \text { years } \\
\text { (4 at 50x) }\end{array}$ & $\begin{array}{l}1 \text { condition } \\
\text { (nominal) }\end{array}$ & $\begin{array}{l}\text { Flux } \\
\text { Temperature } \\
\text { Out-of-reactor } \\
\text { tests }\end{array}$ & $\begin{array}{l}50 \% \text { over } \\
4 \text { years }\end{array}$ & $\begin{array}{l}7 \text { test } \\
4 \text { years }\end{array}$ & 1 \\
\hline $\begin{array}{l}\text { Intermodvle } \\
\text { sea? }\end{array}$ & & $\begin{array}{l}5 \text { hours.- } \\
3 \text { montiss }\end{array}$ & $\begin{array}{l}\text { cond'tion } \\
\text { (nominal) }\end{array}$ & $\begin{array}{l}\text { Flux } \\
\text { Temperature } \\
\text { Dut-of-reactor } \\
\text { tests }\end{array}$ & $50 x$ & $\begin{array}{l}\text { I test, up } \\
\text { to } 6 \text { months }\end{array}$ & 1 \\
\hline
\end{tabular}

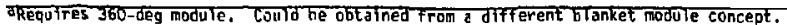


Table 3-63. MARS blanket test categorization. Full power is assumed for the duration of the test.

\begin{tabular}{|c|c|c|c|c|c|c|c|}
\hline Test & $\begin{array}{l}\text { Desired } \\
\text { information }\end{array}$ & Duration & $\begin{array}{l}\text { Number } \\
\text { and } \\
\text { conditions }\end{array}$ & $\begin{array}{l}\text { Paramaters } \\
\text { monitored }\end{array}$ & $\begin{array}{l}\text { Avofle } \\
\text { ability } \\
\text { require- } \\
\text { ments }\end{array}$ & $\begin{array}{c}\text { Total and } \\
\text { tine }\end{array}$ & Modules \\
\hline $\begin{array}{l}\text { Thermal } \\
\text { hydraulics } \\
\text { Trit ium } \\
\text { breeding }\end{array}$ & $\begin{array}{l}\text { Energy mul- } \\
\text { tfplication } \\
\text { Fraction } \\
\text { of high- } \\
\text { temperature } \\
\text { heat } \\
\text { Heat-transfer } \\
\text { properties } \\
\text { Tritium } \\
\text { brecding ratto } \\
\text { Trittum perme- } \\
\text { ation rates } \\
\text { lnttlal burn- } \\
\text { up rates } \\
\text { Short-term } \\
\text { transmutation } \\
\text { products }\end{array}$ & $\begin{array}{l}0.1 \text { to } 3 \text { days } \\
\text { (trithim } \\
\text { steddy state) }\end{array}$ & $\begin{array}{l}\text { Ahout } 5 \text { con- } \\
\text { flgur ations, } \\
\text { severai tests } \\
\text { per con- } \\
\text { figuration } \\
\text { with various } \\
\text { Coolant } \\
\text { tenperature } \\
\text { Lis en- } \\
\text { richnents } \\
\text { lfthlum } \\
\text { quant Ity in } \\
\text { solid breeder }\end{array}$ & $\begin{array}{l}\text { Meutron flux } \\
\text { Temperature } \\
\text { Coolant temperaturc, } \\
\text { flow, pressure } \\
\text { profiles } \\
\text { Transient therms } \\
\text { effects } \\
\text { Tritium level } \\
\text { Trit ium in coolant } \\
\text { Strain } \\
\text { Out-of-reaetor tests } \\
\text { Chentcal composition } \\
\text { Imass spec.) of } \\
\text { coolants }\end{array}$ & $\begin{array}{l}100 \% \text { over } \\
1 \text { to } 3 \text { days } \\
\text { solid }\end{array}$ & $\begin{array}{l}\text { uso tests } \\
150 \text { davs } \\
\text { (one ytar at } \\
50 x \text { duty } \\
\text { cycle }\end{array}$ & $\begin{array}{l}5 \\
\text { Cor 1, con- } \\
\text { figuable }\end{array}$ \\
\hline $\begin{array}{l}\text { Radiation } \\
\text { chemistry } \\
\text { effects }\end{array}$ & $\begin{array}{l}\text { As above with } \\
\text { longer-term } \\
\text { irradiation } \\
\text { tasts } \\
\text { Burnup }\end{array}$ & $\begin{array}{l}3 \text { to } 6 \text { months } \\
\text { (full power) }\end{array}$ & $\begin{array}{l}\text { One condftion } \\
\text { (nominal) }\end{array}$ & $\begin{array}{l}\text { As above, with ori- } \\
\text { ority touard mater- } \\
\text { fals tnteractions } \\
\text { and trittun }\end{array}$ & $\begin{array}{l}50 \% \text { over } 6 \\
\text { to } 12 \text { manths }\end{array}$ & $\begin{array}{l}\text { Ore test, } 6 \\
\text { to } 12 \text { achiths }\end{array}$ & 1 \\
\hline $\begin{array}{l}\text { Longer- } \\
\text { tern } \\
\text { radiation } \\
\text { effects/ } \\
\text { qualifica- } \\
\text { tion }\end{array}$ & $\begin{array}{l}\text { Longer-term } \\
\text { effects of } \\
\text { fuston neu- } \\
\text { tron spectrum } \\
\text { on mater lal } \\
\text { combinstions } \\
\text { Transmutation } \\
\text { products } \\
\text { Chemtcal re- } \\
\text { actions }\end{array}$ & $\begin{array}{l}1 \text { to } 2 \text { years } \\
\text { (full power) }\end{array}$ & $\begin{array}{l}\text { One condittan } \\
\text { (noninal) }\end{array}$ & As above & $\begin{array}{l}50 \% \text { over } 2 \\
\text { to } 4 \text { years }\end{array}$ & $\begin{array}{l}\text { One test, } 2 \\
\text { to } 4 \text { years }\end{array}$ & 1 \\
\hline $\begin{array}{l}\text { intermodule } \\
\text { seal }^{a}\end{array}$ & & $\begin{array}{l}\text { Up to } 3 \\
\text { months }\end{array}$ & $\begin{array}{l}\text { One condition } \\
\text { (nominal) }\end{array}$ & $\begin{array}{l}\text { Flux } \\
\text { Temperature } \\
\text { Out-of-reactor } \\
\text { tests }\end{array}$ & $\begin{array}{l}50 \% \text { up to } \\
6 \text { months }\end{array}$ & $\begin{array}{l}\text { One test. } \\
\text { up to } 6 \\
\text { months }\end{array}$ & 10 \\
\hline
\end{tabular}

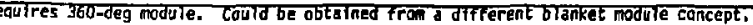


Table 3-64. Fusion Breeder Program blanket test categorization.

\begin{tabular}{|c|c|c|c|c|c|c|c|}
\hline Test & $\begin{array}{l}\text { Desired } \\
\text { information }\end{array}$ & Durat Ion & $\begin{array}{l}\text { Number } \\
\text { and } \\
\text { conditions }\end{array}$ & $\begin{array}{l}\text { Paraneters } \\
\text { monitored }\end{array}$ & $\begin{array}{l}\text { Avail- } \\
\text { ability } \\
\text { require- } \\
\text { ments }\end{array}$ & $\begin{array}{l}\text { Total and } \\
\text { time }\end{array}$ & Hodules \\
\hline $\begin{array}{l}\text { Meutronics } \\
\text { Thertal } \\
\text { hydraulics } \\
\text { Tritium } \\
\text { breeding }\end{array}$ & $\begin{array}{l}\text { Energy mul- } \\
\text { tiplication } \\
\text { Fraction } \\
\text { of high- } \\
\text { tempersius' } \\
\text { heat } \\
\text { Heat-transfer } \\
\text { properties } \\
\text { Tritfum } \\
\text { breeding rat io } \\
\text { Iritium perme- } \\
\text { atton rotes } \\
\text { Initial burn- } \\
\text { up rates } \\
\text { Short-term } \\
\text { transmutation } \\
\text { products }\end{array}$ & $\begin{array}{l}0.1 \text { to } 3 \text { days } \\
\text { (tritium } \\
\text { steady tiute) }\end{array}$ & $\begin{array}{l}\text { About } 5 \text { con- } \\
\text { ffgurotions, } \\
\text { severs) tests } \\
\text { per con- } \\
\text { fiquration } \\
\text { with various } \\
\text { Coolant } \\
\text { temperature } \\
\text { L16 en- } \\
\text { richnents } \\
\text { Lithium } \\
\text { guantity in } \\
\text { solid breeder }\end{array}$ & $\begin{array}{l}\text { Heutron f lux } \\
\text { Temper ature } \\
\text { Coolant temperature. } \\
\text { flow, pressure } \\
\text { profiles } \\
\text { Transient thermal } \\
\text { effects } \\
\text { Trit 1um level } \\
\text { Trit lum in coolant } \\
\text { Strain } \\
\text { Out-nf-reactor tests } \\
\text { Chemical conpositton } \\
\text { (mass spec.) of } \\
\text { coolants }\end{array}$ & $\begin{array}{l}100 \% \text { over } \\
1 \text { to } 3 \text { days } \\
\text { solid }\end{array}$ & $\begin{array}{l}250 \text { tests } \\
150 \text { days } \\
\text { (one year at } \\
507 \text { duty } \\
\text { cycle }\end{array}$ & $\begin{array}{l}5 \\
\text { lor } 1 \text {, con- } \\
\text { figurabie }\end{array}$ \\
\hline $\begin{array}{l}\text { Radiation } \\
\text { chemistry } \\
\text { effects }\end{array}$ & $\begin{array}{l}\text { As above with } \\
\text { longer-term } \\
\text { irradiation } \\
\text { tests } \\
\text { Burnup }\end{array}$ & $\begin{array}{l}3 \text { to } 6 \text { months } \\
\text { (full power) }\end{array}$ & $\begin{array}{l}\text { One condition } \\
\text { (nominal) }\end{array}$ & $\begin{array}{l}\text { As above, with pri- } \\
\text { ortty toward mater- } \\
\text { ials t interactions } \\
\text { and tritium }\end{array}$ & $\begin{array}{l}507 \text { aver } 6 \\
\text { to } 12 \text { months }\end{array}$ & $\begin{array}{l}\text { One test, } 6 \\
\text { to } 12 \text { months }\end{array}$ & 1 \\
\hline $\begin{array}{l}\text { Longer- } \\
\text { tern } \\
\text { radiation } \\
\text { effects/ } \\
\text { qualifica- } \\
\text { tion }\end{array}$ & $\begin{array}{l}\text { Longer-term } \\
\text { effects of } \\
\text { fusion neu- } \\
\text { tron spectrum } \\
\text { on material } \\
\text { combinstions } \\
\text { Transmutation } \\
\text { products } \\
\text { Chenical re- } \\
\text { actions }\end{array}$ & $\begin{array}{l}1 \text { to } 2 \text { years } \\
\text { (ful1 power) }\end{array}$ & $\begin{array}{l}\text { One condtition } \\
\text { (nominal) }\end{array}$ & As above & $\begin{array}{l}50 x \text { over } 2 \\
\text { to } 4 \text { years }\end{array}$ & $\begin{array}{l}\text { One test, }{ }^{2} \\
\text { to } 4 \text { years }\end{array}$ & 1 \\
\hline $\begin{array}{l}\text { intermodule } \\
\text { seald }\end{array}$ & & $\begin{array}{l}\text { Uf to } 3 \\
\text { months }\end{array}$ & $\begin{array}{l}\text { One condition } \\
\text { (nominal) }\end{array}$ & $\begin{array}{l}\text { Flux } \\
\text { Temperature } \\
\text { Out-of-reactor } \\
\text { tests }\end{array}$ & $\begin{array}{l}50 x \text { up to } \\
6 \text { months }\end{array}$ & $\begin{array}{l}\text { One test, } \\
\text { up to } 6 \\
\text { months }\end{array}$ & $j^{\mathbf{a}}$ \\
\hline
\end{tabular}

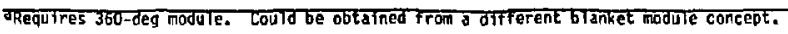


quite aissimilar blanket concepts. Although the most obvious difference between the two designs is the fuel-breeding nature of the hybrid blanket, there are others as well. They include wall loading $\left(5 \mathrm{NW} / \mathrm{m}^{2}\right.$ at $59 \mathrm{~cm}$ for MARS vs $1.5 \mathrm{HiW} / \mathrm{m}^{2}$ at $1.5 \mathrm{~m}$ for FBP), tritium breeding material $\left({ }^{83} \mathrm{~Pb}-17_{\mathrm{Li}}\right.$ and $\mathrm{LiAlO_{2 }}$ for MARS vS $\mathrm{Li}$ for $\left.\mathrm{FBP}\right)$, ano coolant/multiplier $\left({ }^{83_{\mathrm{Pb}-}}{ }^{17} \mathrm{Li}\right.$ coojant/multiplier for MARS vs $L i$ coolant and Be multiplier for FBP). As a result, a number of generic test issues and aesign-dependent blanket factors can be enumerated. The generic issues include:

- Tritium breeding perfarmance.

- Afterheat anu afterheat cooling.

- MHD pressure orop.

- Intermoaule seal performance.

- Heat transfer and heat removal.

- Materials compatibility, chemical effects and transmutation products.

- Structural effects, including thermal stress, differential expansion, LME, DBTT, temper embrittlement, heliun generation, and corrosion.

- Abnormal conditions, incluoing a loss of coolant flow (LOCF) and loss of coolant accident (LOCA).

Uesign-dependent issues for MARS include sintering, tritium solubility and diffusion in solias, and the rate of formation of $\mathrm{T}_{2}$. Specific hybrid issues include the choice of coolant ( $L i$ vs ${ }^{83} \rho_{\rho_{-}-}{ }^{17} \mathrm{Li}$ ), structure (ferritic vs stainless steels), breeding ratio, fission rate, and fuel handing.

In adition to such test issues, the TDF machine and various materials and blanket test nodules must unoergo design verification testing before full $\mathrm{D}-\mathrm{T}$ operation can begin. Although larger, higher-power machines are expected to require up to three years of initial testing, TUF's smaller size and power máy necessitate on ly one year of such tests before reasonably intensive operation can begin. Two factors are important. First, all blanket moduies must undergo extensive testing before they are qualified for use in TDF. They must appear suitable as candidates for machine testing and demonstrate high reliability, to preclude extensive negative impacts on TOF availability. Secono, the test schedules should be coordinated with the overall TDF startup and long-terin test phases to make maximum use of the testing times available. 
The preceeding discussions indicate a wide range of desirable test capabilities for TDF. Although specific TDF capabilities are dependent on many competing design issues, some key features important to the success of TUF as a test facility are:

- Steady-state, as opposed to pulsed, environment.

- Integrated neutron/heat-generation/magnetic conditions.

- Large area and volume relative to other high-flux/fluence 14-MeV neutron sources.

- Realistic fusion neutron spectrum.

Key TOF capabilities relative to testing requirements include $1.4 \mathrm{Niw} / \mathrm{m}^{2}$ wall loading at $25 \mathrm{~cm}$ raoius, $7 \mathrm{~m}^{2}$ of available test arr ( (two 1-m-long blanket regions, anci three long-term materials test regions), high short-term availability, if aesired, neutron flus combined with $10 \mathrm{~W} / \mathrm{cm}^{2}$ surface heat fiux.

With these capabilities, it is possible to compare TDF to other fusionrelevant neutron facilities. Table 3-65 compares the features of several existing and proposed test facilities. The machines considered include, in addition to the current TDF concept and current FMIT, FED, INTOR, and TASKA designs, proposed upgrades of TFTR (TFET-I, TFET-II, ano LITE), an MFTF-E upgrage, and a possible TDF upgrade (TDF-SP). The operational requirements for TFET-I are taken from the Princeton Plasma Physics Laboratory proposal. They incluae pulsed operation with 6-s pulses at a wall loading of $0.2 \mathrm{Mh} / \mathrm{m}^{2}$ once every $600 \mathrm{~s}$ for periods of severa] hours, and a total lifetime of 10,000 D-T shots. The capabilities of TFET-II and LITE are taken from information provided by Paul Reardon to Panel Three of the Magnetic Fusion Advisory Comittee on Juiy 29-30, 1982. We have assumed that TFET-II and LITE wit have the same fluence limit as TFET-I. The MFTF-B+ capabilities are from current stuaies underway at LLNL to examine the feasibility of operating MFTF-B in a D-T mode. In this mode, an upgrade would operate at a steady-state first wall flux of about $1 \mathrm{MW} / \mathrm{m}^{2}$ for continuous runs lasting as long as $10 \mathrm{hr}$. We estimate a facility availability range of 1 to $5 \%$ for this concept. Finaily, the TDF-SP concept represents a possible TDF improvement incorporating stable end plugs, which could give a first wall flux of from 2 to $3 \mathrm{MW} / \mathrm{m}^{2}$ with availabilities between 50 and $70 \%$. 
Table 3-65. Fusion neutron facilities.

\begin{tabular}{|c|c|c|c|c|}
\hline Facility & $\begin{array}{c}\text { Flux } \\
\left(n / \mathrm{cm}^{2}-s\right)\end{array}$ & $\begin{array}{l}\text { Duration } \\
\text { (s) }\end{array}$ & $\begin{array}{l}\text { Area } \\
\left(\mathrm{cm}^{2}\right)\end{array}$ & $\begin{array}{l}\text { Neutron } \\
\text { spectrum }\end{array}$ \\
\hline FMIT & $\begin{array}{r}5 \times 10^{14} \\
1.5 \times 10^{14}\end{array}$ & $\begin{array}{l}\text { SS } \\
\text { SS }\end{array}$ & $\begin{array}{l}100 \mathrm{~cm}^{3} \text { (vol) } \\
1000 \mathrm{~cm}^{3} \text { (vol) }\end{array}$ & $\begin{array}{l}\text { Nonfusion } \\
\text { Nonfusion }\end{array}$ \\
\hline TDF & $6-9 \times 10^{13}$ & SS & 70,000 & Fusion \\
\hline$T D F-S P$ & $9-13 \times 10^{13}$ & SS & 70,000 & Fusion \\
\hline TASKA & $7 \times 10^{13}$ & SS & 78,500 & Fusion \\
\hline INTOR & $5.8 \times 10^{13}$ & $100-200$ & 120,000 & Fusion \\
\hline MFTF-Upgràue & $4.4 \times 10^{13}$ & SS & 10,000 & Fusion \\
\hline FED & $2.2 \times 10^{13}$ & 100 & 120,000 & Fusion \\
\hline \multicolumn{5}{|c|}{ TFTR upgrades: } \\
\hline TFET-I & $0.9 \times 10^{13}$ & 6 & 100,000 & Fusion \\
\hline TFET-II & $4 \times 10^{13}$ & 10 & $\sim 180,000$ & Fusion \\
\hline LITE & $15 \times 10^{13}$ & 10 & $\sim 180,000$ & Fusion \\
\hline
\end{tabular}


From Table 3-65, we can see that TDF conbines a high flux with a large surface area, thereby allowing reasonably sized blanket modules to be tested, at the same time as a large number of small materials samples. However, these basic TDF characteristics must be used carefully in conjunction with blanket module design and test program planning to realize the maximum potential of the TDF concept. Thus, we will consider the previously discussed test requirements and their impact on the design and use of TDF test facilities.

The following characteristics are important with regaro to materials specimen testing within the constraints of the basic Tof concept:

- Because of its small radius, TOF is characterized by the rapid radial falloff of neutron flux and, therefore, of apa and helium production.

- Materials tests tend to be long-term relative to blanket moaule tests.

- Although many materials tests are passive, some have tenperature, stress, or other requirements that will require interconnactiuns with power or instrumentation.

- TUF will have about $10 \mathrm{~W} / \mathrm{cm}^{2}$ of particle flux $(100 \mathrm{eV}$ from the halo, ano $30 \mathrm{keV}$ from the plasma), which must be considered.

These characteristics lead to several design considerations. First, to maximize the test volume of useful materials in light of the rapid raoial falloff, maximum use must be made of all available surface area for testing. For materials tests that can be carried out over the full life of the machine, this means utilizing even relatively inaccessible areas near the first wall. For moderate-term tests, considering that blanket module testing will require more frequent changeout than will materials test specimens, materials specimens can be located in areas adjacent to blanket modules, accessible whenever a blanket module is removed. However, some space must be available to allow for penetrations for electrical interconnections with specific materials test specimens. Finally, shorter-term materials tests may be conaucted as part of the blanket nodule test program itself.

As seen in Figure 3-152, these requirenients result in the use of all space in the central cell region not already used for blanket modules. These areas, denoted by asterisks, will be accessible whenever the appropriate . blariket module is removed. Figure 3-153 shows a more detailea view and inoicates the access for cables or coolant channels running between the magnet 


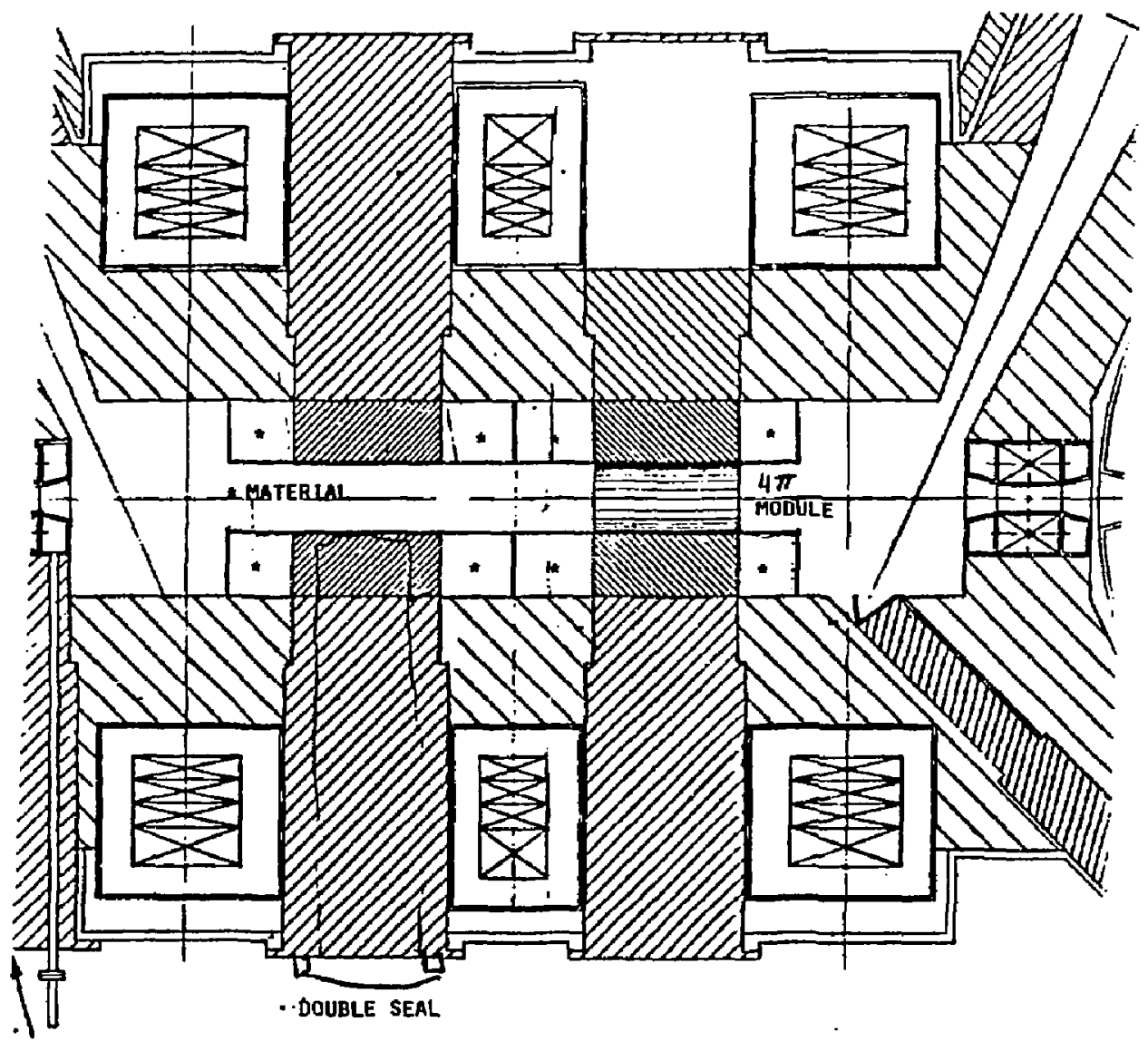

Figure j-152. TDF test configuration. 


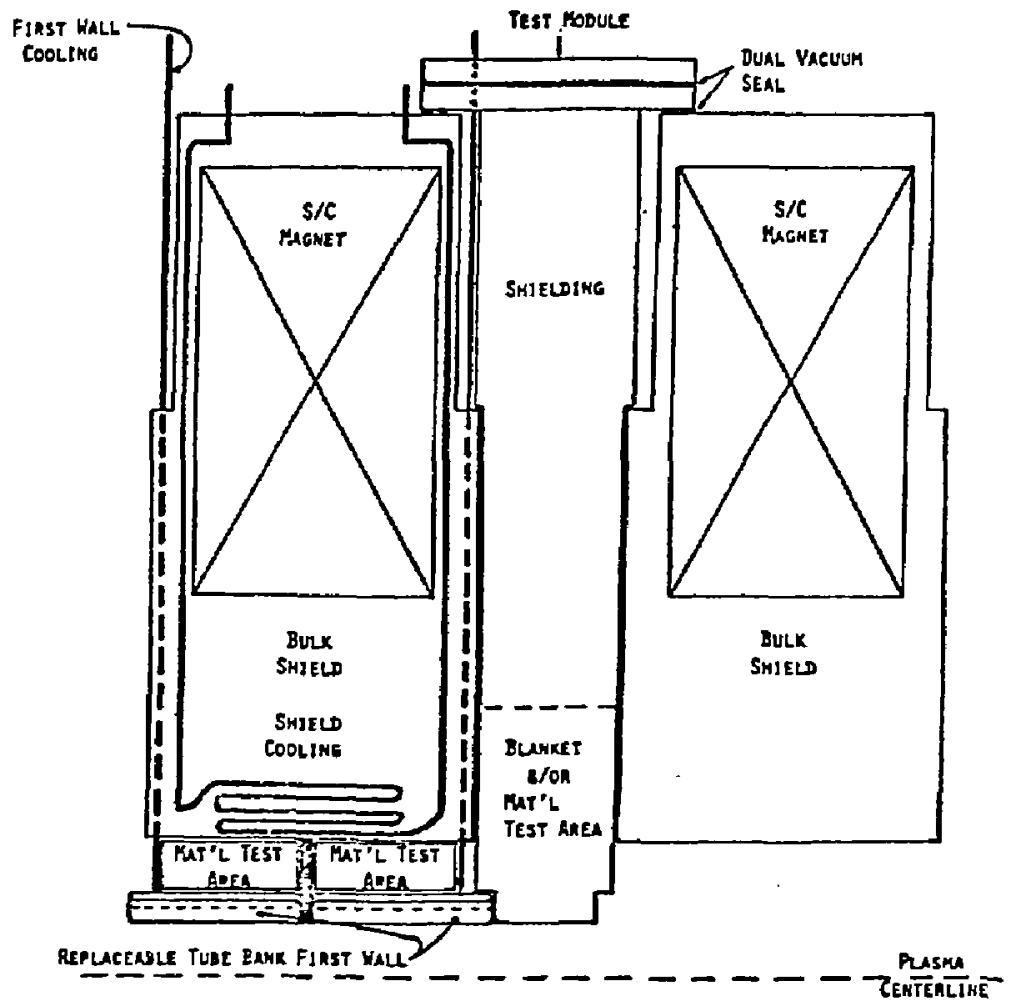

Figure 3-153. Detailed TOF test configuration. 
bulk shields and the blanket modules. It also shows the location of a replaceable tube-bank first wall. Although many materials samples wi]l require shielding from the plasma and halo neutral particle flux, this first wall will be renovable, to allow for testing of unprotected samples, as well as for maintenance and replacement.

It is also important to consider two other factors: wall loaoing and availability. over the long term, $5 r \%$ (or greater) availability is highly desirable to naximize fluence capabilities for end-of-iife testing. Altarnatively, the same effect can be obtained by increasing the ultimate wall loaving at a lower availability. In either case, short-term materials tests will also benefit because tests requiring lower fluence could be run more quickly; this would make the results available earlier and have a greater potential for impacting the choice of future materials to be tested. The shorter-term materials tests would also benefit from the quickest possible attainment of high ayailabilities early during the life of TDF. The key design considerations that result from these materiais test requirements are thus

(1) maximizing the attainable wall loading (up to $2 \mathrm{MW} / \mathrm{m}^{2}$ needed to attain about 100 dpa over the machine lifetime) and

(2) capitalizing on the small size and power of TOF and incorporating redundancies to realize a high availability over the machine lifetime.

Issues more relevant to the design of the materials test capsules and specimens themselves than to the design of TDF inciude temperature control, stress, pressure, instruntentation, etc. Although these issues must be addressed during the specific design of materials tests themselves, they must be pointed out here because they affect TOF design. For example, temperature control of materials specinens is expected to require temperatures constant to about 1 to $2 \%$ over a range of 50 to $700{ }^{\circ} \mathrm{C}$. This will entail both heating anc cooling and, therefore, require both electrical connections and piping and the pumping of coolants. Appropriate access must be provided for these lines and provisions made in case of failure, especially for pipe breaks. Finally, for both materials and blanket module tests, remote handling capabilities must be developed consistent with the testing requirements out lined here and with those determined later. 
blanket module test requirements place an entirely different set of constraints on TuF test facilities and test program considerations. The two basic requirements are short-term availability and turnaround times. The short-term availability requirenents are set by the following:

- Large numbers of tests will entail less than about $30 \mathrm{hr}$ of cont inuous testing.

- Many of these tests will tolerate 1 to 2 hr of downtime, especially early in the test period.

- A single hour of downtime late in the test period could necessitate a 4- to 5-hr increase in test length, especially near the end of a 30-hr test.

- Therefore, in determing machine availabilities, 1 to $2 \mathrm{hr}$ of downtime in 30 might be tolerable. However, a 4- to 5-hr downt imie (auring a 30-hr test) would probably necessitate a restart af ter allowing recovery to pre-test conditions.

- Shorter tests, although proportionately more stringent, should be more easily accomnooated.

Some considerations impacting turnaround times follow:

- To maximize total machine availability, turnaround times should be no longer than the requirea test times.

- Many tests need run only on the order of $30 \mathrm{hr}$.

- To maximize the usefulness of TOF for blanket module tests, mooules should be replaceable after 10 to $60 \mathrm{hr}$ in place.

- Therefore, turnaround times shoula be no Tonger than $60 \mathrm{hr}$.

These considerations impact the TDF cesign in two ways: (1) the blanket modules must be easily accessible, and (2) the vacuum seal design is a key aesign feature. The considerations resulted in a significant number of trade-offs in vacuum seal location, with a final design incorporating the features of seai locations both within and outside the test area. Gverall design considerations made an external seal highiy desirable (see Fig. 3-154). For blanket tests requiring rapid turnarouna, a blanket port concept with a dual vacuum seal at the top was devised. This concept allows a test module to be inserted into a port through the dual seal without breaking the overall machine vacuum; however, it requires a first wall vacuum seal in the blanket 


\section{NUCLEAR TESTING REQUIREMENTS AND FACILITY CAPABILITIES PEF RUN}

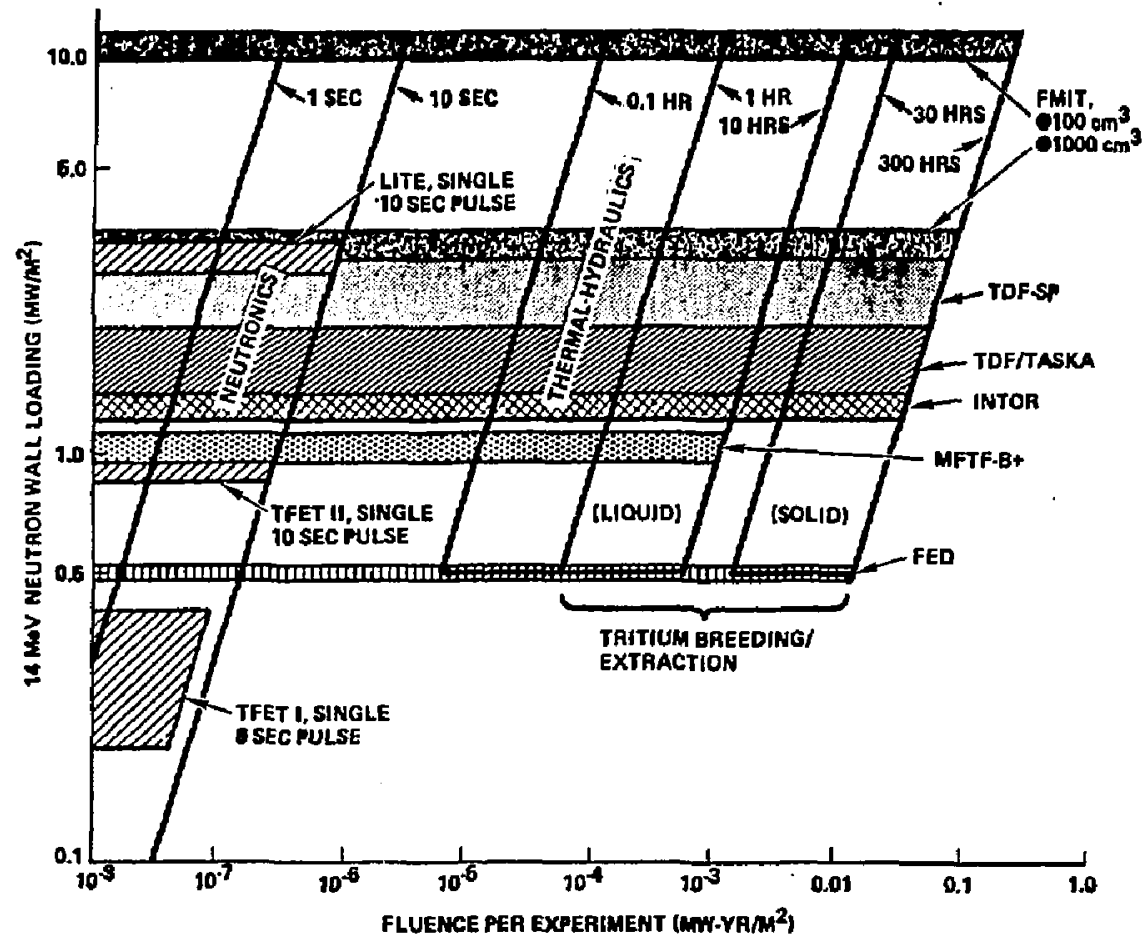

Figure 3-154. Neutron fluence requirements and capabilities per run. 
oort itself. Because this extra wall is not desirable for some blanket tests, doditional blanket modules will be designed to fit directly into the mocule test space with the port removed.

The resulting blanket module concept incorporates the following teatures:

- Two 360-deg module test areas, each with a useful width of about $1 \mathrm{~m}$.

- Circumferential mooule use, including (1) one 360-deg module, (2) one 180-aeg module and two smaller modules, and (3) three 120-deg moaules.

- An optional port concept to allow rapid changeout for short-term tests.

- An optional first wall to allow protected and unprotected module testing.

Two additional figures indicate how TDF performance matcnes the design requirements of the five categories described earlier and compares with the capabilities of several other fusion facilities. Figure 3-154 illustrates the wall loading and fluence requirements per test for the five test categories and is based on the data in Table 3-61. The TDF capabilities assume a wall loading of $1.4-2.0 \mathrm{mw} / \mathrm{m}^{2}$. Figure $3-155$ similarly plats wall loading ano total fluence over the machine lifetime. The large boxes in each figure represent approximate limits to the requirements for each of the five test categories, with specific machine capabilities superimposed over them. As can be seen, TDF and TASKA are the only machines that closely match almost all of the requirements; however, structural materials danage testing for prototype qualification coula be accomplished only with a higher wall loading (in the range 3 to 4 mil, m $^{2}$ ) or an extencied lifet ime machine with imcreased availability (up to $70 \%$ ).

\subsubsection{Key Technical Issues}

Two key items remain: the development of actual test plans and schedules and the gesign of specific materials test configurations and blanket test mooules. Both issues are, of course, closely related. Specifically, the test plans must be closeiy coordinated with the aesign capabilities of the blankat mocules themiselves ano with the capabilities and operating schedule of TDF. 


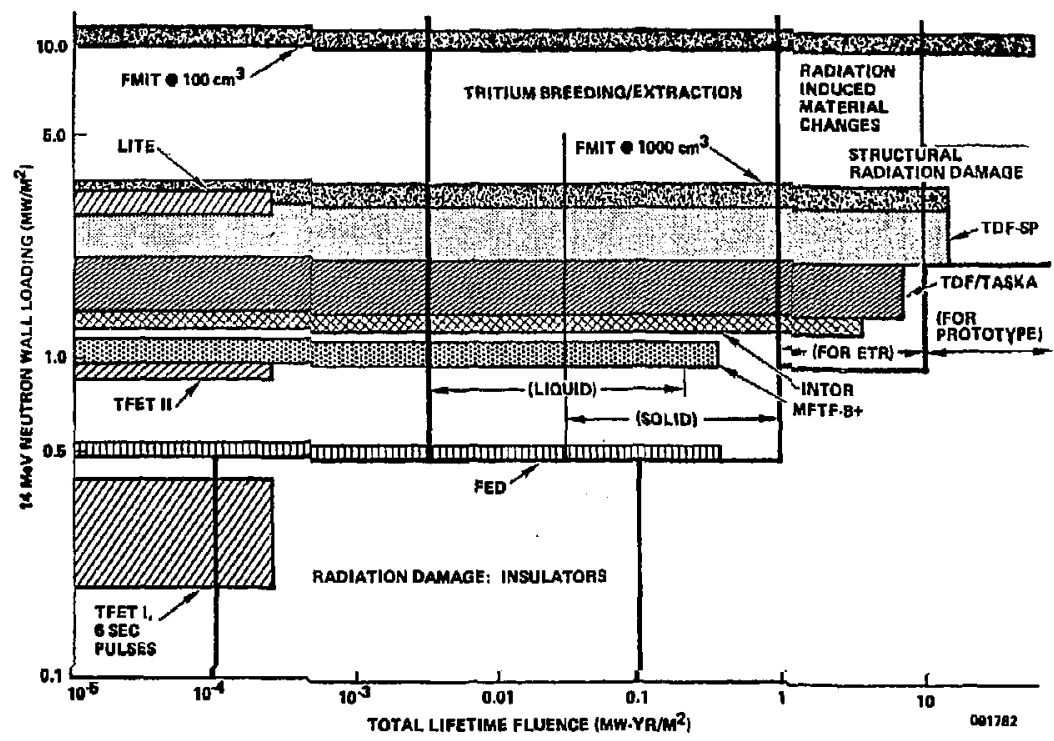

Figure 3-155. Total integrated neutron fluence requirements and capabilities. 
To develop appropriate test plans, we must match the test-length factors iscussed previousiy with the final TOF operating plan to ensure that tests are coordinated with expected machine availabilities and wall loading. This is also subject to the timing ana sequence requirements of the tesis themselves. In gtneral, however, it should be possible to mun many short-term tests (e.g., neutronics, thermal hydraulics) early in the TDF lifetime, when availabilities are expected to be low. In fact, a natural progression from many short-term tests and initial integrated blanket module tests to final long-term blanket qualification testing woula coordinate well with the expected increase in availability over the lifetime of TUF.

Finally, specific blanket modules must be designed on the Lasis of several factors. First, machine anc blanket designs aesirable for testing in IDF must be selected. This will almost certainly change with time, ano new desigus may become desirable for testing during the liretime of TOF. In addition, as early designs are tested in TOF, modifications or complete cesign changes may be indicated by test results. The requirements for each blanket concept must be considered in light of the various blanket test configurations available for TDF (90- to 360-deg circumferential module or blanket port). Further, since TOF is a small machine relative to a DEMO or prototype device, the issue of act-alike vs look-alike modules is important. Clearly, TOF caniogt test full-sized (or "look-alike") blanket modules; therefore, to ensure a test moaule that closely represents the expected performance of a full-scale moaule under actual operating conditions, accurate scaling is extremely important. For exanple, since heating profiles anc thermal hyoraulics are important test consioerations, the effects of geometrical scaling down of piping, flow channels, etc., must be carefully considerea. This requires scaling flow velocities to preserve Reynolds ano Prandtl liumbers in steady-state operation. Simulation of transient conditions will require detailed coniceration of many other factors.

As tests on preliminary blanket concepts are completed, final blanket concepts for long-term testing within TUF must be jeveloped on the basis of a careful screening of earlier rasults. In this way, maximum use can be mace of the TDF test facilities, with a minimal potential of aamage to either the modules or the TOF facilities themselves. 


\subsection{VACUUM PUMPING SUBSYSTEM SUMMARY}

The TDF vacuum pumping system is comprised of three types of pumps, each with a different function. (1) Deuterium, tritium, and impurities are pumped on LHe-cooled, $\mathrm{LN}_{2}$-shielded cryocondensing panels that are deployed within the main vacuum envelope of the eno cells and the neutral beamlines. (2) The helium gas resulting from the D-T fusion reactions is pumped on LHe-cooled, $\mathrm{LN}_{2}$-shielded cryosorption panels that are contained in small vessels appendeo to the end cells with isolation valves. The sorption material is argon gas. (3) The initial purpdown or vesse 1 "rough pumping" is accomplished by an external mechanical pumping systen that also pumps degassed effluent from the D-T and helium cryopumps to the external fuel cleanup systern.

\subsubsection{General Vacuum Requirements}

The general vacuum requirements for TDF include:

- Neutral censities.

- Neutral beam arift spaces.

- End cell regions.

- Continuous operation.

- Minimization of the D-T inventory within the vacuum envelope.

- Uelivery of pumped gases in a manner compatible with the fuel processing system.

- Compatibility with TUF environment and availability.

- Ease of maintenance.

- Installation/removal.

- Leak-checking/repair.

Because TDF is meant to run in the steacy-state mode, the purping system must also have that capability. The main cryocontensing (D-T pumps) pane1s are designed to be degassed in discrete, sequential groups while the remaining panels continue to operate unperturbed by the degassing process. Degassed effluent from the cryopanels passes through an internal (to the cryopanel) isolation valve, where it is collected on a much smaller LHe-coolea panel enclosed in a $\mathrm{LN}_{2}$-cooled gas-tight shroud. Then the isolation valve is closed, the collector panel is degassed, and the gas is discharged to the 
xternal mechanical pump and into the fuel cleanup system. The appended welium pumps operate in a similar manner; half of thes are open to the vessel and pumping while the other half are isolated from the vessel and degassing into the external, mechanical pumping system.

\section{$\therefore$ 9.2. Discussion}

For such a scheme to work, the installed pumping capacity must be greater than that needed to maintain the required system pressure. Tne excess capacity must degas in a continuous, sequential manner at a rate equal to the throughput of the reactor. Further, because the degassing flow rate niust be equal to the gas input and only a fraction of the total pumping system is oegassing at any time, the degassing process must take place at a pressure several oraers of magnitude higher than the required system pressure.

The quantity of gas accumulated on the cryopanels and collector panels (degassing pumps) during steady-state rperation (gas inventory in the vacuum vessel) is related to the gas throughput, degassing cycle time, excess capacity fraction, and gas leakage into the vessel during degassing. Assuming the degas leakage is negligible (pressure rise less than a factor of two), the gas inventory can be determined from

$I=Q t_{d}(3 / 2 \gamma-1) / \gamma-1$,

where $Q$ is the gas throughput, $t_{d}$ is the degassing cycle time, and $\gamma$ equals 1 plus the fraction of excess capacity.

A constraint on the regeneration of cryopanels for TDF (or any fusion reactor) is the amous of tritium allowed to accumulate on the panels. Although this amount has not been fixed for TDF, the objective is to make it the minimum practicable.

\subsubsection{Design}

The specific oesign requirements set by TDF physics for the end cell vacuumi pumping system were to maintain the neutral gas pressure at less than $5 \times 10^{-6}$ Torr at a gas flow rate of $124 \mathrm{Torr}-1$ iters/s for ceuterium, 
47.6 Torr-liters/s for tritium and 0.17 Torr-7iters/s for helium under steady-state conditions. Table 3-66 shows the significant parameters of the end celi vacuum pumping system.

The TDF continuous cryopumping system is based on a regenerable design developed and tested at LLNL. It can be regenerated without reintroducing an unacceptable portion of the pumpeo gas back into the high-vacuum space. A photograph of the prototype tested at LLNL is shown in Fig. 3-156. The pump features a set of LHe-cooled panels for condensing the ceuterium. Thermal shielding for the panels is provided by a series of " $Z$ "-shaped double chevrons that, when open; allow deuterjum to pass to the condensing panels from two sides. For regeneration, closed louvers isolate the condensing surfaces from the high-vacuum space. The liquid helium is then purged from the condensing panels, releasing the condensed deuterium. The deuterium flows through an isolation value between the closed panel and the LHe-cooled collector purip, which can be periodically degassed to the exterior mechanical pumping system.

Figure 3-157 shows how this regenerable pumping technique is applied to TDF. The 2-by-4-m panel shown is one of 72 , haif of which are deployed in each end cell. A degassing unit is comprised of six panels, each having an isolation valve leading to a common collector pump shroud. The louver actuator is also common so that the six panels can be simultaneousiy negassea into their collector pump. A six-panel assembly is shown in Fig. 3-158. This assembly is designed as a self-contained unit with all of its service penetrations mounted on its vacuum cover plate. Thus, it can be installed, operated, anc removed as a separate entity. Uf the 12 six-panel units for pumping deuterium and tritium in the end cells, 10 are always pumping while 2 are degassing.

Figure 3-159 shows the helium-appendage pumping installation in the end cells. There are four separata appendage pairs ( $\theta$ pumps), two pairs in each end cell. Each punip contains two argon-cryotrapping panels having a total pumping area of $3.1 \mathrm{~m}^{2}$. Each pair share a common flapper valve that alternately opens and closes the 1.3-m-diam opening to the appended vessels, allowing four to pump while the other four are degassing.

Figure 3-160 shows the details of the helium-argon cryotrapping pane?. The louvers on these panels are fixed because the flapper valve serves to isolate them during the degassing cycle. 
Table 3-66. End cell vacuum parameters.

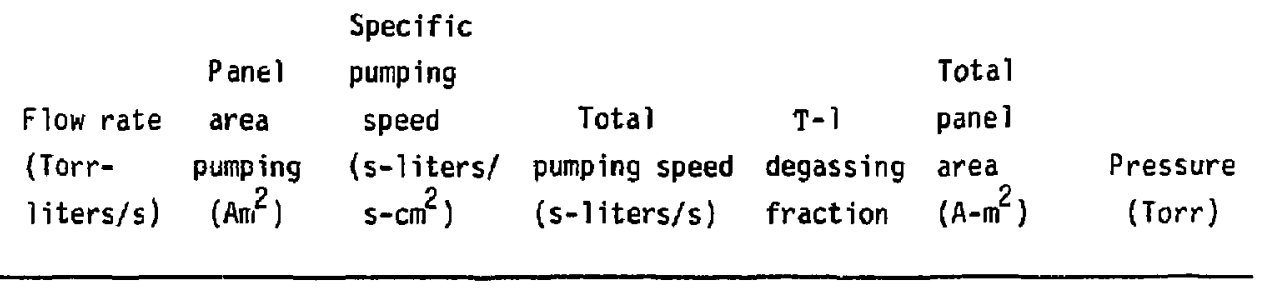

\begin{tabular}{lllllllll}
\hline & 47.6 & 960 & 6.4 & $6.1 \times 10^{7}$ & 0.2 & 1152 & $7.8 \times 10^{-7}$ \\
\hline
\end{tabular}

\begin{tabular}{lllllllll} 
D $\quad 124$ & 960 & 7.75 & $7.44 \times 10^{7}$ & 0.2 & 1152 & $1.7 \times 10^{-6}$ \\
\hline
\end{tabular}

$\begin{array}{rrrrrrr}\text { He } \quad 0.17 & 28.4^{\mathrm{a}} & 3.2^{\mathrm{a}} & 9 \times 10^{5 \mathrm{a}} & 1 & 56.8^{\mathrm{a}} & \cdots \\ 5.31^{\mathrm{b}} & 11.1^{\mathrm{b}} & 5.9 \times 10^{5 \mathrm{~b}} & 1 & 10.62^{\mathrm{b}} & 2.9 \times 10^{-7} \\ & & & \end{array}$

Cryopanel area.

$\mathrm{b}_{\text {Appended pump-entrance area. }}$ 


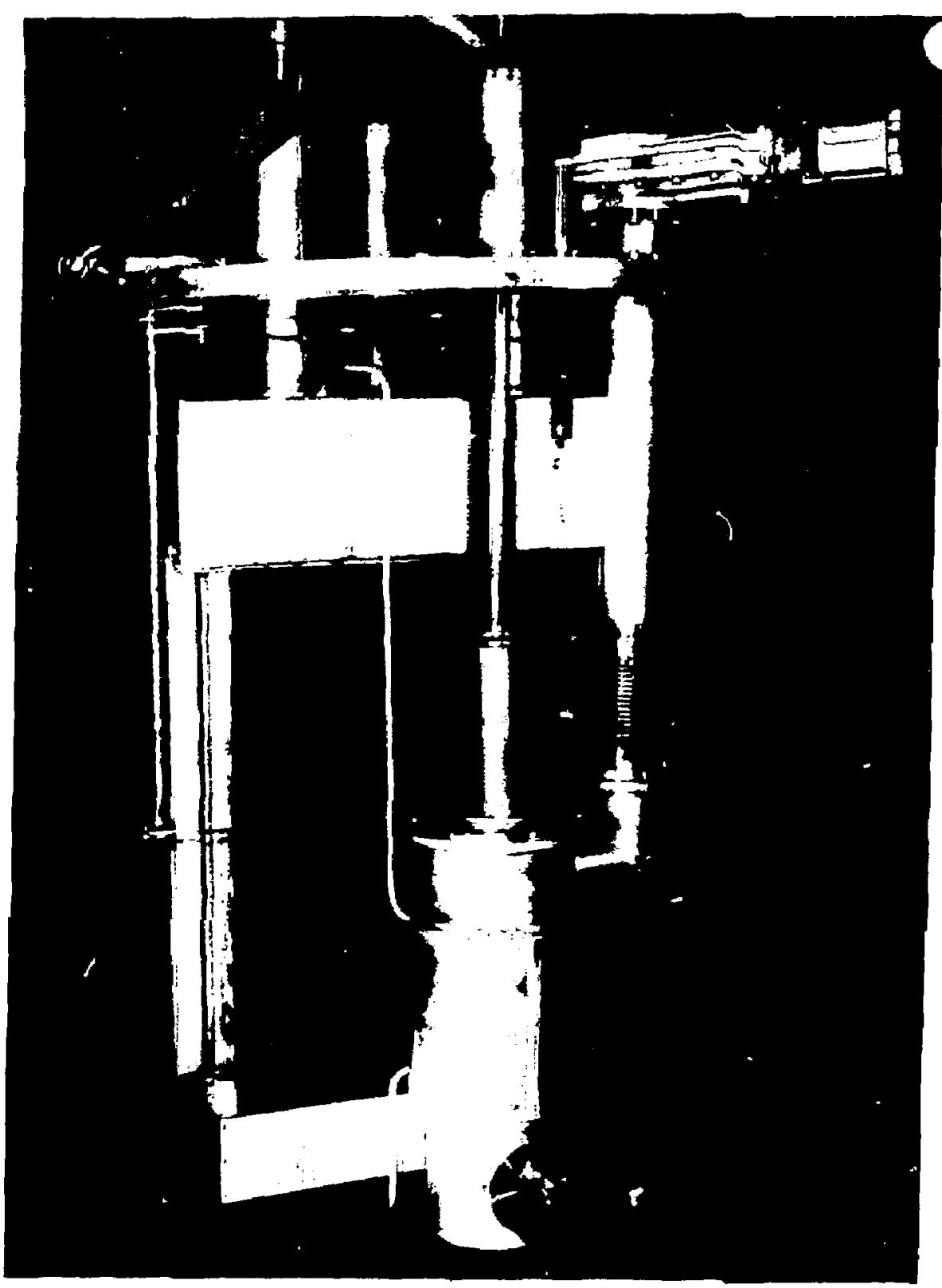

Figure 3-156. Prototype of the continuous cryopumping system tested at LLNL. 

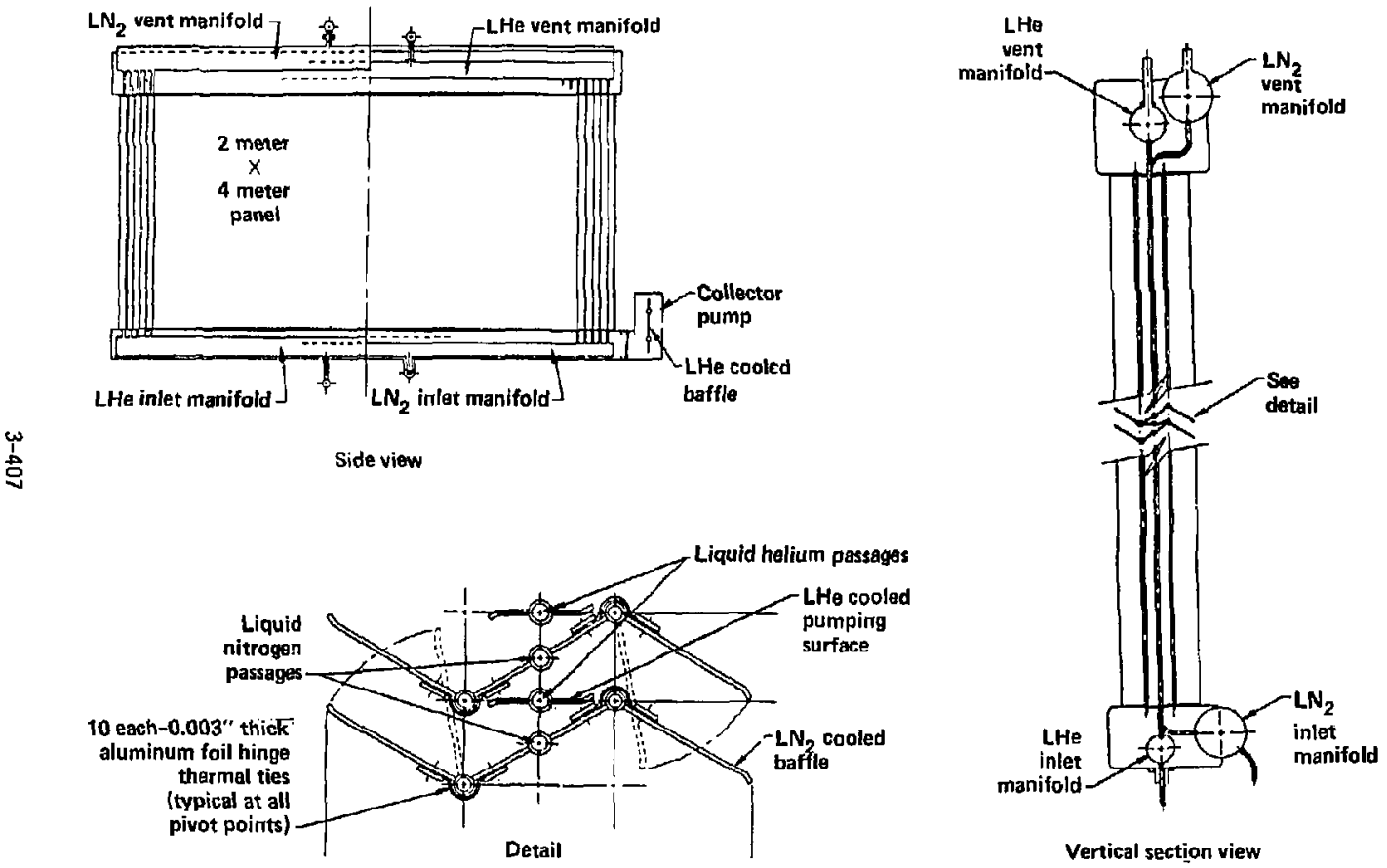

Vertical section view

Figure 3-157. Regenerable cryopumping panel concept for TDF. 


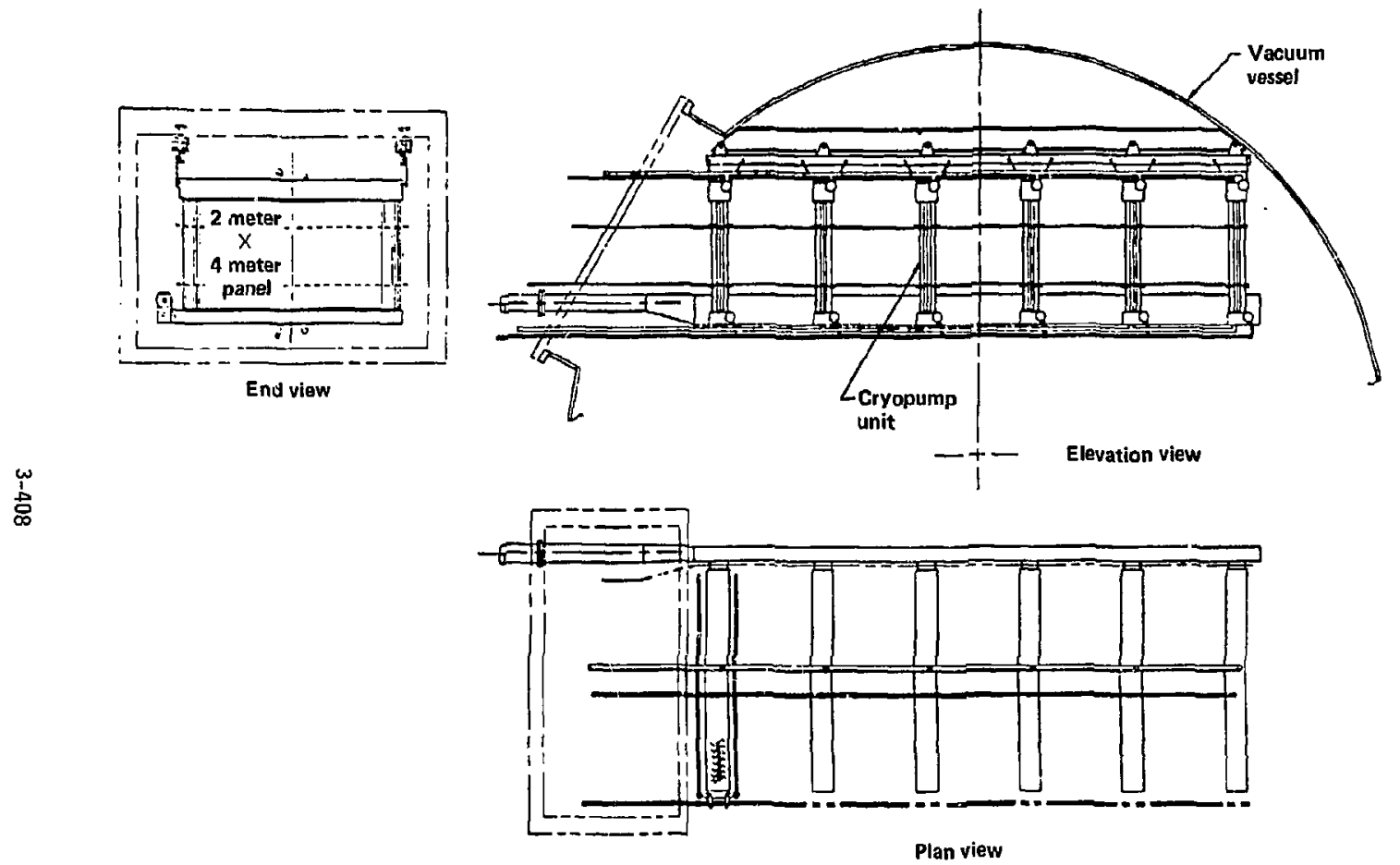

Figure 3-758. One of 12 six-panel degassing assemblies (36 panels in each end cell for a total of 72 ). 


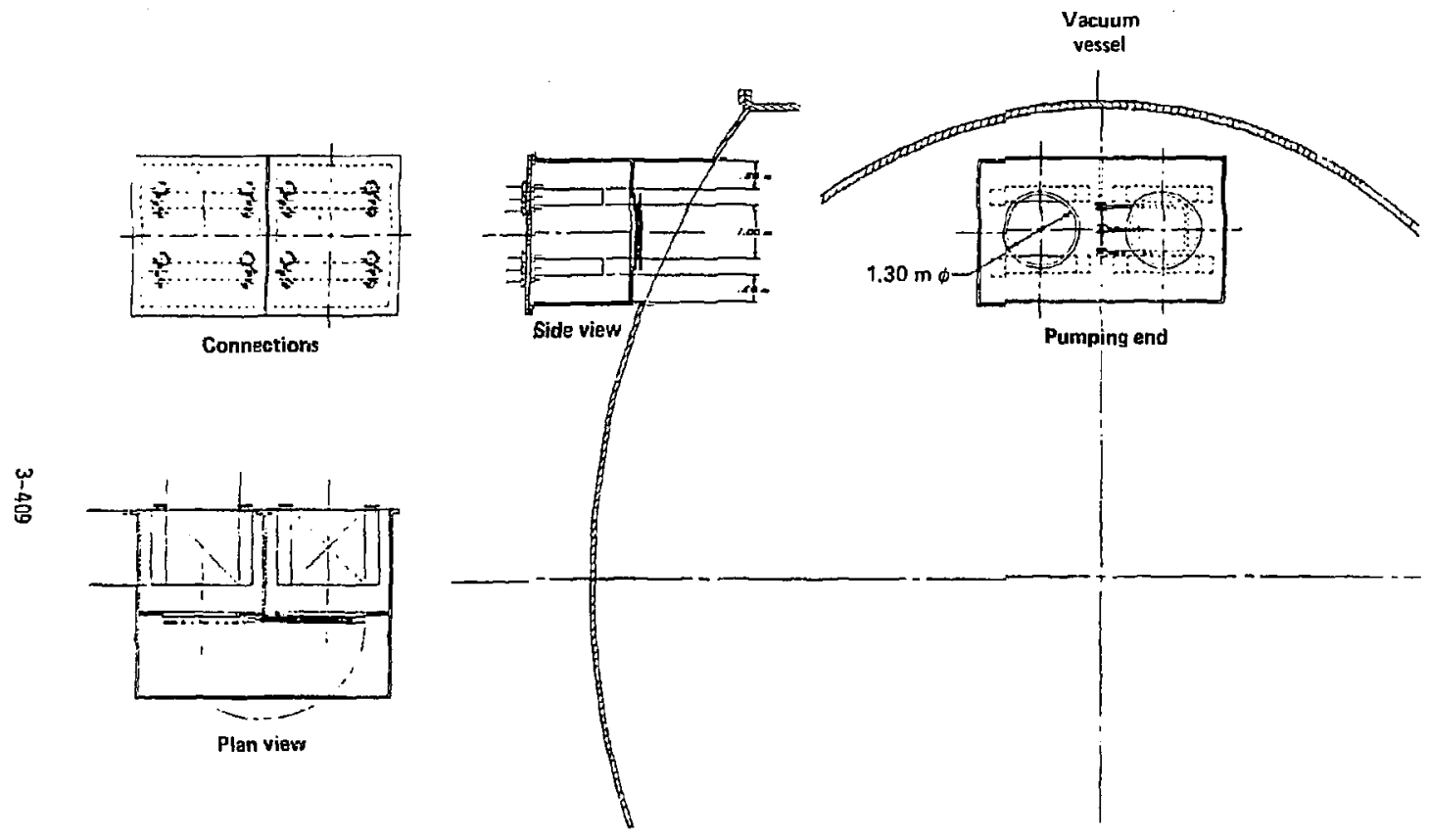

Figure 3-150. Heisum-appendage pumping instaliztion in the end cel3s. There are four appendage pairs (8 pumps), two pair in each end cell. 

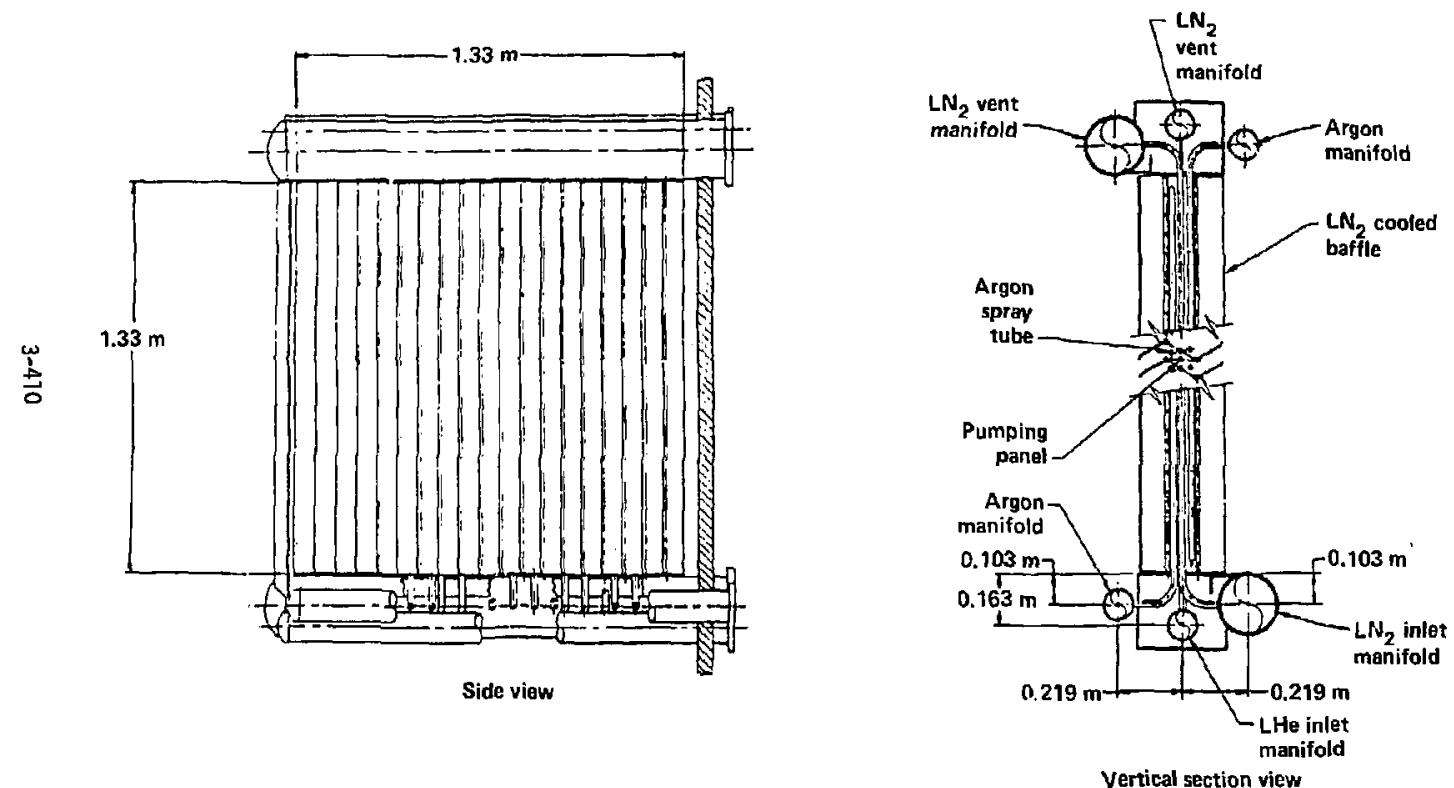

Figure 3-160. Details of the helium-argon cryotrapping panel. $\therefore$ 
The LHe supply and return circuits for the entire cryopumping system, as we]) as for the $L_{2}$ supply and return circuits, will operate by natural convection in the manner of MFTF-B. ${ }^{27}$ In addition to the LHe supply and return circuits to the cryopanels, there will be a warm helium gas system for purging Lhe from the panels auring degassing.

The cryopumps for the neutral beam injectors will operate essentialiy the same as the end cell pumps. However, they will be configured djfferently to conform with neutral-beam injection design constraints (see Section 3.2).

The operational characteristics of the ena cell continuous cryopump system can be analyzed as follows:

$Q=$ reacEor throughput $T_{2}+\sigma_{2}$ in Torr-liters/s.

$P_{a}=$ average allawable reactor pressure in Torr.

$S=Q / P_{a}=S A$ - required pumping speed in liters $/ s$, where $s=$ the specific pumping speed in liters $/ \mathrm{s}-\mathrm{cm}^{2}$ and $A=$ pumping area required to yiela $s-\operatorname{con}^{2}$.

$\gamma=$ total installed pumping area in $\mathrm{cm}^{2}$.

$A(r-1)=\operatorname{area}\left(\mathrm{cm}^{2}\right)$ of pump available for degassing at any given time.

$\Gamma / \Gamma-1=$ ratio of installed pump area to avainable degassing area.

$T_{c}=$ total cycle time of an a $(r-1)$ - pump area (pumping time + degassing $t$ ime) in seconds.

$T_{d}=$ degassing time of an $A(\gamma-1)$ in pump-area in seconds.

Because the gas flow into the reactor $Q$ must equal the gas flow out, 
$Q=Q_{\text {out }}$ or $S A P_{a}=S_{d} P_{d}$,

where $S_{d}$ is the degassing pump speed (]iters/s) and $P_{d}$ is the degassing pump pressure (Torr). Further, the amount of gas degassed during the time $t_{d}$ must equal the amount of gas pumped during the time $\left(t_{c}-t_{d}\right)$ for a given $A(\gamma-1)$ pump area and

$S_{0} P_{d} t_{d}=S A(r-1) P_{d}\left(t_{c}-t_{d}\right)$, or

$\gamma=t_{c} /\left(t_{c}-t_{d}\right)$

Summing up all the gas in the panels at any given time (the pumping panels inventory) as a function of yielas,

$T_{p}=\operatorname{sAP}{ }_{a} / 2\left(t_{c}-t_{d}\right)$ or $I_{p}=Q / 2\left(t_{c}\right)$.

Finally, since the inventory in the collector pumps for any a( $\gamma-1)$ degassing unit would equal the gas collecteo on that unit during its punping time:

$I_{C}=Q\left(t_{C}-y_{d}\right) /(\gamma / \gamma-1)-1=Q t_{d}$.

Operation of the LLNL prototype, regenerable, continuous cryopurri shows that 10 minutes is a reasonable time for degassing a cryopump and returning it to service. Thus, if $t_{a}$ is set at $600 \mathrm{~s}$, the inventory of gas in the pumping system can be plotted as a function of the excess capacity $(\gamma-1)$, where

$I_{\text {tot }}+I_{p}+I_{c}=Q t_{d}(3 / 2 \gamma-1) /(Y-1)$.

Figure 3-161 shows a plot of tritium inventory against exces s capacity for the TLF end-ce 11 pumping system. The $20 \%$ excess capacity set for TOF gives an end cell inventory of $40.3 \mathrm{~g}$ for tritium. Because the tritium source originates in the central cell neutral beams and the source's gas efficiency is $n 30 \%$, tritium inventory in the neutral beam cryopumps is twice that in 


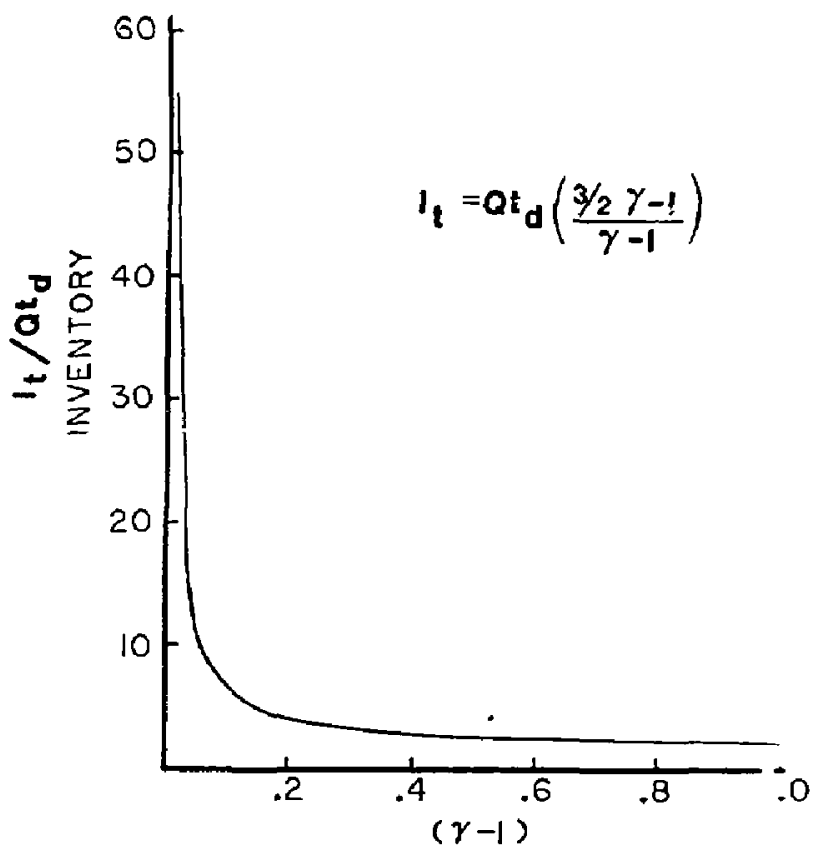

EXCESS CAPACITY

Figure 3-161. Plot of tr a :um inventory vs excess capacity for the TDF end cell purnping system. 
the end cells. Thus, the total tritium inventory in the TDF vacuum envelope is $3 \times 40.3=121 \mathrm{~g}$.

The cryogen requirements for the end cells were estimated as follows:

- Liquid-helium-cooled heat load due thermal raciation:

$A=1152 \mathrm{~m}^{2}$ of panels $+40 \%$ for auxiliary systems $=1612 \mathrm{~m}^{2}$. Effective emissivity between $L_{2}$ cooled shield and LHe-cooled surfaces is 20.4 , allowing for long-term degradation.

$$
\begin{aligned}
Q_{R} & ={ }_{A \sigma E} \quad T_{L N_{2}}^{4}-T_{L H e}^{4} \\
Q_{R} & =\left(1612 \times 10^{4}\right)\left(5.67 \times 10^{-12}\right)(0.4)(\times 4 / 80-\times 4 / 4.5) \\
& =1.5 \times 10^{3} \mathrm{~W} \\
& =1.5 \mathrm{~kW} .
\end{aligned}
$$

- Neutron heating of LHe-cooled structures:

Volume of aluminum $-3.75 \times 10^{6} \mathrm{cril}^{3}$. Neutron heating $-0.4 \times$ $10^{-3} \times 3.75 \times 10^{6}=1.5 \mathrm{~kW}$.

- Liquid helium loss due to degassing purge gas:

Estimated at $21.5 \mathrm{~kW} . Q=Q_{R}+Q_{N}+Q_{d}=4.5 \mathrm{~kW}=6500$

liters/hr.

Estimating $\mathrm{LN}_{2}$ consump.ion, using a thernal radiation emissivity of 0.7 and the same specific neutron heating but no degassing losses, gives

$$
Q_{L N_{2}}=585 \mathrm{~kW}-11,700 \text { liters } / \mathrm{hr} \text {. }
$$

The external vacuum pumping system will be used to evacuate the vacuum chamber and to transfer the gas from the collector pumps to the fuel cleanup systen. Designed for tritiun service, it will have an oil-free scroll-type pump, suitable manifolaing, and valving to both "rough pump" the vessel and transfer the collector pump gas.

The scroll pump will have a capacity of 560 liters/s, with a base or blank-aff pressure of less than $10^{-2}$ Torr. Assuming a vacuum chamber volume 
of $6 \times 10^{6}$ liters, the rumpdown would be accomplished as follows: From atmosphere to 0.5 Torr, the scroll pump would take approximately $24 \mathrm{hr}$.

$t=V / s(f)$ in seconas,

where $V=$ is the vessel volume, $s$ is the displacement speeo of the pump, and $f$ is the pumpdown factor from 760 to 0.5 Torr.

$t=8.1\left(6 \times 10^{6} / 580\right)=86,786 \mathrm{~s}=24 \mathrm{hr}$.

From 0.5 to $10^{-4}$ Torr, one or several helium cryosorption pumps could be made available.

$t=v / s\left[\ln r\left(0.5 / 10^{-4}\right]\right.$,

where $s=10^{5}$ iiters/s (the air speed of one helium cryosorption pump) and $v=2 \times 10^{6}$ ititers.

$t=\left(6 \times 10^{6} / 10^{5}\right) \ln 5000=500 \mathrm{~s}=0.14 \mathrm{hr}$.

Thus, the pumpdown time would be about $25 \mathrm{hr}$.

Key Technical Issues. Two key technical issues require further investigation:

- Panel leakage ouring degassing.

- Degassing characteristics of argon adsorbent loaded with helium and D-T.

The leakage of the LLliL prototype panel appears to be on the order of 25 to $30 \%$ of the gas pumpea. No attempt was made to optimize this test panel for leakage; the point was to deternine operational characteristics and concept feasibility, both of which appear to be acceptable.

An acceptable leakage level can be set by noting that the allowable total pressure in the end cells. (fron physics) is $5 \times 10^{-6}$ Torr and the design pressure is $2.77 \times 10^{-6}$ Torr $\left(7.8 \times 10^{-7} \mathrm{~T}_{2}, 1.7 \times 10^{-6} \mathrm{D}_{2}\right.$, and $\left.2.9 \times 10^{-7} \mathrm{He}\right)$. There will be no leakage during degassing of the helium pumps, but there will be $D_{2}$ and $T_{2}$ leakage during degassing of the six 
panel units in the end cell $0-T$ pumping system and in the NBI pumping system. Because we can increase the totat pressure by $\left(5 \times 10^{-6}-2.77 \times 10^{-6}\right)$ or $2.23 \times 10^{-6}$ to $5 \times 10^{-6}$, the sum of the partial pressure of $T_{2}$ and $D_{2}$ can be:

$P_{T_{2}}+P_{D_{2}}=P_{a}-P_{H e}=\left(5 \times 10^{-6}-2.9 \times 10^{-7}\right)$

or

$P_{T_{2}}+P_{D_{2}}=4.7 \times 10^{-6}$ Torr.

From this information, we can determine worst-case degassing leakage for the TDF end cell pumping system.

The total U-T gas flow to the end-cell pumps includes plasma fan flow, $\mathrm{Q}_{\mathrm{D}_{2 \mathrm{P}}}$ and $\mathrm{Q}_{\mathrm{T}_{2 \mathrm{P}}}$, plus the panel-degass leakage, $\mathrm{Q}_{\mathrm{L}_{2} \ell^{\mathrm{l}}}$ and $\mathrm{Q}_{\mathrm{T}_{2}}$.

$Q_{D_{2}}+{ }^{Q} T_{2}=Q_{U_{2 P}}+Q_{D_{2} \ell}+{ }_{T} Q_{2 P}+{ }_{T} Q_{2}$

and the total $0-7$ pressure

$P_{D T}=P_{D_{2}}+P_{T_{2}}=\frac{{ }_{D_{2}}}{S_{D_{2}}}+\frac{{ }^{Q_{T}}}{S_{T_{2}}}$,

where S/D/2 ano S/T/2 are total pumping speeds for deuterium and tritium, respectively. From Table 3-66, we know that

$\frac{\mathrm{Q}_{\mathrm{D}_{2}}}{\mathrm{Q}_{\mathrm{T}_{2}}}=\frac{124}{47.6}=2.6$

ano

$\frac{\mathrm{S}_{\mathrm{D}_{2}}}{\mathrm{~S}_{\mathrm{T}_{2}}}=1.2$

ano, from above,

$P_{D_{2}}+P_{T_{2}}=4.7 \times 10^{-6}$ Torr. 
Solving for $Q_{\mathrm{D}_{2}}$ and $Q_{\mathrm{T}_{2}}$, we get

$4_{2}=235.4$ Torr-1iters/s

ano

$\mathrm{Q}_{\mathrm{T}_{2}}=90.53$ Torr-1iters/s.

The $\mathrm{D}_{2}$ accumulated ( $W$ Torr-liters) on two six-panel pumping-degassing units:

$W=\frac{{ }^{2 Q} U_{2}}{10} T_{c}-T_{u}+\left(\frac{2 O_{2 R_{2}} t_{t}}{10} \frac{t}{10}\right) 5$.

The first term is the $D_{2}$ gas accumulated on two six-panel units in one pumping cycle because of the flow from the olasma fans. The second term is $\mathrm{D}_{2}$ gas accumulated on the same two units as a result of degas leakage from

the five pair of six-panel units that degas during the $\left(T_{c}-T_{d}\right)$ pumping time. The time to transfer the gas from the panels to the collector pump (during which tinie leakage occurs) is $\left(t_{t}\right)$, which was determined on the LLML prototype pump. Thus,

$w=2\left[\frac{124(3600-600)}{10}\right]+\left[\frac{(2)(111.4)(30)}{10}\right] 5$

and

$W=7.77 \times 10^{4}$ Torr-7iters $D_{2}$.

The leakage per pair of six-panel units, then, is

$L=\frac{2}{10} Q_{D_{2}{ }^{t}}{ }^{t}=\frac{2}{10}(111.4)(30)=668.4$ Torr-liters.

The percent leakage to maintain the peak pressure at less than $5 \times 10^{-6}$ Torr is

$\frac{L}{W}=\frac{668.4}{77700} \cong 1 \%$.

While it may not be possible to achieve such a low leakage rate for a cont inuous regenerable pumping system, it can be closely approached by minimizing the leakage conductance. This can be done by clase-fitting extruded lcuvers and maximizing the collector pumping speed. Further the 
probability of the tweive 4-m $\times$ 2-m panels, in a pair of six-panel degassing units, transferring their gas load to the collector pumps simultaneously is very low. Considering this, the time-averaged peak pressure due to aegas leakage would be much lower, probabiy 5 to $10 \%$.

The second technical issue--the degassing characteristic of an argon-adsorbent helium pump--is simply a matter of gaining the necessary experience. 
?10. PARTICLE UUMPS

3.10.1. Overview

The particle dumps must absorb about $100 \mathrm{MW}$ of power, which is generated by the jon sources. This power is adsorbed by the apertures, the ion or neutral dumps, or the plasma ena aumps. The beamline section of this report aescribes the actual location and configuration of the ion oumps and the apertures. This section discusses the basic particle dump oesign, including the neutral and plasma end oumps.

The neutral dumps have a tested configuration as a baseline design. It is shown by Fig. 3-162. A plate of Amzirc (LriC copper, copper with $0.2 \%$ zirconium) has machined fins and a backing plate brazeo onto the same siae. This provides a channel for the water coolant. Nucleate boiling takes place between the fins with the coolant continuously mixing water at the $f$ in tip. This process allows very high heat transfer. The basic oesign has been tested to $2 \mathrm{~kW} / \mathrm{cm}^{2}$ over a panel area $20 \times 20 \mathrm{~cm}$. The design heat flux for Tur is 1 $\mathrm{kH} / \mathrm{cml}^{2}$. The low flux is required to maintain a life of about 0.5 full-power years. The front surface is thicker than that tested, because material is removed by sputtering. Figure 3-163 shows panels of this design assembled onto a frame. This subassembly is inserted into the central cell.

The plasma end dumps use a much simpler and cheaper technology than that of the neutral dumps. Two designs are proposed. For heat flux below $700 \mathrm{~W} / \mathrm{cm}^{2}$, the panel material is stainless steel. For heat flux above 100 to $300 \mathrm{~W} / \mathrm{cm}^{2}$, the material required is TZM (made of titanium, zirconium, and moiybdenum). As Fig. 3-164 shows, the power density at the center of the plasma $f a n$ is $350 \mathrm{k} / \mathrm{sm}^{2}$ at a location $28 \mathrm{~m}$ from the machine's center. This power density falls off quickly further from the center. As Fig. 3-165 shows, the panel is constructed of a corrugated sheet welded onto a flat sheet. This forms several channels for the coolant. The surface facing the plasma has enough thickness to allow for sputtering. 


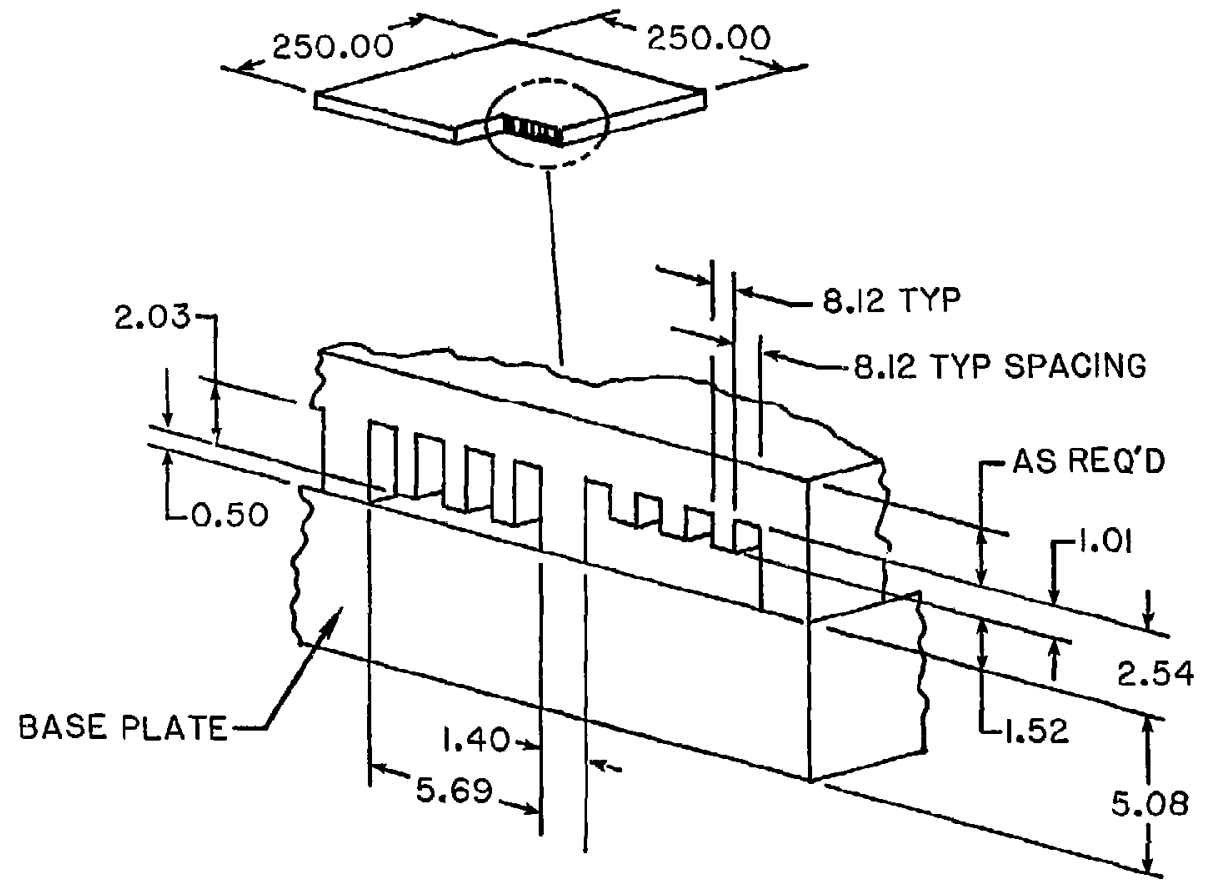

DIMENSIONS IN MILLIMETERS

Figure 3-762. Baseline panel design. 


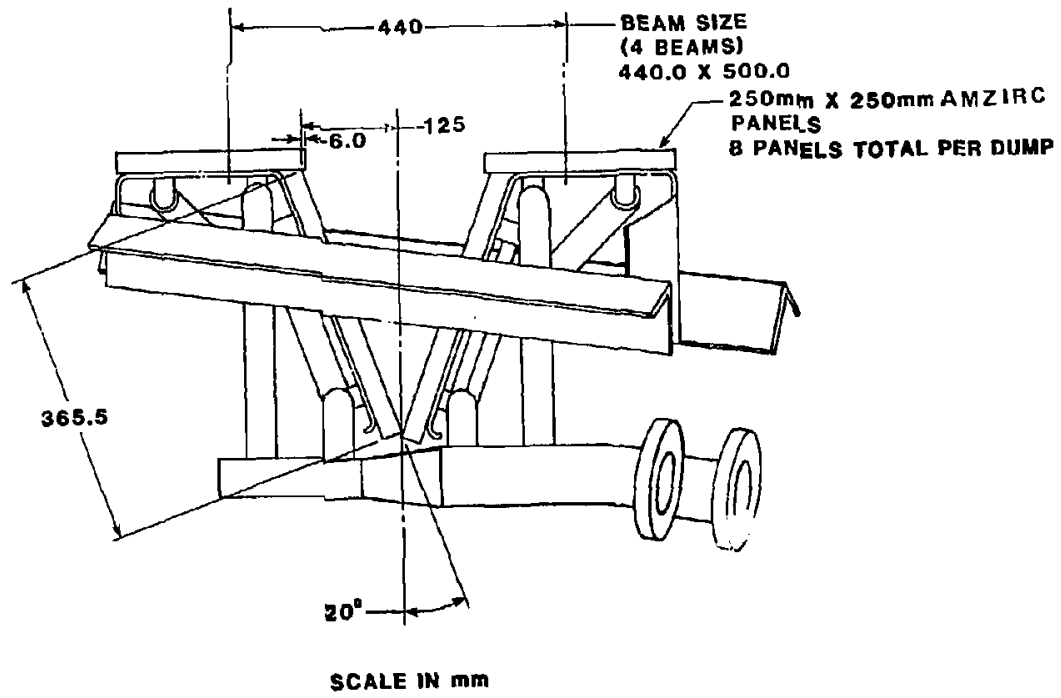

Figure 3-163. Uump panei assembly. 


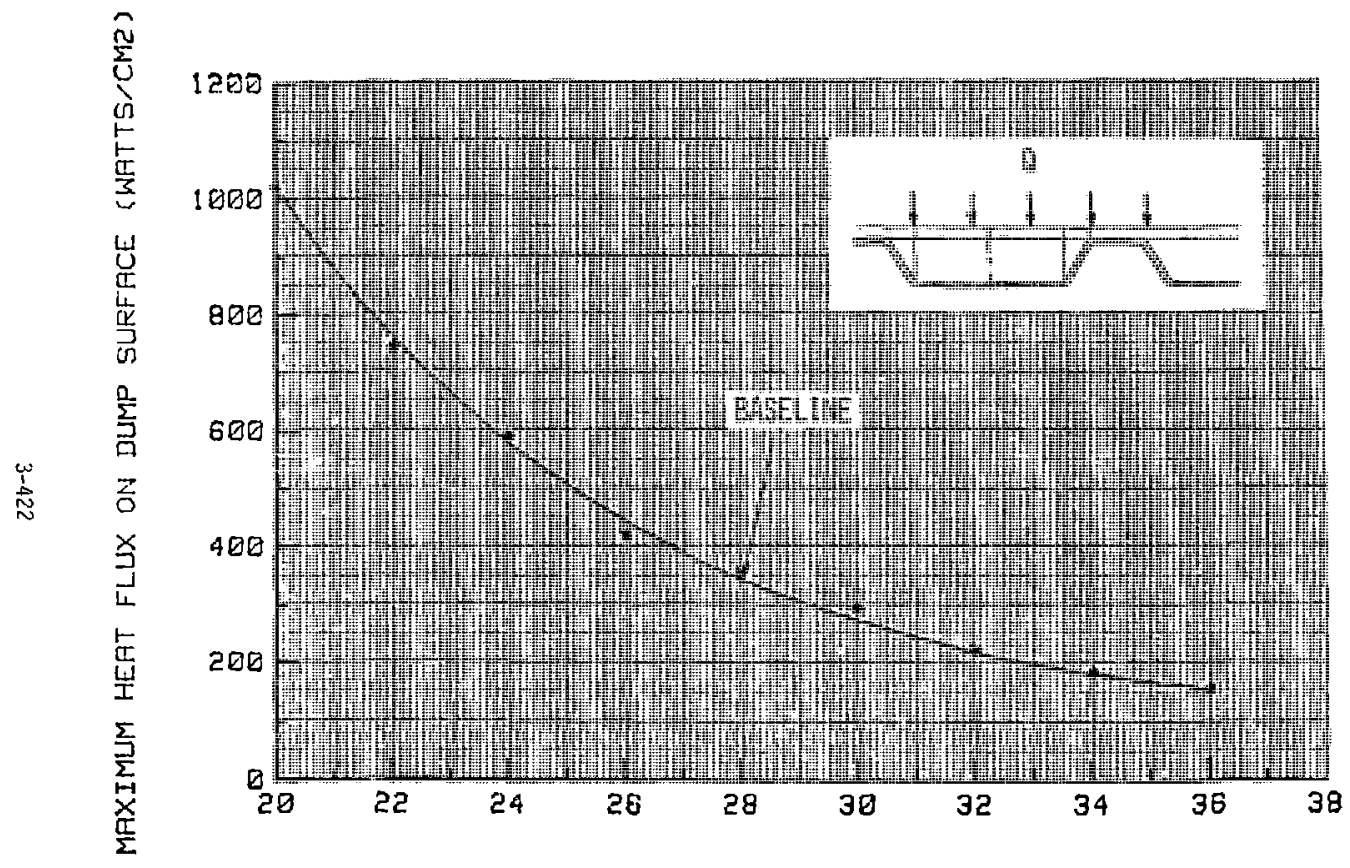

Figure 3-164. Maximum heat $f 7 u x$ on plasma end dump surface at various dump locations. 


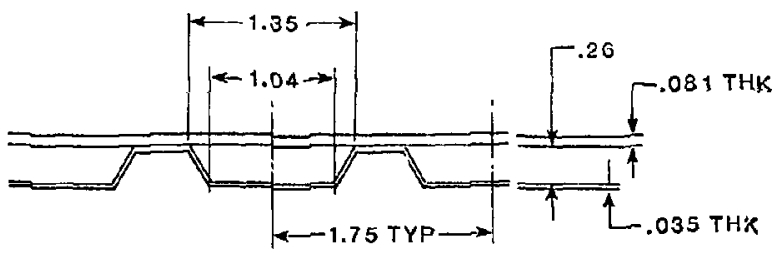

Sec $A \cdot A$

TRANVERSE

SUPPORTS

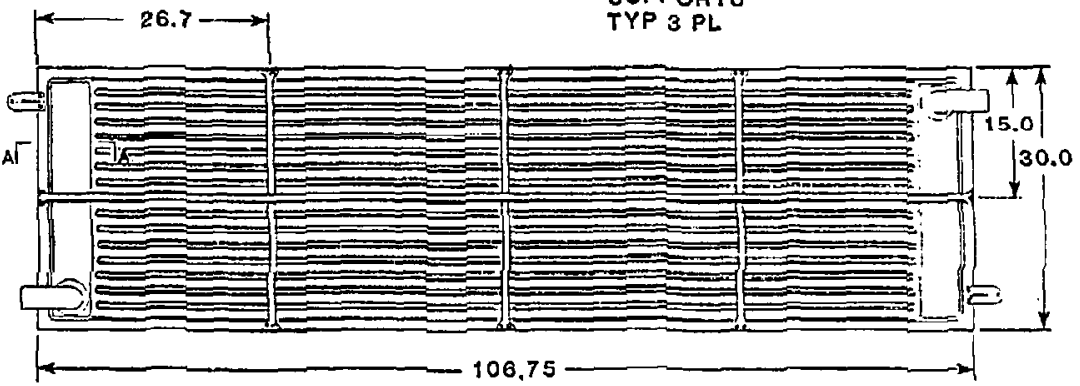

DIMENSIONS IN INCHES

Figure 3-165. Basic end dump panel. 


\subsubsection{Requirements}

The particle dumps must absorb the power generated by the ion sources either as an aperture, ion or neutral oump surface, or at the plasma end oump. The neutral dumps must absorb about $30 \mathrm{MW}$ of deuterium or tritium particles with an average energy of $60 \mathrm{keV}$. We chose the angle of incidence of the incoming particles with respect to the pump surface to limit the power flux to $1 \mathrm{kw} / \mathrm{cm}^{2}$. This limit is due to the large amount of sputtering expected, which requires a thick surface on the dunips with a corresponding temperature increase at the front surface. The life of 0.5 ful1-power years is a requirement for both fatigue and sputtering :ife. The dump design should not require development, but shoula be within the state of the art for high heat fiux absorption. We considered coolant requirements for minimizing the total system costs.

\subsubsection{Neutral Beam Dump Design}

Design. Our design is based on a study by MicDonnell Doiglas under contract to the Lawrence Berkeley Laboratory (LBL) ${ }^{38}$ Several materials for a front surface were considered in this stuay: OFHC copper, Amzirc, tungsten, and molybcienum. Amzirc was chosen due to its higher conduct,ivity and strength than $O F H C$ copper at the expected operating temperature. A $20 \times 20 \mathrm{~cm}$ panel was tested on a beamline at LBL and performed well for at least 600 shots at 2 $\mathrm{kH} / \mathrm{cm}^{2}$. For IDF, the requirement for a long life has great?y affected thermal requirements. A General Dynamics study recomendeo that the curve (Fig. 3-166) of sputtering rate angle of incidence be used for life calculation. As shown, the sputtering enhancement increases faster than the decrease in incident power with an increase in angle of ir:idence. This is why, for a given input power censity, the front surface temperature continues to increase with the angle of incidence.

The life of a given thickness of material can be expressed by:

$L=\frac{h E_{p} K}{3600 P \cos \alpha Z S(\alpha)}$, 


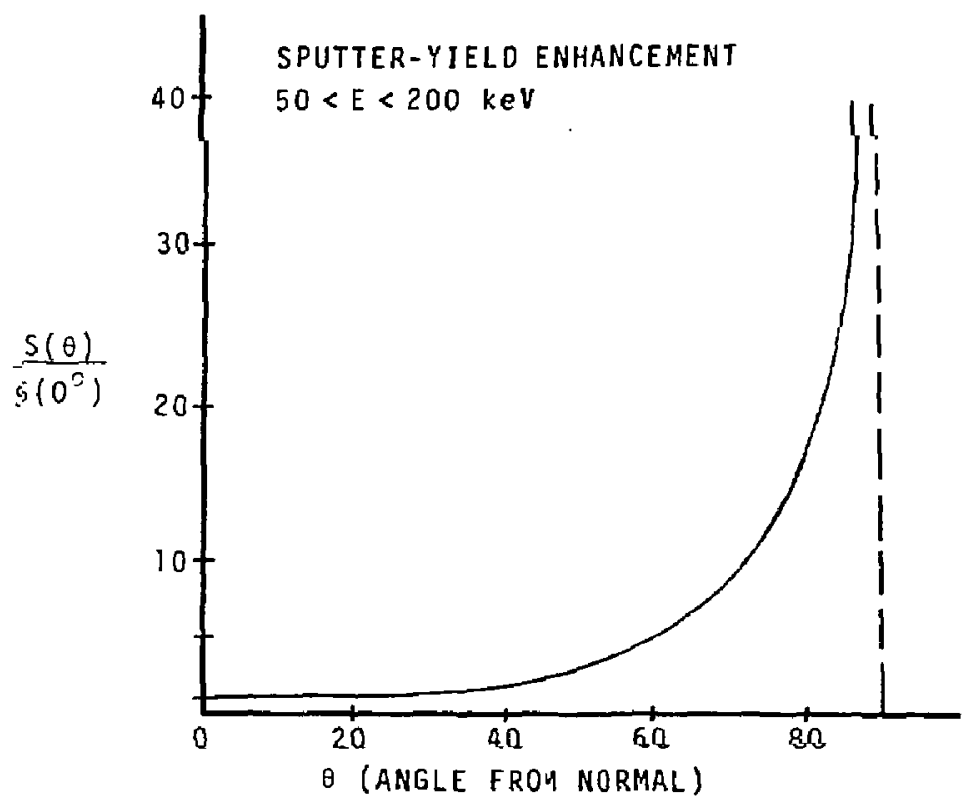

Figure 3-166. Sputtering rate enhancement vs angle of incidence from normal. 
where

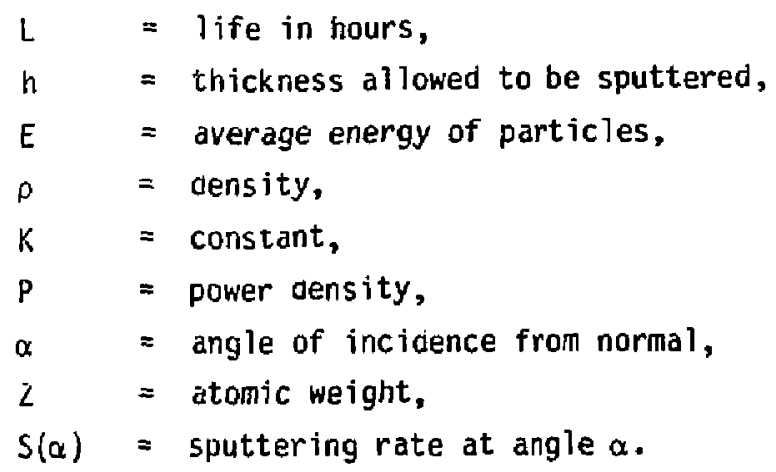

The front surface temperature of a given thickness of material can be expressed as

$$
T_{F . S .}=T_{w}+\frac{\ell q}{k A} \text {, }
$$

where

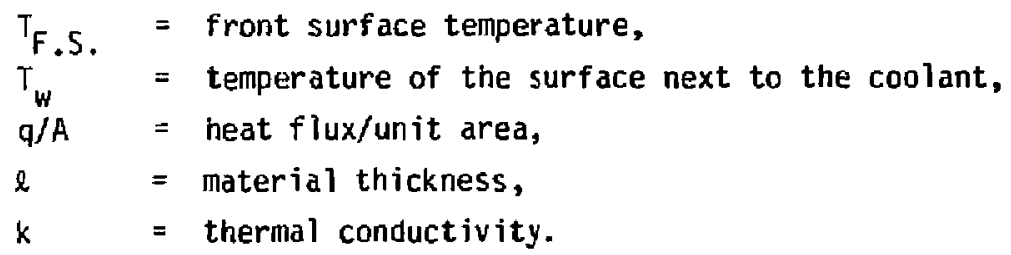

The power derisity on the neutral dump surface depends on the source characteristics, the distance from the source $g^{r}$ id to the dump, the geometry of the four sources in the beamlines, and the absorption characteristics of the plasma. Figure 3-167 shows the calculated power aensity on the oump slirface when : re source 15 on and the plasma is at full density; Fig. 3-168 shows the power density when four are on at the same time. Note that the region of highest power density is a small area, within which the calculated power density on the angled dump surface is about $1.2 \mathrm{~kW} / \mathrm{cm}^{2}$. The front surface temperature of the dump is about $875^{\circ} \mathrm{C}$. At this temperature, the strength of the Amzirc orops and the induced stress exceeds the elastic limit of the material. To reduce this temperature, the front surface must be made 


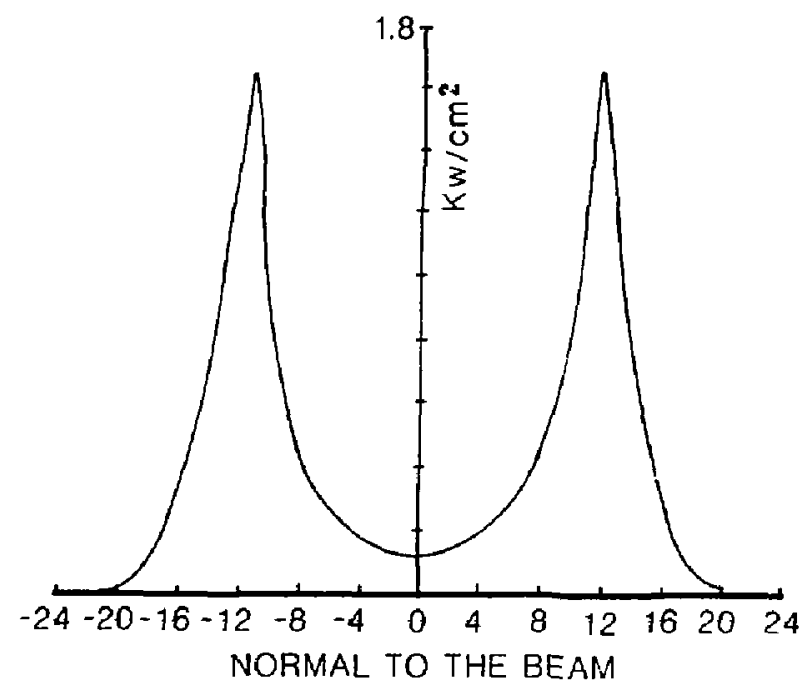

Figure 3-167. Calculated power density normal to the beam after passing through the piasma. 


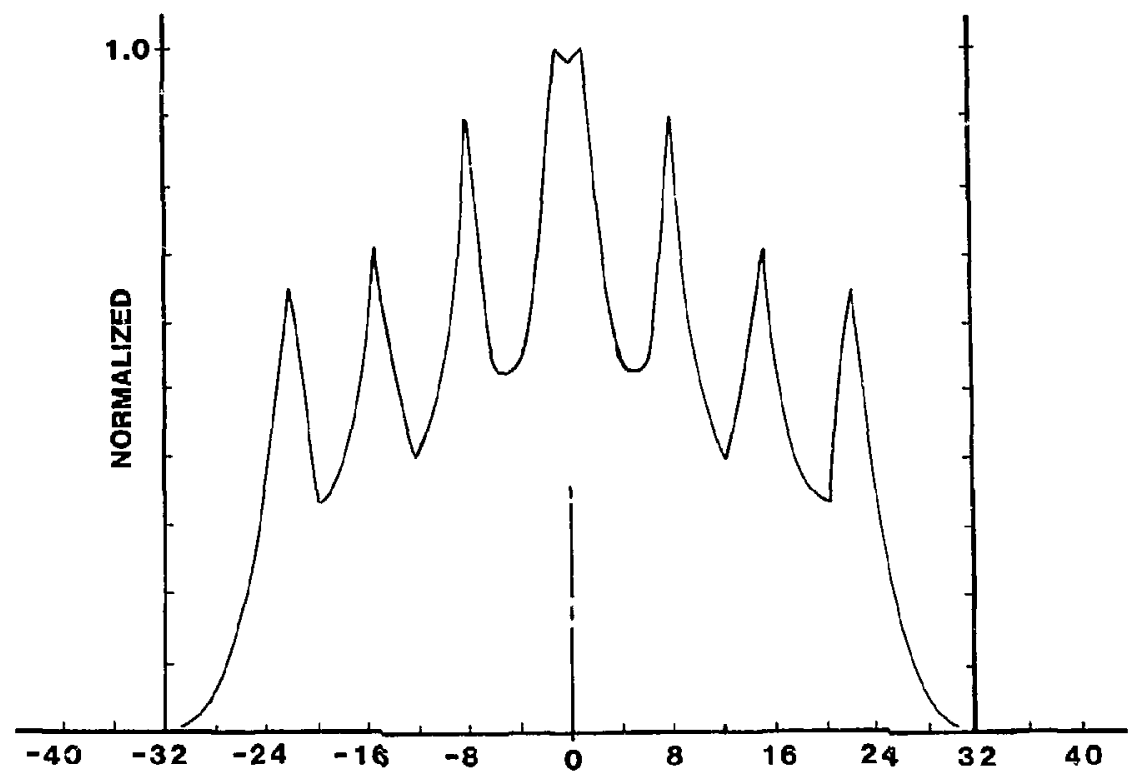

Figure 3-168. Calculated power density normal to the beam for four sources on the dump. 
thinfer than necessary far the 0.5 full-pawer-year requirement. Several consioerations make this a reasonatie deviation. The view factor from one panel to the otner is about $60 \%$. This means that most of the sputtered atoms are redeposited onto the opposite panel. In addition, the panels can be inspected at the end of each experimental phase. If the small region appears to be losing material at an unacceptable rate, the panel can be moved or eachanged. He expect the panels to require repiacenent only after three calendar years of operation.

The life of the panels is based on steady-state sputtering consiaerations. General Dynamics made a study to ensure that the panels could handle the high heat flux during startup, when the plasma density is low. They assumed the density buildup of the plasma to be linear, increasing from 0 to a maximum in $0.5 \mathrm{~s}$. The four sources were turned on $0.125 \mathrm{~s}$ apart, as illustrated in Fig. 3-169. As the plasma builds up, the power density on the surface of the neutral dump goes down, but as each source comes on there is an overlap and the power oensity goes up. The results of lhis study are discussed in Section 3.10.4, but to sumarize, the maximum front surface temperature reaches about $330{ }^{\circ} \mathrm{C}$ for the area where two sources overlap. This is below that expected for the steady-state case.

McDonnell Douglas conductea a thermal stress analys is on the panel's design using two cases. The first was for a front surface thic'ness of $1.8 \mathrm{~cm}$ of Amzirc, and the second was for a thickness of $1.0 \mathrm{~cm}$. They found that, because of the high surface temperature, the stress near the front surtace exceeded the yiela strength of the Amzirc. In adaition, there were scme regions near the panel's back side that had high stresses induced by the particular configuration used in the analysis. The study thus indicated a reaesign of this region. It also predicteo a very short panel fatigue life; however, thick copper plates have been used as beam-dump surfaces in sevaral operating beamlines with little evidence of fatigue cracks or other types of failure. Since the plate is very thick at the beginning of the experimeits, its response is a thick-plate response, and the high strain shoulo not cituse failure. As the plate sputters, the thickness between the front surface and the cooling channels is reduced and the temperature of the front surface goes down. We know that the panel can absorb $2 \mathrm{kH} / \mathrm{cm}^{2}$ for at least 600 shots with no evidence of jegradation. 


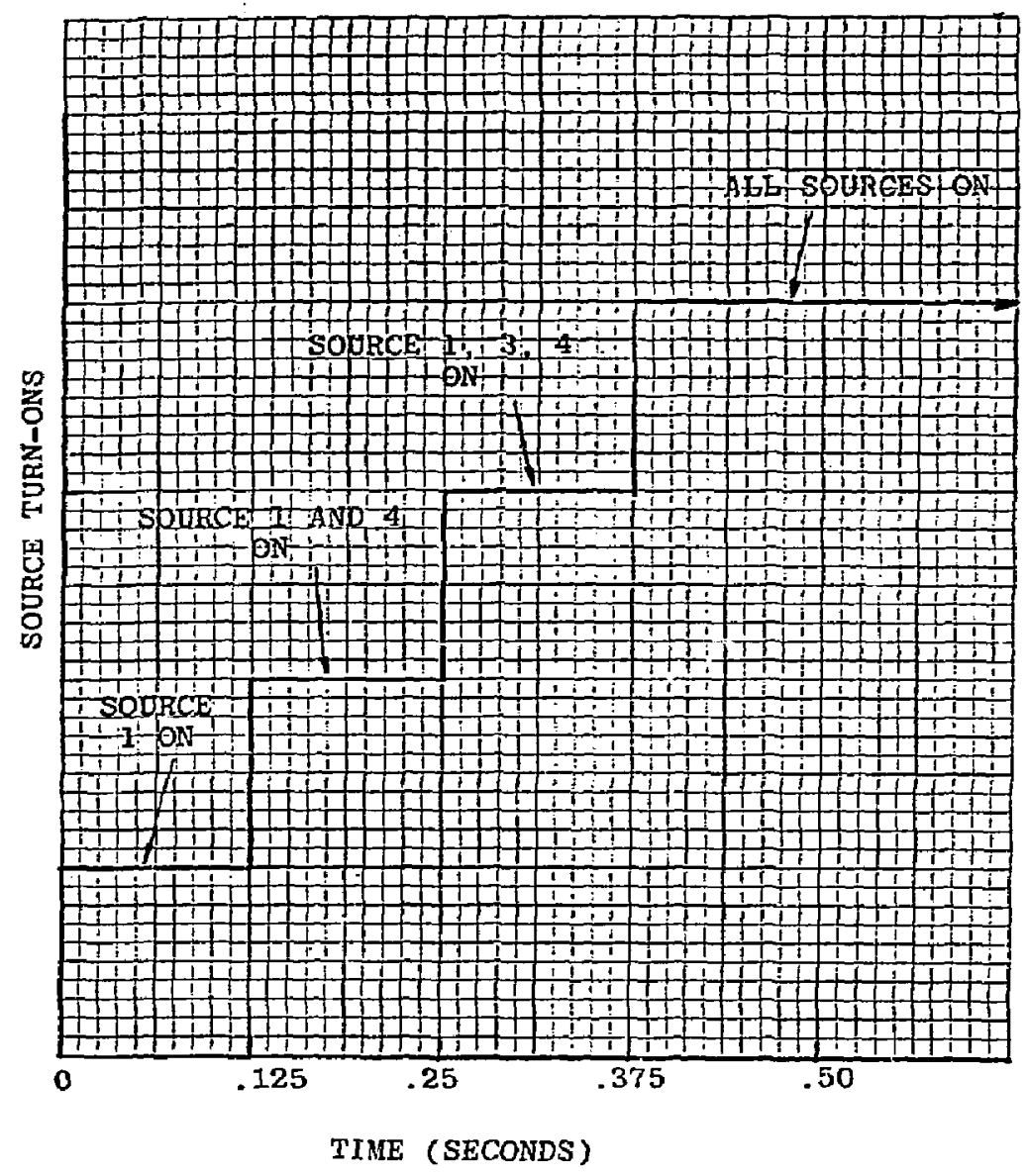

Figure 3-169. TDF source startup sequence for central cell neutral-beam injectors. 
The power density on the plasma end dump is:

$$
P_{\text {end }}=\frac{P_{c c}}{K} \frac{B_{\text {erig }}}{B_{c c}} \text {, }
$$

where:

$\begin{array}{ll}P_{\text {end }} & =\text { power density on the end dunip, } \\ P_{c c} & =\text { power density in the central cell on the same flux re, } \\ B_{\text {end }} & =\text { magnetic field strength at the eno, } \\ B_{c c} & =\text { magnetic field strength on same flix line in the cents l ceil, } \\ K & =\text { constant dependent on beta. }\end{array}$

At 28 m from the machine's center, the calculated power density at the center of the plasma end dump is $350 \mathrm{~W} / \mathrm{cm}^{2}$. The size of the fan footprin is about $2 \times 17 \mathrm{~m}$ after passing through a halo scrapper. Because the powe density on the plasma end dump decreases rapidly away from the center of $t$. $f a n$, we have designed two panels of different materials. Those we considered were: A16061, Amzirc, S5304, and TZM. The properties we considereo were suuttering rate, resistance to coolant corrosion, ease or fabricaticn, strength vs temperature, and thermal characteristics. The sputtering thickness for one full-power year vs heat flux for these materials is shown in Fig. 3-170. The maxinum front surface temperature vs heat flux for these materials is shown in Fig 3-171. Based on these results and the calculated strength required for the cọolant pressure, we chose TZM for the baseline design for a heat flux over $100 \mathrm{~W} / \mathrm{cm}^{2}$. Below this value, a 55304 panel satisfies the requirements. Since it costs about 40 times less than TZM, we would use it wherever we expect low heat flux.

We calculated the water flow rate and pressure required using the Dittus-Boelter Nusselt number relation:

$W_{u}=0.023(\mathrm{Re})^{0.8}(\mathrm{Pr})^{0.4}$

where $\mathrm{K}_{\mathrm{e}}$ is the Reynolas number and $\mathrm{P}_{r}$ is the Prandt l number. As the heat fiux increases, the local pressure required to suppress boiling aiso increases, as shown in Fig. 3-172. We assume an inlet temperature of $40^{\circ} \mathrm{C}$ 


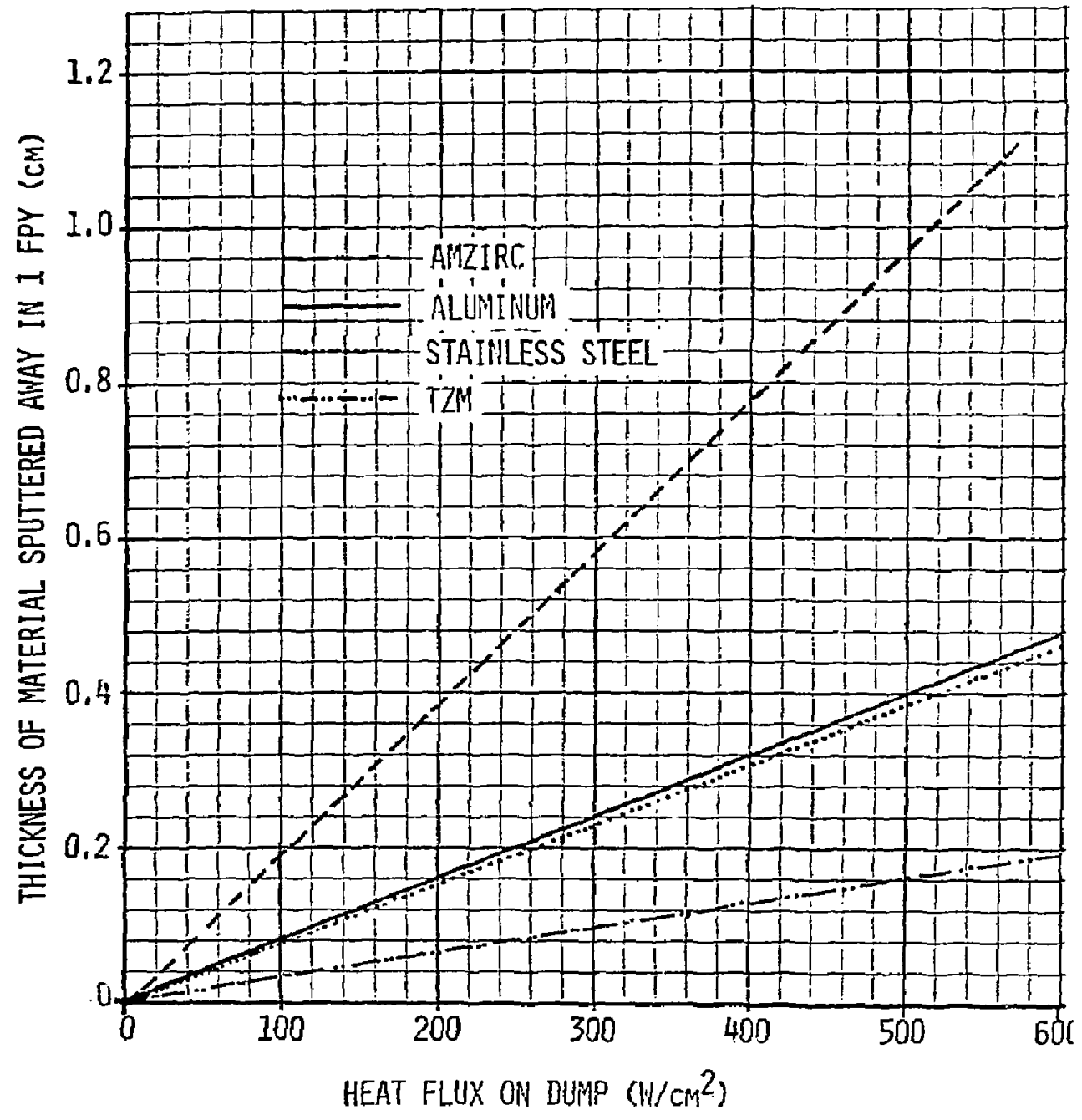

Figure 3-170. Sputtering thickness for 1 ful1-power year for various materials at increasing heat flux. 


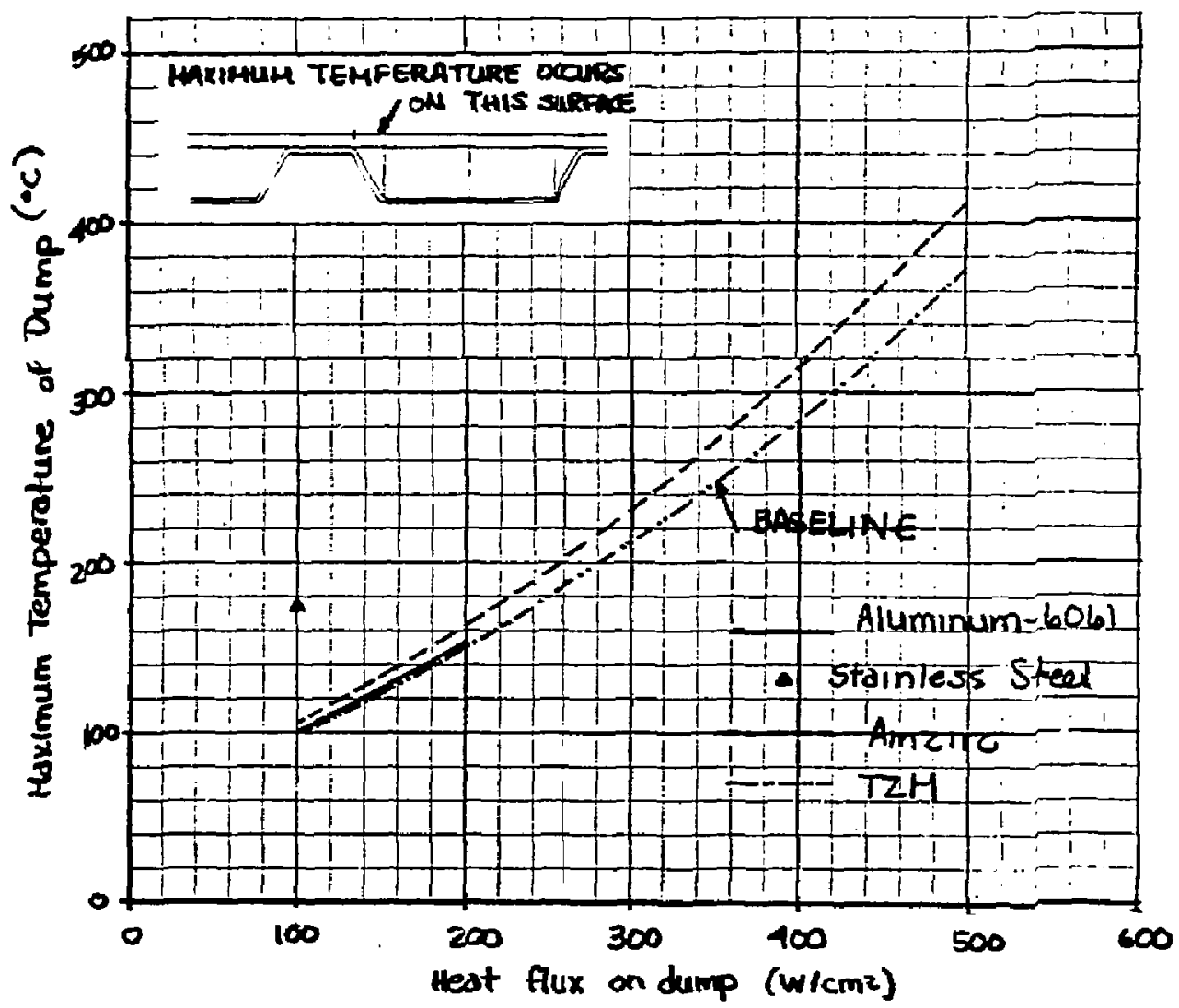

Figure 171. Maximum temperatures of the bean dump front surface. 
LOCAL PRESSURE REQUIRED TO SUPPRESS BOILING (PSI)

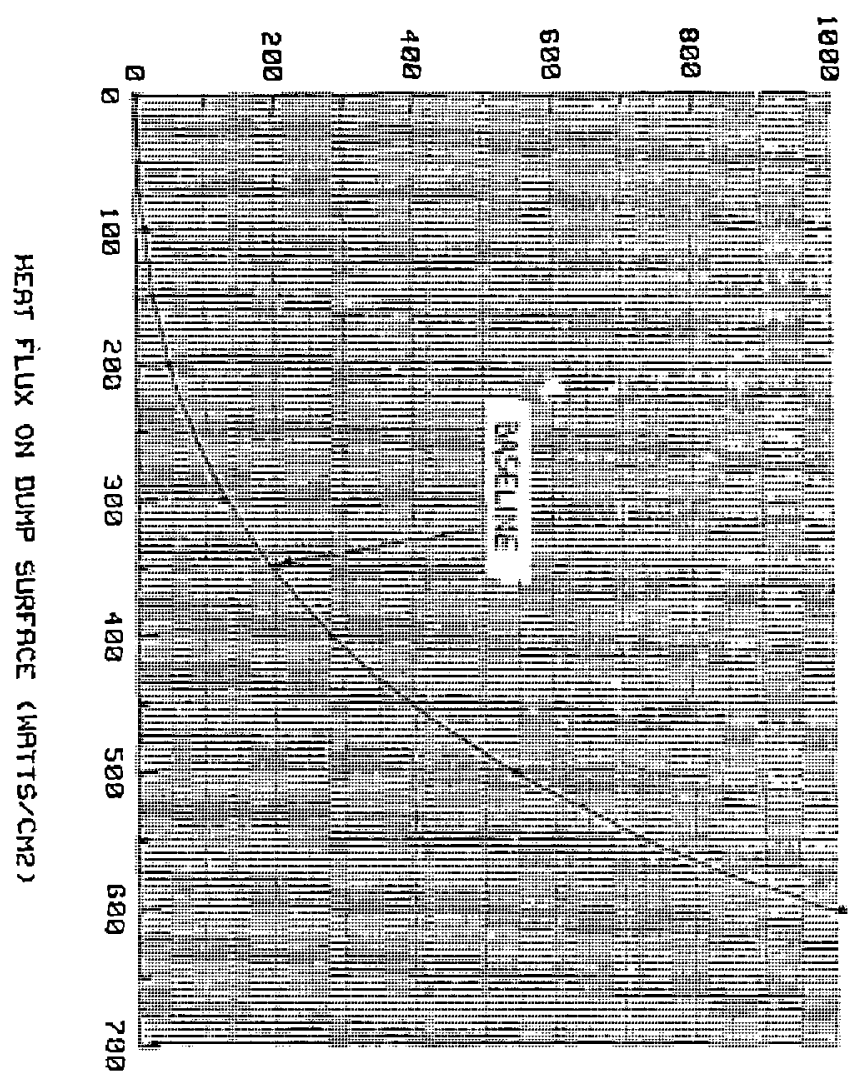


and allow a $15^{\circ}$ rise in water temperature. The minimum coolant flow for chis case for both plasma oumps is $10,340 \mathrm{gpr}$ with an inlet pressure of 250 psi.

We determined the amount of material required on the front surface (Fig. 3-170) to allow for sputtering assuming that $60 \%$ of the incident fiux is 20-keV oevterium ana $40 \%$ was a $50 / 50$ mixture of $27-k e V$ D-T particles.

We then dia a structural analysis of a panel constructed cf the canoioate inaterial using the ASME boiler Coae 2 as a requirement. Membrane ano bending stresses were computed at various locations for a given pressure witn a joirit efficiency of $0 . B$ for the weided regions and an idealized configuration (Fig. 3-173). For a heat flux greater than $100 \mathrm{~W} / \mathrm{cm}^{2}$, only Amzirc and $\mathrm{T} \angle \mathrm{M}$ met the requirements for strength. Because of the higher sputtering rate of Amzirc, we chose TZM for the higher heat flux areas.

The indivioual panels are assembled onto a frame and placed inside the yessel. The assembled configuration is shown in Fig. 3-174. We added small box-like protrusion to the vacuum vessel to allow removal ano replacement of four to five panels while protecting personnel from high radiation. These panels will be manifolded together to allow such maintenance. Additional details on the plasma end pump design can be found in section 3.10.9.

Tritium. One-half of the beamlines in the central ceil inject tritium particles into the plasma. Some of these particles diffuse through the oump surfaces into the cooling water. We perforineo an analysis to determine the amount that may have to be recovered from the coolant.

We assumed that the analysis applies to both the neutral and ion aumps. Figure 3-175 shows the configuration used. Because aiffusion rates are a function of temperature, we calculated the temperature profile through the thickness of the aump surface using the equation shown in $\vec{F}$ ig. 3-175.

Figure $3-176$ shows the initial concentration of tritium particles, caiculated by the University of California-Los Angeles (LCLA). This information was input to a computer code that calculated the aniount of tritium expected to enter into the cooling water. It was calculated at

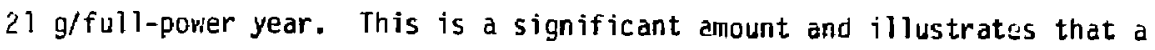
tritiun recovery system must be usea for the cooling water. 


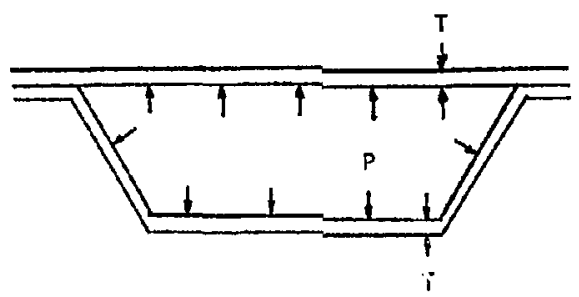

ACTUAL COIL GEOMETRY

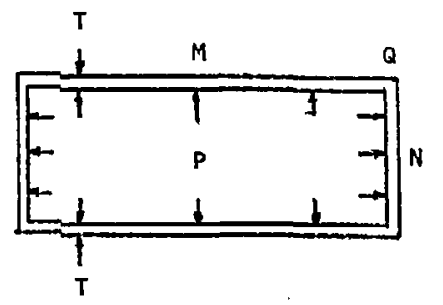

IDEALIZED RECTANGIULAR SECTION WITH THE SAME FLON AREA

Figure 3-173. Panel coii geometry. 

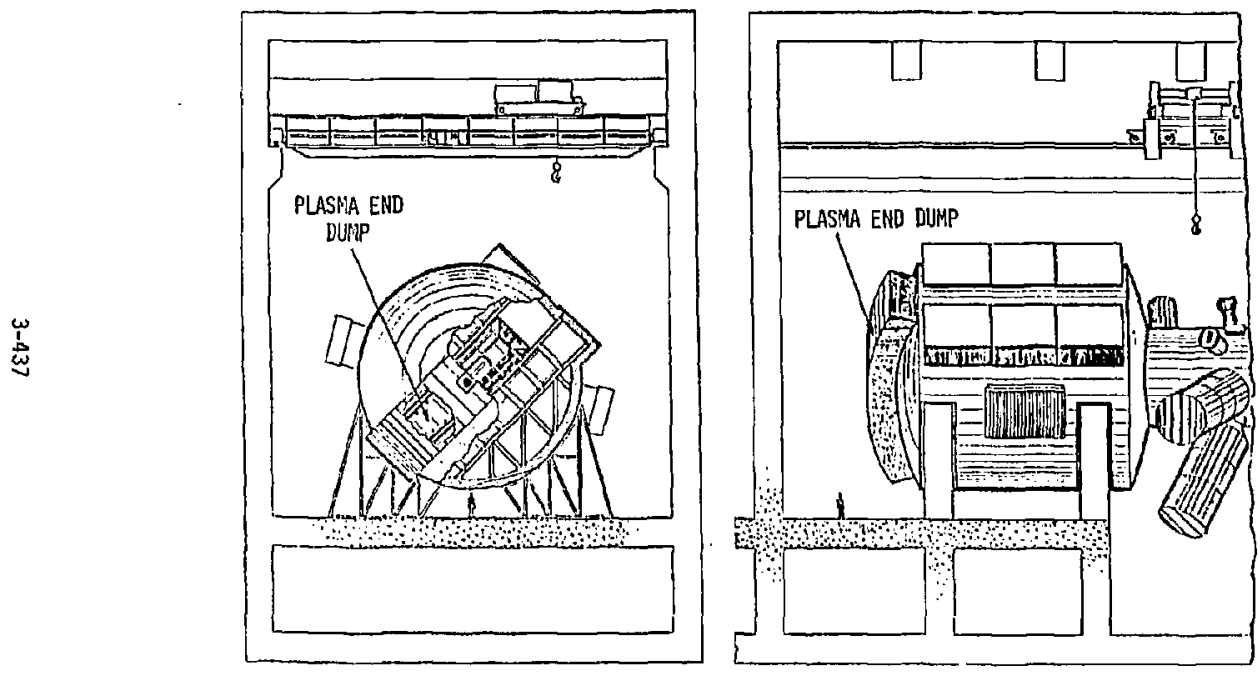

figure 3-174. TOF end cell configuration. 


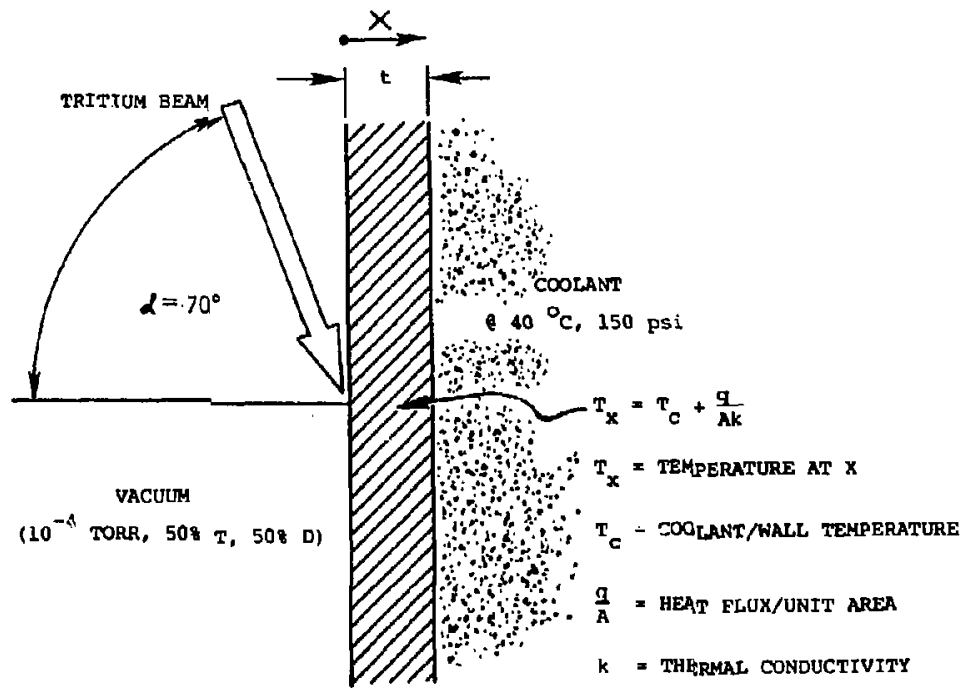

Figure 3-175. Tritium diffusion model. 
NEUtral beAM deposition IN METAL SURFACE

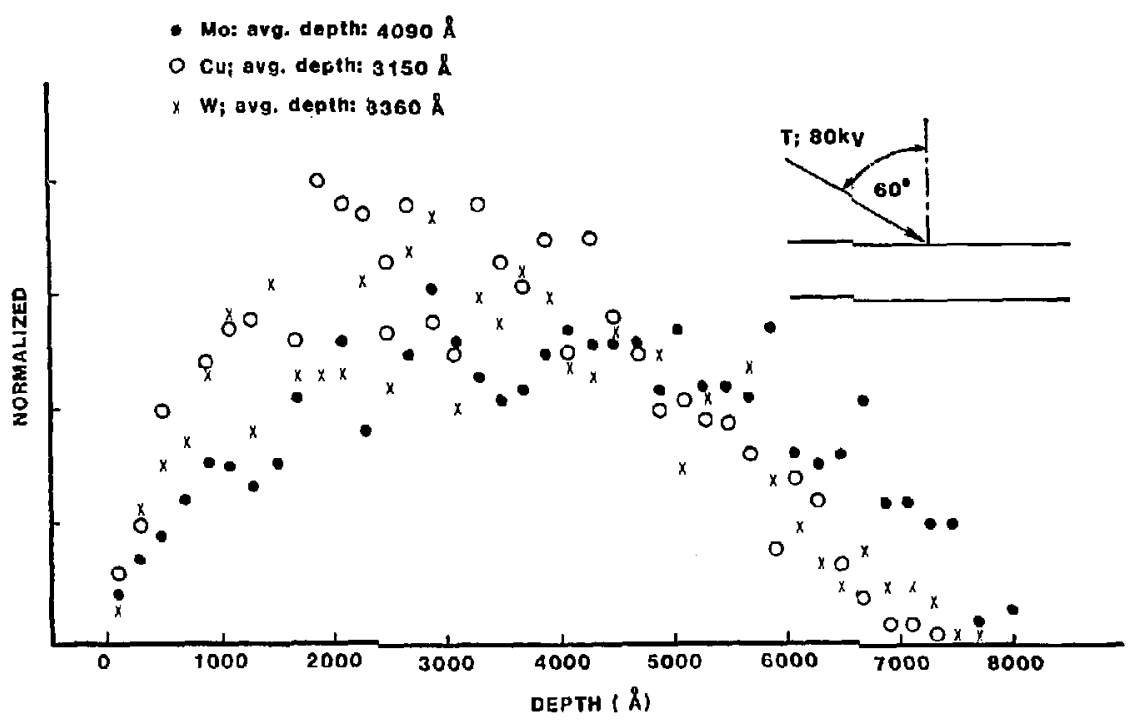

Figure 3-176. Neutral-beam deposition in metal surface. 
Key Technical Issues

Particle Dumps. Although the baseline configuration for the particle dumps has been testea, the thicker required surface required has not been used as an actively cooled surface. If it acts as a true thick plate, experience shows that the aesign will furction satisfactorily. Yielding at the hot thin layers at the surface has not been a problem for the large number of flat, thick, copper beam dumps used to oate in experiments at LLNL, LBL, the Uoublett III Experiment, and Oak Ridge National Laboratory (URNL). Cracks that developed on the thinner surface do not appear to grow into the thick plate. Copper plates have been melted on the front surface and have continued to be useful dunps. Before a Jecision is made on a TOF a configuration, several particle dumps will have been tested on beamlines now planned for long-pulse operation. These are the Mirror Fusion Source Test Facility (JFTF) at LLNL, the Neutral Beam Engineering Test Facility at the LBL, and the test facility at ORNL. Present plans for MFTF call for testing an Amzirc panel with the basaline design with a front plate $1.0 \mathrm{~cm}$ thick rather than the 1.8 Cm required for TDF. It may be possible to reduce the required thickness of the panel due to the view factors between panels. As the plate gets thinner, the front surface temperature goes down, and the design becomes mere conservative from fatigue life considerations.

The data we used to caiculate the thickness of the panel front surface is not taken from direct measurement. It. is available for low energ. particles only. Since the data indicates a rapid increase of sputtering enhancement with increasing angle of incidence, the accuracy of such calculations becomes very important. Uata fisr high-energy-deuterium particle sputtering as a function of inciaent angle is vitally needed, because the factor of a 9 tines sputtering enhancement at $70 \%$ incident arives the pane 1 design.

Although the stress analys is indicates very high stresses both on the front surface of the pane 1 and close to the water channels, neither may be as serious as it appears. Based on what we have already discussed, the front surface high stress may not be a prcblem, and with a slightly different design we can significantly reduce the stresses near the channels. 
If sputtering and stresses are used as an absolute determination of pane 1 seleciion, either another material must be used or the Amzirc surface must be laminated with material such as tungsten. We considered several means of laminating, including duffusion welding, explosion bonding, and plasma spraying. The usual question of bond survivability make these laminated designs more risky than the baseline panel. Laminated surfaces are presently being developeo as part of the High ieat Flux Component Development Program (HHFCUP).

One technical issue for the plisma end duilp is the cost of moving the cump in relation to the machine's -enter. Experinents on the two baseline oesigns have not been carrieo out to determine their heat-transfer capability. Another is the ariount of tritium tat diffuses through the material selecteo, which again depends on the aistance from the machine center. Although a panel made of TZM appears to be relatively easy to nanufacture, we have not made one.

\subsubsection{Neutrāl-beamı Uump--Startup Analys is}

Problem. The startup method chosen for the neutral beam injectors ariu the plasma growth profile will greatly affect the beam aump's heat load. During startup, the surface heat flux on the dump may be much larger than ouring the normal oump operating mooe. This is because it takes time for the plasma trapping fraction $f+$, to increase to the steady-state value. The baseline heat-transfer panels used as the oump surface are designed to operate uncier steaoy-state conditions. Thus, the temperature and thermal gradient experienced by the panel during the startup phase must be evaluated for determining if that is a worse case.

Assumptions. Figure 3-17? shows the Amzirc heat-transfer panel under consideration. The baseline panel is commercially available from MDAC. Small internal channels with turbulent water flow promote forced convection and nucleate-boiling heat transfer. For Tof, the original McDonnell Douglas oesign has been modified by increasing the front surface thickness to allow for a one-half $f(u)$-power year of sputtering. At the beginning of life, the front plate thickness is $1.8 \mathrm{~cm}$, while the end-life thickness is $0.2 \mathrm{~cm}$ a loss of $1.6 \mathrm{~cm}$ of inaterial. 
WATER COOLING

CHANNELS

FLUX FRON NEUTRAL BEAM INJECTOR

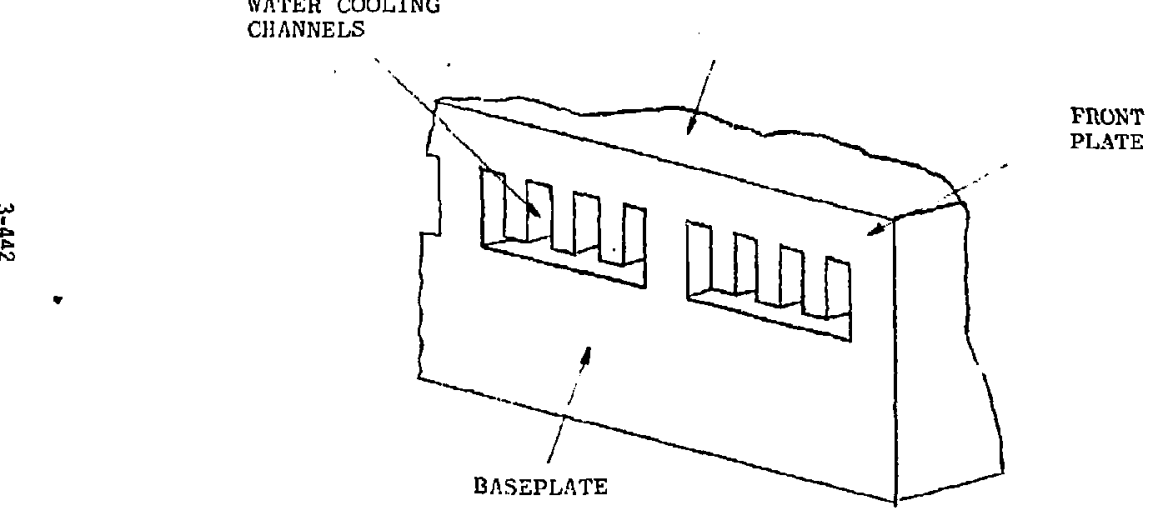

Figure 3-177. Internaliy-finned, water-cooled dump. 
Eoch central cell neutral beam injector has four ion sources with beams that overlap during start-up to distribute the oeposited energy spatially on the dump surface, as shown in Fig. 3-178. The TDF rectangular stancara source is $12 \times 40 \mathrm{~cm}^{2}$, has a divergence of $0.5 \mathrm{deg}$ in both directions, has a current of 60 to $70 \mathrm{~A}$, ano is located $9.5 \mathrm{~m}$ from the front surface of the dump.

The neutral-beam source turn-on sequence is shown on Fig. 3-179. The beams are turned on at $0.125 \mathrm{~s}$ intervals in the following order: Source 1 , Source 4, Source 3, and Source 2. In this manner, the heat tlux overlap at the worst lacation is delayed.

Figure 3-180 shows the assumed $f+$ vs time. At time zero, no plasma is present; therefore, $f+$ is zero. At this time the full-beam intensity is incident on the dump. At $0.5 \mathrm{~s}$ the plasma concentration reaches its steady-state mode; an $f+$ of 0.79 is present continually from this point on, ano only $27 \%$ of the beam intensity strikes the dump.

Discussion. We simulated four startup cases: Two begin-life (front plate thickness of $1.8 \mathrm{~cm}$ ) and two end-life (tront plate thickness of $0.2 \mathrm{~cm}$ ) scenarius. We had to check two worst-case locations, (1) the maximum heat flux location of source 1 , because this transient flux occurs over the longest period of time, and (2) the maximum heat-fiux location caused by the intersection of Sources 3 and 4 , because this is the highest heat flux present over the longest period of time.

The heat flow to the dump at a given time is calculated as follows:

$\operatorname{DHF}(x, t)=[\Sigma \perp \operatorname{SHF}(x, t)][1-\operatorname{PTF}(t)] \sin \theta$,

where:

$\operatorname{DHF}(x, t)=$ the resultant dump heat flux at point $x$ at time $t\left(k w / \mathrm{cm}^{2}\right)$ $\mathcal{L S H F}(x, t)=$ the norma $]$ heat flux from source at time $t$ at point $\times\left(\mathrm{kW} / \mathrm{cm}^{2}\right)$

$\operatorname{PTF}(t)=$ the plasma trapping $f+$,

$\theta \quad=$ angle of dump inclination i.e., angle between beam and cump surface, (20 deg for TDF). 


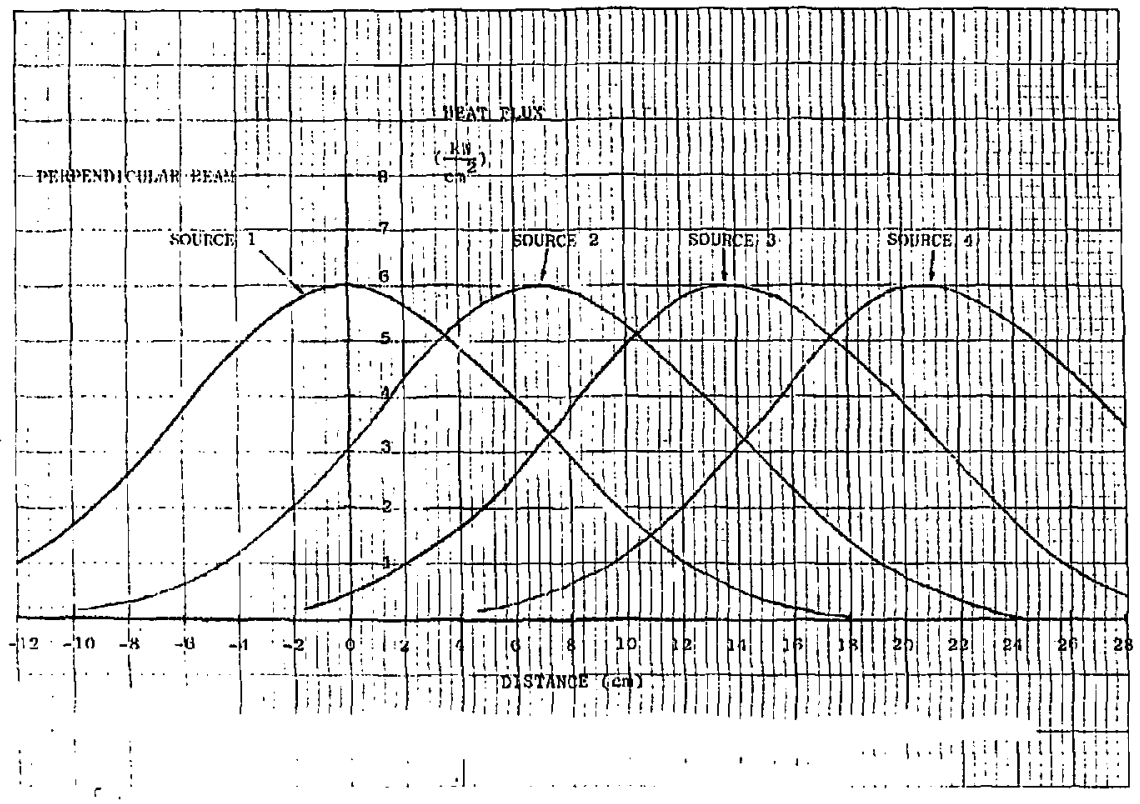

Figure 3-178. The central cell neutral-beam energy overlap on a normal aump surtace with no plasma trapping. 


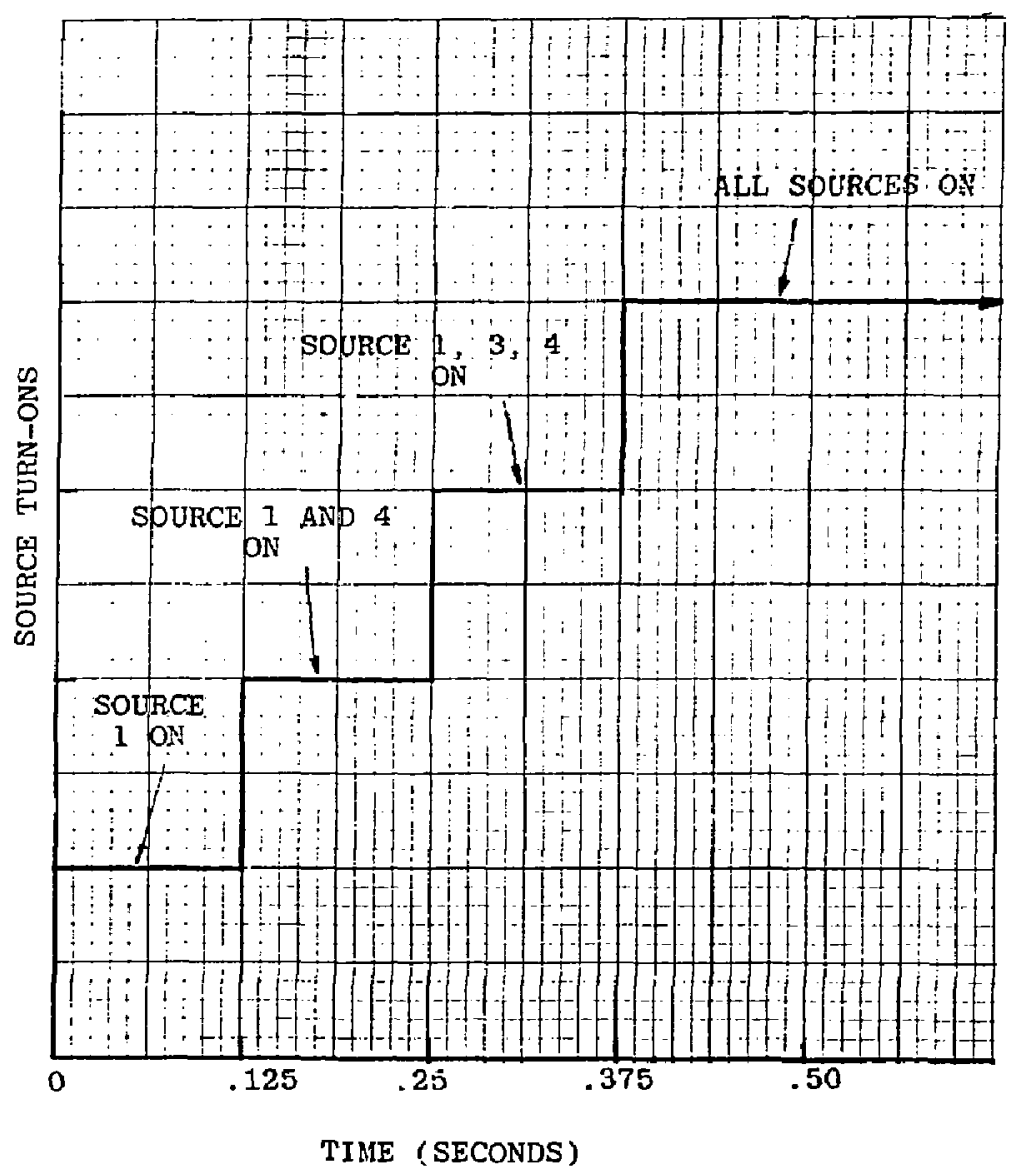

Figure 3-179. Source startup sequence for the central cell neutral-beam injectors. 


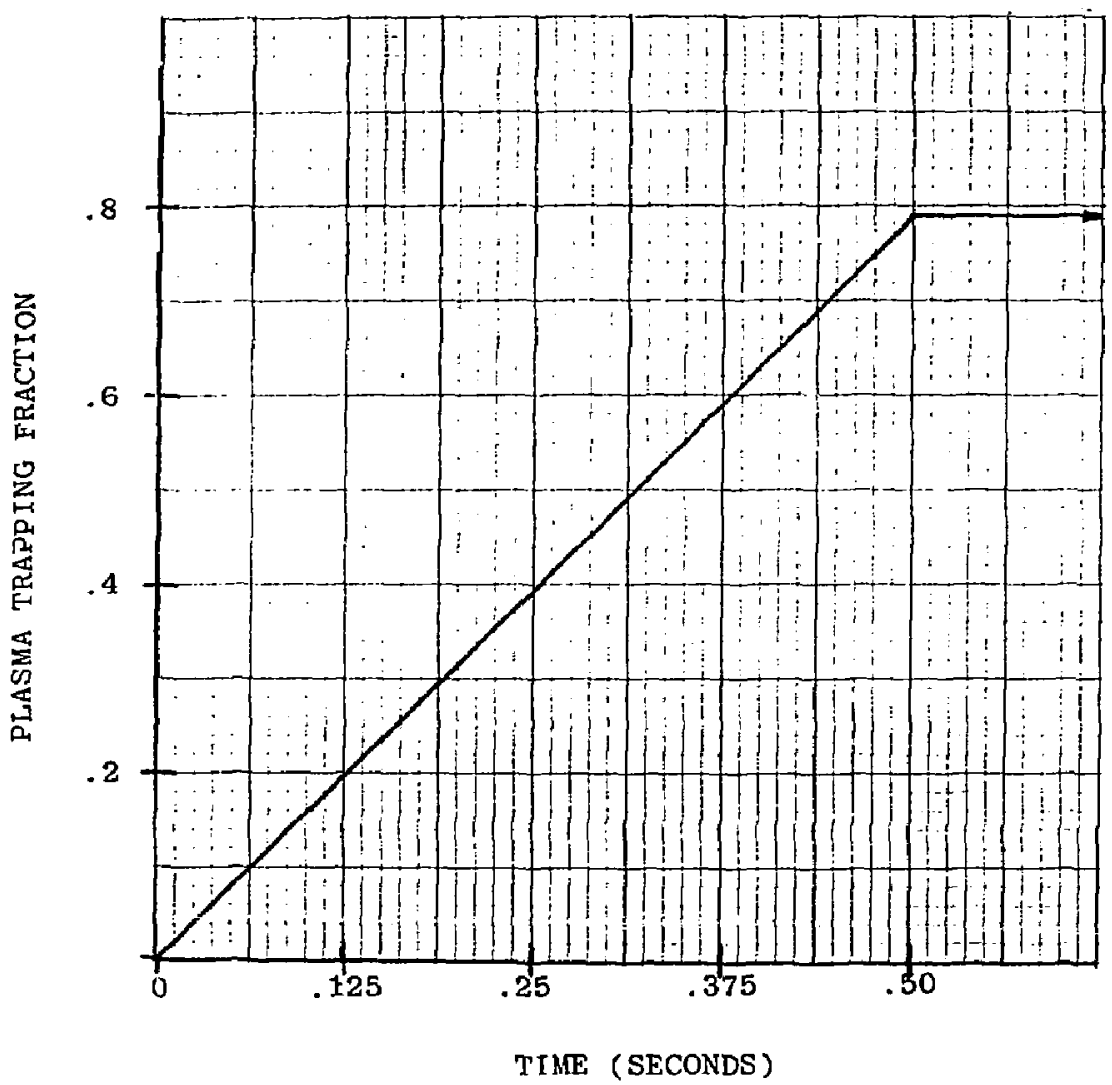

Figure 3-180. TDF plasma trapping fraction increase at startup. 
Figure 3-181 shows transient resultant heat fluxes on the dump surface for the two locations studied.

The transient temperature profile at the front plate of the cump was aetermined with a one-dimensional model using the Convair thermal-analyzer finite-aifference computer program. ${ }^{39}$ The model nodes are shown in Fig. 3-182.

ine front plate of the panel assembly was divided into 36 , nodes with the heat flux from the beam injectors impinging on the surface of noae 1 . We assumed the plate to be a semi-infinite solid for the begin-life cases. This is because the front plate is thick and the transient condition is short (l.5 s).

The plate was too thin in the end-life study to be considered a semi-infinite solid; therefore, we simulated cooling at the back surface. The McAdams forced-boiling correlation, ${ }^{40}$ conservative in our interest range, was suggested by the LLNL to predict the heat flux to the water:

$\frac{\underline{U}}{A}=2.256 \times 10^{-7}\left(T_{W}-T_{S A T}\right)^{3.86}$,

where

$Q=$ the heat flux $\left(\mathrm{kW} / \mathrm{cm}^{2}\right)$,

$T_{W}=$ the wall temperature $\left({ }^{\circ} \mathrm{C}\right)$,

$T_{S A T}=$ the saturation temperature at local pressure $\left({ }^{\circ} \mathrm{C}\right)$.

We took the local pressure to be 150 psi. Ait rodes were iflitiaijzed to $60^{\circ} \mathrm{F}\left(15.6^{\circ} \mathrm{C}\right)$.

Results. Resulting profiles of the front surface of the beam dump are shown in Figs. 3-183 through 3-186. The maximum temperature the front surface experiences, is $270{ }^{\circ} \mathrm{C}$ for begin-life ( $\mathrm{Fig} .3-187$ ) and $330{ }^{\circ} \mathrm{C}$ for the end-life (Fig. 3-188) cases. These temperatures are well below the $1083^{\circ} \mathrm{C}$ melting temperature of copper. The maximum temperature, at the 0.2-cai front surface delta, is $15^{\circ} \mathrm{C}$ for the begin-life and $90^{\circ} \mathrm{C}$ for the end-life case. This front surface must maintain structural integrity. 

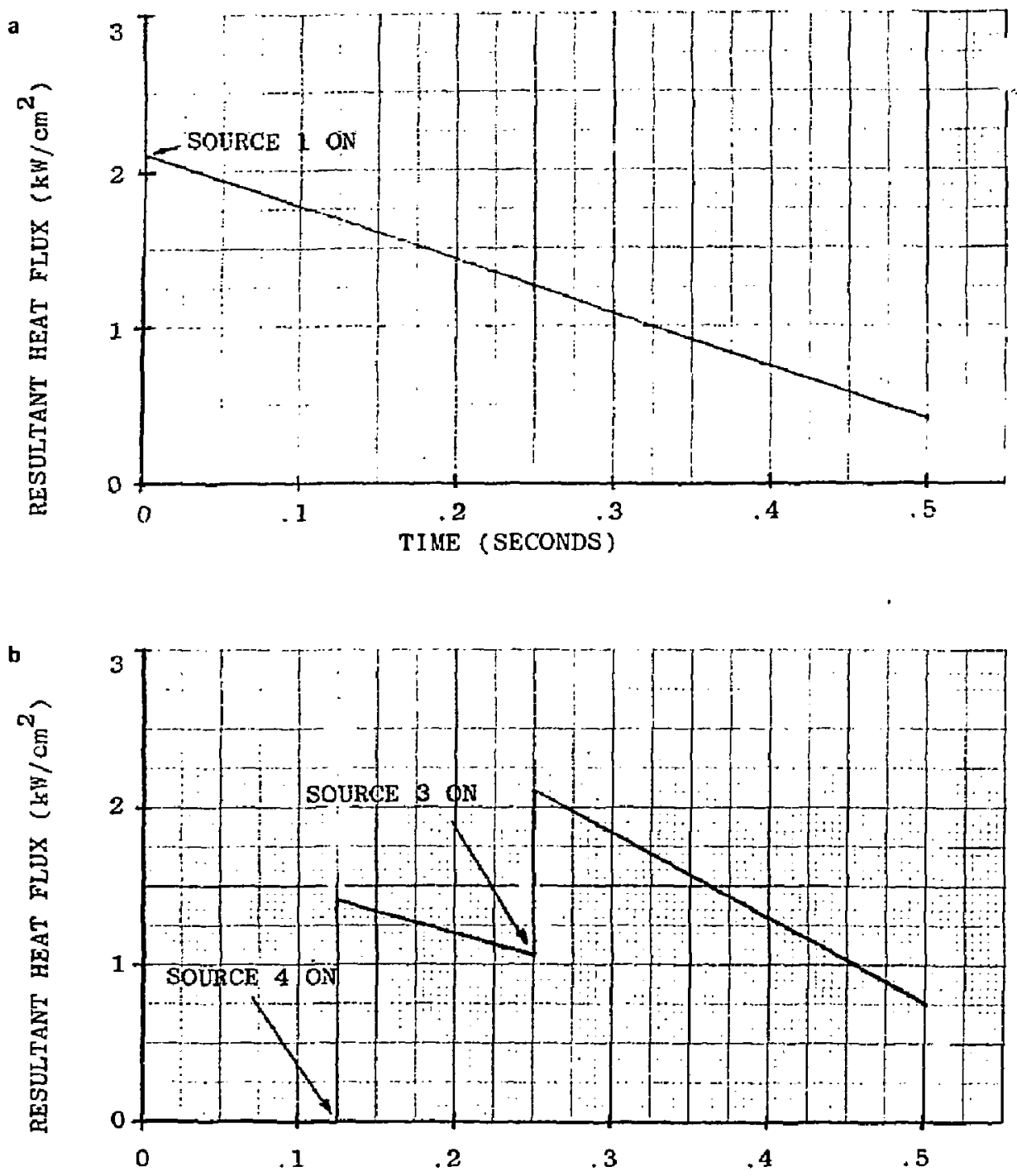

TIME (SECONDS)

Figure 3-181. The heat flux scenario for the reactor startup phase study of the neutral dump at various maximum flux locations. Shown are (a) location of source 1 and (b) locations of sources 3 and 4 . 


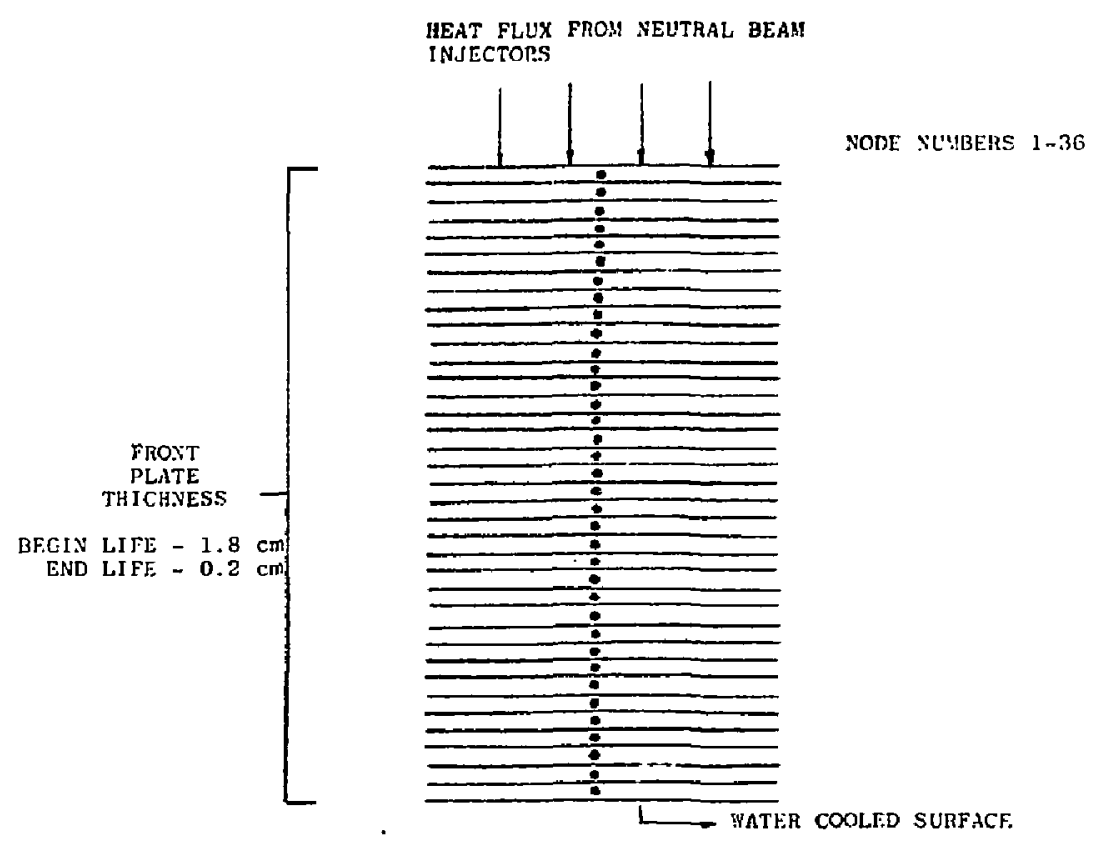

Figure 3-182. The thermal analyzer front plate nodalization used to predict thermal gradients in the front plate of the neutral-beam dump curing system startup. 


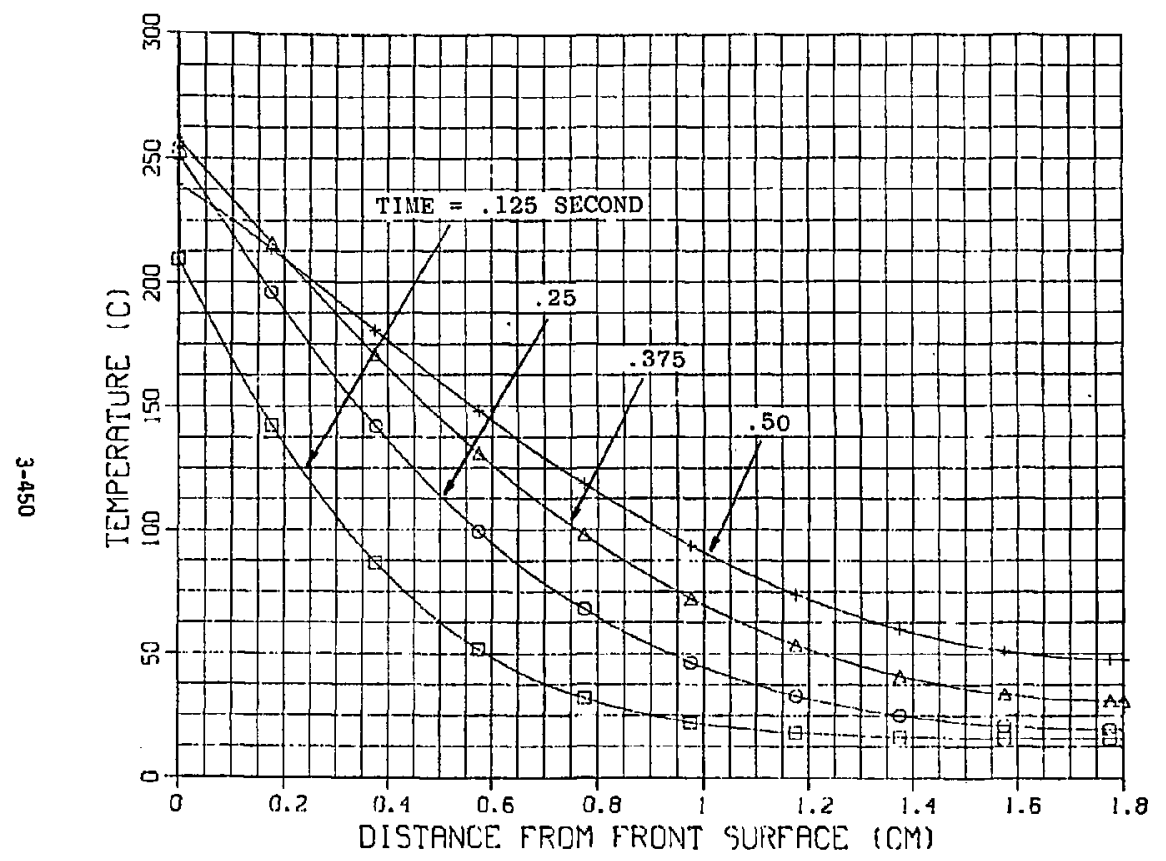

wone No. Otstrance

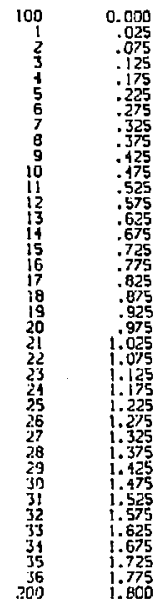

Figure 3-183. The begin-life temperature profile or the beam dump's front surface during reactor startup at the peak heat flux location of suurce 1 . 


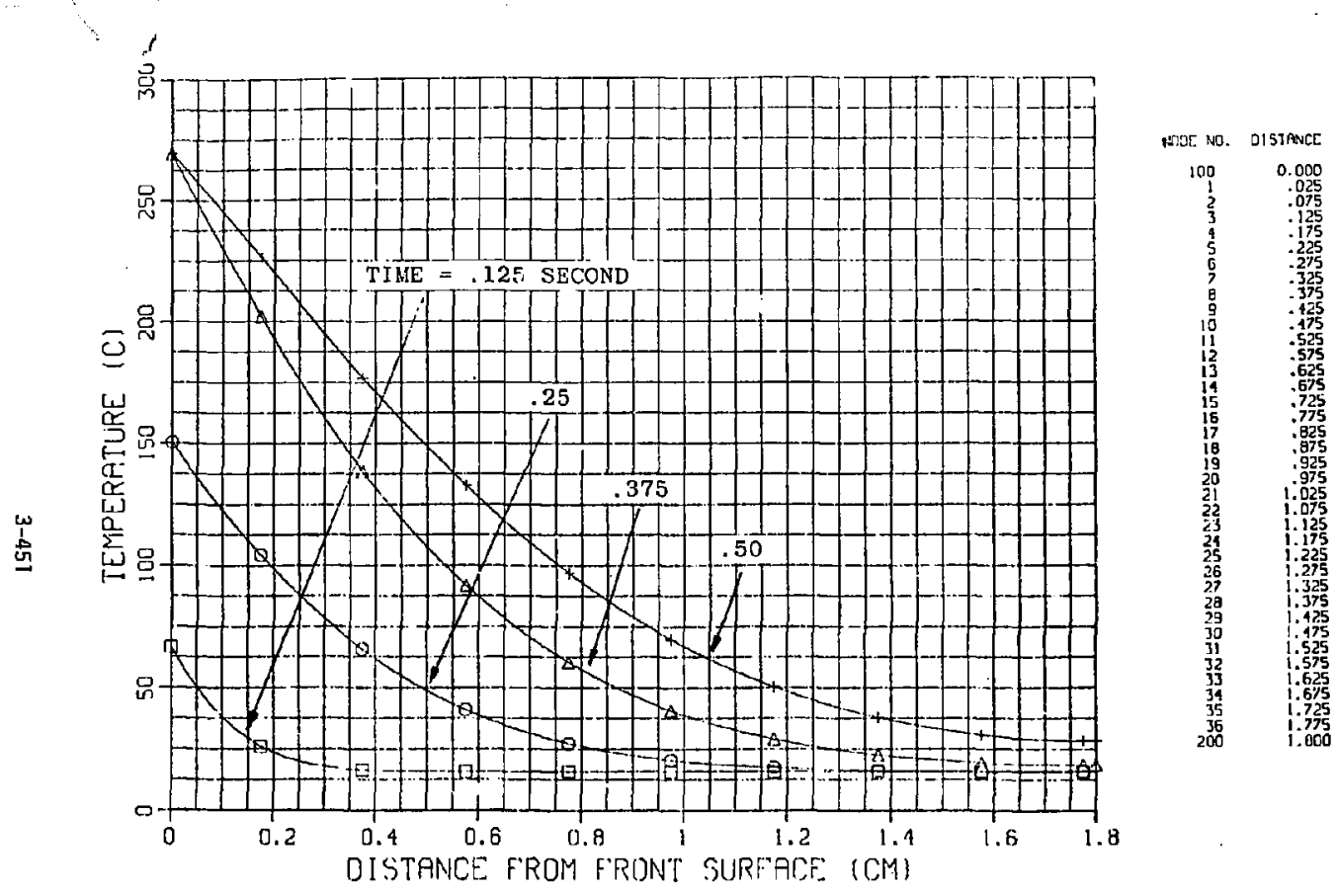

Figure 3-184. The begin-life temperature profile of the beam dump's iront surface ouring reactor startup at the peak heat flux at the intersection of sources 3 and 4 . 


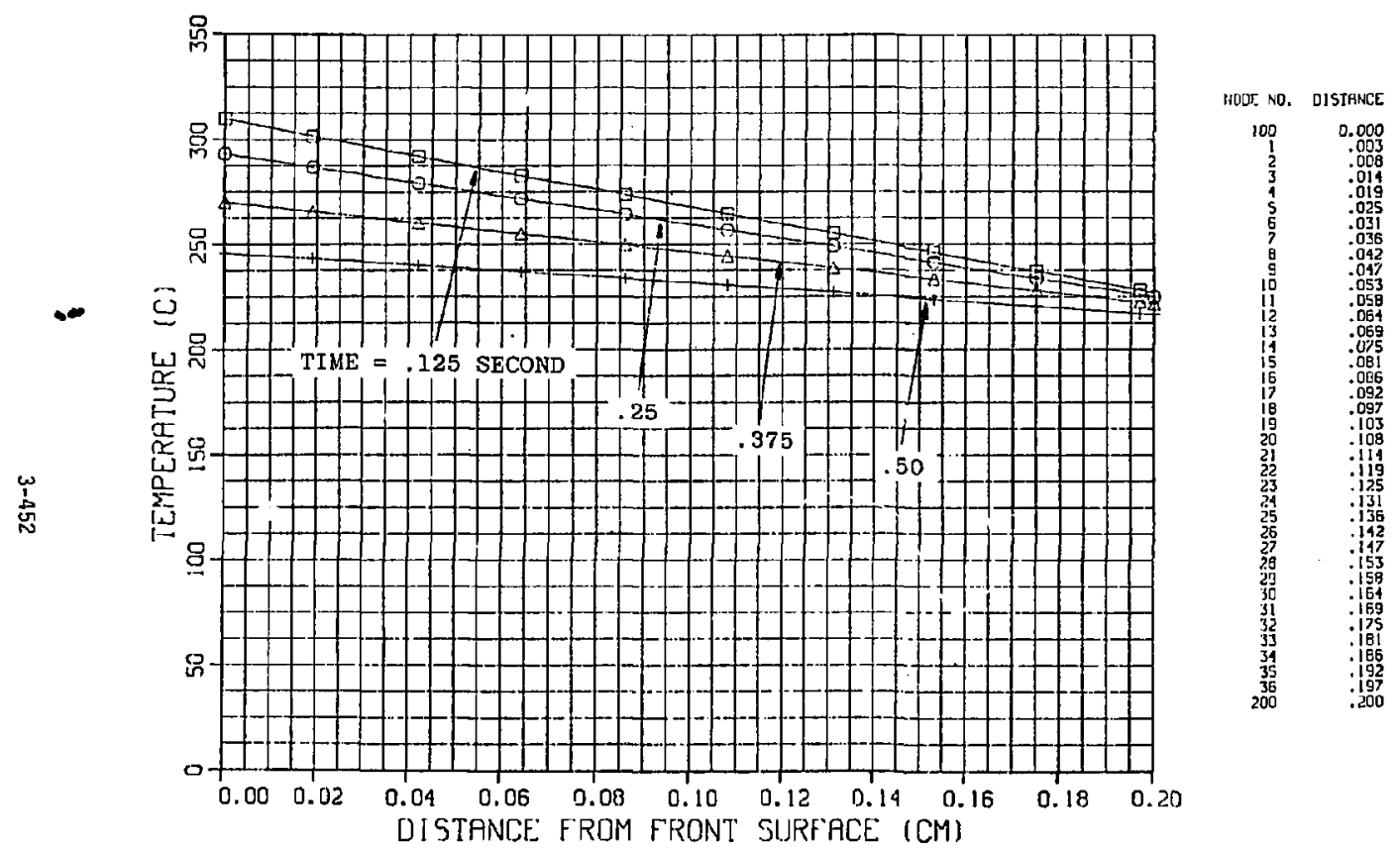

Figure 3-185. The end-life temperature profile of the beam dump's front

surface during reactor startup at the peak heat flux location of source 1 . 


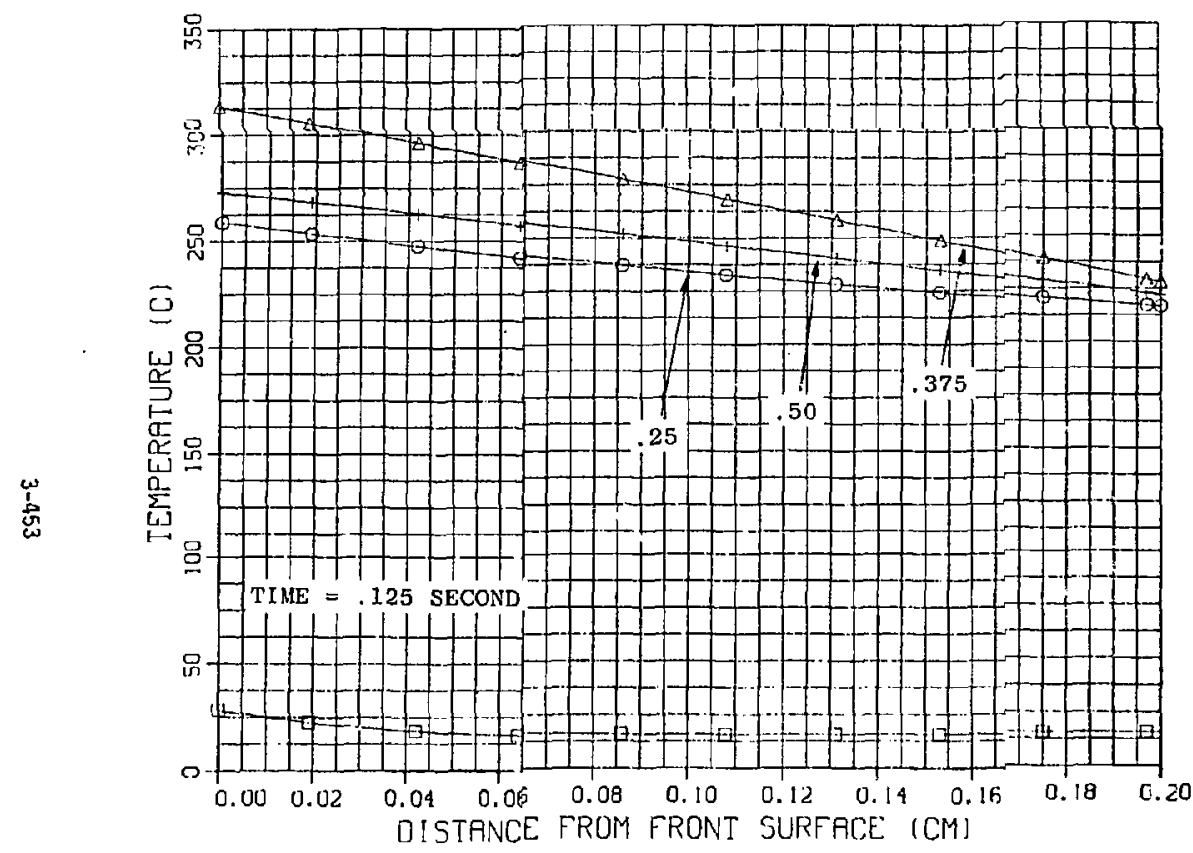

NODE NO. DISTANCE

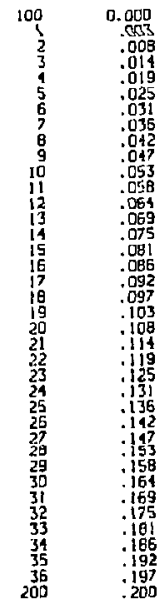

Figure 3-186. The end-1ife temperature profile of the beam dump's front surface during reactor startup at the peak heat flix at the intersection of sources 3 and 4 . 


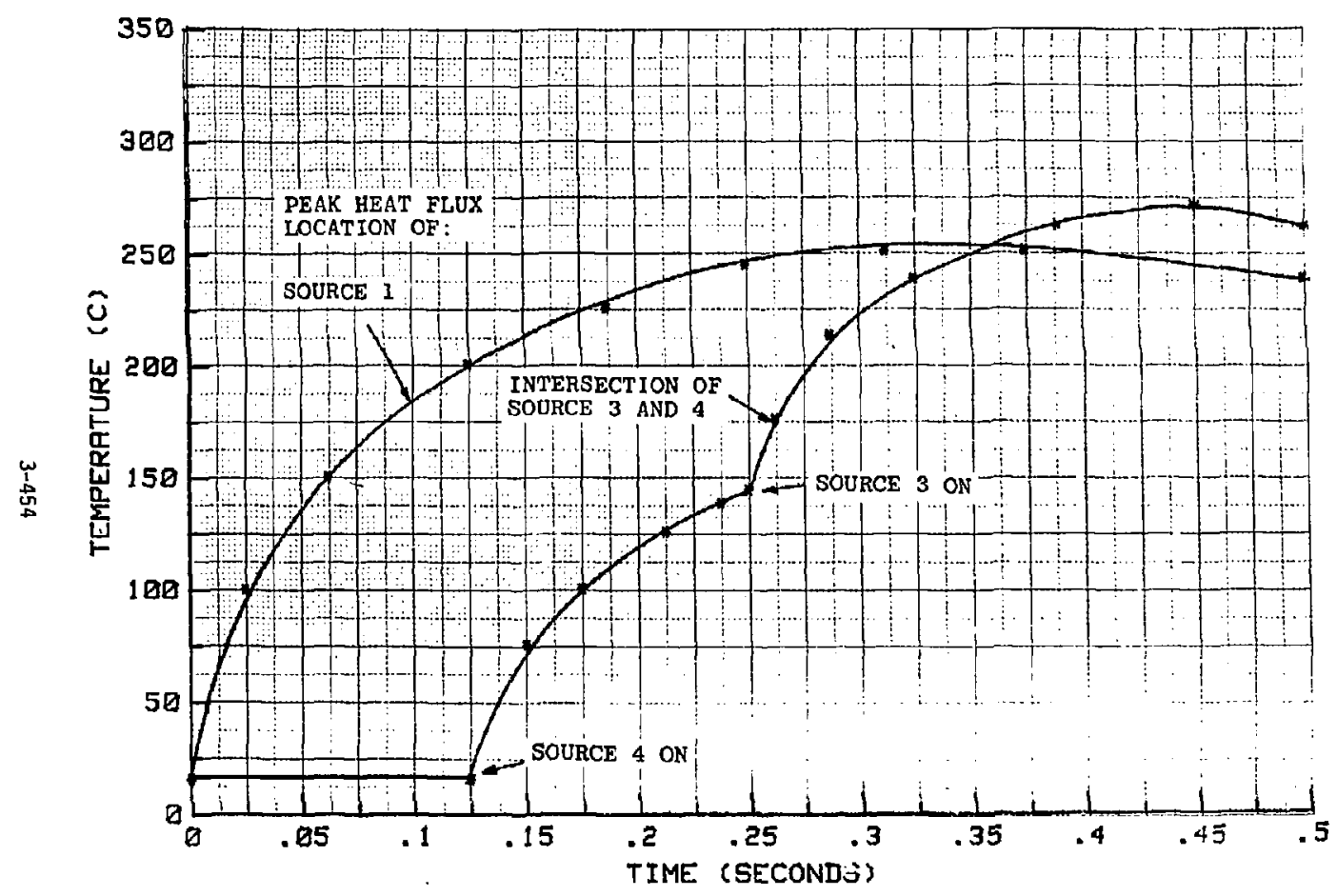

Figure 3-187. The begin-life maximum temperature of the bean dump's front surface during reactor startup.

1 


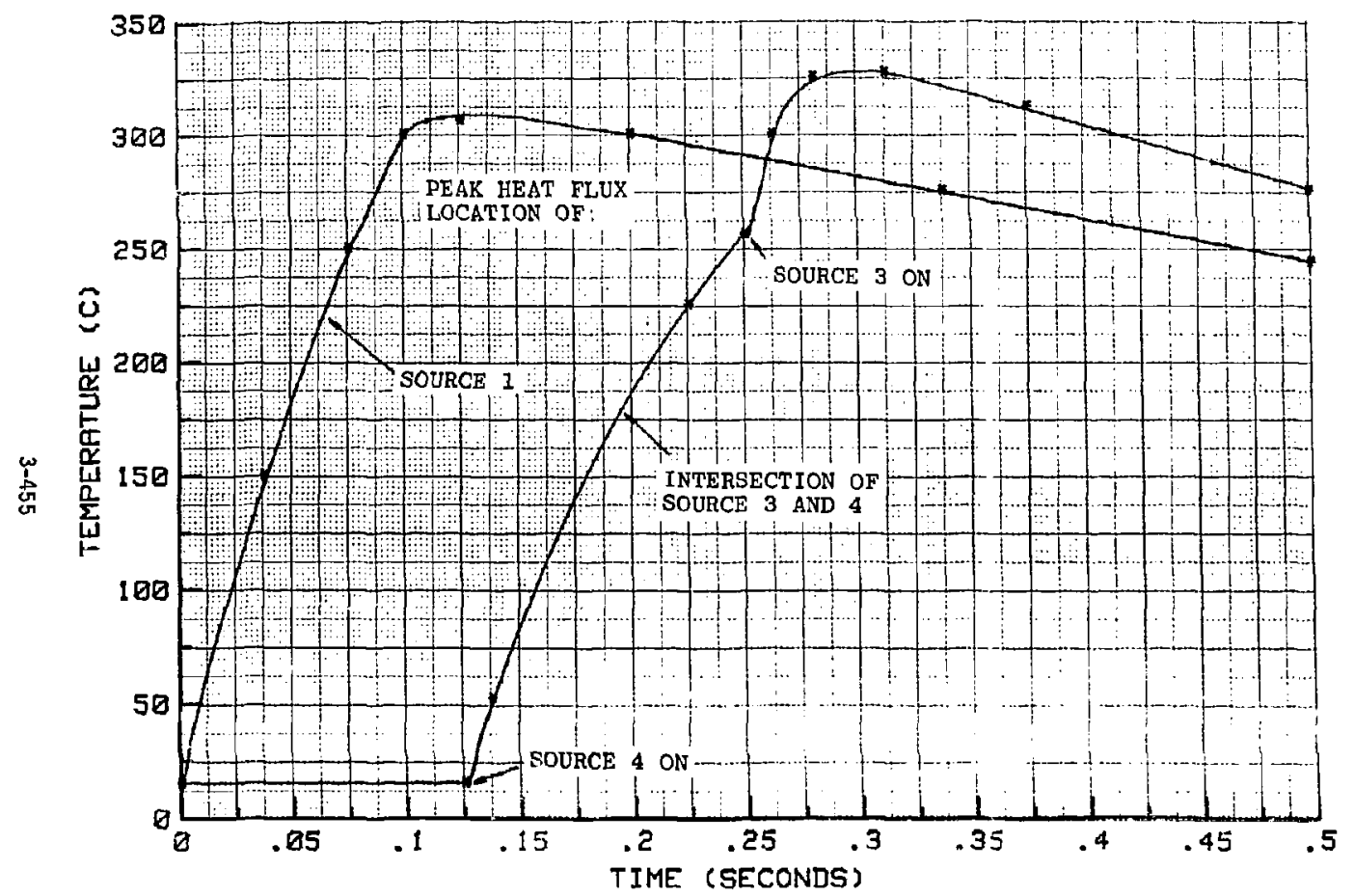

Figure 3-188. The end-life maximum temperature of the beam dump's front surface during reactor startup. 
Conclusions. The temperatures of the panel during reactor startup are well below melt temperatures even though the beam intensity is larger than steacy-state during the $0.5 \mathrm{~s}$. Stress aue to these temperature gradients must be checked to assure the panel's integrity during startup.

\subsubsection{Central Cell Beam Dumps--Tradestudy and Dff-Normal Conditions}

Qverview. We conducted a trade study comparing three orientations of the central-cell beam dump to the incident beams and evaluating heat load and thermal performance. The orientations were: flat-vertical (15 to 25 deg angles between surface and incident beams), contoured-vertical (with a constant $15 \mathrm{deg}$ between surface and incident beams), and flat-horizontal (with -60 deg between surface and incident beams).

The peak heat load on a flat-horizontal beam dump (normal to the beamline) is greater than $1.8 \mathrm{~kW} / \mathrm{cm}^{2}$. The pumping power required is more than an order of magnitude higher than that required for the other two configurations. For the contoured-vertical, a single-phase water flow coolant system can be used to maintain the beam dump surface below its upper temperature limit.

We also considered effects of two off-normal conditions a beam offset from the center of the plasma and a loss of plasma.

Discussion. Beanline attenuation by the circular plasma in the central cell results in heat flux peaks on the dump near the edges of the plasma. We calculated heat flux distributions the three orientations, as shown in Fig. 3-189. Both vertically dumps resulted in heat loads less than the oesign criterion of $1 \mathrm{kw} / \mathrm{cm}^{2}$.

Dur analysis assumed that the dump was a panel of AMZIRC, and that water flow was single phase. The resulting pumping power is shown as a function of systein pressure in Fig. 3-190.

For the central cell beam heat flux calculations, we assumed that the beam centerlines ccincided with the centerline of the plasma. This best matches the attenuation capability of the beam with the plasm heat distribution and results in the minimum possible neat flux to the dump. To determine the sensitivity of peak beam-dump heat flux to a misalignment 


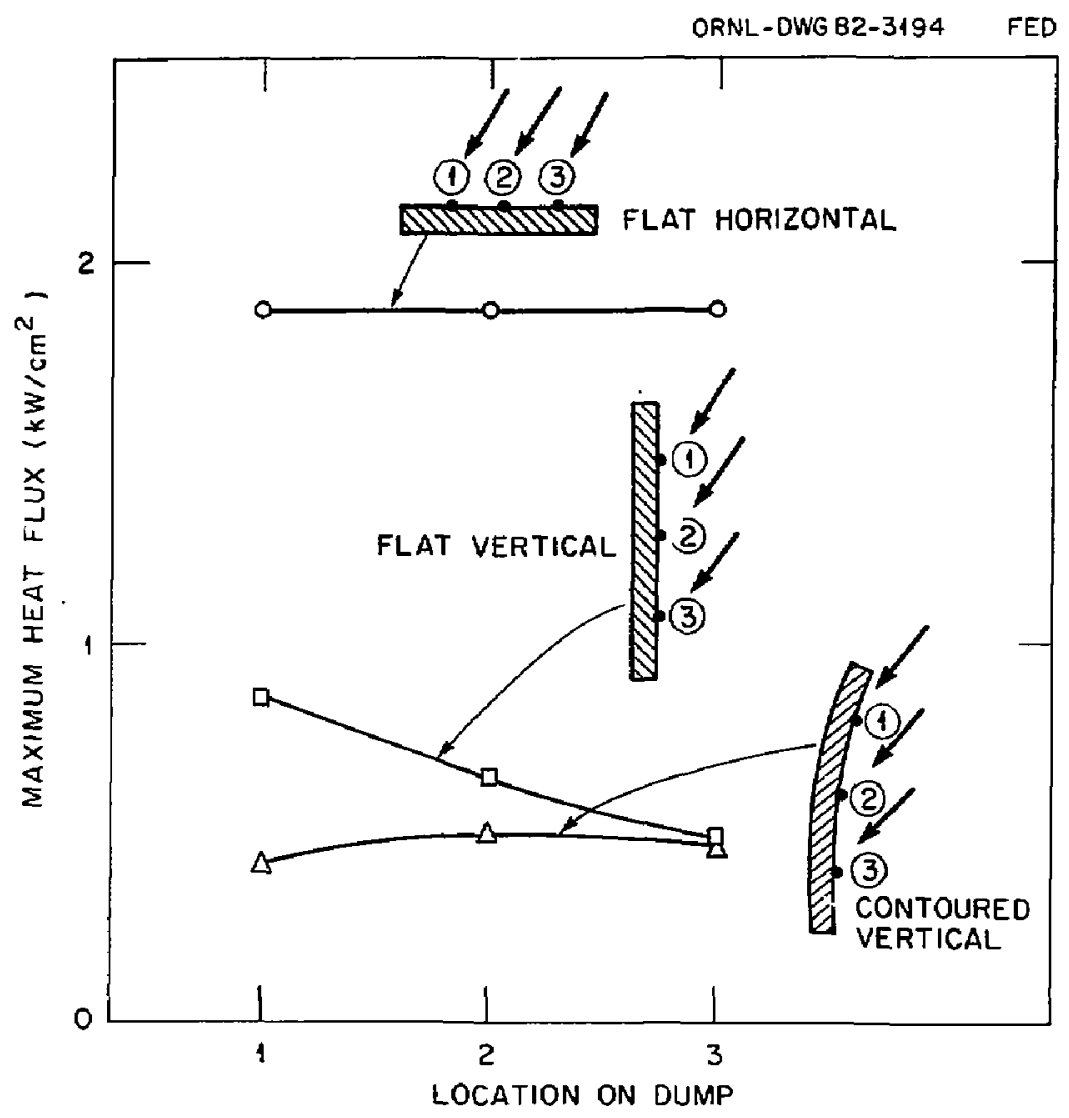

Figure 3-189. Peak heat fluxes on central cell beam dumps. 


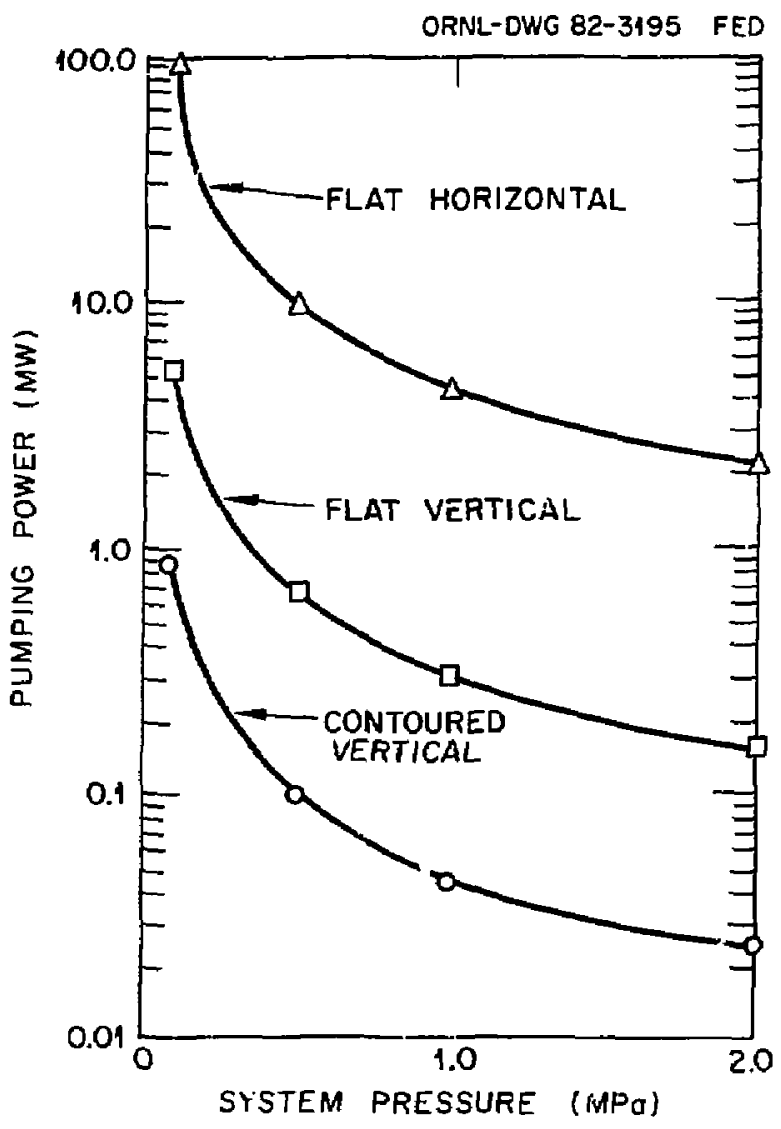

Figure 3-190. Pumping power for TDF central cell beam dumps. 
ibetween the center 1 ines of the beam and the plasma, we calculated the neat, flux aistribution for an of set of $1.0 \mathrm{cin}$. The resulting distribution is shown in Fig. 3-191. The $1.0 \mathrm{crn}$ off set corresponos to only a 0.1 deg deviation of the beam angle and results in the $30 \%$ increase in peak heat flux to the dump indicated in the figure. This indicates a likely requirement for actively controlling the beam direction.

Anotfier off-normal condition for the beam dump occurs when the plasma disappears and the beam is allowed to impinge on the dump surface without attenuation. In this case the surface teinperature of the dump rises very rapidly. Resulting surface temperature risz is shown in Fig. 3-192 for several values of flux. Times needed to increase the surface temperature by $500{ }^{\circ} \mathrm{C}$ range from 0.02 to $0.10 \mathrm{~s}$ for the three beam dump orientations, which inaicates the need for a very rapid response for beam shutdown.

\subsubsection{Beam Dumps for the Transition Region}

Overview. There are three ion beam dumps in each of the anchor cell regions, the anchor pumping beam, anchor sloshing beam and the transition pumping beam. Their locations are shown in Fig. 3-193.

Table 3-67 sumarizes heat loads, associated orientations, dump size, ano Tife.

For each dump, the objective was to minimize surface heat loads consistent with geometry and mantenance considerations. The maximum allowea heat flux is $1.0 \mathrm{kH} / \mathrm{cm}^{2}$ for both the anchor sloshing beam oump and the transition pumping beam dump, but the heat loads at normal incidence to the incoming beam exceeded that; therefore, we angled the dumps. The surfare thickness and the erosion rates are similar to those for the central cell beam dumps. Their life is approximately 0.4 full-power years. The maxinum heat ?0a0 is $260 \mathrm{~W} / \mathrm{cm}^{2}$ for the anchor pumping beam dump, and it; life is significantly longer ( 7 ful]-power years) than those of ine other aumps because of the increased surface thickness allowed and the decreased erosion rate. The results shown in the table are for a maximum surface thickness of $1.0 \mathrm{~cm}$ at a heating rate of $1.0 \mathrm{~kW} / \mathrm{cm}^{2}$. This is based on fatigue considerations for 5000 cycles. Erosion lives are based on erosion rates at $1.0 \mathrm{~kW} / \mathrm{cm}^{2}$. (This is given as a function of incident angle in Section 3.10.1.) 
ORNL-DWG 82-3197 FED

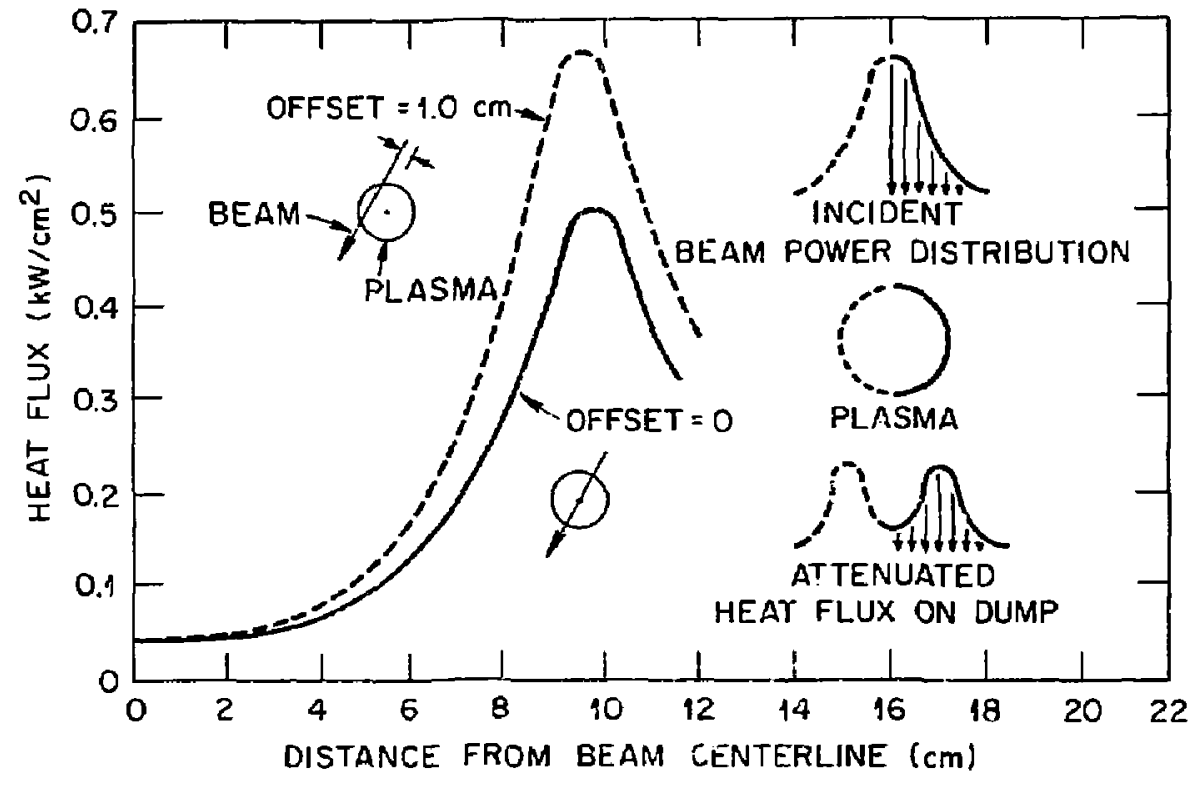

Figure 3-191. Efrect of misalignment between beam and plasma. 


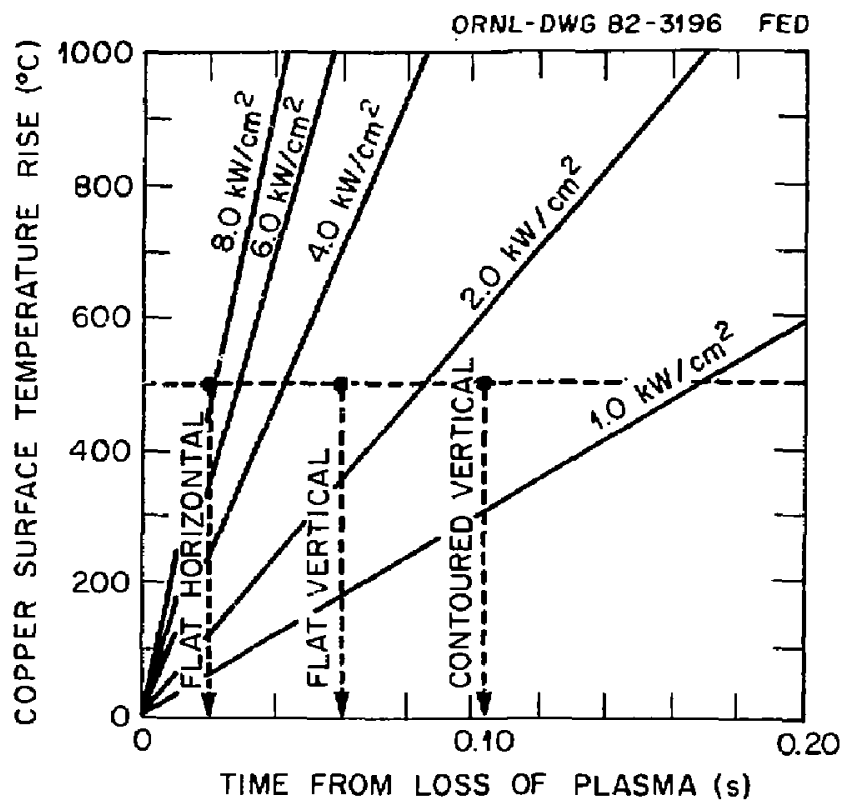

Figure 3-192. Effect of plasma loss on the beam dump surface. 
ORNL - DWG 82-3271 FED

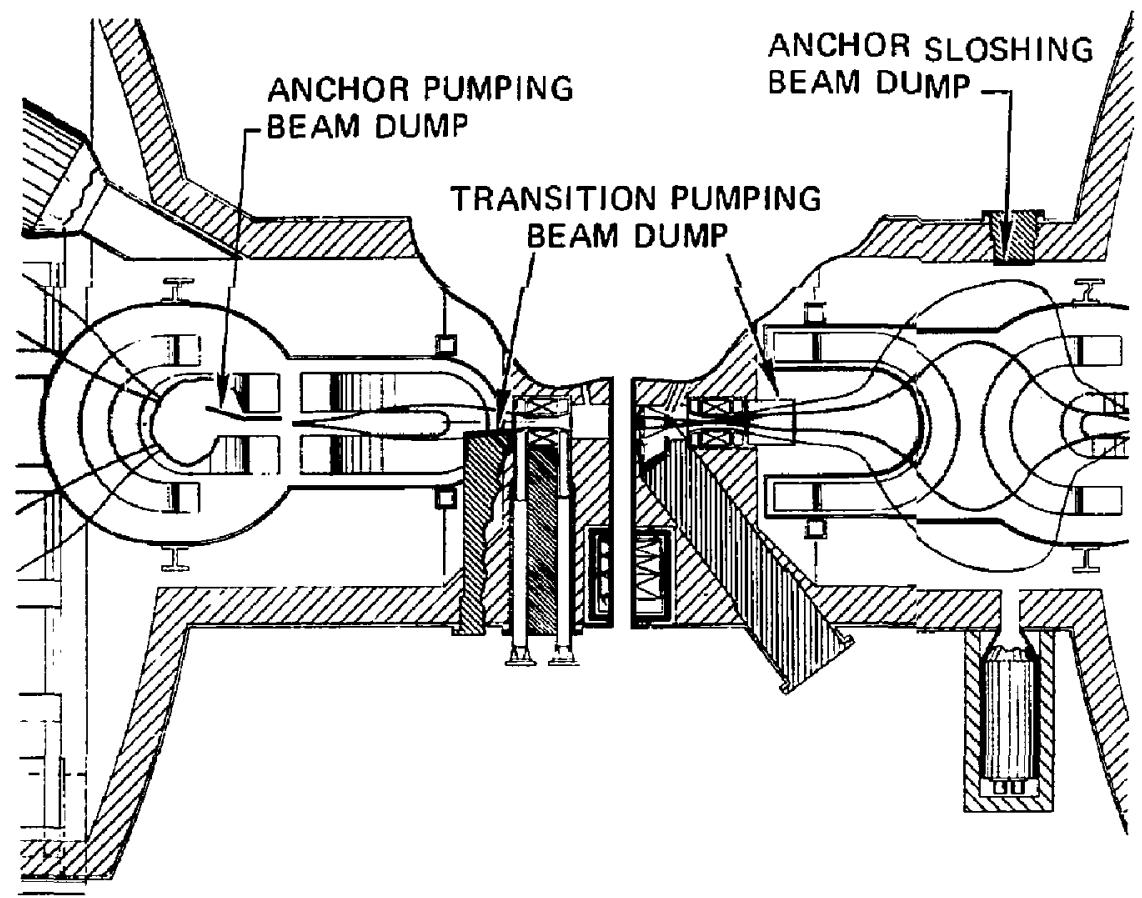

Figure 3-193. Transition region beam dump locations. 
Table 3-67. Summary of beam dump heat flux, size, and life.

\begin{tabular}{|c|c|c|c|}
\hline & Anchor pumping & Anchor sloshing & Transition pumping \\
\hline $\begin{array}{l}\text { Peak heat flux } \approx H / \mathrm{cm}^{2} \\
\text { (Angle) }\end{array}$ & $\begin{array}{l}260 \\
\left(75^{\circ} / 53^{\circ}\right)\end{array}$ & $\begin{array}{l}1000 \\
\left(60=54^{\circ}\right)\end{array}$ & $\begin{array}{l}1000 \\
\left(30^{\circ} / 60^{\circ}\right)\end{array}$ \\
\hline Surface thickness $(\mathrm{cml})$ & 4.0 & 1.0 & 1.0 \\
\hline Erosion per FPY & 0.49 & 1.6 & 1.8 \\
\hline Life ${ }^{a}$ (FPY) & 27 & 0.4 & 4 \\
\hline Dump size $(\mathrm{cm})$ & $40 \times 120$ & $30 \times 60$ & $130 \times 50$ \\
\hline
\end{tabular}

\footnotetext{
a Ena of life thickness $-0.3 \mathrm{~cm}$

b 5000 cycles
} 
Neutral beam parameters we used to obtain the heat loaus are provided in Section 3.3.

\section{Discussion}

Anchor Purnping Beam Dump. The anchor pumping beam dump lies along the edge of the plasma fan between 8.45 and $9.6 \mathrm{~m}$. This location and the dump's resulting surface heat load distribution are shown in Fig. 3-194. Seventy percent of the $116 \mathrm{~kW}$ of beam power delivered to the plasma is transmitted through to the oump. The edges of the beam are scrapeo off as it leaves the beam line. The result is essentially zero heat flux at a point greater than $40 \mathrm{~cm}$ in diameter and normal to the beam at the dump. The beam is incident at $15 \mathrm{deg}$ and $37 \mathrm{deg}$ in the two sections of the dump shown. This change of angle results in the step change in heat load distribution.

Anchor Sloshing Beam Dump. This dump is attached to the wall opposite the sloshing beam. In the beamline these are redundant beams $25-\mathrm{cm}$ apart. (This is accommodated by increasing the azimuthal dimension of the beam oump.)

The beam delivers a peak heat flux of $1.24 \mathrm{kH} / \mathrm{cm}^{2}$ normal to the beam at the dump. To reduce this heat load, the dump is oriented at 54 deg off-normal to the beam. Figure $3-195$ shows the resulting heat flux cistribution. The required dump dimensions are about $30 \mathrm{~cm} \times 60 \mathrm{~cm}$ and the predicted erosion life is about the same as that for the central-cell aumps 0.4 full-power years.

Transition Pumping Beam Dump. This dump is adjacent to the choke coil. This dump takes a total of $2.3 \mathrm{NW}$ delivered by six overlaping beams. The heat flux oistribution normal to the beam and the resulting peak heat load are shown in Fig. 3-196. The peak heat 7 lux of $1.2 \mathrm{~kW} / \mathrm{cm}^{2}$ is reduced to the maximum of $1.0 \mathrm{~kW} / \mathrm{cm}^{2}$ by orienting it as shown. However, the dump and its shield plug must clear the coil support structure radially and are, therefore, a Inaximum of $80 \mathrm{~cm}$ in axial length. This requires that a portion of the aump be oriented in the radial direction.

The transition beam dump surface area is $130 \mathrm{~cm} \times 50 \mathrm{~cm}$. Based on erosion, its life is 0.4 full-power years, which is similar to that for the sloshing beam dump. 


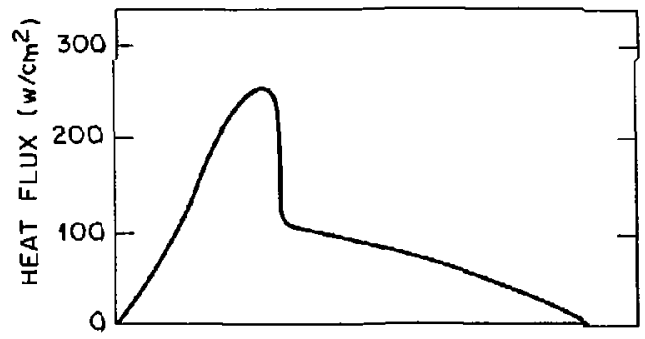

$w$
學

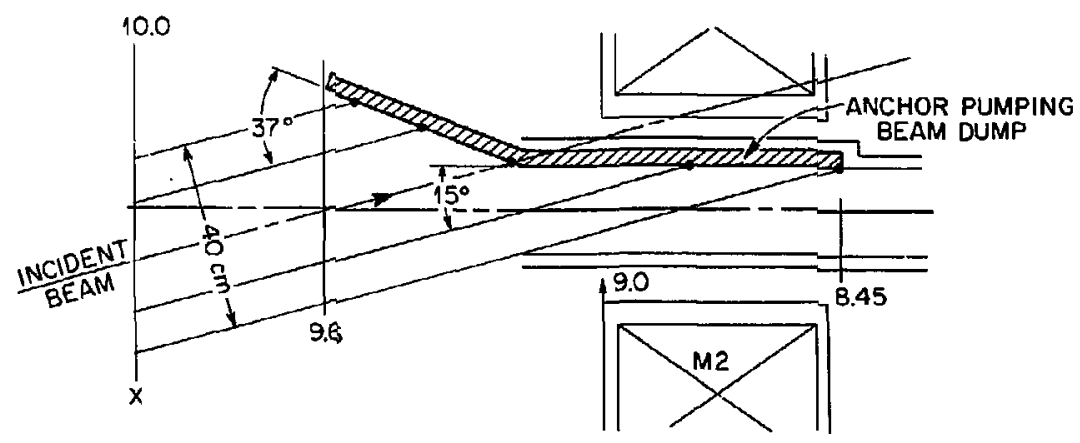

Eigure 3-799. Surface heat iudu ont tire amotur pumpting beant ưump. 


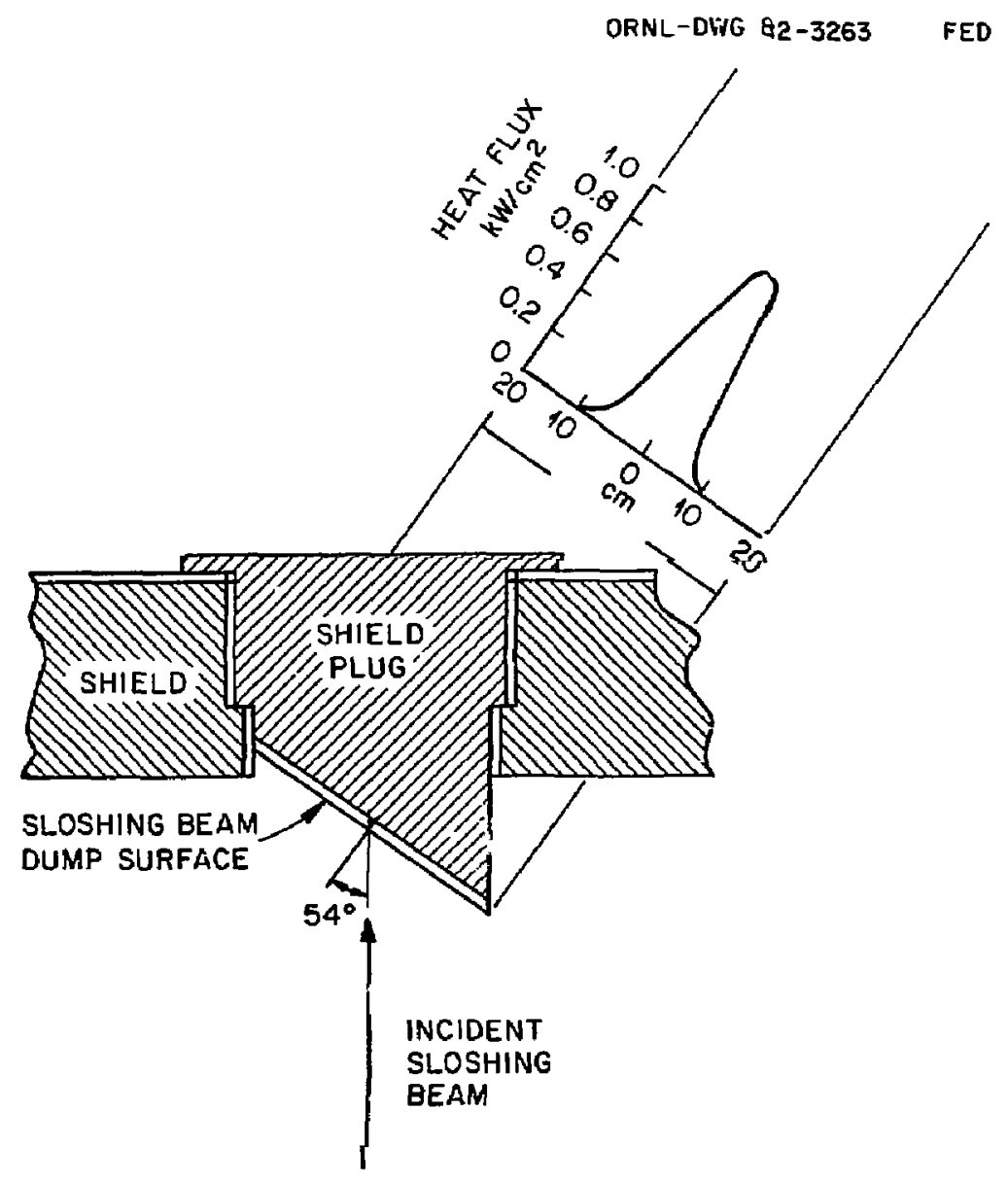

Figure 3-195. Surface heat loao on the anchor sloshing beam qump. 


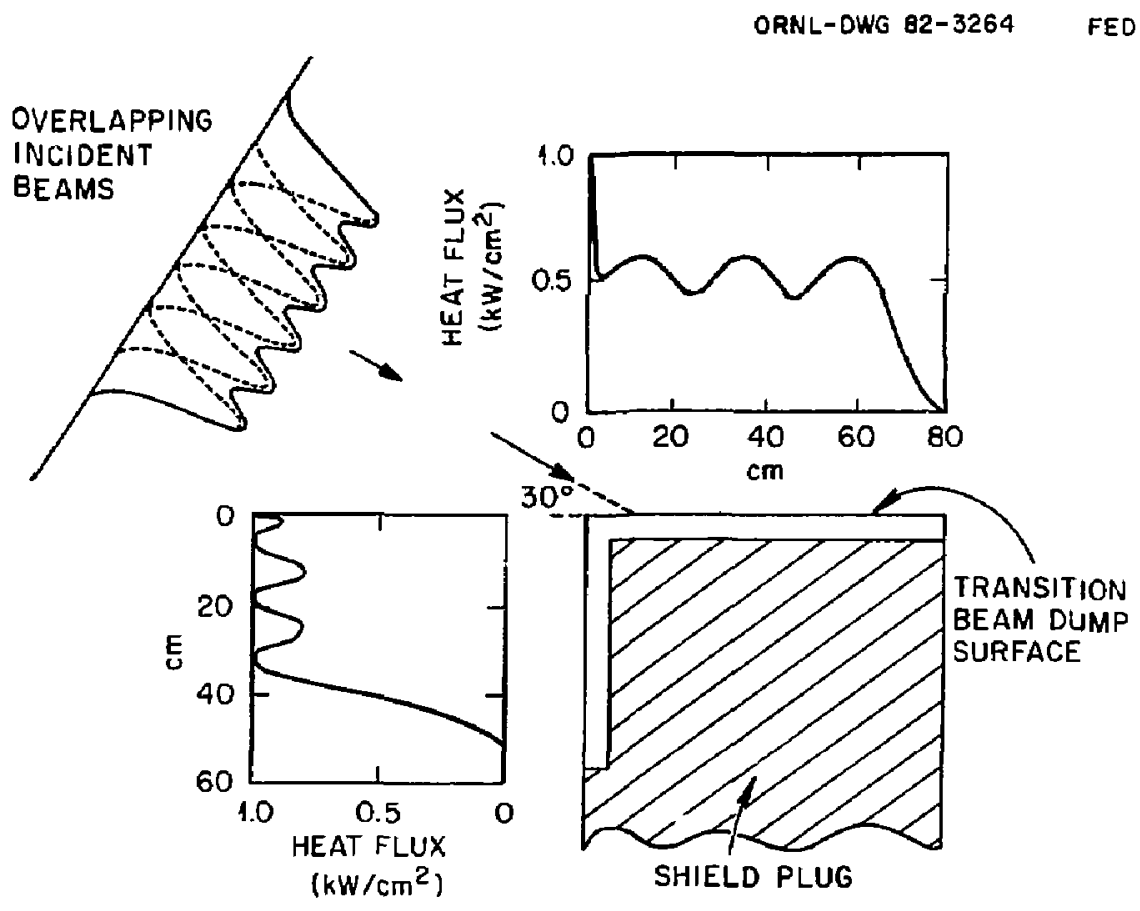

Figure $3-196$. Surtace heat load on the transition pumping beam dump. 
End Cell Cost Trade Study. We parformed an end ceil trade study to determine the sensitivity of end cell cost to end cell size. Because it occurred midway through the TDF effort, our study was based on the initial conical end celt corfiguration shown in Fig. 3-197. Four elements were considered: the vacuum vessel, shiela, eno dump, and building.

Results showed that the incremental cost of the device mer meter addeo to the half length is approximately $\$ 5 \mathrm{~m}$. The vacuum vessel ala builoing costs were the major ones; that of the end oump decreases with increasing end cell length. However, this component accounted for only about $1 \%$ of the total. Differential costs are shown in Fig. 3-198 ano summarize the effect of increasing machine half-ier.

We calculated costs for each of the components for three end cell lengths. These corresonded to comes on the conical end cell at $27 \mathrm{~m}, 31 \mathrm{~m}$, and $35 \mathrm{~m}$ from the half-length as shown in Fig. 3-199. Also shown is the vessel surface area as a function of length. The vertical iines at the three reference lengths represent the arca of the end dome addea to the areas for the cylinderical ano conical portion of the she11. Lalculated thicknesses for the vacuum vessel are based on stability requirements for a cylinuerical shell subjected to a uniform external pressure. We used the maximum end cel] radius for each of the reference end cell lengths. Resulting thicknesses are shown as a function of external pressure in Fig. 3-200. Relative costs of the vacuum yessel are baseo on scaling structural material volumes--the product of surface area (from Fig. 3-199) and snell thickness (Fig. 3-200) at 0.10 MPa (one atmosphere). Resulting volumes are conservative, but they do provide a bas is for relative cost. The reference cost was the oefined vessel cost at the time of the trade study: $\$ 12 \mathrm{ll}$ at a length of $31 \mathrm{~m}$. Relative material volumes were 0.48 and 1.75 at 27 and $35 \mathrm{~m}$ machine half-lengths, respective $1 y$, resuiting in costs of $\$ 5.8 \mathrm{M}$ and $\$ 21 \mathrm{M}$.

Shield cost was based on the volume of ordinary concrete at $\$ 480 / \mathrm{m}^{3}$. The differences in volume required were based on the vacuum vessel surface areas shown in Fig. 3-199 and a canstant 60-cm thickness of concrete. We cliose the thickness based on neutronics estimates pei :ormed early in the program. Resulting cost increments over the $27 \mathrm{~m}$ length are $\$ 274 \mathrm{~K}$ and $\$ 610 \mathrm{~K}$ for $37 \mathrm{~m}$ and $35 \mathrm{~m}$ machine hilf-lengths, respectiveiy. 


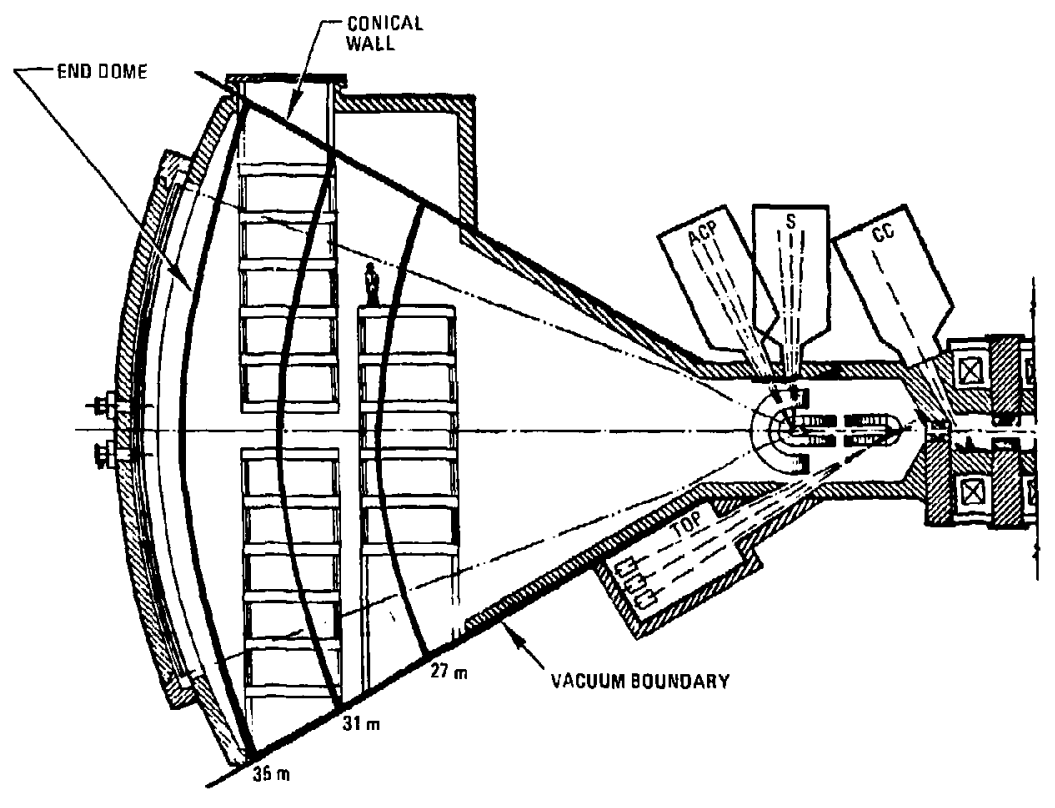

Figure 3-197. End cell plan layout used in cost trade study. 


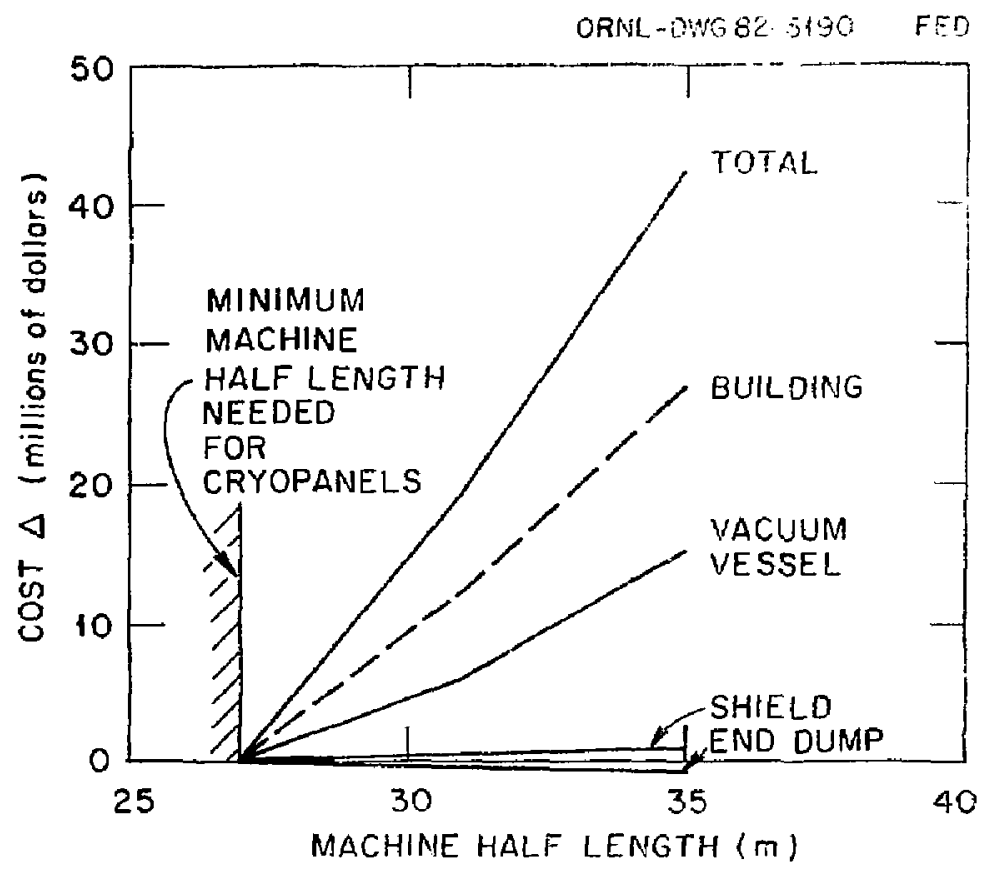

Figure 3-198. Summary of end cell cost study. 


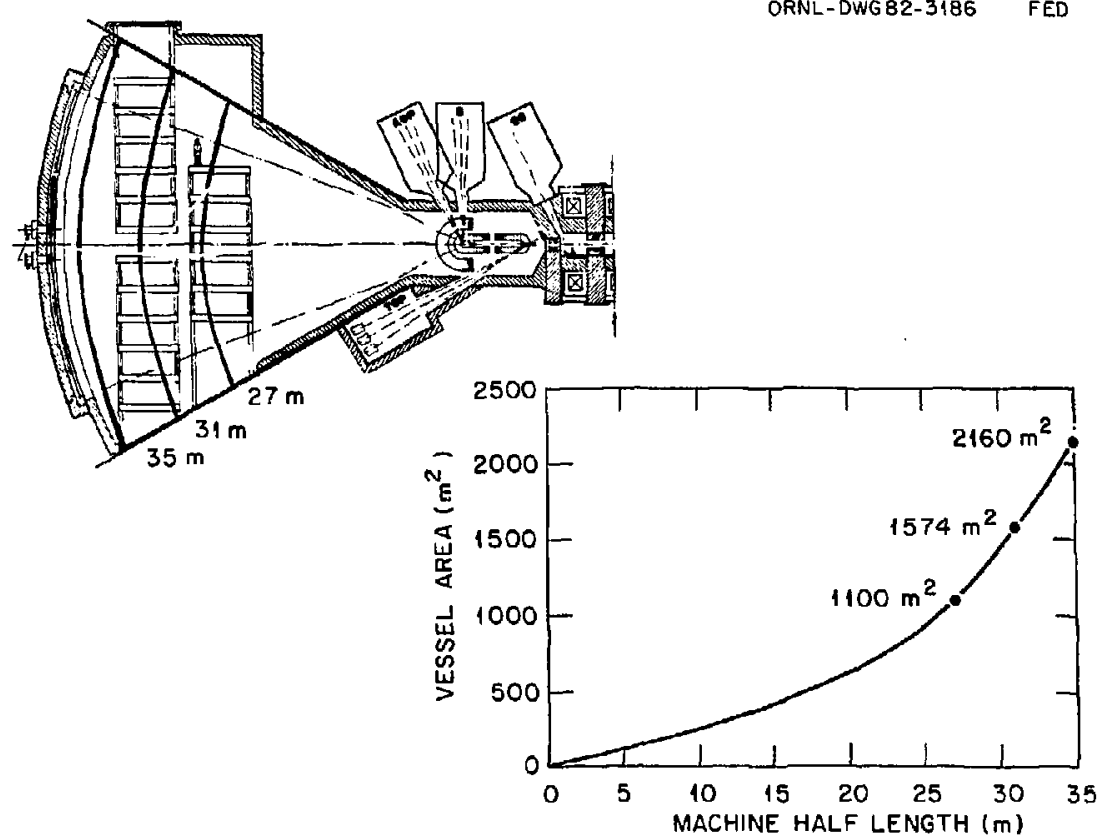

Figure 3-199. Vacuum vessel surface area. 


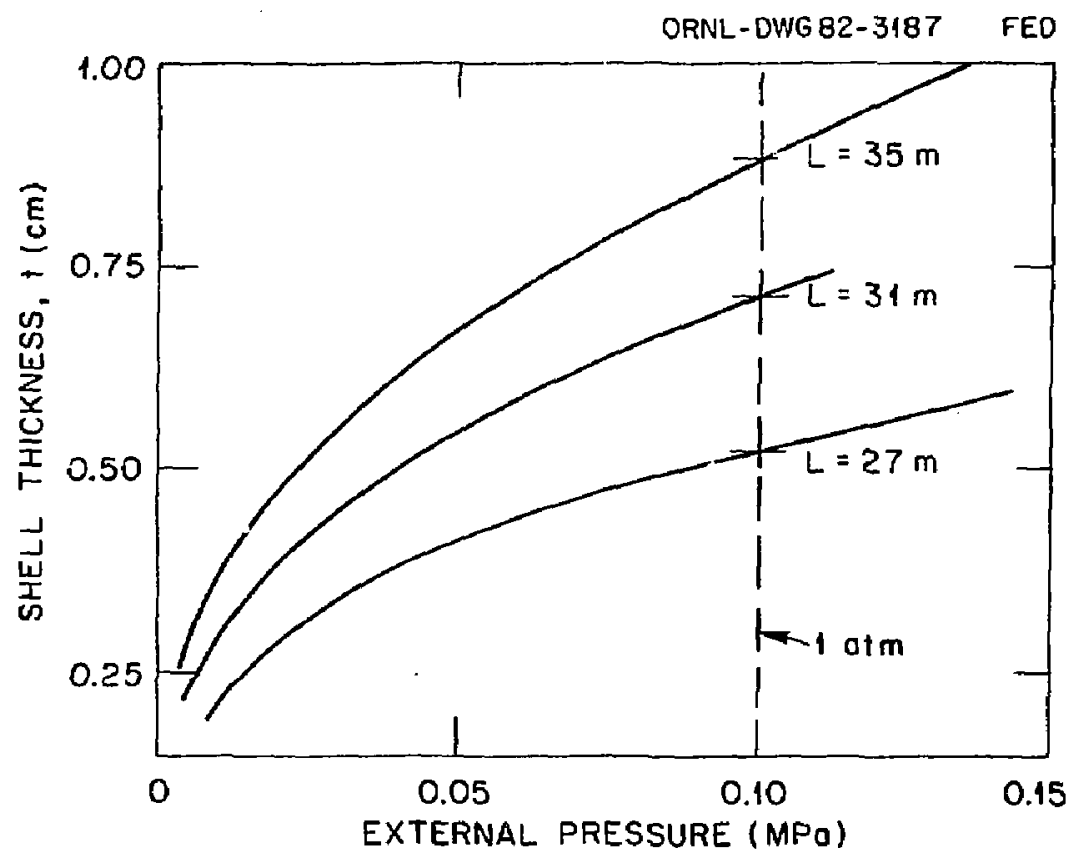

Figure 3-200. End cell vacuum vessel thicknesses used for trace study relative volumies. 
We determined the end aump cost based on $\$ 246 \mathrm{~K}$, the value we initially defined as necessary for a machine half-length of $31 . \mathrm{m}$. It was assumed that pumping cost at this length was equal to the panel cost. For shorter half-lengths, the heat loads on the dump increase, as shown in Fig. 3-201. From this data we determineo that heat loaos for the 27 and $31 \mathrm{~m}$ lengths were proportionally larger than the $150 \mathrm{w} / \mathrm{cm}^{2}$ heat load at $35 \mathrm{~m}$. We calculated requireo pumping power for each machine half-length ano assumed that costs would be proportional.

Table 3-68 lists end dump parameters and pane 1 and pumping power costs. Although the panel size and cost is reduced with decreasing machine halflength, the total cost increases because of lrigher pumping power; therefore, it shows up as a negative increment. The final cost is on the order of that for the sitielo.

We also determined reactor building costs for the three lengths, incluoing appropriate increases in machine diameter. They were $\$ 47.7 \mathrm{M}$, $\$ 60.4 \mathrm{M}$, and $\$ 74.9 \mathrm{M}$ for 27,31 , and $35 \mathrm{~m}$ lengths respectively. The differential cost, as inoicated in $F$ ig. 3-198, was the largest contributor $t_{0}$ the overall cost.

\subsubsection{First Wall of the Central Cell}

Uverview. The first wall regions of the central cell near the bean dumps (Fig. 3-202) are subjected to surface heat loads of approximately $200 \mathrm{~W} / \mathrm{cm}^{2}$. They will require a cooled surface that should be separate from the shield so that replacement is relatively easily. The cooled first wall unit must surround the beam dumps and accommodate other components, such as those requireo for fueling. Access is made available when test modules and their shield plugs are removed.

Analysis of an array of cooling tubes showed that the first wall can be cooled with single-phase flow, either axial or circumferential. Stamless steel tubes with 1.2-mm thick walls will have a maximum wall temperature of $400^{\circ} \mathrm{C}$. Copper AMZIRC is more promising than stainless as a first wall material because the wall thicknesses can be more easily increased, allowing for repair of possible local erosion. 


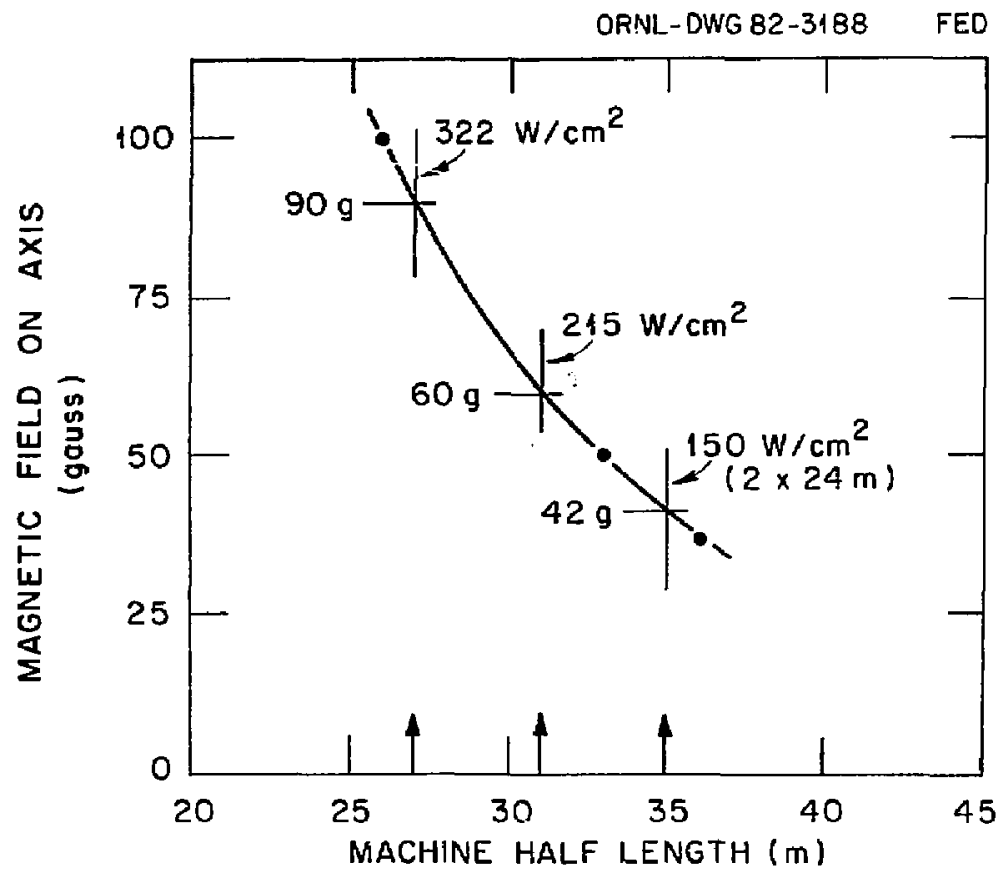

Figure 3-201. End dump surface heat loads baseo on magnetic fielo strength. 
Table 3-68. End oump parameters and costs.

\begin{tabular}{llll}
\hline Half length $(\mathrm{m})$ & 27 & 31 & 35 \\
Dump size $(\mathrm{m})$ & $1.4 \times 17$ & $1.7 \times 20.5$ & $2.0 \times 24$ \\
Dump area $\left(\mathrm{m}^{2}\right)$ & 24 & 35 & 48 \\
$\rho_{\text {anel cost }(\$)}$ & 169,000 & 246,000 & 337,000 \\
q $_{\text {AvE }} / \mathrm{q}_{\text {PEAK }}\left(\mathrm{W} / \mathrm{cm}^{2}\right)$ & $130 / 322$ & $80 / 215$ & $60 / 150$ \\
Pumping power $(\mathrm{kW})$ & 850 & 290 & 110 \\
Pumping cost $(\$)$ & 721,000 & 246,000 & 93,000 \\
Total cost $(\$)$ & 890,000 & 492,000 & 430,000 \\
$\Delta$ cost & $-\ldots$ & $(398,000)$ & $(460,000)$ \\
\hline
\end{tabular}



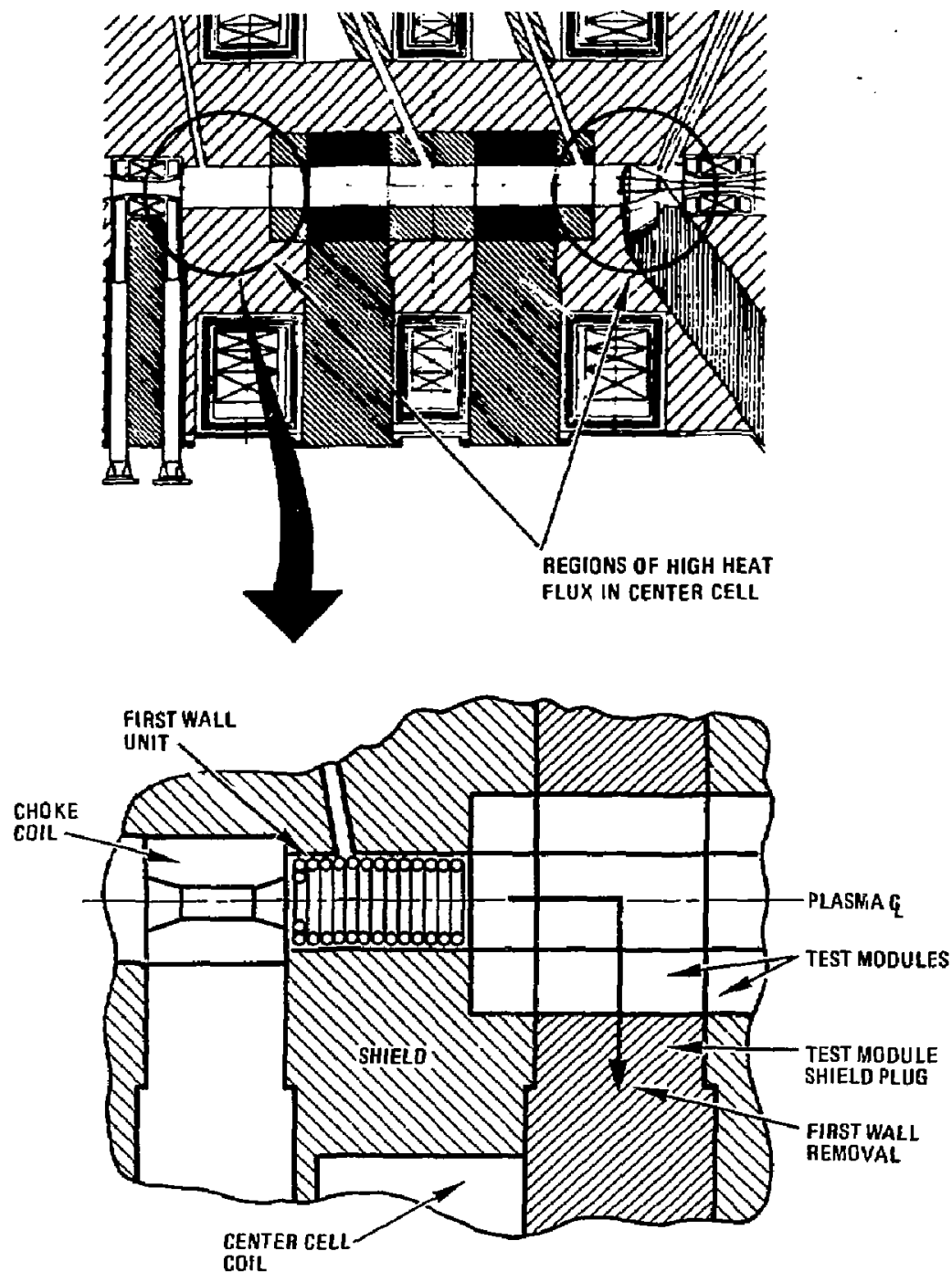

Figure 3-202. First wall of the central cel1. 
ijcussion. We made heat transfer calculations for water coolant flowing in . ixial ano circumferential directions, using a first wall region $1.0 \mathrm{~m}$ in diameter and $1.0 \mathrm{~m}$ long.

We used a "one-region mode?" in which the physical properties were taken to be constant, allowing us to treat the whole coolant passage as one region. our equations were as follows:

For conduction in the solio wall, we solved

$T_{w, \text { out }}=T_{w, \max }-q_{s} \cdot t_{w} / k_{w}$,

where

$T_{w, \max }=$ outsiae surface temperature

$T_{w, \text { out }}=$ temperature at inner wall,

$i_{s}=$ heat flux,

$t_{w}=$ wall thickness,

$k_{w}=$ thermal conauctivity.

For the heat balance in coolant, we used

$\rho U C_{p} A\left(T_{\text {out }}-T_{\text {in }}\right)=q_{S} \cdot S$,

where

$\rho=$ coolant density,

$U=$ coolant velocity,

$A=$ îlow area,

$S$ = surface area.

Heat transfer from the wall to coolant was

$q_{s}=h\left(T_{w, \text { out }}-T_{\text {out }}\right)$ 
where

$T_{\text {out }}=$ coolant exit temperature,

$h=$ fi $m$ coefficient,

$\mathrm{h}=0.023 \operatorname{Re}{ }^{0.8} \mathrm{Pr}^{0.4} \cdot \mathrm{k} / \mathrm{d}_{\mathrm{e}}$

where

$R_{e}=$ Reynolds number,

$\mathrm{Pr}=$ Prandt 1 number,

$k=$ thermal conductivity,

$\mathbf{d}_{\mathbf{E}}=$ oiameter.

Pressure drop is

$\Delta p=\left(1.5+\lambda L / d_{e}\right) \rho U^{2} / 2$,

where

$L=$ Length of pipe,

$\lambda=0.3164 \mathrm{Re}^{-1 / 4}$.

Pumping power is $W_{p}=$ AUAp.

We solved Eqs. (3-92) and (3-93) simultaneously, giving the coolant outlet temperature and coolant velocity.

We assumed the first wall consisted of an array of tubes with $2.0 \mathrm{~cm}$ outer diameters. The wall temperature was limited so that either the heated surface temperature did not exceed the maximum allowable temperature or the cooled surface temperature dio not exceed the coolant saturation temperature.

Table 3-69 shows the results for circumferential coolant flow; those for axial flow are similar.

hot only are maximum temperatures of the coolant and structure limited by thermal stress; so is the temperature difference in the structure. We used differences of $150^{\circ} \mathrm{K}$ for $316 \mathrm{SS}, 100^{\circ} \mathrm{K}$ for copper, and $70^{\circ} \mathrm{K}$ for aluminum. This limited the SS316 wall thickness to be less than $1.2 \mathrm{~mm}$. 
Table 3-69. First wall parameters for circumferential coolant flow. The heat load was $2.0 \mathrm{Mk}^{-\mathrm{m}^{-2}}$.

\begin{tabular}{|c|c|c|c|c|}
\hline Material & AMLIRC & Aluminum & S\$316 & \\
\hline$a(\mathrm{~mm})$ & 12.0 & 12.0 & 17.6 & 15.0 \\
\hline$t_{w}(m m)$ & 4.0 & 4.0 & 3.2 & 2.5 \\
\hline$k_{w}^{\prime \prime}\left(w \cdot m^{-1} k^{-1}\right)$ & 320 & 200 & 16 & 16 \\
\hline$P_{\text {olit }}(M P a) / T_{\text {sat }}\left({ }^{\circ} \mathrm{C}\right)$ & $1.0 / 180$ & $1,0 / 180$ & $1.0 / 180$ & $1.0 / 180$ \\
\hline $\mathrm{T}_{\mathrm{w}, \text { al lowable }}\left({ }^{\circ} \mathrm{C}\right)$ & 320 & 200 & 400 & 400 \\
\hline$T_{w, \max }\left({ }^{\circ} \mathrm{C}\right)$ & 205 & 200 & 330 & 400 \\
\hline$T_{w, \text { out }}\left({ }^{\circ} \mathrm{C}\right)$ & 180 & 160 & 180 & 87.5 \\
\hline$\Delta T_{W}\left\langle{ }^{0} C\right\rangle$ & 25 & 40 & 150 & 312.5 \\
\hline $\mathrm{T}_{\text {in }} / \mathrm{T}_{\text {out }}\left({ }^{\mathrm{O}} \mathrm{C}\right)$ & $60 / 95.2$ & $60 / 88.5$ & $60 / 78.6$ & $60 / 64.2$ \\
\hline$\left.U(\pi) \cdot s^{-1}\right)$ & 3.90 & 4.82 & 3.43 & 20.7 \\
\hline $\operatorname{Re}$ & $1.57 \times 10^{5}$ & $1.94 \times 10^{5}$ & $2.02 \times 10^{5}$ & $1.04 \times 10^{6}$ \\
\hline Pressure drop $(\mathrm{kPa})$ & 26.1 & 38.7 & 16.5 & 523 \\
\hline Pumping power $(\mathrm{kH})$ & 1.15 & 2.11 & 1.37 & 192 \\
\hline
\end{tabular}




\subsubsection{Blistering of the Beam Dumps}

Overview. The avajlable data on b7istering unoer hydrogen-species ion bombarcment suggest that the TUF beam dump will not blister. At most, a few olister layers less than $0.5 \mu \mathrm{m}$ thick will form and spall early in operation. Continued operation will not result in furtner generations as the metal reaches an equilibrium gas content. A single blister layer will be atout $0.4 \mu \mathrm{m}$ in copper and $0.3 \mu \mathrm{m}$ in molybdenum or tungsten.

The data we surveyed does not include the projected conditions for the beam dump, and verification experiments will be required. The very high particle flux, energy distribution in the beam, and device operating cycle need to be simulated to evaluate the expected operating performance.

1. Beam Conditions. The beam will be a 50:50 D-T mixture at $80 \mathrm{keV}$. The average energy per particle at the beam dump is 67 $\mathrm{keV}$, and the design heat load on the beam oump is $1 \mathrm{~kW} / \mathrm{cm}^{2}$. This is equivalent to a current particle density of $1.02 \times 10^{17}$ $\mathrm{cm}^{2} \cdot 5$.

2. Beam Dump Material. Candidate materials are copper AMZIRC, molybdenum, and tungsten. Water cooling can limit the surface temperature of a copper al loy beam dump to $320{ }^{\circ} \mathrm{C}$. The water in let temperature is $\sim 50{ }^{\circ} \mathrm{C}$.

Background Information. Draft review articles by Langley ${ }^{41}$ ana Behrisch ${ }^{42}$ sumnarize the available blistering data. We have surmarized the material.

Experimental bombaroment of copper, molyboenum, and tungsten with $\mathrm{H}^{+}$or $\mathrm{D}+$ in a range of conditions has caused blistering. Most of the experiments have been at or below $20^{\circ} \mathrm{C}$, with few data for higher temperatures. The blistering resuits from relatively low hydrogen solubility, strong nydrogen-defect interactions, and relatively high hydrogen diffusion rates. As a consequence, the blistering is a strong function of bombarament temperature and_has a modest ion flux dependence.

The approximate maximum temperature at which bisters form in hydrogen bombarded copper is $75^{\circ} \mathrm{C}$, while for molybdenum it is $20^{\circ} \mathrm{C}$. The critical fluence for bi isters to form ranges from $l$ to $10 \times 10^{18}$ particles $/ \mathrm{cm}^{2}$. The thickness of blister lids that exfoliate at the critical fluence approximately equals to the mean prujected range of the particle. For $80 \mathrm{keV}$ 
$\mathrm{D}+$, this distance is $0.43 \mu \mathrm{m}$ for copper, $0.28 \mu \mathrm{m}$ for molybdenum and 0.30 um for tungsten (see Table 3-70). The range is less for $80-k e V ~ H+$, and probably lightly greater for $80 \mathrm{keV} \mathrm{T+}$.

Blistering is repetitive unoer helium bombardment for some experimental conditions, but there is no evidence for repetitive blistering under hydrogen isotope bombardment.

blistering is usually reauced or eliminated by the following conoitions:

- Higher temperatures.

- Bombarament with distributed energies, rather than monoenergetic ions.

- Achieving high fluences $\left(>10^{19}\right.$ ions $/ \mathrm{cm}^{2}$ ).

All of these conditions prevail for the JUF beam dump so any blistering should be a transient phenomeron. It is imperative to note, however, that the projected service fluences of $1 \times 10^{17}$ particles/ $/ \mathrm{cm}^{2} . \mathrm{s}$ for long-term, continuous service are totally unexplored conditions. The fluence for j000-hour operation would be $-4 \times 10^{23}$ particles $/ \mathrm{cm}^{2}$, about three orders of magnitude beyond the available experimental data.

\subsubsection{P]asma End Lump}

Overview. In the tandem mirror fusion reactor configuration ions leak out along the anchor cell magnetic field lines into the end cell regions. This plasma fan must be caught by an energy collector, such as a heat exchanger. The heat exchanger for. TDF is the plasma end dump. The plasma is oumped onto its surface and the waste heat is carried away by a cooling medium. Although there are methoos of using the heat (e.g. direct electrical conversion), they are not considereo because of high capital cost.

The overall configuration and spatial orientation of the plasma end aump is shown in Fig $3-174$. Each dump is located $28 \mathrm{~m}$ axially from the center of the machine and must accommoate maximum plasma heat flux of $350 \mathrm{w} / \mathrm{cm}^{2}$ and a total power of $27.25 \mathrm{~kW}$ per end cell. The gimensions of the plasma footprint at the $28-\mathrm{m}$ point are approximately 2 by $17 \mathrm{~m}$. The dump lifet ime requirement is $\geq 0.5$ full-power years.

Design Summary. The cump is an array of heat-exchanger panels joined in serjes to comon inlet and outlet headers. The total area of the panel array is larger than the plasma fan footprint by approximately $1 / 4$ to $1 / 2 \mathrm{~m}$ on all sides. 
Table 3-70. Projectea range of duterium ions in beani-aump materials. a

\begin{tabular}{|c|c|c|c|c|}
\hline \multirow[b]{3}{*}{ Material } & \multicolumn{4}{|c|}{$\begin{array}{r}\text { Range for given energy } \\
(\mu \mathrm{m})\end{array}$} \\
\hline & \multicolumn{2}{|c|}{$80 \mathrm{keV}$} & \multirow{2}{*}{$\begin{array}{c}40 \mathrm{keV} \\
\text { Deuterium }\end{array}$} & \multirow{2}{*}{$\begin{array}{c}27 \mathrm{keV} \\
\text { Deuterium }\end{array}$} \\
\hline & Deuterium & Hyoirogen & & \\
\hline Coppar & $0.43^{b}$ & 0.36 & 0.23 & 0.17 \\
\hline Molyodenum & $0.28^{C}$ & 0.24 & 0.15 & 0.11 \\
\hline Tungsten & 0.30 & 0.25 & 0.16 & 0.11 \\
\hline
\end{tabular}

${ }^{\text {a }} \mathrm{bata}$ is taken from Stopping Powers \&nd Range of Ions in Matter. 3 , edited by H. H. Anciersen \& J. F. Ziegler (Pergaman Press, 1977).

${ }^{\mathrm{b}} \mathrm{The}$ standard deviation is $0.13 \mathrm{um}$; the full width at half-maximum of distribution is $0.30 \mu \mathrm{m}$.

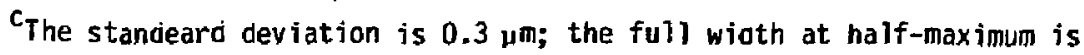
$0.2 \div \mu \mathrm{m}$. 
Each Danel consists of a corrugated sheet of metal weloed to a flat sheet forming several channeis through which water flows. Each panel has one inlet and one outlet and a manifold at the top and bottom so that the water flurs past the plasma impingement zone once. The basic configuration is shown in Fig. 3-165.

Initially, this oump was located about $30 \mathrm{~m}$ from the machine's center. The heat deposition and fan size there required a 28-panel array spanning the $24 \mathrm{~m}$ of the plasila footprint. In a later effort to reduce the size of the machine, we moved the oump inward to $Z=28 \mathrm{~m}$. Here, the heat flux is higher and low conductivity materials, such as stainless steel, are unsuitable. Thus, we chose TZM, a molybdenum alloy. Since TZM panels are not available commercially, they were designed by General Dynamics based on an availatle panel of stainless stee 1.43

After analyzing the design, we addeo braces to proviae structural rigidity and prevent warping caused by the thermal stresses present during operation. With this reinforcement, the panel's design life is one full-power year before needing replacement. The dump assembiy is located in the end cell on the rear wall. Since we did not want to remove the end dome to access them, we added a small box-like protrusion to the end wall to house the assembly. Sections of this house (uisible in Fig. 3-173) are removable to provide access: In designing the dump for accessibility we also considered that the heat flux is higher in the center of the plasma fan than at the edges. This means that the center panels of the assembly could neeo replacement, while-the end panels were still usable. To solve this problem, the array woulo be made in four or five sections, individually replaceable.

Several factors oictated the overall size. First, the panel must extend beyond the $2 \mathrm{~m}$ wiath of the plasma fan. Second, the channel cross sectional area and the overall flow rate are oictated by cooling requirements. Since the panel has one inlet and one outlet, the manifcld requirements limit the number of channels per panel. Commercially available materials determine the width of the panel. Rolled sheet stock is a maximum of $36 \mathrm{in}$. wide. Thus, we chose a panel width of $34 \mathrm{in.}$ with 16 channels per panel. The panel length of 107 inches is not a problem. The main inlet/outlet manifold for the panel bank assembly must carry the full cooling water volume flow. The four- or five-section array allows for as many inlet and outlet headers, each carrying $1 / 4$ or $1 / 5$ the total flow. This gives a more complex supply/return water system outside the machine. 
The manifolds run paralle] to the plasma fan on both ends of the panel assembly and are set slightly back from the plane of the aump panel. Structural frame support is mounted on the manifold piping between inlets and outlets. Brackets are mounted on the inside wall of the housing on which the panels are hung.

The inlet and outlet piping, as well as support brackets, boit onto flanges on the manifold piping. This facilitates changing indivioual panels. All the piping used in the water cycle of these dumps is commercially available, so little manufacturing is required beyand the paneis.

Installation is simple. Sections on the side of the house are removed, and the array is lowered into place by an overheaa crane and hung on the brackets. The only o:her installation involves attaching the header pipes to the main water supply and return lines.

System Requirements. Systen requirements for the plasma end dump are: summarized in Table 3-71.

Design Analysis

Thermooynamic Characteristics of End Dumps. It is advantageous to move the end dump toward the center of the reactor to reduce the size and thus the cost of the end-cell region. However, as the beam dump toward the machine center, the maximum heat flux on the dump increases, and some practical factors limit the magnitude the oump can handle. At some location, a simple heat transfer panel will not accommodate the heat flux, and a high-cost, high-heat transfer panel must be incorporated. The maximum heat flux occurs along the reactor center iine, therefore, we calculate it at various distances along the axis.

The following conservation of flux equation is applicable:

$B_{E N D} A_{E N D}=A_{C C} B_{C C}(1-B)^{l / 2}$,

where $B_{c c} \quad=$ the central cell magnetic field with out plasma $(T)$,

$1-B=$ correction of the central cell magnetic field to account for reduction of field when the plasma is introduced (dimensionless). 
Table 3-71. Plasma end-dump system requirements.

Parameter Value

Total power (both ends) (MW)

Species (\%)/Energy flux (keV)

Maximuni power density at $28 \mathrm{~m}$

Maximun power density at $28 \mathrm{~m}$

Coolant requireo for (both ends) (gpm) 10,340

haximum inlet water temperature $\left({ }^{\circ C}\right)$

Life (yr)

Water pressure (psi)

1

54.5

See Table 3-75

See Table 3-75

350

1

$200-250$ 
$\mathrm{B}_{\text {END }} \quad=$ the magnetic field at the dump $(T)$,

$A_{C C^{2}} A_{E N D}=$ the area of the plasma the dump in the end cell $\left(\mathrm{cm}^{2}\right)$.

From conservation of energy, it is also true that:

$\left(P_{C C}\right)\left(A_{C C}\right)=\left(P_{E N D}\right)\left(A_{E N D}\right)$

where $P_{c C}$ and $P_{E W O}\left(\mathrm{~kW} / \mathrm{cm}^{2}\right)$ are the heat fluxes of the plasma at the central cell and at the beam dump location, respectively.

Noting the area ratio present in both Eqs. (3-95) and (3-96), one can arrive at the following heat flux relation:

$P_{E N D}=P_{C C} \frac{B_{E N D}}{B_{C C}(1-B)^{1 / 2}}$.

The maximum central cell flux of $162.9 \mathrm{~kW} / \mathrm{cmi}^{2}$ occurs at the central axis of the reactor. The central cell field is $4.5 \mathrm{~T}$, and the average field strength is $0.24 \mathrm{~T}$. Using Eq. (3-97) we can calculate the maximum incident power flux on the panel anywhere along the center axjal reactor line, provided the field is known at that location. Figure 3-764 shows the resultant heat flux map calculated from $\mathrm{Eq}$. (3-97). Centerline magnetic field strength values calculated by LLNL were used.

As the heat flux increases, the local pressure requireo to suppress boiling also increases as depicted in Fig. 3-172. We assumed an in let temperature of $40^{\circ} \mathrm{C}$ and allowed a minimum temperature rise of $15^{\circ} \mathrm{C}$. Then we calculated the film-drop temperature of the turbulent water rlow using the following Dittus-Bolter Nusselt number relation:

$N u=0.023(R e)^{0.8}(\mathrm{Pr})^{0.4}$,

where

Nu = Nusselt number,

$R e=$ Reynolds number ,

$\mathrm{Pr}=$ Prandt 1 number. 
Sputtering of End-Dump Surfaces. Sputtering caused by plasma particles can limit the life of the plasma dumps. In this section we outline our procedure for calculating the surface thickness sputtered away from the end aumps without aooressing the question of lifetime.

The surface thickness sputtered, $d$ in $\mathrm{cm}$, is given by the relation:

$d=\frac{t}{n} \sum_{j} \frac{N\left(V_{j}\right)}{e V_{j}} \sum_{k} f_{k} s_{k}$,

where

$N\left(V_{j}\right)$ is the particle energy flux $\left(\mathrm{kW} / \mathrm{cm}_{2}\right)$ ev $v_{j}$ is incident particle energy, $v_{j}$ is the particle voltage $(k V), e$ is the electron charge (C), n is the atomic number density of the surface material, $t$ is the exposure time $(s) . S_{k}$ is the sputter yield in atums per inciaent particle, $f_{k}$ is the traction of particles at the particular energy level. The sumation factor over the plasma species contains this sputter yielo and fraction. Since the exact angle of incidence of the incicent particles is not known, we assume normal incidence and use $\mathrm{Eq}$. (3-99) without modification.

Table 3-72 contains some of the input quantities used to compute sputtered thickness. Sixty percent of the incident energy flux is composeo of $20-k V D$ and $40 \%$ of a $50 / 50$ mixture of $27=k V D$ and $T$ particles. Substitution of these quaritities into Eq. (3-99) results in the expression

$$
\begin{aligned}
a=\frac{t}{n} & \left\{1.15 \times 10^{17}(27 \mathrm{kV}) S_{0}(27 \mathrm{kV})+S_{T}(27 k V)\right. \\
& \left.+3.12 \times 10^{17}(20 \mathrm{kV}) S_{D}(20 \mathrm{kV})\right\},
\end{aligned}
$$

that can be directly applied to the end dumps. Explicit results for d require input values for the remaining unspecified quantities in Eq. (3-100). Listed in Table 3-73 are the surface number densities and sputter-yields of the more pronising material candidates: beryllium, aluminum, iron, copper, molybuenum, and tungsten. Results for iron, copper, and molybdenum can be applied to stainless steel, AMIIRC, and TZM surfaces respectively. 
Table 3-72. Particles incident on end dumps.

\begin{tabular}{cccc}
$\begin{array}{c}\text { Percent of } \\
\text { energy flux }\end{array}$ & $\begin{array}{c}\text { Particle } \\
\text { Voltage } V(k v)\end{array}$ & $\begin{array}{c}\text { Particle } \\
k\end{array}$ & $\begin{array}{c}\text { Particle fraction } \\
f_{k}\end{array}$ \\
\hline 60 & 20 & $D$ & 1.0 \\
40 & 27 & $D$ & 0.5 \\
& & $T$ & 0.5 \\
\hline
\end{tabular}


Table 3-73. Atomic number densities and sputter yielas.

Dump surface material

\begin{tabular}{lllllll} 
Quantity & Be & A] & Fe & Cu & Mo & W \\
\hline$n\left(\mathrm{~cm}^{3}\right)$ & 1.24 & 6.02 & 8.47 & 8.47 & 5.65 & 6.32 \\
$\mathrm{~S}_{D}(20 \mathrm{kV})$ & 5.0 & 5.3 & 7.1 & 1.7 & 2.0 & 4.4 \\
$\mathrm{~S}_{D}(27 \mathrm{kV})$ & 4.1 & 4.2 & 5.8 & 1.4 & 1.7 & 3.6 \\
$\mathrm{~S}_{\mathrm{T}}(27 \mathrm{kV})$ & 6.5 & 6.9 & 9.5 & 2.3 & 2.8 & 6.3 \\
\hline
\end{tabular}


Figure 3-170 shows resultant sputtering thickness for one full power year of panel operation for various materials as a function of reat.

Structural Analysis, Panel Coil Thickness and Life Time Analysis. The plasma end-dump panel is subjected to coolant pressure and temperature gravients that gepenc on the magnitude of the impinging heat flux. The temperature gradient also depends on the panel thickness. To make a conservative analysis for the material selection and scoping, we assunie the panel thickness as the final thickness after sputtering erosion. Tables 3-74 through 3-78 list the candidate materials, their mechanical properties at various temperatures, and 10,000 hour creep-rupture stress.

The panel coil flow area selected from thernial consicuerations has a trapezoidal cross section of uniform thickness with a flow area of approximately $0.31 \mathrm{in}^{2}$. For analysis purposes, we assumed the two panel plates to have the same thickness. The structural design must meet the requirements of the ASME Boiler Code. In Ref. 44, 10 panei coils are analyzed by computer codes basea on finite element modeling methoos. As our current program is a feasibility study, we felt that we could make our analys is without resorting to a finite element program. References 44 and 45 adoress problems of a similar nature. The ASHE Boiler Code does not discuss pressure vessels of trapezoidal cross section, however, analys is procedures are outlined for noncircular, specifically rectangular pressure vessels. Figure 3-173 shows the actual coil geometry and the idealized rectangular section having the same flow area, depth and the same trial thickness $T$. The idealized section also shows locations $N, N$, and $Q$, where the stresses are to be computeo for internal pressure per the Boiler Code, Section VIII Div I UA 706. At these three locations we computed the membrane and bending stresses for a given pressure and assumed thickness, using a joint efficiency factor of 0.8 .

Thermal stress analysis requires knowledge of the temperature gradient through the coil-wall thickness. We computed gradient for an assumed thickness based on heat flux and conductivity of the chosen material. For a more detailed and complete thermal stress analysis, we must use a computer code based on a finite element model of the pane?.

To assess the magnitude of the thermal stresses for preliminary sizing of structural components, it is customary to compute the thermal stresses based 
Table 3-74. Mechanical properties of candidate materials at $200^{\circ} \mathrm{F}$.

\begin{tabular}{|c|c|c|c|c|c|c|}
\hline AL6061 & 9.8 & 0.3 & 13.0 & 35 & 39 & 35 \\
\hline $\begin{array}{c}\text { AMZIRC } \\
(54 \% \text { CW })\end{array}$ & 18.7 & 0.3 & 9.8 & 46 & 60 & 50 \\
\hline SS304 & 28.0 & 0.3 & 8.4 & 60 & 120 & 90 \\
\hline TZM & 46.0 & 0.3 & 2.7 & 127 & 148 & $138^{*}$ \\
\hline
\end{tabular}

*ESTIMATED VALUE 
Table 3-75. Mechanical properties of candidate materials at $300^{\circ} \mathrm{F}$.

\begin{tabular}{|c|c|c|c|c|c|c|}
\hline MATERIAL & $\begin{array}{c}\text { MODULUS OF } \\
\text { ELASTICITY } \\
\mathbf{E}_{1} \\
\text { Msi } 1\end{array}$ & $\begin{array}{c}\text { POISSON'S } \\
\text { RATIO } \\
v^{*}\end{array}$ & $\begin{array}{c}\text { COFPFICIENT } \\
\text { OF THERMAL } \\
\text { EXPANSION } \\
\text { a } \\
\text { In/In/OF }\end{array}$ & $\begin{array}{c}\text { Y IELD } \\
\text { STRESS } \\
\text { S }_{y} \text { KSI }\end{array}$ & $\begin{array}{c}\text { TENSILE } \\
\text { ULTIMATE } \\
\text { STRESS } \\
\mathbf{s}_{\mathbf{u}}, \mathrm{KSI}\end{array}$ & $\begin{array}{l}10,000 \mathrm{lir} \\
\text { CREEP REPTURE } \\
\text { STRESS } \\
{ }^{{ }^{\prime}} \text { ' KSI }\end{array}$ \\
\hline AL6061 & 9.6 & 0.3 & I3. 3 & 30 & 34 & 20 \\
\hline $\begin{array}{c}\text { AHZI I C } \\
\text { (54\% CW) }\end{array}$ & 18.5 & 0.3 & 9.8 & 45 & 58 & 45 \\
\hline SS304 & 27.5 & 0.3 & 8. 2 & 60 & 115 & 87 \\
\hline TZH & 45.0 & 0.3 & 2.8 & 125 & 144 & $135^{*}$ \\
\hline
\end{tabular}

*ESTIMATED VALUE 
Table 3-76. Mechanical properties of candidate materials at $400^{\circ} \mathrm{F}$.

\begin{tabular}{|c|c|c|c|c|c|c|}
\hline MATERIAL & $\begin{array}{c}\text { MODULUS OF } \\
\text { ELASTICITY } \\
\mathbb{E}_{1} \\
\text { Ms1 }\end{array}$ & $\begin{array}{c}\text { POISSON'S } \\
\text { RAT'IO } \\
v^{*}\end{array}$ & $\begin{array}{c}\text { COEPFICIENT } \\
\text { OF THICRHAL } \\
\text { EXPANSION } \\
\alpha \\
1 \mathrm{a} / \mathrm{in} / O \mathrm{~F}\end{array}$ & $\begin{array}{c}\text { YIELD } \\
\text { STRESS } \\
\text { S }_{y}, \text { KSI }\end{array}$ & $\begin{array}{c}\text { TENSILE } \\
\text { ULTIMATE } \\
\text { STRESS } \\
\text { S }_{\mathbf{u}} \text {, KSI }\end{array}$ & $\begin{array}{c}10,000 \text { Hr. } \\
\text { CREBP RBPTURE } \\
\text { STRESS } \\
{ }^{\sigma_{R} \cdot \text { KSI }}\end{array}$ \\
\hline AL6061 & 9.0 & 0.3 & 13.6 & 15 & 17 & 7.5 \\
\hline $\begin{array}{l}\text { AMZIRC } \\
(54 \% \text { CW })\end{array}$ & 18.0 & 0.3 & 9.9 & 44 & 55 & 43 \\
\hline 55304 & 27.0 & 0.3 & 9.6 & 56 & 110 & 83 \\
\hline
\end{tabular}

*ESTIMATEd VALUE 
Table 3-77. Hechanical properties of canoidate materials at $500^{\circ} \mathrm{F}$.

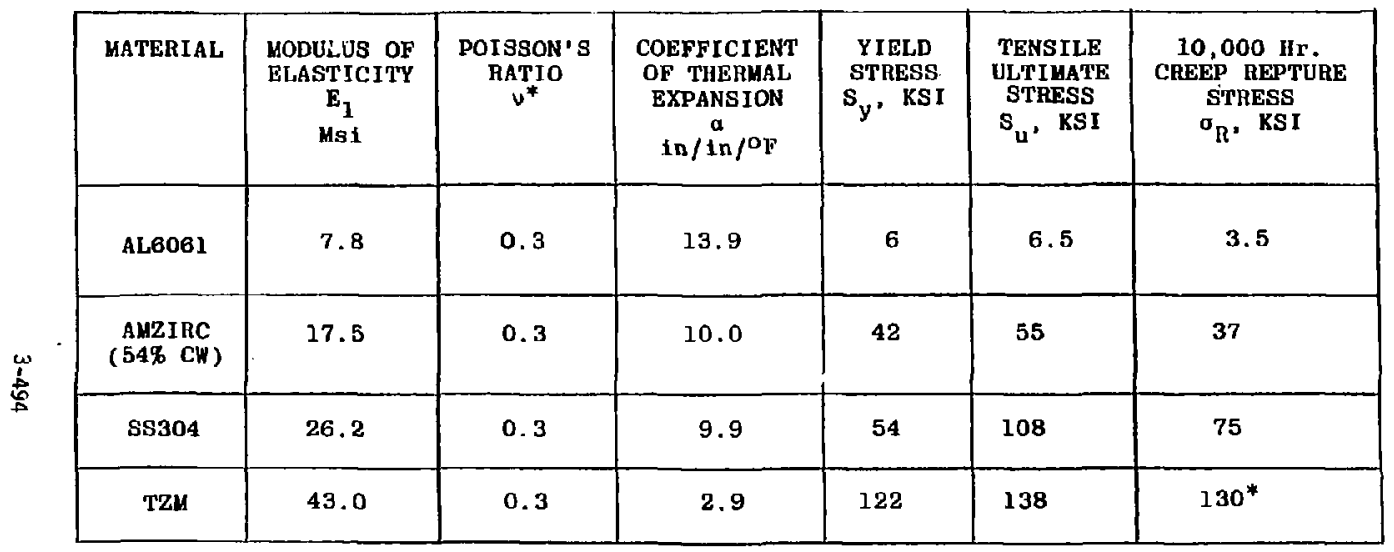

*ESTIMATED V ILUE 
Table 3-78. Mechanical properties of candidate materials at $600^{\circ} \mathrm{F}$.

\begin{tabular}{|c|c|c|c|c|c|c|}
\hline MATERIAL & $\begin{array}{c}\text { UODULUS OF } \\
\text { ELAS:ICITY } \\
\text { E }_{1} \\
\text { UsI }\end{array}$ & $\begin{array}{c}\text { POISSON'S } \\
\text { RATIO } \\
v^{*}\end{array}$ & $\begin{array}{c}\text { COEFFICIENT } \\
\text { OF THERMAL } \\
\text { EXPANSION } \\
\alpha \\
1 n / 1 n / /^{\circ}\end{array}$ & $\begin{array}{c}\text { YIELD } \\
\text { STÉESS } \\
\mathbf{s}_{y}, \mathrm{KSI}\end{array}$ & $\begin{array}{l}\text { TENSILE } \\
\text { ULTIMATE } \\
\text { STRESS } \\
\text { S }_{u} \cdot \mathrm{KSI}\end{array}$ & $\begin{array}{c}10,000 \mathrm{Hr} . \\
\text { CREEP REPTURE } \\
\text { STRESS } \\
{ }^{\circ}{ }^{\prime} \text { KSI }\end{array}$ \\
\hline AL6061 & 7.0 & 0.3 & 14.2 & 2.7 & 3 & 1.5 \\
\hline $\begin{array}{c}\text { AMZIRC } \\
(54 \% \text { CW })\end{array}$ & 16.5 & 0.3 & 10.1 & 40 & 53 & 30 \\
\hline S3304 & 25.5 & 0.3 & 10.3 & 50 & 106 & 71 \\
\hline TZM & 42.0 & 0.3 & 2.9 & $120^{\circ}$ & 135 & $127^{*}$ \\
\hline
\end{tabular}

*ESTIMATED VALUE 
on complete constraint. As discusied in Ref. 46, the maximum compressive stress causeo by a temperature gradient of $\Delta T$ is

$$
Q(t)=\frac{\alpha E A T}{T-v},
$$

where

$$
\begin{aligned}
& Q(t)=\text { stress, } \\
& \alpha \quad=\text { the thermal coefficient of expansion, } \\
& E \quad=\text { Youns's mooulus of elasticity, } \\
& v \quad=\text { Poisson's ratio. }
\end{aligned}
$$

The stresses allowable by the Boiler Code for each stress $c^{7}$ assification are usually given in terns of the stress intensity $S_{m t}$, which is calculated from the tensile and creep properties of the candidate inaterials. Table 3-79 gives the criteria for calculating it. The Boiler Code considers primary and seconciary applicable for the structure under consideration. Table 3-80 lists maximum allowable stress intensities based on a conservative design approach as Refs. 44 and 45 suggest. Using Tables 3-75 through 3-80, we established various panel coil thicknesses for heat fluxes from 100 to $600 \mathrm{~W} / \mathrm{cm}^{2}$. These thicknesses are 1isted in Table 3-81. Figure 3-171 shows the maximum temperature of the dump surface sputtering for one full-power year.

Conclusions. We computed plasma end dump panel coil thicknesses for oifferent heat fluxes. From the stress analys is point of view, AMZIRC and TZM could meet the Boiler Code requirements for the heat fluxes shown in Table 3-81.

Material Selection for Plasma End bump. The candioate materiais should have the following material properties:

- A low sputtering rate,

- High thermal conductivity,

- Adequate strength for the operating temperature,

- Resistance to corrosion caused by coolant flow, and

- Ease of fabrication. 
Table 3-79. Panel-coil structural design criteria.

\begin{tabular}{|c|c|c|c|}
\hline $\begin{array}{l}\text { TIME-DEPENDENT } \\
\text { ALLOWABLE STRESS : }\end{array}$ & $\mathbf{s}_{t}$ & $\begin{array}{l}\text { LOWER } \\
\text { VALUE OT }\end{array}$ & $\begin{array}{l}\text { 2/3 OF STRESS TO CAUSE CREEP RUPTURE IN TIME } t \\
\text { MINIMUM STRESS TO PRODUCE 1\% STRAIN IN TIME } t\end{array}$ \\
\hline $\begin{array}{l}\text { GENERAL PRIMARY- } \\
\text { MEMBRANE ALIOHABLE } \\
\text { STRESS: }\end{array}$ & ${ }^{S m_{t}}$ & $\begin{array}{l}\text { LOWER OF } \\
\text { Sm AND } s_{t}\end{array}$ & . \\
\hline
\end{tabular}


Táble 3-80. Stress categories and maximum allowable stress intensities.

\begin{tabular}{|c|c|c|}
\hline CATEGORY & INCLUDED IN CATEGORY & $\begin{array}{l}\text { MAXIMUM ALLOWABLE } \\
\text { STRESS INTENSITY }\end{array}$ \\
\hline$s_{1}$ & Pr: & $\mathrm{Sm}_{t}$ \\
\hline$S_{2}$ & $\mathrm{Pm}+\mathrm{Pb}$ & $1.15 \mathrm{Sm}_{\mathrm{t}}$ \\
\hline$s_{3}$ & $2 \mathrm{Pm}+\mathrm{Pb}+\mathrm{Q}(\mathrm{t})$ & $S m_{t}$ \\
\hline
\end{tabular}

Pm = Primary Mean Stress

$\mathrm{Pb} \quad=$ Primary Bending stress

$Q(t)=$ Maximum Thermal Stress Based on Complete Constraint = $-\frac{\alpha \mathrm{E} \Delta \mathrm{T}}{1-v}$ 
Table j-81. Plasma end dump heat flux versus panel thickness.

\begin{tabular}{|c|c|c|c|c|c|c|c|c|c|c|}
\hline \multirow[b]{2}{*}{$\begin{array}{l}\text { HEAT } \\
\text { FLUX } \\
w / \mathrm{cm}^{2}\end{array}$} & \multirow[b]{2}{*}{$\begin{array}{l}\text { MAX. } \\
\text { SURFACE } \\
\text { TEMP. } \\
\text { OF }\end{array}$} & \multirow[b]{2}{*}{$\begin{array}{c}\text { WATER } \\
\text { IPRESSUNE } \\
\text { REQUI ILAMENT } \\
\text { PS I }\end{array}$} & \multicolumn{2}{|r|}{ AL6061 } & \multicolumn{2}{|r|}{$\begin{array}{l}\text { AHZIRC } \\
54 \% \text { CW }\end{array}$} & \multicolumn{2}{|r|}{ Ss304 } & \multicolumn{2}{|r|}{ THZ } \\
\hline & & & $\begin{array}{l}\Delta T \\
\Delta F\end{array}$ & $\begin{array}{c}\text { THICKNESS } \\
t \\
\text { IN }\end{array}$ & $\begin{array}{l}\Delta T \\
\Delta_{F}\end{array}$ & $\begin{array}{c}\text { THI CKNESS } \\
t \\
\text { IN }\end{array}$ & $\begin{array}{l}\Delta T \\
\Delta_{F}\end{array}$ & $\begin{array}{c}\text { THICKNESS } \\
t \\
\text { IN }\end{array}$ & $\begin{array}{l}\Delta T \\
O_{F}\end{array}$ & $\begin{array}{c}\text { THI CKNESS } \\
\mathrm{t} \\
\text { IN }\end{array}$ \\
\hline 600 & 543 & 1000 & -- & -- & 90.0 & 0.18 & -- & - & -- & - \\
\hline 500 & 477 & 540 & -- & -- & 62.8 & 0.10 & -- & - & 107 & 0.06 \\
\hline 400 & 408 & 277 & -- & -- & 34.6 & 0.07 & -- & - & 57 & 0.04 \\
\hline 300 & 342 & 121 & -- & -- & 18.0 & 0.05 & -- & -- & 32 & 0.03 \\
\hline 200 & 275 & 45 & 19.0 & 0.04 & 10.0 & 0.03 & -- & - & 15 & 0.02 \\
\hline 100 & 200 & 13 & 5.0 & 0.02 & 2.5 & 0.02 & 56.0 & 0.02 & 7 & 0.02 \\
\hline
\end{tabular}


Täble 3-80. Stress categories and maximum allowable stress intensities.

\begin{tabular}{|c|c|c|}
\hline CATEGORY & INCLUDED IN CATEGORY & $\begin{array}{l}\text { MAXIMUM ALLOWABLE } \\
\text { STRESS INTENSITY }\end{array}$ \\
\hline$s_{1}$ & $P_{m}$ & $S m_{t}$ \\
\hline $\mathrm{S}_{2}$ & $\mathrm{Pm}+\mathrm{Pb}$ & $1.5 \mathrm{Sm}_{t}$ \\
\hline$s_{3}$ & $2 P_{n}+P b+Q(t)$ & $3 \quad S m_{t}$ \\
\hline
\end{tabular}

Pm = Primary Mean Stress

$\mathrm{Pb} \quad=$ Primary Bending Stress

$Q(t)=$ Maximum Thermal Stress Based on Complete Constraint = $-\frac{\alpha E \Delta T}{1-v}$ 
Táble j-81. Plasma end aump heat flux versus panel thickness.

\begin{tabular}{|c|c|c|c|c|c|c|c|c|c|c|}
\hline \multirow[b]{2}{*}{$\begin{array}{l}\text { HEAT } \\
\text { FLUX } \\
W / \mathrm{cm}^{2}\end{array}$} & \multirow[b]{2}{*}{$\begin{array}{l}\text { MAK, } \\
\text { SURFACE } \\
\text { TEMP. } \\
\text { OF }\end{array}$} & \multirow[b]{2}{*}{$\begin{array}{c}\text { HATCR } \\
\text { PRESSURE } \\
\text { REQUINCHENT } \\
\text { PSI }\end{array}$} & \multicolumn{2}{|r|}{ AL60G1 } & \multicolumn{2}{|r|}{$\begin{array}{l}\text { AMZIRC } \\
54 \% \mathrm{CW}\end{array}$} & \multicolumn{2}{|r|}{ SS304 } & \multicolumn{2}{|r|}{ THZ } \\
\hline & & & $\begin{array}{l}\Delta T \\
O F\end{array}$ & $\begin{array}{c}\text { THICKNESS } \\
t \\
\text { IN }\end{array}$ & $\begin{array}{l}\Delta T \\
\mathrm{O}_{\mathrm{F}}\end{array}$ & $\begin{array}{c}\text { THICKNESS } \\
t \\
\text { IN }\end{array}$ & $\Delta_{F} \mathbf{T}$ & $\begin{array}{c}\text { TIICKRESS } \\
t \\
\text { IN }\end{array}$ & $\begin{array}{l}\Delta \mathbf{T} \\
\mathbf{O F}\end{array}$ & $\begin{array}{c}\text { THICKNESS } \\
\text { IN } \\
\text { IN }\end{array}$ \\
\hline 600 & 643 & 1000 & -- & -- & 90.0 & 0.12 & - & -- & -- & -- \\
\hline 500 & 477 & 540 & -- & -- & 62.8 & 0.10 & - & -- & 107 & 0.06 \\
\hline 400 & 408 & 277 & - & -- & 34.6 & 0,07 & -- & -- & 57 & 0.04 \\
\hline 300 & 342 & 121 & -- & -- & 18.0 & 0.05 & -- & $\cdots$ & 32 & 0.03 \\
\hline 200 & 275 & 45 & 19.0 & 0.04 & 10.0 & 0.03 & -- & -. & 15 & 0.02 \\
\hline 100 & 206 & 13 & 5.0 & 0.02 & 2.5 & 0.02 & 56.0 & 0.02 & 7 & 0.02 \\
\hline
\end{tabular}


No single material has all of these properties. Molybdenum has a low sputtering rate, aird precipitation-strengthened alloys of copper like AMZIRC have high thermal conductivity, moderate strength at elevated temperatures, reasorable resistance to corrosion, and ease of fabrication. Thus, we consicered a molybdenum front surface bonded to AMZIRC. The bonding methods consigered were:

- Plasma arc ceposition of molybdenum on copper

- Chemicar vapor deposition of molybdenum on copper

- Explosive welding.

Molyboenum and copper have very aifferent coefficients of thermal linear expansion: $\alpha=4.9 \times 10^{-6} /{ }^{\circ} \mathrm{C}$ for molybdenum and $\alpha=17.6 \times 10^{-6},{ }^{\circ} \mathrm{C}$ for AHżLRC. In addition, we need a thick coating of 5 to $10 \mathrm{~mm}$ of molybdenum on copper. Both the plasma arc and chenical vapor processes deposit molybdenum on copper at about 400 to $500{ }^{\circ} \mathrm{C}$, and after cooling, we found that the surface did not have a strong bond. However a weak bond is expected between copper-molybdenum because they have different coefficients of thermal linear expansion. We have not tried the explosive welding process.

Molybdenum can be brazed to copper, but the brazing temperature is about $800^{\circ} \mathrm{C}$ ano the process would anneal the copper alloy to very low mechanical strength levels, e.g., to that of annealed copper.

Thus our choice reduces to pure materials. The canaidates considered were:

- TZM (0.5 Ti, $0.25 \mathrm{Zr}$, bàlance molybdenum),

- amzirc (0.15 Zr, balance ofhr copper),

- Al 6061 T-6, and

- Stainless steel 304 .

We found that stainless steel had too low a thermal conductivity, which produces large thermal gradients. Al 6061 had good thermal conductivity but very little mechanical strength at 300 to $400^{\circ} \mathrm{C}$. That leaves the choice of AMZIRC or TZM.

TZM has both a low sputtering rate and low thermal conductivity, in direct contrast to AMZIRC. AMZIRC could would have to be joined by low-temperature soldering, which would be undesirable. High-temperature brazing would soften the materiai. On the other hand, TZM or molybdenum can be Electron-beam welded and have good fabricability. If thermel gradients can be tolerated in TZM, then it would be the preferred material for plasma end 
Jumps. TZM has the advantage of very low swelling caused by neutron irradiation below $500{ }^{\circ} \mathrm{C}$ (Ref. 47), but it does become embrittled by neutron irridation. Copper swells most between $300^{\circ} \mathrm{C}$ to $400^{\circ} \mathrm{C}$ (Ref. 48 ).

Key technical issues. Two issues must be adoressed. As Fig. 3-164 shows, the maximum heat flux on the panel increases as the oump moves toward the center of the reactor. Although the cost of the vacuum vessel decreases with a smaller end cell, the dump cost increases because a higher technology panel is required.

The magnitude of tritium diffusion into the cooling water for the baseline panel should be calculated accurately. Separation of tritium from water can be a very expensive procedure. Running the dump surface at higher tenuperatures aggravates the problem, since the diffusion rate increases with temperature. There are expensive methods to oesign around the problem, e.g., using two bonced materials with aifferent aiffusion rates for the front surface of the dump. A cost analysis on the trade might warrant the more complex panel design. 


\subsection{TRITIUM STUDIES}

\subsubsection{Sumiary}

On the basis of fueling requirements and probable operating characteristics of the TUF reactor and its auxiliary components, which were obtained from various program participants, we calculated a material balance for aeuterium and tritium in the rof system. The tritium system capacity and performance requirements :yere based on the determineo material balance for a 20-MN, full-power, continuous operation. The technological approach for the tritium subsystems was based primarily on the Tritium Systems Test Assembly (TSTA) development program at Los Alamos National Laboratory but also included results from the TF?R program at Princeton University, the Mouno Laboratory tritium-treatment systems, and the design studies for research and commercial fusion systems, including the Fusion Energy Design Center.

Most of the effort in oesigning the TDF tritium systems went into evaluating the capabilities of subsystems for allowing safe operation of the TOF reactor and for safe handling of its estimated tritium inventories. Models were developed to evaluate the capability of the probable cleanup systems to timely clean up postulated facility accijents. This analysis indicates that the proposed designs for tritium systems will be adequate for maintaining tritium release values well within the design goals for credible accident conditions. The major uncertainty involves the tritium permeation rates through the beam dumps to the cooling water.

\subsubsection{System Requirements}

The purpose of the TDF tritiu'il systems is to allow the tritium fuel for the fusion cevice to be receiveo, stored, purified, and recycled but be sufficiently contained so that the operating personnel or local population in the vicinity of the facility are not subjected to potenicially harmful exposures of tritium. The tritiun-related subsystems for accomplishing this include the following: 
- Tritium Receiving and Storage (TRS).

- Fuel Cleanup (FCU).

- Isotope Separations (ISS).

- Fuel Preparation (FPS).

- Tritium Haste Treatment (TWT).

- Tritiated Water Recovery (TWR).

- Facility Air ljetritiation (FAD).

- Glovebox Gas Treatment (GGT).

- Tritium Systems Data Acquisition (TDA).

The tritium systems must maintain airborne concentrations of tritium below $5 \mu \mathrm{Ci} / \mathrm{m}^{3}$ in spaces occupied by unprotected operating personnel and below $500 \mu \mathrm{Ci} / \mathrm{m}^{3}$ for protected (in bubble suit) aperating personnel. In adition, the tritium systems are designed *o satisfy the following tritium release goals:

$\begin{array}{lll}\text { Normal tritium loss from faci]ity } & \leq 10 \mathrm{Ci} / \mathrm{d} & \leq 1000 \mathrm{Ci} / \mathrm{y} \\ \text { Maximum off-normal loss from facility } & \leq 30 \mathrm{Ci} / \mathrm{d} & \leq 5000 \mathrm{Ci} / \mathrm{y} \\ \text { Maximum accident release } & <10 \mathrm{~g} & <50,00 \mathrm{Ci}\end{array}$

\subsubsection{Tritium Material Balance}

We calculated a tritium material balance to provide a basis for the design of the various tritium-related subsystems incluaing tritium inventories. Calculations were based on the aata of Table 3-82.

The calculated burn-up rates for deuterium and tritium unaer these conditions were 2.05 and $3.07 \mathrm{~g} / \mathrm{d}$, respectively, with the generation of $4.08 \mathrm{~g} / \mathrm{d}$ of $\mathrm{He}-4$. The calculated material balance is shown in Table 3-83. The design of the tritium systems is based on flow rates shown in the table, but these are only approximate values. In the mass balance calculations, the efficiencies of the neutral-beam injectors and the back pumping of the NBI vacuum pumps were considered. On the basis of calculations performed at TRW, about $24.6 \%$ of the NBI feed gas will be injected and $85.3 \%$ of the injected gas wil] enter the plasma. The close proximity of some of the NBI beam dumps to NBI ducts will result in some of the injected gas being removed from the reactor chamber by the NBI vacuum pumps. Conductance calculations by the 
Table 3-82. Fuel and plasma stability requirements for a 2-MW fullpower operation of the fusion device.

\begin{tabular}{lcc}
\hline System & $\begin{array}{c}\text { Species } \\
\text { (T or D) }\end{array}$ & $\begin{array}{c}\text { Current } \\
(A)\end{array}$ \\
\hline Central cell NBIs & $T$ & 540 \\
Central cell NBIs & $D$ & 540 \\
Anchor and transition NBIs & 0 & 66.3 \\
Central cell fuel injectors & 0 & 800 \\
\hline
\end{tabular}


Table 3-83. Reactor fueling rates and vacuum pump exhaust rates for ful1-power (20 MW) operation.

Flow rates

System

Species

(atom moles/s) (g/d)

Fuel ing rates

Central cell NBIs

T $\quad 2.677 \times 10^{-2} \quad 6976$

Central rell NBIs

D $\quad 2.677 \times 10^{-2}$

4659

Anchor $\therefore$ transition NBIs

0

Central cell fuel injectors

D

$3.286 \times 10^{-2}$

5718

$8.291 \times 10^{-3}$

1443

Exhaust rates $^{a}$

Central cell tritium

NBI vacuum pumps

$\begin{array}{llr}D & 1.010 \times 10^{-3} & 176 \\ T & 2.121 \times 10^{-2} & 5526\end{array}$

Central cell deuterium

NBI vacuum pumps

D $\quad 2.121 \times 10^{-2} \quad 3690$

Anchar and transition

NBI vaculim pumps

D $\quad 2.480 \times 10^{-2} \quad 4316$

Plasma end cell vacuum pumps

$\begin{array}{llr}\text { T } & 1.239 \times 10^{-5} & 3 \\ \text { D } & 2.089 \times 10^{-2} & 3636\end{array}$

T $\quad 4.531 \times 10^{-3} \quad 1181$

Assumes 24.6 NBI efficiency and $85.3 \%$ plasma penetration of the injected neutral beam particles; assumes $10 \%(5 \% \mathrm{~T}$ and $5 \% \mathrm{D})$ of the injected gas will be arawn back up the NBI ducts by the NBI vacuum pumps because of the close proximity of the NBI beam aumps to some of the ducts and the relatively high conductance of these ducts. Only $0.1 \%$ of the injected gas is assumed to be drawn back through the anchor and transition NBI ducts because of the remote lacation of the beam oumps and the low duct conductance. 
designer indicated that $10 \%$ of the gas injected in the central cell will be removed by the NBI vacuum pumps, approximately $5 \% \mathrm{D}$ and $5 \% \mathrm{~T}$. Because the NBI* beam aumps in. the anchor and transition cell are further removed from their injection ducts and because the duct conductances will be much smaller, we assume orly $0.1 \%$ of the injected gas will be drawn back into the NBI vacuum pumps in the anchor and transition cells, again a $50 \%$ split of $D$ and $T$.

A summary of the mass balance calculations and the distribution of various gas streams to the tritium systems is shown schematically in Fig. 3-203. The 1sotope Separations System (ISS) separates the various hydrogen isotopes priur to recycling of the recovered fuel so that the fuel can be blended to the desired mix prior to reinjection. Because condensable contaminant gases collect in the ISS cryogenic distillation columns, we must maintain the contaminant gas levels to less than about $1 \mathrm{ppm}$ (vol.) to minimize colunin cleanout. Thus, the contaminant gases are removed in the fuel cleanup system.

\subsubsection{Fuet Cleanup System}

When the fusion device reaches its maximum operating temperature, a number of contaminants, most of which will become gaseous, are expected to be liberated from the various materials of construction. These contaminant gases, along with trace amounts from in-leakage, are removed by the fuel cleanup (FCU) system prior to entering the ISS unit. Little information is available on the probable contaminant concentration from the fusion device. Personnel of ISTA surveyed fusion-device researchers to obtain a consensus of the most probable contaminant concentrations in order to estimate concentrations in the end dump vacuum pump gas strearms of the TDF plasma ( $\mathrm{see}$ Tab]e 3-84).

A schematic flow diagram of the fuel cleanup system is shown in Fig. 3-204. The exhaust gas from the plasma end dump vacuum purips is first passed through a catalytic reactor operated at about $450 \mathrm{~K}$ to convert free oxygen to an appropriate oxide. Then the gas is cooled and the contaminants are removed by cryogenic adsorption on molecular-sieve beds at a temperature of about $75 \mathrm{~K}$. At this temperature all of the oxides, hydrocarbons, ammonia, and argon will concense and be adsorbed on the molecular-sieve beds. The only gaseous species that will not condense at these temperatures will be hyorogen isotopes and helium, which will eventualiy pass directly to the ISS unit, although there is some holdup of the hydrogen isotopes early in the cycle. 


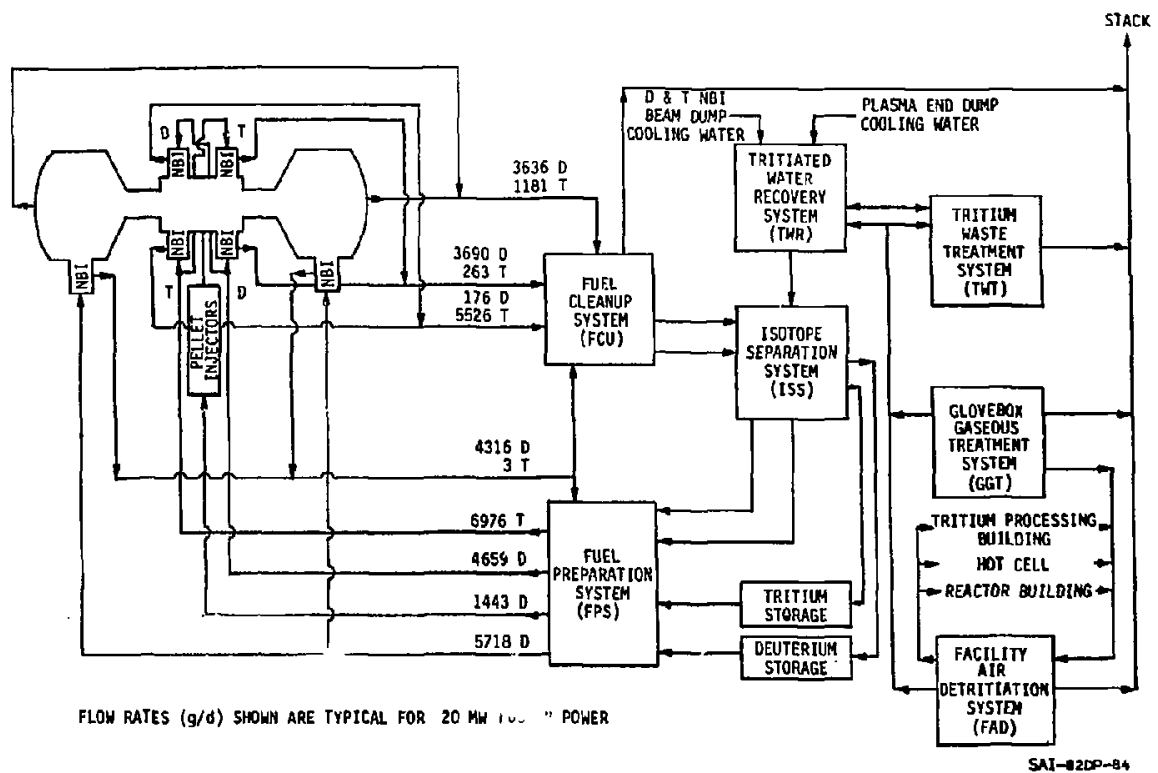

Ffgure 3-203. Tritium processing and treatment systems for TDF. 
Table 3-84. Estimated contaminant-gas concentrations in the vacuum-pump exhaust for the plasma vessel of TDF.

\begin{tabular}{lccl}
\hline $\begin{array}{c}\text { Gaseous } \\
\text { Component }\end{array}$ & $\begin{array}{c}\text { Atomic } \\
\%\end{array}$ & $\begin{array}{c}\text { Flow rate } \\
(\mathrm{g} / \mathrm{d})\end{array}$ & $\begin{array}{c}\text { Probable chemical } \\
\text { species }\end{array}$ \\
\hline $\mathrm{He}$ & 0.04 & 4.1 & $\mathrm{He}$ \\
$\mathrm{Ara}$ & 1.17 & 1221 & $\mathrm{Ar}$ \\
$\mathrm{C}$ & 0.02 & 6.3 & $\mathrm{C}\left(\mathrm{O}_{1} \mathrm{~T}_{1} \mathrm{H}\right)_{4}$ \\
$\mathrm{~N}$ & 0.10 & 36.7 & $\mathrm{~N}\left(\mathrm{D}_{1} \mathrm{~T}_{1} \mathrm{H}\right)_{3}$ \\
0 & 0.50 & 209.7 & $\left(\mathrm{O} \mathrm{T}_{1} \mathrm{H}\right)_{2} \mathrm{O}$, \\
$\mathrm{H}$ & & $\mathrm{CO}, \mathrm{CO}_{2}$ \\
$\mathrm{O}$ & 1.00 & 25.4 & $\mathrm{OT}, \mathrm{HO}_{2} \mathrm{HT}_{1} \mathrm{OT}_{2}$, \\
$\mathrm{T}$ & 75.54 & 3989 & $\mathrm{~T}_{2}, \mathrm{and} \mathrm{H}_{2}$ \\
\hline
\end{tabular}

aAgon at about 30 times the He ash concentration is introduced in the vacuum pumps to pump the helium. 

The condensed containment gases are removed from the molecular-sieve beds by heating the beos to about $475 \mathrm{~K}$ and purging with dry nitrogen followeo by $0^{2}$. The purged contaminant gas is heated and passed through another catalytic reactor operated at about $800 \mathrm{~K}$. A slight excess of oxygen is added to ensure complete decomposition of ammonia ano hydrocarbons and to convert the hyorogen isotopes to water. The water is racovered in a freeze-out trap operated at ahout $160 \mathrm{~K}$; the argon, carbon dioxide, oxygen, and $n$ itrogen will pass through. These gases are oirected to the tritiated waste treatment (TWT) systen for trace tritium removal before being oischarged. At $160 \mathrm{~K}$, the vapor pressure of water is at least 10,000 times lower than that of the other species. The hydrogen isoiopes, collected as water in the freeze-out trap, are heateo to a vapor form and passed through a hot metal getter bed of activated uranium at a temperature of about $750 \mathrm{~K}$. This strips the oxygen and allows the $H, D$, and $T$ to pass through to the plasma end dump vacuum pump feed stream of the first catalytic reactor. Because there are two molecular-sieve adsorbent beds, two freeze-dut traps, and at least two hot metal beas, these systems can be operated continously.

The exhaust gases from the deuterium and tritium central cell neutralbeam vacuum pumps pass through a molecular-sieve adsorbent bed cooled to $75 \mathrm{~K}$. This removes contaminant gases, including ammonia, argon, oxygen, and the hydrocarbons. These species are periodicalily removed and recycled to the primary fuel cleanup portion of the system. The recovered ceuterium from the neutrai-beam vacuum pumps of the anchor and transition cells bias only trace impurities, including tritium, so only a portion of this gas stream neeas to be sent to the FiU system. Most of this gas stream can be returned directly to the fuel preparation system.

\subsubsection{Isotope Separation System}

The purified hydrogen isotopes from the FCU are sent to the middle of the first distillacion column in the ISS vit, as shown in Fig. 3-205 and Table 3-85. The distillation columns are packed with commercial, stainless-steel packing material and are operated at about $20 \mathrm{~K}$.

In Column $T, H$ and $D$ are fractionated from $T$ and are sent to column ? through a catalytic, rom-temperature equilibrator. The equilibrators allow the reestablishment of equilibrium between the six hydrogen-isotope species 


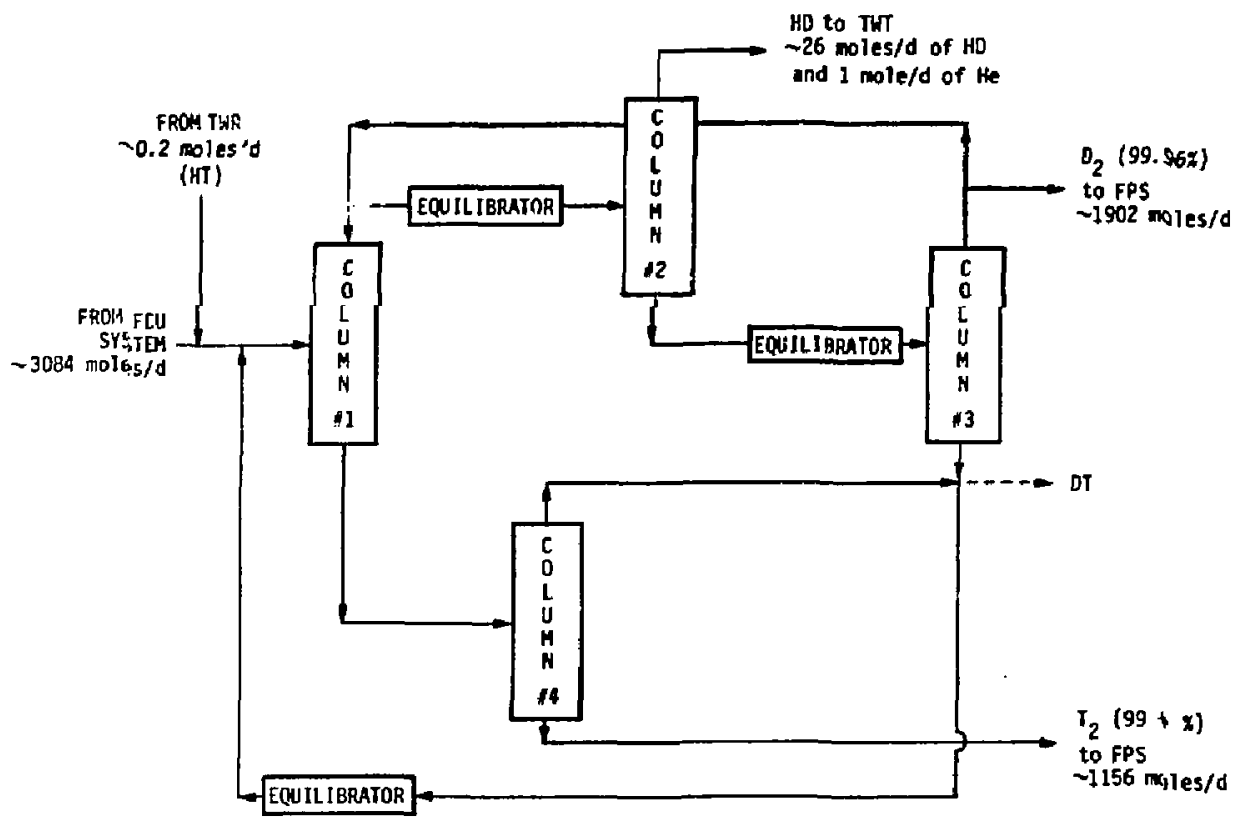

5n1-1200-6

Figure 3-205. Isotope separation syster for TDF. 
Table 3-85. The approximate dimensions of the ISS distillation columns of TOF.

\begin{tabular}{ccc}
\hline Column & Packed height (m) & i.d. (cm) \\
\hline 1 & 4.1 & 8.2 \\
2 & 4.1 & 5.6 \\
3 & 3.2 & 7.0 \\
4 & 4.1 & 11.1 \\
\hline
\end{tabular}


$\left(H^{2}, D^{2}, T^{2}, H D, H T\right.$, and $\left.O T\right)$, which allows a cleaner separation. The protium specie is fractionated from $D^{2}$ and any remaining trace $T$ species in Column 2 and sent to the TWT system for further cleanup and for discharge or oisposal. The materials at the bottom of Column 2 are sent to the middle of Column 3 by means of another equilibrator. Here, the $0^{2}$ is fractionated and a portion of the product is pumped to the fuel preparation system (FPS) while the remainder recycled to the top of Column 1 . At this point, the $0^{2}$ is about $99.96 \%$ pure.

The materials at the bottom of Column 3 contain trace contaminants of $T$ and are returned to the midale of column 1 for a recycie, again through an equilibrator. The tritium, which is concentrated in the bottom of Column ], is directed to the middle of Column 4 where the tritium is purified to greater than 99\% (isotopically pure) and is sent to the FPS. The overheads of column 4 , which contain some of the $U$ species, are returned for recycle with the bottoms from Column 3 .

The ISS is designed basically in accordance with the TSTA design concept ano equipment. The probadle flow rates for TUF, as determined in the material balance calculations, are also shown in Fig. 3-204.

\subsubsection{Fuel Preparation System}

The fuel preparation system consists of a series of transfer pumps, tanks, and fuel blenders. Feed gas for this system comes from the tritium storage beds, deuterium storage tanks, the ISS unit, and the anchor and transition neutra]-beam vacuum pumps. Separate portions of this system are discussed in the various fueling systems for the neutral-beam injectors and the pellet injector.

\subsubsection{Tritiated-Waste Treatment System}

The tritiated waste treatment system collects the various gaseous waste streams in TDF that may be contaminated with tritium, separates the tritium and either recycles the tritium to the ISS unit (by way of the tritiated water recovery--TWR--system) for isotopic purification and reuse as fuel or solidifies the tritiated-containing compounds for solid waste aisposal. The 
potential tritium-contaminated-waste gases from the FCU, ISS, TKR, and the facility air aetritiation (FAO) are collected with the use of a compressor and temporarily storeo in a pressurized holding tank. The contaminated air is then treated with a cleanup system similar to that shown in Fig. 3-206. In this treatment scheme, the contaminated gas is heated and passed through a catalytic reactor at about $810 \mathrm{~K}$ to convert the hyarogen isotopes to water (axygen is aoded as necessary). The gas is cooled to condense the water, and the resulting tritiated water, along with the nontritiated water, is adsorbed, at about ambient temperature on two beds of molecular-sieve zeolite in series. With heated catalyst reactors, oxide conversion factors of $10^{5}$ are obtainable. For water removal, decontamination factors (DFs) (where the DF is aefined as the inlet tritium concentration divided by the out let tritium concentration) of $>10^{6}$ are also obtainable with adequately sized molecular sieve beds.

As an added measure of safety, steam injection is provided between the first and secund molecular-sieve dryer beds. The objective is to add nontritiated water, which as a carrier will increase the removal of tritiated water in the secono dryer bed. The steam injection may or may not be used depending on the tritiatea-water concentration in the effluent of the secona aryer bea. Two sets of dual molecular-sieve dryer beds are used in a parallel (rather than series) arrangement so that one of each set can be regenerated separately while the other is in service. Regeneration is accomplished by passing heated air in the reverse direction through the molecular-sieve dryer beds, recovering must of the water in the regeneration gas with a cooler-condenser, and renloving the remaining water in a smaller molecularsieve uryer. The beds can also be regenerated with a hot air recirculating system using the cooler-condenser to remove the water. The recovered tritium-contaminated water in the TWT system can either be sent to the TWR system for tritium removal or solidifed for waste disposal. The TWT system has a gaseous treatment capacity of about $85 \mathrm{~m}^{3} / \mathrm{h}(50 \mathrm{cfm})$.

\subsubsection{Tritiated-Water Recovery System}

The primary purpose of the tritiated-water recovery system is to remove tritium from the plasma end oump and from the cooling of the tritium neutral-beam dump water. Tritium permeates through the beam dumps into the 


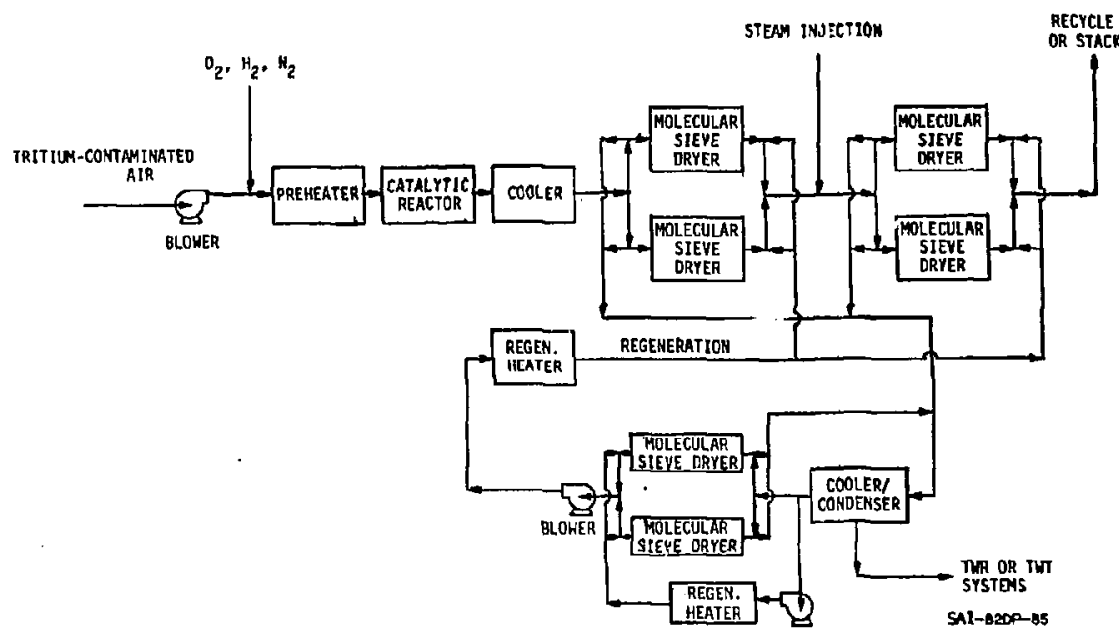

Figure 3-206. Contaminated air distribution system for TDF. 
cooling water at significant rates because of implantation energy and the relatively high temperature of the beam dump material. It has been estimated that for a full-power year of operation about $21 \mathrm{~g}$ of tritium will enter the cooling water through the neutral-beam aumps and about $98 \mathrm{~g}$ of tritium will enter through the plasma end dump.

Because leaks in the beam dump cooling system are virtually impossible to prevent, we must reouce the tritium in the coolant to a tolerable concentration. Experience with nuclear reactors and other processes using tritiated water indicates that a tolerable level of tritium coolant is about $1 \mathrm{Ci} /$ liters or less. It can be shown that to maintain the tritium concentration in the beam aump caoling water below about $1 \mathrm{ci} / \mathrm{liter}$, tritiated water must be removed at a rate equivalent to the curies per liter of tritium entering the cooling water. Because the fusion device will only be operated with a maximun availability of about $50 \%$ full power per year, the THR system should have a process capacity of about 1600 liters/d. With the TWR system in operation, about 50 days of ful1-power operation by the fusion devise would be required before the tritium concentration in the cooling water reaches $0.9 \mathrm{C}$ /liter.

The Thik system is shown schematically in Fig. 3-207. The process involves combined electrolysis and catalytic exchange technology. The tritiated water is injectea into the middle of the exchange column that is filled with a catalyst material to promote the exchange of gaseous hyorogen isotopes with water. Because the equilibrium at the column operating temperature is near ambient, formation of tritiated water (HTO) is tavored, and elriched HTO concentrates in the bottom of exchange column. A portion of this enriched HTO is removed ano electrolyzed to form hydrogen isotopes and oxygen. Most of the electrolyzed hydrogen isotopes are returned to the botton of the exchange column to further enrich the water in tritium, while the remaining portion of the gas is airected to a cryogenic aistillation column for adoitional isotopic enrichmerit. The overheads from the oistillation column are returned to the top (stripping portion) of the column for exchange with the tritium-aepleted water and stripping of any remaining tritium from the gas. The tritium-free hydrogen gas, mixed with oxygen from the electrolyzer, then exits through the top of the exchange column, recombine to form water, and returns to the cooling-water system. 


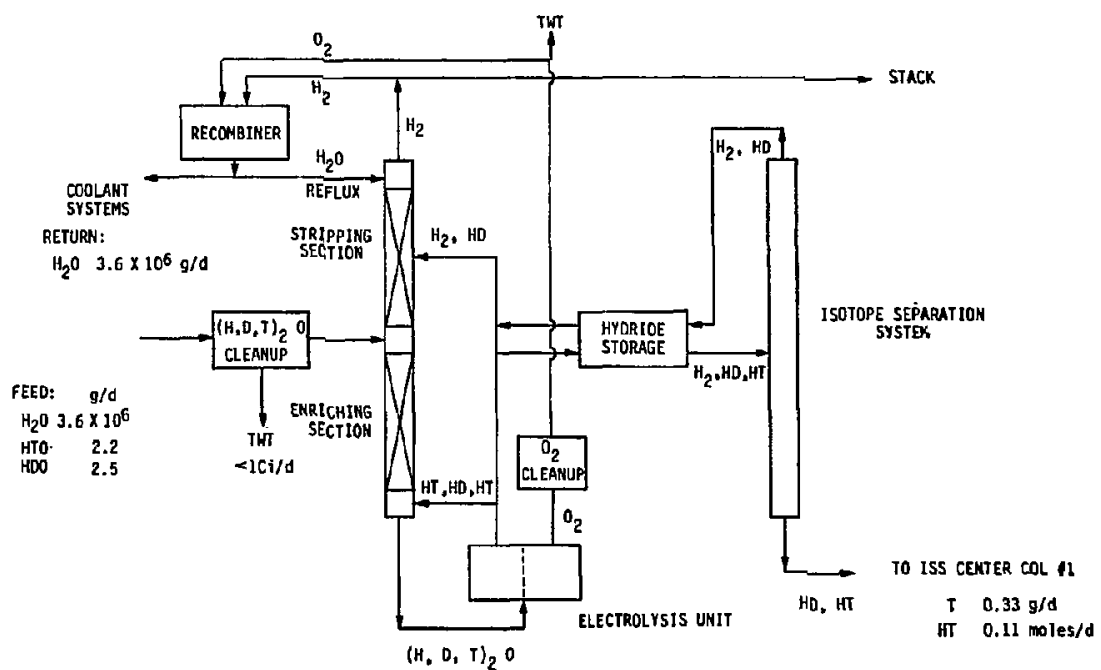

Figure 3-207. TUF tritiated-water recovery systen. 
Because the tritiated water is enriched in the exchange column by a factor of about 5000, the feed to the cryogenic distillation column is relatively small. The deuterium in the cooling water will be enriched in a similar manner to tritium but to a lesser extent and will follow it through the process oe partially mixed with the protium isotope.

\subsubsection{Facility Air Detritiation System}

The facility air cetritiation system consists of ihree identical $8500 \mathrm{~m}^{3} / \mathrm{h}$ ( $5000 \mathrm{cfm}$ ) treatment systems for removing tritium-contaminated gaseous wastes from the reactor building, the tritium processing building, and the hot cell. Any one or all of the units can be directed to the cleanup of one or more of these spaces. The process flow sheet for each of these treatment systems is shown in $\mathrm{Fig}$. 3-206. The removal process involves the oxidation in a catalytic reactor of all airborne tritium and its compounds to tritiated water and their removal on molecular-sieve dryer beds. Reoundant aryer beas provide regeneration capabilities that allow continuous operation of all three of the $8500 \mathrm{~m}^{3} / \mathrm{h}$ systems. The regeneration procedures for the aryer beds are the same as described for the gaseous TWI treatment system.

\subsubsection{Glovebox Gaseous Treatment System}

For tritium systems in which large inventories of tritium are likely, portions are enclosed in a secondary contajnment system called the Glovebox Gaseous Treatment System. These include the TRS, FCU, ISS, FPS, and the TWR systems. Argon is in the secondary containment system at $a$ slightly positive pressure at about $500 \mathrm{~Pa}$ or $2 \mathrm{in}$. of water. The total volume of the glovebox gaseous treatment (GGT) system is about $850 \mathrm{~m}^{3}$. The argon is constantly recirculated and constantiy monitored for the presence of either hydrogen isotopes or oxygen that woulo indicate a leak in one or more of the enclosed units. Enough sampling ports are provided to localize a potential leak. A $85-m^{3} / \mathrm{h}$ (50 cfm) gaseous treatment system will remove any hydrogen isotopes or oxygen that may leak into the secondary containment. The GGT system consists of essentially the same elements as the TWT and FAO systems (see Fig. 3-206). If for any reason the GGT becomes inoperative, the argon in the secondary containment $c$ an be processed with the TWT system and fresh argon replenished from storage. 
Isolation valves will also be included in the system so that, should a leak Gevelop in one of the components, the entire system will not become contaminated.

\subsubsection{Tritium Systems Data Acquisition}

Each inoividual tritium treatment system has redundant tritium monitoring instruments as part of the ficroprocessor-based operational control system. There are a number of other stack and area tritium ano combustible gas monitors in various facility locations. The indicated presence of high tritium or conibustible gas concentrations by these monitors woulo cause the automatic operation of one or more of the gas treatment systems or a venting operation. These comprise the tritium systems data acquisition (TDA) system which aTso provides backup control for the various processes and provides the communication link with the master computer control system for the facility.

\subsubsection{Response of Tritium Systems to Accidents}

The response time of the tritium cleanup systems for an acciaental release clearly aepends, among other variables, on the source term and treatment system capacity. Based on the calculated material balance and inaivioual componerit design, we estimated the probable tritium inventories at various locations in the TUF (sbe Table 3-86). In adajtion, we analyzed accioent scenarios for the most propable serious accident and the worst-case accident involving the release of the tritium inventory to their respective spaces. Models were developed for each scenario and the response of the variolis systems to a postulated accident was deternined. The assumed tritium conditions for each space are summarized in Table 3-87.

Reactor Building. The most probable serious accidental release in the reactor building would be the loss of one of the tritium neutral-beam injector vacuum pumps with the subsequent release of its tritium inventory, about $100 \mathrm{~g}$, into the reactor building. Figure 3-208 we show the calculated tritium concentration remaining as a function of time and of various FAD system cleanup rates. In these calculations we assurned a mixing efficier $y$ of $70 \%$, that is, 
Table 3-86. Estimated tritium inventories at various locations in TDF.

\begin{tabular}{lcc}
\hline & \multicolumn{2}{c}{ Tritium $^{\mathrm{a}}$} \\
\cline { 2 - 3 } & $(\mathrm{g})$ & $(\mathrm{Ci})$ \\
\hline Plasma & $<0.1$ & $<30$ \\
Vessel wails and beam dumps & $<10^{\mathrm{b}}$ & $<\mathrm{i} \times 10^{5 b}$ \\
Plasma end beam dump vacuum pumps & 66 & $6.4 \times 10^{5}$ \\
Tritium NBI vacuum pumps & 307 & $3.0 \times 10^{6}$ \\
Fuel preparation system & $<100^{\mathrm{b}}$ & $<10 \times 10^{6 \mathrm{~b}}$ \\
Fuel cleanup system & 100 & $10 \times 10^{6}$ \\
Isotope separation system & 600 & $5.8 \times 10^{6}$ \\
Tritiated water recovery system & 2 & $0.2 \times 10^{4}$ \\
Tritiated waste treatment system & 20 & $2 \times 10^{5}$
\end{tabular}

abaseo on full-power operation and represents maximum values.

bery uncertāin estimates. 
Tah1e 3-87. Factlity characterlstics and assumed condttions for sertous accident cases involving release of trit ium inventor les to their respective spates.

\begin{tabular}{|c|c|c|c|c|c|}
\hline Butlding/Systen & $\begin{array}{l}\text { Bullding/syster } \\
\text { volume (m) } \\
\text { (volx/o)) }\end{array}$ & $\begin{array}{l}\text { Butlding/system } \\
\text { leak rate }\end{array}$ & $\begin{array}{l}\text { Cleanup } \\
\text { rate } \\
\left(\mathrm{n}^{3} / \mathrm{d}\right)\end{array}$ & $\begin{array}{l}\text { Source } \\
\text { tern }\end{array}$ & $\begin{array}{c}\text { Initial Trittun } \\
\text { concentrition } \\
\left(\left[1 / n^{3}\right)\right.\end{array}$ \\
\hline Reactor bullding & 82,500 & 1 & 825 & 1009 & 11.8 \\
\hline $\begin{array}{l}\text { Tritium processing } \\
\text { building }\end{array}$ & 18,500 & 1 & 185 & $300 \mathrm{~g}$ & 158 \\
\hline Hot cell & 28,000 & 1 & 280 & $100 \mathrm{ct} / \mathrm{d}$ & 0 \\
\hline $\begin{array}{l}\text { Glovebox gaseous } \\
\text { treatment }\end{array}$ & 852 & $10^{-3}$ & $8.5 \times 10^{-3}$ & 6009 & $6.8 \times 10^{3}$ \\
\hline
\end{tabular}

assumes instantaneous, complete mixing. 


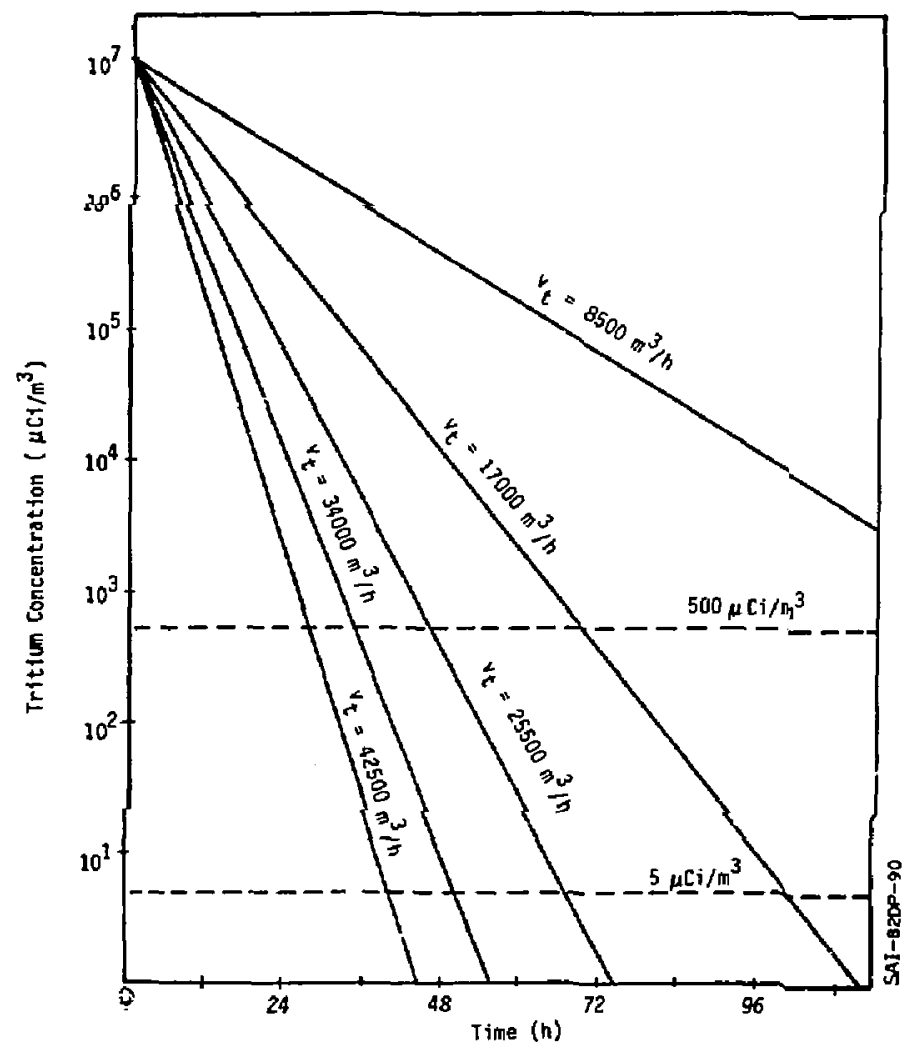

Figure 3-208. Tritium concentrations remaining in the reactor building as a function of time and of cleanup rates for an accidental release of $100 \mathrm{~g}$ of tritium. 
the actual cleanup rate $v_{t}$ was $30 \%$ less than that shown. This approach is not exact but should be a fairly close approximation. As seen from

Fig. 3-208, little is gained by using a total cleanup rate greater than about $25,500 \mathrm{~m}^{3} / \mathrm{h}$ (approximately $18,000 \mathrm{~m}^{3} / \mathrm{h}$ actual). With the use of three $8500-m^{3} / h$ cleanup units, the time required to reduce the tritium concentration in the reactor building to $500 \mu \mathrm{Ci} / \mathrm{m}^{3}$ will be about $46 \mathrm{~h}$ for the assumed conaitions. About $67 \mathrm{~h}$ of continuous operation will be needed to reduce the concentration to about $5 \mu \mathrm{Ci} / \mathrm{m}^{3}$. In these caiculations, we assumed that any tritiated water formed in the reactor building atmosphere would not be aosorbed on the building walls to any appreciable extent and therefore would not change the removal rate. Calculations regariding the counterdiffusion of tritium through the building leakage paths indicated that loss of tritium through this mechanism is completely insignificant $(<0.1 \mathrm{C} i)$ proviaed there was a negative pressure differential of several centineters of water in the reactor building compared with the surrounoing atmosphere. I $r$ this pressure affferential were lost, namely through the loss of all cleanup systems, then the loss of tritium per aay was estimated to be $0.21 \mathrm{i} i / d$ fior a leak rate per day of 1 vol\% and $1.1 \mathrm{Ci} / \mathrm{j}$ with a 10 vol\% leak rate per day.

The loss of tritium through atmospheric discharge fron the cleanup system was also determineu to be trivial. Nearly all exhaust from the cleanup system is recirculatea. Only the amount of gas needed to compensate for the in-leakage required to maintain a slight negative-pressure differential is discharged to the atmosphere. Even if a decontamination factor (DF) of only $10^{4}$ is assumed for the tritium removal system, the amount of tritium lost through this path would only be about $0.13 \mathrm{Cj}$ for a leak rate per coly of 1 vol\% and about $1.3 \mathrm{Ci}$ for a 10 vol\%/d leak rate with all three FAD systems operating. The DF for the tritium cleanup system will probably be in the range of $10^{5}$ to $10^{6}$, depending on the actual design.

If for some reason the tritium inventory in $\mathbf{0} 11$ of the vaculam pumps in the reactor building were released at one time, the tritium cleanup times and builaing losses would be somewhat greater than those indicated.

Tritium Processing Building. Although the estimated total tritium inventory in the tritium processing building (about $720 \mathrm{~g}$ ) is greater than for the reactor building $(375 \mathrm{~g})$, the probability for an accidental release $i$.; less 
because of the extensive use of double containment. In addition, provisions have been made to recover the tritium in the event of a failure. For example, in the ISS a high pressure storage tank is provided to accepi most of the column inventory in case of a sudden loss of cooling. In addition, spare powdered uranium-filled storage tanks are provided that will allow storage of the entire column inventory as a hydride. To lose any appreciable amout of tritium to the tritium processing building also requires a simultaneous major rupture in the GGT system enclosure with the failure of the primary system. It was concluded that the most probable serious accident would release no more than about $300 \mathrm{~g}$ of tritium to the tritium processing building.

The time required from one to three of the FAD units to cleanup the atmosphere of the tritium processing building after contamination with $300 \mathrm{~g}$ of tritium is presented in Fig. 3-209. With all three units in operation, the times required to reduce the tritium concentration to levels of 500 and $5 \mu \mathrm{ci} / \mathrm{m}^{3}$ are about 13 and $18 \mathrm{~h}$, respectively.

Hot Cel1. Little information is available on the source term magnitudes for contaminated materials that may require maintenance or oisposal. Source terms as high as $100 \mathrm{ci} / \mathrm{d}$ can be handed in the hot cell if the airborne tritium concentration is maintained below $500 \mu \mathrm{C} i / \mathrm{m}^{3}$ by the operation of two or more of the FAD units (see Fig. 3-210), assuming a constant source term. Assuming uniform hot cell mixing, the largest source term that can be handled while maintaining the average tritium concentration at no more that $500 \mu \mathrm{Cj} / \mathrm{m}^{3}$ is about $276 \mu \mathrm{Ci} / \mathrm{a}$ when all three FAD units are in use. This calculation also assumes $70 \%$ mixing of the recirculated, cleanup system, exhaust gas. If the average airborne-tritium concentration is to be maintained below $5 \mu \mathrm{Ci} / \mathrm{m}^{3}$, the largest source term that can be handled in the hot cell is about $2.2 \mathrm{Ci} / \mathrm{d}$.

Glovebox Gaseous Treatment System. For the GGT system, we assumed the entire tritium inventory of the ISS unit (about $600 \mathrm{~g}$ ) could be lost to the argon gas. If this were the case, about 7 days would be required to reduce the tritium concentration in this system to $500 \mu \mathrm{Ci} / \mathrm{m}^{3}$ with the $85-\mathrm{m}^{3} / \mathrm{h} \mathrm{GGT}$ cleanup system and about 9 days would be requireo to reduce it to 5 $\mu \mathrm{Ci} / \mathrm{m}^{3}$. Because this system is small and can be tightly sealed, the leak rate is assumed to be less than $10^{-3}$ vol\%/d or $8.5 \times 10^{-3} \mathrm{~m}^{3} / \mathrm{d}$. 


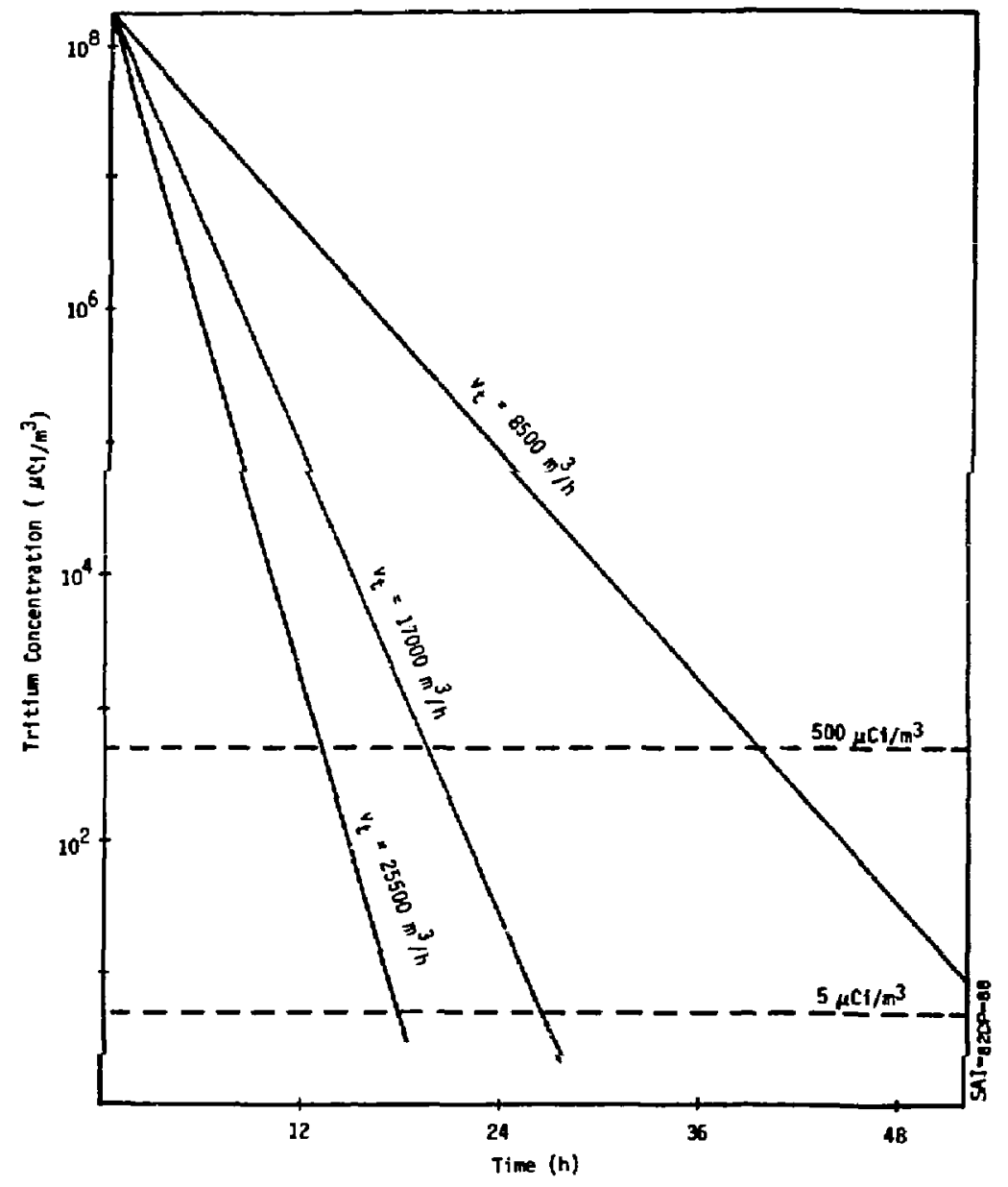

Figure 3-209. Tritium concentration in tritium processing building as a function of time and of cleanup rate for an accidental release of $300 \mathrm{~g}$ of tritium. 


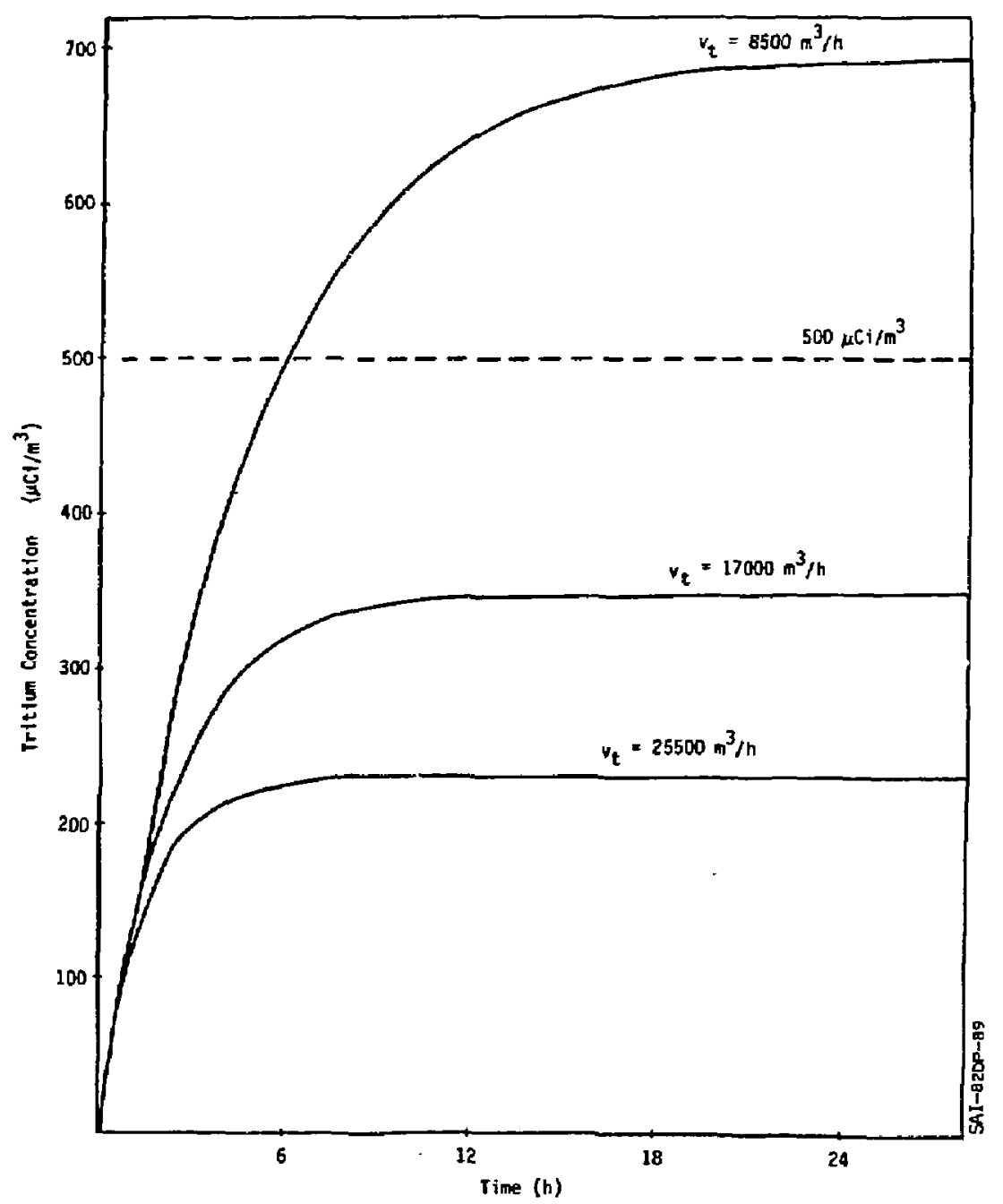

Figure 3-210. Tritium concentration in hot cell as a function of time and of cleanup rate for a $100 \mathrm{Ci} / \mathrm{d}$ source term. 


\subsubsection{Conclusions}

The capabilities of the facility cleanup systems for the assumeo conditions and inventories are presented in Tab?e 3-88. The estimated total tritiun release for even the worst-case accident scenarios is well within the design goals. Provioed the facility integrity is maintainea and electrical power is not lost for more than a day or so, the tritium cleanup systems shoula keep the tritium release beiow the design goals for all postulated accioent scenarios.

\subsubsection{Uncertain Technical Issues}

The inajor technical uncertainties that coula significantly affect the oesign ano operation of the TDF are the following:

- Rate of tritium permeation into the beam-dump cooling water.

- Presence of residual tritium in reactor components that require maintenance in the hut cell.

- Actual leak rates from the facility.

A large scale demonstration of the technology for removing tritium from large volumes of cooling water has not yet been demonstrated and will unooubtedly be very costly unless arastic technologicai breakthroughs occur. In addition, considerable uncertainty exists in the assumed tritium firmeation rates through the beam dump materials unoer the operating conditions. If the estimates made with regard to both of these uncertainties turn out to be too low, the operation of the TUF and the cost of the tritium sysitems coulo be appreciably affected. However, the aevelopment of techniques to refuce the tritium permeation rates and the development of improved ano less costly techniques for purifjcation of tritiated water are considerably more likely than not.

The accuniulation of tritium and its subsequent release during disassembly ano mainterlance of certain reactor components may not be a prodem at all, but insufficient information was collected in this brief study to assure that such will be the case. 
Table 3-88. Responses of facility cleanup systems to accidents and estimates of tritium losses to the atnosphere.

\begin{tabular}{|c|c|c|c|c|c|c|}
\hline \multirow[b]{2}{*}{ Butlding/5ystem } & \multirow[b]{2}{*}{$\begin{array}{l}\text { lnvent ory } \\
\text { released } \\
\text { (g of T) }\end{array}$} & \multirow[b]{2}{*}{$\begin{array}{l}\text { Assumed } \\
\text { leak rate } \\
\text { (volx/d) }\end{array}$} & \multicolumn{2}{|c|}{$\begin{array}{l}\text { Time (h) to reduce airhorne } \\
\text { trit tum concentration to: }\end{array}$} & \multicolumn{2}{|c|}{ Iritfum lost to atmosphere } \\
\hline & & & $500 \mathrm{uCi} / \mathrm{m}^{3}$ & $5 \mathrm{uClim}^{3}$ & $\begin{array}{l}\text { Through cleanup- } \\
\text { systen dischargea }\end{array}$ & $\begin{array}{l}\text { Dut - leak oge } \\
\text { loss of cleanup } \\
\text { system }(\mathrm{Cl} / \mathrm{d})^{\mathrm{b}}\end{array}$ \\
\hline \multicolumn{7}{|l|}{ Most prabable ser tous accident } \\
\hline Reactor bullding & 100 & 1 & 46 & 67 & 0.2 & 0.2 \\
\hline Tritium processing butlding & 300 & 1 & 13 & 18 & 0.1 & 0.8 \\
\hline $\begin{array}{l}\text { Glovebox gaseous maste } \\
\text { treatment }\end{array}$ & 50 & $10^{-3}$ & 140 & 185 & 0.1 & $0.1^{\mathrm{c}}$ \\
\hline \multicolumn{7}{|l|}{ Norst-case accident } \\
\hline Reactor buitding & 375 & 10 & 52 & 73 & 7 & 4.0 \\
\hline Tritlun processing butlding & 600 & 10 & 14 & 19 & 2.5 & 1.7 \\
\hline $\begin{array}{l}\text { Glovebox gaseous waste } \\
\text { treatment }\end{array}$ & 600 & $10^{-2}$ & 164 & 210 & 0.1 & $0.15^{C}$ \\
\hline \multicolumn{7}{|l|}{ Earthquake } \\
\hline Reactor butldingt & 100 & 10 & 46.6 & 67.7 & 20 & $-2000^{e}$ \\
\hline
\end{tabular}

assimes a cleanup system of of $10^{4}$ and all three FAO units aperating for reactor and for tritlum processing bulldings.

bassumes no pressurization.

cosses are to the trit!im processing butiding and 4 at $1-1 n$. water gauge assumed.

Assumes instantaneous pressurization to $3 \mathrm{psig}$ and a leak rate of $10 \mathrm{vol} / \mathrm{d}$

at. this pressure.
eTakes 37 min to reduce pressure to ambient with all three FAO systems

operating and discharging to the atmosphere.

Some uncertainty remains with respect to how well such a large factlity

can be sealed to minimize leak rates, It appears that inflatable seals for

the large access doors can be designed to maintain the leat rates helow the

design goai. This is not a major issue as the cleanup systems as designed

will handle considerably isrger leakage rates while still meeting the tritiun

release goats of the design. 


\subsection{PLANT FACILITIES}

\subsubsection{Introouction end Summary}

The plant for TOF includes features common to any large industrial facility as well as features unique to fusion. For example, the heat rejection system that uses natural draft cooling towers is generic to power plants ano to many industrial facilities. On the other hand, hot cell tacilities with capabilities for remote repair and mantenance functions and tritium-processing facilities with capabilities for separating larse quantities of tritium from other gases are both unique to a fusion plant. The cost estimate of such special plant faciljties (Section 5) shows their economic imoortance to the overali plant.

The various elements of the plant are designed and arranged to achieve a smoothly functioning and economic arrangement. Facilities that are functionally interdependent are located close to one another to provide operational ease and flexibjlity and to reduce cost.

Most of the conceptual design effort is focused on the facilities unique to fusion while a less detailed definition is given for the remaining facilities. These efforts have resulted in an estimate of approximate sizes and an identification of the general characteristics of the major facilities, the principal elements which are summarized in the following paragraphs.

Mechanical Systems. The heat transport system removes heat from the reactor ani from plant auxiliary components and vischarges this heat to the atmosphere by means of a mechanical draft cooling tower. Maximum continuous heat discharged is $258 \mathrm{MW}$.

The emergency cooling water system provides cooling water to the safetyrelated components (e.g., atmospheric tritium-cleanup units, diesel generators, control room) in the event of a loss of off-site power supply.

The handling system for activated structures provides for off-site disposal of structures activated as a result of reactor operation. The activated structures incluae spent test modules, beam dumps, and end cell cumps. 
The radioactive-waste handling system provides for collecting, processing, and off-site oisposal of radioactive solid, liquid, and gaseous wastes generated in the plant. These wastes include aemineralizer resin slurry, effluent from equipment and building floor drains, and vented gases from equipment and storage tanks.

In addition, a number of other mechanical systems support safe operation and maintenance of the plant.

Alternating Current Power System. The ac power system consists af a heavy-duty and : light-outy substation, each receiving power from two independent utility lines and distributing the power through protecteo electrical feeders to all the electrical loads. It also includes stanaby ciesel power generators and the noninterruptible battery inverter power supplies that proviae backup power for essential loads if the utility power fails. The heavy-duty line provides the TUF operating power to the major Traas listed in Table 3-89. If the heavy-duty power fails, the light-duty system would continue to provioe power to the loads listed in Table 3-89 and to provide enough backup power to heavy-duty systems to provide for testing ana mairtenance operations. In general, the ac power system includes all electrical equipment that receives or yenerates ac power, transforms the power to voltage levels needed by ToF loads, and distributes the power through protected electrical feeders to the various TDF loads.

Buildings and Structures. ine major buildings and structures that have interoependent functions, that require close proximity, and that are plant safety related are located on a common floor mat and on multiple levels. This arrangement is cost effective and does not affect operational flexibility. These builaings are the reactor building, the hot cell building, and the tritium builoing. The power supply and cryogenics building is not a safety-related building. To reduce cost, this building is structurally separate from the reactor builaing but is in close proximity and has a common wall with the reactor building. Although a major building, the control building is located approximately $100 \mathrm{~m}$ away from the reactor building to reauce magnetic field effects on the computers.

In addition to the above major buildings, other buildings and structures are proviaed to support safe operation and maintenance of the plant. 
Table 3-89. Major electrical power loads.

MH MV.A

- Tritium neutral-beam power supplies

$75 \quad 85$

- Ueuterium neutral-bean power supplies

- ECKH power supplies

$95 \quad 105$

- hater-cooiea copper-coil power supples

55

- Cryogenic system power

5260

- Cooling system power

$12 \quad 15$

- Fueling, pracessing, and tritium-cleanup power

- Vacuitm-system power

$10 \quad 12$

- Superconducting-coil power supplies

- Facilities (Table?-94)

$8 \quad 10$

23

12

$15 \quad 18$

Total

275

315 
Key Issues. The oesign of the hot cell builoing is a key facility design issue. The remote maintenance requirements of the reactor, though not yet adequately identified will determine the hot cell size and may significantiy increase the building size.

\subsubsection{Facility Requirements}

The plant facilities requirements can be classified under four categories--functional, design, operation and maintenance, and safety. The design requirements derive from all the other requirements and specify the criteria to be used while designing a system or a component.

Furctional Requirements. The functional requirements provide the support functions for one plant as a whole. Thus plant facilities must provide the following:

- Housing and structural support for all the equipment, components, and services of the plant.

- Protection from excessive release of radiation to th ? environment (allowable release limits are discussed in Section 4 , Safety Assessment).

- Protection for safety of public and plant personnel from natural phenomena, incluoing tornado ana seismic events.

- Features for safe and efficient movement of material, personnel, and equipment as required for plant operation and maintenance.

- All facilities and services that will support safe functioning of the plant, e.g., supplies of cooling water, electricity, radioactive-waste handing facilities.

Operation and Maintenance Requirements. These requirements are discussed ir Section 3.16 of this report (Maintenance).

Safety Requirements. These requirements specify the upper bounds on the consequences of operation of a system or a component in order to maintain safety of public and plant personnel. (See discussion in Section 4.) 
nesign Requirements. As already mentioned, the design requirements derive rom all other requirements. Although many are required for a detailed design of plant facilities, orly a few major requirements are considered for the present conceptual design effort.

- Neutron ano gamma radiations--The walls, floors, and ceilings of the reactor building ano hot cell building shall be adequately shielded (approximately 2-m-thick concrete) to limit release of these radiations.

- Tritiull permeation--The internal surfaces of the reactor building (including radwaste and heat-exchanger areas), hot cell builaing, tritium building, and the cryogenics building, are lined with epoxy coating to liwit tritium permeation into concrete.

- building design leak rate--Tc limit leakage of radioactive gases (primariiy tritium) from the reactor builaing, hot cell building, and tritium-processing building, we will assume a leak rate of less than $10 \%$ per day. The basis for this rate is discussed in Section 4, Safety Assessment.

- Plant normal and emergency cooling--During normal operation, a total of $258 \mathrm{Mh}$ of thermal power must be removed from the TOF device, its support systems, and miscellaneous plant facilities. In addition, several components and facilities require emergency cooling water in case the normal cooling water supply is disrupted because of a loss of off-site power supply. The total emergency cooling load is expected to be less than 1 MH. The components and facilities requiring emergency cooling water are the atmospheric tritium cleanup units, the diesel generators, and the controt building.

Structural Design Cyiteria. These criteria govern the design of the buildings and structures for the TDF plant. In general, the design criteria comply with a) 1 appropriate federal regulations as well as with all industry coues and standarss. State and local government regulations, codes, and standaras must be added to these criteria after a site is selected.

The criteria specified herein are based largely on those established for the breeder plant and, as a result, are more detailed than usual for this TDF study. The information is readily available, and will provide a useful base 
for future studies. It is implicitly assumed that most of the structural design criteria applicable to breeder and light-water-reactor plants will also apply to fusion plants.

Codes and Stanoards. Unless stated otherwise, the design of all buildings ana structures is tentatively based on applicable portions of the following codes, specifications, industry standards, regulations, and other reference documents. Where conflict occurs between criteria, the more restrictive will apply. Where no date is shown, the latest issue will apply.

Governing Codes, Specif ications, and Industry Standards.

- International Conference of Building Officials, "Uniform Building Code" (UBC).

- American Institute of Steel Construction (AISC), "Specification for the Design, Fabrication, and Erection of Structural Steel for Buildings," 8th Edition, 1978.

- American Welding Society (AWS), "Structural Weloing Code", AkS 01.1 - 79; and "Reinforcing Steel Welding Code," AWS D12.1 - 75.

- American Concrete Institute (ACI), "Building Code Requirements for Reinforced Concrete" ACI 318 - 77 and Commentary; and "Code Requirements for Nuclear Safety Related Cincrete Structures," - Ai I 349 - 76 .

- American National Standards Institute (ANSI), "Building Code Requirements for Minimum Design Loars in Buildings and Other Structures," ANSI A58.1.

- American Society for Testing and Materials (ASTM). Applicable standards of ASTM specifications are referred to in Section A.7. Construction Materialc.

U.S. Nuclear Regulatory Commission (USNRC) Regulations and Standard Revie Plans.

- Code of Federal Regulations (CFR), Title 10 - Atomic Energy, Part 50, "Licensing of Production and Utilization Facilities," inciuding applicable Appenoices $A$ through $M$. 
- Code of Federal Regulations (CFR), Title 10 - Atomic Energy, Part 100, "Reactor Site Criteria," including Appendix A thereto, "Seismic and Geologic Criteria for Nuclear Power Plants," adopted ivovember 27, 1973.

- USNRC - Regulatory Guides as follows:

1.59 Design Basis Floods for Nuclear Power Plants

1.60 Design Respanse Spectra for Seismic Design of Nuclear Power Plants

1.61 Damping Values for Seismic Design of Nuclear Power Plants

1.76 Uesign basis Tornado for Nuclear Power Plants

1.92 Combination of Modes and Spatial Components in Seismic Response Analysis

- USNRC - Standard Review Plans

\subsubsection{0ther Seismic Category I Structures}

Site Characteristics. A hypothetical site for the TDF was selected for the purposes of this study. This hypothetical site is not only generally compatible with installation at an established government nuclear reservation, such as laaho Nationa] Engineering Laboratory (INEL), but also is conpatible with a significant number of potentia? U.S. sites. General information about the site is given in Table 3-90.

Seismic Design Classificaton of Structures. From the standpoint of their importance to safety, the plant structures, systems, and components are classified into three categories as follows:

Seismic Category I Siructure. Seismic Category I structures, systems and components are necessary to ensure the capability of preventing or mitigating the consequences of accidents that could result in potential off-site exposures comparatle to the guideline exposures of DOE (see Section 4). These structures shall be designed to remain functional in the event of an earthquake of maximum intensity. The following are Seismic Category 1

Structures: reactor building (including heat exchanger and radwaste areas), hot cell building, tritium building, diesel-generator building, control building, control tunnel. 
Table: 3-90. Characteristics of hypothetical site for TDF.

Characteristic

Value assumed

Geology/seismology

MIE $^{\text {a }}$ ground acceleration

$-0.25 g$

PAPE ${ }^{\mathrm{a}}$ ground acceleration

$-0.13 \mathrm{~g}$

Ground response spectra

Use Regulatory Guide

1.60 assumptions

$U B C^{a}$ Seismic Zone 2

Zone 2

Foundation material

Net allowable bearing capacity

Static

$500 \mathrm{KPa}$

Dynamic

$700 \mathrm{kPa}$

Damp ing

Use Regulatory Guide

1.61 assumptions

"s" wave velocity

$1200 \mathrm{~m} / \mathrm{s}$

Depth-to-bedrock

At surface

Location of the nearest surface

The site is located more than $8 \mathrm{~km}$ capable of faulting

from a surface capable of faulting more than 305 in in length.

Snow and ice load

$1 \mathrm{kPa}$

Wind load for safety-related structures

Velocity at $9 \mathrm{~m}$ above ground

$145 \mathrm{~km} / \mathrm{h}$

Tornado loading

Region

Use regulatory guide 1.76 ,

Region III, Design Basis Tornado

Characteristics

Maximum wind speed
Rotational speed
Maximum rotational speed
Radins of maximum rotational speed
Pressure drop
Rate of pressure drop

$386 \mathrm{~km} / \mathrm{h}$

$306 \mathrm{~km} / \mathrm{h}$

$80 \mathrm{~km} / \mathrm{h}$

$46 \mathrm{~m}$

$10 \mathrm{kPa}$

$4.1 \mathrm{kPa} / \mathrm{s}$ 
- Non-Seismic Category I Safety Related Structure. These structures are not necessary to ensure the capability of prevent or mitigate the consequences of accidents which could result in potential offsite exposures comparable to the DOE guidline exposures, but whose damage coulo affect the integrity of adjacent safety related systems, structures, and equipment. The following are the Non-Seismic Category I Safety Related Structures: power supply and cryogenics bujlding, vent stack.

- Non-Seismic Category I Non-Safety Related Structures. These are structures whose damage could not affect the integrity of safety-related systems, structures, and equipment. A11 plant structures not listed in the previous two categories are in this group. Non-Seismic Category I Non-Safety Related Structures include but are not limited to the following: administration builaing, plant services building, cooling tower, demineralized-water storage tank, air-compressor stacion, water-treatment plant, pump house, raw-water storage tank, fire-water storage tank, off-site raw-water supply line, off-site power supply, guard house.

Plant Internal Loads. Dead loads include, first, the wejght of all permanent. structural elements and, second, the wejght of all permanently attached equipment, piping, etc.

Live loads include, first, floor area loads (occupancy and laydown) and, second, loads from moveable equipment or structures.

Internal Pressure Load for the Design Basis Accident. For the design basis accident the internal pressure of the reactor building is $120 \mathrm{kPa}$. The positive pressure is the result of an accident involving cryogen release and nelium pressurization.

Thermal Load. Accident temperatures are unspecified at this time.

Loaas Not Included. For simplicity, the following loads are not included in the preconceptual structural design criteria: missiles (other than tornado), normat and accioent pipe loads, dynamic earth pressures, ano conpartment pressurization. 
Design Bases. In the following sections, factors of safety, codes, and loading combinations, which will be used for all structures, are established.

Factors of Safety. A minimum factor of safety shall be provided for all structures as shown in Table 3-91.

Seismic Category I Structures. Concrete structures in Seismic Category I are designed in accordance with ACI-349 and supplemented by ACI-318. The steel structures will be designed in accordance with the AISC code. The applicable loading combinations will be as specified in the ARC Standard Review Plan 3.8.4.

Non-Seismic Category I Safety-Related Structures. Reinforced concrete structures in this category are designed in accordance with ACI-318 for concrete structures and will be in accordance with the loaoing combinations in Chapter 9 of $\mathrm{ACI}-318$. The steel structures are desigried in accorance with the AISC code. Wind and sejsmic loadings are in accordance with ANSI A-58.1 and the Uniform Building Code (UBC), respectively.

In addition to the above loading combinations, Non-Seismic Category I Safety Related structures are checked and strengthened as required to assure they do not collapse on a Category I structure or system during a design basis tornaco or maximum intensity earthquake (MIE) event.

For concrete structures under these conditions, the load factor for a 11 loads shall be 1.0. For steel structures the allowable stresses shall be increased by $60 \%$ and the plastic section modulus of steel shapes sha11 be used. For these load combinations, only the main structural members are considered. The design criteria allows these siructures to be damaged during these events; for example, permanent deformation of the structures may result from these extreme loadings.

Non-Seismic Category I Non-Safety Related Structures. These structures will be designed in accordance with ACI-318 for concrete structures. Steel structures will be designed in accordance with the AISC code, and wind and seismic loadings wilT be in accordance with ANSI A-58.7 and the $U B C$, respectively. 
Table 3-91. Safety factors assumed for various loading conditions.

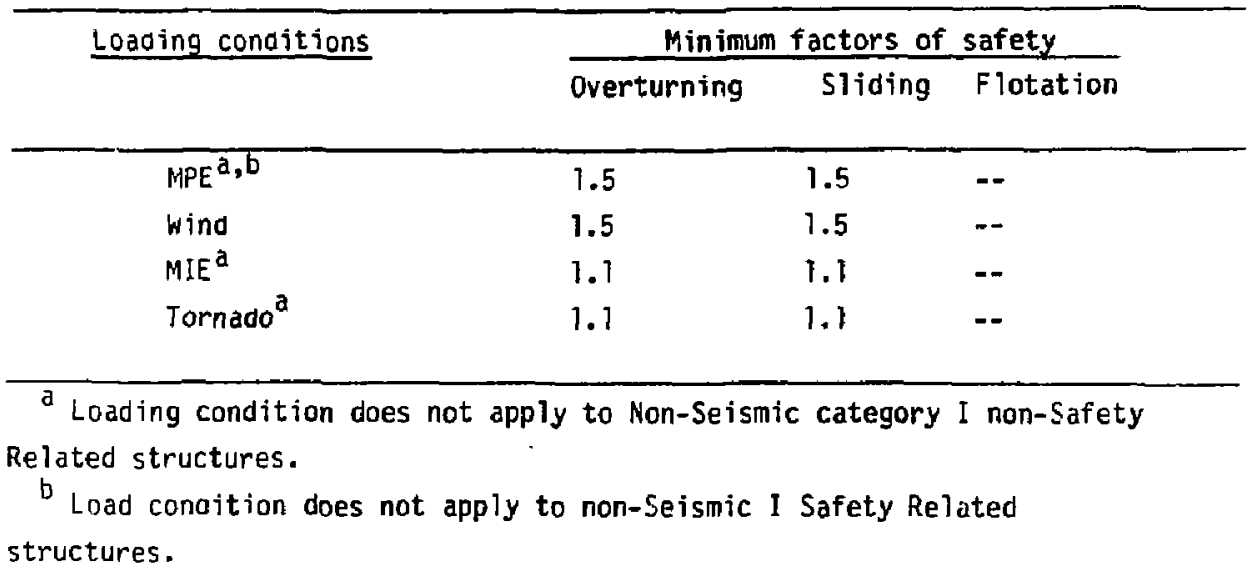


Construction Materials. The principal construction materials for Seismic Category I and Non-Seismic Categary I structures are concrete, reinforcing steel, structural steel, masonry, and metal decking and are noted below:

- Concrete design compressive strength are for all plant structures is $27 \mathrm{MPa}$, and for lean concrete backfill and for electrical duct encasement is $17 \mathrm{MPa}$. (Compressive strength ( $\mathrm{fc}^{\prime}$ ) refers to compressive strength at 28 days if oniy cement is used and to compressive strength at 90 days if pozzolan replaces part of the cement.

- Keinforcing steel shall be deformed billet steel, conforming to ASTM Designation A-615 grade 60.

- Welded steel-wire fabric sha]1 conform to ASTM A-185 (plain wire) or A-497 (deformed wire).

- All structural steel shali be ASTM A-36.

- Fasteners shal1 conform to ASTM A-307. High-strength bolts shall conform to ASTM A-325 or A-490.

- Anchor bolts shall conform to ASTM A-307, A-36, or A-449.

- Unless otherwise required by special conditions, such as the contro] room penetration areas, metal decking shall be used and considered in the design for forming concrete slabs.

AC Power. The as power system must perform the following functions:

- Provide ample power for all electrical loads to TDF, such as those ident if jed in Tables 3-91 and 3-92.

- Provide enough independent power to the facility to allow maintenance and test work if the main power system fails.

- Provide standby backup power to essential loads if all utility power is Tost.

- Provide coorainated fault protection to the feeders at all voltage reveis.

Electrical equipment will be designed for continuous operation with limited low-cost redundancy to improve the system availability. Based on the data of Tables 3-91 and 3-92, the ac power lines and backup power units will require the capacities and voltage regulations given in Table 3-93. 
Table 3-92. Estimate of facility power loads.

\begin{tabular}{|c|c|c|}
\hline \multirow[b]{2}{*}{ Load description } & \multicolumn{2}{|c|}{ Power } \\
\hline & kW & $k V \cdot A$ \\
\hline Building and ground lighting & 500 & 500 \\
\hline HVAC $^{\mathrm{a}}$ for all buildings & 1500 & 3000 \\
\hline Fuel reporcessing & 5000 & 6000 \\
\hline Shop and service equipment & 2500 & 3000 \\
\hline Laboratory and office equipment & 900 & 1000 \\
\hline Diagnostics & 1500 & 1800 \\
\hline Data acquisition & 90 & 100 \\
\hline Process $C \& I^{b}$ & 90 & 100 \\
\hline Emergency tritium removal ${ }^{b, c}$ system (1) & 1300 & 1400 \\
\hline system (2) & 1300 & 1400 \\
\hline Emergency cooling & 500 & 600 \\
\hline Essential lights & 50 & 50 \\
\hline Uninterruptible power ${ }^{b}$ & 40 & 50 \\
\hline Backup power for testing and maintenance & 8000 & 10000 \\
\hline
\end{tabular}

\footnotetext{
${ }^{a}$ Heating, ventilating, air conditioning.

b Transferable to diesel generator plant.

C Only one of the two systems needed for cleanup.
} 
Table 3-93. Capacity requirements for ac power system.

Uescription

Power

Two indepentent utility lines

Heavy-duty power line

Light-duty facility power line

$400 \mathrm{MV} \cdot \mathrm{A}, 230 \mathrm{kV}$

$50 \mathrm{MV} \cdot \mathrm{A}, \quad 69 \mathrm{kV}$

Heavy-duty line to provide

Voltage regulation

$\pm 10 \%$ daily variation

Power to major loads

$300 \mathrm{NV} \cdot \mathrm{A}, 13.8 \mathrm{kV}$

Backup power to facility line

$20 \mathrm{MV} \cdot \mathrm{A}, 13.8 \mathrm{kV}$

Light-duty line to provide

Voltage regulation

$\pm 5 \%$ daily variation

FaciTity power

$\pm 7 \%$ normal transient

Backup power to heavy-duty 7 ine loads

$15 M V \cdot A, \quad 13.8 \mathrm{kV}$

$15 \mathrm{MV} \cdot \mathrm{A}, \quad 13.8 \mathrm{kV}$

Two standby diesel generators to provide

Backup power to essential loads

$2500 \mathrm{kV} \cdot \mathrm{A}, 2.4 \mathrm{kV}$

Four noninterruptible power supplies to provide

Power to noninterruptible loads.

$40 \mathrm{KV} \cdot \mathrm{A}$ 


\subsubsection{Design Description}

The systems, buildings, and structures of the plant facilities are designed with the plant operation and functional requirements in mind in order to achieve the most desirable relationship between the systems and components and to provioe building locations, shapes, and sizes that are consistent with the space required by their functions and contained equipment. Arrangement of the overall site facilities is shown on the TOF Plot Pian (Fig. 3-211). All of the faciities, except the river water pump house and possibly an operator training facility, are enclosed within the exclusion area boundary fence. However, the administration, control, ano security buildings are located just outside the radiation safety zone. The exclusion area is set at a radius of 500 in for public safety reasons (see Section 4). Brief design descriptions of the major individual systems, buildings, and structures are given in the following subsections.

Site Facilities. The following paragraphs and Fig. 3-2ll pertain to the site facilities provideo for the JDF plant.

Design Basis. A?trough the TOF plant is designed for a wide range of potential sites throughout the continental United States, the following assumptions are made for the purpose of this study:

- Location: A site with characteristics equivalent to the Iaaho National Engineering Labaratory (INEL) site.

- Seismic zone: UBC Zone 2

- Tornado zone: Zone 3

- Exclusion Area Boundary: 500-m radius

- Water Supply: Assumed adequate to meet all normal requirenients

- Electric Utility: Assimico high-voltage supply (>l60 kV) available

Design Description. Principal site elements include buildings, structures, access roads, on-site roadways, security fencing, and surface and subsurface mechanical and electrical equipment and utilities. The mechanical draft cooling tower is located at the rear of the site consistent with the 


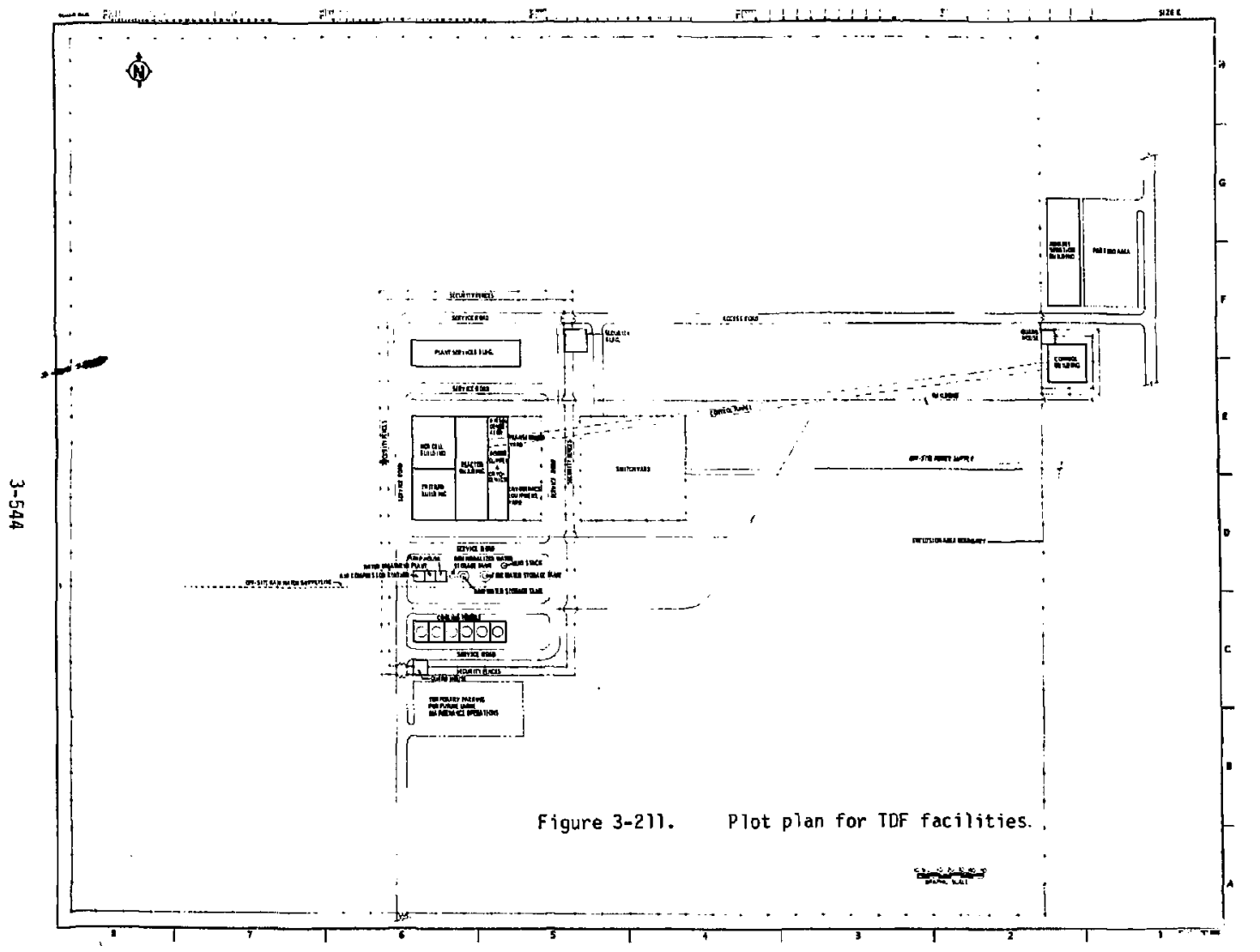


prevalent wind direction of the site. All the site elements are located and arranged on the basis of safety, functional and operational flexibility, constructability, and economic considerations.

Aaministration, Control, and Security Buildings. All traffic to and from the site is controlled at the security building located just outside the exclusion area fence. All administrative and plant control personnel are houseo in this building complex. Visitors are allowed to observe the operation of the reactor through closed-circuit TV from the control building. This arrangement restricts non-maintenance personnel and visitors to areas outside the radiation safety exclusion area.

Experimental Complex. The heart of the TDF plant is located in the building complex referred to as the "experimental complex" for convenience. This complex consists of the reactor building, iincluding heat exchanger and radwaste areas), hot cell building, tritium-processing building, and power supply and cryogenics building, including the diesel generator area.

Switchyard. The switchyard is a fenced area that contains all the electrical equipment necessary to accomodate incoming high-voltage power. It is located close to the power supply and cryogenics building and the transformer yard.

0ther Site Facilities. Other major facilities provided include a river-water-intake pump house (which supplies all normal plant water requirements), raw water reservoir, raw water tieatment equipment, fire water storage tank, and plant fire protection equipment.

Mechanical Systems.

Heat Transport System. The heat transport system (HTS) provides the equipment and facilities for supplying high-purity jemineralized and deionized cuoling water to remove heat from the reactor components and other auxiliary components of the plant during normal operation. The components served by the system and the corresponding heat loads are indicated in Table 3-94. 
Table 3-94. Major plant cooling loads.

\begin{tabular}{lc}
\hline Systems and components: & Cooling load, MW \\
Neutral dump & 16 \\
Neutral-beam internal & 65 \\
End-cell dump & 44 \\
Subtotal & \\
Reactor components: & 52 \\
Copper coils & \\
Test module 28 & 4 \\
Shield & 4 \\
Vacuum vessel & \\
ECKH & \\
Fuel processing and cleanup & 4 \\
Subtotal & \\
Facilities and other systems: & 12 \\
Cryogenics system & 16 \\
Power conversion & \\
Coolant pumping & -- \\
HVAC and miscellaneous & \\
Subtotal & 258 \\
Total & \\
\hline
\end{tabular}


A schematic diagram of the heat-transport system is shown in Fis. 3-2.12. As seen in this figure, the HTS cansists of three primary heat transport loops ano a heat rejection loop. The heat rejection loop disposes of the heat to the atmosphere through a mechanical draft wet cooling tower. The reason for separating the primary heat transport into three loops is twofold. First, this separates the components operating in a tritium and neutron environment from those not operating in a similar environment. As seen in Fig. 3-212, the components in the reactor cooling ano power absorption cooling loops operate in a tritium and neutron environment. Secondly, the components in the power absorption cooling loop require high coolant pressure to avoid boil-off of the coolant which may be caused by high-surfare heat load. The peak coolant pressure in the power absorption cooling loop is $2.4 \mathrm{MPa}$ while that in the reactor and facility cooling loops is $1.0 \mathrm{MPa}$. For all the three primary cooling loops, the hot and cold leg temperatures are 60 and $43^{\circ} \mathrm{C}$, respectively. The hot and cold leg temperatures in the heat rejection loop are 45 ana $28^{\circ} \mathrm{C}$, respectively.

\section{Ernergency Cooling Water System. This system provides cooling water} primarily to the atmospheric tritium cleanup units (ATCU), solid waste system, aiese? generators, and control building heating, ventilating, and air conditioning (HVAC) during aDnormal operating conaitions. Operation of the system nccurs, for example, during an incident when the reactor has been shut down and there is a large tritium leakage; the off-site power is unavailable, and the plant is using an on-site power source (diesel generator). The ATCUs are the major components requiring emergency cooling water, and it is assumed that no more than one of the ATCUs will be operating at full capacity ouring she abnormal inciaent. The largest ATCU cooling water load occurs when the -eactor building ATCU is in the emergency operating mode. A schematic aiagram If the emergency cooling water system is shown in Fig. 3-213. The system is a losed cooling loop with a forceo-draft water-to-air heat exchanger for ejecting the heat to the atmosphere.

Activated-Structures Handling System. The periodic replacement (as a result of neutron activation) of the reactor components (viz., beam dumps, choke coil structure, test-module first wall, etc.) generate activated, solid, 


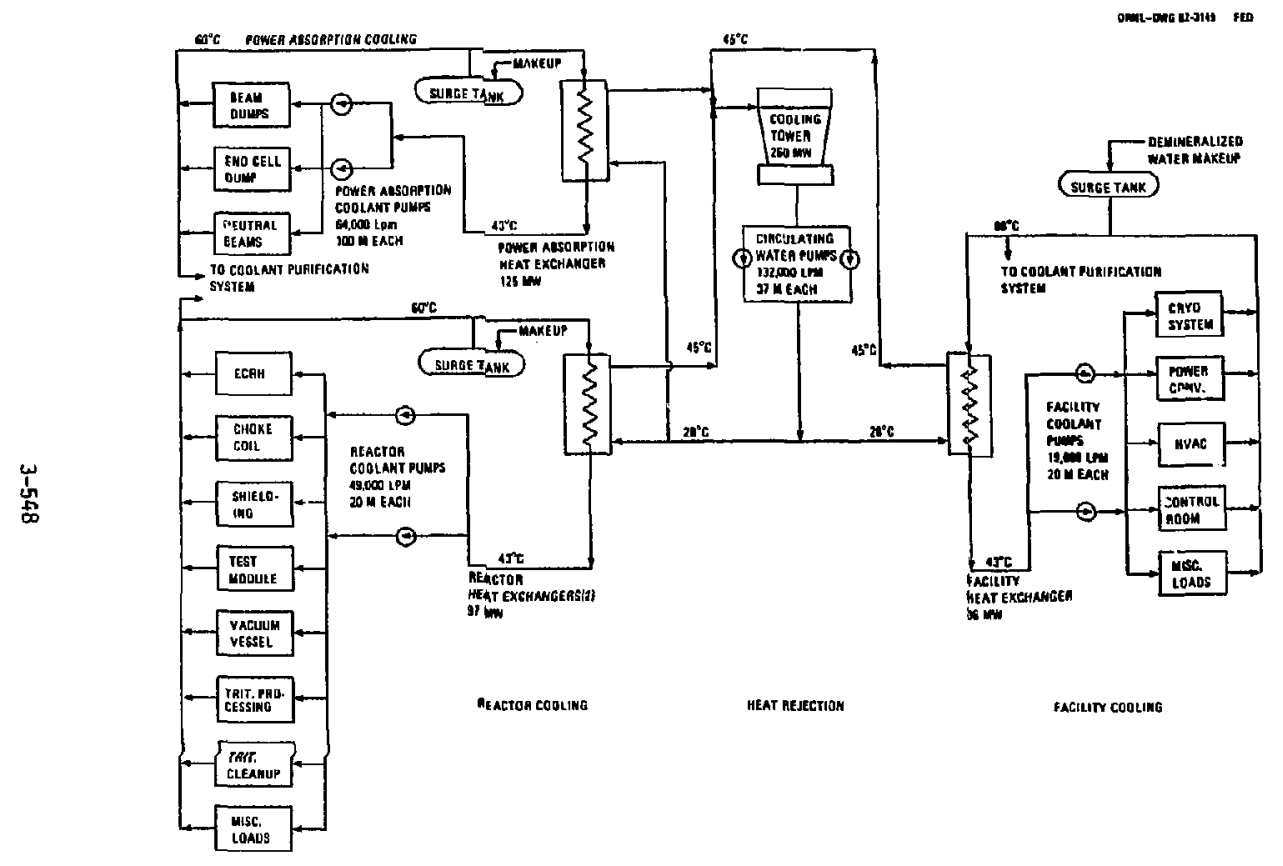

Figure 3-212. Heat transport system. 


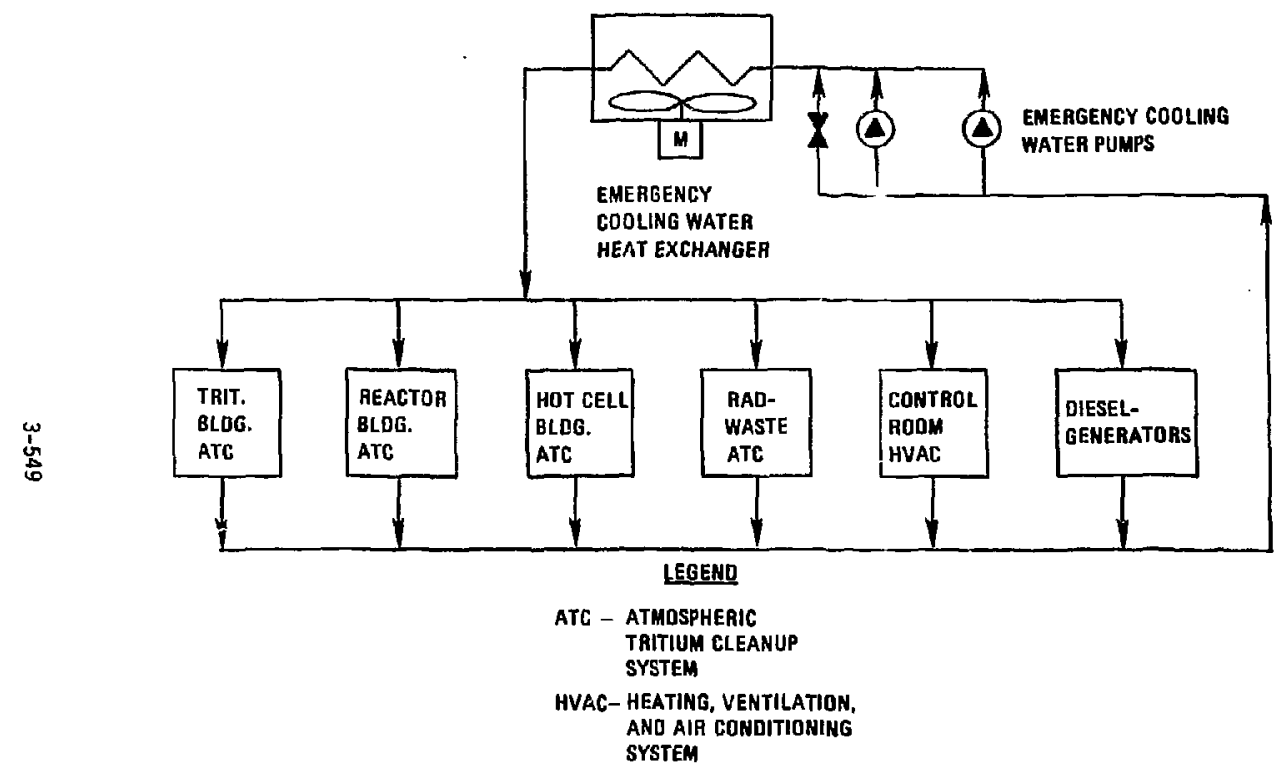

Figure 3-213, Emergency-cooling water systenl. 
radioactive wastes. A conceptual scheme for handing and disposing of these wastes is discussed here.

To put the magnitude (mass) and characteristics (activity and afterheat) of the wastes in perspective, the activated-structures' radioactive waste for three fusion reactor concepts are listed in Table 3-95. The informaton was obtained from Refs. 49 and 50. Similar information for the TDF reactor is not available at the present stage of design development; however, it is anticipated that the mass and afterheat of the TDF wastes will be much srialler, because Tor operates at a much lower thermal power level and does not have any blanket. Also there may be significant differences in waste characteristics, depending on the choice of structural materials and component design.

The processing and disposal of activated structures is strongly affectea by the initial level and time-dependent behavior of induced activity anc nuclear af terheat. The initial Tevels of activity and afterheat dictate the shielding and cooling requirements, respectively. The decay characteristics determine the storage required to reduce the levels of activity and af terheat prior to processing and off-site disposal. To determine the shielding, cooling, and storage requirements for the irradiated structures, it is necessary to generate decay curves for activity and afterheat. To date, aoequate information has not been developed to generate these curves. Baseo on preliminary information, ${ }^{49}$ order-of-magnitude decay characteristic curves were generated (Figs. 3-214 and 3-215) for the helium-cooled tandem-mirror hybria reactor (TMHR-He). Similar curves for UWMAK-III reactor 50 are also included to provide some perspective on the decay characteristics of several structural materials. Sinilar decay characteristics are expected for TOF although the initial level of activity will be much less because of much lower neutron fluence.

Figure 3-216 is a schematic of a typical on-site process for the removal and preparatory work required for off-site disposal of activated structura] materials. After the component requiring replacement is removed from the reactor, it is transferred to the hot cell where decontamination and aisassembly operations are performed. Here the reusable (if any) portion of the component is separated from the nonreusable portion and held for assembly 
Table 3-95. Amaunt and characteristics of radioactive waste for three reactor types. (Data obtained from Refs. 56 and 53.$)^{a}$

\begin{tabular}{|c|c|c|c|c|c|c|c|}
\hline Reactor & Source & $\begin{array}{l}\text { Constituent } \\
\text { matertals }\end{array}$ & $\begin{array}{l}\text { Annual } \\
\text { radwaste } \\
\left(10^{3} \mathrm{~kg}\right)\end{array}$ & $\begin{array}{l}\text { Specific } \\
\text { activityb } \\
\text { at shutdown } \\
\text { (CI/CM) }\end{array}$ & $\begin{array}{l}\text { Tota] } \\
\text { octivity } \\
\text { at shutdown } \\
\text { (CI) }\end{array}$ & $\begin{array}{l}\text { Speciffo } \\
\text { afterheat } \\
\text { at shutdown } \\
\left(\mathrm{H}_{\mathrm{T}} / \mathrm{GM}\right)\end{array}$ & $\begin{array}{l}\text { Total } \\
\text { afterheat } \\
\text { et shutdown } \\
\left(b_{T}\right)\end{array}$ \\
\hline $\begin{array}{l}\text { INAMAK-II] } \\
\text { (2000 MNE) }\end{array}$ & $\begin{array}{l}\text { First wall } \\
\text { Blaniket }\end{array}$ & TZH & $B 2$ & 24 & $2 \times 10^{9}$ & 0.1 & $8 \times 10^{6}$ \\
\hline $\begin{array}{l}\text { THHR } \\
\text { HeTfum cooled } \\
\text { (400 MNA) }\end{array}$ & $\begin{array}{l}\text { First wall } \\
\text { B Ianket }\end{array}$ & $\begin{array}{l}\text { Inconel } \\
718 \\
\text { HT-9 }\end{array}$ & 12.6 & 80 & $1 \times 10^{9}$ & 0.3 & $4 \times 10^{6}$ \\
\hline $\begin{array}{l}\text { THHR } \\
\text { molten-salt } \\
\text { cooled } \\
\text { (4000 MHA) }\end{array}$ & Blanket & $\stackrel{\text { T2M }}{\cdot}$ & 7.1 & 53 & $3 . B \times 10^{B}$ & 0.2 & $1,5 \times 10^{6}$ \\
\hline
\end{tabular}

For TQF, mass and afterfeat are expected to he much less than figures show here.

bafter one year of operation.

cTMHR is Tanden Mirror Hybrid Reactor. 


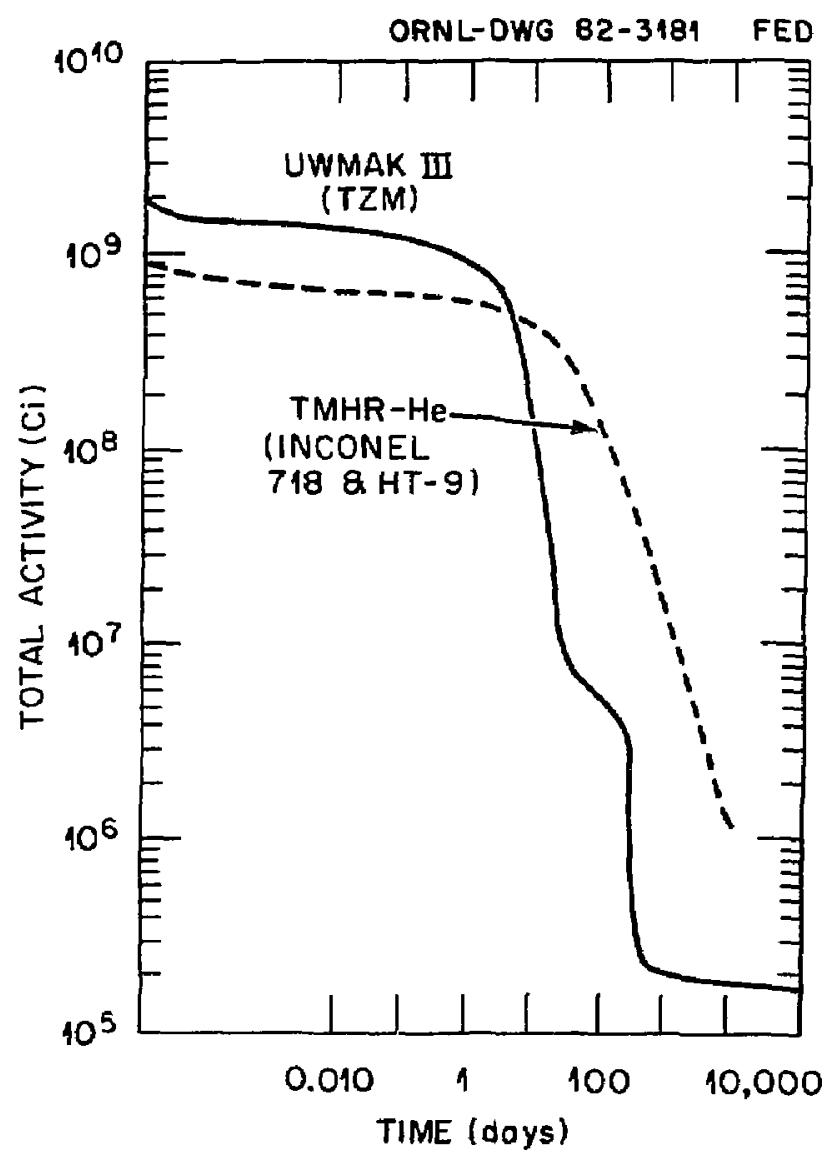

Figure 3-214. Activity as a function of time after reactor shutdown. 


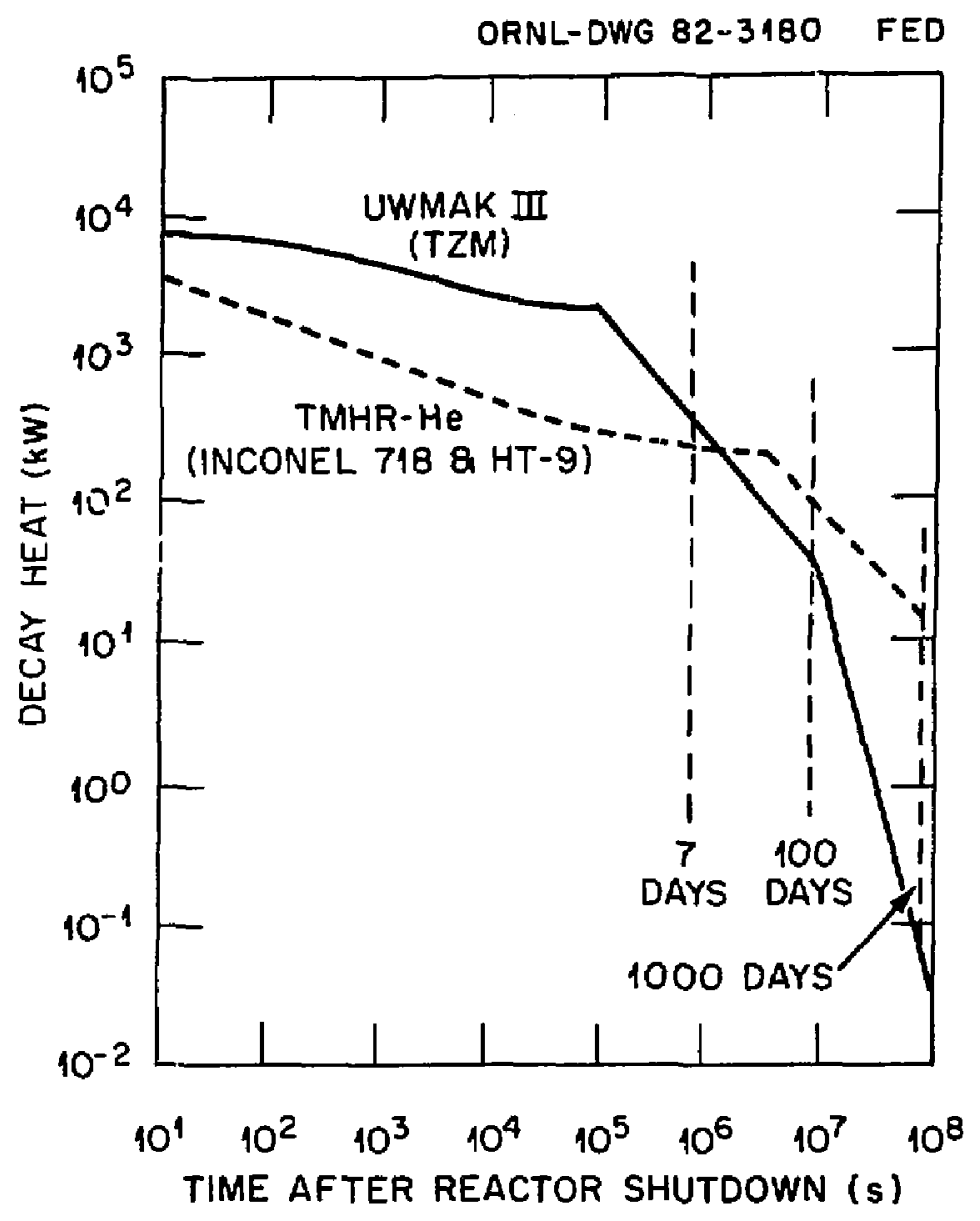

Figure 3-215. Decay heat vs time after reactor shutdown. 


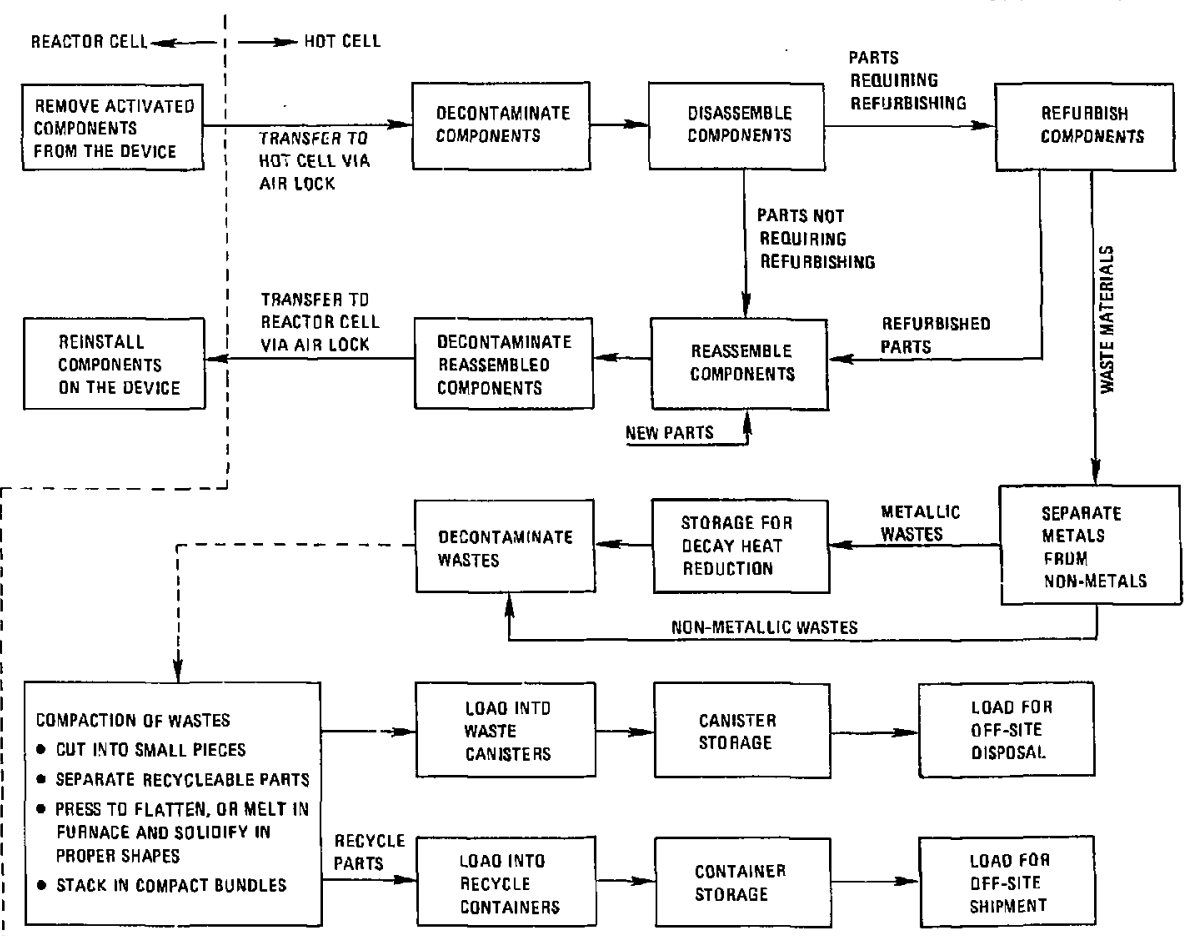

Figure 3-216. Processing of activated structure wastes. 


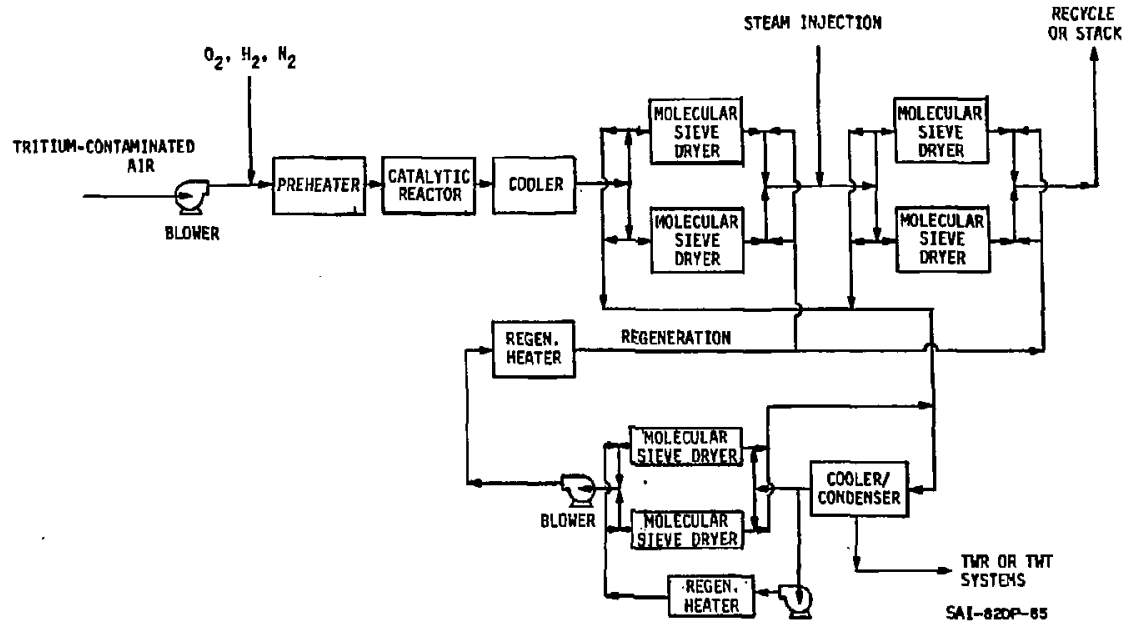

Figure 3-206. Contaminated air distribution system for TDF. 
cooling water at significant rates because of implantation energy and the relatively high temperature of the beam dump material. It has been estimated that for a full-power year of operation about $21 \mathrm{~g}$ of tritium will enter the cooling water through the neutral-beam aumps and about $98 \mathrm{~g}$ of tritium will enter through the plasma end dump.

Because leaks in the beam dump cooling system are virtually impossible to prevent, we must reduce the tritium in the coolant to a tolerable concentration. Experience with nuclear reactors and other processes using tritiated water indicates that a tolerable level of tritium coolant is about I Ci/liters or less. It can be shown that to maintain the tritium concentration in the beam oump cooling water below about $1 \mathrm{Ci} / 1$ iter, tritiated water must be removed at a rate equivalent to the curies per liter of tritium entering the ccoling water. Because the fusion device will only be operated with a maximum availability of about $50 \%$ full power per year, the TWR system should have a process capacity of about 1600 liters/d. With the TWR system in operation, about 50 days of full-power operation by the fusion cievise would be required before the tritium concentration in the cooling water reaches 0.9 [i/liter.

The TwR system is shown schematically in Fig. 3-207. The process involves combined electrolysis and catalytic exchange technology. The tritiated water is injected into the middle of the exchange column that is filled with a catalyst material to promote the exchange of gaseous hydrogen isotopes with water. Because the equilibrium at the column operating temperature is near ambient, formation of tritiated water (HTO) is favored, and enriched HTO concentrates in the bottom of exchange column. A portion of this enriched HTO is removed ano electrolyzed to form hydrogen isotopes and oxygen. Most of the electrolyzed hydrogen isotopes are returned to the bottom of the exchange column to further enrich the water in tritium, while the remaining portion of the gas is airected to a cryogenic aistillation column for adaitional isotopic enrichment. The overheads from the aistillation column are returned to the top (stripping portion) of the column for exchange with the tritium-aepleted water and stripping of any remaining tritium from the gas. The tritium-free hydrogen gas, mixed with oxygen from the electrolyzer, then exits through the top of the exchange column, recombine to form water, and returns to the cooling-water system. 
with new components. The assembied components pass through the decontamination process to remove loose contamination that may otherwise spread in the reactor building.

The need to store activated structures for decay heat remova? depends on the waste shipping requirements, which include limiting the shipping container heat load, geometry of cask, Department of Transportation (DOT) packaging requirements, etc. The next step in the processing of activated structure, after it has decayed to the required level, is compacting for disposal. The compaction process generally involves cutting the structure into small pieces followed by separating the recyclable parts. The recyclable parts are shipped off-site for further processing, if needed. The unrecycleable parts are either pressed to flatten or are melted in a furnace if the pieces are highly irregular in shape. Finally, the compacteo waste is loaded in canisters for shipment to the disposal site.

The hot cell facilities needed for the activated structures handing cannot be accurately determined until more is known about the merall process requirements. Most of the remote handing equipment needed is identifjed and discussed in Section 3.16, Maintenance. The equipment needed for the compaction process and subsequent preparation for off-site shipment inciudes:

- Hydraulic/pneumatic press for flattening.

- Furnace to melt the irregularly shaped pieces.

- Molds to cast the molten material into desired shapes.

- Remote manipulators to facilitate above operations and to load the compacteo pieces into canisters.

For the process describeo above for handing activated structures and with the hot cell facjities provided for the purpose we assume that the largest component to be handled is the central cell magnet. If, in an unlikely event an activated component larger than the central cell magnet requires replacement and or disposal, special facilities will be required and will be provided when the need arises. However, long-term storage facilities for radioactive decay of these comonents are provided in the hat cell buifding as well as in the reactor building.

Radioactive-Waste Handling Systen. The radioactive waste handling system, or the ragwaste system as it is commonly called, provides equipment 
anc facilities for collecting, storing, processing, monitoring, and disposing of liquid, solid, and gaseous radioactive wastes. No attempt has yet been made to quantify the amount of radwastes generated and, as a result, no estimate of total amount of radioactivity associateo with these wastes is available. Thus only a scheme for handling radioactive wastes and the nature of the equipment and facilities needed are outlined here.

Liquid wastes originate from demineralizer wastes, valve and pump seal leaks, laboratory processing fluids, equipment drains, shower and laundry drains, Jecontamination and washdown operations. Gaseous wastes originate nlainly from dissolved gases in the coolant, radiolytic decomposition of water, gases added to the plant such as helium, nitrogen, sulfur hexafluoride, anc vent air circulated through storage tanks. Solid wastes arise from a variety of wiscellaneaus sources. The three miar saurses are the sisent res in from the various demineralizer:, the used filter cartridges, and the prepared radioactive concrete. In addition, there are such things as blotters, worn clothes, shoe covers, gloves, broken tools, decontaminaton materials, worn-out equipment, and other'miscellaneous solid items.

A schematic diagram of the radwaste system is shown in fig. 3-217. Gaseous wastes can be processed on a batch basis, rather thar on a continuous basis, because of the anticipated small quantity of gases involved. Waste gases are collected, compressed, and stored in decay tanks for 30 to 45 days before their controlled release to the atmosphere. Kadiatior, monitors measure gas activity before any release is made from the plant. When the gas in the decay tank reaches the desired level of activity, it is pass6.d through high-efficiency filters. The gas is then diluted in the stack for control of spectific oxtivity".

Liquid waste handing has two goals: first, to release innocuous wastes to the environment after'suitable monitoring ano, second, to reduce the volume of waste by separating out the pure water and converting the concentrated active wastes in to a stable form for long-term storage before release from the facility. Wastes are classified as "clean" or "dirty", according to the chemical content of the liquid. The clean wastes have a much lower total dissoiveo and suspended solid than the dirty wastes. Wastes originating in the primary system are clean, but wastes originating in the showers or decontaminaton process are dirty. Liquid wastes are coliected in holdup tanks 


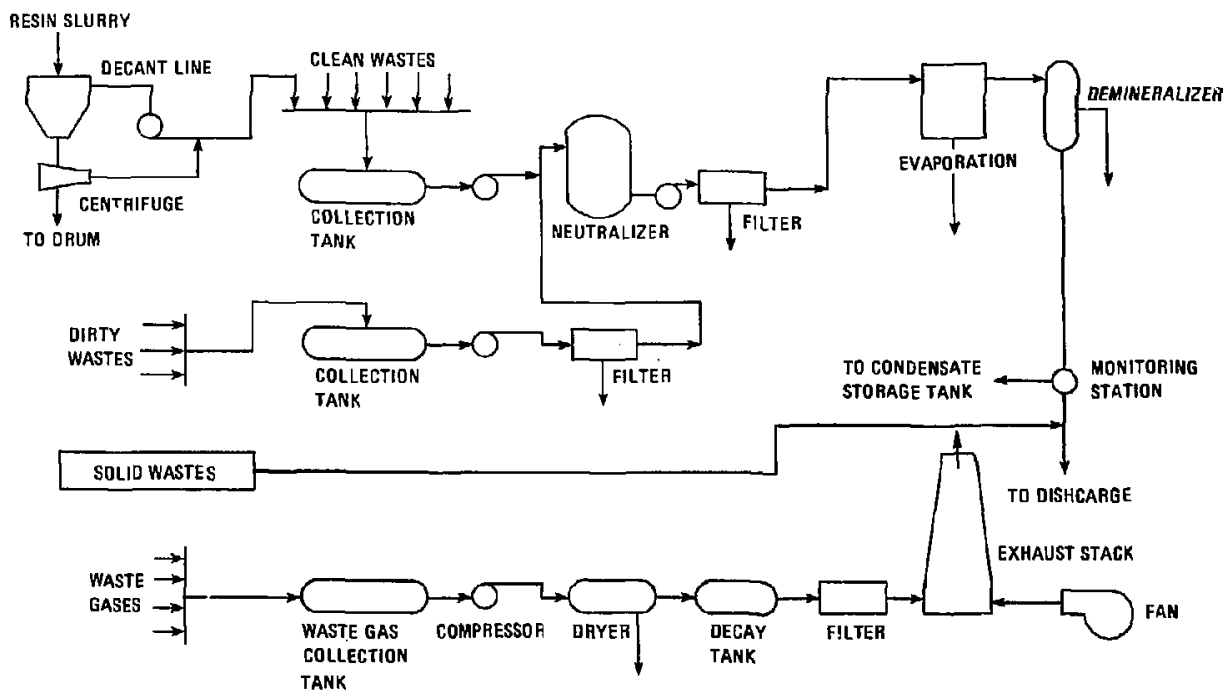

Figure 3-217. Radioactive waste handling system. 
for batch processing (because of anticipated low waste volumes). Chemically clean wastes are treated for $\mathrm{pH}$ adjustment, while the dirty wastes are first passed through filters before they are mixed with the clean liquid stream. The nilxed stream is then treated by evaporation and demineralizaton to separate out the pure water and to reduce the volume of radioactive liquid. The active concentrate, along with the solio radwastes from the plant, is then mixed with concrete in drums and shipped off-site for disposal. To meet DOT shipping requirements, the activity of the wastes is monitored before shipping off-site.

Other Mechanical Systems. In adition to the major systems described above, a large number of other mechanical systems are needed to support the operation of the device. Although these systems are not oescribed in this report, the cust of providing these systems is inciuded in the plant cost. Some of these systems are:

- Demineralized water system

- Service water system

- Potable water system

- Raw water systeri

- Fire water system

- Fuel oil system

- HVAC (heating, ventilating, and air conditioning) system

- Compressed air system

- Cover gas (inert) supply system

- Helium and nitrogen gas supply system

- SF6 supply system

- Radiation tonitoring system

A facility interface diagram (Fig. 3-218) shows all the mechanical systems and their interfaces with the reactor and with its auxiliary systems.

Buildings and Structures.

Building Arrangement. The general arrangement of the major buildings ana structures are presented in Figs. 3-219 through 3-226. 


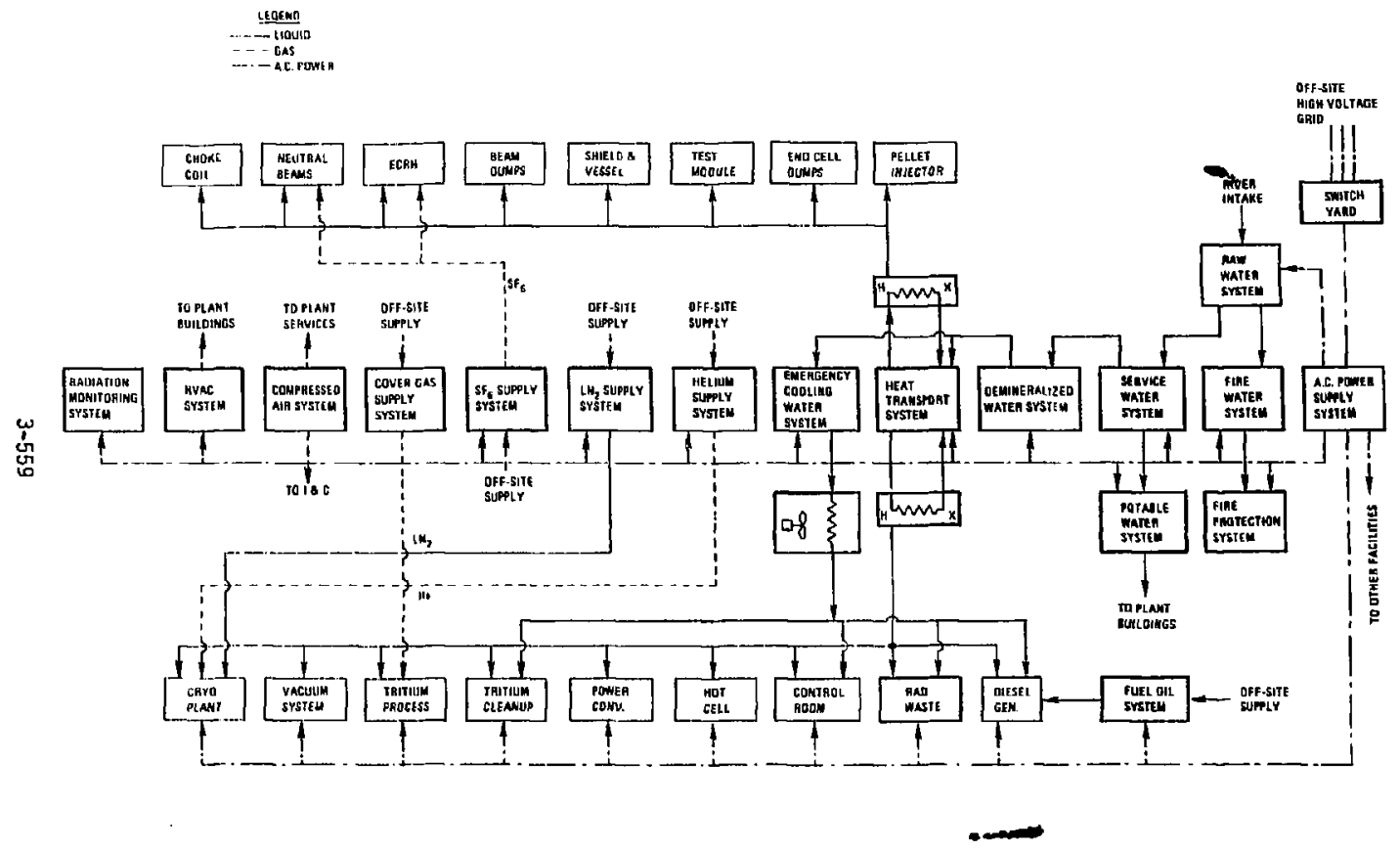

Figure 3-218. Interface of facility systems. 


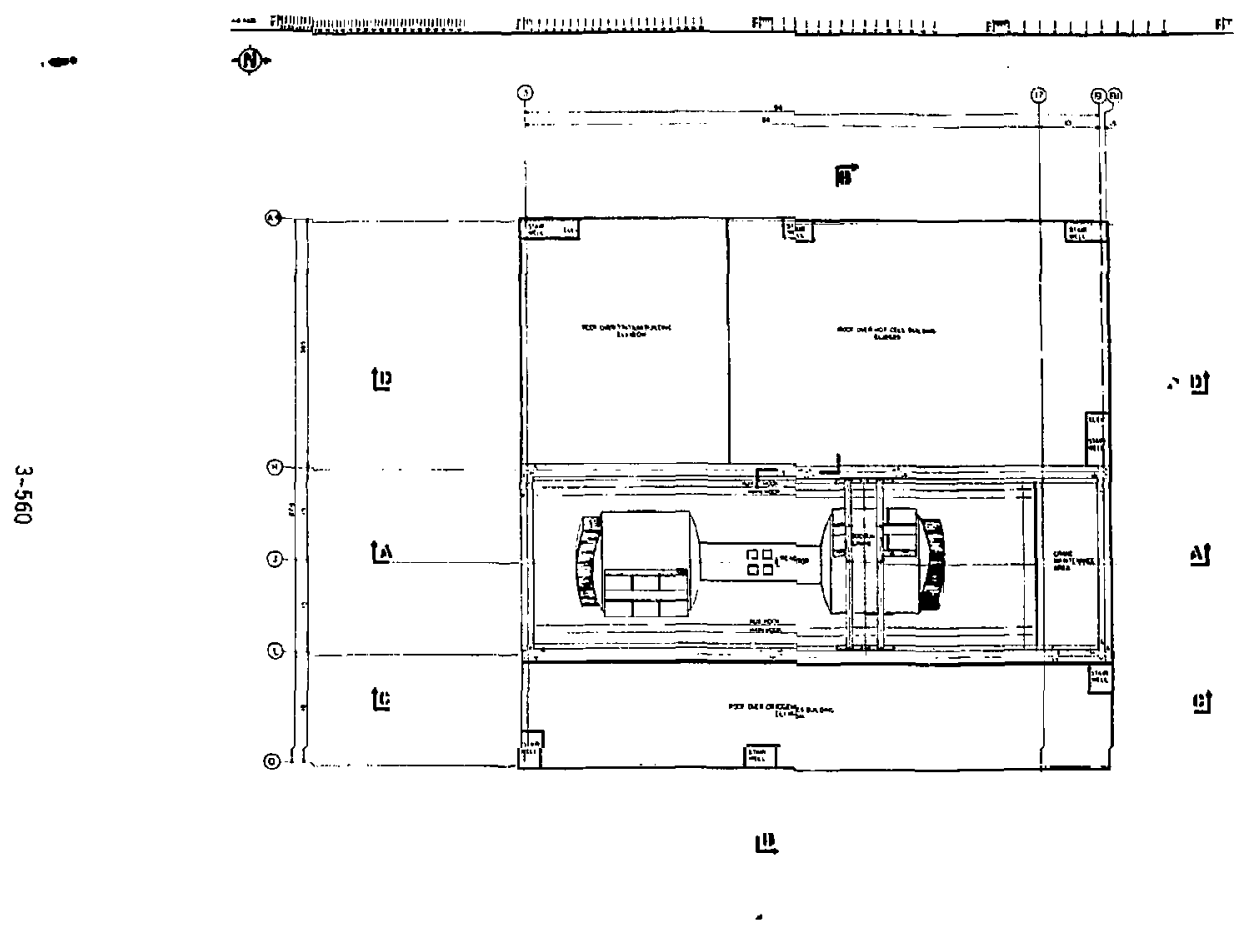

Figure 3-219. Reactor and support buildings ( $p$ lan below el. $130 \mathrm{~m}$ ). 


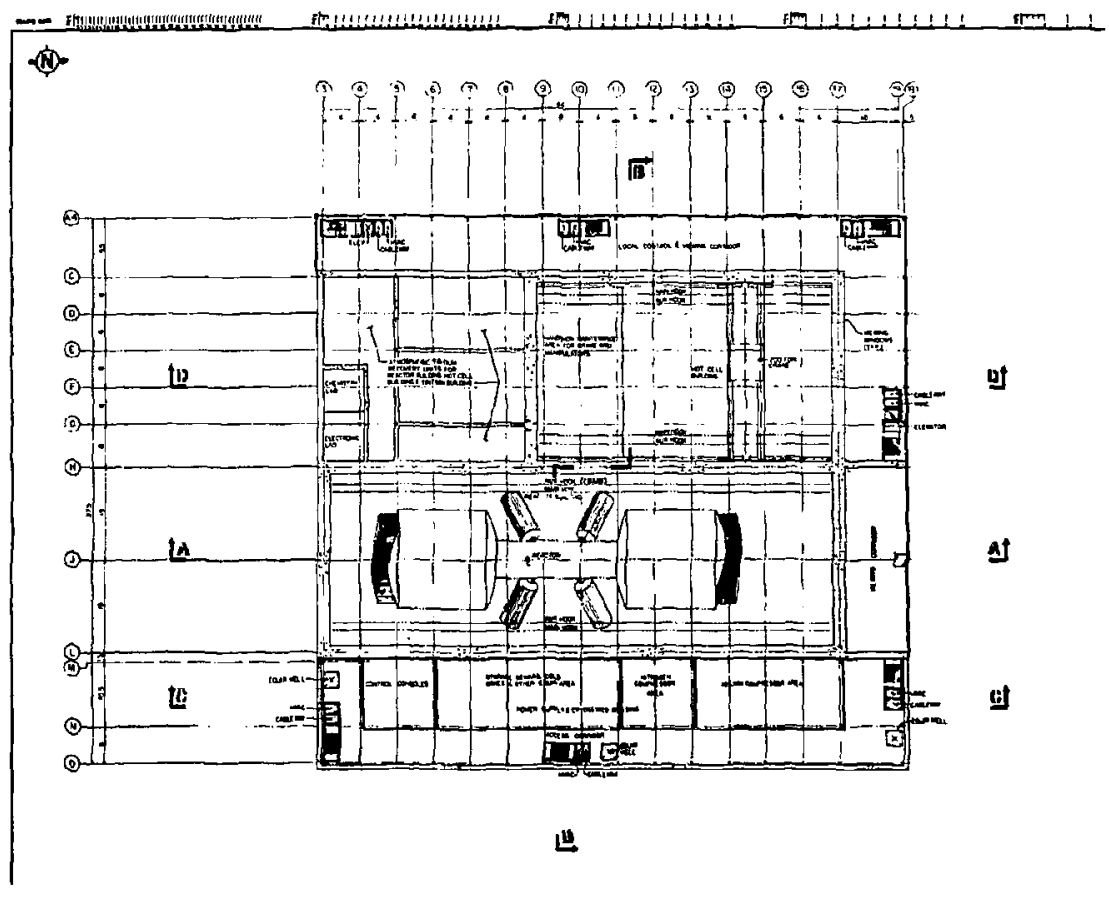

Figure 3-220. Reactor and support bujldings (plan beluis el. $113 \mathrm{~m}$ ). 


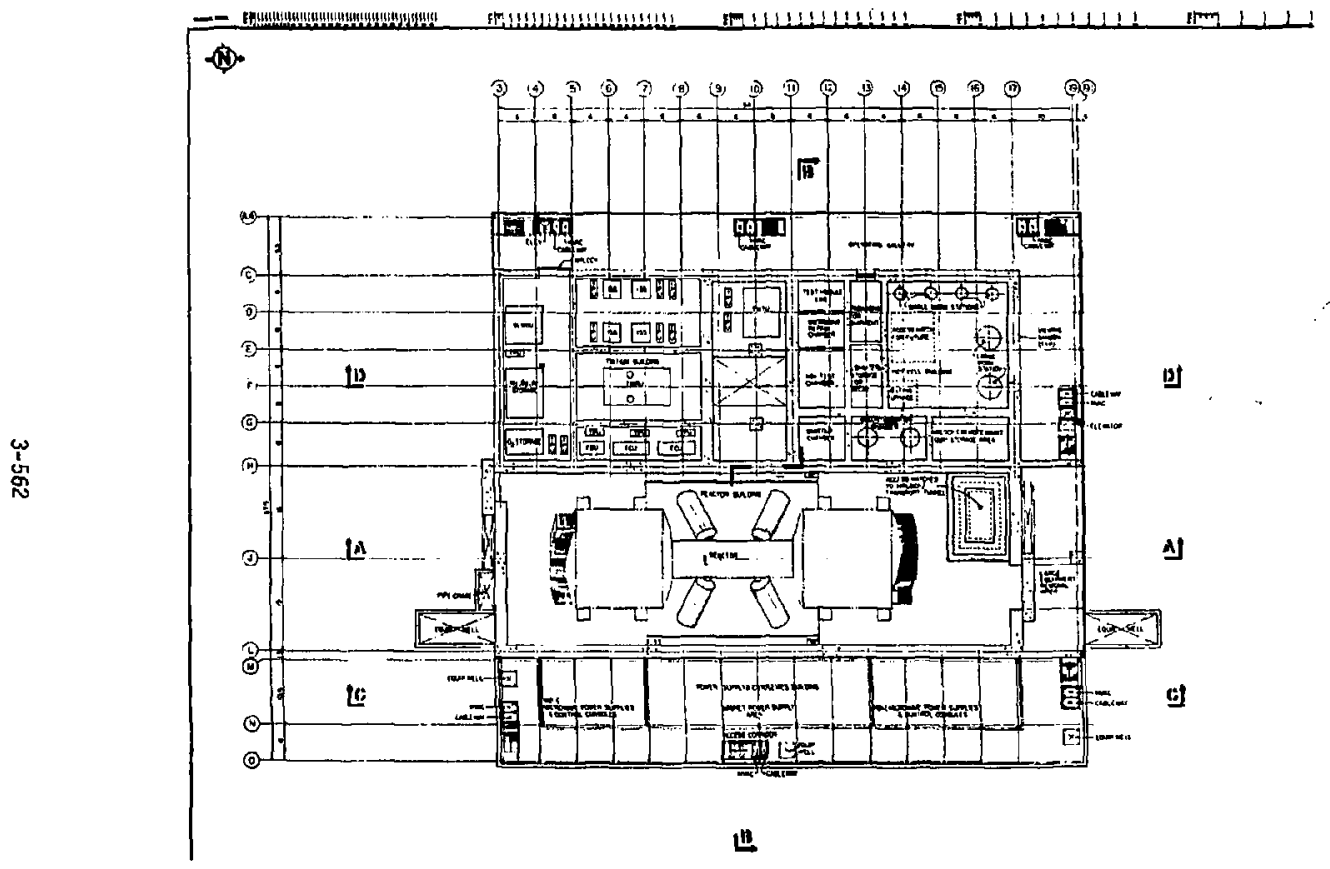

Figure 3-221. Reactor and support buildings ( $p$ lan below el. $106 \mathrm{~m}$ ). 


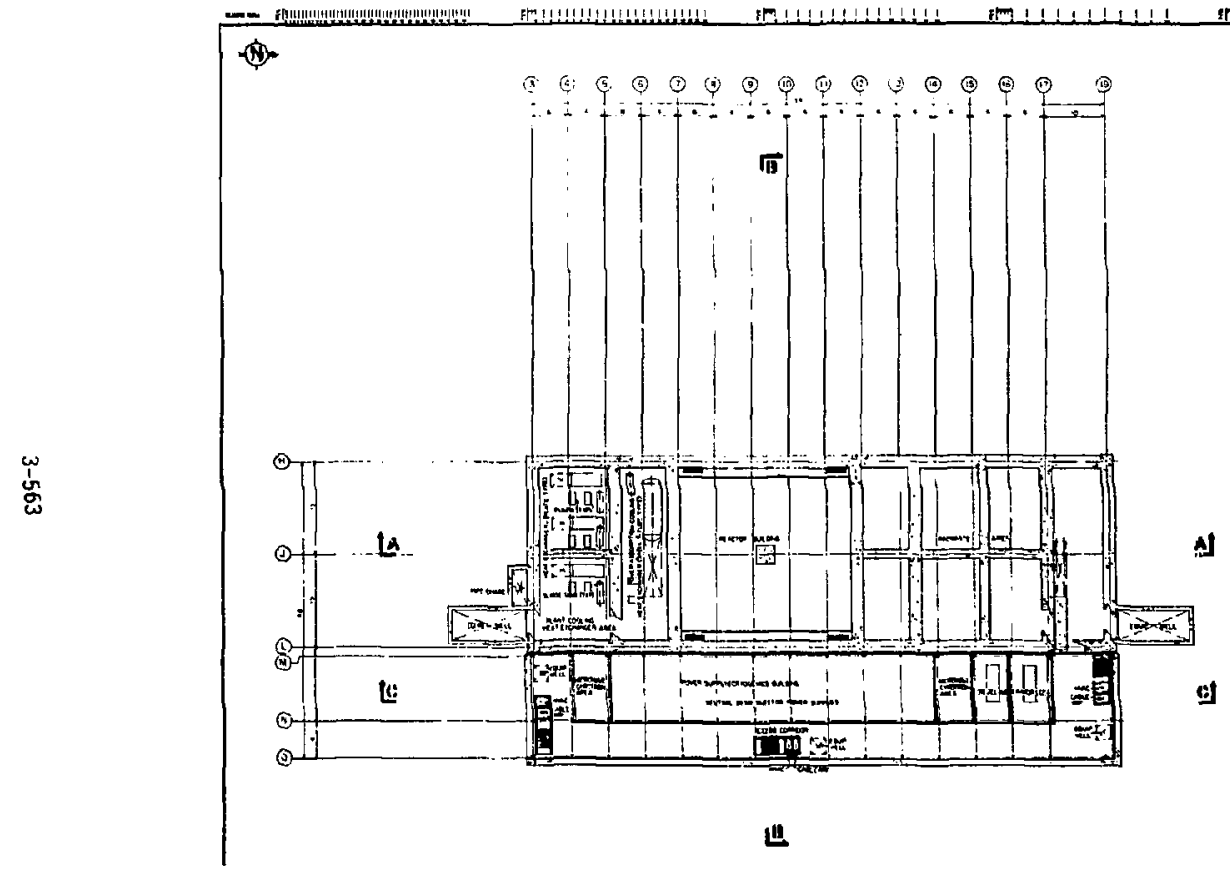

Figure 3-222. Reactor and support buildings (plan below el. $98 \mathrm{~m}$ ). 


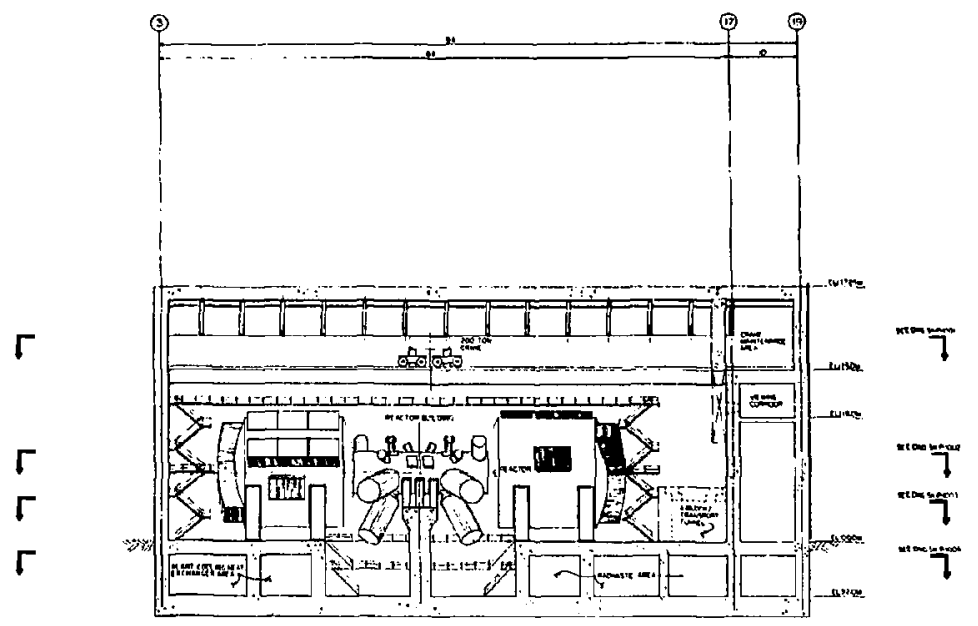

Seciun A-A

Figure 3-223. Reactor building (el, $100 \mathrm{~m}$ ) in more detail (Section A-A from

Fig. 3-219). 


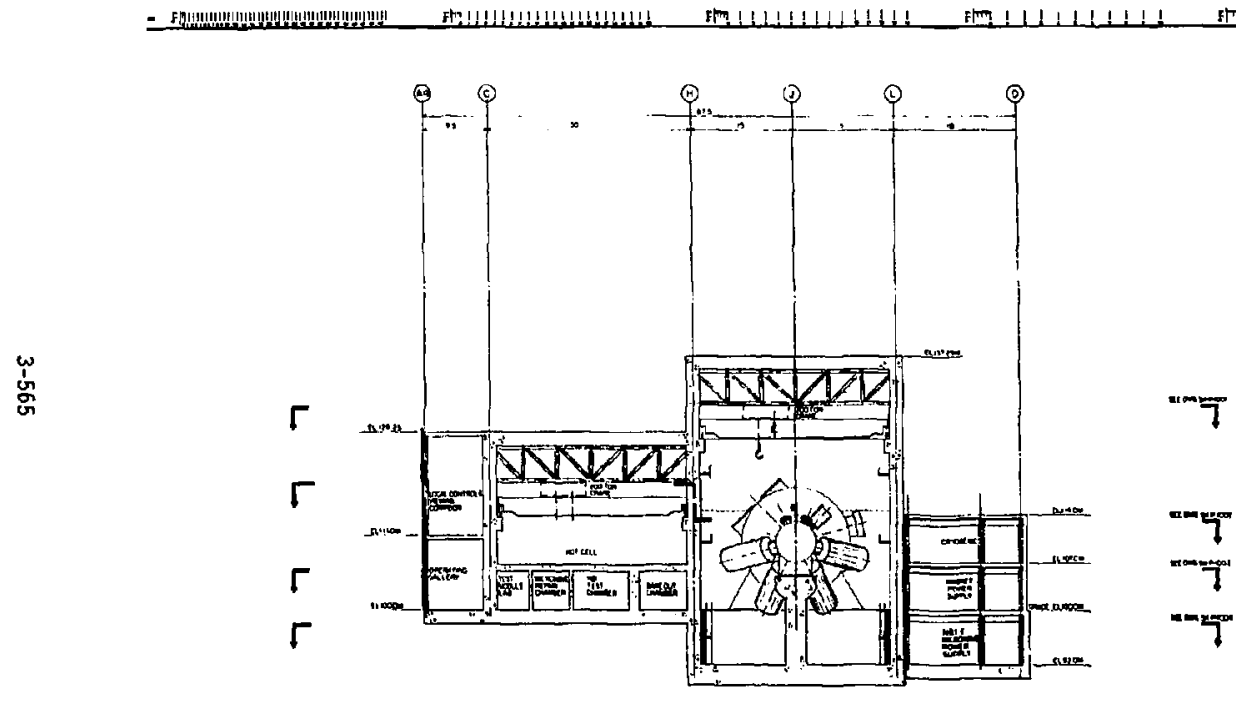

SECTON IS-13

Figure 3-224. Reactor complex (Section B-B from Fig. 3-219). 


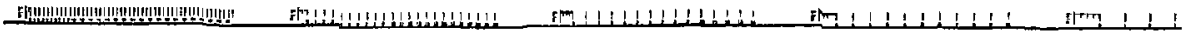

誃

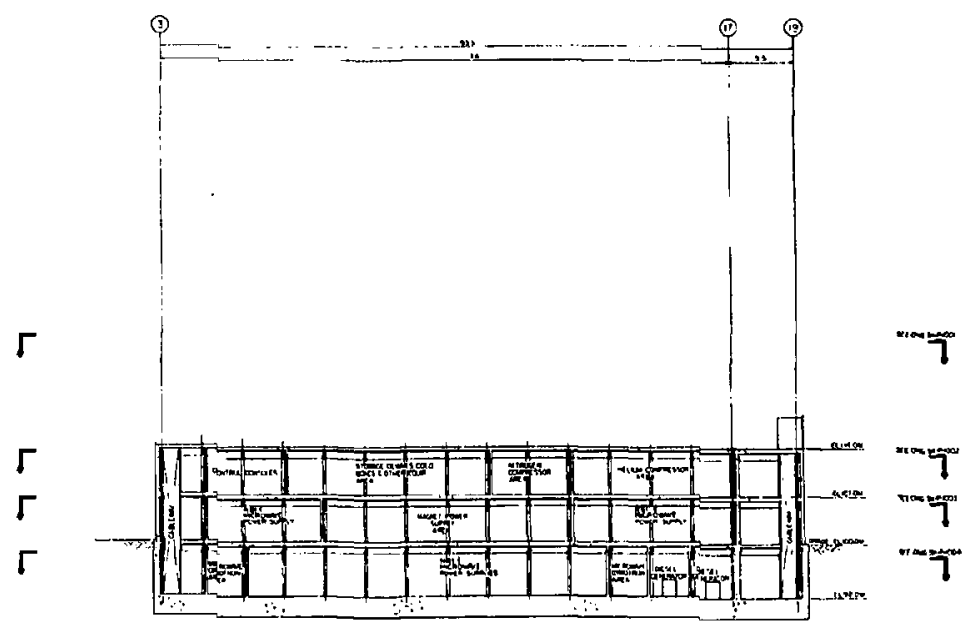

Secton $6: \mathbf{t}$

Figure 3-225. Cryogenics building of reactor complex (Section C-C from

fig. 3-219). 
$\omega$
1
0

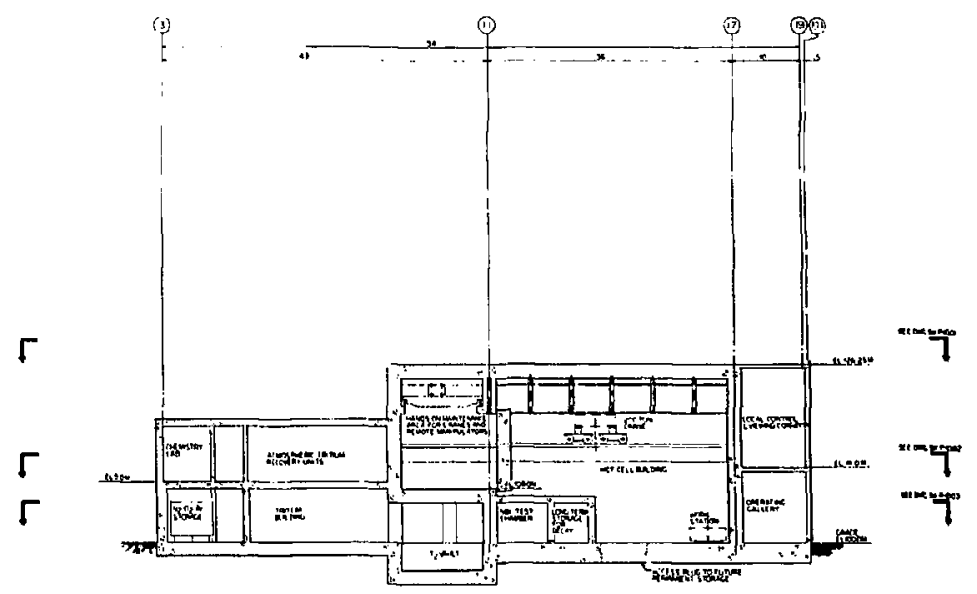

$\operatorname{sectaN} \boldsymbol{D} \cdot \boldsymbol{b}$

Figure 3-226. Tritium and hot-cell buildings (Section D-D from Fig. 3-219). 
The structural design approach encompasses functional arrangement, safety considerations, and construction economies. In addition, the construction and circulation plan of the building envelope follows appropriate builaing code standards and industry practices. All major builoings are required to be geveloped in accordance with the load combinations, allowable stresses, and safety factors specified by DOE and/or NRC building design criteria. The reactor buifding pressure boundary design is in accordance with Section III of the ASME Boiler and Pressure Vessel Code. Other building aesigns, in general, follow the codes and standards of the American Concrete Institute (ACI) and the American Institute of Steel Construction (AISC).

Reactor Building. The major requirements of the reactor building are Dutlined in Section 3.12.2. The inside dimensions of the building are $80 \mathrm{~m}$ long, $28 \mathrm{~m}$ wide and $36 \mathrm{~m} \mathrm{~h}$ igh. The buriding has a certrai pit that is $28 \mathrm{~m}$ long, $26 \mathrm{~m}$ wide, and $10 \mathrm{~m}$ aeep. The dimensions of the building are determineo on the following basis:

- The builaing length is set by the overall length of the device and allowing for maintenance access from the ends, for lay oown space, and for the transport tunnel that connects the reactor building with the hot cell building.

- The building width is set by the overall width of the device, and allowing for maintenance of the centrat cell neutral-beam injectors. This maintenance allowance has a major economic impact on the reactor building. Thus, much effort was placed to keep the building width to a minimum. However, detailed analyses of the maintenance requirements may necessitate a wider building.

- The height of the building is estabished by the device end cell diameter, end cell support requirement from the floor, the device disassembly and maintenance requirements, crane bridge and trolley size, and the size of the trusses needed to support the static and dynamic loading of the bulaing roof. A 200 metric-tan crane is used to handle the heaviest single component, the central cell magnet, which weighs approximately 170 metric tons. The overall height needed for the crane, including hook sling height, is $7 \mathrm{~m}$. The depth of trusses is estimated to be approximately $5 \mathrm{~m}$. 
- The central pit is needed to accommodate the central cell neutral-beam injectors and their maintenance.

- The thickness $(2 \mathrm{~m})$ of the floor and ceiling is needed to provide shielding against neutron and gamma radiation during operation and maintenance. No detailed analyses were made to identify more accurate shieloing requirements. These analyses, if made, are expected to identify less stringent requirements.

Several other considerations that have affected the design of the reactor builoing stem from the safety and maintenance functions of this building.

- Protection from a seismic event requires a $3 \mathrm{~m}$ thick basemat to prevent overturning and vertical displacement of the building. Tornacio and missile protection is also considered in the building design.

- The overpressure requirement $(120 \mathrm{kPa}$ overpressure from cryogen release) leads to a consioeration for stiffening the corners of the building and providing stiffening ribs to the side walls.

- Reducing the leakage of radioactive gases, primarily tritium, from the reactor building leads to considerations for making the building leaktight. Since the primary source of leakage is penetrations, it has been assumed that the building can be designed for a leak rate of less than $70 \%$ per day (by volume) by providing adequate seals at the penetrations. Leak rates of light-water reactor buildings range from $0.1 \%$ to $1 \%$ per day.

- To provide for maintenance of the overhead crane and some of the remote manipulators, an extension of the reactor builoing is provided at the north end of the building.

- A local operating ana viewing gallery is provided along the north wall of the reactor building to oversee the maintenarce operation of the reactor.

- Access to the reactor building is provided through the heat exchanger area and through the radwaste area, which are located below the reactor building.

- The ventiiation system provided for the building maintains a subatmospheric pressure ( 0.5 Torr below atmospheric) to prevent out-leakage from the building. The ventilation system also maintains pressure differentials with the adjacent buildings to prevent any in-leakage of contamination from these buildings. 
- All internal surfaces (floor, waIIs, and ceiling) of the building are coated with epoxy paint to minimize permeation of tritium into concrete. While very little experimental data is available regarding effectiveness of epoxy paint, Tritium Systems Test Assembly (TSTA) at LOS Alamos is using this type of paint in its builaings. A test conducted at Los Alamos where an epoxy coated surface was exposed to highly concentrated (much higher than expected in a reactor buildingl, elemental tritium gas for several uays, showed that the paint is highly impervious to tritium gas and is easy to decontaminate. The major advantages of an epoxy paint over a steel liner are that it is inexpensive and does not enhance formation of oxides. Stainless steel. works as a catalyst to enhance the formation of oxides making it difficult to decontaminate the surface.

- A transport tunnel is provided at the reactor building floor level to transport components for maintenance to and from the hot cell building. This tunnel also serves as an airlock between the two buildings and provides shielaed storage area for the remote manipulators during reactor operation. For better sealing, all transfer operations in and out of the tunnel are made through hatches provided in the roof slab of the tunnel.

Hot Cell building. The basic function of the hot cell facility is to support the maintenance of TDF. Major components are removed from the oevice and taken into the hot cell facility for repair and adjustment. The hot cells must provide adequate working space and a controlled environment to support the maintenance of the major components.

The TDF is expected to have an availability on the order of 25 to $50 \%$. At this level of availability, many maintenance activities may be performed in the reactor building without significantly impacting availabi]ity. Very quick repair of failed comonents is not expected to be as important as it might be for power reactors with goals of higher availability. Thus, general purpose, as opposed to highly specialized, hat cells are currently being suggested for the hot cell facility. Large areas are provided to allow disassembly of large comonents while smaller cells provide repair space for smaller camponents and 
gisassembled pieces. In addition to general purpose areas, the following are required: an anechoic chamber (without echoes) to test and adjust ECRH components; a decontamination area to allow removal of surface contamination from components to be repaired; a bakeout area for outgassing the components before assembly; a test chamber for neutral-beam injectors; a laboratory for examining the test modules; a long-term storage area for radioactive decay; and a packaging room to prepare for final off-site disposal.

The design of the hot cell is determined by the requirements and by applying engineering judgment to estimate an appropriate configuration. While the identification of specific maintenance requirements is still in an embryonic stage, the general philosophies of maintenance procedure will be incorporated in the hot cell building aesign.

A number of design options are considered. One option is to use a number of special purpose celis within the hut cell building. Special purpose cells would be more responsive to quick repair times needed to support high availability. Each special purpose cell could be equipped to handle and repair only one component type. While repair time could be greatly decreased with special purpose cells, the cost of building and equipping such cells would greatly increase the cost of the hot cell facility.

At the other extreme, another design option is the use of the reactor building for maintenance so that no hot cell facility would be needed. While this would eliminate the need for one facility, it would significantly increase the size and cost of the reactor building. The repair and maintenance in the reactor building of all radioactive components would great $1 y$ increase repair times because of increased restraints on working conditions and on maintenance equipment, which in turn would decrease plant availability.

The selected design is a compromise between the two extremes. A hot cell facility is provided with general purpose areas for maintaining the major radioactive conponents. Some component mainenance within the reactor builaing is still expected. The compromise allows a reasonable chance of attaining the aesireu availability without greatly expanding the cost of the plant. Several unique features of the hot cell bulding are described in the following paragraphs. 
A decontamination cell equipped to measure radioactivity and contamination, is positioned so that all contaminated components moving in and out of the hot cell must pass through a control station where each item can be checked for contamination and cleaned if necessary. The purpose of this is to prevent contamination from passing between the hot cell and other buildings.

The maintenance of the hot cell facility influences the hot cell configuration. Two types of work stations in the hot cell area are considered. One provides several individual cells to allow personnel entry for hands-on or glovebox procedures. The individual cells are shielded and isolated from each other so that radioactive component maintenance could continue in adjacent cells while other cell maintenance is being performed. A cell must be decontaminated before personnel entry. The most desirable feature of this approach is that hands-on (or bubble-suit) maintenance is perceived to be faster and more efficient than the alternative approach.

The alternative is to have all the work stations in one large area and perform all the cell maintenance operations remotely. Tools and equipment would be removed, decontaminateo, and repaired in a separate glovebox type cell, but no personnel entry would occur in the hot cell area. The advaritages of this approach are that the work stations do not need to be decontaminated for maintenance operations, and the reduced shielding requirements may reduce the cost and complexity of the facility.

The first approach has many oisadvantages. Decontamination of hot cells for personnel entry is very difficult and time-consuming. Thus, the advantage of personnel entry is largely negated. Miovement of cranes and components through contaminated areas after decontaminaton would likely lead to recontamination of the component and the work stations, even though they are isolated mast of the time.

Totally remote maintenance of hot cells has been demonsirated in a number of facilities. Thus, while not easy, the use of the non-entry cell is feasible and is the approach selected for TDF. Unfortunately, the size of the TDF hot cell and of the components to be repaired are substantially larger than most of those already using totally remote hot cell maintenance. Thus, the hot cell for TDF represents a significant extrapolation of current techno $\log y$. 
Maintenance of the overhead crane is of particular concern and is likely to be a major difficulty in any totally remote design. However, the crane is high enough above the sources of contamination to allow glovebox type maintenance of the components. A shielded area is provided for this purpose.

The ventilation system plays a key role in the control of contamination in hot cells. The most successful flow pattern appears to be one in which the fresh air enters near the top of the cell and flows out through gratings in the floor. Each work station has a section of the outlet grating within its bouncary. This flow pattern tends to pin the contamination to the floor and sweep it to the filters beneath the floor without contaminating other areas of the hot cell.

The use of an inert gas atmosphere for the hot cell area has been suggested by some. The asvantages of an inert gas atmosphere are that fire hazards are greatly reduced, and the formation of ozone is prevented. 0zone is formed from the oxygen in the air by high levels of radiation. ozone is highly corrosive to metals ana attacks many of the plastics that are desirable for use in hot cells. Additional advantages of an inert atmosphere include the possibility of performing cutting and welding operations without special inert gas sources and the possible easier rerroval of any tritium released to the hot cell atmosphere. These advantages tend to offset the disadvantages of additional cost and complexity of maintaining an inert gas atmosphere. However, as long as a non-entry hot cel] area is employed, the use of an inert gas has little influence on the hot cell design and the impact on overall cost is negligible.

Tritium Building. The tritium facility based on TSTA prototype is a full scale processing plant with high reliability. It is designed to be completely separate from the reactor building, but it is located nearby to minimize the length of the tritium feedlines, thus reducing the tritiun-accident potential. The building is designed for contact maintenance; it has an air environment and is free of gamma and neutron radiation. All tritium-handing units are under remote, independent, hard-wire, computer control; the computer is equipped with an uninterruptible power supply to ensure that all tritium systems can ve shut down in the event of a power failure. The tritium starage area is in the tritium facility and is a shielded, barricaded vault holding an 
inert cover gas for fire protection. Because of space and safety benefics, the majority of the tritium is stored as UTX, but some low-pressure gaseous storage in tanks is also provided.

The tritium building is 30 by 50 by $16 \mathrm{~m}$ high; within it a slightly negative pressume is maintained by a self-contained heating, ventilation, air conaitioning system (HVAC) interfaced to the atmospheric tritium cleanup (ATC) system to reduce tritium out-leakage.

Two personnel airlocks go into the tritium building; the one designed for routine use connects the healtn physics area with the lower level of the building. The other is intenaea for emergency use. Within the tritium building, space is provided for the ATC units and the interfaced HVAC system. The placement of all units is designed to provide good accessibility. The tritiated waste recovery unit, the tritrated waste treatment unit, and the solid waste disposal unit all of which generate and/or process tritiated waste, are located for easy waste removal. The isotope separation units are high; the 4-m-high vacuum sheli around each unit is removed vertically and downward. (Space is provided in the basenrent but not shown.) Storage space is provided for nontritiated gaseous deuterium and hydrogen as well as for helium ano nitrogen. Within the tritium building are three service areas. The first is an analytical laboratory for ooing routine analyses on catalysts, molecular sieves, etc; the secono is an electronics laboratory in which ronactivated tritium monitors are serviced and tested; the thira is a maintenance area where tritium units are repaired.

In the tritium building, which is designed for contact maintenance, certain procedures are followed to reduce worker contact with tritium. Atmospheric levels are maintainea at $<5 \mathrm{uCi} / \mathrm{m}_{3^{*}}$. Normal fire prevention practices for hycirogen are exercised.

Heat Exchanger and Radwasie Areas. The area directly beneath the TDF reactor floor is devoted to the reactor support systems. These support systems include the heat transport system, radioactive waste handling system, and facilities for access control to the reactor building. For the location of equipment containing radioactive fluids, full advantage is taken of the proximity of the area to the reactor. 
A.1 penetrations and openings between the reactor building and the areas Lelow it are equipped with seals to prevent diffusion of tritium and activated ajr. As a further precaut;ion, the KVAC system maintains variea negative-pressure differentials between these areas and other adjacent buildings. A separate partitioned room in the radwaste handling area contains a safety check area for personnel. Tritium proof sealed doors and airlocks permit access to the reactor building from this area.

The heat exchanger area contains equipment for cooling various reactor components and auxiliary facilities. This equipment includes circulating pumps, heat exchangers, surge tanks, water chillers, and treatinent equipment. since the cooling water in these components may become radioactive after circulating through the reactor during operation, closed loop cooling systems minimize environmental and personnel hazards by restricting activated flow to the heat exchanger area. Activation is shortilved, and access to this area is prohibited during reactor operation. Some of the components in this area may require local shielding where more intense ano persistent radiation exists. This local shielding will be put in place at a later cate after actual measurements of radioactivity have been taken.

The radioactive waste area mainly houses the radwaste system components of the three waste-processing facilities:

- Liquid waste processing facility

Decanting equipment

Collection tanks

Neutralizer

Evaporator and demineralizer

Filters, pumps, radiation monitoring equipment, etc.

- Solid waste processing facitity

Cement filling station

Drum processing station

Drum storage area

Radiation monitoring equipment

- Gaseous waste processing facility

Collection tank

Compressor and aryer

Decay tank

Particulate filter

Radiation monitoring equipment 
No equipment arrangement nas been developed for the radioactive waste area, but the space provided is considered adequate. Because the radioactive materials are handled by the cumponents, the radwaste area is located in an adequately shielded building. Because this area (aiso the heat exchanger area) is located in the basement of the reactor building, it is provided with 2-m thick walls, floor, and roof to conform to structural design requirements. To provide as low as reasonably achievable (ALARA) radiation protection, equipment will be arranged in a hianner that isolates the operating ana maintenance areas from the processing equipment.

The heat exchanger area is $28 \mathrm{~m}$ by $22 \mathrm{~m}$ by $6 \mathrm{~m}$ high. The radwaste area, including the personnel access control area, is $28 \mathrm{~m}$ by $28 \mathrm{~m} 6 \mathrm{~m} \mathrm{high}$.

Potur Supply Building. The power supply builaing contains the equipment for ECRH power conversion and supply, NbI power conversion and supply, and magnet power conversion and supply. No detailed equipment arrangement has been developed for this building; only general areas are ioentified in the layout crawings. The building has two floors. The first floor contains the ECRH power generators (gyrotrons), the compensation and transient suppression networks and modulator/regulator modules which supply high-voltage dc power for the reutrai-beam ion sources. The second floor contains the magnet power conversion and supplies, consisting of rectifiers and switches, local controls and interface to central controls, and HVAC for the power supply building. All transformers, capacitors, and auxiliary substation equipment are located outdoors.

Because of high electrical potential and the possibility of arcing relative to the transient suppression networks, each unit is housed in a separate enclosure on the first floor. Eacin of the enclosures has a grounded metal lining to further protect equipment and personnel.

The pover supply bujlding measures $80 \mathrm{~m}$ by $16 \mathrm{~m}$ by $14 \mathrm{~m}$ high. The building has a wall common with the reactor building, which helps to reauce the length of the high-voltage dc power cables to the ion source supplies.

Cryogenics Building. This building is the vertical extension of the power supply building and consists of one floor only, measuring $80 \mathrm{~m}$ by $16 \mathrm{~m}$ by $7 \mathrm{~m} \mathrm{high.} \mathrm{The} \mathrm{helium} \mathrm{and} \mathrm{nitrogen} \mathrm{cryogenic} \mathrm{supply} \mathrm{equipment} \mathrm{is} \mathrm{located} \mathrm{in}$ 
this builaing. No detailed equipment arrangement has been developed for this Lujluing; only yeneral areas are identified in the layout orawings. These areas are the helium cumpressor and oil removal unit area; nitrogen compressor and oil removal unit area; storage dewar, cold box, and other equipment area; ano cuntrol console area. The high-pressure gaseous helium and nitrogen storage tanks are located outooors for convenience of off-site supply of these gases.

Control Building. The central control building is remotely locateo from the experimental complex. The computers, used both for the TDF systems ana for handing experimental data, are housed in the same building.

The control building is outside the radiation safety exclusion area. The TUF device is remotely controllea, and personnel are subjected only to the lower radiation dose outside the exclusion area. The extra path length of signal carriers is not significant because all control and experimental data acquisition signals are transmitted through optical data links. A spinal coro concept carries ali services to and from the TUF, and the aata links are carried in a utility tunnel. Safety-related signals and interlocks are carrieo in electrical cables, which are also routed through the utility tunnel.

A gallery is envisaged above the control building in anticipation of the many visitors expeçted at TLF. Visitors, who will not need to wear film badges, may observe central control room operations without aisturbance and can see inside the experimental area on closed-circuit TV.

Structural Design. A preconceptual structural design is presented here on the most important TOF buildings and structures. For a few key areas of the TDF structures, a very limited structural analysis is performed in accordance with the structural criteria discussed in Section 3.12.2. Possible alternate oasigns for some key areas and recommenoed future studies are also aiscusseo.

Reactor juilding Complex. The reactor building (including heat exchanger and radwaste areas), hot cell tujlding, and tritium building have been combined into one large complex sharing a common basemat. For the most part, these buildings are massive reinforced-concrete structures that use structural 
stee 1 members only to support concrete weight during forming, thereby elininating expensive and time-consuming shearing and form removal operations. The most significant exceptions to this are the roofs of the reactur and hot cell buildings, which use steel trusses to carry both dead weight and seismic loads. Concrete roof and floor slabs are designed to act as a oiaphragm to transfer lateral loads to the concrete walls that are designed as shear-resisting elements. The founoation mat consists of slabs on in elastic foundation. The thickness of walls and slabs surrounding rauioactive areas is generally governed by shielding requirenents.

The most significant structural features of the complex are the 2-m-thick walls, floors, and roofs required for shielding in the reactor building ano hot cell. These, combined with the large distances over which these structural elements must span, constitute a very significant cost factor. The large thickness requirea for shielding increases structural mass, which results in high gravity and seismic loads.

Areas of high sensitivity to these loads are the roofs of the reactor building and of the hot cell, which have large clear spans. The design selected is a ceep steel truss system with rolled beams for roof purline supporting a steel aeck. This design is selected because of its relative ease of construction: First, the trusses are placed on the walls. Then the seconoary framing and steel decking are erected on the trusses. Next, the reinforced-concrete roof slab is placed.

The steel truss concept is selected over the following two alternate methoos:

The first uses post tensioned T-beams with a reinforced concrete slab placed on top. The main disadvantage is the large crane capacity neeced and the time required to lift the heavy T-beams into position. This lifting problem is most critical on the high reactor building as opposed to the hot cell roof. Even with this disadvantage, the post-tensioned T-beam concept is the more attractive of the two alternate concepts consicered because of its low fabrication cost. A future cost-comparison study (T-beam vs stee) truss) is recommended.

The second alternate concept is the barrel vault (arch-shaped roof). because the large concrete thickness is required for shielding, not for strength, its thickness must be held constant. Thus the usual economies of a barrel vault cannot be utilized. Also, because of curvature of the vault, 
more concrete is required, compared to the flat roof system. Moreover, the barrei vauit requires a steel truss for form support.

Reactor Support. The reactor has three support regions: north eno cell support, central cell support, and south end cell support.

The rorth and south end cell supports are attached directly to the operating floor $(\mathrm{e} 1.100 \mathrm{~m})$ of the reactor building, which has been reinforced by walls directly below the supports, as shown in Fig. 3-223. Horizontal loass are taken by the operating floor acting as a diaphragm.

For the purposes of this study, it has been assumed that the south end cell support provides north south, east west, and vertical restraints. The north end cell support provides only east west and vertical restraints, and the central cel] support provides only vertical restraint. This arrangement allows machine thermal growth.

The central cell support is the most critical from a structural viewpoint, because it must to be small in plan to permit maintenance of the neutral-beam injectors, and yet must be $13 \mathrm{~m}$ high. This design concept relies on the structural strength of the reactor so that the central cell and anchor cells transmit their full north-south, and a part of the east-west, seismic loads to the south end cell support. Note that since the south end cell support is assumed fixed, the central and anchor cells must also transmit the north south sejsmic load of the north end cell to the south end cell support. It is not known at this time if this is feasible. If the central cell support also requires east-west restraint, then the single cantilevered column support will most likely not be acceptable because it is probably too flexible horizontally compared to the stiffness of the central cell and anchor cell structures with respect to east-west loading. In this case, in future stuoies we should investigate the following two alternate concepts:

- A concrete column (see Fig. 3-223) with a horizontal reinforced-concrete east-west strut to the west wall at the 100-m elevation and an optional similar strut in the north-south direction to the floor at 100-m elevation. This will horizontally stisfen the center anchor support significantly.

- The same structural configuration described above except that each columin and strut(s) is made of four wide flange steel menbers laceo together to form box sections. 
Power-Supply Complex. This complex consists of power supply, cryogenics, and diesel generator buildings. This is a structurally separate building from the reactor building complex. The power supply complex has no radiation shielding requirement. Tornado protection requires much thinner walls than the 2-ill walls of the adjacent reactor building complex. If the two structures were joined into one large structure, the power supply complex would require significant strengthening to take the large soil pressure reaction which would be induceo in the basemat of the power suppiy complex. As a separate builaing it can be an economical light steel structure with relatively thin reinforced-concrete floors and walls and basemat. The piping and electrical connections between the two complexes, however, must withstand relative seismic and differentiai settiement dispiacements.

AC Power System. The proposed heavy-duty substation employs three single phase transformers, each having two windings, as shown in Figure 3-227. A spare transformer, located on a stancby pad at the substation, is recominended so that full-power service can be restored rapidly if a transformer failure occurs. An alternate approach is to use two large three-phase transformers with high impedance windings to reduce the short-circuit fault currents and the cost of the $13.8-\mathrm{kV}$ circuit breakers. The spare transformer, if provided, would be more expensive than a single-phase high-impedance transformer. To reduce the cast of the substation, no bank circuit breakers are provided in either $13.8 \mathrm{kV}$ circuit. For this purpose, differential c'urent measurements between the primary and secondary of the transformers are used to trip the high-voltage circuit breakers connected to the utility line, which is assumed to be $230 \mathrm{kV}$ for cost estimating purposes. Service disconnect switches are provided to isolate the $230 \mathrm{kV}$ and $13.8 \mathrm{kV}$ circuits for maintenance and backup-power operation.

Figure 3-228 is a one-line diagram of the 13.8-kV substation distribution feeaers, which identify the major loaas. Mnemonics used in the alagram are identified in Table 3-96. The two $33.8 \mathrm{kV}$ high-inpedance windings were selected in 1 ieu of one $13.8-\mathrm{kV}$ winding to reduce the short-circuit fault currerit ano the cost of the distribution circuit breakers. The numbers in parentheses refer to the number of $13.8-\mathrm{kV}$ circuit breakers associated with a given load. The nominal load size is given by the number inside the circles. 
230 kV, 3ф, $60 \mathrm{~Hz}$ UTiLITY LINE

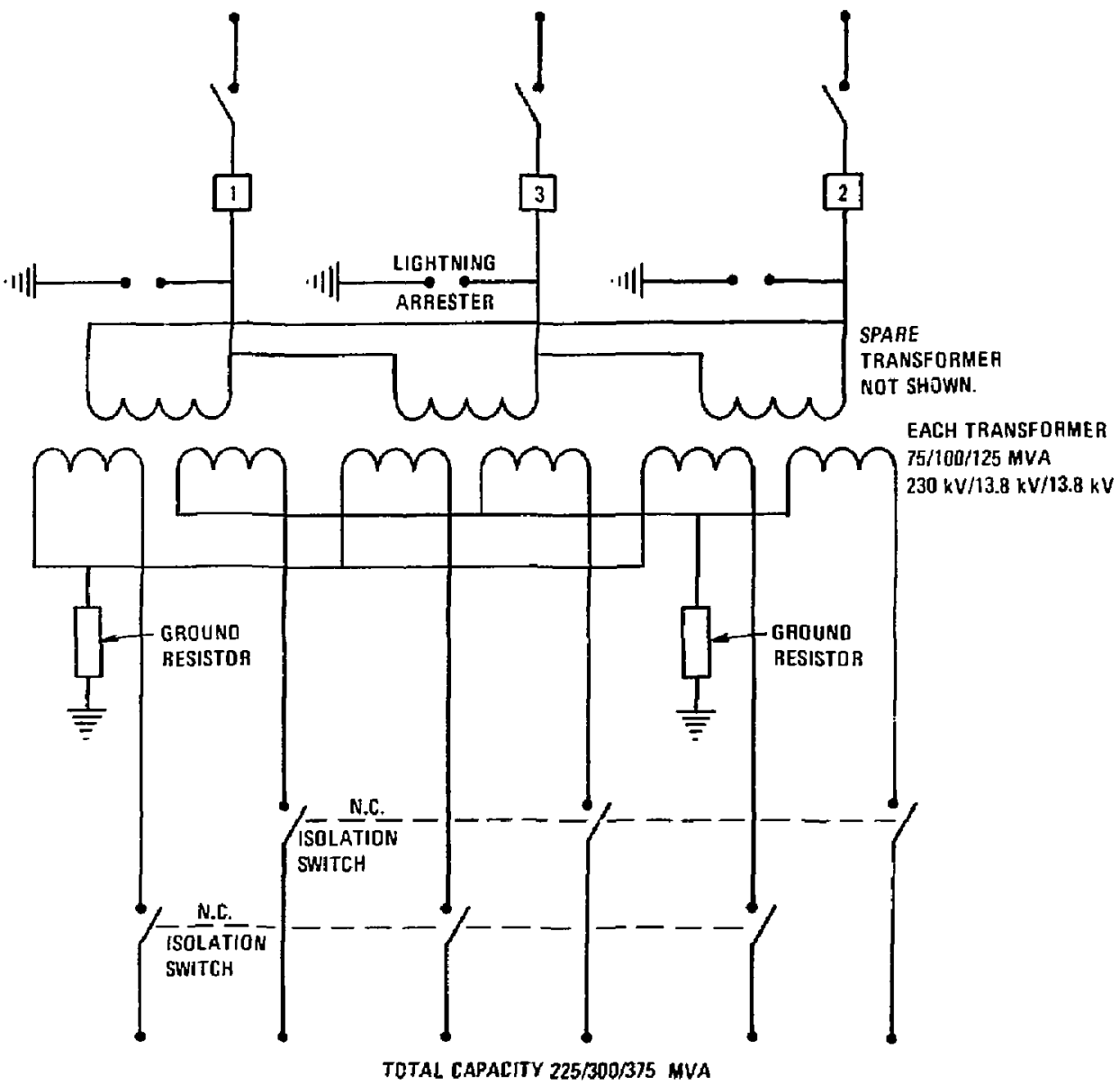

Figure 3-227. High-voltage side of heavy-duty substation with single-phase transformers. 


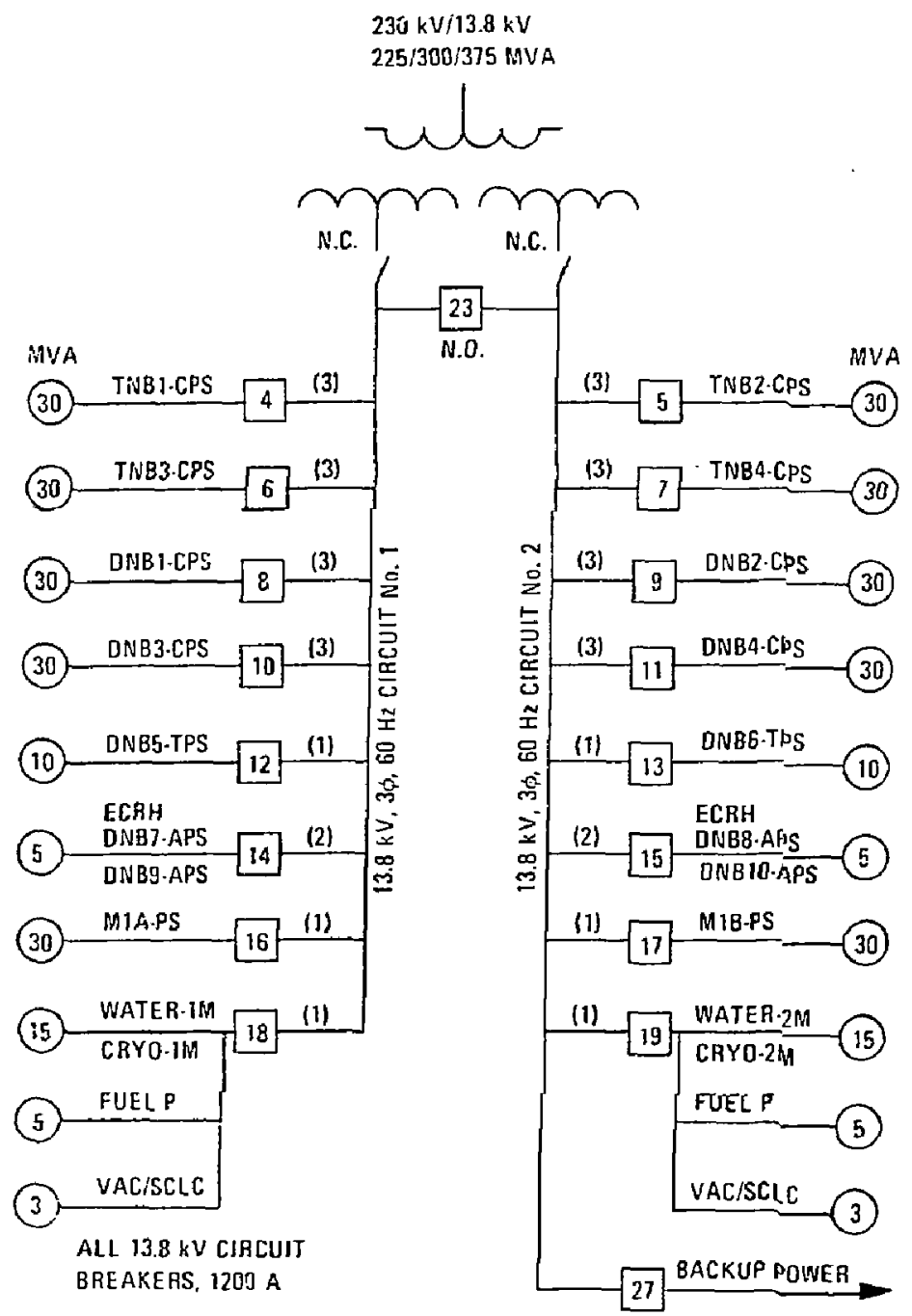

Figure 3-228. Heavy-duty substation $33.8-k V$ power-distribution system. 
Table 3-96. Power system nomenclature.

TNBX-CPS

ONBX-CPS

DNEX-]PS

ONBX-APS

M1A-PS, end $A$, MIE-PS, end $B$,

SCLC

FUEL- $\rho$

CRYU-PYi

WATER M

VAC
Tritium neutral-beam line No. $X$ sentral power supply, $x=1,2,3$, or 4 .

Deuterium neutral- beam line No. $x$ central cell power suppiy, $x-1,2,3$, or 4 .

Deuterium neutral-beam line No. $x$ transition cell power supplies, $x=5$ and 6 .

Deuterium neutra]-beam line anchor-cell power supplies, $X=7$ through 10 .

M- 1 choke coil power supply;

otherwise like M-lA-PS.

Load center for superconducting-coil power supplies.

Fuel process and tritium cleanup systems.

Cryogenic system power, predominately ac power to motors.

Water-coo]ing system power, predominately ac power to pump motors and cooling tower fans. Vacuum system ac power to mechanical pumps. 
As an example, there are four tritium neutral-beam lines (TNB] through TNB4) and four deuterium neutral-beam lines ([NBI through DNB4). Each neutral-beam line has three sources operating simultaneosisly and each source has separate filament, arc, and grid power supplies that receive 13.8-kV power from a ceoicated circuit breaker. Therefore, there are three circuit breakers per beam line, one for each source. Al] 13.8-kV circuit breakers have the nlaximum capacity for carrying $1200 \mathrm{~A}$. Current trip levels can be set at any current from $100 \mathrm{~A}$ to $1200 \mathrm{~A}$.

The 13.8-kV circuit breakers 18 and 19 provide power to many smaller laads at lower voltage levels, shown in the more detajled one-line diagram of Fig. 3-229. Transformers reduce the voltage from $13.8 \mathrm{kV}$ to $2.4 \mathrm{kV}$ for operation of the cooling-pump motors, PMI through PM4, and for the cryogen compressor motors, $C M T$ through $C M 4$. The $2.4-\mathrm{kV}$ power is further reauced to $480 \checkmark$ for the superconducting coil power supplies, PS1 through PS9. Double-ended load centers provide power to the motors and superconducting coil power supplies.

keferring again to Fig. 3-228, we see circuit breaker 27 provides power to the light-duty substation if its independent power line should fail. Circuit breakers 16 and 17 provide power to the water-cooled choke coil power supplies by means of voltage-controlled tap-changing auto transformers described here under the magnet section, 3.2 .

The light-duty substation (Fig. 3-230) provides power to loads requiring better voltage regulation. Heavy-duty pulsed loads such as neutral-beam power supplies are not operated off this line, except for test and maintenance of one source at a time when the heavy-duty line is out of service. The light-auty substation provides power to the normal and transferrable loaos identified in Table 3-90 above and provides backup power by means of circuit breaker 23 if needed when the heavy-duty system is out of service. The 2.4-kV system distributes power among the TDF buildings through load centers where the voltage is further reduced to $480 \mathrm{~V}$ and $120 \mathrm{~V}$. These facility load centers and their distribution power cables were not identified and included in a detajled cost estimate, but an allowance was maoe in the building cost to cover the cost of the low-voltage power distribution system.

Referring to Fig. 3-230, we note two diesel generators are sized to provide power to the essential ioads needed after the machine is shut down. These loaos are identified in Table 3-92. Either diesel generator can supply 


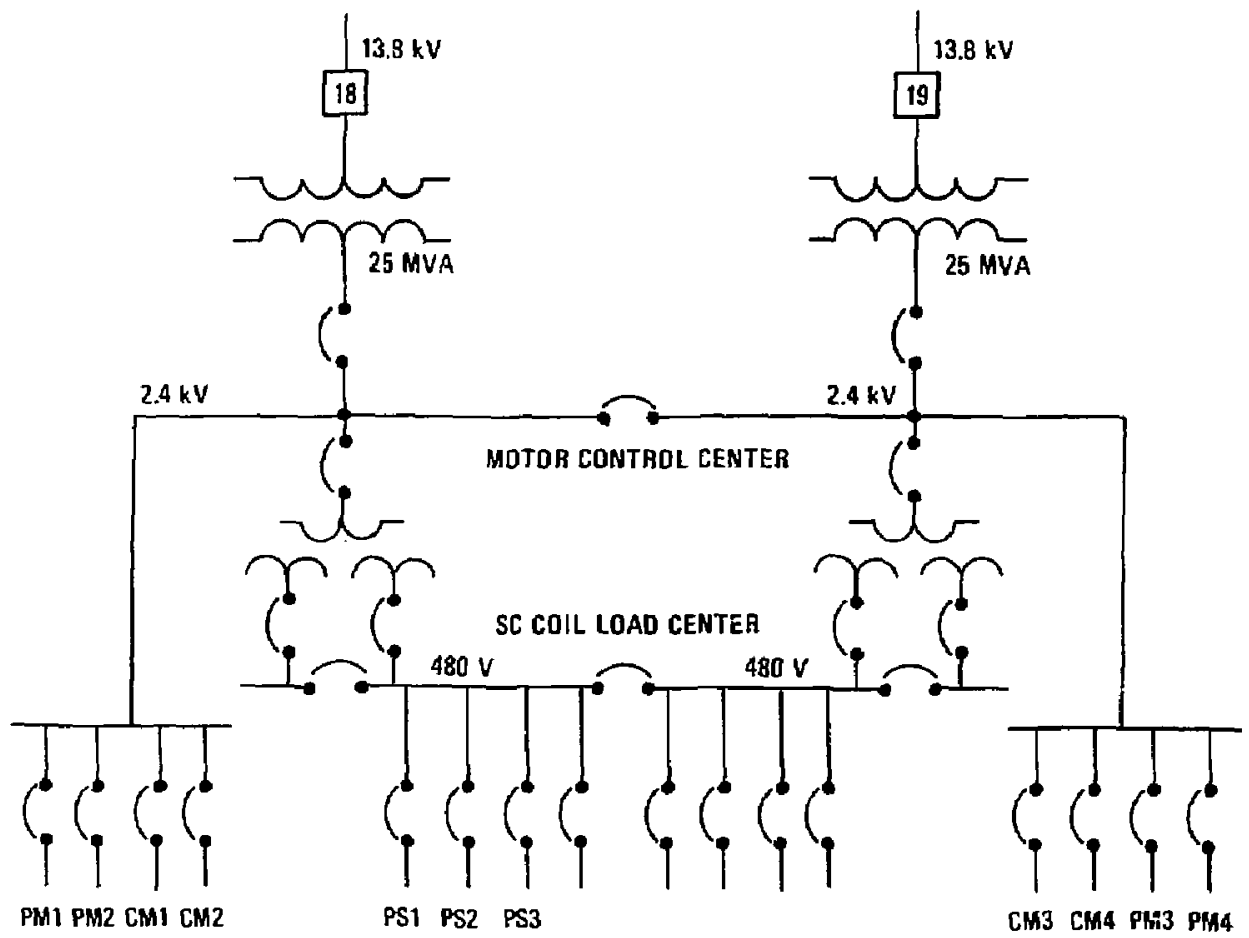

Figure 3-229. Motor-control and load-control centers for the superconductor coil power supplies. 


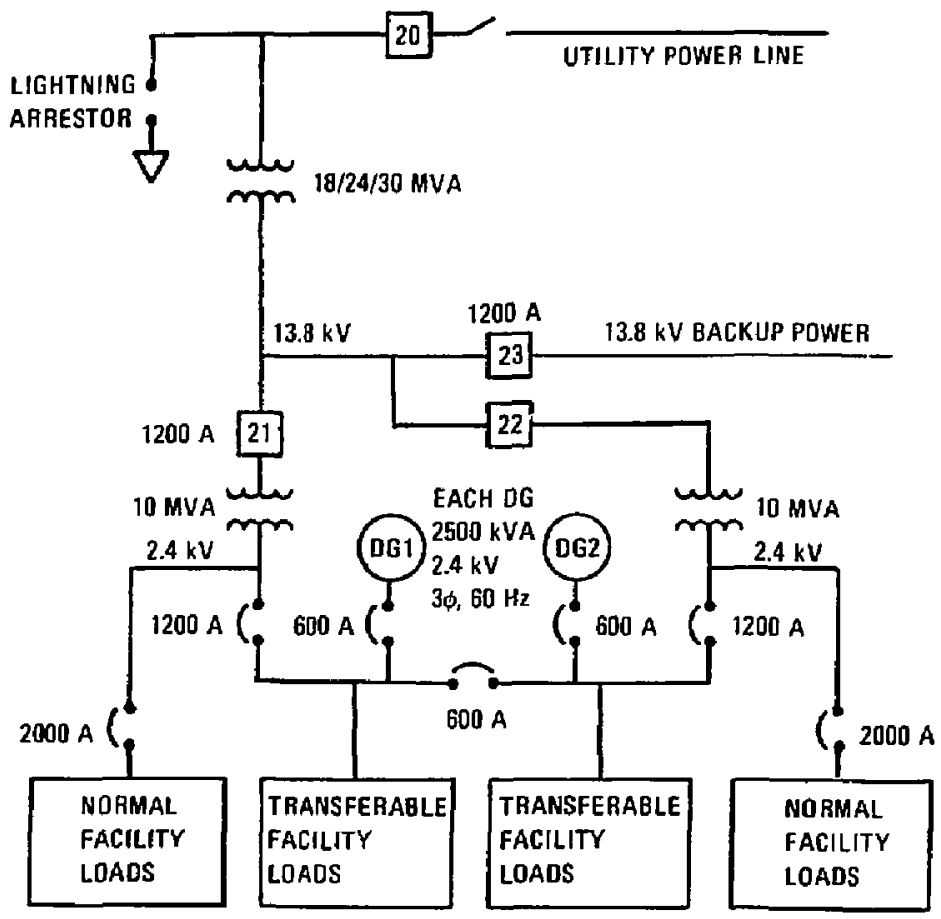

Figure 3-230. Light-duty substation and essential power-distribution system. 
enough power to meet the minimum required. If all utility power is lost, the esel generators start up automatically. Some manual assistance may be needed to connect the essential loads or a few normal facility loads if both diesel generators are on-line.

Figure 3-23) is a one-line diagram of the battery inyerter system that can supply very limited power to noninterruptible power loads until the batteries are discharged. The diesel generators should be on-line, charging the batteries long before they are discharged. This system proviues power to safety related protection equipment.

\subsubsection{Key Issues}

The key issues associated with the design of plant facilities have two major characteristics: first, they are primarily economic (as opposed to technical) and, second, they are not unique to TDF but are generic to currently envisioned neutron producing fusion devices. The major key issues involve the design requirements for:

- The hot cell facility.

- The reactor building.

- The tritium processing building.

Because the cost of the plant facilities is a significant part of the overall TDF cost, these key issues deserve some attention. Uncertainties in these design requirements leave the cost of plant facilities indefinite at best.

The design of the hot celi facility has not yet received adequate attention. The maintenance requirements of the TDF device will greatly influence this design. The major uncertainties that will significantly affect the cost of the hot cell facility include:

- The number, nature, frequency, and sequence of maintenance operations will dictate the size of the facility.

- The use of a number of special purpose cells rather than one large ce? will affect the complexity and cost of the facility.

- The use of metal plates rather than epoxy paint for cell wall lining will also influence the facility cost.

The other major uncertainties that will have significant impact on the cost of the buildings housing the hot cell, the reactor, and tritium processing include: 


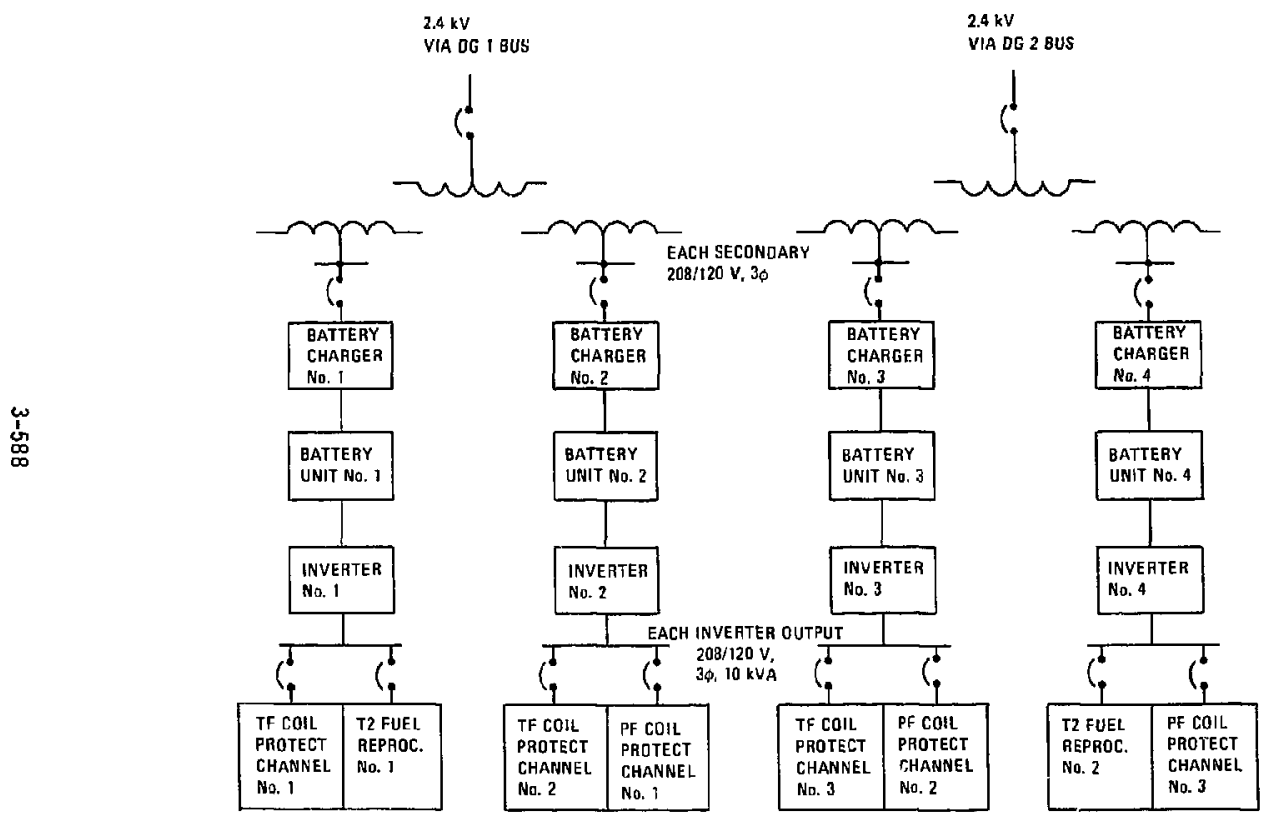

Figure 3-231. No-break control power for the superconductor-coil protection and fuel reprocessing systems. 
- Shielding requirements--Currently the wall, fioor, and roof thicknesses are assumed to be two meters, based on neutron- and gamma-shielding requirements for the hot cell and reactor buildings. No definite calculations exist to valioate these assumptions. Any reduction in the thicknesses save building costs.

- Safety criteria for facilities handing large amounts of tritium have yet to be established. These criteria will dictate the designability, constructability, and cost of these three buildings with respect to leak tightness, internal pressure, and seismic and tornado loading requirements.

- Hands-on and remote maintenance requirements inside the reactor building will influence the size of this building.

The following structural design issues relaie to heavy weights of walls and doors:

- One area of high sensitivity to the heavy building weight caused by the required shielding thickness is the limited span that can be tolerated by the basemat because of soil pressure. This requires that the basemat be stiffened by internal walls (and the first floor where possible) to keep basemat thickness and reinforcement at reasonable levels. In future studies, we should investigate the trade off between incorporating such walls into the building layout as opposed to eliminating some of them and providing more basemat thickness and/or more reinforcement.

- Another area of high sensitivity to the required shielding thickness are the seismic loads induced on the walls of the reactor building. Most of these heavy walls span large distances in the absence of stiffening from adjacent floors or walls. This configuration appears feasible, even though, the reinforcement levels required for seismic loads are very high in the longer walls. Future studies should consider alternate designs in which these walls are stiffened with pilasters.

- The weight of the shielaing doors to crane maintenance bays in the reactor and hot cell buildings should be minimized. To reauce the door mass, the door opening should only be large enough to allow the crane bridge to pass, with a larger opening provioed at one location 
for accomodating the crane trolley. Also, the door should be constructed of several sections (vertical joints) to further reduce the lifted,weight. The same relatively small lifting mechanisn could then move irom section to section and slowly lift the entire door by sections.

No key technical issues are associated with the ac power system since reliable equipment is comercially available. The usual electrical safety practices allowing for leakage of hydrogen isotope gases that may cause explosive mixtures in local zones must to be observed.

An economic issue involves the amount of redundant equipment needed. Is it possible to operate the TOF without a better voltage-regulated light-duty power substation? Are the oiesel generators needed and if so, how many?

In this mirror-experiment study, we followed the gujdelines established for tokamak studies, assuming these guidelines also apply to mirror machines fueled with tritium.

To summarize, we note that the major facilities are currently being designed on the basis of very optimistic assumptions. The cost estimate of these facilities may increase when a detailed evaluation of the key issues is undertaken. 
3.13.]. Summary

The instrumentation and control (I\&C) system is a distributed, hierarchical computer control ano instrumentation system, organized functionally into three major oivisions:

- The Supervisory Control and Diagnostic System (SCOS). Responsible for the overall control and direction of the I\&C system, it acquires and stores both diagnostic and facility data, and provides the operator/machine interface. The SCDS consists of nine 32-bit minicomputers with peripherals, operator consoles, and interfaces to the Local Control and Instrumentation System.

- The Local Control and Instrumentation Systen (LCIS). Provides the interface between the SCDS and TDF equipment. The LCIS consists of about 65 subsystems, each with a local control computer (micro-processor) linked by fiber optics to a muliticrate Computer-Automated Measurement and Control (CAMAC) system which interfaces directly to the facility hardware.

- The Safety Interlock System (SIS). Continuously monitors all safety critical input/output points. The SIS consists of two programmable coritrollers with backup hardware, sensors, and alarms. Normal1y, the system operates independently of the SCDS and the LCIS, but data is available to the SCOS (via the LCIS) for display or storage.

\subsubsection{Requirements}

The I\&C system is responsible for monitoring and controlling TDF. A11 operations of the facility's hardware must be initiated through the I\&C. Besides monitoring all TDF parameters, the system must also allow for displaying and storing data. The cesign is influenced by the following considerations:

The scope of the system; the enormous number of input/output points to be monitored and/or controlled. Based on MFTF, there will be about 3000 control points, and about 7000 that will need monitoring. 
- The extended layout of equipment. Due to the remote location of the control center, there may be distances of up to several hundred meters between the controlling computers and the facility hardware.

- The need for flexibility. The design must be able to continualiy accomodate new sensors and functions, or even entire systems.

- The need for redundancy and system reliability. It must be possible to switch over readily to backup hardware, should equipment failures occur.

- Modularization is highly desirable. Extensive use should be made of comon (interchangable) hardware and software.

\subsubsection{Discussion}

The current design is based on the I\&C system for the MFTF, with modifications as necessary. This approach was taken, rather than design "from the bottom up", due to the similarities of the I\&C requirements for the two systems and to the natural unavailability at this stage of quantitative, detailed signal and control point lists for many of TDF's subsystems. Because there are many similarities between the two facilities, an I\&C design done in this manner should be accurate with respect to system structure and should also provide a reliable estimate of the scope and size of the system.

The next three sections describe the systems.

The Supervisory Control and Diagnost ic System (SCDS). This distributea, hierarchical computer control and instrumentation system will consist of nine Perkin Elmer 3230/3250 minicomputer systems (or their equivalent), each controlling one or more major TDF systems (e.g., the vacuum system, or the plasma streaming guns). Al1 interprocessor communications are accomplished $\checkmark$ ia a shared or common memory. The nine minicomputers are logically arranged around the shared memory in a star configuration, as shown in Fig. 3-232. Although each computer is dedicated to controlling a particular set of hardware, access to other data and control functions (if permitted) will be possible via interprocessor communication. The functions for the nine Scos computers are: 


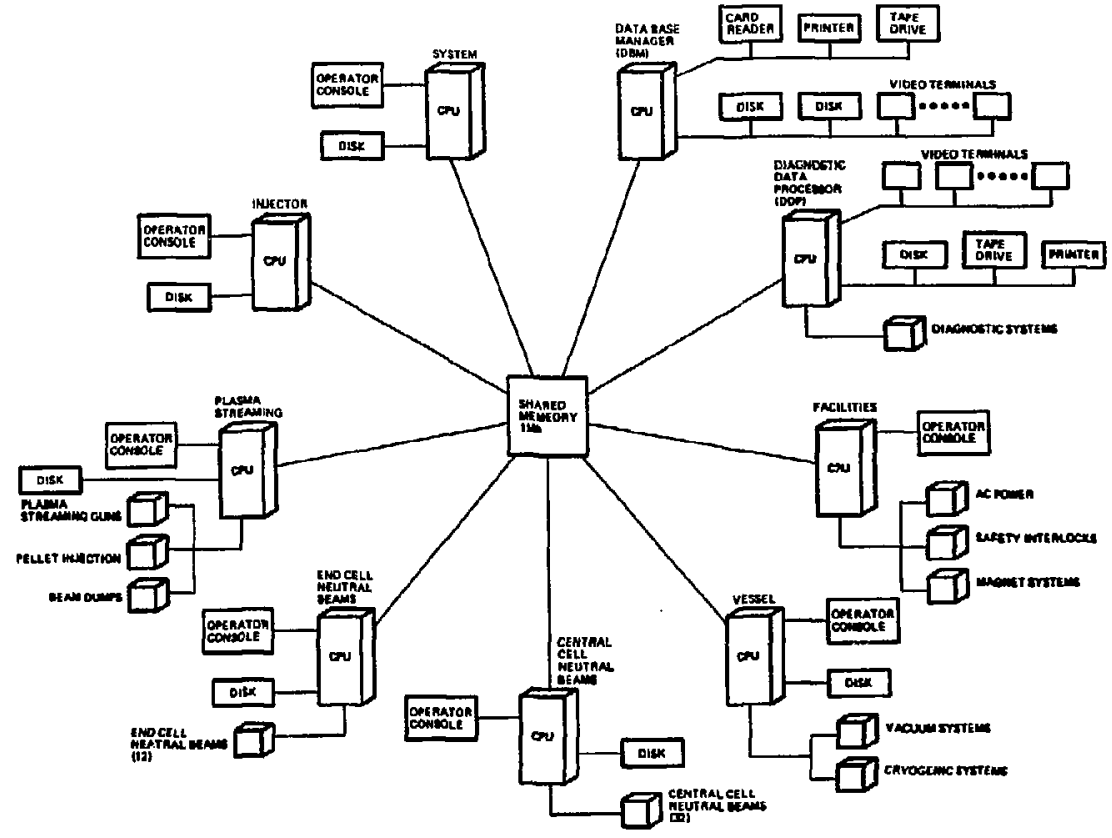

Figure 3-232. The supervisory control and oiagnostics system (SCOS). 
- System Supervisor. This computer controls all the other SCDS computers. Its console would theoretically be manned by the chief operator, who would have access to all the lower-tier computers and would thus have complete control over all TDF operations.

- Neutral Beam Injector Supervisor. Coordinates the operation of the next-lower tier of SCOS computers, such as the neutral-beam subsysteris.

- Plasma Streaming Supervisor. Coorainates operation of the plasma streaming guns, pellet injection, and monitoring of the beam dumps.

- Central Cell Neutral Beam Supervisor. Coordinates operation of the central cell neutral-beam injector sources. Supervisor controls 32 ilicroprocessor-based subsystems, one for each neutral beam source (rour sources per dealrt itrite for eright beam? imes\}.

- End Cell Neutral Beam Supervisor. Coordinates operation of the end celi neutral-beam sources. One subsystem per source is used, for a total of 12 subsystems: four each for the anchor pumping beams, the anchor sloshing beanis, and the transition pumping beams.

- The Vessel Supervisor. Responsible for the vacuum and tryogenics systems.

- The Facilities Supervisor. Responsible for the ac power, the safety interlocks, ana the magnet systems.

- The Diagnostic Data Processor. Controls operation of all the plasma diagnostics.

- The Data Base Manager. Updates and maintains a data base of current values for all the TDF's parameters. Recent (but superceeded data remiains on disk for a certain time, after which it is stored on magnetic tape and is available by request.

The six SCOS subsystems used for facility control have multiple CRT displays, touch-sensitive panels for operator/computer interaction, and disk storage units. The diagnostic data processor and data base manager, which will be used for software ae: elopment in addition to their regular duties, will have a full set of computer peripherals. These will include a magnetic tape orive, Tine printers, graphics terminals, and large disk storage units. 
LCIS Interface. Six of the SCOS computers communicate with the local control ano instrumentation system. The LCIS consists of about 65 microprocessor-based subsystems, which interface directly to the facility's hardware. (The LCIS is described in more detail later in this section.) There are two types of LCIS/SCOS-computer interfaces. Commands and most of the data are transmitted by standand 9600 baud, bi-directiona1, RS-232 serial interfaces. Certain diagnostic instruments transfer data at a higher rate, and this is done by a direct memory access.

Diagnostics. The aiagnostic data processor is responsible for controlling all of TDF's diagnostics. Since a large ana continually growing set of diagnostic tools will assuredly be needed, the present I\&C design includes the ability to handle diagnostics in general. The specific instruments or devices to be used will be defined later.

The diagnostic system is patterned after MFTF. Instead of using one computer for diagnostics and the other for data base managenent, it might be better (for reasons of system reliability) to divide the diagnostic channels between them, and provide each computer with access to the aata base. This arrangement, an alternative to a dedicated aiagnostic data processor and data base manager, is pictureo in Fig. 3-233. Note that the use of 13 diagnostic systems has been assumed. The interfaces between the SCDS and LCIS computers meritioned previously are also shown. The line communication multiplexer provjges a bi-directional RS-232 modem for several diagnostic systems, and a quad synchronous adapter provides a uni-directional, high-speed, direct-memory access for four diagnostics. Although not specificaliy defined at this time, some possible diagnostics (as used on the MFTF) are:

- Backgrouna gas pressure. An assortment of vacuum gauges for measuring the neutral particle pressure.

- Charged particle flux. A Faraday-cap collector for measuring ion current, and a disk-shaped probe for measuring the net electronic current.

- Electromagnetic fluctuations. A high-impedance electrostatic probe and a group of nested single-turn loops for measuring fluctuations.

- Far infra-red laser interferometry. To measure the phase shift of radiation with wavelengths of from 0.1 to $0.5 \mathrm{~mm}$, as they pass through the plasma cross section.

- Jon spectroscopy. Similar in diagnostic function to the laser but operates at wavelengths of from 1 to $4 \mathrm{~mm}$. 
फ

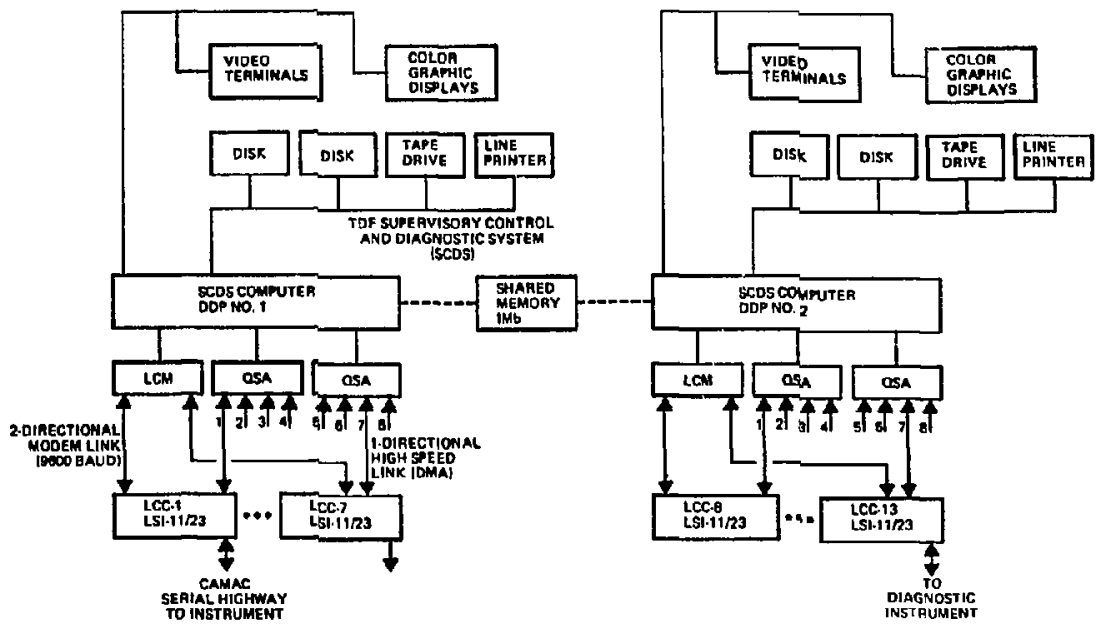

Figure 3-233. The plasma diagnostics data processing and display system. 
- Neutral-beam transmission. A spatial array of secondary electron detectors that are used to deduce the radial profile of plasma line density.

- Neutron counters. Liquid, organic, and thin plastic scintillators, or fast-fission proporticnal counters, depending on the range of fluxes to be covered.

- Neutral-flux spectroscopy. Surface barrier detectors that are used for measuring the energy distributions of neutral (deuterium) particles emanating from various plasma regions.

- Plasma-current sensor. An assortment of diamagnetic loops, pickup coils, and pickup loops that respond to the changing magnetic flux linkages caused by plasma currents.

- Sloshing-ion sensors. Individual secondary electron detectors that are used for measuring the angular distribution of neutral particle flux from thermal barrier region.

- Ultraviolet spectroscopy. A self-scan image sensor for measuring the absolute intensity of the ultraviolet flux emitted by a plasma.

- $X$-ray spectroscopy. High-purity germanium detectors and/or inorganic scintillators (such as $\mathrm{NaI}$ or $\mathrm{C}_{s} F$ ) used for measuring the $x$-ray energy spectrum, from which plasma electron temperatures can be deduced.

One of the key design requirements is protection against single-point failures. Accordingly, each SCDS computer has a designated backup. In other words, one of the other computers can perform the identica? functions. The system and injector supervisors back up one another, as do the facilities and vessel supervisors, and the data base manager and diagnostic processors. The LCIS nicroprocessors (LCCS) that are connected to a particular SCDS computer are also connected to the back-up computer. If an SCOS computer fails, control of the LCCs that are affected switches to the backup computer. This can be done automatically (by computer control) or manualiy. Furthermore, the data base manager and the diagnostic data processor computers can each be switched to assume the duties of the other. In particular, the disk containing the oata base $c$ an be switched to the functioning computer, so that new data can continue to be stored. If the disk itself fails, data can still be written oirectly on nagnetic tape by one of two tape drives. 
Therefore, the facility can continue operating despite the loss of any single SCUS computer, although perhaps with some performance degradation.

Operator-Machine Interface. The chief aesign goals for the operator's consoles are the operator's convenience, and flexibility for future change. The maxinum possibie use will be made of current technology, such as using touch-sensitive panels for command and data entry. Several CRT calor monitors will be used per console. Color will be used to convey a maximum amount of information per display, and to highlight data and/or requests for action by priority ievel. For example, alarms could be displayed as flashing reo.

All operations will be "soft-key" controlled, so that all an operator has to do to change one display to another is to press keys. A hierarchical systent is employed whereby the operator can assume controt of any lower tier system. However, access will be key-coded: only certain individuals will be allowed to have control over any given system. Key coding coula be by badge number, or by a similar means, and all accesses to the system (plus any actions that are taken) will become part of the historical record.

All consoles will have the appropriate sof tware support for any selectable function. At any time, it will only be necessary to update the software to ada new functions, rather than having to make expensive hardware reconfigurations.

Exception Handling. A protocol for exception handling will be aeveloped. For all the monitored signal;, information such as high- and low-tolerance limits and criticality will be stored. A violation of these limits (exceptions) will initiate actions by the SCOS, which will depend on what category the exception is in:

- Category 1. Requires only that the operator be not ifieo and the exception recorued. Notification might be aone by a flashing Gisplay screen. No action by the operator is required.

- Category 2. Requires a response from the operator. Some action by the operator might be necessary, followed by an acknowleogement by the operator that the problem has been corrected or that it is alright to proceeo.

0 Category 3. Requires that the SCDS take an immediate corrective action, such as aborting an operation, or shutting something oown. 
In adition, some exceptions can change their category. For instance, in combination with other exceptions, they can become more serious. One of the advantages of a computer-based exception handling system is that it can easily be programmed to look for such correlations.

Local Control and Instrumentation System. The local control and instrumentation system (LCIS) is responsible for acquiring data from, ana controlling, all the hardware in the TDF. The LCIS is an array of microprocessor-based subsystems, which together form the interface between the scos cornputers and the facility's equipment. Each subsystem has several elements in common: a local control computer (an LSI-11 microprocessor), fiber-optic links, and either a CAMAC system or Modicon programmable controller. Most of the subsystems utilize the CAMAC system, which is described later in this section. the Modicon programmable controllers are used in the safety interlock system, and also are described later.

There will be about 65 LCIS subsystems (scaling from the MFTF, and assuining the use of 13 aiagnostic systems), one for each major piece of TDF equipment. (For example, the central cell neutral-beam Scos computer will contral 32 LCIS subsystems, one for each central cell neutral-bean source. The ena cell neutral-beam SCDS computer will control 12 LCIS subsystems: four anchor sloshing beams, four anchor pumping bearns, and four transition pumping beanl sources.)

A 7 though there are a large number of subsystems, they perform many common tasks. If the hardware is developed in functional modules, common tasks will naturally lead to the use of common hardware. Thus, different subsystems will require only using different combinations of the same modules. The results are greater cost savings, the avajiability of more backup hardware, and better system reliability.

The LCIS Computer. The basic configuration of an LCIS subsystem is shown in Fig. 3-234. The Local Control Computer (LCC) is assumed to be the LSI-1//2, a 16-bit microcomputer made by the Digital Equipment Corporation (DEC). This was the LCIS computer selected for the MFTF. At that time it represented current technology, but it is now somewhat dated. Presumabiy, some newer microcomputer would be selected for the TDF, one which would have up-to-date technology at the time of the final design. 
The LSI-11 includes 32 kbytes of random access memory (RAM), with no disk storage. Programs are down-loaded from the SCOS computers. The interface to the SCDS computer is the OLVLL-E, also made by DEC. This is a standaro RS-232 serial-line interface (a 9600-baud moderi line). The serial-highway driver provides the interface to the CAl,AC serial highway. It supports programmed input/output operation, as well as block data transfer oirectly to memory (DMA). Optical transmitters and receivers convert our message format to the bi-phase manchester code used for serial highway transmissions.

Fiber optics. Due to the extended layout of the I\&C system, fiber-optic links are required. The LCC's will be located in the central control room, which is assumed to be in a separate building from that housing the facility's hardware. The CAMAC crates are located adjacent to the power supplies, or in a specially screened room, in the facility bujlding. The separation invoived may be 300 meters, well within the capability of current fiber-optics tecimology. This technology is developing rapid?y and is becoming increasingly more reliable, both at higher da $\neq a$ rates and over greater transmission distances. Optical transmissions have low attenuations and large bandwidths, are immune to electromagnetic interference, and al how electrical and ground isolation between the control room and the facility's haraware buildings. Using fiber-optics communication networks in facilities such as this has become commonplace.

Computer-Automated Measurement and Control (CAMAC). CAMAC is a standardized (IEEE STU 583) data acquisition and control system used extensively in nuclear and particle physics research facilities, and in industrial applications. The haroware configuration of a CAMAC system is shown in Fig. 3-234. The fundamental unit, the CAMAC crate, is basically an input/output-card cage, which houses and provides power for plug-in CAMAC modules, each of which performs some specific operation (usually an input/output). Extrapolating from MFTF, we estimate that approximately 220 CAMAC crates will be needed. In general, 2 to 4 crates will be used for control systems and 7 to 10 crates for diagnostic systems.

The U-port module consists of an optical transmitter/receiver which converts the bi-phase manchester code (used for serial highway transmission) to the D-port format (for use in the CAMAC crate). The serial crate 


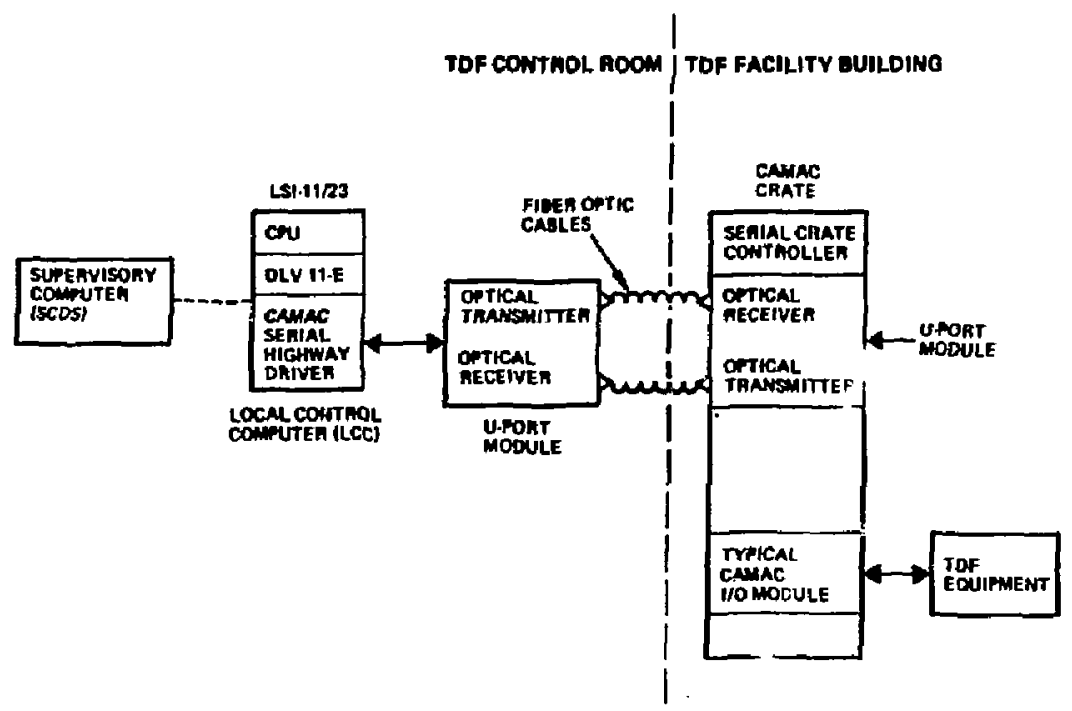

Figure 3-234. The local control and insirumentation system (LCIS) subsystem. 
controller provides the interface to the CAMAC I/O modules and controls all operations within the crate. The plug-in modules are subsysten specific, but provide combinations of standard functions, such as digital and analog input/output. Thus, an appropriately selected small number of different modules can be used for a wide variety of applications. Because of the high degree of standardization and interchangability, any component can be readily replaced, thus minimizing oowntime aue to equipment failure. For the most part hardware is commercially available from a number of vendors. However, depending on the appplication, some modules may have to be designed and built, either in-house or by a vendor.

Software. A single purpose data acquisition and control program, such as the MFTF's plasma diagnostic and local control executive, will be used in all of the LCCS. The command language is based on a small number of primitives, each one causing some simple operation to be performeo. Lifferent sets of commanos run oifferent subsystems, but all the commands aree made up of the same primitives and are run by the same executive program.

Hardware interface details are buried in a aevice tabie, which contains the information needed for a particular operation. The saftware set also includes a series of input/output routines, one for each defined function. These routines are invoked by the executive when a command is executed. The software is thus readily adaptable to hardware reconfiguration. Adding a new input/output point requires only that a new entry be made in the device table. Only if a new function is introduced would a new input/output routine be required.

Test/calibration. There is a need to operate the TOF's subsystems and diagnostics independently of the SCUS, for debugging and calibrating. An LSI-11 computer, located in the control room area, will be used to emulate the SCDS. When connected to this emulator by fiber optics, one or more special test computers (or terminals) can check or calibrate the TOF's hardware from anywhere in the facility. 
Safety Incerlock System (SIS). This system will monitor all personnel safety and equipment protection signals. The SIS consists of:

- Two programmable controllers with hardware backup.

- Various sensors for detecting the status of interlocks and other safety inicators, such as critical temperatures ano pressures, radiation, and other hazards.

- Alarm indicators, which include audio/visual warning devices, such as horns, beepers, and warning lights.

Use of the Modicon 584 programmable controller, the choice for MFTF, has been assumed for the present design. (The final design would, presumably, make use of the latest technology, ano would thus use another, newer controller.) This unit is similar to the CAMAC crate aiready discussed, in that it functions as an input/output card cage. However, unlike the CAMAC crate, the intelligence is built-in rather than being supplied by an external computer. Plug in moaules provioe a given number of input/output channels per module, and allow flexibility in configuring the mainframe. The system configuration is shown in Fig. 3-235. The programable controllers are 7 inked by fiber optics and mooems to an LCIS computer, ano hence to the SCDS facilities computer. Some representative inputs and outputs are shown. (Note that many of the input sensors monitoring the Tof's subsystem equipment are not themselves part of the SIS. Even though they provide information to the SIS, they are considered part of their respective subsystems.) The outputs include permissives and status signals to other systems, lights indicating go/ro-go conditions, and the expected assorted bells and whistles for audio/visual warnings. Programmable controllers have nonvolatile menories, but no local disk storage. Programs have to be supplied via the down-linic from the LC1S/SCUS computers, or from a special programming terminal located in the controi rooil area. During nomal operations, the programable controliers will function independentiy from the LCIS/SCOS, although data will be sent to the SCUS. The program stored in memory is in complete control, with no provision for remote control by the LCIS/SCOS. Specifically, this means that any SIs-generated interlocks or system shutdowns cannot be bypassed by the higher-level computer systems.

Programmable controllers have certain advantages over both computer-controlled and hardwired systems. Their primary advantage over a 


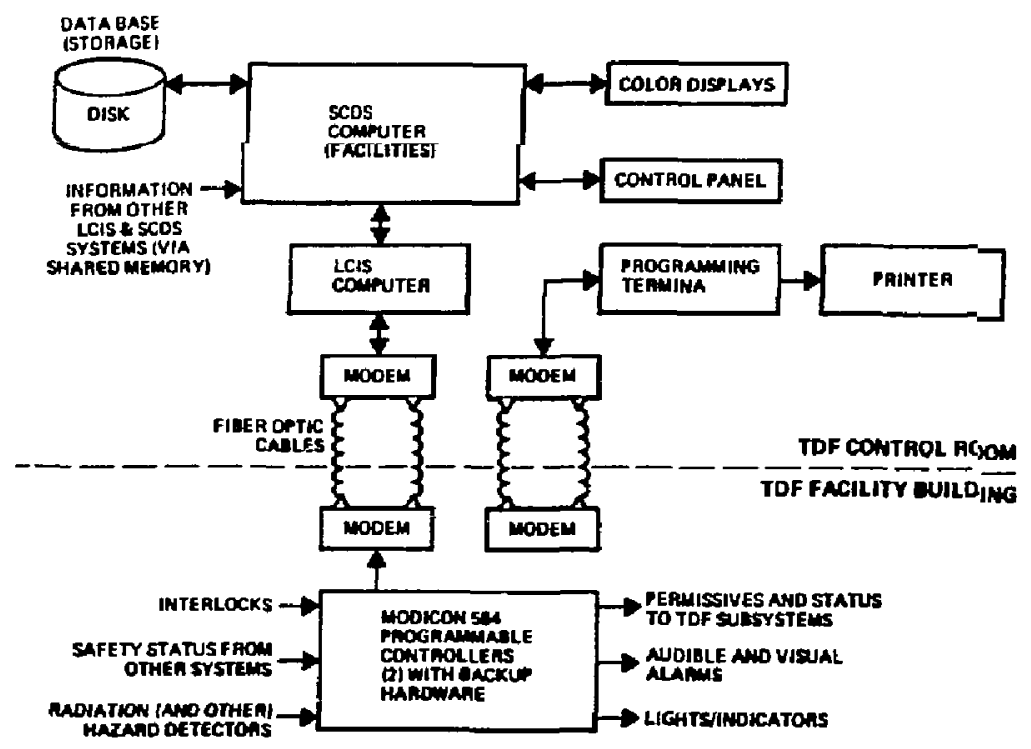

Figure 3-235. The safety interlock system (SIS). 
totally computer-controlled system is speed. Computer processing time is naturajly proportional to the total number of input/output points. For very iarge systems, depending on the other duties and consequent avajlability of the computer, the time between consecutive servicings of any given input/output point (and therefore, the response time) may be degraded to an unacceptable level. One solution is a computer dedicated to do just this task. However, if only relatively simple logic is required, a programable controller provides faster response time with a lower cost. A programmable controller can be reconfigured simply by plugging in new boards and changing its software. The engineering and installation costs are less. These costs are estimated at $\$ 50$ per input/output point (including programing), which contrasts with an estimated $\$ 100$ per input/output point for designing and installing hardwire relays. Furthermore, hardwire relays would (most likely) be useless after any significant reconfiguration, and would have to be replaced from scratch.

The proposed system has extensive built-in test capabilities and is extensively protected against single point failures. This incluaes dedicated inputs, which can be switcheo in to test critical outputs on-line, and dedicated outputs for testing critical input channels. Hardware backup relays ensure that power will be removed from critical outputs in case of an input/output module failure, or in case of an improper controller operation. Checking is bujlt into the software to test for invalid program sequences, A watchoog timer is also used to pinpoint response time delays and outright failures. The system's integrity is protected by keylocked cabinets, which restrict access to the controller's hardware and the programming terminal. Keylocked switches will also to used to protect the program's memory. Without using these keys, the controlier cannot be reprogrammed. 


\subsection{CRYOPLANT}

\subsubsection{Summary}

A cryogenic system will be required to satisfy TOF's helium and nitrogen refrigeration and liquefaction demands. A preliminary requirements survey and analysis has shown that a cryogenic system very similar to that proposed for the MFTF-B project will meet TDF's cryogenis demands.

The cryoplant study investigated five major elements in a conceptua? cesign:

- The $L H e$ and $L_{2}$ requirements.

- The plant size requirements.

- The utility requirements.

- The cost of major componerits.

- The incremental operating and capital costs.

The information presented here for all these areas, except for the LHe and $\mathrm{LN}_{2}$ requirements, is based on an MFTF-B plant design proposal supplied by Koch Process Systems.

Several TDF team organizations were consulted during the requirements survey to assess the cryoplant denands as accurately as possible. These included: the Fusion Engineering Design Center (FEDC), LLNL, TRW, and Science Applications, Inc. (SAI).

\subsubsection{Requirement Survey and Analys is}

There are five major TOF systems that require $\mathrm{LHe}$ and $\mathrm{LN}_{2}$ refrigeration/liquefaction. These denands, which must be met with a reasonable safety margin, are sumarized in Table 3-97. The design must also provide for 8 hours of operation (from storage dewars) in a standby mode (such as during refrigerator maintenance).

Magnet Cryogenic Requirements. The superconducting magnets for the TDF require LHe and $L_{2}$ during all their operational mooes: cooldown, steady-state, stañdby, warmup, and emergency shutdown (the magnet goes normal and quenching follows). The FEDC currently has responsibility for the anchor 
Table 3-97. Estimated crycgen requirements.

\begin{tabular}{lcr}
\hline \multicolumn{1}{c}{ Subsystem/team member } & $\begin{array}{c}\text { He } 7 \text { ium } \\
\text { (W at } 4.4 \mathrm{k} / \text { liters/hr) }\end{array}$ & $\begin{array}{c}\text { Nitrogen } \\
\text { (W at } 78 \mathrm{~K})\end{array}$ \\
\hline Superconducting magnets/GD,FEOC & $2500 / 253$ & 21,600 \\
Vacuum system and cryopanels/LLNL & 2050 & 579,000 \\
Beam lines/TRW & 900 & 12,500 \\
Tritium processing/SAI & 400 & 4,500 \\
Peliet injection/FEOC & 2 & 10 \\
Total estimate & $6 \mathrm{~kW} / 253$ liters/hr & $618 \mathrm{~kW}$ \\
\hline
\end{tabular}


cell coils, while General Oynamics was assigned the design of the central and barrier cells. Table 3-98 summarizes the anchor cell requirements, 51 and the LHe and $L N_{2}$ heat loads, as supplied by the FEDC. Table 3-99 summarizes the requirements for the solenoid coils $(S-0,5-1)$. These are in-house estimates, based on MFTF-B solenoid heat-load analyses. Table 3-99 also lists the scaling factors used to assess the solenoid heat loads, as aerived from the MFTF- $B$ final design review and analysis. The LHe scaling factors were based on heat loads obtained from a detailed thermal analysis of the MFTF-B solenoid. The heat load is normalized to the parameters that have the most effect on the heat load. For example, the conduction heat load for an IIFTF-B solenoid is proportional to the total cold mass (magnet pack and insulation) weight; i.e., the greater the weight, the larger and more numerous are the struts that support the magnet. Similarly, the magnitude of the centerline magnetic field directly affects the size and number of interconnecting struts that support the loads induced by the magnetic field in a fault moae, and the number ana size of these struts directly affect the heat loads. A typical MFTF-B solenoid weighs 30,000 lbs, produces a magnetic field of $1 \mathrm{~T}$, and gains $13.07 \mathrm{~W}$ by conduction. 52 Therefore, the conduction scaling factor, normalized to weight and field would be:

$\frac{13.07 \mathrm{~W}}{300001 \mathrm{~b} \times 1 T}=0.4356 \frac{\mathrm{MW}}{\mathrm{Tb} \cdot \mathrm{T}}$

The remaining Llie scaling factors are derived by similar methods. These scaling factors are:

- Radiation from the $\mathrm{LN}_{2}$ shield to the LHe-temperature magnet case.

- Conduction by the instrumentation wires.

- Conduction and radiation losses by the fluid transfer lines.

- $I^{2} R$ losses at splices.

- Neutronic heating.

All solenojd magnet $L N_{2}$ radiation loads are based on $83.9 \mathrm{~W} / \mathrm{m}^{2}$ of magnet shield surface area and are calculated accordingly.

These magnet cryogen heat loads are only preliminary estimates, and must be upaated as the aesign progresses. The calculated total $L$ He and $\mathrm{LN}_{2}$ magnet heat loads are estimated as being $2596 \mathrm{~W}$ and $21,614 \mathrm{~W}$, respectively for nine superconducting coils. 
Table 3-98. Anchor ce. 1 region cryogen requirements as provided by the FEDC cryoplant study.
Component heat loads
LHe (w)
$L N_{2}(W)$

Stack and vapor cooled leads

96

(conduction, vaporization)

Splices

( $l^{2} R$ heating)

12

$\mathrm{LN}_{2}$ shield to LHe magnet case

54

(Radiatior)

Neutron hẹating

306

Struts/supports

20

(conduction)

Vacuum vessel to $L N_{2}$ shield

9000

LHe requirement

(vapor coGled leads at 0.35 liters/hr

and $100 \mathrm{~A}$

136

Total est inate (ali six coils)

$288+136$ liters $/ \mathrm{hr}$

9000 
Table 3-99. Cryogenic requirements as determined from heat load estimates for solenoid coils $S-0$ and $S-1 .^{a}$

\begin{tabular}{|c|c|c|c|c|}
\hline \multirow[b]{2}{*}{ Components } & \multicolumn{2}{|c|}{ S-0 solenoid } & \multicolumn{2}{|c|}{ S-1 solenoid } \\
\hline & LHe $(H)$ & $\mathrm{LN}_{2}(\mathrm{~W})$ & LHe $(W)$ & $\mathrm{LN}_{2}(\mathrm{~W})$ \\
\hline Struts (conduction) & 408 & & 639 & \\
\hline Radiation & 50 & 3356 & 58 & 4615 \\
\hline Instrumentation (cab)e conduction & 9 & & 10 & \\
\hline \multicolumn{5}{|l|}{ Fluid line transfer } \\
\hline (radiaion/conduction) & 7 & & 7 & \\
\hline Splice ( $I^{2} R$ heating) & 4 & & 5 & \\
\hline Neutronic heating (TRW analysis) & 78 & 8 & 107 & 10 \\
\hline \multicolumn{5}{|l|}{ Liquid helium requirement for } \\
\hline yapor cooled leads & 31.7 & & 27.0 & \\
\hline Total estimate per coil & $556+$ & 3364 & $826+$ & 4625 \\
\hline & $31.7 \mathrm{l} / \mathrm{hr}$ & & $27 \mathrm{l} / \mathrm{hr}$ & \\
\hline
\end{tabular}


Vacuum Pumping System. The 36 cryopanels in the end cell region maintain the rUF at a vacuum of $5 \times 10^{-6}$ Torr and are the second largest user of LHe and $L N_{2}$. LiNL is responsible for the cryopanel design and analysis. Cryogen requirements for the unirradiated system were obtained from Ref. ${ }^{53}$. The LHe and $\mathrm{LN}_{2}$ loads reported were 1,500 and $575,000 \mathrm{~W}$, respectively.

Total adoitional heat deposition from neutron irrudiation was calculated assuming a volumetric neutron heat flux of $4 \mathrm{~mW} / \mathrm{cm}^{3}$ at the plasma beam center in the vicinity of the cryopanel $5^{54}$, along with an "e folding distance" of $6.8 \mathrm{~cm}^{55}$ it was also assumed that the $\mathrm{LHe}^{5}$ and $\mathrm{LN}_{2}$ portions of the cryopanels consisted of $3.2 \times 10^{6} \mathrm{~cm}^{3}$ and $2.33 \times 10^{7} \mathrm{~cm}^{3}$ of non-transparent material, respectively. 54

Based on the preceding assumptions, neutronic heat integration analys is was conpleted. The total neutron heat deposition rates for the LHe and $\mathrm{LN}_{2}$ portions are $554 \mathrm{~W}$ and $4032 \mathrm{~W}$, respectively. These additional loads increase. the tota! LHe and $L \mathrm{~N}_{2}$ vacuum system cryopanel loads to $2054 \mathrm{~W}$ and $579,032 \mathrm{~W}$, respectively.

Main-Vessel Beamlines. The eight main-vessel beamlines are being designed by TRW. They contāin small superconducting magnets, internal beam dumps, neutralizer ducts, and cryopanels that require Lhe and $\mathrm{LN}_{2}$. Information regarding cryogenic requirements ${ }^{56}$ gives $L$ He and $L N_{2}$ requirements of $896 \mathrm{~W}$ and $12,500 \mathrm{w}$, respectively. These estimates include neutron heating contributions.

Tritium Systems/Processing. The TOF tritium systems are being oesigned by $S A I$, and represent a relatively minor load on the cryoplant; $400 \mathrm{~W}$ of LHe and $4480 \mathrm{~W}$ of $L N_{2}$ cryogens are required for the separation, c]eanup, ana processing of deuteriuln and tritium within the TDF. ${ }^{57}$ In addition, $301 \mathrm{iters} / \mathrm{hr}$ of LHe liquefaction is required.

Pellet Injection. The pellet injector system requires very little Life or $\mathrm{LN}_{2}$ refrigeration. The $\mathrm{FEOC}$ is designing the pellet injector, and supplied the following cryogen requirements ${ }^{58}: 2 \mathrm{~W}$ of LHe and $10 \mathrm{~W}$ of $\mathrm{LN}_{2}$. 


\subsubsection{Cryoplant System Concepts}

Helium $-y s L_{i j l}$. The system requirements for the latest TOF configuration are $584 \mathrm{~b} \mathrm{~W}$ of LHe refrigeration at $64 \mathrm{~kW}$, and 253 liters/hr of LHe liquefaction. A helium system experiences two types of loads from TDF.

- A liquid-helium refrigeration load--Defined as a cycle in which the helium is returned to the refrigerator as a saturated vapor at $4.35 \mathrm{~K}$, arid only needs cooling (at a constant temperature) to the saturated liquid state at $4.35 \mathrm{~K}$.

- A liquid-helium liquefaction load--Definea as a cycle in which the helium is either returned to the liquefiers (or is lost completely) as a superheated vapor at a temperature greater than $4.35 \mathrm{~K}$. Thus, it must first be cooled to the saturated vapor temperature $(4.35 \mathrm{~K}$ ) and then cooled at a constant temperature (as with refrigeration) to the saturated liquid state at $4.35 \mathrm{~K}$.

A typical liquefaction load would result fram the LHe boiling off through a vapor-cooled magnet current lead, which might or might not be returned to the system. If it is not returned, it still represents a liquefaction load since gaseous helium at room temperature must be liquefaction to make up the loss. A helium system therefore receives two ratings. One rating describes the available refrigeration and liquefaction capacity while the systern is operating in a combined mode; the other rating is the system's capacity when it is useo only as a liquefier.

The requirements just described for LHe are substantially below those of the MFTF-B cryoplant system, with its capacity of $9,000 \mathrm{~W}$ of LHe refrigeration at $4.35 \mathrm{~K}$, and $530 \mathrm{Titers} / \mathrm{hr}$ of LHe liquefaction in the combined cycle mode. These capacities allow a 50\% safety margin for the refrigeration load and $109 \%$ for liquefaction. It is not overly conservative to design a cryoplant with such margins, since the actual demands are generally at least 30 to $40 \%$ greater than expected, due to unforeseen heat losses in the system.

Cold Box (Refrigerator). The cold box is the heart of the LHe systein. It contains several heat exchangers, and Joule-Thomson type thrattling valves. It utilizes $L N_{2}$ precooling, which loads the $L N_{2}$ refrigerator/liquefier. 
Compressors. There are a total of six oil-injected-screw type compressors in the primary compression system. The first stage consists of four, and the second stage of two, units.

Helium Storage and Supply Dewers. The helium system must include provisions for supplying LHe during a cryoplant shutdown period of eight hours. In addition, the systern must be capable of storing all the gaseous helium boiled off during this time by the major TDF systems. When the cryoplant is ance again operational, a recovery system must pressurize and store this helium at intermediate pressures, before it is refrigerated and liquefieo.

The systeml's liquia helium storage capacity is 65,000 liters, which is sufficient to supply all of the TDF's systems with their LHe cryogenic requirements for the 8 -hour standby period during cryoplant inaintenance. A large gas bag will hold all the gaseous helium boiled off by equipment ouring the standby, for pressurization, refrigeration, and liquefaction at a later time.

Nitrogen System. The nitrogen reliquefier/subcooler provides cooling for the TDF systems' $L N_{2}$ shields, and also precools the heliuin gas before it is refrigerated and liquefied. It has a cooling capacity of $500 \mathrm{~kW}$, and includes a 150,000 liter licuid storage dewar, which is capable of providing at least 8 hours of service during a cryoplant standby for maintenance.

Bujlding/Utility Requirements. The floor space required for the TOF cryoplant will be comparable to the MFTF-6 cryoplant, but it wi]l have a slightly difierent arrangment due to differences in reactor dimensions. This study assumed that the TDF's cryoplant will be completely separate from the reactor building (which housed part of MFTF-B's cryoplant). It was assumed that the entire TUF cryoplant (including the colo box, and all the storage tanks and dewars not housed in the MFTF-B configuration) could be placed in a builoing with twice the floor space of that proposed for MFTF-B. Assuming an MFTF-B builaing size of $6300 \mathrm{ft}^{2}$, the TOF building will therefore require approximately $12,600 \mathrm{ft}^{2}$. Since certain components of a cryoplant (such as storage tanks) do not require housing, it is probable that the actual building requirement for TDF may be only slightly larger than the MFTF-B building requirement. This cannot be accurately assessed until more detailed TDF designs have been cormpleted. 
The estimated electrical utility requirements for a cryoplant of this size are: LHe $5400 \mathrm{~kW}, \mathrm{LN}_{2} 6000 \mathrm{~kW}$. The total power requirement is $11.4 \mathrm{MW}$. These power requirements may be used to illustrate the relative utility cost for LHe and $\mathrm{LN}_{2}$ refrigeration. The LHe system, capable of removing $9000 \mathrm{~W}$ of heat at liquid helium temperature $(4.35 \mathrm{~K}$ ), requires $5400 \mathrm{~kW}$ to operate. Therefore, this "costs:"

$\frac{5400 \mathrm{~kW}}{9 \mathrm{~kW}}$ or $\frac{600 \mathrm{~W} \text { of electric power }}{\text { watt of heat removed at } 4.35 \mathrm{~K}}$.

Similarly, for every watt of heat removed at $L_{2}$ temperature $(78 \mathrm{~K})$ it "costs:"

$\frac{6000 \mathrm{~kW}}{500 \mathrm{~kW}}$ or $\frac{12 \mathrm{~W} \text { of electric power }}{\text { watt of heat removed at } 78 \mathrm{~K}}$.

3.14.4. Incremental Capital and Operation Costs

Given the TDF/MFTF-B cryoplant capital and operating costs, it is possible to derive the incremental cost data. These data can be very valuable for system comparisons, pre-design analysis, and rough cost estinates. The operating costs reflect the electric power and water utijity requirements. The liquio helium incremental operating costs are $\$ 37.50 / \mathrm{kW}-\mathrm{hr}$. The Lie capital costs include building, installing, preoperational testirig, and original equipment costs. The incremental capital costs for LHe are $\$ 1333 / \mathrm{W}$ at $4.35 \mathrm{~K}$. At $78 \mathrm{~K}$, the operating and capital costs for liquid nitrogen are $\$ 0.75 / \mathrm{kW}-\mathrm{hr}$ and $\$ 75.90 / \mathrm{H}$, respectively. 


\subsection{MAGNET POWER CONVERSICN AND PROTECTION}

\subsubsection{Sumgary}

All the magnet coils, except for the $M-1$ choke coils, are superconoucting. They are charged slowly and operate in a steady state for a long time. During a normal shutdown, the coils are discharged slowly (slow dump). However, if the coil protection system detects an imminent quench, the coils are all discharged rapidly (fast dump). The fast discharge transfers most of the energy stored in the coils to dump resistors, so that the miaximum temperature rise of a cojl is $<300 \mathrm{~K}$ for credible faults, accidents, or operator errors. The coil protection system obtains its power from the noninterruptabie supplies to ensure its continued operation should the utility power $f a i l$ and to prevent inadvertent fast shutdowns from occurring.

Each superconducting coil has a low-voltage, high-current power supply for charging the coils and supplying bussing and cable losses ouring steaoy-state operation. The choke coil magnets are normal resistance, watercuoled copper coils connected in series to ensure that the same current flows through each of them during normal and faulted operation. Medium-voltage power supplies are needed to supply their relatively large power losses. If the water flow to the M-l coils or the liquid level in their pressurized water storage vessel falls below a safe level, a fast aischarge of all coits is initiated, which turns off the power to the magnet power supplies.

Thyristors in the primary circuits of the rectifier transformers of the superconducting coils control the coil currents. Voitage-controlled tapped auto transformers control the choke coil currents.

\subsubsection{Requirements}

The data for the magnet field-coil power supply and protection system requirements are given in Table 3-100. The charging time should not exceed $A$ hours, and the power supplies must be able to provide the steady-state currents shown in the table with sufficient potentials to overcome the bus, cable, ana terminal voltage órops, and the $L$ (di/dt) needed for startup. The $\mathrm{M}-1$ choke coils are water-cooled copper coils, and their pawer supplies must also provide additional voltage to overcome the voltage drops across these coils during steady-state operation. 
Table 3-100. The TDF's coil power conversion and protection requirements.

\begin{tabular}{|c|c|}
\hline Parameter & Value \\
\hline Maximum coil charge time $(\mathrm{hr})$ & 4 \\
\hline Maximum coil discharge time constant & \\
\hline - Fast discharge (s) & 200 \\
\hline - Slow aischarge (hr) & 2 \\
\hline $\begin{array}{l}\text { Maximum discharge voltages } \\
\text { (Pool boiling SC coils): }\end{array}$ & \\
\hline - Cuil to grouna (kv) & 2 \\
\hline - Coil terminal-to-terminal (kV) & 2 \\
\hline $\begin{array}{l}\text { Steady-state coil currents and } \\
\text { stored energs ( } \mathrm{kV} / \mathrm{Md})\end{array}$ & Current/energy \\
\hline $\begin{array}{l}\operatorname{Coi1}(\mathrm{KA} / \mathrm{MJ}) \\
S-0 \\
S-1 \\
M-1 \\
M-2 \\
M-3 \\
T-1\end{array}$ & $\begin{array}{l}9.75 / 406 \\
7.84 / 896 \\
14.22 / 71.8 \\
6.55 / 17.0 \\
6.55 / 13.9 \\
5.71 / 13.3\end{array}$ \\
\hline Maximum temperature rise during a coil quench $(K)$ & $<300$ \\
\hline
\end{tabular}


- The central cell solenoidal cails, S-O and S-1, have a large amount of stored energy in their magnetic fields. Because these coils use pool boiling helium to maintain cryogenic temperatures, the voltage across the coils and from coil to ground are limited to about $2 \mathrm{kV}$. To prevent magnet damage due to a quench, it is necessary to transfer a large part of the stored energy to dump resistors in a short $t$ ime. This requires that the fast discharge time constant be no greater than some upper limit yet to be determined. Based on previous analysis, this limit is cstimated to be $200 \mathrm{~s}$.

\subsubsection{Design description}

Design data for the magnet power supplies are given in Table 3-110. Compared to the other coils, $M-2, M-3$, and $T-7$ have a low anount of stored energy. Because of their low inductance, very little additional voltage is needed to meet their startup requirements. Previous experience has shown that $12 v$ is adequate for covering the resistive circuit voltage drops and the small additional voltage needed for charging these superconducting coils. The central cell coils have a larger amount of stored energy and associated inductances. To overcome their higher inductance voltages during startup, their power supply terminal voltages must be several volts higher than for the other coils. The ac input voltages and amperages depend soniewhat on the power supply design. The data in Table 3-101 are typical for a good design, ano provide the basic requirements for designing the ac power and coil protection load center for the superconducting coils.

From Table 3-101, it is evident that the resistive choke coils require a tota] of $52 \mathrm{Mih}$ for steady-state operation. Most of the voltage is needed to cover the coil voltage orop. The circuit voitage orop and inductive startup voltages are small in comparison. The large power load for these coils require: that their ac power be taken airectiy from $13.8 \mathrm{kV}$ feeders. A generic one-line diagram, applicable to all the superconducting coil power supplies, is shown in Fig. 3-236. The coil current control is achieyed with silicon controlled rectifiers (SCRs) in the primary of the rectifier transformer. The voltages and currents in the coils are controlled by varying the firing angles to the SCRs (thyristors). Due to the large currents involved, the power supply losses are appreciable and require water cooling. The geita and wye 6-pulse bridges provide 12-pulse dc power to the coils. The free-wheeling aiodes provide a low impedance drop for a very slow shutdown, when the ac breakers are open. 
Table 3-707. Magnet power supply aesign data.

\begin{tabular}{lllllll}
\hline Coil ID & $S-0$ & $S-1$ & $M-1$ & $M-2$ & $M-3$ & $T-1$ \\
\hline $\begin{array}{l}\text { Type of coit } \\
\begin{array}{l}\text { Number of power } \\
\text { supplies }\end{array}\end{array}$ & $S C^{\mathrm{a}}$ & $S C^{\mathrm{a}}$ & $N W C^{\mathrm{b}}$ & $S C^{\mathrm{a}}$ & $S C^{\mathrm{a}}$ & $S C^{\mathrm{a}}$ \\
& 1 & 2 & 2 & 2 & 2 & 2
\end{tabular}

\section{Power supply ratings}

- $A C$ input

$\begin{array}{lrrrrrr}V & 480 & 480 & 13,800 & 480 & 480 & 480 \\ A & 216 & 216 & 1,250 & 120 & 120 & 110 \\ \text { KVA } & 180 & 180 & 30,000 & 100 & 100 & 90\end{array}$

- $\underline{D C}$ output

\begin{tabular}{|c|c|c|c|c|c|c|}
\hline$v$ & 15 & 18 & 1,800 & 12 & 12 & 12 \\
\hline A & 9,800 & 8,000 & 15,000 & 6,800 & 6,800 & 6000 \\
\hline $\mathrm{kW}$ & 147 & 144 & 26,000 & 82 & 2 & 72 \\
\hline
\end{tabular}


OANi-DWG 81-2849R FED

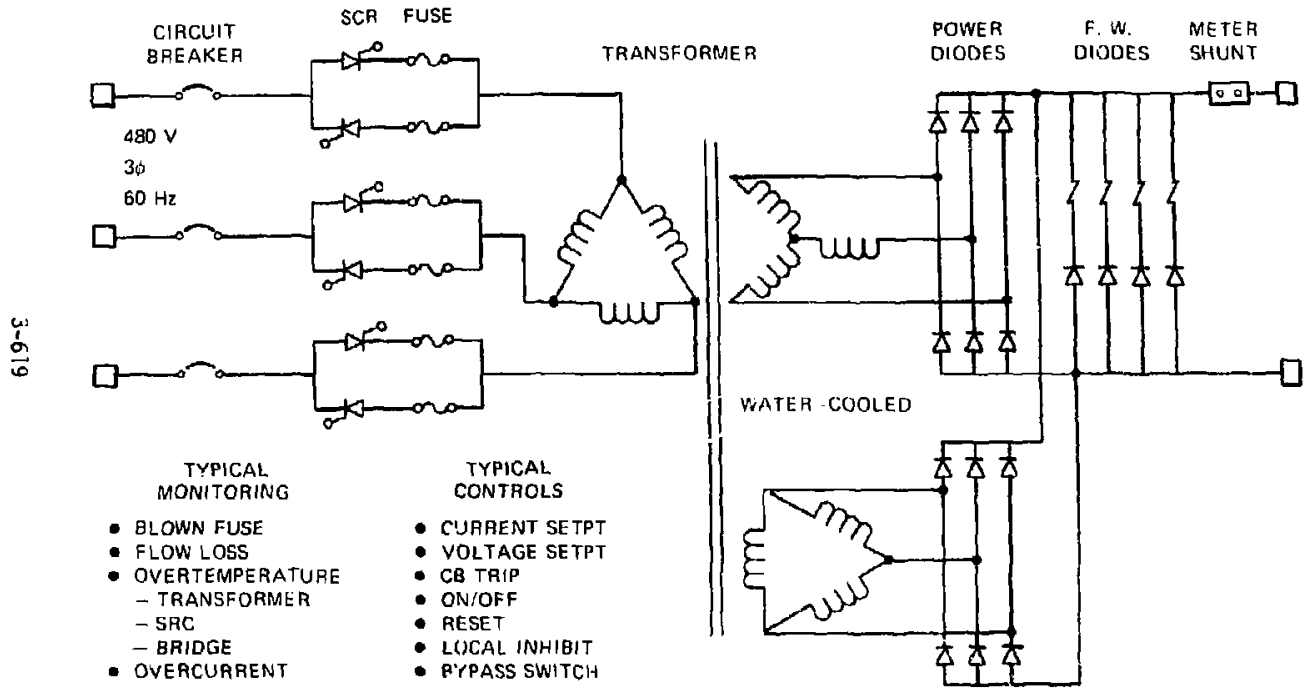

Figure. 3-236. A typical primary power supply for the superconducting coils: consisting of a silicon-controlled rectifier with low voltage and high curr, 
Figure 3-237 is a one-line diagram of the choke coil power supplies. Two $13.8 \mathrm{kV}$ feeders supply the motor ariven tapped auto transformers to provide? nominal $6.9-\mathrm{kV}$ power to the rectifier transformers. The auto transformer tap. provide voltage control for startups, and for establishing the desired operating current. The secondaries of the rectifier transformer are connected in a cielta and wye configuration, as shown in Fig. 3-236, for 12-pulse operation. The rectifier symbols in the squares of Fig. 3-237 represent six-pulse bridge rectifier circuits. The choke coil currents are about twice those of the superconducting coils, so a choke coil requires two 12-pulse units connected in parallel. The choke coils must be connected in series, so that the same amount of current will flow through each one under both norinal and faulted operation. The coils could be interleaved among the power supplies, which woula require four $15 \mathrm{kA}$ busses; or, tiley can be connected as shown in $F$ ig. $3-237$ to reduce the bussing cost.

The superconoucting coil protection equipment requires aump resistors, switches, and circuits such as those shown in Fig. 3-233. When an imininent quench condition is detected, a fast energy dump is initiated by opening the circuit breakers to interrupt the current flow from the power supplies. The inouctive voltage rises and orives the current through the dump resistors. Their maximum resistance (ano hence the coil discharge time) is limiteo by the permissible voltage drop between the coils and ground, and across the coits" terminals. A slow energy dump can be initiated by opening pneumatically controlled switches to dissipate the coil energy much more slowly through the 0.0015-ohm slow-dump resistors.

Each superconducting coil will have all the cojl protection equipment shown in Fig. 3-238, and listed, with their ratings, in Table 3-102. The discharge voltages for the anchor cell and the central cell coils will be limited to 500 ano $1000 \mathrm{~V}$, respectively, which is sufficient to transfer most of the storea energy to the dump resistors.

Figure 3-239 shows a conceptual plan view for the electrical section of the builoing, ano Fig. 3-240 is a conceptual elevation view for the magnet electrical support equipment in this section. The large transformers and medium-voltage circuit breakers are lacateo outsige, but close to, the builoing. The power supplies (PS), switches (SW), and circuit breakers (BR) are located in cabinets, conveniently grouped inside the electrical building, 


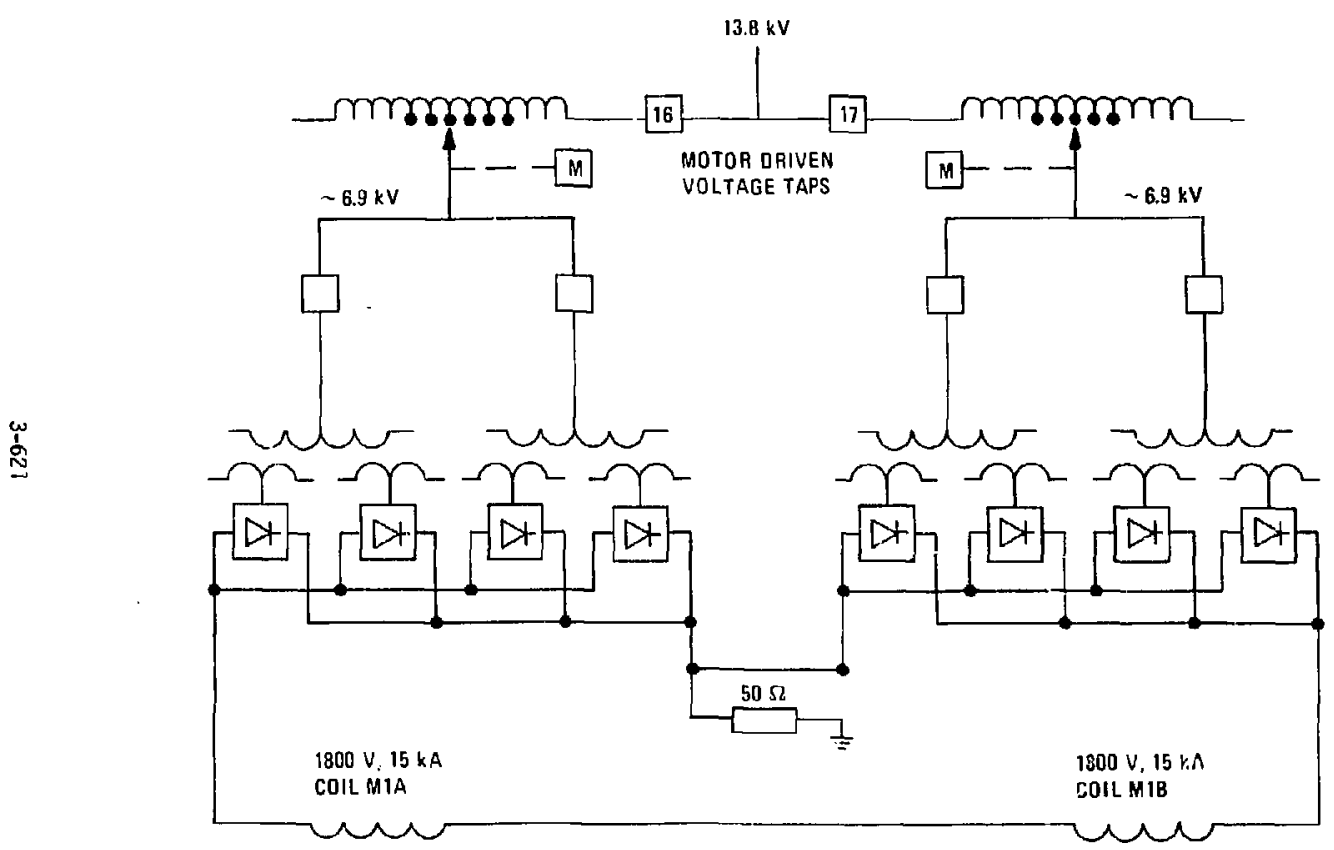

Figure 3-237. The magnet power conversion systtin for the series-connected choke coils, $M-1$. 


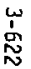

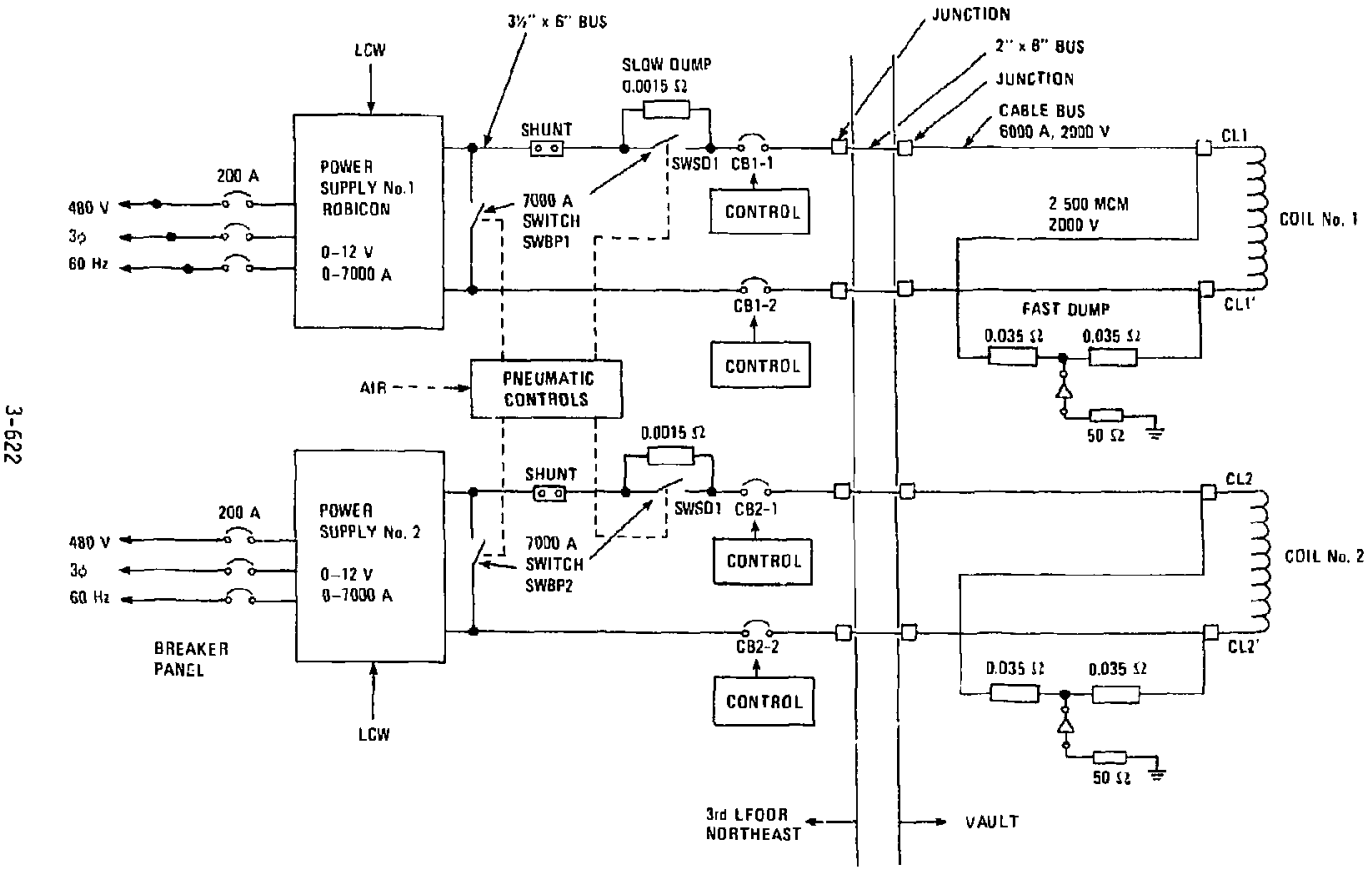

Figure 3-238. The magnet power supply and coil protection system for the $M-2$ and $M-3$ pair of superconducting coils. 
Tabie 3-102. Design data for the magnet protection system.

\begin{tabular}{|c|c|c|c|c|c|c|}
\hline \multirow[b]{2}{*}{ Párameter } & \multicolumn{5}{|c|}{ Coi] } & \multirow[b]{2}{*}{$T-1$} \\
\hline & $S-0$ & $S-1$ & $M-1$ & $M=2$ & $M-3$ & \\
\hline Number of coils & 1 & 2 & 2 & 2 & 2 & 2 \\
\hline \multicolumn{7}{|l|}{ Operating stored } \\
\hline energy/coil (MJ) & 406 & $89 b$ & 11.8 & 17.0 & 13.9 & 13.3 \\
\hline \multicolumn{7}{|l|}{ uperating } \\
\hline current $(\mathrm{k} A)$ & 9.75 & 7.85 & 14.22 & 6.55 & 0.55 & 5.71 \\
\hline \multicolumn{7}{|l|}{ Equivalent } \\
\hline inductance $(H)$ & 8.5 & 29.1 & 0.12 & 0.79 & 0.65 & 0.82 \\
\hline \multicolumn{7}{|l|}{ Quench discharge } \\
\hline voltage (kv) & 1.0 & 1.2 & $N / A$ & 0.5 & 0.5 & 0.5 \\
\hline \multicolumn{7}{|l|}{ Quench decay tine } \\
\hline constant $(s)$ & 85 & 194 & 0.91 & 11.3 & 9.3 & 11.7 \\
\hline \multicolumn{7}{|l|}{$D C$ circuit breakers } \\
\hline Nuniber/coil & 2 & 2 & $N / A$ & 2 & 2 & 2 \\
\hline Volts (kV) & 1.0 & 1.2 & & 0.5 & 0.5 & 0.5 \\
\hline Current $(k A)$ & 10.0 & 8.0 & & 7.0 & 7.0 & 6.0 \\
\hline \multicolumn{7}{|l|}{ Fast-Dump Resistors } \\
\hline Number/coil & 8 & 16 & & 2 & 2 & 2 \\
\hline Resistance (onms) & 0.20 & 0.60 & & 0.035 & 0.035 & 0.035 \\
\hline Peak power (kW) & 1200 & 600 & & 1500 & 1500 & 1150 \\
\hline Energy (Md) & 50 & 56 & & 17.0 & 13.9 & 13.3 \\
\hline \multicolumn{7}{|l|}{ Slow-Dump Resistors } \\
\hline Number/coil & 1 & 1 & & 1 & $\mathbf{i}$ & 1 \\
\hline Res istance (ohms) & 0.0015 & 0.0045 & & 0.00015 & 0.00015 & 0.00015 \\
\hline Peak power (kW) & 150 & 300 & & 6.5 & 6.5 & 5 \\
\hline \multicolumn{7}{|l|}{ Ground Resistors } \\
\hline Number/coil & 4 & 8 & & 1 & 1 & 1 \\
\hline Resistance (ohms) & 50 & 50 & & 50 & 50 & 50 \\
\hline Power (kW) & 1.0 & 1.0 & & 1.0 & 1.0 & 1.0 \\
\hline
\end{tabular}



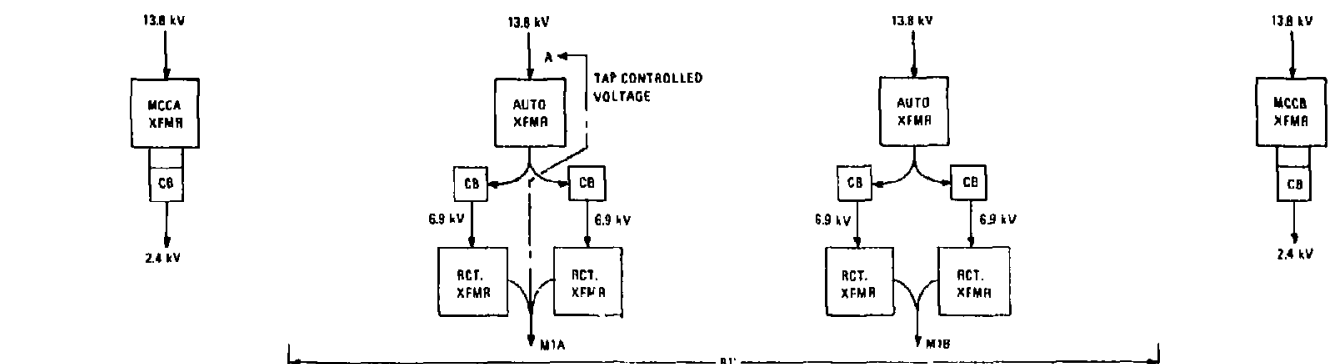

$c$
1
0
0

Figure 3-239. A conceptual plan view of the power conversion ar magnet protection equipment. 


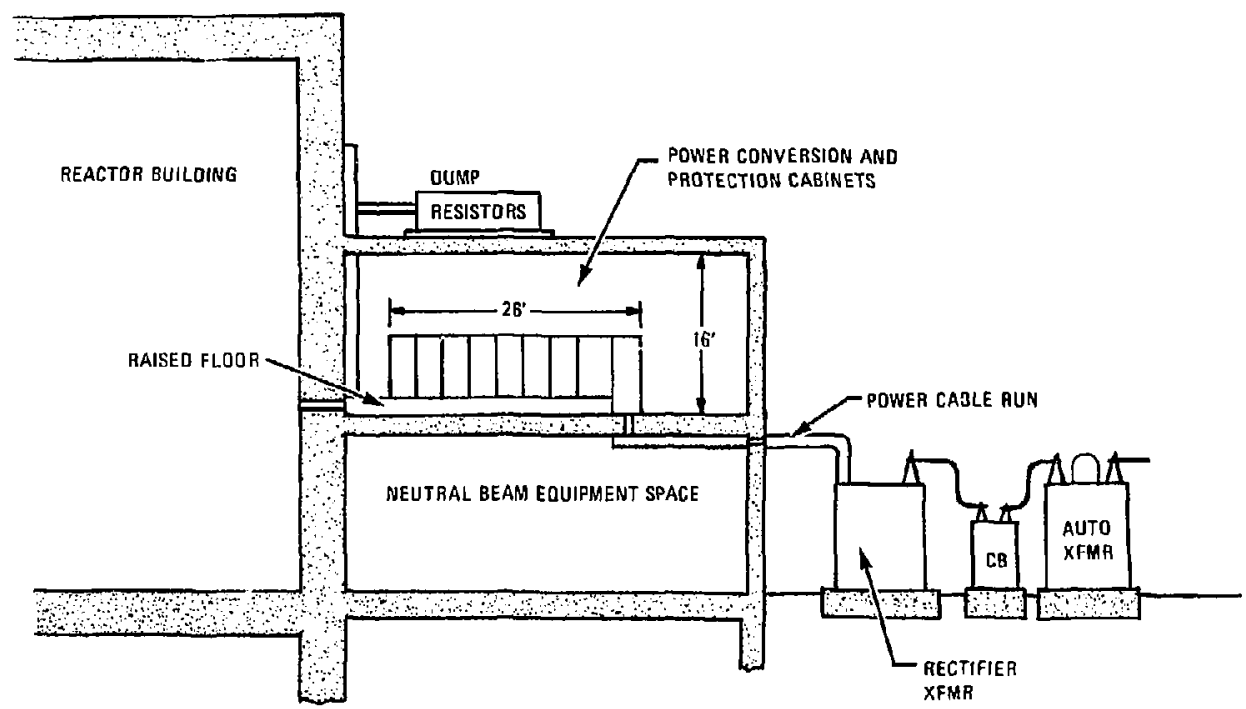

Figure 3-240. Section A-A elevation view of the power conversion and coil protection equipment, from Figure 3-239. 
in close proximity to their associated coils in the reactor cell. Each group also contains one ac power transition cabinet (AC), which connects to the load centers, the instrumentation and controi cabinets (IC), and the water cooling system (WiC) cabinets. Cabinets are arranged in a "U"-configuration so that the back sice is accessible for installation and maintenance. Cabinets can be disconnected from their electrical cables and busses, ano their water cooling lines (located under the center raised floor), and jacked by fork-iift trucks into the 9-ft aisle for removal and replacement. Front panels along the main corridor of the building are the local control centers.

The many large dump resistors are located on top of the electrical building. Vertical busses connect them to horizontal busses that pass under the raised floor and penetrate the reactor builoing through cylindrical steel pipes. An irsulating epaxy-type filler separates the busses from each other and the large pipe, which is secured by the concrete wall. The pipe also ser'ies as a magnetic shield. Electrical cable conduit is also expoxied into the large pipes. The conauit cables are also secured with epoxy, so that any tritium that shoulo leak into the reactor building will not reaoily escape through the electrical wall penetrations.

The two load control centers, LCCA ano LCCB, provide ac power ano circuit protection for the superconducting-coil power supplies. Figure 3-24l shows the one-line ciagrams of these load centers, which also supply 2.4-kV power to the coolant pump and cryogen compressor motors. Additional $480-V$ power from the load center supplies smaller motors and the building's lighting neeos.

\subsubsection{Key issues.}

Magnet power supplies and their load centers can be designed with proven, highly reliable commercial components. The coil protection system developed for the MFTF-B is also applicable to the TDF. The usual electrical safety practices need to be incorporated into the design. No technical problems exist. 


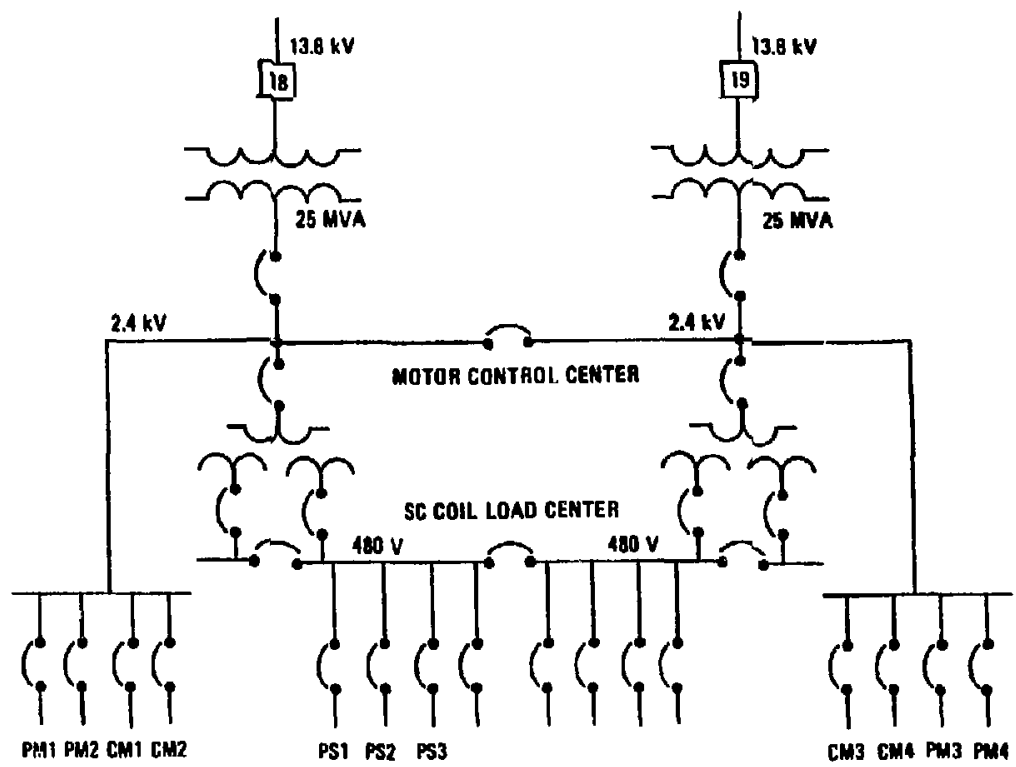

Figure 3-241. The motor and load control centers for the superconducting coil power suppities. 


\subsection{NAINTENANCE}

\subsubsection{Overview}

Maintenance and disassembly of the niajor systems were prime arivers in developing the TDF configuration. Wherever it was appropriate, these considerations influenced component aesigns with regard to access, handing, and loration. Early in the project, we established a maintenance philosophy by which to develop the baseline configuration and its components. The major aspects are:

- Contacting maintenance operdtions at the shield boundary 24 hours after device shutdoinn.

- Being able to accomplish all maintenance and disassembly operations remotely under emergency conditions.

- Having inodularized component installations.

- Using prover remote-maintenance equipment technology.

Establishing a philosophy for maintaining a D-T fusion device requires taking several considerations into account. These include:

- Prior to any disassembly, personnel should be able to routinely do hanos-on inspections, perform maintenance equipment setups, and supervise maintenance activities in the reactor buiding.

- Since activation levels within the shield boundary are too high for hands-on maintenance, remote operations are required.

- Because of these requirements, the bulk shieid is designed to 7 imit activation at its outer surface to a level that wili permit contact maintenance in the reactor building (outside the device shield boundary) after a suitabie cooldown period.

- In adaition, the shield is designed to specifically protect certain components (e.g., the superconducting coils) from material damage and excessive net.ron heating.

In light of these requirements, TDF is designed to have a maintenance approach which maximizes the flexibility of mainterance operations. Personnel access into the reactor builoing is permitted after a reasonable shutoown period, within the limits discussed in Section 3.16.2. 


\subsubsection{Requirements and Performance}

The maintenance appraach is three-fold, considering the requirements for contact and remote maintenance, component disassembly, and the technology available for remote handing equipment.

Contact and Kemote Maintenance. Generally, beginning one oay after shutoown. all areas outside the oevice shield are maintained by contact operations. This assumes that the plasma chamber is :ncpened, and that ali vessel penetrations are properiy shielded. In adoition, these contact tasks miust also be remotely accumplishable in emergency situations where personnel wou?a be prohibited from entering the reactor building.

Component Lisassembly. Wherever practical, subsystems and components are designed for replacement as individual modules having minimal impact on acjacent systems. This concept is particularly applicable to components kriown to neeo more than one replacement during the device's lifetime. During the baseline's gevelopment, ease of access and simplicity of handling were configuration requirements.

Remote Maintenance Equipment Technology. Because TDF is a near-term device, component maintenance and replacements planning specifically assumes the use of existing remote maintenance equipment and technology in the manipulator, viewing, and transport systems. Technological developments are considered to be near-tern, if they are expected to occur within several years.

In summary, the requirements and limitations for both contact and remote mäinteriance are:

- Permissible dose rate. The surface dose rate at the shield bouncary will be $2.5 \mathrm{mr} / \mathrm{h}$. At this level, ouring a 40-hour work week next to the device, the exposure to personnel will not exceed the federal limitation of $5 \mathrm{rems} / \mathrm{yr}$. (It might be possible to increase the aose rate limit by reaucing the shield thickness, if it were assumed that there are no limits on the numbers of ayailable maintenance personnel or that contact operations will not require extenced 
perioos of downtime. Neither of these assumptions was used.) Furthermore, a dose rate limit of $2.5 \mathrm{mr} / \mathrm{h}$ is consistent with othe recent reactor studies.

Shutcown time. The shield's thickness was designed to allow personnel to have access to the shield boundary 24 hours after shutaown. Because the short-livea radioisotopes in the shield material will decay to permissible leveis, this waiting period will reduce the surface dose rate to $2.5 \mathrm{mr} / \mathrm{h}$. This time can also be used by personnel for such general maintenance preparations as de-energizing the coils, draining coolant, and preparing equiphent. Contact maintenance boundary. Contact maintenance will be limited to operations outside the device shield boundary, provioed that the shielo is intact, and that the major penetrations are shielded. The contact maintenance sounaary is shown in Fig. 3-242. (Generally speaking, contact maintenance operations are defined as operations done without a protective suit. Therefore, disassembiy procedures in the reactor building include provisions for the decontamination of tritium and activated particulate matter. A design goal for TOF is to have contact operations done without suits. However, if contamination leveis exceed the maximum permissible concentrations, the use of suits with portable life-support systems is an acceptable mode for hands-on operations.) The shield materials and thicknesses that meet tine biological requirement for a $2.5 \mathrm{mr} / \mathrm{h}$ exposure rate are also shown in Fig. 3-242. The central cell consists of $115 \mathrm{~cm}$ - of stainless steel, with $5 \mathrm{~cm}$ of lead cladding on the outer surface. The superconducting solenoid coils require $90 \mathrm{~cm}$ of stainless steel between their bores and the plasma. The anchor cell is shielded with $60 \mathrm{~cm}$ of borated (heavy) concrete, and the end cell shiela is $60 \mathrm{~cm}$ of ordinary concrete.

- Hiscellaneous. Several other operational constraints must also be consicered in a maintenance context. Personnel and maintenance equipment will not be permitted in the reactor building aliring the device's operation. The biological hazard for personnel is obvious. For equipment, this policy will avoid neutron-induced activation, which would result in having to remotely maintain the 


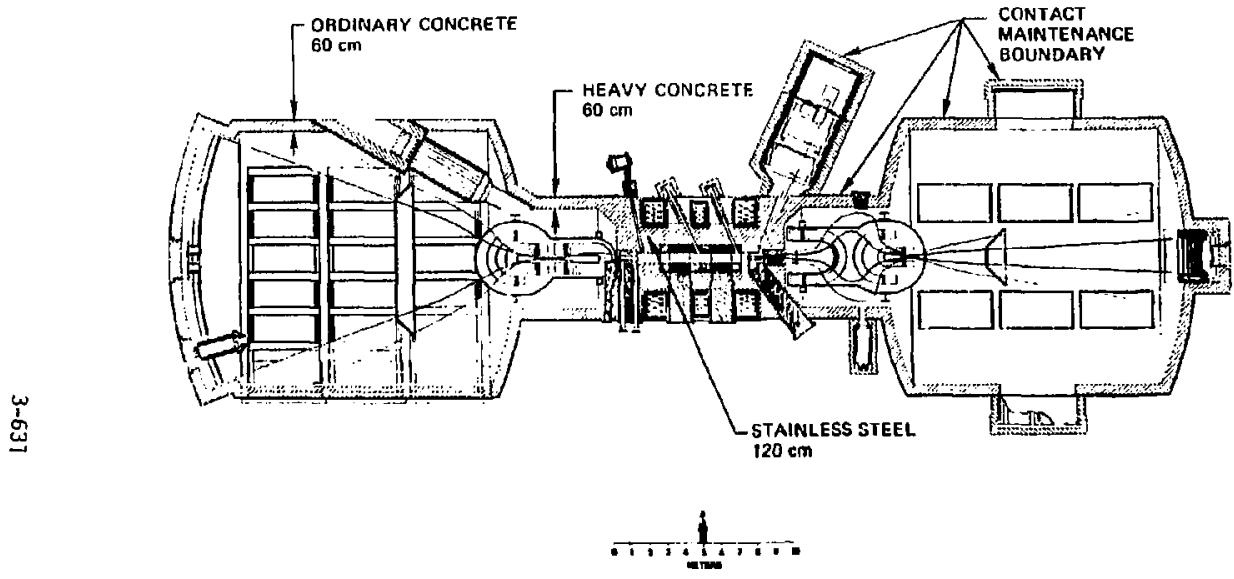

Figure 3-242. The contact maintenance boundary and shield thicknesses. 
maintenance equipment. This is why the overhead crane systems, which must remain in the reactor bulding, are stored behind a shielded enclosure at one end of the facility.

The magnetic coils must be de-energized during both remote and contact maintenance operations. This is primarity a safety consideration for personnel, maintenance equipment, and the magnets. The concern relates to two factors:

- The energy stored in the larger coils is significant, being measured in the megajoule ( $M J)$ range.

- The attractive force of the magnets is significant, and can affect

the operation of electric motors in the manipulator systems, or turn small magnetizable tools into projectiles. (A plot of the magnetic

flux arounci the device is in Section 3.2 .

However, the coils can be kept at their operating temperature if the maintenance operations oo not involve disturbing the magnet systemis. A test mocule replacement is an example where the coils would be de-energized, but could still be kept at liquid helium temperature.

\subsubsection{Maintenance Equipment}

The remote maintenance equipment basically consists of manipulators, viewing systems, and transporters. Because of the near-term time frame for TLF, only present? $y$ available equipment is being considereo. Nanipulator systems are used to accomplish the functions of a human. In general, these systems consist of closed-circuit TVs (CCTVS), available in color or black ano white with two- or three- dimensional imagery, coupleo to a manipulator system. The transport systems include the overhead cranes and other transport. devices in the reactor building used for removing and installing components on the oevice, and for moving components to the hot cell. A preliminary listing of special-purpose, remote handing equipment is presented in Table 3-103.

\subsubsection{Scheauled and Unscheouled uperations}

Most of the major components will require scheauled maintenance or repiacement during the device's lifetime, and all of them may require 
Table 3-103. Preliminary list of speciat-purpose remote hanaling equipment.

Particle oump hanoling aevice

Helium dump handling device

Cryopane] assembly hanaling device

Beaml ine handling device

(Anchor pumping, transition pumping, and sloshing)

beamline handling service (central cell)

Overhead handling fixtures for:

Cryopanels

Sloshing beamlines

Choke coil

Transition ano anchor coil assembly

Test module

Pellet injector

Test maaule hardling device

Beam dump hanaling device 
unscheduled replacement. Provisions for these unscheduled replacements has been considered in developing the baseline configuration, although the emphas is has been placed on scheduled maintenance operations. A number of components have been identified as requiring periodic replacement. The design of these components, as well as their position on the device, was very much influenced by maintenance considerations, such as accessibility and modularity. Table 3-104 is a list of these components, along with pertinent comments, that compiles the best estimates of their expected lifetimes. Readers are cautioned against using these estimates as absolute values, since they represent only a first cut. The value of this table lies in the making of relative comparisons for identifying maintenance drivers, and for assessing maintenance equipment needs.

Using the lifetime data from Table 3-104, a chart of scheduled component replacements can be developed. Figure 3-243 is just such a chart. It shows the first replacement times for various components plotted against calendar years and equivalent ful1-power operating years (FPY). For reference, the availability distribution is also shown. The first components requiring replacenient will be the ion sources, with an average expected lifetime of approximately $0.2 \mathrm{FPY}$, or 1.5 calendar years. The superconducting coils are iifetime components, and are therefore shown as having no scheduled replacements, even after 15 calendar years. The choke coil has an expected lifetime of 0.25 FPY, and it is assumed that its failure will be dependent on neutron-inauced degradation. Therefore, noting that the first year of device operation is with hydrogen only, the accumulated operating time to failure will begin after the first calendar year. This is assumed to be true for most of the components. Note that the expected lifetimes are given in FPY, which is directly related to actual operating time for the device. For example, based on the assumed availability distribution for TDF, the 15-year calendar life of the device is equivalent to $5.4 \mathrm{FPY}$.

Two important points can be made by using Fig. 3-243. The first is that maintenance procedures and equipment must be in place ea: ly in the operating phase of the program. Slightly more than $1-1 / 2$ years after startup, the first use of remote-handling equipment occurs, implying that maintenance considerations must be factored into the designs at an early stage. The second is that it behooves the component designer to seek extended lifetimes, 
Table 3-104. Expecteo lifetimes of components in the central cell, anchor cells, ario end cells.

\begin{tabular}{|c|c|c|}
\hline Component & $\begin{array}{l}\text { Expected Lifetime } \\
\text { (full power years) }\end{array}$ & Comments \\
\hline \multicolumn{3}{|l|}{ Central Cell } \\
\hline$S-0 \operatorname{coti}$ & 5.4 & Full lifetine \\
\hline$S-1 \operatorname{coj} 1$ & 5.4 & Full lifetime \\
\hline Test modules & $0.1-1$ & Heek ly, monthly, yearly \\
\hline CC beamlines & 0.2 & \\
\hline Sources & $<0.1^{b}$ & \\
\hline Uumps & $0.2^{\mathrm{a}}$ & System driver \\
\hline Cryopanels & 0.5 & \\
\hline magnets & 5.4 & Full lifetime \\
\hline CL beam dumps & 0.2 & \\
\hline Choke coil & 1 & $\begin{array}{l}\text { Design objective; } \\
0.25 \text { may be actual }\end{array}$ \\
\hline Pellet injector & 0.3 & \\
\hline \multicolumn{3}{|l|}{ Anchor [e]1 } \\
\hline Transition coil & 5.4 & Full lifetime \\
\hline Anchor coils & 5.4 & Full lifetime \\
\hline$\$$ beamline & $0.5^{b}$ & \\
\hline $\begin{array}{l}\text { Sources } \\
\text { Dumps }\end{array}$ & $\begin{array}{l}0.2^{\circ} \\
0.5^{\mathrm{a}}\end{array}$ & System driver \\
\hline Cryopanels & 1.0 & \\
\hline Magnets & 2.0 & \\
\hline TCP beam dump & $<0.2$ & \\
\hline$\$$ beam oump & $<0.2$ & \\
\hline ACP beam dump & $<0.2$ & \\
\hline
\end{tabular}


Tadle 3-104. Cont'a.

\begin{tabular}{|c|c|c|}
\hline End Cell & & \\
\hline Particle dunp & 0.5 & \\
\hline Cryopanel assemblies & 1.1 & \\
\hline Helium pumps & 1.7 & \\
\hline TCP beamline & 0.2 & \\
\hline Sources & $<0.1^{b}$ & \\
\hline Dunips & $0.2^{\mathrm{a}}$ & System ariver \\
\hline Cryopanels & 1.0 & \\
\hline Magnets & 2.0 & \\
\hline ACP beamline & 0.5 & \\
\hline Sources & $0.2^{b}$ & \\
\hline Dumps & $0.5^{\mathrm{a}}$ & System driver \\
\hline Cryopanels & 1.0 & \\
\hline Magnets & 2.0 & \\
\hline
\end{tabular}

The system driver is that component with the shortest life, which requires the entire system (mooule) to be removed for maintenance.

$c$ Beamline sources are not system orivers, even though their expected lifetimes are the shortest in the beamlines, because they are designed to be replaced as independent submodules within the beamline system. 
ORNL-DWG 82-3331 FED

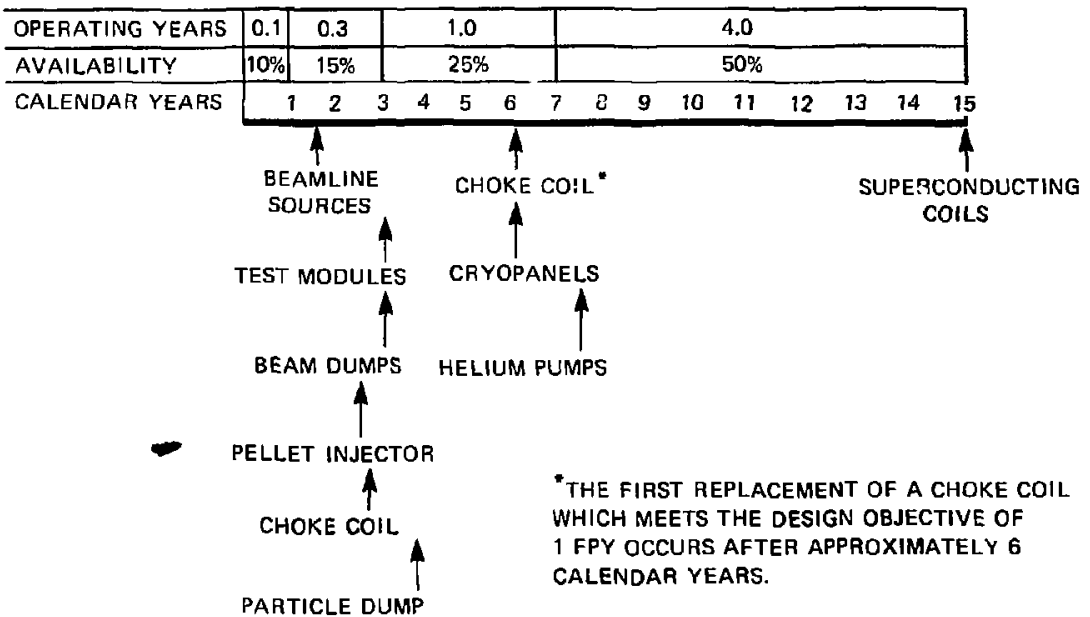

Figure 3-243. An estimate of first-time replacements for selected components. 
where they can be realistically achieved. For instance, Fig. 3-243 shows that the first choke coil changeout will occur after six calenciar years instead of 2.5, if the choke coil's operating lifetime can be extended from 0.25 to $1 \mathrm{FPY}$.

The chart in Fig. 3-244 examines the scheduled replacement history of one component over the device lifetime. Only the choke coil is shown, but a similar chart can be developed for any component. For a choke coil with an operating lifetime of $0.25 \mathrm{FPY}$, there are 21 scheduled replacements. During the last phase of the device's operation, with a 50\% availability, the changeouts will occur approximately twice each year.

\subsubsection{Component Replacements}

Here are abbreviated descriptions of some of the component replacement scenarios that were consiaured during the configuration development. In each case, it is assumed that hanos-on maintenance operations will be done whenever possible. Therefore, maintenance personnel are involvea as long as the device is unopened. They are responsible for setting up equipment such as scaffolding, manipulators, and fixtures, and they provide the on-site inspection prior to starting replacement operations.

Test Module. Figure 3-245 shows the removal of a test module, which is located at the upper centerline of the central cell. Note that this module includes the test specimen as an integral part of the shield plug. After removing the service connections and unfastening the vacuum flange interface between the module and the vacuum boundary of the central cell, a lifting fixture is assembled ontc the module flange for overhead lifting. All of the operations done before the test module is lifted out are manualiy assisteo. The module's weight is estimated as being about 50 to 70 tons, with overal dimensions of $1.2 \times 1.6 \times 4.3 \mathrm{~m}$. Reinstalling the replacement module requires that an alignment fixture be indexed to and mounted onto the central cell. The re-installation process is totally remote, until the module is again seatea on the vacuum flange.

Choke Coil. Figure 3-246 shows a choke coil being removed. This operation is essentially the same as that for the test moaule, because of the coil's upper 
OPERATING LIFE OF A CHOKE COIL $=0.25 \mathrm{FPY}$

ORNL-DWG B2-3330

FEO

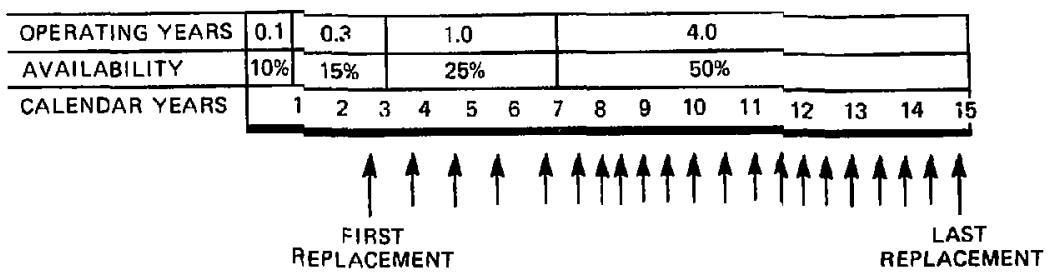

Figure 3-244. The choke coil replacements scheduled for the device's lifetime. 


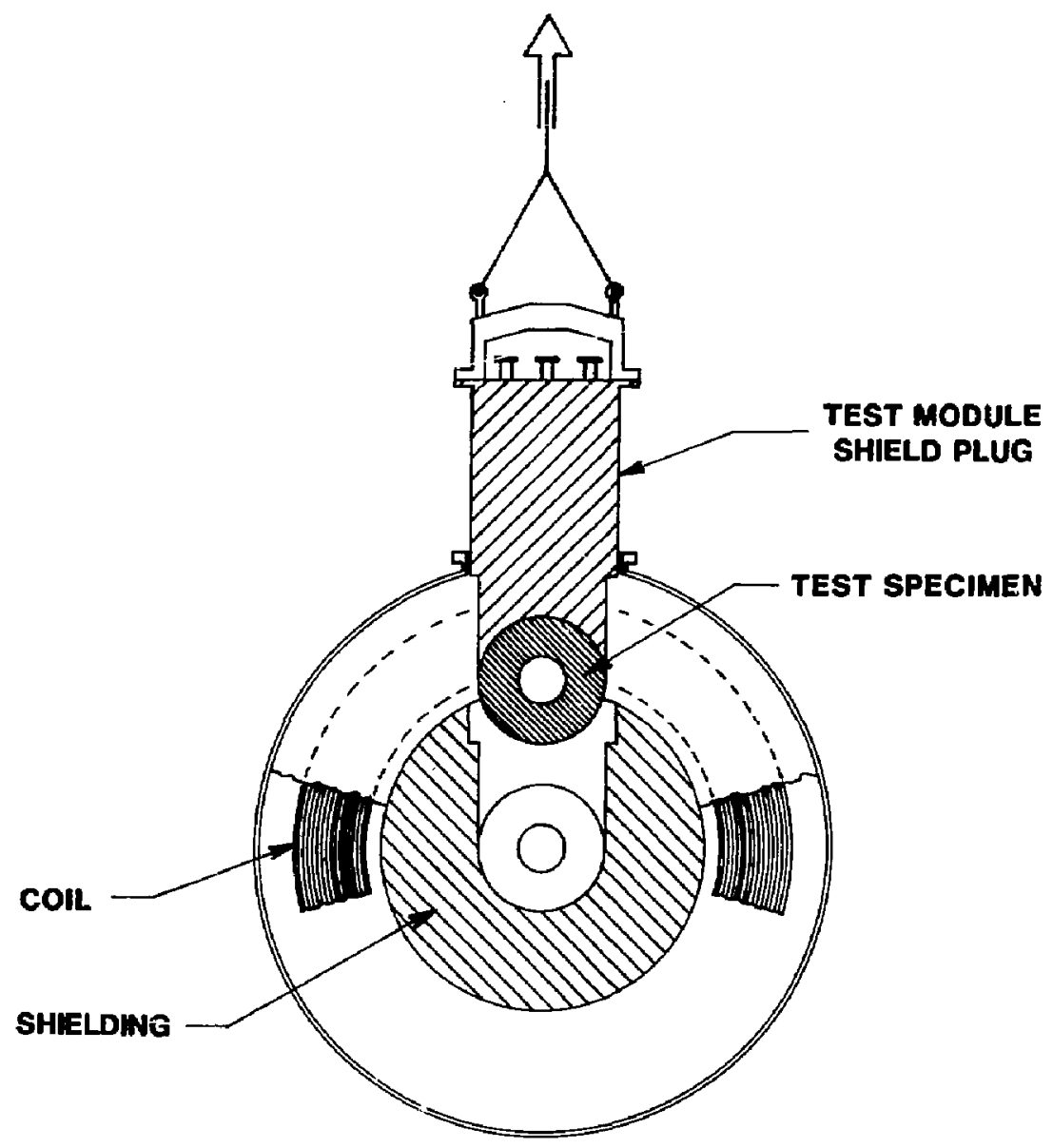

Figure 3-245. Test module removal. 


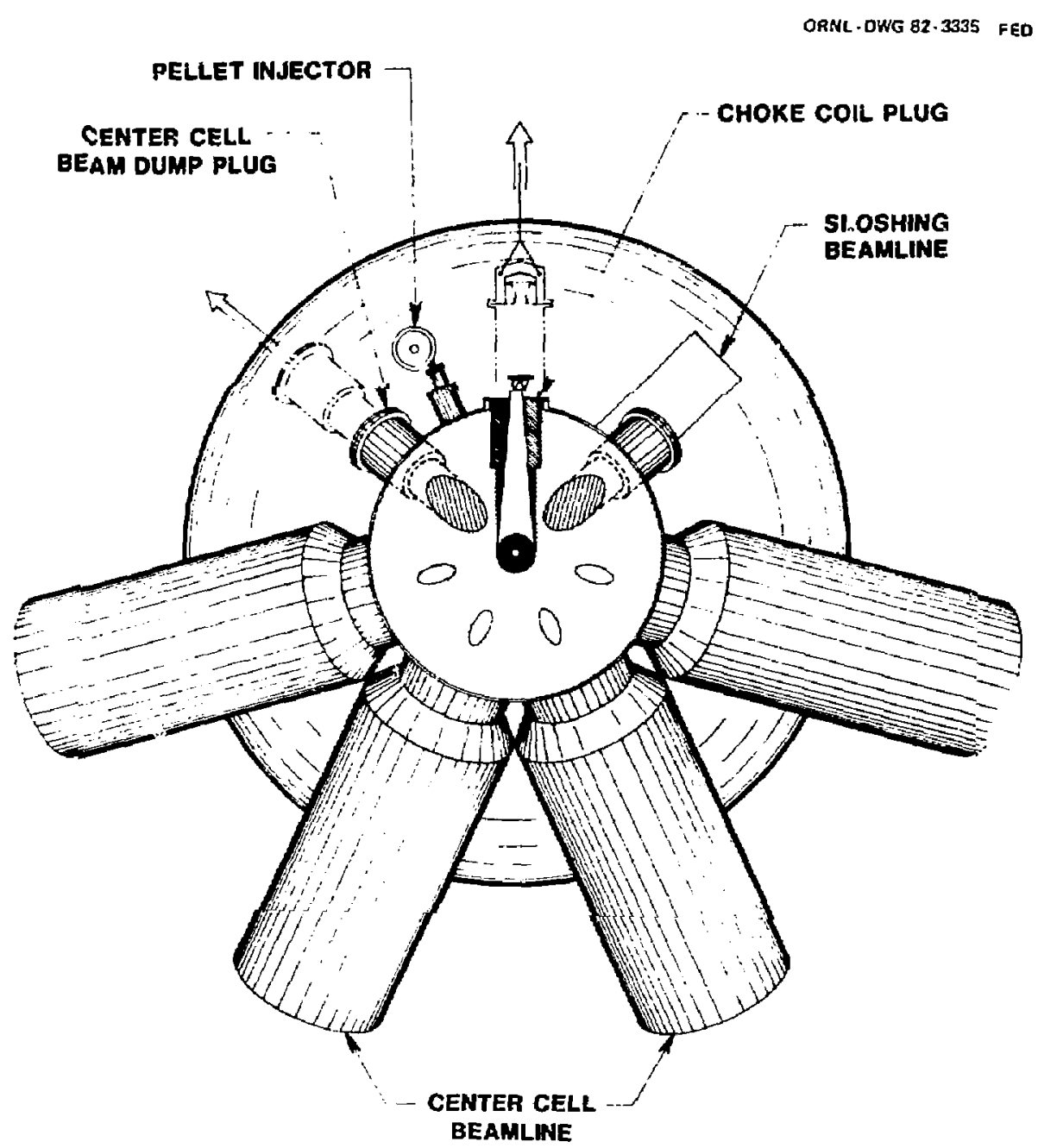

Figure 3-246. Choke coil and heam oump removal. 
centerline location. The choke coil is expected to be one cf the most frequently replaced components, so it was located for an easy overhead access. Before this mudule (the shield plug and magnet) is lifted out, all operations are contact. The choke coil modyle veighs approximately 20 tons, ano it also requires an alignment fixture for reinstallation.

Central Ce1] Beam Dump. Figure 3-246 also shows the orientation and removal of a beam dump. To remove these components, handling equipment must be used that can accomplish a motion away from the vertical, which precludes using the overhead crane. This type of maintenance equipment is shown in subsequent figures.

Anchor-Sloshing Beamline. Figure 3-247 illustrates removing the beimline components as a rail-mounted pallet assembiy, enabling the entire system to be hanaled as one large module. This approach is taken because of the limited access available for the beamlines. The ion source replacement is the one component which can be indepenoently changed after removing its service connections and the shield cover. A rail-mounted extraction device is requirea to pull the pallet after the vacuum flange interface is disconnected. Figure 3-247 is also typical for the anchor and transition pumping beamlines. The weight of the largest pal?et for these beamlines is estimated to be less than 50 tons.

Central Cell Beamline. The disassembly procedure for the central cell beamlines is more complex than that for the other beamlines. This results from minimizing the clearance between to the reactor builaing's walls.

Figure 3-248 shows the arrangement for their disassembly. The first component to be remcied is the module consisting of the nuclear shield and the rear superconducting magnetic shield. This first module interfaces with the beamline at the rear yacuum bulkhead, where the sources are mounted. (This module is also removed to gain access to the ion sources, when they need replacement.) The second mooule that must be removed is the rear half of the nuclear shield, which provides ample access to the pallet-mounteo beamline components. The nuclear and magnetic shields that remain are essentialiy fixed in place. 


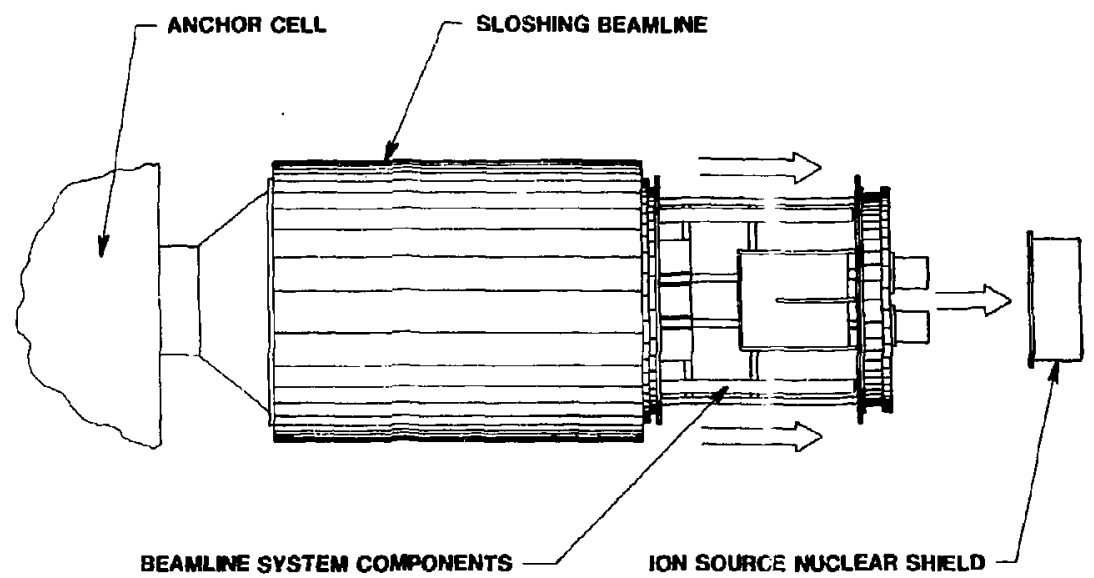

Figure 3-247. Beamline removal: typical for anchor sloshing, transition pumping, or anchor pumping. 
ORNL.DWG 82.3337 FED

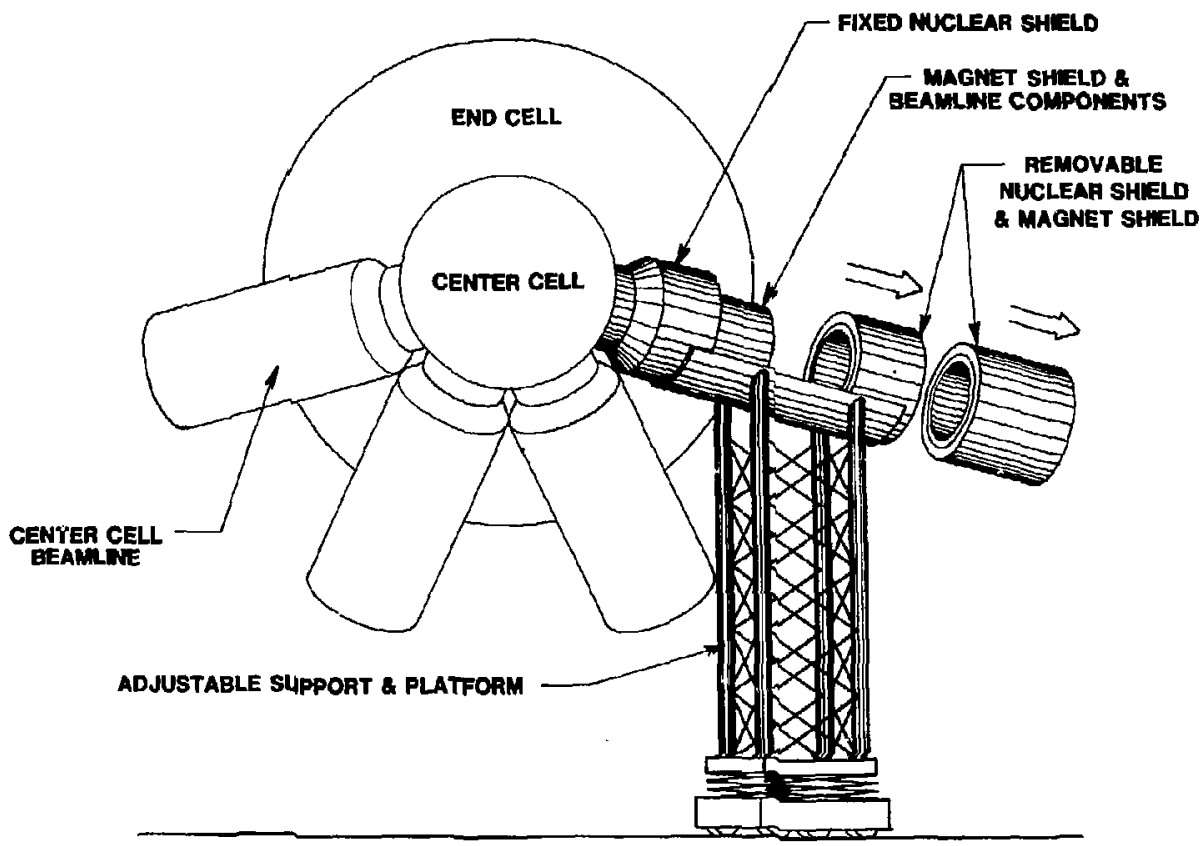

Figure 3-248. Central cell beamline removal. 
The adjustable support platform is used to assist an extraction device (not shown), which handles the pallet assembly prior to its being lifted by the overhead crane. The extraction device for the central cell beaml ine would be mounted to the building wall in a manner simitar to that shown for the cryopanel removal in Fig. 3-249.

Cryopanel. Each end cell consists of thirty-six $2 \times 4 \mathrm{~m}$ cryopanel pumps, which are arranged in modular assemblies of three panels each, as shown in Fig. 3-249. This module is a manageable size that will not change the builoing wall oimensions, which were establisised by the central cell beamine removal.

This figure shows one of the six lower panel assemblies being removeo. First, the cryopanel handing device removes the shielded access cover to gain entrance into the end cell. It then pulls the track-mounted assembiy out onto an inclined plane. It is possible to adjust this handling device vertically and horizontality by using the jack-pads and wheets of the trolley bed, respectively. Finally, the overhead crane is used to oeliver the component to the hot cell entrance.

Particle Dump. The particle dump consists of a number of panel modules, which are connected to common inlet and outlet coolant manifolds. Figure 3-250 shows one of these panels being removed with a mobile handling aevice. Access to the panels is gained by removing a lower shielded cover.

Helium Pump. Figure 3-25l show's a helium pump module being removed with a hanaling device similar to that used for removing the particle dump.

Magnet Vacuum Vessel. The anchor and transition coils are inside a common vacuun boundary, which is supported within the anchor cell. The vacuum vessel (with the anchor and transition coils) weighs approximately 80 tons and is the largest modular component of TDF.

Initially, there were severai ways to install this module into the anchor cell ano severai ways of renoving it, should its unscheduled replacement be required. However, to keep within the constraints established for a minimum 


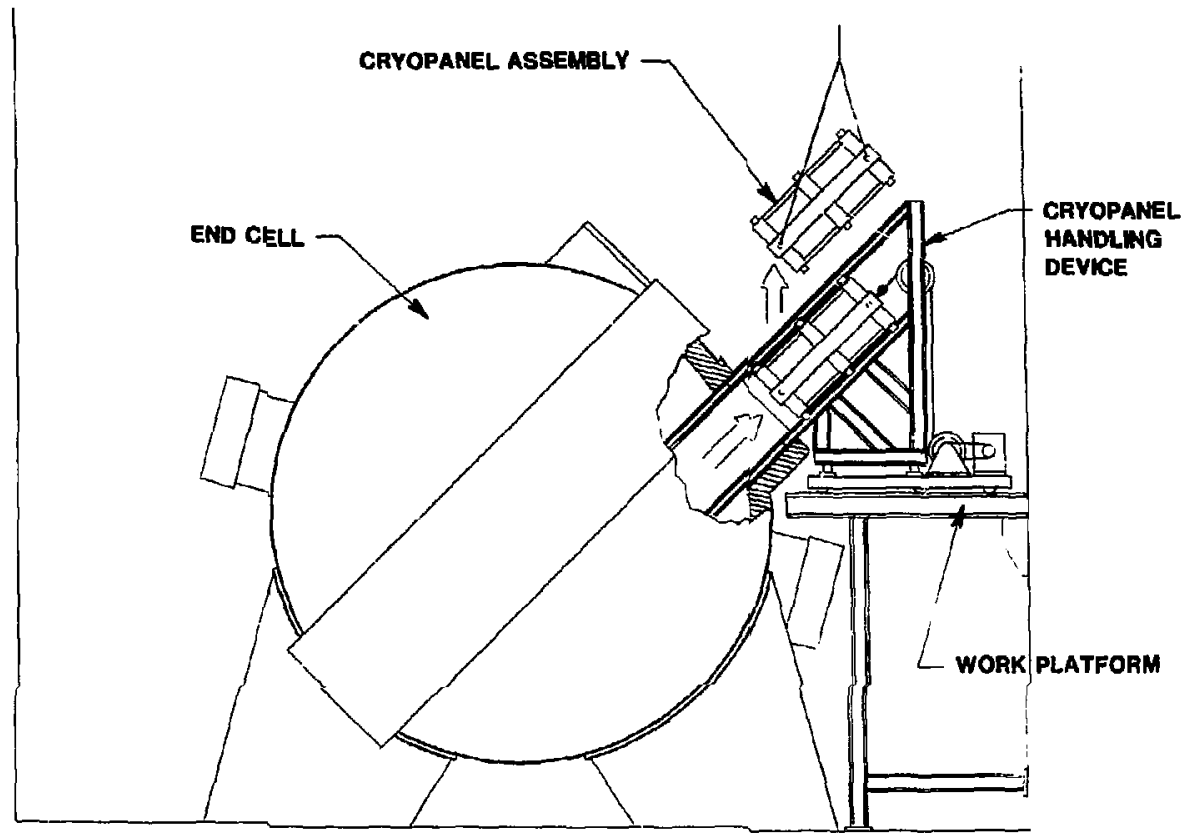

Figure 3-249. Removal of cryopanel modular assemblies. 
ORNL.DWG 82 - 3340 FED

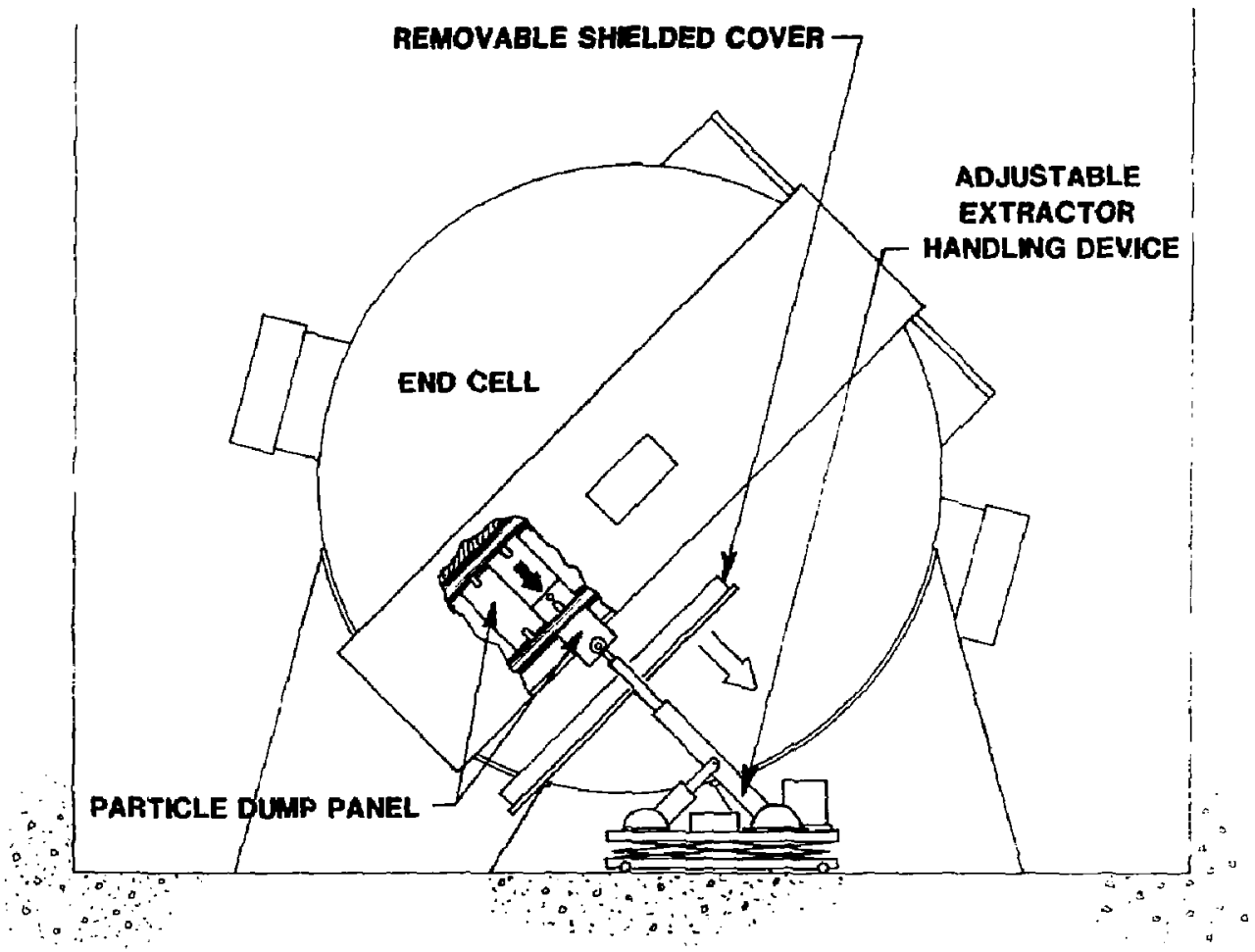

Figure 3-250. Particle dump panel removal. 


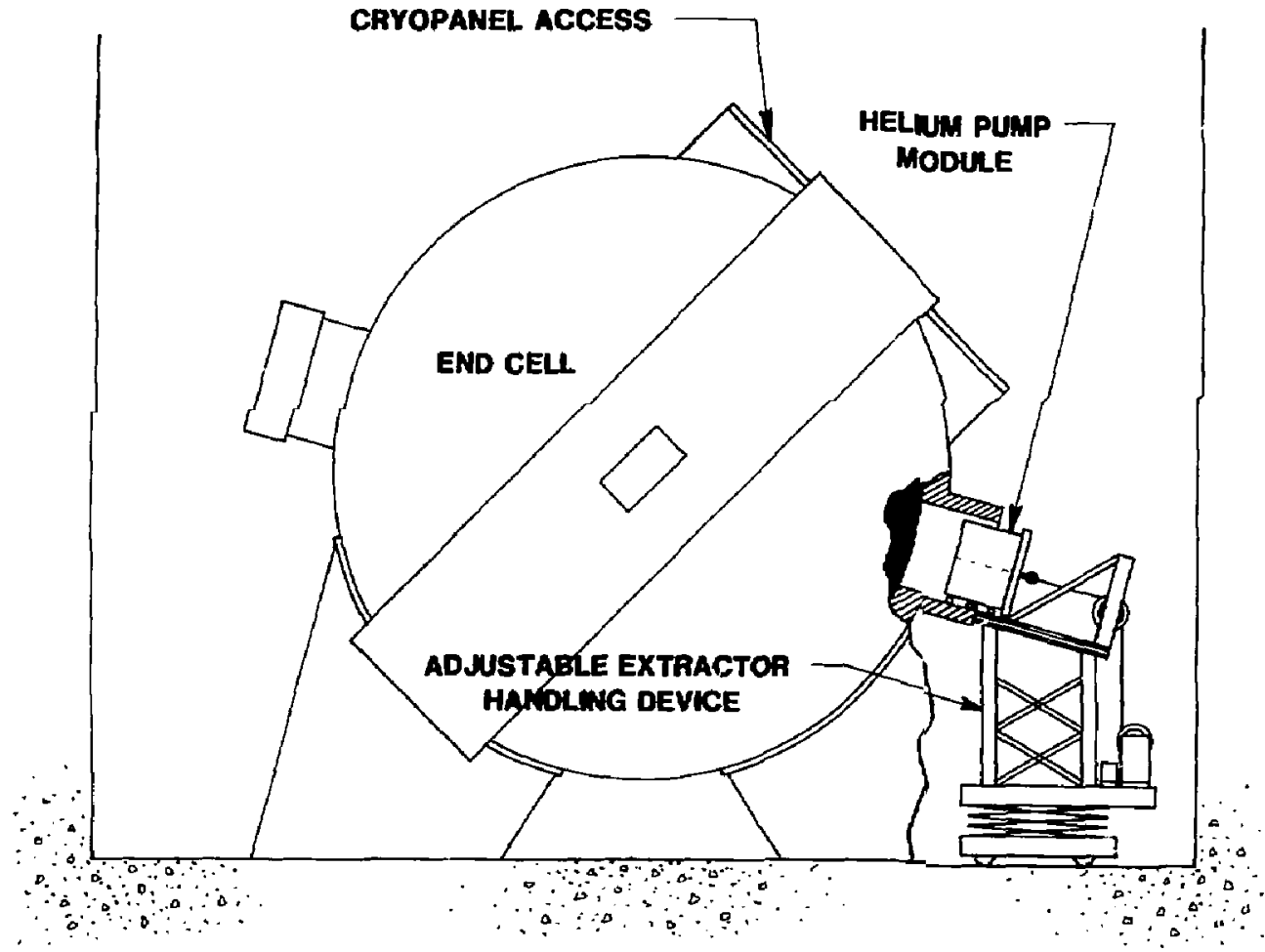

Figure 3-251. Helium pump mooule removal. 
reactor building size, the installation and replacentent are accomptished

through the rear of the end cel1. Figure 3-252 shows the sequential removal of the vessel assembly.

This operation requires that the cryopanel assemblies and the end dome be removea. A rail system mounted at the top centerline of the end cell cylinder is one way to support the vacuim vessel during its removal. The rail(s) and a cable and hoist mechanism also appear to be a straightforward way for initially installing this component. 


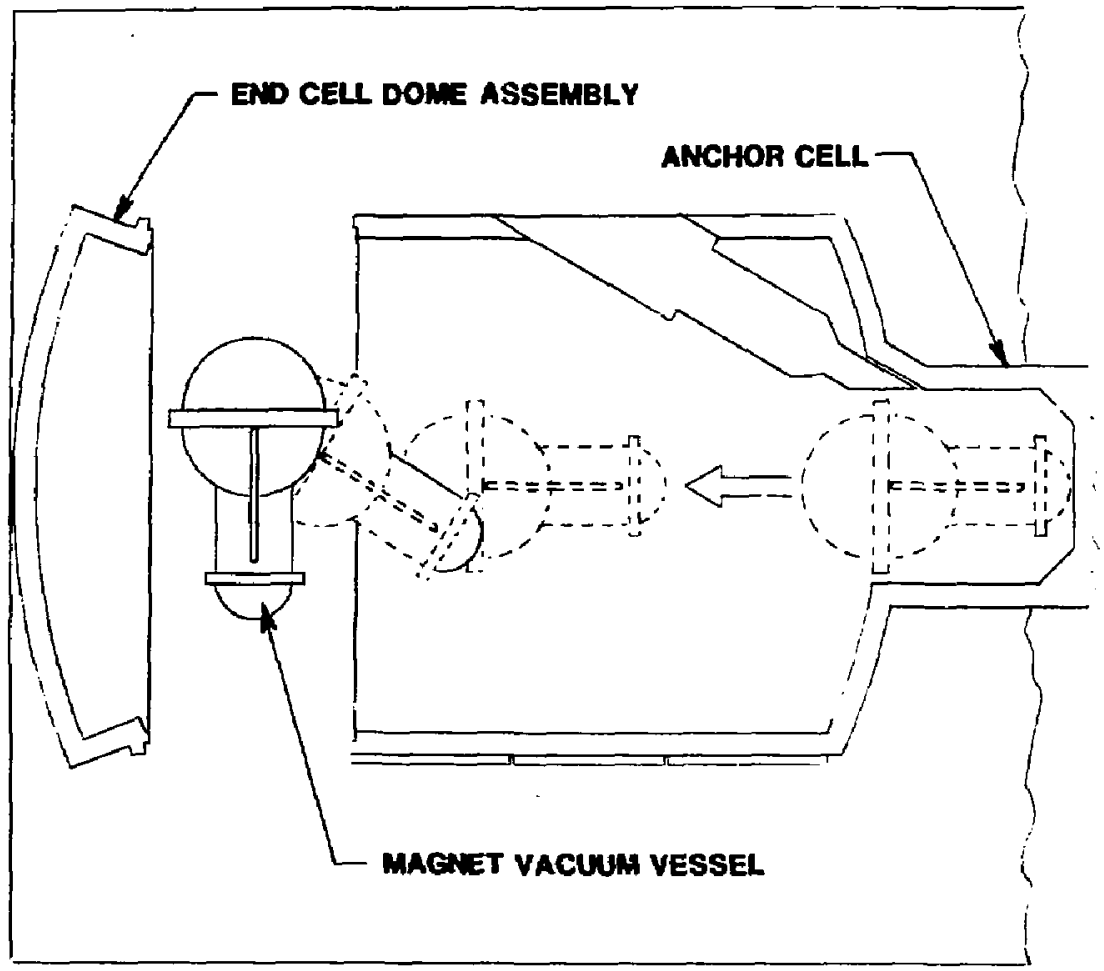

Figure 3-2.52. Magnet vacuum vessel assembiy removal. 
1. "The Structural Analysis of Nonhomogeneous Solenoids Using the Stansol Computer Program," Mechanics Research, Inc., MRI-C2754-TR-3, (1975).

2. M. Kohler, Am. Physik 5(32), 211 (1938).

3. R. Van Konynenburg and M. Guinaen, Radiation Effects on MFTF-B Magnets, Lowrence Livermore Nationdl Laboratory, Livermore, $C A$, interdepartment menio (June 1981).

4. Final Design Review for the A-Cell, Transition, and Solenoid Coils for the Mirror Fusion Test Facility-B (MFTF -B), General Dynamics Corporation and the Lawrence Livermore National Laboratory, Livermore, $C A$, GUC-MFTF-B-87-04 (1982), p. 5-7.

5. J. M. Williams, C. E. Klabunue, J. D. Redman, R. R. Coltman, Jr., and R. L. Chaplin, "The Effects of Irradiation on the Copper Norma Metal of a Conposite Superconouctor," IEEE Transaction on Magnetics 15 ( 1 ) (1939).

6. joel Schuitz, If You Must Run Water Past Your Insulation, Plasma Fusion Center, Massachusetts Institute of Technology, Cambridge, MA, MITN-82-06 (1982).

7. R. Chaplin and R. Cultman, Jr., "Defects and Transmutations in Reactor Irradiated Copper," in Proc. Intern. Conf. Meutron Irrad. Effects, Argonne, IL, 1981 (Argonne National Laboratory, Argonne, IL, 1981).

8. 0. B. Montgomery, Solenoid Magnet Design: The Magnetic and Mechanical Aspects of Resistive and Superconducting Systems, (Robert E. Krieger Publishing Co., Melbourne, FL, 32901, (1980).

9. Mirror Advanced Reactor System Study, Lawrence Livermore National Laboratory, Livermore, CA, UCRL 53333 (1983).

REFERENCES--Section 3.3

10. E. F. Marguarat, H. H. Hazelton, M. M. Menon, D. E. Schechter, W. L. Shirling, and C. C. Tsai, "Advanced Design of Positive Ion Sources for iveutral Beam Applications," in 8th Symp. Energy Problems Fusion, San Francisco) CA, November 1979 (IEEE Service Center, Single Publication Sales Lept., 445 Hoes Lane, Piscataway, N.J. 08854 1979). 
11. C. C. Tsai, M. M. Menor, P. M. Ryan, D. E. Schechter, and H. H. Hazelton, "Long-Pulse lon Source for Neutral Beam Applications," Kev. Sci. Instrum. $\underline{53}(4)(7982)$.

12. H. H. Hazelton, private communication.

13. R. A. Dewitt, "Coil Design Considerations and Analysis for Neutral Beam Magnets," in 7th 5ymp. Eng. Problems of Fusion, Knoxville, TN, 1977 (IEEE Service Center, Single Publjcation Sales Dept., 445 Hoes Lane, Piscataway, N.J. 08854, 1977).

14. L. D. Stewart, Studies of Neutral Beam Power Loss in Stray Magnetic Fields.

15. H. W. Kugel, H. F. Oylla, E. P. Eubank, T. A. Kozub, R. Moore, G. Schilling, L. D. Stewart, A. von Halle, and M. D. Williams, "PDX Neutral Beam Deionization Losses," in Proc. 28th Symp. Amer. Vacuum Soc., Anaheim, CA, Nov. 1981 (Journal of Vacuum Science and Technology 20, 3-4, March-April, 1982).

16. A. C. Riviera and J. Sheffield, "Transfer Efficiency of Intense Neutra] Beams, " Nucl. Fusion 15, 944, (1975).

17. R. S. Hemsworth, Enhanceo Reionization Loss for Neutral Beams in Magnetic Fields, United Kingdom Atomic Energy Agency, Culham Laboratory, Culham, Abington, Oxforoshire, Erigland, CLM-R162 (1976).

18. M. Pirani and J. Yarwood, Yacuum Technology (Cambridge Press, Lonoon, 1960).

19. L. D. Stewart, H. P. Eubank, L. R. Grisham, G. Schilling, R. W. Stooksberry, ano M. Uirikson, "Regionization Losses in Neutral-Beam Ducts," in 8th Symp. Engineering Problems Fusion, San Francisco, CA, Nov. 1979 (IEEE Service Center, Single Publication Sales Dept., 445 Hoes Lane, Piscataway, N.J. 08854, 1979).

20. L. C. Pittinger, Power Density Calculations for Beams from Diffuse, Astigmatic Rectangular Sources, Lawrence Livermore National Laboratory, Livermore, CA, Engineering Note ENC 77-] (1977).

21. R. Conn University of California-Los Angeles, 405 Hilgard Ave., Los Angeles, CA, 90024, private communication (1982).

22. A. W. Molvik, E. D. Baird, and K. H. Berkner, A Compact Bo-keV Neutral-Beam Module, Lawrence Livermore National Laboratory, Livermore, CA, UCRL-79682 (1977).

23. J. W. Shearer, T. A. Cutler, and L. W. Dilgard, Lawrence Livermore hational Laboratory, Livermore, CA, UCRL 52824 (1979). 
24. Henning, et al., Engineering the Baseba]1 Magnet System, Lawrence Livermore National Laboratory, Livermore, CA, UCRL-73067 (1971).

25. A. K. Chargin and C. J. Anderson, Solving Problems of Neutral-Beam Injectors at LLNL, Lawrence Livermore National Laboratory, Livermore, CA, UCRL-75023 (1973).

26. J. Kim, W. L. Stirling, and W. K. Dagenhart, An Ion Energy Recovery Experiment Based on Magnecic Electron Suppression, Oak Ridge National Laboratory, Oak Ridge, TN, ORNL/TM-7283 (1980).

27. Model conveyed in a privace communication from John Conrad, University of Wisconsin Madison, WI, to Larry Stewart, Exxon Nuclear/PPPL 4/21/80 for institution into EFTNB code.

REFERENCES--Section 3.7

28. A. Jung and M. A. Abdou, Mucl. Tech. 41, 71 (1978).

29. M. Ragheb, A. Klein, and C. W. Naynard, Nuc1. Tech./Fusion 1, 99 (1981).

30. L. El-Guebaly and C. W. Maynard, Irans Am. Nucl. Soc. 42, 612 (1982).

31. MCNP--A General Monte Carlo Code for Neutron and Photon Transport, Los Alamos National Laboratory, Albuquerque, NM, LA-7396-M (1981).

32. B. Browin, T. B Tewitt, T. Scott, and A. Klank, J. Nucl. Mater. 52, 215 (1974).

33. M. Sawan, Radiation Limits in Copper Stabilizer of Superconducting Magnets, MARS project memo, University of Wisconsin, Madison, WI, WIS-MARS-82-029 (1982).

34. R. Coltman, Ir., ano C. Klabunde, J. Nucl. Mater. 103-104, 717 (1981).

35. M. Sol1, J. Nucl. Mater. 72, 168 (1978).

36. J. Fowler, Jr., G. Hurley, J. Kennedy, and F. Clinard, Jr., J. Nucl. Mater. 103-104, 755 (1981).

REFERENCES--Section 3.9

37. K. 1. Thomlmassen, V. N. Karpenko, An Axicell Design for the End Plugs of MFTF-B, Lawrence Livernore National Laboratory, Livermore, CA, UC1D-19318, (1982). 
38. Heat Adsorption Pane? Deve lopment Design Sumary Report, McDonne?l Douglas Astronautics Company, St. Louis, Mo., Report 4511010 (1981).

39. O'Neill, R. F., et al., Convair Thei mal Analyzer Computer Progranı No. P45600, San Diego, CA, 696-0-T-80-737, 30, (1980).

40. McAdans, W.H., "Heat Transfer at High Rates to Water with Surface Boiling," Industrial and Engineering Chemistry, 41, 1945-1955 (1949).

41. R. A. Langley et al., "Blistering," Nucl. Fusion, to be published (1983).

42. R. Behrisch, "Sputtering by Farticle Bombardment II," (to be publisheo by Springer-Verlag, Berlin, 1983).

43. Parlelcoil Heat Transfer Handbook, (Dean Products, inc., Brooklyn, New York, 1975) Technical Data No. 358A.

44. D. L. Smith, et al., Starfire - A Commercial Tokamak Fusjon Power Plant Study, Argonne National Laboratory, Argonne, IL, Ani/F, Vol 1, pp 80-81 (1980).

45. D. Burgreen, Design Methods for Power Plant Structures (C.P. Press, Jamaica, N.Y., 1975).

46. J. S. Przeemieniecki, et al., "Thermal Stresses" in Introauction To Structural Prob lems in Nuc lear Reactor Enginering: (Pergamon Press, New York, 1962).

47. D. S. Gelles, et al., "Void Swelling in the Molybdenum Allay TZM Irridated to High Fluences," $\underline{\mathrm{J}}$. of Nucl. Mater., 103-104, 1141-1146 (1981).

48. M. Gomolinski and G. Brebec, "Mode pour la croissence des cavities sous irradiation," …․․ of Nucl. Mater., 43, 59 (1972).

REFERENCES--Section 3.12

49. Clement Wong, GA Technologies, Los Angeles, CA, private communication (April 1980).

50. UWMAK-III, A Noncircular Tokamak Power Reactor, University of Wisconsin, Madison, WI, UWFDM-150 (1976). 
51. B. L. Hunter, Fusion Engineering Design Center, Oak Ridge, TN, private comunication (May 1982).

52. Dr. I-K Kim, General Oynamics, San Diego, CA, private communication (May $1982)$.

53. T. Batzer, Lawrence Livermore National Laboratory, Livermore, $C A$, private omunication (June 1982).

54. J. Osher, Lawrence Livermore National Laboratory, Livermore, $C A$, private commun ication (July 1982).

55. M. Sawan, University of Wisconsin, Madison, W1, private communication (Juiy 1982).

56. P. Kidd, TRW, Redondo Beach, CA, private communication (June 1982).

57. D. Pence, Science Applications, Inć., La Jolla, CA, private commurication (June, 1982).

58. G. Gorker, Fusion Engineering Design Center, Oak Ridge, TN, private communication (June 1982). 


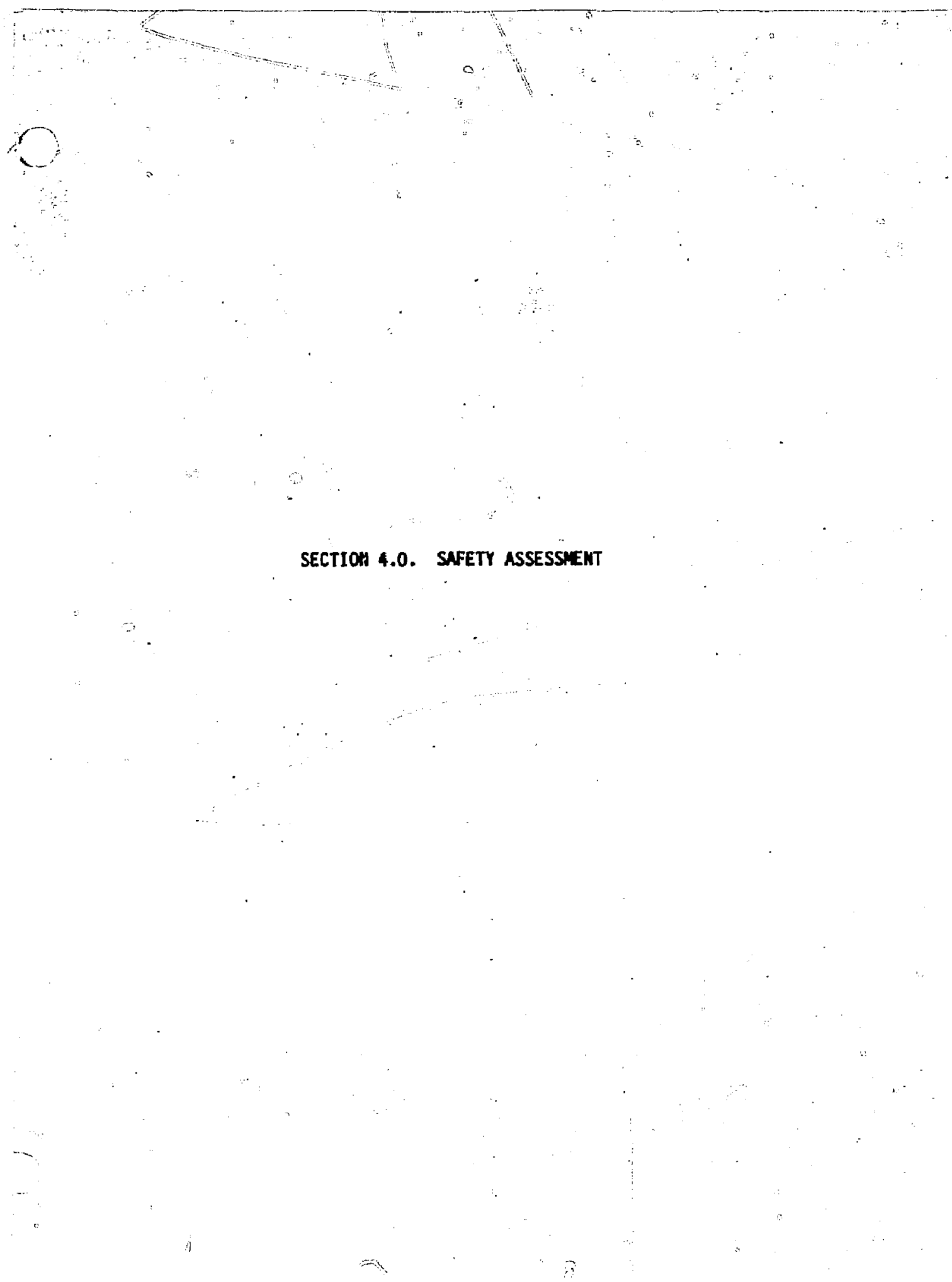


A.1. INTKODUCTION

Here we present a preliminary assessment of the safety aspects of TDF. our objectives are to identify the safety issues, assist with design trade-offs, and ensure that safety considerations are incorporated in the aesign at a very early stage. A detaileo safety analysis is neither practical nor warranted at this stage of TDF design. However, preliminary safety considerations have played a major role in TOF design.

For an overall safety assessment, the total fuel cycle must ue examined. TUF uses a U-T fuel cycle, and a smail quantity of lithium is used in the test mocules. The deuterium and lithium involved are not radiaactive. Urily a 5 mall quantity of tritium, approximately $45 \mathrm{~g} /$ month (at $50 \%$ availability), needs to be shipped to the plalit. The rest of the tritium fuel cycle, with an inventcry of less than $1 \mathrm{~kg}$, is contained within the plant. Thus, the rajoactive hazard associated with fuel shipment is minimal.

In performing our assessment, we used the approach of the Fusion Engineering Device (FEO) ${ }^{i}$ and STARFIRE ${ }^{2}$ aesign studies. Our safety standaras, criteria, and radiological gijidelines are basea on DOE orders ana procecures. These suggest that, under normal operations, the radiation aose rate at the site boundary shoula be limited to $<10 \mathrm{mR}$ per year to an individual, and under an off-normal or accident condition, the total dose shoula be limited to $<1$ Rem over 2 hours. Potential accidents and their sources in the TDF design are listed in Table 4-1.

Tritium is the primary source of radioactivity release. A preliminary estimate of potential radiological consequences of tritium release, based on the above guidelines, incicates the following:

If the tritium leakage rate to the environment is to be kept to $10 \mathrm{C} i$ day under normal operating conditions, an exclusion area bourdary located $45 \mathrm{~J}$ in away from the buildings is needed; TDF is provided with a site boundary at $500 \mathrm{~m}$.

- An accidental release of tritium inside a building and on accompanying building overpressurization result in tritium leakage because builoings are not 100\% leak-tight. Thus, leak-tightness, together with the site-bounoary distance, determines the upper limit on the amount of tritium that can be released inside a builaing without exceeding the dose limit at the site bourdary. 
Table 4-1. Sources of safety hazards in TDF design.

\begin{tabular}{ll} 
Potential hazard & Possible result \\
\hline Tritium inventory & Tritium release \\
$\begin{array}{l}\text { Induced activity in the shield, magnet, } \\
\text { structural material, and building structures }\end{array}$ & $\begin{array}{l}\text { Release of activated } \\
\text { structural material }\end{array}$ \\
Corrosion products in the primary coolant & $\begin{array}{l}\text { Loss of collant flow to the } \\
\text { heat dumps }\end{array}$ \\
Cryogenics & $\begin{array}{l}\text { Release of liquid helium } \\
\text { rf neaters }\end{array}$ \\
Lithium in test modules & rf-heater failure
\end{tabular}


With the site boundary at $500 \mathrm{~m}$, and assuming the reactor and tritium buildings have a minimum leak rate of $1 \%$ per day, the maximum allowable tritium release is approximately $700 \mathrm{~g}$. A cursory analys is indicates

that the largest amount of vulnerable tritium for release is approximately $200 \mathrm{~g}$.

We assessed the impact of an accidental release of cryogens inside the reactor building. The maximum allowable liquid-helium release is approximately $2500 \mathrm{~kg}$, without exceeding the $120-\mathrm{kPa}$ building design pressure. The cryogenics system can be designed to provide appropriate partitioning and valving arrangements so that the maximum potential release is well below the limit.

Our preliminary safety assessmient does not identify any aspect that may make the plant unsafe for the public or for personnel.

\subsection{SAFETY CRITERIA AND REQUIREMENTS}

The DOE Order $5480^{3}$ and tne former DOE Manua $]^{4}$ provide the basis for establishing safety criteria and give severai applicable guidelines. The $D O E$ policy is that radiation exposure of individuals and population groups be limiteo to "as low as reasonably achievable (ALARA)". For DOE projects, most of the burden of interpreting the ALARA policy falis under the DOE safety analysis and review system ${ }^{5}$ described in DOE Order 5487.1. This review system determines if the proposed design meets the intent of the ALARA policy.

\subsubsection{Radiological Guideline}

It is important to establish some safety guidelines so that design activities may proceed before any safety review is undertaken. In particular, thuse guioelines that may affect design and construction of the reactor building must be identified and their applicability to TDF determired, because this issu: affects the basic configuraton of the facilities. Limiting the amount of radioactive release to the general public has a major effect on the reactor building design. The various operating conditions that require safety guidelines are suggested in Table 4-2. Proposed radiation-exposure guidelines for TDF are preseited in Table 4-3.

The radiation exposure criteria have been considered by 00 . $^{3-8}$ The DOE Order 5481.?, "Safety Analysis and Review Systems," establishes uniform 
Table 4-2, Safety cuidelines for Tof nperatino conditions.

\begin{tabular}{|c|c|c|c|}
\hline $\begin{array}{l}\text { Operation } \\
\text { condition }\end{array}$ & Deseription & $\begin{array}{l}\text { Propantlity nf } \\
\text { nceorrence (P) } \\
\left(y r^{-1}\right)\end{array}$ & $\begin{array}{l}\text { Safety } \\
\text { gutdelines }\end{array}$ \\
\hline 1 & $\begin{array}{l}\text { Reular planned events } \\
\text { (e.4., normal fecility operatlons) }\end{array}$ & $p=1$ & $\begin{array}{l}\text { Normally operat ing mattes } \\
\text { shouid provide heaith and } \\
\text { safety protection }\end{array}$ \\
\hline
\end{tabular}

II

Events that may nccur several $\quad$ Ixpsio-1

Facility should he able to times during life of factitity return to operation with(e.q., minar incldents) nut extens tue corrective action

III

Events thot may nceur infrequently durina life of

D facility (e.c., more severe incidents)

$10^{-1}>p>10^{-3}$

Faclitiy should he obie to return to operatian followino extensive corrective act inns

Limiting fault events that are $10^{-3}, \mathrm{P}>10^{-7}$ Facility damsge may nrt. expected durina facility Iife hut are postulated hecause of oreclude retiornina to potential significant radioactive operat ion

release and, second, they represent upper haunds an fallires or accidents with sifficient. prohahtilty $\left(>10^{-7}\right.$ per veor) to requitre constderation to destion.

Doperat ing condition descriptions were taten from Ref, 6. 
Table 4-3, Dose-71nit gufdelines for TDF.

\begin{tabular}{|c|c|c|c|c|}
\hline \multirow[b]{2}{*}{ Dperat ing conditions } & \multicolumn{2}{|r|}{ Off-stte } & \multicolumn{2}{|r|}{ Cn-site } \\
\hline & Init & Desian ohject fve & limit & Desion ohfective \\
\hline $\begin{array}{l}\text { 1. lirrmal operation } \\
\text { (nrem/yr) }\end{array}$ & $\begin{array}{l}500 \text { per } \\
\text { individual }\end{array}$ & $\begin{array}{l}\text { slo/per individuat } \\
\text { sloo/per individual" }\end{array}$ & $\begin{array}{l}\text { soo in } \\
\text { oncontrolled } \\
\text { dreo }\end{array}$ & $\begin{array}{l}\text { sioo in uncontrolled } \\
\text { area }\end{array}$ \\
\hline $\begin{array}{l}\text { It Incidents of } \\
\text { frequency (mren/yr) }\end{array}$ & $\begin{array}{l}170 \text { per } \\
\text { average } \\
\text { ponulation } \\
\text { menter }\end{array}$ & $\begin{array}{l}\text { s35 averaqe population } \\
\text { memher }{ }^{h}\end{array}$ & $\begin{array}{l}\text { sono in } \\
\text { controlled } \\
\text { area }\end{array}$ & $\begin{array}{l}\text { slooo in controlled } \\
\text { ared }\end{array}$ \\
\hline $\begin{array}{l}\text { IJ Infreauent } \\
\text { incidonts (rem) }\end{array}$ & 5 & $\leq$ & 5 & $\leq 5$ \\
\hline $\begin{array}{l}\text { IV Limiting falts not } \\
\text { expeted during Tof } \\
\text { life (rem) }\end{array}$ & 25 & $\leq 5$ & 25 & $\leq 25$ \\
\hline
\end{tabular}

"For aperating conditton I, (normal operat 1ans)

${ }^{h}$ For aperatinq condition II, (Incident of moderate freauency) 
requirements for the preparation and review of safety analyses for al] DOE operations. The DOE Order 5480.1, Chapter V, "Safety at Nonreactor Nuclear Facilities," establishes additional requirements for an Environmental, Safet ano Health program. The intent of DOE policy is to assure that enyironmental protection, safety, and health matters are comprehensively addressed and that identifiable risks are reduced to acceptably low levels.

The design of UOE-owred nuclear facilities is based on a philosophy that the facility be capable of being safely shut down following postulated accidents. In addition, it must be designed to provide a defense-in-depth against an uncontrollea release of radioactivity to the enviranment.

The radiolagical consequence guidelines in Table 4-3 are to be interpreted as minimum guidelines to protect health and safety. In addition, UOE policy requires that radiation exposures meet ALARA palicy. The dosage guidelines shown in Table 4-3 are based on review of appropriate DOE Urders, DOE Manual chapters, ano Tritium Systems Test Assembly and Tokamak Fusion Test Reactor reports on preliminary safety analyses. The exposure guidelines suggested in Table 4-3 apply only to TDF and should not be construed as applying to otiuer present or future fusion test facilities, research facilities, or reactor projects. The values quoted are for whole body exposures; however, the UOE values for other organs (hands, arms, bone, etc.) and other time periods (quarterly) are also applicable.

Magnetic Fie?ds. We have adopted a suggested criterion ${ }^{9}$ as an interim exposure guideline. It suggests that personnel exposure be limited to magnetic fields less that $100 \mathrm{G}$. The magnetic-field background of earth is approximately $0.5 \mathrm{G}$. This will probabiy make it necessary to de-energize all the magnets before personnel are allowed to enter the reactor building. It will be also necessary to magnetically shield the control room or locate it far enough away from the reactor building so that the magnetic fields will be acceptably small.

Confinement System Criteria. The confinement of radioactive releases nornally involves developing a system to provide the desired level of pritection. The guide in Ref. 6 contains a discussion of continemerit systems that have beer; reviewed and adapted to the TDF situation. Physical barriers are normally provided to prevent an uncontrolled release of radioactivity to the environment and to prevent or minimize the spread of contaminaton within the 
facility. These barriers should be provioed either between the environment and those areas of TDF that contain radioactive material or between areas in the facility that are normally expecteo to have contamination levels lower than those for which the barrier is designed. Such physical barriers and their associated systems (e.g., ventilation, atmosphere cleanup, etc.) make up the confinernent systems.

Confinement systems shoula prevent or mitigate the effects of an uncontrolied release of radioactive material so that on-site and off-site coses and effluent concentrations are maintained within recommended guidelines (1able a-3) during all facility operating canditions. Current estimates of iritium-release consequences confirm that a confinement system is necessary.

The TIJF cesign considers the use of primary, secondary, and tertiary confinenent boundaries to provide a defense-in-depth against a release of radioacfive material to the environment. When warranted by lesser potential hazaros, portions of the facility could be cesigned with fewer confinement boundaries (e.g., primary and seconoary confinement or, in some cases, primary cunfinement alone). These confinement boundaries are defined as follows:

- Primary confinement refers to the barrier or enclosure and its associated systems for ventilation and atmosphere cleanup or off-gas treatment that will be exposed directly to potential sources of high-level radioactive contamination (e.g., hot cells, glove boxes, tanks, process piping, funie hoods, etc.).

- Secondary confinement refers to the barrier and associated ventilation system that enclose the primary confinement and that separate potentially contaminateo operating areas from normally noncontaminated areas of the faci?ity.

- Tertiary confinement refers to the structure and associated ventilation system that enclose the primary and secondary confinements and include other facility areas that are not expected to become contaminated. This is the final barrier between the outsice environment and the potential contamination.

Typical characteristics of these three confinement barriers, including safety-classification recommendations, are summia: ized in Table 4-4. These do not establish the safety importance of any potentiā confinement systems. 
Table 4-4, Characteristics of TOF harrlers for confinement of radioactive astertals.

\begin{tabular}{|c|c|c|c|}
\hline $\begin{array}{l}\text { Suhject of conf Inement } \\
\text { conf Inement }\end{array}$ & Primary cnnfinement & Secondary conf inement & Tertiary \\
\hline $\begin{array}{l}\text { Type of structere } \\
\text { huildings-: } \\
\text { process plping tank, } \\
\text { fump hond, etc. }\end{array}$ & $\begin{array}{l}\text { Hot cell, glove hox, } \\
\text { cell, e.a., vacuim pump } \\
\text { or fueling-machine efil. }\end{array}$ & $\begin{array}{l}\text { Operating compartinent or } \\
\text { reactor, hot cell, } \\
\text { tritium hufiding. }\end{array}$ & Facility \\
\hline $\begin{array}{l}\text { Design-hasis events } \\
\text { edrthquakes }\end{array}$ & Accidents, earthquakes. & $\begin{array}{l}\text { Accidents, earthquakes. } \\
\text { and other severe } \\
\text { conditinns. }\end{array}$ & Accifents, \\
\hline $\begin{array}{l}\text { Safetv classification" } \\
\text { Setsmic desion }\end{array}$ & $=$ & $\ddot{-\infty}$ & $\begin{array}{l}-4 \\
-\infty\end{array}$ \\
\hline Contamination & Nornally contaninated. & $\begin{array}{l}\text { Potentially contaninated. } \\
\text { contaminated. }\end{array}$ & Sormally not \\
\hline $\begin{array}{l}\text { Operat ing pressure } \\
\text { respect }\end{array}$ & Hegative with respect & Hegative with respect to & Hegative with \\
\hline to secondary canfineme. & ent. & tert tary conf irement. & to stmosphere. \\
\hline $\begin{array}{l}\text { Atmosphere contral } \\
\text { ventilatinn }\end{array}$ & Sepsrate rentilation & Redundant vent llation & Redundont \\
\hline $\begin{array}{l}\text { or nff-qas treotment } \\
\text { systom, atmospherp } \\
\text { cleanup. Inert oas } \\
\text { dtmnsphere. }\end{array}$ & system with air clpanap. & $\begin{array}{l}\text { system with dir clesn- } \\
\text { up. }\end{array}$ & \\
\hline
\end{tabular}

The reguired safety-class conf inement harriers should he determined from the potentilal racinactive hazards acsnciatod with pach part of the facility. The fallisro of any nonsafetymelnss harrfer should not causp a loss of function in any remaining safety-class harrers.

$h$ Safety-class barriers should be ipsigned to special safety-related selsmic standards. Monsafety-class confinement barriers may he destaned to mormal setsmie standards. 
Safety Classification. We suggest the following definitions, based on aiscussion in Ref. 6 , for establishing the safety importance of various parts of TDF.

- Safety class applies to those components, systems, and structures that are required to prevent or mitigate the consequences of a postulated release of radioactive material that has the potential for causing off-site doses in excess of 0.5 rem to the whole bouy, or the equivalent dose to any part of the body.

- Nonsafety class components, systems, and structures are those not cefined as safety class. The maximum off-site dose resulting from the failure of a nonsafety class system shoulo not exceed 0.5 rem to the whole body, ar the equivalent dose to any part of the body. The aetermination of saftety class requires accident analysis. Because these andyses are just beginning for most fusion components and systems, no firil determinations are made at this tinie. The class carries with it special quality and reliability criteria to establish a high level of assurance that safety class systems will perform their safety-related functions as designea. These special attributes include the ability to withstand severe natural phenomena. The insplications of safety class designation on design are considerable ani need to be worked out specifically and as needed.

Uesign Implications. Confinement capabilities are based on the ability of ventilation systems to maintain a controllea, continuous airflow pattern from the environment into the confinement building and then from noncontaminated areas of the building to potentially contaminated areas and then to normally contaminateo areas. This airflow sequence, in conjunction with other design and operating features, shoulo prevent the spread of contamination with in the facility ouring normal operations, minimize the spread of contamination during abnormal conoitions, and ensure adequate cleanup of exhaust-gas streams before they are releaseo.

The TUF conditions may require some confinement barriers to be pressure-containing. If a barrier is not so designed, it cannot control the release of radioactive material whenever the pressure exceeds a minimum negative pressure. For periods when this happens, reauced credit will be given for cleanup systems in the normal exhaust path. The confinement 
ventilation shoula be able to maintain desired air-flow characteristics when access doors or cell hatches are open. When necessary, air locks should be used to minimize the impact of facility access requirements.

Safety class confinement ventilation systems should have duplicate features to ensure airflow characteristics that will provide the necessary confinement under all creoible circumstances. If necessary, the ventilation system should be supplied with safety class electric power and be operable on both on- and off-site power sources.

Confinement air cleanup systems should be provided within the major confinement ventilation-exhaust system. The air-filtration units should be functional throughout postulated accidents and should retain collectea radioactive material after such accidents. The number of air-filtration stages required for any area should be aetermined by the quantity and type of radioactive materials to be confined. Air-filtration units should be installeo as close as practical to the source of contaminantion to minimize ductwork contamination. Off-gas stream pretreatment should be proviaed upstream of the off-gas treatment system to remove or reduce the chemica 1 concentrations that would adversely affect system operation.

The air-filtration units should be located and shielded to maintain occupacional doses within the recommended guidelines Guring operations and maintenance. The system should facilitate periodic inspection and testing during operation and shutauwn.

The level of radioactivity in the exhaust shoulo be continuousiy monitored and alarmed to detect high activity. Appropriate protective actions should be taken to prevent an uncontrolled release of radioactivity to the environment and to minimize the spread of contaminaton within the facility.

\subsection{POTENTIRL RADIOLOGICAL CONSEQUENCES}

\subsubsection{Tritium Release Considerations}

The radiological aspects of released tritium are discussed in Ref. 10. Since our basic concern is the impact of released tritium on the general public, the potential concentration and dose rate of accidenta?y released tritium at the exclusion boundary are the most important parameters. These 
concentrations and oose rates depend on a variety of variables, many of which are site-dependent. For example, the distance to the site-exclusion boundary, the wind, atmospheric, and topographical conditions are all important to the analysis, as are the building and ventilation designs. These variables make the calculation of dose rates very complex.

A conservative estimate (upper bound) of allowable tritium relcase from the plant (both normal and accidental) is given in later sections, using the oose guidelines given in Table 4-3. Actual allawable limits wili be higher. Uur conservatism originates from using the most stringent atmospheric and topographical conditions. In adition, no creoit has been taken for an elevateo exhaust from a stack.

\subsubsection{Nornal Releases}

During normal operations, tritiun can be released in liquid or gas.

Gaseous releases to the atmosphere originate from permeation through components and from leakages of valves and pumps within the plant (see Fig. 4-1). These releases would eventually exit to the atmosphere through the ventilation systems. Also, releases to the acmosphere could originate from the radidactive-waste treatment systems. The liquid releases also originate from the radioactive-waste treatment systems. Raoioactive-waste water and the cooling water would be mixed at the cooling-tower blowdown before being discharged into the receiving body of water. Figure 4-2 shows the release pathway available.

We consider only the consequences of gaseous release. We anticipate the coses from liquid release to be minimal, as the quantity and potential for release are both small.

\subsubsection{Normal Gaseous Release}

Airborne releases of tritium are carried and diluted by the atmosphere. A certain portion is inhaled by humans, and some is depasited on the vegetation and works its way through the food chain.

The doses from all airborne pathways are calculated using an average of actual-site meteorological conditions from several light-water reactor (LWR) 


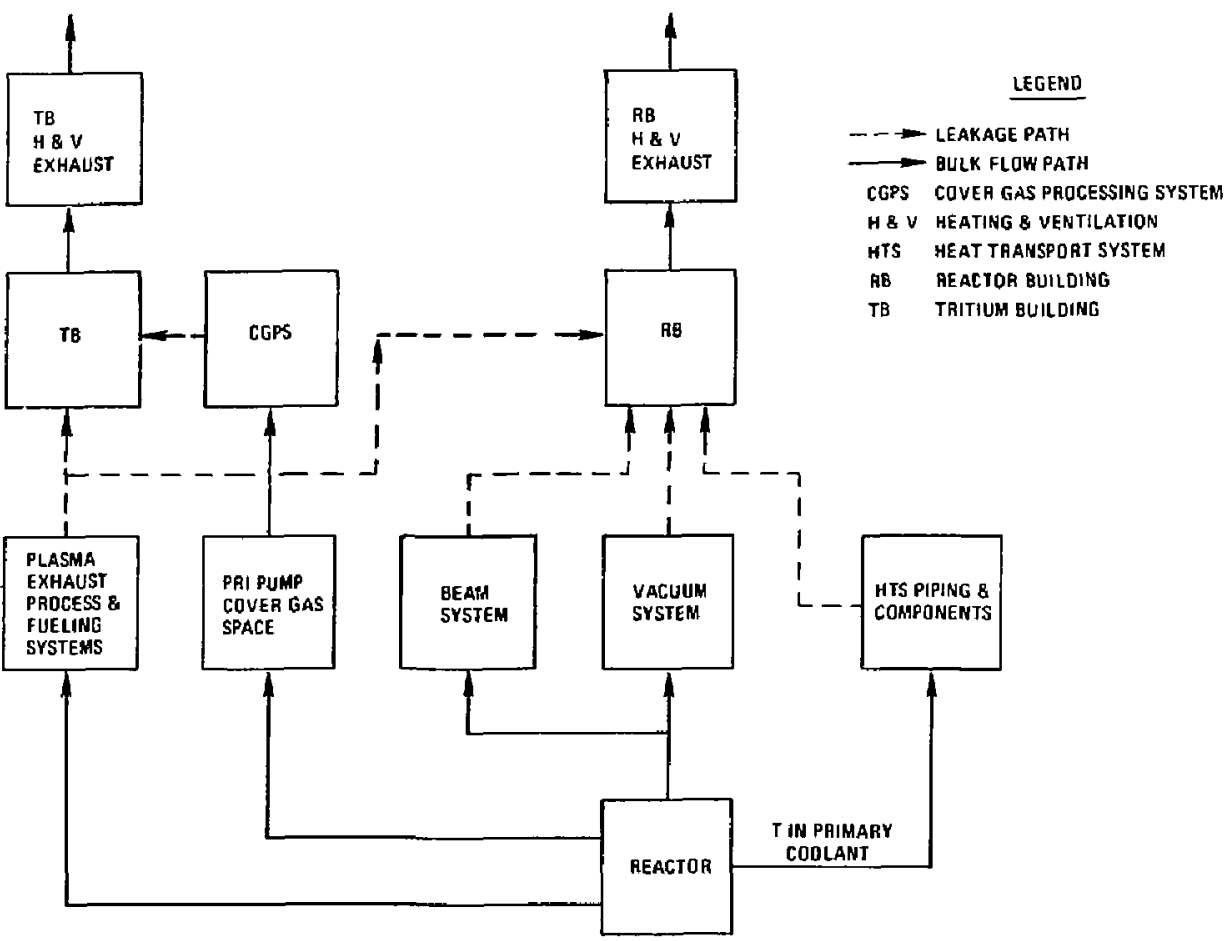

Figure 4-1. Major gaseous tritium leakage pathways. 


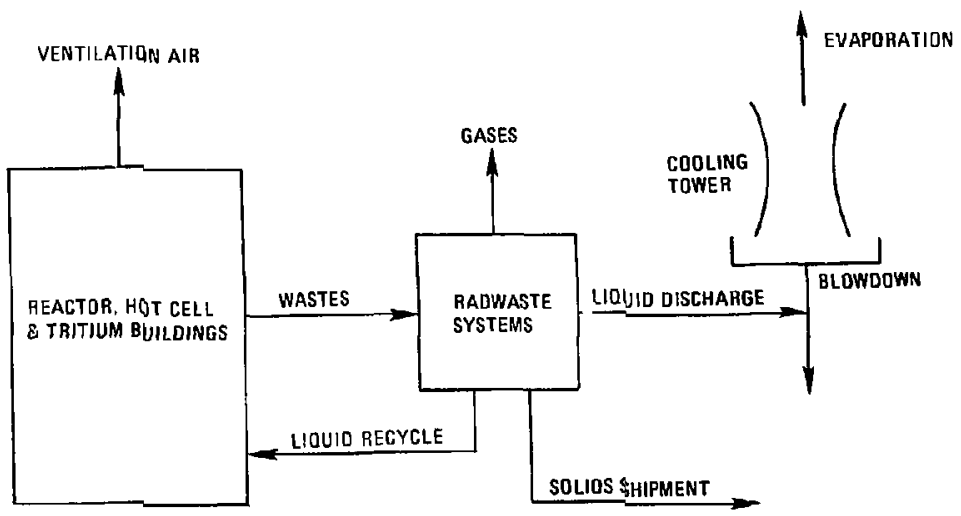

Figure 4-2. Tritium-re iease pathways to environs. 
plants. '3 All dose pathways are assumed present at the same location. Using the models of Ref. 12, we can characterize ine dose from all pathways as:

$D_{A}=C Q[X(x)] / Q$,

where

$D_{A} \quad=$ dose $(m R / y r)$ from all airborne pathways,

$4=$ airborne release rate from the $p$ ? ant $(\mathrm{Ci} / \mathrm{yr})$,

$x(x) / 0=$ atmospheric dilution $f$ actor $\left(\mathrm{s} / \mathrm{m}^{3}\right)$ at a distance $x$ from the point of release,

$c=$ a constant converting release rate $\left(\right.$ Rem $\left.\cdot m^{3} / C i \cdot s\right)$ to dose for all pathways.

e evaluated the constant $C$ by using the recommended values and models of Kef. 12. For the atmospheric dilution factors we used an average of 29 LWR sites 11 as a furction of distance. A functional dependence of $x / Q$ with respec, to distance was derived from the data of Ref. 11. This dependence is of the form

$x(x) / 4=0.078 \cdot x^{-1.37}$,

where: is the aistance in meters.

Equation (4-2) is valid for the region from 800 to $80,000 \mathrm{~m}$ from the plant oata from Ref. 11 covers this region on $1 y$ ). As the aistance from the plant decreases to less than $800 \mathrm{~m}_{2}$ the building wake effects begin to dominate the $X / 4$; thus $E q .(4-2)$ tends to be overconservative.

The dose calculation using the models of Ref. 12 indicates that the ingestion of vegetation, inhalation, and the drinking of milk are the most important contamination pathways $(53 \%, 25 \%$, and $16 \%$, respectively). Since tritius does not contribute to the external whole-body dose or skin dose, only the organ-dose guideline ( $<10 \mathrm{mR}$ per year) applies. Equation (4-1) is compared to this guideline, and the release rate is calculated for different Gistanc es and plotted on Fig. 4-3.

F gure 4-3 can be used in two ways. With a given exclusion area bounuary, one can obtain the allowable tritium-release rate from the plant 


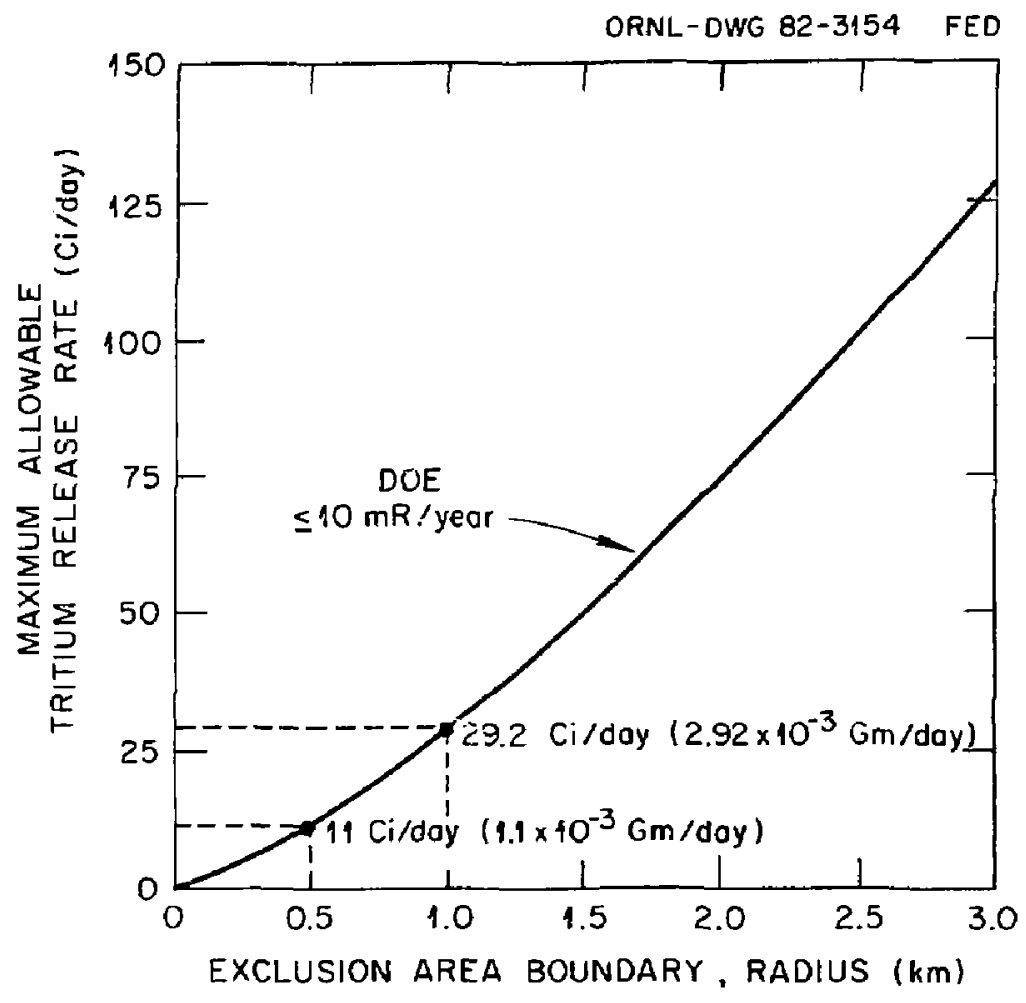

Figure 4-3. Maximum-allowable tritium-release rate from plant buildings under normal conditions. 
- through the ventilation system and, in turn, oetermine the ventilation flow rate from the building. Alternatively, with a given release rate (design goai), one can cbtain the required bourıdary. Thus, with a boundary $500 \mathrm{~m}$ from the experimental complex, the allowable tritium release is -11 Ci per aay.

A]ternatively, using a release rate of $10 \mathrm{Ci}$ per day of tritium as an example, Fig. 4-3 indicates that an exclusion-area boundary of $\sim 450 \mathrm{~m}$ results in an acceptable dose (<10 $\mathrm{mR}$ per year) at the site boundary. The tritium-cleanup and ventilation-system oesigns are targeted for this goal.

\subsubsection{Hccidental Keleases}

As soon as it is known that an accident has occurred in the plant, all normal operatior; are suspended and emergency procedures are put into operation. Normal ventilation exhaust of the buildings is discontinued and liquid discharges are stopped, leaving atmospheric leakage as the only release pathway avaitabl? for tritium.

The dose levels for mandatory evacuation (1 rem whole-body dose) are used to evaluate the short-term effects of accidental tritium release. of the dose pathways available to man, only inhalation is important for the zero to 2-hour dose. Immersion in a cloud of tritium does not contribute to the dose, because tritium is a weak beta emitter; clothing stops it. The other pathways can be avoided by importing food from outside the affected area.

The dose from an accidental release is evaluated as a function of exclusion area boundary for several builoing leakage rates. The oose is then compared to the manoatory evacuation whole-body oose, ano the maximum tritium inventory is caiculated.

The inhalation alose from tritium is given by

$D=x(x) / Q \cdot B R \cdot A_{R} \cdot D C F$,

where

$4=$ ojose in reni

$X(x) / Q=2$-hour atmosphere-dispersion factor $\left(\mathrm{s} / \mathrm{m}^{3}\right)$,

$A_{R} \quad=$ tritium activity (Ci) released from building,

$\mathrm{BR} \quad=$ breathing rate $\left(\mathrm{m}^{3} / \mathrm{Cj}\right)$

DCF = dose conversion fartor $\left(\mathrm{rem} / \mathrm{C}_{i}\right)$. 
The accioent $x / 0$ as a function of aistance is calculated using Hef. 12 and is evaluated for distarices ranging from 200 to $70,000 \mathrm{NJ}$.

In calculating the activity we assume that no removal (i.e., cleanup) systems exist to renove tritium from the building atmosphere. The activity released is given by

$A_{R}=A_{0}\left(1-e^{-\lambda} L^{t}\right)$,

where

$A_{0}=$ total activity ( $\left.C i\right)$ released inside the building,

$\lambda_{L}=$ builoing leakage rate, $\left(\mathrm{hr}^{-1}\right)$,

$\mathrm{L}$ = time (2 hrs) after accidental release.

The initial activity available for release $\left(A_{0}\right)$ is conservatively assumed to be instantly available for release at the start of the accident. In reality it would be released over time ranging from a tew seconas to several hours, depenaing on the severity of the accident.

Equations (4-3) and (4-4) are solved for $A_{0}$ and are shown in Fig. 4-4 as a function of exclusion-area distance for different building leakage rates. A)so shown is the tritium concentration in the $80,000-m^{3}$ reactor bujloing. We make several conservative assumptions to obtain a conservative upper bound of allowable tritiun release in a building. A value of $5.0 \times 10^{-4} \mathrm{~m}^{3} / \mathrm{s}$ is used for breathing rate, the race for yery strenuous activity at the site boundary. The dose conversion factor used, $158 \mathrm{rem} / \mathrm{Ci}$, is also very conservative. No credit is taken for an elevatea release from a stack, which allows increasea oispersion and reduced doses at the site boundary. The atmospheric dispersion factor $(x / Q)$ assumes a grouna level release, which is more realistic than an elevated release because all the ventilation systenis are closed after an acciuent.

Figure 4-4 shows that when the site bounaary is locateo at $500 \mathrm{~m}$, the maxinum allowable tri:ium release in a building designed for a leak rate of $1 \%$ per cay is $-700 \mathrm{~g}$. If the building is designed for a $10 \%$ per cay leak rate, a release of about $70 \mathrm{~g}$ results in a 1 -rem dose in 2 hours at the site bounoary. 


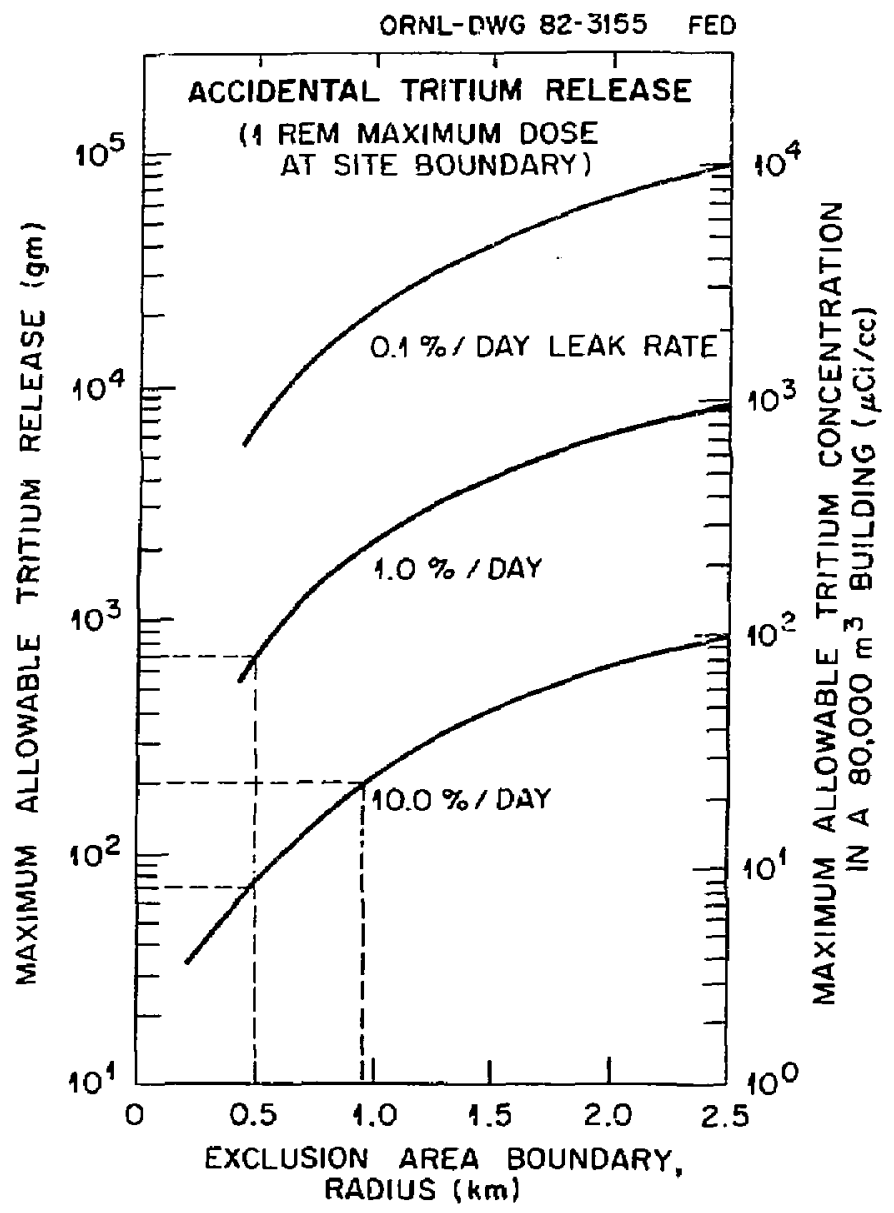

Figure 4-4. Niaximum-allowable tritium-release in plant buildings from an accidental release. 
Thus, by controlling the three variables--the builoing leak rate, the location of the site bounoary, and the maximum credible anount of accioental tritium release--the TDF plant can be designed to keep the dose consequences of an accidential tritium release within the acceptable limit. The site boundary is usually the most controllable of the three parameters. We discuss the remaining two in the following subsections.

\subsubsection{Builoing Leak Rate}

Two major sources of leakage from a building are first, penetrations of pipings, conduits, and the like, and, second, cracks in the concrete. Preventive Ileasures generally used to reduce leak rates include sealing the penetrations, prestressing the concrete, and steel-lining the inside surfaces of floor, wall, and ceiling. All these measures are used in LWR containment buildings, and a volume leak rate ranging from 0.1 to $1.0 \%$ per day is obtained when the accidental internal-pressure buildup is about $500 \mathrm{kPa}$. For the TOF reactor building, neither prestressing nor steel lining is envisioned. Epoxy coating is used for the building internal surfaces and is considered adequate for tritium confinement (reducea tritium permeation into concrete). As a result, it is aifficult to ascertain TUF's leak rate. However, considering that the accidental pressure buildup is not expected to exceed $120 \mathrm{kPa}$, the leak rate should be limited to a range of $1 \%$ to $10 \%$ per day.

\subsubsection{Creuible Tritium Release}

Without a detailed analysis identifying potential accident scenarios, we cannot determine the amount of tritium release in the worst credible situation. As a cursory assessment, however, the following seven major cases are examineo:

- Case 1. Fajlure of fueling system and escape of tritium into reactor building.

- Case 2. Vacuum-vessel leak.

- Case 3. Loss of cryogen supply to the end cell cryopanels.

- Case 4. Rupture of duct connecting the end cell and the tritium processing system.

- Case 5. Combination of Case 3 and Case 4 in a seismic event. 
- Case 6. Loss of cryogen supply to the tritium-processing system.

- Case 7. Rupiure of isotopic primary vessel of separation unit (ISS)

Case 1: Tritium is pumped through the neutral beam injector at the rate of $136 \mathrm{~g} / \mathrm{hr}$. In an accident, isolating valves can be closed in a matter of seconds resulting in a release of approximately 10 to $20 \mathrm{~g}$, which is we 11 below the allowable limit.

Case 2: A vacuum-vessel leak will not result in mach tritium release because the pressure inside the vessel is less than the building atmosphere. The plasma from the vessel can be evacuated ano tritium feeding sioppeo before the pressure is equalized.

Case 3: About $200 \mathrm{~g}$ of tritium is available for outgassing from the cryopanels in each end cell. In case of a complete loss of cryogens, all the tritiurn may be outgassed and will be pumped into the tritiun-processing system without any release into the building.

Case 4: If a duct connecting the end cell and the tritium-processing system ruptures, events will be similar to those of Case 2 . No significant tritiuni release is anticipated.

Case 5: If we postulate a complete loss of cryogens (see Case 3) and the rupture of the connecting auct (Case 4), the entire inventory of one end cell $(200 \mathrm{~g})$ is available. How much of this will be released into the reactor builoin'j and how much will be pumped into the tritium-procassing system are difficult to estimate without detailed analyses. In the worst case, the entire amount will be released.

Case 6: In a loss of cryogen supp, to the tritiun-processing system, no tritium release is anticipated, as the system is designed to transfer the tritium from the major components (e.g., ISS units) to uranium beos.

Case 7: In the tritium-processing system, the components containing tritium are provided with three confinement barriers. The inner vessel serves as the primary containment, while a glove box surrounding the inner vessel serves as secondary and the tritium-processing building serves as tertiary confinement. The probability of simultaneous failure of the primary ano secondary confinements is extremely smal1. Even if both confinements fail, the maximun release will not exceed $200 \mathrm{~g}$ as each of the ISS units contains that amount.

In suimary, assiming $200 \mathrm{~g}$ as the maximum credible amount of accidental release of tritium, aither of the following design alternatives is adequate to 
mitigate the consequences of normally and accidentally released tritium:

- Alternative 1. A design building-leak rate of $10 \%$ per day ana an exclusion area boundary location of $950 \mathrm{~m}$.

- Alternative 2. A design buílding-leak rate of $5 \%$ per day and an exc'usion area bounaary location of $500 \mathrm{~m}$.

If for some yet-unidentified reasons, a leak rate of less than 16 per day is rieeded, two possibilities exist: a leak-tight steel lining or a double-confinement building, consisting of a steel enciosure surrounded by a concrete builaing (Fig. 4-5). A detailed study specific to TDF is needed to determine which is more economical.

\subsubsection{Activated Structural Materials}

In addition to tritium, there is induced radioactivity in the first wall, the shiela, and the heat-dump materials. This activity is much less mobile than that of tritium. No credibie mechanism that would cause melting of the structural materiai has been identified. Since there is a bulk shield arouno the plasna chanber, the likelihood of this activity becoming mobile, even without plasnia shutdown, is not considered likely. Nevertheless, because solie of this activity is in the form of corrosion products, it impases constraints on maintenance and repair activities, and therefore strongly influences design and choice of structural materials. though not present at the beginning of reactor operation, this radiaactivity will increase with tilia and after several weeks of operation reach a level that is significant for access consiaerations. However, the associated afterheat is small and is distributea through a large volume, thus mạking it easier to ensure adequate cooling in emergency shutdowns.

Special effort is given to the materials selection to reduce both long-terin radioactivity and radwaste storage. The reactor is also designed to be accessible within 24 hours after shutdown for hands-on maintenance and iater for maintenance of reactor internals by remote operations.

\subsubsection{Loss-of-Flow Accident}

The pressurized water coolant for removing heat in the beam and end cell jumps is subcooled to a minimum of $100^{\circ} \mathrm{C}$. If the plasma is not shut down 
ORNL-DWG 82-3156

FEO
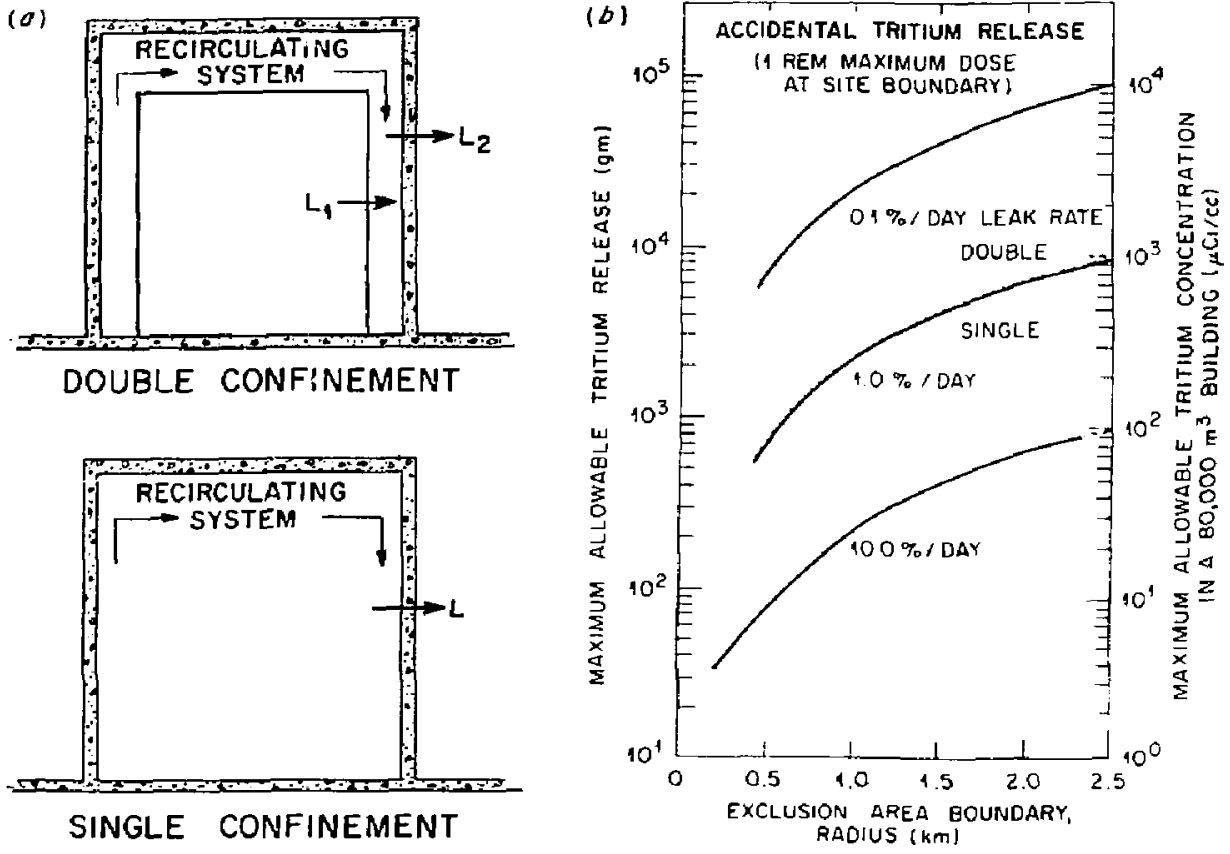

Figure 4-5. Single conf inement compared to double conf inement. 
in the event of a loss-of-flow accident, such as a loss of pumping power or a loss of pressurization, the coolant will go first into nucleate boiling, then into film boiling, and finally into burnout. Although the beam and end cell oumps have a large thermal inertia, if the plasma is not shut down within a short time after loss of flow, the wall material will ablate and will extinguish the plasma. However, the decay heat is not sufficient to cause melting.

\subsubsection{Liquia Heliuin}

Liquid helium is used for maintaining cryogenic temperatures in the superconducting coils and the cryopumps. In the event of coil break or helium pipe break, the liquid helium will be spilled into the reactor building, whicn will be maintained at a slightly negative difterential pressure. Un exposure, liquid heliun will flash into a tro-phase vapor-liquid mixture. The volume of the reactor builoing is large, but a certain degree of pressurization will result from the helium vapor; we must ensure that the design pressure of the reactor builiding will be greater. Furthermore, if the supercunducting coils are in operation, the loss of helium will drive the conductors normal and could lead to melting of coils, which in turn could aggravate the effects of the helium spill.

A preliminary calculation shows that a liquid-helium release exceeding $2500 \mathrm{~kg}$ is needed to exceed the $120-\mathrm{kPa}$ oesign pressure of the reactor building. The cryogenics system may be designed so that a single failure does not result in a release larger than this.

\subsubsection{Rf Heating}

We have made a preliminary assessment of the biological hazard from the rf heating system ${ }^{2}$. The permissible exposure to $r f$ waves is $10 \mathrm{~mW} / \mathrm{cm}^{2}$. Leakage will not exceed this unless the rf system plumbing cracks, and even so, the effect of a crack would be readily detectable from changes in system behavior. In aodition, $r f$ generation requires high voltages, and these produce $x$ rays. The TDF design will incorporate features for reducing these biological hazaras. 


\subsubsection{Lithium Fire}

The current design does not require the use of lithium, but relatively small quantities may be needed for test modules. Violent reactions may be expected if lithium contacts water (or concrete, which contains water). However, if it is spilled on steel in air, the lithium reacts with the nitrogen in the air and several minutes elapse before the reaction becomes severe. Flooding the atmosphere with an inert gas such as argon stops the reaction. The TUF design criteria milist contain any spill from a test moduie in a steel sump or catch basin that can be so flooded. Concrete surfaces that could be contacted by the lithium should be lined with steel, ana lithium piping should have doubie walls to reduce chances of a leak. We will design the facjijty to accomodate whatever breeding-blanket test materjals are chosen, rather than attempt to retrofit the facility.

\subsection{SAFETY FEATURES}

To develop a safe design for the plant, we take several steps to reduce or eiminate each safety-related problem. The most important are the use of a leak-tight, neutron- and gamma-shielded, and seismic- and tornado-protected reactor building. The major safety-related design steps are listed in Tab?e 4-5, together with their rationale. The following paragraphs discuss the mast important safety features.

\subsubsection{Pressure Vesse? Building}

One technique for reducing the consequences of radioactive release is to oesign the building as a pressure vesse? that can withstand the accidental release. The pressure vessel will contain all the radioactivity from an unexpected release until slower acting systems can be useo to reduce the bujloing pressure ano remove the radioactivity. Our rectangular reactor builaing can withstand some low amount of overpressure (about $120 \mathrm{KPa}$ ) ano still maintain a low leakage rate (on the order of 1 to lok of the building volume per aay). 
Tahle 0-5. Nesion steps for reducind safety-related hazards.

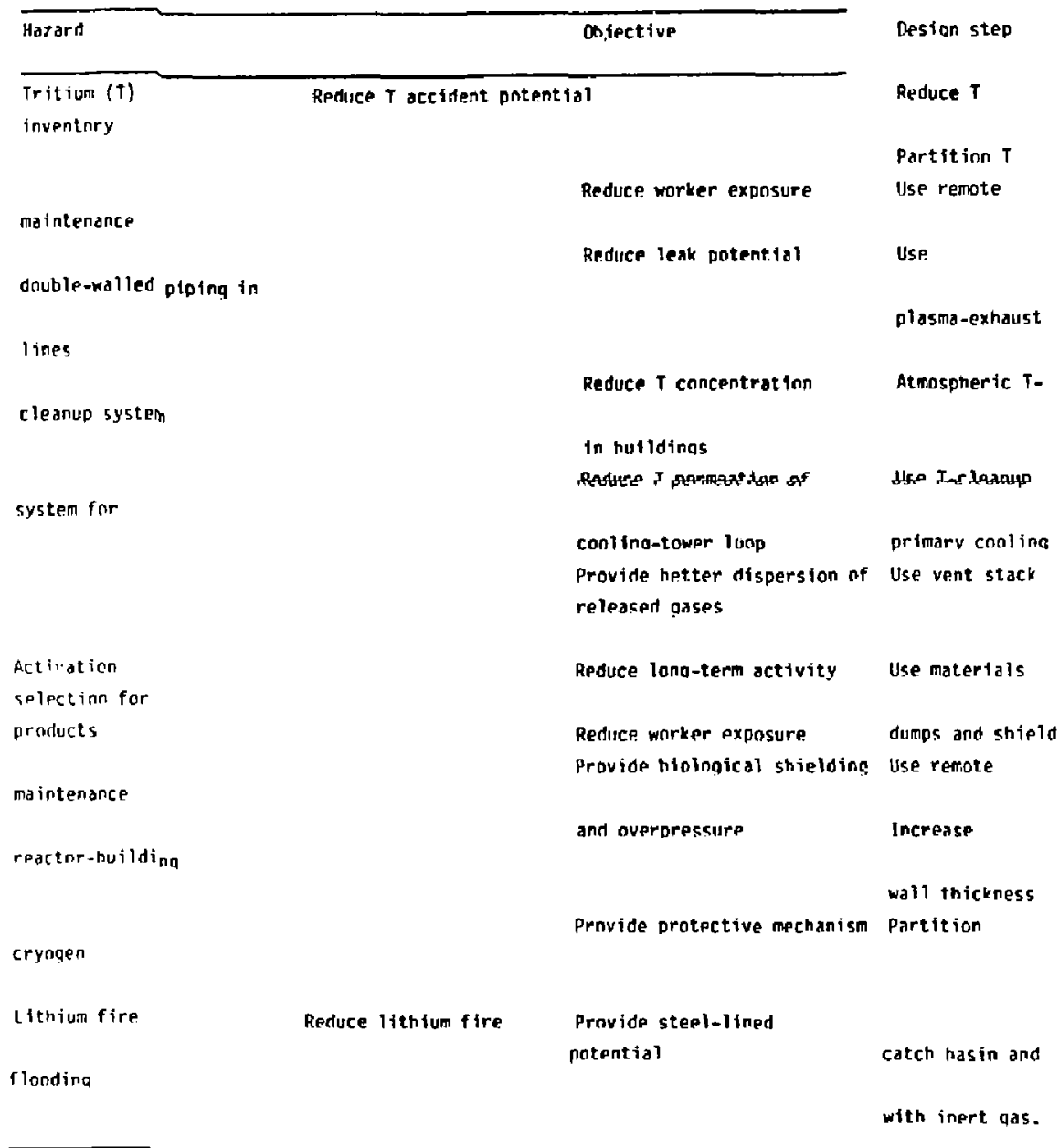




\subsubsection{Reduce Vuinerable Tritium}

One design objective of the TDF systems is to reduce the amount of tritiun that can be released in an accident. The amount of vulnerable tritium is made as low as practical by reducing the inventories in the processing equipment, e.g., recycling cryovacuum pumps more often. The tritium system is also partitioneo so that an accident that causes the release of tritium from one part of the system ooes not cause a release from other parts of the system or from other equipment. For example, isolation valves between the fueling device and the plasma chamber are used to provent tritium release from the fueling oevice in the event that the integrity of the plasma-chamber vacuum is rost.

\subsubsection{Partition Cryogens}

Since the potencial impact of cryogen evaporation is quite significant, another design objective is ta reduce the amount of cryogen that could be released by a single accident. This is accomplished with piping arrangements and valving so that a major cryogen leak in one part of the system does not cause the whole system to blow down. For exalniple, each superconductor coil is piped separately from the storage Dewar.

\subsubsection{Vent Stack}

A vent stack can significantly reduce the consequences of tritium relazse. By rolazsing radiactite effiluats well above ground leve?, we achieve better mixing and greater dispersion, which results in a significantly lower dose at the site boundary. The stack-effluent consequences are further reouced by processing or diluting the effluent stream before release.

\subsubsection{Site Characteristics}

Because site characteristics can have a major influence on the consequences of radioactive releases, site selection is very iniportant. Criteria such as site-exclusion boundary and the population density of the surrounding areas directly affect the cose rate at the boundary as well as the 
postulated average population dose. For example, if the distance from the plant to the site boundary is long, the consequences of tritium release will be significantly reduced. The exclusion area boundary of $500 \mathrm{~m}$ appears adequate for TOF.

\subsubsection{Uiscussion}

Several techniques are available to reduce the probability, ano hence consequences, of radioactivity release. Incorporating these techniques into to provide adequate protection from tritium release appears feasible. Alternatives can be selected baseo on potential cost and scheoule impact.

\subsection{ENVIRONMENTAL CONSIDERATIONS}

An environmental review is needed to identify potential environmental hazards. All elements, from the procurement of raw materials to the handing and disposing of radioactive wastes, should be considered. Under DOE auspices, we are preparing a report to assess the technicat status of environinental concerns related to the total fusion energy program. Through our study we will provide guidance for minimizing hazards in this areá. Environmental studies for TOF should be consistent with this generic effort.

The major environmental concerns for TUF are exposure to radiation and inagnetic fields. Radiation exposure has the greatest potential for impact both within and outside the plant, under all conditions. Since first-generation fusion machines will use the $D-T$ fuel cycle, technology for tritium control and cleanup are being further developed to lessen any possibility of release to the environment. The ISTA project will demonstrate the effectiveness of these technologies.

Exposure to magnetic fields is a potentia concern to on-site personnel. Present information about the biological effects of magnetic fields is sparse but generaliy suggests that health effects are not significant.

Aside from these, the environmental impacts of TDF are expected to be either less than or equal to those of other large fusion projects. Potential impacts stem from thermal discharge, land use, and toxic chemical-waste effluent. None of these is expected to cause abnormal concern or delay the project, and all can be handled with current technology. 
Following is a list of the key environmental hazaros in approximately their order of importance. As usual, it is difficult to distinguish between safety and environmenta? issues. Gur study did not specifically address all of them.

- Radionuclide releases (routine and accidental),

- Radoactive waste transportation and disposal,

- Magnetic fielas,

- Land use,

- Thermal discharge,

- Radioactive liquid and gas releases,

- Chemical releases,

- Socioeconomic impact.

\subsection{KEY SAFETY ISSUES}

The key issue is the potential release of radioactive materials from the plant, and the basic concern is the impact of this release (primarily of tritium) on the general public. We use a deterministic method to obtain information on the upper bounds of the consequences of radioactivity release. But a detailed probabilistic-risk assessment is needed in the future, because the events that lead to accidents or failures of the reactor and its systems are probabilistic. The causes of accidents and sources of radicactivity release will be identified. Regardless of whether the evaluation is probabilistic or deterministic, tritium is the major source of concern. Utmost care is neevea to design systems and components for transport, storage, pellet formation, and tritium processing to prcuide the required multiple barriers against its release. These barriers will include the following: double-walled piping system for tritium transport; glove boxes around the tritium-processing equipment; redundant systems and components, e.g., atmospheric tritium-recovery system, emergency detritiation system. 


\section{REFERENCES--Section 4}

3. Fusion Engineering Device Design Description, Vol. 2, Oak Ridge National Laboratory, 0ak Ridge, TN ORNL/TM-7948/V2 (1981).

2. STARF IRE - A Commercial Tokamak Fusion Power Plant Study, Vol. II, Argonne National Laboratory, Argonne, IL, ANL/FPP-80-i (1980).

3. "Safety of Non Reactor Nuclear Facilities," DOE Order 5480.1, Chapter V, Department of Energy, Washington, D. C. (1981).

4. "Standards for Radiation Protection," DOE Manual, Chapter 0524, (from Energy Researcli Developinent Agency Manua1, Chapter 0524, approved March 30, 1979.)

5. "Safety Anałys is and Review System," DOE Order 5481.1, Department of Energy, Washington, D. C. (1979).

6. Draft Nonreactor Nuclear Facilities: Standards and Criteria Guide, U.S. vepartment of Energy, Washington, D.C. (1981).

7. Tritium System Test Assembly, Preliminary Safety Analys is Report, Los Alamos Scientific Laboratory, Los Alamos, NM (1979).

8. Tokamak Fusion Test Reactor, Preliminary Safety Analys is Report, Princeton Fiasma Physics Laboratory, Princeton, NJ (1978).

9. E. L. Alpen, U.S. Department of Energy, washington, B.C. private communication (1980).

10. P. S. Rohwer and W. H. Wi Icox, "Radiological Aspects of Environmental Tritium," Nuclear Safety, 17, (2) (1976).

11. SWESSAR-P1, Section 2.3, Stone and Webster Standard Safety Analys is Report (Stone and Webster, Boston, MA, 1974).

12. "CalcuTation of Annual Doses to Man from Routine Releases of Reactor Effluent for the Purpose of Evaluating Compliance with 10 CFR Part 50," Kegulatory Guide 1.109, Appendix 1, U.S. Nuclear Regulatory Commission, Washingion, O. C. (1977). 


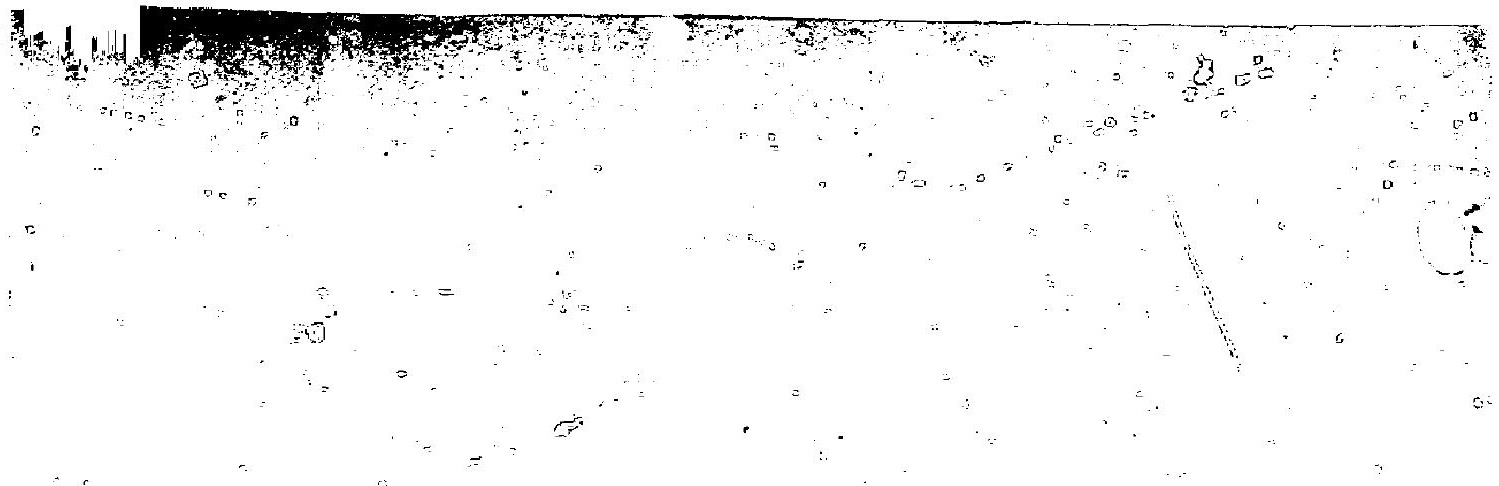

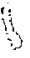

27

SECTIOW 5.0. COST ESTIMATES

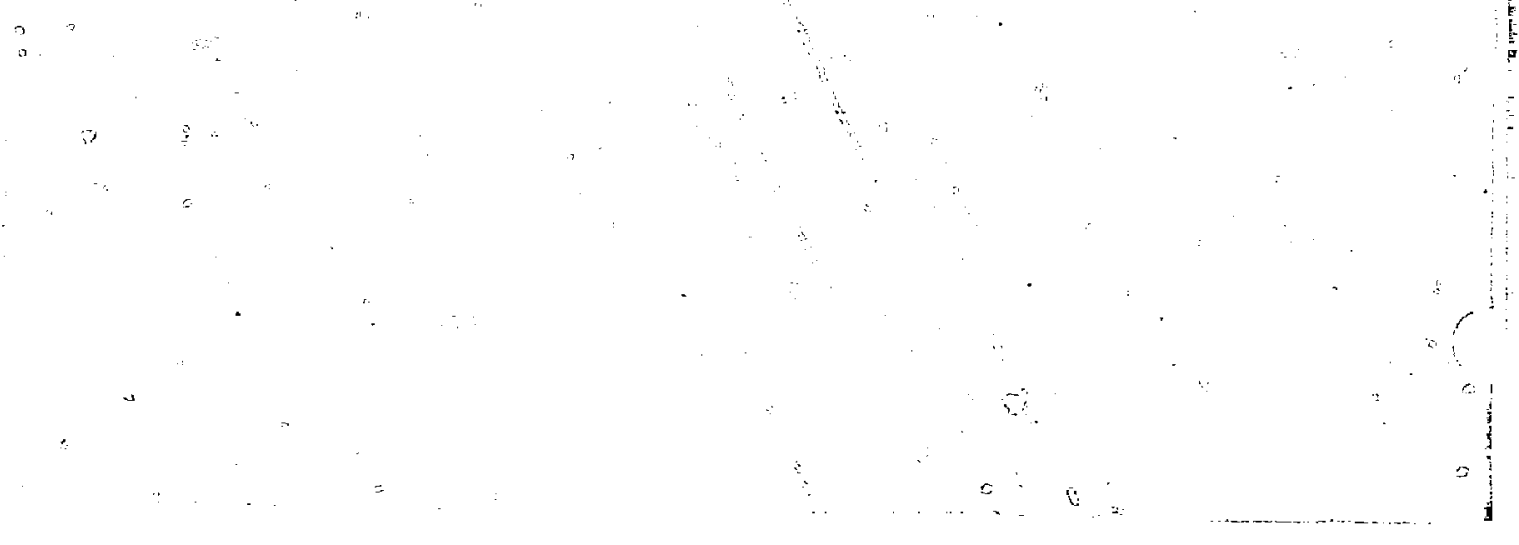




\subsection{INTRODUCTIUN}

One of the goals of the engineering design effort in the TDF program was to describe each subsystem in enough detail to allow the preparation of a creoible cost estimate. An estimate of total capital cost was generated using three different methoos. All three methods used the same direct costs with the add-ans varying to represent the experience of the three costing organizations (Bechtel Group, FEUC, and LLNL). The costs presented here were assembled by Bechtel, with the raw aata provided by each subsystem oesign group.

The resulting total capital costs are, in millions of aollars: Bechtel $\$ 1,290$, FEDC - $\$ 1,147$, LLNL $-\$ 960 \pm 20 \%$. Although there are significant differences in these estimates, they are all withili the uncertaity associated with completeriess of the design work. Our assesment is that the cost is about what should be eapected of a device about the size of MFTF with the adoition of requirements for tritium, activation, and high-duty cycle.

\subsection{TOTAL CAPITAL COSTS}

The total estimated capital costs are shown in Table 5-1. The estimate assumes that:

- Uur scope will be that of a prime contractor responsible to the owner for engineering, procurement, and construction.

- Equipment and materiais will be procured from U.S. sources, and lead times will be able to support the project schedule without cust penalties.

- Sufficient manual and nonmanual personnel to complete the project on schedule is available in the project vicinity.

- Existing water sources and power will be adequate for the projects requirements.

\subsubsection{Direct Costs}

The direct costs shown in Table 5-2 include the plant equipment, materials and subcontracts, and installation labor associated with the permanent facilities, systems, and equipment. 
Table 5-1. TDF capital cost summary, as of mid-1982. Dollars are in millions.

\begin{tabular}{lr}
\hline Description & Cost \\
\hline Birect cost & 653 \\
Indirect cost & $\underline{110}$ \\
Tota] fiela cost & 191 \\
Home office cost & 38 \\
Owner's cost (operating entity) & $\underline{298}$ \\
Contingency & $\underline{1,290}$ \\
Total capital cost & \\
\hline
\end{tabular}


Tahie 5-2. TPF direct cost sumary, as of mid-19A2. Domars are in thousands.

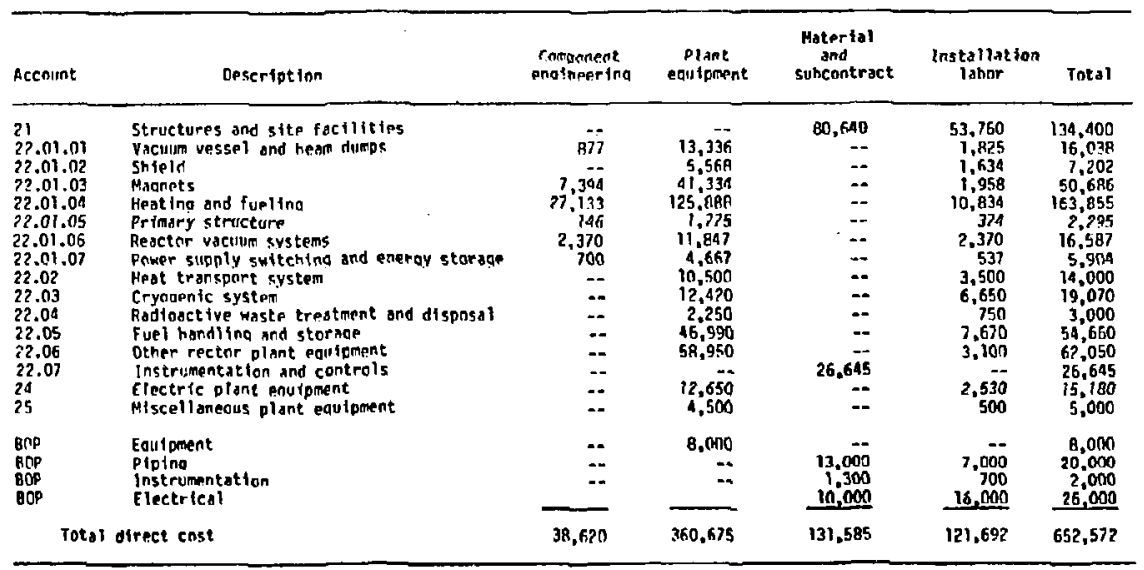


The engineers and organizations responsible for the design and specification of various equipment estimated their own involvements as shown in Table 5-3. A cost aata collection methodology was developea to ensure that. all components ano systems were incluoed, ano to ensure that all cost data had a common basis.

The work breakown structure was first arranged to correspond to the stanuard accounts for fusion cost est imates oeveloped by Eattelle. ${ }^{l}$ The engineer responsible for the account cieveloped the work breakdown details below the level shown on Table 5-2. (By reviewing these detailed account breakdowns before actual estinating began, the cost estimating group was able to resolve omissions and redunaancies early in the project.)

The responsible engineers were then provided with equipnient cost forms, which were used to transmit cost data on equipnent, component engineering, and installation. The equipment cost includes the base mechanical equipment plus all required orives, piping, instrumentation, and electrical materials furnished with the equipment before delivery to the jobsite. It exciudes installation, freight, vendor representatives, contingency, and component engineering. The component engineering cost, included as a separate item, is the cost associated with the design of a first-of-a-kind component. Installation costs were reported as direct installation hours excluding nonmanual supervision. Allovances for vendor representatives, freight, and installation materials were developed by the cost estimating group. Materials include all permanent plant materials usually purchased in bulk quantities, such as earthworh, formwork, rebar and embedded metal, concrete, steel, pipe, valves and connectors, electrical equipment, wire and cable, conauit and cable tray, computers and control modules, fiela instruments and bulks.

To simplify the estimate's preparation and accurately account for all field labor, subcontracts are generally not inciuded. Instead, we assume that all field labor is performed by the prime contractor's work forces and results in essentially the sane total estimated construction cost as that based on some subcontractor work. The instrumentation and control (1\&C) system is the only exception to this approach. TRW estimated the I\&C package on a subcontract basis; this made it easier to integrate the I\&C estimate into the total plant estimate. Bechtel personnel prepared the materials estimate based on the scope definition aeveloped at the FEDC. 
Tabie 5-3. TOF capital cost accounts.

\begin{tabular}{|c|c|c|}
\hline Account & Description & Respensible Organization \\
\hline 21 & Structures and site facilities & $\begin{array}{l}\text { Fusion Engineering } \\
\text { Design Center (FEDC) }\end{array}$ \\
\hline 22 & Reactor plant equirment & \\
\hline 22.01 .01 & Vacuum resse 1 & General Dynamics (GD), LLNL \\
\hline 22.01 .02 & Shield & TRW \\
\hline 22.01 .03 & riagnets & GD, FEDC \\
\hline 22.01 .04 .01 & Beam heating & TRW, FEDC \\
\hline 22.01 .04 .02 & RF heating & FEDC \\
\hline 22.07 .04 .03 & Fueling & FEDC \\
\hline 22.01 .05 & Primary structure and support & GD \\
\hline 22.01 .06 & Reactor vaculin system & LLNL \\
\hline 22.01 .07 & $\begin{array}{l}\text { Power supply switching and } \\
\text { energy storage }\end{array}$ & FEDC \\
\hline 22.02 & Heat transport system & FEDC \\
\hline 22.03 & Cryogenic system & Gu \\
\hline 22.04 & $\begin{array}{l}\text { Radioactive waste treatment } \\
\text { and aisposal }\end{array}$ & FEDC \\
\hline 22.05 & $\begin{array}{l}\text { Fuel handling and storage } \\
\text { Systems }\end{array}$ & Science Applications, Inc. \\
\hline 22.06 & Other reactor plant equipment & FEOC \\
\hline 22.07 & Instrumentation and control & TRW \\
\hline 24 & Electric plant equipment & FEDC \\
\hline 25 & $\begin{array}{l}\text { Miscellaneous plant equipment } \\
\text { BOP Bulk materials }\end{array}$ & $\begin{array}{l}\text { FEDC } \\
\text { Bechte? }\end{array}$ \\
\hline
\end{tabular}


Installation labor includes all manual labor directly involved in erecting the permanent plant facilities, systens, and equipment. Typically, the study participants provided installation manhours and Bechtel reviewed them. Where the manhour estimates were omittec or appeared to be inconsistent with generai construction experience, Bechtel developed appropriate a) lowances. We assumed an average U.S. Tabor rate of $\$ 22.00 / \mathrm{hr}$ including all applicable premiums, fringes, caxes, and insurance addit ves based on a review of current U.S. craft labor agreenients.

\subsubsection{Indirect Costs}

Indirect costs are those that cannot be directly identified with specific permarient plant facilities, systems, and equipment. This incluaes

- Temporary construction facilities: temporary buildings, working areas, roads, parsing areas, utility system, and general purpose scaffolding .

- Miscellaneous construction services: general job cleanup, maintenance of construction equipment and tool maintenance, material handing, surveying, and watchmen and guards.

- Construction equipment and supplies: construction equipment, small tools, consumable supplies, and purchased utilities.

- Fiela office: craft supervision, engineering, procurement, scheduling, personnel administration, warehousing, first aid, and field office operating costs.

- Preliminary checkout and acceptance testing: material and equipment testing.

- Project insurance: public liability, property damage, and builder's risk insurances.

The costs of the facilities, supplies, and services were estimated as a percent of direct labor costs based on an evaluation of Bechtel experience with similar projects. This analysis resulted in ar allowance for indirect costs equal to $90 \%$ of airect labor.

Home Office Costs. Home office costs include preliminary, definitive, and detail design engineering, cost engineering, scheduling, procurement, and project and construction management services. 
The estimate of home office costs is based on an analysis of experience with nuclear facilities and simjlar projects. This resulted in an allowance for home office costs equal to $25 \%$ of total field cost.

Owner's Costs. Owner's costs are those incurred by the project during engineering and construction that are not directly attributable to the prime contractor. They include such items as

- Program administration and managemerit,

- Preliminary engineering,

- Site investigation,

- Research ana development,

- Initial charges, inventory, and spares,

- Startup and trainirig.

Because TUF will be a government-owned and -operated facility, we have in: luded a relatively small allowance of $4 \%$ of the total field and home office cost.

Contingency. This is an alrowance for the design's uncertainty in quantity, pricing, or productivity that is under the control of the engineer/constructor ano within the defined scope of the project. Implicitly, the allowance will be spent during design and construction, and it cannot be considered as a source of funds for overruns or additions to the project scope.

Contingency excludes factors outside the engineer's/constructor's control, such as scope changes that force major regulatory, operating, safety, and environmental standards; site selection; and political and finaincial market conditions. Contingency is $30 \%$ of the total of field, home office, and awner's costs.

\subsubsection{Discussion of Estimate}

Cost comparisons. The total capital cost summary assumes that TDF will be constructed at an undeveloped site with the engineer/constructor as the prime contractor. TOF may also be constructed at an existing national laboratory, with the laboratory as prime contractor, ard existing facilities may be used. To estimate the indirect costs, we employed the factors developed at the Fusion Engineering Design Center (FEOC).? 
The FEDC definition of total airect cost excludes component engineering and installation labor for the reactor plant equipment. However, Jabor costs for facilities that would normally be subcontracted to incustry are included as oirect costs in the FEOC algorithms. These include

- Structures ano site facilties (account 21)

- Heat transport system (account 22.02)

- Radioactive waste treatment ano disposal (account 22.04)

- wther reactor plant equipment except remote maintenance equipment (account 22.06), and

- Miscellaneous piant equipment (account 25).

The total direct cost of TOF, compatiole with FEOC is shown in

Table 5-4. This is the cost that should be used in conparing TDF to the FED designs developed by the FEDC. The total cost, aeveloped by the FEDC method, is shown in Table 5-5.

The factors are comparable to the costs shown in Tables $5-1$ and $5-2$ if engineering ana management is jaentified as the component engineering, home office and owner's cost, and if installation is aefined as the labor not included in the FEOC oirect cost. The aifference between the two total cost estimates is the indirect (fieid) cost. This will vary according to the applicability and availability of existing facilities for TDF construction.

The capital cost was also estimated by LLN, based on construction at LLNL and using existing facilities ano services. Using the direct cost from in Table 5-2, LLNL applies the following factors:

- Construction equipment ano services-10\%,

- LLNL project engineering--5\%,

- Systems checkout and acceptance--5\%,

- Project management--2.5\%.

At this stage in the study, an uncertainty in direct cost of $\pm 20 \%$ is appliea to account for oesign ana scope changes before a comitment to construct. LLML estimates the contingency is estimated to be $20 \%$. The capital cost is then estimated as shown in Table 5-6.

Birect cost elements. To oisplay the major cost elements, and to provide a basis for scating the TOF component costs, we show a further breakoown of the cost accounts in Table 5-7. We used the oirect costs from Table 5-2, including equipment, material, component engineering, and labor. 
Table 5-4. Total oirect cost of TDF, compatible with FEDC. This is the cost that should be used in comparing TDF to the FED designs developed by the FEDC.

\begin{tabular}{lc}
\hline Item & $\operatorname{Cost~(SN)}$ \\
\hline Equiprnent cost & 360.7 \\
Material and subcontract & 131.6 \\
Subcontract labor & $\frac{58.8}{551.7}$ \\
Total direct cost & \\
\hline
\end{tabular}

Table 5-5. Total cost develpped by the FEOC.

\begin{tabular}{lc}
\hline Item & Cost (SM) \\
\hline Total direct cost & $55 \mathrm{~J}$ \\
Engineering and management (45\%) & 248 \\
Installation (15\% of total direct cost) & $\frac{83}{882}$ \\
Subtotal & $\underline{265}$ \\
Contingency (30\%) & 1147 \\
Total cost
\end{tabular}


Table 5-6. Estimated capital cost.

\begin{tabular}{llc}
\hline \multicolumn{1}{c}{ Item } & Cost $\$(M)$ \\
\hline Direct cost & 653 & \pm 131 \\
Indirect cost $(22.5 \%)$ & $\frac{147}{800}$ & \pm 29 \\
Total field cost & $\frac{160}{960}$ & \pm 160 \\
Contingency $(20 \%)$ & \pm 32 \\
Total capital cost & \pm 192 \\
\hline
\end{tabular}


Tatle 5-7. Direct cost elements, as of mid-1982. Dollars are in thousanas.

\begin{tabular}{|c|c|}
\hline Item & Cost \\
\hline $\begin{array}{l}\text { Reactor vessel total } \\
\text { Centre? cell vessel } \\
\text { Anchar/ena cell vessel } \\
\text { Central cell shield } \\
\text { Anchor/end cell shield } \\
\text { Support structure }\end{array}$ & $\begin{array}{r}\frac{23,41 i}{210} \\
13,944 \\
6,202 \\
1,000 \\
2,061\end{array}$ \\
\hline $\begin{array}{l}\text { Magrets total } \\
\text { Central cell coils } \\
\text { Anchor/transition coils } \\
\text { Choke coils } \\
\text { Anchor wacusw wesse] } \\
\text { Central intercoil support }\end{array}$ & $\begin{array}{r}50,920 \\
32,995 \\
8,001 \\
2,265 \\
7,425 \\
234\end{array}$ \\
\hline $\begin{array}{l}\text { Heating total } \\
\text { Centrai cell NBI } \\
\text { Anchor } / \text { transition NBI } \\
\text { RF heating } \\
\text { Beam qumps }\end{array}$ & $\begin{array}{r}162,372 \\
121,758 \\
24,980 \\
13,750 \\
1,884\end{array}$ \\
\hline $\begin{array}{l}\text { Fuel system total } \\
\text { Fue? injection } \\
\text { Purification and preparation } \\
\text { water cleanup systems } \\
\text { Air cleanup systems } \\
\text { uther tritium processing }\end{array}$ & $\begin{array}{r}58,027 \\
3,367 \\
14,153 \\
19,868 \\
11,978 \\
8,66]\end{array}$ \\
\hline $\begin{array}{l}\text { Maintenance equipment total } \\
\text { HeactGr building } \\
\text { Hot çll } \\
\text { Special purpose }\end{array}$ & $\begin{array}{l}\frac{59,045}{22,637} \\
24,551 \\
11,857\end{array}$ \\
\hline $\begin{array}{l}\text { Reactor services total } \\
\text { Vacuum system } \\
\text { LHe system } \\
\text { LN system } \\
\text { Reactor cooling } \\
\text { Facjlity cooling } \\
\text { Heat rejection }\end{array}$ & $\begin{array}{r}\frac{49,657}{16,587} \\
11,470 \\
7,600 \\
6,200 \\
1,300 \\
6,500\end{array}$ \\
\hline
\end{tabular}


Table 5-7 (cont'd)

\begin{tabular}{lr}
\hline Item & Cost \\
\hline Electrical systems total & 21,089 \\
Magnet power supplies & 4,397 \\
Magnet control systems & 1,518 \\
Pulsed power substation & 17,508 \\
Facility power substation & 2,040 \\
Emergency power & $1,63 ?$ \\
& \\
Instrumentation and control iotal & 26,645 \\
Supervisory control/aata system & 13,055 \\
Local control instrumentation & 10,601 \\
Personnel safety/interlocks & 2,989 \\
& \\
Facilities total & 201,400 \\
Reactor building & 40,000 \\
Hot cel1 & 24,200 \\
Reactar auxiliary bulding & 17,200 \\
Power supply building & 6,000 \\
Other buildings & 30,000 \\
Site improvements & 14,400 \\
Cooling system structures & 2,600 \\
Radioactive waste treatnent system & 3,000 \\
Miscellaneous plant equipment & 8,000 \\
Bulk materiais & 56,000 \\
& 652,572 \\
Total direct cost & \\
\hline Includes tritiut building & \\
\hline
\end{tabular}

ancludes irjtium building. 


\section{REFERENCES--Section 5}

1. S.C. Schuite, T.L. Willke, and J.R. Young, Fusion Reactor Design Studies - Standard Accounts for Cost Estimates, Bat telle-Pacif ic liorthwest Laboratory, Fusion Engineering Desígn Center Oak Ridge, TN, PNL-2648 (1978).

2. Fusion Engineering Device Design Description, Fusian Engineering Design Center, Oak Kidge, TN, ORML/TM-7948 (T98T).

$\mathrm{CJ} / \mathrm{kt}$ 